\title{
Stress, mood, and cortisol dynamics in daily life
}

Citation for published version (APA):

van Eck, M. (1996). Stress, mood, and cortisol dynamics in daily life. [Doctoral Thesis, Maastricht University]. Universiteit Maastricht. https://doi.org/10.26481/dis.19961018me

Document status and date:

Published: 01/01/1996

DOI:

10.26481/dis.19961018me

Document Version:

Publisher's PDF, also known as Version of record

\section{Please check the document version of this publication:}

- A submitted manuscript is the version of the article upon submission and before peer-review. There can be important differences between the submitted version and the official published version of record.

People interested in the research are advised to contact the author for the final version of the publication, or visit the DOI to the publisher's website.

- The final author version and the galley proof are versions of the publication after peer review.

- The final published version features the final layout of the paper including the volume, issue and page numbers.

Link to publication

\footnotetext{
General rights rights.

- You may freely distribute the URL identifying the publication in the public portal. please follow below link for the End User Agreement:

www.umlib.nl/taverne-license

Take down policy

If you believe that this document breaches copyright please contact us at:

repository@maastrichtuniversity.nl

providing details and we will investigate your claim.
}

Copyright and moral rights for the publications made accessible in the public portal are retained by the authors and/or other copyright owners and it is a condition of accessing publications that users recognise and abide by the legal requirements associated with these

- Users may download and print one copy of any publication from the public portal for the purpose of private study or research.

- You may not further distribute the material or use it for any profit-making activity or commercial gain

If the publication is distributed under the terms of Article $25 \mathrm{fa}$ of the Dutch Copyright Act, indicated by the "Taverne" license above, 
STRESS, MOOD, AND CORTISOL DYNAMICS IN DAILY LIFE 


\section{UPSCR}

\section{IPSER Series in Ecological Psychiatry}

M.W. deVries

\section{Editor}

$* * *$

\section{Vol.1 Assessing Schizophrenia in Daily Life}

- The Experience Sampling Method Philippe A.E.G. Delespaul

Vol.2 (Genetic) Epidemiology as a Tool to Identify Risk Factors for Emergence and Persistence of Illness in the Functional Psychosis Jim J. Van Os

Vol.3 Stress, Mood, and Cortisol Dynamics in Daily Life

Marleen M.M. Van Eck

The International Institute for Psycho-social and Socio-ecological Research (IPSER)

Collaborating Center of the World Federation of Mental Health Associated with the Rijksuniversiteit Limburg

Cover design: Mark. Delahay

Druk: Datawyse Maastricht

van Eck. Marleen

Stress, mood, and cortisol dynamics in daily life/Marleen yan Eck.

Thesis University of Limburg. Maastricht. - With ref.-

With summary in Dutch.

ISBN 90-9009783-X

Subject headings: perceived stress/experience sampling/cortisol/mood. 
Promotor

Prof. Dr. M.W. deVries

\section{Co-promotor}

Dr. N.A. Nicolson

\section{Beoordelingscommissie}

Prof. Dr. H.M. van Praag (voorzitter)

Prof. Dr. A.P.W.M. Appels

Prof. Dr. M.A. van den Hout

Prof. Dr. J.F. Orlebeke (Vrije Universiteit Amsterdam)

Prof. Dr. A.A. Stone (State University of New York)

Funding was provided by the Netherlands Organization for Scientific Research (NWO; grant 900-564-024) and the University of Limburg. 
It is not the large things that send a man to the madhouse... No, it's the continuing series of small tragedies that send a man to the madhouse Not the death of his love but a shoelace that snaps with no time left.

Charles Bukowski, 'The shoelace'. Bukowski Reads His Poetry, Takoma Records, Santa Monica, Calif., 1980. 

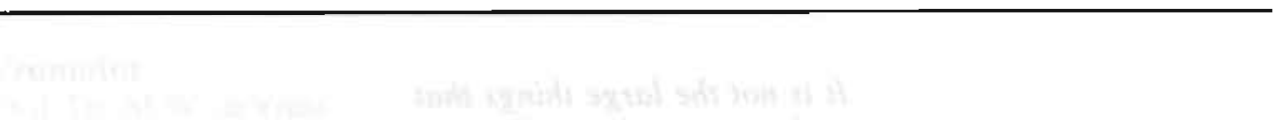

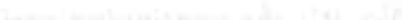




\section{Contents}

page

Chapter 1

Chapter 2

Chapter 3

Chapter 4

Chapter 5

Chapter 5.1

Chapter 5.2

Chapter 6

Chapter 7

Chapter 8

References

Appendices

Summary

Samenvatting

Dankwoord

Curriculum vitae

Introduction $\quad 3$

$\begin{array}{ll}\text { Methods } & 21\end{array}$

The nature of stress in daily life: events, appraisals, and activities

The effects of perceived stress and stressful daily events on mood states

Submitted for publication

Cortisol and catecholamine excretion in relation to perceived stress

Perceived stress and salivary cortisol in daily life Annals of Behavioral Medicine 16(3): 221-227 (1994) Four-teen hour urinary catecholamine excretion in relation to perceived stress

and stressful daily events on salivary cortisol

Psychosomatic Medicine (in press)

Individual differences in cortisol responses to a laboratory 127 speech task and their relationship to responses to stressful daily events

Biological Psychology (43): 09-84 (1996)

Concluding remarks 


\section{Chapter 1}

Introduction 


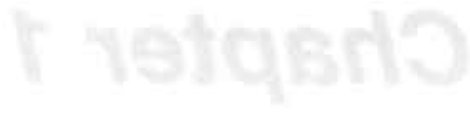

noilsuboyln 
Our lives can be characterized as constant adaptation to sudden or more gradual changes of our environment. Sometimes these changes are small and we can adapt to them without even noticing them, but at other times, these changes can be too frequent or severe and thereby threatening. In any case, at some point in life every human being will experience 'stress'; the day when inexplicably nothing seems to go right is a universal experience. In modern society, stress has become a popular concept and has gained a common everyday usage. We use it, for instance, to explain a wide variety of outcomes, mostly negative: it may serve to explain an upcoming headache or a bad mood, an upset stomach, 'nerves' or a lack of motivation. It is also common practice to attribute eccentric or incomprehensible behavior of friends and acquaintances to the fact that they are 'under a lot of stress'. Stress is even referred to as an 'epidemic disease".

One reason for the popularity of the stress concept may be its increasing linkage to disease and well-being. Research in the field of stress has indeed indicated that in addition to its role in the development and course of a wide range of somatic diseases (e.g. cardiovascular disease, infectious diseases, diabetes mellitus), psychosocial stress plays an important role in the etiology and maintenance of various psychiatric illnesses, including depression, anxiety, and psychosomatic disorders (Brown, 1993; Chrousos \& Gold, 1992; Paykel \& Dowlatshahi, 1988). During the last decade there has been an increase in the Netherlands in the number of mental disorders among those eligible for work disability benefits, as well as an increase in the duration of sickness absences because of mental disorders (Schroer, 1993). Of all disabled workers, more than $28 \%$ (227,000 individuals) received a benefit in 1990 after a mental disorder had been diagnosed; this in contrast to $21 \%$ in 1981. The total percentage of work disability due to mental disorder among civil servants increased over the same period from 35\% to 50\% (Knepper, 1991). In more than $80 \%$ of these cases, the mental disorder was described as "overstrain " (Schroer, Nijhuis, \& van Zutphen, 1988); in 40\% of mental disorder disabilities 'stress at work' was specifically mentioned. These figures are alarming not only for the individual (ex)worker, but also for the corporate community and society as a whole. Companies have to dea! with a considerable loss of work time, resulting in higher production costs and a pressure toward greater work productivity, which may lead again to an increase in absenteeism and disability due to a higher workioad. The costs for society due to stress-related absenteeism and disablement (including medication and health care utilization) have been estimated in 1988 at 9 billion Dutch guilders (Laan, 1989). In an effort to reduce feelings of stress and to increase physical fitness and improve mental health, fitness programs (Bruijn, 1988) and stress management courses (Marcelissen, 1989) at or near the work place have rapidly increased in popularity in the Netherlands, further encouraged by laws placing greater responsibility for the employee's health and well-being on the employer (ARBO-law). The main aims of introducing these programs at the work place are to increase the employees" physical health, thereby reducing absenteeism and work-related stress, and increasing work performance (Cox, Gotts, Boot, \& Kerr, 1988; Falkenberg, 1987).

Although the word stress seems to be implicitly understood by all, it is given substance by an infinite number of different things. For one person stress is having a fight with his or her spouse, for the other it is a busy day at work, and again for another stress may mean having nothing to do. Earlier research emphasized the impact of major disruptions in personal and social life (e.g. death of spouse, unemployment) (Holmes \& Rahe, 1967), but more recent studies, have alerted us to the fact that the minor but much more frequent occurring hassles of daily living also 
play a role in both somatic and psychological health outcomes (DeLongis, Coyne. Dakof, Folkman, \& Lazarus, 1982). These minor but often chronic stresses at work, in the family, and in other social relationships are also much closer to the lay person's concept of stress, as described above, and to problems that may lead to absenteeism and disablement at the work place. Although responses to acute experimental stress have been studied extensively, little is known about the nature and effects of the stresses of daily living. Knowledge about the nature of psychological and physiological responses to daily life stress is possibly of great theoretical importance for an understanding of the etiology and course of both physical and psychological pathology, and is the object of the present thesis. The first goal of the study is to describe the nature and scope of daily life stress, contrasting the experiences of individuals who perceive themselves to be stressed with those who do not. Second, this research investigates affective and neuroendocrine responses in relation to stressful daily events and tries to explain individual differences in such responses. Before explicitly going into the major research questions and how they will be addressed, we will provide a brief overview of various theoretical approaches to stress, followed by a discussion of the stress-illness relationship. Next, the available evidence concerning the influence of daily stress on psychological and physiological functioning will be discussed.

\section{THE CONCEPT OF STRESS: PSYCHOLOGICAL VIEWS}

Although the term 'stress' has been widely used since the beginning of this century (Cannon, 1929), controversy concerning its definition remains. Three distinct definitions of stress can be found in the literature, which vary in their emphasis on stimulus, response or intervening process and which are closely related to ways in which stress can be measured: in terms of a stimulus or a situation (e.g. noise, death of a spouse, mathematics task), in terms of a behavioral or physiological response (e.g. performance decrements, increased heart rate, or elevated cortisol), and stress as a state of imbalance. when the (perceived) demands on the person tax or exceed his (perceived) capabilities to deal with these demands (Goldberger \& Breznitz, 1982). These definitions also vary in their emphasis on physiological/endocrinological versus psychological/behavioral processes. For the sake of simplicity, the various approaches to the concept of stress will be divided below into psychological and physiological stress traditions, starting with the psychological tradition.

\section{Life change approach}

In psychology and medicine, the term stress has traditionally been used to refer to a stimulus or situation which produce certain (behavioral, psychological, and/or physiologicai) responses (Hinkle. 1974; Wolff \& Goodell, 1968). Since the development of a method for the quantification of major life changes by Holmes and Rahe (the Social Readjustment Rating Scale [SRRS]; (Holmes \& Rahe, 1967)), the association between stress and disease has been extensively investigated. The hypothesis guiding the Holnes-Rahe approach was that an increased number of life changes requiring considerable adjustment would precede the onset of illness. This implies that life events are, in and of themselves, the precipitating cause of illness. The SRRS self-report checklist allows the respondent to indicate which events (both positive and negative, such as divorce, birth or death of a close family member, loss of job) were experienced within a specified time period. To assess the significance of 
these events, life change units were obtained by having different groups of subjects indicate the amount of readjustment (independent of the desirability or emotions induced by the event) they thought would be required. The item 'marriage' was used as anchor point in these ratings. In this way, the total number of events or a summation of the life change units could be obtained and then related to measures of illness and health. Since its development, the SRRS has been used in numerous studies to investigate the association between life changes and illness, and life changes have, for instance, been related to sudden cardiac death (Rahe \& Arthur, 1978; Rahe \& Lind, 1971). In general, life events have been shown to be related to various somatic disorders, including heart disease, sudden cardiac death, and infectious diseases (Holmes \& Masuda, 1974), and to various psychiatric disorders, including acute schizophrenia (Brown, 1974) and depression (Paykel, 1974).

\section{Criticism of the life event approach}

The life event approach, in which stressors are basically treated as objective environmental stimuli, has received considerable criticism (Aagaard, 1984; Rabkin \& Streuning, 1976; Schroeder \& Costa, 1984). Some points of criticism are: only low to modest correlations have been found between life event scores and health outcomes: the exclusive use of the category of major life events as a synonym of stress and the use of 'social readjustment' as the sole dimension to define stress; psychometric problems including reliability and content validity; inclusion of many items which may have been confounded with the outcome measures studied; and the use of retrospective study designs, which could lead to an exaggeration of the importance attributed to past events from a need to justify subsequent illness.

Later life event checklists (e.g. the Life Experience Checklist (Sarason, Johnson \& Siegel, 1978) and the PERI lists of life events (Dohrenwend, Krassnoff, Askenasy, \& Dohrenwend, 1978)) addressed some of these issues. Both negative and positive life change scores were included to be able to investigate whether positive and negative life events exhibit the same relationship with physical and emotional outcomes. Also included were additional subjective evaluations of the life events, like desirability, impact, controllability, and predictability. The basic objections, however, remained: retrospective research design, limited and predetermined sample of life events, too little consideration of the subjective evaluation of the event or the ongoing relationship between person and environment. One exception is the laborintensive and time-consuming semi-structured interview (the Bedford Life Events and Difficulties Scale [LEDS] (Brown, 1974)) method developed by Brown and colleagues (Brown \& Harris, 1989) for the measurement of life events and chronic difficulties. Here, in addition to the use of objective criteria for what can be considered a life event or enduring problem, this approach incorporates the influence of psychosocial factors (the biographical context) on the subjective experience of events.

\section{Transactional theory of stress, appraisal and coping}

The transactional stress theory developed by Lazarus and colleagues (Lazarus, 1966) places particular emphasis on the importance of an individual's subjective evaluation ('appraisal') of stressful events. In this now very widely accepted approach to the concept of stress, psychosocial stress is defined as "a relationship between the person and the environment that is appraised by the person as taxing or exceeding his or her resources and endangering his or her well-being"' (Lazarus \& Folkman, 1984b, p. 19). In the present study we will use the term 'stress' 
in this context. This approach states that whether a state of stress develops depends both on external conditions in the environment as well as on the constitutional vulnerability and the adaptive capacity of the person. It therefore may explain why identical events do not lead to stress perceptions in all persons, since differences in personality, social support, prior experience with the stressor, and so on, may effect the person's ability to deal with the stressor. Central to the transactional approach are two processes that act as mediators between the behavior of the person and the environment: first, the subjective evaluation or appraisal of potentially stressful events or situations, and second, the process of coping that follows after an event has been appraised as stressful.

The concept of appraisal refers to three distinct types of appraisal: primary appraisal, secondary appraisal, and reappraisal. Primary appraisal refers to the significance of the situation for a person's well-being ('What is at stake?'). Three broad and basic categories of evaluations of well-being can be distinguished (Lazarus \& Launier, 1978): 'irrelevant', meaning that the situation has no subjective relevance and can therefore be ignored, 'benign-positive' when the person regards an event as signifying a positive state of affairs, and 'harmful-stressful'. Three types of stressful appraisals are mentioned: 'harm/loss', which refers to harm already done, 'threat', which refers to a future potential for harm or loss, and 'challenge', which is defined as a potential for growth or positive gain. Stress can thus have both positive and negative effects. These categories of stressful appraisals do not have to be mutually exclusive, a loss, for example, can at the same time entail a future threat. Secondary appraisal refers to the evaluation of the coping recourses and options available to deal with a stressful transaction ('What can I do about it?'). Consequently, it follows that secondary appraisal determines to a large extent whether one feels threatened as opposed to challenged, and it will shape the coping strategies of the person under stress. Reappraisal points to the feedback process from the ongoing interaction between the person and the environment, which may lead to changes in primary and secondary appraisals.

The concept of coping refers to "the process of managing external and/or internal demands that tax or exceed the resources of the person" (Lazarus \& Launier, 1978. p. 311). Two broad categories of coping nechanisms or strategies have been distinguished, each serving a major adaptive function: problem-focused coping is directed at actively modifying the self or the stressful situation, while emotionfocused coping is directed at managing feelings of distress. Both are used in almost every stressful situation. Coping and appraisal mutually influence each other throughout a stressful encounter, since if the person-environment relation changes as a result of coping efforts the evaluation of the situation will change as well.

Individual differences in stress, appraisal, and coping responses are determined at least in part by an individual's adaptive capacity at the physiological, psychological, and social level (Lazarus \& Folkman, 1984b). Examples at the physiological level are genetic or constitutional factors like physical fitness and genetically determined or acquired immunity. At the psychological level, there are personal goals and commitments, and personality characteristics like self-esteem. neuroticism, and sense of control. At the social level, important antecedent variables are social economic status, social support networks, and sociocultural norms and values. Stressful events or situations can be described with respect to both immediate and long-ternn effects. Short-term effects at the physiological level include somatic or physiological changes and, in extreme cases, acute illness. Effects at the psychological level include positive or negative emotions, and at the social level they 
may entail social disturbances or group alienation. The general categories of longterm outcomes are: physical health (e.g. chronic illness, impaired physiological functioning); subjective well-being (e.g. depression, anxiety, other psychological symptoms); and social functioning (e.g. socoal failure, change in social role). All levels as described ahove are assumed to he interrelated.

\section{Criticism of the transactional approach}

According to the transactional model, stress is best regarded as a complex rubric, a convenient term to refer to the operation of many variables and processes in situations in which the demands tax or exceed the person's resources. This complexity and diversity of aspects related to the transactional definition of stress forces one to make choices about which classes of variables to study. Lazarus and Folkman's definition of stress has also received its share of criticism. The main point of critique has been that it is overly subjective, in that both stress and coping resources are defend and measured in terms of appraisal processes (Dohrenwend \& Shrout, 1985). The choice between obtaining subjective or relatively objective indices of stress is an important one and should depend on the purposes of the study. If the goal is to investigate the etiologic role of life circumstances as precursors of pathology, one should try to reduce the subjectivity of event measures to reduce possible confounding of the stress-pathology relationship. Even then, knowledge about the context of the event, about the circumstances surrounding the event, may still be important for an understanding of why the disorder developed. If not prediction of pathology but understanding the process of stress per se is the main aim. then the subjective experience of stress is worth studying in and of itself (Wagner, 1990). Although a certain degree of circularity (the same process is being measured in the independent and the dependent variables) is inevitable with relational definitions of stress, this can be limited by asking what it is about the person, in interaciion with a given environmental situation, that generates appraisals of harm, threat, or benefit (Lazarus, De Longis, Folkman, \& Gruen, 1985).

\section{THE CONCEPT OF STRESS: PHYSIOLOGICAL VIEWS}

Since most psychological or behavioral signs of stress are often of a subjective nature (such as subjective self-reports of negative affect, low job-satisfaction, anxiety, or agitation), a substantial body of stress research has focused on more objective indicators of stress. Characteristic of both humans and animals is that they respond to stressful events with various well-defined and quantifiable physiological responses, such as the release of stress hormones in the blood, increased heart rate and blood pressure. Moreover, repeated or chronic physiological activation is held to be an important factor in the link between stress and disease. Therefore, we will provide a brief review of the physiological stress tradition.

\section{Short history of early studies}

Early stress research in animals emphasized that responses of the organism to a wide variety of stimuli were non-specific. Selye (1936, p. 32) defined stress as "the nonspecific response of the body to any noxious stimulus". This nonspecific response to stimuli such as heat, cold, or exercise, has been called the General Adaptation Syndrome; it is characterized by increased adreno-cortical activity, degeneration of the thymus and lymphatic structures; and hemorrhage and ulceration of the stomach 
and other parts of the gastrointestinal tract. Three temporal phases can be distinguished: the alarm phase, the stage of resistance, and the stage of exhaustion. According to the theory, conditioning factors (e.g. age, genetic predisposition, diet, drugs) will determine which specific disease of adaptation will manifest itself after prolonged exposure to a stressor. The pituitary-adrenal-cortical axis regulates the release of corticosteroids from the adrenal cortex, with cortisol as the most important glucocorticoid homone in humans, and with the potential of exerting its effects on practically all normal body cells and tissues. Some of the main functions of cortisol are: stimulation of gluconeogenesis, inhibition of glucose uptake, suppression of inflammation and suppression of numerous immune functions (Munck, Guyre, \& Holbrook, 1984). Whereas Selye especially emphasized the activity of the pituitaryadrenal-cortical axis, Cannon (1929) investigated the role of the sympathetic-adrenalmedullary system, which regulates secretion of the catecholamines adrenaline and noradrenaline. Cannon showed that besides physical stimuli, non-physical or emotional stimuli could also activate the physiological system. He formulated the concept of 'physiological homeostasis', which means that those physiological responses will be triggered which meet the demands of the environment. The increased activity of the central nervous system (CNS) and release of catecholamines were interpreted as an emergency' reaction. In this way, the organism is prepared for appropriate action aimed at coping with the stressor and restoring control: "fight or flight'. Enhanced sympathetic activity leads to increases in glucose, respiratory rate, blood pressure, and heart rate, and to a blood flow away from the intestines to the muscles, where oxygen and nutrients are most needed. It was Mason (1968) who pointed out, after reviewing earlier studies on animals, healthy humans, and psychiatric patients, that many of the stressful experiences that had been investigated shared one common characteristic: the induction of emotional arousal. For instance, when an animal is exposed to a novel or unfamiliar environment, it is the psychological relevance of the stressor that determines the stress response and not the particular physical stressor. Important conditions for eliciting responses were found to be novelty, uncertainty, unpredictability, involvement, and anticipation of unpleasant experiences. The importance of emotional arousal as a trigger of the stress response and the fact that those specific responses are triggered that meet the demands of the environment are not compatible with a non-specific stress response as formulated in Selye's theory; the physiological stress response is therefore best regarded as a differentiated response of the organism.

Another important finding was that marked individual differences exist $1 \mathrm{n}$ the adrenal-cortical response to a given stressful situation (Mason, 1968; Rose, 1984). These differences seem to be related to individual coping styles or defense mechanisms and to how the event is appraised. Rose (1984) noted that cortisol responses extinguish rapidly in individuals upon re-exposure to most kind of events, reflecting adaptation.

\section{Recent physiological stress models}

More recent models have integrated the major physiological concepts described above. In the animal model proposed by Henry and Stephens (1977) it was suggested that the nature of behavioral and accompanying physiological reactions are determined by the outcome of the appraisal process, which in turn results in different coping strategies. Two extremes can be differentiated with respect to behavioral, physiological, and CNS activities. When the appraisal process results in fear or anxiety but the animal still tries to eliminate the threat by active effortful 
coping, this will lead to sympathetic activation, for example a pronounced increase in adrenaline release and heart rate. This resembles the 'fight/flight' response described by Cannon (1929), with territorial control (mobility, display, aggression) as behavioral components. However, when the appraisal results in uncertainty or loss of control and the animal sees no escape or has no adequate coping response available, the hypothalamus-pituitary-adrenocortical axis is activated to release ACTH and cortisol, as was originally described by Selye. At the behavioral level this response is accompanied by inhibition (freezing or doing nothing), subordination, and decreased sexual and maternal behavior ('conservation-withdrawal'). Frankenhaeuser's effortdistress model (1983), based on studies of healthy humans (e.g. Frankenhaeuser, Lundberg, \& Forsman, 1980; Lundberg \& Frankenhaeuser, 1980), is consistent with the model of Henry and Stephens (1977). Both focus on two components of psychological arousal, effort and distress (experienced either singly or in combination), which seem to be differentially related to catecholamine and cortisol secretion. A state of 'effort without distress' (a positive affective state) is accompanied by catecholamine secretion, while under this condition cortisol secretion is low or may be actively suppressed. On the other hand, a state of 'effort with distress' (which may characterize many stressful events) is associated with an increased secretion of both catecholamines and cortisol. An example of this state is mental work carried out under conditions of stimulus overload. The state of 'distress without effort', which may be reflected in giving up or feelings of helplessness, is accompanied by an increase in cortisol secretion, and resembles the "conservationwithdrawal' response as described by the model of Henry and Stephens. Personal control seems to be an important modulating factor here, with less control leading to more distress. In summary, cortisol, in comparison to other stress-labile hormones (e.g. catecholamines, growth hormone, prolactin, testosterone), appears to show the most specific relationship to subjective distress as opposed to effort or general arousal (e.g. Lundberg \& Frankenhaeuser, 1980), and is thought to reflect the adequacy of coping behavior (e.g. Vickers, 1988).

\section{STRESS, HEALTH, AND DISEASE}

As described above, in most cases the psychological and physiological responses to stress reflect normal, adaptive processes which prepare the body to cope with the situation. Negative emotions alert people to deal with or avoid difficuli situations, and physiological reactions prepare the body for 'fight or flight' responses (Chrousos \& Gold, 1992). It has also been noted that stress does not always lead to negative effects like poor performance or illness, but may result in training or toughening effects as well (Dienstbier, 1989; Ursin, 1980). Thus in the normal course of events, when coping is successful and the stressful experience is of short duration, the benefits largely outweigh the costs of the stress response. Only when behavioral or cognitive coping strategies for dealing with stressful experiences are not effective or not available and stress endures are psychological and physiological responses likely to become exaggerated or prolonged, thereby increasing the risk for pathology to develop. In humans only extreme stressors will evoke responses like fighting or fleeing, while the majority of events experienced in daily life ask for more subtle solutions. Since rapid availability of oxygen, nutrients, glucose, and so on is not necessary to meet the demands of contemporary daily challenges, disruption of bodily homeostasis may occur. 
question in this regard is whether individual differences in laboratory reactivity reflect those occurring during stressful situation in real-life (Turner, Ward, Gellman, Johnston, Light, \& van Doornen, 1994). The usefulness of laboratory assessments rests, in part, on this assumption. Later in this chapter we will deal with these subjects in more detail.

\section{FROM MAJOR LIFE EVENTS TO MINOR DAILY EVENTS}

As already described previously, the major life event approach has received considerable criticism relating to aspects of both design and method. A basic objection has been the reliance on retrospective research designs. The retrospective assessment of summaries of life events over many months obscures the temporal, dynamic interplay between environmental demands, appraisals of demands, and outcomes (Stone \& Shiffman, 1992), which is central in the transactional theory of stress and coping. More details of the stress process are needed to unravel the mechanisms underlying the relationship between events and disease. The dynamic processes between demands, appraisals, and outcomes are presumed to change so quickly that they can only be captured through frequent assessment. Of course, the frequency of measurement should follow the fluctuations of the phenomena under study; fluctuations in heart rate would ask for minute to minute assessments over periods less than a day, while if we were interested in the relationship between stress and premenstrual symptoms, daily measurements over several months would be more appropriate.

Another disadvantage of retrospective research designs is that the recall of events over relatively long periods may be influenced by biases. Recall of events and mood over long time periods is obviously limited by forgetting, but research suggests that this is not a random process. For instance, it has been shown that recall of past mood and both positive as well as negative events is strongly influenced by the respondent's mood at the time of recall (Bower, 1981; Teasdale \& Forgarty, 1979); the current mood state makes mood-congruent memories more accessible and moodincongruent memories less accessible. Although this applies especialily to global ratings of past experiences, it is also likely that recall of specific events may be affected; emotionally important or salient events are more likely to be remembered than less salient ones (Strongman \& Russell, 1986). By reducing the recall time interval, these biases can be also reduced.

Taken together, these points have lead to the conclusion that other categories of events, such as chronic stressors and the nnilder but much more frequent stresses of daily living, should also be investigated concerning their relationship to health. Since the events of everyday life eccur musch more frequently than the major life events, they might be even more important to well-being. The investigation of minor daily events may also help to delineate the stressful features of chronic experiences, such as work stress. Indeed, an increasing number of studies have shown that daily life events are related to lower psychological well-being and increased somatic symptomatology, even after the possible confounding effect of major life events has been controlled for (e.g. DeLongis et al., 1982; Stone, Neale, \& Shiffman, 1993; Zarski, 1984). Daily events or 'hassles' are defined as "the experiences and conditions of daily living that have been appraised as salient and harmful or threatening to the endorser's wellbeing" (Lazarus, 1984, p.376). Examples of situations that can be hassles are: getting caught in a traffic jam, having to wailt, quarreling with your child. There are several 
question in this regard is whether individual differences in laboratory reactivity reflect those occurring during stressful situation in real-life (Turner, Ward, Gellman, Johnston, Light, \& van Doornen, 1994). The usefulness of laboratory assessments rests, in part, on this assumption. Later in this chapter we will deal with these subjects in more detail.

\section{FROM MAJOR LIFE EVENTS TO MINOR DAILY EVENTS}

As already described previously, the major life event approach has received considerable criticism relating to aspects of both design and method. A basic objection has been the reliance on retrospective research designs. The retrospective assessment of summaries of life events over many months obscures the temporal, dynamic interplay between environmental demands, appraisals of demands, and outcomes (Stone \& Shiffman, 1992), which is central in the transactional theory of stress and coping. More details of the stress process are needed to unravel the mechanisms underlying the relationship between events and disease. The dynamic processes between demands, appraisals, and outcomes are presumed to change so quickly that they can only be captured through frequent assessment. Of course, the frequency of measurement should follow the fluctuations of the phenomena under study; fluctuations in heart rate would ask for minute to minute assessments over periods less than a day, while if we were interested in the relationship between stress and premenstrual symptoms, daily measurements over several months would be more appropriate.

Another disadvantage of retrospective research designs is that the recall of events over relatively long periods may be influenced by biases. Recall of events and mood over long time periods is obviously limited by forgetting, but research suggests that this is not a random process. For instance, it has been shown that recall of past mood and both positive as well as negative events is strongly influenced by the respondent's mood at the time of recall (Bower, 1981; Teasdale \& Forgarty, 1979); the current mood state makes mood-congruent memories more accessible and moodincongruent memories less accessible. Although this applies especialily to global ratings of past experiences, it is also likely that recall of specific events may be affected; emotionally important or salient events are more likely to be remembered than less salient ones (Strongman \& Russell, 1986). By reducing the recall time interval, these biases can be also reduced.

Taken together, these points have lead to the conclusion that other categories of events, such as chronic stressors and the nnilder but much more frequent stresses of daily living, should also be investigated concerning their relationship to health. Since the events of everyday life eccur musch more frequently than the major life events, they might be even more important to well-being. The investigation of minor daily events may also help to delineate the stressful features of chronic experiences, such as work stress. Indeed, an increasing number of studies have shown that daily life events are related to lower psychological well-being and increased somatic symptomatology, even after the possible confounding effect of major life events has been controlled for (e.g. DeLongis et al., 1982; Stone, Neale, \& Shiffman, 1993; Zarski, 1984). Daily events or 'hassles' are defined as "the experiences and conditions of daily living that have been appraised as salient and harmful or threatening to the endorser's wellbeing" (Lazarus, 1984, p.376). Examples of situations that can be hassles are: getting caught in a traffic jam, having to wailt, quarreling with your child. There are several 
forms of daily life stress. Some stressors are chance events, and usually rare in occurrence (e.g. flat tire when in a hurry, out of toilet paper just when needed, unexpected phone calls), while others are repeated, either because a person remains in the same ongoing social situation (e.g. work, marriage) with consistent demands (e.g. high work load, conflicts with spouse), or because of certain personality dispositions, such as a person's ineffective style of coping with common situations (Kanner, Coyne, Schaefer, \& Lazarus, 1981). Since no one lives a life completely without stress, the impact of hassles on physical and mental health is thought to depend on factors such as a chronically high frequency of stressful daily events, an increase in hassles during a given period (the 'last straw'), the presence of hassles with compelling psychological importance, or an underlying (biological) vulnerability to stress or to a specific illness (Kanner et al., 1981; Zautra, Guarnaccia, Reich, \& Dohrenwend, 1988).

The current study investigates the impact of minor daily events on mood and the HPA system. In the next two sections we present a review of the available literature concerning the relationship between daily events, on the one hand, and mood and cortisol responses, on the other hand. The following related subjects will be of special interest: individual differences in mood and cortisol responses, the context of the event, and event appraisa!s.

\section{STRESSFUL DAILY EVENTS AND MOOD STATES}

A common approach to the study of daily stress examines the relationship between stressful events and mood by assessing both once a day for several weeks (Stone \& Shiffman, 1992); others have assessed these phenomena on a monthly basis (e.g. Lewinsohn \& Libet, 1972). The simplest approach is open-ended: subjects are asked to describe the day's most stressful events (Rehm, 1978) or asked to describe anything that went wrong during the day (Caspi, Bolger, \& Eckenrode, 1987; Eckenrode, 1984). Responses are then classified into discrete categories. The most often used method for assessing daily events is the event checklist, where, from a list of events, subjects are asked to identify which events have occurred over a specified time period, ranging from one day to one month. Examples of monthly checklists are the Unpleasant Events Schedule and the Pleasant Events Schedule (Lewinsohn \& Amenson. 1978), the Hassles and Uplifts scale (Kanner et al., 1981), and the Inventory of Small Life Events (Zautra \& Guarnaccia, 1986). Items on these checklists were formulated by the researchers or taken from existing scales and constructed to cover events in major life domains, such as family, work, leisure, and household maintenance. Examples of daily checklists are the Daily Life Experience checklist (Stone \& Neale, 1982), the Daily Stress Inventory (Brantley, Waggoner, Jones, \& Rappaport, 1987), and the Daily Stress Scale (Bolger, DeLongis, Kessler, \& Schiling, 1989a). Here. by using an open-ended format. a more representative domain of events was first obtained by sampling events which conform to certain criteria (e.g. 'stressfulness', (Brantley et al., 1987)) from the participant population in which the checklist was to be used. Elicited events were then grouped into various categories to form the checklist.

Regardless of what approach has been used to study daily stress, several studies have shown a same-day association between daily stress and negative mood (Affleck, Tennen, Urrows, \& Higgins, 1994; Bolger et al., 1989a; Clark \& Watson, 1988; DeLongis, Folkman, \& Lazarus, 1988; Eckenrode, 1984; Larsen, Diener, \& Emmons, 1986; Lewinsohn \& Libet, 1972; Rehm, 1978; Stone \& Neale, 1984; Stone et 
al., 1993). Results with respect to positive mood are inconclusive; most studies have not differentiated between negative and positive mood, but in those that have, positive mood was either lower (Neale, Hooley, Jandorf, \& Stone, 1987; Repetti. 1993; Stone \& Neale, 1984) or unchanged (Watson, 1988) on days when many stressful events occurred.

Evidence for large individual differences in the magnitude and the direction of the association between daily stress and mood has been found in a number of studies. Examples of factors related to such differences are self-esteem (Campbell, Chew, \& Scratchley, 1991), social support (Affleck et al., 1994; Barling \& Kryl, 1990; Caspi et al., 1987; DeLongis et al., 1988), major life events (Affleck et al., 1994; Caspi et al., 1987), and neuroticism (Affleck et al., 1994; Bolger \& Schilling, 1991; Eckenrode, 1984). The moderating role of chronic stress or long-term difficulties on the relationship between daily life stress and mood has received relatively little attention. In a study by Caspi et al. (Caspi et al., 1987), the chronic stress of living in a low quality neighborhood increased the immediate effects of stressful daily events on mood and also increased the likelihood that daily stressors had an enduring effect on next day's mood. Investigating the influence of perceived stress levels on the daily stress-mood relationship may be important, since individuals experiencing high perceived stress feel their lives to be unpredictable, uncontrollable, and overloaded, and can be seen as at risk for the development of stress-related somatic and mental health problems (Cohen, Kamarck, \& Mermelstein, 1983). Personality factors probably play a very important role in perceived stress. Self-report measures of stress have been found to correlate significantly with measures of negative affectivity or neuroticism (Watson, 1988; Watson \& Pennebaker, 1989). Negative affectivity reflects a general disposition to focus on the negative aspects of self and others and to experience events as negative or distressing (Eysenck \& Eysenk, 1968; Watson \& Clark, 1984). In stress research, negative affectivity has been treated in two different ways. First, it is thought of as a confounding variable, spuriously inflating the relationship among stressors, stress symptoms, and affective outcomes (Chen \& Spector, 1991: Costa \& McCrae, 1987; Schaubroeck, Ganster, \& Fox, 1992; Watson \& Pennebaker, 1989). However, negative affectivity has also been investigated as a moderator variable. influencing individuals' reactivity to stressful events. Several studies demonstrated that individuals higher in negative affect show greater negative mood reactivity to stressors (Affleck et al., 1994; Bolger \& Schilling, 1991; Marco \& Suls, 1993). Differences between high and low negative affect individuals in how events are appraised or coped with may possibly account for these differences in mood reactivity.

Little is known about how long the effects of daily events on mood persist. although this would be important for understanding the consequences of minor events. Heaith chances may only occur when daily events are very frequent and negative mood responses persist in time, for instance while coping has been unsuccessful or incomplete. The available literature from end-of-day studies suggests that daily stressors affect same-day mood but do not necessarily affect mood on subsequent days (Bolger et al., 1989a; DeLongis et al., 1988; Lewinsohn \& Libet, 1972; Neale et al., 1987; Rehm, 1978; Stone \& Neale, 1984), but more research is necessary to clarify this subject.

Since people experience a large range of stressful events in daily life, several studies have been employed to try to increase our understanding of what categories of events and which event characteristics are most strongly related to well-being. Several studies indeed seem to indicate that the psychological impact of daily events 
depends on the context in which they occur. Particularly undesirable work events and interpersonal conflicts appear to have the strongest relationship to negative mood states (Bolger et al., 1989a; Bolger \& Schilling, 1991; Clark \& Watson, 1988; Repetti, 1993; Stone, 1987). Work events have also been associated with lower positive mood (Stone, 1987). Despite the important role of the concept of appraisal in contemporary stress theory in determining whether a daily event is experienced as stressful or not, little research has been done in relation to appraisals of naturally occurring daily events. There is evidence from numerous laboratory studies and several naturalistic studies that subjects' personal ratings of events, like frequency, demand, controllability, and predictability of events, improve prediction of outcomes such as performance, anxiety, depression, negative affect, and tension (Averill, 1973; Cohen, 1980; Dewe, 1991; Folkman, 1984; Katz \& Wykes, 1985; McGrath \& Beehr, 1990; Miller, 1979; Peeters, Buunk, \& Schaufeli, 1995; Sarason et al., 1978; Thompson, 1981). Additional research is needed that studies cognitive appraisals of daily events in a natural environment and investigates how these appraisal dimensions relate to mood.

While end-of-day assessments of daily hassles have important advantages compared to the major life event approach, several drawbacks still remain; these will be discussed in the final section of this chapter.

\section{STRESSFUL DAILY EVENTS AND CORTISOL DYNAMICS}

Until recently, the two main approaches to the investigation of the impact of stressful events on the HPA axis in humans have been laboratory reactivity studies and 'natural experiments'. While both approaches have been of great value for our understanding of the stress process, each has limitations. We will see that neither approach is adequate if one adopts the dynamic conceptualization of stress as a process, which changes over time and in relation to the environment.

Most studies of psychophysiological reactivity have used standardized laboratory tasks such as physical strain or exercise, mental stress tasks (e.g. reactiontime tasks, arithmetics, computer games), cold pressor tests, and mood induction, all with questionable relevance to real life (Berger, Bossert, Krieg, Dirlich, Ettmeier, Schreiber, et al., 1987; Brown, Sirota, Niaura, \& Engebretson, 1993; Bullinger. Naber, Pickar, Cohen, Kalin, Pert, et al., 1984; Forsman \& Lundberg. 1982; Mason, Hartley, Kotchen, Mougey, Ricketts, \& Jones, 1973). Ecological valid information about the sources of stress people experience in their daily lives, and the responses to it, seem difficult to obtain in laboratory experiments (Lazarus \& Launier, 1978). One can question whether the responses to such tasks are relevant or valid reflections of habitual reactivity to naturally occurring stressors - in other words, to what extent does laboratory-assessed reactivity generalize to the field? With regard to hormonal reactivity, little is known about the generalizability of laboratory studies to field studies. Results from cardiovascular studies indicate quite strong associations between chronic levels of cardiovascular activity across settings, but little support has been found for the relationship between laboratory reactivity and field reactivity (Turner et al., 1994). Moreover, it is impossible, from a practical and ethical point of view, to generate in the laboratory the stressful events and responses of the type and severity found in real-life (van. Doornen \& Turner, 1992). Although experimental control and the possibility of isolating and manipulating variables have important advantages and may be the optimal strategy for the evaluation of certain aspects of 
stress theory, it seems more important at this stage to investigate and try to describe what is happening in everyday life with regard to stress processes. Some have tried to increase ecological validity by mimicking daily life situations in the laboratory: for example public speaking (Bassett, Marshall, \& Spillane, 1987; Kirschbaum \& Hellhammer, 1993), or stress inducing films (Hubert \& de Jong-Meyer, 1989).

Natural experiments take advantage of the occurrence of real-life, potentially stressful events, of either an acute or of a more chronic nature. With regard to acute stressors, increases in cortisol excretion were, for example, found in situations such as forcing non-swimmers to jump in the deep-end of a swimming pool (Vaernes, Ursin, Darragh, \& Lambe, 1982), an anticipated surgical procedure (Ben-Aryeh, Roll, Kahana, \& al., 1985; Knight, Atkins, Eagle, Evans, Finkelstein, Fukushima, et al., 1979), academic examinations (Allen, Batty, Dodd, Herbert, Hugh, Moore, et al., 1985; Jones, Copolev, \& Outsch, 1986; Nicolson, 1992), and parachute (Schedlowski, Jacobs, Stratmann, Richter, Hadicke, Tewes, et al., 1993; Ursin, 1978) or bungee jumping (Hennig, Laschefski, \& Opper, 1994). Some examples of the kind of chronic stressors studied in the field are living near the damaged power plant at Three Mile Island (Schaeffer \& Baum, 1984), the period of bereavement after the loss of a child (Hofer, Wolff, Friedman, \& Mason, 1972a; Hofer, Wolff, Friedman, \& Mason, 1972b), enforced captivity (Dekaris, Sabioncello, Mazuran, Rabatic, Svoboda-Beusan, Racunica-, et al., 1993; Rahe, Karson, Howard, Rubin. \& Poland, 1990), combal exposure or related post-traumatic stress disorder (Bourne, Rose, \& Mason, 1967; Piman \& Orr, 1990), and work stress (e.g. Arnetz, Brenner, Levi, Hjelm. Petterson, Wasserman, et al., 1991: Caplan, Cobb, \& French, 1979; Coeck, Jorens, Vandevivere, \& Mahler, 1991; Lundberg \& Paim. 1989; Rose \& Fogg, 1993; Theorell, 1989; Timio \& Gentili, 1976). The effects of chronic or intermittent stress has received far less attention in the literature than the effects of acute stress, and data. on cortisol levels during prolonged stress have: been inconsistent, with enhanced (Arnetz et al., 1991; Coeck et al., 1991; Hofer et al., 1972a; Rahe et al., 1990; Rose \& Fogg, 1993; Schaeffer \& Baum, 1984; Timio \& Gentili, 1976) as well as decreased (Bourne et al., 1967; Caplan et al., 1979; Dekaris et al., 1993: Pitman \& Orr, 1990) concentrations reporled, and large variability among individuals.

A major drawback of the natural experiment is that, since the researcher generally has to be present in the field to collect cortisol measures, only anticipated events can be investigated. Additionally, mosi of the studies have examined stress under rather extreme or unusual situations, approaching major life events in terms of severity. Only few studies have investigated whether less severe but much more frequent daily events also have an impact on cortisol secretion, and fundings have been inconsistent, with reports of lowered (Caplan et al., 1979), elevated (Brantley. Dietz, McKnight, Jones, \& 'Tulley, 1988; Lundberg, Granqvist, Hansson, Magnusson, \& Wallin, 1989), and unchanged (Cummins \& Gevirtz, 19913) cortisoll levels.

Since individua! characteristics may influence how stressfu! situations are appraised they are thought to be important determinants of the emotional response to a given situation; mood states are, in turn, likely to mediate the endocrine response to the situation. Available information on the relationship between personality uaits, mood states, and event characteristics on the one hand and on cortisol levels on the other hand has until now been based on the traditional approaches discussed above. Several personality characteristics (e.g. trait anxiety, depression, anger, coping style, personality) have been associated with basal cortisol levels (Brandtstädter. BaltesGotz, Kirschbaum, \& Hellhammer, 1991; Pope \& Smith, 1991; Schaeffer \& Baum, 1984), as well as with cortisol responses, to stress (Bohnen, Nicolson, Sulon, \& Jolles, 
1991; Demyttenaere, Nijs, Evers-Kiebooms, \& Koninckx, 1989; Kirschbaum, Hellhammer, Strasburger, Tiling, Kamp, \& Luddecke, 1989; Nicolson, van Poll, \& deVries, 1992). Other studies, however, have found no association between personality traits, coping styles, and cortisol laboratory baseline levels or responsiveness to laboratory stressors (Bosserts, Berger, Krieg. Schreiber, Junker, \& von Zerssen, 1988; Kirschbaum, Bartussek, \& Strasburger, 1992a). Although there is abundant evidence that cortisol increases in response to distress or negative mood (Arnetz et al., 1991; Lundberg \& Frankenhaeuser, 1980; Mason, 1968), the effects of positive mood are less clear. Positive affective states, have been associated with decreases (Hubert \& de Jong-Meyer, 1989; Kugler \& Kalveram, 1989) as well as increases (Brown et al., 1993) in cortisol levels. Several laboratory studies have investigated the differential effects of various event appraisals (e.g. novelty, predictability, controllability) on cortisol (Mason, 1968). To our knowledge, this has not been done with regard to daily stress yet. In addition, there is no research that investigated whether different types of daily events have a different impact on cortisol. Since the context of an event has been found to relate to the magnitude of its psychological impact, this would be an interesting research question.

A major disadvantage of the studies described above is that often only a small or a few cortisol sample were obtained per subject or per day. In the study by Rose and Fogg (Rose \& Fogg, 1993), where repeated measurements of cortisol responses to work stress in air traffic controllers were obtained, a subgroup of subjects was found to respond to an increase in workload with large increases in cortisol. Repeated measurement of cortisol not only increases reliability, but also allows the investigation of dynamic relationships between stressful daily experiences and neuroendocrine activity. Additional research is necessary to see how the results obtained so far relate to those obtained with repeated measurements designs in the context of real life, and with daily events as the focus of interest.

\section{PRESENT STUDY: AIMS AND RESEARCH QUESTIONS}

Due to the hypothesized role of appraisal as a mediator between the environment and the person in determining the occurrence of a stressful experience and the responses to it, stress should be measured in a context were intrinsically motivating events and emotional involvement are most likely to happen; the natural environment of the subject seems to be the ideal research setting. Here, individuals can be observed in their normal social networks, settings, and activities, and stressful events can be studied in the life setting where they actually occur. Additionally, since stress is currently conceptualized as a dynamic process, which changes over time and in relation to the environment, it is necessary to include repeated measurements of stress, hormone levels, and emotional states to investigate the relationship between stress and affective and neuroendocrine responses (Dimsdale, 1984). The advantages of the repeated measurement approach can be further enhanced by simultaneously collecting information on individual traits, event characteristics and cognitive appraisals, which can then be investigated for their effect on mood and cortisol excretion.

Compared to the major life event approach, end-of-day assessments of minor stressors have important advantages, but even within a day, much of the dynamic interplay between events, appraisals of events, and outcomes remain hidden. Recall biases of events and mood are still likely to occur, and same-day associations between 
daily events and mood remain causally ambiguous. With this we mean that not only daily stress may have an effect on mood, but that a bad mood may increase the likelihood that daily events will occur or will be perceived as stressful.

In the present study, two relatively new approaches were employed to investigate the relationship between stress, affective states, and cortisol: i.e. the Experience Sampling Method (ESM) and ambulatory monitoring of salivary cortisol. The Experience Sampling Method has been specifically designed to study the subjective everyday experiences of people in their natural environments (Csikszentmihalyi \& Larson, 1987; de Vries, 1992). Typically, an electronic signaling device is used to alert subjects to fill in a self-report questionnaire at preselected but randomized time-points, providing information about an individual's mental status or symptoms within the context and flow of experience. When, how often, and for how long subjects are signaled depends on the goal of the study. The prospective assessment procedure is an important characteristic of ESM; since the variables of interest are assessed at frequent intervals during the day and close to the moment at which they actually occur, we are able to look at more dynamic stress processes, with a minimum of confounding due to biased recall or forgetting (Bower, 1981). ESM therefore also provides better estimates of the frequency, distribution, and intensity of psychological variables than cross-sectional designs (Larson \& Csikszentmihalyi. 1983).

The choice of cortisol as a physiological stress indicator was based on both theoretical and practical grounds. From a theoretical point of view, cortisol is a sensitive indicator of the stressfulness and adequacy of psychological responses to person-environment transactions, an important regulator of vital physiological processes, and a possible mediator of physical responses leading to disease. From a practical viewpoint, the opportunity of measuring cortisol reliably in saliva instead of blood makes repeated, stress-free sampling in real-life contexts possible (Kirschbaum \& Hellhammer, 1994; Nicolson et al., 1992). Saliva samples can be reliably collected and stored by subjects themselves, enabling them to carry out their daily routine without undue interference. Although our main emphasis was on cortisol, 14-hour urinary catecholamine levels were also determined twice, as possible indicators of long-term or more chronic stress.

The present study design compares two groups of male white collar workers. with high versus low levels of perceived stress, monitored during their normal daily activities. Subjects completed ESM self-reports concerning activities, mood states, and recent stressful events, and collected saliva samples for cortisol determination in response to signals emitted by a preprogrammed watch ten times a day for five consecutive days. Our study focused on four main topics: (1) the nature and experience of stress in daily life; (2) the relationship between perceived stress, stressful daily events and mood states; (3) the relationship between perceived stress. stressful daily events and cortisol levels; and (4) the generalizability of stress responses from the laboratory to real life. As outlined below, each of these topics will be addressed in separate chapters.

Following a description of the subjects and methods in Chapter 2, Chapter 3 is devoted to the description of quantitative as well as qualitative aspects of stressful daily events. Various questions are addressed, including: How frequently are stressful events experienced? What kind of events are experienced as stressful in daily life? Do stressful experiences differ with respect to frequency, kind, and appraisal in subjects with a high versus low level of perceived stress? How do major life events and chronic difficulties relate to the pattern of stressful daily events? 
Chapter 4 examines the influence of daily events on both negative and positive mood states. In addition to the immediate effects of daily stress on mood, we also examine temporal patterns of the effects of events on mood states. We examine whether average mood levels and mood responses to daily events were related to individual differences in perceived stress level. Finally, various kinds of events (work demands, negative social interactions) and event appraisals (e.g. unpleasantness, predictability, controllability) are investigated for their possible differential effects on mood states.

In Chapter 5 we address the question whether high perceived stress is associated with elevated cortisol levels. We also investigate the extent to which individual characteristics (trait anxiety, depression, anger, psychosomatic complaints, mood states, number of stressful events) contribute to differences in cortisol levels. Lastly, we examine the relationship between perceived stress level and urinary catecholamine excretion following work versus weekend days.

Chapter 6 focuses on the possible impact of minor daily events on cortisol secretion. We also examine the association between an individual's affective state and cortisol changes in response to such events. Finally, we test whether perceived stress and other individual characteristics are related to cortisol reactivity to stressful events.

Chapter 7 describes emotional and cortisol responses to a stress-inducing speech task in the same group of subjects. Beyond assessing the relationship between individual trait characteristics, current mood states, and cortisol responses to the stress task, a main objective of the laboratory experiment was to explore the consistency between laboratory and field cortisol measures, particularly with regard to the usefulness of laboratory measures in predicting cortisol levels and response to stressful events in the field. To this end, cortisol levels and responses measured in the laboratory were compared with those measured in real life.

Chapter 8 summarizes the main research findings and attempts to place them in a larger perspective. In addition it discusses limitations of the study and possible future research directions. 
Chapter 2

Methods 

The research questions presented in Chapter 1 necessitated a diversity of methodological approaches, including questionnaires, the Experience Sampling Method, monitoring of hormone levels in saliva and urine, and an experimental stress task. Chapter 2 describes first the subject selection procedure and demographic characteristics of the sample. Next, the measures and data collection procedures are described in detail. Methodological issues involved in the analysis of ESM data are discussed, including the coding of ESM responses, compliance, and assessment of possible biases and / or experimental effects of ESM procedures.

\section{SUBJECTS}

The sample consisted of 92 male white collar workers who had been first screened and then selected from six local industries and government agencies (see 'procedure' section), based on their perceived stress scores in the upper or lower tertiles of the screening sample distribution (PSS score $<10$ or 216). Subjects who reported a history of serious chronic illness, endocrine disorder, medications known to affect cortisol levels, or treatment (past or current) for mental health problems were excluded. During subject intake, each high stress subject was matched for age group, marital status, and household composition with a low stress subject to insure that the two groups did not differ on demographic characteristics that might affect exposure to certain classes of stressors.

Table 2.1. Demographic data for 'High' and 'Low' stress subjects.

\begin{tabular}{cc}
\hline Low Stress Subjects & High Stress Subjects \\
$\mathrm{N}=46$ & $\mathrm{~N}=42$ \\
\hline
\end{tabular}

Age

mean

s.d.

range
42.7

7.7

27.57
41.5

6.0

28-52

\section{Marital status}

$\begin{array}{lrr}\text { married } & 41(89.1 \%) & 37(88.1 \%) \\ \text { unmarried } & 2(4.3 \%) & 2(4.8 \%) \\ \text { living together } & 2(4.3 \%) & 2(4.8 \%) \\ \text { divorced } & 1(2.2 \%) & 1(2.4 \%)\end{array}$

\section{Household composition}

living alone

couple with children

couple without children
3 (6.5\%)

$37(80.4 \%)$

$6(13.0 \%)$
$3(7.1 \%)$

$34(81.0 \%)$

$5(11.9 \%)$

'High' and 'low' perceived stress groups were defined as follows: the mean of the first and the second PSS assessment was used to categorize subjects as above or below the screening sample median score (12). Four subjects (two from each group). 
One week before the field study, subjects received a second questionnaire battery to be filled in at home and returned at the briefing session. The questionnaires concerned psychosocial stress (including the PSS again), personality and psychological symptoms (depression, anger) (see next section).

\section{CROSS-SECTIONAL INSTRUMENTS}

An overview is presented of the questionnaires used. The questionnaires administered during the screening phase of the study will be described first (Questionnaire I), followed by the questionnaires completed the week before ESM took place (Questionnaire II). Questionnaires which have not been widely used will be described more thoroughly than more familiar ones.

\section{Questionnaire I}

Demographic information: A demographic questionnaire requesıed information concerning respondent's age, marital status, type of household, number of children (for the matching procedure), chronic diseases and medication use (possible exclusion criteria), and alcohol use, smoking habits, and participation in active sports (possible confounders of hormone concentrations).

Perceived Stress: Subjects were selected according to their perceived stress level. Perceived stress was measured by means of the Perceived Stress Scale (PSS: Cohen et al., 1983). The PSS is a global measure of the degree to which situations in one's life are appraised as stressful. ltems were designed to tap the extent to which individuals feel their life to be unpredictable, uncontrollable, and overloaded. These themes are important components of the experience of stress (Averill, 1973; Cohen, 1978; Seligman, 1975). The PSS measures cognitions and emotions relating to general stress levels rather than specific events or situations (see Table 2.2.). The items are rated on a 5-point frequency scale, ranging from 'never' $(0)$ to 'very often' (4). A total score is obtained by reversing the scoring on the positive items and then summing across the 10 items.

Table 2.2. Items Perceived Stress Scale.

1. In the last month, how often have you been upset because of something that happened unexpectedly?

2. In the last month, how often have you felt that you were unable to control the important things in your life?

3. In the last month, how often have you felt nervous and 'stressed'?

4. In the last month, how often have you felt confident about your ability to handle your personal problems?

5. In the last month, how often have you felt that things were going your way?

6. In the last month, how often have you found that you could not cope with all the things that you had to do?

7. In the last month, how often have you been able to control irritations in your life?

8. In the last month, how often have you felt that you were on top of things?

9. In the last month, how often have you been angered because of things that happened that were outside of your control?

10. In the last month, how often have you felt difficulties were piling up so high that you could not overcome them? 
One week before the field study, subjects received a second questionnaire battery to be filled in at home and returned at the briefing session. The questionnaires concerned psychosocial stress (including the PSS again), personality and psychological symptoms (depression, anger) (see next section).

\section{CROSS-SECTIONAL INSTRUMENTS}

An overview is presented of the questionnaires used. The questionnaires administered during the screening phase of the study will be described first (Questionnaire I), followed by the questionnaires completed the week before ESM took place (Questionnaire II). Questionnaires which have not been widely used will be described more thoroughly than more familiar ones.

\section{Questionnaire I}

Demographic information: A demographic questionnaire requesıed information concerning respondent's age, marital status, type of household, number of children (for the matching procedure), chronic diseases and medication use (possible exclusion criteria), and alcohol use, smoking habits, and participation in active sports (possible confounders of hormone concentrations).

Perceived Stress: Subjects were selected according to their perceived stress level. Perceived stress was measured by means of the Perceived Stress Scale (PSS: Cohen et al., 1983). The PSS is a global measure of the degree to which situations in one's life are appraised as stressful. ltems were designed to tap the extent to which individuals feel their life to be unpredictable, uncontrollable, and overloaded. These themes are important components of the experience of stress (Averill, 1973; Cohen, 1978; Seligman, 1975). The PSS measures cognitions and emotions relating to general stress levels rather than specific events or situations (see Table 2.2.). The items are rated on a 5-point frequency scale, ranging from 'never' $(0)$ to 'very often' (4). A total score is obtained by reversing the scoring on the positive items and then summing across the 10 items.

Table 2.2. Items Perceived Stress Scale.

1. In the last month, how often have you been upset because of something that happened unexpectedly?

2. In the last month, how often have you felt that you were unable to control the important things in your life?

3. In the last month, how often have you felt nervous and 'stressed'?

4. In the last month, how often have you felt confident about your ability to handle your personal problems?

5. In the last month, how often have you felt that things were going your way?

6. In the last month, how often have you found that you could not cope with all the things that you had to do?

7. In the last month, how often have you been able to control irritations in your life?

8. In the last month, how often have you felt that you were on top of things?

9. In the last month, how often have you been angered because of things that happened that were outside of your control?

10. In the last month, how often have you felt difficulties were piling up so high that you could not overcome them? 
We used the 10 item version of the scale (PSS10) because it has been shown to have as good or better psychometric properties than the original 14 item version (Cohen \& Williamson, 1988). Research by Cohen and others showed acceptable levels of validity and reliability. See Appendix I for the reliability and validity of the PSS in the present sample.

Coping: Coping conceptualized as a fairly stable characteristic ("coping style') was assessed with the 47-item Utrecht Coping List (UCL), developed for the Dutch population by' Schreurs et al. (Schreurs \& van de Willige, 1988). The seven factor-analytically derived subscales are: 'active problem solving', 'palliative responding', 'avoidance', 'seeking social support', 'depressive reaction', 'expression of emotions', and. 'comforting cognitions'.

Psychological and somatic complaints: The Symptom Checklist (SCL-90; Derogatis, Lipman, \& Cori, 1973) is a 90-item self-report questionnaire developed for measuring psychopathology in ambulatory psychiatric patients, but also in community samples cq non-patients. The questionnaire was adapted and translated into Dutch by Arrindell and Ettema (1981; 1986) and measures, on a five-point scale, both somatic and psychological complaints experienced during the last week. The instrument consists of eight subscales: (1) sleeping problems, (2) hostility, (3) depression, (4) somatic complaints, (5) distrust and interpersonal sensitivity, (6) insufficiency of thought and performance, (7) agoraphobia, and (8) anxiety. The SCL90 total score can be interpreted as an indication of the general level of psychological and/or somatic dysfunctioning during the past week. Reliability and validity data for the Dutch SCL-90 have been reported by Arrindell and Ettema (1986) and by Koeter et al. (1988).

Psychosomatic symptoms: The revised version of the SUNYA Psychosomatic Symptom Checklist (PSC; Attanasio, Andrasik, Blanchard, \& Arena, 1984) contains 17 common psychosomatic complaints, lie headaches, backaches and nausea. Subjects rate each item on frequency $(O=$ not, $4=$ occurs daily) and intensity $(0=$ not a problem, 4 = extremely bothersome) of occurrence, using a five point scale. A Total. score, reflecting the overall level of psychosomatic distress, is obtained by summing the cross-products of each item's frequency by intensity. Factor analyses of PSC data. obtained from college students revealed one general psychosomatic distress factor with little overlap with other commonly used measures of psychological distress such as the Beck depression Inventory or the State-Trait Anxiety Inventory (Attanasio et: al., 1984).

\section{Questionnaire II}

Life events: Life events were recorded with the questionnaire form of the List of Threatening Experiences (LTE.Q: Brugha, Bebbington, Tennant, \& Hurry, 1985). Subjects were asked about the occurrence of 12 categories of events (e.g. death of a partner, child, parent: got divorced, unemployed) during the last year. This list of event categories originated from a large series of life events sampled in a UK community epidemiological survey and in psychiatric outpatients with affective disorders (Bebbington, Tennant, \& Hurry, 1981). Each event in the original list was. rated for long-term contextual threat, according to the methods of Brown and Harris (1978). Reliability and validity data for the LTE-Q have been reported by Brugha and Conroy (1985) and Brugha and Cragg (1990).

Long-rerm Difficulties: Chronic stress was assessed with the Long-term Difficulties Questionnaire (LLM; Hendriks, Ormel, \& van de Willige, 1990; Ormel, 
1985). This inventory is a Dutch adaptation of the Biographical Problem Inventory List (BIOPRO; Hosman, 1983) and focuses on chronic and environmental stress, including problems in relation to work and study, housing, physical environment, leisure, finance and social relationships (partner, family, friends, neighbours). Of the original 32 items, 16 items concerning health were omitted, a question about leisure activities was added. Subjects rate each item on a four-point intensity scale with the anchors (1) none, (2) some, (3) quite, and (4) serious (difficulties). A total score is obtained by summing across all items.

Personality: Personality was assessed with the Dutch abridged MMPI (NVM; Luteijn \& Kok, 1985). This questionnaire consists of 83 items forming 5 scales: Negativism, Somatization. Timidity, Serious Psychopathology and Extraversion. Reliability and validity of the NVM have been described by Luteijn and Kok (1985).

Depression: Depression as a complex of symptoms was assessed with the validated Dutch translation of the Self-Rating Depression Scale (SRDS: Dijkstra 1974: Zung, 1965).

Anxiety: Trait anxiety was measured with the validated Dutch version of the State-Trait Anxiety Inventory (ZBV; van der Ploeg, Defares, \& Spielberger, 1980)

Anger: Trait anger was measured with the validated Dutch version of the Spielberger Trait Anger Scale (ZAV; van der Ploeg, Defares, \& Spielberger, 1982). This questionnaire consists of two subscales: 'anger-temperament' (the general disposition of experiencing anger and giving voice to it) and 'anger-reaction' (the disposition of expressing anger, for instance when provocated or criticized).

\section{THE EXPERIENCE SAMPLING METHOD}

The Experience Sampling Method (ESM) was designed to study the subjective, everyday experiences of people in their natural environments. Typically, an electronic signaling device is used to alert subjects to fill in a self-report questionnaire at preselected but randomized time-points, providing information about an individual's mental status or symptoms within the context and flow of experience. The self-reports request a range of information about subjects' current thoughts, moods, complaints, activities, and physical and social context. Signaling devices (e.g. pagers, wristwatch terminals), schedules of signals (e.g. random, fixed or fixed-random time-sampling; event-sampling) and ESM self-report forms may vary depending on the particular objects and goals of the study (see Csikszentmihalyi \& Larson, 1987; Delespaul, 1992).

During the last two decades, a large number of researchers have contributed, often independently, to the development of the Experience Sampling Method. One of the earliest lines of investigation under the name of ESM was started at the University of Chicago in 1975 by Czikszentimihalyi and associates. They obtained data on daily activities and experiences of adolescents (Csikszentmihalyi \& Larson, 1984). Similar techniques were developed or adapted by Hurlburt (e.g. Hurlburt, 1990) for the study of thought content, by Klinger et al. (1980) who studied the stream of conscious thought in daily life, by Massimini et al. (e.g. Massimini, Csiksentmihalyi, \& Carli, 1987 ) for the study of optimal experience, and by deVries and associates for the study of psychopathology (de Vries, 1987; de Vries, 1992). Many others could be added to this list. (see for a review Delespaul, 1995). The research group in Maastricht has studied a range of psychiatric disorders: schizophrenia (e.g. de Vries \& Delespaul, 1992; Delespaul, 1995), anxiety (e.g. Dijkman-Caes \& deVries, 1991), depression 
(Kraan, Meertens, Hilwig, Volovics, Dijkman-Caes, \&. Portegijs, 1992; van Diest, 1992), and somatization. They have also used ESM to study acute stress (Nicolson et al., 1992), pain (Lousberg, Schmidt, Groenman, Vendrig, \& Dijkman-Caes, 1995), and addiction (Kaplan, 1992).

The above indicates that ESM can be used with a variety of populations, provided that the research subjects can read and write and that a viable research alliance can be established. In spite of the success of ESM in describing mental disorders in context, there are limitations. Difficulties have been encountered in sampling elderly subjects with dementia, and individuals with acute and severe depression.

Early research focused on methodological aspects of ESM such as reactivity effects, self-selection biases, compliance, and validity and reliability of self-reports, and provided important information on the reliability and validity of ESM data (e.g. Csikszentmihalyi \& Larson, 1987; Delespaul, 1995; Hormuth, 1986). These aspects will continue to be of importance in every particular ESM study conducted. Controls and checks on the subjects should be built into the Experience Sampling designs, like checking on missed signals, the timeliness of responses (does the subject respond to the signal on time?) and on the influence of the method on the objective and subjective experiences of the subjects (Are the subject's objective circumstances influenced by participation? Is the subjective perception of a situation influenced by the method?) (see 'compliance and reactivity issues' section).,

\section{Description of the ESM instrument}

The ESM booklets used in the current study consisted of forms to be completed following each signal ('beep level' forms) and forms to be completed at the beginning or the end of each day ('day level' forms). These forms will be described separately below.

\section{ESM form: beep level}

At every 'beep' subjects completed a self-report form (see Appendix II), containing several questions about the subject's mental state and physical and social. context. Subjects were asked what they were brooding about, what they were doing, and where and with whom they were at the moment of the beep. Daily stress or hassles were assessed with the open question 'Did any stressful event or situation take place between the present and the previous beep?'. Thus, the assessment of daily events had a more retrospective character than the other questions at the beeplevel. The ESM forms also included Likert scales (from I 'not at all' to 7 'very much') for the evaluation of thoughts, mood, physical well-being, individually defined psychosomatic complaints, current activity, and stressful events. Activities were evaluated on the dimensions: enjoyed doing, skill, effort, and challenge. The following appraisais of stressful events were also rated on Likert scales: unpleasantness, importance, predictability, controllability, and frequency of prior occurrence. Subjects also reported when the event started and when it ended, or that the event was still going on. This information gives an indication of the duration of the event and the time elapsed since termination. Additional items were included to help in the interpretation of the hormonal data: occurrence and intensity of physical exertion and any ingestion of food, coffee, alcohol, cigarettes or medication which may have taken. place since the last signal. 


\section{ESM form: day level}

At the end of each Experience Sampling day, subjects were asked to rate (Likert-scales) the extent to which they were bothered by their individual complaint that day, how stressful the day had been and how their general mood had been. Also included was a short checklist of 15 events (Daily Stress Scale; Bolger et al., 1989a). Except for transportation and financial problems, this list consisted entirely of items concerning demands (e.g. a lot of work at home, at work, a lot of demands made by family) or interpersonal conflicts (e.g. argument with spouse, child, colleague). Subjects indicated if an event on the list had occurred to them that day and, if so, what the impact of that event had been (from 1 'not at all bothersome' to 7 'extremely bothersome').

Additional information was obtained each day immediately after waking (sleep quality, sleep pattern previous night) and again before going to bed (effects of participation in the study, timing of meals, work and time to bed).

\section{ESM procedures}

Most participants find the ESM rewarding but also taxing. A crucial part of the Experience Sampling process is therefore the creation of an alliance and a mutual understanding about the research procedures and aims of the study. Cooperation and compliance depend on this alliance.

During an initial semi-standardized interview one or two days before the start of ESM, called the 'briefing', the purpose of the ESM research and the additional saliva and urine sampling was explained and informed consent was obtained. We communicated our interest in learning about their daily experiences. At this time we also gathered additional information concerning possible recent diseases and related medication use, and individualized complaints were chosen. Subjects were instructed to select one complaint (either of a psychological or somatic nature) that was important to them and that occurred rather frequently. Instructions about the use of the watch and the ESM booklets were given. Subjects were instructed to fill out selfreports as soon as possible after a signal (within 15 minutes). Anticipated difficulties in the use of ESM (e.g. driving a car, attending a meeting) and possible solutions to it were discussed. The subjects were given control of the signaling device only in that they could turn it off if they did not want to be disturbed (e.g. during a daytime nap). As a reminder, instructions were also printed on the inside cover of the booklet. The subjects were discouraged from showing their completed booklets to others and were asked not to look back through previously completed forms.

The ESM period encompassed five days, from Thursday to Monday (three workdays and two weekend days). Tuesdays and Wednesdays were reserved for (de)briefing sessions. On ESM days, subjects were signaled 10 times a day between 7:30 a.m. and 10:30 p.m., with an average interval of 90 minutes between consecutive 'beeps'. This was done according to a preprogrammed schedule chosen to maximize interpretability of cortisol values without being predictable by the subject. The 'beeps' were randomly distributed around fixed time points (e.g. 8:15 a.m., 9:45 a.m., and so on throughout the day), with a maximum deviation of $20 \mathrm{~min}$. After each signal subjects filled in an ES form, which took about 2 minutes, and collected a saliva sample (see section below).

One or two days after the five days of ESM, a final 'debriefing' session took place, which started with the Stress Inducing Speech Task (SIST; see 'Stress Inducing Speech Task' section). After the stress task, ESM booklets were checked. Subjects 
were asked to explain reasons for any missing responses and to clarify illegible or unclear responses to facilitate later coding. The 'debriefing' session was closed with a structured interview about the possible impact of the study on the daily life of the subject. Subjects received a small financial compensation (fl. 20,-) as well as an information booklet about stress. 'Briefing' and 'debriefing' sessions each took about one hour. An individual subjects was briefed and debriefed by the same person.

\section{SALIVA SAMPLING}

Cortisol levels were determined in saliva. Cortisol in saliva is a reliable and valid indicator of the free cortisol in plasma, which is considered to be the biologically active hormone, and cortisol concentrations are independent of the flow rate of saliva (Vining, McGinley, \& Symons, 1983). The rate of equilibrium of cortisol between saliva and blood is very fast. Cortisol responds within less than five minutes to increases in stress (Vining et al., 1983) and has a half life in blood or saliva of about an hour (Fredrikson, Sundin, \& Frankenhaeuser, 1985). These characteristics of saliva cortisol also provide an appropriate time frame for studying its relationship to experiences in daily life. It is assumed that a sample, collected within 20 minutes after signaling, reflects adrenal cortical activity during the time period between that last signal and the previous signal. Cortisol, in comparison to other stress-labile hormones (e.g. the catecholamines, growth hormone, prolactin, testosterone), shows the most specific relationship to subjective distress, in contrast to general arousal, effort, or trauma (Delahunt \& Mellsop, 1987; Lundberg \& Frankenhaeuser, 1980; Rose, 1984). The ability to measure cortisol reliably in saliva instead of blood, makes repeated, stress-free sampling possible. Measuring cortisol in blood is usually stressful in itself (Rose \& Hurst, 1975) and interrupts ongoing activities, which makes repeated measurement extremely difficult, especially over longer time periods.

Subjects collected saliva by holding a dental cotton roll in the mouth for 1 to 2 minutes. Saturated dental rolls were then placed in a capped plastic vial (Salivette; Sarstedt). which subjects labeled with the time of day and then stored with the ESM booklet in a specially designed wallet. This wallet contained ten Salivette vials and an ESM booklet with ten seli-report forms. Subjects placed their saliva samples in their freezer at the end of each day. We have found no differences in cortisol levels in uncentrifuged samples frozen immediately or kept at room temperature for two days (Nicolson et al., 1992); others report no differences up to two to four weeks (Kahn, Rubinow, Davis, 1988; Kirschbaum \& Hel!hammer, 1989). Uncentrifuged samples were kept at a temperature of $-20 \mathrm{C}$ until they were analyzed. A maximum of 50 samples per subject was thus obtained.

Analysis Salivary cortisol levels were determined in duplicate by direct. radioimmunoassay (Ansseau, Sulon, Doumont, Cerfontaine, Legros, Sodoyez, et al., 1984), using ${ }^{125}$ I-cortisol (Farmos diagnostica, Finland) and antiserum made against the 3-CMO-BSA conjugate by Dr. J. Sulon, University of Liege, Belgium. The lower detection limit of the assay was $12 \mathrm{ng} / \mathrm{dl}$, with a mean intra-assay coefficient of variation of $4.8 \%$ (range: $2.2 \%-7.5 \%$ for 4 assays). Each subject's samples were analyzed in the same assay to reduce sources of variability. 


\section{URINE SAMPLING}

Levels of the catecholamines adrenaline (A) and noradrenaline (NA) were determined in 14-hour urine. Compared to plasma levels which are highly liable with a half-life of less than three minutes, urinary levels represent estimates of sympatheticadrenomedullary activity integrated over extended time periods (usually 1 to 3 hours) and they can be used to determine long-term (14- to 24-hour) changes in levels of catecholamines (CA) (Frankenhaeuser, 1975b). The collection of urine samples is also relatively easy, noninvasive and well-suited for naturalistic field studies (Frankenhaeuser \& Gardell, 1976). Because the catecholamine in urine constitute a small but relatively constant fraction of liberated amines in the body, the direction of change or the relative levels are meaningful, but absolute numbers have limited value. NA levels are somewhat difficult to interpret because NA is secreted by both nerves and the adrenal medulla and is also subject to rapid neuronal reuptake; A levels give a more reliable estimate of adrenal medullary activity because the adrenal medulla is the sole source of circulating A (Frankenhaeuser, 1975b). The 14-hour samples collected in this study were chosen to reduce the effects of variation between subjects with different circadian rhythms and to minimize the biasing effects of idiosyncratic events occurring during the day. For example, a person who runs up the stairs will probably show elevated CA levels at the next voiding, but such one-time elevations should be mitigated in a 14-hour collection. Overnight CA excretions can be regarded as baseline levels and are therefore useful for assessing physiological changes associated with chronic stress (Baum, Lundbeg, Grunberg, Singer, \& Gatchel, 1985).

Subjects collected two overnight urine samples, one after a workday (Thursday to Friday) and one after a weekend day (Sunday to Monday). Urine was collected by the subject from 6.00 p.m. to $8.00 \mathrm{a} . \mathrm{m}$. the following day ( 14 hours), in 2 liter urine containers (Sarstedt). Subjects emptied their bladder in the toilet at 6.00 p.m. and from that moment collected subsequent urine in the container. At $8.00 \mathrm{a} . \mathrm{m}$. the next day they emptied their bladder for the last time in the container. Subjects kept their container in the refrigerator during the collection period. The urine samples were collected by the researcher or research-assistant at $9.00 \mathrm{a} . \mathrm{m}$. at the workplace of the subject. From the time the subject left home until collection of the container at the workplace, samples were kept in an insulated bag with a cooling element. Immediately after the collection of the container we added HCL $(37 \%)$ to the total volume of urine until a $\mathrm{pH}$ of $<3$ was reached, to prevent oxidation. From the total volume, $10 \mathrm{ml}$. samples were extracted and immediately frozen at a temperature of -20 $\mathrm{C}$ until analyses.

Analysis Adrenaline and noradrenaline excretion rates were determined by means of high-pressure liquid chromatography (HPLC) with electrochemical detection (Kissinger, Riggin, Alcorn, \& Rau, 1975). ${ }^{\text {. CA levels were corrected for }}$ creatinine excretion $(g / l)$ and expressed in $\mu \mathrm{g} / \mathrm{g}$ of creatinine. Nine samples with creatinine levels below $0.60 \mathrm{~g} / 1$ were considered unreliable and were not used in the analysis. Due to practical reasons, three subjects did not collect any urine, and another four subjects collected urine only once.

Analyses of adrenaline and noradrenaline were performed under the supervision of Dr. Rahman and Dr. Duvivier, University Hospital of Liege, medical chemistry service, Belgium. 


\section{STRESS INDUCING SPEECH TASK (SIST)}

A disadvantage of conducting a study in the natural environment is the idiosyncratic nature of events and activities and the heterogeneous stressful circumstances subjects encounter in daily life. Individuals' responses to these varying circumstances are difficult to compare. Therefore, all subjects in this study were exposed to a standard stress test in an experimental context in order to get more insight into individual differences in neuroendocrine responses. Additionally, we investigated whether cortisol activity as assessed in the laboratory generalized to cortisol activity as measured in the field. An important consideration was that the information provided by the standardized stressor should be relevant for the understanding of the endocrine response to stimuli in claily life; in other words, the stressor should have 'ecological validity'. We chose to use at speech task, similar to that described by Steiner and associates (1988).

The test took place at the beginning of the ESM debriefing session, between $11 \mathrm{a} . \mathrm{m}$. and $13.00 \mathrm{p} . \mathrm{m}$. This test was unanticipated by the subjects, who believed that the final session would only be used for 'debriefing' the ESM study. The experimenter (blind to whether a subject belonged to the high stress or the low stress group) read the written SIST instructions aloud for the subject. The instructions were to prepare (10 minutes) and present ( 5 minutes) a videotaped speech about their personal strengths and weaknesses for later evaluation by a team of psychologists. After the 10 minutes of preparation, subjects received a signal, the video recorder was started, and the subjects delivered their presentation while looking directly into the camera. Afterwards, subjects relaxed for 15 minutes in neutral activities (e.g. reading magazines). At 4 time points subjects filled in a short mood questionnaire and provided a saliva sample: (T1) upon arrival, (T2) after the 10 minutes of preparation, (T3) after the 5-minute presentation, and (T4) after 15 minutes of relaxation. During the course of the experiment we decided to lengthen the recovery period to get a clearer picture of the cortisol response profile. For 49 subjects, a fifth saliva sample (T5) was taken on average 50 minutes after the first assessment. This way we could look at endocrine responses during the anticipation, reactivity and recovery phase of the stressor.

\section{CODING OF ESM MEASURES}

Information about activities ('What were you doing?'), location ('Where were you?'), social context ('With whom were you?'), and stressful events ('Did any stressful experience or event occur?') was obtained by open-ended questions. In order to be able to identify, for instance, typical patterns of time use or stressful daily events, the total range of activities and daily events has to be reduced. The specific method used for coding these experiences is very important because it entails decisions about which events or experiences can be differentiated and which will be combined. An important question to be answered before using these data in further analyses concerns the reliability of the coding procedure. This was assessed by calculating the agreement between two independent observers on the coding of all variables to be used in the analysis (see Appendix III). 


\section{Coding procedure}

In the literature are examples of ESM studies where responses to open-ended questions were coded by the subjects themselves (e.g. Brandstatter, 1983), by other participants (e.g. Campbell et al., 1991) or by trained coders (e.g. Wong \& Csikszentmihalyi, 1991). In this study we used trained coders for several reasons: first, if coded by subjects themselves they would need more time to fill out the ESM form thereby endangering study compliance (e.g. activities were coded under 57 fine grained but reliable categories which would take up too much time, and too many instructions needed), and second, because we wanted to start without preconceived notions of what a 'stressful event' might be. Pre-coded responses ('menus') require limited numbers of simple categories which are predefined to be of importance.

Responses were coded by two independent raters, according to an established coding system. The two raters first coded three ESM booklets and discussed differences in the results to clarify problematic categories and definitions. Next, they coded a subset of ESM booklets (five booklets for each of 27 subjects) without discussing them with each other. These codings were used for the reliability analysis. All data actually used in further analyses (beyond the reliability analysis) were coded by both raters with differences being replaced by consensus ratings.

\section{Coding of activities, location and social context}

Classification of activities was based on the list of events in the International Time Budget Study (Szalai, 1972). This list contained 96 codes which were later reduced to 47 codes for use in several Experience Sampling Method studies at the University of Limburg. In the current analyses, the 47 activities were collapsed into 8 broader categories: inactivity/rest, work (which also included volunteer work, regular classes, homework, special courses, and study breaks), household/maintenance (also including child care, care to adults, shopping and general services, personal care services, personal hygiene and personal medical services), leisure (including activities like sports, hobbies, attending a film, reading a book, or watching television), social interaction (conversations, visits to or by friends, parties etc.), meals (including regulai meals, special meals and snacks), transport, and other activities (including personal mental or emotional activities, political and civic activities, and other activities not falling in one of the other activities).

With respect to location ('Where are you?') and social context ('Are you together with someone?'), we again used the standard coding manual developed for ESM studies at the University of Limburg, later collapsing the specific codes into six broader categories: at home, nefwork (homes of family, friends, and acquaintances). work, public places (street, shop, cafe, sports hall, health care settings), transport (in car, bus, train, airplane, on bike), and other places.

Responses to the question 'Are you together with someone?' were coded and then collapsed into 7 categories: alone, household members (including partner, children, parents, brothers and sisters and other household members), non-resident family, friends, colleagues, neighbours/acquaintances, and strangers.

Responses were coded missing/invalid if a question was not filled in (missing) or if a response was not legible or invalid (e.g. filled in too late); responses were rated as can't code if something valid had been written down, but it was not clear which category did apply (for instance when important background information is missing). This category can give us a good idea about the quality of the coding system. 


\section{Coding of stressful events}

In contrast to the domains described above, there was no pre-existing coding system for stressful events. Until now, there have been few studies investigating the effects of different types of minor stressors on health and well-being (Bolger et al., $1989 \mathrm{a} ;$ Stone, 1987). It is common practice to aggregate daily stressors into a summary measure for analytic purposes. This may, however, conceal important variation in the microprocesses underlying somatic and psychological well-being. For this reason, we differentiated events reported according to the following domains: context of the event, who was involved in the event (if someone was involved), whether the event was internal or external (observable or not), and whether the event entailed a social interaction or a task demand.

The context categories were based largely on the ESM activity codes, which was expanded to include the categories personal heaith-somatic and personal healthpsychological. We wanted to be able to identify events conceming health, especiaily mental health, because of their possible confounding to psychiatric and somatic states.

The context domain contained the following 8 categories: work (events concerning boss, supervisor, co-worker, clients, general happenings concerning self at work, events concerning study etc.), network (events concerning spouse, partner, children, relatives, friends, neighbours or acquaintances), household/financial (events concerning general housework, family related duties, errands, loans, selling, buying. financial problems etc.), leisure (events concerning hobbies, sports, outings, vacation etc.), personal health-somatic (events concerning illness, injury of the subject himself), personal health-psychological (e.g. nerves, anxiety, worries about health), transport (e.g. missed bus, traffic jam, unusual traffic), and an 'other' category (minor irritants like noise, the weather, broken glass, ESM. cold shower. combinations of different events etc.).

The coding of events under a 'social interaction' domain and a 'task demands' domain paralleled the checklist we used in the ESM booklet at the end of the day, which consisted mainly of events concerning task demands or interpersonal conflicts. A diary study on the influence of daily stress on mental health, based on a community sample of 166 married couples, showed that interpersonal conflicts and tensions were by far the most distressing events (Bolger et al., 1989a). Task demands were also of interest to us because overload is an important theme in a sample of working people. These people have in general more than one social role (family, work eic.) and are therefore more exposed to various role related demands. A study by Stone (1987) showed greater psychological impact of work-related demands.

With respect to the 'social interaction' domain, responses were coded into three categories: not applicable (event is not a social interaction); negative interaction (e.g. argument, conflict) or discussion/conversation.

The 'task demands' domain consisted of five categories: not applicable (clearly no demands involved); problematic task (e.g. difficulties, problems with..); $a$ lot of work/tasks or extra work/tasks; time pressure, deadlines; failure at task.

For coding who was involved in the event we used the same categories as described under the ESM social context domain: alone, household members, nonresident family, friends, colleagues, neighbours/acquaintances and strangers.

Events were coded as external or internal in an effort to separate the more or less 'observable' events from entirely 'subjective' events. Criteria for coding events as external were: 1) they should be observable (i.e. theoretically verifiable) and 2) 
they should have happened in the interval since the last beep. The opposite applies to internal events: they are not observable. Examples of internal events are introspections, worries, internal evaluations, anticipations. The internal/external distinction does not refer to responsibility for causation.

A 'can't code' category was included in every domain for event descriptions which could not be classified due to the absence of sufficient information.

\section{Interrater agreement}

Before using the qualitative information in further analyses, we have to investigate if the coding system we used is a useful one. Generality is important here in demonstrating that the obtained ratings are not the idiosyncratic results of one rater's subjective judgment (Tinsley \& Weiss, 1975). Regarding the interrater agreement we are interested in the following question: Is it possible to classify the information under the various categories and can we do this reliably? Information gathered under the activity, location and company domains is clear and straightforward. The categories belonging to these domains are also very concrete and well-defined. Problems should not be expected here. But with regard to the coding domains for stressful events, we can expect some variance because of interpretation differences and difficulties. Additionally, the subject's description of the event may be lacking in the types of detail necessary to classify it into one of several related categories. In Appendix III we describe how the interrater agreement was calculated (Cohen's unweighted Kappa) and what the Kappa's for the different codes were.

With respect to the reliability of coded responses, we can conclude that the open-ended responses could be classified very reliably (see Appendix III). Even in categories with only a small number of observations the degree of agreement was generally substantial. We did find that some specific event domains were more difficult to rate. This was the case for the domains 'internal/external', 'social interaction' and 'task demands', where Kappa's varied from moderate to almost perfect $(.46-.89)$, but where a substantial number of responses fell into the 'can't code' category. This means that the obtained information was often difficult to interpret, leading to a substantial loss of information. Because these analyses were done after the first 27 subjects were sampled, more attention was paid in subsequent debriefing sessions to the clarification of reported information on stressful events, in order to keep loss of information due to uncodable responses to a minimum.

\section{DATA ANALYSES}

The ESM dataset is characterized by a large number of repeated observations ('beeps') on a relatively small number of individuals, with a large number of variables measured at each beep. As a consequence, Experience Sampling data have a complexity which often make traditional analytic approaches unsatisfactory. Depending on the type of research question, ESM data can be analyzed either at the subject level, at the beep level, or at both levels at the same time (Larson \& Delespaul, 1992). At the beep level, the repeated measurements are used as the unit of analysis, with a maximum of 50 measurements per subject in the current study. At the subject level, the individual is used as the unit of analysis, including one time measurements as well as aggregated data. One time measurements are, for instance, scores from crosssectional questionnaires (e.g. perceived stress score) and briefing and debriefing data. 
Aggregated data are, for instance, mean mood or event scores per subject, or the percentage of total beeps a subject spent on a certain activity (e.g. work).

Although in certain cases beep level analyses may be the only possible approach (e.g. when one is dealing with relatively rarely occurring categories of experience like going to a party'), there are some important problems that are inherent in such an approach (Larson \& Delespaul, 1992). Generally, when beep level analyses are carried out, the number of units in the analyses is inflated and dependency exists between adjacent data points. Significance tests, therefore, do not provide a valid estimate of probabilities. In addition, beep level analyses give: equal weight to all instances of a category of experience. This means that subjects with more valid responses in that category are given more weight and will have more influence: on the outcome. These problems of inflated number of units, dependency between observations, and unequal weighting can be diminished by employing subject level analysis, where aggregate scores are computed for each individual and the subject is used as the unit of analysis (Larson \& Delespaul, 1992). Aggregated scores, which are based on repeated measurements, also have the advantage of increasing the reliability of measurement. Therefore, this is an appropriate approach when research guestions about the chronic level of variables are of interest (see for example Chapter 3 and Chapter 5).

Nonetheless, subject level analyses are also subject to several pitfalls. Besides the fact that they ignore the rich data base available, this approach can introduce aggregation bias, which occurs when unmeasured or unspecified variables exist that distort the causal relationship between variables (e.g. "when heterogeneous groups [or sub-units] are combined into a single group [unit], and the combined group analysis yield conclusions that are misleading about the sub-units") (Jaccard \& Wan, 1993, p.43). Another restriction on the use of subject level analysis is that a substantial number of subjects with at least more than one observation is required when one is interested in making comparisons across several situations (e.g. work. home; work days, weekend days) using a repeated measures analysis of variance, since this technique does not tolerate missing data. Most like $l y$, a number of subjects will not have enough observations in each situation, leading to a substantial loss of information. Two other important shortcomings of this technique are: it may provide misleading estimates of the effects of an independent variable (data are treated as if there were an equal number of observations for each level of the independent variable, but in many occasions this will not be the case), and it ignores the fact that many of the independent variables are correlated in ESM: the data are treated as if the variables are orthogonal. For example, certain stressful events (e.g. network events) tend to occur only in certain locations (e.g. at home rather than at work).

Besides the problems described above, aggregated data obscures the interplay between experientiai and psychological or physiological states and processes which momentary measures try to capture. To gain more insight into the relationship between perceived stress, stressful events, cortisol and / or mood states over time, we will have to apply another analytic method. A more effective approach for analyzing both subject and beep level data is by using random-coefficient regression models (Bryk \& Raudenbush, 1992; Goldstein, 1987). This approach is a variant of the multiple linear regression model, applicable for data with a. hierarchical nesting structure as is the case with ESMI data. This approach can account for the dependency of data within a subject and for residual dependency, it can deal with the problem of missing data, and it allows for individual differences in intercepts, slopes, and error structures. Additionally, by using random regression models, the outcomes 
at the beep level can be modeled as a function of both the beep and the subject level variables. The model allows us to obtain estimates of, for example, cortisol reactivity to events both as a function of psychological state (e.g. mood state) and trait (e.g. level of perceived stress) variables over all subjects and for each individual. The number of observations need not be the same for all persons, and observations may be unequally spaced over the time interval. In the present study, random regression models have been applied in Chapter 4, Chapter 6, and Chapter 7. Greater detail on theoretical and technical aspects of this analytic approach can be found under the method sections of these chapters.

\section{COMPLIANCE AND REACTIVITY ISSUES}

The general purpose of ESM is to study the subjective experiences of persons interacting in their natural environments. This means that the variables measured should be a representative sample of those in the person's environment and that they should be measured with as little distortion in retrospective recall as possible. ESM's primary weaknesses lie mainly in the responsibility given to the subject in collecting not only subjective data, such as feelings and appraisals, but also objective ones like the description of situations. Therefore it is very important to check on compliance of subjects (e.g. How many responses are missing and for what reason?), timeliness of responses (Are responses made on time?), and on effects of ESM on the objective and subjective experiences of the subjects (e.g. Does ESM influence subject's choice of activities, mood?). Because: ESM asks more of its participants than more conventional research methods it is also important to check on how taxing the task of being a subject was fellt to be.

\section{Compliance of subjects}

Particularly for people with relatively high levels of appraised stress, it is important to determine if participants were motivated and able to fill in a sufficient number of ESM booklets within an acceptable time limit after the beep occurred. The choice for this time limit is rather arbitrary; a compromise has to be found between leaving enough time for the subject to comply to his tasks (filling in the booklet and taking a saliva sample) and keeping the delay between the beep and response time as smali as possible.

In this study, a response was considered valid and maintained for analyses when a response was given within 5 minutes before and 20 minutes after the beep occurred. Only subjects with at least 20 valid responses and with not a single day completely without valid responses were included in the analyses. In our sample, 4 subjects did not meet our criteria. Two subjects in the low stress group completed a sufficient number of valid beeps, but both missed a whole day (one subject went abroad for a day with a business client). In the high stress group, one subject had only 16 valid response, and another subject missed all responses at the weekend because he was ill. Analyses were based on 88 subjects. 
Table 2.3. Delay between beep and response time.

ESM formats Saliva Samples

Nr. of responses after: $1 \mathrm{~min}$.

$5 \mathrm{~min}$.

$477 \quad 11 \%$

$168238 \%$

$10 \mathrm{~min}$.

$249857 \%$

$303769 \%$

$15 \mathrm{~min}$.

$328575 \%$

$346479 \%$

$20 \mathrm{~min}$.

$352980 \%$

$360382 \%$

$363283 \%$

$365683 \%$

\begin{tabular}{llllll}
\hline Invalid & 144 & $3 \%$ & 107 & $2 \%$ \\
Missing & 624 & $14 \%$ & 637 & $15 \%$ \\
\hline
\end{tabular}

Total number of responses

4400

4400

Of all programmed beeps, $83 \%$ were responded to within the time limit of 5 minutes before and 20 minutes after the beep occurred (see Table 2.3). For all valid responses the mean delay between beep and response time was 5 minutes $(s, d=4.0)$, and in $3 \%$ of all cases the delay between beep and response time was larger than -5 and +20 minutes (invalid responses). Finally, $14 \%$ of all beeps were not recorded. Although the saliva samples were not included in our 'validity' criteria, we also checked on the compliance of saliva samples. Here we found almost the same distribution of valid, invalid and missing responses: $83 \%$ valid responses, $2 \%$ invalid responses and 15\% missing responses. Mean delay between beep and response time for saliva samples was 3 minutes $(s . d .=4.8)$.

The number of valid, invalid and missing responses in the low versus high stress groups are shown in Table 2.4. The mean number of valid responses completed per subject was 41 . In the low stress group subjects completed an average of 42 (s.d.= 5.7) valid ESM responses compared to $40($ s.d. $=6.8$ ) in the high stress group. The difference between groups was not significant (Mann-Whitney U test; two-sided, $p=.1)$. The mean number of saliva samples was $43(s . d .=5.8)$ in the low stress group and $40($ s.d. $=6.6)$ in the high stress group (Mann-Whitney $U$ test; two-sided, $p=.1$ ).

Table 2.4. Number of valid, invalid, and missing responses in 'High' and 'Low' stress, groups.

\begin{tabular}{|c|c|c|c|c|}
\hline \multirow[b]{2}{*}{ Responses } & \multicolumn{2}{|c|}{$\begin{array}{l}\text { Low Stress Group } \\
\qquad \mathrm{n}=46\end{array}$} & \multicolumn{2}{|c|}{$\begin{array}{c}\text { High Stress Group } \\
n=42\end{array}$} \\
\hline & ESM & Saliva & ESM & Saliva \\
\hline Valid (mean) & $42^{\prime}(84 \%)$ & $43(86 \%)$ & $40(80 \%)$ & $40(80 \%)$ \\
\hline Valid (total) & $1947(85 \%)$ & $1960(85 \%)$ & $1685(80 \%)$ & $1696(81 \%)$ \\
\hline Invalid (total) & $50(2 \%)$ & 32 (1\%) & $94(5 \%)$ & $75(4 \%)$ \\
\hline Missing (total) & 303. (13\%) & $308(13 \%)$ & $321(15 \%)$ & $329(16 \%)$ \\
\hline
\end{tabular}

In summary, four subjects were discarded from the study, because they did not meet the criteria of having filled out at least 20 ESM reports within a time limit of -5 and +20 minutes, and without a complete day missing. The final sample completed $83 \%$ of all possible responses within the time limit, providing an average of 41 
responses per subject. The same applied for compliance of saliva samples. The delay between beep and response time was generally small; $57 \%$ of the responses were given within 5 minutes after the beep. High stress subjects were not less compliant than low stress subjects.

\section{Reasons for missing responses}

Missing responses can be due to a variety of reasons (e.g. sleep, lack of motivation, interference of beeps with important, stressful, or private activities). For the reliability and validity of ESM, it is important to ascertain that there is no systematic underreporting of specific situations. Figure 2.1.a. displays the number of valid responses during the day. The least valid responses were obtained in the morning and late at night, with the first beep (between 8:07 and 8:30 a.m.) receiving the least valid responses $(73 \%)$. One possible reason for missing or out of range responses in the morning and evening hours was that subjects were still or already asleep. Since subjects were asked to fill out the time they woke up in the morning and went to sleep in the evening, the percentage of missing and invalid responses due to sleep could be calculated. Of all missing and invalid responses, $10.4 \%$ were known to be due to the fact that the beep occurred before the subject woke up in the morning. while $0.4 \%$ was due to going to sleep before the last beep occurred. Sleep was the reason for missing and invalid responses in $45 \%$ of all first beeps in the morning (all in the weekend). The small decrease in valid responses at beep 7 was probably related to the fact that subjects were leaving the work place and were on their way home which complicated compliance. Notes in booklets suggested this.

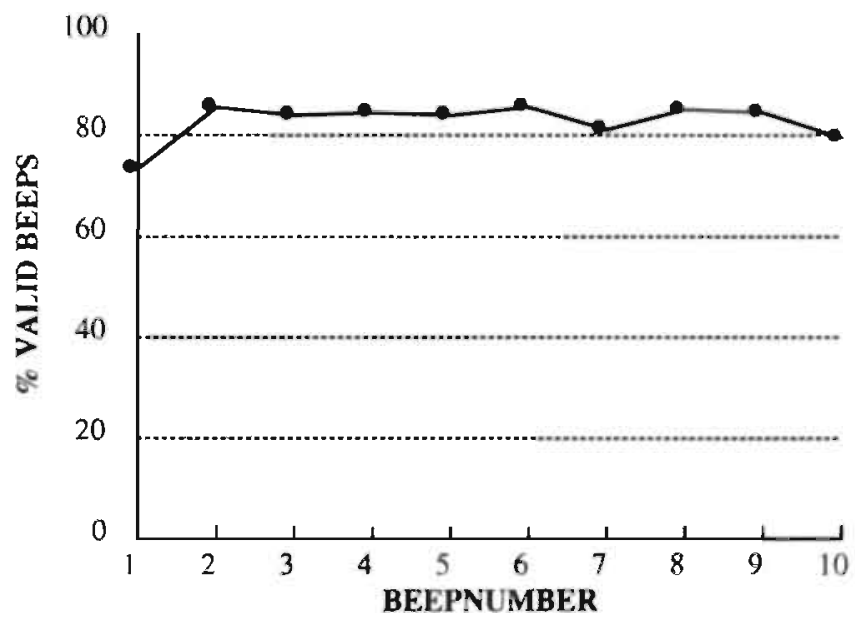

Figure 2.1.a. Percentages of valid responses during the day. 


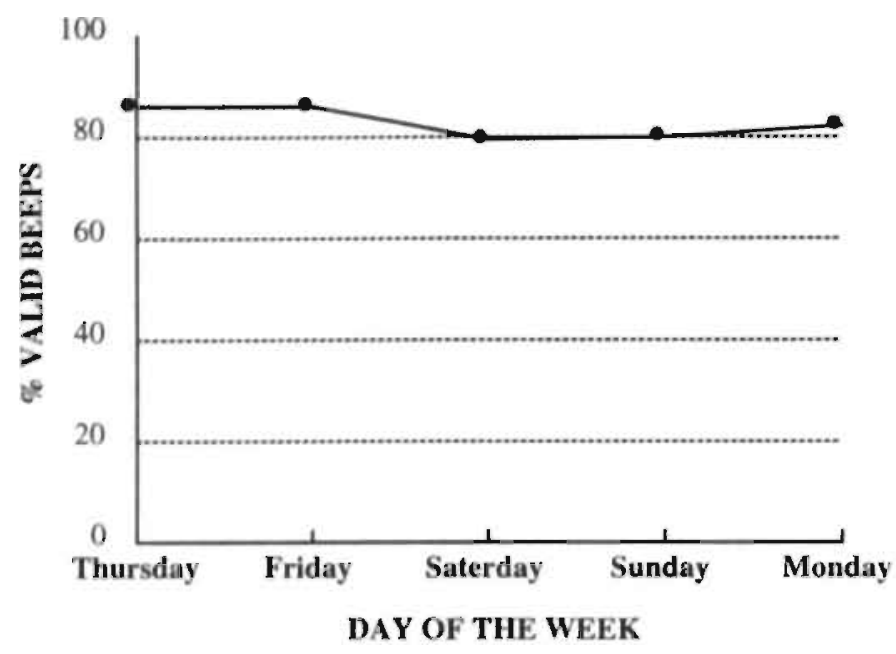

Figure 2.1.b. Percentages of valid responses, at different research days.

Fig. 2.1.b. shows that the percentage of valid responses did not vary much by research day. Although a decline in valid responses might be expected at the end of the research week because of a decrease in motivation, we found this small decline to be most prominent during the weekend. ANOVA-repeated measures over the mean number of valid responses (aggregated by subject) showed a significant withinsubject effect for 'DDay of the Week' $(F(4,344)=4.2 ; p<.01)$, but no berweensubjects effect for 'Group' (high vs. low stress) was found $(\mathrm{F}(1,86)=2.7$; ns), and no significant interaction between 'Day of the Week' and 'Group' $(F(4,344)=.4)$. Significantly more responses were missed during the weekend than during work days $(t(87)=4.4 ; \mathrm{p}<.001)$. In the weekend, $74 \%$ of all missing and invalid responses could be related to the fact that subjects were still asleep.

Notes in booklets revealed various other reasons for missing and out of range responses. Reasons mentioned relatively often were: not hearing the watch in a noisy environment (e.g. sports hall, party) or mistaking the alarm beep in the early morning for the first beep (leading to a missing response at the end of the day), problems with the watch (put off by mistake, not working properly), forgetting to take the ESM format along when leaving the house or work place, engaging in sports and other hobbies, business appointments, meetings or speeches at work, and driving a car or bicycle. In summary, the percentage of missing responses does not vary dramatically during the day and during different research days. The most important reason for missing and out of range responses in the early morning was that subjects were still asleep. The remaining missing and out of range responses were due to a variety of reasons making systematic underreporting of events unlikely.

\section{Representativeness of recorded days}

It is hoped that the five research days provide a representative sample of the subject's daily life. But, since these days were just five days in a subject's daily life, it is quite possible that they, either by chance or as a react of the procedure, differed from 'normal' days. Therefore, we asked subjects during the ESM debriefing 
interview whether the research days had been unusual in some respect, and if so, for what reason. During the ESM period, subjects also evaluated at the end of each day (just before bed time), whether that day could be considered as a normal one. This was measured with a Lickert scale, ranging from 1 ('not at all normal') to 7 ('very much normal').

Fifty one subjects $(58 \%)$ reported during the debriefing that the research days gave a good impression of their normal daily life. Eleven subjects (13\%) were more doubtful, reporting that the research days were a little bit unusual. Twenty-six subjects $(29 \%)$ ) found the research days to be unusual in some respect. No differences were found between the two subject groups. Twenty-one subjects provided explanations for what was unusual: parties, birthdays in family $(n=9)$; more or unusual work $(n=6)$; less work/stress than usual $(n=8)$; health complaints of self or family $(n=4)$; a day off on a usual work day $(n=3)$. 'Less work/stress' as an unusual factor was mentioned by six subjects from the high stress group compared to two subjects from the low stress group. At the day level, the mean rating of how normal the day had been was $4.3(\mathrm{~s} . \mathrm{d}=1.2)$ (aggregated by subject over 5 days). On the whole, then, subjects experienced their research days as normal. High stress subjects appraised research days as being a little less normal than low stress subjects (3.9 versus 4.8 . respectively; $t(84)=4.0 ; p<.001)$, but they still thought that the ESM sampling gave a good impression of their normal daily experiences.

In summary, although both subject groups reported that the research days were occasionally unusual for one reason or another, a majority indicated that the research days were representative of their normal daily routine. In the high stress group, the level of stress or work pressure experienced during the sampling period seems to be particularly salient, as this was frequently mentioned as the reason why the research days were unusual (e.g. "this week was unusual in that I had more work/fewer pressing deadlines than usual" etc.).

\section{Inconvenience and task difficulty of ESM}

To get an indication of how taxing and difficult the ESM procedure was for subjects, we asked during the debriefing interview whether ESM was 'bothersome or annoying' and whether any question in the ESM booklets was difficult to interpret or to answer. Additionally, we inquired whether they were willing to participate in an ESM study again. Subjects gave an evaluation of how disturbing individual beeps were in the ESM reports at the moment they were beeped.

Table 2.5. Annoyance with RSM as, recorded during debriefing interviews.

Low Stress Subjects High Stress Subjects

Did you find ESM

\begin{tabular}{lrr} 
'bothersome'? & $\mathrm{N}=46$ & $\mathrm{~N}=42$ \\
\hline No & $23(50 \%)$ & $21(50 \%)$ \\
A little/sometimes & $20(44 \%)$ & $14(33 \%)$ \\
Yes & $3(6 \%)$ & $7(17 \%)$ \\
\hline
\end{tabular}

As Table 2.5. shows, a vast majority of subjects (89\%) reported that ESM was either slightly or not at all bothersome. Only $6 \%$ of the low stress subjects and $17 \%$ of the high stress subjects said the method was troublesome. The differences between groups were not significant. The ten subjects who found ESM bothersome gave the 
following explanations: the filling in of ESM reports becomes particularly annoying toward the end of the sampling period (three subjects), during weekend leisure time (two subjects), or because it disrupts activities or occurs in the company of other people (five subjects).

Seventy-three percent of the subjects reported to be willing to participate another time with ESM, 14\% would be willing under certain conditions (e.g. only after some time, different questions in the ESM booklet), and $13 \%$ did not want to take part again (e.g. once is enough, too intensive, too long, too many beeps). There were no differences between the two groups in willingness to participate in a future ESM study.

In response to the question whether there were any difficult questions in the ESM forms, $72 \%$ of the subjects had no probiems at all, $19 \%$ had only minor problems, and $9 \%(n=8)$ of the subjects had problems with one or a few specific questions only (e.g. what is a stressful event?; sometimes difficult to score mood items; sometimes appraisals of activities not applicable). Again, there were no group differences.

At the beep-level, subjects rated whether the beep was disturbing on a scale from 1 'not at all' to 7 'very'. Of all valid responses, $48 \%$ were rated as 'not disturbing' (score 1) and $14 \%$ as 'disturbing' (score 5 or higher). 'High' stress subjects rated the beeps as slightly more disturbing on average than low stress subjects (mean score of 2.5 versus 2.2; Mann-Whitney $U$ test; $p=.04$; two-sided). It was further striking, and logical, that invalid beeps were rated as more disturbing on average than valid beeps (mean score $[n=48]$ of 3.0 versus 2.3 ; Mann-Whitney $U$ test; $\mathrm{p}<.01$; two-sided).

In summary, most subjects were not unduly inconvenienced by the ESM procedure and were generally positive in their evaluations of the method. Only $13 \%$ of the subjects stated unwillingness to participate again in an ESM study. Half of the beeps were rated as not disturbing at all. High stress subjects rated the beeps as a little bit more disturbing than low stress subjects, but their average score was still low. These data show that ESM can be used with good results in samples of working men with different levels of perceived stress. Our results compare well with results obtained in other studies. in a study on German adults, $22 \%$ reported that ESM disrupted daily routine and $75 \%$ were willing to participate again with ESM (Hormuth, 1985). In samples of Dutch subjects with a panic or other neurotic disorder, about $28 \%$ said the method was bothersome and $72 \%$ was willing participate again (Dijkman-Caes, 1993).

\section{Study effects}

In order to obtain a representative sample of the experiences of people in their daily life, we made repeated measurements with ESM within a day and during several days. Because of these repeated measurements, which means intense involvement of the subject, we wanted to know if participating in ESM research produced study effects which would reduce the ecological validity of the study. Therefore, debriefing interviews included questions about the possible influence of the method on the subject's daily life. 
Table 2.6. The method's Influence on thoughts, moods, activities and social contacts.

\begin{tabular}{|c|c|c|c|c|}
\hline Study effects & $\begin{array}{l}\text { Low-High. } \\
\text { Subjects } \\
\text { Thoughts } \\
\text { (\%) }\end{array}$ & $\begin{array}{l}\text { Low-High } \\
\text { Subjects } \\
\text { Moods } \\
\text { (\%) }\end{array}$ & $\begin{array}{l}\text { Low-High } \\
\text { Subjects } \\
\text { Activities } \\
\text { (\%) }\end{array}$ & $\begin{array}{l}\text { Low-High } \\
\text { Subjects } \\
\text { Social contacts } \\
\text { (\%) }\end{array}$ \\
\hline Lirtle or none & 13 & 5 & 4 & 9 \\
\hline Yes & 31 & 19 & 4 & 7 \\
\hline No & $\begin{array}{ll}63 & 62\end{array}$ & 76 & 92 & 91 \\
\hline
\end{tabular}

A majority of subjects said the method had no influence on their thoughts, moods, activities and social contacts (see Table 2.6). The small differences found between groups were not significant. The ESM method had the largest influence on subject's thoughts. Effects on thoughts mentioned during debriefing interviews were: thinking about ESM during the day and waiting for beeps to come $(n=10)$, more aware of thoughts $(n=10)$, confrontation with thoughts $(n=3)$, giving a moment thought to things you normally take for granted $(n=l)$, and it helps to relativize experiences $(n=2)$ and to concentrate $(n=1)$. The main effects on mood were: higher awareness of feelings $(n=4)$, and writing down negative feelings makes them disappear $(n=1)$. Irritation $(n=7)$ or being bored $(n=2)$ were mentioned as negative effects of ESM. Effects on activities were: giving more thought to activities than. usual $(n=2)$, less participation in sports $(n=1)$, little adjustments in planned activities during the weekend $(n=3)$, and some people said they waited to engage in a certain activity till after the beep $(n=3)$. The most often mentioned effect of ESM on social contacts was: having to explain ESM all the time to other people ( $n=8$ ), what some people liked and others did not. One subject thought the beeper scared others off, and another subject thought that his family behaved differently (no arguments).

In summary, on average $80 \%$ of the subjects said the method had no influence on daily life. If study effects were reported, there were both positive and negative effects of participating in ESM. 



\section{Chapter 3}

The nature of stress in daily life: events, appraisals, and activities 



\section{INTRODUCTION}

Stressful daily events have been found to be related to lower psychological well-being and increased somatic symptomatology (Del-ongis et al., 1982; Stone et al., 1993). These studies, most of them characterized by an a priori labeling of events as stressors, provided only limited information about the types of events that are experienced as stressful by varying populations and in varying contexts. They did, however, show that large individual differences exist in the vulnerability to minor events as well as in the impact of such events. The meaning and impact of an event is to a large degree determined by one's perceptions and reactions to such events. They may depend, for instance, on earlier experiences, personality social support, personal goals and beliefs. The current transactional approach to psychosocial stress defines minor events or 'hassles' as experiences and conditions of daily living that are experienced as salient and harmful or threatening to the endorser's well-being (Lazarus \& Folkman, 1984b). A central feature of this approach is the importance it places on these cognitive appraisal processes that intervene between the occurrence of an event and the individual's reaction to it. Despite the acknowledged importance of the transactional nature of stress, the significance of the appraisal process, and the many laboratory and animal studies that have been performed on this topic, few studies have investigated the nature of suressful daily events and the meaning individuals give to stressful events in a real-life setting. The present study aims to examine in the natural environment of the individual what types of events are experienced as stressful and how stressful events are appraised. We further investigated whether individuals who perceive themselves to be stressed differ in the types and appraisals of reported stressful events from individuals who do not.

With respect to the nature of stressful events, qualitative measures of stressful daily events may increase our insight into the type of situations or experiences which are most problematic and emotionally difficult for certain populations or for the individual. By exploring recurrent themes or patterns of hassles in reports of everyday events as described by individuals themselves, instead of focusing only on frequencies of event occurrence, we can more reliably characterize those events (e.g. work demands, conflicts with the partner) that form the basis of chronic stressful life conditions (e.g. work problems, family problems) (Wagner, 1990). Research suggests that patterns of hassles vary with developmental stage and sociodemographic characteristics (Kanner et al., 1981). In the study by Kanner et al., groups of middleaged people, students, and health professionals could be differentiated according to certain thematic patterns of hassles. Middle-aged subjects reported more hassles with economic issues (e.g. rising cost of living, property, investments, taxes), health professionals reported more hassles with anxieties and high pressure (e.g. too many things to do, not enough time, too many responsibilities), and college students tended to have more academic and social hassles (e.g. meeting standards, wasting time, and loneliness).

With regard to the meaning and significance of an event, it is possible that although people encounter an enormous range of events in daily life, events are. stressful for only a limited number of reasons. For an understanding of what makes certain events stressful for certain kinds of people, we not only have to know what (is. perceived to have) happened, but also how an individual gives meaning to the event, through cognitive appraisal processes (Lazarus \& Launier, 1978). There is evidence, for instance, that individuals' personal ratings of events improve prediction of outcomes such as anxiety, depression, negative affect, tension, and grade point 
average (Dewe, 1991; Peeters et al., 1995; Sarason et al., 1978). Although the transactional stress theory makes a distinction between primary and secondary appraisal processes ('what is at stake', and 'what can be done about the situation'. respectively), in many cases these processes are not separable (Holroyd \& Lazarus, 1982). For this reason, we have not tried to distinguish between primary and secondary appraisal in the present study. Based on the literature (e.g. Cohen, 1980; Dewe, 1991; Katz \& Wykes, 1985; Lazarus \& Folkman, 1984b; McGrath \& Beehr, 1990; Miller, 1979; Thompson, 1981), we selected five dimensions that appear to be important in how individuals evaluate stressful experiences: Unpleasantness ('How unpleasant was this event for you?'), importance ('How important was this event for you?'), predictability ('To what degree did you foresee that this event was going to happen?'), controllability ('How much controll did you have over the course of the event?'), and frequency of prior event occurrence ('How often has such an event happened to you before?').

The main objectives of this chapter are, firstly, to provide both quantitative and qualitative descriptions of daily life stress in white collar men, and secondly, to contrast the experiences of individuals who perceive themselves to be stressed with those who do not. Considering our first objective, we examine 1) how often stressful situations occurred, 2) what kinds of situations were experienced as stressful, and 3) evaluations of events, as discussed above. Regarding our second objective, we first examine 4) the amount of time spent on different activities in high and low stress subjects. Information on the frequencies of activities that people engage in on a daily basis can provide the necessary framework to understand the experience of stress in daily life. In addition, patterns of daily time use are compared between groups to assess whether the two groups had a similar chance of exposure to various kinds of stressful situations. We next examine 5) whether high stress subjects differed from low stress subjects in how they appraised daily activities in terms of enjoyment, challenge, required skill and effort. Here, we expect that appraisal of activities may reflect the stressfulness of daily life experiences that were not identified by the individual as discrete events. Next, we investigate differences between high and low stress groups in 6) frequency, 7) content and 8) evaluation of stressful experiences. We further examine whether life events and chronic difficulties were related to the frequency of stressful events reported during the five days of ESM. Both life events (for example, divorce) and chronic difficulties (for example, problematic relationship with spouse) could affect the person's pattern of stressful daily events or their personal significance (Kanner et al., 1981). Finally, we investigate a more methodological issue, that is, how do repeated within-day event reports compare with checkilist results obtained at the end of the day? Since most studies measure stress on a daily basis, it is important to investigate the influence of the frequency of measurements on event reports. For instance, do open-ended probes for events within-days elicit more stressful events than end-of-day event checklists (for example, due to forgetting events at the end of the day or increased attention for events within-days), or do we find report of fewer stressful events within-days compared to end-of-day checklist (for example, due to passive recognition of checklist events rather than active recall, or due to a more general evaluation of the demands of the day instead of the more discrete events occurring during the day)?

In the past. several methodological approaches have been used to elicit information about daily events, ranging from open-ended questions to structured checklisis. The advantages and disadvantages of the various methods have been discussed by Stone et al. (1991). The simplest approach has been the open-ended one, 
where subjects were asked about the day's most stressful event (Rehm, 1978) or asked if anything went wrong during the day (Caspi et al., 1987; Eckenrode, 1984). If a respondent answered 'yes', she or he was then asked to describe what had happened. Responses were then classified into discrete categories. Strongly influenced by the work of Holmes and Rahe on major life events (Schedule of Recent Events; Holmes \& Rahe, 1967), the most often used method of assessing daily events is the event checklist, where, from a list of events, subjects are asked to identify those that have occurred to him or her over a specified time period, ranging from one day to one month. Checklists covering the last month include the Unpleasant Events Schedule and the Pleasant Event Schedule (Lewinsohn \& Amenson, 1978; Lewinsohn \& Talkington, 1979), the Hassles and Uplifts scale (Kanner et al., 1981). the Inventory of Small Life Events (Zautra \& Guarnaccia, 1986), and the Everyday Problem Checklist (Vingerhoets \& Menges, 1989). Items on these scales were formulated or taken from existing scales to cover events in major life domains such as family, work, leisure, and household maintenance. The much shorter checklists designed to be used on a daily basis include the Daily Life Experience checklist (DLE; Stone \& Neale, 1982), the Daily Stress Inventory (DSI; Brantley et al., 1987), and the Daily Stress Scale (DSS; Bolger et al., 1989a). To develop daily checklists with representative events, individuals from the population in which the checklist was later intended to be used were asked to provide open-ended descriptions of daily events and situations that fit certain criteria (for example, 'stressfulness", (Brantley et al., 1987)). Elicited events were then grouped into various major life domains (e.g. DLE: work, leisure, family, friends, financial, and other; DSS: overloads. interpersonal conflicts) or chosen on the basis of reported event frequency (DSI).

In the present study, we used the Experience Sampling Method (ESM) (Csikszentmihalyi \& Larson, 1987; de Vries, 1987; de Vries, 1992) to investigate the types and cognitive appraisals of stressful events in the everyday life of white collar men. In the context of their normal social networks, settings and activities, 88 subjects completed ESM self-reports at semi-random intervals ten times a day over 5 consecutive days, including three work and two non-work days. Stressful daily events were assessed by the open-ended question 'did anything stressful take place since the last assessment?' Our choice for the open-ended approach was based on the fact that most daily event checklists are not suitable for frequent use during a single day. Also, we did not want to restrict a priori the definition of 'stressful' to certain categories of problems, being more interested in how white collar men themselves describe their stressful daily events. The ESM affords several advantages for investigating stress from a transactional perspective. Since events are assessed at frequent intervals each day, not long after the occurrence of the event, we are able to look at stress processes more dynamically as they evolve in time, but also more dynamically in the sense that not only the person but also the natural environment of the person is taken into account in the assessment of a stressful experience. These processes can be empirically assessed with a minimum of confounding due to biased recall (e.g. 'effort after meaning') or forgetting (Bower, 1981). In this way, we are able to elicit information about the appraised meaning of an event at the time it occurs, and about the social or physical context at that moment. Using ESM, even very minor sources of stress can be recorded, which might otherwise be forgotten at the end of the day. Therefore, more reliable estimates of rates of event occurrences are possible. 


\section{SUBJECTS}

The subject selection procedure has been described in Chapter 2 (see 'Subjects' and 'Procedure' sections). As shown in Table 3.1., this procedure resulted in two groups (42 High Stress 'HS' subjects and 46 Low Stress 'LS' subjects) that were very similar in sociodemographic characteristics. This means that any differences between the groups in the use of time or in exposure to stressful events are unlikely to reflect differences in age, marital status, or family composition.

Table 3.1. Demographic characteristics of the study participants.

$\begin{array}{ll}\text { LS } & \text { HS } \\ \mathrm{n}=46 & \mathrm{n}=42\end{array}$

p-value

(2-tailed)

\section{demographic variables}

\begin{tabular}{|c|c|c|c|}
\hline age & mean (st.dev.) & mean (st.dev.) & \\
\hline mean & $42.7(7.7)$ & $41.5(6.0)$ & ns \\
\hline range & $27-57$ & $28-52$ & \\
\hline \multicolumn{4}{|l|}{ marital status } \\
\hline married & $41(89.1 \%)$ & $37(88.1 \%)$ & ns \\
\hline unmarried & $2(4.3 \%)$ & $2(4.8 \%)$ & \\
\hline living together & $2(4.3 \%)$ & $2(4.8 \%)$ & \\
\hline divorced & $\mathrm{i}(2.2 \%)$ & $1(2.4 \%)$ & \\
\hline \multicolumn{4}{|l|}{ household composition } \\
\hline living alone & $3(6.5 \%)$ & $3(7.1 \%)$ & ns \\
\hline couple with children & $37(80.4 \%)$ & $34(81.0 \%)$ & \\
\hline couple without children & $6(13.0 \%)$ & $5(11.9 \%)$ & \\
\hline
\end{tabular}

\section{METHODS}

Measures relating to stressful events and to daily activities are derived from ESM reports, as described in Chapter 2 (see 'Description of the ESM instrument' section). End-of-the-day measures used in the current analyses include the Daily Stress Scale (Bolger et al., 1989a) and a global rating of the day's stressfulness. Also included in this analysis are measures of life events, recorded with the List of Threatening Experiences (LTE-Q:Brugha et al., 1985) and chronic difficulties, assessed with the Long-term Difficulties Questionnaire (LLM: Hendriks et al., 1990) (see chapter 2 'Cross-sectional instruments' section).

Analyses were done with SPSS/PC+ 4.0 on Macintosh. Unless stated otherwise, differences in variables were tested with non-parametric tests, using twotailed significance tests. In the analysis of the ESM data, measures were aggregated so that the subject was used as the unit of analysis (Larson \& Delespaul, 1992). 


\section{A CASE EXAMPLE}

The following case study of a subject who experiences high levels of stress in his. life illustrates the level of detail this assessment method provides about the ebb and flow of an individual's stressful experiences during the course of five days.

Jan, 41 years old, married with child (one year old), works for the municipal government as a building and housing inspector. He experiences his life as somewhat overloaded and uncontrollable lately (at least for the past 3 months; PSS score $=17$ ) and describes the following chronic difficulties: work is demanding due to regulatory changes, he has no time to study and is therefore falling behind, there is not enough time for home upkeep and garden maintenance, and too little real leisure time. When asked which psychosomatic complaint bothers him the most, he mentions stomach ache. The five days of ESM for Jan are shown in Figure 3.1. Here we see that on Thursday he has a busy day at work, with difficult assignments. Several work-related stressful events are reported, like settling a petition and dealing with a request for subsidy, with a concurrent increase in feelings of agitation and stomach pain. Positive affect also seems to be below average during that day at work. While driving home he seems to unwind, he starts to feel more relaxed, is less bothered by stomach pain and his mood improves. The next day, Jan describes a similar pattern, with several stressful events in the morning at work, increasing agitation, stomach pain, and a slight dip in positive affect. In the afternoon and evening, several events are described that relate to his child's health and to household maintenance, but these events seem to have hardly any effect on mood state or physical complaint, since after leaving work Jan reports less agitation, stomach pain and higher positive mood. In contrast with the work days, the weekend is spent with relatively few stressful experiences and on the whole he feels relaxed, in a good mood, and not bothered by stomach pain. Most of the time is spent with wife, child and other family members: e.g. playing with son, visiting family, entertaining family, working on car, and watching t.v. Although Monday -back at work- no events are reported, we can see that Jan gets slightly more agitated again and is more bothered by stomach pain than during the weekend. ESM data indicated that work is the major source of stress for Jan, as was also described in the chronic difficulties questionnaire. Household maintenance was also reported as stressful on several occasions. but had less influence on his emotional state or physical complaint. 
Figuur 3.1. Example of one subject's stressful events. emotional states (Agitation, Positive Affect) and psychosomatic complaint reported during five days of ESM
Apitacioa

Complaint

Poritive mood



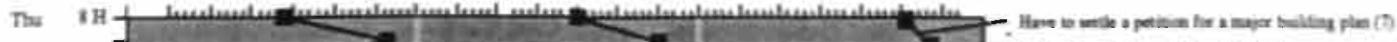
12 H] 年

Fin
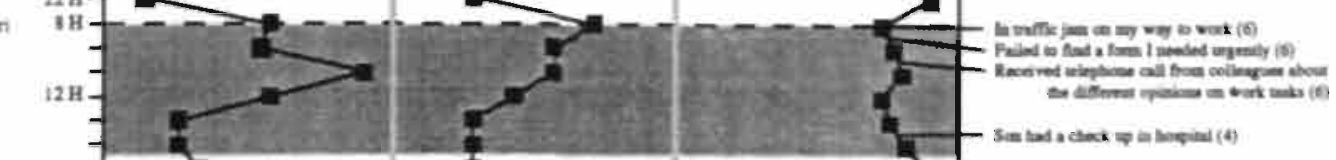

se
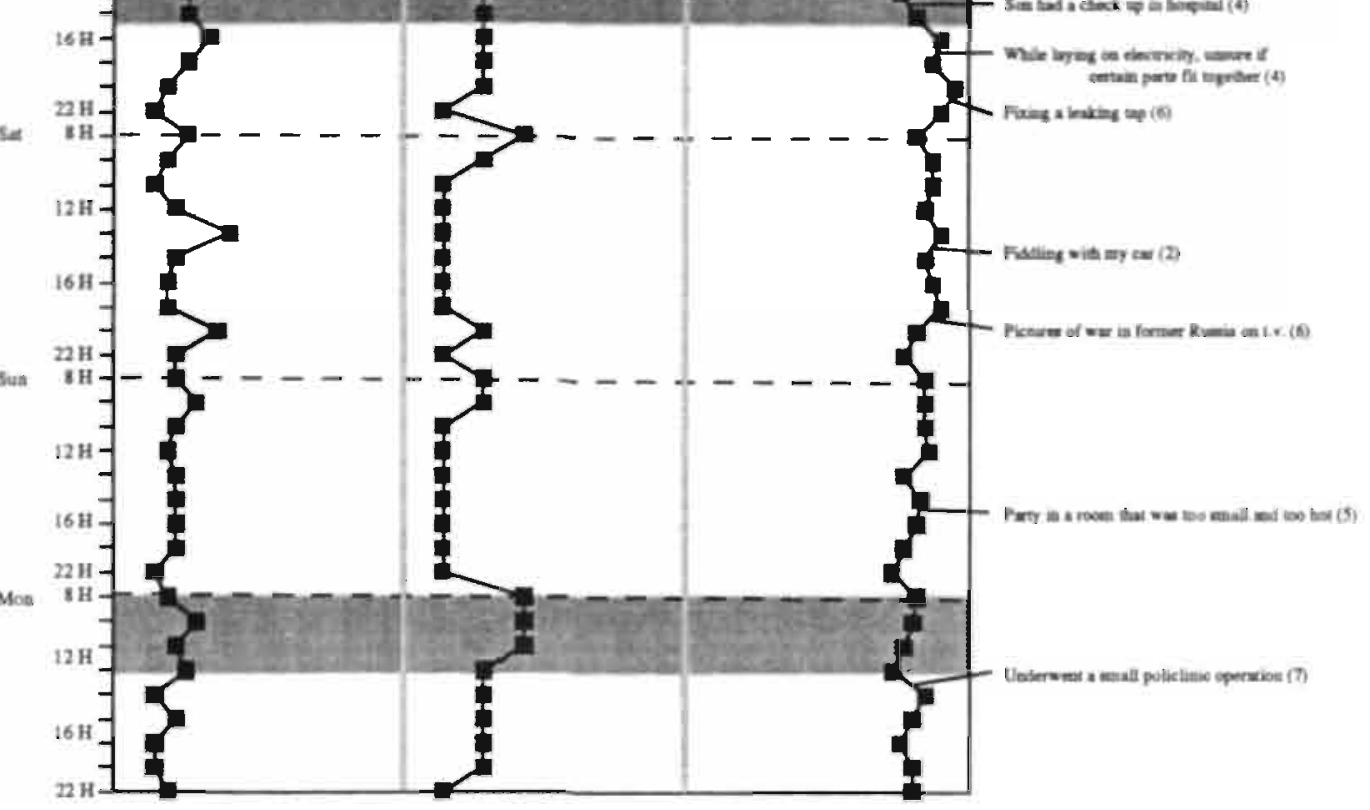


\section{STRESSFUL EVENTS IN DAILY LIFE: FREQUENCY, CONTEXT AND APPRAISAL}

We first examine the frequency, nature, and evaluation of events reported as stressful by this sample of white collar men. The ESM forms asked subjects to describe any stressful events or situations which may have taken place in the interval since the last ESM report (on average 90 minutes). Subjects' descriptions of stressful daily events were coded according to context, with categories work, nefwork (events. concerning family, friends, and acquaintances), household/financial, leisure, personal health (somatic and psychological health), transport, and other. In addition, events were classified according to whether or not they involved a social interaction and/or a task demand (see chapter 2 'Coding of ESM measures section). Subjects also rated the events they reported on the dimensions unpleasantness, imporiance, predictability, controllability, and frequency of prior occurrence.

\section{Event frequency}

Subjects reported a total of 626 stressful events during the five days of ESM, with a mean of 7.1 events per subject (median $5.0 ; \mathrm{s.d} .=7.3)$. Events as a percentage of total beeps averaged $16.8 \%(\mathrm{~s} . \mathrm{d} .=16.5)$. Of all stressful events reported, $33 \%$ were rated as highly stressful (score of $\geq 5$ ).

\section{Event context}

The distribution of events by life domain (expressed as frequency of each type of stressor divided by total beeps x 100) is shown in Figure 3.2. Subjects reported stressful events related to work and to social network most often. Of all events reported, $48 \%$ were work-related. Since the frequency of work events could relate to the amount of time spent at work, we also examined the probability of the occurrence of a work event given the amount of time spent at work. On average, subjects reported being at work on $31 \%$ of total beeps. 52 of the subjects reported a higher percentage of work-related events than would be expected based on the amount of time they spent at work (Wilcoxon Matched-Pairs Signed-Ranks test, $\mathrm{p}<.001$ ). This indicates that work-related events were not only absolutely but also relatively high in frequency. Next, we examined the correlations between the percentages of events reported in the various contexts (Table 3.2). The strongest correlations were between network and household events ( $r h o=.35 ; \mathrm{p}<.01$ ) and between network and work events $(r h o=.35 ; \mathrm{p}<.01)$. These correlations indicate that the experience of stressful events was not constrained to a particular life domain for an individual.

Table 3.2. Correlations (Spearman) between the percentages of events in the various contexts ${ }^{\mathrm{a}}$.

\begin{tabular}{lllllll}
\hline & 1 & 2 & 3 & 4 & 5 & 6 \\
\hline 1. Work & & & & & & \\
2. Network & $.35^{* *}$ & & & & & \\
3. Household & $.29^{* *}$ & $.35^{* *}$ & & & & \\
4. Leisure & $.21^{*}$ & .20 & .14 & & & \\
5. Personal Health & -.18 & .01 & .04 & -.16 & & \\
6. Transport & .15 & .07 & .10 & -.10 & .12 & .12 \\
7. Other & .05 & $.29 * *$ & .20 & .10 & -.10 & \\
\hline
\end{tabular}

*p<.05; * *p<.01; ${ }^{a} \mathrm{n}=88$ 




Figure 3.2. Frequency of stressful daily events by life domain.

In Figure 3.3. the percentage of events coded as task demand, as social interaction and as internal or external are shown. A minority of stressful events were characterized as social interaction or task demand events, and the large majority of events were (theoretically) observable or verifiable. Internal events (e.g. worries, anxieties) represented only $6.6 \%$ of all reported events. Examples of reported stressful events for each category are shown in Table 3.3.



Figure 3.3. Frequency of stressful daily events: social interaction, task demand, and external/internal events. 
Table 3.3. Examples of stressful events.

\section{Work}

(events concerning boss, supervisor, clients. co-worker, general happenings at work)

'behind on computer work'. 'while working getting a lot of crazy telephone calls". "difficult discussion at work meeting'. 'job evaluation conversation'. 'a lot of work, a lot of different things'. 'can not fix computer programme error'. 'discussion about changes in organisation'. 'writing an account that has to be finished today". "making appointments under high time-pressure'.

\section{Network'}

(events concerning spouse, partner, children, relatives, friends, other acquaintances)

'discussion with spouse about arriving too late at friends' house'. 'getting children ready in time for school', 'children are annoying', "argument with spouse about household affairs". 'received message that sister has been in car accident' 'whole family wants attention at the same moment'. 'baby-sitter cancelled appointment:

\section{Houschold}

(events concerning general housework, errands,

family related duties, financial problems, etc.)

having to do a lot of errands in a short time period'. 'have to make a decision about buying a couch'. 'working in garden under high timepressure'. 'having to do a lot of dishes'. 'trying to fix a dripping tab'. 'while repairing bicycle chain breaks'. 'cleaning-up the mess without any support'.

\section{Leisure}

(events concerning hobbies, sports, outings. vacation, etc.)

'playing tennis match'. 'driving in a sports car'. "watching soccer: favoritc team missed a lot of chances'. 'performance with choir'. 'music band played out of tune' 'jogging', 'watching the Euro cup soccer game (Holland-Scotland)'

Personal Health

(events concerning illness, injury of the subject

himself, worries about health, nerves)

'headache'. 'nervous". 'underwent a small operation'.

\section{Transport}

(e.g. missed bus, traffic jam, unusual traffic)

'heavy traffic while driving home'. 'missed bus'. 'cycling in pouring rain'. 'had to hit the brakes suddenly', 'could not find a place to park the car'. "almost run over by a car while on my bike".

\section{Social Interaction}

(c.g. argument, conflict, conversation)

'squabble with children'. 'discussion with colleague about problems in the organisation'. 'conflict with partner about upcoming visit parents'. 'meeting with department'. 'discussion with spouse about furure childcare'. 'argument with boss'. 'had to give bad news to a client'. 'argument with spouse about how to act in certain situation'.

\section{Task Demands}

(e.g. difficult or problematic tasks, lime pressure, deadlines, a lot of work, failure at task)

'pressured by unexpected visit of client at work'. 'have to hurry washing, shaving, and dressing because woke up too late'. 'difficulties painting the wall', 'failed to saw up the wood into pieces'. 'had to take care of a lot of errands during my lunch break'. 'the usual hectic Saturday: running back and forth'. 'too much work to do'.

\section{Event appraisals}

Correlations among event appraisals are shown in Table 3.4. As would be expected, the strongest correlation was between event stressfulness and unpleasantness. Events that were less predictable or less controllable were also more unpleasant. Next we examined whether the various types of stressful events differed on the six appraisal dimensions. Figure 3.4 shows the mean scores for the types of stressful events on the different appraisal dimensions. Surprisingly, Leisure events were rated as most stressful, but they were the least unpleasant by far and the most. expected. An explanation could be that certain Leisure events (e.g. sport games) are experienced as stressful and exciting but that the stress is of a positive and 
challenging nature. Whether the types of events differed significantly from each other with regard to the appraisal dimensions was only tested for a subset of events, since not all event types were reported by all subjects thus resulting in small sample sizes for certain event types. Comparisons were made for Work versus Network, Interactions versus Non-Interaction, and Demand versus Non-Demand events. Work events were reported as occurring significantly with greater regularity than Network events (frequency: Wilcoxon Matched-Pairs Signed-Ranks test, p<.05), but no significant differences were found for the other appraisal dimensions. Interaction events were appraised as significantly more important $(\mathrm{p}<.01)$, more controllable $(\mathrm{p}<.001)$, and occurring more often $(\mathrm{p}=.05)$ than Non-Interaction events. Demands were appraised as significantly less important than Non-Demands events. The other appraisal dimensions showed no significant differences here.

Table 3.4. Correlations (Spearman) between event Appraisais ${ }^{\mathrm{a}}$ and mean appraisal scores ${ }^{\mathrm{b}}$.

\begin{tabular}{|c|c|c|c|c|c|c|c|}
\hline & I & 2 & 3 & 4 & 5 & $\bar{M}$ & $S D$ \\
\hline 1. Unpleasantness & & & & & & 3.6 & 1.4 \\
\hline 2. Stressfulness & $.41 * *$ & & & & & 3.9 & 1.0 \\
\hline 3. Importance & .03 & $.22 * * *$ & & & & 4.1 & 1.5 \\
\hline 4. Controllability & $-.23 * * 0$ & -.03 & .03 & & & 3.7 & 1.5 \\
\hline 5. Chronicity & -.01 & -.03 & $-18 * *$ & $.22 * * *$ & & 3.1 & 1.8 \\
\hline 6. Predictability & $-.31 * *$ & -.03 & $19 * * *$ & .07 & $18 * * *$ & 3.4 & 1.4 \\
\hline
\end{tabular}

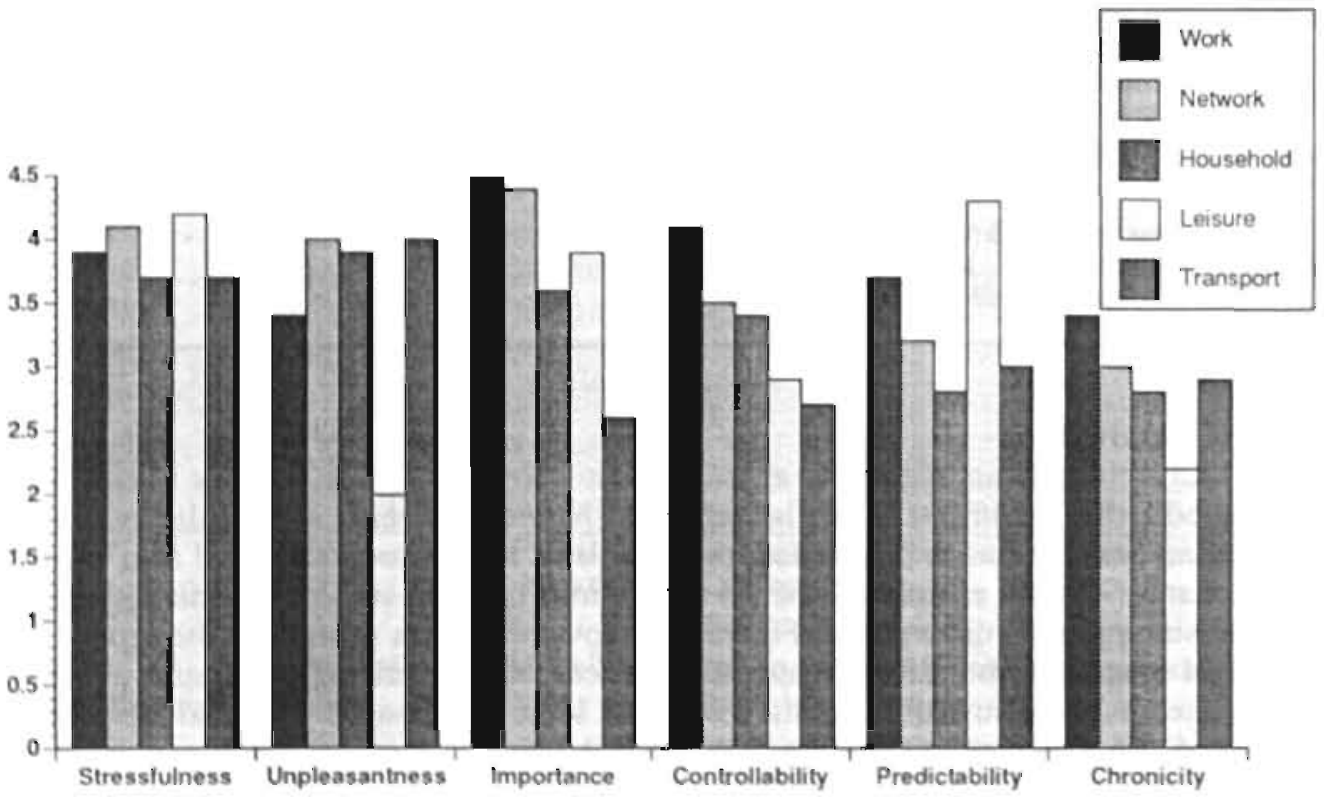






Figure 3.4. Mean appraisal scores calculated for events in the context of $(\mathbf{A})$ work $(n=65)$. network $(n=36)$, household $(n=22)$, leisure $(n=19)$, transport $(n=29)$, and for $(B)$, no-interaction

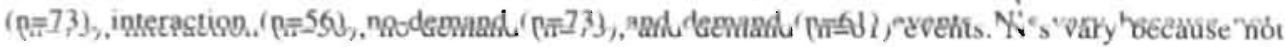
all event types were reported by all subjects.

In summary, subjects reported stressful work events most often, followed by social network events, and the experience of stressful events was not limited to a certain context. In general, events that were appraised as less predictable and less controllable were the most unpleasant. Stressful work events differed from stressful network events only in the appraised frequency of past occurrence, as was confirmed by the current event frequency scores. Negative social interactions were appraised as most important, most controllable, but also as occurring with greater regularity in the past, when compared to all other events, although current frequency scores revealed that social interaction events were actually reported relatively rarely during ESM sampling.

\section{RELATIONSHIP OF DAILY EVENTS TO LIFE EVENTS AND CHRONIC DIFFICULTIES}

It has been suggested (e.g. Hinkle, 1974; Kanner et al., 1981; Kaplan, 1979) that life events may initiate a cascade of daily stressors; for example, a divorce may lead to a series of new hassles in the context of keeping house, making meals, or keeping up social contacts, which did not have to be dealt with previously. As described in Chapter 2, life events were recorded with the questionnaire form of the List of Threatening Experiences (LTE-Q; Brugha et al., 1985), and chronic siress was assessed with the Long-term Difficulties Questionnaire (LLM; Hendriks et al., 1990), which focuses on problems in relation to work/study, housing, physical environment, leisure, finance, and social relationships (see 'Cross-sectional instruments' section). 
Table 3.5. Rate of daily event occurrence by increasing number of life-events and chronic. difficulties.

Number of Life-Events

\begin{tabular}{|c|c|c|c|c|c|c|c|}
\hline & \multicolumn{2}{|c|}{$0(n=51)$} & \multicolumn{2}{|c|}{$!(n=22)$} & \multicolumn{2}{|c|}{$\geq 2(n=15)$} & \multirow[t]{2}{*}{$p$} \\
\hline & mean & sd & mean & sd & mean & sd & \\
\hline $\begin{array}{l}\text { Frequency of } \\
\text { Daily Events }\end{array}$ & $15.7 \%$ & $15.4 \%$ & $17.4 \%$ & $17.9 \%$ & $19.4 \%$ & $18.8 \%$ & ns \\
\hline
\end{tabular}

Chronic Difficulties

\begin{tabular}{|c|c|c|c|c|c|c|c|}
\hline & \multicolumn{2}{|c|}{ None $(n=6)$} & \multicolumn{2}{|c|}{ Minor $(n=39)$} & \multicolumn{2}{|c|}{ Severe $(n=33)$} & p \\
\hline Daily Eventsa & $11.5 \%$ & $17.8 \%$ & $10.9 \%$ & $10.9 \%$ & $24.1 \%$ & $20.3 \%$ & $<.01$ \\
\hline Unpleasantness & & & & & & & \\
\hline of Daily Events ${ }^{b}$ & 2.3 & 1.0 & 3.3 & 1.6 & 4.0 & 1.3 & $<.01$ \\
\hline Freq $\times$ Unpleasant ${ }^{c}$ & 34.2 & 60.6 & 36.9 & 41.4 & 92.4 & 76.0 & $<.01$ \\
\hline
\end{tabular}

a Mean frequency of events as a percentage of total beeps

b Mean unpleasantness rating of events

${ }^{c}$ Mean frequency by unpleasantness rating

When we compared subiects who resoorted no. one. or two or mote life-pevents in the past year on the percentage of stressful daily events reported during the ESM period, however, no differences in rate of stressful daily events occurrences were observed. Subjects with more life-events did not report more daily events (Table 3.5.). Dividing the total scores on the chronic difficulties scale into three categories (none, minor [average or below average in number], or severe [above average in number], we found that subjects with higher scores on the chronic difficulties scale reported more daily events (Kruskal-Wallis one-way anova; p<.001) and rated events as more unpleasant $(\mathrm{p}<.05)$. The sum of frequency by unpleasantness ratings was significantly higher as the chronic difficulties scores increased ( $p<.001)$ (Table 3.5.).

In summary, not life events but chronic difficulties were positively related to the frequency and intensity of stressful daily events reported during the ESM period.

\section{COMPARISON OF WITHIN-DAY EVENT AND END-OF-DAY MEASURES}

We next examined how two different methods of daily event assessment compared in terms of the number and type of stressors reported; in other words, do repeated within-day event reports provide different information than checklist results obtained at the end of the day? Subjects were asked at the end of each Experience Sampling day how stressful the day had been and completed a short checklist, the Daily Stress Scale (Bolger et al., 1989a). This instrument (as described in 'Description of ESM instrument' section chapter 2), consists mainly of items concerning demands (e.g. a lot of work at home, at work) or interpersonal conflicts (e.g. argument with spouse, child, colleague), with two additional items concerning transportation and financial problems. 
Table 3.6. Prevalence of events obtained by within-day (ESM) assessments compared to end-ofday checklist assessments.

Did (at least 1) event occur?

According to:

\begin{tabular}{lccc}
\hline Sources of Stress & ESM & Checklist & Chi-Square \\
\hline $\begin{array}{c}\text { Home stress } \\
\text { Overload }\end{array}$ & $7.1 \%$ & $36.8 \%$ & $<.001$ \\
Argument & $8.6 \%$ & $17.5 \%$ & $<.00 !$ \\
Work stress & & & \\
Overload & $15.3 \%$ & $40.0 \%$ & $<.001$ \\
Argument & $19.6 \%$ & $7.0 \%$ & $<.001$ \\
Home Total & $14.1 \%$ & $43.9 \%$ & $<.001$ \\
Work Total & $26.8 \%$ & $40.7 \%$ & $<.001$ \\
\hline
\end{tabular}

The number of ESM events reported each day was positively correlated with the number of events reported on the checklist at the end of the day ( $r h o=.43$. $\mathrm{p}<.001: \mathrm{n}=439$ ) and with the end of the day global rating of the day's stressfulness ( $r h o=.52, \mathrm{p}<.001 ; \mathrm{n}=404$ ). We compared the frequency of reports of home stress and work stress (rated as overloads or as arguments) within-days with the reports of these events at the end of the day. Scores were rated 0 if no event was reported and 1 if at least one event was reported. Results are shown in Table 3.6. Except for work arguments, a higher frequency of events was reported with the checklist at the end of the day compared to the within-day measurements. All differences were significant. Similar results were found when only checklist events that were at least somewhat unpleasant were analyzed. We can conclude: that by recognition (as with checklists) many more events are reported than when actively asked for (open-ended question). Since some checklist items were of a more global nature (e.g. 'a lot of work at home', 'a lot of work at work'), it is also possible that when summarizing one's experiences at the end of the day these items are more easily endorsed, while during the day events are more discrete and too small in itself to be reported. Perceptual biases (e.g. effort after meaning) may he more likely to occur at the end of the day than at the momentary ESM assessments.

In summary, many more events were reported with the checklist at the end of the day compared to the open-ended, within-day measurements.

\section{DIFFERENCES BETWEEN HIGH AND LOW STRESS GROUPS}

To investigate whether individual perceptions of high versus low levels of stress are associated with personal characteristics and daily life experiences, differences between the two stress groups were tested with respect to psychological characteristics, the distribution of daily activities, and measures of stressful daily events. 


\section{Psychological characteristics}

The two groups were compared on the following measures: life events (LTEQ), chronic difficulties (LLM), psychological and somatic complaints (SCL-90), psychosomatic symptoms (PSC), depressive symptomatology (Zung), trait anxiety (STAI), trait anger (STAS), personality characteristics (Dutch abridged MMPI: NVM), and coping style (UCL). These instruments have been described in Chapter 2 (see section 'Cross-sectional instruments').

Results are shown in Table 3.7. HS subjects reported significantly more chronic difficulties during the last year than LS subjects, but no more life events. HS subjects also exhibited higher scores on both psychological (anxiety, anger, and depression) and psychosomatic complaints questionnaires than LS subjects and scored higher on the personality dimensions negativism, somatization, timidity and serious psychopathology. Additionally, HS subjects seem to use passive coping styles (palliative reaction, depressive reaction, expression of emotion) more often than LS subjects and an active, problem-focused coping style less often. Where possible, mean scores for both samples were compared to published norms for the Dutch population. The general pattern found was that LS subjects scored average or below average compared to norm scales, while HS subjects scored above average or high.

Frequency and intensity scores for the seven different categories of chronic difficulties are shown in Table 3.8. HS subjects reported significantly more chronic difficulties for all categories except for the category Family (i.e. parents, children, brothers / sisters, in-laws). Work and Housing difficulties had the highest prevalence in both groups, and HS subjects reported relational difficulties with the partner relatively frequently. The two groups were similar in the kind and ranking order of frequency of work difficulties mentioned: 1) high work pressure or work load, 2). conflicts or differences in opinion with boss or colleagues, 3) little appreciation and 4) changed or indistinct job functions due to reorganization. With regard to difficulties with partner, again several specific problems were mentioned by both groups: 1) conflicts or differences in opinion (for example, concerning household tasks or children), 2) low frequency of sexual contact (not in the mood, too busy), and 3) diminished interest in each other or doubts about choice of partner. Housing difficulties were more diverse, with problems such as conflicts with neighbours, criminality (vandalism, theft, drugs) and pollution. With regard to the intensity of chronic difficulties, only work difficulties were scored as more intense by HS subjects $($ mean $=2.0$ (s.d. 0.5) for HS subjects versus mean= 1.7 (s.d. 0.4) for LS subjects, Mann-Whitney $U$ test, $\mathrm{p}<.05$ ), and a trend in the same direction was found for difficulties concerning partner (mean=2.1 (s.d. 0.7) for HS subjects versus mean=1.7 (0.3) for LS subjects, $p=.06$ )

As shown in Table 3.9, psychosomatic complaints occurred with regularity (at least once a week) in a higher percentage in HS than in LS subjects and occurred more frequently overall in the HS group. Significant differences between groups in frequency of complaints (using the full range of frequency ratings) were found for: fatigue, depression, backache, headache, insomnia, general weakness, stomach pain, heart palpitations, and nausea. The same psychosomatic complaints were also rated as significantly more severe in HS subjects (p-values all <.01). The nine subjects who reported no complaint at all were all from the LSi group. 
Table 3.7. Characteristics of high and low stress groups.

\begin{tabular}{|c|c|c|c|}
\hline & $\begin{array}{l}\text { LS } \\
\mathrm{n}=46 \\
\text { mean (st.dev) }\end{array}$ & $\begin{array}{l}\text { HS } \\
\mathrm{n}=42 \\
\text { mean (st.dev) }\end{array}$ & $\begin{array}{l}\text { p-value } \\
\text { (2-tailed) }\end{array}$ \\
\hline Life-events & $.5(.7)$ & $.8(.9)$ & ns \\
\hline Chronic difficulties & $19.5(2.3)$ & $23.3(4.0)$ & $\cdots$ \\
\hline SCL-90 (total) & $102.5(11.1)$ & $147.4(39.3)$ & $\cdots$ \\
\hline Trait anxiety & $28.3(4.4)$ & $39.8(7.6)$ & $\cdots$ \\
\hline Depression & $36.5(5.5)$ & $48.4(7.7)$ & $\cdots$ \\
\hline Trait anger & $14.6(4.1)$ & $18.0(4.6)$ & $\cdots$ \\
\hline Psychosomatic symptoms & $6.1(5.6)$ & $27.5(23,2)$ & $\cdots$ \\
\hline \multicolumn{4}{|l|}{ Personality: } \\
\hline Negativism & $10.0(5.5)$ & $19.0(6.8)$ & $\cdots$ \\
\hline Somatization & $2.8(2.8)$ & $9.5(7.3)$ & $\cdots$ \\
\hline Timidity & $8.3(6.3)$ & $11.3(7.4)$ & • \\
\hline Serious Psychopathology & y $1.3(1.9)$ & $2.8(2.5)$ & $\cdots$ \\
\hline Extraversion & $15.4(5.3)$ & $16.5(5.6)$ & ns \\
\hline \multicolumn{4}{|l|}{ Utrecht Coping List: } \\
\hline Active coping & $21.1(4.0)$ & $17.3(3.4)$ & $\cdots$ \\
\hline Palliative reaction & $14.6(3.0)$ & $17.2(3.3)$ & $\cdots$ \\
\hline Avoidance coping & $14.1(2.5)$ & $15.1(3.6)$ & ns \\
\hline Social support & $11.7(2.7)$ & $12.5(2.7)$ & ns \\
\hline Depressive reaction & $8.8(1.6)$ & $12.6(3.4)$ & $* *$ \\
\hline Expression emotions & $5.8(1.3)$ & $6.9(1.7)$ & $* *$ \\
\hline Comforting cognitions & $10.8(2.6)$ & $11.7(2.8)$ & $\mathrm{ns}$ \\
\hline
\end{tabular}

* $p<05 ; \cdots p<01 ; \cdots * p<001$

Table 3.8. Percentage of HS and LS subjects reporting chronic difficulties in each life domain.

\begin{tabular}{|c|c|c|c|}
\hline & $\begin{array}{l}\text { low PSS } \\
\mathrm{n}=40\end{array}$ & $\begin{array}{l}\text { high PSS } \\
n=42\end{array}$ & $\begin{array}{l}\text { p-value } \\
\text { (2-tailed })\end{array}$ \\
\hline 1. Work difficulties & $39 . i \%$ & $88.1 \%$ & $* *$ \\
\hline $\begin{array}{l}\text { 2. Housing/Finance difficulties } \\
\text { Relational difficulties }\end{array}$ & $43.5 \%$ & $71.4 \%$ & $* *$ \\
\hline 3. Partner & $23.9 \%$ & $53.7 \%$ & $*$ \\
\hline 4. Family & $23.9 \%$ & $41.5 \%$ & ns \\
\hline 5. Friends/Acq. & $19.6 \%$ & $38.1 \%$ & $*$ \\
\hline 6. Leisure difficulties & $19.6 \%$ & $39.1 \%$ & * \\
\hline
\end{tabular}

p<05: ** p<.01: "*** p<001; Group differences in the percentage of reported chronic difficulties were tested by
means of chi-square tests. 
Table 3.9. Psychosomatic complaints in HS and LS subjectsa.

\begin{tabular}{|c|c|c|c|}
\hline & low PSS & high PSS & $\begin{array}{l}\text { p-value }{ }^{b} \\
\text { (2-tailed) }\end{array}$ \\
\hline I. Fatigue & $39.2 \%$ & $66.7 \%$ & $* * *$ \\
\hline 2. Depression & $2.2 \%$ & $42.9 \%$ & $* * *$ \\
\hline 3. Backache & $17.3 \%$ & $38.1 \%$ & * \\
\hline 4. Headache & $8.7 \%$ & $35.7 \%$ & $* * *$ \\
\hline 5. Insomnia & $10.9 \%$ & $31.0 \%$ & $* * *$ \\
\hline 6. Weakness & $0.0 \%$ & $19.1 \%$ & $* * *$ \\
\hline 7. Eye pain & $8.7 \%$ & $16.6 \%$ & ns \\
\hline 8. Stomach pain & $2.2 \%$ & $11.9 \%$ & $* * *$ \\
\hline 9. General stiffness & $2.2 \%$ & $11.9 \%$ & ns \\
\hline 10 Heart palpitations & $2.2 \%$ & $11.9 \%$ & $*$ \\
\hline 11 Dizziness & $4.3 \%$ & $11.9 \%$ & ns \\
\hline 12. Diarrhea / constipation & $0.0 \%$ & $9.5 \%$ & ns \\
\hline 13. Nausea & $0.0 \%$ & $7.2 \%$ & $* *$ \\
\hline 14. Spastic colitis & $0.0 \%$ & $7.1 \%$ & ns \\
\hline 15. High blood pressure & $2.2 \%$ & $4.8 \%$ & ns \\
\hline 16. Asthma & $0.0 \%$ & $2.4 \%$ & ns \\
\hline 17. Gastric ulcer & $0.0 \%$ & $0.0 \%$ & $\mathrm{~ns}$ \\
\hline \multicolumn{4}{|l|}{ " $p<05, \cdots p<01, \cdots p<001$} \\
\hline $\begin{array}{l}\text { a In the first two columns, the per } \\
\text { a week are reported. In the th } \\
\text { psychosomatic complatints are sh }\end{array}$ & and LS & $\begin{array}{l}\text { osomatic ci } \\
\text { he differe }\end{array}$ & teported \\
\hline
\end{tabular}

\section{Distrlbution of dally activities}

Next, we examined differences between high and low stress groups in time spent in various activity contexts. One might suspect that HS subjects worked more, or relaxed less, than their LS counterparts; daily stress might be reflected in imbalances in work and maintenance versus rellaxation, recreational and social activities. In addition, if, for instance, HS subjects spent more of their time working, then they would have an increased chance of exposure to stressful work events. This analysis was based on ESM activity codes (see Chapter 2. 'Coding of ESM measures section).

Figure 3.5. shows the mean percentage of time spent in the various activities for LS and HS subjects over all study days. HS subjects did not differ significantly from LS subjects in the percentage of time spent in different activities. When we subdivided the category Leisure into Passive (e.g. watching television) and Active (e.g. sports, hobbies) Leisure, no significant differences were found between the HS and LS group (Passive Leisure: $73.9 \%$ versus $79.1 \%$, Active Leisure: $26.1 \%$ versus $20.9 \%$ respectively; ns). Nor did the percentage of time spent in various activities outside working hours (weekend days and after 5 p.m. during work days) show significant between group differences. In summary, we found no differences between the two groups in patterns of time use. 


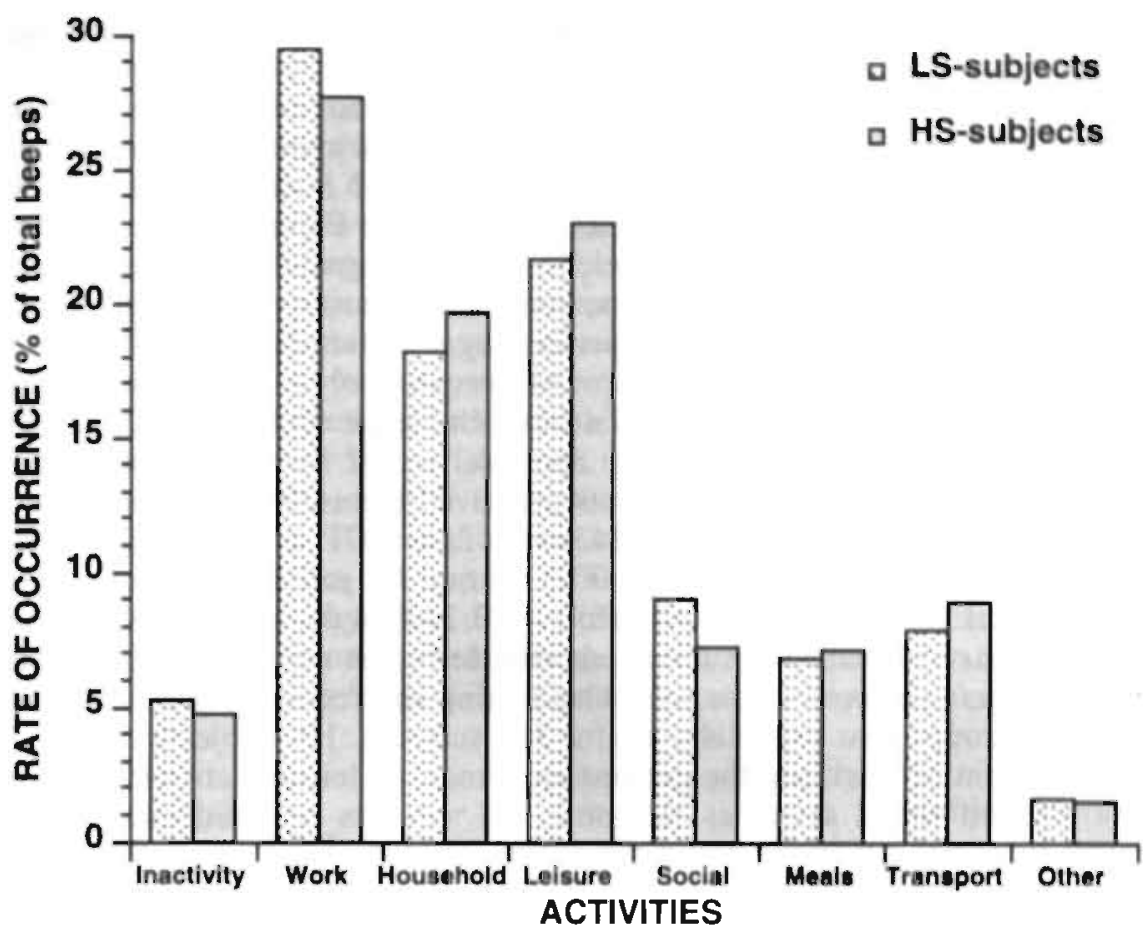

Figure 3.5. Use of time: frequency of activities.

\section{Appraisals of daily activities}

Since appraisals of daily activities were expected to reflect the stressfulness of daily life experiences other than stressful events, we investigated differences between stress groups in how they evaluated their daily activities. Data for this analysis were the ESM ratings of how current activity was experienced in terms of enjoyment, skill, effort, and challenge. As shown in Table 3.10., these dimensions were interrelated.

Table 3.10. Correlations (Pearson) among activity appraisals ${ }^{\mathrm{a}}$.

$1 \quad 2 \quad 3$

\footnotetext{
1. Motivation

2. Skill

3. Effort

$42 * * *$

4. Challenge

$-.32 * * * \quad-.36 * * *$

*...p $<$ <.001

.03

$-.22 * * * \quad .40 * * *$

a Peastson correlations were computed across all beeps $(n=3540)$.
}

Clear differences in how the two group evaluated their activities were found. HS subjects reported that they enjoyed activities less $(4.9$ versus $5.5 ; \mathrm{p}<.001)$, that they were less skillful $(5.7$ versus $6.1 ; \mathrm{p}<.001)$, and that the activities required more 
effort to perform ( 1.8 versus $1.6 ; \mathrm{p}<.01)$ than LS subjects. The two groups did not differ in their assessment of how challenging activities were.

Next, we tested by means of analyses of variance for repeated measures for significant differences in the degree to which the various appraisals were dependent on different activities (Work, Household, Leisure and Social Activities). Mean appraisal scores for the different activities are shown in Figure 3.6a-e. Confirming univariate analyses, the two groups differed in their ratings of the various activities: HS subjects enjoyed doing activities less, perceived themselves as less skillful, and they felt that more effort was required in performing activities $(\mathrm{F}(1,81)=13.46, \mathrm{p}<.001$; $\mathrm{F}(1,81)=6.86, \mathrm{p}<.05 ; \mathrm{F}(1,81)=7.14, \mathrm{p}<.01$, respectively), while there were no differences between groups for the appraisal of challenge $(\mathrm{F}(1,81)=.02$; ns). There were significant differences in the mean appraisal scores for the various activities, with the exception of how skillfull subjects perceived themselves to be in relation to the various activities (enjoyment: $\mathrm{F}(3,243)=45.12, \mathrm{p}<.001$; effort: $\mathrm{F}(3,243)=20.27$, $\mathrm{p}<.001$; challenge: $\mathrm{F}(3,243)=35.81, \mathrm{p}<.001)$. Group by activity interaction effects were significant for enjoyment and effort $(\mathrm{F}(3,243)=4.07, \mathrm{p}<.01 ; \mathrm{F}(3,243)=6.6$, $\mathrm{p}<.001$, respectively), with HS subjects scoring lower on enjoyment and higher on effort. Work was perceived as the most challenging and requiring the most effort in both subject groups, but especially so for HS subjects. HS subjects reported the lowest enjoyment of work and the greatest enjoyment of leisure activities. While LS subjects enjoyed social activities the most, HS subjects reported relatively little enjoyment in social situations and rated social interactions as more demanding in terms of effort than LS subjects.


Figure 3.6.a-d Mean appraisal of required skill, enjoyment, effort, and challenge for work. household, leisure, and social activities. 


\section{Frequency, context and appraisal of stressful daily events}

In this final section, differences between stress groups in the frequency, context, and evaluation of stressful daily events are tested.

Event frequency: LS subjects reported a mean of 5.4 events (s.d. $=5.5$ ) and HS subjects 9.0 events (s.d. = 8.6) during the five days of Experience Sampling (MannWhitney U test, p<.05). Seven subjects (all LS) reported no event at all. Event reports as a percentage of total beeps averaged $12.1 \%$ for the LS group (s.d. $=11.5$ ) versus $21.9 \%$ for the HS group (s.d. = 19.5) (Mann-Whitney U test, p<.01). Since a major category of reported events was work-related, we expected more frequent reports of stressful events during weekdays than on weekends. Multivariate analyses of variance for repeated measures indicated a highly significant main effect on event frequency ( $\%$ of total beeps) for the within-subject factor day ( $S$ days) $(\mathrm{F}(4,340)=16.02 ; \mathrm{p}<.001)$, as well as for the between-subject factor group (HS.LS) $(\mathrm{F}(1,85)=8.02 ; \mathrm{p}<.01)$. The interaction of day by group was also significant $(\mathrm{F}(4,340)=3.45 ; \mathrm{p}<.01)$. As can be seen in Figure 3.7., subjects indeed reported more stressful events on workdays than on weekend days. The HS subjects reported more events than LS subjects on each day of the week, but especially so on Thursday, Saturday and Monday. On Sunday, both groups reported relatively few events.

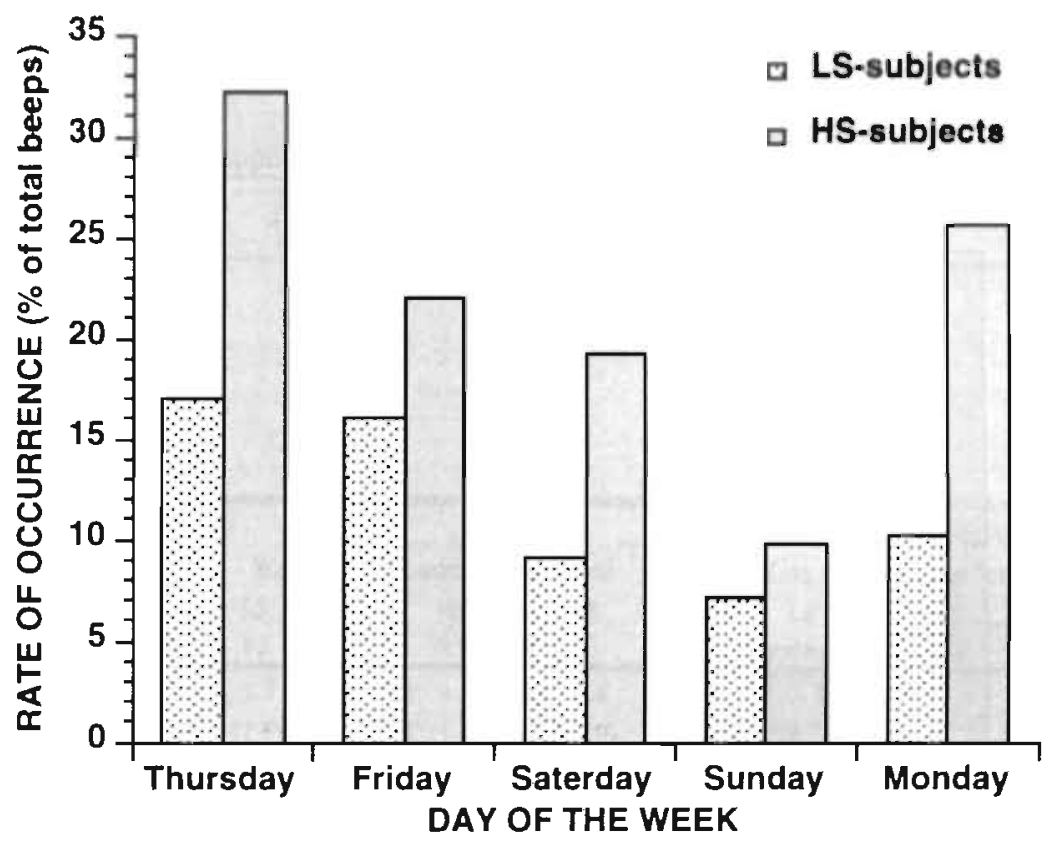

Figure 3.7. Frequency' of stressful daily events for HS and LS groups by day of the week.

Event context: As shown in Figure 3.8, HS subjects reported significantly more stressful work and network events than LS subjects, across all beeps. LS subjects, on the other hand, reported significantly more transportation events. HS subjects aiso reported significantly more social interaction events than LS subjects $(7.0 \%$ versus $3.2 \%, \mathrm{p}<.01)$, but not significantly more task demands $(6.9 \%$ versus $4.4 \%, \mathrm{~ns})$. As 
shown in Table 3.11, task demands as compared to social interactions are reported with equal frequency at home and at work in both groups. Both groups reported more task demands and more social interactions at work than at home. HS subjects reported significantly more events entailing negative social interactions (but not task demands) than LS subjects, both at home and at work.

Social context of event: We also coded who was involved in a reported event (social context) using the following categories: alone, household members, non-resident family, friends, colleagues, neighbours/acquaintances, and strangers (see Chapter 2 'Coding of ESM measures' section). HS subjects reported significantly more daily events concerning household members $(4.1 \%$ versus $1.6 \%, \mathrm{p}<.01)$, colleagues $(4.0 \%$ versus $2.5 \%, \mathrm{p}<.05)$, and other acquaintances $(1.6 \%$ versus $0.5 \%, \mathrm{p}<.05)$ than LS subjects across all beeps. There were no group differences for the categories alone, non-resident family, friends or strangers. Within the category household members, HS subjects reported significantly more events concerning partner (1.6\% versus $0.8 \%$, $\mathrm{p}<.01)$ and children $(2.6 \%$ versus $0.8 \%, \mathrm{p}<.01)$.

Next, we examined who was involved in events coded specifically as social interactions. In both groups, most negative interactions were with colleagues. Although such events were relatively rare, HS subjects had significantly more negative social interactions with colleagues $(3.3 \%$ versus $1.9 \%, \mathrm{p}<.01)$ and with the partner $(1.1 \%$ versus $0.3 \%, \mathrm{p}<.05)$, across all beeps.

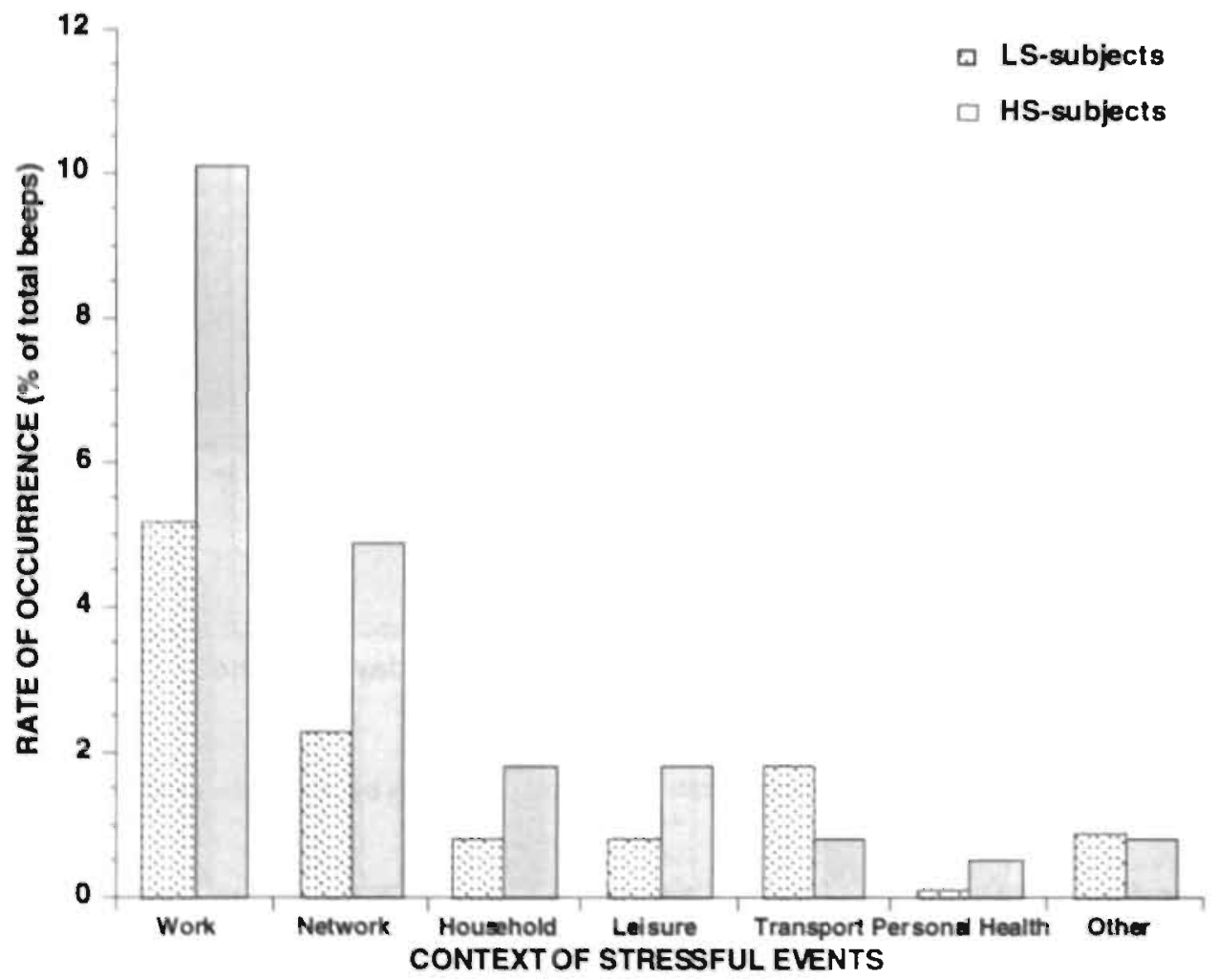

Figure 3.8. Frequency of stressful daily events for HS and LS groups by context. 
Table 3.11. Mean rate of occurrence $(+\mathrm{sd})$ of task demands and social interactions at home and at work (\% of total beeps).

\begin{tabular}{|c|c|c|c|}
\hline & $\begin{array}{l}\mathrm{LS} \\
\mathrm{n}=46\end{array}$ & $\begin{array}{l}\mathrm{HS} \\
\mathrm{n}=42\end{array}$ & $\begin{array}{l}\text { p-value } \\
\text { (2-tailed) }\end{array}$ \\
\hline \multicolumn{4}{|l|}{ at Home } \\
\hline Task demand & $2.6 \%(6.0)$ & $3.5 \%(5.8)$ & ns \\
\hline Social interaction & $1.8 \%(4.4)$ & $4.3 \%(7.5)$ & .05 \\
\hline \multicolumn{4}{|l|}{ at Work } \\
\hline Task demand & $7.3 \%(1[.4)$ & $12.4 \%(16.2)$ & ns \\
\hline Social interaction & $7.7 \%(10.4)$ & $13.2 \%(14.0)$ & .01 \\
\hline
\end{tabular}

Event appraisals: On average. HS subjects appraised events as more stressful and less controllable than LS subjects (Table 3.12). There were no differences between groups in the appraisals of event severity, importance, predictability, and chronicity. Event stressfulness and controllability were further examined for different types of events (work, network, household, leisure, social interactions, task demands). Statistical tests were done only for work events, task demands, and social interactions, because of the small sample sizes related to the other types of events. Results indicated that work events were significantly more stressful for HS than for LS subjects (see Table 3.12.).

Table 3.12. Mean event appraisals (+sd) for HS and LS subjects.

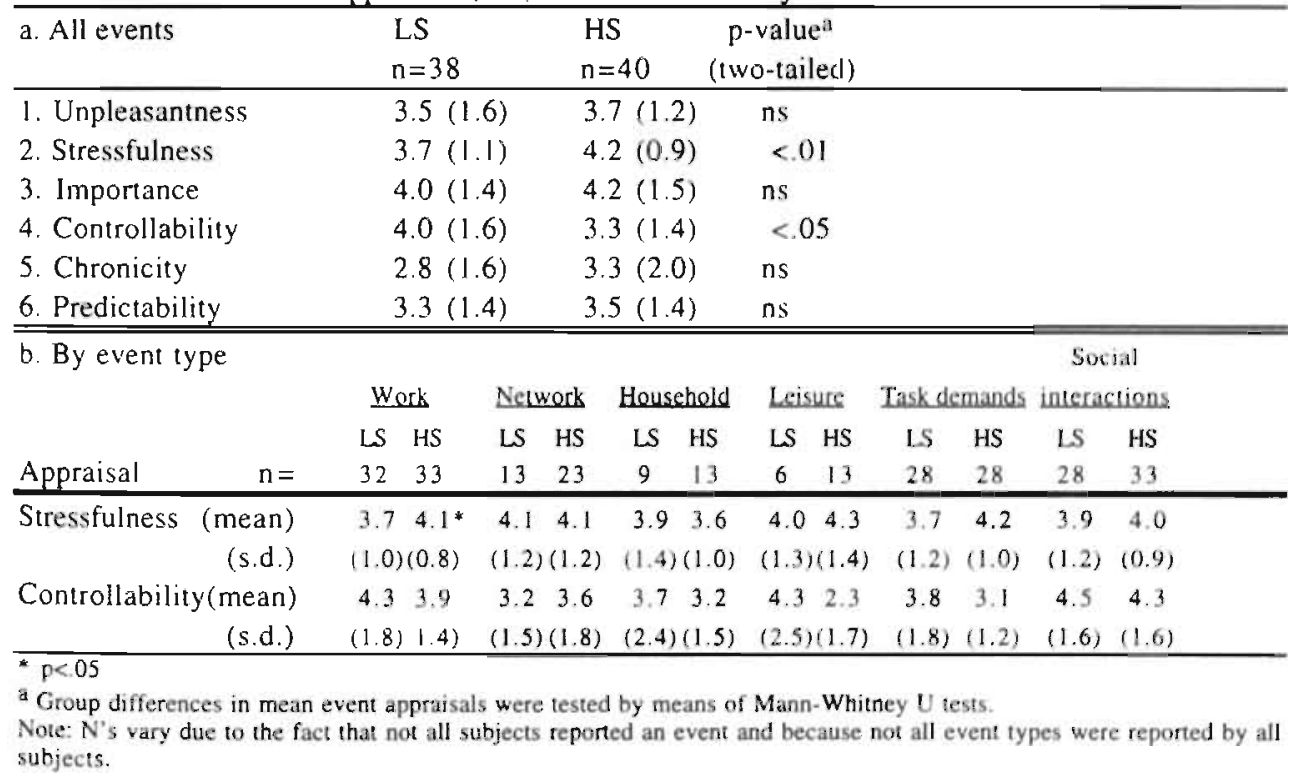

\section{DISCUSSION}

The aim of this chapter was to provide descriptive data on both quantitative and qualitative aspects of stressful events and activities in daily life, to increase our understanding of the construct stress as expressed in daily life. Using ESM, repeated 
measurements of stressful events, activities and their characteristics were made several times a day on several successive days in a sample of male white collar workers with varying stress levels.

Subjects reported a stressful event on $17 \%$ of all beeps. Whether this figure is high or low cannot be said; the frequency of reported stressful daily events cannot be compared to other studies since only a few have used an open-ended format to elicit events (Eckenrode, 1984; Rehm, 1978), and when used, different time-frames (events reported at the end of the day) together with different study populations (students, women) make comparisons irrelevant. Results indicated that in our sample the major source of stress in daily life was work, followed by the social network. These stress contexts make sense, given the developmental and demographic profile of the subjects. their most important social roles were those of employee, husband, and father. In line with the transactional definition of minor stressful events (Lazarus \& Folkman, 1984b, p.376), stressful daily events in this study can be characterized by its salience (importance) and unpleasantness. The finding that unpredictable and uncontrollable events were especially unpleasant is in accordance with results of many laboratory studies (Katz \& Wykes, 1985; Miller, 1979; Solomon, Holmes, \& McCaul, 1980; Thompson, 1981). Although subjective appraisals, as described above, appear to be important factors for understanding the stressfulness of daily events or situations, other dimensions should also be investigated for their relevance to the experience of stressful events, for example the experience of loss associated with an event (e.g. loss of a person, object, status, role, self-esteem) (Brown, 1989), future threat or feelings of uncertainty about how to cope with the event.

As might be expected, a high perceived stress level was related to higher scores on cross-sectional measures of psychological (anxiety, depression, anger) and psychosomatic (fatigue, depression, backache, headache, and insomnia) complaints, to the personality traits negativism, somatization, timidity and psychopathology, and to the more frequent use of passive and less frequent use of active (problem-focused) coping styles. Although HS subjects did not experience significantly more life events in the past year, they did report more chronic difficulties. Since HS subjects were healthy and actively employed, the pronounced group differences are somewhat surprising. From these data, the HS group emerges as a group of vulnerable, somewhat neurotic individuals, troubled by various psychological and psychosomatic complaints, experiencing several chronic environmental and relational difficulties, and using inadequate coping styles. An interesting question in this regard is how these characteristics would influence their lives in the future, if we could follow them, for example, another 10 years. In addition to the subjective measures, we would need to measure objective indices of health and work status (e.g. frequency of absenteeism from work, number of visits to health professionals, health records), to gain insight into the long-term consequences of high perceived stress. The pronounced group differences may also be related to the fact that the LS group contained subjects which seem to be more stress-resistant than on average. Compared to norm scores, LS subjects scored below average on psychological complaints, below average on the personality traits negativism, somatization, timidity, psychopathology, and average on extraversion (NPV). LS subjects also used more adequate coping styles indicated by high (norm) scores on problem-focused coping and low scores on depressive reaction.

Subjects with more chronic difficulties, but not with more life-events, reported significantly more daily events. Since chronic difficulties are much more dependent on the subjective judgment of a person than the more objective life-events, it is not 
surprising that chronic difficulties were related to the highly subjective stressful daily events. With respect to the major life events, it should be noted that life events, as occurred in the past year, were very rare in our sample, making it difficult to find an association between life events and daily events. HS subjects did report more life events, but not significantly more. Chronic difficulties seem not only related to increased perceptions of minor demands, but, as has been suggested for major life events (Kaplan, 1979), also seem related to the affective significance of minor events for the person, since with increasing chronic difficulties, events were rated as increasingly unpleasant. Subjects with high levels of chronic difficulties may experience events that are of themselves more unpleasant, but it is also possible that events. that relate to specific chronic difficulties (for example " an argument with spouse about household chores in the case of chronic marital problems) may be more salient and threatening, and thereby more unpleasant, for the person than the same event occurring in the absence of a chronic problem.

With respect to daily activities, the two groups had similar patterns of time use, and both groups perceived work activities as the most challenging but also requiring. the most effort. HS subjects did differ from LS subjects in how they evaluated their activities. Although in absolute terms HS subjects reported considerable enjoyment in various activities, perceived themselves to be quite skillful, and did not feel that activities required much effort to perform, they evaluated activities significantly less positively on these dimensions than LS subjects. This indicates that perceived stress levels are not only expressed in stressful events, but aiso in the evaluation and experience of daily activities. The differences between stress groups in the appraisal of activities were not restricted to a specific activity but were observed in several contexts.

With respect to daily events, work emerged as the major source of stress. Overall, HS subjects reported twice as many stressful daily events; they were also more likely to report events during work days than LS subjects. They reported twice as many work-related stressful events as LS subjects, as well as significantly more social network events. Within these life domains, HS subjects reported significantly more events related to colleagues, partner, children, and other acquaintances. In addition to an increased (subjective) exposure to stressful daily events, HS subjects appraised events as more stressful and less controllable. Although no group differences were found for task demands, HS subjects had significantly more negative social interactions, both at work and at home, and especially so with their colleagues and their partners. These results suggest that, besides the overall frequency of events, what differentiates HS from LS subjects may not so much be found in the stresses of family or work overloads, time-pressure, recurrent deadlines, but in stress resulting from problems with interpersonal relationships. Although social interaction events were reported too infrequently for firm conclusions, the finding of increased stressful interpersonal events in HS subjects is interesting in light of reports that interpersonal conflicts provoke the strongest negative mood states (Bolger, Delongis, Kessler, \& Wethington, 1989b; Bolger \& Schilling, 1991; Clark \& Watson, 1988; Repetti, 1993). In addition, stressful interpersonal events have been found to be related to neuroticism (Bolger \& Schilling, 1991). It would be interesting to investigate if and through which mechanisms interpersonal conflicts are related to differences in wellbeing between HS and LS subjects. A limitation of the current study is that we did not assess positive events and are thus unable to address the important question of the extent to which positive events influence well-being or compensate for the effects of negative events in HS and LS groups. 
An important next step toward understanding the relationship between stress in daily life and individual health outcomes is to examine short-term psychological and physiological responses to daily events. As we have seen, daily events are heterogeneous with respect to both the context in which they occur and the way in which they are appraised by the individual. Such characteristics of events may be equally if not more important determinants of immediate responses than simple event occurrence. Chapter 4 describes the impact of stressful daily events and their characteristics on negative and positive mood states. 


\section{Chapter 4}

\section{THE EFFECTS OF PERCEIVED STRESS AND STRESSFUL EVENTS ON MOOD STATES}

Submitted for publication

Marleen van Eck ${ }^{1}$. Nancy Nicolson ${ }^{1}$, Hans Berkhof ${ }^{2}$

1 Department of Psychiatry \& Neuropsychology, Social Psychiatry and Psychiatric Epidemiology section, University of Limburg, the Netherlands

2 Department of Statistics and Measurement Theory, University of Groningen, the Netherlands 



\begin{abstract}
This study examined the effects of perceived stress on the relationship between stressful daily events and mood states. Forty 'high stress' (HS) and 46 "low stress' (LS) subjects were selected on the basis of Perceived Stress Scale scores from a. sample of male white collar workers. Subjects completed Experience Sampling self-reports ten times a day over five consecutive days. Multilevel analysis revealed that current stressful events were associated with significant increases in negative mood (Agitation), but not with significant changes in positive mood. HS subjects showed significantly stronger negative mood reactivity (both Agitation and Negative Affect) in response to current events than LS subjects. The effects of events on negative mood persisted for at least $90 \mathrm{~min}$ after an event was reported, both in HS and LS subjects. A future event was associated with higher current Agitation in both subject groups, but with higher Negative Affect in HS subjects only. Results suggest increased vulnerability to daily events in HS subjects, and highlight the need for longitudinal studies for establishing long-term health effects
\end{abstract}

\title{
INTRODUCTION
}

In an effort to understand the effects of environmental stressors on health and psychological distress, there has been a shift from studying the effects of major life events to the effects of minor daily events, or daily hassles (Kanner et al., 1981: Zautra et al., 1988). During the last decade it has become increasingly clear that daily life stress is associated with lower psychological well-being and increased somatic symptomatology (DeLongis et al., 1988; Stone et al., 1993). The recent emphasis on daily life events is due, in part, to the extensive criticism directed at the traditional approaches to the study of psychosocial stress. An important drawback of the major life event approach has been the frequent reliance on retrospective research designs, with related problems of biased recall and forgetting (e.g. Bower, 1981). Additionally, the retrospective assessment of life events over several months obscures the temporal, dynamic interplay between environmental demands, perceptions of demands, and outcomes (Stone \& Shiffman, 1992), a central tenet of current transactional theories of stress and coping (Lazarus \& Folkman, 1984a). End-of-the-day assessments of minor events have been designed to address these problems. Measurement biases can be minimized by measuring the variables related to the stress process closer to the moment they actually occur. Prospective daily designs also offer better opportunities to identify the temporal patterns between events and outcomes, a first step in establishing causal relationships.

But even within the time frame of a day, much of the temporal interplay between events, appraisals of events, and outcomes remains out of sight. In addition, forgetting of less salient events and a bias towards remembering more recent events is still likely to occur. Moreover, the recall of daily mood is probably influenced by the subject's mood at the time of recording (Stone et al., 1991). Same-day associations between daily stress and mood remain causally ambiguous: a bad mood, for example, may increase the likelihood that daily events will occur or will be perceived as stressful. Finally, previous studies suggest that the effects of minor stressful events may be largely confined to the day on which they occur (e.g. Bolger et al., 1989a; Rehm, 1978; Stone \& Neale, 1984). These problems all point to the need to narrow the time frame even further to clarify within-day patterns of association between stressful events and mood. 
In the current study, a prospective within-day design was used, with subjects recording event occurrence and mood states several times a day over several consecutive days (Experience Sampling Method), to examine the effects of daily events on mood. The major issue addressed concerns interindividual variability in the daily stress process. More specifically, we investigated how perceived stress, as a measure of chronic stress, translates into the day-to-day experience of and response to minor daily events. First, we examined whether subjects with a high perceived stress level experience more stressful events. Second, we investigated whether high perceived stress magnifies the effects of daily stressors on mood. Third, we investigated temporal patterns of mood reactivity to events. In this context we hypothesized that the mood responses of high stress subjects might be of a longer duration than those of low stress subjects, and that high stress subjects might show greater mood changes in anticipation of future events. Finally, we explored the relationship between event characteristics, such as the context in which an event occurred and event appraisals, and mood states.

End-of-day diary studies have demonstrated a same-day association between daily stress and negative mood (Affleck et al., 1994; Bolger et al., 1989a; Clark \& Watson, 1988; DeLongis et al., 1988; Eckenrode, 1984; Larsen et al., 1986; Lewinsohn \& Libet, 1972; Rehm, 1978; Stone \& Neale, 1984; Stone et al., 1993). The results with respect to positive mood are less clear. Many studies do not differentiate between positive and negative mood; in those that do, positive mood was either lower (Neale et al., 1987; Repetti, 1993; Stone \& Neale, 1984) or unchanged (Watson, 1988 ) on days when many stressful events occurred. Only one study has previously investigated event and mood associations within days (Marco \& Suls, 1993), and here, daily stress was accompanied by "worse mood". There is evidence for large individual differences in the magnitude and the direction of the association between daily stress and mood. Factors which contribute to such differences include selfesteem and self-complexity (Campbell et al., 1991), social support (Affleck et al., 1994; Barling \& Kryl, 1990; Caspi et al., 1987: DeLongis et al., 1988), recent major life events (Affleck et al., 1994; Caspi et al., 1987) and neuroticism (Affleck et al., 1994; Bolger \& Schilling, 1991; Marco \& Suls, 1993). Little research has been done on the effects of chronic stress or long-term difficulties. In a study by Caspi et al. (1987), the chronic stress of living in a low quality neighborhood exacerbated the immediate effects of daily events on mood and also increased the likelihood that daily stressors had an enduring effect on next day's mood.

In the present study, we investigated the effect of perceived stress level on the relationship between daily events and mood. Individuals with a high perceived stress level feel their life to be unpredictable, uncontrollable and overloaded, and can be seen as at risk for the development of stress-related somatic and mental health problems (Cohen et al., 1983), resulting, for instance, in increased sick leave from work. Personality factors probably play a very important role in perceived stress; research indicates that self-report measures of stress correlate significantly with measures of Negative Affectivity (NA) or neuroticism (Watson, 1988: Watson \& Pennebaker, 1989). Several studies demonstrated that individuals higher in NA exhibit increased negative mood reactivity to minor stressors (Affleck et al., 1994; Bolger \& Schilling, 1991: Marco \& Suls, 1993). Considering the above, we hypothesized that individuals with high perceived stress would show greater mood reactivity to daily events than those with low perceived stress. Differences in mood reactivity might for instance be due to differences in the appraisal of events or differences in coping strategies. In order to examine differences in mood reactivity to 
reported stressful events, it is important to control for possible differences in baseline mood between high and low stress subjects. This way we can distinguish between differences in overall levels of negative mood (when events are absent) and added effects of events.

Little is known about the duration of effects of daily events or about the influence of individual differences on this process, although this would be important for understanding the consequences of daily events and might possibly reveal mechanisms related to the longer-term effects of chronic stressful experiences (Eckenrode \& Bolger, 1995). Possibly only when negative moods persist following stressful events, in addition to a high frequency of events, negative health changes may follow (e.g. accumulation of distress). One interpretation for the persistence of negative mood after an event has ended is that coping has been unsuccessful or still ongoing (e.g. persistence of worrying about event). In the current study we investigated if an event reported one assessment earlier (from now on called "prior event") has an (enduring) effect on current mood, and whether perceived stress influences the relationship between prior event and current mood. Most studies have found no relationship between daily stress and mood on the following day (e.g. Lewinsohn \& Libet, 1972; Neale et al., 1987; Rehm, 1978; Stone \& Neale, 1984); in fact, two studies found that on days following stressful events, mood scores were actually better than on other stress free days (Bolger et al., 1989a; DeLongis et al., 1988). A possible explanation for these findings is that feelings of relief predominated after the resolution of stressful events. Two other studies did document worse moods on the day following a suessful event, but oniy in subjects with a low fevel of social support (Affleck et al., 1994; Caspi et al., 1987). In the only within-day study to date (Marco \& Suls, 1993) prior events had no effect on current mood. We also investigated whether future events have an effect on current mood states and if these effects vary with differences in perceived stress level. Future events may influence current moods due to an increased worrying or attention for future events (anticipation effect). Also for an adequate test of the influence of prior event on current mood state it is necessary to control for possible effects of a future event at $t+1$ on current mood states. In this way, we are able to differentiate between an effect on current mood state due to the influence of a prior event and one duc to an anticipated future event.

Of the enormous range of stressful events people experience in daily life, which categories of events have the strongest psychological impact? Results from end-of-the-day studies indicate that the psychological impact of daily events does indeed depend on the context in which they occur. In particular, undesirable events at work (i.e. feeling under a lot of pressure and having negative interactions with coworkers) were associated with both lower positive and higher negative moods (Repetti, 1993; Stone, 1987). In other studies, interpersonal conflicts were found to provoke the strongest negative mood states (Bolger et al., 1989a; Bolger \& Schilling, 1991; Clark \& Watson, 1988; Repetti, 1993). We therefore hypothesized that, within days, stressful work events, task overloads at work or at home, and negative social interaction events would show a larger effect on mood states than other types of events.

According to contemporary stress theory, the subjective appraisal of a stressful event may be more important than the occurrence of an event per se (Lazarus \& Folkman, 1984b). There is evidence for instance, that subjects' personal ratings of events improve prediction of outcomes such as anxiety, depression, negative affect, tension, and grade point average (Dewe, 1991; Peeters et al., 1995; Sarason et al., 
1978). Despite the theoretical importance of the appraisal process, little research has been done on appraisal dimensions of naturally occurring dailly events in relationship to mood. Results of one study where appraisals of daily events were investigated (Neale et al., 1987) led to the conclusion that a simple score based on the sum of event desirability ratings did as well in predicting mood as more complex appraisal measures. A limitation of this study was that the results were based on summary measures over four days; analysis of measurements within days might reveal more subtle relationships. On theoretical grounds, then, we expected that the more unpleasant and important, and the less predictable and controllable the event, the stronger the mood responses. In addition, we predicted that events that had occurred relatively frequently in the past would have less effect on mood than novel stressors.

\section{METHOD}

\section{Subjects}

Local industries and government agencies were approached via their personnel departments to participate in the study. A decision as to which categories of employees fell under the definition of 'white-collar' was made by each personnel department on the basis of standard job function descriptions. Questionnaires were distributed among these employees, accompanied by a letter explaining the goals of the study. Participation was voluntary, and care was taken to insure anonymity. 316 male employees from six different industries or agencies completed the screening questionnaire. The mean score on the Perceived Siress Scale (PSS) for this sample was 12.7 (s.d. 6.0), similar to US norms (mean 13.02, s.d. 6.45) (48).

Ninety-two subjects with scores in the upper or lower tertiles of the screening sample distribution (PSS-10 score $<10$ or 216 ) were recruited, excluding individuals who reported a history of serious chronic illness or treatment (past or current) for mental health problems. Exclusion criteria were reassessed during an initial interview, study aims and procedures were explained, and informed consent obtained. During subject intake, each 'high stress' (HS) subject was matched for age group, marital status, and household composition with a 'low stress' (LS) subject to insure that the two groups did not differ on demographic characteristics that might affect exposure to certain classes of daily stressors. Six subjects were later excluded from analysis: Four due to failure to meet ESM compliance criteria (see 'Daily Experience' section, below), one because he became so acutely stressed that he was unable to work during the sampling period, and another one was excluded due to missing data on the mood variables. Of the 86 remaining. subjects, 40 subjects comprised the 'high stress' (HS) group and 46 subjects the 'low stress' (LS) group. Mean age was 42.1 years (range 27 to 57 years). $89 \%$ were married, and $81 \%$ had children living at home.

\section{Assessment of perceived stress}

The 10-item version of the PSS was translated into Dutch. The PSS is a global measure of the degree to which situations in one's life are appraised as stressful. Items were designed to tap the extent to which individuals feel their life to be unpredictable, uncontrollable and overloaded. The items were rated on a 5-point frequency scale, ranging from 'never' $(0)$ to 'very often' (4) in the last month. Validity and reliability data have been reported by Cohen and others (Cantor, Norem, Langston, Zirkel, Fleeson, \& Cook-Flannagan, 1991: Cohen, 1986; Cohen et al., 1983; Cohen, Tyrrell, \& Smith, 1991). The mean of the two PSS scores was 7.3 (st.dev. 2.2) for the low stress group and 17.9 (st.dev. 3.3) for the high stress group. 


\section{ESMI procedure}

The Experience Sampling Method (ESM) (Csikszentmihalyi \& Larson, 1987: de Vries, 1992) was used to collect data from subjects at selected moments during their normal daily activities. Subjects received auditory signals ('beeps'), after which they filled in a questionnaire. After a 'briefing' session, in which all procedures were explained in detail and informed consent obtained, subjects were sampled for a period of five consecutive days (Thursday through Monday). A wristwatch was programmed to emit beeps ten times each day, at semi-random intervals of approximately 90 minutes between the hours of 8 am and 10 PM. Beeps were clustered around the midpoint of each time block (i.e., $8.15 \mathrm{am}, 9.45,11.15$, and so on), with the exact time sequence of beeps varied each day to decrease predictability. In a final 'debriefing' session, subjects were asked to clarify reasons for missing data. Compliance with the procedures was generally good. The criteria set for subject inclusion in the analysis (> 20 ESM reports completed within 20 minutes after being signalled and no missing data for entire days) were met by all but four subjects (two from each group). The remaining 88 subjects completed an average of $83 \%$ of all possible responses within the time limit, for an average of 41 responses per subject. HS and LS groups did not differ significantly in compliance rates ( 40.1 vs 42.3 responses per subject, Mann-Whitney U-test, p>.05). Compliance was lowest for the first beep (at approximately $8.15 \mathrm{am})$, with an average of $73 \%$ valid reports. On Saturdays, response rate for first beeps was $61 \%$, and on Sundays, $59 \% .74 \%$ of all missing and invalid responses on weekends could be attributed to the fact that subjects were still asleep when signalled.

\section{ESM measures of streseful evente and mood}

The ESM form contained open-ended questions concerning thought content (worries), the physical and social context, and what the individual was doing when signalled. The forms also included 7-point Likert scales (from 1 'not at all' to 7 'extremely') for rating aspects of thoughts, mood, physical well-being, individually defined (psycho)somatic complaints, current activity, and stressful events.

Stressful events: Subjects were asked to describe any stressful event or situation which may have taken place in the interval since the last ESM report, in order to obtain as complete as possible a record of each subject's stressful experiences. Although we refer to these as 'current' events. it should be noted that they took place during the interval between two beeps (i.e., within a time-interval of approximately 90 minutes) and were not necessarily still ongoing at the moment subjects were signalled. 'Prior' events were defined as events which were reported at beep $t-1$. while 'future' events were defined as events which were reported at beep $t+1$.

Event context: Subjects' descriptions of stressful daily events were coded according to context, with the categories work, network (events concerning family, friends and acquaintances), householdfinancial, leisure, personal health-somatic, personal health-psychological, transport, and other. The twelve events that were coded in one of the personal health categories were excluded from the analysis to avoid possible confounding with the mood outcome measures. Of all reported events, $48 \%$ work-related, with the next most common type (network events) representing $18 \%$ of the total events. For this reason, analysis differentiated between the contexts work (1) vs. non-work (0) events.

Some examples of reported work events are: "unclear $/$ vague assignment at work", 'too much to organize, not enough time', 'difficult conversation with boss about job performance', 'chairing a big meeting'. Reported non-work events included: 'having a fight with my wife about household duties'. 'conflict with spouse about how to raise our son', 'child who will not listen', and 'making dinner, child crying, other child taking a bath, and this all at the same time".

In addition, events were scored according to whether or not they involved a social interaction or a task demand (e.g. overloads, deadlines). The codes for work domain, interaction, 
and demand were not mutually exclusive; for example, an argument with the boss about a missed work deadline would be coded as present for each variable. ${ }^{2}$

Event appraisals: Subjects rated reported events on the following dimensions: unpleasantness, importance, predictability, controllability and frequency of prior occurrence. (chronicity).

Mood measures: The 17 ESM mood items were reduced to three mood measures, based on the results of a principal component analysis with varimax rotation, which accounted for $78 \%$ of the total variance when subject mean scores were used. Ratings on the items cheerful, satisfied, relaxed, energetic, self-assured, concentrated and enthusiastic were summed to form a 'Positive Affect' (PA) scale. (Cronbach's alpha $=.95$ ). Two separate components of negative mood were identified: 'Negative Affect (NA)', including the items depressed, anxious, worried, lonely, tired, and miserable (alpha $=87$ ), and 'Agitation $(\mathrm{Ag})$ ', with the items restless, irritated, hurried, and nervous (alpha $=.93$ ). The sums of the scale items were divided by the number of items in the scale, so that ali mood measures have ranges from i to 7 . Within-subject associations among the mood scales were investigated by first standardizing responses on each scale within a subject ( $\mathrm{z}$ scores) and then calculating Spearman rank correlations over all reports. The resulting correlations were low to negligible (NA - Ag: rho $(3569)=.21, \mathrm{p}<.001 ; \mathrm{NA}-\mathrm{PA}$ : rho (3564) $=$ $-.1 \mathrm{i}, \mathrm{p}<.001 ; \mathrm{Ag}-\mathrm{PA}$; rho $(3563)=-.07, \mathrm{p}<.001$; all tests one-tailed), supporting the notion that the three mood dimensions are relatively independent.

\section{Statistical analysis}

For the estimation of the effects of daily stress on mood we used the hierarchical linear model (HLM) or multilevel model (Bryk \& Raudenbush, 1987; Goldstein, 1987) using the programm MIXREG (Hedeker, 1993). The HLM is a variant of a multiple regression model, for the situation in which the data can be structured in a hierarchical way. As the beep-level observations are nested within persons, the HLM can be used to analyze the present data.

A major advantage of the HLM above more conventional methods like ANOVA for repeated measurements is that HLM deals flexibly with the problem of missing observations, which are inevitable in ESM datasets, and the observations do not need to be evenly spaced over the time interval (Gibbons, Hedeker, Elkin, Waternaux, Kraemer, Greenhouse, et al., 1993). Furthermore, the HLM allows for flexible specification of the dependency among the observations within the same person. In the mood dataset, the dependency consists of more than one component. First, as the average mood level is assumed to vary across persons, two observations are more similar if they are from the same person. Second, two mood observations from the same person tend to be more similar if they are taken at points close in time. For the sake of simplicity, many previous studies have ignored this autocorrelation. This biases the standard errors of the regression coefficients downwards and may consequently lead to incorrect test results. Another attractive feature of the HLM is that both time-varying and time-invariant covariates can be added simultaneously to the model (see below).

The two nesting levels present in the data will be called measurement level and person level. The variables added at the measurement level (e.g. events) vary with time, while the variables added at the person level represent individual characteristics (e.g. perceived stress level). The effect of a time-varying variable can be decomposed in two parts: a fixed effect that is constant across persons and a person-specific random effect that can vary from person to person.

2 The reliability of the coding system was assessed by comparing the classifications of 345 events by two independent coders. Interrater agreement was determined by means of Cohen's kappa. On the whole, the qualitative information could be classified with a high degree of agreement (Laundis \& Koch, 1977), especially for the content categories. The overall Kappa for content was .90. Kappa's for social interaction and for task demand were .73 and .65 , respectively. 
At the measurement level, the relationship between events and mood state for person $i$ can he explicitly modelled. Here, we describe a model with stressful event as the only explanatory variable. The model generalizes in an obvious way to the situation with more time-varying covariates. The model at the measurement level has the following form:

$$
(\text { Mood })_{i j}=\alpha_{i}+\beta_{i} *(\text { Event })_{i j}+\varepsilon_{i j}
$$

where $(\text { Mood })_{i j}$ is the mood state at the $j$-th measurement of person $i$, (Event) ${ }_{i j}$ indicates whether a stressful event occurred to person $i$ at the $j$-th measurement (dummy coded as 1 or 0 ), $\alpha_{i}$ is the intercept of person $i$, the regression coefficient $\beta_{i}$ is the reactivity of person $i$ to a stressful event. and the $\varepsilon_{i j}$ 's are normally distributed residual terms with mean zero and variance $\sigma^{2}$. The residual terms within a person are postulated to be first order autocorrelated. This means that the correlation between two residuals that lie $k$ time units apart equals $\rho^{k}$

The coefficients $\alpha_{i}$ and $\beta_{i}$ are allowed to vary across persons, as indicated by the person index $i$. Instead of modelling the variation across persons by estimating $\alpha_{i}$ and $\beta_{i}$ for each person, these coefficients are written as a function of person-specific covariates. The covariate perceived stress (dummy coded as 1 or 0 ) indicates whether an individual belongs to the high or to the low stress group. The parameters $\alpha_{i}$ and $\beta_{i}$ can be modelled at the person level as:

$$
\begin{aligned}
& \alpha_{i}=\gamma_{\alpha}+\delta_{\alpha} \text { (Perceived stress) } i+u_{\alpha, i} \\
& \beta_{i}=\gamma_{\beta}+\delta_{\beta} \text { (Perceived Stress) } i+u_{\beta, i}
\end{aligned}
$$

where the $\gamma^{\prime}$ 's and $\delta$ 's denote fixed effects, which are constant across persons. Since perceived stress might not explain all variation in the $\alpha_{i}$ 's and $\beta_{i}$ 's across persons, person-specific random terms $u_{\alpha, i}$ and $u_{\beta, i}$ were added to the righthand side of the above equations. The $u_{\alpha, i}$ and $u_{\beta, i}$ are assumed to be normally distributed with mean vector zero, variances $\sigma^{2} \alpha$ and $\sigma^{2} \beta$, and covariance $\sigma_{\alpha \beta}$.

Separate models were built to estimate effects on each of the three mood states; Negative Affect, Agitation, and Positive Affect. In each model, the explanatory variables were selected by a backward selection approach. This means that we started with a model that contained all possible explanatory variables and then excluded variables with a non-significant effect. Two-tailed significance of a fixed effect was tested by dividing the estimated effect by its standard error. This ratio is approximately normally distributed. For testing the significance of the random effects, we applied the likelihood ratio test (one-tailed) (Bryk \& Raudenbush, 1992). Variables with nonsignificant main effects were reiained in the model only when interaction effects were significant. Because the variables prior event (event at beep $t-I$ ) and future event (event at beep $t+l$ ) were missing at the first or the last observation, respectively, of each day, these observations were deleted from the data. This resulted in a reduction from 3452 to 2358 observations. To check that the deletion of observations did not lead to a significant change in the regression coefficients, we started by estimating each model on the basis of all observations, thus without the variables prior and future events. With one exception (for NA; see discussion), deletion of observations did not significantly change the values of the regression coefficients of the remaining variables. We therefore present only the final models, including the prior and future variables. For the eventcontext variable we used effect coding (for example, $0=$ no event, 1 = work event, -1 = non-work event). The event-appraisal variables were assigned the value zero if no event was reported. The other values of the event-appraisal variables were centered around the mean for each subject, so that observed effects represent within-person effects. 


\section{RESULTS}

High stress subjects reported stressful events more frequently and had, on average, higher negative affect and agitation and lower positive affect than low stress subjects (see Table 4.1.).

Table 4.1. Frequency's of stressful events and mood states in low and high stress groups.

\begin{tabular}{|c|c|c|c|}
\hline & $\frac{\text { Low PS }}{(n=46)}$ & $\underset{(n=40)}{\text { High PS }}$ & \\
\hline & mean (st.dev.) & mean (st.dev.) & $\frac{\text { p-value }^{\mathrm{i}}}{(2 \text {-tailed) }}$ \\
\hline $\begin{array}{l}\text { Frequency of stressful events }{ }^{2} \\
\text { Mood States }^{3}\end{array}$ & $12.3 \%(11.9)$ & $20.6 \%(17.8)$ & $<.01$ \\
\hline Positive Affect & $5.7(0.6)$ & $5.3(0.3)$ & $<.001$ \\
\hline Negative Affect & $1.2(0.1)$ & $1.4(0.4)$ & $<.001$ \\
\hline Agitation & $1.3(0.3)$ & $1.7(0.5)$ & $<.001$ \\
\hline
\end{tabular}

${ }^{1}$ Differences in frequency and mood states were tested by means of Mann-Whitney U tests

2 Stressful event reports as a percentage of total ESM reports

${ }^{3}$ Subject means, aggregated fover all ESM reports

For each mood state, results will bo presented in the folivwing orcier: firsi, we will describe the effect of stressful daily events on mood state (both fixed as well as random effect), and whether this effect was related to perceived stress. Second, the results on the relationship between prior and future events on the one hand and current mood state on the other hand will be reported, followed by the effect of perceived stress on this relationship. Third, the effects of event type and event appraisals will be described. For each step, the significant effects of variables will be presented first, followed by the non-significant effects.

\section{Effects of events on Negative Affect}

The effect of daily events on Negative Affect differed significantly between subjects (Random Effects CE in Table 4.2.). Although the occurrence of an event was followed by slight elevations in Negative Affect in both groups, the significant Current Event $\times$ Perceived Stress interaction indicates that stressful events increased Negative Affect mainly in HS subjects. This pattern is shown in Figure 4.1. There was also a positive association between the intercept variance and event variance (Covariance Intercept/CE in Table 4.2.); this indicates that controlling for perceived stress, subjects with the highest average scores on Negative Affect showed the greatest increases in this mood state following events.

A small but marginally significant main effect was present for prior event., showing that the effect of an event on Negative Affect persist on average longer than the interval between two ESM reports. Level of perceived stress did not influence the magnitude of this carryover effect. Similar to the pattern observed for current events, a stressful event occurring in the interval following the current report (future event) had no significant effect over all subjects, but in HS subjects, Negative Affect was elevated preceding a future event. 
Table 4.2. Multilevel Results for Negative Affect (NA).

\begin{tabular}{|c|c|c|c|}
\hline Fixed Effects & Estimate & S.E. & $\mathrm{p}<$ (one-tailed) \\
\hline Intercept (mean NA) & 1.140 & 0.041 & $* * *$ \\
\hline Perceived Stress Group (PS) & 0.170 & 0.055 & $\cdots$ \\
\hline Current Event (CE) & 0.046 & 0.033 & ns \\
\hline CE $\times$ PS & 0.151 & 0.046 & $\cdots$ \\
\hline Prior Event (PE $\left.{ }^{1}\right)$ & 0.032 & 0.018 & .07 \\
\hline Future Event $\left(\mathrm{SE}^{2}\right)$ & 0.030 & 0.026 & ns \\
\hline FE $\times$ PS & 0.066 & 0.037 & .07 \\
\hline Event Unpleasantness & 0.029 & 0.010 & $\cdots$ \\
\hline Controllability Event & -0.019 & 0.009 & - \\
\hline Chronicity Event & -0.019 & 0.010 & * \\
\hline
\end{tabular}

\begin{tabular}{|c|c|c|c|}
\hline Random Effects & Estimate & S.E. & $\mathrm{p}<$ \\
\hline \multicolumn{4}{|l|}{ Person level: } \\
\hline Var (Intercept) & 0.055 & 0.010 & $* *$ \\
\hline Var (Current Event) & 0.012 & 0.006 & • \\
\hline Cov (CE, Intercept) & 0.015 & 0.006 & $\bullet$ \\
\hline \multicolumn{4}{|l|}{ Measurement level: } \\
\hline Var (Residual term) & 0.078 & 0.002 & $* *$ \\
\hline
\end{tabular}

\footnotetext{
Autocorrelation

$0.443 \quad 0.021$

$\mathrm{n}=\mathbf{2 3 5 8}$ observations (including 342 events) nested within 85 subjects ( $39 \mathrm{HS}, 46 \mathrm{LS}$ )

1 event at $1-1 ;{ }^{2}$ event at $1+1 ;{ }^{*} p \leq .05 ;{ }^{* *} p \leq .01 ;{ }^{* \vec{*} *} p \leq .001$

Von-signitican fixed effecis:

Event ryps: Work, Social Interaction, Task Demands

Event appraisals: Importance, Predictability

Interdcrions: PE $\times$ PS

Nore. The variable 'time over days' (automatically estimated when autoconelations are corrected for) was excluded from Table $4.2,4.3$, and 4.4 since estimated effects were negligible (all $<-0.002$ ) and not relevant with regard to hypotheses being tested.
}

As expected, events that were rated as more unpleasant were associated with larger increases in NA, whereas events that were relatively controllable had smaller effects on NA than uncontrollable events. In addition, the more frequently a stressful event had occurred in the past ("chronicity"), the smaller the observed effect on NA. Further differentiation of events by context did not improve the model; in other words, work and non-work events did not differ in their effects on Negative Affect. With respect to the event appraisals, no significant effects were found for event importance or event predictability. 


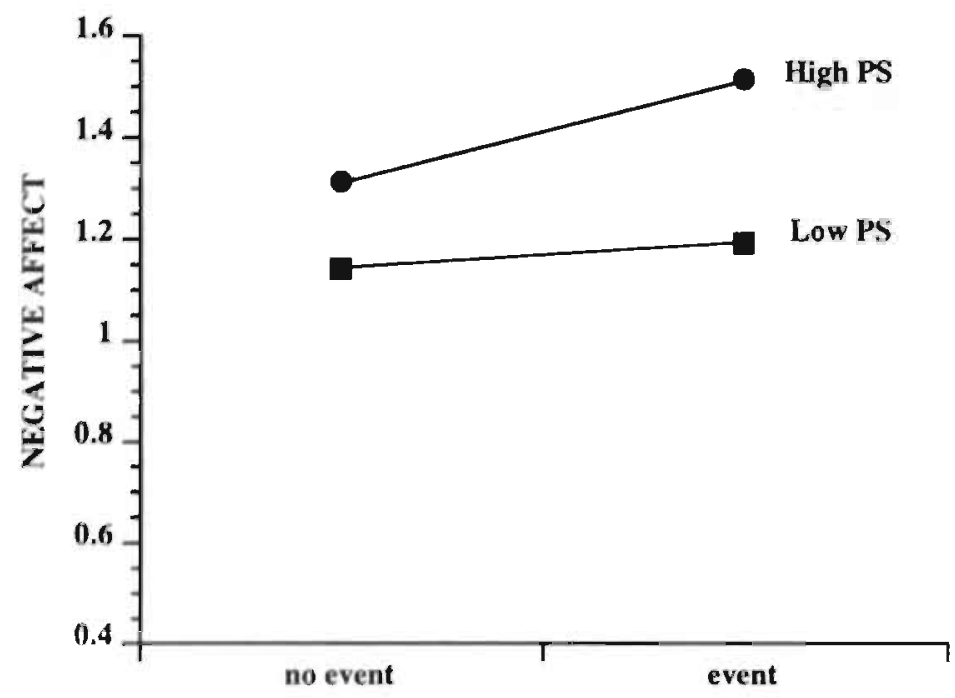

Figure 4.1. Modelled change in Negative Affect in 'high' and 'low' stress subjects in response to a stressful event (controlling for all other variables).

\section{Effects of events on Agitation}

In both HS and LS groups, current stiessful events were associated with significant elevations in Agitation levels (see Table 4.3.). Again we found a random effect for events, meaning that the influence of stressful events on Agitation differed significantly between subjects. Part of this between-subject variance could be explained by perceived stress level: HS subject reacted significantly more strongly to current stressful events than LS subjects, as evidenced by the significant Current Event $x$ Perceived Stress interaction. These results are presented graphically in Figure 4.2. As with Negative Affect, there was a positive association between the intercept variance and event variance (Covariance InterceptiCE in Table 4.3.), indicating that subjects with higher average scores on Agitation showed higher reactivity to current events (after controlling for perceived stress).

With respect to the temporal processes of mood reactivity, the significant effect of prior event on current Agitation indicates a carryover effect of events on mood. The presence of a future event also had a significant positive effect: current Agitation was higher when a stressful event was reported in the interval following the current report. There was no evidence for a moderating effect of perceived stress level on these carryover or anticipation effects.

Current events characterized as task demands were associated with significant increases in Agitation compared to non-task demand events. With respect to the event appraisals we found that, similar to the findings for Negative Affect, when events were more unpleasant than average. Agitation was higher, whereas events that were more controllable than average had a smaller effect on Agitation. Whether or not an event involved a negative social interaction or was work-related had no specific effect on this mood variable. No additional effects were found for event importance, predictability or chronicity. 
Table 4.3. Multilevel Results for Agitation (Ag).

\begin{tabular}{|c|c|c|c|}
\hline Fixed Effects & Estimate & S.E. & ps (one-tailed) \\
\hline Intercept (mean Ag) & 1.247 & 0.058 & $\cdots$ \\
\hline Perceived Stress Group (PS) & 0.323 & 0.077 & $\cdots$ \\
\hline Current Event (CE) & 0.373 & 0.098 & $\cdots$ \\
\hline CE $\times$ PS & 0.433 & 0.140 & $*$ \\
\hline Prior Event (PE ${ }^{l}$ ) & 0.099 & 0.032 & $\cdots$ \\
\hline Future Event $\left(\mathrm{SE}^{2}\right)$ & 0.213 & 0.034 & $\cdots$ \\
\hline Task Demands & 0.132 & 0.033 & $\cdots$ \\
\hline Event Unpleasantness & 0.056 & 0.019 & $\cdots$ \\
\hline Controllability Event & -0.045 & 0.016 & $\cdots$ \\
\hline
\end{tabular}

Random Effects

Estimate S.E. $\mathrm{p}<$

Person level:

$\begin{array}{llll}\text { Var (Intercept) } & 0.101 & 0.019 & * * \\ \text { Var (Current Event) } & 0.255 & 0.058 & * * \\ \text { Cov (CE, Intercept) } & 0.049 & 0.025 & *\end{array}$

Measurement level:

Var (Residual term)

$0.257 \quad 0.008$

Autocorrelation

$0.352 \quad 0.022$

$\mathrm{n}=2358$ observations (including 342 events) nested within 85 subjects ( $39 \mathrm{HS}, 46 \mathrm{~L}$ )

${ }^{1}$ event at $t-1 ;{ }^{2}$ event at $1+1 ;{ }^{*}$ ps.05; ${ }^{* *}$ p $\leq .01 ;{ }^{* * *}$ p $\leq .001$

Non-significant fixed effects:

Event lype: Work, Social Interaction

Event appraisals: Importance, Predictability, Chronicity

Interactions: $\mathrm{PE} \times \mathrm{PS}$. FE $\times$ PS

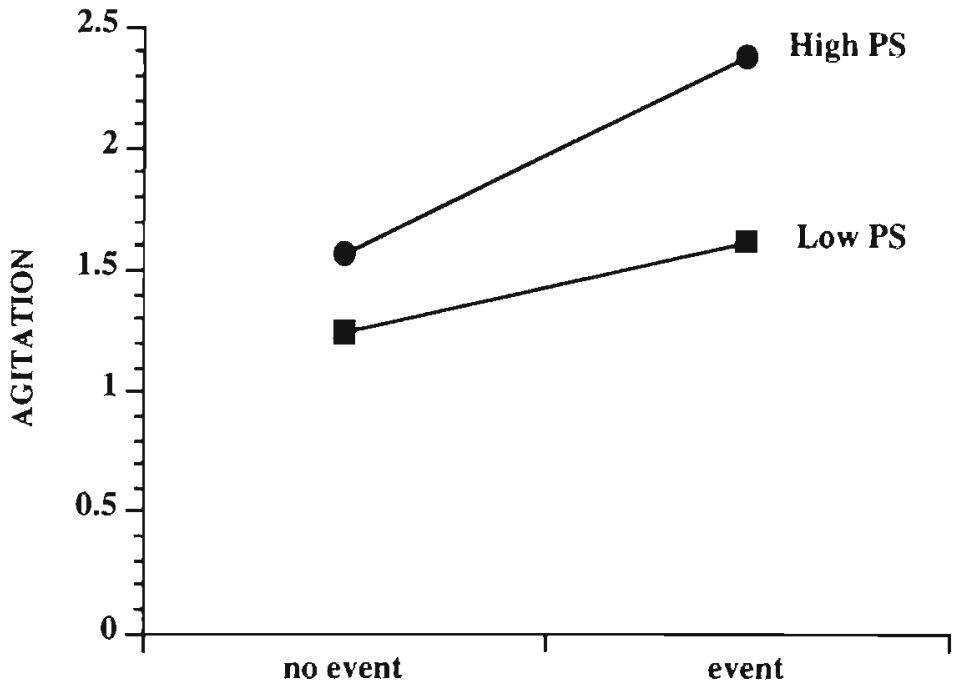

Figure 4.2. Modelled change in Agitation in 'high' and 'low' stress subjects in response to a stressful event (controlling for all other variables). 


\section{Effects of events on Positive Affect}

The multilevel results for Positive Affect are shown in Table 4.4. Occurrence of. a stressful event was not associated with any change in Positive Affect in either HS or LS subjects. In addition, no significant association was found between intercept variance and event variance (Covariance Intercept/CE; see Table 4.4.).

However, a carryover effect for a prior event on current Positive Affect was found: current positive mood was less positive when preceded by a prior event. The significant Prior Event x Perceived Stress interaction indicated a small rebound effect for HS subjects: when a prior event was present, HS subjects had relatively higher current Positive Affect than when a prior event was absent. A future event had no observable effect on current Positive Affect, regardless of perceived stress level. Following events that were more unpleasant than average, a significant decrease in Positive Affect was observed. Positive Affect was no more likely to change in response to a work than to a non-work event, or following a social interaction or a task demand event when compared to no social interaction and non-task demand events.

Table 4.4. Multilevel Results for Positive Affect (PA)

\begin{tabular}{lrcc}
\hline Fixed Effects & Estimate & \multicolumn{1}{c}{ S.E. } & $\mathrm{p}<$ (one-tailed) \\
\hline Intercept (mean PA) & 5.790 & 0.076 & $*$ \\
Perceived Stress Group (PS) & -0.409 & 0.108 & \\
Current Event (CE) & -0.038 & 0.029 & $\mathrm{~ns}$ \\
Prior Event (PE ${ }^{1}$ ) & -0.085 & 0.037 & $*$ \\
$\quad$ PE x PS & 0.101 & 0.052 & $*$ \\
$\quad$ Event Unpleasantness & -0.044 & 0.015 & $* *$
\end{tabular}

\begin{tabular}{lrrr} 
Random Effects & Estimate & S.E. & $\mathrm{p}<$ \\
\hline Person level: & & & \\
$\quad$ Var (Intercept) & 0.241 & 0.039 & $* * *$ \\
$\quad$ Vat (Current Event) & 0.006 & 0.009 & $\mathrm{~ns}$ \\
$\quad$ Cov (CE. Intercept) & -0.017 & 0.015 & $\mathrm{~ns}$ \\
Measurement level: & & & \\
$\quad$ Var (Residual term) & 0.175 & 0.005 & $* *$ \\
& & & \\
\hline Autocorrelation & 0.266 & 0.023 & $* * *$
\end{tabular}

$\mathrm{n}=2358$ observations (including 342 events) nested within 85 subjects ( 39 HS, 46 L.S)

1 event at t-1: "ps.05: "* ps.01: "*at ps.001

Non-significant fixed effects:

Event type: Work, Social Interaction, Task Demands

Event appraisals: Importance. Predictability, Chronicity, Controllability

Future Event

Interactions: $\mathrm{CE} \times \mathrm{PS}, \mathrm{FE} \times \mathrm{PS}$ 


\section{DISCUSSION}

Our main objectives were to investigate whether stressful daily events resulted in short-term changes in mood states, and whether differences in perceived stress level were related to the magnitude of mood changes. Results differed for each of the three mood dimensions investigated. With respect to Agitation, we found that daily events were associated with higher levels of Agitation in both HS and LS groups, with HS subjects showing the strongest mood reactivity. For Negative Affect, events were associated with worse moods in HS subjects only. Current events were not associated with changes in Positive Affect.

These results are consistent with those found in individuals scoring high in trait negative affectivity or neuroticism. Trait negative affectivity has not only been linked to higher overall levels of negative mood (Watson \& Clark, 1984) but also to increased reported exposure to stressful events (e.g. Bolger \& Schilling, 1991: Martin, Ward, \& Clark, 1983) and to increased mood reactivity to stressors (e.g. Bolger \& Schilling, 1991; Eysenck \& Eysenck, 1985; Marco \& Suls, 1993). Level of perceived stress was indeed strongly correlated with trait anxiety in the present study (rho (85) $=.77, \mathrm{p}<.001$ ), and HS subjects not only reported more stressful events, but also higher negative mood levels across all situations and stronger negative mood reactivity to events. Since other analyses indicated that the events reported by HS subjects were rated as no more severe than events reported by LS subjects (mean intensity of events in HS subjects is 3.5 \{st.dev. $=1.3$ \} versus $3.2\{$ st.dev. $=\{.7\}$ in LS subjects; ns), differences in appraised severity of events cannot explain the observed differences in mood responses. A possible explanation for increased mood reactivity among $\mathrm{HS}$ subjects is the use of less effective coping strategies (see (Bolger, 1990)). In our study, as in all studies based on self-report information, we cannot determine whether HS subjects were actually exposed to more stressful situations, or whether they were more likely to perceive events as stressful, had a heightened recall of negative situations, or were more likely to generate stressful situations, for example by failing to anticipate and thereby prevent events from happening. Laboratory studies have shown that neuroticism, which is significantly related to self-report measures of stress (Watson \& Pennebaker, 1989), increases the recall of negatively toned information (Martin et al., 1983), thereby possibly accounting for some of the positive association that has been found between neuroticism and exposure to stressors (e.g. Fergusson \& Horwood, 1987). Contrary to these reports, neuroticism was not related to increased event reporting in a recent within-day field study (Marco \& Suls, 1993). It seems possible that recall biases play less a role when the time intervals between assessments are short. One way of getting more objective event measures is to obtain confirmation of event occurrence from partners (Stone et al., 1991); this option is not realistic, however, when (as in our study) a large percentage of events occur in the workplace. Although it might be possible to obtain confirmation of event occurrence from co-workers or closest colleagues, this option does not seem very practical. In addition, many events (for example, demand situations) are very difficult to observe.

In LS subjects, we found that current events were associated with higher Agitation levels but not with higher Negative Affect levels. Given the specific items of the Agitation scale (restless, irritated, hurried and nervous), it makes sense that this mood scale would be especially sensitive to short-term effects of daily stressors. The Negative Affect scale items (depressed, anxious, worried, lonely, tired and miserable) are more likely to reflect longer term or intense reactions. HS subjects seem to react in 
a more passive and depressive way to stressful events then LS subjects, which may relate to differences in coping strategies, but also to the more chronic nature of stress in HS subjects ('giving up').

In contrast to results of a number of end-of-day diary studies (Neale et. al., 1987; Repetti, 1993; Stone \& Neale, 1984), current stressful events did not lower Positive Affect in the present study. Differences in mood scales and frequency in assessments might account for the lack of an effect of current events on Positive Affect. Associations between stressful events and mood do not have to be the same within and across days. It is possible that an accumulation of several events experienced during the day lowers PA at the end of the day, but that the occurrence of one stressful event does not have an irnmediate effect on Positive. Affect. Lack. of an association between stressful events and Positive Affect is more in line with several studies that suggest that Positive Affect and Negative Affect reflect two separate affective systems and were Positive Affect was found to be unresponsive to perceived daily stress but increased in relation to pleasant social events (Clark. \& Watson, 1988; Kanner et al., 1981; Watson, 1988). A possibility is that people's positive moods are not easily affected by minor stressors, but only, as suggested by the current findings, when events are more unpleasant than average.

To increase our knowledge about how long minor daily events continue to exert their influence on mood we investigated whether stressful events reported on average 90 minutes earlier still influenced current. mood states. Events at beep (t-I) were indeed associated with a persistent elevation in Agitation at beep (t0) and a marginally significant elevation in Negative Affect. In other words, the effects of events on mood states persisted for at least 90 minutes (the average interval between two ESM reports) after a stressful event was reported at (t0). These carryover effects for the negative moods were equivalent in both HS and LS groups. The results contrast with those of Marco and Suls (1993), who found no 'carryover' of the negative mood effects of prior events. Two methodological differences between the two studies may explain the inconsistencies: firstly, Marco and Suls' negative mood scale was composed of different items (tense, unhappy, and angry), and secondly, with eight beeps per day, time intervals between successive reports would have been somewhat longer on average than the 90 minute interval in the current study. Here, the occurrence of a future event was accompanied by higher current Agitation in both HS and LS subjects, but by higher Negative Affect in HS subjects only. The: effect sizes for subsequent events were larger than for prior events (especially for Agitation, and, in HS subjects. Negative Affect), indicating that a future event influenced current negative mood more than prior events. These results confirm the common observation that the anticipation of an event can increase current negative mood. More detailed analysis are necessary to determine whether anticipation effects are limited to certain types of events or certain event appraisals. This was beyond the scope of the present study.

Of the various types of events (work vs non-work events, social interaction vs non-social events and, task demands vs non-task demands), we found that task. demand situations (e.g. overloads, time pressure or deadlines at home or at work) were accompanied by significant higher Agitation levels. This association makes intuitive sense, especially in a white-collar sample. It is not clear, however, why we failed to replicate the finding in several other studies of a strong effect of interpersonal conflicts on negative mood (Bolger et al., 1989a; Bolger \& Schilling, 1991: Clark \& Watson, 1988; Repetti, 1993). The fact that these earlier studies investigated the relationship between interpersonal conflicts and mood across days. 
may in part explain the difference. For instance, the occurrence of repeated social interaction events during the day may be necessary to have a measurable effect on mood. Moreover, retrospective biases may have a greater influence in daily than in within-day studies; for example, a bad mood at the end of the day may be ascribed to a interpersonal conflict earlier that day. Another factor may be that we have coded events as interaction events ourselves, while in the other studies subjects indicated on a checklist whether a personal conflict had occurred or not. It is possible that interpersonal conflicts indicated by subjects on a checklist are of a more serious nature than the events coded by us. Finally, the loss of a considerable number of events reported due to the inclusion of the prior and future event variables (538 events vs. 342 events) may have resulted in a low statistical power. This is supported by the fact that in the full dataset (without the lagged variables), social interaction events and task demands did significantly increase Negative Affect.

Individuals' subjective appraisals of daily events had additional effects on mood responses. The more unpleasant an event was rated, the greater its effect on Agitation and Negative Affect, whereas only events that were more unpleasant than average were associated with lower Positive Affect. The perception of having control over a stressful event lowered its effect on both Agitation and Negative Affect levels. Negative Affect levels were also lower when an event had occurred more frequently than average in the past. No significant effects were found for event importance or event predictability. This does not necessarily mean that these variables are not important. Firstly, within-subject samples of events may have been too heterogeneous or too small in the current study to reveal consistent and reliable effects of all the specific appraisal variables, and secondly, since the appraisal variables were to some degree correlated with each other, the strongest predictor variables will have masked the weaker ones when all variables were entered into the model simultaneously.

Some caveats should be mentioned with respect to interpretation and generalization of these results. Firstly, subjects were well-educated, white collar males; their assessments of what constitutes a 'stressful' event or situation is not necessarily based on the same criteria as other groups (for example females, blue collar workers, students, or retired people) might use. The same applies to their mood ratings. Men tend to report lower, less extreme levels of negative emotions as well as positive emotions than women (Cameron, 1975; Diener, Sandvik, \& Larsen, 1985), and daily stress has been found to be more upsetting to women than to men (Bolger et al., 1989a). Men also appear to expend greater effort in limiting emotional distress (at the expense of physiological distress), resulting in lower emotional reactivity (Gottman \& Levenson, 1988). We also cannot rule out the possibility that current mood influenced the reporting of recent stressful event occurrence. However, the finding that prior events were associated with persistent increases in Agitation and Negative Affect supports the assumption that events influenced mood and not vice versa. Another limitation is that we did not assess positive events and are thus unable to address the important question of the extent to which positive events compensate for, or neutralize, the effects of negative events in HS and LS groups.

In summary, results support the interpretation that individuals with high perceived stress are more vulnerable to 'daily hassles' and therefore may be at greater risk for the development of stress-related health problems. Individuals with high perceived stress levels were more trait anxious, more likely to experience daily stressors, higher in overall negative mood, and more reactive, in terms of negative. mood states, to daily events. This suggests a reciprocal relationship between perceived stress, events and mood reactivity, where increases in event exposure and 
high mood reactivity maintain the appraisal of high stress. Only longitudinal studies with prospective and repeated measurements of both trait and state measures, will be able to clarify causal pathways, including the long-term effects of stressful daily events. Analytic strategies will have to be used that can estimate the relative contribution of each predictor variable, since many variables related to the stress process tend to be highly intercorrelated to each other. Another important subject for future research is the investigation of why HS individuals or trait anxious individuals exhibit greater negative mood reactivity to minor events. Exploration of differences between HS and LS groups in the actual coping strategies used when confronted with stressful events seems to be a good start.

\section{ACKNOWLEDGEMENTS}

We thank C. Dijkman for assistance in all aspects of the study. We also thank Dr. D. Hedeker for his advice regarding the use of the MIXREG program. 


\section{Chapter 5}

\section{Cortisol and catecholamine excretion in relation to perceived stress}

Section S.I: Annals of Behavioral Medicine 16(3): 221-227 (1994)

Marleen van Eck ${ }^{1}$, Nancy Nicolson ${ }^{1}$

Department of Psychiatry \& Neuropsychology, Social Psychiatry and

Psychiatric Epidemiology section, University of Limburg, the Netherlands 
Chapter 5 addresses the relationship between perceived stress and salivary cortisol and urinary catecholamines levels in the context of everyday life. In general we test the hypothesis that high perceived stress is associated with both elevated cortisol as well as elevated catecholamine levels. In section 5.1, two groups of male white collar workers differing in levels of perceived stress were compared on subjective self-report measures of distress and on levels of salivary cortisol, as measured ten times a day over five days. Here, the effect of perceived stress on cortisol is tested, either in general, or during certain phases of the circadian cycle or certain days of the week (work versus non-work days. In section 5.2, the same groups of subjects were compared on 14-hour levels of catecholamines. Urine samples were collected twice overnight, one after a work day and one after a weekend day. Here, we test the effect of perceived stress on catecholamine levels, either in general or during certain days of the week (work versus non-workdays). 



\title{
Section 5.1
}

\section{Perceived stress and salivary cortisol in daily life}

\begin{abstract}
Clarifying the nature of endocrine responses to chronic or intermittent stress in daily life requires repeated measurements of stress, hormone levels and emotional states. In this study, 42 'high stress' (HS) and 46 'low stress' (LS) subjects were selected on the basis of Perceived Stress Scale scores from a larger sample of male white-collar workers. Subjects completed self-reports (Experience Sampling, ESM) and collected saliva samples for cortisol determination 10 times a day over 5 consecutive days, including 3 work and 2 non-work days. Here we test the hypothesis that high perceived stress is associated with elevated cortisol.

The HS group scored higher than the LS group on measures of trait anxiety, depression, anger and psychosomatic complaints, as well as on ESM measures of positive and negative affect and stressful daily events. Although negative affect was higher and stressful events more frequent on workdays than weekends, especially for HS subjects, no difference in workday vs. weekend cortisol levels was found in the subsample of subjects with sufficient data. On workdays, HS subjects had higher mean cortisol levels than LS subjects at each of the 10 sampling times between 8 am. and $10 \mathrm{pm}$ (repeated measures ANOVA, p<.02). Mean workday cortisol was correlated with higher trait anxiety, depression and the low arousal dimension of ESM negative affect.
\end{abstract}

\section{INTRODUCTION}

Over the last 20 years, a wide range of studies has established an association between psychosocial stress and a variety of psychological and physical disorders. The strength of these associations are usually small and inconsistent, but stressful life events, for instance, have been linked to depression, neurotic impairment and other psychological symptomatology (Brown \& Harris, 1989; Dohrenwend \& Dohrenwend, 1974; Dohrenwend \& Dohrenwend, 1978). Stress has also been linked empirically with increased susceptibility to infectious disease (Cohen et al., 1991), with other immune-related disorders like recurrences of herpes infections (Hoon, Hoon, Rand, Johnson, Hall, \& Edwards, 1991) and asthmatic exacerbations (Isenberg, Lehrer, \& Hochron, 1992), with coronary heart disease (Bassett, 1982), and with diabetes mellitus (Goetsch, Wiebe, Veltum, \& Van Dorsten, 1990). The diversity of stress concepts and research methodologies used in these studies makes it difficult to integrate all of the findings.

Since the beginning of this century, the endocrine system has received much attention in stress research (Mason, 1968; Selye, 1936), with an emphasis on the hypothalamic-pituitary-adrenal (HPA) axis, since this system forms a theoretically promising pathway for mediating the relationships between psychosocial stress and subsequent disorder (Curtis, 1972). Cortisol secretion appears to play an important role in the regulation of physiological and behavioral responses under stressful situations (Mason, 1975; Rose, 1984). Cortisol increases after strenuous physical exercise (Mason et al., 1973) and mental task load, specifically in response to distress, as opposed to effort or general arousal (Fibiger \& Singer, 1989; Lundberg \& 
Frankenhaeuser, 1980). Cortisol is also the most important glucocorticoid, with the potential of exerting its effects on practically all normal (body) cells and tissues. Some of the most important effects of cortisol are: stimulation of gluconeogenesis, inhibition of glucose uptake, suppression of inflammation and suppression of numerous immune functions. Glucocorticoids almost invariably suppress rather than enhance biological defense mechanisms. They are thought to protect against the normal defense reactions that are activated by stress, by preventing these defense reactions from overshooting and by' preserving the specificity of immune reactions (Munck et al., 1984).

Indeed, most of the physiological reactions to stress are thought to be adaptive, i.e. they counter the effects of physical stress (trauma, bleeding) or are seen as a preparation for fight or flight responses to acute danger (Cannon, 1929). The question now is, why and when could this adaptive mechanism be a pathway to disease? There are indications that the same suppressive effects of glucocorticoids on several functions of the immune-system may leave a subject more susceptible to infections or even tumors (Claman, 1977). Other studies indicated that increased levels of cortisol may be involved in the etiology of coronary heart diseases (Troxler et al., 1977). The general idea is that stress causes an overproduction of antiinflammatory and immune suppressive action, leading to adverse consequences. (e.g. break down of immune system) (Gaillard \& Al-Damluji, 1987; Selye, 1976). There is also evidence from animal studies for a gradual loss of adaptation within the pituitarycorticoadrenal response. If stress is prolonged, hypophyseal receptors appear to lose their sensitivity to cortisol and as a consequence, prolonged increases in cortisol tend to further slow the recovery of cortisol after stress because of the ineffectiveness of the negative feedback loop (Bassett \& Cairncross, 1977; Kloet \& Reul, 1987). Recent theoretical overviews by Henry and Stephens (1977) and Siegrist (1989) are more or less in line with this concept of loss of adaptation.

It is reasonable to assume that stress reactions will only lead to disease when they are prolonged, or occur very often. The effects of chronic or intermittent stress on the HPA-axis, however, have received far less attention in the literature than the effects of acute stressors, and studies on naturally occurring stressors and their effects are even more rare. Data on cortisol levels during chronic psychosocial stress are inconsistent, with enhanced as well as decreased concentrations reported, and large variability among individuals. Elevated levels of cortisol were found in subjects living near the damaged nuclear power plant at Three Mile Island (Schaeffer \& Baum, 1984). Higher levels of urinary cortisol correlated significantly with urinary catecholamines, self-reports of physical and mental symptoms, and decrements in task performance. A study following parents of leukemic children into the period of bereavement, for as long as 3 years after the death of the child, showed that 17 hydroxycorticosteroid excretion rates were related to the effectiveness of subjects' psychological defenses (Hofer et al., 1972a; Hofer et al., 1972b). Elevated levels of plasma and salivary cortisol were found in Iran hostages after release from captivity, reflecting distress, anxiety and elation. Salivary cortisol was the only physiological measurement that demonstrated a significant correlation with psychiatrists' ratings of the hostages' psychological disturbance (Rahe et al., 1990).

The above studies have examined stress under rather extreme or unusual situations. With respect to more normal settings, studies of air traffic controllers suggest that occupational stress is associated with increased cortisol production (Rose \& Fogg, 1993; Rose, Jenkins, Hurst, Kreger, Barrett, \& Hall, 1982). Caplan et al. (1979) studied white-collar workers in a relatively everyday organizational setting. 
While there was no main effect of work load or personality traits on mean cortisol, high perceived work load was associated with lower morning cortisol values.

The results presented here are part of a larger study concerning emotional and hormonal responses to naturally occurring stress in daily life. Stress is currently conceptualized as a dynamic process, which changes over time and in relation to the environment. It is therefore necessary to include repeated measurements of stress. hormone levels and emotional states to investigate the stress-hormone relationship. This relationship should ideally be studied in naturalistic settings and over time, so that we can begin to understand how adaptation occurs in response to intermittent daily hassles, in individuals who are observed in their normal social networks, settings and activities (Dimsdale, 1984). For these reasons, we chose to use two relatively new techniques, Experience Sampling (ESM) (Csikszentmihalyi \& Larson, 1987; de Vries, 1992) and monitoring of salivary cortisol (Kirschbaum \& Hellhammer, 1989), to assess the relationship between an individual's affective state and neuroendocrine changes over time. Subjects were selected on the basis of Perceived Stress Scale scores (Cohen et al., 1983) from a large sample of male white-collar workers and were then monitored during their daily activities. Subjects completed self-reports. (ESM) and collected saliva samples for cortisol determination at semi-random intervals ten times a day over five consecutive days, including three work and two non-work days.

Here we test the hypothesis that high perceived stress is associated with elevated cortisol, either in general, or during certain phases of the circadian cycle or certain days of the week (e.g. work versus non-work days). With respect to diurnal patterns, no specific hypothesis was formulated. The few studies in which cortisol has been measured at more than one time of day in healthy subjects report significant relationships between cortisol and psychological trait measures at some time points and not at others. For example, in a large German survey study in which salivary cortisol was measured three times on a single day, only 8 am levels showed clear associations with psychological and demographic variables (Brandtstädter et al., 1991). In a similar fashion, perceived work load had an effect on cortisol levels in white-collar workers that was dependent on the time of day when blood was sampled (Caplan et al., 1979), and hostility was associated with high cortisol in daytime, but not in evening or overnight urine samples (Pope \& Smith, 1991). Depressive symptoms could also be related to increased cortisol secretion at specific times of day. Concerning day of the week effects, we hypothesized that cortisol would be elevated on workdays compared to the weekend, possibly in interaction with perceived stress. Although subject groups were selected according to a global measure of stress and not on the basis of work-related stress, we reasoned that work might be a major source of stress. Studies by Frankenhaeuser and colleagues (1989) have demonstrated that cardiovascular and neuroendocrine activity are higher at work than at home, and that slow recovery of responses, or 'unwinding', after work might represent a long-term health risk.

Finally, we examined the extent to which psychological variables (trait anxiety, depression, anger and psychosomatic complaints; ESM measures of mood and stressful events) might contribute to individual differences in workday cortisol level. 


\section{METHOD}

\section{Subjects}

Local industries and government agencies were approached via their personnel departments to participate in the study. A decision as to which categories of employees fell under the definition of 'white-collar' was made by each personnel department on the basis of standard job function descriptions. Questionnaires were distributed among these employees, accompanied by a letter explaining the goals of the study. Participation was voluntary, and care was taken to insure anonymity. 316 male employees from six different industries or agencies completed the screening questionnaire. The mean score on the Perceived Stress Scale (PSS) for this sample was 12.7 (s.d. 6.0), similar to US. norms (mean 13.02, s.d. 6.45) (Cohen et al., 1983).

92 Subjects with scores in the upper or lower tertiles of the screening sample distribution (PSS-10 score $<10$ or 216 ) were recruited to participate in the study, excluding any individuals with a history of endocrine disorder, medications known to affect cortisol levels, psychopathology (major affective disorder, psychoses), or currently in treatment for mental health problems.

'High-' and 'low-' stress subjects were matched for age group, marital status, and household composition. Because it was not possible to match all subjects, numbers of subjects in the final two groups differ slightly.

Four subjects failed to meet Experience Sampling compliance criteria (see 'Daily Experience' section, below) and were therefore excluded from further analysis. Of the 88 remaining subjects, 42 subjects comprised the 'high stress' (HS) group and 46 subjects the 'low stress' (LS) group. Mean age was 42.1 years (range 27 to 57 years), with no significant difference between the two groups. $89 \%$ were married, and $81 \%$ had children living at home.

\section{MEASURES}

\section{Questionnaires}

In addition to the PSS, questionnaires concerning psychosocial stress, coping style, and psychological and physical symptoms were completed. Self-report instruments were chosen on the basis of their theoretical relevance to the stress process and psychometric reliability and validity. The following meastires are used in the cuirent analysis:

Perceived Stress: The 10-item version of the PSS was translated into Dutch by the first author and then back-translated as a check into English by the second author. The items were rated on a 5-point frequency scale, ranging from 'never' (0) to 'very often' (4) in the last month. A total perceived stress score was obtained by reversing the scoring on the positive items and then summing across the 10 items. All subjects completed the PSS twice, during the initial screening and again immediately preceding Experience Sampling. The two PSS scores were highly correlated $(r h o=73, \mathrm{p}<.001)$; the mean score will be used in the analysis.

Psychosomatic symptoms: The SUNYA Psychosomatic Symptom Checklist (PSC) includes 17 common psychosomatic complaints (e.g., headaches, backaches and nausea ). The scale was originally developed by Cox, Freundlich, \& Meyer (1975) and revised by Attanasio et al. (1984). Subjecis rate each complaint on 5 -point scales for frequency ( 0 "never or rarely occurs " to 4 "occurs daily") and intensity ( 0 "not bothersome" to 4 "extremely bothersome"). A Total Score, reflecting the overall level of psychosomatic distress, is obtained by summing the cross-products of each item's frequency by intensity.

Depression: Depressive symptomatology was assessed with the Dutch translation (Dijkstra, 1974) of the Zung Self-Rating Depression Scale (Zung, 1965).

Anxiety; Trait anxiety was measured with the Dutch version (van der Ploeg et al., 1980) of the State-Trait Anxiety Inventory (STAI). 
Anger: Trait anger was measured with the Dutch version (van der Ploeg et al., 1982) of the Spielberger Trait Anger Scale. Although two subscales ('anger-temperament' and 'angerreaction') exist, only the total' score is used here.

\section{Daily experience}

The Experience Sampling Method (ESM) (Csikszentmihalyi \& Larson, 1987; de Vries, 1987; de Vries, 1992) was used to collect data from subjects at selected moments during their normal daily activities. Subjects received auditory signals, after which they filled in a questionnaire and collected a saliva sample. Although additional reports were completed at the end of each day, for the purpose of this analysis we will refer only to the 'beep level' data.

After a 'briefing' session, in which all procedures were explained in detail and informed consent obtained, subjects were sampled for a period of 5 consecutive days, beginning early on a Thursday morning and ending late Monday. In this sample, the weekends were non-workdays. A Seiko wristwatch was programmed to emit beeps 10 times each day, at semi-random intervals of approximately 90 minutes between the hours of 8 am and $10 \mathrm{pm}$. Beeps were clustered around the midpoint of each time block (e.g., 8.15 am, 9.45, 11.15, and so on); the exact time sequence of beeps was varied each day to decrease predictability. In a final 'debriefing' session, ESM forms were checked for legibility, and subjects were asked to clarify reasons for missing data.

Compliance with the procedures was generally good. The criteria we set for inclusion in the analysis ( 220 ESM reports, completed within 20 minutes after being signalled and no missing data for entire days) were met by all but four subjects ( 2 from each group). The remaining 88 subjects completed an average of $83 \%$ of all possible responses within the time limit, for an average of 41 responses per subject. HS and LS groups did not differ in compliance rates (40.1 vs 42.3 responses per subject, Mann-Whitney U-test, p>.05). Compliance was lowest for the first beep (at approximately $8.15 \mathrm{am}$ ), with an average of $73 \%$ valid reports. On Saturdays, response rate for first beeps was $61 \%$, and on Sundays, $59 \%$. $74 \%$ of all missing and invalid responses on weekends could be attributed to the fact that subjects were still asleep.

The ESM form contained open-ended questions concerning thought content, the physical and social context, and what the individual was doing when signalled. The forms also included 7 point Likert scales (from I 'not at all' to 7 'very much') for rating aspects of thoughts, mood, physical well-being, individually defined (psycho)somatic complaints, and present activity. Subjects were asked to describe any stressful events or situations which may have taken place in the interval since the last ESM report and to rate these events on a number of dimensions. Information about smoking (Wust, Kirschbaum, \& Hellhammer, 1990), food (Quigley \& Yen, 1979), coffee (Pincomb, Lovallo, Passey, Brackett, \& Wilson, 1987), and alcohol intake, medications, and physical exertion (Cook, Ng. Read, Harris, \& Riad-Fahmy, 1987) since the last beep was also obtained, to help control for possible confounding influences on cortisol secretion.

\section{Salivary cortisol}

Salivary cortisol is a reliable and valid indicator of the free cortisol in plasma, which is considered to be the biologically active hormone; cortisol concentrations are independent of the flow rate of saliva (Vining et al., 1983). Salivary cortisol increases within minutes in response: to acute stressors (Hellhammer, Kirschbaum, \& Belkien, 1987) and has a half-life of approximately one hour (Fredrikson et al., 1985). We have found no difference in cortisol levels in saliva samples either frozen immediately or kept at room temperature for 2 days (Nicolson et al., 1992); others report no change in unfrozen samples up to 30 days (Kirschbaum \& Hellhammer, 1990).

At the same time ESM forms were being completed, subjects collected saliva by holding a cotton dental roll in the mouth for approximately 1 minute. The saturated roll was placed in a capped plastic vial (Salivette; Sarstedt), which was stored in a specially designed wallet. At the end of each day, subjects placed the vials in their home freezers. At the end of the sampling period, 
uncentrifuged samples were stored at $-20 \mathrm{C}$. Compliance with the saliva sampling procedure was good in both high and low stress groups, with approximately the same mean response rate (83\%) as for the ESM reports.

Salivary cortisol levels were determined in duplicate by direct radioimmunoassay (Ansseau et al., 1984), using ${ }^{125}$ I-cortisol (Farmos diagnostica, Finland) and antiserum made against the 3CMO-BSA conjugate by Dr. J. Sulon, University of Liege, Belgium. The lower detection limit of the assay was $12 \mathrm{ng} / \mathrm{dl}$, with a mean intra-assay coefficient of variation of $4.8 \%$ (range: $2.2 \%$ $7.5 \%$ for 4 assays). Each subject's samples were analysed in the same assay, to reduce sources of variability.

\section{Stallstical analysis}

The 16 ESM mood items were reduced to three mood measures, based on the results of a principal component analysis with varimax rotation, which accounted for $78 \%$ of the total variance when subject mean scores were used. Ratings on the items cheerful, satisfied, relaxed, energetic, self-assured, concentrated and enthusiastic were summed to form ai 'positive affect' (PA) scale (Cronbach's alpha $=.95$ ). Two separate components of negative affect were identified: "NA-low arousal', including the items depressed, anxious, worried, lonely, tired, and miserable (alpha = 87), and 'NA-high arousal', with the items restless, irritated, hurried, and nervous (alpha $=.93$ ). The sums of the scale items were divided by the number of items in the scale, so that all mood measures have ranges from 1 to 7 .

Because the distribution of cortisol values was positively skewed, transformation to natural logarithms of cortisol concentrations was performed prior to statistical analysis. Nonparametric tests were used for univariate group (Mann-Whitney U-test) and within-subject (Wilcoxon matched-pairs signed-rank test) comparisons. Unless noted otherwise, significance tests are twotailed. Analysis of variance (SPSS procedure MANOVA) was used to test differences (and interactions) between HS and LS groups, workdays and weekends, and the 10 times of day sampled. Analyses were performed with SPSS -Macintosh version.

\section{RESULTS}

Given the general nature of the PSS, which was used to define high and low stress groups in this study, it is useful to contrast the two groups on more specific measures of distress. In addition to trait measures, we compared groups on a number of aggregated state measures derived from the ESM data. Mean scores on each of the 3 ESM mood scales were computed across all valid beeps for each subject. Frequency of stressful daily events was calculated as the percentage of total beeps for a subject on which a stressful event was reported, regardless of rated severity. 
Table 5.1.1. Differences in psychological measures between low and high stress groups

$$
\text { low PSS high PSS }
$$

mean (st.dev) mean (st.dev.) D-value

(2-tailed)

Trait measures

Perceived stress (PSS)

Trait Anxiety

Trait Anger

Zung Depression

Psychosomatic

symptoms (PSC)

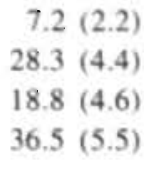

$6.1(5.6)$
$18.1(3.4)$

$39.8(7.6)$

$23.0(5.2)$

$48.4(7.7)$

$27.5(23.2)$
$<.0001$

$<.0001$

$<.000 \|$

$<.0001$

$<.0001$

\section{ESM measures*}

\begin{tabular}{lccl} 
PA & $5.7(0.6)$ & $5.3(0.3)$ & $<.001$ \\
NA-low arousal & $1.2(0.1)$ & $1.4(0.4)$ & $<.0001$ \\
NA-high arousal & $1.3(0.3)$ & $1.8(0.6)$ & $<.0001$ \\
frequency of stressful events & $12.3 \%(11.9)$ & $22.0 \%(20.0)$ & $<.01$ \\
\hline
\end{tabular}

" subject means, aggregated from beep level data

As shown in Table 5.1.1., subjects with a high level of perceived stress were significantly more trait anxious and depressed than subjects with a relatively lower level of perceived stress. They also scored higher on trait anger and reported more psychosomatic symptoms. HS subjects had significantly lower ESM positive affect, higher negative affect-low arousal and higher negative affect-high arousal scores. They were almost twice as likely to report stressful daily events as LS subjects. In absolute terms, the mean number of stressful events reported over the five days of ESM was 9.0 for the HS and 5.3 for the LS group.

We next examined whether high perceived stress was associated with elevated salivary cortisol. Mean cortisol levels were first computed for each subject at each of the 10 time blocks for workdays and again for weekend days. To test for main effects on cortisol levels as well as interactions, analysis of variance was performed with the between-subject factor group (HS, LS) and within-subject factors time of day ( 10 beeps) and day type (workday, weekend). 39 of the 88 subject cases were rejected because of missing data for any of the 20 (10 time blocks $\times 2$ day types) measures. The remaining 49 subjects included $28 \mathrm{LS}$ and $21 \mathrm{HS}$ subjects.

Cortisol concentrations showed the well-defined circadian rhythm, with peak salivary concentrations in the early morning samples. declining values in the afternoon and lowest concentrations at the end of the day; the main ( ffect for time of day was highly significant $(F(9,423)=182.04, p<.001)$, with no significant interactions with either group or day type. Contrary to our hypothesis, no significant main effect for group was found. Further, there was no workday-weekend diffierence in cortisol levels, either as a main effect or in interaction with group. 




Figure 5.1.1. Mean cortisol (ng/dl; $+1-$ s.e.) during workdays for the 'high' $(n=34)$ and the 'low' stress' group $(\mathrm{n}=39)$.

As noted earlier (see 'Daily Experience', above), the percentage of missing responses was higher on weekends than on workdays, especially for early morning samples. Inclusion of weekend data in the model is thus largely responsible for the considerable data attrition. We therefore chose to examine workdays and weekends again separately. While this does not address the issue of workday versus weekend differences, it allows us to test for group differences in cortisol in a larger sample.

For workdays, 15 subjects were forced out of the analysis due to missing data, leaving $39 \mathrm{LS}$ and $34 \mathrm{HS}$ subjects. Time of day again showed the Iargest effect on cortisol levels $(\mathrm{F}(9.639)=262.98, \mathrm{p}<.001)$, but here the difference between HS and LS groups was also significant $(\mathrm{F}(1.71)=6.02, \mathrm{p}<.02)$. The interaction effect was not significant $(F(9,639)=.67, p>.05)$. Cortiso! concentrations for HS and LS groups on workdays are shown in Figure 5.1.1.

In the analysis of differences in cortisol levels on weekends, only 56 subjects (32 LS and 24 HS) had sufficient data for inclusion. For this subsample, the usual time of day effect was found $(F(9,486)=85.80, p<.001)$, but there was no consistent difference between the two stress groups $(F(1,54)=.79, p>.05)$. The group by time of day interaction approached significance $(F(9,486)=1.83, p=.06)$, with a tendency for the high stress group to have higher cortisol levels in the morning through early afternoon (beeps 2, 3, 4 and 5, between 9.30 am and 2.30 PM) and in the late evening 
(beeps 9 and 10, after 8 PM) only. Again, it is important to note that this (nonsignificant) pattern is based on a relatively small subset of the entire sample.

To summarize, cortisol levels showed the expected strong diurnal pattern in all analyses we performed. More interestingly, 'high stress' subjects had elevated cortisol throughout the day on workdays, confirming our hypothesis, at least for these days. It would seem logical to assume that such a pattern would be mediated by group differences in stress exposure or, more specifically, in emotional response to daily stress. Questions concerning reactivity of mood and cortisol to stressful events are beyond the scope of this paper. However, measures of workday stress and distress could provide additional insights into the perceived stress - cortisol relationship.

Within subjects, workdays in comparison to weekends were associated with a higher rate of stressful events (Wilcoxon matched-pairs test, p<.0001) and greater NA-high arousal ( $<<.0001)$, with no differences in either NA-low arousal or PA. The coded descriptions of reported stressors suggested that the work environment was a major arena for such daily hassles, with stressors more often occurring in work than in household, leisure or social network contexts. $43 \%$ of events in LS subjects and $47 \%$ of events in HS subjects were work-related. While this general pattern of workday distress was found in both groups, within-subject differences in workday vs. weekend NA-high arousal were greater in HS than in LS subjects (Mann-Whitney, $\mathrm{p}<.001)$; that is, HS subjects were disproportionately more negatively aroused on workdays. Workday/weekend differentials in NA-low $(.05<p<.10)$ and stressful events $(.05<p<.10)$ did not distinguish the two groups, although results were in the expected direction of relatively greater work-related stress/distress in HS subjects.

Since HS subjects scored higher than LS subjects on trait anxiety, depression, anger and psychosomatic complaints, as well as on ESM measures of positive and negative affect, we expected positive relationships between these variables and cortisol levels, at least on workdays. Mean levels of cortisol over the three workdays were determined by first calculating the mean at each of the 10 time blocks for every subject and then averaging the 10 values for each subject. This aggregated measure of mean cortisol was slightly higher in the HS (116 ng/dl, sd 37.5) compared to the LS group (101 ng/dl, sd 28.2) (one-tailed Mann-Whitney test, $\mathrm{p}<.05$ ).

Spearman rank order correlations between mean workday cortisol and both psychological measures and ESM mood sciales are shown in Table 5.1.2. Small but significant (one-tailed tests) positive correlations were found between cortisol and trait anxiety, depressive symptomatology and NA-low arousal.

Table 5.1.2. Correlations between psychological variables and mean salivary cortisol during workdays

\begin{tabular}{lcl}
\hline & $\mathrm{r}$ & $\mathrm{n}$ \\
\hline Trait anxiety & $.18^{*}$ & 88 \\
Trait anger & .08 & 88 \\
Zung Depression & $.18^{*}$ & 87 \\
Psychosomatic & & \\
symptoms (PSC) & .14 & 88 \\
PA & -.10 & 87 \\
NA - low arousal & $.20^{*}$ & 87 \\
NA - high arousal & .08 & 87 \\
\hline
\end{tabular}

"pe.05 (Spearman; one-tailed tests) 
Although these results indirectly support the conclusion that daily stress and distress lead to increases in cortisol, the role of possible confounding factors should be explored. If, for example, HS subjects smoked more than LS subjects, this might explain the observed differences in cortisol secretion. HS and LS groups were therefore compared on the percentage of workday beeps with reported smoking, strenuous physical exertion (rated 7 on the 7-point scale), coffee, food, and alcohol intake - all activities that could theoretically result in elevated cortisol secretion (see 'Daily Experience', above). No significant differences were found, with mean rates for HS and LS groups as follows (HS/LS): smoking $17 \% / 16 \%$, exertion $0.7 \% / 1.3 \%$, coffee $46 \% / 41 \%$, food $42 \% / 39 \%$, and alcohol $8.0 \% / 7.8 \%$. Although we cannot conclude that these behaviors had no influence on cortisol secretion, effects seem equally likely to have occurred in either group.

\section{DISCUSSION}

In this study, two groups of male white-collar workers differing in levels of perceived stress were compared on subjective, self-report measures of distress and on levels of salivary cortisol, as measured repeatedly over five days. The 'high stress' group scored higher than the 'low stress' group on measures of trait anxiety, depression, anger and psychosomatic complaints, as well as on ESM measures of positive and negative affect and stressful daily events. Cortisol showed a clear diurnal secretory pattern in both groups, with values in the normal range for healthy men (Kirschbaum \& Hellhammer, 1989). On workdays, cortisol was elevated throughout the day in the 'high stress' relative to the 'low stress' group. Several measures of distress, including both trait (anxiety, depression) and concurrent state (NA-low arousal) measures, correlated significantly with mean workday cortisol.

The observed pattern of higher workday cortisol levels in the 'high stress' group is consistent with our initial hypothesis; a straightforward interpretation would be that mild chronic or intermittent stress in situations like those subjects reported during the ESM sampling period was sufficient to increase secretion of the hormone. Since the half-life of cortisol in saliva is shorter than the intervals between beeps, consistently elevated cortisol from morning through late evening indicates continued secretion. More detailed temporal analyses are needed, however, to understand. cortisol dynamics in response to daily stress.

Of the few studies of stress and cortisol in everyday environments to date. results have been inconsistent. Caplan et al. (1979) tested the effect of perceived work load (a summary measure of quantity, deadlines and calls and office visits) on plasma cortisol and found that high work load was associated with low morning cortisol levels. The authors suggested that this finding might reflect a shift in the circadian rhythm of cortisol as a result of job stress. A major disadvantage of this study is the cross-sectional design, in which blood samples were drawn at various times of day, with only one cortisol determination per subject. In other words, comparisons between morning, midday and afternoon cortisol concentrations were comparisons between subjects and not within subjects. Although perceived stress may differ in important ways from perceived work load, our results clearly contradict those of Caplan and colleagues; we found higher early morning cortisol in high stress subjects and no evidence at all for a circadian shift. Brandtstädter et al. (1991), who measured salivary cortisol concentration in 767 adults three times over the course of one day, found higher morning cortisol concentrations in men with high life 
satisfaction, high psychological well-being and high level of employment. Cortisol levels in the afternoon or early evening were not predicted by psychological variables. Again, these results are difficult to reconcile with our findings.

Trait negative affectivity (NA) is conceptualized as the tendency to experience a wide range of negative emotions (Watson \& Clark, 1984). In studies of stress-illness relationships, trait NA has been held responsible for inflated correlations between stressors and self-reported health symptoms (Costa \& McCrae, 1987; Watson \& Pennebaker, 1989). In general, NA has not been found to predict objective health problems or physiological stress responses and has therefore been considered a nuisance in the analysis of stress data (Schaubroeck et al., 1992). The 'high stress' group in our study was defined on the basis of scores on a very clearly subjective self-report measure, the Perceived Stress Scale, and the fact that HS subjects scored higher on state and trait measures of distress as well as self-reported stress exposure suggests the pervasive influence of trait NA. In this light, our finding of an association between subjective stress, trait and state NA measures, and salivary cortisol levels is particularly noteworthy.

As is usually the case in psychophysiological studies, the psychological variables we measured accounted for only a small percentage of the variability in cortisol. Moreover, the observed elevations in workday cortisol secretion in HS subjects were slight, relative to pathophysiological levels seen in Cushing's disease or some cases of major depression. Since cortisol levels are not routinely measured in prospective epidemiological studies of healthy individuals, we have no guidelines at present for assessing the clinical relevance or health implications of these findings.

Analyses in this study were done on aggregated data, which has the advantage of increasing the reliability of measurement when only the chronic level of variables is of interest. On the other hand, aggregated measures may be biased (Jaccard \& Wan, 1993), and they obscure the important interplay between experiential and physiological states and processes which momentary measures are intended to clarify. To gain more insight into the relationship between perceived stress and cortisol, we need to move to beep-level analyses. We can then investigate fluctuations over time in stressful events, mood, psychosomatic complaints and determine how these fluctuations relate to endocrine activity (concurrent and lagged relationships). One promising method for the analyses of temporal data is hierarchical linear modeling, or multi-level analysis (Bryk \& Raudenbush, 1992). This approach can account for the dependency of data within a subject, can deal with the problem of missing data, and allows for individual differences in intercepts, slopes and error structures. We are currently undertaking multi-level analyses, in the belief that these new methods will help realize the enormous research potential afforded by momentary measures.

\section{ACKNOWLEDGEMENTS}

We thank Dr. J. Sulon, University of Liege, for performing RIA analyses of salivary cortisol, and C. Dijkman for assistance in all aspects of the study. 



\section{Section 5.2}

\section{Four-teen hour urinary catecholamine excretion in relation to perceived stress.}

\section{INTRODUCTION}

It has been well documented that, besides the pituitary and adrenal cortex, the adrenal medullary system also plays a central role in stress and arousal (Cannon, 1929; Mason, 1975). Increased catecholamine (CA) secretion has been associated with exposure to a wide variety of psychological and physical stressors (Frankenhaeuser, 1975a); for example to failure, loss, challenge, mental effort, uncertainty, and threat situations, as well as to physical stimuli such as cold, pain, anoxia, exercise, and heat (Frankenhaeuser, 1976; Frankenhaeuser, 1979). The magnitude of CA responses to potentially stressful situations or events is usually determined by the intensity of the appraisal of threat as related to one's perceived ability to meet the demands of the situation (Lazarus \& Folkman, 1984b).

Personal control appears to be an important factor in relation to CA excretion; high personal control (perceived self-efficacy) was accompanied by low levels of plasma adrenaline $(\mathrm{A})$ and noradrenaline (NA) during interactions with a phobic object, while moderate control resulted in substantial increases in plasma CA (Bandura, Taylor, Williams, Mefford, \& Barchas, 1985). With respect to occupational stress, a lack of control over the pace of one's work and extreme workloads (both high and low) were associated with high levels of CA (Frankenhaeuser \& Johansson, 1976; Frankenhaeuser, Nordheden, Myrsten, \& Post, 1971; Timio \& Gentili, 1976). On the other hand, high CA output has also been associated with successful coping and with the ability to maintain a high performance level during stressful conditions, probably as a result of high mental effort (Frankenhaeuser, 1979; Siegrist et al., 1989; Ursin, 1978). There is some evidence to suggest that the two CA are differentially sensitive to behavioral and situational factors. NA relates to active, aggressive emotional states, whereas excessive A levels relate to passive, anxious states (Henry, 1982), but also to states involving high mental effort (Fibiger, Singer, \& Miller, 1984; Frankenhaeuser et al., 1971).

Although a positive relation seems to exist between CA levels and adaptive functioning. frequent and excessive elevations and/or long-term elevation of $\mathrm{CA}$ levels are assumed to have damaging effects on various bodily organs and the cardiovascular system (Gruchow, 1979; Madden \& Livnat, 1991; Surwit, Williams, \& Shapiro, 1982). However, in contrast with the numerous studies on acute stress situations, studies concerning habitual CA excretion as related to enduring characteristics of the social environment or of the individual are more scarce. With respect to long-term occupational stress, elevated A excretion during periods of working 'overtime' was observed; not only during working hours but also in the evening at home (Rissler, 1977). In a study by Baum and colleagues (1986), the stress of being unemployed was related to higher levels of both $\mathrm{CA}$, and levels increased as the length of employment increased. Forsman (1980) also found a positive correlation between habitual A excretion and experiences of distress (measuring day-to-day stress) as measured in the field. As another example, the continuing uncertainty and perceived threat of living near the site of the nuclear power plant accident at Three 
Mile Island was associated with chronic elevations in overnight samples of both $\mathrm{A}$ as well as NA (Schaeffer \& Baum, 1984). Finally, in another study investigating the effects of long-term stress on CA, it was found that urinary CA levels in American hostages freed from captivity in Iran were highly elevated and appeared to reflect three affects: distress, anxiety, and elation (Rahe et al., 1990).

In the present study, urinary CA were determined to investigate the relationship between perceived stress and catecholamine excretion. It was hypothesized that high levels of perceived stress, as a measure of more chronic stress, are associated with elevated CA levels, either in general or related to the type of day (work day versus weekend). Additionally, several person characteristics (trait anxiety, depression, anger, and psychosomatic symptoms), and mood states (Fibiger, Singer, Miller, Armstrong, \& Datar, 1984) were explored for their possible relationship with CA levels. As opposed to plasma CA levels, urinary CA levels ( 14 to 24 hour samples) are typically used in studies for the measurement of long-term, chronic stress, and are particularly well-suited for studying psychosocial influences of everyday life (Frankenhaeuser, 1975a). Because urine samples show a slower rate of change than plasma samples, they can be applied to determine long-term changes in CA levels. The collection of urine samples is also relatively easy, non invasive and very well suited for field studies during which subjects' normal habits and ordinary daily activities should nol be changed (Frankenhaeuser \& Gardell, 1976).

\section{METHODS}

\section{Subjects and questlonnaires}

For details about subject recruitment, procedures and questionnaires see section 5.1.

\section{Urine sampling}

Catecholamine (CA) levels were determined in urine. Because the $\mathrm{CA}$ in urine constitute a small but relatively constant fraction of liberated amines in the body, the direction of change or the relative levels are meaningful, but absolute numbers have limited value. NA levels are somewhat difficult to interpret because NA is secreted by both nerves and the adrenal medulla and is also subject to rapid neuronal reuptake, but $\mathrm{A}$ can be considered a reliable estimate of adrenal medullar activity because the adrenal medulla is the sole source of circulating A (Frankenhaeuser, 1975b). The 14-hour samples we used in this study, suppress (not eliminate) the effects of variation between subjects with different circadian thythms and minimize the biasing effects of idiosyncratic events (Baum et al., 1985).

Subjects collected two overnight urine samples (from 6 p.m. to 8 a.m.), one after a workday (Thursday to Friday) and one after a weekend day (Sunday to Monday). Subjects emptied their bladder in the toilet at 6 p.m. and from that moment collected subsequent urine in a 2 liter container (Sarstedt) until 8 a.m. the next day at which time they emptied their bladder for the last time in the container. Subjects refrigerated their samples during the collection period. Immediately after collection of the container by the research staff we added HCL. $(37 \%)$ to the total volume of urine until a $\mathrm{pH}$ of $<3$ was reached, to prevent oxidation. From the total volume, $10 \mathrm{ml}$. samples were extracted and immediately frozen at a temperature of $-20 \mathrm{C}$ until analyses.

Adrenaline and noradrenaline excretion rates were determined by means of high-pressure liquid chromatography (HPLC) with electrochemical detection. ${ }^{3} \mathrm{CA}$ levels were corrected for

Analyses of adrenaline and noradrenaline were performed in the laboratory' of Dr. Rahman and Dr. Duvivier, University Hospital of Liege. Belgium.. 
creatinine excretion, and expressed in $\mu \mathrm{g} / \mathrm{g}$ of creatinine. Samples of subjects with creatinine levels below $0.60 \mathrm{~g} / \mathrm{l}$ were discarded $(\mathrm{n}=9)$, because they might give artificial too high concentrations (low levels could be suspect of e.g. hyperthyroidism, advanced renal disease). Due to practical reasons, three subjects did not collect any urine, and another four subjects collected urine only once.

\section{Statistical analysis}

Analysis of variance was used (SPSS procedure MANOVA) was used to test differences and interactions between HS and LS groups and work day and weekend day samples. Nonparametric Spearman correlations were calculated the various psychological trait and state measures and the catecholamines. Significance tests are two-tailed. Analyses were performed with SPSS-Macintosh version.

\section{RESULTS AND DISCUSSION}

Mean values for adrenaline (A) as well as noradrenaline (NA) after a work day and after a weekend day are shown in Table 5.2.1. As can been seen, CA concentrations were highly comparable in high stress versus low stress subject groups.

Table 5.2.1. Mean adrenaline and noradrenaline concentrations ( $\mu \mathrm{g} / \mathrm{g}$ creatinine) plus standard deviation in high stress (HS) and low stress (LS) groups after a work day and after a weekend day.

\begin{tabular}{lcccc}
\hline & \multicolumn{2}{c}{ Work day } & \multicolumn{2}{c}{ Weekend day } \\
& LS & HS & LS & HS \\
& $\mathrm{n}=42$ & $\mathrm{n}=37$ & $\mathrm{n}=41$ & $\mathrm{n}=37$ \\
\hline & & & & \\
Adrenaline & $4.3(2.4)$ & $4.7(35)$ & $4.3(2.5)$ & $4.3(37)$ \\
Noradrenaline & $21.4(12.4)$ & $19.9(9.3)$ & $19.6(10.5)$ & $19.2(8.3)$ \\
\hline
\end{tabular}

To test for main effects of perceived stress and day type on CA levels as well as interaction effects, analysis of variance were performed with the between-subject factor group (HS, LS) and the within-subject factor day type (workday, weekend). 13 of the 88 subject cases were rejected because of missing data. The remaining 75 subjects included 37 HS and 38 LS subjects. For A, no main effect for group was found $(\mathrm{F}(1,73)=0.34, \mathrm{p}>.05)$. There also was no work day / weekend difference in $\mathrm{A}$ levels, either as a main effect or in interaction with group $(F(1,73)=0.16, p>.05 ; F(1$, $73)=1.59, p>.05$ ). For NA, again no main effects for group or day type were observed, and also no interaction effect (group: $F(1,73)=0.24, p>.05$; day type: $F(1,73)=0.66$, $p>05)$; group $x$ day type: $F(1,73)=0.07, p>.05)$.

Spearman rank order correlations between mean A and NA and several psychological measures (trait anxiety, depression, anger, psychosomatic symptoms) and mean ESM mood states (positive affect, negative affect, agitation) were next computed. None of the correlations reached significance (all correlations <.21). A trend was found for positive affect to be positively related to both $\mathrm{A}$ as well as NA (A: $r h o=.21, \mathrm{p}=.06$; NA: $r h o=.19, \mathrm{p}=.08$ ).

The results do not support our hypothesis that high perceived stress is reiated to high CA levels, either in general or related to the day of the week, and do not 
replicate the findings reviewed above of increased catecholamine excretion in the context of long-term stress. In fact, none of the variables investigated showed any relationship to both adrenaline as well as noradrenaline levels. The trend found for positive affect to be related to both catecholamines is in line with the general finding of a weak but positive correlation between CA secretion and indices of emotional stability and adaptation (Johansson, Frankenhaeuser, \& Magnusson, 1973). In the present study, urine samples were collected between 6 p.m. and 8 a.m. and is comparable in design to the study by Schaeffer and Baum (1984) where, in contrast with our study, they did find elevated catecholamine levels in chronically stressed subjects. Most other studies sampled urine during the day, and in the study by Forsman (1980), where urine was sampled in the morning, afternoon, evening, and at night ( 11 p.m. to 8 a.m.), only the morning and afternoon CA levels were related to the experience of stress as measured in daily life. Therefore it is possible that in our study of healthy subjects, where samples were obtained mostly overnight (constituting a more or less baseline level) which reduces the impact of specific environmental influences during the day, complete unwinding took place during the night. More extreme stress levels are perhaps necessary to observe an impact on CA baseline levels. It would be interesting to investigate whether perceived stress and the other person and mood variables would relate to $\mathrm{CA}$ levels during the day as opposed to overnight, when specific environmental circumstances are more influential.

An important limitation of our study was that no restrictions were put on the subjects concerning feeding and drinking patterns (including alcohol), exercise, and cigarette smoking. This was done to increase compliance because of the already intensive and time consuming nature of our ESM field study. CA levels are, besides stress, sensitive to all the factors mentioned above, and the absence of a control for these factors may have confounded the results. HS and LS groups were therefore compared on the percentage of ESM beeps (see paragraph 'daily experience' of section 5.1) with reported smoking. coffee, food, alcohol intake, and physical exertion reported during both evenings (work and weekend) when urine collection took place (between 6.45 p.m. and 21.45 ). No significant differences were found between groups suggesting that, although we can not rule out the possibility that these factors had an influence on CA excretion, it should not have confounded the group comparisons. 


\section{Chapter 6}

\section{The effects of perceived stress, traits, mood states, and stressful events on salivary cortisol}

\section{Psychosomatic Medicine (in press)}

Marleen van Eck ${ }^{1}$, Hans Berkhof ${ }^{2}$, Nancy Nicolson', Jose Sulon ${ }^{3}$

1 Department of Psychiatry \& Neuropsychology, Social Psychiatry and Psychiatric Epidemiology section, University of Limburg, the Netherlands

2 Department of Statistics and Measurement Theory, University of Groningen, the Netherlands

3 Faculty of Veterinary Medicine, University of Liege, Belgium 

ABSTRACT This study examined the effects of perceived stress and related individual characteristics, mood states, and stressful daily events on salivary cortisol levels. Forty-one 'high stress' and 46 'low stress' subjects were selected on the basis of Perceived Stress Scale scores from a sample of male white collar workers. Subjects completed Experience Sampling self-reports and collected saliva samples ten times a day over five consecutive days. Multilevel analysis revealed that trait anxiety and depression, but not perceived stress, were associated with small but statistically significant cortisol elevation. No effects on cortisol were found for recent life events, chronic difficulties, trait anger, or psychosomatic symptoms. Distress, as reflected by the mood states Negative Affect and Agitation, was associated with higher cortisol levels, whereas Positive Affect had no statistically significant effect. Stressful daily events were associated with increased cortisol secretion, the magnitude of the effect depending on whether the event was still ongoing and on how frequently a similar kind of event had occurred previously. Although perceived stress, anxiety and depression did not increase cortisol reactivity to daily events, we found evidence for reduced habituation to recurrent events in subjects scoring high on these traits. Mood appeared to play a mediating role in the relationship between stressful events and cortisol secretion. These results suggest that negative affectivity is not just a confounder, but is related to elevated cortisol secretion during normal daily activities. The finding that even minor events and fluctuations in mood states were associated with increased adrenocortical activity points to a possible mechanism linking subjective experience to health outcomes.

\section{INTRODUCTION}

The neuroendocrine system has long been thought to play an important role in the causal pathway linking stress and ill health (Cannon, 1929; Selye, 1976; Weiner, 1992). The hypothalamic-pituitary-adrenal (HPA) axis, which is involved in the regulation of a wide range of physiological and behavioral responses to stress, has been implicated in numerous illness processes (Chrousos \& Gold, 1992; De La Torre, 1994; Tsigos \& Chrousos, 1994), including the etiology of psychiatric disorders (Checkley, 1992; Dinan, 1994; Gold et al., 1988). Over the past decade, it has become clear that not only major life events (Brown \& Harris, 1989; Holmes \& Rahe, 1967), but also minor daily stressors or "hassles" can have negative effects on health and well-being (DeLongis et al., 1982; Ivancevich, 1986; Zarski, 1984). In contrast to the wealth of information concerning the neuroendocrine effects of major real-life stressors, there is relatively little known about the effects of chronic stress and even less about the effects of minor daily events. The goal of the current study was to increase our understanding of the impact of daily life stress on the HPA axis.

While a growing number of studies have examined the relationship between daily events and mood (Bolger et al., 1989a; Clark \& Watson, 1988; Goplerud \& Depue, 1985; Lundberg et al., 1989; Neale et al., 1987; Repetti, 1993; Stone, 1987; Stone \& Neale, 1984) or physical symptoms (DeLongis et al., 1988; Goreczny, Brantley, Buss, \& Waters, 1988; Jandorf, Deblinger, Neale, \& Stone, 1986), few have investigated whether stressful daily experiences have an effect on cortisol excretion. Findings have been inconsistent, which is not surprising in light of the large differences in cortisol measurement procedures and definitions of daily stress or distress. Cortisol levels have often been based on a single measurement per subject or per day. To illustrate the diversity of results, greater work demands were associated 
with lower cortisol levels in subjects sampled in the morning (but not in the afternoon) in one study (Caplan et al., 1979); another study found that feelings of irritation, tenseness, and tiredness in assembly line workers were associated with elevated cortisol levels on workdays and that cortisol levels were absolutely higher on 'bad' compared to 'normal' or 'good' workdays (Lundberg et al., 1989). Examining within-subject associations over several days, one study found elevated afternoon urinary cortisol on high stress compared to low stress days (Brantley et al., 1988), while in another, no relationship between the number of undesirable events reported at the end of the day and cortisol levels measured in evening urine could be demonstrated (Cummins \& Gevirtz, 1993). With more frequently measured responses to work stress in air traffic controllers, Rose and colleagues (1993) found that a subgroup of subjects responded to an increase in the number of planes they had to manage with large increases in cortisol.

New approaches are needed for investigating cortisol responses to daily events. Repeated measurement of cortisol not only increases reliability, but provides a clearer picture of the temporal relationship between stressful events and neuroendocrine responses. This is especially important if the events of interest occur unpredictably (as is usually the case in the natural environment) and therefore cannot be directly monitored by the researcher. Additional insights into the stress process can be gained by including measures of the context in which events take place and measures of subjective responses, including mood and cognitive appraisals (Folkman \& Lazarus, 1985).

The current study therefore combines Experience Sampling (ESM (Csikszentmihalyi \& Larson, 1987; de Vries, 1992) methodology and frequent salivary cortisol sampling to address two main questions about daily life stress and cortisol:

(I) Are high perceived stress and distress associated with elevated cortisol levels? Despite the lack of consistent results in the literature on the effects of chronic stress on the HPA axis, we reasoned that healthy individuals experiencing persistent but not overwhelming levels of distress and difficulty in coping with daily demands would have higher overall cortisol than persons who experience fewer problems. In addition to the effects of perceived stress level, we examined the contributions of conceptually related measures of stress exposure and chronic distress. These included recent life events, chronic difficulties, trait anxiety. depression. hostility, and psychosomatic complaints. Several of these variables have previously been linked to cortisol levels (Brandtstädter et al., 1991; Pope \& Smith, 1991; Schaeffer \& Baum, 1984).

In addition to trait-like measures of stress and distress, we assessed the effects of negative and positive mood states on cortisol. Although there is abundant evidence that cortisol increases in response to negative states (Arnetz \& Fjellner, 1986; Lundberg \& Frankenhaeuser, 1980: Mason, 1968; Nicolson. 1992), the effects of positive mood are less clear. Positive affect has been associated with lower (Hubert \& de Jong-Meyer, 1989; Kugler \& Kalveram, 1989) as well as higher (Brown et al., 1993) cortisol levels.

(2) Do stressful daily events influence cortisol levels?

In general, we hypothesized that even minor stressors could result in increased cortisol secretion. We further hypothesized that such effects might be dependent on an individual's chronic level of perceived stress. Based on results of previous studies showing greatei psychological impact of work-related events (Stone, 1987) and negative social interactions (Bolger et al., 1989a), we expected that these categories of events would have the largest impact on cortisol. Similarly, events that subjects 
rated as more unpleasant, more important, less predictable, and less controllable were expected to have larger effects, and ongoing events were expected to have more effect on cortisol than recently terminated events. Finally, we hypothesized that events reported to occur relatively infrequently in daily life would, due to their novelty, have a greater effect on cortisol levels than recurrent events.

The study design compares two groups of male white collar workers, with high versus low levels of perceived stress. A total of 87 subjects completed ESM selfreports and collected saliva for cortisol determination at frequent intervals over a period of five days. A previous analysis of a subset of these data, based on subject mean cortisol levels, provided some support for the hypothesis of elevated cortisol levels in the high stress group, at least on workdays (van Eck \& Nicolson, 1994). The current analysis adds to these preliminary findings by assessing the separate contributions of "trait" (e.g., perceived stress level, trait anxiety, depression) and "state" (e.g., mood, appraisal) variables, at the same time controlling for diurnal and possible confounding influences on cortisol secretion. Most importantly, the application of hierarchical linear modeling, or multilevel analysis (Bryk \& Raudenbush, 1992), enables us to investigate the neuroendocrine effects of the wide variety of stressful events experienced in daily life.

\section{METHODS}

\section{Subjects}

Local industries and government agencies were approached via their personnel departments to participate in the study. A decision as to which categories of employees fell under the definition of 'white-collar' was made by each personnel department on the basis of standard job function descriptions. Questionnaires were distributed among these employees, accompanied by a letter explaining the goals of the study. Participation was voluntary, and care was taken to insure anonymity. 316 male employees from six different industries or agencies completed the screening questionnaire. The mean score on the Perceived Stress Scale (PSS) for this sample was 12.7 (s.d. 6.0), similar to US norms (mean 13.02, s.d. 6.45) (Cohen et al., 1983).

Ninety-two subjects with scores in the upper or lower tertiles of the screening sample distribution (PSS-10 score $\leq 10$ or $\geq 16$ ) were recruited, excluding individuals who reported a history of serious chronic illness, endocrine disorder, medications known to affect cortisol levels, or treatment (past or current) for mental health problems. Fxclusion criteria were reassessed during an initial interview, study aims and procedures were explained, and informed consent obtained. During subject intake, each 'high stress' (HS) subject was matched for age group. marital status, and household composition with a 'low stress' (LS) subject to insure that the two groups did not differ on demographic characteristics that might affect exposure to certain classes of daily stressors. Five subjects were later excluded from analysis: four due to failure to meet ESM compliance criteria (see 'Daily Experience' section, below) and one because he became so acutely stressed that he was unable to work during the sampling period. Of the 87 remaining subjects, 41 subjects comprised the HS group and 46 subjects, the LS group. Mean age was 42.1 years (range 27 to 57 years). $89 \%$ were married, and $81 \%$ had children living at home

\section{Questionnaires}

The following measures were used in the current analysis:

Perceived stress: The 10-item version of the PSS was translated into Dutch. The ittms are rated on a 5-point frequency scale, ranging from 'never' (0) to 'very often' (4) in the last month. 
Life events: Life events were recorded with the questionnaire form of the List of Threatening Experiences (LTE-Q; Brugha et al., 1985). Subjects were asked about the occurrence of 12 categories of events (e.g. death of a partner, child, parent; got divorced) during the last year.

Long-term difficulties: Chronic stress was assessed with the Long-term Difficulties Questionnaire (LLM; Hendriks et al., 1990). This inventory focuses on problems in relation to work/study, housing, physical environment, leisure, finance, and social relationships (partner, family, friends, neighbors). Subjects rate each of the 16 items on a four point intensity scale with the anchors (1) none, (2) some, (3) quite, (4) serious (difficulties). A total score is obtained by summing across all items.

Psychosomatic symptoms: The revised version of the SUNYA. Psychosomatic Symptom Checklist (PSC) (Attanasio et al., 1984) includes 17 common psychosomatic complaints (e.g., headaches, backaches, nausea). Subjects rate each complaint on 5-point scales for frequency (0 "never or rarely occurs " to 4 "occurs daily") and intensity ( 0 "not bothersome" to 4 "extremely bothersome"). A, total score is obtained by summing the cross-products of each item's frequency by intensity.

Depression: Depressive symptomatology was assessed with the Dutch translation (Dijkstra, 1974) of the Zung Self-Rating Depression Scale.

Anxiety: Trait anxiety was measured with the Dutch version (van der Ploeg et al., 1980) of the State-Trait Anxiety Inventory (STAI).

Anger: Trait anger was measured with the Dutch version (van der Ploeg et al., 1982) of the Spielberger Trait Anger Scale. The scale has two subscales: 'anger-temperament' and 'angerreaction'.

\section{Daily experience}

The Experience Sampling Method (ESM) (Csikszentmihalyi \& Larson, 1987; de Vries, 1992) was used to collect data from subjects during their normal daily activities. Subjects received auditory signals ('beeps'), after which they filled in a questionnaire and collected a saliva sample. After receiving detailed instructions, subjects. were sampled for a period of 5 consecutive days (Thursday through Monday). A Seiko wristwatch was programmed to emit. 'beeps' 10 times each day, at semi-random intervals of approximately 90 minutes, between the hours of 8 am and 10 PM. Beeps were clustered around the midpoint of each time block e.g. $8.15 \mathrm{am}, 9.45,11.15$, and so on), with the exact time sequence of 'beeps' varied each day to decrease predictability. in a final 'debriefing' session, subjects were asked to clarify reasons for missing data.

The criteria we set for subject inclusion in the analysis ( $\geq 20$ ESM reports completed within 20 minutes after being signalled and no missing data for entire days) were met by all but foui subjects ( 2 from each group). The remaining 88 subjects completed an average of $83 \%$ of all possible responses within the time limit, for an average of 41 responses per subject. HS and LS groups did not differ in compliance rates (40.1 vs 42.3 responses per subject, Mann-Whitney Utest, $\mathrm{p}>.05$ ). Compliance was lowest for the first 'beep' (at approximately $8.15 \mathrm{am}$ ), with ant average of $73 \%$ valid reports. On Saturdays, response rate for first 'beeps.' was $61 \%$ and on Sundays, $59 \%$. On weekends, $74 \%$ of all missing and invalid responses could be attributed to the fact that subjects were still asleep when signalled.

The ESM form contained open-ended questions concerning thought content, the physical and social context, and what the individual was doing when signalled. The forms also included Likert scales (from 1 'not at all' to 7 'very much') for rating aspects of thoughts, mood, physical well-being, individually defined (psycho)somatic complaints, present activity, and stressful events. Subjects were asked to describe any stressful events or situations which may have taken place in. the interval since the last ESM report and to rate these events on a number of dimensions: unpleasantness, importance, predictability. controllability , and frequency of prior occurrence.. Subjects were also asked to indicate at what time the event had started, and if and when it had 
ended at the moment they were 'beeped'. Information concerning maximum level of physical exertion, smoking, food, coffee, and alcohol intake since the last 'beep' was also obtained.

The 17 ESM mood items were reduced to three mood measures, identified by means of principal component analysis with varimax rotation, which accounted for $78 \%$ of the total variance when subject mean scores were used. Ratings on the items cheerful, satisfied, relaxed, energetic, self-assured, concentrated, and enthusiastic were summed to form a 'Positive Affect' scale (Cronbach's alpha $=.95$ ). Two separate components of negative affect were identified: 'Negative Affect', including the items depressed, anxious, worried, lonely, tired, and miserable (alpha = .87 ), and 'Agitation', with the items restless, irritated, hurried, and nervous (alpha $=.93$ ). The sums of the scale items were divided by the number of items, so that all mood measures have ranges from 1 to 7 .

Subjects' descriptions of stressful daily events were first coded according to content, with categories work, network (events concerning family, friends and acquaintances). household/financial, leisure, personal health-somatic, personal health-psychological, transport, and other. Twelve events coded in the two personal health categories were excluded from analysis because of possible confounding with psychological and somatic state measures. In the current analysis, the remaining events were collapsed into the categories. work ( $48.0 \%$ of events) and nonwork $(50.5 \%)$. Some examples of reported work events were: 'unclear / vague assignment at work', 'too much to organize, not enough time', 'difficult conversation with boss about job performance', 'leading a big meeting'. Reported non-work events included: 'having a fight with: my wife about household duties', 'conflict with spouse about how to raise our son', 'child who will not listen', and 'making dinner, child crying, other child taking a bath, and this all at the same time'. In addition to content, events were classified according to whether or not they involved a social interaction and/or a task demand. ${ }^{4}$

\section{Salivary cortisol}

Salivary cortisol is a reliable indicator of the free cortisol in plasma, which is considered to be the biologically active hormone (Vining et al., 1983). Salivary cortisol increases within minutes in response to acute stressors and has a half-life off approximately one hour (Hellhammer et al., 1987). We have found no differences in cortisol levels in uncentrifuged samples frozen immediately or kept at room temperature for 2 days (Nicolson et al., 1992); others report no differences up to 2-4 weeks (Kahn et al., 1988; Kirschbaum \& Hellhammer, 1989).

At the same time ESM forms were being completed, subjects collected saliva by holding a cotton dental roll in the mouth for approximately 1 minute. The saturated roll was placed in a capped plastic vial ("Salivette", Sarstedt), which subjects stored in their home freezers each evening. At the end of the sampling period, uncentrifuged samples were stored at -20 degrees Celsius until analysis. Compliance with saliva sampling was good in both high and low stress groups, with the same mean response rate $(83 \%)$ as for the ESM reports. Five extreme cortisol values $(>1200 \mathrm{ng} / \mathrm{dl})$ were deleted from the dataset before analysis.

Salivary cortisol levels were determined in duplicate by direct radioimmunoassay (Ansseau et al., 1984), using ${ }^{125}$ I-cortisol (Farmos diagnostica, Finland) and antiserum made against the 3CMO-BSA conjugate by Dr. J. Sulon, University of Liege, Belgium. The lower detection limit of the assay was $12 \mathrm{ng} / \mathrm{dl}$, with a mean intra-assay coefficient of variation of $4.8 \%$ (range: $2.2 \%$ -

4 The reliability of the coding system was assessed by comparing the classifications of 345 events by two independent coders. Interrater agreement was determined by means of Cohen's kappa. On the whole, the qualitative information could be classified with a high degree of agreement (Laundis \& Koch, 1977), especially for the content categories. The overall Kappa for content was .90 , with intra-category Kappa's ranging from .60 to .96 ; Kappa's for social interaction and for task demand were .73 and .65 . respectively. 
$7.5 \%$ for 4 assays). All samples from an individual subject were analysed in the same assay to reduce sources of variability.

\section{Statistical analysis: multilevel or hierarchlcal linear model}

The multilevel model or the hierarchical linear model (Bryik \& Raudenbush, 1992; Goidstein, 1987) is a variant of the multiple linear regression model, applicable for data with a hierarchical nesting structure. In the present dataset, the measurements made at the 'beeps' are nested within persons. The two nesting levels will be called measurement level and person level. At each level of the hierarchy, explanatory variables can be added to the model. The variables that are added at the measurement level (e.g. mood states, events) vary with time, while the added variables at the person level represent characteristics of individual respondents (e.g. high or low perceived stress, trait anxiety).

An advantage of the hierarchical linear model is that it allows for missing observations. In addition, the observations do not need to be evenly spaced over the time interval.

At the measurement level, the relationship between cortisol and time of the day can be modeled explicitly. If, for example, this relation is linear, the model would be of the following form:

$$
(\text { CORT })_{i t}=b_{\mathrm{o} i}+b_{1 i}(\mathrm{TIME})_{i t}+\mathrm{e}_{i t} \text {, }
$$

where $(\text { CORT) })_{i t}$ is the level of cortisol at the th theasurement of person $i$, (TIME) $)_{i r}$ represents the time of the day at which this measurement is taken, $b_{\mathrm{o} i}$ and $b_{1 i}$ are the intercept and slope of person $i$, and the $e_{i l}$ 's are normally distributed error terms with mean zero and variance $\sigma^{2}$. The resulting equation can be extended with time-varying explanatory variables. For example, to study the effect of stressful event occurrence on cortisol, a dummy-coded variable (EVENT) ir can be added to the above equation as follows:

$$
(\mathrm{CORT})_{i t}=b_{\mathrm{o} i}+b_{1 i}(\mathrm{TIME})_{i t}+b_{2 i}(\text { EVENT })_{i t}+\mathrm{e}_{i t} .
$$

where $b_{2 i}$ is the effect of a stressful event for person $i$. The regression coefficients $b_{j i}(j=0,1,2$, ....) are allowed to vary across individuals. Therefore, we can split $b_{j i}$ into two components: a fixed component $d_{0 i}$ that is constant across persons (fixed effect $l$ and a random component $u_{j i}$ that varies across persons (random effeci). This gives the following person-level model for $b_{j i}(j=0,1$. $2, \ldots)$ :

$$
b_{j i}=d_{\mathrm{o} j}+u_{j i}
$$

Instead of estimating $u_{j i}$ for each person, we postulate that the $u_{j i}$ 's $(i=1,2,3, \ldots)$ are randlom draws from a normal distribution (explaining the term random effect). The mean of $u_{j i}$ is zero and the variance of $u_{j i}$ is $\tau_{j j}$. The value of $\tau_{j j}$ indicates how much the value of $u_{j i}$ differ across: persons: the higher the value of $\tau_{j j}$ the more the values of $u_{j i}$ differ. The covariance between $u_{j i}$ and $u_{k i}$ ( $j$ not equal to $k$ ) is $\tau_{j k}$. A positive value of $\tau_{j k}$ implies that a person with a high value of $b_{j}$ tends to have a high value of $b_{k}$ as well.

Suppose that part of the variation in $b_{j i}(j=0,1,2, \ldots)$ across persons can be explained by the person variable (GROUP) that indicates whether person $i$ belongs to the high or low stress group. The regression coefficients $b_{j i}(j=0,1,2)$ can now be modeled as:

$$
b_{j i}=d_{\mathrm{oj}}+d_{1 j}(\text { GROUP })_{i}+\mathrm{u}_{j i},
$$

where $d_{y}(j=0,1,2, \ldots)$ denotes the fixed effect of $(\text { GROUP })_{i}$ on $b_{j i}$. The $u_{j i}(j=0,1,2, \ldots)$ is now a random effect of person $i$ after controlling for (GROUP);

Estimates of the fixed effects $d_{0 j}$ and $d_{1 j}(j=0,1,2)$ as well as of the (co)variances $\sigma^{2}$ and $\tau_{j k}(j, k=0,1,2)$ were obtained with the program ML3e (Prosser, Rasbach, \& Goldstein, 1991). For model selection we started with an empty model and added main and interaction effects of the theoretically important variables. Significance of fixed effects was tested by dividing the estimated effect by its standard error. This ratio is approximately normally distributed. For testing the 
significance of the (co)variances. $\tau_{j k}(j, k=0,1,2)$, we applied the likelihood ratio test (Bryk \& Raudenbush, 1992). This test compares a model with and a model without a random effect. Hence, the (co)variances that are added to the model because of the specification of an extra random effect are tested simultaneously. Non-significant effects were excluded from the models. The postulated normality of the random effects was checked by inspecting normal probability plots of the individual estimates of $b_{j i}(j=0,1,2)$, which were obtained by the empirical Bayes approach (Bryk \& Raudenbush, 1992; Goldstein, 1987). The normality of the measurement level empirical Bayes residuals was also checked by inspection of the normal probability plot. The observed cortisol values for each person were plotted by time of day to check for the presence of outlying cortisol curves (see also Results, below).

The estimation of effects on cortisol entailed four steps, presented here as separate models. Since the analysis of repeated assessments of salivary cortisol is complicated by the hormone's strong diurnal rhythm and secretory peaks (which lead to a decline in variance from moming to evening), the first step involved accurate fitting of the diurnal curve, in order to allow comparisons of cortisol values across the day. Next, possible confounding factors, such as smoking (Kirschbaum \& Hellhammer, 1994), exercise (Cook et al., 1987), coffee (Pincomb et al., 1987) and food intake (Quigley \& Yen, 1979), were included as explanatory variables in the same model. All variables with significant fixed effects identified in this model were included in ail subsequent models.

The remaining three models test our main hypotheses. First, the effects of level of perceived stress, mood states, and individual trait characteristics on overall cortisol level were estimated. Workday vs. weekend differences and interactions between stress level and diurnal variability were also examined. The next model estimated effects of stressful events and event characteristics on cortisol, excluding the contribution of mood variables. In the final model, mood state variables were re-entered in order to evaluate the relative contributions of events, mood, and trait characteristics to cortisol levels.

Instead of a model with two levels, we could have formulated a three-level model, where measurements are nested within days, which in turn are nested within persons. However, when a three-level model was evaluated the p-values were approximately the same as the $p$-values of the two-level model. Because the more complex model did not change the conclusions, we decided to present the simpler model. The similarity of the results of the two models can be explained by the observation that the variance at the day level, although present, was small compared to the variance at the person and the measurement levels. Again, for the sake of simplicity, we did not extend the model with an autocorrelation term, since its inclusion did not change the model results.

\section{RESULTS}

\section{Characteristics of high and low stress groups}

High and low stress groups differed on almost all measures of stress and distress, as assessed with questionnaires and ESM reports (see Table 6.1.). Only the number of life events experienced in the past year did not differentiate the two groups. 
Table 6.1. Characteristics of high and low stress groups.

\begin{tabular}{|c|c|c|c|}
\hline & $\begin{array}{l}\text { LS } \\
\text { mean (st.dev.) }\end{array}$ & $\begin{array}{l}\text { HS } \\
\text { mean (st.dev.) }\end{array}$ & $\frac{D \text {-value }}{\text { (2-tailed) }}$ \\
\hline Total $\mathbf{n}$ & 46 & 42 & \\
\hline Age & $42.7(7.7)$ & $41.5(5.9)$ & ns \\
\hline \multicolumn{4}{|l|}{ Questionnaires } \\
\hline Perceived stress & $7.2(2.2)$ & $18.1(3.4)$ & $<.0001$ \\
\hline Trait anxiety & $28.3(4.4)$ & $39.8(7.6)$ & $<.0001$ \\
\hline Trait anger & $18.8(4.6)$ & $23.0 .(5.2)$ & $<.0001$ \\
\hline Depression & $36.5(5.5)$ & 48.4. (7.7) & $<.0001$ \\
\hline Psychosomatic symptoms & $6.1(5.6)$ & $27.5(23.2)$ & $<.0001$ \\
\hline Life events & $.5(.7)$ & $.8(.9)$ & ns \\
\hline Chronic difficulties & $19.5(2.3)$ & $23.3 \cdot(4.0)$ & $<.0001$ \\
\hline \multicolumn{4}{|l|}{ ESM reports* } \\
\hline Positive affect & $5.7(0.6)$ & 5.3. $(0.3)$ & $<.00 !$ \\
\hline Negative affect & $1.2(0.1)$ & $1.4(0.4)$ & $<.0001$ \\
\hline Agitation & $1.3(0.3)$ & 1.8. $(0.6)$ & $<.0001$ \\
\hline Frequency of stressful events & $12.3 \%(11.9)$ & $22.0 \%(20.0)$ & $<.01$ \\
\hline
\end{tabular}

- subject means, aggregated from 'beep' level data

\section{Controlling for diurnal and external influences on cortisol secretion}

Because the measurement level residuals in a model fitted with raw cortisol data were highly skewed to the right, with decreasing variance from morning to evening, data transformation was necessary to meet standard model assumptions. Log transformation, the usual remedy for cortisol skewness, resulted in increasing variance over the day. We therefore used a fifth root transformation (cortisol ${ }^{0.2}$ ), which gave normally distributed residuals with a homoscedastic pattern throughout the day.

The observed cortisol curve was best described by a two-piece third-degree polynomial (spline function (Smith, 1979)), with the node at $12.25 \mathrm{p} . \mathrm{m}$. The spline was created by adding a truncated term (defined as (TIME -12.25$)^{3}$ after $12.25 \mathrm{pm}$ and as zero before $12.25 \mathrm{pm}$ ) to a third-degree polynomial, which improved fit in the morning hours when cortisol levels dropped most sharply. Random terms were added. to allow each subject to have his own intercept and slope. Random terms for TIME ${ }^{2}$. TIME $^{3}$, and the truncated term did not change the magnitude of the fixed effects and were therefore excluded from the model for the sake of simplicity. All time effects (fixed and random) included in the model were highly significant. As shown in Figure 6.1 , the estimated contisol time curve closely approximates the observed mean values at the "beeps" and is clearly superior to a simple linear curve. Plots of the observed cortisol curve for each individual revealed no outlying curves; further inspection of the normal probability plots of individual cortisol means, slopes, and measurement level residuals, also indicated no significant violation of normality assumptions, supporting the appropriateness of the mixed effects approach.

The model was next extended with the possible confounders (alcohol, coffee, food intake, smoking, and physical exertion). Effects of any food intake and lunch were included separately (Follenius, Brandenberger, Hietter, Simeoni, \& Reinhardt, 1982; Quigley \& Yen, 1979). Maximum recent physical exertion was coded on a 7 point scale. The other variables were coded 1 if reported to have occurred in the interval since the previous 'beep' and 0 otherwise. Recent food, coffee, and alcohol 
intake were reported on $43 \%, 40 \%$, and $12 \%$ (respectively) of total ESM 'beeps', and recent smoking was reported on $16 \%$ of all 'beeps'. The median level of maximum physical exertion in the interval preceding a 'beep' was 3 , with levels of 6 or 7 reported on fewer than $3 \%$ of the total 'beeps'.

In the resulting multilevel model, recent smoking and food intake were associated with higher cortisol levels, with an additional positive effect of lunch on cortisol above food intake in general. No significant effects were found for coffee consumption, alcohol intake, or physical exertion. Model estimates for diurnal and external effects on salivary cortisol levels are shown in Table 6.2. (Column 1).

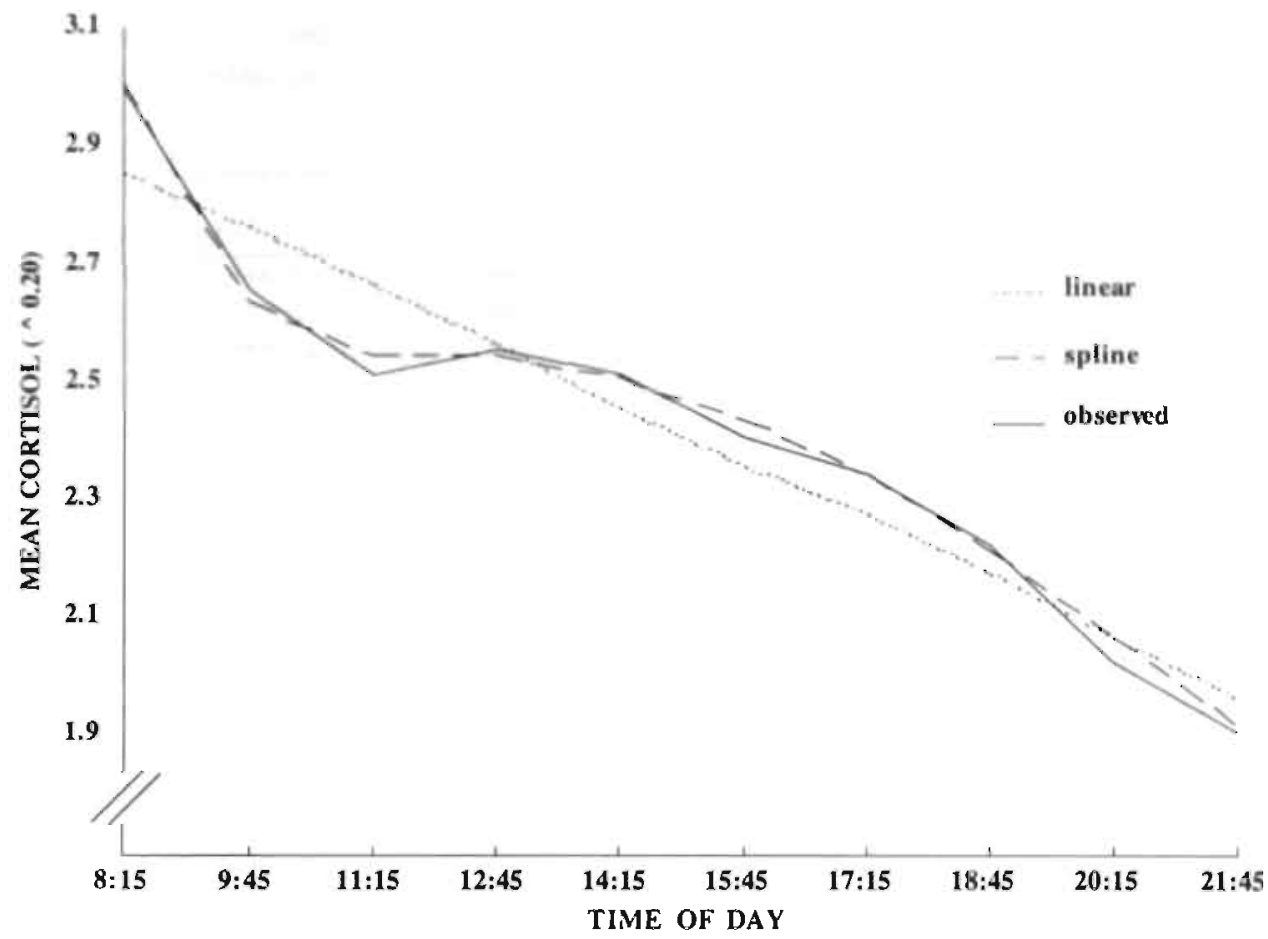

Figure 6.1. Observed and estimated cortisol curves.

Effects of individual characteristics and mood states on cortisol levels In the next model, we tested for effects of psychological trait and state variables on cortisol levels. Results are summarized in Table 6.2. (Column 2). Perceived Stress (PS Group, dummy-coded as 1 for HS and 0 for LS subjects) was entered into the model first, to test our hypothesis that high stress is associated with elevated cortisol. The effect of perceived stress, however, was not significant. The Perceived Stress by Time of Day (TIME) interaction term was also non-significant, indicating that the diurnal pattern of cortisol secretion was similar in HS and LS subjects. Adding Day Type (Work, coded as 1, vs Weekend, coded 0) and its interaction term with Perceived Stress did not improve the model; thus, contrary to expectation, cortisol levels were no higher on workdays than on weekends in either subject group. These variables were therefore excluded from the model. 
We next examined the effects of individual differences in trait anxiety, depression, anger, psychosomatic complaints, number of recent life events, and chronic difficulties. Anger, psychosomatic complaints, life events, and difficulties had no significant effects on cortisol. Trait anxiety and depression were each associated with significant elevations in cortisol levels.

Table 6.2. Effects of individual characteristics " mood states, and stressful daily events on cortisol levels.

The table summarizes the results of steps followed in the development of the full multilevel model. Model 1 provides estimates of diurnal and extraneous effects on cortisol. Model 2 adds estimates for trait characteristics and mood states. Model 3 shows the added contribution of stressful events (with mood effects excluded). In Model 4, all previously significant variables are included simultaneously.

\begin{tabular}{|c|c|c|c|c|}
\hline Fixed Effects ${ }^{\mathrm{a}}$ : & \multicolumn{4}{|c|}{ Estimate + (s.e.) } \\
\hline Interçept & $3.395(0.040) \cdots$ & $3.230(0.080) \cdots$ & $3.260(0.079) \cdots$ & $3.234(0.080)^{* \cdots}$ \\
\hline Time of day (TIME) ${ }^{b}$ & $.0 .577(0.036)^{\cdots}$ & $.0 .584(0.036)^{\cdots}$ & $-0.578(0.037)^{* *}$ & $-0.583(0.036)^{\cdots \cdots}$ \\
\hline TIME $^{2}$ & $0.128(0.010)^{\cdots}$ & $0.130(0.010)^{\cdots *}$ & $0.128(0.010)^{\cdots \cdot}$ & $0.129(0.010)^{\cdots}$ \\
\hline $\mathrm{TIME}^{3}$ & $-0.010(0.001)^{\cdots}$ & $-0.010(0.001)^{* \cdots}$ & $.0 .010(0.001)^{\cdots}$ & $-0.010(0.001)^{\cdots}$ \\
\hline Truncated function & $0.010(0.001) \cdots$ & $0.010(0.001)^{* \cdots}$ & $0.010(0.001)^{\cdots}$ & $0.010(0.001)^{\cdots}$ \\
\hline Food intake & $0.025(0.011)^{\cdots}$ & $0.027(0.010)^{* *}$ & $0.024 ;(0.011)^{\prime \prime}$ & $0.026(0.011)^{* *}$ \\
\hline Lunch & $0.037(0.019)^{\circ}$ & $0.037(0.019)^{*}$ & $0.043(0.019)^{2}$ & $0.043(0.019)^{*}$ \\
\hline Smoking. & $0.046(0.021)^{\circ}$ & $0.048(0.021)^{*}$ & $0.046(0.021)^{*}$ & $0.048(0.021)^{*}$ \\
\hline Trait anxiety & & $0.004(0.002)^{\circ}$ & $0.0039(0.002)^{\circ}$ & $0.004(0.002)^{*}$ \\
\hline Agitation & & $0.027(0.011)^{* *}$ & & $0.022(0.011)^{\prime \prime}$ \\
\hline Stressful event & & & $0.033(0.013)^{*}$ & $0.019(0.014)$ \\
\hline Ongoing event & & & $0.027(0.013)^{*}$ & $0.022(0.013)$ \\
\hline Prior frequency of event & & & $-0.072(0.030)^{*}$ & $-0.076(0.030)^{*}$ \\
\hline Prior frequency $\times$ trait an & & & $0.002(0.0008)^{*}$ & $0.002(0.001)^{\circ}$ \\
\hline
\end{tabular}

Random Effects: (Co)variance + (s.e.)

\begin{tabular}{|c|c|c|c|c|}
\hline \multicolumn{5}{|l|}{ Person level: } \\
\hline Var (Intercept) & $0.031(0.006)^{\cdots}$ & $0.039(0.009)^{2.0 .}$ & $0.031(0.006)^{n \ldots}$ & $0.037(0.009)^{\cdots}$ \\
\hline Var (Time) & $0.0002(0.0001)^{\cdots}$ & $0.0002(0.0001)^{* \cdots}$ & $0.0002(0.0001)^{* \cdots}$ & $0.0002(0.001)^{\cdots}$ \\
\hline Cov (Time, Intercept) & $.0 .001(0.0004)$ & $-0.001(0.0005)$ & $.0 .001(0.0004)$ & $-0.00 !(0.0004)$ \\
\hline \multicolumn{2}{|l|}{ Var (Agitation) } & \multicolumn{2}{|l|}{$0.003(0.001)^{*}$} & $0.003(0.001)^{\cdots *}$ \\
\hline \multicolumn{2}{|c|}{ Cov (Agitation, Intercept) } & \multicolumn{2}{|l|}{$-0.005(0.003)$} & $-0.004(0.003)$ \\
\hline \multicolumn{2}{|c|}{ Cov (Agitation, Time of day) } & \multicolumn{2}{|l|}{$0.0001(0.0002)$} & $0.0001(0.0002)$ \\
\hline \multicolumn{5}{|l|}{ Measurement level: } \\
\hline Var (Residual term) & $0.060(0.0015) \cdots$ & $0.058(0.0015)^{\cdots} \cdots$ & $0.059(0.0015)^{\cdots} \cdots$ & $0.058(0.0015)^{\cdots}$ \\
\hline \multicolumn{5}{|c|}{$\begin{array}{l}\text { Model } 1 \text { and Model 2:3157 observations nested within } 86 \text { subjects ( } 40 \mathrm{HS}, 46 \mathrm{LS} \text { ). } \\
\text { Model } 3 \text { and Model 4:3108 observations, including } 591 \text { stressful events, nested within } 86 \text { subjects ( } 40 \text { HS, } 46 \text { LS). }\end{array}$} \\
\hline \multicolumn{5}{|c|}{$\begin{array}{l}\text { a Non-significant fixed effects were excluded from the model. Model 1: Coffee intake. Alcohol intake, Physical } \\
\text { exertion. Model 2: PS Group, PS Group by Time of day. Day type, PS Group by Day type, Trait anger. Psychosomatic } \\
\text { symptoms, Life-events, Chronic difficulties. Positive affect. Model 3: Event content - Work. Social interaction, Task } \\
\text { demand-; Event appraisal - Unpleasantness. Predictability, Importance. Controllability -- Event by PS Group. Event by } \\
\text { Trait anxiety, Event by Depression. Model 4: Positive affect. Event by Agitation, Event by Negative affect. }\end{array}$} \\
\hline
\end{tabular}


Due to multicollinearity, it was necessary to add these variables separately to the model. In Table 6.2 (Column 2), the effect of trait anxiety is shown. The effect of depression, when entered separately, was of a similar magnitude (estimate Depression: $0.003, \mathrm{p}=.05$ ).

To test the association between mood state and cortisol levels, the variables Positive Affect, Negative Affect and Agitation were next added to the model. Positive Affect was not significantly related to cortisol. In contrast, both Negative Affect and Agitation were associated with higher cortisol. Because Negative Affect and Agitation were intercorrelated ( $r=0.51 ; p<.0001$, over 3569 reports), the effect of Negative Affect was masked when entered simultaneously with Agitation into the equation. When entered separately, standardized effects were approximately the same for both variables. In Table 6.2. (Column 2), the effect of Agitation is shown (estimate Negative Affect: $0.032, \mathrm{p}<.01$ ).

Finally, random effects for Negative Affect and Agitation were included in the model. The random effect for Agitation was significant, meaning that the effect of Agitation on cortisol varied across persons. In Figure 6.2., the individual estimates of the effect of Agitation on cortisol are plotted for HS and LS subjects. Here, it is evident that some individuals displayed a considerably closer relationship between cortisol and Agitation than others, but that these individual differences were not associated with perceived stress level. 




INDIVIDUAL ESTIMATES (SLOPES) OF AGITATION EFFECTS

Figure 6.2. Distribution of the individual estimates of the effects of the mood state Agitation on cortisol for HS and LS subjects. These estimates were calculated by an empirical Bayes approach, using multilevel analyses (see Method section).

To provide a clearer picture of effect magnitudes, the estimate for each variable can be translated back into the original measurement unit. A standard deviation increase in trait anxiety, for example, was associated with a cortisol increase of 5.4 $\mathrm{ng} / \mathrm{dl}$ above the mean level of $81.3 \mathrm{ng} / \mathrm{dl}$. Regarding Agitation, the mood state effect estimate shown in Table 6.2. (Column 2) indicates the increase in transformed cortisol associated with a one unit increase on the mood scale (from 1 to 2 on the scale ranging from 1 to 7). Translated back into raw cortisol, this one unit increase was associated with a cortisol increase of $4.7 \mathrm{ng} / \mathrm{dl}$ above the mean cortisol level. When a subject scores, for instance, 5 on the negative mood scale, the mood estimate (see Table 6.2., Column 2) should be multiplied by 4, resulting in a higher cortisol level. A 4 unit increase on the mood scale was associated with a cortisol increase of $20 \mathrm{ng} / \mathrm{dl}$ above the mean cortisol level. 


\section{Reactivity of cortisol to stressful events}

In the third model, the occurrence of a stressful event since the last ESM report (Event, dummy-coded as $\mathrm{I}$ or 0 ) and variables representing the coded content of the event (work-related or not, social interaction or not, task demand or not; all coded as I or -1 ) and rated characteristics of the event were added to the model. ${ }^{5}$ Results are summarized in Table 6.2. (Column 3). Cortisol levels were significantly elevated following stressful daily events. No random effect for Event was found, meaning that the influence of a stressful event on cortisol did not differ significantly between subjects. Although most of the Event effect was determined by HS subjects, level of perceived stress did not have a significant effect on cortisol reactivity to stressful events. Next, we controlled for the time-frame of the event: was the event already finished when a subject took a saliva sample ( $71 \%$ of all events), or was it still going on ( $29 \%$ of events) ${ }^{6}$ When a stressful event was ongoing at the time the ESM report was completed, the level of cortisol was significantly higher than if the event had already ended. Translated back into the original units, ongoing events were associated with mean cortisol increases of $10.6 \mathrm{ng} / \mathrm{dl}$ and terminated events with increases of $5.7 \mathrm{ng} / \mathrm{dl}$, relative to no event.

The various event content categories (work vs non-work events, social interactions vs non-social events and task demands vs non-task demands) were not significantly associated with cortisol. Contrary to expectation, event unpleasantness, importance, predictability, and controllability contributed no additional effects. Prior frequency did have an independent effect: when similar kinds of events were reported to have occurred relatively frequently in the past, the cortisol response to the current event was lower. Here, significant interactions with trait anxiety were found (see Table 6.2. Column 3). Significant interactions were also found for depression and perceived stress (when entered separately to the model, replacing trait anxiety): cortisol levels were less likely to show this apparent habituation effect to recurrent events in individuals scoring high on these traits (estimate Prior frequency * Depression: $0.0017, \mathrm{p}<.01$; estimate Prior frequency * PSS: $0.033, \mathrm{p}<.01$ ).

\section{Relationship between cortisol reactivity to events and mood state}

In our final model, we examined whether mood states mediated the cortisol response to stressful daily events. Positive Affect had no effect on cortisol and was therefore excluded from the model. When entered separately into the model, the two negative mood states, Negative Affect and Agitation, were each positively related to cortisol. With Agitation in the model, the effect of stressful event occurrence on cortisol was lowered to a non-significant level. A similar pattern was observed for Negative Affect: the effect of Event was reduced (although in this case it remained significant) after addition of Negative Affect to the model. This indicates that negative mood states were stronger predictors of cortisol level than stressful events. Finally, the lack of significant interactions between the two negative mood states and Event indicates that mood effects on cortisol were no greater in the presence than in the absence of an Event. Table 6.2. (Column 4) summarizes model parameters, with

5 For each rated characteristic of the event, the subject's mean was subtracted from the actual rating, thus keeping the Event effect unchanged; this allowed us to assess the withiri-person effects of the various event appraisals above and beyond the effect of the event itself.

6 First coded -0.5 (event finished), 0 (no event), or 0.5 (ongoing event), and then weighted for the number of events that were 'finished' compared to the number of events 'ongoing' in order to keep the effect of Event unchanged after addition. 
Agitation included. Similar results were obtained when Negative Affect was included instead of Agitation (estimate Negative Affect: 0.024, p<.05).

\section{DISCUSSION}

\section{Etfects of tralt measures of stress and distress on salivary cortisol}

The current study provides evidence that trait negative affectivity can result in moderate cortisol elevation. Trait anxiety and depression showed small but statistically significant positive associations with cortisol. To date, evidence for elevated cortisol in association with anxiety and depression has come mainly from studies of more severely or chronically distressed subjects (Rahe et al., 1990; Schaeffer \& Baum, 1984) or psychiatric patients (Linkowski et al., 1985), while several studies of healthy adults have failed to demonstrate an association between basal cortisol and these or other trait measures (Brandtstädter et al., 1991; Ockenfels, Porter, Smyth, Kirschbaum, Hellhammer, \& Stone, 1995). It should be noted that subjects in the current study, having been selected on the basis of extreme perceived stress scores, displayed a broader distribution of depression and anxiety scores than would have been likely in an unselected sample of white collar workers. Moreover, the large number of cortisol samples collected per subject at different times of day provided more reliable estimates of hormone levels than is usually the case. Anxiety and depression were highly correlated in this sample, which made it impossible to assess the relative contributions of each. Further research is necessary to clarify this issue.

We had hypothesized that cortisol levels would be elevated in subjects with high perceived stress (see Introduction, Question 1). A repeated measures analysis of variance of a subset of these data (van Eck \& Nicolson, 1994), with cortisol values aggregated over each subject and time of day, had shown higher cortisol levels in HS compared to LS subjects when only the three workdays were included. Given the lack of a statistically significant effect of PSS on cortisol in the current multilevel analysis, however, we conclude that perceived stress is not a strong predictor of cortisol secretion, at least in this sample. Nor did we find significant interaction effects between PSS and the time of the day that would support a stress-related disruption in the circadian rhythm of secretion, as has been suggested by other authors (Caplan et al. 1979; Ockenfels et al. 1995). Additional measures of life stress, including an objective life event measure as well as a measure of chronic difficulties over a wide range of life domains, similarly showed no statistically significant associations with cortisol. The intercorrelations among the various measures of stress and distress argue for cautious conclusions about the relative influence of each on cortisol levels. The apparently disparate results of a recent study (Ockenfels et al., 1995), which showed an effect of perceived stress, but not of anxiety or depression, on cortisol levels should be seen in this light.

\section{Effects of state affect measures and stressful daily events}

The two negative mood states, Agitation and Negative Affect, were both associated with higher cortisol levels. The estimated effects were relatively small: a one unit increase in Agitation (from 1 to 2 on a scale ranging from 1 to 7 ) was associated with a cortisol increase of $4.7 \mathrm{ng} / \mathrm{dl}$ above the mean level of $81.3 \mathrm{ng} / \mathrm{dl}$. At more extreme scores on the mood scale, however, cortisol increases were more pronounced. Highly negative states were reported infrequently in daily life contexts 
by both HS or LS subjects, resulting in highly skewed distributions and small standard deviations (Agitation was scored as 1 'not at all' on $56 \%$ of all 'beeps', Negative Affect on $51 \%$ of all 'beeps') (see also Table 6.1). The low mean levels of negative states reported by both HS and LS subjects are characteristic of normal samples, with men tending to report even lower, less extreme levels of negative emotions as well as positive emotions than women (Cameron, 1975; Diener et al., 1985).

Positive mood showed no association with cortisol. The results of previous studies are inconsistent with respect to the effects of positive mood states: in some cases, higher positive affect was accompanied by lower cortisol (Hubert \& de JongMeyer, 1990; Hubert \& de Jong-Meyer, 1991), in others, by higher cortisol (Brown et al., 1993; Hubert, Möller, \& de Jong-Meyer, 1993). One of the few naturalistic studies revealed an inverse relationship between positive mood states and cortisol levels (Kugler \& Kalveram, 1989), but this analysis, by aggregating mood states for each subject, tested only between-subject and not within-subject associations. In the present study, the Positive Affect scale included both high (e.g. 'energetic') and low arousal (e.g. "relaxed') items. If, as might be expected on theoretical grounds (Hennessy \& Levine, 1979), high arousal states are associated with elevated cortisol levels and low arousal states with lower cortisol, including both types of items in one scale could cancel out any effect. This possibility should be addressed in future studies of mood effects on cortisol.

Daily events or situations experienced as stressful were associated with increased cortisol secretion (see Introduction, Question 2). Surprisingly, the rated unpleasantness of an event had no additional effect, nor did other appraisal measures. We had expected that events involving negative social interactions might have more pronounced effects on cortisol, but categorization of events on this dimension, or on relationship to work, or task demands, did not improve the model. Significant effects may be difficult to find because of the heterogeneity of responses resulting from the variety of stressful events encountered. Responses will be modulated by all kinds of objective and subjective event characteristics as well as by their interactions with each other.

We did find a significant effect for the time course of an event, with ongoing events having the expected greater effect on cortisol than terminated events. This is in line with temporal patterns of cortisol excretion observed in response to laboratory stressors; after termination of stress exposure, cortisol levels typically return to baseline within 1-2 hours (Kirschbaum \& Hellhammer, 1989). In addition, events that were reported to be recurrent had a smaller effect on cortisol than more novel events, suggesting that habituation can occur. Although perceived stress, trait anxiety, and depression were not significantly associated with elevated cortisol reactivity to stressful events, cortisol habituation to recurrent daily stressors did appear to be less likely to occur in individuals scoring high on these measures. A similar pattern was observed in a recent laboratory study, in which subjects were asked to perform the same stressful task on $\mathrm{S}$ consecutive days. Here, the majority showed a significant cortisol response on the first day only, while a sub-group of individuals with low selfesteem, depressed mood, and physical symptoms showed persistent cortisol responses to the task over all exposures (Kirschbaum, Pruessner, Stone, Federenko, Gaab, Lintz, et al., 1995).

We found no statistically significant individual differences. ("random effects" in the model) in cortisol reactivity to events. While laboratory studies of responses to a battery of stress tasks provide some evidence for individual differences in reactivity 
(Forsman \& Lundberg, 1982), such differences appear to be less stable than differences in basal levels, which have a larger genetic component (Kirschbaum, Wust, Faig, \& Hellhammer, 1992b). It is possible that either the total sample size was too small or that the within-subject samples of events were too heterogeneous in the current study to reveal consistent and reliable individual response patterns.

The effect of stressful events on cortisol appears to be mediated to a large extent by associated increases in negative mood, since addition of Agitation or (to a lesser extent) Negative Affect to the model resulted in a decrease in the Event effect to a non-significant level. This interpretation is consistent with most views of the stress process and is supported by unpublished results from the same study, showing that both Negative Affect and Agitation increase following stressful events. More detailed temporal analyses of these data may help clarify whether an emotional response to an event is necessary to trigger a hormonal response.

\section{LImitations and conclusions}

For practical reasons, the study focused on a relatively homogeneous group of male white collar employees. We are not able to assess the extent to which the results are generalizable to other populations. Given the evidence for sex differences in neuroendocrine reactivity to psychosocial stress (Collins \& Frankenhaeuser, 1978; Kirschbaum, Wust, \& Hellhammer, 1992c), a replication of this study with inclusion of female subjects would be informative. Another limitation is that we did not assess positive daily events, which could theoretically affect cortisol levels via their influences on mood (Stone, 1987), possibly mitigating the effects of negative events. However, since positive events have their greatest effect on positive affect (Stone, 1987), it is important to recall that Positive Affect was not associated with cortisol in the present study.

In conclusion, this study suggests that neuroticism is not simply a confounder in psychosomatic research (McCrae, 1990), but is associated with increased adrenocortical activity during normal daily activities. Self-report measures of anxiety and depression, negative mood states and daily stressors, all known to be strongly related to trait negative affectivity (Watson \& Pennebaker, 1989), were associated not only with increased perceptions of chronic stress, but also with elevated cortisol levels. The finding that even minor everyday events and fluctuations in mood states have an impact on cortisol secretion points to a possible mechanism linking subjective experience to health outcomes. Further research is needed to determine whether mild, chronic cortisol elevation contributes to disease processes in somatic illness or psychiatric disorder, in particular depression.

\section{ACKNOWLEDGEMENTS}

We thank C. Dijkman for assistance in all aspects of the study. We also thank Prof. T.A.B. Snijders and four anonymous reviewers for substantial comments on earlier versions. 


\section{Chapter 7}

\section{Individual differences in cortisol responses to a laboratory speech task and their relationship to responses to stressful daily events}

Biological Psychology 43(1): 69-84 (1996)

Marleen van Eck ${ }^{1}$, Nancy Nicolson ${ }^{1}$, Hans Berkhof ${ }^{2}$, Jose Sulon ${ }^{3}$

Department of Psychiatry \& Neuropsychology, Social Psychiatry and

Psychiatric Epidemiology section, University of Limburg, the Netherlands

2 Department of Statistics and Measurement Theory, University of Groningen, the Netherlands

3 Faculty of Veterinary Medicine, University of Liege, Belgium 

A Stress Inducing Speech Task (SIST) was used to investigate the contribution of perceived stress, individual traits, and current mood states to individual differences in salivary cortisol responses. Additionally, we examined the correspondence between laboratory baseline cortisol levels and overall levels in daily life, and between cortisol responses to the SIST and a measure of stress reactivity to stressful events in daily life. Forty-two 'high stress' and forty-five 'low stress' whitecollar males completed the SIST and an Experience Sampling study, in which stressful daily events and cortisol levels were monitored for five days. No association was found between perceived stress, trait anxiety, anger, depression, psychosomatic symptoms, coping style or personality and cortisol responses to the SIST. Negative mood state at baseline was associated with higher cortisol levels at baseline, just before, and just after the speech. Laboratory and field cortisol levels were moderately correlated, but no association was found between laboratory and field response measures. Laboratory baseline levels, but not responses to the speech task, were significant predictors of field cortisol levels.

\section{INTRODUCTION}

Changes in cortisol levels have been shown to be sensitive indicators of psychosocial stress and coping patterns in both laboratory and natural situations (Frankenhaeuser, 1986; Mason, 1968; Rose, 1984). Although adrenocortical responses to psychological stress have been found to differ between individuals, sources of these differences are far from clear. For a better understanding of the potential link between cortisol responses and disease (Bassett, 1982; Cohen et al., 1991; Gaillard \& Al-Damluji, 1987; Troxler et al., 1977), more insight into individual differences in cortisol reactivity is needed. Since it is reasonable to assume that stress reactions will only lead to disease when they are prolonged or occur very often, it is likely that the pathogenic influence of cortisol hyperreactivity (or hyporeactivity) depends on whether this reflects a stable individual characteristic. Evidence from animal studies indicates that cortisol hyporesponders may be more prone to autoimmune disorders (Sternberg et al., 1989), while cortisol hyperresponders might be more vulnerable to infectious diseases (Mason, 1991). Research on psychological stress and cortisol indicates that the subjective appraisal of apprehension and emotional involvement can lead to increased cortisol secretion (Kirschbaum \& Hellhammer, 1989; Mason, 1975; Rose, 1984). Personality and other traits may influence how stressful situations are appraised and may thus have predictive value for understanding individual differences in emotional and physiological responses to apparently identical situations (Arnetz \& Fjellner, 1986; Vaernes et al., 1982).

The goals of the current study were twofold. Our first objective was to determine whether perceived stress level (as a measure of mild chronic stress) was related to emotional and cortisol responses to an experimental stressor. From existing data, it is not clear whether chĩonic stress affects an individual's pattern of response to acute stressors. There is some evidence for an attenuated cortisol response in chronically stressed human subjects (Bourne et al., 1967; Friedman, Mason, \& Hamburg, 1963) but these studies focused on responses to highly traumatic situations (e.g., acute medical complication in a fatally ill family member, combat exposure). Experiments in rats indicate that the effects of chronic or repeated stress exposure on later glucocorticoid responses depend to a large extent on stressor intensity (Ottenweller, Natelson, Pitman, \& Drastal, 1989). 
There is considerable overlap between perceived stress and other subjective measures of distress, negative affectivity, and ineffective coping. For this reason, we also investigated the contribution of trait anxiety, depression, psychosomatic symptoms, anger, coping style and personality to individual differences in cortisol response. Such traits have been associated with basal cortisol levels (Brandtstädter et al., 1991; Pope \& Smith, 1991; Schaeffer \& Baum, 1984) as well as with stress responses (Bohnen et al., 1991; Demyttenaere et al., 1989; Kirschbaum et al., 1989; Nicolson et al., 1992) in a number of previous studies. Other studies, however, have found no association between personality traits, coping styles, and cortisol responsiveness to laboratory stressors (Bossert et al., 1988; Kirschbaum et al., 1992a).

As noted above, individual characteristics are thought to be important determinants of the emotional response to a given situation; mood states are, in turn, likely to mediate the endocrine response to the situation. Although there is abundant evidence that cortisol increases in response to distress or negative mood (Arnetz \& Fjellner, 1986; Lundberg \& Frankenhaeuser, 1980; Mason, 1968; Nicolson, 1992), the effects of positive mood are less clear. Positive affective states have been associated with decreases (Hubert \& de Jong-Meyer, 1989; Kugler \& Kalveram, 1989) as well as increases (Brown et al., 1993) in cortisol levels.

Our second objective was to compare cortisol responses measured in the laboratory with those measured in real life. As Turner, Ward, Gellman, Johnston, Light, \& van Doornen (Turner et al., 1994) have stated: "if exaggerated responses do play a role in disease development, it is via everyday challenging situations that they will exert their pathogenic influence. The usefulness of laboratory assessment rests, in part, on the assumption that laboratory responses reflect those occurring during natural situations". The widespread availability of ambulatory monitoring techniques has stimulated research on the relationship between laboratory and daily life cardiovascular activity. Evidence from several studies indicates that blood pressure levels obtained in the field are better predictors of subsequent hypertension, complications from hypertension and mortality than measurements obtained in the doctor's office (Devereux, Pickering, Harshfield, 1983; Perloff, Sokolow, \& Cowan, 1983). A recent review (Turner et al., 1994) concluded that moderate evidence exists for laboratory-field generalization of cardiovascular activity. The evidence differs in strength, however, for different types of laboratory-field associations. The associations between basal, chronic levels of cardiovascular activity across settings are the strongest, while inconsistent support has been found for the relationship between laboratory reactivity and field reactivity, and between laboratory reactivity and field levels.

Especially with regard to endocrine reactivity, the generalizability of laboratory studies to field studies has been underexplored. In a study of plasma catecholamine reactivity in a small sample of healthy young men (Dimsdale, 1984), no correlation was observed between endocrine responses to laboratory mental arithmetic and those to real-life public speaking. Although van Doornen and Blokland (1992) observed a correlation between pre-task adrenaline levels in the laboratory (reaction time and tracking task; cold pressor test) and the level preceding a real-life siressor (public defense of $\mathrm{PhD}$ thesis), urinary catecholamine responses to the laboratory tasks did not correlate with responses to the field stressor. To our knowledge, only one siudy has investigated laboratory-field associations of cortisol excretion patterns (Houtman \& Bakker, 1987). In a sample of nine student teachers, saliva cortisol responses were compared in a real and a standardized lecturing situation. Most correlations were low and nonsignificant, but this may have been due 
to the small sample size and the poor standardization of time at which the subjects started lecturing in the real life situation.

In the current study, a Stress Inducing Speech Task (Steiner \& Levine, 1988) was employed to investigate trait and state correlates of salivary cortisol responses to stress. Although studies of psychophysiological reactivity often employ standardized laboratory tasks such as mental arithmetic, computer games or cold pressor tests, one can question whether the reactions observed are relevant or valid reflections of habitual reactivity to naturally occurring psychosocial stressors. Speech tasks have a greater ecological validity and reliably induce endocrine and cardiovascular responses as well as moderate distress (Bassett et al., 1987; Dimsdale, 1984; Kirschbaum \& Hellhammer, 1993; Steiner \& Levine, 1988). In addition to the laboratory experiment, subjects participated in a naturalistic field study of daily stress and cortisol dynamics. This allowed us to evaluate the consistency of laboratory and field cortisol measures, particularly with regard to the usefulness of the laboratory measures in predicting cortisol levels and responses to naturally occurring stressors in the field situation.

\section{METHODS}

\section{Subjects}

Local industries and government agencies were approached via their personnel departments. 316 male white collar workers from six different industries or agencies completed the screening questionnaire. The mean score on the Perceived Stress Scale (PSS) for this sample was 12.7 (s.d. 6.0), similar to US. norms (mean 13.02, s.d. 6.45) (Cohen et al., 1983). Individuals scoring in either the upper or the lower tertile of the screening sample PSS distribution (PSS-10 score $\leq 10$ or $\geq 16$ ), and with no history of serious chronic illness, endocrine disorder, or medications known to affect cortisol levels (as ascertained by self-report) were later approached to participate in the main study, until 92 subjects, balanced for 'low' and 'high' stress states, were recruited ( $85 \%$ of those approached). Of the 92 subjects who took part in the study, 5 were excluded from the analysis due to missing data. The final sample included 42 subjects in the 'high stress' (HS) group and 45 subjects in the 'low stress' (LS) group. Mean age was 42.1 years (range 27 to 57 years): $89 \%$ were married, and $81 \%$ had children living at home.

\section{Experimental stressor: Stress Inducing Speech Task (SIST)}

The SIST took place between 11 am and 1 pm (before lunch), one or two days after subjects completed participation in an Experience Sampling study (see below). On arrival, subjects were unaware that they would be asked to deliver a speech. After completing baseline measures, subjects were asked to prepare ( 10 minutes) and present (5 minutes) a videotaped speech describing their personal strengths and weaknesses for later evaluation by a team of psychologists. Following the preparation period. subjects, received a signal to begin their presentation while looking directly into the camera. After the speech, subjects relaxed for 15 minutes in neutral activities (e.g. reading magazines). At four timepoints subjects filled in a mood questionnaire and provided a saliva sample: (T1) upon arrival, (T2) after the 10 minutes of preparation, (T3) after the 5-minute presentation, and (T4) after 15 minutes of relaxation. During the course of the study, the recovery period was lengthened to provide a clearer picture of the cortisoll response profile; for 49 subjects a fifth saliva sample (T5) was taken on average 50 minutes after the first assessment.

The mood questionnaire consisted of 15 five-point Likert scale items (Folkman \& Lazarus, 1985). Subjects indicated to what extent $(0=$ not at all; $5=$ a great deal $)$ they felt: 
worried, anxious, nervous, tense, confident, hopeful, eager, irritated, sad, disappointed, guilty, exhilarated, pleased, happy and relieved. The 15 mood items formed two distinct dimensions: Negative Affect (NA, 8 items) and Positive Affect (PA, 7 items), with Cronbach's alpha's at T1 of .79 for NA and .75 for PA.

\section{Fleld study: cortisol dynamics In daily life}

The Experience Sampling Method (ESM) (Csikszentmihalyi \& Larson, 1987; de Vries, 1992) was used to collect data from subjects at selected moments during their normal daily activities. Subjects received auditory signals ('beeps') to complete a questionnaire and a saliva sample. Subjects were sampled for a period of 5 consecutive days ( 3 work and 2 non-work days), 10 times each day, at semi-random intervals of approximately 90 minutes between the hours of 8 am and $10 \mathrm{pm}$. Compliance with the procedures was generally good: $83 \%$ of all possible responses were completed within the time limit of 20 minutes. For a more detailed description of the Experience Sampling Method procedure and compliance issues, see van Eck \& Nicolson (1994).

In addition to items concerning current mood, activity, social and physical context, the ESM form contained a 7-point Likert scale (from 1 'not at all stressful' to 7 'very stressful') for the rating of any stressful event or situation which had taken place in the interval since the last ESM report. Subjects reported a total of 626 stressful events during the five days of ESM, with a mean of 7.1 events per subject (median 5.0; st.dev. 7.3). Events were reported on an average of $16.8 \%$ (st.dev. 16.5) of total "beeps". Information concerning smoking, food, coffee, and alcohol intake, and maximum level of physical exertion since the last beep was also obtained.

\section{Paychological moasures}

The following measures were used in the current analysis:

Perceived Stress: The 10-item version of the PSS was translated into Dutch. The PSS is a global measure of the degree to which situations in one's life are appraised as stressful. Items tap the extent to which individuals feel their life to be unpredictable, uncontrollable, and overloaded. The items were rated on a 5-point frequency scale, ranging from 'never' $(0)$ to 'very often' (4) in the last month. All subjects completed the PSS twice, during the initial screening and again immediately preceding Experience Sampling. The two PSS scores were highly correlated (rho=73, $<<.001$ ). The mean of the two PSS scores was 7.2 (st.dev. 2.2) for the low stress group and 18.1 (st.dev. 3.4) for the high stress group.

Psychosomatic symproms: The revised version of the SUNYA Psychosomatic Symptom Checklist (PSC; Attanasio et al., 1984) includes 17 common psychosomatic complaints (e.g., headaches, backaches and nausea). Subjects rated each complaint on 5-point scales for frequency ( 0 'never or rarely occurs' io 4 "occurs daily') and intensity ( 0 'not bothersome' to 4 'extremely bothersome'). A Total Score is obtained by summing the cross-products of each item's frequency by intensity.

Depression: Depressive symptomatology was assessed with the Dutch translation (Dijkstra, 1974) of the Zung Self-Rating Depression Scale (Zung, 1965).

Anxiery: Trait anxiety was measured with the Dutch version (van der Ploeg et al., 1980) of the State-Trait Anxiety Inventor (STAI).

Anger: Trait anger was measured with the Dutch version (van der Ploeg et al., 1982) of the Spielberger Trait Anger Scale. The scale has two subscales: 'anger-temperament' and 'angerreaction :

Personality: Personality characteristics were assessed with the Dutch abridged MMPI (NVM: Luteijn \& Kok, 1985), which consists of five scales: Negativism, Somatization, Timidity, Major Psychopathology, and Extraversion. 
Coping: Coping style was assessed with the 47-item Utrecht Coping List (UCL; Schreurs \& van de Willige, 1988). The seven factor-analytically derived subscales are: 'active problem solving', 'palliative responding', 'avoidance', 'seek social support', 'depressive reaction", 'expression of emotions' and 'comforting cognitions'.

HS subjects scored significantly higher than LS subjects on psychosomatic symptoms ( 27.5 vs $6.1, \mathrm{p}<.001)$, depression $(48.4$ vs $36.5, \mathrm{p}<.001)$, anxiety $(39.8$ vs $28.3, \mathrm{p}<.001)$, anger (18.0 vs 14.6, $\mathrm{p}<.001$ ), the personality scales negativism ( 19.0 vs $10.0, \mathrm{p}<.001)$, somatization $(9.5$ vs $2.8, \mathrm{p}<.001)$, timidity $(11.3$ vs $8.3, \mathrm{p}<.05)$, and psychopathology $(2.8$ vs $1.3, \mathrm{p}<.01)$, and on the coping styles active coping (17.3 vs $21.1, \mathrm{p}<.001)$, palliative reaction (17.2 vs 14.6. $\mathrm{p}<.001$ ), depressive reaction (12.6 vs 8.8, $p<.001$ ), and expressed emotions (6.9 vs 5.8, p<.01). Where possible, mean scores for both samples were compared to published norms for the Dutch population. The general pattern found was that LS subjects scored average or below average compared to the norm scales, while HS subjects scored above average or high. No significant differences between HS and LS groups were found for the personality scale extraversion (16.5 vs $15.4)$ and the coping styles avoidance (15.1 vs 14.1), seek social support (12.5 vs 11.7), and comforting cognitions (11.7 vs 10.8 ).

\section{Saliva sampling and biochemical analysis}

Subjects collected saliva by holding a cotton dental roll in the mouth for approximately 1 minute. The saturated roll was placed in a capped plastic vial ("Salivette", Sarstedt). Uncentrifuged samples were stored at -20 degrees $C$, until analyses. Salivary cortisol levels were determined in duplicate by direct radioimmunoassay (Ansseau, Papart, Pitchot, Timsit-Berthier, Legros, \& von Frenckel, 1992), using ${ }^{125} \mathrm{I}$-cortisol and antiserum made against the 3-CMO-BSA conjugate. The lower detection limit of the assay was $12 \mathrm{ng} / \mathrm{dl}$, with a mean intra-assay coefficient of variation of $4.8 \%$ (range: $2.2 \%-7.5 \%$ for 4 assays).

\section{Statistical analysis}

A fifth root transformation of the raw cortisol data (cortisol ${ }^{0.2}$ ) was used to obtain normally distributed residuals throughout the day in the cortisol field data. For comparability, the same transformation was applied to the laboratory cortisol data. Multivariate analysis of variance for repeated measures (MANOVA procedure, SPSS) was performed to test for effects on cortiso! levels during the SIST; in addition to the repeated measures factor Time (TI through T4 and, for a subsample, T5), Group (PSS high versus low) and Group x Time interaction effects were tested. Baseline cortisol was included as a covariate. A separate MANOVA analyses was repeated on the mood measures. Significance levels are based on multivariate (F) tests. Forward linear stepwise regression analyses were performed to estimate the proportion of variance in cortisol (dependent variable) explained by various (independent) person variables.

Cortisol responses to the SIST were computed as area-under-the-concentration-time curve (AUC), using the trapezoidal integration. The value of the laboratory sample at TI is reported as laboratory baseline (L.BASAL). L.RESPONSE was defined as the portion of the AUC above L.BASAL. Because the SIST always took place between 11.00 a.m. and 1.00 p.m., prior to lunch, field cortisol level (F.BASAL) was defined as the mean level over 5 days with the third ESM beep (at approximately $11.15 \mathrm{a.m}$.) as our reference point. The mean (within-subject) coefficient of variation for the five cortisol values was $4.1 \%$, ranging from $1.5 \%$ to $7.2 \%$.

The model used for the analysis of the cortisol field data has been described in detail elsewhere (van Eck, Berkhof, Nicolson, \& Sulon, in press). The multilevel model is a variant of the multiple linear regression model applicable for data with a hierarchical nesting structure (here: repeated measurements within persons), where the dependent variable (here: cortisol) is defined at the lowest level of the hierarchy. In this model, field cortisol level (F.LEVEL) was defined as the 
mean cortisol level over the five days in the field after controlling for diurnal patterns and the other factors (smoking, lunch and other food intake) found to have significant effects.

Individual cortisol response estimates to stressful events in daily life were calculated by an empirical Bayes, approach, using multilevel analyses (Bryk \& Raudenbush, 1992). These response estimates are weighted averages of an overall regression estimate (based on the whole data set) and a person-specific regression estimate. The weight of the person-specific estimate, relative to the overall estimate, depends on its reliability as an estimate of the individual responses. Events were dummy coded as $\mathrm{l}=$ event, $0=$ no event, since use of the 7-point scale did not improve the prediction of cortisol. Stress response scores in the field (F.RESPONSE) were estimated after diurnal patterns and other factors (listed above) with significant effects on cortisol levels were controlled for in the multilevel model. As reported elsewhere (van Eck et al., in press), stressful daily events were associated with significantly higher cortisol secretion.

The association between laboratory and field cortisol was tested with Spearman rank-order correlations between: 1) L.BASAL and F.BASAL, 2) L.RESPONSE and F.BASAL, and 4) L.RESPONSE and F,RESPONSE. As a last step, the contributions of L.BASAL and L.RESPONSE as predictors of F.LEVEL were evaluated with multilevel analysis.

\section{RESULTS}

\section{Effects of percelved stress level on cortisol and mood response to the experimental stressor}

Salivary cortisol responses to the SIST are shown in Figure 7.1. Most subjects responded to the task with increased cortisol secretion; 76 of the 87 subjects showed a cortisol response greater than L.BASAL (T1). On average, peak cortisol levels were observed at T4, approximately 30 minutes after the SIST preparation period began and 15 minutes after the speech ended. The maximum increase in cortisol during the SIST averaged $159 \mathrm{ng} / \mathrm{dl}$ (a percentual increase of 109\%) above L.BASAL.

MANOVA analysis revealed significant effects for the L.BASAL covariate $(B=$ $82, t(l, 84)=16.25, p<.001$ ) and for the repeated measurement factor Time (T! through T4: $F(3,255)=59.39 ; p<.001)$. The cortisol response pattern of the high stress group did not differ significantly from that of the low stress group, as evidenced by nonsignificant effects for both the PSS main effect $(F(1,84)=.02$; ns $)$ and the PSS by Time interaction $(F(3,255)=.18$; ns). MANOVA analysis for the sub-sample of 49 subjects with a final cortisol measurement at T5 yielded similar results: a significant main effect for the factor Time (T1-T5; $F(4,18.8)=16.83 ; p<.001)$, no main effect for PSS $(F(1,46)=.06$; ns $)$, and no interaction effect for PSS by Time $(F(4,188)=1.46$; ns). On average, cortisol at T5 (268 ng/dl) had significantly declined relative to peak levels $(335 \mathrm{ng} / \mathrm{dl}$ ) (Wilcoxon test, $p<.001$ ), although it was still elevated in relation to L.BASAL ( $167 \mathrm{ng} / \mathrm{dl}$ ). Comparing the recovery process in high vs. low stress groups, post-hoc comparisons showed no difference between the groups in either the magnitude of the cortisol elevation above L.BASAL persisting at T5 (high PSS $(n=26): 109 \mathrm{ng} / \mathrm{dl}$; low PSS $(\mathrm{n}=23): 95 \mathrm{ng} / \mathrm{dl}$; Mann-Whitney test, $\mathrm{n} . \mathrm{s}$.$) or the$ magnitude of the decline in cortisol from maximum response to T5 (high PSS: decline $=54 \mathrm{ng} / \mathrm{dl}$; low PSS: decline $=82 \mathrm{ng} / \mathrm{dl} ;$ Mann-Whitney test, n.s. $)$. 


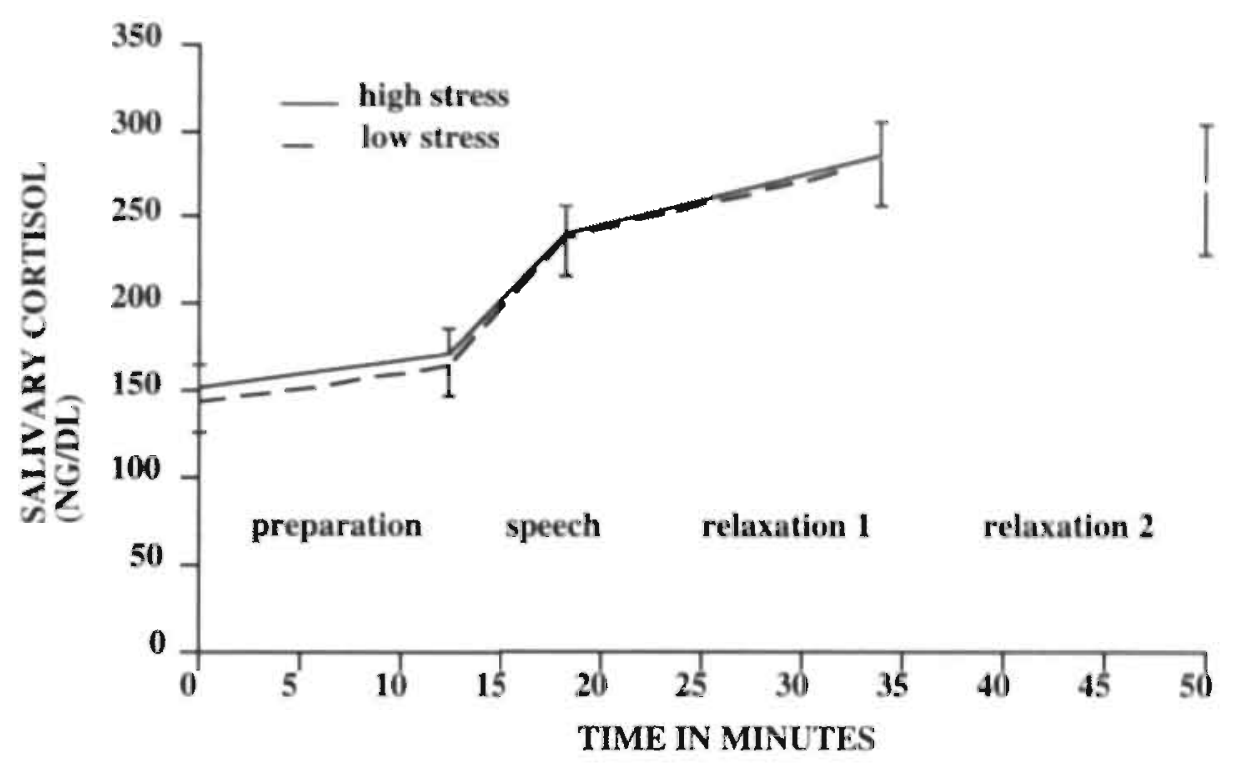

Figure 7.1. Cortisol response to speech task in high and low stress subjects. Data at T1 - T4 based on 87 subjects, data at T5 based on 49 subjects.

Mood states also changed in response to the stress task, with significant Time effects for both PA $(F(3,255)=14.53 ; p<.001)$ and NA $(F(3,255)=42.11 ; p<.001)$. Post-hoc comparisons revealed a significant NA increase $(p<.0001)$ and PA decrease $(p<.001)$ from $\mathrm{T} 1$ to $\mathrm{T} 2$, after preparation but before speech delivery. As can be seen in Figure 7.2., mood had recovered to baseline by 'T3. 'High stress' subjects had a higher overall level of NA than 'low stress' subjects $(F(1,85)=22.15 ; p<.001)$; the level of PA did not differ between groups $(F(1,85)=.55 ; p>.05)$. Time by PSS interactions were not significant; in other words, high stress subjects showed no greater increase in NA or greater decrease in PA in response to the task than low stress subjects. 


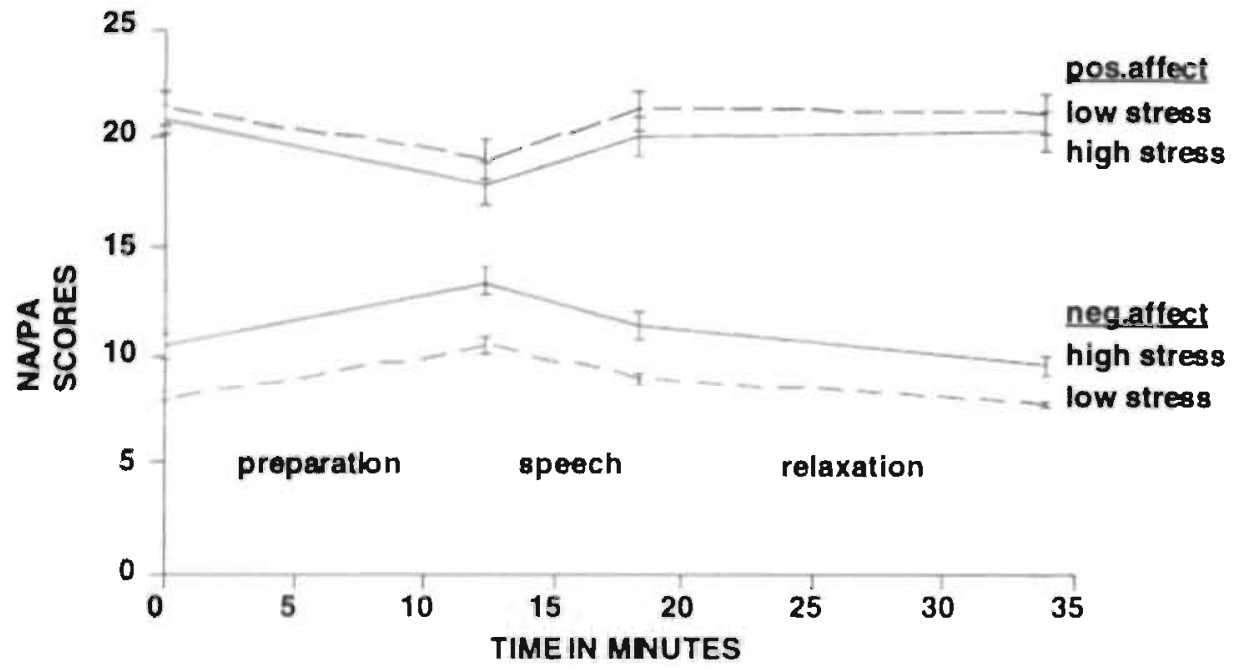

Higure 7.2. Response of NA and PA to speech task in high and low stress subjects.

\section{Relationship between mood state and cortisol response to the SIST}

To investigate whether subjects' emotional responses to the SIST were related to their cortisol responses, Spearman rank correlation coefficients were computed between the four repeated cortisol measurements and the summary measure L.RESPONSE, on the one hand, and the four mood measurements of both mood scales, on the other hand. No significant correlations were found between either NA or PA and L.RESPONSE. Of all correlations between the four PA values and the four cortisol values (T1 through T4), not a single coefficient was significant. However, NA at baseline was positively associated with cortisol at L.BASAL (T1), with cortisol just before the speech task (T2) and with cortisol just after the speech task (T3) (rho $=.29$; $\mathrm{p}<.01, r h o=.31 ; \mathrm{p}<.01, r h o=.25 ; \mathrm{p}<.05$, two-tailed tests).

\section{Relationship between trait characteristics and cortisol response}

Possible influences on cortisol L.RESPONSE of trait anxiety, depression, anger, coping styles, total psychosomatic complaints, and personality scales were tested with forward stepwise multiple regressions. None of the above traits was a significant predictor of L.RESPONSE (all higher $F$ probabilities than .05).

\section{Laboratory to fleld generalization}

L.BASAL was significantly higher than F.BASAL (147 ng/dl versus $116 \mathrm{ng} / \mathrm{dl}$; p<.001, Wilcoxon Matched-Pairs Signed-Rank Test, two-tailed test). The difference beiween these two measures was similar for high and low stress groups (HS: 36.9 ng/dl, LS: $24.7 \mathrm{ng} / \mathrm{dl} ;$ ns). The correlation between L.BASAL and F.BASAL cortisol levels was moderately strong ( $r h o=.56 ; \mathrm{p}<.001)$. No relationship was found between L.RESPONSE and F.BASAL ( $r h o=-.08 ; n s$ ). Next we compared cortisol responses to. the SIST (L.RESPONSE) with estimates of cortisol reactivity to naturally occurring stressful events (F.RESPONSE); no significant relationship was found ( $r h o=-.13$, ns). 
Table 7.1. Effects of lab baseline levels (L.BASAL) and lab response values (L.RESPONSE) on field cortisol level.

\begin{tabular}{lrrr}
\hline Fixed Effects & Estimate & S.E. & $\mathrm{p}<$ \\
\hline Intercept & 2.94 & 0.144 & 0.001 \\
Time of day (TIME) & -0.581 & 0.037 & 0.001 \\
TIME $^{2}$ & 0.129 & 0.010 & 0.001 \\
TIME $^{3}$ & -0.010 & 0.001 & 0.001 \\
Truncated function & 0.010 & 0.001 & 0.001 \\
Lunch & 0.040 & 0.020 & 0.03 \\
Other food intake & 0.025 & 0.01 .1 & 0.01 \\
Smoking & 0.043 & 0.021 & 0.02 \\
\hline L-BASAL & 0.172 & 0.050 & 0.001 \\
LRESPONSE & 0.001 & 0.003 & $n s$ \\
Random Effects & (Co)variance & S.E. & p< \\
\hline Person level: & & & 0.001 \\
intercept & 0.024 & 0.005 & 0.001 \\
Time of day & 0.0002 & 0.00004 & \\
Time of day/Intercept & -0.001 & 0.0004 & 0.001 \\
\hline Measurement level: & & & \\
Residual term & 0.059 & 0.002 & \\
\hline
\end{tabular}

* 3082 observations nested within 85 subjects (41 HS, 44 LS)

Finally, we estimated a multilevel model in which L.BASAL and L.RESPONSE were used to predict cortisol F.LEVEL. This analysis has the advantage of making use of all of the cortisol field data. Controlling for significant external influences should increase both the reliability of the dependent cortisol measure and its comparability to the laboratory assessments. As shown in Table 7.1., when both laboratory measures were entered simultaneously into the model, only L.BASAL explained a significant amount of variance in F.LEVEL.

\section{DISCUSSION}

The first aim of the present study was to evaluate the contribution of perceived stress and trait characteristics to individual differences in cortisol responses to a laboratory stress task. Associations between emotional and cortisol responses to the speech task were also examined. To summarize the main findings, the SiST elicited a strong cortisol response in a large majority of the subjects, which was unrelated to differences in perceived stress level. Trait anxiety, depression, psychosomatic symptoms, anger, coping style and personality similarly failed to predict the cortisol response. Neither negative nor positive emotional responses to the speech task showed any association with the cortisol response. High negative affect at baseline, however, was associated with higher total cortisol levels at baseline, just before the speech task, and again just after the speech task.

This suggests that individual differences in current distress (especially anticipatory distress) may be more important determinants of cortisol secretion than PSS, which is a measure of more chronic distress. Although high PSS was associated 
with high NA, no direct relationship was found between PSS and cortisol secretion. It is worth noting that subjects with the highest NA at lab baseline (T1) had, at the same time, the highest cortisol baselines relative to their own $11 \mathrm{a} . \mathrm{m}$. field levels ( $r h o=.26$, $\mathrm{p}<.01$ ). An elevated laboratory baseline, possibly due to anticipatory anxiety, could explain the lack of a relationship between NA and the additional cortisol response measured during the SIST. Moreover, the fact that high stress subjects had higher baseline NA than low stress subjects suggests that there may be a tendency toward higher cortisol in response to the SIST in this group, which was masked by the anticipation response.

As summarized in an overview of the published data (Kirschbaum et al., 1992a), most studies have found either no association or only a weak association between cortisol responses to stress and personality measures. The hypothesis linking personality factors with cortisol responses rests on the assumption that cortisol reactivity is a stable individual characteristic. There is some evidence in support of this assumption. Forsman and Lundberg (1982) found significant individual consistency in urinary cortisol responses to repeated tasks; correlations were higher between responses to more or less identical tasks (males: $r=65$; females: $r=.38$ ) than between responses to different kind of tasks (males: $r=13$; females: $r=19$ ). Consistency in cortisol responses to different tasks was also noted by Berger et al. (1987). Another study (Kirschbaum et al., 1992c) found significant intercorrelations among maximum cortisol responses to three stimulation procedures (mental arithmetic, public speaking, bicycle ergometry) in males, but not in females. In a more recent study in the same laboratory, significant correlations (ranging from .38 - .60) were found between cortisol responses to the same task repeated on 5 days (Kirschbaum et al., 1995) Although some response consistency seems to exist, at least in males, more research is necessary.

Even if stable individual differences in cortisol reactivity exist, it is not clear that they reflect psychological traits (Bossert et al., 1988). Genetic factors, for instance, have been shown to play a role in cortisol reactivity (Kirschbaum et al., 1992b). Recently, some support for an association between personality factors and cortisol reactivity was reported (Kirschbaum et al., 1995); low self-esteem, negative self-concept, depressed mood and physical health problems were found to differentiate high and low cortisol responders. Twenty healthy males were exposed on five consecutive days to the same psychological stress task (public speaking and mental arithmetic in front of an audience), with low responders showing evidence of habituation after the first day and high responders continuing to show large cortisol responses on successive task exposures. If only Day 1 cortisol responses had been analyzed (as in the present study), only one out of 12 correlations between cortisol responses and personality scales would have reached significance. This indicates that measuring a stress response once may be insufficient for the manifestation of individual differences in cortisol reactivity. This appears to be an important point for further research.

The second aim of the study was to investigate the relationship between laboratory and field cortisol activity. A moderately strong correlation was found between laboratory baseline levels and 11 a.m. field cortisol levels, and laboratory baseline levels also predicted some of the variance in overall field cortisol levels. In contrast, no association was found between laboratory responses and the field reactivity measure, and laboratory responses were not useful in predicting overall cortisol levels in the field. The finding of a relationship between laboratory and field levels of cortisol, but not between. laboratory and field reactivity, is more or less in 
accordance with results of cardiovascular studies, where levels are found to be more stable than reactivity / variability scores across settings (Turner et al., 1994). Similarly, greater consistency has been found for repeated lab baseline cortisol levels than for cortisol reactivity measures to different laboratory tasks (Berger et al., 1987; Forsman. 1982). As with personality traits, the temporal stability of the cortisol response is an important issue. To the extent that 'reactivity' is not a stable trait, a correspondence between laboratory and field cortisol responses will be unlikely. Results of our field study on the effects of stressful daily events on cortisol levels (van Eck et al., in press), did not provide support for stable individual differences in cortisol responses to daily events; cortisol responses were more dependent on the current mood state.

Another possible explanation for the lack of laboratory to field generalization of cortisol responses is the different timing of cortisol response measurement in the lab compared to the field study. Although in the lab cortisol was assessed parallel to the stress task, in the field, stressful events could have occurred at any time in the 90 minutes interval prior to cortisol assessment. Substantial post-stress recovery of cortisol levels might have taken place by the time the saliva sample was collected. However, when we repeated the analysis presented above with a new field response measure based on the subset of stressful events that were still ongoing when cortisol was assessed, again no association was found between laboratory and field reactivity.

Although real life pubiic speaking was reported as a stressful event during ESM sampling by a few subjects, any similarity between lab and field stressors was coincidental. One could argue that the predictive validity of a laboratory stressor would increase if one were to choose a specific field stressor with a strong correspondence in psychological meaning to the laboratory stressor (Houtman \& Bakker, 1987; van Doornen \& Turner, 1992). However, even if the researcher succeeds in matching a field stressor with a relevant laboratory task, a severe drawback of this approach is that only a small subset of the enormous range of stressors people encounter in their daily lives can be simulated in the laboratory. Furthermore, the importance of a laboratory - field association would be lessened dramatically if only linkings of specific lab - field stressors were to show an association (Turner et al., 1994; van Doornen \& Turner, 1992). Significant correlations may be difficult to find because of the heterogeneity of field responses resulting from the variety of stressful experiences encountered. Even more than in the laboratory (Strube, 1989), responses to daily life stressors will be modulated by objective and subjective event characteristics (e.g. novelty, severity, duration, content), interacting with each individual's characteristics and prior experiences (Forsman, 1982). In conclusion, while we found no evidence for an association between cortisol responses in laboratory and field, the paucity of studies on this subject and the difficulties (conceptual, methodological, and statistical) associated with its investigation point to the need for additional research.

\section{ACKNOWLEDGEMENTS}

We thank C. Dijkman for assistance in all aspects of the study. 



\section{Chapter 8}

\section{Concluding remarks}





\section{RECAPITULATION OF THE MAIN RESEARCH AIMS AND FINDINGS}

In an effort to increase our understanding of the stress process as it relates to health, the present research investigated the impact of minor daily events on mood and the HPA system. Our first aim was to describe the nature and scope of daily life stress in a group of white collar men and to contrast the experiences of individuals who perceive themselves to be stressed with those who do not. The second aim was to determine how mood and cortisol levels change in response to stressful daily events. A final aim pertained to the stability and generalizability of individual stress responses. We examined whether the cortisol responses to a laboratory stress task reflect those occurring during stressful situations in real life.

Since it is reasonable to assume that stress responses will only lead to disease when they are prolonged or occur very often, it is likely that the pathogenic influence of these stress responses depends on whether they reflect a stabie individual characteristic or not. Therefore, we also investigated whether more or less stable person characteristics like perceived stress level, trait anxiety, and depressive symptomatology were related to individual differences in responses to daily stress. Special attention was also paid to the possible influence of the context and the appraisal of an event on outcome measures. The Experience Sampling Method was used to collect data from subjects during their normal daily activities. In addition, subjects participated in a laboratory stress task, in which they were unexpectedly asked to deliver a speech. In this final chapter, the main findings of the study are briefly reviewed and discussed, including potential clinical implications and suggestions for future research.

The results showed that work was the major source of daily stress in our sample, followed by problems related to the social network. These event contexts reflect the sample's life stage and demographic characteristics; their most important social roles were those of full-time employee, spouse, and father. In general, stressful daily events could be characterized by their salience and unpleasantness; especially events that were appraised as less predictable and less controllable were experienced as unpleasant. The high stress (HS) group could be characterized as vulnerable, trait anxious individuals, who suffered from a variety of psychological and psychosomatic complaints, experienced chronic environmental and relational difficulties, which they coped with using generally less effective styles. HS subjects reported twice as many events as low stress (LS) subjects, especially more work and social interaction events (involving colleagues or the partner). The HS group differed from the LS group in how both daily activities and stressful events were perceived: HS subjects evaluated their activities as requiring more effort, while they were less motivated and less skillful in doing them, while events were appraised as more stressful and less controllable.

From the data presented in chapter 4,5 , and 6 , we can conclude that high perceived stress was not only related to higher Agitation and higher Negative Affect levels and lower Positive Affect levels across all situations, but also magnified the effects of daily events on negative mood states. Positive mood was only affected by very unpleasant events. The effects of events on negative mood states persisted for at least 90 minutes in both HS and LS groups. A future event also increased current Agitation in both groups, but higher current Negative Affect was only observed in the HS group. The findings also indicated that certain kind of events (task demands) and event appraisals (unpleasantness, controllability, chronicity) were more likely to influence mood states. 
With respect to cortisol, it was not perceived stress but trait anxiety and depressive symptomatology that exhibited small but significant positive associations with cortisol levels during the day. Stressful daily events were found to be associated with increased cortisol secretion, the magnitude of the effect depending on whether the event was still ongoing and on how frequently a similar kind of event had occurred previously. Type of event or type of appraisal had no additional effect on cortisol. Although perceived stress, anxiety, and depression did not increase cortisol reactivity to daily events, evidence was found for reduced habituation to recurrent events in individuals scoring high on these traits. Consistent with most views of the stress process, the effect of stressful events on cortisol appeared to be mediated to a large extent by associated increases in negative mood states.

From the data presented in chapter 7 we conclude that the laboratory response measures do not necessarily reflect those obtained in the field.

The main findings of the study can be briefly summarized as follows:

(1) Individuals with high perceived stress reported twice as many daily events, with significantly more work and social interaction events. Events were also appraised as more stressful and less controllable (Chapter 3 ).

(2) Individuals with high perceived stress not only showed higher negative mood levels and lower positive mood levels throughout the day, but also exhibited higher negative mood states in response to stressful daily events (Chapter 4).

(3) In both groups, minor daily events had small but significant positive effects on salivary cortisol levels, which were largely mediated by negative mood states (Chapter 6).

(4) Individuals scoring high on trait anxiety or depressive symptomatology had higher cortisol secretion throughout the day and showed less habituation of cortisol responses to recurrent daily stressors (Chapter 5 \& 6).

(5) Laboratory and field cortisol levels were found to be moderately correlated, but there was no significant association between laboratory and field cortisol response measures. Laboratory baseline levels, but not responses to the laboratory stressor, were significant predictors of field cortisol levels (Chapter 7).

\section{MEASUREMENT OF STRESS IN DAILY LIFE}

For the measurement of daily stress, various options are available to the researcher, each with its own specific advantages and disadvantages. Laboratory studies try to simulate minor stressful events by exposing subjects to various tasks. like computer games, mental arithmetic, films or speeches. The experimental situation has the major advantage that the stressor can be standardized. thereby controlling for the objective component of the stressor. In addition, laboratory stress models can be of great value for basic research questions (e.g. for studying the mechanisms related to individual differences in response to various standardized stressors, or the influence of coping strategies on response to various stressors). However, one can 
question the relevance of the laboratory stressor for the stress that people experience in everyday life. In the laboratory most stressors are of a voluntary nature, of short duration, often anticipated, and they usually require active, effortful coping. It is not difficult to see that these kind of stressors cannot be as intrinsically motivating, threatening, challenging, or demanding of complex coping strategies as are most of the events people experience in their daily lives. Therefore, the validity of the dominant use of laboratory studies to increase our understanding of stress in daily life is questionable. Although the objective component of psychosocial stress is important for an understanding of the stress process, the study of how people perceive, report, and respond to feelings of stress, irrespective of its objective base, is of great importance in itself. For individuals, their idiosyncratic perceptions of stress and their subjective responses to it are most critical in determining its consequences. They may cause people to alter their work habits or their social relationships, change health behavior, or seek medical or psychological support.

Most studies designed to assess individual's subjective perceptions of daily stress have been limited to checklist questionnaires handed out to large populations at one point in time. Although understandable in light of time and cost factors, both psychological but also physiological stress measures collected at one point in time are subject to various biases. Besides a number of methodological problems with available checkilists (related, for instance, to item content or item sample), event checklists are often handed out long after the events have actually taken place. Therefore, less salient events will be forgotten, and the successful or unsuccessful resolution of the event ('effort after meaning') will determine to a large degree endorsement of an event item and scores on distress measures. These problems may still be profound when events are measured on a daily bases. One-time assessments of physiological parameters will probably be unreliable, for instance in that they include a lot of 'noise' due to normal fluctuations in levels of activity, or in that they reflect test or evaluative anxiety more than daily stress. One-time assessments of most physiological parameters also leave the temporal relationship between psychosocial stress and physiology unclear.

The ESM, in the present study characterized by repeated measurements of events, subjective mood and cortisol, has several advantages over cross-sectional approaches to the study of daily stress. First, it increases assessment reliability and provides better estimates of the frequency, distribution, and intensity of psychological variables. Subjects could not only report events occurring at the moment that they were beeped but also any event that had occurred during the past interval. By using an open-ended approach to elicit stressful events we did not restrict a priori the definition of 'stressful' to certain specific items or classes of events. Since events were assessed not long after their occurrence, we were able to elicit information about the sources of stress and the appraised meaning of an event with a minimum of confounding due to biased recall or forgetting. Using semi-random time intervals, anticipation effects were kept to a minimum.

With the ESM we were able to show, for example, that certain demographic characteristics (age, life stage, marital status) of our study sample were reflected in the pattem of significant events they reported. If one would use a single checklist for various samples or populations, important events with specific salience for a specific population may be missed. Events like school exams or cancelled dates, for instance, may be of great significance for a student population, but of minor relevance for a group of elderly people. Checklists should preferably be developed from data obtained from the population in which the checklist is going to be used. We also 
showed that the experience of stressful events is not static; the frequency of reported events varied according to the day of the week (more events during the week than in the weekend) and the time of the day (relatively more events during work hours). This also indicates the importance of the context, here the work environment, for eliciting events. With ESM, we were also able to compare subjects' immediate level of experience (e.g. ESM events reported during the day) with their short-term retrospective assessments (e.g. events reported at the end of the day). Here we observed that with end-of-day reflections many more events were reported than within days. It would be interesting to further investigate influence of response method (checklist versus open-ended) and possible biases in the process of recollection and appraisals of events, which may be more likely to occur at the end of the day.

Second, ESM provides a clearer picture of the dynamic and temporal nature of the relationship between stressful minor events and affective and neuroendocrine responses. This is especially important if the events of interest occur unpredictably (as is usually the case in the natural environment) and therefore cannot be directly monitored by the researcher. The correspondence between stressful daily events, selfreporis, and physiological measures can only be assessed on a within-subject basis. The results demonstrated the potential value of ESM in studying stress in the field. We were able to relate current mood and cortisol levels in daily life to stressful experiences within the context of stable individual differences, type of event, event appraisal, and time. Since assessments were made close to the moment at which the variables of interest occurred, we increased the possibility of establishing causal relationships. Although we cannot rule out the possibility that, for instance, current mood influenced the reporting of recent stressful events, the finding that prior stressful events were associated with persistent increases in negative mood states supports the assumption that events influenced mood and not vice versa.

Third, ESM provides a means for investigating both state and trait aspects of the daily stress process. In the present study we saw, for example, that a global measure of long-term difficulties was significantly related to the reported number of daily events. Person characteristics were found not only to be related to mean mood levels but also to the magnitude of mood responses to current stressful events. With respect to state aspects of daily stress. ESM is particularlly appropriate for the study of the quality of experiences. Since appraisal processes are thought to be predictive of emotional and physiological outcomes of stress, this is an important feature of ESM. In the present siudy, results indeed revealed that several appraisals were differentially related to the outcome measures. And although subject groups did not differ in the amount of time spent on various activities, important differences between groups were observed in how these activities were appraised and experienced. The multilevel approach used in chapters 4,6 , and 7 is optimally suited for the analyses of the ESM data set, in which both variables at the person level (trait characteristics) and the measurement level (state characteristics) are obtained. Using multilevel analyses we can adequately test the transactional stress model, in which trait and state aspects are intertwined.

A number of possible limitations of the present ESM study should also be considered. The repeated use of a short checklist instead of an open-ended approach to sample events might have had some practical advantages (e.g., no time consuming coding tasks for the researcher). However, most available checklists are too long to be suitable for frequent use, while a short event list limits dramatically the range of events that can be investigated. A checklist might be preferable, however, if one is 
interested in certain kind of events, or when one has knowledge about which kind of events are most important for a particular population. Another limitation of the current study design is the lack of temporal resolution. Many stressful events occurred in the interval between beeps, which means that by the time the measures were taken, some mood and/or cortisol response recovery may have taken place. Theoretically, an event-sampling approach (in which subjects are instructed to fill out a questionnaire and take a saliva sample at the moment or just after they experience a stressful event) might have some advantages, but such a procedure would very likely influence not only the daily routine of subjects but also how events are defined, experienced, and coped with. Only true ambulatory monitoring of cortisol (continuous measures: in practice infeasible) could really solve this problem.

A drawback of every subjective approach to the study of (daily) stress. including ESM, is that we are not able to differentiate between the relative contribution of the person and the environment in the experience and response to stress. In particular, since the present study relied on self-reports of stressful events, it was not possible to determine whether HS subjects were actually more exposed to stressful events or that high perceived stress levels (or other individual characteristics, like trait negative affectivity) merely increased reports of stressful events. Our measurement of daily events might possibly improve if we adapted the approach developed by Brown and colleagues (1974;1978), in which clear definitions have been formulated for what should and what should not be: considered a stressful event. The assessment of events is based as much as possibie on the facts of the event, their salience, and the context in which an event occurred (e.g. loss, or longterm contextual threat), rather than on how the respondent perceived them. In addition, a distinction is made between events which the respondent might have brought upon himself ('dependent' events) and those which simply happened to him ('independent' events). Although interviewers can be trained to reliably code major events, it seems highly unlikely that daily events can reliably be coded as 'dependent' or 'independent'. Another disadvantage is that the interviews necessary for the assessment of events are very time-consuming and therefore costly. One useful approach might be to study the daily stress process in those contexts in which one can anticipate the source(s) of stress and in which the potential stressor is identical for every subject, thereby controlling for the objective stimulus situation (e.g. driving exam, job loss, last weeks of the tax year for accounting firms). But as we can see from these examples, most stressors would then approximate major life events in terms of intensity and in frequency and might therefore not be relevant for an understanding of the much more frequent but minor stresses of day-to-day life.

\section{PERCEIVED STRESS AND NEGATIVE AFFECTIVITY}

Personality factors undoubtedly play an important role in perceived stress. Self-report measures of stress and distress have been found to correlate significantly with measures of trait negative affectivity or neuroticism: the disposition to accentuate the negative aspects of the self, other people, and the world in general, and to experience aversive emotional states. High negative affect individuals are more likely to experience discomfort at all times and across situations, and this trait has been shown to be reflected in higher overall levels of negative mood (Watson \& Clark, 1984). Negative affectivity also appears to be a vulnerability factor for the development of anxiety and depressive disorders, indicates poor prognosis, and seems 
to reflect, both the current influence of state affect and the residual effect of earlier depressive episodes (Clark, Watson, \& Mineka, 1994). Negative affectivity has been found to be highly stable over a 20 year period (Costa \& McCrae, 1980; Costa \& McCrae, 1986; Ormel, 1983), and there is evidence for a genetic compound (Lykken, 1982; Scarr, Webber, Weinberg, \& Wittig, 1981; Shields, 1962). While most theoretical models argue that dysphoric mood states result from an interplay between stressful events and certain vulnerability factors such as dysfunctional cognitions, Watson and colleagues (Watson, Clark, \& Harkness, 1994, p.29) have offered a different explanation: "The same individuals are likely to perceive innocuous or ambiguous events as stressful (negativistic appraisal), to respond poorly to actual stressors in their lives (stress overreactivity), to have a negativistic and dysfunctional cognitive style (e.g. self-criticism and self-blame), and to experience intense episodes of dysphoria (e.g. anxiety, depression, guilt, anger)." In other words, dysphoria, rather than being caused by stress and dysfunctional cognitions, may simply be another subcomponent of the same general construct 'negative affectivity' or neuroticism. It has been shown, for instance, in several prospective studies that a chronic pattern of general emotional and/or physical distress exists in about $25 \%$ of the respondents in the various populations investigated (Depue \& Monroe, 1986). General distress levels in these groups appeared to be determined to a large extent by characteristics of the individual and not by environmental (life events) or psychosocial (social support) variables. It is highly likely that a substantial portion of this chronically distressed group will consist of individuals scoring high on the personality dimension negative affectivity, since most scales of psychological disorder in the stress literature assess general negative affect, life dissatisfaction, and a lack of positive well-being (Depue \& Monroe, 1986).

Perceived stress did not overlap completely with measures of psychological distress in the present study; for example, we found that perceived stress, chronic difficulties, trait anxiety, and depressive symptoms independently predicted psychosomatic symptoms (Appendix I). Level of perceived stress was, however, highly correlated with trait anxiety, which is often used as a measure of trait negative affectivity and may explain at least partly the differences in overall mood found between HS and LS subjects. It is important to note that subjects with high perceived stress levels not only had higher negative mood levels across days, but high perceived stress also magnified the effect of stressful daily events on negative mood states within days. In other words, perceived stress does not just spuriously inflate the relationship among stressors, stress symptoms and affective outcomes, but acts as a moderator variable influencing an individual's reactivity to stressful events. The increased negative mood reactivity to minor events may be an important factor in the maintenance of chronic distress in the high perceived stress group. Therefore it seems important in the future to investigate variables potentially related to stress reactivity. such as coping behavior, and social support.

Global perceived stress was not a strong predictor of cortisol secretion in this sample. However, the intercorrelations among the various measures of stress and distress argue for cautious conclusions about the relative contribution of each to cortisol levels. The fact that self-report measures of anxiety and depression were associated not only with increased perceptions of chronic stress but also with elevated cortisol levels also suggests that negative affectivity is not simply a confounder in psychosomatic research. Several other studies have provided support for a relationship between negative affectivity and objective measures of stress; for instance, trait negative affectivity has been positively associated with salivary 
immunoglobulins (Stone, Cox, Valdimarsdottir, Jandorf, \& Neale, 1987; Ursin, Mykletun, Tonder, Vaernes, Relling, Isaksen, et al., 1984) and systolic blood pressure (Harburg, Julius, McGinn, McLeod, \& Hoobler, 1964). Although perceived stress, trait anxiety, and depression did not increase cortisol reactivity to stressful events, we did find evidence for reduced cortisol habituation to recurrent events in subjects scoring high on these traits. Besides these trait measures, the state measures of negative mood were found to be important mediators of the relationship between stressful events and cortisol secretion. Following the transactional stress model, a subject's appraisal of an event with respect to 'what is at stake' and 'what can be done about it' determines the level of emotional involvement, which in turn influences outcome processes. This study indeed showed that the subjective appraisal of apprehension and emotional involvement can lead to increased cortisol secretion. Since high stress subjects reacted more strongly with negative mood to stressful events, and since the effect of stressful events on cortisol was mediated to a large extent by associated increases in negative mood, the apparent lack of a significant association between perceived stress and cortisol reactivity to stressful events is unclear, although we did observe a trend in the expected direction.

Results of the present study are consistent with three related mechanisms thought to play a role in the modifier effect of negative affectivity on the relationship between life stress and psychobiological distress (Ormel \& Wohlfarth, 1991). First, negative affectivity may influence the appraisal of daily events and therefore their consequences for well-being, with subjects scoring high on measures of negative affectivity appraising identical events as more demanding and threatening (Lazarus \& Folkman, 1984b; Watson \& Clark, 1984). Second, negative affectivity may be associated with differential psychobiological sensitivity to stressors (Bolger \& Schilling, 1991; Eysenck, 1967; Tellegen, 1985). Third, negative affectivity may be related to less adequate coping with daily events (McCrae \& Costa, 1986). Although in the present study we had no control over the kind of events experienced, HS subjects perceived events as equally unpleasant as LS subjects, but these events were appraised as more stressful and less controllable by HS subjects. Moreover, activities (especially work activities) were appraised as more demanding and less enjoyable. With respect to psychobiological sensitivity to stressors, HS subjects showed increased negative mood reactivity to daily events and also exhibited less cortisol habituation to recurrent events when compared to LS subjects. Finally, HS reported using an active coping style less often in general, and the coping styles palliative reaction and expression of emotions more often. These latter coping styles are generally considered to be less effective. HS subjects scored higher on the scale depressive reaction, indicating that they experience more feelings of helplessness in response to daily events than LS subjects. We have to keep in mind, though, that scores on general coping styles are not necessarily predictive of the actual coping strategies used in relation to specific events. However, the fact that HS subjects appraised daily events as less controllable than LS subjects also seems to indicate that they were less certain of their coping skilis in relation to daily events.

The fact that self-reports of stress, symptoms, and negative mood are highly correlated with measures of negative affectivity has important implications for the measurement of stress (Pennebaker \& Watson, 1988). Since there are large individual differences in negative affectivity which seem to have a heritable component and seem to be stable over time and situation, measures of what is presumed to be transient work stress will be strongly correlated with negative affectivity. This means that, for the study of the impact of stressful events, any between-subject design will 
have to gather data from a very large sample to be able to control for individual differences in negative affectivity. Another problem related to the concept of negative affectivity is that of causality; any causal interpretation will be meaningless since the process related to the perception of stress is the same process related to the perception of symptoms. Therefore, the optimal design is a within-subject design with repeated measurements over time, where both within- as well as between-subject relationships can be investigated prospectively, as is the case with ESM. As discussed before, the association between subjective self-reports and physiological measures can also only be determined on a within-subject basis. There is also a great need for longitudinal research, as clearly formulated by Clark et al. (1994, p.113): "Until prospective studies have followed individuals - whose premorbid personality and environmental characteristics are known - over sufficiently long periods of time to yield a sufficient base rate of disorder, we will not be able to distinguish causal from concomitant, confounding, or residual factors."

\section{GENERALIZABILITY FROM THE LABORATORY TO THE FIELD}

The positive relationship between trait anxiety and depressive symptonatology and contisol secretion throughout the day was not icplivated in the laboratory; no association was found between trait anxiety or depression and laboratory baselines (Chapter 7). With respect to stress reactivity, trait characteristics were related neither to elevated cortisol reactivity to stressful daily events in the field nor to the magnitude of the cortisol responses to the laboratory stress task. The field results regarding reduced cortisol habituation to recurrent events in subjects scoring high on perceived stress, trait anxiety, and depression could not be investigated in the laboratory since the laboratory stress task was not a recurrent but a novel event. In the laboratory, only negative mood state at baseline was associated with higher cortisol levels at baseline, just before, and just after the speech. In the field, we also found that current negative mood state was an important mediator of cortisol responses. Since it is reasonable to assume that personality traits influence how stressful situations are appraised and may therefore have predictive value for understanding individual differences in responses to stressful situations (as was the case for emotional responses), the lack of an association between the trait characteristics and cortisol responses, either to the SIST or to naturally occurring events in the field (except for recurrent events), is somewhat disappointing although certainly not an exception in the literature. Several methodological and statistical issues may possibly obscure this relationship. In the laboratory, the absence of a relationship between person characteristics and cortisol baseline levels and cortisol reactivity could be partly explained by an elevated laboratory cortisol baseline in both groups, possibly due to anticipatory anxiety, which also masks the cortisol response. This laboratory baseline was significantly higher than cortisol field levels assessed at the same time. It is also possible that strong novelty or experimental effects of the stressor override any individual differences in cortisol reactivity when laboratory stress responses are assessed only once. The fact that almost $90 \%$ of the subjects reacted with a substantial cortisol response to the SIST task supports this interpretation. Additional support for this argument was also found from a recent study by Kirschbaum and colleagues (1995), where some evidence for an association between personality traits and cortisol reactivity in healthy males was reported. In this study, subjects were exposed on five consecutive days to the same psychological 
stress task; low self-esteem, negative self-concept, depressed mood, and physical health problems differentiated high from low cortisol responders. If only Day 1 cortisol responses had been analyzed, results would have been negative; low responders showed habituation after the first day, while high responders continued to show large cortisol responses over the five days. These results cast great doubt on the value of one-time laboratory stress assessments; they seem to be insufficient for the manifestation of individual differences in cortisol reactivity. Therefore, in future laboratory studies cortisol responses should be measured at least twice; not only to repeated identical tasks, but preferably also to different tasks. In the field, repeated assessments of events were made, but it is possible that the within-subject samples of events were too heterogeneous to reveal consistent and reliable individual response patterns to events in general.

It has been widely assumed that by measuring stress reactivity in the laboratory we can say something about stress reactivity in general, which would help clarify individual vulnerability to real-life stress. The use of laboratory cortisol reactivity as a risk factor for stress-related disease depends on the extent to which reactivity is indeed a reliable, stable, and generalizable individual characteristic. Although several laboratory studies have provided some evidence in support of response consistency across identical and, to a lesser degree, between different kinds of tasks (at least in males) (Forsman, 1982; Kirschbaum et al., 1995; Kirschbaum et al., 1992c), the evidence is far from conclusive, and more research is necessary. Given the above, the generalizability of reactivity from the lab to the field also seems questionable; it is certain that the variety of stressors experienced in real life will be much larger than in the laboratory. Results indeed showed that laboratory and field response measures were not associated.

Even if laboratory response measures have no predictive value for reactivity to stressful events in daily life, it has been hypothesized that they might be good predictors of overall levels of hormonal secretion throughout the day (Manuck et al., 1989). According to this theory, overall levels reflect the cumulative effects of daily life stressors rather than the reactivity to a transient stressful episode. This idea was also not supported, however, by our results; laboratory baseline levels, but not responses to the speech task, were significant predictors of field cortisel levels. Once the pre-task level was known, no additional predictive information was gained from measuring the laboratory stress response. These results are comparable to those for cardiovascular parameters, where levels have been found to be more stable than reactivity scores across settings. Only a weak relationship has been found between laboratory reactivity and daily heart rate and blood pressure levels; once the resting level is known there is no additional gain from task level or stress responses (Turner et al., 1994).

The low predictive validity of laboratory cortisol reactivity may to some extent be ascribed to methodological problems, like elevated baseline cortisol in the lab or the timing of cortisol response measurements in the lab compared to the field. The laboratory procedure could possibly be improved by allowing more time for the 'baseline' level to stabilize. With respect to the timing of response measures, better matching of lab and field measures is probably only possible in a study of predictable real-life stressors. Again, this would drastically curtail the range of real-life stressors that can be investigated. In addition, this study showed that unanticipated events tended to be experienced as particularly unpleasant.

An even more serious problem may be the heterogeneity of stressful experiences in real life. In daily life it is not possible to standardize stressful events to 
control for the influence of factors like novelty, task difficulty, content, duration. These factors could all contribute to low response consistency. In daily life, stressful events, coping, and emotional-behavioral responses are related to each other in an ongoing process. For instance, although a certain work event may be dealt with successfully on Monday, the same event may become stressful again on Friday, if support from colleagues is lacking. Due to the heterogeneity of stressors and the ever changing and complex coping processes, reactivity to stressors in daily life may not show high stability over time. Results of our field study did not provide support for significant individual differences in cortisol responses to daily events; cortisol responses were more dependent on current negative moodl states. Since the current study was one of the first to investigate the generalizability of cortisol reactivity from the lab to the field, more research is necessary to answer this important guestion.

\section{CLINICAL IMPLICATIONS}

Results indicated that the experience of distress in daily life situations can indeed lead to mild HPA hyperactivity. Whether these mild elevations of cortisol levels could be a marker of increased risk for disease processes can only be speculated upon. In general, the physiological function of stress-induced increases in glucocorticoid levels is thought to be adaptive. Although there are several theories linking adrenocortical responses following psychosocial stress to later pathology, there is a general lack of knowledge about the mechanisms involved in the stressillness relationship, and we do not know, for example, how stable the cortisol elevations are, or whether such elevations are substantial enough to have clinical relevance. There are currently no epidemiological studies in which the progressive course and consequences of HPA-axis abnormalities have been investigated. The available data are largely based on animal studies or on correlational designs. There is, for instance, evidence from animal studies which suggests that genetic variation in the stress response plays an important role in illness; while low HPA responsiveness is linked to autoimmune disease, high HPA responsiveness appears to be related to infectious diseases (Mason, 1991; Sternberg et al., 19891).

The costs of chronic elevated cortisol levels may turn out to be more important in predisposing the organism to disease than acute reactivity. Possibly only when stress is frequent and prolonged, without the opportunity to fully recover, will the association between stress and disease become apparent in subsequent processes of adaptation or exhaustion, when suppression of various defense mechanisms proceed far beyond physiologicai needs. With respect to somatic pathology, chronically elevated cortisol levels cause many adverse effects (McEwen \&. Stellar, 1993), with main effectors the immune system (Cohen et al., 1991; Kiecolt-Glaser \&, Glaser, 1991), the cardiovascular system (Brindley \& Rolland, 1989; Jacobs, Friedman, \& Mittleman, 1992), and the adipose tissue and muscle (Cox \& Gonder-Frederick, 1991). With regard to psychiatric disorders, elevated cortisol levels and other HPA disturbances are associated with major depression (Linkowski et al., 1985). In some cases, HPA. abnormalities persist after clinical recovery of depression and are indicative of poor prognosis (Charles, Schittecatte, Rush, Panzer, \& Wilmotte, 1989). On the other hand. low cortisol levels have been associated with post-traumatic stress disorder, which is thought to reflect a chronic adaptation in stress-induced. HPA activation in the form of a heightened feedback sensitivity (Yehuda et al., 1993). It remains unclear. however, whether these HPA disturbances are involved in the etiology of the 
disorder. Interesting in this regard are theories concerning how acute stressful events can result in long lasting biological effects on the organism. It has been postulated that psychosocial stressors may result in alterations at the level of gene regulation and expression; here, the HPA axis is thought to play an important mediating role (Checkley, 1992; Gold, 1988; Holsboer, 1992; Post, 1992). Evidence is mounting from animal studies that the nervous system, especially the hippocampus, is subject to wear and tear as a result of stressful experiences (Jacobson \& Sapolsky, 1991: Sapolsky, Krey, \& McEwen, 1986). Since the hippocampus is very important for mood, learning. and memory processes and subtle regulation of the HPA axis during mental stress, changes in hippocampal functioning may be extremely important for understanding the mechanisms in stress-related disorders, especially psychiatric disorders.

Although chronic levels may be more important than acute reactivity for future disease, the effects of acute stress may synergize with the actions of chronic stress, as has been shown in cardiovascular disease (Jacobs et al., 1992). Persistent increases in cortisol levels may indicate heightened biological vulnerability that increases the probability of dysregulation. This probability of dysregulation is thought to increase substantially when the vulnerable system is challenged by environmental and psychosocial challenges (Axelrod \& Reisine, 1984). Since stressful daily events are very common, especially in the HS group, one might expect frequent provocation of biological instability from these events in vulnerable individuals, and particularly when stress responses fail to habituate upon repeated exposure. Although it seems unlikely that minor events may initiate acute disorder in a stably regulated system, they may lead to disorder in the long run or may help maintain chronic disorder in dysregulated systems (Depue \& Monroe, 1986). Given the relationship between negative affectivity and increased cortisol levels, and between perceived stress (being strongly related to negative affectivity) and increased mood reactivity, it seems possible that stable individual characteristics may contribute to a more general susceptibility or a heightened vulnerability to the development of disorder.

\section{DIRECTIONS FOR FUTURE RESEARCH}

In this final section, a number of suggestions will be made for possible future research into the (daily) stress-distress relationship. As emphasized earlier, there is a general need for prospective longitudinal research, with repeated measurements of the stress-distress relationship over sufficiently long periods of time. It may be useful to combine ESM and traditional research methods (Alliger \& Williams, 1993), since both immediate and long-term assessments of stress variables are meaningful, and ESM can increase our understanding of the mechanisms underlying long-term outcomes as assessed by traditional methods (e.g. prospective surveys). Results obtained in the field should then be investigated in laboratory experiments to see if these results can be replicated. In addition, an interdisciplinary approach will have to be used (McEwen \& Stellar, 1993), involving various psychological, behavioral, neurobiological, endocrine, and immunologic measures, as well as their interactions with each other. Person factors (for example negative affectivity, coping style), stress measures (perceived stress, life events, chronic difficulties, daily events), state. measures (mood states, psychosomatic symptoms), and physiological measures (e.g. neuroendocrine parameters) can than be investigated simultaneously. From the present study, it may seem that all important hormonal effects of psychosocial stress relevant for health are related to cortisol, but this is certainly not true. Stress can not. 
only induce increases in cortisol or catecholamine levels, but has also been related to changes in levels of vasopressin (Axelrod \&. Reisine, 1984), prolactin (Meyerhof, Oleshansky, \& Mougey, 1988), b-endorphin. (Meyerhof et al., 1988), testosterone (Rose, 1984), and other hormones.

Follow-up assessments of the subjects investigated in the present study could shed light on some important but complex questions. For example: How stable is negative affectivity over time and how stable is the negative affectivity - perceived stress, negative mood, cortisol relationship? How stable are cortisol levels and cortisol responses to events and how does stability relate to person characteristics? What are the factors predicting disease, absenteeism, disability versus health? With respect to the health consequences of stress, up till now, most life stress studies included only general indicators of emotional or somatic disorder. Moving away from general models, it may be important to include measures of specific disorders like atherosclerosis, angina pectoris, or respiratory infections, since the mechanisms underlying stress-disorder interactions may vary across disorder and may only be understood by analyzing specific disorders (Depue \& Monroe, 1986). For instance, while personality and coping behavior may play a dominant role in the onset or maintenance of certain clisorders, other disorders may be more strongly related to environmental factors or health behaviors.

In general, representative samples of the general population and, more importantly, of high risk groups (e.g. subjects with a genetic vulnerability for psychiatric disorders, subjects in high risk jobs like health and educational employees) should preferably be observed pre-morbidly and then followed longitudinally. Further research should also focus on women, since there is considerable evidence to suggest that there are differences not only in the kind of daily' stressors women experience (Folkman \& Lazarus, 1980: Frankenhaeuser et al., 1989), but also in physiological (including neuroendocrine) responses to stress (Frankenhaeuser, 1980; Kirschbaum et al., 1992c). In addition, several factors of theoretical importance to the stress process lacking in the present study should ideally be included in such a study: protective factors, such as positive events, social support. and coping strategies; health parameters, such as records of absenteeism, disability and health status, and other physiological measures like cardiovascular measures, day-time catecholamines, and immunological parameters.

Such a study may shed additional light on the relative impact of temperamental and environmental influences on psychological states (anxiety, depression), HPA and immune function, and somatic health (Ormel \& Wohlfarth, 1991). For instance, how do life events, chronic difficulties, and daijy events predict change and stability in psychological distress levels and physiological levels, controiling for important person characteristics like negative affecuvity? Despite a paucity of longitudinal data, several models for the relationship between personality, stressful life events, and well-being have been proposed (Headey \&. Wearing. 1989). Personality models state that wellbeing is solely a function of personality and will therefore be highly stable over time (Costa \& McCrae, 1980). Adaptation level models stipulate that adaptation to events is so rapid and so complete that well-being is not measurably affected by events (Brickman, Coates, \& Janoff-Bulman, 1978; Diener, 1984). Other models have been proposed that treat life events as wholly exogenous stressors with allegedly damaging effects on physical and mental health (Block \& Zautra, 1981). Criticism relating to methodology and model specification as well as meager empirical support for these models have led to the development of other models. A model that has received some empirical support is the dynamic equilibrium model (Duncan-Jones. 
Fergusson, Ormel, \& Horwood, 1990; Headey \& Wearing, 1989). In this model each person is regarded as having 'normal' equilibrium levels of life events and well-being, dependent on stable psychobiological and environmental characteristics. These stable characteristics vary from person to person. Only deviations from normal event levels (internal or external) change the normal level of well-being. Deviations are usually temporary because stable personality traits, time, and support (for example treatment) play an equilibrating function. Another model is the dynamic-vulnerability model (Ormel, 1995), which differs from the previous mentioned model in that it postulates that vulnerability can increase or decrease considerably during the life course following certain internal or external events. Cumulation of vulnerability can occur as a consequence of earlier episodes (Post, 1992). The validity of this model has not yet been thoroughly investigated. Although these models emphasize major life events, they could easily be extended to include chronic difficulties and minor daily events. Only longitudinal studies can test the validity of the various models. It should be noted here that with assessing the relative predictive power of environmental and psychosocial factors on the one hand, and stable person attributes on the other hand, it is not meant that both can be measured independently or that this is a necessity. Although care should be taken that events are not merely reflections of symptomatic impairment (through use of symptom-free event measures and attempts to determine the independence of events), personality factors will influence the generation of most events and vice versa. As formulated by Lazarus and Folkman (1986, p.77): "there is simply no way to define an event as a stressor without referring to the properties of persons that make their well-being in some way vulnerable to that event."

From a practical point of view, knowledge about the relative contribution of personality or environmental factors on well-being may provide important information for intervention programs designed to reduce personal distress. In cases where environmental factors appear to play no role; interventions should primarily focus on strategies that are not tied to stressful situations but that are solely directed at the person (for example relaxation techniques, reducing worry-proness). In the other case, we need to pay more attention to teaching the person how to deal with his psychosocial environment (for example increasing control by teaching adaptive coping strategies, restructuring of dysfunctional cognitions, increasing assertiveness, time-management, and avoiding stressors by stimulus control). Since the evidence so far points to a combination of a vulnerable personality structure and objectively stressful situations as determinants of well-being, intervention programs should be directed at both the person and his environment. By using diaries like those in the present study, valuable information can be obtained about a person's idiosyncratic vulnerabilities relating to his environment (stressful events), personality (event appraisals, stress reactivity), adaptive capacities (coping strategies), and well-being (anxiety, depression, somatic complaints). These personalized diaries provide a rich source of data on which effective interventions for that specific individual can be based, as well as a means of evaluating their effects. Although this last paragraph emphasizes personal responsibility for health and well-being and seems the most sensible and successful strategy in the short term, an interesting question for the future relates to the responsibility of society as a whole. It may be that by increasing our demands on the individual as a result of growing economic pressures, an increasing number of people will not be able to meet the new requirements. The question here is whether these people should be considered ill and deviating from 'normal', or whether society has lost sight of what is 'normal'. 



\section{REFERENCES}

Aagaard, J. (1984). Stressful life events and illness: A review with special reference to a criticism of the life event method. In J. Cullen \& J. Siegriest (Eds.), Breakdown inhuman adaptation to stress. Towards a multidisciplinary approach. Boston: Martinus Nijhof Publishers.

Abelson, J. L.. \& Curtis, G. C. (1996). Hypothalamic-Pituitary-Adrenal axis activity in panic disorder: prediction of long-term outcome by pretreatment cortisol levels. American Journal of Psychiatry, 153(1), 69-73.

Affleck, G.. Tennen, H., Urrows, S., \& Higgins, P. (1994). Person and contextual features of daily stress reactivity: Individual differences in relations of undesirable daily events with mood disturbance and chronic pain intensity. Journal of Personality and Social Psychology, 66(2), 329-340.

Allen. P. I. M., Batty, K. A., Dodd, C. A. S., Herbert, J., Hugh, C. J., Moore, G. F., Seymour, M. J., Shiers, H. M., Stacey, P. M., \& Young. S. K. (1985). Dissociation between emotional and endocrine responses preceding an academic examination in male medical students. Journal of Endocrinology, 107, 163-170.

Alliger, G. M., \& Williams, K. J. (1993). Using signal-contingent experience sampling methodology to study work in the field: A discussion and illustration examining task perceptions and mood. Personnel Psychology, 46, 525-549.

Ansseau, M., Papart, P., Pitchot, W., Timsit-Berthier, M., Legros, J. J., \& von Frenckel, R. (1992). Dexamethasone suppression test and prediction of treatment response to selective antidepressants. European Psychiatry. 7, 191-194.

Ansseau, M., Sulon, J., Doumont. A., Cerfontaine, J. L., Legros, J. J., Sodoyez, J. C., \& DemeyPonsart. E. (1984). The use of saliva cortisol in the dexamethasone suppression test. Psychiatry Research, 13, 201-211.

Ametz, B. B., Brenner, S.-O., Levi, L., Hjelm, R., Petterson, I.-L., Wasserman, J., Petrini, B., Eneroth, P., Kallner, A., Kvetnansky, R., \& Vigas, M. (1991). Neuroendocrine and immunologic effects of unemployment and job insecurity. Psychotherapy and Psychosomatics.

Ametz, B. B., \& Fjellner, B. (1986). Psychological predictors of neuroendocrine responses to mental stress. Journal of Psychosomatic Research, 30, 297-305.

Arrindell, W. A., \& Ettema, H. (1981). Dimensionele structuur, betrouwbaarheid en validiteit van de Nederlandse bewerking van de Symptom Checklist (SCL-90); gegevens gebaseerd op een fobische en een 'normale' populatie. Nederlands Tijdschrift voor Psychologie, 36, 77-108.

Arrindell, W. A., \& Ettema, H. (1986). SCL-90: Handleiding bij een muitidimensionele psychopathologie indicator. Lisse: Swets \& Zeitlinger B.V.

Attanasio, V., Andrasik, F., Bilanchard, E. B., \& Arena, J. G. (1984). Psychometric properties of the SUNYA revision of the Psychosomatic Symptom Checklist. Journal of Behavioral Medicine, $7(2), 247-2.58$.

Averill, J. R. (1973). Personal control over aversive stimuli and its relationship to stress. Psychological Bulletin, 80(4), 286-303.

Axelrod, J., \& Reisine, T. D. (1984). Stress hormones: Their interaction and regulation. Science, 224. 452-459.

Bandura, A., Taylor, C. B., Williams, S. L., Mefford, I. N., \& Barchas, J. D. (1985), Catecholamine secretion as a function of perceived coping self-efficacy. Journal of Consulting and Clinical Psychology, 53(3), 406-414.

Barling, J., \& Kryl, I. P. (1990). Moderators of the relationship between daily work stressors and mood. Work \& Stress, 4(4), 319-329.

Bassett, J. R. (1982). Psychological stress and the coronary artery in ischemic heart disease. In S. Kalsner (Eds.), The coronary artery (pp. 474-500), London: Croom Helm. 
Bassett, J. R., \& Cairncross, K. D. (1977). Changes in the coronary vascular system following prolonged exposure to stress. Pharmacology Biochemistry \& Behavior, 6, 311-318.

Bassett, J. R., Marshall, P. M., \& Spillane, R. (1987). The physiological measurement of acute stress (public speaking) in bank employees. International Journal of Psychophysiology, 5(4), $265-273$.

Baum, A., Fleming, R., \& Reddy, D. M. (1986). Unemployment stress: loss of control, reactance and learned helplessness. Social Science and Medicine, 22(5), 509-516.

Baum, A. S., Lundbeg, U., Grunberg, N. E., Singer. J. E., \& Gatchel, R. J. (1985). Urinary catecholamines in behavioral research on stress. In C. R. Lake \& M. G. Ziegler (Eds.), The Catecholamines in Psychiatric and Neurologic Disorders (pp. 55-72). Stoneham: Butterworth Publishers.

Bebbington, P. E., Tennant, C., \& Hurry, J. (1981). Adversity and the nature of psychiatric disorder in the community, Journal of Affective Disorders, 3, 345-366.

Ben-Aryeh, H., Roll, R., Kahana, L., \& al., e. (1985). Saliva as an indicator of stress. International Journal of Psychosomatics, 32, 3-8.

Berger, M., Bossert, S., Krieg. J. C., Dirlich, G., Ettmeier, W., Schreiber, W., \& von Zerssen, D. (1987). Interindividual differences in the susceptibility of the cortisol system: An important factor for the degree of hypercortisolism in stress situations? Biological Psychiatry, 22, 1327. 1339.

Block, J., \& Zautra, A. (1981). Satisfaction and distress in a community: a test of the effect of life events. American Journal of Community Psychology, 9, 165-180.

Bohnen, N., Nicolson, N., Sulon, Jl., \& Jolles, J. (1991). Coping style, trait anxiety and cortisol reacivity during mental stress. Journal of Psychosomatic Research, 35(2/3), 141- 147.

Bolger, N. (1990). Coping as a personality process: A prospective study. Journal of Personality and Social Psychology, 59, 525-537.

Bolger, N., DeLongis, A.. Kessler, R. C., \& Schilling, E. A. (1989a). Effects of daily stress on negative mood. Journal of Personality and Social Psychology, 57(5), 808-818.

Bolger, N., Delongis. A., Kessler, R. C., \& Wethington, E. (1989b). The contagion of stress across multiple roles. Journal of Marriage and the Family, 51, 175-183.

Bolger, N.. \& Schilling, E. A. (1991). Personality and the problems of everyday life: The role of neuroticism in exposure and reactivity to daily stressors. Journal of Personality, 59(3), 355386.

Bossert, S., Berger, M., Krieg, J. C., Schreiber, W., Junker, M., \& von Zerssen, D. (1988). Cortisol response to various stressful situations: Relationship to personality variables and coping styles. Neuropsychobiology, 20, 36-42.

Bourne, P. G., Rose, K. M., \& Mason, J. W. (1967). Urinary 17-OHCS levels. Data on seven helicopter ambulance medics in combat. Archives of General Psychiatry, 17, 104- I 10.

Bower, G. H. (1981). Mood and memory. American Psychologist, 36(2), 129-148.

Brandstatter. H. (1983). Emotional responses to other persons in everyday life situations. Journal of Personality and Social Psychology. 45(4), 871-883.

Brandtstädter, J., Baltes-Gotz, B., Kirschbaum, C.., \& Hellhammer, D. (1991). Developmental and personality correlates of adrenocortical activity as indexed by salivary cortisol: Observations in the age range of 35 to 65 years. Journal of Psychosomatic Research, 35(2/3), 173- 185.

Brantley, P., Dietz, L., McKnight, G. T., Jones, G. N., \& Tulley, R. (1988). Convergence between the Daily Stress Inventory and endocrine measures of stress. Journal of Consulting and Clinical Psychology, 56, 549-551.

Brantley, P. J., Waggoner, C. D., Jones, G. N., \& Rappaport, N. B. (1987). A daily stress inventory: Development, reliability, and validity. Joumal of Behavioral Medicine, 10(1), 61-74.

Brickman, P.. Coates, D., \& Janoff-Bulman, R. (1978). Lottery winners and accident victims: Is happiness relative? Journal of Personality and Social Psychology, 36, 917-927. 
Brindley, D., \& Rolland, Y. (1989). Possible connections between stress, diabetes, obesity, hypertension and altered lipoprotein metabolism that may result in atherosclerosis. Clinical Science, 77, 453-461.

Brown, G. W. (1974). Meaning, measurement, and stress of life events. In B. S. Dohrenwend \& B. P. Dohrenwend (Eds.), Stressful Life Events: Their Nature and Effects (pp. 217-243). New York: Willey \& Sons.

Brown, G. W. (1989). Life events and measurement. In G. W. Brown \& T. O. Harris (Eds.), Life events and illness (pp. 3-45). London: Guilford Press.

Brown, G. W. (1993). Life events and affective disorder: Replications and limitations. Psychosomatic Medicine, 55, 248-259.

Brown, G. W., \& Harris, T. (1978). Social origins of depression: A study of psychiatric disorder in women. London: Tavistock.

Brown, G. W., \& Harris, T. O. (Ed.). (1989). Life Events and lllness. London: Guilford Press.

Brown, W. A., Sirota, A. D., Niaura, R., \& Engebretson, T. O. (1993). Endocrine correlates of sadness and elation. Psychosomatic Medicine, 55, 458-467.

Brugha, T., Bebbington, P., Tennant, C., \& Hurry, J. (1985). The list of threatening experiences: A subset of 12 life event categories with considerable long-term contextual threat. Psychological Medicine, 15, 189-194.

Brugha, T. S., \& Conroy, R. (1985). Categories of depression: Reported life events in a controlled design. British Journal of Psychiatry, 147, 641-646.

Brugha, T. S., \& Cragg, D. (1990). The list of threatening experiences: the reliability and validity of a brief life events questionnaire. Acta Psychiatrica Scandinavica, 82, 77-81.

Bruijn, J. V. d. (1988). Bedrijfsfitness, vorm van secundaire arbeidsvoorwaarde. Arbeidsomstandigheden, 64(7), 474-477.

Bryk, A. S., \& Raudenbush, S. W. (1987). Application of hierarchical linear models to assessing change. Psychological Bulletin, 101(1), 147-158.

Bryk, A. S., \& Raudenbush, S. W. (1992). Hierarchical Linear Models: Applications and Dafa Analysis Methods. London: Sage Publications.

Bullinger, M., Naber, D., Pickar, D., Cohen, R. M., Kalin, N. H., Pert, A., \& Bunney, W. E. (1984). Endocrine effects of the cold pressor test: Relationship to subjective pain appraisal and coping. Psychiatry Research, 12, 227-233.

Calabrese, J. R., Kling, M. A., \& Gold, P. W. (1987). Alterations in immunocompetence during stress, bereavement, and depression: Focus on neuroendocrine regulation. The American Journal of Psychiatry, 144(9), 1123- 1134.

Cameron, P. (1975). Mood as an indicator of happiness: Age, sex, social class, and situational differences. Journal of Gerontology, 30, 216-224.

Campbell, J. D., Chew, B., \& Scratchley, L. S. (1991). Cognitive and emotional reactions to daily events: The effects of self-esteem and self-complexity. Journal of Personality, 59(3), 473-505.

Cannon, W. B. (1929). Organization for physiological homeostasis. Physiological Review, 9. 399.

Cantor, N., Norem, J., Langston, C., Zirkel, S., Fleeson, W., \& Cook-Flannagan, C. (1991). Life tasks and daily life experience. Journal of Personality. 59(3), 425-451.

Caplan, R. D., Cobb, S., \& French, J. R. P., Jr. (1979). White collar work load and cortisol: Disruption of a circadian rhythm by job stress? Journal of Psychosomatic Research, 23 , $181-192$.

Carroll, B. J. (1985). Dexamethasone suppression test: a review of contemporary confusion. Journal of Clinical Psychiatry, 46(2), 13-24.

Caspi, A., Bolger, N., \& Eckenrode, J. (1987). Linking person and context in the daily stress process. Journal of Personality and Social Psychology, 52(1), 184-195. 
Charles, G. A., Schittecatte, M., Rush, A. J., Panzer, M., \& Wilmotte, J. (1989). Persistent cortisol non-suppression after clinical recovery predicts symptomatic relapse in unipolar depression. Journal of Affective Disorder, 17, 271-278.

Checkley, S. (1992). Neuroendocrine mechanisms and the precipitation of depression by life events. British Journal of Psychiatry, 160(suppl. 15), 7-17.

Chen, P. Y.. \& Spector, P. E. (1991). Negative affectivity as the underlying cause of correlations between stressors and strains. Journal of Applied Psychology. 76(3), 398-407.

Chrousos, G. P., \& Gold, P. W. (1992). The concepts of stress and stress system disorders. Overview of physical and behavioral homeostasis. Journal of the American Medical Association, 267(9), 1244-1252.

Claman, H. N. (1977), Corticosteroids and lymphoid cells. New England Journal of Medicine, $287,388-397$.

Clark, L. A., \& Watson, D. (1988). Mood and the mundane: Relations between daily life events and self-reported mood. Journal of Personality and Social Psychology, 54(2), 296-308.

Clark, L. A., Watson, D., \& Mineka, S. (1994). Temperament, personality, and the mood and anxiety disorders. Journal of Abnormal Psychology, 103(1), 103- 116.

Coeck, C., Jorens, P. G., Vandevivere, J., \& Mahler, C. (1991). ACTH and cortisol levels during residency training. The New England Journal of Medicine, 325(10), 738.

Cohen . S. (1978). Environmental load and the allocation of attention. In A. Baum. J. Singer, \& S. Valins (Eds.), Advances in environmental psychology. Hillsdale: Lawrence Erlbaum.

Cohen. S. (1980). Aftereffects of stress on human performance and social behavior: A review of research and theory. Psychological Bulletin, 82-108.

Cohen, S. (1986). Contrasting the Hassles Scale and the Perceived Stress Scale: Who's really measuring appraised stress? American Psychologist, 41, 716-718.

Cohen, S., Kamarck, T., \& Mermelstein, R. (1983). A global measure of perceived stress. Journal of Health and Social Behavior, 24, 385-396.

Cohen, S., Tyrrell, D. A. J., \& Smith, A. P. (1991). Psychological stress and susceptibility to the common cold. The New England Journal of Medicine, 325(9), 606-612.

Cohen, S., \& Williamson, G. M. (1988). Perceived stress in a probability sample of the United States. In S. Spacepan \& S. Oskamp (Eds.). The Social Psychology of Health (pp. 31-67). Newbury Park, CA: Sage Publications.

Collins, A. C.. \& Frankenhaeuser, M. (1978). Stress responses in male and fernale engineering studenis, Journal of Human Stress, 4(2), 43-48.

Cook, N. I. Ng, A., Read, G. F., Harris, B., \& Riad-Fahmy, D. (1987). Salivary cortisol for monitoring adrenal activity during marathon runs. Hormone Research, 25, 18-23.

Costa, P. T., \& McCrae, R. R. (1980). Influence of extraversion and neuroticism on subjective well-being: Happy and unhappy people. Journal of Personality and Social Psychology, 38(4), 668-678.

Costa, P. T. \& McCrae, R. R. (1986). Personality stability and its implications for clinical psychology. Clinical Psychological Review, 6, 407-423.

Costa, P. T., \& McCrae, R. R. (1987). Neuroticism, somatic complaints, and disease: Is the bark worse than the bite? Journal of Personality, 55(2), 299-316.

Cox, D. J., Freundlich, A., \& Meyer, R. G. (1975). Differential effectiveness of electromyograph feedback, verbal relaxation instructions and medication placebo with tension headaches. Journal of Consulting and Clinical Psychology. 43, 892-898.

Cox, D. J., \& Gonder-Frederick, L. A. (1991). The role of stress in diabetes mellitus. In P. McCabe,N. Schneidermann,T. M. Field, \& J. S. Skyler (Eds.), Stress, Coping and Disease (pp. 118-134). New York: L Erlbaum Assoc,

Cox, T., Gotts, G., Boot, N., \& Kerr, J. (1988). Physical exercise, employee fitness and the management of health at work. Ergonomics, 24, 795-806. 
Csikszentmihalyi, M., \& Larson, R. (1984). Being adolescent: Conflict and growth in the teenage years. New York: Basic Books.

Csikszentmihalyi, M., \& Larson, R. (1987). Validity and reliability of the experience-sampling method. The Journal of Nervous and Mental Disease, 175(9), 526-536.

Cummins, S. E., \& Gevirtz, R. N. (1993). The relationship between daily stress and urinary cortisol in a normal population: An emphasis on individual differences. Behavioral Medicine, 19, 129. 134.

Curtis, G. C. (1972). Psychosomatics and chronobiology: Possible implications of neuroendocrine rhythms. Psychosomatic Medicine 34(3), 235-256.

De La Torre, B. (1994). Psychoendocrinologic mechanisms of life stress. Stress Medicine, 10. 107-114.

de Vries, M. W. (1987). Introduction: Investigating mental disorders in their natural settings. Journal of Nervous and Mental Disease, 175(9), 509-513.

de Vries, M. W. (1992). The Experience of Psychopathology: Investigating Mental Disorders in their Natural Settings. Cambridge: University Press.

de Vries, M. W., \& Delespaul, P. A. E. G. (1992). Variability of schizophrenia symptoms. In M. W. de Vries (Eds.). The experience of psychopathology: Investigating mental disorders in their natural settings (pp. 97-109). Cambridge: Cambridge University Press.

Dekaris, D., Sabioncello, A., Mazuran, R., Rabatic, S., Svoboda-Beusan, I., Racunica, N. L., \& Tomasic, J. (1993). Multiple changes of immunologic parameters in prisoners of war. Assessments after release from a camp in Manjaca, Bosnia. Journal of the American Medical Association, 270(5), 595-599.

Delahunt, J. W., \& Mellsop, G. (1987). Hormone changes in stress. Stress Medicine, 3, 123-134.

Delespaul, P. A. E. G. (1992). Technical note: Devices and time-sampling procedures. In M. W. deVries (Eds.), The experience of psychopathology: Investigating mental disorders in their natural settings (pp. 363-376). Cambridge: Cambridge University Press.

Delespaul, P. A. E. G. (1995) Assessing schizophrenia in daily life-The Experience Sampling Method-. Thesis, University of Limburg, Maastricht, The Netherlands.

DeLongis, A., Coyne, J. C., Dakof, G., Folkman, S., \& Lazarus, R. S. (1982). Relationship of daily hassles, uplifts, and major life events to health status. Health Psychology, I(2), 119-136.

DeLongis, A., Folkman, S., \& Lazarus, R. S. (1988). The impact of daily stress on health and mood: Psychological and social resources as mediators. Journal of Personality and Social Psychology, 54(3), 486-495.

Demyttenaere, K., Nij̧s, P., Evers-Kiebooms, G., \& Koninckx, P. R. (1989). The effect of a specific emotional stressor on prolactin, cortisol, and testosterone concentrations in women varies with their trait anxiety. Fertility and Steriliry, 52(6), 942-948.

Depue, R. A., \& Monroe, S. M. (1986). Conceptualization and measurement of human disorder in life stress research: The problem of chronic disturbance. Psychological Bulletin, 99(1), 36-51.

Derogatis, L. R., Lipman, R. S., \& Cori, L. (1973). SCL-90, an outpatient rating scale -preliminary report. Psychopharmacology Bulletin, 9, 13-27.

Devereux, R. B., Pickering, T. G., Harshfield, G. A., (1983). Left ventricular hypertrophy in patients with hypertension: Importance of blood pressure response to regularly occurring stress. Circulation, 68, 470-476.

Dewe, P. J. (1991). Measuring work stressors: The role of frequency, duration and demand. Work \& Stress, 5, 77-91.

Diener, E. (1984). Subjective well-being. Psychological Bulletin, 45, 542-575.

Diener, E., Sandvikt E., \& Larsen, R. (1985). Age and sex effects for affect intensity. Developmental Psychology, 21, 542-546.

Dienstbier, R. A. (1989). Arousal and physiological toughness: Implications for mental and physical health. Psychological Review, 96(1), 84-100. 
Dijkman-Caes, C., \& deVries, M. W. (1991). Daily life situations and anxiety in panic disorder and agoraphobia. Journal of Anxiety Disorders, 5, 343-357.

Dijkman-Caes, C. I. M. (1993) Panic disorder and agoraphobia in daily life. Thesis, University of Limburg, Maastricht, The Netherlands.

Dijkstra, P. (1974). De zelfbeoordelingsschaal voor depressie van Zung. In: H. M. van Praag \& H. G. M. Rooymans (Eds.), Stemming en Ontstemming (pp. 98-120). Amsterdam; De Erven Bohn.

Dimsdale, J. E. (1984). Generalizing from laboratory to field studies of human stress physiology. Psychosomatic Medicine, 46(5), 463-469.

Dinan, T. G. (1994). Glucocorticoids and the genesis of depressive illness: A psychobiological model. British Journal of Psychiarry, 164, 365-371.

Dohrenwend, B. P., \& Shrout, P. E. (1985). "Hassles" in the conceptualization and measurement of life stress variables. American Psychologist, 40, 780-785.

Dohrenwend, B. S., \& Dohrenwend, B. P. (1974). Overview and prospects, for research on stressful life events. In B. S. Dohrenwend \& B. P. Dohrenwend (Eds.), Stressful Life Events: Their Nature and Effects (pp. 313-331). New York: Wiley, J. \& Sons.

Dohrenwend, B. S.. \& Dohrenwend, B. P. (1978). Some issues in research on stressful life events. The Journal of Nervous and Mental Disease, 166(1),7-15.

Dohrenwend, B. S.. Krassnoff, L., Askenasy, A. R., \& Dohrenwend, B. P. (1978). Exemplification of a method for scaling life events: The PERI Life Events Scale. Journal of Health and Social Behavior, 19, 205-229.

Duncan-Jones, P., Fergusson, D., Ormel, J., \& Horwood, L. (1990). A model of stability and change in minor psychiatric symptoms: results from three longitudinal studies. Psychological Medicine, Monograph Supplements, nr. 18. Cambridge:Cambridge University Press.

Eckenrode, J. (1984). Impact of chronic and acute stressors on daily reports of mood. Journal of Personality and Social Psychology, 46(4), 907-918.

Eckenrode, J., \& Bolger, N. (1995). Daily and within-day event measurement.. In S. Cohen, R. C. Kessler, \& L. Underwood Gordon (Eds.). Measuring stress: A guide for health and social scientists (pp. 80-101). New York: Oxford University Press.

Eysenck, H. J., \& Eysenck, S. B. G. (1985). Personality and' individual differences. New York: Plenum:

Eysenck, H. I.. \& Eysenk, S. B. (1968). Eysenck Personality Inventory. San Diego: CA: Educational and Industrial Testing Service.

Eysenck. H. L. (1967). The Biological Basis of Personality. Springfield, lllinois: Charles C Thomas.

Fulkenberg, L. E. (1987). Employee fitness programs: Their impact on the employee and the prganizationt. Academy Management Review, 12, 511-522.

Fergusson, D. M.. \& Horwood, L. J. (1987). Vulnerability to life event exposure. Psychological Medicine, 17, 739-749.

Fibiger, W., \& Singer, G. (1989). Biochemical assessment and differentiation of mental and physical effort. Work \& Stress. 3(3), 237-247.

Fibiger, W., Singer, G., Miller, A. J., Asmstrong. S., \& Datar, M. (1984). Cortisol and catecholamines changes as functions of time-of-day and self-reported mood. Neuroscience \& Biobehavioral Review.8, 523-530.

Fibiger, W. Singer, G. \& Miller, A. J. (1984). Relationships between catecholamines in urine and physical and mental effort. International Journal of Psychophysiology, I, 325-333.

Folkman. S. (1984). Personal control and stress and coping processes: a theoretical. analysis. Journal of Personality and Social Psychology, 46(4), 839-852.

Folkman, S., \& Lazarus, R. S. (1980). An analysis of coping in a middle-aged community sample. Journal of Health and' Social Behavior, 21, 219-239. 
Folkman, S., \& Lazarus, R. S. (1985). If it changes it must be a process: Study of emotion and coping during three stages of a college examination. Journal of Personality and Social Psychology, 48, 150-170.

Follenius, M., Brandenberger, G., Hietter, B., Simeoni, M., \& Reinhardt, B. (1982). Diurnal cortisol peaks and their relationship to meals. Journal of Clinical Endocrinology and Metabolism, 55, 757-761.

Forsman, L. (1980). Habitual catecholamine excretion and its relation to habitual distress. Biological Psychology, 11, 83-97.

Forsman, L. (1982). Consistency in catecholamine excretion in laboratory and natural settings: Correlational and variance components analysis. Scandinavian Journal of Psychology, 23, 99106.

Forsman, L., \& Lundberg, U. (1982). Consistency in catecholamine and cortisol excretion in males and females. Pharmacology Biochemistry \& Behavior, 17, 555-562.

Frankenhaeuser, M. (1975a), Experimental approaches to the study of catecholamines and emotion. In L. Levi (Eds.), Emotions: Their parameters and measurement (pp. 209-234). New York: Raven Press.

Frankenhaeuser, M. (1975b). Sympathetic-adrenomedullary activity, behavior, and the psychosocial environment. In P. H. Venables \& M. J. Christie (Eds.), Research in psychophysiology (pp. 71-94). New York: Wiley.

Frankenhaeuser, M. (1976). The role of peripheral catecholamines in adaptation to understimulation and overstimulation. In G. Serban (Eds.), Psychopathology of haman adaptation (pp. 173-191). New York: Plenum Press.

Frankenhaeuser, M. (1979). Psychoendocrine approaches to the study of emotion as related to stress and coping. In H. E. Howe \& R. A. Dienstbier (Eds.), Nebraska symposium on motivation 1978. Lincoln: University of Nebraska Press.

Frankenhaeuser, M. (1980). Psychoneuroendocrine approaches to the study of stressful personenvironment transactions. Selye's Guide to Stress Research, 1, 46-70.

Frankenhaeuser, M. (1983). The sympathetic-adrenal and pituitary-adrenal response to challenge: Comparison between the sexes. In T. M. Dembroski (Eds.), Behavioral Bases of Coronary Heart Disease (pp. 91-105). Basel: Karger.

Frankenhaeuser, M. (1986). A psychobiological framework for research on human stress and coping. In M. Appley \& R. Trumbull (Eds.), Dynamics of Stress (pp. 101-116). New York: Plenum.

Frankenhaeuser, M., \& Gardeli, B. (1976). Underload and overload in working life: Outline of a multidisciplinary approach. Journal of Human Stress, 2(3), 35-46.

Frankenhaeuser. M., \& Johansson, G. (1976). Task demand as reflected in catecholamine excretion and heart rate. Journal of Human Stress, 2, 15-23.

Frankenhaeuser, M., Lundberg, U., \& Forsman, L. (1980). Dissociation between sympatheticadrenal and pituitary-adrenal responses to an achievement situation characterized by high controllability. Comparison between type $\mathrm{A}$ and type $\mathrm{B}$ males and females. Biological Psychology, 10, 79-91.

Frankenhaeuser, M., Lundberg, U., Fredrikson, M., Melin, B., Tuomisto, M., Myrsten, A., Hedman, M., Bergman-Losman, B., \& Wallin, L. (1989). Stress on and off the job as related to sex and occupational status in white-collar workers. Journal of Organizational Behavior, 10 , 321-346.

Frankenhaeuser, M., Nordheden, B., Myrsten, A. L., \& Post, B. (1971). Psychophysiological reactions to understimulation and overstimulation. Acta Psychologica, 35, 298-308.

Fredrikson, M., Sundin, O., \& Frankenhaeuser, M. (1985). Cortisol excretion during the defense reaction in humans. Psychosomatic Medicine, 47(4), 313-319. 
Friedman, S. B., Mason, J. W., \& Hamburg, D. A. (1963). Urinary 17-hydroxycooid levels in parents of children with neoplastic disease. Psychosomatic Medicine, 25, 364-376

Gaillard, R.-C., \& Al-Damluji, S. (1987). Stress and the pituitary-adrenal axis. In A. Grossman (Eds.), Balliere's Clinical Endocrinology and Metabolism: Neuroendocrinology of Stress (pp. 319-354). London etc.: Balliere Tindall.

Gibbons, R. D., Hedeker, D., ELkin, I., Waternaux, C., Kraemer, H. C., Greenhouse, J. B., Shea, M. T., Imber, S. D., Sotsky, S. M., \& Watkins, J. T. (1993). Some conceptual and statistical issues in analysis of longitudinal psychiatric data. Archives of General Psychiatry, 50, 739-750.

Goetsch, V. L., Wiebe, D. J., Veltum, L. G., \& Van Dorsten, B. (1990). Case history and shorter communication. Behavior Research and Therapy, 28(6), 531 -537.

Gold, P. W., Goodwin, F. K., \& Chrousos, G. P. (1988). Clinical and biochemical manifestations of depression: Relation to the neurobiology of stress. Part II. New England Journal of Medicine, 3I9(7), 413-420.

Gold. W. (1988). Stress-responsive neuromodulators. Biological Psychiatry, 24" 371-374.

Goldberger, L., \& Breznitz, S. (1982). Handbook of stress. Theoretical and clinical aspects. New York: The Free Press.

Goldstein, H. (1987). Multilevel Models in Educational and Social Research. London: Charles Griffin \& Co.

Goplerud, E., \& Depue, R. A. (1985). Behavioral response to naturally occurring stress in cyclothymia and dysthymia. Journal of Abnormal Psychology, 94(2), 128-139.

Goreczny, A. J., Brantley, P. J., Buss, R. R., \& Waters, F. (1988). Daily stress and anxiety and their relation to daily fluctuations of symptoms in asthma and chronic obstructive pulmonary disease (COPD) patients. Journal of Psychopathology and Behavioral Assessment, 10(3), 259. 267.

Gottman, J. M., \& Levenson, R. W. (1988). The social psychophysiology of marriage. In P. Noller \& M. A. Fitzpatrick (Eds.), Perspectives on marital interaction (pp. 182-200). Philadelphia, PA: Multilingual Matters.

Gruchow, H. W. (1979). Catecholamine activity and infectious disease episodes. Journal of Human Siress, 5, 11-17.

Harburg, E., Julius, S., McGinn, N. F., McLeod, J., \& Hoobler, S. W. (1964). Personality traits and behavioral patterns associated with systolic blood pressure levels in college males. Journal of Chronic Diseases, 17, 405-414.

Headey, B., \& Wearing, A. (1989). Personality, life events, and subjective well-being: Toward' a dynamic equilibrium model. Journal of Personality and Social Psychology, 57(4), 731-739.

Hedeker, D. (1993). MIXREG, a Fortran prograrn for mixed-effects linear regression models. University of Illinois: Prevention Research Center.

Hellhammer, D. H., Kirschbaum, C., \& Belkien, L. (1987). Measurement of salivary cortisol under psychological stimulation. In J. Hingtgen, D. Hellhammer, \& G. Huppmann (Eds.), Advanced Methods in Psychobiology (pp. 281-289). Toronto: C. J. Hogrefe Inc.

Hendriks, A. A. J., Ormel, J., \& van de Willige, G. (1990). Langdurige moeilijkheden gemeten volgens zelfbeoordelingsvragenlijst en semi-gestructureerd interview. Gedrag en Gezondheid. 18(6), 273-283.

Hennessy. J. W., \& Levine, S. (1979). Stress, arousal, and the pituitary-adrenal system: A psychoendocrine hypothesis, Progress in Psychobiology and Physiological Psychology, 8. 133-178.

Hennig, J., Laschefski, U., \& Opper, C. (1994). Biopsychological changes after bungee jumping: beta-endorphin immunoreactivity as a mediator of euphoria? Neuropsychobiology, 29(1), 28-32.

Henry, J. P. (1982). The relation of social to biological processes in disease. Social Science and Medicine, 16, 369-380. 
Henry, J. P., \& Stephens, P. M. (1977). Stress, Health and the Social Environment: a Sociobiologic Approach to Medicine. New York: Springer-Verlag.

Hinkle, L. E. (1974). The concept of 'stress' in the biological and social sciences. International Journal of Psychiatry in Medicine, 5, 335-357.

Hofer, M. A., Wolff, C. T., Friedman, S. B., \& Mason, J. W. (1972a). A psychoendocrine study of bereavement PartI. 17-Hydroxycorticosteroid excretion rates of parents following death of their children from leukemia. Psychosomatic Medicine, 34(6), 481-491.

Hofer, M. A., Wolff, C. T., Friedman, S . B., \& Mason, J. W. (1972b). A psychoendocrine study of bereavement. Part II. Observations on the process of mourning in relation to adrenocortical function. Psychological Medicine, 34(6), 492-504.

Holmes, T. H., \& Masuda, M. (1974). Life change and illness susceptibility. In B. S. Dohrenwend \& B. P. Dohrenwend (Eds.), Stressful life events: Their nature and effects (pp. 45-72). New York: John Wiley \& Sons.

Holmes, T. H., \& Rahe, R. H. (1967). The social readjustment rating scale. Journal of Psychosomatic Research, 11, 213-218.

Holroyd, K. A., \& Lazarus, R. S. (1982). Stress coping and somatic adaptation. In L. Goldberger \& S. Breznitz (Eds.), Handbook of Stress: Theoretical and Clinical' aspects (pp. 21-35). New York: Free Press.

Holsboer, F. (1987). Psychoneuroendocrine strategies. Advances in Psychosomatic Medicine, 17. 185-233.

Holsboer, F. (1992). The hypothalamic-pituitary-adrenocortical system. In E. S. Paykel (Eds.), Handbook of affective disorders (pp. 267-287). New York: Churchill Livingstone Inc.

Hoon, E. F., Hoon, P. W., Rand, K. H., Johnson, J., Hall, N. R., \& Edwards, N. B. (1991). A psychobehavioral model of genital herpes recurrence. Journal of Psychosomatic Research, 35, 25-36.

Hormuth, S. E. (1985). Methoden für psychologische Forschung im Fels (Diskussionspapier Nr. 43). Heidelberg: Psychologischen Institut der Universitat Heidelberg.

Hormuth, S. E. (1986). The sampling of experiences. Journal of Personality, 54(1), 262-293.

Hosman, C. M. H. (1983). Psychosociale problematiek en hulpzoeken. Een sociaalepidemiologische studie ten behoeve van de preventieve geestelijke gezondheidszarg. Lisse: Swets \& Zeitlinger.

Houtman, I. L. D., \& Bakker, F. C. (1987). Stress in student teachers during real and simulated, standardized lectures. Journal of Human Stress, 13(4), 180-187.

Hubert, W., \& de Jong-Meyer, R. (1989). Emotional stress and saliva cortisoll response. J Clin Chem Clin Biochem, 27(4), 235-237.

Hubert, W., \& de Jong-Meyer, R. (1990). Psychophysiological response patterns to positive and negative film stimuli. Biological Psychology, 31, 73-93.

Hubert, W., \& de Jong-Meyer, R. (1991). Autonomic, neuroendocrine, and subjective responses to emotion-inducing film stimuli. Intermational Journal of Psychophysiology, 11, 131-140.

Huber, W. H., Moller, M., \& de Jong-Meyer, R. (1993). Film-induced amusement changes in saliva cortisol levels. Psychoneuroendocrinology, 18(4), 265-272.

Hurlburt, R. T. (1990). Sampling normal and schizophrenic inner experience. New York: Plenum Press.

Isenberg. S. A., Lehrer, P. M., \& Hochron, S. (1992). The effects of suggestion and emotional arousal on pulmonary function in asthma: a review and a hypothesis regarding vagal mediation. Psychosomatic Medicine, 54, 192-216.

Ivancevich, J. M. (1986). Life events and hassles as predictors of health symptoms, job performance, and absenteeism. Journal of Occupationai Behaviour, 7, 39-51.

Jaccard, J., \& Wan, C. K. (1993). Statistical analysis of temporal data with many observations: issues for behavioral medicine data. Annals of Behavioral Medicine, 15(1), 43-49. 
Jacobs, S. C., Friedman, R., \& Mittleman, M. (1992). 9-Fold increased risk of myocardial. infarction following psychological stress as assessed by a case-control study. Circulation, 86 (suppl), 198. Abstract.

Jacobson, L., \& Sapolsky, R. (1991). The role of the hippocampus in feedback regulation of the hypothalamic-pituitary-adrenocortical axis. Endocrine Review, 12, 118-134.

Jandorf, L., Deblinger, E., Neale, J. M.. \& Stone, A. A. (1986). Daily versus major life events as predictors of symptom frequency: A replication study. Journal of General Psychology, II(3), 205-218.

Johansson, G., Frankenhacuser, M., \& Magnusson, D. (1973). Catecholamine output in school children as related to performance and adjustment. Scandinavian Journal of Psychology, 14. 20-28.

Jones, K. V.. Copolev, D. L., \& Outsch, K. H. (1986). Type A, test performance and salivary cortisol. Journal of Psychosomatic Research, 30, 699-707.

Kahn, J.-P., Rubinow, D. R., Davis, C. L., \& al., e. (1988). Salivary cortisol: A practical methodi for evaluation of adrenal function. Biological Psychiatry, 23, 335-349.

Kanner, A. D., Coyne, J. C. Schacfer, C., \& Lazarus, R. S. (1981). Comparison of two modes of stress measurement: Daily hassles and uplifts versus. major life events. Journal of Behavioral Medicine, 4, 1-39.

Kapian, C. D. (1992). Drug craving and drug use in the daily life of heroin addicts, In M. W. deVites (Eds.). The experience of psychopathology: Investigating mental disorders in their natural settings (pp. 193-218). Cambridge: Cambridge Press.

Kaplan, H. D. (1979). Social psychology of disease. In H. G. Freeman,S. Levine, \& L. F. Reeder (Eds.), Handbook of Medical Sociology (pp. 53-70). Englewood Cliffs, N.J: PrenticeHall.

Kathol, R. G., Noyes, R., \& Lopez, A. (1988). Similarities in hypothalamic-pituitary-adrenal axis. acivity between patients with panic disorder and those experiencing external stress. Psychiatric Clinics of North America, II, 335-348.

Katz, R., \& Wykes, T. (1985). The psychological difference between temporally predictable and unpredictable stressiul events: evidence for information control theories. Journal of Personality and Social Psychology: 48(3), 781-790.

Kiecoll-Glaser, J. K. \& Glaser, R. (1991). Stress and immune function in humans. In R. Ader, D. L. Felten, \& N Cohen (Eds.), Psychoneuroimmunology (pp. 849-867), San Diego, California: Academic Press, Inc.

Kirschbaum, C., Bartussek, D., \& Strasburger, C. J. (1992a). Cortisol responses to psychologicar stress and correlations, with personality traits. Personality and Individual Differences, 13(12), 1353-1357.

Kirschbaum, C., \& Hellhammer. D. H. (1989). Salivary cortisol in psychobiological research: An overview. Neuropsychobiology, 22, 150-169.

Kirschbaum, C., \& Hellhammer, D. H. (1990). Methodological aspects of salivary cortisol measurement and its application in stress research (abstract). Journal of Clinical Chemistry and Clinical Biochemistry, 28, 649-066.

Kirschbaum, C., \& Helihammer, D. H. (1993). The 'Trier Social Stress Test' - a tool for investigating psychobiological stress responses in a laboratory setting. Neuropsychobiology, 28, 76-81:

Kirschbaum, C., \& Hellhammer, D. H. (1994). Salivary cortisol in psychoneuroendocrine research: Recent developments and applications. Psychoneuroendocrinology, 19(4), 313-333

Kirschbaum, C., Hellhammer, D. H., Strasburger. C. J., Tiling, E., Kamp, R., \& Luđdecke, H. (1989). Relationships between salivary cortisol, electrodermal activity and anxiety under mild experimental stress in children. In H. Weiner,I. Forin, R. Murison, \& D. Hellhammer (Eds.), Frontiers in Stress Research (pp. 383-387). Toronto: Hans Huber. 
Kirschbaum, C., Pruessner, J., Stone, A. A., Federenko, I., Gaab, J., Lintz, D;, Schommer, N, \& Hellhammer, D. (1995). Persistent high cortisol responses to repeated psychological stress in a subpopulation of healthy men. Psychosomatic Medicine, 57, 468-474.

Kirschbaum, C., Wust, S., Faig, H. G., \& Hellhammer, D. H. (1992b). Heritability of cortisol responses to human corticotrophin-releasing hormone, ergometry, and psychological stress in humans. Journal of Clinical Endocrinology and Metabolism. 75(6), 1520-1530.

Kirschbaum, C., Wust, S., \& Hellhammer, D. (1992c). Consistent sex differences in cortisol responses to psychological stress. Psychosomatic Medicine, 54, 648-657.

Kissinger, P. T., Riggin, R. M., Alcom, R. L., \& Rau, L.-D. (1975), Estimation of catecholamines in urine by high performance liquid chromatography with electrochemical detection. Biochemical Medicine, 13, 299-306.

Klinger, E., Barta, S., \& Maxeimer, M. (1980). Motivational correlates of thought content, frequency and commitment. Journal of Personality and Social Psychology, 39, 1222- 1237.

Kloet, E. R. d., \& Reul, J. M. H. M. (1987). Feedback action and tonic influence of corticosteroids on brain function: a concept arising from the heterogeneity of brain receptor systems. Psychoneuroendocrinology, 12, 85- 105.

Knepper, S. (1991). De epidemiologie van psychische arbeidsongeschiktheid. feiten en achtergronden. In R. Bijl \& D. Bauduin (Eds.), Categorie V. Arbeidsongeschiktheid wegens psychische stoornissen (pp. 7-26). Utrecht: NcGv.

Knight, R. B., Atkins, A., Eagle, C. J., Evans, N., Finkelistein, J. W., Fukushima, D., Katz, J., \& Weiner, H. (1979). Psychological stress, ego defenses, and cortisol production in children hospitalized for elective surgery. Psychosomatic Medicine, 41(1), 40-49.

Koeter, M. W. J., Ormel, J., \& van den Brink, W. (1988). Totaalscore op de SCL-90 als maal voor de ernst van psychopathologie. Nederlands Tijdschrift! voor de Psychologie. 43, 381-391.

Kraan, H., Meertens, H., Hilwig, M., Volovics, L., Dijkman-Caes, C., \& Portegijs, P. (1992). Selecting measures, diagnostic validity and scaling in the study of depression. in M. W. deVries (Eds.), The experience of psychopathology: Investigating mental disorders in their naturat settings (pp. 324-336). Cambridge: Cambridge Press.

Kugler, J., \& Kalveram, K. T. (1989), Is salivary cortisol related to mood states and psychosomatic symptoms? In H. Weiner (Eds.), Frontiers of Stress Research (pp. 389-391). New York Toronto: Hans Huber Publishers.

Laan, M. (1989, 20.04.89). De lange weg naar beter werk (3). Gezondmaking van bedrijven maakt stress tot volksziekte. Het Parool.

Larsen, R. J., Diener, E., \&. Emmons, R. A. (1986). Affect intensity and reactions to daily life events. Journal of Personality and Social Psychology, 5i, 803-814,

Larson, R., \& Delespaul, P. (1992). Analyzing experience sampling data: A guidebook for the perplexed. In M. W. de Vries (Eds.), The experience of psychopathology: Investigating mental disorders in their natural settings (pp. 58-78). Cambridge: Cambridge University Press.

Larson, R. S., \& Csikszentmihalyi, M. (1983). The experience sampling method. In H. R. Reis (Eds.), New directions for methodology of social and behavioral science: naturalistic approaches to studying social interaction. (pp. 41-46). San Francisco: Jossey-Bass.

Laundis, J. R., \& Koch, G. G. (1977). The measurement of observer agreement for categorical data. Biometrics, 33, 396-398.

Lazarus, R. S. (1966). Psychological stress and the coping process. New York: McGraw-Hill.

Lazarus, R. S. (1984). Puzzles in the study of daily hassies. Journal of Behavioral Medicine, 7(4), 375-389.

Lazarus, R. S., De Longis, A., Folkman, S., \&. Gruen, R. (1985). Stress and adaptational outcomes: The problem of confounded. measures. American Psychologist, 40, 770-779.

Lazarus, R. S., \& Folkman, S. (1984a). Coping and adaptation. In. W. D. Gentry (Eds.), Handbook of Behavioral Medicine (pp. 282-325). New York: Guilford Press. 
Lazarus, R. S., \& Folkman, S. (1984b). Stress, appraisal, and coping. New York: Springer.

Lazarus, R. S., \& Folkman, S. (1986). Cognitive theories of stress and the issue of circularity. In M. H. Appley \& R. Trumbull (Eds.), Dynamics of Stress. (pp. 63-80). New York: Plenum.

Lazarus, R. S., \& Launier, R. (1978). Stress-related transactions between person and environment. In L. A. Pervin \& M. Lewis (Eds.), Perspectives in Interactional Psychology (pp. 287-327). New York: Plenum.

Lewinsohn, P. M., \& Amenson, C. S. (1978). Some relations between pleasant and unpleasant mood-related events and depression. Journal of Abnormal Psychology, 87(6), 644-654.

Lewinsohn, P. M., \& Libet, J. (1972). Pleasant events, activity schedules, and depressions. Journal of Abnormal Psychology, 79(3), 291-295.

Lewinsohn, P. M., \& Talkington, J. (1979). Studies on the measurement of unpleasant events and relations with depression. Applied Psychological Measurement, 3, 83-101.

Linkowski, P., Mendlewicz, J., Leclereq, R., Brasseur, M., Hubain, P., Golstein, J., Copinschi, G., \& van Cauter, E. (1985). The 24-hour profile of adrenocorticotropin and cortisol in major depressive illness. Journal of Clinical Endocrinology and Metabolism, 61(3), 429-438.

Lousberg, R., Schmidt, A. J. M., Groenman, N. H., Vendrig, L., \&. Dijkman-Caes, C. (1995). Validating the MPI-DLV with Experience Sampling data. In H. B. R. Lousberg (Eds.), Chronic pain: Multiaxial assessment and behavioral mechanisms. Thesis, University of Limburg, Maastricht, The Netherlands.

Lundberg. U., \& Frankenhaeuser, M. (1980). Pituitary-adrenal and sympathetic-adrenal correlates of distress and effort. Journal of Psychosomatic Research, 24, 125-130.

Lundberg. U., Granqvist, M., Hansson, T., Magnusson, M., \& Wallin. L. (1989). Psychological and physiological stress responses during repetitive work at an assembly line. Work \& Stress, 3(2). 143-153.

Lundberg. U., \& Palm, K. (1989). Workload and catecholamine excretion in parents of preschool children. Work \& Stress, 3(3), 255-260.

Luteijn, F., \& Kok, A. R. (1985). Handleiding NVM. Herziene uitgave. Lisse: Swets \& Zeitlinger.

Lykken, D. T. (1982). Research with twins: the concept of emergenesis. Psychophysiology, 1.1. 249-270.

Madden, K. S., \& Livnat, S. (1991). Catecholamine action and immunologic reactivity. In R. Ader,D. L. Felten, \& N. Cohen (Eds.), Psychoneuroimmunology (pp. 283-310). San Diego. California: Academic Press, Inc.

Manuck, S. B., Kasprowicz, A. L., Monroe, S. M., Larkin, K.. T., \& Kaplan, J. R. (1989). Psychophysiologic reactivity as a dimension of individual differences. In $\mathbf{N}$. Schneiderman, S. M. Weiss, \& P. G. Kaufman (Eds.), Handbook of research methods in cardiovascular behavioral medicine (pp. 365-382), New York: Plenum.

Marcelissen (1989). Onderzoek naar stress in de werksituatie. In Verslag van het symposium 'Stress in de werksituatie: Beleid en preventie.14 december 1988, Den Haag (pp. 43-56). Leiden: NIPG/TNO.

Marco, C. A., \& Suis, J. (1993), Daily stress and the trajectory of mood: Spillover, response assimilation, contrast, and chronic negative affectivity. Journal of Personality and Social Psychology, 64(6), 1053-1063.

Martin, M., Ward, J. C., \& Clark. D. M. (1983). Neuroticism and the recal! of positive and negative personality information. Behaviour Research and Therapy, 21, 495-503.

Mason, D. (1991). Genetic variation in the stress response: susceptibility to experimental allergic encephalomyelitis and implications for human inflammatory disease. Immunology Todiay, 12, 57-60.

Mason. J. W. (1968). A review of psychoendocrine research on the pituitary-adrenal cortical system. Psychosomatic Medicine, 30, 576-607.

Mason, J. W. (1975). A historical view of the stress field. Journal of Human Stress, I(1), 7-12. 
Mason, J. W., Giller, E. L., Kosten, T. R., \& Harkness, L. (1988). Elevation of urinary norepinephrine/cortisol ratio in post-traumatic stress disorder. Journal of Nervous and Mental Disease, I76(8), 498-502.

Mason, J. W., Hartley, H., Kotchen, T. A., Mougey, E. H., Ricketts, P. T., \& Jones, L. G. (1973). Plasma cortisol and norepinephrine responses in anticipation of muscular exercise. Psychosomatic Medicine, 35(5), 406-414.

Massimini, F., Csiksentmihalyi, M., \& Carli, M. (1987). The monitoring of optimal experience. A tool for psychiatric rehabilitation. Journal of Nervous and Mental Disease, 175(9), 545-549.

McCrae, R. R. (1990). Controlling neuroticism in the measurement of stress. Stress Medicine, 6 , 237-241.

McCrae, R. R., \& Costa, P. T. (1986). Personality, coping, and coping effectiveness in an adult sample. Journal of Personality, 54(2), 385-405.

McEwen, B. S., \& Stellar, E. (1993). Stress and the individual. Mechanisms leading to disease. Archives of International Medicine, 153, 2093-2101.

McGrath, J. E., \& Beehr, T. A. (1990). Time and the stress process: Some temporal issues in the conceptualization and measurement of stress. Stress Medicine, 6, 93-104.

Meyerhof, J. L., Oleshansky, M. A., \& Mougey, E. H. (1988). Psychological stress increases plasma levels of prolactin, cortisol, and POMC-derived peptides in man. Psychosomatic Medicine, 50, 295-303.

Miller, S. M. (1979). Controllability and human stress: Method, evidence and theory. Behavior Research and Therapy, 17, 287-305.

Munck, A., Guyre, P. M., \& Holbrook, N. J. (1984). Physiological functions of glucocorticoids in stress and their relation to pharmacological actions. Endocrine Reviews, 5(1), 25-44.

Neale, J. M., Hooley, J. M., Jandorf, L., \& Stone, A. A. (1987). Daily life events and mood. In C. R. Snyder \& C. E. Ford (Eds.), Coping with Negative Life Events: Clinical and Social Psychological Perspectives (pp. 161-189). New York: Plenum.

Nicolson, N. A. (1992). Stress, coping and cortisol dynamics in daily life. In M. W. de Vries (Eds.), The Experience of Psychopathology: Investigating Mental Disorders in their Natural Settings (pp. 219-232). Cambridge: Cambridge University Press.

Nicolson, N. A., van Poll, R., \& deVries, M. (1992). Ambulatory monitoring of salivary cortisol and stress in daily life. In C. Kirschbaum,G. F. Read, \& D. Hellhammer (Eds.), Assessment of Hormones and Drugs in Saliva in Biobehavioral Research (pp. 163-173). Seattle: Hogrefe \& Huber.

Ockenfels, M. C., Porter, L., Smyth, J., Kirschbaum. C., Hellhammer, D. H., \& Stone, A. A. (1995). Effect of chronic stress associated with unemployment on salivary cortisol: Overall cortisol levels, diurnal rhythm, and acute stress reactivity. Psychosomatic Medicine, 57, 460-467.

Ormel, J. (1983). Neuroticism and well-being inventories: Measuring traits or states. Psychological Medicine, 13, 165-176.

Ormel, J. (1985). Her Groningse eerstelijnsproject: Opzet en aanpak. (Intern Rapport), Rijksuniversiteit Groningen, Afdeling Sociale Psychiatrie.

Ormel J. (1995). Etiologie van angst en depressie: kwetsbaarheid en losmakende factoren. In E. R. de Kloet \& V. M. Wiegant (Eds.), Stress en neuropathologie. Leiden: Boerhaave Commissie voor Postacademisch Onderwijs in de Geneeskunde, Rijksuniversiteit Leiden.

Ormel, J., \& Wohlfarth, T. (1991). How neuroticism, long-term difficulties, and life situation. change influence psychological distress. Journal of Personality and Social Psychology, 60(5), 744-755.

Ottenweller, J. E., Natelson, B . H., Pitman, D. L., \& Drastal, S. D . (1989) . Adrenocortical and behavioral responses to repeated stressors: toward an animal model of chronic stress and stressrelated mental illness. Biological Psychiatry, 26(8), 829-41. 
Paykel, E. S. (1974). Life stress and psychiatric disorder: Application of the clinical approach. In B. S. Dohrenwend \& B. P. Dohrenwend (Eds.), Stressful life events: Their narure and effects. (pp. 135-149), New York: John Wiley \& Sons.

Paykel, E. S., \& Dowlatshahi, D. (1988). Life events and mental disorder. In S. Fisher \& J. Reason (Eds.), Handbook of life stress, cognition and health (pp. 241-263). New York: Wiley.

Peeters, M. C. W., Buunk, B. P.. \& Schaufeli, W. B. (1995). A micro-analytic exploration of the cognitive appraisal of daily stressful events at work: the role of controllability. Anxiery, Stress, and Coping, 8, 127-139.

Pennebaker, J. W., \& Watson, D. (1988). Self-reports and physiological measures in the workplace. In J. J. Hurrell, L. R. Murphy, S. L. Sauter, \& C. L. Cooper (Eds.), Occupational Stress: Issues and Developments in Research (pp. 184-199). New York: Taylor \& Francis.

Perloff, D., Sokolow, M., \& Cowan, R. (1983). The prognostic value of ambulatory blood pressures. Journal of the American Medical Association, 249, 2793-2798.

Pfohl, B., Herman, B., \& Schlechte, J. (1985). Pituitary-adrenal axis rhythm disturbances in psychiatric depression. Archives of General Psychiatry, 42, 897-903.

Pincomb, G. A., Lovallo, W. R., Passey, W. R., Brackett, R. B., \& Wilson, M. F. (1987). Caffeine enhances the physiological' response to occupational stress in medical students. Health Psychology, 6, 101-112.

Pitman, R. K., \& Orr, S. P. (1990). Twenty-four hour urinary cortisol and catecholamine excretion in combat-related posttraumatic stress-disorder. Biological Psychiatry, 27, 245-247.

Pope, M. K., \& Smith, T. W. (1991). Cortisol excretion in high and low cynically hostile men. Psychosomatic Medicine, 53, 386-392.

Post, R. M. (1992). Transduction of psychosocial stress into the neurobiology of recurrent affective disorder. American Journal of Psychiatry, 149(8). 999-1011.

Prosser, R., Rasbach, J., \& Goldstein, H. (1991). ML3 Software for Three-level Analyses. Users" Guide for V.2. In London: Institute of Education, University of London.

Quigley, M. E., \& Yen, S. S. C. (1979). A. midday surge in cortisol levels. Journal of Clinical Endocrinology and Metabolism. 49, 945-947.

Rabkin, J. C., \& Streuning. E. L. (1976). Life events, stress, and illness. Science, 194, 1013-1020.

Rahe, R. H., \& Arthur, R. J. (1978). Life change and illness studies: past history and future directions. Journal of Human Stress, 4(2), 3-15.

Rahe, R. H., Karson, S., Howard, N. J., Rubin, R. T., \& Poland, R. E. (1990), Psychological and physiological assessments on American hostages freed from captivity in Iran. Psychosomatic Medicine, 52(1), 1-16.

Rahe, R. H., \& Lind, E. (1971). Psychosocial factors and sudden cardiac death: a pilot study. Journal of Psychosomatic Research, 6, 15-24.

Rehm, L. (1978). Mood, pleasant events, and unpleasant events: Two pilot studies. Journal of Consulting and Clinical Psychology, 46, 854-859.

Repetti, R. L. (1993). Short-term effects of occupational stressors on daily mood and health complaints. Health Psychology, 12(2), 125-131.

Rissler, A. (1977). Stress reactions at work and after work during a period of quantitative overload. Ergonomics, 20, 13-16.

Rose, R. M. (1984). Overview of endocrinology of stress. In G. M. Brown,S. H. Koslow, \& S. Reichlin (Eds.), Neuroendocrinology and Psychiatric Disorder (pp. 95-122), New York: Raven Press.

Rose, R. M., \& Fogg. L. F. (1993). Definition of a responder: analyses of behavioral, cardiovascular, and endocrine responses, to varied workload in air traffic controllers. Psychosomatic Medicine, 55, 325-338.

Rose, R. M., \& Hurst, M. W. (1975). Plasma cortisol and growth hormone responses to intravenous catheterization. Journal of Human Stress, $I(1), 22-36$. 
Rose, R. M., Jenkins, C. D., Hurst, M., Kreger, B. E., Barrett, J., \& Hall, R. P. (1982). Endocrine activity in air traffic controllers at work.III. Relationship to physical and psychiatric morbidity. Psychoneuroendocrinology, 7(2), 125-134.

Sapolsky, R., Krey, L., \& McEwen, B. S. (1986). Neuroendocrinology of stress and aging: the glucocorticoid cascade hypothesis. Endocrine Review, 7, 284-301.

Sarason, 1. G.. Johnson, J. H., \& Siegel, J. M. (1978). Assessing the impact of life changes: Development of the Life Experiences Survey. Journal of Consulting and Clinical Psychology, 5. 932-946.

Scarr, S., Webber, P. L., Weinberg, R. A., \& Wittig, M. A. (1981). Personality resemblance among adolescents and their parents in biologically related and adoptive families. Journal of Personality and Social Psychology, 40, 885-898.

Schaeffer, M. A., \& Baum. A. (1984). Adrenal cortical response to stress at Three Mile Island. The Journal of Nervous and Mental Disease, 46(3), 227-237.

Schaubroeck, J., Ganster, D. C., \& Fox, M. L. (1992). Dispositional affect and work-related stress. Journal of Applied Psychology, 77(3), 322-335.

Schedlowski, M., Jacobs, R., Stratmann, G., Richter, S., Hadicke, A., Tewes, U., Wagner, T. O., \& Schmidt, A. U. (1993). Changes of natural killer cells during acute psychological stress. Journal of Clinical Immunology, 13(2), 119-126.

Schreurs, P. J. G., \& van de Willige, G. (1988). De Utrechtse Copinglijst (UCL). Lisse, the Netherlands: Swets \& Zeitlinger.

Schroeder, D. H., \& Costa, P. T. (1984). Influence of life event stress on physical illness: Substansive effects or methodological flaws. Journal of Personality and Social Psychology, 46. 853-863.

Schroer, C. A. P., Nijhuis, F. J. N., \& van Zutphen, W. (1988). Gebruik en betekenis van de term overspanning in geneeskundige literatuur. Tijdschrift voor Sociale Gezondheidszorg, 60 , 163-167.

Schroer, K. (1993) Verzuim wegens overspanning. Een onderzoek naar de aard van overspanning, de hulpverlening en het verzuimverloop. Thesis, University of Limburg. The Netherlands.

Seligman, M. E. P. (1975). Helplessness: On depression, development and death. San Francisco: Freeman, W.H.

Selye, H. (1936). A syndrome produced by diverse noxious agents. Nature, 138, 32-36.

Selye, H. (1976). The Stress of Life. (revised edition). New York: McGraw Hill.

Shields, J. (1962). Monozygotic twins. Oxford: Oxford University Press.

Siegrist, J., Klein, D., \& Matschinger, H. (1989). Occupational stress, coronary risk factors, and cardiovascular responsiveness. In I. Weiner,R. Florin,Murison, \& D. Hellhammer (Eds.). Frontiers of stress research (pp. 323-335). Toronto: Huber.

Smith, P. L. (1979). Splines as a useful and convenient statistical tool. The American Statistician, 33(2), 57-62.

Solomon, S., Holmes, D. S., \& McCaul, K. D. (1980). Behavioral control over aversive events: Does control that requires effort reduce anxiety and physiological arousal? Journal of Personality and Social Psychology, 39(4), 729-736.

Steiner, H., \& Levine, S. (1988). Acute stress response in anorexia nervosa. A pilot study. Child Psychiatry and Human Development, 18(4), 208-218.

Sternberg, E. M., Hill, J. M., Chrousos, G. P., \& al., e. (1989). Inflammatory mediator-induced hypothalamic-pituitary-adrenal axis activation is defective in streptococcal cell wall arthritissusceptible Lewis rats. Proc Natl Acad Sci USA, 86, 2374-2378.

Stone, A. A. (1987). Event content in a daily survey is differentially associated with concurrent mood. Journal of Personality and Social Psychology, 52(1), 56-58. 
Stone, A. A., Cox, D. S., Valdimarsdottir, H., Jandorf, L., \& Neale, J. M. (1987). Evidence that secretory IgA antibody is associated with daily mood. Journal of Personality and Social Psychology, 52(5), 988-993.

Stone, A. A., Kessler, R. C., \& Haythornthwaite, J. A. (1991). Measuring daily events and experiences: Decisions for the researcher. Journal of Personality, 59(3), 575-607.

Stone, A. A., \& Neale, J. M. (1982). Development of a methodology for assessing daily experiences. In A. Baum \& J. E. Singer (Eds.), Advances in environmental psychology (pp. 49-83). Hillsdale, New Jersey: Lawrence Erlbaum Associates, Publishers.

Stone, A. A., \& Neale, J. M. (1984). Effects of severe daily eventsi on mood. Journal of Personality and Social Psychology, 46(1), 137-144.

Stone, A. A., Neale, J. M., \& Shiffman, S. (1993). Daily assessments of stress and coping and their association with mood. Annals of Behavioral Medicine, 15(1), 8-16.

Stone, A. A., \& Shiffman, S. (1992). Reflections on the intensive measurement of stress, coping, and mood, with emphasis on daily measures. Psychology and Health, 7, 115-129.

Strongman, K., \& Russell, P. (1986). Salience of emotion in recall. Bulletin of the Psychonomic Society, 24, 25-27.

Strube, M. J. (1989). Assessing subjects' construal of the laboratory situation. Ini N. Schneidermann,S. M. Weiss, \& P. G. Kaufmann (Eds.), Handbook of Research Methods in cardiovascular Behavioral Medicine (pp. 527-542). New York / London:" Plenum Press.

Surwit, R. S., Williams, R. B., \& Shapiro, D. (1982), Behavioural approaches to cardiovascular disease. New York: Academic Press.

Szalai, A. (Ed.). (1972). The use of time: Daily activities of urban and suburban populations in rwelve countries. Den Haag: Mouton.

Teasdale, J., \& Forgariy, S. (1979). Differential effects of induced mood on retrieval of pleasant and unpleasant events from episodic memory. Journal of Abnormal Psychology, 88, 248-257.

Tellegen, A. (1985). Structures of mood and personality and their relevance to assessing anxiety; with an emphasis on self-report. In A. H. Tuma \& J. D. Maser (Eds.), Anxiety and the anxiety disorders (pp. 681-706). Hillsdale, NJ: Erlbaum.

Theorell, T. (1989). Spontaneously occurring stressors. In H. e. a. Weiner (Eds.). Frontiers of Stress Research (pp. 111-121). New York, Toronto: Huber, H.

Thompson, S. C. (1981). Will it hurt less if I can control it? A complex answer to a simple question. Psychological Bulletin, 90(1),89-101.

Timio, M., \& Gentili, S. (1976). Adrenosympathetic overactivity under conditions of work stress. British Journal of Preventive and Social Medicine, 30, 262-265.

Tinsley, H. E. A., \& Weiss, D. J. (1975). Interrater reliability and agreement of subjective judgments. Journal of Counseling Psychology, 22(4), 358-376.

Troxler, R. G., Sprague, E. A., Albanese, R. A., Fuchs, R., \& Thompson, A. J. \{1977). The association of elevated plasma cortisol and early atherosclerosis as demonstrated by coronary angiography. Atherosclerosis, 26, 151-162.

Tsigos, C., \& Chrousos, G. P. (1994). Physiology of the hypothalamic-pituitary-adrenal axis, in heaith and dysregulation in psychiatric and autoimmune disorders. Endocrinology and Metabolism Clinics of North America, 23(3), 451-466.

Tumer, J. R., Ward, M. M., Gellman, M. D., Johnston, D. W., Light, K. C., \&: van Doomen, L. J. P. (1994). The relationship between laboratory and ambulatory cardiovascular activity: Current evidence and future directions. Annals of Behavioral Medicine, 16(1), 12-23.

Uhde, T. W., Joffe. R. T., Jimerson, D. C., \& Post. R. M. (1988). Normal urinary free cortisol and plasma MHPG in panic disorder: Clinical and theoretical implications. Biological Psychiatry, 23. 575-585.

Ursin, H. (1978). Activation, coping, and psychosomatics. In H. Ursin. E. Baade, \& S. Levine (Eds.), Psychobiology of Stress (pp. 201-228). New York: Academic Press. 
Urșin, H. (1980). Personality, activation and somatic health. In S. Levine \& H. Ursin (Eds.), Coping and health (pp. 259-279). New York: Plenum Press.

Ursin, H., Mykletun, R., Tonder, O., Vaemes, R., Relling, G., Isaksen, E., \& Murison, R. (1984). Psychological stress factors and concentrations of immunoglobulins and complement components in humans. Scandinavian Journal of Psychology, 25, 340-347.

Vaernes, R., Ursin, H., Darragh, A., \& Lambe, R. (1982). Endocrine response patterns and psychological correlates. Journal of Psychosomatic Research, 26, 123-131.

van der Ploeg, H. M., Defares, P. B., \& Spielberger, C. D. (1980). Handleiding bij de ZelfBeoordelings Vragenlijst - ZBV - een nederlandstalige bewerking van de Spielberger State . Trait Inventory (a manual for the STAI). Lisse: Swets \& Zeitlinger.

van der Ploeg, H. M., Defares, P. B., \& Spielberger, C. D. (1982), Handleiding bij de Zelf-Analyse Vragenlijst - ZAV - een nederlandstalige bewerking van de Spielberger State - Trait Anger Scale. Lisse: Swets \& Zeitlinger.

van Diest, R. (1992). Vital exhaustion or depression: a study of daily mood in exhausted male subjects at risk for myocardial infarction. In M. W. deVries (Eds.), The experience of psychopathology: Investigating mental disorders in their natural settings (pp. 233-239). Cambridge: Cambridge Press.

van Doornen, L. J. P., \& Turner, J. R. (1992). The ecological validity of laboratory testing. In J. R. Turner,A. Sherwood, \& K. C. Light (Eds.), Individual differences in cardiovascular response to stress (pp. 63-83). New York: Plenum.

van Doornen, L. J. P., \& van Blokland, R. W. (1992). The relationship between cardiovascular and catecholamine reactions to laboratory and real-life stress. Psychophysiology, 29(2), 173-181, van Eck, M. M., Berkhof, H., Nicolson, N., \& Sulon, J. (in press). The effects of perceived stress, traits, mood states, and stressful daily events on salivary cortisol. Psychosomatic Medicine.

van Eck, M. M., \& Nicolson, N. A. (1994). Perceived stress and salivary cortisol in daily life. Annals of Behavioral Medicine, 16(3), 221-227.

Vickers, R. R. (1988). Effectiveness of defenses: a significant predictor of cortisol excretion under stress. Journal of Psychosomatic Research, 32, 2! -29.

Vingerhoets, A. J. J. M., \& Menges, L. J. (1989). Psychosocial load and symptoms: An inquiry into their relationship with coping styles. Stress Medicine, 5, 189. 194.

Vining, R. F., McGinley, R. A., \& Symons, R. G. (1983). Hormones in saliva: Mode of entry and consequent implications for clinical interpretation. Clinical Chemistry, 29(i0), 1752-1756.

Wagner, B. M. (i990). Major and daily stress and psychopathology: On the adequacy of the definitions and methods. Stress Medicine, 6, 217-226.

Watson, D. (1988). Intraindividual and interindividual analyses of positive and negative affect: Their relationship to health complaints, perceived stress, and daily activities. Joumal of Personality and Social Psychology, 54(6), 1020- 1030.

Watson, D., \& Clark, L. A. (1984). Negative affectivity: The disposition to experience aversive emotional states. Psychological Bulletin, 96(3), 465-490.

Watson, D., Clark, L. A., \& Harkness, A. R. (1994). Structures of personality and their relevance to psychopathology. Journal of Abnormal Psychology, 103(1), 18-31.

Watson, D., \& Pennebaker, J. W. (1989). Health complaints, stress, and distress: Exploring the central role of negative affectivity. Psychological Review, 96(2), 234-254.

Weiner, H. (1992). Perturbing the Organism: The Biology of Stressful Experience. Chicago: University of Chicago Press.

Wolff, S., \& Goodell, H. (1968). Harold G. Wolffs stress and disease (2 ed.). Springfield (III.): Charles C. Thomas.

Wong, M. M., \& Csikszentmihalyi, M. (1991). Motivation and academic achievement: The effects of personality traits and the quality of experience. Journal of Personality. 59(3), 539-574. 
Wüst, S., Kirschbaum, C., \& Hellhammer, D. (1990). Smoking increases salivary cortisol. In C. Kirschbaum \& D. Hellhammer (Ed.), Proceedings of the 2nd European Symposium on Hormone and Drug Assessment in Saliva. Trier.

Yehuda, R., Resnick, H., Kahana, B., \& Giller, E. L. (1993). Long-lasting hormonal alterations to extreme stress in humans: Normative or maladaptive? Psychosomatic Medicine, 55, 287-297.

Zarski, J. J. (1984). Hassles and health: A replication. Health Psychology, 3(3), 243-251.

Zautra, A. J., \& Guarnaccia, C. A. (1986). Measuring small life events. American Journal of Community Psychology, 14(6), 629-655.

Zautra, A. J., Guarnaccia, C. A., Reich, J. W., \& Dohrenwend, B. P. (1988). The contribution of small events to stress and distress. In L. H. Cohen (Eds.), Life Events and Psychological Functioning. Theoretical and methodological issues (pp. 123-148). Newbury Park, California: Sage Publication.

Zung, W. W. K. (1965). A self-rating depression scale. Archives of General Psychiatry, 12, 63-70. 


\section{Appendices}

Appendix I: Reliability and validity of the Perceived Stress Scale in a sample of Dutch white collar workers.

Appendix II: ESM report.

Appendix III: Interrater agreement on codings for ESM measures. 


\section{Appendix I}

\section{Reliability and validity of the Perceived Stress Scale in a sample of Dutch white collar workers}

\section{INTRODUCTION}

Measures of stress (life events, daily events, or chronic stressful situations), can be broadly divided into subjective and objective stress measures, what points to two conceptually different approaches to the concept of stress. First, the transactional model of psychosocial stress which states that "stress lies not in the environmental input but in the person's appraisal of the relationship between that input and its demands and the person's agendas (e.g. beliefs, commitments, goals) and capabilities to meet, mitigate, or alter these demands in the interest of well-being", p.770 (Folkman \& Lazarus, 1985; Lazarus, De Longis, Folkman, \& Gruen, 1985; Lazarus \& Launier. 1978; Mason, 1975). This view contrast with a second, more objective approach to stress, in which stressors are treated as environmental stimuli, inputs, that are much more independent of the reaction or state of mind of the person (Dohrenwend, Link. Kern, Shrout, \& Markowitz, 1990; Dohrenwend \& Shrout, 1985; Dohrenwend, Dohrenwend, Dodson, \& Shrout, 1984). This approach implies that events are, in and of themselves, the precipitating cause of pathology and illness behavior. From the transactional point of view, the impact of these 'objectively' stressful events is, to a large degree, determined by one's perceptions of their stressfulness.

In research on stress, we would like to be able to identify individuals who are at risk for developing stress-related psychological or somatic problems. We know that only a small percentage of individuals exposed to objective stressful (life) events will fail to cope effectively and thereby develop symptoms. We also know that health complaints (objective and subjective) have multifactorial causes, of which stress is only one. A measure of perceived (appraised) stress would be very useful for screening populations for individuals at risk. Such an instrument should ideally be short; it must also be reliable and have construct validity: that is, it should be able to predict stress symptomatology / pathology, while not simply measuring subjective distress alone.

A subjective stress measure, the Perceived Stress Scale (PSS), was developed by Cohen and colleagues (Cohen, Kamarck, \& Mermelstein, 1983), for the measurement of a global level of perceived stress with the goal of providing additional information about the relation between stress and pathology. The PSS measures cognitions and emotions relating to general stress levels rather than specific events or situations. Items tap the extent to which individuals feel their life to be. unpredictable, uncontrollable and overloaded: important components of the experience of stress (Averill, 1973; Cohen.1978; Lazarus, 1966; Miller, 1979; Seligman, 1975). An example of a PSS item is: 'In the last month, how often have you felt that you were unable to control the important things in your life?' The scale attempts to represent situations where persons perceive that the denands exceed their ability to cope. Because the items are of a general nature they are free of content specific to any subpopulation. Another advantage of the generality of the scale is that it is sensitive to the nonoccurrence of events, to chronic life circumstances, to anticipation of future events and to events occurring in the lives of friends and relatives. In the case of scales measuring event-specific levels of perceived stress, 
respondents are: asked to rate the stressfulness or impact of each experienced event. This means that perceived stress levels will be limited by the specific list of events in the scale. In these scales it is also not possible for a single event to have the impact of three or four less salient ones.

A scale measuring global levels of perceived stress can have various valuable functions (Cohen et al., 1983). At the first place, the PSS can be used when the major aim of the study is the role of appraised stress, as opposed to objective stress. It can, for instance, be used to determine whether appraised stress is an etiologic (or risk) factor in psychological or physical disorders (e.g. when the objective sources of stress are diffuse or difficult to measure). Second, it can be used together with an objective scale to study whether appraised stress mediates the relationship between objective stress and illness. In other words, it can provide information about the processes through which stressful events influence pathology. Similarly, it can be used to look more closely at the process by which various moderators of the objective stressor/pathology relationship operate (e.g. social support, coping, personality). Third, the PSS can be used as an outcome measure, i.e. assessing experienced stress as a function of objective stressful events, coping processes, personality characteristics eIc. A final valuable function of the PSS that will be examined in this article, could be its use as an economical tool for screening purposes. When administered more than

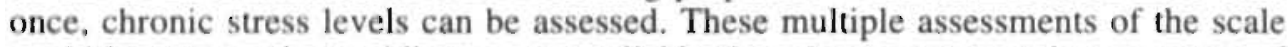
could be averaged, providing a more reliable (based on more samples) measure of chronic stress, and as well a predictor representing a longer time period than the onemonth period covered by the PSS.

Research by Cohen and others showed acceptable levels of validity and reliability of the PSS in samples of the United States. Cronbach's alpha coefficients for the internal reliability of the PSS were high in the various studies (ranging between .75 and .86) (Cohen et al., 1983; Cohen \& Williamson, 1988), and test-retest reliability over a period of two days was also high $(r=85)$. For longer periods (one month, 6 weeks), test-retest correlations were moderate (.50 and .55 respectively) (Cantor, Norem, Langston, Zirkel, Fleeson, \& Cook-Flannagan, 1991; Cohen et al., 1983). Perceived stress seems to be an important mediator of the relationship between stressful life events and symptomatology. Work by Cohen et al. (Cohen. 1986; Cohen et al., 1983; Cohen \& Williamson, 1988) showed for instance that the PSS was a better predictor than life-event scales of psychological symptoms, somatic symptoms and utilization of health services and that stress perception was also related to selfreports of health behaviors. Higher PSS scores were also prospectively associated in a dose-response manner with an increased risk of acute infectious respiratory illness (Cohen. Tyrrell, \& Smith. 19917. The PSS was found to measure a different and independently predictive construct than a depressive symptomatology scale. Cohen (Cohen, 1986) demonstrated that even after controlling for the possible overlap of the PSS and psychopathology, the PSS prospectively predicts psychological symptoms, physical symptoms and health behaviors.

Biy presenting psiychometric and descriptive data on the PSS in a Dutch sample of white collar workers, we want to show that the PSS is a reliable and valid instrument that could be used for screening purposes. The shortness and generality of the scale (containing only 10 items) is a major advantage above the usually long and specific event scales that are used for measuring stress levels, especially in large scale screening where various other instrument are usually included too. As described above, the reliability and validity of the scale has been proven within a culture, i.e. in various samples in the Unites States (students, adult populations, diabetics), but we do 
not have information about the qualities of the scale across cultures. At face value the individual items seem to be aspecific to a particular culture, but this is certainly not enough a base to rely on. Therefore we looked at the reliability and consiruct validity of the scale in a Dutch sample of white collar workers, where the PSS was used as a screening instrument. Besides the internal reliability, we investigated the stability of the PSS over time in a subsample of subjects who filled out the PSS twice. Scores on the scale in the Dutch sample were also compared to US. norms. The construct validity of the PSS was examined through comparison with various indices related to stress: life events, chronic stress, coping style, health behavior, and psychological and somatic complaints. We expected the PSS to be related to the number of life events because perceptions of stress should generally increase with increases of objective stress levels. We expected a higher correlation of the PSS with chronic difficulties though, because in this scale respondents were asked to indicate the intensity of he problems they were facing. The intensity scores should reflect some of the same stress appraisal as measured by the PSS. Perceptions of stress were also expected to be positively related to anxiety, depression, less effective coping styles, and poorer self reported health and health-related behavior. Besides these measures, also cortisol levels were determined in saliva as a physiological measure of subjective stress. Salivary cortisol is a reliable and valid indicator of the free cortisol in plasma, which is considered to be the biologically active hormone. Cortisol concentrations are independent of the flow rate of saliva. Cortisol was measured only once in our respondents, so we did not look for reactive but rather for basal levels of cortisol and the possible influence of (ongoing) perceived stress on that level. Results from studies on cortisol levels in cases of chronic psychosocial load are inconsistent. Sometimes enhanced concentrations have been found (Hofer, Wolff, Friedman, \& Mason, 1972; Jacobs, Mason, Kosten, Kasl, Ostfeld, \& Wahby, 1987, Kiecolt-Glaser, Ricker, George, Messick, Speicher, Gamer, et al., 1984) but decreased levels as well (Caplan, Cobb, \& French, 1979). We also looked at the predictive ability off appraised stress opposed to alternative measures of stress. Parallel to other studies, the PSS was: expected to be the strongest predictor of health variables, because it is presumably the level of appraised stress and not the objective occurrence of an event, that causes one's reaction to a stressor(s).

Subjects were first screened and then a subgroup was selected from various local industries and government agencies according to their perceived stress leve!. Reliability and validity data were collected in these two samples. Although our samples are not representative of the Dutch population in general, we believe that our data should give us a good indication of the usefulness of the Perceived Siress Scale. as a screening instrument in Dutch populations.

\section{METHODS}

\section{Subjects and procedures}

Both the screening sample ( $n=316$ for males, and $n=60$ for females) and the (sub)sample that had been selected for the ESM study ( $n=92$, all males) were used to investigate the reliability and valididty of the PSS. For details about subject recruitment and procedures see: chapter 2 . 


\section{Questionnaires}

\section{Screening sample}

In addition to the PSS, questionnaires concerning coping style (Utrecht Coping List), and psychological and physical symptoms (Symptom Checklist 90; Psychosomatic Symptom Checklist) were completed by the screening sample $(n=376)$. Demographic information was obtained concerning respondents' age, marital status, household composition, completed education, chronic diseases, medications, alcohol use, smoking habits, and participation in active sports. For details about the questionnaires see chapter 2 .

\section{Subsample}

The 92 subjects who participated in the main study completed additional questionnaires concerning psychosocial stress (life events: List of Threatening Experiences; chronic stress: Longterm Difficulties Questionnaire), and psychological symptoms (anxiety: State-Trait Anxiety Inventory; depression: Self-Rating Depression Scale). For details about the questionnaires see chapter 2 .

\section{Cortisol}

When subjects in the screening sample handed in the completed questionnaires, they also provided a single saliva sample for cortisol determination. All samples were taken between 11.00 and 13.00 hours, before lunch. Subjects collected saliva by holding a polyester dental roll in the mouth for 1 to 2 minutes; the roll was then placed in a capped plastic vial (Salivette; Sarstedt). Information about use of coffee and tobacco in the last hour and use of medication in the last 24 hours was simultaneously obtained to control for possible confounding influences.

Uncentrifuged samples were stored at $-20 \mathrm{C}$. Salivary cortisol levels were determined in duplicate by direct radioimmunoassay (Ansseau, Sulon, Doumont, Cerfontaine, Legros, Sodoyez, et al., 1984), using ${ }^{125}$ ]-cortisol (Farmos diagnostica, Finland) and antiserum made against the 3CMO-BSA conjugate by Dr. J. Sulon, University of Liege, Belgium. The lower detection limit of the assay was $12 \mathrm{ng} / 100 \mathrm{ml}$, with a mean intra-assay coefticient of variation of $4.8 \%$ (range: $2.2 \%$ $-7.5 \%$ for 4 assays).

\section{Statistical analysis}

Unless otherwise stated, statistical tests are non-parametric and two-tailed.

\section{RESULTS}

\section{Unldimensionality and reliability of the PSS}

Principal components extraction with varimax rotation was performed on the 10 items of the Perceived Stress Scale. One component was identified which accounted foi $58.7 \%$ of the total variance. All items had positive loadings of .45 or higher. A total scale score was therefore obtained by summation of the 10 item scores.

For the screening sample, Crohnbach's alpha as a measure of the internal reliability of the PSS was .86 , or slightly higher than that of the original US. scale (Cohen \& Williamson, 1988). Item-total correlations fell between .38 and .74. For the 92 subjects who completed the PSS a second time, Crohnbach's alpha for the scale was .85 , with item-total correlations ranging from .29 to .73 .

The PSS was completed for a second time by the subsample of 92 subjects, after an average interval of 116 days (range 13-213 days). We looked at the test-retest reliability of the PSS to examine its stability. PSS scores from the first and second measurements were highly correlated. ( $r h o=.73, p<001$ ), with a mean decrease of 2.0 
(s.d. 4.7, range -9 to +14 points). Given that the PSS is based on reports for the last month, one would expect larger differences in scores as the time interval between test and retest increases. However, linear regression showed no greater change in PSS score with increasing intervals between test and retest $(F(1,90)=.739$; ns).

\section{Cross-cultural generalizability of the PSS: comparison of results with US. normative data}

Table 1 presents the means and standard deviations of scores on the PSS for the various demographic variables represented in our sample. These results can be compared with published norms from a large $(n=2270)$, representative US. sample (Cohen \& Williamson, 1988), to assess the cross-cultural validity of the Dutch version of the PSS.

Table 1. Mean PSS scores and standard deviations for demographic categories.

\begin{tabular}{lrll}
\hline Category & N & PSS mean & SD \\
\hline Sex male & 316 & 12.6 & 5.9 \\
female & 60 & 13.6 & 6.5 \\
Age & & & \\
$20-29$ & 47 & 12.51 & 5.5 \\
$30-39$ & 136 & 12.6 & 6.0 \\
$40-49$ & 128 & 13.2 & 6.1 \\
$50-59$ & 62 & 12.5 & 6.3
\end{tabular}

Household composition

living with parents

single

10.8

12.2

couple/no children

12.6

couple/children

12.9

single parent

23.0

other

$\begin{array}{rr}16 & 10.8 \\ 32 & 12.2 \\ 91 & 12.6 \\ 227 & 12.9 \\ 1 & 23.0 \\ 9 & 13.3\end{array}$

4.8

6.8

5.7

6.1

6.0

Number of people in household

$\begin{array}{lrrr}\text { one } & 33 & 12.1 & 6.7 \\ \text { two } & 98 & 12.5 & 5.7 \\ \text { three } & 77 & 12.2 & 6.0 \\ \text { four or more } & 126 & 14.7 & 7.2 \\ \text { mber of children in household } & & & \\ \text { none } & 189 & 12.3 & 5.7 \\ \leq 2 & 159 & 12.9 & 6.1 \\ >2 & 27 & 14.5 & 6.9\end{array}$

Education

\begin{tabular}{lrrr} 
lower & 7 & 17.7 & 4.2 \\
middle & 132 & 13.1 & 5.7 \\
higher & 232 & 12.5 & 6.2 \\
\hline
\end{tabular}

The mean score for the Dutch sample $(12.7+$ - s.d. 6.0 ; range $2-35)$ is comparable to US. norms (mean $=13.02$, s.d. $=6.45$ ), especially when the selected characteristics of our white collar sample are taken into account. Scores for US. managerial and clerical professions were 12.0 and 13.5, respectively. In agreement 
with US. norms for females and males, women reported somewhat higher mean PSS scores than men, but this difference failed to reach significance in our sample $(F(374)=1.29$, ns; one-way ANOVA). We found no significant association between respondent's age and perceived stress ( $r=-.02$, n.s.), although a negative relationship between these two variables was reported for the US. sample. Respondents still living with their parents had the lowest levels of perceived stress, but one-way ANOVA revealed no significant effect for household composition on level of perceived stress. In the US. sample, PSS was weakly related to household size and the number of children in the household. In our sample a similar pattern was observed for household size $(r h o=10, p<.05)$, but perceived stress level did not increase significantly in households with more children $(<18$ years $)(F(372)=1.86$, ns; one-way ANOVA) probably because of the small number of people with more than 2 children in our sample. As in the US., higher education was associated with lower perceived stress. Probably due to the small number of people with lower education in our sample, however, this effect just failed to reach significance $(F(368)=2.72, p=.06$ : one-way ANOVA.

\section{The construct valldity of the PSS}

\section{Association of PSS with self-reported health and health-related behaviors.}

Fifty-seven respondents (15.2\% of the sample) reported suffering from one or more chronic condition, including high blood pressure $(n=14)$, cardiovascular disease $(n=8)$, respiratory problems $(n=7)$, and diabetes mellitus $(n=1)$. In the open-ended category 'other chronic diceases' $(n=40)$ the most freguently reported conditions were allergies, hay-fever and eczema. As shown in Table 2, subjects with a chronic illness reported no higher perceived stress level than those with no illness.

Table 2. PSS scores in relation to health and health-related behaviors

\begin{tabular}{lcccc}
\hline Measure & & N & PSSinean & SD \\
\hline Do you have a chronic disease? & yes & 57 & 13.3 & 6.3 \\
& no & 319 & 12.6 & 6.0 \\
Do you take medication? & & & & \\
& yes & 53 & $15.0 * *$ & 6.9 \\
Do you exercise regularly? & no & 321 & 12.4 & 5.8 \\
& yes & 248 & 12.5 & 5.8 \\
Do you smoke? & no & 127 & 13.3 & 6.3 \\
& & & & \\
& yes & 104 & 12.1 & 6.0 \\
& no & 270 & 13.0 & 6.0 \\
\hline
\end{tabular}

$* p<0.0$ H, two-tailed; Mann Whitney U-test

Respondents who used medications, however, had significantly higher PSS scores than those who did not. Medications most frequently used were from the categories: circulatory tract $(n=17)$, respiratory tract $(n=12)$, analgesics $(n=10)$, CNS/psychological (e.g. hypnotics, anxiolytics, anti-depressants) $(n=8)$. There was no difference in the PSS scores of respondents who exercised once a week or more 
versus those who reported no regular exercise, and smokers had no higher PSS scores than non-smokers.

Psychosomatic complaints, as measured with the PSC, were examined as another aspect of self-reported health. Frequency, Intensity and Total (frequency $\mathrm{x}$ intensity) scores on the PSC were all positively correlated with perceived stress ( $r h o=.54, .45$, and .59 , respectively; $p<.001$ ). Correlations remained significant even after the more obvious psychological symptoms (fatigue, depression and insomnia) were omitted from the scale ( $r h o=.44, .36$, and .50 , respectively; $p<.001$ ).

\section{Psychological complaints}

Mean scores on the SCL-90 scales $(n=336)$ were within the normal ranges established for the Dutch population (Koeter, OrmeI, \& van den. Brink, 1988). PSS scores were positively correlated with all eight SCL-90 scales: agoraphobia $(r h o=35)$, sleep problems $(r h o=41)$, hostility $(r h o=47)$, sonatic complaints $(r h o=47)$, insufficiency of thought and performance $(r h o=.56)$, distrust and interpersonal sensitivity ( $r h o=.57$ ), anxiety $(r h o=.62)$, depression $(r h o=.66)$, as well as with the total score $(r h o=.68)($ all tests $p<.001)$.

\section{Coping style}

Scores on the Utrecht Coping List scales $(n=215)$, were similar to norms published for the Dutch population (Schreurs \& van de Willige, 1988). The following Spearman correlations between PSS scores and the assessed coping styles were found: 'depressive reactions" ( $r h o=.59, p<.001)$, 'active problem-solving' (rho=-.37. $p<.001$ ), 'palliative' (rho=.22, p<.01), 'expression of emotions' ( $r h o=.13, p<.05$ ), 'avoidance' ( $r h o=11, p<.06$ ), 'seek social support' ( $r h o=.07, \mathrm{~ns}$ ), and 'comforting cognitions' ( $r h o=.06, \mathrm{~ns}$ ).

\section{Cortisol}

We had expected that high perceived stress would be associated with elevated cortisol levels. However, there was no significant correlation between PSS and prelunch salivary cortisol in either males ( $r h o=07$, ns; $n=295$ ) or females ( $r h o=.01$, ns; $\mathrm{n}=58)$. Mean salivary cortisol was $170 \mathrm{ng} / \mathrm{dl}(\mathrm{sd}=80.3)$ for males and $189 \mathrm{ng} / \mathrm{dl}$ for females $(s d=73.9)(p=.05$, Mann-Whitney $U)$.

\section{High PSS vs low PSS comparisons}

The analysis described in the following paragraphs were done in the subsample of our study. In the subsample, high and low perceived stress groups were defined as follows: the mean of the first and second PSS assessments was used to categorize subjects as above or below the screening sample median score (12). For the resulting group of 44 'high stress' subjects, mean PSS score was $18.0(\mathrm{~s} . \mathrm{d}=3.4)$, compared to a mean PSS of $7.3($ s.d. $=2.2)$ in the group of 48 'low stress' subjects.

The construct validity of the PSS was further examined through two indices of distress: anxiety disposition and depressive symptomatology. Perceptions of stress were expected to be positively related to psychological symptoms. Group differences in anxiety (mean: 22.9 high stress vs 18.8 low stress group) and depression (48.4 vs $36.8)$ were both significant $\left(p^{\prime} s<.001\right)$ and in the expected direction. 
Aelationship between PSS and number of life events and chronic difficulties

We found a small but significant correlation between the number of life events and the PSS ( $r h o=.20, p=.03$ ). The difference in mean number of life events between the 'high stress' group (.73 events) and the 'low stress' group. (.46 events) was not significant ( $p=19$; Mann-Whitney U). As expected, the correlation between chronic difficulties and the PSS was markedly higher $(r h o=.56, p<.001)$. There was a significant difference in number of long-term difficulties between the two groups, with respondents in the 'high stress' group experiencing more difficulties than those in the 'low stress' group (23.3 vs $19.5 ; p<, 001)$.

\section{The PSS versus life event and chronic difficulty measures in predicting symptomatology}

Following the analysis presented by Cohen et al. (Cohen et al., 1983), we looked at the PSS compared to the number of life events as predictors of psychosomatic and depressive symptoms. Perceived stress was expected to be a stronger predictor of symptomatology than the number of life events (Lazarus \& Launier, 1978: Mason, 1971), and also, although to a lesser extent, expected to be a stronger predictor than long-term difficulties (though limited by the specific sample of the various difficulties, this measure does account for the appraised severity of difficulties). The data in Table 3 lend support to these assumptions.

Table 3. Spearman correlations of stress measures with psychosomatic and depressive symptomatology.

\begin{tabular}{lcc}
\hline & Psychosomatic Symptoms & Depression \\
\hline Perceived stress & $.60^{* * *}$ & $.76^{* * *}$ \\
Number of life events & .07 & .06 \\
Long-term difficulties & $.47 * * *$ & $.52 * * *$ \\
\hline
\end{tabular}

$\cdots p<.001$

Of the three stress measures, perceived stress showed the highest correlations to both psychosomatic and depressive symptomatology. The number of life-events did not correlate with either psychosomatic or depressive symptomatology. So, although subjects who experienced more life events also experienced more stress, they did not have more symptoms.

Because both perceived stress and chronic difficulties were strongly related to symptomatology it is desirable to show that both stress scales are not measuring the same things. Therefore, chronic difficulties were partialled out of the correlation between perceived stress and psychosomatic symptoms $s_{n}$ and the PSS was partialled out of the correlation between chronic difficulties and psychosomatic symptoms. Partial correlations were calculated with ranked variable scores for reasons of not normally distributed variables. The correlation between the PSS and psychosomatic symptoms, controlling for chronic difficulties, was .45 (p<.001). The correlation between chronic difficulties and psychosomatic symptoms, controlling for perceived stress was .21 $(p<.05)$. Chronic difficulties and perceived stress both independently predicted psychosomatic complaint. Although overlap exists, the scales are not measuring the same thing.

For the validity of the PSS it was also important to demonstrate that perceived stress did not overlap completely with measures of psychological distress. Relations 
among these measures may be due to the common influence of negative affectivity. Self-report stress scales and self-report health measures have been found to reflect a pervasive mood disposition of negative affectivity, thus overestimating the true association between stress and health (Watson \& Clark, 1992; Watson \& Pennebaker. 1989). In our study we found a high correlation between perceived stress and depressive symptomatology $(r h o=.76)$ and between perceived stress and trait-anxiety $(r h o=77)$, probably indicating some overlap again since stress perception could be a symptom of depression or of high trait anxiety. So again partial correlations were calculated on ranked variable scores: results were compared when depressive symptomatology was partialled out of the relation between perceived stress and psychosomatic symptoms and when perceived stress was partialled out of the relation between depressive symptoms and psychosomatic complaints. In the case of the PSS and psychosomatic symptomatology, the correlation was .27 $(\mathrm{p}<.01)$ and in the case of the ZUNG and psychosomatic symptoms, the correlation was .28 (p<.01). It thus appears that both scales independently predict psychosomatic symptomatology. Similarly, both trait anxiety and stress perception independently predicted psychosomatic complaints (partial correlations: $r=.24, p<.05$; and $r=.29, p<.01$. respectively).

\section{DISCUSSION}

The Dutch version of the PSS showed adequate internal reliability and sufficient stability in a sample of white collar workers. The test-retest correlation was high and there was no systematic tendency for scores to fall or rise, reflecting that although the questionnaire is sensitive to temporal change in experienced stress, it also reflects a rather stable individual characteristic. These results are comparable to the results found by Levenstein et al. (Levenstein, Prantera, Varvo, Scribano, Berto, Luzi, et al., 1993) who developed The Perceived Stress Questionnaire (PSQ) which is highly correlated and comparable to the PSS (.73). Here, the sensitivity of this scale to change was tested over 6 months (scale was filled in monthly) and they also found that the mean ratio between a subjects highest and lowest PSQ score was quit small. The fact that the PSS is quite stable but still sensitive to changes in experienced stress is an advantage considering its potential usage as a screening instrument; it is possible to assess chronic levels of perceived stress (when measured more than once) but environmental influences can be studied for its effects as well. Possible causes for the observed test-retest effect are: statistical regression (twice as many people had a lower scale score the second time in stead of a higher score, but this could possibly be explained by the fact that those people with low scale scores the first time were the better adjusted people and therefore less likely to have an increased stress score the second time), and social desirability (subjects selected for the field study wanted to look 'good', stress resistant).

Our sample means and standard deviations of perceived stress were comparable with the US. norms. Especially their means for managerial and clerical professions compared very well with our means for white collar workers. These results give support to the notion that our sample, although not a representative one, does not deviate strongly from a normal probability sample. Means on other crosssectionals like the UCL and the SCL90, when compared to norm scores for the Dutch population, also showed normal population scores. Although correlations between various demographic characteristics and level of perceived stress were in the 
expected direction, most of them did not reach significance. Only household size was positively related to perceived stress. This was probably due to the fact that the correlations described by Cohen and Williamson (Cohen \& Williamson, 1988), which were also small (between .10 and .13), were based on a very large sample $(n=2270)$. Cohen et al.(Cohen et al., 1983) also failed to find a relation between the PSS and sex or age in samples comparable to the size of our white collar sample. It must be said that our sample was also quite homogeneous, in the sense that it consisted of only white collar workers. For instance, differences in socioeconomic status, not measured here, could be very well related to levels of perceived stress.

If the PSS is to be used as a screening instrument it should be able to discriminate subjects on theoretically interesting characteristics. Our data give support for the construct validity of the Dutch version of the PSS; perceived stress was related in the expected way to other stress measures (life events and chronic difficulties), to coping styles, and psychological (anxiety, depression), and psychosomatic complaints. The results on coping are in concordance with studies suggesting that an active-offensive mode of coping (dominated by a tendency of active problem solving and optimism) is more effective in avoiding and solving problems than a reactive-defensive mode of coping (dominated by a tendency of avoidance and depressive reactions).

Our only objective measure of distress, i.e. cortisol, was not related to perceived stress. A major drawback in our study is that we measured cortisol only once. Caplan et al. (Caplan et al., 1979), who studied the effect of perceived white collar workload on cortisol, also did not find a main effect of perceived workload on mean cortisol, but did find an effect of stress on the circadian rhythm of cortisol. Cortisol levels in the morning were significantly lower for employees with high work load. Although our measure of perceived stress is sensitive for more sources of stress than only work load, it would be interesting to study the possible effect of perceived stress on the circadian rhythm. This will be done in our ESM study were we have repeated measures of cortisol.

An important point is, that although the PSS correlates substantially with altemative measures of stress/distress. it assesses a different and independently predictive construct. Some overlap in the various scales is almost inevitable, but the PSS does not measure the same thing as for instance chronic difficulties or depressive symptomatology. The correlation between perceived stress and psychosomatic symptoms, controlling for depressive symptoms, remained significant.

A limitation in our study is that, besides cortisol, we did not have any objective measures of health (e.g. absenteeism at work, visits to health practitioner). Investigating the ability of the PSS to predict such outcomes would be a strong validation. We emphasize that the present correlational analyses do not warrant any inferences of causality. Although perceived stress may have (at least partly) caused both somatic and psychological symptoms, it is also possible that complainis elevated stress perceptions, or that a third factor (e.g. personality) influenced both stress and health. Another important point to be made is that stress scales should be chosen to address specific research questions. The choice for a specific scale will depend on the specific research design used in the study. This also means that the perceived stress scale is not always the appropriate scale. For instance, perceived stress scales should not be used as the only scale in cross-sectional studies of the relationship between stress and psychological distress (Cohen \& Williamson, 1988).

In conclusion, the PSS seems to be a valuable instrument for screening purposes. The scale has high internal reliability. stability, sensitivity and shown 
construct validity. Its subjective character maximizes sensitivity to ongoing stress. Its generality makes this scale less culture and population specific and of special value in populations were the stressful events are hard to pin down. Its short length is also an advantage when used concurrently with various other questionnaires or when used repeatedly over time in longitudinal studies. The PSS seems to be an adequate measure to assess which people are at risk for the development of stress related somatic and mental health problems.

\section{REFERENCES}

Ansseau, M., Sulon, J., Doumont, A., Cerfontaine, J. L., Legros, J. J., Sodoyez, J. C., \& Demey. Ponsart, E. (1984). The use of saliva cortisol in the dexamethasone suppression test. Psychiatry Research, 13, 201-211.

Averill, J. R. (1973). Personal control over aversive stimuli and its relationship to stress.

Psychological Bulletin, 80(4), 286-303.

Cantor, N., Norem, J., Langston, C., Zirkel, S., Fleeson, W., \& Cook-Flannagan, C. (1991). Life tasks and daily life experience. Journal of Personality, 59(3), 425-451.

Caplan, R. D., Cobb, S., \& French, J. R. P., Jr. (1979). White collar work load and cortisol: Disruption of a circadian rhythm by job stress? Journal of Psychosomatic Research, 23, 181. 192.

Cohen, S. (1978). Environmental load and the allocation of attention. In A. Baum, J. Singer, \& S. Valins (Eds.), Advances in environmental psychology. Hillsdale: Lawrence Erlbaum.

Cohen, S. (1986). Contrasting the Hassles Scale and the Perceived Stress Scale: Who's really measuring appraised stress? American Psychologist, 41, 716-718.

Cohen, S., Kamarck, T., \& Mermelstein, R. (1983). A global measure of perceived stress. Journal of Health and Social Behavior, 24, 385-396.

Cohen, S., Tyrrell, D. A. J., \& Smith, A. P. (1991). Psychological stress and susceptibility to the common cold. The New England Journal of Medicine, 325(9), 606-612.

Cohen, S., \& Williamson, G. M. (1988). Perceived stress in a probalitity sample of the United States. In S. Spacepan \& S. Oskamp (Eds.), The Social Psychology of Health (pp. 31-67). Newbury Park, CA: Sage Publications.

Dohrenwend, B. P., Link, B. G., Kern, R., Shrout, P. E., \& Markowitz, J. (1990). Measuring life events: The problem of variability within event categories. Stress Medicine, 6, 179-187.

Dohrenwend, B. P., \& Shrout, P. E. (1985). "Hassles" in the conceptualization and measurement of life stress variables. American Psychologist, 40, 780-785.

Dohrenwend, B. S., Dohrenwend, B. P., Dodson, M., \&. Shrout, P. E. (1984). Symptoms, hassles, social supports, and life events: Problems of confounded measures. Journal of Abnormal Psychology, 93(2), 222-230.

Folkman, S., \& Lazarus, R. S. (1985). If it changes it must be a process: Study of emotion and coping during three stages of a college examination. Journal of Personality and Social Psychology, 48, 150-170.

Hofer, M. A., Wolff, C. T., Friedman, S. B., \& Mason, J. W. (1972). A psychoendocrine study of bereavement PartI. 17-Hydroxycorticosteroid excretion rates of parents following death of their children from leukemia. Psychosomatic Medicine, 34(6), 481-504.

Jacobs, S. C., Mason, J., Kosten, T. R., Kasl, S. V., Ostfeld, A. M., \& Wahby, V. (1987). Urinary free cortisol and separation anxiety early in the course of bereavement and threatened loss. Biological Psychiatry, 22, 148-152. 
Kiecolt-Glaser, J. K., Ricker, D., George, J., Messick, G., Speicher, C. E., Garner, W., \& Glaser, R. (1984). Urinary cortisol levels, cellular immunocompetency, and loneliness in psychiatric inpatients. Psychosomatic Medicine, 46(1), 15-23.

Koeter, M. W. J., Ormel, J., \& van den Brink, W. (1988). Totaalscore op de SCL-90 als maat voof de ernst van psychopathologie. Nederlands Tijdschrift voor de Psychologie, 43, 381-391.

Lazarus, R. S. (1966). Psychological stress and the coping process. New York: McGraw-Hill.

Lazarus, R. S., De Longis, A., Folkman, S., \& Gruen, R. (1985). Stress and adaptational outcomes: The problem of confounded measures. American Psychologisi, 40, 770-779.

Lazarus, R. S., \& Launier, R. (1978). Stress-related transactions between person and environment. In L. A. Pervin \& M. Lewis (Eds.), Perspectives in Interactional Psychology (pp. 287-327). New York: Plenum.

Levenstein, S., Prantera, C., Varvo, V., Scribano, M. L., Berto, E., Luzi, C., \& Andreoli, A. (1993). Development of the perceived stress questionnaire: a new tool for psychosomatic research. Journal of Psychosomatic Research, 37(1), 19-32.

Mason, J. W. (1971). A re-evaluation of the concept of 'non-specificity' in stress theory. Journal of Psychiatric Research, 323-333.

Mason, J, W. (1975). A historical view of the stress field. Journal of Human Stress, 1(1), 7-12.

Miller, S. M. (1979). Controilability and human stress: Method, evidence and theory. Behavior Research and Therapy, 17, 287-305.

Schreurs, P. J. G., \& van de Willige, G. (1988), De Utrechtse Copinglijst (UCL): Lisse, the Netherlands: Swets \& Zeitlinger.

Seligman, M. E. P. (1975). Helplessness: On depression, development and death. San Francisco: Freeman. W.H.

Watson, D., \& Clark, L. A. (1992). Affects separable and inseparable: On the hierarchical arrangement of the negative affects. Journal of Personality and Social Psychology, 62(3), 489-505.

Watson, D., \& Pennebaker, J. W. (1989). Health complaints, stress, and distress: Exploring the central role of negative affectivity. Psychological Review, 96(2), 234-254. 


\section{Appendix II}

\section{ESM report}

\begin{tabular}{lccccccc}
\hline Op het moment van de piep: & niet & \multicolumn{3}{c}{ 'n beetje } & tamelijk & \multicolumn{2}{c}{ zeer } \\
Kon u zich concentreren ? & 1 & 2 & 3 & 4 & 5 & 6 & 7 \\
Piekerde u ? & 1 & 2 & 3 & 4 & 5 & 6 & 7 \\
& & & & & & & \\
Zo ja, waarover..........................................................................................................................................
\end{tabular}

\section{Voelde u zich op dat moment:}

$$
\text { niet 'n beetje tamelijk zeer }
$$

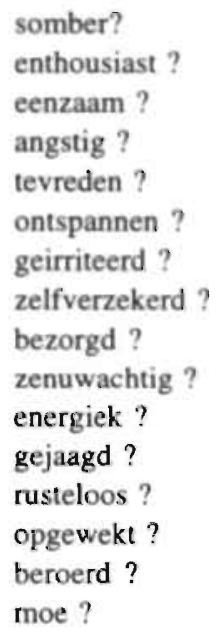

$\begin{array}{lllllll}1 & 2 & 3 & 4 & 5 & 6 & 7 \\ 1 & 2 & 3 & 4 & 5 & 6 & 7 \\ 1 & 2 & 3 & 4 & 5 & 6 & 7 \\ 1 & 2 & 3 & 4 & 5 & 6 & 7 \\ 1 & 2 & 3 & 4 & 5 & 6 & 7 \\ 1 & 2 & 3 & 4 & 5 & 6 & 7 \\ 1 & 2 & 3 & 4 & 5 & 6 & 7 \\ 1 & 2 & 3 & 4 & 5 & 6 & 7 \\ 1 & 2 & 3 & 4 & 5 & 6 & 7 \\ 1 & 2 & 3 & 4 & 5 & 6 & 7 \\ 1 & 2 & 3 & 4 & 5 & 6 & 7 \\ 1 & 2 & 3 & 4 & 5 & 6 & 7 \\ 1 & 2 & 3 & 4 & 5 & 6 & 7 \\ 1 & 2 & 3 & 4 & 5 & 6 & 7 \\ 1 & 2 & 3 & 4 & 5 & 6 & 7 \\ 1 & 2 & 3 & 4 & 5 & 6 & 7\end{array}$

\begin{tabular}{lcccccccc} 
Was uw belangrijkste klacht aanwezig ? & niet & & 'n beetje & tamelijk & \multicolumn{2}{c}{ zeer } \\
Had u last van andere klachten ? & 1 & 2 & 3 & 4 & 5 & 6 & 7 \\
& 1 & 2 & 3 & 4 & 5 & 6 & 7
\end{tabular}




\section{Wat deed u op het moment van de piep ?}

niet 'n beetie tameliik zeer

$\begin{array}{lllllllll}\text { Deed u het graag ? } & 1 & 2 & 3 & 4 & 5 & 6 & 7\end{array}$

$\begin{array}{llllllll}\text { Kostte het u moeite ? } & 1 & 2 & 3 & 4 & 5 & 6 & 7\end{array}$

$\begin{array}{llllllll}\text { Was deze aktiviteit voor u een uitdaging ? } 1 & 2 & 3 & 4 & 5 & 6 & 7\end{array}$

$\begin{array}{llllllll}\text { Kon } u \text { het? } & 1 & 2 & 3 & 4 & 5 & 6 & 7\end{array}$

$\begin{array}{lllllllll}\text { Vond } \mathrm{u} \text { deze aktiviteit belastend? } & 1 & 2 & 3 & 4 & 5 & 6 & 7\end{array}$

\section{Waar was u op het moment van de piep?}

Was u samen met anderen ?

Nee / Ja, met 1_1_1 mensen.

Naam

Wie is dat?

Wilde u nu graagalleen zijn ?

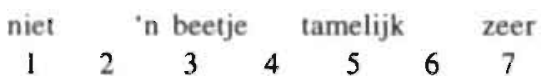

\section{Was er tussen deze en de vorize piep sprake van:}

$\begin{array}{lccccccc} & \text { niet } & \text { 'n beetje } & \text { tamelijk } & \text { zeer } \\ \text { een stressvolle gebeurtenis/situatie ? } & 1 & 2 & 3 & 4 & 5 & 6 & 7\end{array}$

Zo ja, geef een korte omschrijving:

Begintijd:

Eindtijd

Nog niet afgelopen $\mathrm{O}$

Was deze gebeurtenis:

- vervelend?

- verwacht?

- belangrijk voor $\mathrm{u}$ ?

K.on u het verloop ervan beinvloeden ? $\quad \begin{array}{llllllll}2 & 2 & 3 & 4 & 5 & 6 & 7\end{array}$

Heeft deze (of een soortgeliike) situatie zich vaker voor gedaan?






\begin{tabular}{lcccccccc} 
Had $\mathbf{u}$ in de tussentijd last van: & niet & & 'n beetje & \multicolumn{3}{c}{ tamelijk } & zeer \\
Uw belangrijkste klacht? & 1 & 2 & 3 & 4 & 5 & 6 & 7 \\
Andere klachten? & 1 & 2 & 3 & 4 & 5 & 6 & 7
\end{tabular}

Zo ja, welke?

\section{Gebruikte $u$ iets tussen deze en de vorige piep ?}
$\mathrm{O}$ niets
$O$ voedsel
O tabak
O mediciinen, n.I.
O koffie
O alcohol
1_1 glazen

$\begin{array}{lllllllll}\text { Wat was het hoogste niveau van aktiviteit? } & 1 & 2 & 3 & 4 & 5 & 6 & 7\end{array}$

voorbeelden: 1=rusten, 2 =zitten (actief), 3=lopen, $4=$ =stofzuigen, $S=f i e t s e n, 6=t e n n i s s e n, 7=r e n n e n$

Stoorde deze piep u ?



Hoe laat heeft u het speekselmonster genomen ? 


\section{Appendix III}

\section{Interrater agreement on codings for ESM measures}

\section{INTRODUCTION}

An appropriate measure of agreement for nominal data is the Kappa statistic ( $\mathrm{k}$ ) (Cohen, 1960). This statistic is an improvement on the simpler measure, per cent of agreement (Po), because it discounts the proportion of agreement which is expected by chance alone $(\mathrm{Pe})$. The formula for the (unweighted) $k a p p a$ is: $k=(\mathrm{Po}-\mathrm{Pe}) /(1-\mathrm{Pe})$. Because we are dealing with more than two categories we used the intra category kappa (agreement within a category). The 'overall Kappa' is a weighted average of the 'individual Kappa's' for all the alternative dichotomies which can be made by preserving one category and combining all others (Maclure \& Willett, 1987). Values for Kappa vary between 0 and 1.00. When Kappa has a value of 0 there is agreement as expected by chance alone. A Kappa of 1.00 means perfect agreement. We used the criteria given by Landis and Koch (Laundis \& Koch, 1977, p.265) for deciding if the Kappa we found was sufficiently high:

$\begin{array}{ll}\frac{\text { Kappa }}{<0.00} & \text { Degree of Agreement } \\ .00-.20 & \text { 'poor' } \\ .21-.40 & \text { 'flight' } \\ .41-.60 & \text { 'moir' } \\ .61-.80 & \text { 'substante' } \\ .81-1.00 & \text { 'almost perfect' }\end{array}$

\section{RESULTS}

The results on interrater agreement are presented in Table 1 and Table 2. First we will discuss the results for 'activities', 'location' and 'social context', and then for 'stressful events'.

\section{Interrater agreement for the activlty, locatlon and soclal context domains}

Reliability was determined for all the valid cases coded by the two raters. In every domain, the 'can't code' and the 'missing values' category were left out of analysis, because there were only a few observations in these categories: 'can't code' between 0 and 3 observations and 'missing values' between 6 and 14 observations. The fact that so few observations fell into the 'can't code' category means that the qualitative information was generally easy to interpret and to code into one of the available categories. We may conclude that the Kappa's we found were acceptably high (see Table 1). According to the criteria of Laundis and Koch ((Laundis \& Koch, 1977), p.265) the degree of agreement between raters is almost perfect, with all intra category Kappa's $\geq .89$, except for the activity category 'other' (Kappa .58) which had a moderate degree of agreement. So, the qualitative information was coded very reliably and seems easy to interpret. Also the fact that the various 'other' categories 
had so few observations gives support to the notion that the coding system we used was quite exhaustive.

Table 1. Interrater agreement for the activity, location and social context domains.

\begin{tabular}{|c|c|c|}
\hline & Total Observations & Kappa \\
\hline Activity ('what') & 2494 & .91 \\
\hline -leisure/social interaction & 722 & .90 \\
\hline -work & 709 & .95 \\
\hline -household/maintenance & 452 & .94 \\
\hline -transport & 252 & .96 \\
\hline -meals & 188 & .92 \\
\hline -inactivity/rest & 107 & .93 \\
\hline -other & 64 & .58 \\
\hline Location (Where') & 2491 & .94 \\
\hline -at home & 1185 & .97 \\
\hline -work & 788 & .96 \\
\hline -public places & 220 & .90 \\
\hline -transport & 189 & .92 \\
\hline -network & 72 & .91 \\
\hline -other & 37 & .70 \\
\hline Social Context ('Whol') & 2485 & .96 \\
\hline -household members & 964 & .99 \\
\hline -alone & 756 & .99 \\
\hline -colleagues & 546 & .97 \\
\hline -neighbours/acquaintances & 114 & .89 \\
\hline ofriends & 40 & .95 \\
\hline -non resident family & 36 & .89 \\
\hline -strangers & 29 & .90 \\
\hline
\end{tabular}

\section{Interrater agreement for stressful events}

The results for stressful events are presented in table 2. A total of 345 events were described by the 27 subjects. Interrater agreement was determined on all the completed subject responses. The context of events was rated with a high degree of agreement between the two raters. Kappa's varied between .60 and .96 . Only the 'other' category had a moderate degree of agreement, but this category was obviously also the most ambiguous one. It was also possible to use the event information to determine in a reliable way if someone was involved in the event and then who was involved in the event. The Kappa's lay between .76 and .96 . Most of the time no one was involved in the reported event. It was not possible to code in more than a moderately reliable way whether an event was 'external' or 'internal'. Only the 'internal' category had a substantially high Kappa (.76); the other categories had only a moderate degree of agreement. The 'can't code' category contained many observations $(21 \%)$ which suggests that it was very difficult for raters to interpret the qualitative information. Only the more straightforward internal events (e.g. worries, somatic complaints) were rated with a high reliability. The other categories can only be used with caution in further analyses. 
The intra category Kappa's in the social interaction domain were all satisfactory, varying from substantial to almost perfect, except for the can't code category for which the degree of agreement was only moderate. As shown in table 2 . a large number of observations could not be coded: $+10 \%$ of the total. This means that the various categories were not well defined, or that the information gathered was too vague. This second point seems to be more important here; often more detailed information was needed before a reliable judgment could be made. One way to improve on this is by paying more attention to the debriefing session where more information can be obtained on reported information. In the task demands domain, the categories 'problematic task' and 'failure at task' were aggregated into I category and the same accounts for the categories 'a lot of work' and 'time pressure'. This decision was based on the fact that the different subcategories were very interrelated. The Kappa's for the various categories ranged from moderate to almost perfect. Here again, a substantial percentage of responses could not be coded $(+22 \%)$.

Table 2. Interrater agreement for stressful events.






\section{REFERENCES}

Cohen, J. A. (1960). A coefficient of agreement for nominal scales. Educational and Psychological Measurement, 20, 37-46.

Laundis, J. R., \& Koch, G. G. (1977). The measurement of observer agreement for categorical data. Biometrics, 33, 396-398.

Maclure, M., \& Willett, W. C. (1987). Misinterpretation and misuse of the kappa statistic. American Journal of Epidemiology, 126(2), 161-169. 


\section{Summary}

During the last decades, there has been an increasing interest in the stressful aspects of contemporary society and their relationship to the development and maintenance of a wide range of somatic and psychological disorders. While earlier research focused on the impact of major life events or acute experimental stress, little is known about the nature of and the psychological and physiological responses to the minor but much more frequently occurring stresses of daily living. To increase our understanding of the stress process as it relates to health, the present thesis investigated the impact of minor daily events on mood and the HPA system. The aims of the research were 1) to describe the nature and scope of daily life stress in a group of white collar men and to contrast the experiences of individuals who perceive themselves to be stressed with those who do not, 2) to investigate affective and neuroendocrine (cortisol) responses in relation to stressful daily events, and 3) to investigate whether the cortisol responses to a laboratory stress task are generalizable to those occurring during stressful situations in real life. We also investigated whether more or less stable person characteristics like perceived stress level, trait anxiety, and depressive symptomatology were related to individual differences in responses to daily stress.

Chapter I presents an overview of psychological and physiological approaches to the concept of stress and discusses the role of psychosocial stress in health and disease. The existing literature on the impact of daily events on mood and cortisol is briefly reviewed. The following related issues are discussed: individual variability in mood and cortisol, the contexts in which daily events occur, appraisals of events, and the generalizability of laboratory-assessed stress reactivity to real life. Finally, the chapter presents the rationale for the study design and introduces the research questions.

Chapter 2 describes subjects, methods and procedures. Two groups of white collar men, with high versus low levels of perceived stress, were recruited as subjects. The Experience Sampling Method was used to collect data on stressful events and mood from subjects during their normal daily activities. Ten times a day for five consecutive days, subjects received auditory signals (beeps), after which they filled in a questionnaire and collected a saliva sample for cortisol determination. The same subjects also participated in a laboratory stress task, in which they were unexpectedly asked to deliver a speech. Stress responses were operationalized âs increases in cortisol secretion, increases in negative mood, and decreases in positive mood.

Chapter 3 describes quantitative as well as qualitative aspects of the reported stressful daily events. A total of 626 events were reported (on $17 \%$ of all ESM reports). Unpredictable and uncontrollable events were rated as the most unpleasant. Although the majority of stressful events were work-related, events were also frequently reported in a wide variety of life domains. High stress subjects scored high on trait anxiety and depressive symptoms, were bothered by various psychological and psychosomatic complaints, experienced relatively many chronic difficulties, and used more passive and less active coping styles than low stress subjects. High stress subjects reported twice as many stressful events, and they appraised events as more stressful and less controllable. They also reported twice as many work-related events and more events related to their social network. In particular, high stress subjects experienced more negative social interactions, both at work and at home, especially with their colleagues and their wives. 
Chapter 4 investigates the relationship between stressful events and negative and positive mood states, in particular examining the influence of perceived stress level on mood responses. Results showed that stressful events were associated with increases in negative mood. Positive mood only decreased when events were very unpleasant. Subjects scoring high on perceived stress showed significantly stronger negative mood reactivity in response to stressful events. Perceived stress did not influence the duration of the effects of events on mood. Events which involved performance demands and those appraised as more unpleasant, more novel, and less controllable were more likely to influence mood states. Results suggest increased vulnerability to daily events in individuals with high perceived stress.

Chapter 5 addresses the question of whether high perceived stress or related individual characteristics are associated with elevated cortisol or catecholamine levels. Analysis of cortisol values aggregated over each subject and time of day showed higher cortisol levels in high stress compared to low stress subjects when only the workdays were included. Trait anxiety, depressive symptomatology, and negative mood state were also associated with higher cortisol on workdays. Recent life events, chronic difficulties, trait anger, or psychosomatic symptoms, however, showed no relationship to cortisol levels. These results suggest that the kinds of mild chronic or intermittent stress in daily life situations reported during the ESM sampling period were sufficient to increase secretion of cortisol. In contrast, overnight catecholamine levels were unrelated to perceived stress, trait anxiety, depression, anger, psychosomatic symptoms or mood states.

Chapter 6 describes the estimated effects of perceived stress, personality traits, mood states, and stressful daily events on cortisol excretion. A random regression data analysis method revealed that trait anxiety and depression were associated with small but statistically significant cortisol elevations. Although results from the previous chapter had indicated a relationship between perceived stress and mean workday cortisol levels, the random regression analyses, which made use of the full dataset, did not replicate this finding; perceived stress was not associated with cortisol elevation. Cortisol levels were also no higher on workdays than on weekends in either subject group. Stressful daily events were indeed associated with increased cortisol secretion, the magnitude of the effect depending on whether the event was still ongoing and on how frequently a similar kind of event had occurred previously. Type of event or appraisal measures had no additional effects on cortisol. In addition, we found no significant individual differences in cortisol reactivity to events. Although perceived stress, anxiety, and depression did not increase cortisol reactivity to daily events, evidence was found for reduced habituation to recurrent events in subjects scoring high on these traits. Both negative mood states, Agitation and Negative Affect, were also associated with higher cortisol levels. Consistent with most views of the stress process, the effect of stressful events on cortisol appeared to be mediated to a large extent by associated increases in negative mood. The finding that even minor everyday events and fluctuations in mood states have an impact on cortisol secretion may point to a possible mechanism linking subjective experience to health outcomes.

Chapter 7 examines the generalizability of cortisol measures obtained in the laboratory to those obtained in real life. Results showed that the laboratory and reallife (ESM), cortisol levels were moderately correlated, but no association was found between laboratory and real-life stress response measures. Possible implications with respect to the predictive validity of laboratory stress reactivity for stress reactivity in general are discussed. 
In Chapter 8 the major findings of the study are summarized. Both methodological and theoretical issues related to the present research and to stress research in general are discussed, and results are evaluated in the context of possible health implications. Finally, several suggestions for future research are described. 



\section{Samenvatting}

De laatste decennia gaat steeds meer en meer aandacht uit naar de stressvolle elementen van onze huidige maatschappij en de negatieve gevolgen van deze elementen op het ontstaan en het in stand houden van allerlei somatische en psychische stoornissen. Terwijl vroeger onderzoek zich voornamelijk concentreerde op de gevolgen van belangrijke levensgebeurtenissen en acute experimentele stress, is er momenteel nog maar weinig bekend over de aard en de psychologische en fysiologische reacties op de kleine maar meer frequent voorkomende stressvolle gebeurtenissen in het dagelijkse leven. Ter vergroting van ons inzicht in de relatie tussen stress en gezondheid, bestudeert deze dissertatie de effecten vañ stressvolle dagelijkse gebeurtenis op de stemming en het hypofyse-bijnierschors systeem. De doelstellingen van het onderzoek zijn 1) het beschrijven van de aard en de omvang van dagelijkse stress in een groep van werkende mannen ('witte boorden') en het vergelijken van de ervaringen van individuen met een hoog ervaren stressniveau met die van individuen met een laag ervaren stressniveau, 2) het bestuderen van de affectieve en neuro-endocriene (cortisol) reacties op stressvolle dagelijkse gebeurtenissen, en 3 ) het onderzoeken in hoeverre de cortisol reacties op een laboratorium stresstaak corresponderen met de reacties op stressvolle gebeurtenissen in het dagelijkse leven. Tevens onderzochten we of er een relatie was tussen min of meer stabiele persoonskenmerken zoals ervaren stressniveau, angstdispositie en depressieve symptomatologie en individuele verschillen in reacties op dagelijkse. stress.

Hoofdstuk I geeft een overzicht van psychologische en fysiologische benaderingen van het begrip stress en evalueert de rol van psychosociale stress in gezondheid en ziekte. De beschikbare literatuur met betrekking tot de relatie tussen dagelijkse stress enerzijds en stemming en cortisol anderzijds wordt kort besproken, met speciale aandacht voor: individuele variatie in stemming en cortisol, de context waarin stressvolle gebeurtenissen plaatsvinden, de evaluatie (appraisal) van de stressvolle gebeurtenis en de generalisatie van stress-reactiviteit gemeten in het laboratorium naar het veld. Tenslotte wordt de rationale voor het onderzoeksdesign gepresenteerd en worden de onderzoeksvragen geintroduceerd.

In Hoofdstuk 2 worden de subjecten, methoden en procedures beschreven. Er werden twee groepen 'witte boorden' mannen gevormd: een met een hoog en een met een laag ervaren stressniveau. De Experience Sampling Methode werd gebruikt om gegevens van subjecten betreffende stressvolle gebeurtenissen en stemming te verzamelen op geselecteerde momenten tijdens hun normale dagelijkse activiteiten. Subjecten ontvingen tien keer per dag en gedurende vijf dagen signalen (beeps) via een voorgeprogrammeerd horloge, waarna ze een vragenlijst invulden en een speekselmonster namen, voor de cortisolbepaling. Dezelfde subjecten namen ook deel aan een laboratorium stresstaak, waarbij ze onverwacht gevraagd werden een speech te houden. Een stressreactie werd geoperationaliseerd als een stijging in cortisolsecretie, een toename in negatieve stemming en een afname in positieve stemming.

Hoofdstuk 3 beschrijft zowel kwantitatieve als kwalitatieve aspecten van de gerapporteerde stressvolle dagelijkse gebeurtenissen. In totaal werden er 626 gebeurtenissen gerapporteerd (op $17 \%$ van alle ESM beeps) en werden onvoorspelbare en oncontroleerbare gebeurtenissen het meest vervelend bevonden. Alhoewel de voornaamste bron van stress werk-gerelateerd was, kwamen stressvolle gebeurtenissen ook veelvuldig in verschillende andere levensdomeinen voor. 
Subjecten met een hoog stressniveau scoorden hoog op angstdispositie en depressieve symptomen, werden gehinderd door verschillende psychologische en psychosomatische klachten, ervaarden relatief veel langdurige moeilijkheden en gebruikten in het algemeen meer passieve en minder actieve coping stijlen dan subjecten met een laag stressniveau. Hoge stress subjecten rapporteerden twee keer zo veel dagelijkse gebeurtenissen als lage stress subjecten en ervaarden deze als meer stressvol en minder controleerbaar. Zij vermeldden tevens twee keer zoveel werk. problemen en meer problemen in relatie tot hun sociaal netwerk. In het bijzonder ervaarden hoge stress subjecten meer negatieve sociale interacties, zowel thuis als op het werk, en wel speciaal met hun collega's en echtgenotes.

Hoofdstuk 4 onderzoekt de relatie tussen stressvolle gebeurtenissen en negatieve en positieve stemming. Speciale aandacht ging uit naar de invloed van individuele verschillen in ervaren stressniveau op de stemmingsreacties. De resultaten laten zien dat stressvolle gebeurtenissen geassocieerd zijn met een toename in negatieve stemming. Positieve stemming nam alleen af wanneer de gebeurtenis zeer vervelend was. Subjecten met een hoog ervaren stressniveau vertoonden een significant sterkere toename in negatieve stemming als reactie op stressvolle gebeurtenissen. Het ervaren stressniveau had echter geen invloed op de duur van de effecten van stressvolle gebeurtenissen op stemming. Gebeurtenissen, die te maken hadden met de werkdruk of werklast en gebeurtenissen die als vervelender. onbekender en minder controleerbaar werden ervaren, hadden relatief meer invloed op de stemming. De resultaten lijken erop te wijzen dat individuen met een hoog ervaren stressniveau een verhoogde k.wetsbaarheid hebben in relatie tot stressvolle dagelijkse problemen.

Hoofdsıuk 5 is gewijd an de vraag of een hoog ervaren stressniveau of hiermee samenhangende persoonskenmerken geassocieerd zijn met verhoogde cortisol en/of catecholamineniveaus. Analyses van cortisolwaardes geaggregeerd over elk subject en de tijd van de dag heen laten een verhoogd cortisolniveau zien voor hoge stress subjecten in vergelijking met lage stress subjecten, echter alleen tijdens werkdagen. Angst dispositie, depressieve klachten, en negatieve stemming waren ook geassocieerd met hogere cortisolniveaus op werkdagen. Recente levensgebeurtenissen, chronische moeilijkheden, boosheidsdispositie en psychosomatische klachten vertoonde echter geen relatie met cortisol. De resultaten suggereren dat deze vormen van milde chronische of periodieke stress, ervaren in hett dagelijkse leven, in staat zijn de cortisolsecretie te verhogen. Echter, de catecholamineniveaus die werden gemeten gedurende de nacht waren niet geassocieerd met het ervaren stressniveau, angstdispositie, depressie. boosheidsdispositie, psychosomatische klachten, of stemming.

In Hoofdstuk 6 worden de geschatte effecten van het ervaren stressniveau, persoonskenmerken, stemming, en stressvolle gebeurtenissen op de cortisolsecretie beschreven. Een random regression data analyse methode liet zien dat angstdispositie en depressie gerelateerd waren met kleine maar significante verhogingen in. cortisolsecretie. Alhoewel de resultaten van het vorige hoofdstuk een positieve relatie tussen het ervaren stressniveau en het gemiddeld cortisolniveau op werkdagen aangaven, werden deze resultaten niet gerepliceerd wanneer via random regression. analyses gebruik gemaaki werd van alle gegevens; het ervaren stressniveau vertoonde geen relatie met cortisol. De cortisolniveaus waren tevens in beide groepen niet hoger op werkdagen dan in het weekend. De resultaten lieten verder zien dat stressvolle gebeurtenissen inderdaad geassocieerd waren met een verhoogde cortisolsecretie. De grootte van dit effect was afhankelijk van het feit of de stressor 
nog steeds aan de gang was op het moment van de meting en van de frequentie waarmee een gelijksoortige stressor zich reeds eerder had voorgedaan. Het type stressor of de evaluatie van de stressor had geen additioneel effect op cortisol. Tevens vonden we geen individuele verschillen in cortisol reactiviteit op stressvolle gebeurtenissen. Alhoewel het ervaren stressniveau, angstdispositie, en depressieve klachten de cortisol reactiviteit op stressvolle gebeurtenissen niet vergrootten, vonden we wel een verminderde habituatie aan stressoren die reeds herhaaldelijk eerder voorgekomen waren in subjecten die hoog scoorden op deze persoonskenmerken. Beide maten voor negatieve stemming (Agitatie en Negatief Affect) waren ook geassocieerd met hogere cortisolniveaus. In overeenstemming met de meeste opvattingen over het stressproces werd het effect van stressvolle gebeurtenissen op cortisol voornamelijk gemedieerd door de geassocieerde stijging in negatieve stemming. De bevinding dat zelfs kleine, dagelijkse stressoren en fluctuaties in stemming van invloed zijn op de cortisolsecretie wijst op een mogelijk mechanisme wat subjectieve ervaringen met gezondheid en ziekte verbindt.

Hoofdstuk 7 is voornamelijk gewijd aan de vraag of cortisolwaardes gemeten in het laboratorium generaliseerbaar zijn naar cortisolwaardes gemeten in het dagelijkse leven. De resultaten lieten zien dat de cortisolniveaus in het laboratorium en in het dagelijks leven redelijk met elkaar gecorreleerd waren, maar dat er geen relatie was tussen de reactiviteitsmaten in de experimentele setting en de reactiviteitsmaten in het dagelijks leven. De mogelijke implicaties van deze resultaten voor de predictieve validiteit van laboratorium stress-reactiviteit voor stressreactiviteit in het algemeen wordt besproken.

In Hoofdstuk 8 worden de belangrijkste resultaten van het onderzoek samengevat. Zowel methodologische als theoretische aspecten met betrekking tot deze dissertatie en met betrekking tot stress onderzoek in het algemeen worden bediscussieerd en de mogelijke implicaties van de resultaten voor de gezondheid worden nader bekeken. Het hoofdstuk wordt afgesloten met enkele suggesties voor vervolgonderzoek. 



\section{Dank}

Het uitvoeren van dit onderzoek was voor mij van tijd tot tijd vergelijkbaar met een fietstocht door het Limburgse landschap: al fietsend de heuvels op denk je "waar ben ik toch aan begonnen?", maar al snel bij het afdalen verandert deze gedachte in "ik ben blij dat ik het gedaan heb", totdat de volgende heuvel in zicht komt. Mijn dank gaat uit naar diegenen die ervoor gezorgd hebben dat ik in het heuvellandschap bleef en niet in de Alpen terechtkwam.

Daarmee bedoel ik op de eerste plaats de vele proefpersonen die zich bereid verklaarden om zich een kleine week lang door een horloge te laten achtervolgen en hun wel, wee en speeksel aan ons toe te vertrouwen. Zonder hen was dit boek er niet geweest. Op de tweede plaats bedoel ik Nancy Nicolson, mijn co-promotor. Nancy, ik dank je voor je grote betrokkenheid en steun bij het soms eenzame en lange traject van promotie-onderzoek. Met veel deskundigheid, geduld en optimisme voorzag je mijn diverse kladteksten van commentaar. Ik heb veel van je geleerd. Marten de Vries, mijn promotor, wil ik vooral bedanken voor alle vrijheid en vertrouwen die ik van hem kreeg, zowel tijdens als na mijn aanstelling als onderzoeker in opleiding.

Carolien Dijkman dank ik voor de efficiënte en fijne samenwerking tijdens de periode van dataverzameling. Op de vele vroege maandagochtenden was het een troost te weten dat ik niet als enige met de fiets op 'jacht' was naar (urine) monsters. Dr. Jose Sulon, Dr. Rahman en Dr. J. Duvivier ben ik zeer erkentelijk voor de bepalingen van respectievelijk de speeksel -en urinemonsters. De geweldige hulp van Hans Berkhof bij de vele gecompliceerde statistische analyses was onmisbaar. Zonder hem waren vele vraagstellingen onbeantwoord gebleven. Hans heeft laten zien dat een statisticus ook begrip voor de inhoud van de vraagstelling kan hebben, een zeldzaam verschijnsel. Professor Arthur Stone, tevens lid van de beoordelingscommissie, wil ik bedanken voor de motiverende discussies (zowel in Maastricht als in de US) en voor zijn komst naar Maastricht op 18 oktober.

Mijn collega's van de sectie Sociale Psychiatrie en Psychiatrische Epidemiologie wil ik bedanken voor hun morele ondersteuning maar vooral ook voor het gezellig geleuter tijdens de vele lunches en de fanatieke competities als er weer eens een nieuw computerspelletje was. In het bijzonder denk ik hierbij aan: Alex, Bart, Marie-Jose, Monique, Sarah, en Trees. Alhoewel ik 'de club' heb verlaten zal ik ze zeker nog lang missen. Bart Leunissen, mijn kamergenoot, ben ik speciaal erkentelijk voor het herhaaldelijk aanhoren van mijn onderzoeksfrustraties en het (meestal) stilzwijgend accepteren van de wanorde op mijn bureau('s). Bart, ik wens je meer succes bij de volgende kamergenoot. Philip Delespaul wil ik bedanken voor de fantastische hulp bij aspecten van de lay-out.

Mijn paranimfen Monique Pennings en Désirée Nijst bedank ik voor de hulp bij de organisatie van de 18-de. Monique: veel succes bij de 'vernieuwingen' in je leven, zowel privé als in de werksfeer. Desiree: "Hoe zit het nu met de bolderkar?"

Mijn moeder bedank ik voor de vrijheid en het vertrouwen die ze me altijd gegeven heeft om datgene te doen wat ik graag wou doen, om me vervolgens daarin dan ook te stimuleren. Last but not least: Rob. Bedankt voor je steun als ik weer eens met een pestbui thuiskwam. Het feit dat je (gelukkig) vaak niks begreep van mijn onderzoeks -en computer problemen, maakte dat ik de boel weer snel kon relativeren. Je motto "gewoon doorgaan" bracht me steeds weer een stapje verder. 



\section{Curriculum vitae}

Marleen van Eck werd geboren op 10 december 1965 te Wassenaar. In 1984 behaalde zij het VWO diploma aan het Griftland College te Soest. Na een verblijf van een jaar in het buitenland (Israël, Engeland), begon zij met de studie Gezondheidswetenschappen, afstudeerrichting Geestelijke Gezondheidkunde, aan de Rijksuniversiteit Limburg. Deze studie rondde zij in 1990 af. Aansluitend was zij als onderzoeker in opleiding verbonden aan de vakgroep Psychiatrie en Neuropsychologie, sectie Sociale Psychiatrie en Psychiatrische Epidemiologie, van de Rijksuniversiteit Limburg. Hier was zij werkzaam op een project dat gefinancierd werd door de Nederlandse Organisatie voor Wetenschappelijk Onderzoek (NWO), Deze dissertatie is een neerslag van dit werk. Tijdens dit dienstverband volgde zij tevens de opleiding tot gedragstherapeut. Na een tijdelijke aanstelling als toegevoegd onderzoeker bij dezelfde vakgroep is zij sinds 15 oktober 1995 werkzaam bij het RIAGG OZL te Heerlen, afdeling Psychotherapie. 


\section{mood,}

\section{cortisol}
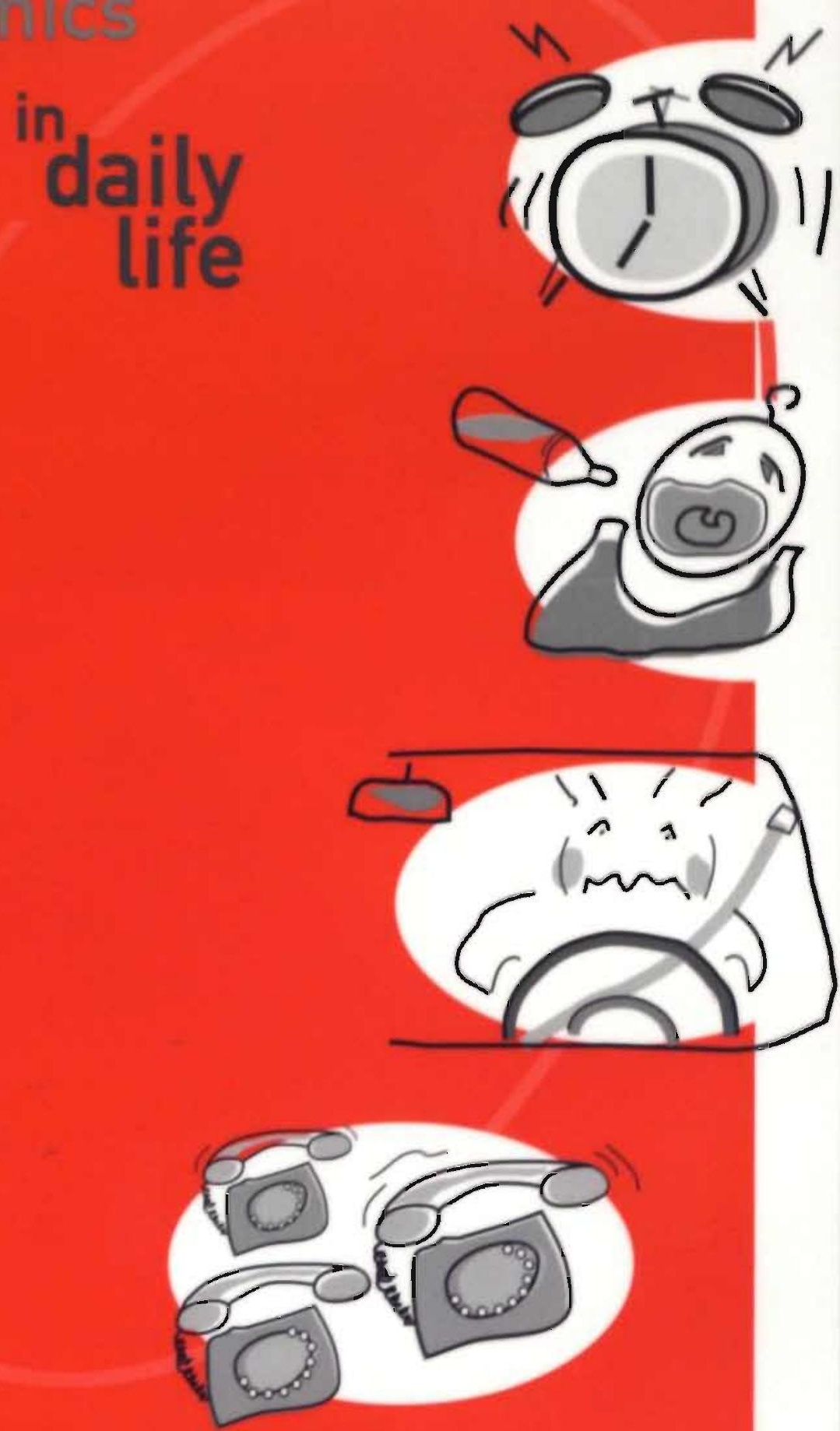

M. van Eck 
STRESS, MOOD, AND CORTISOL DYNAMICS IN DAILY LIFE 


\section{UPSCR}

\section{IPSER Series in Ecological Psychiatry}

M.W. deVries

\section{Editor}

$* * *$

\section{Vol.1 Assessing Schizophrenia in Daily Life}

- The Experience Sampling Method Philippe A.E.G. Delespaul

Vol.2 (Genetic) Epidemiology as a Tool to Identify Risk Factors for Emergence and Persistence of Illness in the Functional Psychosis Jim J. Van Os

Vol.3 Stress, Mood, and Cortisol Dynamics in Daily Life

Marleen M.M. Van Eck

The International Institute for Psycho-social and Socio-ecological Research (IPSER)

Collaborating Center of the World Federation of Mental Health Associated with the Rijksuniversiteit Limburg

Cover design: Mark. Delahay

Druk: Datawyse Maastricht

van Eck. Marleen

Stress, mood, and cortisol dynamics in daily life/Marleen yan Eck.

Thesis University of Limburg. Maastricht. - With ref.-

With summary in Dutch.

ISBN 90-9009783-X

Subject headings: perceived stress/experience sampling/cortisol/mood. 
Promotor

Prof. Dr. M.W. deVries

\section{Co-promotor}

Dr. N.A. Nicolson

\section{Beoordelingscommissie}

Prof. Dr. H.M. van Praag (voorzitter)

Prof. Dr. A.P.W.M. Appels

Prof. Dr. M.A. van den Hout

Prof. Dr. J.F. Orlebeke (Vrije Universiteit Amsterdam)

Prof. Dr. A.A. Stone (State University of New York)

Funding was provided by the Netherlands Organization for Scientific Research (NWO; grant 900-564-024) and the University of Limburg. 
It is not the large things that send a man to the madhouse... No, it's the continuing series of small tragedies that send a man to the madhouse Not the death of his love but a shoelace that snaps with no time left.

Charles Bukowski, 'The shoelace'. Bukowski Reads His Poetry, Takoma Records, Santa Monica, Calif., 1980. 

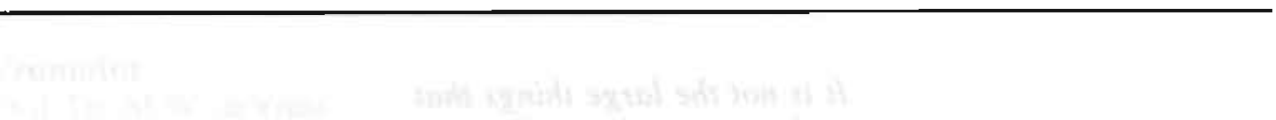

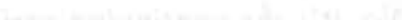




\section{Contents}

page

Chapter 1

Chapter 2

Chapter 3

Chapter 4

Chapter 5

Chapter 5.1

Chapter 5.2

Chapter 6

Chapter 7

Chapter 8

References

Appendices

Summary

Samenvatting

Dankwoord

Curriculum vitae

Introduction $\quad 3$

$\begin{array}{ll}\text { Methods } & 21\end{array}$

The nature of stress in daily life: events, appraisals, and activities

The effects of perceived stress and stressful daily events on mood states

Submitted for publication

Cortisol and catecholamine excretion in relation to perceived stress

Perceived stress and salivary cortisol in daily life Annals of Behavioral Medicine 16(3): 221-227 (1994) Four-teen hour urinary catecholamine excretion in relation to perceived stress

and stressful daily events on salivary cortisol

Psychosomatic Medicine (in press)

Individual differences in cortisol responses to a laboratory 127 speech task and their relationship to responses to stressful daily events

Biological Psychology (43): 09-84 (1996)

Concluding remarks 


\section{Chapter 1}

Introduction 


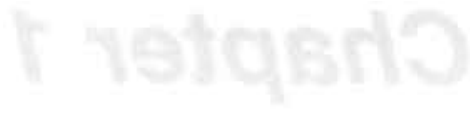

noilsuboyln 
Our lives can be characterized as constant adaptation to sudden or more gradual changes of our environment. Sometimes these changes are small and we can adapt to them without even noticing them, but at other times, these changes can be too frequent or severe and thereby threatening. In any case, at some point in life every human being will experience 'stress'; the day when inexplicably nothing seems to go right is a universal experience. In modern society, stress has become a popular concept and has gained a common everyday usage. We use it, for instance, to explain a wide variety of outcomes, mostly negative: it may serve to explain an upcoming headache or a bad mood, an upset stomach, 'nerves' or a lack of motivation. It is also common practice to attribute eccentric or incomprehensible behavior of friends and acquaintances to the fact that they are 'under a lot of stress'. Stress is even referred to as an 'epidemic disease".

One reason for the popularity of the stress concept may be its increasing linkage to disease and well-being. Research in the field of stress has indeed indicated that in addition to its role in the development and course of a wide range of somatic diseases (e.g. cardiovascular disease, infectious diseases, diabetes mellitus), psychosocial stress plays an important role in the etiology and maintenance of various psychiatric illnesses, including depression, anxiety, and psychosomatic disorders (Brown, 1993; Chrousos \& Gold, 1992; Paykel \& Dowlatshahi, 1988). During the last decade there has been an increase in the Netherlands in the number of mental disorders among those eligible for work disability benefits, as well as an increase in the duration of sickness absences because of mental disorders (Schroer, 1993). Of all disabled workers, more than $28 \%$ (227,000 individuals) received a benefit in 1990 after a mental disorder had been diagnosed; this in contrast to $21 \%$ in 1981. The total percentage of work disability due to mental disorder among civil servants increased over the same period from 35\% to 50\% (Knepper, 1991). In more than $80 \%$ of these cases, the mental disorder was described as "overstrain " (Schroer, Nijhuis, \& van Zutphen, 1988); in 40\% of mental disorder disabilities 'stress at work' was specifically mentioned. These figures are alarming not only for the individual (ex)worker, but also for the corporate community and society as a whole. Companies have to dea! with a considerable loss of work time, resulting in higher production costs and a pressure toward greater work productivity, which may lead again to an increase in absenteeism and disability due to a higher workioad. The costs for society due to stress-related absenteeism and disablement (including medication and health care utilization) have been estimated in 1988 at 9 billion Dutch guilders (Laan, 1989). In an effort to reduce feelings of stress and to increase physical fitness and improve mental health, fitness programs (Bruijn, 1988) and stress management courses (Marcelissen, 1989) at or near the work place have rapidly increased in popularity in the Netherlands, further encouraged by laws placing greater responsibility for the employee's health and well-being on the employer (ARBO-law). The main aims of introducing these programs at the work place are to increase the employees" physical health, thereby reducing absenteeism and work-related stress, and increasing work performance (Cox, Gotts, Boot, \& Kerr, 1988; Falkenberg, 1987).

Although the word stress seems to be implicitly understood by all, it is given substance by an infinite number of different things. For one person stress is having a fight with his or her spouse, for the other it is a busy day at work, and again for another stress may mean having nothing to do. Earlier research emphasized the impact of major disruptions in personal and social life (e.g. death of spouse, unemployment) (Holmes \& Rahe, 1967), but more recent studies, have alerted us to the fact that the minor but much more frequent occurring hassles of daily living also 
play a role in both somatic and psychological health outcomes (DeLongis, Coyne. Dakof, Folkman, \& Lazarus, 1982). These minor but often chronic stresses at work, in the family, and in other social relationships are also much closer to the lay person's concept of stress, as described above, and to problems that may lead to absenteeism and disablement at the work place. Although responses to acute experimental stress have been studied extensively, little is known about the nature and effects of the stresses of daily living. Knowledge about the nature of psychological and physiological responses to daily life stress is possibly of great theoretical importance for an understanding of the etiology and course of both physical and psychological pathology, and is the object of the present thesis. The first goal of the study is to describe the nature and scope of daily life stress, contrasting the experiences of individuals who perceive themselves to be stressed with those who do not. Second, this research investigates affective and neuroendocrine responses in relation to stressful daily events and tries to explain individual differences in such responses. Before explicitly going into the major research questions and how they will be addressed, we will provide a brief overview of various theoretical approaches to stress, followed by a discussion of the stress-illness relationship. Next, the available evidence concerning the influence of daily stress on psychological and physiological functioning will be discussed.

\section{THE CONCEPT OF STRESS: PSYCHOLOGICAL VIEWS}

Although the term 'stress' has been widely used since the beginning of this century (Cannon, 1929), controversy concerning its definition remains. Three distinct definitions of stress can be found in the literature, which vary in their emphasis on stimulus, response or intervening process and which are closely related to ways in which stress can be measured: in terms of a stimulus or a situation (e.g. noise, death of a spouse, mathematics task), in terms of a behavioral or physiological response (e.g. performance decrements, increased heart rate, or elevated cortisol), and stress as a state of imbalance. when the (perceived) demands on the person tax or exceed his (perceived) capabilities to deal with these demands (Goldberger \& Breznitz, 1982). These definitions also vary in their emphasis on physiological/endocrinological versus psychological/behavioral processes. For the sake of simplicity, the various approaches to the concept of stress will be divided below into psychological and physiological stress traditions, starting with the psychological tradition.

\section{Life change approach}

In psychology and medicine, the term stress has traditionally been used to refer to a stimulus or situation which produce certain (behavioral, psychological, and/or physiologicai) responses (Hinkle. 1974; Wolff \& Goodell, 1968). Since the development of a method for the quantification of major life changes by Holmes and Rahe (the Social Readjustment Rating Scale [SRRS]; (Holmes \& Rahe, 1967)), the association between stress and disease has been extensively investigated. The hypothesis guiding the Holnes-Rahe approach was that an increased number of life changes requiring considerable adjustment would precede the onset of illness. This implies that life events are, in and of themselves, the precipitating cause of illness. The SRRS self-report checklist allows the respondent to indicate which events (both positive and negative, such as divorce, birth or death of a close family member, loss of job) were experienced within a specified time period. To assess the significance of 
these events, life change units were obtained by having different groups of subjects indicate the amount of readjustment (independent of the desirability or emotions induced by the event) they thought would be required. The item 'marriage' was used as anchor point in these ratings. In this way, the total number of events or a summation of the life change units could be obtained and then related to measures of illness and health. Since its development, the SRRS has been used in numerous studies to investigate the association between life changes and illness, and life changes have, for instance, been related to sudden cardiac death (Rahe \& Arthur, 1978; Rahe \& Lind, 1971). In general, life events have been shown to be related to various somatic disorders, including heart disease, sudden cardiac death, and infectious diseases (Holmes \& Masuda, 1974), and to various psychiatric disorders, including acute schizophrenia (Brown, 1974) and depression (Paykel, 1974).

\section{Criticism of the life event approach}

The life event approach, in which stressors are basically treated as objective environmental stimuli, has received considerable criticism (Aagaard, 1984; Rabkin \& Streuning, 1976; Schroeder \& Costa, 1984). Some points of criticism are: only low to modest correlations have been found between life event scores and health outcomes: the exclusive use of the category of major life events as a synonym of stress and the use of 'social readjustment' as the sole dimension to define stress; psychometric problems including reliability and content validity; inclusion of many items which may have been confounded with the outcome measures studied; and the use of retrospective study designs, which could lead to an exaggeration of the importance attributed to past events from a need to justify subsequent illness.

Later life event checklists (e.g. the Life Experience Checklist (Sarason, Johnson \& Siegel, 1978) and the PERI lists of life events (Dohrenwend, Krassnoff, Askenasy, \& Dohrenwend, 1978)) addressed some of these issues. Both negative and positive life change scores were included to be able to investigate whether positive and negative life events exhibit the same relationship with physical and emotional outcomes. Also included were additional subjective evaluations of the life events, like desirability, impact, controllability, and predictability. The basic objections, however, remained: retrospective research design, limited and predetermined sample of life events, too little consideration of the subjective evaluation of the event or the ongoing relationship between person and environment. One exception is the laborintensive and time-consuming semi-structured interview (the Bedford Life Events and Difficulties Scale [LEDS] (Brown, 1974)) method developed by Brown and colleagues (Brown \& Harris, 1989) for the measurement of life events and chronic difficulties. Here, in addition to the use of objective criteria for what can be considered a life event or enduring problem, this approach incorporates the influence of psychosocial factors (the biographical context) on the subjective experience of events.

\section{Transactional theory of stress, appraisal and coping}

The transactional stress theory developed by Lazarus and colleagues (Lazarus, 1966) places particular emphasis on the importance of an individual's subjective evaluation ('appraisal') of stressful events. In this now very widely accepted approach to the concept of stress, psychosocial stress is defined as "a relationship between the person and the environment that is appraised by the person as taxing or exceeding his or her resources and endangering his or her well-being"' (Lazarus \& Folkman, 1984b, p. 19). In the present study we will use the term 'stress' 
in this context. This approach states that whether a state of stress develops depends both on external conditions in the environment as well as on the constitutional vulnerability and the adaptive capacity of the person. It therefore may explain why identical events do not lead to stress perceptions in all persons, since differences in personality, social support, prior experience with the stressor, and so on, may effect the person's ability to deal with the stressor. Central to the transactional approach are two processes that act as mediators between the behavior of the person and the environment: first, the subjective evaluation or appraisal of potentially stressful events or situations, and second, the process of coping that follows after an event has been appraised as stressful.

The concept of appraisal refers to three distinct types of appraisal: primary appraisal, secondary appraisal, and reappraisal. Primary appraisal refers to the significance of the situation for a person's well-being ('What is at stake?'). Three broad and basic categories of evaluations of well-being can be distinguished (Lazarus \& Launier, 1978): 'irrelevant', meaning that the situation has no subjective relevance and can therefore be ignored, 'benign-positive' when the person regards an event as signifying a positive state of affairs, and 'harmful-stressful'. Three types of stressful appraisals are mentioned: 'harm/loss', which refers to harm already done, 'threat', which refers to a future potential for harm or loss, and 'challenge', which is defined as a potential for growth or positive gain. Stress can thus have both positive and negative effects. These categories of stressful appraisals do not have to be mutually exclusive, a loss, for example, can at the same time entail a future threat. Secondary appraisal refers to the evaluation of the coping recourses and options available to deal with a stressful transaction ('What can I do about it?'). Consequently, it follows that secondary appraisal determines to a large extent whether one feels threatened as opposed to challenged, and it will shape the coping strategies of the person under stress. Reappraisal points to the feedback process from the ongoing interaction between the person and the environment, which may lead to changes in primary and secondary appraisals.

The concept of coping refers to "the process of managing external and/or internal demands that tax or exceed the resources of the person" (Lazarus \& Launier, 1978. p. 311). Two broad categories of coping nechanisms or strategies have been distinguished, each serving a major adaptive function: problem-focused coping is directed at actively modifying the self or the stressful situation, while emotionfocused coping is directed at managing feelings of distress. Both are used in almost every stressful situation. Coping and appraisal mutually influence each other throughout a stressful encounter, since if the person-environment relation changes as a result of coping efforts the evaluation of the situation will change as well.

Individual differences in stress, appraisal, and coping responses are determined at least in part by an individual's adaptive capacity at the physiological, psychological, and social level (Lazarus \& Folkman, 1984b). Examples at the physiological level are genetic or constitutional factors like physical fitness and genetically determined or acquired immunity. At the psychological level, there are personal goals and commitments, and personality characteristics like self-esteem. neuroticism, and sense of control. At the social level, important antecedent variables are social economic status, social support networks, and sociocultural norms and values. Stressful events or situations can be described with respect to both immediate and long-ternn effects. Short-term effects at the physiological level include somatic or physiological changes and, in extreme cases, acute illness. Effects at the psychological level include positive or negative emotions, and at the social level they 
may entail social disturbances or group alienation. The general categories of longterm outcomes are: physical health (e.g. chronic illness, impaired physiological functioning); subjective well-being (e.g. depression, anxiety, other psychological symptoms); and social functioning (e.g. socoal failure, change in social role). All levels as described ahove are assumed to he interrelated.

\section{Criticism of the transactional approach}

According to the transactional model, stress is best regarded as a complex rubric, a convenient term to refer to the operation of many variables and processes in situations in which the demands tax or exceed the person's resources. This complexity and diversity of aspects related to the transactional definition of stress forces one to make choices about which classes of variables to study. Lazarus and Folkman's definition of stress has also received its share of criticism. The main point of critique has been that it is overly subjective, in that both stress and coping resources are defend and measured in terms of appraisal processes (Dohrenwend \& Shrout, 1985). The choice between obtaining subjective or relatively objective indices of stress is an important one and should depend on the purposes of the study. If the goal is to investigate the etiologic role of life circumstances as precursors of pathology, one should try to reduce the subjectivity of event measures to reduce possible confounding of the stress-pathology relationship. Even then, knowledge about the context of the event, about the circumstances surrounding the event, may still be important for an understanding of why the disorder developed. If not prediction of pathology but understanding the process of stress per se is the main aim. then the subjective experience of stress is worth studying in and of itself (Wagner, 1990). Although a certain degree of circularity (the same process is being measured in the independent and the dependent variables) is inevitable with relational definitions of stress, this can be limited by asking what it is about the person, in interaciion with a given environmental situation, that generates appraisals of harm, threat, or benefit (Lazarus, De Longis, Folkman, \& Gruen, 1985).

\section{THE CONCEPT OF STRESS: PHYSIOLOGICAL VIEWS}

Since most psychological or behavioral signs of stress are often of a subjective nature (such as subjective self-reports of negative affect, low job-satisfaction, anxiety, or agitation), a substantial body of stress research has focused on more objective indicators of stress. Characteristic of both humans and animals is that they respond to stressful events with various well-defined and quantifiable physiological responses, such as the release of stress hormones in the blood, increased heart rate and blood pressure. Moreover, repeated or chronic physiological activation is held to be an important factor in the link between stress and disease. Therefore, we will provide a brief review of the physiological stress tradition.

\section{Short history of early studies}

Early stress research in animals emphasized that responses of the organism to a wide variety of stimuli were non-specific. Selye (1936, p. 32) defined stress as "the nonspecific response of the body to any noxious stimulus". This nonspecific response to stimuli such as heat, cold, or exercise, has been called the General Adaptation Syndrome; it is characterized by increased adreno-cortical activity, degeneration of the thymus and lymphatic structures; and hemorrhage and ulceration of the stomach 
and other parts of the gastrointestinal tract. Three temporal phases can be distinguished: the alarm phase, the stage of resistance, and the stage of exhaustion. According to the theory, conditioning factors (e.g. age, genetic predisposition, diet, drugs) will determine which specific disease of adaptation will manifest itself after prolonged exposure to a stressor. The pituitary-adrenal-cortical axis regulates the release of corticosteroids from the adrenal cortex, with cortisol as the most important glucocorticoid homone in humans, and with the potential of exerting its effects on practically all normal body cells and tissues. Some of the main functions of cortisol are: stimulation of gluconeogenesis, inhibition of glucose uptake, suppression of inflammation and suppression of numerous immune functions (Munck, Guyre, \& Holbrook, 1984). Whereas Selye especially emphasized the activity of the pituitaryadrenal-cortical axis, Cannon (1929) investigated the role of the sympathetic-adrenalmedullary system, which regulates secretion of the catecholamines adrenaline and noradrenaline. Cannon showed that besides physical stimuli, non-physical or emotional stimuli could also activate the physiological system. He formulated the concept of 'physiological homeostasis', which means that those physiological responses will be triggered which meet the demands of the environment. The increased activity of the central nervous system (CNS) and release of catecholamines were interpreted as an emergency' reaction. In this way, the organism is prepared for appropriate action aimed at coping with the stressor and restoring control: "fight or flight'. Enhanced sympathetic activity leads to increases in glucose, respiratory rate, blood pressure, and heart rate, and to a blood flow away from the intestines to the muscles, where oxygen and nutrients are most needed. It was Mason (1968) who pointed out, after reviewing earlier studies on animals, healthy humans, and psychiatric patients, that many of the stressful experiences that had been investigated shared one common characteristic: the induction of emotional arousal. For instance, when an animal is exposed to a novel or unfamiliar environment, it is the psychological relevance of the stressor that determines the stress response and not the particular physical stressor. Important conditions for eliciting responses were found to be novelty, uncertainty, unpredictability, involvement, and anticipation of unpleasant experiences. The importance of emotional arousal as a trigger of the stress response and the fact that those specific responses are triggered that meet the demands of the environment are not compatible with a non-specific stress response as formulated in Selye's theory; the physiological stress response is therefore best regarded as a differentiated response of the organism.

Another important finding was that marked individual differences exist $1 \mathrm{n}$ the adrenal-cortical response to a given stressful situation (Mason, 1968; Rose, 1984). These differences seem to be related to individual coping styles or defense mechanisms and to how the event is appraised. Rose (1984) noted that cortisol responses extinguish rapidly in individuals upon re-exposure to most kind of events, reflecting adaptation.

\section{Recent physiological stress models}

More recent models have integrated the major physiological concepts described above. In the animal model proposed by Henry and Stephens (1977) it was suggested that the nature of behavioral and accompanying physiological reactions are determined by the outcome of the appraisal process, which in turn results in different coping strategies. Two extremes can be differentiated with respect to behavioral, physiological, and CNS activities. When the appraisal process results in fear or anxiety but the animal still tries to eliminate the threat by active effortful 
coping, this will lead to sympathetic activation, for example a pronounced increase in adrenaline release and heart rate. This resembles the 'fight/flight' response described by Cannon (1929), with territorial control (mobility, display, aggression) as behavioral components. However, when the appraisal results in uncertainty or loss of control and the animal sees no escape or has no adequate coping response available, the hypothalamus-pituitary-adrenocortical axis is activated to release ACTH and cortisol, as was originally described by Selye. At the behavioral level this response is accompanied by inhibition (freezing or doing nothing), subordination, and decreased sexual and maternal behavior ('conservation-withdrawal'). Frankenhaeuser's effortdistress model (1983), based on studies of healthy humans (e.g. Frankenhaeuser, Lundberg, \& Forsman, 1980; Lundberg \& Frankenhaeuser, 1980), is consistent with the model of Henry and Stephens (1977). Both focus on two components of psychological arousal, effort and distress (experienced either singly or in combination), which seem to be differentially related to catecholamine and cortisol secretion. A state of 'effort without distress' (a positive affective state) is accompanied by catecholamine secretion, while under this condition cortisol secretion is low or may be actively suppressed. On the other hand, a state of 'effort with distress' (which may characterize many stressful events) is associated with an increased secretion of both catecholamines and cortisol. An example of this state is mental work carried out under conditions of stimulus overload. The state of 'distress without effort', which may be reflected in giving up or feelings of helplessness, is accompanied by an increase in cortisol secretion, and resembles the "conservationwithdrawal' response as described by the model of Henry and Stephens. Personal control seems to be an important modulating factor here, with less control leading to more distress. In summary, cortisol, in comparison to other stress-labile hormones (e.g. catecholamines, growth hormone, prolactin, testosterone), appears to show the most specific relationship to subjective distress as opposed to effort or general arousal (e.g. Lundberg \& Frankenhaeuser, 1980), and is thought to reflect the adequacy of coping behavior (e.g. Vickers, 1988).

\section{STRESS, HEALTH, AND DISEASE}

As described above, in most cases the psychological and physiological responses to stress reflect normal, adaptive processes which prepare the body to cope with the situation. Negative emotions alert people to deal with or avoid difficuli situations, and physiological reactions prepare the body for 'fight or flight' responses (Chrousos \& Gold, 1992). It has also been noted that stress does not always lead to negative effects like poor performance or illness, but may result in training or toughening effects as well (Dienstbier, 1989; Ursin, 1980). Thus in the normal course of events, when coping is successful and the stressful experience is of short duration, the benefits largely outweigh the costs of the stress response. Only when behavioral or cognitive coping strategies for dealing with stressful experiences are not effective or not available and stress endures are psychological and physiological responses likely to become exaggerated or prolonged, thereby increasing the risk for pathology to develop. In humans only extreme stressors will evoke responses like fighting or fleeing, while the majority of events experienced in daily life ask for more subtle solutions. Since rapid availability of oxygen, nutrients, glucose, and so on is not necessary to meet the demands of contemporary daily challenges, disruption of bodily homeostasis may occur. 
question in this regard is whether individual differences in laboratory reactivity reflect those occurring during stressful situation in real-life (Turner, Ward, Gellman, Johnston, Light, \& van Doornen, 1994). The usefulness of laboratory assessments rests, in part, on this assumption. Later in this chapter we will deal with these subjects in more detail.

\section{FROM MAJOR LIFE EVENTS TO MINOR DAILY EVENTS}

As already described previously, the major life event approach has received considerable criticism relating to aspects of both design and method. A basic objection has been the reliance on retrospective research designs. The retrospective assessment of summaries of life events over many months obscures the temporal, dynamic interplay between environmental demands, appraisals of demands, and outcomes (Stone \& Shiffman, 1992), which is central in the transactional theory of stress and coping. More details of the stress process are needed to unravel the mechanisms underlying the relationship between events and disease. The dynamic processes between demands, appraisals, and outcomes are presumed to change so quickly that they can only be captured through frequent assessment. Of course, the frequency of measurement should follow the fluctuations of the phenomena under study; fluctuations in heart rate would ask for minute to minute assessments over periods less than a day, while if we were interested in the relationship between stress and premenstrual symptoms, daily measurements over several months would be more appropriate.

Another disadvantage of retrospective research designs is that the recall of events over relatively long periods may be influenced by biases. Recall of events and mood over long time periods is obviously limited by forgetting, but research suggests that this is not a random process. For instance, it has been shown that recall of past mood and both positive as well as negative events is strongly influenced by the respondent's mood at the time of recall (Bower, 1981; Teasdale \& Forgarty, 1979); the current mood state makes mood-congruent memories more accessible and moodincongruent memories less accessible. Although this applies especialily to global ratings of past experiences, it is also likely that recall of specific events may be affected; emotionally important or salient events are more likely to be remembered than less salient ones (Strongman \& Russell, 1986). By reducing the recall time interval, these biases can be also reduced.

Taken together, these points have lead to the conclusion that other categories of events, such as chronic stressors and the nnilder but much more frequent stresses of daily living, should also be investigated concerning their relationship to health. Since the events of everyday life eccur musch more frequently than the major life events, they might be even more important to well-being. The investigation of minor daily events may also help to delineate the stressful features of chronic experiences, such as work stress. Indeed, an increasing number of studies have shown that daily life events are related to lower psychological well-being and increased somatic symptomatology, even after the possible confounding effect of major life events has been controlled for (e.g. DeLongis et al., 1982; Stone, Neale, \& Shiffman, 1993; Zarski, 1984). Daily events or 'hassles' are defined as "the experiences and conditions of daily living that have been appraised as salient and harmful or threatening to the endorser's wellbeing" (Lazarus, 1984, p.376). Examples of situations that can be hassles are: getting caught in a traffic jam, having to wailt, quarreling with your child. There are several 
question in this regard is whether individual differences in laboratory reactivity reflect those occurring during stressful situation in real-life (Turner, Ward, Gellman, Johnston, Light, \& van Doornen, 1994). The usefulness of laboratory assessments rests, in part, on this assumption. Later in this chapter we will deal with these subjects in more detail.

\section{FROM MAJOR LIFE EVENTS TO MINOR DAILY EVENTS}

As already described previously, the major life event approach has received considerable criticism relating to aspects of both design and method. A basic objection has been the reliance on retrospective research designs. The retrospective assessment of summaries of life events over many months obscures the temporal, dynamic interplay between environmental demands, appraisals of demands, and outcomes (Stone \& Shiffman, 1992), which is central in the transactional theory of stress and coping. More details of the stress process are needed to unravel the mechanisms underlying the relationship between events and disease. The dynamic processes between demands, appraisals, and outcomes are presumed to change so quickly that they can only be captured through frequent assessment. Of course, the frequency of measurement should follow the fluctuations of the phenomena under study; fluctuations in heart rate would ask for minute to minute assessments over periods less than a day, while if we were interested in the relationship between stress and premenstrual symptoms, daily measurements over several months would be more appropriate.

Another disadvantage of retrospective research designs is that the recall of events over relatively long periods may be influenced by biases. Recall of events and mood over long time periods is obviously limited by forgetting, but research suggests that this is not a random process. For instance, it has been shown that recall of past mood and both positive as well as negative events is strongly influenced by the respondent's mood at the time of recall (Bower, 1981; Teasdale \& Forgarty, 1979); the current mood state makes mood-congruent memories more accessible and moodincongruent memories less accessible. Although this applies especialily to global ratings of past experiences, it is also likely that recall of specific events may be affected; emotionally important or salient events are more likely to be remembered than less salient ones (Strongman \& Russell, 1986). By reducing the recall time interval, these biases can be also reduced.

Taken together, these points have lead to the conclusion that other categories of events, such as chronic stressors and the nnilder but much more frequent stresses of daily living, should also be investigated concerning their relationship to health. Since the events of everyday life eccur musch more frequently than the major life events, they might be even more important to well-being. The investigation of minor daily events may also help to delineate the stressful features of chronic experiences, such as work stress. Indeed, an increasing number of studies have shown that daily life events are related to lower psychological well-being and increased somatic symptomatology, even after the possible confounding effect of major life events has been controlled for (e.g. DeLongis et al., 1982; Stone, Neale, \& Shiffman, 1993; Zarski, 1984). Daily events or 'hassles' are defined as "the experiences and conditions of daily living that have been appraised as salient and harmful or threatening to the endorser's wellbeing" (Lazarus, 1984, p.376). Examples of situations that can be hassles are: getting caught in a traffic jam, having to wailt, quarreling with your child. There are several 
forms of daily life stress. Some stressors are chance events, and usually rare in occurrence (e.g. flat tire when in a hurry, out of toilet paper just when needed, unexpected phone calls), while others are repeated, either because a person remains in the same ongoing social situation (e.g. work, marriage) with consistent demands (e.g. high work load, conflicts with spouse), or because of certain personality dispositions, such as a person's ineffective style of coping with common situations (Kanner, Coyne, Schaefer, \& Lazarus, 1981). Since no one lives a life completely without stress, the impact of hassles on physical and mental health is thought to depend on factors such as a chronically high frequency of stressful daily events, an increase in hassles during a given period (the 'last straw'), the presence of hassles with compelling psychological importance, or an underlying (biological) vulnerability to stress or to a specific illness (Kanner et al., 1981; Zautra, Guarnaccia, Reich, \& Dohrenwend, 1988).

The current study investigates the impact of minor daily events on mood and the HPA system. In the next two sections we present a review of the available literature concerning the relationship between daily events, on the one hand, and mood and cortisol responses, on the other hand. The following related subjects will be of special interest: individual differences in mood and cortisol responses, the context of the event, and event appraisa!s.

\section{STRESSFUL DAILY EVENTS AND MOOD STATES}

A common approach to the study of daily stress examines the relationship between stressful events and mood by assessing both once a day for several weeks (Stone \& Shiffman, 1992); others have assessed these phenomena on a monthly basis (e.g. Lewinsohn \& Libet, 1972). The simplest approach is open-ended: subjects are asked to describe the day's most stressful events (Rehm, 1978) or asked to describe anything that went wrong during the day (Caspi, Bolger, \& Eckenrode, 1987; Eckenrode, 1984). Responses are then classified into discrete categories. The most often used method for assessing daily events is the event checklist, where, from a list of events, subjects are asked to identify which events have occurred over a specified time period, ranging from one day to one month. Examples of monthly checklists are the Unpleasant Events Schedule and the Pleasant Events Schedule (Lewinsohn \& Amenson. 1978), the Hassles and Uplifts scale (Kanner et al., 1981), and the Inventory of Small Life Events (Zautra \& Guarnaccia, 1986). Items on these checklists were formulated by the researchers or taken from existing scales and constructed to cover events in major life domains, such as family, work, leisure, and household maintenance. Examples of daily checklists are the Daily Life Experience checklist (Stone \& Neale, 1982), the Daily Stress Inventory (Brantley, Waggoner, Jones, \& Rappaport, 1987), and the Daily Stress Scale (Bolger, DeLongis, Kessler, \& Schiling, 1989a). Here. by using an open-ended format. a more representative domain of events was first obtained by sampling events which conform to certain criteria (e.g. 'stressfulness', (Brantley et al., 1987)) from the participant population in which the checklist was to be used. Elicited events were then grouped into various categories to form the checklist.

Regardless of what approach has been used to study daily stress, several studies have shown a same-day association between daily stress and negative mood (Affleck, Tennen, Urrows, \& Higgins, 1994; Bolger et al., 1989a; Clark \& Watson, 1988; DeLongis, Folkman, \& Lazarus, 1988; Eckenrode, 1984; Larsen, Diener, \& Emmons, 1986; Lewinsohn \& Libet, 1972; Rehm, 1978; Stone \& Neale, 1984; Stone et 
al., 1993). Results with respect to positive mood are inconclusive; most studies have not differentiated between negative and positive mood, but in those that have, positive mood was either lower (Neale, Hooley, Jandorf, \& Stone, 1987; Repetti. 1993; Stone \& Neale, 1984) or unchanged (Watson, 1988) on days when many stressful events occurred.

Evidence for large individual differences in the magnitude and the direction of the association between daily stress and mood has been found in a number of studies. Examples of factors related to such differences are self-esteem (Campbell, Chew, \& Scratchley, 1991), social support (Affleck et al., 1994; Barling \& Kryl, 1990; Caspi et al., 1987; DeLongis et al., 1988), major life events (Affleck et al., 1994; Caspi et al., 1987), and neuroticism (Affleck et al., 1994; Bolger \& Schilling, 1991; Eckenrode, 1984). The moderating role of chronic stress or long-term difficulties on the relationship between daily life stress and mood has received relatively little attention. In a study by Caspi et al. (Caspi et al., 1987), the chronic stress of living in a low quality neighborhood increased the immediate effects of stressful daily events on mood and also increased the likelihood that daily stressors had an enduring effect on next day's mood. Investigating the influence of perceived stress levels on the daily stress-mood relationship may be important, since individuals experiencing high perceived stress feel their lives to be unpredictable, uncontrollable, and overloaded, and can be seen as at risk for the development of stress-related somatic and mental health problems (Cohen, Kamarck, \& Mermelstein, 1983). Personality factors probably play a very important role in perceived stress. Self-report measures of stress have been found to correlate significantly with measures of negative affectivity or neuroticism (Watson, 1988; Watson \& Pennebaker, 1989). Negative affectivity reflects a general disposition to focus on the negative aspects of self and others and to experience events as negative or distressing (Eysenck \& Eysenk, 1968; Watson \& Clark, 1984). In stress research, negative affectivity has been treated in two different ways. First, it is thought of as a confounding variable, spuriously inflating the relationship among stressors, stress symptoms, and affective outcomes (Chen \& Spector, 1991: Costa \& McCrae, 1987; Schaubroeck, Ganster, \& Fox, 1992; Watson \& Pennebaker, 1989). However, negative affectivity has also been investigated as a moderator variable. influencing individuals' reactivity to stressful events. Several studies demonstrated that individuals higher in negative affect show greater negative mood reactivity to stressors (Affleck et al., 1994; Bolger \& Schilling, 1991; Marco \& Suls, 1993). Differences between high and low negative affect individuals in how events are appraised or coped with may possibly account for these differences in mood reactivity.

Little is known about how long the effects of daily events on mood persist. although this would be important for understanding the consequences of minor events. Heaith chances may only occur when daily events are very frequent and negative mood responses persist in time, for instance while coping has been unsuccessful or incomplete. The available literature from end-of-day studies suggests that daily stressors affect same-day mood but do not necessarily affect mood on subsequent days (Bolger et al., 1989a; DeLongis et al., 1988; Lewinsohn \& Libet, 1972; Neale et al., 1987; Rehm, 1978; Stone \& Neale, 1984), but more research is necessary to clarify this subject.

Since people experience a large range of stressful events in daily life, several studies have been employed to try to increase our understanding of what categories of events and which event characteristics are most strongly related to well-being. Several studies indeed seem to indicate that the psychological impact of daily events 
depends on the context in which they occur. Particularly undesirable work events and interpersonal conflicts appear to have the strongest relationship to negative mood states (Bolger et al., 1989a; Bolger \& Schilling, 1991; Clark \& Watson, 1988; Repetti, 1993; Stone, 1987). Work events have also been associated with lower positive mood (Stone, 1987). Despite the important role of the concept of appraisal in contemporary stress theory in determining whether a daily event is experienced as stressful or not, little research has been done in relation to appraisals of naturally occurring daily events. There is evidence from numerous laboratory studies and several naturalistic studies that subjects' personal ratings of events, like frequency, demand, controllability, and predictability of events, improve prediction of outcomes such as performance, anxiety, depression, negative affect, and tension (Averill, 1973; Cohen, 1980; Dewe, 1991; Folkman, 1984; Katz \& Wykes, 1985; McGrath \& Beehr, 1990; Miller, 1979; Peeters, Buunk, \& Schaufeli, 1995; Sarason et al., 1978; Thompson, 1981). Additional research is needed that studies cognitive appraisals of daily events in a natural environment and investigates how these appraisal dimensions relate to mood.

While end-of-day assessments of daily hassles have important advantages compared to the major life event approach, several drawbacks still remain; these will be discussed in the final section of this chapter.

\section{STRESSFUL DAILY EVENTS AND CORTISOL DYNAMICS}

Until recently, the two main approaches to the investigation of the impact of stressful events on the HPA axis in humans have been laboratory reactivity studies and 'natural experiments'. While both approaches have been of great value for our understanding of the stress process, each has limitations. We will see that neither approach is adequate if one adopts the dynamic conceptualization of stress as a process, which changes over time and in relation to the environment.

Most studies of psychophysiological reactivity have used standardized laboratory tasks such as physical strain or exercise, mental stress tasks (e.g. reactiontime tasks, arithmetics, computer games), cold pressor tests, and mood induction, all with questionable relevance to real life (Berger, Bossert, Krieg, Dirlich, Ettmeier, Schreiber, et al., 1987; Brown, Sirota, Niaura, \& Engebretson, 1993; Bullinger. Naber, Pickar, Cohen, Kalin, Pert, et al., 1984; Forsman \& Lundberg. 1982; Mason, Hartley, Kotchen, Mougey, Ricketts, \& Jones, 1973). Ecological valid information about the sources of stress people experience in their daily lives, and the responses to it, seem difficult to obtain in laboratory experiments (Lazarus \& Launier, 1978). One can question whether the responses to such tasks are relevant or valid reflections of habitual reactivity to naturally occurring stressors - in other words, to what extent does laboratory-assessed reactivity generalize to the field? With regard to hormonal reactivity, little is known about the generalizability of laboratory studies to field studies. Results from cardiovascular studies indicate quite strong associations between chronic levels of cardiovascular activity across settings, but little support has been found for the relationship between laboratory reactivity and field reactivity (Turner et al., 1994). Moreover, it is impossible, from a practical and ethical point of view, to generate in the laboratory the stressful events and responses of the type and severity found in real-life (van. Doornen \& Turner, 1992). Although experimental control and the possibility of isolating and manipulating variables have important advantages and may be the optimal strategy for the evaluation of certain aspects of 
stress theory, it seems more important at this stage to investigate and try to describe what is happening in everyday life with regard to stress processes. Some have tried to increase ecological validity by mimicking daily life situations in the laboratory: for example public speaking (Bassett, Marshall, \& Spillane, 1987; Kirschbaum \& Hellhammer, 1993), or stress inducing films (Hubert \& de Jong-Meyer, 1989).

Natural experiments take advantage of the occurrence of real-life, potentially stressful events, of either an acute or of a more chronic nature. With regard to acute stressors, increases in cortisol excretion were, for example, found in situations such as forcing non-swimmers to jump in the deep-end of a swimming pool (Vaernes, Ursin, Darragh, \& Lambe, 1982), an anticipated surgical procedure (Ben-Aryeh, Roll, Kahana, \& al., 1985; Knight, Atkins, Eagle, Evans, Finkelstein, Fukushima, et al., 1979), academic examinations (Allen, Batty, Dodd, Herbert, Hugh, Moore, et al., 1985; Jones, Copolev, \& Outsch, 1986; Nicolson, 1992), and parachute (Schedlowski, Jacobs, Stratmann, Richter, Hadicke, Tewes, et al., 1993; Ursin, 1978) or bungee jumping (Hennig, Laschefski, \& Opper, 1994). Some examples of the kind of chronic stressors studied in the field are living near the damaged power plant at Three Mile Island (Schaeffer \& Baum, 1984), the period of bereavement after the loss of a child (Hofer, Wolff, Friedman, \& Mason, 1972a; Hofer, Wolff, Friedman, \& Mason, 1972b), enforced captivity (Dekaris, Sabioncello, Mazuran, Rabatic, Svoboda-Beusan, Racunica-, et al., 1993; Rahe, Karson, Howard, Rubin. \& Poland, 1990), combal exposure or related post-traumatic stress disorder (Bourne, Rose, \& Mason, 1967; Piman \& Orr, 1990), and work stress (e.g. Arnetz, Brenner, Levi, Hjelm. Petterson, Wasserman, et al., 1991: Caplan, Cobb, \& French, 1979; Coeck, Jorens, Vandevivere, \& Mahler, 1991; Lundberg \& Paim. 1989; Rose \& Fogg, 1993; Theorell, 1989; Timio \& Gentili, 1976). The effects of chronic or intermittent stress has received far less attention in the literature than the effects of acute stress, and data. on cortisol levels during prolonged stress have: been inconsistent, with enhanced (Arnetz et al., 1991; Coeck et al., 1991; Hofer et al., 1972a; Rahe et al., 1990; Rose \& Fogg, 1993; Schaeffer \& Baum, 1984; Timio \& Gentili, 1976) as well as decreased (Bourne et al., 1967; Caplan et al., 1979; Dekaris et al., 1993: Pitman \& Orr, 1990) concentrations reporled, and large variability among individuals.

A major drawback of the natural experiment is that, since the researcher generally has to be present in the field to collect cortisol measures, only anticipated events can be investigated. Additionally, mosi of the studies have examined stress under rather extreme or unusual situations, approaching major life events in terms of severity. Only few studies have investigated whether less severe but much more frequent daily events also have an impact on cortisol secretion, and fundings have been inconsistent, with reports of lowered (Caplan et al., 1979), elevated (Brantley. Dietz, McKnight, Jones, \& 'Tulley, 1988; Lundberg, Granqvist, Hansson, Magnusson, \& Wallin, 1989), and unchanged (Cummins \& Gevirtz, 19913) cortisoll levels.

Since individua! characteristics may influence how stressfu! situations are appraised they are thought to be important determinants of the emotional response to a given situation; mood states are, in turn, likely to mediate the endocrine response to the situation. Available information on the relationship between personality uaits, mood states, and event characteristics on the one hand and on cortisol levels on the other hand has until now been based on the traditional approaches discussed above. Several personality characteristics (e.g. trait anxiety, depression, anger, coping style, personality) have been associated with basal cortisol levels (Brandtstädter. BaltesGotz, Kirschbaum, \& Hellhammer, 1991; Pope \& Smith, 1991; Schaeffer \& Baum, 1984), as well as with cortisol responses, to stress (Bohnen, Nicolson, Sulon, \& Jolles, 
1991; Demyttenaere, Nijs, Evers-Kiebooms, \& Koninckx, 1989; Kirschbaum, Hellhammer, Strasburger, Tiling, Kamp, \& Luddecke, 1989; Nicolson, van Poll, \& deVries, 1992). Other studies, however, have found no association between personality traits, coping styles, and cortisol laboratory baseline levels or responsiveness to laboratory stressors (Bosserts, Berger, Krieg. Schreiber, Junker, \& von Zerssen, 1988; Kirschbaum, Bartussek, \& Strasburger, 1992a). Although there is abundant evidence that cortisol increases in response to distress or negative mood (Arnetz et al., 1991; Lundberg \& Frankenhaeuser, 1980; Mason, 1968), the effects of positive mood are less clear. Positive affective states, have been associated with decreases (Hubert \& de Jong-Meyer, 1989; Kugler \& Kalveram, 1989) as well as increases (Brown et al., 1993) in cortisol levels. Several laboratory studies have investigated the differential effects of various event appraisals (e.g. novelty, predictability, controllability) on cortisol (Mason, 1968). To our knowledge, this has not been done with regard to daily stress yet. In addition, there is no research that investigated whether different types of daily events have a different impact on cortisol. Since the context of an event has been found to relate to the magnitude of its psychological impact, this would be an interesting research question.

A major disadvantage of the studies described above is that often only a small or a few cortisol sample were obtained per subject or per day. In the study by Rose and Fogg (Rose \& Fogg, 1993), where repeated measurements of cortisol responses to work stress in air traffic controllers were obtained, a subgroup of subjects was found to respond to an increase in workload with large increases in cortisol. Repeated measurement of cortisol not only increases reliability, but also allows the investigation of dynamic relationships between stressful daily experiences and neuroendocrine activity. Additional research is necessary to see how the results obtained so far relate to those obtained with repeated measurements designs in the context of real life, and with daily events as the focus of interest.

\section{PRESENT STUDY: AIMS AND RESEARCH QUESTIONS}

Due to the hypothesized role of appraisal as a mediator between the environment and the person in determining the occurrence of a stressful experience and the responses to it, stress should be measured in a context were intrinsically motivating events and emotional involvement are most likely to happen; the natural environment of the subject seems to be the ideal research setting. Here, individuals can be observed in their normal social networks, settings, and activities, and stressful events can be studied in the life setting where they actually occur. Additionally, since stress is currently conceptualized as a dynamic process, which changes over time and in relation to the environment, it is necessary to include repeated measurements of stress, hormone levels, and emotional states to investigate the relationship between stress and affective and neuroendocrine responses (Dimsdale, 1984). The advantages of the repeated measurement approach can be further enhanced by simultaneously collecting information on individual traits, event characteristics and cognitive appraisals, which can then be investigated for their effect on mood and cortisol excretion.

Compared to the major life event approach, end-of-day assessments of minor stressors have important advantages, but even within a day, much of the dynamic interplay between events, appraisals of events, and outcomes remain hidden. Recall biases of events and mood are still likely to occur, and same-day associations between 
daily events and mood remain causally ambiguous. With this we mean that not only daily stress may have an effect on mood, but that a bad mood may increase the likelihood that daily events will occur or will be perceived as stressful.

In the present study, two relatively new approaches were employed to investigate the relationship between stress, affective states, and cortisol: i.e. the Experience Sampling Method (ESM) and ambulatory monitoring of salivary cortisol. The Experience Sampling Method has been specifically designed to study the subjective everyday experiences of people in their natural environments (Csikszentmihalyi \& Larson, 1987; de Vries, 1992). Typically, an electronic signaling device is used to alert subjects to fill in a self-report questionnaire at preselected but randomized time-points, providing information about an individual's mental status or symptoms within the context and flow of experience. When, how often, and for how long subjects are signaled depends on the goal of the study. The prospective assessment procedure is an important characteristic of ESM; since the variables of interest are assessed at frequent intervals during the day and close to the moment at which they actually occur, we are able to look at more dynamic stress processes, with a minimum of confounding due to biased recall or forgetting (Bower, 1981). ESM therefore also provides better estimates of the frequency, distribution, and intensity of psychological variables than cross-sectional designs (Larson \& Csikszentmihalyi. 1983).

The choice of cortisol as a physiological stress indicator was based on both theoretical and practical grounds. From a theoretical point of view, cortisol is a sensitive indicator of the stressfulness and adequacy of psychological responses to person-environment transactions, an important regulator of vital physiological processes, and a possible mediator of physical responses leading to disease. From a practical viewpoint, the opportunity of measuring cortisol reliably in saliva instead of blood makes repeated, stress-free sampling in real-life contexts possible (Kirschbaum \& Hellhammer, 1994; Nicolson et al., 1992). Saliva samples can be reliably collected and stored by subjects themselves, enabling them to carry out their daily routine without undue interference. Although our main emphasis was on cortisol, 14-hour urinary catecholamine levels were also determined twice, as possible indicators of long-term or more chronic stress.

The present study design compares two groups of male white collar workers. with high versus low levels of perceived stress, monitored during their normal daily activities. Subjects completed ESM self-reports concerning activities, mood states, and recent stressful events, and collected saliva samples for cortisol determination in response to signals emitted by a preprogrammed watch ten times a day for five consecutive days. Our study focused on four main topics: (1) the nature and experience of stress in daily life; (2) the relationship between perceived stress, stressful daily events and mood states; (3) the relationship between perceived stress. stressful daily events and cortisol levels; and (4) the generalizability of stress responses from the laboratory to real life. As outlined below, each of these topics will be addressed in separate chapters.

Following a description of the subjects and methods in Chapter 2, Chapter 3 is devoted to the description of quantitative as well as qualitative aspects of stressful daily events. Various questions are addressed, including: How frequently are stressful events experienced? What kind of events are experienced as stressful in daily life? Do stressful experiences differ with respect to frequency, kind, and appraisal in subjects with a high versus low level of perceived stress? How do major life events and chronic difficulties relate to the pattern of stressful daily events? 
Chapter 4 examines the influence of daily events on both negative and positive mood states. In addition to the immediate effects of daily stress on mood, we also examine temporal patterns of the effects of events on mood states. We examine whether average mood levels and mood responses to daily events were related to individual differences in perceived stress level. Finally, various kinds of events (work demands, negative social interactions) and event appraisals (e.g. unpleasantness, predictability, controllability) are investigated for their possible differential effects on mood states.

In Chapter 5 we address the question whether high perceived stress is associated with elevated cortisol levels. We also investigate the extent to which individual characteristics (trait anxiety, depression, anger, psychosomatic complaints, mood states, number of stressful events) contribute to differences in cortisol levels. Lastly, we examine the relationship between perceived stress level and urinary catecholamine excretion following work versus weekend days.

Chapter 6 focuses on the possible impact of minor daily events on cortisol secretion. We also examine the association between an individual's affective state and cortisol changes in response to such events. Finally, we test whether perceived stress and other individual characteristics are related to cortisol reactivity to stressful events.

Chapter 7 describes emotional and cortisol responses to a stress-inducing speech task in the same group of subjects. Beyond assessing the relationship between individual trait characteristics, current mood states, and cortisol responses to the stress task, a main objective of the laboratory experiment was to explore the consistency between laboratory and field cortisol measures, particularly with regard to the usefulness of laboratory measures in predicting cortisol levels and response to stressful events in the field. To this end, cortisol levels and responses measured in the laboratory were compared with those measured in real life.

Chapter 8 summarizes the main research findings and attempts to place them in a larger perspective. In addition it discusses limitations of the study and possible future research directions. 
Chapter 2

Methods 

The research questions presented in Chapter 1 necessitated a diversity of methodological approaches, including questionnaires, the Experience Sampling Method, monitoring of hormone levels in saliva and urine, and an experimental stress task. Chapter 2 describes first the subject selection procedure and demographic characteristics of the sample. Next, the measures and data collection procedures are described in detail. Methodological issues involved in the analysis of ESM data are discussed, including the coding of ESM responses, compliance, and assessment of possible biases and / or experimental effects of ESM procedures.

\section{SUBJECTS}

The sample consisted of 92 male white collar workers who had been first screened and then selected from six local industries and government agencies (see 'procedure' section), based on their perceived stress scores in the upper or lower tertiles of the screening sample distribution (PSS score $<10$ or 216). Subjects who reported a history of serious chronic illness, endocrine disorder, medications known to affect cortisol levels, or treatment (past or current) for mental health problems were excluded. During subject intake, each high stress subject was matched for age group, marital status, and household composition with a low stress subject to insure that the two groups did not differ on demographic characteristics that might affect exposure to certain classes of stressors.

Table 2.1. Demographic data for 'High' and 'Low' stress subjects.

\begin{tabular}{cc}
\hline Low Stress Subjects & High Stress Subjects \\
$\mathrm{N}=46$ & $\mathrm{~N}=42$ \\
\hline
\end{tabular}

Age

mean

s.d.

range
42.7

7.7

27.57
41.5

6.0

28-52

\section{Marital status}

$\begin{array}{lrr}\text { married } & 41(89.1 \%) & 37(88.1 \%) \\ \text { unmarried } & 2(4.3 \%) & 2(4.8 \%) \\ \text { living together } & 2(4.3 \%) & 2(4.8 \%) \\ \text { divorced } & 1(2.2 \%) & 1(2.4 \%)\end{array}$

\section{Household composition}

living alone

couple with children

couple without children
3 (6.5\%)

$37(80.4 \%)$

$6(13.0 \%)$
$3(7.1 \%)$

$34(81.0 \%)$

$5(11.9 \%)$

'High' and 'low' perceived stress groups were defined as follows: the mean of the first and the second PSS assessment was used to categorize subjects as above or below the screening sample median score (12). Four subjects (two from each group). 
One week before the field study, subjects received a second questionnaire battery to be filled in at home and returned at the briefing session. The questionnaires concerned psychosocial stress (including the PSS again), personality and psychological symptoms (depression, anger) (see next section).

\section{CROSS-SECTIONAL INSTRUMENTS}

An overview is presented of the questionnaires used. The questionnaires administered during the screening phase of the study will be described first (Questionnaire I), followed by the questionnaires completed the week before ESM took place (Questionnaire II). Questionnaires which have not been widely used will be described more thoroughly than more familiar ones.

\section{Questionnaire I}

Demographic information: A demographic questionnaire requesıed information concerning respondent's age, marital status, type of household, number of children (for the matching procedure), chronic diseases and medication use (possible exclusion criteria), and alcohol use, smoking habits, and participation in active sports (possible confounders of hormone concentrations).

Perceived Stress: Subjects were selected according to their perceived stress level. Perceived stress was measured by means of the Perceived Stress Scale (PSS: Cohen et al., 1983). The PSS is a global measure of the degree to which situations in one's life are appraised as stressful. ltems were designed to tap the extent to which individuals feel their life to be unpredictable, uncontrollable, and overloaded. These themes are important components of the experience of stress (Averill, 1973; Cohen, 1978; Seligman, 1975). The PSS measures cognitions and emotions relating to general stress levels rather than specific events or situations (see Table 2.2.). The items are rated on a 5-point frequency scale, ranging from 'never' $(0)$ to 'very often' (4). A total score is obtained by reversing the scoring on the positive items and then summing across the 10 items.

Table 2.2. Items Perceived Stress Scale.

1. In the last month, how often have you been upset because of something that happened unexpectedly?

2. In the last month, how often have you felt that you were unable to control the important things in your life?

3. In the last month, how often have you felt nervous and 'stressed'?

4. In the last month, how often have you felt confident about your ability to handle your personal problems?

5. In the last month, how often have you felt that things were going your way?

6. In the last month, how often have you found that you could not cope with all the things that you had to do?

7. In the last month, how often have you been able to control irritations in your life?

8. In the last month, how often have you felt that you were on top of things?

9. In the last month, how often have you been angered because of things that happened that were outside of your control?

10. In the last month, how often have you felt difficulties were piling up so high that you could not overcome them? 
One week before the field study, subjects received a second questionnaire battery to be filled in at home and returned at the briefing session. The questionnaires concerned psychosocial stress (including the PSS again), personality and psychological symptoms (depression, anger) (see next section).

\section{CROSS-SECTIONAL INSTRUMENTS}

An overview is presented of the questionnaires used. The questionnaires administered during the screening phase of the study will be described first (Questionnaire I), followed by the questionnaires completed the week before ESM took place (Questionnaire II). Questionnaires which have not been widely used will be described more thoroughly than more familiar ones.

\section{Questionnaire I}

Demographic information: A demographic questionnaire requesıed information concerning respondent's age, marital status, type of household, number of children (for the matching procedure), chronic diseases and medication use (possible exclusion criteria), and alcohol use, smoking habits, and participation in active sports (possible confounders of hormone concentrations).

Perceived Stress: Subjects were selected according to their perceived stress level. Perceived stress was measured by means of the Perceived Stress Scale (PSS: Cohen et al., 1983). The PSS is a global measure of the degree to which situations in one's life are appraised as stressful. ltems were designed to tap the extent to which individuals feel their life to be unpredictable, uncontrollable, and overloaded. These themes are important components of the experience of stress (Averill, 1973; Cohen, 1978; Seligman, 1975). The PSS measures cognitions and emotions relating to general stress levels rather than specific events or situations (see Table 2.2.). The items are rated on a 5-point frequency scale, ranging from 'never' $(0)$ to 'very often' (4). A total score is obtained by reversing the scoring on the positive items and then summing across the 10 items.

Table 2.2. Items Perceived Stress Scale.

1. In the last month, how often have you been upset because of something that happened unexpectedly?

2. In the last month, how often have you felt that you were unable to control the important things in your life?

3. In the last month, how often have you felt nervous and 'stressed'?

4. In the last month, how often have you felt confident about your ability to handle your personal problems?

5. In the last month, how often have you felt that things were going your way?

6. In the last month, how often have you found that you could not cope with all the things that you had to do?

7. In the last month, how often have you been able to control irritations in your life?

8. In the last month, how often have you felt that you were on top of things?

9. In the last month, how often have you been angered because of things that happened that were outside of your control?

10. In the last month, how often have you felt difficulties were piling up so high that you could not overcome them? 
We used the 10 item version of the scale (PSS10) because it has been shown to have as good or better psychometric properties than the original 14 item version (Cohen \& Williamson, 1988). Research by Cohen and others showed acceptable levels of validity and reliability. See Appendix I for the reliability and validity of the PSS in the present sample.

Coping: Coping conceptualized as a fairly stable characteristic ("coping style') was assessed with the 47-item Utrecht Coping List (UCL), developed for the Dutch population by' Schreurs et al. (Schreurs \& van de Willige, 1988). The seven factor-analytically derived subscales are: 'active problem solving', 'palliative responding', 'avoidance', 'seeking social support', 'depressive reaction', 'expression of emotions', and. 'comforting cognitions'.

Psychological and somatic complaints: The Symptom Checklist (SCL-90; Derogatis, Lipman, \& Cori, 1973) is a 90-item self-report questionnaire developed for measuring psychopathology in ambulatory psychiatric patients, but also in community samples cq non-patients. The questionnaire was adapted and translated into Dutch by Arrindell and Ettema (1981; 1986) and measures, on a five-point scale, both somatic and psychological complaints experienced during the last week. The instrument consists of eight subscales: (1) sleeping problems, (2) hostility, (3) depression, (4) somatic complaints, (5) distrust and interpersonal sensitivity, (6) insufficiency of thought and performance, (7) agoraphobia, and (8) anxiety. The SCL90 total score can be interpreted as an indication of the general level of psychological and/or somatic dysfunctioning during the past week. Reliability and validity data for the Dutch SCL-90 have been reported by Arrindell and Ettema (1986) and by Koeter et al. (1988).

Psychosomatic symptoms: The revised version of the SUNYA Psychosomatic Symptom Checklist (PSC; Attanasio, Andrasik, Blanchard, \& Arena, 1984) contains 17 common psychosomatic complaints, lie headaches, backaches and nausea. Subjects rate each item on frequency $(O=$ not, $4=$ occurs daily) and intensity $(0=$ not a problem, 4 = extremely bothersome) of occurrence, using a five point scale. A Total. score, reflecting the overall level of psychosomatic distress, is obtained by summing the cross-products of each item's frequency by intensity. Factor analyses of PSC data. obtained from college students revealed one general psychosomatic distress factor with little overlap with other commonly used measures of psychological distress such as the Beck depression Inventory or the State-Trait Anxiety Inventory (Attanasio et: al., 1984).

\section{Questionnaire II}

Life events: Life events were recorded with the questionnaire form of the List of Threatening Experiences (LTE.Q: Brugha, Bebbington, Tennant, \& Hurry, 1985). Subjects were asked about the occurrence of 12 categories of events (e.g. death of a partner, child, parent: got divorced, unemployed) during the last year. This list of event categories originated from a large series of life events sampled in a UK community epidemiological survey and in psychiatric outpatients with affective disorders (Bebbington, Tennant, \& Hurry, 1981). Each event in the original list was. rated for long-term contextual threat, according to the methods of Brown and Harris (1978). Reliability and validity data for the LTE-Q have been reported by Brugha and Conroy (1985) and Brugha and Cragg (1990).

Long-rerm Difficulties: Chronic stress was assessed with the Long-term Difficulties Questionnaire (LLM; Hendriks, Ormel, \& van de Willige, 1990; Ormel, 
1985). This inventory is a Dutch adaptation of the Biographical Problem Inventory List (BIOPRO; Hosman, 1983) and focuses on chronic and environmental stress, including problems in relation to work and study, housing, physical environment, leisure, finance and social relationships (partner, family, friends, neighbours). Of the original 32 items, 16 items concerning health were omitted, a question about leisure activities was added. Subjects rate each item on a four-point intensity scale with the anchors (1) none, (2) some, (3) quite, and (4) serious (difficulties). A total score is obtained by summing across all items.

Personality: Personality was assessed with the Dutch abridged MMPI (NVM; Luteijn \& Kok, 1985). This questionnaire consists of 83 items forming 5 scales: Negativism, Somatization. Timidity, Serious Psychopathology and Extraversion. Reliability and validity of the NVM have been described by Luteijn and Kok (1985).

Depression: Depression as a complex of symptoms was assessed with the validated Dutch translation of the Self-Rating Depression Scale (SRDS: Dijkstra 1974: Zung, 1965).

Anxiety: Trait anxiety was measured with the validated Dutch version of the State-Trait Anxiety Inventory (ZBV; van der Ploeg, Defares, \& Spielberger, 1980)

Anger: Trait anger was measured with the validated Dutch version of the Spielberger Trait Anger Scale (ZAV; van der Ploeg, Defares, \& Spielberger, 1982). This questionnaire consists of two subscales: 'anger-temperament' (the general disposition of experiencing anger and giving voice to it) and 'anger-reaction' (the disposition of expressing anger, for instance when provocated or criticized).

\section{THE EXPERIENCE SAMPLING METHOD}

The Experience Sampling Method (ESM) was designed to study the subjective, everyday experiences of people in their natural environments. Typically, an electronic signaling device is used to alert subjects to fill in a self-report questionnaire at preselected but randomized time-points, providing information about an individual's mental status or symptoms within the context and flow of experience. The self-reports request a range of information about subjects' current thoughts, moods, complaints, activities, and physical and social context. Signaling devices (e.g. pagers, wristwatch terminals), schedules of signals (e.g. random, fixed or fixed-random time-sampling; event-sampling) and ESM self-report forms may vary depending on the particular objects and goals of the study (see Csikszentmihalyi \& Larson, 1987; Delespaul, 1992).

During the last two decades, a large number of researchers have contributed, often independently, to the development of the Experience Sampling Method. One of the earliest lines of investigation under the name of ESM was started at the University of Chicago in 1975 by Czikszentimihalyi and associates. They obtained data on daily activities and experiences of adolescents (Csikszentmihalyi \& Larson, 1984). Similar techniques were developed or adapted by Hurlburt (e.g. Hurlburt, 1990) for the study of thought content, by Klinger et al. (1980) who studied the stream of conscious thought in daily life, by Massimini et al. (e.g. Massimini, Csiksentmihalyi, \& Carli, 1987 ) for the study of optimal experience, and by deVries and associates for the study of psychopathology (de Vries, 1987; de Vries, 1992). Many others could be added to this list. (see for a review Delespaul, 1995). The research group in Maastricht has studied a range of psychiatric disorders: schizophrenia (e.g. de Vries \& Delespaul, 1992; Delespaul, 1995), anxiety (e.g. Dijkman-Caes \& deVries, 1991), depression 
(Kraan, Meertens, Hilwig, Volovics, Dijkman-Caes, \&. Portegijs, 1992; van Diest, 1992), and somatization. They have also used ESM to study acute stress (Nicolson et al., 1992), pain (Lousberg, Schmidt, Groenman, Vendrig, \& Dijkman-Caes, 1995), and addiction (Kaplan, 1992).

The above indicates that ESM can be used with a variety of populations, provided that the research subjects can read and write and that a viable research alliance can be established. In spite of the success of ESM in describing mental disorders in context, there are limitations. Difficulties have been encountered in sampling elderly subjects with dementia, and individuals with acute and severe depression.

Early research focused on methodological aspects of ESM such as reactivity effects, self-selection biases, compliance, and validity and reliability of self-reports, and provided important information on the reliability and validity of ESM data (e.g. Csikszentmihalyi \& Larson, 1987; Delespaul, 1995; Hormuth, 1986). These aspects will continue to be of importance in every particular ESM study conducted. Controls and checks on the subjects should be built into the Experience Sampling designs, like checking on missed signals, the timeliness of responses (does the subject respond to the signal on time?) and on the influence of the method on the objective and subjective experiences of the subjects (Are the subject's objective circumstances influenced by participation? Is the subjective perception of a situation influenced by the method?) (see 'compliance and reactivity issues' section).,

\section{Description of the ESM instrument}

The ESM booklets used in the current study consisted of forms to be completed following each signal ('beep level' forms) and forms to be completed at the beginning or the end of each day ('day level' forms). These forms will be described separately below.

\section{ESM form: beep level}

At every 'beep' subjects completed a self-report form (see Appendix II), containing several questions about the subject's mental state and physical and social. context. Subjects were asked what they were brooding about, what they were doing, and where and with whom they were at the moment of the beep. Daily stress or hassles were assessed with the open question 'Did any stressful event or situation take place between the present and the previous beep?'. Thus, the assessment of daily events had a more retrospective character than the other questions at the beeplevel. The ESM forms also included Likert scales (from I 'not at all' to 7 'very much') for the evaluation of thoughts, mood, physical well-being, individually defined psychosomatic complaints, current activity, and stressful events. Activities were evaluated on the dimensions: enjoyed doing, skill, effort, and challenge. The following appraisais of stressful events were also rated on Likert scales: unpleasantness, importance, predictability, controllability, and frequency of prior occurrence. Subjects also reported when the event started and when it ended, or that the event was still going on. This information gives an indication of the duration of the event and the time elapsed since termination. Additional items were included to help in the interpretation of the hormonal data: occurrence and intensity of physical exertion and any ingestion of food, coffee, alcohol, cigarettes or medication which may have taken. place since the last signal. 


\section{ESM form: day level}

At the end of each Experience Sampling day, subjects were asked to rate (Likert-scales) the extent to which they were bothered by their individual complaint that day, how stressful the day had been and how their general mood had been. Also included was a short checklist of 15 events (Daily Stress Scale; Bolger et al., 1989a). Except for transportation and financial problems, this list consisted entirely of items concerning demands (e.g. a lot of work at home, at work, a lot of demands made by family) or interpersonal conflicts (e.g. argument with spouse, child, colleague). Subjects indicated if an event on the list had occurred to them that day and, if so, what the impact of that event had been (from 1 'not at all bothersome' to 7 'extremely bothersome').

Additional information was obtained each day immediately after waking (sleep quality, sleep pattern previous night) and again before going to bed (effects of participation in the study, timing of meals, work and time to bed).

\section{ESM procedures}

Most participants find the ESM rewarding but also taxing. A crucial part of the Experience Sampling process is therefore the creation of an alliance and a mutual understanding about the research procedures and aims of the study. Cooperation and compliance depend on this alliance.

During an initial semi-standardized interview one or two days before the start of ESM, called the 'briefing', the purpose of the ESM research and the additional saliva and urine sampling was explained and informed consent was obtained. We communicated our interest in learning about their daily experiences. At this time we also gathered additional information concerning possible recent diseases and related medication use, and individualized complaints were chosen. Subjects were instructed to select one complaint (either of a psychological or somatic nature) that was important to them and that occurred rather frequently. Instructions about the use of the watch and the ESM booklets were given. Subjects were instructed to fill out selfreports as soon as possible after a signal (within 15 minutes). Anticipated difficulties in the use of ESM (e.g. driving a car, attending a meeting) and possible solutions to it were discussed. The subjects were given control of the signaling device only in that they could turn it off if they did not want to be disturbed (e.g. during a daytime nap). As a reminder, instructions were also printed on the inside cover of the booklet. The subjects were discouraged from showing their completed booklets to others and were asked not to look back through previously completed forms.

The ESM period encompassed five days, from Thursday to Monday (three workdays and two weekend days). Tuesdays and Wednesdays were reserved for (de)briefing sessions. On ESM days, subjects were signaled 10 times a day between 7:30 a.m. and 10:30 p.m., with an average interval of 90 minutes between consecutive 'beeps'. This was done according to a preprogrammed schedule chosen to maximize interpretability of cortisol values without being predictable by the subject. The 'beeps' were randomly distributed around fixed time points (e.g. 8:15 a.m., 9:45 a.m., and so on throughout the day), with a maximum deviation of $20 \mathrm{~min}$. After each signal subjects filled in an ES form, which took about 2 minutes, and collected a saliva sample (see section below).

One or two days after the five days of ESM, a final 'debriefing' session took place, which started with the Stress Inducing Speech Task (SIST; see 'Stress Inducing Speech Task' section). After the stress task, ESM booklets were checked. Subjects 
were asked to explain reasons for any missing responses and to clarify illegible or unclear responses to facilitate later coding. The 'debriefing' session was closed with a structured interview about the possible impact of the study on the daily life of the subject. Subjects received a small financial compensation (fl. 20,-) as well as an information booklet about stress. 'Briefing' and 'debriefing' sessions each took about one hour. An individual subjects was briefed and debriefed by the same person.

\section{SALIVA SAMPLING}

Cortisol levels were determined in saliva. Cortisol in saliva is a reliable and valid indicator of the free cortisol in plasma, which is considered to be the biologically active hormone, and cortisol concentrations are independent of the flow rate of saliva (Vining, McGinley, \& Symons, 1983). The rate of equilibrium of cortisol between saliva and blood is very fast. Cortisol responds within less than five minutes to increases in stress (Vining et al., 1983) and has a half life in blood or saliva of about an hour (Fredrikson, Sundin, \& Frankenhaeuser, 1985). These characteristics of saliva cortisol also provide an appropriate time frame for studying its relationship to experiences in daily life. It is assumed that a sample, collected within 20 minutes after signaling, reflects adrenal cortical activity during the time period between that last signal and the previous signal. Cortisol, in comparison to other stress-labile hormones (e.g. the catecholamines, growth hormone, prolactin, testosterone), shows the most specific relationship to subjective distress, in contrast to general arousal, effort, or trauma (Delahunt \& Mellsop, 1987; Lundberg \& Frankenhaeuser, 1980; Rose, 1984). The ability to measure cortisol reliably in saliva instead of blood, makes repeated, stress-free sampling possible. Measuring cortisol in blood is usually stressful in itself (Rose \& Hurst, 1975) and interrupts ongoing activities, which makes repeated measurement extremely difficult, especially over longer time periods.

Subjects collected saliva by holding a dental cotton roll in the mouth for 1 to 2 minutes. Saturated dental rolls were then placed in a capped plastic vial (Salivette; Sarstedt). which subjects labeled with the time of day and then stored with the ESM booklet in a specially designed wallet. This wallet contained ten Salivette vials and an ESM booklet with ten seli-report forms. Subjects placed their saliva samples in their freezer at the end of each day. We have found no differences in cortisol levels in uncentrifuged samples frozen immediately or kept at room temperature for two days (Nicolson et al., 1992); others report no differences up to two to four weeks (Kahn, Rubinow, Davis, 1988; Kirschbaum \& Hel!hammer, 1989). Uncentrifuged samples were kept at a temperature of $-20 \mathrm{C}$ until they were analyzed. A maximum of 50 samples per subject was thus obtained.

Analysis Salivary cortisol levels were determined in duplicate by direct. radioimmunoassay (Ansseau, Sulon, Doumont, Cerfontaine, Legros, Sodoyez, et al., 1984), using ${ }^{125}$ I-cortisol (Farmos diagnostica, Finland) and antiserum made against the 3-CMO-BSA conjugate by Dr. J. Sulon, University of Liege, Belgium. The lower detection limit of the assay was $12 \mathrm{ng} / \mathrm{dl}$, with a mean intra-assay coefficient of variation of $4.8 \%$ (range: $2.2 \%-7.5 \%$ for 4 assays). Each subject's samples were analyzed in the same assay to reduce sources of variability. 


\section{URINE SAMPLING}

Levels of the catecholamines adrenaline (A) and noradrenaline (NA) were determined in 14-hour urine. Compared to plasma levels which are highly liable with a half-life of less than three minutes, urinary levels represent estimates of sympatheticadrenomedullary activity integrated over extended time periods (usually 1 to 3 hours) and they can be used to determine long-term (14- to 24-hour) changes in levels of catecholamines (CA) (Frankenhaeuser, 1975b). The collection of urine samples is also relatively easy, noninvasive and well-suited for naturalistic field studies (Frankenhaeuser \& Gardell, 1976). Because the catecholamine in urine constitute a small but relatively constant fraction of liberated amines in the body, the direction of change or the relative levels are meaningful, but absolute numbers have limited value. NA levels are somewhat difficult to interpret because NA is secreted by both nerves and the adrenal medulla and is also subject to rapid neuronal reuptake; A levels give a more reliable estimate of adrenal medullary activity because the adrenal medulla is the sole source of circulating A (Frankenhaeuser, 1975b). The 14-hour samples collected in this study were chosen to reduce the effects of variation between subjects with different circadian rhythms and to minimize the biasing effects of idiosyncratic events occurring during the day. For example, a person who runs up the stairs will probably show elevated CA levels at the next voiding, but such one-time elevations should be mitigated in a 14-hour collection. Overnight CA excretions can be regarded as baseline levels and are therefore useful for assessing physiological changes associated with chronic stress (Baum, Lundbeg, Grunberg, Singer, \& Gatchel, 1985).

Subjects collected two overnight urine samples, one after a workday (Thursday to Friday) and one after a weekend day (Sunday to Monday). Urine was collected by the subject from 6.00 p.m. to $8.00 \mathrm{a} . \mathrm{m}$. the following day ( 14 hours), in 2 liter urine containers (Sarstedt). Subjects emptied their bladder in the toilet at 6.00 p.m. and from that moment collected subsequent urine in the container. At $8.00 \mathrm{a} . \mathrm{m}$. the next day they emptied their bladder for the last time in the container. Subjects kept their container in the refrigerator during the collection period. The urine samples were collected by the researcher or research-assistant at $9.00 \mathrm{a} . \mathrm{m}$. at the workplace of the subject. From the time the subject left home until collection of the container at the workplace, samples were kept in an insulated bag with a cooling element. Immediately after the collection of the container we added HCL $(37 \%)$ to the total volume of urine until a $\mathrm{pH}$ of $<3$ was reached, to prevent oxidation. From the total volume, $10 \mathrm{ml}$. samples were extracted and immediately frozen at a temperature of -20 $\mathrm{C}$ until analyses.

Analysis Adrenaline and noradrenaline excretion rates were determined by means of high-pressure liquid chromatography (HPLC) with electrochemical detection (Kissinger, Riggin, Alcorn, \& Rau, 1975). ${ }^{\text {. CA levels were corrected for }}$ creatinine excretion $(g / l)$ and expressed in $\mu \mathrm{g} / \mathrm{g}$ of creatinine. Nine samples with creatinine levels below $0.60 \mathrm{~g} / 1$ were considered unreliable and were not used in the analysis. Due to practical reasons, three subjects did not collect any urine, and another four subjects collected urine only once.

Analyses of adrenaline and noradrenaline were performed under the supervision of Dr. Rahman and Dr. Duvivier, University Hospital of Liege, medical chemistry service, Belgium. 


\section{STRESS INDUCING SPEECH TASK (SIST)}

A disadvantage of conducting a study in the natural environment is the idiosyncratic nature of events and activities and the heterogeneous stressful circumstances subjects encounter in daily life. Individuals' responses to these varying circumstances are difficult to compare. Therefore, all subjects in this study were exposed to a standard stress test in an experimental context in order to get more insight into individual differences in neuroendocrine responses. Additionally, we investigated whether cortisol activity as assessed in the laboratory generalized to cortisol activity as measured in the field. An important consideration was that the information provided by the standardized stressor should be relevant for the understanding of the endocrine response to stimuli in claily life; in other words, the stressor should have 'ecological validity'. We chose to use at speech task, similar to that described by Steiner and associates (1988).

The test took place at the beginning of the ESM debriefing session, between $11 \mathrm{a} . \mathrm{m}$. and $13.00 \mathrm{p} . \mathrm{m}$. This test was unanticipated by the subjects, who believed that the final session would only be used for 'debriefing' the ESM study. The experimenter (blind to whether a subject belonged to the high stress or the low stress group) read the written SIST instructions aloud for the subject. The instructions were to prepare (10 minutes) and present ( 5 minutes) a videotaped speech about their personal strengths and weaknesses for later evaluation by a team of psychologists. After the 10 minutes of preparation, subjects received a signal, the video recorder was started, and the subjects delivered their presentation while looking directly into the camera. Afterwards, subjects relaxed for 15 minutes in neutral activities (e.g. reading magazines). At 4 time points subjects filled in a short mood questionnaire and provided a saliva sample: (T1) upon arrival, (T2) after the 10 minutes of preparation, (T3) after the 5-minute presentation, and (T4) after 15 minutes of relaxation. During the course of the experiment we decided to lengthen the recovery period to get a clearer picture of the cortisol response profile. For 49 subjects, a fifth saliva sample (T5) was taken on average 50 minutes after the first assessment. This way we could look at endocrine responses during the anticipation, reactivity and recovery phase of the stressor.

\section{CODING OF ESM MEASURES}

Information about activities ('What were you doing?'), location ('Where were you?'), social context ('With whom were you?'), and stressful events ('Did any stressful experience or event occur?') was obtained by open-ended questions. In order to be able to identify, for instance, typical patterns of time use or stressful daily events, the total range of activities and daily events has to be reduced. The specific method used for coding these experiences is very important because it entails decisions about which events or experiences can be differentiated and which will be combined. An important question to be answered before using these data in further analyses concerns the reliability of the coding procedure. This was assessed by calculating the agreement between two independent observers on the coding of all variables to be used in the analysis (see Appendix III). 


\section{Coding procedure}

In the literature are examples of ESM studies where responses to open-ended questions were coded by the subjects themselves (e.g. Brandstatter, 1983), by other participants (e.g. Campbell et al., 1991) or by trained coders (e.g. Wong \& Csikszentmihalyi, 1991). In this study we used trained coders for several reasons: first, if coded by subjects themselves they would need more time to fill out the ESM form thereby endangering study compliance (e.g. activities were coded under 57 fine grained but reliable categories which would take up too much time, and too many instructions needed), and second, because we wanted to start without preconceived notions of what a 'stressful event' might be. Pre-coded responses ('menus') require limited numbers of simple categories which are predefined to be of importance.

Responses were coded by two independent raters, according to an established coding system. The two raters first coded three ESM booklets and discussed differences in the results to clarify problematic categories and definitions. Next, they coded a subset of ESM booklets (five booklets for each of 27 subjects) without discussing them with each other. These codings were used for the reliability analysis. All data actually used in further analyses (beyond the reliability analysis) were coded by both raters with differences being replaced by consensus ratings.

\section{Coding of activities, location and social context}

Classification of activities was based on the list of events in the International Time Budget Study (Szalai, 1972). This list contained 96 codes which were later reduced to 47 codes for use in several Experience Sampling Method studies at the University of Limburg. In the current analyses, the 47 activities were collapsed into 8 broader categories: inactivity/rest, work (which also included volunteer work, regular classes, homework, special courses, and study breaks), household/maintenance (also including child care, care to adults, shopping and general services, personal care services, personal hygiene and personal medical services), leisure (including activities like sports, hobbies, attending a film, reading a book, or watching television), social interaction (conversations, visits to or by friends, parties etc.), meals (including regulai meals, special meals and snacks), transport, and other activities (including personal mental or emotional activities, political and civic activities, and other activities not falling in one of the other activities).

With respect to location ('Where are you?') and social context ('Are you together with someone?'), we again used the standard coding manual developed for ESM studies at the University of Limburg, later collapsing the specific codes into six broader categories: at home, nefwork (homes of family, friends, and acquaintances). work, public places (street, shop, cafe, sports hall, health care settings), transport (in car, bus, train, airplane, on bike), and other places.

Responses to the question 'Are you together with someone?' were coded and then collapsed into 7 categories: alone, household members (including partner, children, parents, brothers and sisters and other household members), non-resident family, friends, colleagues, neighbours/acquaintances, and strangers.

Responses were coded missing/invalid if a question was not filled in (missing) or if a response was not legible or invalid (e.g. filled in too late); responses were rated as can't code if something valid had been written down, but it was not clear which category did apply (for instance when important background information is missing). This category can give us a good idea about the quality of the coding system. 


\section{Coding of stressful events}

In contrast to the domains described above, there was no pre-existing coding system for stressful events. Until now, there have been few studies investigating the effects of different types of minor stressors on health and well-being (Bolger et al., $1989 \mathrm{a} ;$ Stone, 1987). It is common practice to aggregate daily stressors into a summary measure for analytic purposes. This may, however, conceal important variation in the microprocesses underlying somatic and psychological well-being. For this reason, we differentiated events reported according to the following domains: context of the event, who was involved in the event (if someone was involved), whether the event was internal or external (observable or not), and whether the event entailed a social interaction or a task demand.

The context categories were based largely on the ESM activity codes, which was expanded to include the categories personal heaith-somatic and personal healthpsychological. We wanted to be able to identify events conceming health, especiaily mental health, because of their possible confounding to psychiatric and somatic states.

The context domain contained the following 8 categories: work (events concerning boss, supervisor, co-worker, clients, general happenings concerning self at work, events concerning study etc.), network (events concerning spouse, partner, children, relatives, friends, neighbours or acquaintances), household/financial (events concerning general housework, family related duties, errands, loans, selling, buying. financial problems etc.), leisure (events concerning hobbies, sports, outings, vacation etc.), personal health-somatic (events concerning illness, injury of the subject himself), personal health-psychological (e.g. nerves, anxiety, worries about health), transport (e.g. missed bus, traffic jam, unusual traffic), and an 'other' category (minor irritants like noise, the weather, broken glass, ESM. cold shower. combinations of different events etc.).

The coding of events under a 'social interaction' domain and a 'task demands' domain paralleled the checklist we used in the ESM booklet at the end of the day, which consisted mainly of events concerning task demands or interpersonal conflicts. A diary study on the influence of daily stress on mental health, based on a community sample of 166 married couples, showed that interpersonal conflicts and tensions were by far the most distressing events (Bolger et al., 1989a). Task demands were also of interest to us because overload is an important theme in a sample of working people. These people have in general more than one social role (family, work eic.) and are therefore more exposed to various role related demands. A study by Stone (1987) showed greater psychological impact of work-related demands.

With respect to the 'social interaction' domain, responses were coded into three categories: not applicable (event is not a social interaction); negative interaction (e.g. argument, conflict) or discussion/conversation.

The 'task demands' domain consisted of five categories: not applicable (clearly no demands involved); problematic task (e.g. difficulties, problems with..); $a$ lot of work/tasks or extra work/tasks; time pressure, deadlines; failure at task.

For coding who was involved in the event we used the same categories as described under the ESM social context domain: alone, household members, nonresident family, friends, colleagues, neighbours/acquaintances and strangers.

Events were coded as external or internal in an effort to separate the more or less 'observable' events from entirely 'subjective' events. Criteria for coding events as external were: 1) they should be observable (i.e. theoretically verifiable) and 2) 
they should have happened in the interval since the last beep. The opposite applies to internal events: they are not observable. Examples of internal events are introspections, worries, internal evaluations, anticipations. The internal/external distinction does not refer to responsibility for causation.

A 'can't code' category was included in every domain for event descriptions which could not be classified due to the absence of sufficient information.

\section{Interrater agreement}

Before using the qualitative information in further analyses, we have to investigate if the coding system we used is a useful one. Generality is important here in demonstrating that the obtained ratings are not the idiosyncratic results of one rater's subjective judgment (Tinsley \& Weiss, 1975). Regarding the interrater agreement we are interested in the following question: Is it possible to classify the information under the various categories and can we do this reliably? Information gathered under the activity, location and company domains is clear and straightforward. The categories belonging to these domains are also very concrete and well-defined. Problems should not be expected here. But with regard to the coding domains for stressful events, we can expect some variance because of interpretation differences and difficulties. Additionally, the subject's description of the event may be lacking in the types of detail necessary to classify it into one of several related categories. In Appendix III we describe how the interrater agreement was calculated (Cohen's unweighted Kappa) and what the Kappa's for the different codes were.

With respect to the reliability of coded responses, we can conclude that the open-ended responses could be classified very reliably (see Appendix III). Even in categories with only a small number of observations the degree of agreement was generally substantial. We did find that some specific event domains were more difficult to rate. This was the case for the domains 'internal/external', 'social interaction' and 'task demands', where Kappa's varied from moderate to almost perfect $(.46-.89)$, but where a substantial number of responses fell into the 'can't code' category. This means that the obtained information was often difficult to interpret, leading to a substantial loss of information. Because these analyses were done after the first 27 subjects were sampled, more attention was paid in subsequent debriefing sessions to the clarification of reported information on stressful events, in order to keep loss of information due to uncodable responses to a minimum.

\section{DATA ANALYSES}

The ESM dataset is characterized by a large number of repeated observations ('beeps') on a relatively small number of individuals, with a large number of variables measured at each beep. As a consequence, Experience Sampling data have a complexity which often make traditional analytic approaches unsatisfactory. Depending on the type of research question, ESM data can be analyzed either at the subject level, at the beep level, or at both levels at the same time (Larson \& Delespaul, 1992). At the beep level, the repeated measurements are used as the unit of analysis, with a maximum of 50 measurements per subject in the current study. At the subject level, the individual is used as the unit of analysis, including one time measurements as well as aggregated data. One time measurements are, for instance, scores from crosssectional questionnaires (e.g. perceived stress score) and briefing and debriefing data. 
Aggregated data are, for instance, mean mood or event scores per subject, or the percentage of total beeps a subject spent on a certain activity (e.g. work).

Although in certain cases beep level analyses may be the only possible approach (e.g. when one is dealing with relatively rarely occurring categories of experience like going to a party'), there are some important problems that are inherent in such an approach (Larson \& Delespaul, 1992). Generally, when beep level analyses are carried out, the number of units in the analyses is inflated and dependency exists between adjacent data points. Significance tests, therefore, do not provide a valid estimate of probabilities. In addition, beep level analyses give: equal weight to all instances of a category of experience. This means that subjects with more valid responses in that category are given more weight and will have more influence: on the outcome. These problems of inflated number of units, dependency between observations, and unequal weighting can be diminished by employing subject level analysis, where aggregate scores are computed for each individual and the subject is used as the unit of analysis (Larson \& Delespaul, 1992). Aggregated scores, which are based on repeated measurements, also have the advantage of increasing the reliability of measurement. Therefore, this is an appropriate approach when research guestions about the chronic level of variables are of interest (see for example Chapter 3 and Chapter 5).

Nonetheless, subject level analyses are also subject to several pitfalls. Besides the fact that they ignore the rich data base available, this approach can introduce aggregation bias, which occurs when unmeasured or unspecified variables exist that distort the causal relationship between variables (e.g. "when heterogeneous groups [or sub-units] are combined into a single group [unit], and the combined group analysis yield conclusions that are misleading about the sub-units") (Jaccard \& Wan, 1993, p.43). Another restriction on the use of subject level analysis is that a substantial number of subjects with at least more than one observation is required when one is interested in making comparisons across several situations (e.g. work. home; work days, weekend days) using a repeated measures analysis of variance, since this technique does not tolerate missing data. Most like $l y$, a number of subjects will not have enough observations in each situation, leading to a substantial loss of information. Two other important shortcomings of this technique are: it may provide misleading estimates of the effects of an independent variable (data are treated as if there were an equal number of observations for each level of the independent variable, but in many occasions this will not be the case), and it ignores the fact that many of the independent variables are correlated in ESM: the data are treated as if the variables are orthogonal. For example, certain stressful events (e.g. network events) tend to occur only in certain locations (e.g. at home rather than at work).

Besides the problems described above, aggregated data obscures the interplay between experientiai and psychological or physiological states and processes which momentary measures try to capture. To gain more insight into the relationship between perceived stress, stressful events, cortisol and / or mood states over time, we will have to apply another analytic method. A more effective approach for analyzing both subject and beep level data is by using random-coefficient regression models (Bryk \& Raudenbush, 1992; Goldstein, 1987). This approach is a variant of the multiple linear regression model, applicable for data with a. hierarchical nesting structure as is the case with ESMI data. This approach can account for the dependency of data within a subject and for residual dependency, it can deal with the problem of missing data, and it allows for individual differences in intercepts, slopes, and error structures. Additionally, by using random regression models, the outcomes 
at the beep level can be modeled as a function of both the beep and the subject level variables. The model allows us to obtain estimates of, for example, cortisol reactivity to events both as a function of psychological state (e.g. mood state) and trait (e.g. level of perceived stress) variables over all subjects and for each individual. The number of observations need not be the same for all persons, and observations may be unequally spaced over the time interval. In the present study, random regression models have been applied in Chapter 4, Chapter 6, and Chapter 7. Greater detail on theoretical and technical aspects of this analytic approach can be found under the method sections of these chapters.

\section{COMPLIANCE AND REACTIVITY ISSUES}

The general purpose of ESM is to study the subjective experiences of persons interacting in their natural environments. This means that the variables measured should be a representative sample of those in the person's environment and that they should be measured with as little distortion in retrospective recall as possible. ESM's primary weaknesses lie mainly in the responsibility given to the subject in collecting not only subjective data, such as feelings and appraisals, but also objective ones like the description of situations. Therefore it is very important to check on compliance of subjects (e.g. How many responses are missing and for what reason?), timeliness of responses (Are responses made on time?), and on effects of ESM on the objective and subjective experiences of the subjects (e.g. Does ESM influence subject's choice of activities, mood?). Because: ESM asks more of its participants than more conventional research methods it is also important to check on how taxing the task of being a subject was fellt to be.

\section{Compliance of subjects}

Particularly for people with relatively high levels of appraised stress, it is important to determine if participants were motivated and able to fill in a sufficient number of ESM booklets within an acceptable time limit after the beep occurred. The choice for this time limit is rather arbitrary; a compromise has to be found between leaving enough time for the subject to comply to his tasks (filling in the booklet and taking a saliva sample) and keeping the delay between the beep and response time as smali as possible.

In this study, a response was considered valid and maintained for analyses when a response was given within 5 minutes before and 20 minutes after the beep occurred. Only subjects with at least 20 valid responses and with not a single day completely without valid responses were included in the analyses. In our sample, 4 subjects did not meet our criteria. Two subjects in the low stress group completed a sufficient number of valid beeps, but both missed a whole day (one subject went abroad for a day with a business client). In the high stress group, one subject had only 16 valid response, and another subject missed all responses at the weekend because he was ill. Analyses were based on 88 subjects. 
Table 2.3. Delay between beep and response time.

ESM formats Saliva Samples

Nr. of responses after: $1 \mathrm{~min}$.

$5 \mathrm{~min}$.

$477 \quad 11 \%$

$168238 \%$

$10 \mathrm{~min}$.

$249857 \%$

$303769 \%$

$15 \mathrm{~min}$.

$328575 \%$

$346479 \%$

$20 \mathrm{~min}$.

$352980 \%$

$360382 \%$

$363283 \%$

$365683 \%$

\begin{tabular}{llllll}
\hline Invalid & 144 & $3 \%$ & 107 & $2 \%$ \\
Missing & 624 & $14 \%$ & 637 & $15 \%$ \\
\hline
\end{tabular}

Total number of responses

4400

4400

Of all programmed beeps, $83 \%$ were responded to within the time limit of 5 minutes before and 20 minutes after the beep occurred (see Table 2.3). For all valid responses the mean delay between beep and response time was 5 minutes $(s, d=4.0)$, and in $3 \%$ of all cases the delay between beep and response time was larger than -5 and +20 minutes (invalid responses). Finally, $14 \%$ of all beeps were not recorded. Although the saliva samples were not included in our 'validity' criteria, we also checked on the compliance of saliva samples. Here we found almost the same distribution of valid, invalid and missing responses: $83 \%$ valid responses, $2 \%$ invalid responses and 15\% missing responses. Mean delay between beep and response time for saliva samples was 3 minutes $(s . d .=4.8)$.

The number of valid, invalid and missing responses in the low versus high stress groups are shown in Table 2.4. The mean number of valid responses completed per subject was 41 . In the low stress group subjects completed an average of 42 (s.d.= 5.7) valid ESM responses compared to $40($ s.d. $=6.8$ ) in the high stress group. The difference between groups was not significant (Mann-Whitney U test; two-sided, $p=.1)$. The mean number of saliva samples was $43(s . d .=5.8)$ in the low stress group and $40($ s.d. $=6.6)$ in the high stress group (Mann-Whitney $U$ test; two-sided, $p=.1$ ).

Table 2.4. Number of valid, invalid, and missing responses in 'High' and 'Low' stress, groups.

\begin{tabular}{|c|c|c|c|c|}
\hline \multirow[b]{2}{*}{ Responses } & \multicolumn{2}{|c|}{$\begin{array}{l}\text { Low Stress Group } \\
\qquad \mathrm{n}=46\end{array}$} & \multicolumn{2}{|c|}{$\begin{array}{c}\text { High Stress Group } \\
n=42\end{array}$} \\
\hline & ESM & Saliva & ESM & Saliva \\
\hline Valid (mean) & $42^{\prime}(84 \%)$ & $43(86 \%)$ & $40(80 \%)$ & $40(80 \%)$ \\
\hline Valid (total) & $1947(85 \%)$ & $1960(85 \%)$ & $1685(80 \%)$ & $1696(81 \%)$ \\
\hline Invalid (total) & $50(2 \%)$ & 32 (1\%) & $94(5 \%)$ & $75(4 \%)$ \\
\hline Missing (total) & 303. (13\%) & $308(13 \%)$ & $321(15 \%)$ & $329(16 \%)$ \\
\hline
\end{tabular}

In summary, four subjects were discarded from the study, because they did not meet the criteria of having filled out at least 20 ESM reports within a time limit of -5 and +20 minutes, and without a complete day missing. The final sample completed $83 \%$ of all possible responses within the time limit, providing an average of 41 
responses per subject. The same applied for compliance of saliva samples. The delay between beep and response time was generally small; $57 \%$ of the responses were given within 5 minutes after the beep. High stress subjects were not less compliant than low stress subjects.

\section{Reasons for missing responses}

Missing responses can be due to a variety of reasons (e.g. sleep, lack of motivation, interference of beeps with important, stressful, or private activities). For the reliability and validity of ESM, it is important to ascertain that there is no systematic underreporting of specific situations. Figure 2.1.a. displays the number of valid responses during the day. The least valid responses were obtained in the morning and late at night, with the first beep (between 8:07 and 8:30 a.m.) receiving the least valid responses $(73 \%)$. One possible reason for missing or out of range responses in the morning and evening hours was that subjects were still or already asleep. Since subjects were asked to fill out the time they woke up in the morning and went to sleep in the evening, the percentage of missing and invalid responses due to sleep could be calculated. Of all missing and invalid responses, $10.4 \%$ were known to be due to the fact that the beep occurred before the subject woke up in the morning. while $0.4 \%$ was due to going to sleep before the last beep occurred. Sleep was the reason for missing and invalid responses in $45 \%$ of all first beeps in the morning (all in the weekend). The small decrease in valid responses at beep 7 was probably related to the fact that subjects were leaving the work place and were on their way home which complicated compliance. Notes in booklets suggested this.

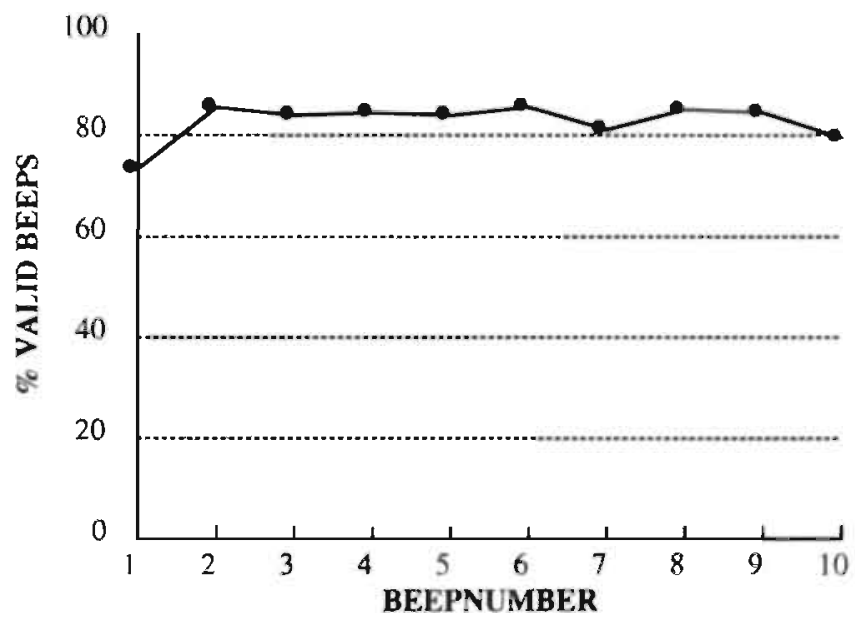

Figure 2.1.a. Percentages of valid responses during the day. 




Figure 2.1.b. Percentages of valid responses, at different research days.

Fig. 2.1.b. shows that the percentage of valid responses did not vary much by research day. Although a decline in valid responses might be expected at the end of the research week because of a decrease in motivation, we found this small decline to be most prominent during the weekend. ANOVA-repeated measures over the mean number of valid responses (aggregated by subject) showed a significant withinsubject effect for 'DDay of the Week' $(F(4,344)=4.2 ; p<.01)$, but no berweensubjects effect for 'Group' (high vs. low stress) was found $(\mathrm{F}(1,86)=2.7$; ns), and no significant interaction between 'Day of the Week' and 'Group' $(F(4,344)=.4)$. Significantly more responses were missed during the weekend than during work days $(t(87)=4.4 ; \mathrm{p}<.001)$. In the weekend, $74 \%$ of all missing and invalid responses could be related to the fact that subjects were still asleep.

Notes in booklets revealed various other reasons for missing and out of range responses. Reasons mentioned relatively often were: not hearing the watch in a noisy environment (e.g. sports hall, party) or mistaking the alarm beep in the early morning for the first beep (leading to a missing response at the end of the day), problems with the watch (put off by mistake, not working properly), forgetting to take the ESM format along when leaving the house or work place, engaging in sports and other hobbies, business appointments, meetings or speeches at work, and driving a car or bicycle. In summary, the percentage of missing responses does not vary dramatically during the day and during different research days. The most important reason for missing and out of range responses in the early morning was that subjects were still asleep. The remaining missing and out of range responses were due to a variety of reasons making systematic underreporting of events unlikely.

\section{Representativeness of recorded days}

It is hoped that the five research days provide a representative sample of the subject's daily life. But, since these days were just five days in a subject's daily life, it is quite possible that they, either by chance or as a react of the procedure, differed from 'normal' days. Therefore, we asked subjects during the ESM debriefing 
interview whether the research days had been unusual in some respect, and if so, for what reason. During the ESM period, subjects also evaluated at the end of each day (just before bed time), whether that day could be considered as a normal one. This was measured with a Lickert scale, ranging from 1 ('not at all normal') to 7 ('very much normal').

Fifty one subjects $(58 \%)$ reported during the debriefing that the research days gave a good impression of their normal daily life. Eleven subjects (13\%) were more doubtful, reporting that the research days were a little bit unusual. Twenty-six subjects $(29 \%)$ ) found the research days to be unusual in some respect. No differences were found between the two subject groups. Twenty-one subjects provided explanations for what was unusual: parties, birthdays in family $(n=9)$; more or unusual work $(n=6)$; less work/stress than usual $(n=8)$; health complaints of self or family $(n=4)$; a day off on a usual work day $(n=3)$. 'Less work/stress' as an unusual factor was mentioned by six subjects from the high stress group compared to two subjects from the low stress group. At the day level, the mean rating of how normal the day had been was $4.3(\mathrm{~s} . \mathrm{d}=1.2)$ (aggregated by subject over 5 days). On the whole, then, subjects experienced their research days as normal. High stress subjects appraised research days as being a little less normal than low stress subjects (3.9 versus 4.8 . respectively; $t(84)=4.0 ; p<.001)$, but they still thought that the ESM sampling gave a good impression of their normal daily experiences.

In summary, although both subject groups reported that the research days were occasionally unusual for one reason or another, a majority indicated that the research days were representative of their normal daily routine. In the high stress group, the level of stress or work pressure experienced during the sampling period seems to be particularly salient, as this was frequently mentioned as the reason why the research days were unusual (e.g. "this week was unusual in that I had more work/fewer pressing deadlines than usual" etc.).

\section{Inconvenience and task difficulty of ESM}

To get an indication of how taxing and difficult the ESM procedure was for subjects, we asked during the debriefing interview whether ESM was 'bothersome or annoying' and whether any question in the ESM booklets was difficult to interpret or to answer. Additionally, we inquired whether they were willing to participate in an ESM study again. Subjects gave an evaluation of how disturbing individual beeps were in the ESM reports at the moment they were beeped.

Table 2.5. Annoyance with RSM as, recorded during debriefing interviews.

Low Stress Subjects High Stress Subjects

Did you find ESM

\begin{tabular}{lrr} 
'bothersome'? & $\mathrm{N}=46$ & $\mathrm{~N}=42$ \\
\hline No & $23(50 \%)$ & $21(50 \%)$ \\
A little/sometimes & $20(44 \%)$ & $14(33 \%)$ \\
Yes & $3(6 \%)$ & $7(17 \%)$ \\
\hline
\end{tabular}

As Table 2.5. shows, a vast majority of subjects (89\%) reported that ESM was either slightly or not at all bothersome. Only $6 \%$ of the low stress subjects and $17 \%$ of the high stress subjects said the method was troublesome. The differences between groups were not significant. The ten subjects who found ESM bothersome gave the 
following explanations: the filling in of ESM reports becomes particularly annoying toward the end of the sampling period (three subjects), during weekend leisure time (two subjects), or because it disrupts activities or occurs in the company of other people (five subjects).

Seventy-three percent of the subjects reported to be willing to participate another time with ESM, 14\% would be willing under certain conditions (e.g. only after some time, different questions in the ESM booklet), and $13 \%$ did not want to take part again (e.g. once is enough, too intensive, too long, too many beeps). There were no differences between the two groups in willingness to participate in a future ESM study.

In response to the question whether there were any difficult questions in the ESM forms, $72 \%$ of the subjects had no probiems at all, $19 \%$ had only minor problems, and $9 \%(n=8)$ of the subjects had problems with one or a few specific questions only (e.g. what is a stressful event?; sometimes difficult to score mood items; sometimes appraisals of activities not applicable). Again, there were no group differences.

At the beep-level, subjects rated whether the beep was disturbing on a scale from 1 'not at all' to 7 'very'. Of all valid responses, $48 \%$ were rated as 'not disturbing' (score 1) and $14 \%$ as 'disturbing' (score 5 or higher). 'High' stress subjects rated the beeps as slightly more disturbing on average than low stress subjects (mean score of 2.5 versus 2.2; Mann-Whitney $U$ test; $p=.04$; two-sided). It was further striking, and logical, that invalid beeps were rated as more disturbing on average than valid beeps (mean score $[n=48]$ of 3.0 versus 2.3 ; Mann-Whitney $U$ test; $\mathrm{p}<.01$; two-sided).

In summary, most subjects were not unduly inconvenienced by the ESM procedure and were generally positive in their evaluations of the method. Only $13 \%$ of the subjects stated unwillingness to participate again in an ESM study. Half of the beeps were rated as not disturbing at all. High stress subjects rated the beeps as a little bit more disturbing than low stress subjects, but their average score was still low. These data show that ESM can be used with good results in samples of working men with different levels of perceived stress. Our results compare well with results obtained in other studies. in a study on German adults, $22 \%$ reported that ESM disrupted daily routine and $75 \%$ were willing to participate again with ESM (Hormuth, 1985). In samples of Dutch subjects with a panic or other neurotic disorder, about $28 \%$ said the method was bothersome and $72 \%$ was willing participate again (Dijkman-Caes, 1993).

\section{Study effects}

In order to obtain a representative sample of the experiences of people in their daily life, we made repeated measurements with ESM within a day and during several days. Because of these repeated measurements, which means intense involvement of the subject, we wanted to know if participating in ESM research produced study effects which would reduce the ecological validity of the study. Therefore, debriefing interviews included questions about the possible influence of the method on the subject's daily life. 
Table 2.6. The method's Influence on thoughts, moods, activities and social contacts.

\begin{tabular}{|c|c|c|c|c|}
\hline Study effects & $\begin{array}{l}\text { Low-High. } \\
\text { Subjects } \\
\text { Thoughts } \\
\text { (\%) }\end{array}$ & $\begin{array}{l}\text { Low-High } \\
\text { Subjects } \\
\text { Moods } \\
\text { (\%) }\end{array}$ & $\begin{array}{l}\text { Low-High } \\
\text { Subjects } \\
\text { Activities } \\
\text { (\%) }\end{array}$ & $\begin{array}{l}\text { Low-High } \\
\text { Subjects } \\
\text { Social contacts } \\
\text { (\%) }\end{array}$ \\
\hline Lirtle or none & 13 & 5 & 4 & 9 \\
\hline Yes & 31 & 19 & 4 & 7 \\
\hline No & $\begin{array}{ll}63 & 62\end{array}$ & 76 & 92 & 91 \\
\hline
\end{tabular}

A majority of subjects said the method had no influence on their thoughts, moods, activities and social contacts (see Table 2.6). The small differences found between groups were not significant. The ESM method had the largest influence on subject's thoughts. Effects on thoughts mentioned during debriefing interviews were: thinking about ESM during the day and waiting for beeps to come $(n=10)$, more aware of thoughts $(n=10)$, confrontation with thoughts $(n=3)$, giving a moment thought to things you normally take for granted $(n=l)$, and it helps to relativize experiences $(n=2)$ and to concentrate $(n=1)$. The main effects on mood were: higher awareness of feelings $(n=4)$, and writing down negative feelings makes them disappear $(n=1)$. Irritation $(n=7)$ or being bored $(n=2)$ were mentioned as negative effects of ESM. Effects on activities were: giving more thought to activities than. usual $(n=2)$, less participation in sports $(n=1)$, little adjustments in planned activities during the weekend $(n=3)$, and some people said they waited to engage in a certain activity till after the beep $(n=3)$. The most often mentioned effect of ESM on social contacts was: having to explain ESM all the time to other people ( $n=8$ ), what some people liked and others did not. One subject thought the beeper scared others off, and another subject thought that his family behaved differently (no arguments).

In summary, on average $80 \%$ of the subjects said the method had no influence on daily life. If study effects were reported, there were both positive and negative effects of participating in ESM. 



\section{Chapter 3}

The nature of stress in daily life: events, appraisals, and activities 



\section{INTRODUCTION}

Stressful daily events have been found to be related to lower psychological well-being and increased somatic symptomatology (Del-ongis et al., 1982; Stone et al., 1993). These studies, most of them characterized by an a priori labeling of events as stressors, provided only limited information about the types of events that are experienced as stressful by varying populations and in varying contexts. They did, however, show that large individual differences exist in the vulnerability to minor events as well as in the impact of such events. The meaning and impact of an event is to a large degree determined by one's perceptions and reactions to such events. They may depend, for instance, on earlier experiences, personality social support, personal goals and beliefs. The current transactional approach to psychosocial stress defines minor events or 'hassles' as experiences and conditions of daily living that are experienced as salient and harmful or threatening to the endorser's well-being (Lazarus \& Folkman, 1984b). A central feature of this approach is the importance it places on these cognitive appraisal processes that intervene between the occurrence of an event and the individual's reaction to it. Despite the acknowledged importance of the transactional nature of stress, the significance of the appraisal process, and the many laboratory and animal studies that have been performed on this topic, few studies have investigated the nature of suressful daily events and the meaning individuals give to stressful events in a real-life setting. The present study aims to examine in the natural environment of the individual what types of events are experienced as stressful and how stressful events are appraised. We further investigated whether individuals who perceive themselves to be stressed differ in the types and appraisals of reported stressful events from individuals who do not.

With respect to the nature of stressful events, qualitative measures of stressful daily events may increase our insight into the type of situations or experiences which are most problematic and emotionally difficult for certain populations or for the individual. By exploring recurrent themes or patterns of hassles in reports of everyday events as described by individuals themselves, instead of focusing only on frequencies of event occurrence, we can more reliably characterize those events (e.g. work demands, conflicts with the partner) that form the basis of chronic stressful life conditions (e.g. work problems, family problems) (Wagner, 1990). Research suggests that patterns of hassles vary with developmental stage and sociodemographic characteristics (Kanner et al., 1981). In the study by Kanner et al., groups of middleaged people, students, and health professionals could be differentiated according to certain thematic patterns of hassles. Middle-aged subjects reported more hassles with economic issues (e.g. rising cost of living, property, investments, taxes), health professionals reported more hassles with anxieties and high pressure (e.g. too many things to do, not enough time, too many responsibilities), and college students tended to have more academic and social hassles (e.g. meeting standards, wasting time, and loneliness).

With regard to the meaning and significance of an event, it is possible that although people encounter an enormous range of events in daily life, events are. stressful for only a limited number of reasons. For an understanding of what makes certain events stressful for certain kinds of people, we not only have to know what (is. perceived to have) happened, but also how an individual gives meaning to the event, through cognitive appraisal processes (Lazarus \& Launier, 1978). There is evidence, for instance, that individuals' personal ratings of events improve prediction of outcomes such as anxiety, depression, negative affect, tension, and grade point 
average (Dewe, 1991; Peeters et al., 1995; Sarason et al., 1978). Although the transactional stress theory makes a distinction between primary and secondary appraisal processes ('what is at stake', and 'what can be done about the situation'. respectively), in many cases these processes are not separable (Holroyd \& Lazarus, 1982). For this reason, we have not tried to distinguish between primary and secondary appraisal in the present study. Based on the literature (e.g. Cohen, 1980; Dewe, 1991; Katz \& Wykes, 1985; Lazarus \& Folkman, 1984b; McGrath \& Beehr, 1990; Miller, 1979; Thompson, 1981), we selected five dimensions that appear to be important in how individuals evaluate stressful experiences: Unpleasantness ('How unpleasant was this event for you?'), importance ('How important was this event for you?'), predictability ('To what degree did you foresee that this event was going to happen?'), controllability ('How much controll did you have over the course of the event?'), and frequency of prior event occurrence ('How often has such an event happened to you before?').

The main objectives of this chapter are, firstly, to provide both quantitative and qualitative descriptions of daily life stress in white collar men, and secondly, to contrast the experiences of individuals who perceive themselves to be stressed with those who do not. Considering our first objective, we examine 1) how often stressful situations occurred, 2) what kinds of situations were experienced as stressful, and 3) evaluations of events, as discussed above. Regarding our second objective, we first examine 4) the amount of time spent on different activities in high and low stress subjects. Information on the frequencies of activities that people engage in on a daily basis can provide the necessary framework to understand the experience of stress in daily life. In addition, patterns of daily time use are compared between groups to assess whether the two groups had a similar chance of exposure to various kinds of stressful situations. We next examine 5) whether high stress subjects differed from low stress subjects in how they appraised daily activities in terms of enjoyment, challenge, required skill and effort. Here, we expect that appraisal of activities may reflect the stressfulness of daily life experiences that were not identified by the individual as discrete events. Next, we investigate differences between high and low stress groups in 6) frequency, 7) content and 8) evaluation of stressful experiences. We further examine whether life events and chronic difficulties were related to the frequency of stressful events reported during the five days of ESM. Both life events (for example, divorce) and chronic difficulties (for example, problematic relationship with spouse) could affect the person's pattern of stressful daily events or their personal significance (Kanner et al., 1981). Finally, we investigate a more methodological issue, that is, how do repeated within-day event reports compare with checkilist results obtained at the end of the day? Since most studies measure stress on a daily basis, it is important to investigate the influence of the frequency of measurements on event reports. For instance, do open-ended probes for events within-days elicit more stressful events than end-of-day event checklists (for example, due to forgetting events at the end of the day or increased attention for events within-days), or do we find report of fewer stressful events within-days compared to end-of-day checklist (for example, due to passive recognition of checklist events rather than active recall, or due to a more general evaluation of the demands of the day instead of the more discrete events occurring during the day)?

In the past. several methodological approaches have been used to elicit information about daily events, ranging from open-ended questions to structured checklisis. The advantages and disadvantages of the various methods have been discussed by Stone et al. (1991). The simplest approach has been the open-ended one, 
where subjects were asked about the day's most stressful event (Rehm, 1978) or asked if anything went wrong during the day (Caspi et al., 1987; Eckenrode, 1984). If a respondent answered 'yes', she or he was then asked to describe what had happened. Responses were then classified into discrete categories. Strongly influenced by the work of Holmes and Rahe on major life events (Schedule of Recent Events; Holmes \& Rahe, 1967), the most often used method of assessing daily events is the event checklist, where, from a list of events, subjects are asked to identify those that have occurred to him or her over a specified time period, ranging from one day to one month. Checklists covering the last month include the Unpleasant Events Schedule and the Pleasant Event Schedule (Lewinsohn \& Amenson, 1978; Lewinsohn \& Talkington, 1979), the Hassles and Uplifts scale (Kanner et al., 1981). the Inventory of Small Life Events (Zautra \& Guarnaccia, 1986), and the Everyday Problem Checklist (Vingerhoets \& Menges, 1989). Items on these scales were formulated or taken from existing scales to cover events in major life domains such as family, work, leisure, and household maintenance. The much shorter checklists designed to be used on a daily basis include the Daily Life Experience checklist (DLE; Stone \& Neale, 1982), the Daily Stress Inventory (DSI; Brantley et al., 1987), and the Daily Stress Scale (DSS; Bolger et al., 1989a). To develop daily checklists with representative events, individuals from the population in which the checklist was later intended to be used were asked to provide open-ended descriptions of daily events and situations that fit certain criteria (for example, 'stressfulness", (Brantley et al., 1987)). Elicited events were then grouped into various major life domains (e.g. DLE: work, leisure, family, friends, financial, and other; DSS: overloads. interpersonal conflicts) or chosen on the basis of reported event frequency (DSI).

In the present study, we used the Experience Sampling Method (ESM) (Csikszentmihalyi \& Larson, 1987; de Vries, 1987; de Vries, 1992) to investigate the types and cognitive appraisals of stressful events in the everyday life of white collar men. In the context of their normal social networks, settings and activities, 88 subjects completed ESM self-reports at semi-random intervals ten times a day over 5 consecutive days, including three work and two non-work days. Stressful daily events were assessed by the open-ended question 'did anything stressful take place since the last assessment?' Our choice for the open-ended approach was based on the fact that most daily event checklists are not suitable for frequent use during a single day. Also, we did not want to restrict a priori the definition of 'stressful' to certain categories of problems, being more interested in how white collar men themselves describe their stressful daily events. The ESM affords several advantages for investigating stress from a transactional perspective. Since events are assessed at frequent intervals each day, not long after the occurrence of the event, we are able to look at stress processes more dynamically as they evolve in time, but also more dynamically in the sense that not only the person but also the natural environment of the person is taken into account in the assessment of a stressful experience. These processes can be empirically assessed with a minimum of confounding due to biased recall (e.g. 'effort after meaning') or forgetting (Bower, 1981). In this way, we are able to elicit information about the appraised meaning of an event at the time it occurs, and about the social or physical context at that moment. Using ESM, even very minor sources of stress can be recorded, which might otherwise be forgotten at the end of the day. Therefore, more reliable estimates of rates of event occurrences are possible. 


\section{SUBJECTS}

The subject selection procedure has been described in Chapter 2 (see 'Subjects' and 'Procedure' sections). As shown in Table 3.1., this procedure resulted in two groups (42 High Stress 'HS' subjects and 46 Low Stress 'LS' subjects) that were very similar in sociodemographic characteristics. This means that any differences between the groups in the use of time or in exposure to stressful events are unlikely to reflect differences in age, marital status, or family composition.

Table 3.1. Demographic characteristics of the study participants.

$\begin{array}{ll}\text { LS } & \text { HS } \\ \mathrm{n}=46 & \mathrm{n}=42\end{array}$

p-value

(2-tailed)

\section{demographic variables}

\begin{tabular}{|c|c|c|c|}
\hline age & mean (st.dev.) & mean (st.dev.) & \\
\hline mean & $42.7(7.7)$ & $41.5(6.0)$ & ns \\
\hline range & $27-57$ & $28-52$ & \\
\hline \multicolumn{4}{|l|}{ marital status } \\
\hline married & $41(89.1 \%)$ & $37(88.1 \%)$ & ns \\
\hline unmarried & $2(4.3 \%)$ & $2(4.8 \%)$ & \\
\hline living together & $2(4.3 \%)$ & $2(4.8 \%)$ & \\
\hline divorced & $\mathrm{i}(2.2 \%)$ & $1(2.4 \%)$ & \\
\hline \multicolumn{4}{|l|}{ household composition } \\
\hline living alone & $3(6.5 \%)$ & $3(7.1 \%)$ & ns \\
\hline couple with children & $37(80.4 \%)$ & $34(81.0 \%)$ & \\
\hline couple without children & $6(13.0 \%)$ & $5(11.9 \%)$ & \\
\hline
\end{tabular}

\section{METHODS}

Measures relating to stressful events and to daily activities are derived from ESM reports, as described in Chapter 2 (see 'Description of the ESM instrument' section). End-of-the-day measures used in the current analyses include the Daily Stress Scale (Bolger et al., 1989a) and a global rating of the day's stressfulness. Also included in this analysis are measures of life events, recorded with the List of Threatening Experiences (LTE-Q:Brugha et al., 1985) and chronic difficulties, assessed with the Long-term Difficulties Questionnaire (LLM: Hendriks et al., 1990) (see chapter 2 'Cross-sectional instruments' section).

Analyses were done with SPSS/PC+ 4.0 on Macintosh. Unless stated otherwise, differences in variables were tested with non-parametric tests, using twotailed significance tests. In the analysis of the ESM data, measures were aggregated so that the subject was used as the unit of analysis (Larson \& Delespaul, 1992). 


\section{A CASE EXAMPLE}

The following case study of a subject who experiences high levels of stress in his. life illustrates the level of detail this assessment method provides about the ebb and flow of an individual's stressful experiences during the course of five days.

Jan, 41 years old, married with child (one year old), works for the municipal government as a building and housing inspector. He experiences his life as somewhat overloaded and uncontrollable lately (at least for the past 3 months; PSS score $=17$ ) and describes the following chronic difficulties: work is demanding due to regulatory changes, he has no time to study and is therefore falling behind, there is not enough time for home upkeep and garden maintenance, and too little real leisure time. When asked which psychosomatic complaint bothers him the most, he mentions stomach ache. The five days of ESM for Jan are shown in Figure 3.1. Here we see that on Thursday he has a busy day at work, with difficult assignments. Several work-related stressful events are reported, like settling a petition and dealing with a request for subsidy, with a concurrent increase in feelings of agitation and stomach pain. Positive affect also seems to be below average during that day at work. While driving home he seems to unwind, he starts to feel more relaxed, is less bothered by stomach pain and his mood improves. The next day, Jan describes a similar pattern, with several stressful events in the morning at work, increasing agitation, stomach pain, and a slight dip in positive affect. In the afternoon and evening, several events are described that relate to his child's health and to household maintenance, but these events seem to have hardly any effect on mood state or physical complaint, since after leaving work Jan reports less agitation, stomach pain and higher positive mood. In contrast with the work days, the weekend is spent with relatively few stressful experiences and on the whole he feels relaxed, in a good mood, and not bothered by stomach pain. Most of the time is spent with wife, child and other family members: e.g. playing with son, visiting family, entertaining family, working on car, and watching t.v. Although Monday -back at work- no events are reported, we can see that Jan gets slightly more agitated again and is more bothered by stomach pain than during the weekend. ESM data indicated that work is the major source of stress for Jan, as was also described in the chronic difficulties questionnaire. Household maintenance was also reported as stressful on several occasions. but had less influence on his emotional state or physical complaint. 
Figuur 3.1. Example of one subject's stressful events. emotional states (Agitation, Positive Affect) and psychosomatic complaint reported during five days of ESM
Apitacioa

Complaint

Poritive mood

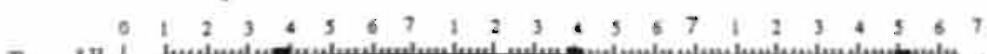

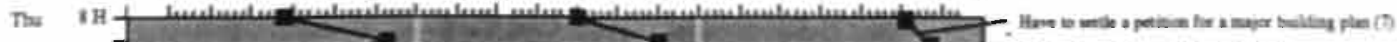
12 H] 年

Fin
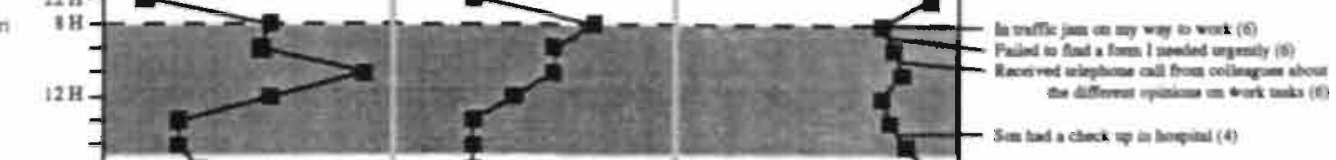

se
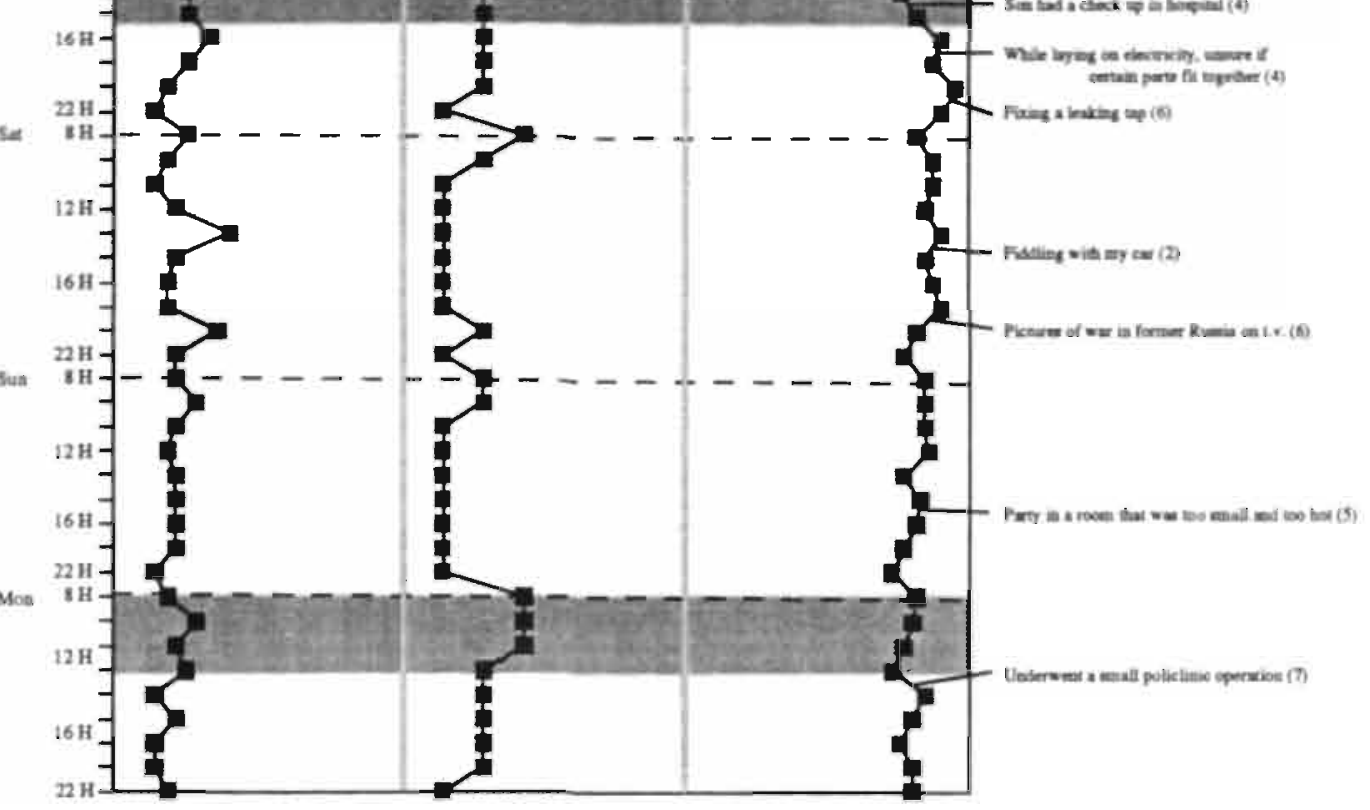


\section{STRESSFUL EVENTS IN DAILY LIFE: FREQUENCY, CONTEXT AND APPRAISAL}

We first examine the frequency, nature, and evaluation of events reported as stressful by this sample of white collar men. The ESM forms asked subjects to describe any stressful events or situations which may have taken place in the interval since the last ESM report (on average 90 minutes). Subjects' descriptions of stressful daily events were coded according to context, with categories work, nefwork (events. concerning family, friends, and acquaintances), household/financial, leisure, personal health (somatic and psychological health), transport, and other. In addition, events were classified according to whether or not they involved a social interaction and/or a task demand (see chapter 2 'Coding of ESM measures section). Subjects also rated the events they reported on the dimensions unpleasantness, imporiance, predictability, controllability, and frequency of prior occurrence.

\section{Event frequency}

Subjects reported a total of 626 stressful events during the five days of ESM, with a mean of 7.1 events per subject (median $5.0 ; \mathrm{s.d} .=7.3)$. Events as a percentage of total beeps averaged $16.8 \%(\mathrm{~s} . \mathrm{d} .=16.5)$. Of all stressful events reported, $33 \%$ were rated as highly stressful (score of $\geq 5$ ).

\section{Event context}

The distribution of events by life domain (expressed as frequency of each type of stressor divided by total beeps x 100) is shown in Figure 3.2. Subjects reported stressful events related to work and to social network most often. Of all events reported, $48 \%$ were work-related. Since the frequency of work events could relate to the amount of time spent at work, we also examined the probability of the occurrence of a work event given the amount of time spent at work. On average, subjects reported being at work on $31 \%$ of total beeps. 52 of the subjects reported a higher percentage of work-related events than would be expected based on the amount of time they spent at work (Wilcoxon Matched-Pairs Signed-Ranks test, $\mathrm{p}<.001$ ). This indicates that work-related events were not only absolutely but also relatively high in frequency. Next, we examined the correlations between the percentages of events reported in the various contexts (Table 3.2). The strongest correlations were between network and household events ( $r h o=.35 ; \mathrm{p}<.01$ ) and between network and work events $(r h o=.35 ; \mathrm{p}<.01)$. These correlations indicate that the experience of stressful events was not constrained to a particular life domain for an individual.

Table 3.2. Correlations (Spearman) between the percentages of events in the various contexts ${ }^{\mathrm{a}}$.

\begin{tabular}{lllllll}
\hline & 1 & 2 & 3 & 4 & 5 & 6 \\
\hline 1. Work & & & & & & \\
2. Network & $.35^{* *}$ & & & & & \\
3. Household & $.29^{* *}$ & $.35^{* *}$ & & & & \\
4. Leisure & $.21^{*}$ & .20 & .14 & & & \\
5. Personal Health & -.18 & .01 & .04 & -.16 & & \\
6. Transport & .15 & .07 & .10 & -.10 & .12 & .12 \\
7. Other & .05 & $.29 * *$ & .20 & .10 & -.10 & \\
\hline
\end{tabular}

*p<.05; * *p<.01; ${ }^{a} \mathrm{n}=88$ 


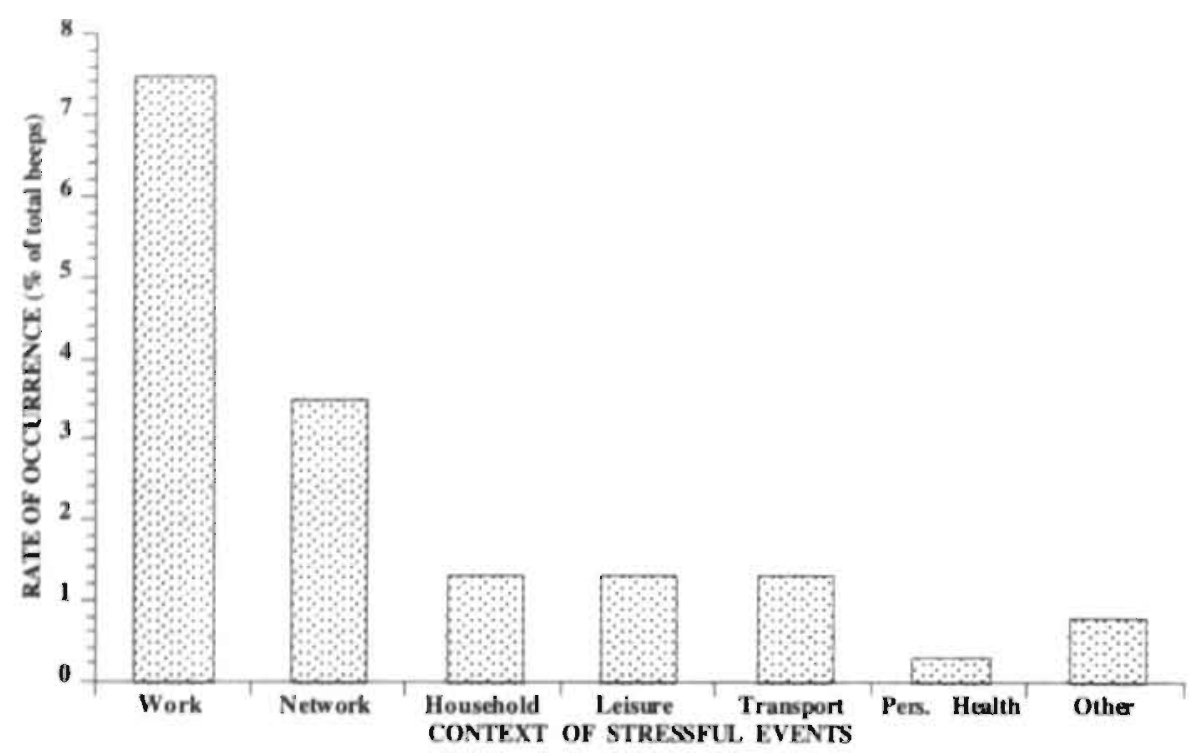

Figure 3.2. Frequency of stressful daily events by life domain.

In Figure 3.3. the percentage of events coded as task demand, as social interaction and as internal or external are shown. A minority of stressful events were characterized as social interaction or task demand events, and the large majority of events were (theoretically) observable or verifiable. Internal events (e.g. worries, anxieties) represented only $6.6 \%$ of all reported events. Examples of reported stressful events for each category are shown in Table 3.3.

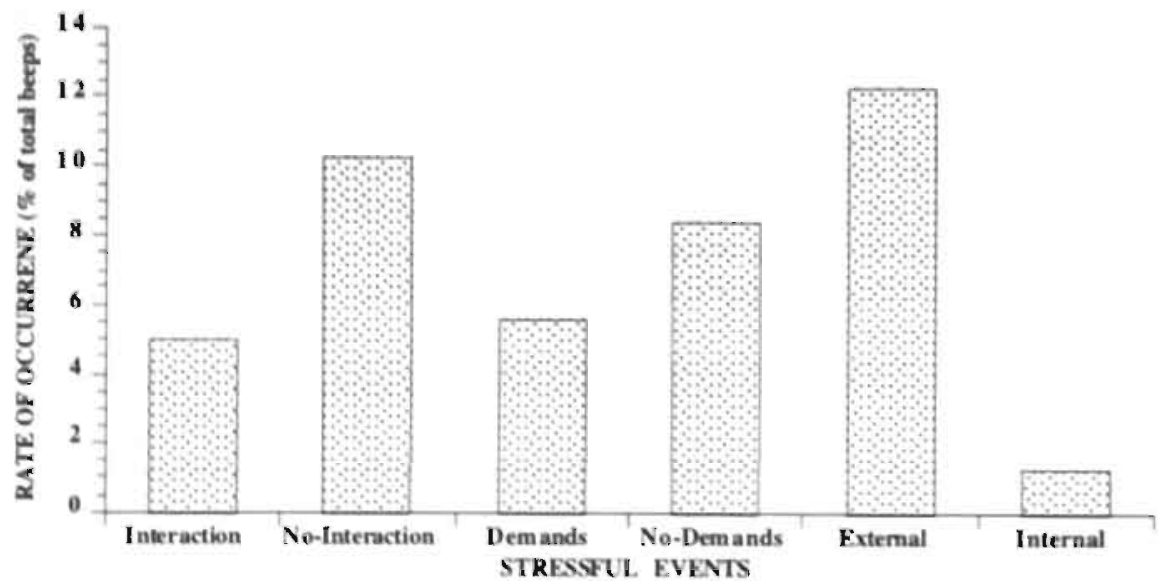

Figure 3.3. Frequency of stressful daily events: social interaction, task demand, and external/internal events. 
Table 3.3. Examples of stressful events.

\section{Work}

(events concerning boss, supervisor, clients. co-worker, general happenings at work)

'behind on computer work'. 'while working getting a lot of crazy telephone calls". "difficult discussion at work meeting'. 'job evaluation conversation'. 'a lot of work, a lot of different things'. 'can not fix computer programme error'. 'discussion about changes in organisation'. 'writing an account that has to be finished today". "making appointments under high time-pressure'.

\section{Network'}

(events concerning spouse, partner, children, relatives, friends, other acquaintances)

'discussion with spouse about arriving too late at friends' house'. 'getting children ready in time for school', 'children are annoying', "argument with spouse about household affairs". 'received message that sister has been in car accident' 'whole family wants attention at the same moment'. 'baby-sitter cancelled appointment:

\section{Houschold}

(events concerning general housework, errands,

family related duties, financial problems, etc.)

having to do a lot of errands in a short time period'. 'have to make a decision about buying a couch'. 'working in garden under high timepressure'. 'having to do a lot of dishes'. 'trying to fix a dripping tab'. 'while repairing bicycle chain breaks'. 'cleaning-up the mess without any support'.

\section{Leisure}

(events concerning hobbies, sports, outings. vacation, etc.)

'playing tennis match'. 'driving in a sports car'. "watching soccer: favoritc team missed a lot of chances'. 'performance with choir'. 'music band played out of tune' 'jogging', 'watching the Euro cup soccer game (Holland-Scotland)'

Personal Health

(events concerning illness, injury of the subject

himself, worries about health, nerves)

'headache'. 'nervous". 'underwent a small operation'.

\section{Transport}

(e.g. missed bus, traffic jam, unusual traffic)

'heavy traffic while driving home'. 'missed bus'. 'cycling in pouring rain'. 'had to hit the brakes suddenly', 'could not find a place to park the car'. "almost run over by a car while on my bike".

\section{Social Interaction}

(c.g. argument, conflict, conversation)

'squabble with children'. 'discussion with colleague about problems in the organisation'. 'conflict with partner about upcoming visit parents'. 'meeting with department'. 'discussion with spouse about furure childcare'. 'argument with boss'. 'had to give bad news to a client'. 'argument with spouse about how to act in certain situation'.

\section{Task Demands}

(e.g. difficult or problematic tasks, lime pressure, deadlines, a lot of work, failure at task)

'pressured by unexpected visit of client at work'. 'have to hurry washing, shaving, and dressing because woke up too late'. 'difficulties painting the wall', 'failed to saw up the wood into pieces'. 'had to take care of a lot of errands during my lunch break'. 'the usual hectic Saturday: running back and forth'. 'too much work to do'.

\section{Event appraisals}

Correlations among event appraisals are shown in Table 3.4. As would be expected, the strongest correlation was between event stressfulness and unpleasantness. Events that were less predictable or less controllable were also more unpleasant. Next we examined whether the various types of stressful events differed on the six appraisal dimensions. Figure 3.4 shows the mean scores for the types of stressful events on the different appraisal dimensions. Surprisingly, Leisure events were rated as most stressful, but they were the least unpleasant by far and the most. expected. An explanation could be that certain Leisure events (e.g. sport games) are experienced as stressful and exciting but that the stress is of a positive and 
challenging nature. Whether the types of events differed significantly from each other with regard to the appraisal dimensions was only tested for a subset of events, since not all event types were reported by all subjects thus resulting in small sample sizes for certain event types. Comparisons were made for Work versus Network, Interactions versus Non-Interaction, and Demand versus Non-Demand events. Work events were reported as occurring significantly with greater regularity than Network events (frequency: Wilcoxon Matched-Pairs Signed-Ranks test, p<.05), but no significant differences were found for the other appraisal dimensions. Interaction events were appraised as significantly more important $(\mathrm{p}<.01)$, more controllable $(\mathrm{p}<.001)$, and occurring more often $(\mathrm{p}=.05)$ than Non-Interaction events. Demands were appraised as significantly less important than Non-Demands events. The other appraisal dimensions showed no significant differences here.

Table 3.4. Correlations (Spearman) between event Appraisais ${ }^{\mathrm{a}}$ and mean appraisal scores ${ }^{\mathrm{b}}$.

\begin{tabular}{|c|c|c|c|c|c|c|c|}
\hline & I & 2 & 3 & 4 & 5 & $\bar{M}$ & $S D$ \\
\hline 1. Unpleasantness & & & & & & 3.6 & 1.4 \\
\hline 2. Stressfulness & $.41 * *$ & & & & & 3.9 & 1.0 \\
\hline 3. Importance & .03 & $.22 * * *$ & & & & 4.1 & 1.5 \\
\hline 4. Controllability & $-.23 * * 0$ & -.03 & .03 & & & 3.7 & 1.5 \\
\hline 5. Chronicity & -.01 & -.03 & $-18 * *$ & $.22 * * *$ & & 3.1 & 1.8 \\
\hline 6. Predictability & $-.31 * *$ & -.03 & $19 * * *$ & .07 & $18 * * *$ & 3.4 & 1.4 \\
\hline
\end{tabular}

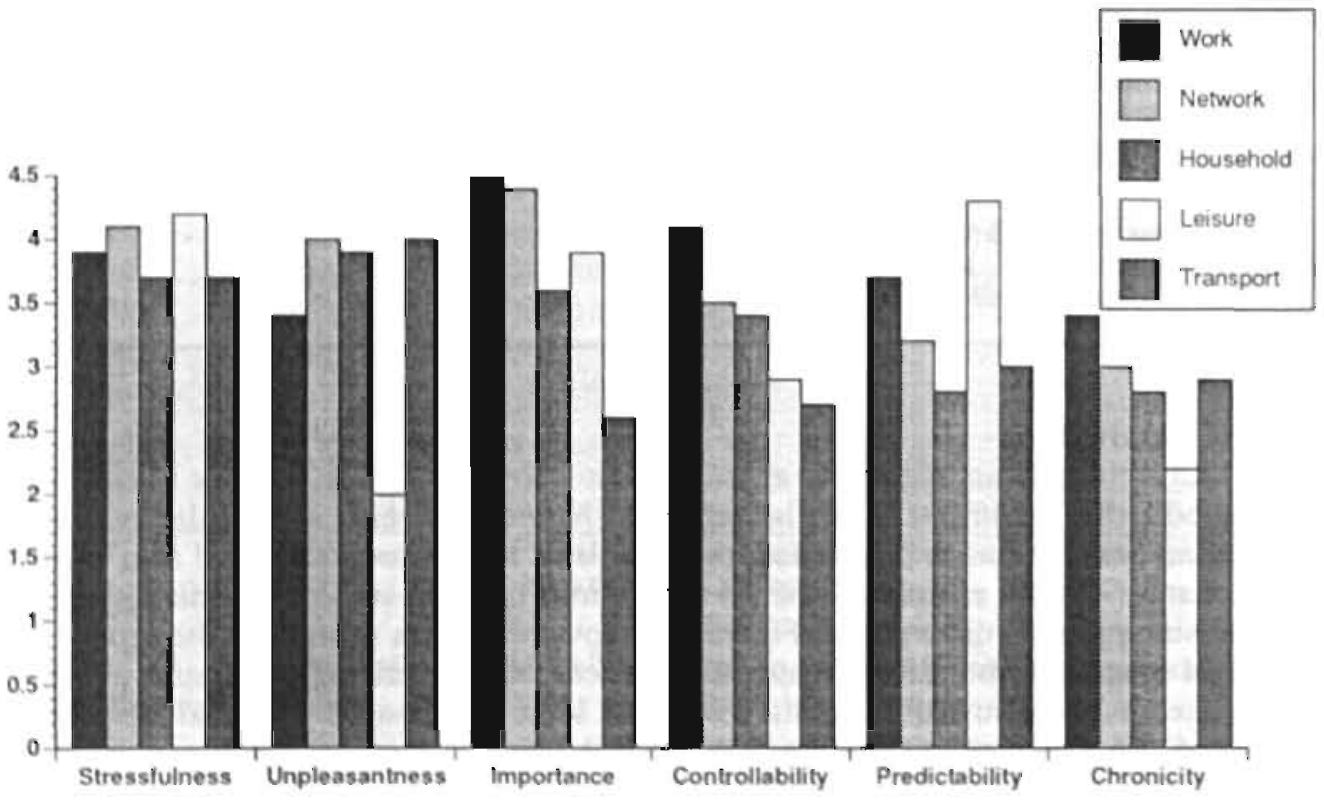






Figure 3.4. Mean appraisal scores calculated for events in the context of $(\mathbf{A})$ work $(n=65)$. network $(n=36)$, household $(n=22)$, leisure $(n=19)$, transport $(n=29)$, and for $(B)$, no-interaction

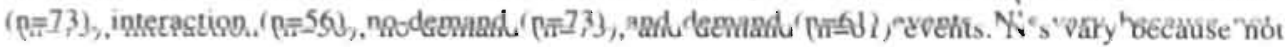
all event types were reported by all subjects.

In summary, subjects reported stressful work events most often, followed by social network events, and the experience of stressful events was not limited to a certain context. In general, events that were appraised as less predictable and less controllable were the most unpleasant. Stressful work events differed from stressful network events only in the appraised frequency of past occurrence, as was confirmed by the current event frequency scores. Negative social interactions were appraised as most important, most controllable, but also as occurring with greater regularity in the past, when compared to all other events, although current frequency scores revealed that social interaction events were actually reported relatively rarely during ESM sampling.

\section{RELATIONSHIP OF DAILY EVENTS TO LIFE EVENTS AND CHRONIC DIFFICULTIES}

It has been suggested (e.g. Hinkle, 1974; Kanner et al., 1981; Kaplan, 1979) that life events may initiate a cascade of daily stressors; for example, a divorce may lead to a series of new hassles in the context of keeping house, making meals, or keeping up social contacts, which did not have to be dealt with previously. As described in Chapter 2, life events were recorded with the questionnaire form of the List of Threatening Experiences (LTE-Q; Brugha et al., 1985), and chronic siress was assessed with the Long-term Difficulties Questionnaire (LLM; Hendriks et al., 1990), which focuses on problems in relation to work/study, housing, physical environment, leisure, finance, and social relationships (see 'Cross-sectional instruments' section). 
Table 3.5. Rate of daily event occurrence by increasing number of life-events and chronic. difficulties.

Number of Life-Events

\begin{tabular}{|c|c|c|c|c|c|c|c|}
\hline & \multicolumn{2}{|c|}{$0(n=51)$} & \multicolumn{2}{|c|}{$!(n=22)$} & \multicolumn{2}{|c|}{$\geq 2(n=15)$} & \multirow[t]{2}{*}{$p$} \\
\hline & mean & sd & mean & sd & mean & sd & \\
\hline $\begin{array}{l}\text { Frequency of } \\
\text { Daily Events }\end{array}$ & $15.7 \%$ & $15.4 \%$ & $17.4 \%$ & $17.9 \%$ & $19.4 \%$ & $18.8 \%$ & ns \\
\hline
\end{tabular}

Chronic Difficulties

\begin{tabular}{|c|c|c|c|c|c|c|c|}
\hline & \multicolumn{2}{|c|}{ None $(n=6)$} & \multicolumn{2}{|c|}{ Minor $(n=39)$} & \multicolumn{2}{|c|}{ Severe $(n=33)$} & p \\
\hline Daily Eventsa & $11.5 \%$ & $17.8 \%$ & $10.9 \%$ & $10.9 \%$ & $24.1 \%$ & $20.3 \%$ & $<.01$ \\
\hline Unpleasantness & & & & & & & \\
\hline of Daily Events ${ }^{b}$ & 2.3 & 1.0 & 3.3 & 1.6 & 4.0 & 1.3 & $<.01$ \\
\hline Freq $\times$ Unpleasant ${ }^{c}$ & 34.2 & 60.6 & 36.9 & 41.4 & 92.4 & 76.0 & $<.01$ \\
\hline
\end{tabular}

a Mean frequency of events as a percentage of total beeps

b Mean unpleasantness rating of events

${ }^{c}$ Mean frequency by unpleasantness rating

When we compared subiects who resoorted no. one. or two or mote life-pevents in the past year on the percentage of stressful daily events reported during the ESM period, however, no differences in rate of stressful daily events occurrences were observed. Subjects with more life-events did not report more daily events (Table 3.5.). Dividing the total scores on the chronic difficulties scale into three categories (none, minor [average or below average in number], or severe [above average in number], we found that subjects with higher scores on the chronic difficulties scale reported more daily events (Kruskal-Wallis one-way anova; p<.001) and rated events as more unpleasant $(\mathrm{p}<.05)$. The sum of frequency by unpleasantness ratings was significantly higher as the chronic difficulties scores increased ( $p<.001)$ (Table 3.5.).

In summary, not life events but chronic difficulties were positively related to the frequency and intensity of stressful daily events reported during the ESM period.

\section{COMPARISON OF WITHIN-DAY EVENT AND END-OF-DAY MEASURES}

We next examined how two different methods of daily event assessment compared in terms of the number and type of stressors reported; in other words, do repeated within-day event reports provide different information than checklist results obtained at the end of the day? Subjects were asked at the end of each Experience Sampling day how stressful the day had been and completed a short checklist, the Daily Stress Scale (Bolger et al., 1989a). This instrument (as described in 'Description of ESM instrument' section chapter 2), consists mainly of items concerning demands (e.g. a lot of work at home, at work) or interpersonal conflicts (e.g. argument with spouse, child, colleague), with two additional items concerning transportation and financial problems. 
Table 3.6. Prevalence of events obtained by within-day (ESM) assessments compared to end-ofday checklist assessments.

Did (at least 1) event occur?

According to:

\begin{tabular}{lccc}
\hline Sources of Stress & ESM & Checklist & Chi-Square \\
\hline $\begin{array}{c}\text { Home stress } \\
\text { Overload }\end{array}$ & $7.1 \%$ & $36.8 \%$ & $<.001$ \\
Argument & $8.6 \%$ & $17.5 \%$ & $<.00 !$ \\
Work stress & & & \\
Overload & $15.3 \%$ & $40.0 \%$ & $<.001$ \\
Argument & $19.6 \%$ & $7.0 \%$ & $<.001$ \\
Home Total & $14.1 \%$ & $43.9 \%$ & $<.001$ \\
Work Total & $26.8 \%$ & $40.7 \%$ & $<.001$ \\
\hline
\end{tabular}

The number of ESM events reported each day was positively correlated with the number of events reported on the checklist at the end of the day ( $r h o=.43$. $\mathrm{p}<.001: \mathrm{n}=439$ ) and with the end of the day global rating of the day's stressfulness ( $r h o=.52, \mathrm{p}<.001 ; \mathrm{n}=404$ ). We compared the frequency of reports of home stress and work stress (rated as overloads or as arguments) within-days with the reports of these events at the end of the day. Scores were rated 0 if no event was reported and 1 if at least one event was reported. Results are shown in Table 3.6. Except for work arguments, a higher frequency of events was reported with the checklist at the end of the day compared to the within-day measurements. All differences were significant. Similar results were found when only checklist events that were at least somewhat unpleasant were analyzed. We can conclude: that by recognition (as with checklists) many more events are reported than when actively asked for (open-ended question). Since some checklist items were of a more global nature (e.g. 'a lot of work at home', 'a lot of work at work'), it is also possible that when summarizing one's experiences at the end of the day these items are more easily endorsed, while during the day events are more discrete and too small in itself to be reported. Perceptual biases (e.g. effort after meaning) may he more likely to occur at the end of the day than at the momentary ESM assessments.

In summary, many more events were reported with the checklist at the end of the day compared to the open-ended, within-day measurements.

\section{DIFFERENCES BETWEEN HIGH AND LOW STRESS GROUPS}

To investigate whether individual perceptions of high versus low levels of stress are associated with personal characteristics and daily life experiences, differences between the two stress groups were tested with respect to psychological characteristics, the distribution of daily activities, and measures of stressful daily events. 


\section{Psychological characteristics}

The two groups were compared on the following measures: life events (LTEQ), chronic difficulties (LLM), psychological and somatic complaints (SCL-90), psychosomatic symptoms (PSC), depressive symptomatology (Zung), trait anxiety (STAI), trait anger (STAS), personality characteristics (Dutch abridged MMPI: NVM), and coping style (UCL). These instruments have been described in Chapter 2 (see section 'Cross-sectional instruments').

Results are shown in Table 3.7. HS subjects reported significantly more chronic difficulties during the last year than LS subjects, but no more life events. HS subjects also exhibited higher scores on both psychological (anxiety, anger, and depression) and psychosomatic complaints questionnaires than LS subjects and scored higher on the personality dimensions negativism, somatization, timidity and serious psychopathology. Additionally, HS subjects seem to use passive coping styles (palliative reaction, depressive reaction, expression of emotion) more often than LS subjects and an active, problem-focused coping style less often. Where possible, mean scores for both samples were compared to published norms for the Dutch population. The general pattern found was that LS subjects scored average or below average compared to norm scales, while HS subjects scored above average or high.

Frequency and intensity scores for the seven different categories of chronic difficulties are shown in Table 3.8. HS subjects reported significantly more chronic difficulties for all categories except for the category Family (i.e. parents, children, brothers / sisters, in-laws). Work and Housing difficulties had the highest prevalence in both groups, and HS subjects reported relational difficulties with the partner relatively frequently. The two groups were similar in the kind and ranking order of frequency of work difficulties mentioned: 1) high work pressure or work load, 2). conflicts or differences in opinion with boss or colleagues, 3) little appreciation and 4) changed or indistinct job functions due to reorganization. With regard to difficulties with partner, again several specific problems were mentioned by both groups: 1) conflicts or differences in opinion (for example, concerning household tasks or children), 2) low frequency of sexual contact (not in the mood, too busy), and 3) diminished interest in each other or doubts about choice of partner. Housing difficulties were more diverse, with problems such as conflicts with neighbours, criminality (vandalism, theft, drugs) and pollution. With regard to the intensity of chronic difficulties, only work difficulties were scored as more intense by HS subjects $($ mean $=2.0$ (s.d. 0.5) for HS subjects versus mean= 1.7 (s.d. 0.4) for LS subjects, Mann-Whitney $U$ test, $\mathrm{p}<.05$ ), and a trend in the same direction was found for difficulties concerning partner (mean=2.1 (s.d. 0.7) for HS subjects versus mean=1.7 (0.3) for LS subjects, $p=.06$ )

As shown in Table 3.9, psychosomatic complaints occurred with regularity (at least once a week) in a higher percentage in HS than in LS subjects and occurred more frequently overall in the HS group. Significant differences between groups in frequency of complaints (using the full range of frequency ratings) were found for: fatigue, depression, backache, headache, insomnia, general weakness, stomach pain, heart palpitations, and nausea. The same psychosomatic complaints were also rated as significantly more severe in HS subjects (p-values all <.01). The nine subjects who reported no complaint at all were all from the LSi group. 
Table 3.7. Characteristics of high and low stress groups.

\begin{tabular}{|c|c|c|c|}
\hline & $\begin{array}{l}\text { LS } \\
\mathrm{n}=46 \\
\text { mean (st.dev) }\end{array}$ & $\begin{array}{l}\text { HS } \\
\mathrm{n}=42 \\
\text { mean (st.dev) }\end{array}$ & $\begin{array}{l}\text { p-value } \\
\text { (2-tailed) }\end{array}$ \\
\hline Life-events & $.5(.7)$ & $.8(.9)$ & ns \\
\hline Chronic difficulties & $19.5(2.3)$ & $23.3(4.0)$ & $\cdots$ \\
\hline SCL-90 (total) & $102.5(11.1)$ & $147.4(39.3)$ & $\cdots$ \\
\hline Trait anxiety & $28.3(4.4)$ & $39.8(7.6)$ & $\cdots$ \\
\hline Depression & $36.5(5.5)$ & $48.4(7.7)$ & $\cdots$ \\
\hline Trait anger & $14.6(4.1)$ & $18.0(4.6)$ & $\cdots$ \\
\hline Psychosomatic symptoms & $6.1(5.6)$ & $27.5(23,2)$ & $\cdots$ \\
\hline \multicolumn{4}{|l|}{ Personality: } \\
\hline Negativism & $10.0(5.5)$ & $19.0(6.8)$ & $\cdots$ \\
\hline Somatization & $2.8(2.8)$ & $9.5(7.3)$ & $\cdots$ \\
\hline Timidity & $8.3(6.3)$ & $11.3(7.4)$ & • \\
\hline Serious Psychopathology & y $1.3(1.9)$ & $2.8(2.5)$ & $\cdots$ \\
\hline Extraversion & $15.4(5.3)$ & $16.5(5.6)$ & ns \\
\hline \multicolumn{4}{|l|}{ Utrecht Coping List: } \\
\hline Active coping & $21.1(4.0)$ & $17.3(3.4)$ & $\cdots$ \\
\hline Palliative reaction & $14.6(3.0)$ & $17.2(3.3)$ & $\cdots$ \\
\hline Avoidance coping & $14.1(2.5)$ & $15.1(3.6)$ & ns \\
\hline Social support & $11.7(2.7)$ & $12.5(2.7)$ & ns \\
\hline Depressive reaction & $8.8(1.6)$ & $12.6(3.4)$ & $* *$ \\
\hline Expression emotions & $5.8(1.3)$ & $6.9(1.7)$ & $* *$ \\
\hline Comforting cognitions & $10.8(2.6)$ & $11.7(2.8)$ & $\mathrm{ns}$ \\
\hline
\end{tabular}

* $p<05 ; \cdots p<01 ; \cdots * p<001$

Table 3.8. Percentage of HS and LS subjects reporting chronic difficulties in each life domain.

\begin{tabular}{|c|c|c|c|}
\hline & $\begin{array}{l}\text { low PSS } \\
\mathrm{n}=40\end{array}$ & $\begin{array}{l}\text { high PSS } \\
n=42\end{array}$ & $\begin{array}{l}\text { p-value } \\
\text { (2-tailed })\end{array}$ \\
\hline 1. Work difficulties & $39 . i \%$ & $88.1 \%$ & $* *$ \\
\hline $\begin{array}{l}\text { 2. Housing/Finance difficulties } \\
\text { Relational difficulties }\end{array}$ & $43.5 \%$ & $71.4 \%$ & $* *$ \\
\hline 3. Partner & $23.9 \%$ & $53.7 \%$ & $*$ \\
\hline 4. Family & $23.9 \%$ & $41.5 \%$ & ns \\
\hline 5. Friends/Acq. & $19.6 \%$ & $38.1 \%$ & $*$ \\
\hline 6. Leisure difficulties & $19.6 \%$ & $39.1 \%$ & * \\
\hline
\end{tabular}

p<05: ** p<.01: "*** p<001; Group differences in the percentage of reported chronic difficulties were tested by
means of chi-square tests. 
Table 3.9. Psychosomatic complaints in HS and LS subjectsa.

\begin{tabular}{|c|c|c|c|}
\hline & low PSS & high PSS & $\begin{array}{l}\text { p-value }{ }^{b} \\
\text { (2-tailed) }\end{array}$ \\
\hline I. Fatigue & $39.2 \%$ & $66.7 \%$ & $* * *$ \\
\hline 2. Depression & $2.2 \%$ & $42.9 \%$ & $* * *$ \\
\hline 3. Backache & $17.3 \%$ & $38.1 \%$ & * \\
\hline 4. Headache & $8.7 \%$ & $35.7 \%$ & $* * *$ \\
\hline 5. Insomnia & $10.9 \%$ & $31.0 \%$ & $* * *$ \\
\hline 6. Weakness & $0.0 \%$ & $19.1 \%$ & $* * *$ \\
\hline 7. Eye pain & $8.7 \%$ & $16.6 \%$ & ns \\
\hline 8. Stomach pain & $2.2 \%$ & $11.9 \%$ & $* * *$ \\
\hline 9. General stiffness & $2.2 \%$ & $11.9 \%$ & ns \\
\hline 10 Heart palpitations & $2.2 \%$ & $11.9 \%$ & $*$ \\
\hline 11 Dizziness & $4.3 \%$ & $11.9 \%$ & ns \\
\hline 12. Diarrhea / constipation & $0.0 \%$ & $9.5 \%$ & ns \\
\hline 13. Nausea & $0.0 \%$ & $7.2 \%$ & $* *$ \\
\hline 14. Spastic colitis & $0.0 \%$ & $7.1 \%$ & ns \\
\hline 15. High blood pressure & $2.2 \%$ & $4.8 \%$ & ns \\
\hline 16. Asthma & $0.0 \%$ & $2.4 \%$ & ns \\
\hline 17. Gastric ulcer & $0.0 \%$ & $0.0 \%$ & $\mathrm{~ns}$ \\
\hline \multicolumn{4}{|l|}{ " $p<05, \cdots p<01, \cdots p<001$} \\
\hline $\begin{array}{l}\text { a In the first two columns, the per } \\
\text { a week are reported. In the th } \\
\text { psychosomatic complatints are sh }\end{array}$ & and LS & $\begin{array}{l}\text { osomatic ci } \\
\text { he differe }\end{array}$ & teported \\
\hline
\end{tabular}

\section{Distrlbution of dally activities}

Next, we examined differences between high and low stress groups in time spent in various activity contexts. One might suspect that HS subjects worked more, or relaxed less, than their LS counterparts; daily stress might be reflected in imbalances in work and maintenance versus rellaxation, recreational and social activities. In addition, if, for instance, HS subjects spent more of their time working, then they would have an increased chance of exposure to stressful work events. This analysis was based on ESM activity codes (see Chapter 2. 'Coding of ESM measures section).

Figure 3.5. shows the mean percentage of time spent in the various activities for LS and HS subjects over all study days. HS subjects did not differ significantly from LS subjects in the percentage of time spent in different activities. When we subdivided the category Leisure into Passive (e.g. watching television) and Active (e.g. sports, hobbies) Leisure, no significant differences were found between the HS and LS group (Passive Leisure: $73.9 \%$ versus $79.1 \%$, Active Leisure: $26.1 \%$ versus $20.9 \%$ respectively; ns). Nor did the percentage of time spent in various activities outside working hours (weekend days and after 5 p.m. during work days) show significant between group differences. In summary, we found no differences between the two groups in patterns of time use. 


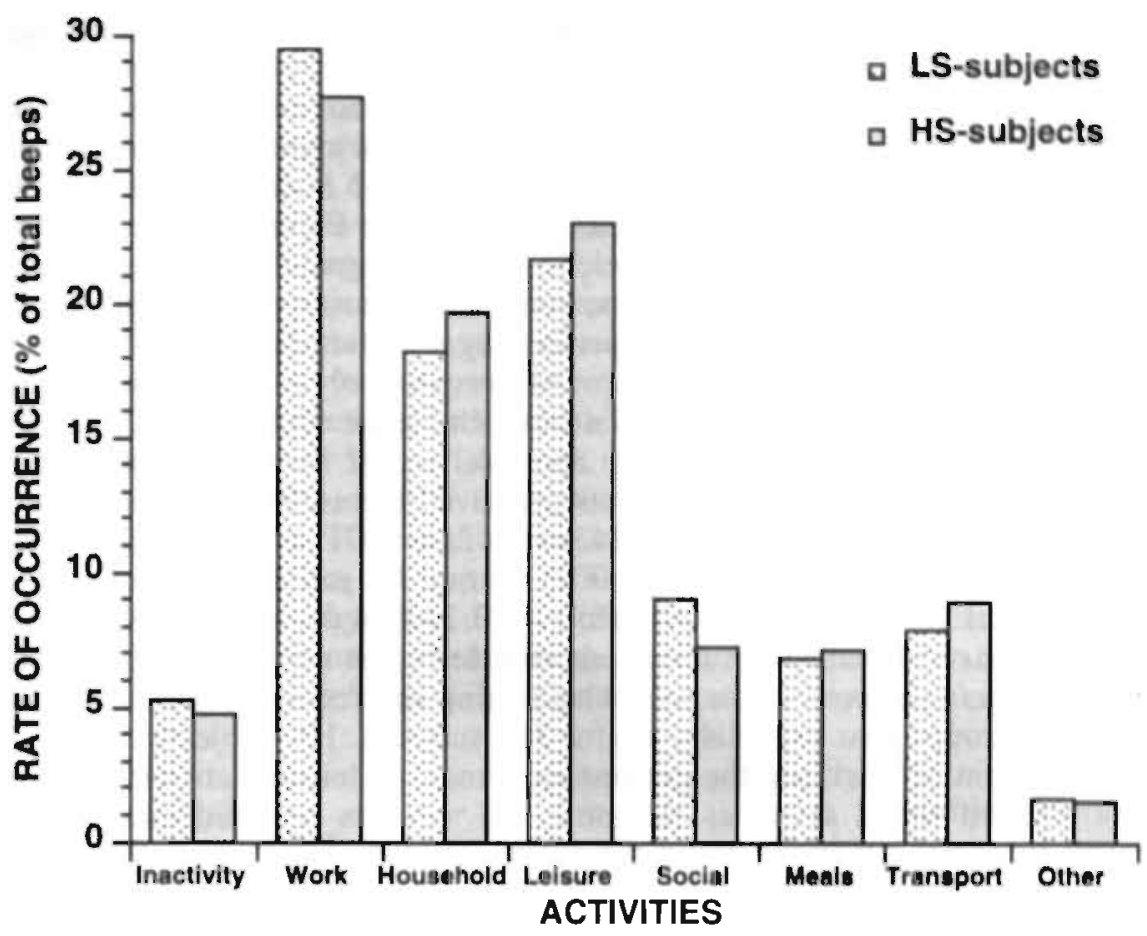

Figure 3.5. Use of time: frequency of activities.

\section{Appraisals of daily activities}

Since appraisals of daily activities were expected to reflect the stressfulness of daily life experiences other than stressful events, we investigated differences between stress groups in how they evaluated their daily activities. Data for this analysis were the ESM ratings of how current activity was experienced in terms of enjoyment, skill, effort, and challenge. As shown in Table 3.10., these dimensions were interrelated.

Table 3.10. Correlations (Pearson) among activity appraisals ${ }^{\mathrm{a}}$.

$1 \quad 2 \quad 3$

\footnotetext{
1. Motivation

2. Skill

3. Effort

$42 * * *$

4. Challenge

$-.32 * * * \quad-.36 * * *$

*...p $<$ <.001

.03

$-.22 * * * \quad .40 * * *$

a Peastson correlations were computed across all beeps $(n=3540)$.
}

Clear differences in how the two group evaluated their activities were found. HS subjects reported that they enjoyed activities less $(4.9$ versus $5.5 ; \mathrm{p}<.001)$, that they were less skillful $(5.7$ versus $6.1 ; \mathrm{p}<.001)$, and that the activities required more 
effort to perform ( 1.8 versus $1.6 ; \mathrm{p}<.01)$ than LS subjects. The two groups did not differ in their assessment of how challenging activities were.

Next, we tested by means of analyses of variance for repeated measures for significant differences in the degree to which the various appraisals were dependent on different activities (Work, Household, Leisure and Social Activities). Mean appraisal scores for the different activities are shown in Figure 3.6a-e. Confirming univariate analyses, the two groups differed in their ratings of the various activities: HS subjects enjoyed doing activities less, perceived themselves as less skillful, and they felt that more effort was required in performing activities $(\mathrm{F}(1,81)=13.46, \mathrm{p}<.001$; $\mathrm{F}(1,81)=6.86, \mathrm{p}<.05 ; \mathrm{F}(1,81)=7.14, \mathrm{p}<.01$, respectively), while there were no differences between groups for the appraisal of challenge $(\mathrm{F}(1,81)=.02$; ns). There were significant differences in the mean appraisal scores for the various activities, with the exception of how skillfull subjects perceived themselves to be in relation to the various activities (enjoyment: $\mathrm{F}(3,243)=45.12, \mathrm{p}<.001$; effort: $\mathrm{F}(3,243)=20.27$, $\mathrm{p}<.001$; challenge: $\mathrm{F}(3,243)=35.81, \mathrm{p}<.001)$. Group by activity interaction effects were significant for enjoyment and effort $(\mathrm{F}(3,243)=4.07, \mathrm{p}<.01 ; \mathrm{F}(3,243)=6.6$, $\mathrm{p}<.001$, respectively), with HS subjects scoring lower on enjoyment and higher on effort. Work was perceived as the most challenging and requiring the most effort in both subject groups, but especially so for HS subjects. HS subjects reported the lowest enjoyment of work and the greatest enjoyment of leisure activities. While LS subjects enjoyed social activities the most, HS subjects reported relatively little enjoyment in social situations and rated social interactions as more demanding in terms of effort than LS subjects.
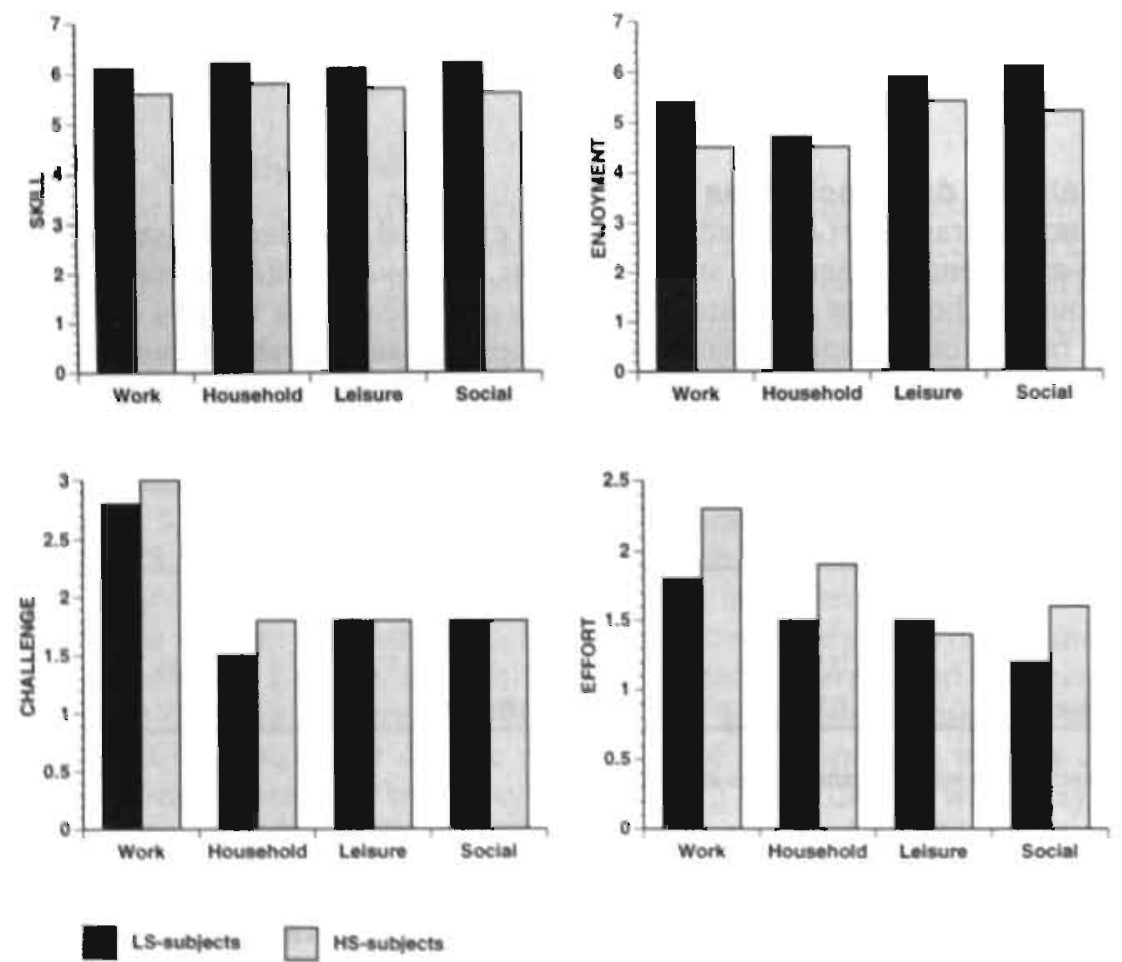

Figure 3.6.a-d Mean appraisal of required skill, enjoyment, effort, and challenge for work. household, leisure, and social activities. 


\section{Frequency, context and appraisal of stressful daily events}

In this final section, differences between stress groups in the frequency, context, and evaluation of stressful daily events are tested.

Event frequency: LS subjects reported a mean of 5.4 events (s.d. $=5.5$ ) and HS subjects 9.0 events (s.d. = 8.6) during the five days of Experience Sampling (MannWhitney U test, p<.05). Seven subjects (all LS) reported no event at all. Event reports as a percentage of total beeps averaged $12.1 \%$ for the LS group (s.d. $=11.5$ ) versus $21.9 \%$ for the HS group (s.d. = 19.5) (Mann-Whitney U test, p<.01). Since a major category of reported events was work-related, we expected more frequent reports of stressful events during weekdays than on weekends. Multivariate analyses of variance for repeated measures indicated a highly significant main effect on event frequency ( $\%$ of total beeps) for the within-subject factor day ( $S$ days) $(\mathrm{F}(4,340)=16.02 ; \mathrm{p}<.001)$, as well as for the between-subject factor group (HS.LS) $(\mathrm{F}(1,85)=8.02 ; \mathrm{p}<.01)$. The interaction of day by group was also significant $(\mathrm{F}(4,340)=3.45 ; \mathrm{p}<.01)$. As can be seen in Figure 3.7., subjects indeed reported more stressful events on workdays than on weekend days. The HS subjects reported more events than LS subjects on each day of the week, but especially so on Thursday, Saturday and Monday. On Sunday, both groups reported relatively few events.

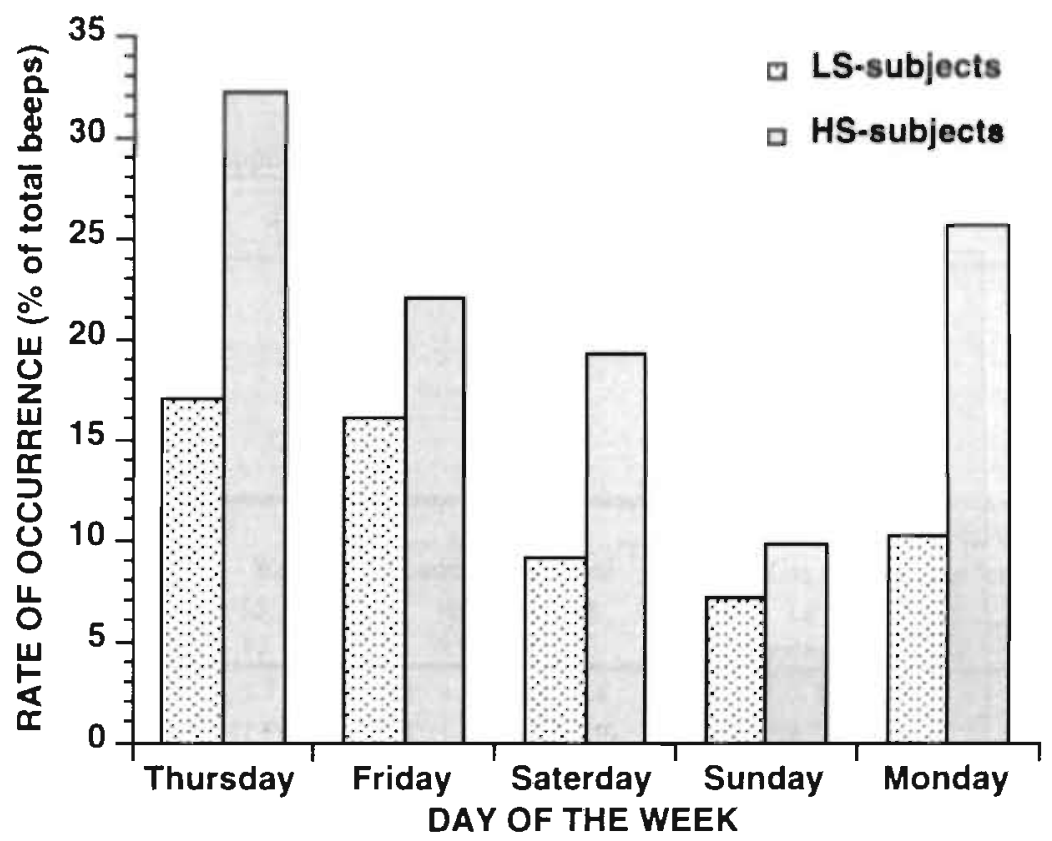

Figure 3.7. Frequency' of stressful daily events for HS and LS groups by day of the week.

Event context: As shown in Figure 3.8, HS subjects reported significantly more stressful work and network events than LS subjects, across all beeps. LS subjects, on the other hand, reported significantly more transportation events. HS subjects aiso reported significantly more social interaction events than LS subjects $(7.0 \%$ versus $3.2 \%, \mathrm{p}<.01)$, but not significantly more task demands $(6.9 \%$ versus $4.4 \%, \mathrm{~ns})$. As 
shown in Table 3.11, task demands as compared to social interactions are reported with equal frequency at home and at work in both groups. Both groups reported more task demands and more social interactions at work than at home. HS subjects reported significantly more events entailing negative social interactions (but not task demands) than LS subjects, both at home and at work.

Social context of event: We also coded who was involved in a reported event (social context) using the following categories: alone, household members, non-resident family, friends, colleagues, neighbours/acquaintances, and strangers (see Chapter 2 'Coding of ESM measures' section). HS subjects reported significantly more daily events concerning household members $(4.1 \%$ versus $1.6 \%, \mathrm{p}<.01)$, colleagues $(4.0 \%$ versus $2.5 \%, \mathrm{p}<.05)$, and other acquaintances $(1.6 \%$ versus $0.5 \%, \mathrm{p}<.05)$ than LS subjects across all beeps. There were no group differences for the categories alone, non-resident family, friends or strangers. Within the category household members, HS subjects reported significantly more events concerning partner (1.6\% versus $0.8 \%$, $\mathrm{p}<.01)$ and children $(2.6 \%$ versus $0.8 \%, \mathrm{p}<.01)$.

Next, we examined who was involved in events coded specifically as social interactions. In both groups, most negative interactions were with colleagues. Although such events were relatively rare, HS subjects had significantly more negative social interactions with colleagues $(3.3 \%$ versus $1.9 \%, \mathrm{p}<.01)$ and with the partner $(1.1 \%$ versus $0.3 \%, \mathrm{p}<.05)$, across all beeps.

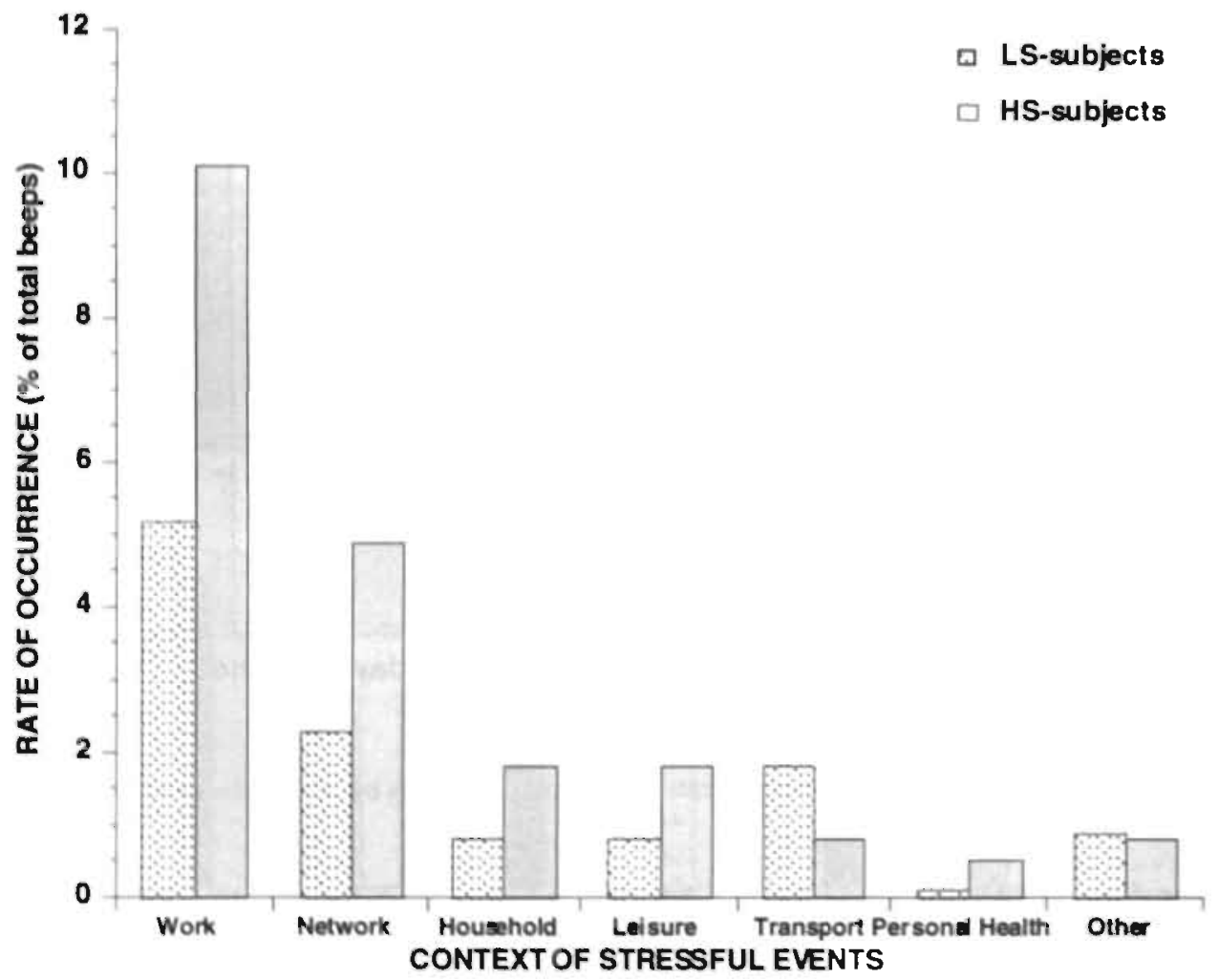

Figure 3.8. Frequency of stressful daily events for HS and LS groups by context. 
Table 3.11. Mean rate of occurrence $(+\mathrm{sd})$ of task demands and social interactions at home and at work (\% of total beeps).

\begin{tabular}{|c|c|c|c|}
\hline & $\begin{array}{l}\mathrm{LS} \\
\mathrm{n}=46\end{array}$ & $\begin{array}{l}\mathrm{HS} \\
\mathrm{n}=42\end{array}$ & $\begin{array}{l}\text { p-value } \\
\text { (2-tailed) }\end{array}$ \\
\hline \multicolumn{4}{|l|}{ at Home } \\
\hline Task demand & $2.6 \%(6.0)$ & $3.5 \%(5.8)$ & ns \\
\hline Social interaction & $1.8 \%(4.4)$ & $4.3 \%(7.5)$ & .05 \\
\hline \multicolumn{4}{|l|}{ at Work } \\
\hline Task demand & $7.3 \%(1[.4)$ & $12.4 \%(16.2)$ & ns \\
\hline Social interaction & $7.7 \%(10.4)$ & $13.2 \%(14.0)$ & .01 \\
\hline
\end{tabular}

Event appraisals: On average. HS subjects appraised events as more stressful and less controllable than LS subjects (Table 3.12). There were no differences between groups in the appraisals of event severity, importance, predictability, and chronicity. Event stressfulness and controllability were further examined for different types of events (work, network, household, leisure, social interactions, task demands). Statistical tests were done only for work events, task demands, and social interactions, because of the small sample sizes related to the other types of events. Results indicated that work events were significantly more stressful for HS than for LS subjects (see Table 3.12.).

Table 3.12. Mean event appraisals (+sd) for HS and LS subjects.

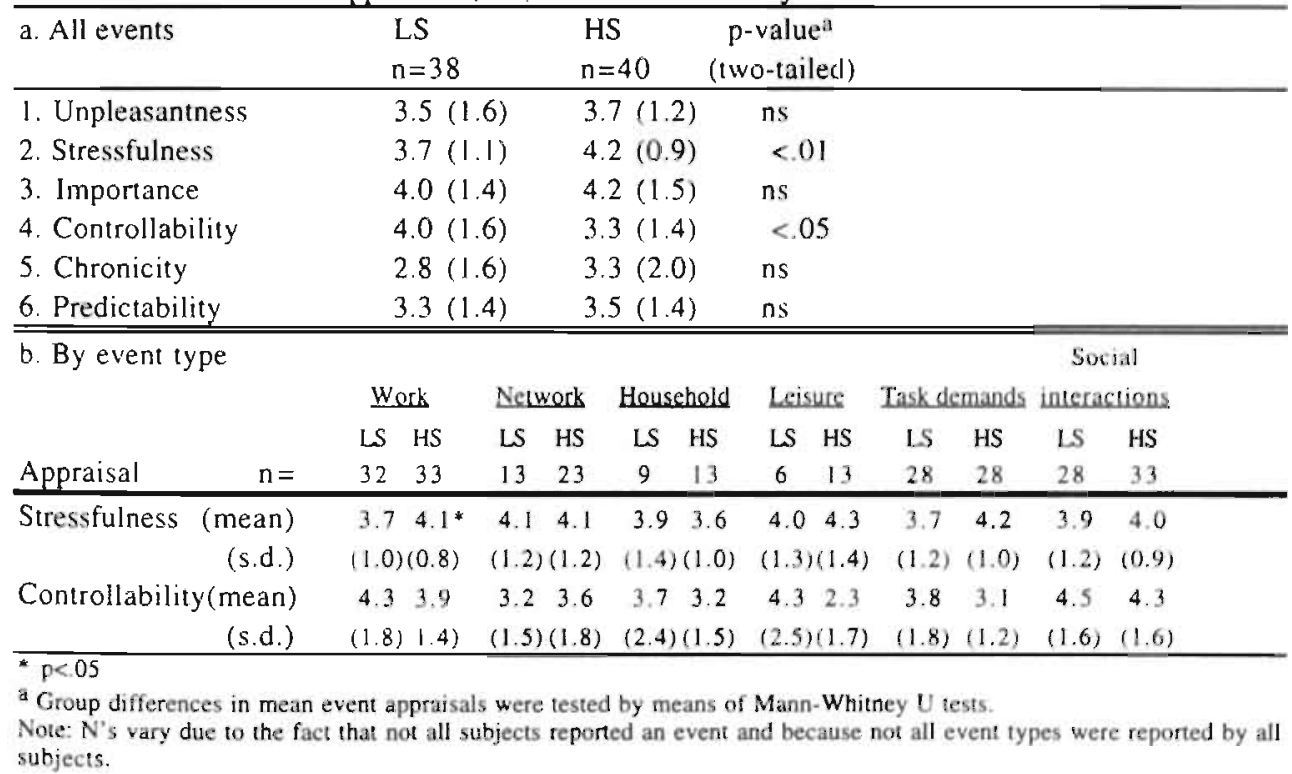

\section{DISCUSSION}

The aim of this chapter was to provide descriptive data on both quantitative and qualitative aspects of stressful events and activities in daily life, to increase our understanding of the construct stress as expressed in daily life. Using ESM, repeated 
measurements of stressful events, activities and their characteristics were made several times a day on several successive days in a sample of male white collar workers with varying stress levels.

Subjects reported a stressful event on $17 \%$ of all beeps. Whether this figure is high or low cannot be said; the frequency of reported stressful daily events cannot be compared to other studies since only a few have used an open-ended format to elicit events (Eckenrode, 1984; Rehm, 1978), and when used, different time-frames (events reported at the end of the day) together with different study populations (students, women) make comparisons irrelevant. Results indicated that in our sample the major source of stress in daily life was work, followed by the social network. These stress contexts make sense, given the developmental and demographic profile of the subjects. their most important social roles were those of employee, husband, and father. In line with the transactional definition of minor stressful events (Lazarus \& Folkman, 1984b, p.376), stressful daily events in this study can be characterized by its salience (importance) and unpleasantness. The finding that unpredictable and uncontrollable events were especially unpleasant is in accordance with results of many laboratory studies (Katz \& Wykes, 1985; Miller, 1979; Solomon, Holmes, \& McCaul, 1980; Thompson, 1981). Although subjective appraisals, as described above, appear to be important factors for understanding the stressfulness of daily events or situations, other dimensions should also be investigated for their relevance to the experience of stressful events, for example the experience of loss associated with an event (e.g. loss of a person, object, status, role, self-esteem) (Brown, 1989), future threat or feelings of uncertainty about how to cope with the event.

As might be expected, a high perceived stress level was related to higher scores on cross-sectional measures of psychological (anxiety, depression, anger) and psychosomatic (fatigue, depression, backache, headache, and insomnia) complaints, to the personality traits negativism, somatization, timidity and psychopathology, and to the more frequent use of passive and less frequent use of active (problem-focused) coping styles. Although HS subjects did not experience significantly more life events in the past year, they did report more chronic difficulties. Since HS subjects were healthy and actively employed, the pronounced group differences are somewhat surprising. From these data, the HS group emerges as a group of vulnerable, somewhat neurotic individuals, troubled by various psychological and psychosomatic complaints, experiencing several chronic environmental and relational difficulties, and using inadequate coping styles. An interesting question in this regard is how these characteristics would influence their lives in the future, if we could follow them, for example, another 10 years. In addition to the subjective measures, we would need to measure objective indices of health and work status (e.g. frequency of absenteeism from work, number of visits to health professionals, health records), to gain insight into the long-term consequences of high perceived stress. The pronounced group differences may also be related to the fact that the LS group contained subjects which seem to be more stress-resistant than on average. Compared to norm scores, LS subjects scored below average on psychological complaints, below average on the personality traits negativism, somatization, timidity, psychopathology, and average on extraversion (NPV). LS subjects also used more adequate coping styles indicated by high (norm) scores on problem-focused coping and low scores on depressive reaction.

Subjects with more chronic difficulties, but not with more life-events, reported significantly more daily events. Since chronic difficulties are much more dependent on the subjective judgment of a person than the more objective life-events, it is not 
surprising that chronic difficulties were related to the highly subjective stressful daily events. With respect to the major life events, it should be noted that life events, as occurred in the past year, were very rare in our sample, making it difficult to find an association between life events and daily events. HS subjects did report more life events, but not significantly more. Chronic difficulties seem not only related to increased perceptions of minor demands, but, as has been suggested for major life events (Kaplan, 1979), also seem related to the affective significance of minor events for the person, since with increasing chronic difficulties, events were rated as increasingly unpleasant. Subjects with high levels of chronic difficulties may experience events that are of themselves more unpleasant, but it is also possible that events. that relate to specific chronic difficulties (for example " an argument with spouse about household chores in the case of chronic marital problems) may be more salient and threatening, and thereby more unpleasant, for the person than the same event occurring in the absence of a chronic problem.

With respect to daily activities, the two groups had similar patterns of time use, and both groups perceived work activities as the most challenging but also requiring. the most effort. HS subjects did differ from LS subjects in how they evaluated their activities. Although in absolute terms HS subjects reported considerable enjoyment in various activities, perceived themselves to be quite skillful, and did not feel that activities required much effort to perform, they evaluated activities significantly less positively on these dimensions than LS subjects. This indicates that perceived stress levels are not only expressed in stressful events, but aiso in the evaluation and experience of daily activities. The differences between stress groups in the appraisal of activities were not restricted to a specific activity but were observed in several contexts.

With respect to daily events, work emerged as the major source of stress. Overall, HS subjects reported twice as many stressful daily events; they were also more likely to report events during work days than LS subjects. They reported twice as many work-related stressful events as LS subjects, as well as significantly more social network events. Within these life domains, HS subjects reported significantly more events related to colleagues, partner, children, and other acquaintances. In addition to an increased (subjective) exposure to stressful daily events, HS subjects appraised events as more stressful and less controllable. Although no group differences were found for task demands, HS subjects had significantly more negative social interactions, both at work and at home, and especially so with their colleagues and their partners. These results suggest that, besides the overall frequency of events, what differentiates HS from LS subjects may not so much be found in the stresses of family or work overloads, time-pressure, recurrent deadlines, but in stress resulting from problems with interpersonal relationships. Although social interaction events were reported too infrequently for firm conclusions, the finding of increased stressful interpersonal events in HS subjects is interesting in light of reports that interpersonal conflicts provoke the strongest negative mood states (Bolger, Delongis, Kessler, \& Wethington, 1989b; Bolger \& Schilling, 1991; Clark \& Watson, 1988; Repetti, 1993). In addition, stressful interpersonal events have been found to be related to neuroticism (Bolger \& Schilling, 1991). It would be interesting to investigate if and through which mechanisms interpersonal conflicts are related to differences in wellbeing between HS and LS subjects. A limitation of the current study is that we did not assess positive events and are thus unable to address the important question of the extent to which positive events influence well-being or compensate for the effects of negative events in HS and LS groups. 
An important next step toward understanding the relationship between stress in daily life and individual health outcomes is to examine short-term psychological and physiological responses to daily events. As we have seen, daily events are heterogeneous with respect to both the context in which they occur and the way in which they are appraised by the individual. Such characteristics of events may be equally if not more important determinants of immediate responses than simple event occurrence. Chapter 4 describes the impact of stressful daily events and their characteristics on negative and positive mood states. 


\section{Chapter 4}

\section{THE EFFECTS OF PERCEIVED STRESS AND STRESSFUL EVENTS ON MOOD STATES}

Submitted for publication

Marleen van Eck ${ }^{1}$. Nancy Nicolson ${ }^{1}$, Hans Berkhof ${ }^{2}$

1 Department of Psychiatry \& Neuropsychology, Social Psychiatry and Psychiatric Epidemiology section, University of Limburg, the Netherlands

2 Department of Statistics and Measurement Theory, University of Groningen, the Netherlands 



\begin{abstract}
This study examined the effects of perceived stress on the relationship between stressful daily events and mood states. Forty 'high stress' (HS) and 46 "low stress' (LS) subjects were selected on the basis of Perceived Stress Scale scores from a. sample of male white collar workers. Subjects completed Experience Sampling self-reports ten times a day over five consecutive days. Multilevel analysis revealed that current stressful events were associated with significant increases in negative mood (Agitation), but not with significant changes in positive mood. HS subjects showed significantly stronger negative mood reactivity (both Agitation and Negative Affect) in response to current events than LS subjects. The effects of events on negative mood persisted for at least $90 \mathrm{~min}$ after an event was reported, both in HS and LS subjects. A future event was associated with higher current Agitation in both subject groups, but with higher Negative Affect in HS subjects only. Results suggest increased vulnerability to daily events in HS subjects, and highlight the need for longitudinal studies for establishing long-term health effects
\end{abstract}

\title{
INTRODUCTION
}

In an effort to understand the effects of environmental stressors on health and psychological distress, there has been a shift from studying the effects of major life events to the effects of minor daily events, or daily hassles (Kanner et al., 1981: Zautra et al., 1988). During the last decade it has become increasingly clear that daily life stress is associated with lower psychological well-being and increased somatic symptomatology (DeLongis et al., 1988; Stone et al., 1993). The recent emphasis on daily life events is due, in part, to the extensive criticism directed at the traditional approaches to the study of psychosocial stress. An important drawback of the major life event approach has been the frequent reliance on retrospective research designs, with related problems of biased recall and forgetting (e.g. Bower, 1981). Additionally, the retrospective assessment of life events over several months obscures the temporal, dynamic interplay between environmental demands, perceptions of demands, and outcomes (Stone \& Shiffman, 1992), a central tenet of current transactional theories of stress and coping (Lazarus \& Folkman, 1984a). End-of-the-day assessments of minor events have been designed to address these problems. Measurement biases can be minimized by measuring the variables related to the stress process closer to the moment they actually occur. Prospective daily designs also offer better opportunities to identify the temporal patterns between events and outcomes, a first step in establishing causal relationships.

But even within the time frame of a day, much of the temporal interplay between events, appraisals of events, and outcomes remains out of sight. In addition, forgetting of less salient events and a bias towards remembering more recent events is still likely to occur. Moreover, the recall of daily mood is probably influenced by the subject's mood at the time of recording (Stone et al., 1991). Same-day associations between daily stress and mood remain causally ambiguous: a bad mood, for example, may increase the likelihood that daily events will occur or will be perceived as stressful. Finally, previous studies suggest that the effects of minor stressful events may be largely confined to the day on which they occur (e.g. Bolger et al., 1989a; Rehm, 1978; Stone \& Neale, 1984). These problems all point to the need to narrow the time frame even further to clarify within-day patterns of association between stressful events and mood. 
In the current study, a prospective within-day design was used, with subjects recording event occurrence and mood states several times a day over several consecutive days (Experience Sampling Method), to examine the effects of daily events on mood. The major issue addressed concerns interindividual variability in the daily stress process. More specifically, we investigated how perceived stress, as a measure of chronic stress, translates into the day-to-day experience of and response to minor daily events. First, we examined whether subjects with a high perceived stress level experience more stressful events. Second, we investigated whether high perceived stress magnifies the effects of daily stressors on mood. Third, we investigated temporal patterns of mood reactivity to events. In this context we hypothesized that the mood responses of high stress subjects might be of a longer duration than those of low stress subjects, and that high stress subjects might show greater mood changes in anticipation of future events. Finally, we explored the relationship between event characteristics, such as the context in which an event occurred and event appraisals, and mood states.

End-of-day diary studies have demonstrated a same-day association between daily stress and negative mood (Affleck et al., 1994; Bolger et al., 1989a; Clark \& Watson, 1988; DeLongis et al., 1988; Eckenrode, 1984; Larsen et al., 1986; Lewinsohn \& Libet, 1972; Rehm, 1978; Stone \& Neale, 1984; Stone et al., 1993). The results with respect to positive mood are less clear. Many studies do not differentiate between positive and negative mood; in those that do, positive mood was either lower (Neale et al., 1987; Repetti, 1993; Stone \& Neale, 1984) or unchanged (Watson, 1988 ) on days when many stressful events occurred. Only one study has previously investigated event and mood associations within days (Marco \& Suls, 1993), and here, daily stress was accompanied by "worse mood". There is evidence for large individual differences in the magnitude and the direction of the association between daily stress and mood. Factors which contribute to such differences include selfesteem and self-complexity (Campbell et al., 1991), social support (Affleck et al., 1994; Barling \& Kryl, 1990; Caspi et al., 1987: DeLongis et al., 1988), recent major life events (Affleck et al., 1994; Caspi et al., 1987) and neuroticism (Affleck et al., 1994; Bolger \& Schilling, 1991; Marco \& Suls, 1993). Little research has been done on the effects of chronic stress or long-term difficulties. In a study by Caspi et al. (1987), the chronic stress of living in a low quality neighborhood exacerbated the immediate effects of daily events on mood and also increased the likelihood that daily stressors had an enduring effect on next day's mood.

In the present study, we investigated the effect of perceived stress level on the relationship between daily events and mood. Individuals with a high perceived stress level feel their life to be unpredictable, uncontrollable and overloaded, and can be seen as at risk for the development of stress-related somatic and mental health problems (Cohen et al., 1983), resulting, for instance, in increased sick leave from work. Personality factors probably play a very important role in perceived stress; research indicates that self-report measures of stress correlate significantly with measures of Negative Affectivity (NA) or neuroticism (Watson, 1988: Watson \& Pennebaker, 1989). Several studies demonstrated that individuals higher in NA exhibit increased negative mood reactivity to minor stressors (Affleck et al., 1994; Bolger \& Schilling, 1991: Marco \& Suls, 1993). Considering the above, we hypothesized that individuals with high perceived stress would show greater mood reactivity to daily events than those with low perceived stress. Differences in mood reactivity might for instance be due to differences in the appraisal of events or differences in coping strategies. In order to examine differences in mood reactivity to 
reported stressful events, it is important to control for possible differences in baseline mood between high and low stress subjects. This way we can distinguish between differences in overall levels of negative mood (when events are absent) and added effects of events.

Little is known about the duration of effects of daily events or about the influence of individual differences on this process, although this would be important for understanding the consequences of daily events and might possibly reveal mechanisms related to the longer-term effects of chronic stressful experiences (Eckenrode \& Bolger, 1995). Possibly only when negative moods persist following stressful events, in addition to a high frequency of events, negative health changes may follow (e.g. accumulation of distress). One interpretation for the persistence of negative mood after an event has ended is that coping has been unsuccessful or still ongoing (e.g. persistence of worrying about event). In the current study we investigated if an event reported one assessment earlier (from now on called "prior event") has an (enduring) effect on current mood, and whether perceived stress influences the relationship between prior event and current mood. Most studies have found no relationship between daily stress and mood on the following day (e.g. Lewinsohn \& Libet, 1972; Neale et al., 1987; Rehm, 1978; Stone \& Neale, 1984); in fact, two studies found that on days following stressful events, mood scores were actually better than on other stress free days (Bolger et al., 1989a; DeLongis et al., 1988). A possible explanation for these findings is that feelings of relief predominated after the resolution of stressful events. Two other studies did document worse moods on the day following a suessful event, but oniy in subjects with a low fevel of social support (Affleck et al., 1994; Caspi et al., 1987). In the only within-day study to date (Marco \& Suls, 1993) prior events had no effect on current mood. We also investigated whether future events have an effect on current mood states and if these effects vary with differences in perceived stress level. Future events may influence current moods due to an increased worrying or attention for future events (anticipation effect). Also for an adequate test of the influence of prior event on current mood state it is necessary to control for possible effects of a future event at $t+1$ on current mood states. In this way, we are able to differentiate between an effect on current mood state due to the influence of a prior event and one duc to an anticipated future event.

Of the enormous range of stressful events people experience in daily life, which categories of events have the strongest psychological impact? Results from end-of-the-day studies indicate that the psychological impact of daily events does indeed depend on the context in which they occur. In particular, undesirable events at work (i.e. feeling under a lot of pressure and having negative interactions with coworkers) were associated with both lower positive and higher negative moods (Repetti, 1993; Stone, 1987). In other studies, interpersonal conflicts were found to provoke the strongest negative mood states (Bolger et al., 1989a; Bolger \& Schilling, 1991; Clark \& Watson, 1988; Repetti, 1993). We therefore hypothesized that, within days, stressful work events, task overloads at work or at home, and negative social interaction events would show a larger effect on mood states than other types of events.

According to contemporary stress theory, the subjective appraisal of a stressful event may be more important than the occurrence of an event per se (Lazarus \& Folkman, 1984b). There is evidence for instance, that subjects' personal ratings of events improve prediction of outcomes such as anxiety, depression, negative affect, tension, and grade point average (Dewe, 1991; Peeters et al., 1995; Sarason et al., 
1978). Despite the theoretical importance of the appraisal process, little research has been done on appraisal dimensions of naturally occurring dailly events in relationship to mood. Results of one study where appraisals of daily events were investigated (Neale et al., 1987) led to the conclusion that a simple score based on the sum of event desirability ratings did as well in predicting mood as more complex appraisal measures. A limitation of this study was that the results were based on summary measures over four days; analysis of measurements within days might reveal more subtle relationships. On theoretical grounds, then, we expected that the more unpleasant and important, and the less predictable and controllable the event, the stronger the mood responses. In addition, we predicted that events that had occurred relatively frequently in the past would have less effect on mood than novel stressors.

\section{METHOD}

\section{Subjects}

Local industries and government agencies were approached via their personnel departments to participate in the study. A decision as to which categories of employees fell under the definition of 'white-collar' was made by each personnel department on the basis of standard job function descriptions. Questionnaires were distributed among these employees, accompanied by a letter explaining the goals of the study. Participation was voluntary, and care was taken to insure anonymity. 316 male employees from six different industries or agencies completed the screening questionnaire. The mean score on the Perceived Siress Scale (PSS) for this sample was 12.7 (s.d. 6.0), similar to US norms (mean 13.02, s.d. 6.45) (48).

Ninety-two subjects with scores in the upper or lower tertiles of the screening sample distribution (PSS-10 score $<10$ or 216 ) were recruited, excluding individuals who reported a history of serious chronic illness or treatment (past or current) for mental health problems. Exclusion criteria were reassessed during an initial interview, study aims and procedures were explained, and informed consent obtained. During subject intake, each 'high stress' (HS) subject was matched for age group, marital status, and household composition with a 'low stress' (LS) subject to insure that the two groups did not differ on demographic characteristics that might affect exposure to certain classes of daily stressors. Six subjects were later excluded from analysis: Four due to failure to meet ESM compliance criteria (see 'Daily Experience' section, below), one because he became so acutely stressed that he was unable to work during the sampling period, and another one was excluded due to missing data on the mood variables. Of the 86 remaining. subjects, 40 subjects comprised the 'high stress' (HS) group and 46 subjects the 'low stress' (LS) group. Mean age was 42.1 years (range 27 to 57 years). $89 \%$ were married, and $81 \%$ had children living at home.

\section{Assessment of perceived stress}

The 10-item version of the PSS was translated into Dutch. The PSS is a global measure of the degree to which situations in one's life are appraised as stressful. Items were designed to tap the extent to which individuals feel their life to be unpredictable, uncontrollable and overloaded. The items were rated on a 5-point frequency scale, ranging from 'never' $(0)$ to 'very often' (4) in the last month. Validity and reliability data have been reported by Cohen and others (Cantor, Norem, Langston, Zirkel, Fleeson, \& Cook-Flannagan, 1991: Cohen, 1986; Cohen et al., 1983; Cohen, Tyrrell, \& Smith, 1991). The mean of the two PSS scores was 7.3 (st.dev. 2.2) for the low stress group and 17.9 (st.dev. 3.3) for the high stress group. 


\section{ESMI procedure}

The Experience Sampling Method (ESM) (Csikszentmihalyi \& Larson, 1987: de Vries, 1992) was used to collect data from subjects at selected moments during their normal daily activities. Subjects received auditory signals ('beeps'), after which they filled in a questionnaire. After a 'briefing' session, in which all procedures were explained in detail and informed consent obtained, subjects were sampled for a period of five consecutive days (Thursday through Monday). A wristwatch was programmed to emit beeps ten times each day, at semi-random intervals of approximately 90 minutes between the hours of 8 am and 10 PM. Beeps were clustered around the midpoint of each time block (i.e., $8.15 \mathrm{am}, 9.45,11.15$, and so on), with the exact time sequence of beeps varied each day to decrease predictability. In a final 'debriefing' session, subjects were asked to clarify reasons for missing data. Compliance with the procedures was generally good. The criteria set for subject inclusion in the analysis (> 20 ESM reports completed within 20 minutes after being signalled and no missing data for entire days) were met by all but four subjects (two from each group). The remaining 88 subjects completed an average of $83 \%$ of all possible responses within the time limit, for an average of 41 responses per subject. HS and LS groups did not differ significantly in compliance rates ( 40.1 vs 42.3 responses per subject, Mann-Whitney U-test, p>.05). Compliance was lowest for the first beep (at approximately $8.15 \mathrm{am})$, with an average of $73 \%$ valid reports. On Saturdays, response rate for first beeps was $61 \%$, and on Sundays, $59 \% .74 \%$ of all missing and invalid responses on weekends could be attributed to the fact that subjects were still asleep when signalled.

\section{ESM measures of streseful evente and mood}

The ESM form contained open-ended questions concerning thought content (worries), the physical and social context, and what the individual was doing when signalled. The forms also included 7-point Likert scales (from 1 'not at all' to 7 'extremely') for rating aspects of thoughts, mood, physical well-being, individually defined (psycho)somatic complaints, current activity, and stressful events.

Stressful events: Subjects were asked to describe any stressful event or situation which may have taken place in the interval since the last ESM report, in order to obtain as complete as possible a record of each subject's stressful experiences. Although we refer to these as 'current' events. it should be noted that they took place during the interval between two beeps (i.e., within a time-interval of approximately 90 minutes) and were not necessarily still ongoing at the moment subjects were signalled. 'Prior' events were defined as events which were reported at beep $t-1$. while 'future' events were defined as events which were reported at beep $t+1$.

Event context: Subjects' descriptions of stressful daily events were coded according to context, with the categories work, network (events concerning family, friends and acquaintances), householdfinancial, leisure, personal health-somatic, personal health-psychological, transport, and other. The twelve events that were coded in one of the personal health categories were excluded from the analysis to avoid possible confounding with the mood outcome measures. Of all reported events, $48 \%$ work-related, with the next most common type (network events) representing $18 \%$ of the total events. For this reason, analysis differentiated between the contexts work (1) vs. non-work (0) events.

Some examples of reported work events are: "unclear $/$ vague assignment at work", 'too much to organize, not enough time', 'difficult conversation with boss about job performance', 'chairing a big meeting'. Reported non-work events included: 'having a fight with my wife about household duties'. 'conflict with spouse about how to raise our son', 'child who will not listen', and 'making dinner, child crying, other child taking a bath, and this all at the same time".

In addition, events were scored according to whether or not they involved a social interaction or a task demand (e.g. overloads, deadlines). The codes for work domain, interaction, 
and demand were not mutually exclusive; for example, an argument with the boss about a missed work deadline would be coded as present for each variable. ${ }^{2}$

Event appraisals: Subjects rated reported events on the following dimensions: unpleasantness, importance, predictability, controllability and frequency of prior occurrence. (chronicity).

Mood measures: The 17 ESM mood items were reduced to three mood measures, based on the results of a principal component analysis with varimax rotation, which accounted for $78 \%$ of the total variance when subject mean scores were used. Ratings on the items cheerful, satisfied, relaxed, energetic, self-assured, concentrated and enthusiastic were summed to form a 'Positive Affect' (PA) scale. (Cronbach's alpha $=.95$ ). Two separate components of negative mood were identified: 'Negative Affect (NA)', including the items depressed, anxious, worried, lonely, tired, and miserable (alpha $=87$ ), and 'Agitation $(\mathrm{Ag})$ ', with the items restless, irritated, hurried, and nervous (alpha $=.93$ ). The sums of the scale items were divided by the number of items in the scale, so that ali mood measures have ranges from i to 7 . Within-subject associations among the mood scales were investigated by first standardizing responses on each scale within a subject ( $\mathrm{z}$ scores) and then calculating Spearman rank correlations over all reports. The resulting correlations were low to negligible (NA - Ag: rho $(3569)=.21, \mathrm{p}<.001 ; \mathrm{NA}-\mathrm{PA}$ : rho (3564) $=$ $-.1 \mathrm{i}, \mathrm{p}<.001 ; \mathrm{Ag}-\mathrm{PA}$; rho $(3563)=-.07, \mathrm{p}<.001$; all tests one-tailed), supporting the notion that the three mood dimensions are relatively independent.

\section{Statistical analysis}

For the estimation of the effects of daily stress on mood we used the hierarchical linear model (HLM) or multilevel model (Bryk \& Raudenbush, 1987; Goldstein, 1987) using the programm MIXREG (Hedeker, 1993). The HLM is a variant of a multiple regression model, for the situation in which the data can be structured in a hierarchical way. As the beep-level observations are nested within persons, the HLM can be used to analyze the present data.

A major advantage of the HLM above more conventional methods like ANOVA for repeated measurements is that HLM deals flexibly with the problem of missing observations, which are inevitable in ESM datasets, and the observations do not need to be evenly spaced over the time interval (Gibbons, Hedeker, Elkin, Waternaux, Kraemer, Greenhouse, et al., 1993). Furthermore, the HLM allows for flexible specification of the dependency among the observations within the same person. In the mood dataset, the dependency consists of more than one component. First, as the average mood level is assumed to vary across persons, two observations are more similar if they are from the same person. Second, two mood observations from the same person tend to be more similar if they are taken at points close in time. For the sake of simplicity, many previous studies have ignored this autocorrelation. This biases the standard errors of the regression coefficients downwards and may consequently lead to incorrect test results. Another attractive feature of the HLM is that both time-varying and time-invariant covariates can be added simultaneously to the model (see below).

The two nesting levels present in the data will be called measurement level and person level. The variables added at the measurement level (e.g. events) vary with time, while the variables added at the person level represent individual characteristics (e.g. perceived stress level). The effect of a time-varying variable can be decomposed in two parts: a fixed effect that is constant across persons and a person-specific random effect that can vary from person to person.

2 The reliability of the coding system was assessed by comparing the classifications of 345 events by two independent coders. Interrater agreement was determined by means of Cohen's kappa. On the whole, the qualitative information could be classified with a high degree of agreement (Laundis \& Koch, 1977), especially for the content categories. The overall Kappa for content was .90. Kappa's for social interaction and for task demand were .73 and .65 , respectively. 
At the measurement level, the relationship between events and mood state for person $i$ can he explicitly modelled. Here, we describe a model with stressful event as the only explanatory variable. The model generalizes in an obvious way to the situation with more time-varying covariates. The model at the measurement level has the following form:

$$
(\text { Mood })_{i j}=\alpha_{i}+\beta_{i} *(\text { Event })_{i j}+\varepsilon_{i j}
$$

where $(\text { Mood })_{i j}$ is the mood state at the $j$-th measurement of person $i$, (Event) ${ }_{i j}$ indicates whether a stressful event occurred to person $i$ at the $j$-th measurement (dummy coded as 1 or 0 ), $\alpha_{i}$ is the intercept of person $i$, the regression coefficient $\beta_{i}$ is the reactivity of person $i$ to a stressful event. and the $\varepsilon_{i j}$ 's are normally distributed residual terms with mean zero and variance $\sigma^{2}$. The residual terms within a person are postulated to be first order autocorrelated. This means that the correlation between two residuals that lie $k$ time units apart equals $\rho^{k}$

The coefficients $\alpha_{i}$ and $\beta_{i}$ are allowed to vary across persons, as indicated by the person index $i$. Instead of modelling the variation across persons by estimating $\alpha_{i}$ and $\beta_{i}$ for each person, these coefficients are written as a function of person-specific covariates. The covariate perceived stress (dummy coded as 1 or 0 ) indicates whether an individual belongs to the high or to the low stress group. The parameters $\alpha_{i}$ and $\beta_{i}$ can be modelled at the person level as:

$$
\begin{aligned}
& \alpha_{i}=\gamma_{\alpha}+\delta_{\alpha} \text { (Perceived stress) } i+u_{\alpha, i} \\
& \beta_{i}=\gamma_{\beta}+\delta_{\beta} \text { (Perceived Stress) } i+u_{\beta, i}
\end{aligned}
$$

where the $\gamma^{\prime}$ 's and $\delta$ 's denote fixed effects, which are constant across persons. Since perceived stress might not explain all variation in the $\alpha_{i}$ 's and $\beta_{i}$ 's across persons, person-specific random terms $u_{\alpha, i}$ and $u_{\beta, i}$ were added to the righthand side of the above equations. The $u_{\alpha, i}$ and $u_{\beta, i}$ are assumed to be normally distributed with mean vector zero, variances $\sigma^{2} \alpha$ and $\sigma^{2} \beta$, and covariance $\sigma_{\alpha \beta}$.

Separate models were built to estimate effects on each of the three mood states; Negative Affect, Agitation, and Positive Affect. In each model, the explanatory variables were selected by a backward selection approach. This means that we started with a model that contained all possible explanatory variables and then excluded variables with a non-significant effect. Two-tailed significance of a fixed effect was tested by dividing the estimated effect by its standard error. This ratio is approximately normally distributed. For testing the significance of the random effects, we applied the likelihood ratio test (one-tailed) (Bryk \& Raudenbush, 1992). Variables with nonsignificant main effects were reiained in the model only when interaction effects were significant. Because the variables prior event (event at beep $t-I$ ) and future event (event at beep $t+l$ ) were missing at the first or the last observation, respectively, of each day, these observations were deleted from the data. This resulted in a reduction from 3452 to 2358 observations. To check that the deletion of observations did not lead to a significant change in the regression coefficients, we started by estimating each model on the basis of all observations, thus without the variables prior and future events. With one exception (for NA; see discussion), deletion of observations did not significantly change the values of the regression coefficients of the remaining variables. We therefore present only the final models, including the prior and future variables. For the eventcontext variable we used effect coding (for example, $0=$ no event, 1 = work event, -1 = non-work event). The event-appraisal variables were assigned the value zero if no event was reported. The other values of the event-appraisal variables were centered around the mean for each subject, so that observed effects represent within-person effects. 


\section{RESULTS}

High stress subjects reported stressful events more frequently and had, on average, higher negative affect and agitation and lower positive affect than low stress subjects (see Table 4.1.).

Table 4.1. Frequency's of stressful events and mood states in low and high stress groups.

\begin{tabular}{|c|c|c|c|}
\hline & $\frac{\text { Low PS }}{(n=46)}$ & $\underset{(n=40)}{\text { High PS }}$ & \\
\hline & mean (st.dev.) & mean (st.dev.) & $\frac{\text { p-value }^{\mathrm{i}}}{(2 \text {-tailed) }}$ \\
\hline $\begin{array}{l}\text { Frequency of stressful events }{ }^{2} \\
\text { Mood States }^{3}\end{array}$ & $12.3 \%(11.9)$ & $20.6 \%(17.8)$ & $<.01$ \\
\hline Positive Affect & $5.7(0.6)$ & $5.3(0.3)$ & $<.001$ \\
\hline Negative Affect & $1.2(0.1)$ & $1.4(0.4)$ & $<.001$ \\
\hline Agitation & $1.3(0.3)$ & $1.7(0.5)$ & $<.001$ \\
\hline
\end{tabular}

${ }^{1}$ Differences in frequency and mood states were tested by means of Mann-Whitney U tests

2 Stressful event reports as a percentage of total ESM reports

${ }^{3}$ Subject means, aggregated fover all ESM reports

For each mood state, results will bo presented in the folivwing orcier: firsi, we will describe the effect of stressful daily events on mood state (both fixed as well as random effect), and whether this effect was related to perceived stress. Second, the results on the relationship between prior and future events on the one hand and current mood state on the other hand will be reported, followed by the effect of perceived stress on this relationship. Third, the effects of event type and event appraisals will be described. For each step, the significant effects of variables will be presented first, followed by the non-significant effects.

\section{Effects of events on Negative Affect}

The effect of daily events on Negative Affect differed significantly between subjects (Random Effects CE in Table 4.2.). Although the occurrence of an event was followed by slight elevations in Negative Affect in both groups, the significant Current Event $\times$ Perceived Stress interaction indicates that stressful events increased Negative Affect mainly in HS subjects. This pattern is shown in Figure 4.1. There was also a positive association between the intercept variance and event variance (Covariance Intercept/CE in Table 4.2.); this indicates that controlling for perceived stress, subjects with the highest average scores on Negative Affect showed the greatest increases in this mood state following events.

A small but marginally significant main effect was present for prior event., showing that the effect of an event on Negative Affect persist on average longer than the interval between two ESM reports. Level of perceived stress did not influence the magnitude of this carryover effect. Similar to the pattern observed for current events, a stressful event occurring in the interval following the current report (future event) had no significant effect over all subjects, but in HS subjects, Negative Affect was elevated preceding a future event. 
Table 4.2. Multilevel Results for Negative Affect (NA).

\begin{tabular}{|c|c|c|c|}
\hline Fixed Effects & Estimate & S.E. & $\mathrm{p}<$ (one-tailed) \\
\hline Intercept (mean NA) & 1.140 & 0.041 & $* * *$ \\
\hline Perceived Stress Group (PS) & 0.170 & 0.055 & $\cdots$ \\
\hline Current Event (CE) & 0.046 & 0.033 & ns \\
\hline CE $\times$ PS & 0.151 & 0.046 & $\cdots$ \\
\hline Prior Event (PE $\left.{ }^{1}\right)$ & 0.032 & 0.018 & .07 \\
\hline Future Event $\left(\mathrm{SE}^{2}\right)$ & 0.030 & 0.026 & ns \\
\hline FE $\times$ PS & 0.066 & 0.037 & .07 \\
\hline Event Unpleasantness & 0.029 & 0.010 & $\cdots$ \\
\hline Controllability Event & -0.019 & 0.009 & - \\
\hline Chronicity Event & -0.019 & 0.010 & * \\
\hline
\end{tabular}

\begin{tabular}{|c|c|c|c|}
\hline Random Effects & Estimate & S.E. & $\mathrm{p}<$ \\
\hline \multicolumn{4}{|l|}{ Person level: } \\
\hline Var (Intercept) & 0.055 & 0.010 & $* *$ \\
\hline Var (Current Event) & 0.012 & 0.006 & • \\
\hline Cov (CE, Intercept) & 0.015 & 0.006 & $\bullet$ \\
\hline \multicolumn{4}{|l|}{ Measurement level: } \\
\hline Var (Residual term) & 0.078 & 0.002 & $* *$ \\
\hline
\end{tabular}

\footnotetext{
Autocorrelation

$0.443 \quad 0.021$

$\mathrm{n}=\mathbf{2 3 5 8}$ observations (including 342 events) nested within 85 subjects ( $39 \mathrm{HS}, 46 \mathrm{LS}$ )

1 event at $1-1 ;{ }^{2}$ event at $1+1 ;{ }^{*} p \leq .05 ;{ }^{* *} p \leq .01 ;{ }^{* \vec{*} *} p \leq .001$

Von-signitican fixed effecis:

Event ryps: Work, Social Interaction, Task Demands

Event appraisals: Importance, Predictability

Interdcrions: PE $\times$ PS

Nore. The variable 'time over days' (automatically estimated when autoconelations are corrected for) was excluded from Table $4.2,4.3$, and 4.4 since estimated effects were negligible (all $<-0.002$ ) and not relevant with regard to hypotheses being tested.
}

As expected, events that were rated as more unpleasant were associated with larger increases in NA, whereas events that were relatively controllable had smaller effects on NA than uncontrollable events. In addition, the more frequently a stressful event had occurred in the past ("chronicity"), the smaller the observed effect on NA. Further differentiation of events by context did not improve the model; in other words, work and non-work events did not differ in their effects on Negative Affect. With respect to the event appraisals, no significant effects were found for event importance or event predictability. 


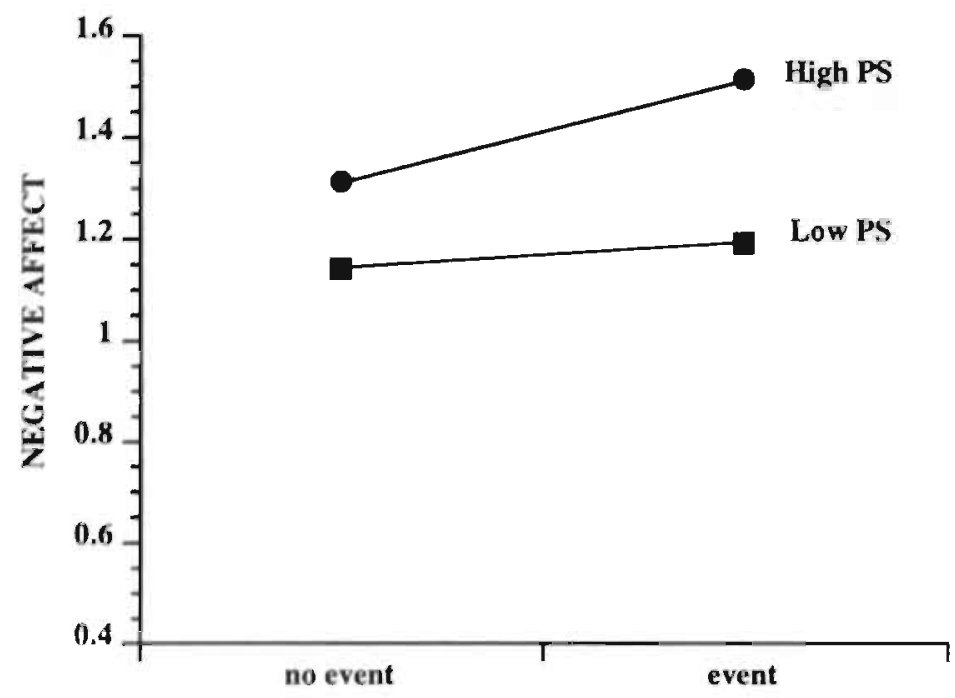

Figure 4.1. Modelled change in Negative Affect in 'high' and 'low' stress subjects in response to a stressful event (controlling for all other variables).

\section{Effects of events on Agitation}

In both HS and LS groups, current stiessful events were associated with significant elevations in Agitation levels (see Table 4.3.). Again we found a random effect for events, meaning that the influence of stressful events on Agitation differed significantly between subjects. Part of this between-subject variance could be explained by perceived stress level: HS subject reacted significantly more strongly to current stressful events than LS subjects, as evidenced by the significant Current Event $x$ Perceived Stress interaction. These results are presented graphically in Figure 4.2. As with Negative Affect, there was a positive association between the intercept variance and event variance (Covariance InterceptiCE in Table 4.3.), indicating that subjects with higher average scores on Agitation showed higher reactivity to current events (after controlling for perceived stress).

With respect to the temporal processes of mood reactivity, the significant effect of prior event on current Agitation indicates a carryover effect of events on mood. The presence of a future event also had a significant positive effect: current Agitation was higher when a stressful event was reported in the interval following the current report. There was no evidence for a moderating effect of perceived stress level on these carryover or anticipation effects.

Current events characterized as task demands were associated with significant increases in Agitation compared to non-task demand events. With respect to the event appraisals we found that, similar to the findings for Negative Affect, when events were more unpleasant than average. Agitation was higher, whereas events that were more controllable than average had a smaller effect on Agitation. Whether or not an event involved a negative social interaction or was work-related had no specific effect on this mood variable. No additional effects were found for event importance, predictability or chronicity. 
Table 4.3. Multilevel Results for Agitation (Ag).

\begin{tabular}{|c|c|c|c|}
\hline Fixed Effects & Estimate & S.E. & ps (one-tailed) \\
\hline Intercept (mean Ag) & 1.247 & 0.058 & $\cdots$ \\
\hline Perceived Stress Group (PS) & 0.323 & 0.077 & $\cdots$ \\
\hline Current Event (CE) & 0.373 & 0.098 & $\cdots$ \\
\hline CE $\times$ PS & 0.433 & 0.140 & $*$ \\
\hline Prior Event (PE ${ }^{l}$ ) & 0.099 & 0.032 & $\cdots$ \\
\hline Future Event $\left(\mathrm{SE}^{2}\right)$ & 0.213 & 0.034 & $\cdots$ \\
\hline Task Demands & 0.132 & 0.033 & $\cdots$ \\
\hline Event Unpleasantness & 0.056 & 0.019 & $\cdots$ \\
\hline Controllability Event & -0.045 & 0.016 & $\cdots$ \\
\hline
\end{tabular}

Random Effects

Estimate S.E. $\mathrm{p}<$

Person level:

$\begin{array}{llll}\text { Var (Intercept) } & 0.101 & 0.019 & * * \\ \text { Var (Current Event) } & 0.255 & 0.058 & * * \\ \text { Cov (CE, Intercept) } & 0.049 & 0.025 & *\end{array}$

Measurement level:

Var (Residual term)

$0.257 \quad 0.008$

Autocorrelation

$0.352 \quad 0.022$

$\mathrm{n}=2358$ observations (including 342 events) nested within 85 subjects ( $39 \mathrm{HS}, 46 \mathrm{~L}$ )

${ }^{1}$ event at $t-1 ;{ }^{2}$ event at $1+1 ;{ }^{*}$ ps.05; ${ }^{* *}$ p $\leq .01 ;{ }^{* * *}$ p $\leq .001$

Non-significant fixed effects:

Event lype: Work, Social Interaction

Event appraisals: Importance, Predictability, Chronicity

Interactions: $\mathrm{PE} \times \mathrm{PS}$. FE $\times$ PS

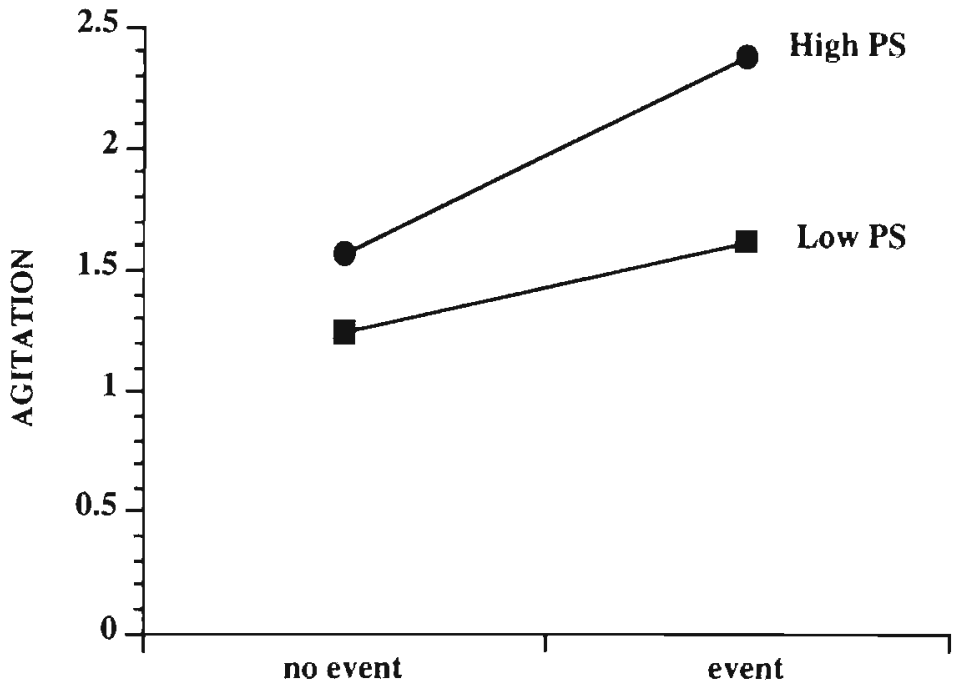

Figure 4.2. Modelled change in Agitation in 'high' and 'low' stress subjects in response to a stressful event (controlling for all other variables). 


\section{Effects of events on Positive Affect}

The multilevel results for Positive Affect are shown in Table 4.4. Occurrence of. a stressful event was not associated with any change in Positive Affect in either HS or LS subjects. In addition, no significant association was found between intercept variance and event variance (Covariance Intercept/CE; see Table 4.4.).

However, a carryover effect for a prior event on current Positive Affect was found: current positive mood was less positive when preceded by a prior event. The significant Prior Event x Perceived Stress interaction indicated a small rebound effect for HS subjects: when a prior event was present, HS subjects had relatively higher current Positive Affect than when a prior event was absent. A future event had no observable effect on current Positive Affect, regardless of perceived stress level. Following events that were more unpleasant than average, a significant decrease in Positive Affect was observed. Positive Affect was no more likely to change in response to a work than to a non-work event, or following a social interaction or a task demand event when compared to no social interaction and non-task demand events.

Table 4.4. Multilevel Results for Positive Affect (PA)

\begin{tabular}{lrcc}
\hline Fixed Effects & Estimate & \multicolumn{1}{c}{ S.E. } & $\mathrm{p}<$ (one-tailed) \\
\hline Intercept (mean PA) & 5.790 & 0.076 & $*$ \\
Perceived Stress Group (PS) & -0.409 & 0.108 & \\
Current Event (CE) & -0.038 & 0.029 & $\mathrm{~ns}$ \\
Prior Event (PE ${ }^{1}$ ) & -0.085 & 0.037 & $*$ \\
$\quad$ PE x PS & 0.101 & 0.052 & $*$ \\
$\quad$ Event Unpleasantness & -0.044 & 0.015 & $* *$
\end{tabular}

\begin{tabular}{lrrr} 
Random Effects & Estimate & S.E. & $\mathrm{p}<$ \\
\hline Person level: & & & \\
$\quad$ Var (Intercept) & 0.241 & 0.039 & $* * *$ \\
$\quad$ Vat (Current Event) & 0.006 & 0.009 & $\mathrm{~ns}$ \\
$\quad$ Cov (CE. Intercept) & -0.017 & 0.015 & $\mathrm{~ns}$ \\
Measurement level: & & & \\
$\quad$ Var (Residual term) & 0.175 & 0.005 & $* *$ \\
& & & \\
\hline Autocorrelation & 0.266 & 0.023 & $* * *$
\end{tabular}

$\mathrm{n}=2358$ observations (including 342 events) nested within 85 subjects ( 39 HS, 46 L.S)

1 event at t-1: "ps.05: "* ps.01: "*at ps.001

Non-significant fixed effects:

Event type: Work, Social Interaction, Task Demands

Event appraisals: Importance. Predictability, Chronicity, Controllability

Future Event

Interactions: $\mathrm{CE} \times \mathrm{PS}, \mathrm{FE} \times \mathrm{PS}$ 


\section{DISCUSSION}

Our main objectives were to investigate whether stressful daily events resulted in short-term changes in mood states, and whether differences in perceived stress level were related to the magnitude of mood changes. Results differed for each of the three mood dimensions investigated. With respect to Agitation, we found that daily events were associated with higher levels of Agitation in both HS and LS groups, with HS subjects showing the strongest mood reactivity. For Negative Affect, events were associated with worse moods in HS subjects only. Current events were not associated with changes in Positive Affect.

These results are consistent with those found in individuals scoring high in trait negative affectivity or neuroticism. Trait negative affectivity has not only been linked to higher overall levels of negative mood (Watson \& Clark, 1984) but also to increased reported exposure to stressful events (e.g. Bolger \& Schilling, 1991: Martin, Ward, \& Clark, 1983) and to increased mood reactivity to stressors (e.g. Bolger \& Schilling, 1991; Eysenck \& Eysenck, 1985; Marco \& Suls, 1993). Level of perceived stress was indeed strongly correlated with trait anxiety in the present study (rho (85) $=.77, \mathrm{p}<.001$ ), and HS subjects not only reported more stressful events, but also higher negative mood levels across all situations and stronger negative mood reactivity to events. Since other analyses indicated that the events reported by HS subjects were rated as no more severe than events reported by LS subjects (mean intensity of events in HS subjects is 3.5 \{st.dev. $=1.3$ \} versus $3.2\{$ st.dev. $=\{.7\}$ in LS subjects; ns), differences in appraised severity of events cannot explain the observed differences in mood responses. A possible explanation for increased mood reactivity among $\mathrm{HS}$ subjects is the use of less effective coping strategies (see (Bolger, 1990)). In our study, as in all studies based on self-report information, we cannot determine whether HS subjects were actually exposed to more stressful situations, or whether they were more likely to perceive events as stressful, had a heightened recall of negative situations, or were more likely to generate stressful situations, for example by failing to anticipate and thereby prevent events from happening. Laboratory studies have shown that neuroticism, which is significantly related to self-report measures of stress (Watson \& Pennebaker, 1989), increases the recall of negatively toned information (Martin et al., 1983), thereby possibly accounting for some of the positive association that has been found between neuroticism and exposure to stressors (e.g. Fergusson \& Horwood, 1987). Contrary to these reports, neuroticism was not related to increased event reporting in a recent within-day field study (Marco \& Suls, 1993). It seems possible that recall biases play less a role when the time intervals between assessments are short. One way of getting more objective event measures is to obtain confirmation of event occurrence from partners (Stone et al., 1991); this option is not realistic, however, when (as in our study) a large percentage of events occur in the workplace. Although it might be possible to obtain confirmation of event occurrence from co-workers or closest colleagues, this option does not seem very practical. In addition, many events (for example, demand situations) are very difficult to observe.

In LS subjects, we found that current events were associated with higher Agitation levels but not with higher Negative Affect levels. Given the specific items of the Agitation scale (restless, irritated, hurried and nervous), it makes sense that this mood scale would be especially sensitive to short-term effects of daily stressors. The Negative Affect scale items (depressed, anxious, worried, lonely, tired and miserable) are more likely to reflect longer term or intense reactions. HS subjects seem to react in 
a more passive and depressive way to stressful events then LS subjects, which may relate to differences in coping strategies, but also to the more chronic nature of stress in HS subjects ('giving up').

In contrast to results of a number of end-of-day diary studies (Neale et. al., 1987; Repetti, 1993; Stone \& Neale, 1984), current stressful events did not lower Positive Affect in the present study. Differences in mood scales and frequency in assessments might account for the lack of an effect of current events on Positive Affect. Associations between stressful events and mood do not have to be the same within and across days. It is possible that an accumulation of several events experienced during the day lowers PA at the end of the day, but that the occurrence of one stressful event does not have an irnmediate effect on Positive. Affect. Lack. of an association between stressful events and Positive Affect is more in line with several studies that suggest that Positive Affect and Negative Affect reflect two separate affective systems and were Positive Affect was found to be unresponsive to perceived daily stress but increased in relation to pleasant social events (Clark. \& Watson, 1988; Kanner et al., 1981; Watson, 1988). A possibility is that people's positive moods are not easily affected by minor stressors, but only, as suggested by the current findings, when events are more unpleasant than average.

To increase our knowledge about how long minor daily events continue to exert their influence on mood we investigated whether stressful events reported on average 90 minutes earlier still influenced current. mood states. Events at beep (t-I) were indeed associated with a persistent elevation in Agitation at beep (t0) and a marginally significant elevation in Negative Affect. In other words, the effects of events on mood states persisted for at least 90 minutes (the average interval between two ESM reports) after a stressful event was reported at (t0). These carryover effects for the negative moods were equivalent in both HS and LS groups. The results contrast with those of Marco and Suls (1993), who found no 'carryover' of the negative mood effects of prior events. Two methodological differences between the two studies may explain the inconsistencies: firstly, Marco and Suls' negative mood scale was composed of different items (tense, unhappy, and angry), and secondly, with eight beeps per day, time intervals between successive reports would have been somewhat longer on average than the 90 minute interval in the current study. Here, the occurrence of a future event was accompanied by higher current Agitation in both HS and LS subjects, but by higher Negative Affect in HS subjects only. The: effect sizes for subsequent events were larger than for prior events (especially for Agitation, and, in HS subjects. Negative Affect), indicating that a future event influenced current negative mood more than prior events. These results confirm the common observation that the anticipation of an event can increase current negative mood. More detailed analysis are necessary to determine whether anticipation effects are limited to certain types of events or certain event appraisals. This was beyond the scope of the present study.

Of the various types of events (work vs non-work events, social interaction vs non-social events and, task demands vs non-task demands), we found that task. demand situations (e.g. overloads, time pressure or deadlines at home or at work) were accompanied by significant higher Agitation levels. This association makes intuitive sense, especially in a white-collar sample. It is not clear, however, why we failed to replicate the finding in several other studies of a strong effect of interpersonal conflicts on negative mood (Bolger et al., 1989a; Bolger \& Schilling, 1991: Clark \& Watson, 1988; Repetti, 1993). The fact that these earlier studies investigated the relationship between interpersonal conflicts and mood across days. 
may in part explain the difference. For instance, the occurrence of repeated social interaction events during the day may be necessary to have a measurable effect on mood. Moreover, retrospective biases may have a greater influence in daily than in within-day studies; for example, a bad mood at the end of the day may be ascribed to a interpersonal conflict earlier that day. Another factor may be that we have coded events as interaction events ourselves, while in the other studies subjects indicated on a checklist whether a personal conflict had occurred or not. It is possible that interpersonal conflicts indicated by subjects on a checklist are of a more serious nature than the events coded by us. Finally, the loss of a considerable number of events reported due to the inclusion of the prior and future event variables (538 events vs. 342 events) may have resulted in a low statistical power. This is supported by the fact that in the full dataset (without the lagged variables), social interaction events and task demands did significantly increase Negative Affect.

Individuals' subjective appraisals of daily events had additional effects on mood responses. The more unpleasant an event was rated, the greater its effect on Agitation and Negative Affect, whereas only events that were more unpleasant than average were associated with lower Positive Affect. The perception of having control over a stressful event lowered its effect on both Agitation and Negative Affect levels. Negative Affect levels were also lower when an event had occurred more frequently than average in the past. No significant effects were found for event importance or event predictability. This does not necessarily mean that these variables are not important. Firstly, within-subject samples of events may have been too heterogeneous or too small in the current study to reveal consistent and reliable effects of all the specific appraisal variables, and secondly, since the appraisal variables were to some degree correlated with each other, the strongest predictor variables will have masked the weaker ones when all variables were entered into the model simultaneously.

Some caveats should be mentioned with respect to interpretation and generalization of these results. Firstly, subjects were well-educated, white collar males; their assessments of what constitutes a 'stressful' event or situation is not necessarily based on the same criteria as other groups (for example females, blue collar workers, students, or retired people) might use. The same applies to their mood ratings. Men tend to report lower, less extreme levels of negative emotions as well as positive emotions than women (Cameron, 1975; Diener, Sandvik, \& Larsen, 1985), and daily stress has been found to be more upsetting to women than to men (Bolger et al., 1989a). Men also appear to expend greater effort in limiting emotional distress (at the expense of physiological distress), resulting in lower emotional reactivity (Gottman \& Levenson, 1988). We also cannot rule out the possibility that current mood influenced the reporting of recent stressful event occurrence. However, the finding that prior events were associated with persistent increases in Agitation and Negative Affect supports the assumption that events influenced mood and not vice versa. Another limitation is that we did not assess positive events and are thus unable to address the important question of the extent to which positive events compensate for, or neutralize, the effects of negative events in HS and LS groups.

In summary, results support the interpretation that individuals with high perceived stress are more vulnerable to 'daily hassles' and therefore may be at greater risk for the development of stress-related health problems. Individuals with high perceived stress levels were more trait anxious, more likely to experience daily stressors, higher in overall negative mood, and more reactive, in terms of negative. mood states, to daily events. This suggests a reciprocal relationship between perceived stress, events and mood reactivity, where increases in event exposure and 
high mood reactivity maintain the appraisal of high stress. Only longitudinal studies with prospective and repeated measurements of both trait and state measures, will be able to clarify causal pathways, including the long-term effects of stressful daily events. Analytic strategies will have to be used that can estimate the relative contribution of each predictor variable, since many variables related to the stress process tend to be highly intercorrelated to each other. Another important subject for future research is the investigation of why HS individuals or trait anxious individuals exhibit greater negative mood reactivity to minor events. Exploration of differences between HS and LS groups in the actual coping strategies used when confronted with stressful events seems to be a good start.

\section{ACKNOWLEDGEMENTS}

We thank C. Dijkman for assistance in all aspects of the study. We also thank Dr. D. Hedeker for his advice regarding the use of the MIXREG program. 


\section{Chapter 5}

\section{Cortisol and catecholamine excretion in relation to perceived stress}

Section S.I: Annals of Behavioral Medicine 16(3): 221-227 (1994)

Marleen van Eck ${ }^{1}$, Nancy Nicolson ${ }^{1}$

Department of Psychiatry \& Neuropsychology, Social Psychiatry and

Psychiatric Epidemiology section, University of Limburg, the Netherlands 
Chapter 5 addresses the relationship between perceived stress and salivary cortisol and urinary catecholamines levels in the context of everyday life. In general we test the hypothesis that high perceived stress is associated with both elevated cortisol as well as elevated catecholamine levels. In section 5.1, two groups of male white collar workers differing in levels of perceived stress were compared on subjective self-report measures of distress and on levels of salivary cortisol, as measured ten times a day over five days. Here, the effect of perceived stress on cortisol is tested, either in general, or during certain phases of the circadian cycle or certain days of the week (work versus non-work days. In section 5.2, the same groups of subjects were compared on 14-hour levels of catecholamines. Urine samples were collected twice overnight, one after a work day and one after a weekend day. Here, we test the effect of perceived stress on catecholamine levels, either in general or during certain days of the week (work versus non-workdays). 



\title{
Section 5.1
}

\section{Perceived stress and salivary cortisol in daily life}

\begin{abstract}
Clarifying the nature of endocrine responses to chronic or intermittent stress in daily life requires repeated measurements of stress, hormone levels and emotional states. In this study, 42 'high stress' (HS) and 46 'low stress' (LS) subjects were selected on the basis of Perceived Stress Scale scores from a larger sample of male white-collar workers. Subjects completed self-reports (Experience Sampling, ESM) and collected saliva samples for cortisol determination 10 times a day over 5 consecutive days, including 3 work and 2 non-work days. Here we test the hypothesis that high perceived stress is associated with elevated cortisol.

The HS group scored higher than the LS group on measures of trait anxiety, depression, anger and psychosomatic complaints, as well as on ESM measures of positive and negative affect and stressful daily events. Although negative affect was higher and stressful events more frequent on workdays than weekends, especially for HS subjects, no difference in workday vs. weekend cortisol levels was found in the subsample of subjects with sufficient data. On workdays, HS subjects had higher mean cortisol levels than LS subjects at each of the 10 sampling times between 8 am. and $10 \mathrm{pm}$ (repeated measures ANOVA, p<.02). Mean workday cortisol was correlated with higher trait anxiety, depression and the low arousal dimension of ESM negative affect.
\end{abstract}

\section{INTRODUCTION}

Over the last 20 years, a wide range of studies has established an association between psychosocial stress and a variety of psychological and physical disorders. The strength of these associations are usually small and inconsistent, but stressful life events, for instance, have been linked to depression, neurotic impairment and other psychological symptomatology (Brown \& Harris, 1989; Dohrenwend \& Dohrenwend, 1974; Dohrenwend \& Dohrenwend, 1978). Stress has also been linked empirically with increased susceptibility to infectious disease (Cohen et al., 1991), with other immune-related disorders like recurrences of herpes infections (Hoon, Hoon, Rand, Johnson, Hall, \& Edwards, 1991) and asthmatic exacerbations (Isenberg, Lehrer, \& Hochron, 1992), with coronary heart disease (Bassett, 1982), and with diabetes mellitus (Goetsch, Wiebe, Veltum, \& Van Dorsten, 1990). The diversity of stress concepts and research methodologies used in these studies makes it difficult to integrate all of the findings.

Since the beginning of this century, the endocrine system has received much attention in stress research (Mason, 1968; Selye, 1936), with an emphasis on the hypothalamic-pituitary-adrenal (HPA) axis, since this system forms a theoretically promising pathway for mediating the relationships between psychosocial stress and subsequent disorder (Curtis, 1972). Cortisol secretion appears to play an important role in the regulation of physiological and behavioral responses under stressful situations (Mason, 1975; Rose, 1984). Cortisol increases after strenuous physical exercise (Mason et al., 1973) and mental task load, specifically in response to distress, as opposed to effort or general arousal (Fibiger \& Singer, 1989; Lundberg \& 
Frankenhaeuser, 1980). Cortisol is also the most important glucocorticoid, with the potential of exerting its effects on practically all normal (body) cells and tissues. Some of the most important effects of cortisol are: stimulation of gluconeogenesis, inhibition of glucose uptake, suppression of inflammation and suppression of numerous immune functions. Glucocorticoids almost invariably suppress rather than enhance biological defense mechanisms. They are thought to protect against the normal defense reactions that are activated by stress, by preventing these defense reactions from overshooting and by' preserving the specificity of immune reactions (Munck et al., 1984).

Indeed, most of the physiological reactions to stress are thought to be adaptive, i.e. they counter the effects of physical stress (trauma, bleeding) or are seen as a preparation for fight or flight responses to acute danger (Cannon, 1929). The question now is, why and when could this adaptive mechanism be a pathway to disease? There are indications that the same suppressive effects of glucocorticoids on several functions of the immune-system may leave a subject more susceptible to infections or even tumors (Claman, 1977). Other studies indicated that increased levels of cortisol may be involved in the etiology of coronary heart diseases (Troxler et al., 1977). The general idea is that stress causes an overproduction of antiinflammatory and immune suppressive action, leading to adverse consequences. (e.g. break down of immune system) (Gaillard \& Al-Damluji, 1987; Selye, 1976). There is also evidence from animal studies for a gradual loss of adaptation within the pituitarycorticoadrenal response. If stress is prolonged, hypophyseal receptors appear to lose their sensitivity to cortisol and as a consequence, prolonged increases in cortisol tend to further slow the recovery of cortisol after stress because of the ineffectiveness of the negative feedback loop (Bassett \& Cairncross, 1977; Kloet \& Reul, 1987). Recent theoretical overviews by Henry and Stephens (1977) and Siegrist (1989) are more or less in line with this concept of loss of adaptation.

It is reasonable to assume that stress reactions will only lead to disease when they are prolonged, or occur very often. The effects of chronic or intermittent stress on the HPA-axis, however, have received far less attention in the literature than the effects of acute stressors, and studies on naturally occurring stressors and their effects are even more rare. Data on cortisol levels during chronic psychosocial stress are inconsistent, with enhanced as well as decreased concentrations reported, and large variability among individuals. Elevated levels of cortisol were found in subjects living near the damaged nuclear power plant at Three Mile Island (Schaeffer \& Baum, 1984). Higher levels of urinary cortisol correlated significantly with urinary catecholamines, self-reports of physical and mental symptoms, and decrements in task performance. A study following parents of leukemic children into the period of bereavement, for as long as 3 years after the death of the child, showed that 17 hydroxycorticosteroid excretion rates were related to the effectiveness of subjects' psychological defenses (Hofer et al., 1972a; Hofer et al., 1972b). Elevated levels of plasma and salivary cortisol were found in Iran hostages after release from captivity, reflecting distress, anxiety and elation. Salivary cortisol was the only physiological measurement that demonstrated a significant correlation with psychiatrists' ratings of the hostages' psychological disturbance (Rahe et al., 1990).

The above studies have examined stress under rather extreme or unusual situations. With respect to more normal settings, studies of air traffic controllers suggest that occupational stress is associated with increased cortisol production (Rose \& Fogg, 1993; Rose, Jenkins, Hurst, Kreger, Barrett, \& Hall, 1982). Caplan et al. (1979) studied white-collar workers in a relatively everyday organizational setting. 
While there was no main effect of work load or personality traits on mean cortisol, high perceived work load was associated with lower morning cortisol values.

The results presented here are part of a larger study concerning emotional and hormonal responses to naturally occurring stress in daily life. Stress is currently conceptualized as a dynamic process, which changes over time and in relation to the environment. It is therefore necessary to include repeated measurements of stress. hormone levels and emotional states to investigate the stress-hormone relationship. This relationship should ideally be studied in naturalistic settings and over time, so that we can begin to understand how adaptation occurs in response to intermittent daily hassles, in individuals who are observed in their normal social networks, settings and activities (Dimsdale, 1984). For these reasons, we chose to use two relatively new techniques, Experience Sampling (ESM) (Csikszentmihalyi \& Larson, 1987; de Vries, 1992) and monitoring of salivary cortisol (Kirschbaum \& Hellhammer, 1989), to assess the relationship between an individual's affective state and neuroendocrine changes over time. Subjects were selected on the basis of Perceived Stress Scale scores (Cohen et al., 1983) from a large sample of male white-collar workers and were then monitored during their daily activities. Subjects completed self-reports. (ESM) and collected saliva samples for cortisol determination at semi-random intervals ten times a day over five consecutive days, including three work and two non-work days.

Here we test the hypothesis that high perceived stress is associated with elevated cortisol, either in general, or during certain phases of the circadian cycle or certain days of the week (e.g. work versus non-work days). With respect to diurnal patterns, no specific hypothesis was formulated. The few studies in which cortisol has been measured at more than one time of day in healthy subjects report significant relationships between cortisol and psychological trait measures at some time points and not at others. For example, in a large German survey study in which salivary cortisol was measured three times on a single day, only 8 am levels showed clear associations with psychological and demographic variables (Brandtstädter et al., 1991). In a similar fashion, perceived work load had an effect on cortisol levels in white-collar workers that was dependent on the time of day when blood was sampled (Caplan et al., 1979), and hostility was associated with high cortisol in daytime, but not in evening or overnight urine samples (Pope \& Smith, 1991). Depressive symptoms could also be related to increased cortisol secretion at specific times of day. Concerning day of the week effects, we hypothesized that cortisol would be elevated on workdays compared to the weekend, possibly in interaction with perceived stress. Although subject groups were selected according to a global measure of stress and not on the basis of work-related stress, we reasoned that work might be a major source of stress. Studies by Frankenhaeuser and colleagues (1989) have demonstrated that cardiovascular and neuroendocrine activity are higher at work than at home, and that slow recovery of responses, or 'unwinding', after work might represent a long-term health risk.

Finally, we examined the extent to which psychological variables (trait anxiety, depression, anger and psychosomatic complaints; ESM measures of mood and stressful events) might contribute to individual differences in workday cortisol level. 


\section{METHOD}

\section{Subjects}

Local industries and government agencies were approached via their personnel departments to participate in the study. A decision as to which categories of employees fell under the definition of 'white-collar' was made by each personnel department on the basis of standard job function descriptions. Questionnaires were distributed among these employees, accompanied by a letter explaining the goals of the study. Participation was voluntary, and care was taken to insure anonymity. 316 male employees from six different industries or agencies completed the screening questionnaire. The mean score on the Perceived Stress Scale (PSS) for this sample was 12.7 (s.d. 6.0), similar to US. norms (mean 13.02, s.d. 6.45) (Cohen et al., 1983).

92 Subjects with scores in the upper or lower tertiles of the screening sample distribution (PSS-10 score $<10$ or 216 ) were recruited to participate in the study, excluding any individuals with a history of endocrine disorder, medications known to affect cortisol levels, psychopathology (major affective disorder, psychoses), or currently in treatment for mental health problems.

'High-' and 'low-' stress subjects were matched for age group, marital status, and household composition. Because it was not possible to match all subjects, numbers of subjects in the final two groups differ slightly.

Four subjects failed to meet Experience Sampling compliance criteria (see 'Daily Experience' section, below) and were therefore excluded from further analysis. Of the 88 remaining subjects, 42 subjects comprised the 'high stress' (HS) group and 46 subjects the 'low stress' (LS) group. Mean age was 42.1 years (range 27 to 57 years), with no significant difference between the two groups. $89 \%$ were married, and $81 \%$ had children living at home.

\section{MEASURES}

\section{Questionnaires}

In addition to the PSS, questionnaires concerning psychosocial stress, coping style, and psychological and physical symptoms were completed. Self-report instruments were chosen on the basis of their theoretical relevance to the stress process and psychometric reliability and validity. The following meastires are used in the cuirent analysis:

Perceived Stress: The 10-item version of the PSS was translated into Dutch by the first author and then back-translated as a check into English by the second author. The items were rated on a 5-point frequency scale, ranging from 'never' (0) to 'very often' (4) in the last month. A total perceived stress score was obtained by reversing the scoring on the positive items and then summing across the 10 items. All subjects completed the PSS twice, during the initial screening and again immediately preceding Experience Sampling. The two PSS scores were highly correlated $(r h o=73, \mathrm{p}<.001)$; the mean score will be used in the analysis.

Psychosomatic symptoms: The SUNYA Psychosomatic Symptom Checklist (PSC) includes 17 common psychosomatic complaints (e.g., headaches, backaches and nausea ). The scale was originally developed by Cox, Freundlich, \& Meyer (1975) and revised by Attanasio et al. (1984). Subjecis rate each complaint on 5 -point scales for frequency ( 0 "never or rarely occurs " to 4 "occurs daily") and intensity ( 0 "not bothersome" to 4 "extremely bothersome"). A Total Score, reflecting the overall level of psychosomatic distress, is obtained by summing the cross-products of each item's frequency by intensity.

Depression: Depressive symptomatology was assessed with the Dutch translation (Dijkstra, 1974) of the Zung Self-Rating Depression Scale (Zung, 1965).

Anxiety; Trait anxiety was measured with the Dutch version (van der Ploeg et al., 1980) of the State-Trait Anxiety Inventory (STAI). 
Anger: Trait anger was measured with the Dutch version (van der Ploeg et al., 1982) of the Spielberger Trait Anger Scale. Although two subscales ('anger-temperament' and 'angerreaction') exist, only the total' score is used here.

\section{Daily experience}

The Experience Sampling Method (ESM) (Csikszentmihalyi \& Larson, 1987; de Vries, 1987; de Vries, 1992) was used to collect data from subjects at selected moments during their normal daily activities. Subjects received auditory signals, after which they filled in a questionnaire and collected a saliva sample. Although additional reports were completed at the end of each day, for the purpose of this analysis we will refer only to the 'beep level' data.

After a 'briefing' session, in which all procedures were explained in detail and informed consent obtained, subjects were sampled for a period of 5 consecutive days, beginning early on a Thursday morning and ending late Monday. In this sample, the weekends were non-workdays. A Seiko wristwatch was programmed to emit beeps 10 times each day, at semi-random intervals of approximately 90 minutes between the hours of 8 am and $10 \mathrm{pm}$. Beeps were clustered around the midpoint of each time block (e.g., 8.15 am, 9.45, 11.15, and so on); the exact time sequence of beeps was varied each day to decrease predictability. In a final 'debriefing' session, ESM forms were checked for legibility, and subjects were asked to clarify reasons for missing data.

Compliance with the procedures was generally good. The criteria we set for inclusion in the analysis ( 220 ESM reports, completed within 20 minutes after being signalled and no missing data for entire days) were met by all but four subjects ( 2 from each group). The remaining 88 subjects completed an average of $83 \%$ of all possible responses within the time limit, for an average of 41 responses per subject. HS and LS groups did not differ in compliance rates (40.1 vs 42.3 responses per subject, Mann-Whitney U-test, p>.05). Compliance was lowest for the first beep (at approximately $8.15 \mathrm{am}$ ), with an average of $73 \%$ valid reports. On Saturdays, response rate for first beeps was $61 \%$, and on Sundays, $59 \%$. $74 \%$ of all missing and invalid responses on weekends could be attributed to the fact that subjects were still asleep.

The ESM form contained open-ended questions concerning thought content, the physical and social context, and what the individual was doing when signalled. The forms also included 7 point Likert scales (from I 'not at all' to 7 'very much') for rating aspects of thoughts, mood, physical well-being, individually defined (psycho)somatic complaints, and present activity. Subjects were asked to describe any stressful events or situations which may have taken place in the interval since the last ESM report and to rate these events on a number of dimensions. Information about smoking (Wust, Kirschbaum, \& Hellhammer, 1990), food (Quigley \& Yen, 1979), coffee (Pincomb, Lovallo, Passey, Brackett, \& Wilson, 1987), and alcohol intake, medications, and physical exertion (Cook, Ng. Read, Harris, \& Riad-Fahmy, 1987) since the last beep was also obtained, to help control for possible confounding influences on cortisol secretion.

\section{Salivary cortisol}

Salivary cortisol is a reliable and valid indicator of the free cortisol in plasma, which is considered to be the biologically active hormone; cortisol concentrations are independent of the flow rate of saliva (Vining et al., 1983). Salivary cortisol increases within minutes in response: to acute stressors (Hellhammer, Kirschbaum, \& Belkien, 1987) and has a half-life of approximately one hour (Fredrikson et al., 1985). We have found no difference in cortisol levels in saliva samples either frozen immediately or kept at room temperature for 2 days (Nicolson et al., 1992); others report no change in unfrozen samples up to 30 days (Kirschbaum \& Hellhammer, 1990).

At the same time ESM forms were being completed, subjects collected saliva by holding a cotton dental roll in the mouth for approximately 1 minute. The saturated roll was placed in a capped plastic vial (Salivette; Sarstedt), which was stored in a specially designed wallet. At the end of each day, subjects placed the vials in their home freezers. At the end of the sampling period, 
uncentrifuged samples were stored at $-20 \mathrm{C}$. Compliance with the saliva sampling procedure was good in both high and low stress groups, with approximately the same mean response rate (83\%) as for the ESM reports.

Salivary cortisol levels were determined in duplicate by direct radioimmunoassay (Ansseau et al., 1984), using ${ }^{125}$ I-cortisol (Farmos diagnostica, Finland) and antiserum made against the 3CMO-BSA conjugate by Dr. J. Sulon, University of Liege, Belgium. The lower detection limit of the assay was $12 \mathrm{ng} / \mathrm{dl}$, with a mean intra-assay coefficient of variation of $4.8 \%$ (range: $2.2 \%$ $7.5 \%$ for 4 assays). Each subject's samples were analysed in the same assay, to reduce sources of variability.

\section{Stallstical analysis}

The 16 ESM mood items were reduced to three mood measures, based on the results of a principal component analysis with varimax rotation, which accounted for $78 \%$ of the total variance when subject mean scores were used. Ratings on the items cheerful, satisfied, relaxed, energetic, self-assured, concentrated and enthusiastic were summed to form ai 'positive affect' (PA) scale (Cronbach's alpha $=.95$ ). Two separate components of negative affect were identified: "NA-low arousal', including the items depressed, anxious, worried, lonely, tired, and miserable (alpha = 87), and 'NA-high arousal', with the items restless, irritated, hurried, and nervous (alpha $=.93$ ). The sums of the scale items were divided by the number of items in the scale, so that all mood measures have ranges from 1 to 7 .

Because the distribution of cortisol values was positively skewed, transformation to natural logarithms of cortisol concentrations was performed prior to statistical analysis. Nonparametric tests were used for univariate group (Mann-Whitney U-test) and within-subject (Wilcoxon matched-pairs signed-rank test) comparisons. Unless noted otherwise, significance tests are twotailed. Analysis of variance (SPSS procedure MANOVA) was used to test differences (and interactions) between HS and LS groups, workdays and weekends, and the 10 times of day sampled. Analyses were performed with SPSS -Macintosh version.

\section{RESULTS}

Given the general nature of the PSS, which was used to define high and low stress groups in this study, it is useful to contrast the two groups on more specific measures of distress. In addition to trait measures, we compared groups on a number of aggregated state measures derived from the ESM data. Mean scores on each of the 3 ESM mood scales were computed across all valid beeps for each subject. Frequency of stressful daily events was calculated as the percentage of total beeps for a subject on which a stressful event was reported, regardless of rated severity. 
Table 5.1.1. Differences in psychological measures between low and high stress groups

$$
\text { low PSS high PSS }
$$

mean (st.dev) mean (st.dev.) D-value

(2-tailed)

Trait measures

Perceived stress (PSS)

Trait Anxiety

Trait Anger

Zung Depression

Psychosomatic

symptoms (PSC)

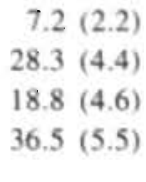

$6.1(5.6)$
$18.1(3.4)$

$39.8(7.6)$

$23.0(5.2)$

$48.4(7.7)$

$27.5(23.2)$
$<.0001$

$<.0001$

$<.000 \|$

$<.0001$

$<.0001$

\section{ESM measures*}

\begin{tabular}{lccl} 
PA & $5.7(0.6)$ & $5.3(0.3)$ & $<.001$ \\
NA-low arousal & $1.2(0.1)$ & $1.4(0.4)$ & $<.0001$ \\
NA-high arousal & $1.3(0.3)$ & $1.8(0.6)$ & $<.0001$ \\
frequency of stressful events & $12.3 \%(11.9)$ & $22.0 \%(20.0)$ & $<.01$ \\
\hline
\end{tabular}

" subject means, aggregated from beep level data

As shown in Table 5.1.1., subjects with a high level of perceived stress were significantly more trait anxious and depressed than subjects with a relatively lower level of perceived stress. They also scored higher on trait anger and reported more psychosomatic symptoms. HS subjects had significantly lower ESM positive affect, higher negative affect-low arousal and higher negative affect-high arousal scores. They were almost twice as likely to report stressful daily events as LS subjects. In absolute terms, the mean number of stressful events reported over the five days of ESM was 9.0 for the HS and 5.3 for the LS group.

We next examined whether high perceived stress was associated with elevated salivary cortisol. Mean cortisol levels were first computed for each subject at each of the 10 time blocks for workdays and again for weekend days. To test for main effects on cortisol levels as well as interactions, analysis of variance was performed with the between-subject factor group (HS, LS) and within-subject factors time of day ( 10 beeps) and day type (workday, weekend). 39 of the 88 subject cases were rejected because of missing data for any of the 20 (10 time blocks $\times 2$ day types) measures. The remaining 49 subjects included $28 \mathrm{LS}$ and $21 \mathrm{HS}$ subjects.

Cortisol concentrations showed the well-defined circadian rhythm, with peak salivary concentrations in the early morning samples. declining values in the afternoon and lowest concentrations at the end of the day; the main ( ffect for time of day was highly significant $(F(9,423)=182.04, p<.001)$, with no significant interactions with either group or day type. Contrary to our hypothesis, no significant main effect for group was found. Further, there was no workday-weekend diffierence in cortisol levels, either as a main effect or in interaction with group. 


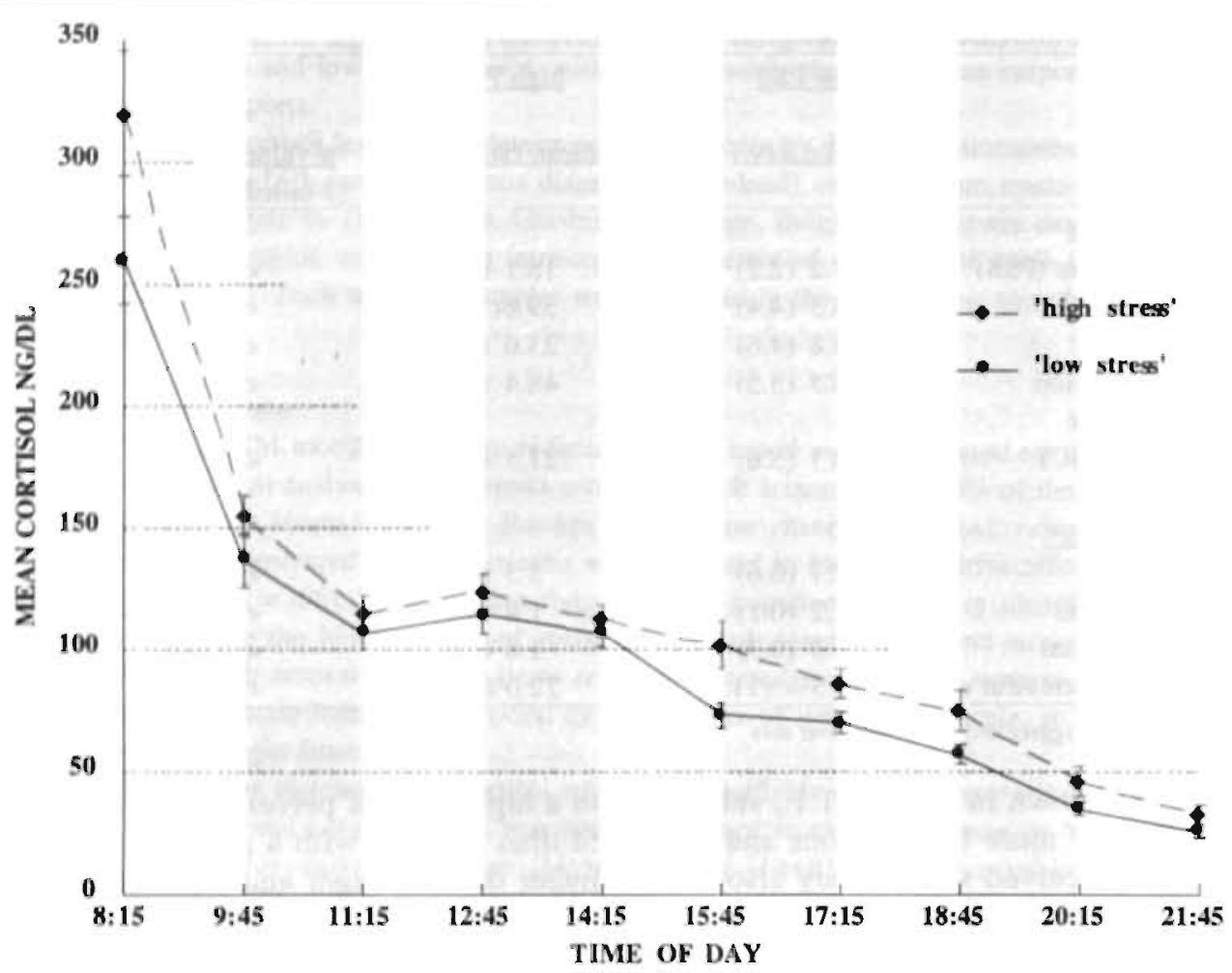

Figure 5.1.1. Mean cortisol (ng/dl; $+1-$ s.e.) during workdays for the 'high' $(n=34)$ and the 'low' stress' group $(\mathrm{n}=39)$.

As noted earlier (see 'Daily Experience', above), the percentage of missing responses was higher on weekends than on workdays, especially for early morning samples. Inclusion of weekend data in the model is thus largely responsible for the considerable data attrition. We therefore chose to examine workdays and weekends again separately. While this does not address the issue of workday versus weekend differences, it allows us to test for group differences in cortisol in a larger sample.

For workdays, 15 subjects were forced out of the analysis due to missing data, leaving $39 \mathrm{LS}$ and $34 \mathrm{HS}$ subjects. Time of day again showed the Iargest effect on cortisol levels $(\mathrm{F}(9.639)=262.98, \mathrm{p}<.001)$, but here the difference between HS and LS groups was also significant $(\mathrm{F}(1.71)=6.02, \mathrm{p}<.02)$. The interaction effect was not significant $(F(9,639)=.67, p>.05)$. Cortiso! concentrations for HS and LS groups on workdays are shown in Figure 5.1.1.

In the analysis of differences in cortisol levels on weekends, only 56 subjects (32 LS and 24 HS) had sufficient data for inclusion. For this subsample, the usual time of day effect was found $(F(9,486)=85.80, p<.001)$, but there was no consistent difference between the two stress groups $(F(1,54)=.79, p>.05)$. The group by time of day interaction approached significance $(F(9,486)=1.83, p=.06)$, with a tendency for the high stress group to have higher cortisol levels in the morning through early afternoon (beeps 2, 3, 4 and 5, between 9.30 am and 2.30 PM) and in the late evening 
(beeps 9 and 10, after 8 PM) only. Again, it is important to note that this (nonsignificant) pattern is based on a relatively small subset of the entire sample.

To summarize, cortisol levels showed the expected strong diurnal pattern in all analyses we performed. More interestingly, 'high stress' subjects had elevated cortisol throughout the day on workdays, confirming our hypothesis, at least for these days. It would seem logical to assume that such a pattern would be mediated by group differences in stress exposure or, more specifically, in emotional response to daily stress. Questions concerning reactivity of mood and cortisol to stressful events are beyond the scope of this paper. However, measures of workday stress and distress could provide additional insights into the perceived stress - cortisol relationship.

Within subjects, workdays in comparison to weekends were associated with a higher rate of stressful events (Wilcoxon matched-pairs test, p<.0001) and greater NA-high arousal ( $<<.0001)$, with no differences in either NA-low arousal or PA. The coded descriptions of reported stressors suggested that the work environment was a major arena for such daily hassles, with stressors more often occurring in work than in household, leisure or social network contexts. $43 \%$ of events in LS subjects and $47 \%$ of events in HS subjects were work-related. While this general pattern of workday distress was found in both groups, within-subject differences in workday vs. weekend NA-high arousal were greater in HS than in LS subjects (Mann-Whitney, $\mathrm{p}<.001)$; that is, HS subjects were disproportionately more negatively aroused on workdays. Workday/weekend differentials in NA-low $(.05<p<.10)$ and stressful events $(.05<p<.10)$ did not distinguish the two groups, although results were in the expected direction of relatively greater work-related stress/distress in HS subjects.

Since HS subjects scored higher than LS subjects on trait anxiety, depression, anger and psychosomatic complaints, as well as on ESM measures of positive and negative affect, we expected positive relationships between these variables and cortisol levels, at least on workdays. Mean levels of cortisol over the three workdays were determined by first calculating the mean at each of the 10 time blocks for every subject and then averaging the 10 values for each subject. This aggregated measure of mean cortisol was slightly higher in the HS (116 ng/dl, sd 37.5) compared to the LS group (101 ng/dl, sd 28.2) (one-tailed Mann-Whitney test, $\mathrm{p}<.05$ ).

Spearman rank order correlations between mean workday cortisol and both psychological measures and ESM mood sciales are shown in Table 5.1.2. Small but significant (one-tailed tests) positive correlations were found between cortisol and trait anxiety, depressive symptomatology and NA-low arousal.

Table 5.1.2. Correlations between psychological variables and mean salivary cortisol during workdays

\begin{tabular}{lcl}
\hline & $\mathrm{r}$ & $\mathrm{n}$ \\
\hline Trait anxiety & $.18^{*}$ & 88 \\
Trait anger & .08 & 88 \\
Zung Depression & $.18^{*}$ & 87 \\
Psychosomatic & & \\
symptoms (PSC) & .14 & 88 \\
PA & -.10 & 87 \\
NA - low arousal & $.20^{*}$ & 87 \\
NA - high arousal & .08 & 87 \\
\hline
\end{tabular}

"pe.05 (Spearman; one-tailed tests) 
Although these results indirectly support the conclusion that daily stress and distress lead to increases in cortisol, the role of possible confounding factors should be explored. If, for example, HS subjects smoked more than LS subjects, this might explain the observed differences in cortisol secretion. HS and LS groups were therefore compared on the percentage of workday beeps with reported smoking, strenuous physical exertion (rated 7 on the 7-point scale), coffee, food, and alcohol intake - all activities that could theoretically result in elevated cortisol secretion (see 'Daily Experience', above). No significant differences were found, with mean rates for HS and LS groups as follows (HS/LS): smoking $17 \% / 16 \%$, exertion $0.7 \% / 1.3 \%$, coffee $46 \% / 41 \%$, food $42 \% / 39 \%$, and alcohol $8.0 \% / 7.8 \%$. Although we cannot conclude that these behaviors had no influence on cortisol secretion, effects seem equally likely to have occurred in either group.

\section{DISCUSSION}

In this study, two groups of male white-collar workers differing in levels of perceived stress were compared on subjective, self-report measures of distress and on levels of salivary cortisol, as measured repeatedly over five days. The 'high stress' group scored higher than the 'low stress' group on measures of trait anxiety, depression, anger and psychosomatic complaints, as well as on ESM measures of positive and negative affect and stressful daily events. Cortisol showed a clear diurnal secretory pattern in both groups, with values in the normal range for healthy men (Kirschbaum \& Hellhammer, 1989). On workdays, cortisol was elevated throughout the day in the 'high stress' relative to the 'low stress' group. Several measures of distress, including both trait (anxiety, depression) and concurrent state (NA-low arousal) measures, correlated significantly with mean workday cortisol.

The observed pattern of higher workday cortisol levels in the 'high stress' group is consistent with our initial hypothesis; a straightforward interpretation would be that mild chronic or intermittent stress in situations like those subjects reported during the ESM sampling period was sufficient to increase secretion of the hormone. Since the half-life of cortisol in saliva is shorter than the intervals between beeps, consistently elevated cortisol from morning through late evening indicates continued secretion. More detailed temporal analyses are needed, however, to understand. cortisol dynamics in response to daily stress.

Of the few studies of stress and cortisol in everyday environments to date. results have been inconsistent. Caplan et al. (1979) tested the effect of perceived work load (a summary measure of quantity, deadlines and calls and office visits) on plasma cortisol and found that high work load was associated with low morning cortisol levels. The authors suggested that this finding might reflect a shift in the circadian rhythm of cortisol as a result of job stress. A major disadvantage of this study is the cross-sectional design, in which blood samples were drawn at various times of day, with only one cortisol determination per subject. In other words, comparisons between morning, midday and afternoon cortisol concentrations were comparisons between subjects and not within subjects. Although perceived stress may differ in important ways from perceived work load, our results clearly contradict those of Caplan and colleagues; we found higher early morning cortisol in high stress subjects and no evidence at all for a circadian shift. Brandtstädter et al. (1991), who measured salivary cortisol concentration in 767 adults three times over the course of one day, found higher morning cortisol concentrations in men with high life 
satisfaction, high psychological well-being and high level of employment. Cortisol levels in the afternoon or early evening were not predicted by psychological variables. Again, these results are difficult to reconcile with our findings.

Trait negative affectivity (NA) is conceptualized as the tendency to experience a wide range of negative emotions (Watson \& Clark, 1984). In studies of stress-illness relationships, trait NA has been held responsible for inflated correlations between stressors and self-reported health symptoms (Costa \& McCrae, 1987; Watson \& Pennebaker, 1989). In general, NA has not been found to predict objective health problems or physiological stress responses and has therefore been considered a nuisance in the analysis of stress data (Schaubroeck et al., 1992). The 'high stress' group in our study was defined on the basis of scores on a very clearly subjective self-report measure, the Perceived Stress Scale, and the fact that HS subjects scored higher on state and trait measures of distress as well as self-reported stress exposure suggests the pervasive influence of trait NA. In this light, our finding of an association between subjective stress, trait and state NA measures, and salivary cortisol levels is particularly noteworthy.

As is usually the case in psychophysiological studies, the psychological variables we measured accounted for only a small percentage of the variability in cortisol. Moreover, the observed elevations in workday cortisol secretion in HS subjects were slight, relative to pathophysiological levels seen in Cushing's disease or some cases of major depression. Since cortisol levels are not routinely measured in prospective epidemiological studies of healthy individuals, we have no guidelines at present for assessing the clinical relevance or health implications of these findings.

Analyses in this study were done on aggregated data, which has the advantage of increasing the reliability of measurement when only the chronic level of variables is of interest. On the other hand, aggregated measures may be biased (Jaccard \& Wan, 1993), and they obscure the important interplay between experiential and physiological states and processes which momentary measures are intended to clarify. To gain more insight into the relationship between perceived stress and cortisol, we need to move to beep-level analyses. We can then investigate fluctuations over time in stressful events, mood, psychosomatic complaints and determine how these fluctuations relate to endocrine activity (concurrent and lagged relationships). One promising method for the analyses of temporal data is hierarchical linear modeling, or multi-level analysis (Bryk \& Raudenbush, 1992). This approach can account for the dependency of data within a subject, can deal with the problem of missing data, and allows for individual differences in intercepts, slopes and error structures. We are currently undertaking multi-level analyses, in the belief that these new methods will help realize the enormous research potential afforded by momentary measures.

\section{ACKNOWLEDGEMENTS}

We thank Dr. J. Sulon, University of Liege, for performing RIA analyses of salivary cortisol, and C. Dijkman for assistance in all aspects of the study. 



\section{Section 5.2}

\section{Four-teen hour urinary catecholamine excretion in relation to perceived stress.}

\section{INTRODUCTION}

It has been well documented that, besides the pituitary and adrenal cortex, the adrenal medullary system also plays a central role in stress and arousal (Cannon, 1929; Mason, 1975). Increased catecholamine (CA) secretion has been associated with exposure to a wide variety of psychological and physical stressors (Frankenhaeuser, 1975a); for example to failure, loss, challenge, mental effort, uncertainty, and threat situations, as well as to physical stimuli such as cold, pain, anoxia, exercise, and heat (Frankenhaeuser, 1976; Frankenhaeuser, 1979). The magnitude of CA responses to potentially stressful situations or events is usually determined by the intensity of the appraisal of threat as related to one's perceived ability to meet the demands of the situation (Lazarus \& Folkman, 1984b).

Personal control appears to be an important factor in relation to CA excretion; high personal control (perceived self-efficacy) was accompanied by low levels of plasma adrenaline $(\mathrm{A})$ and noradrenaline (NA) during interactions with a phobic object, while moderate control resulted in substantial increases in plasma CA (Bandura, Taylor, Williams, Mefford, \& Barchas, 1985). With respect to occupational stress, a lack of control over the pace of one's work and extreme workloads (both high and low) were associated with high levels of CA (Frankenhaeuser \& Johansson, 1976; Frankenhaeuser, Nordheden, Myrsten, \& Post, 1971; Timio \& Gentili, 1976). On the other hand, high CA output has also been associated with successful coping and with the ability to maintain a high performance level during stressful conditions, probably as a result of high mental effort (Frankenhaeuser, 1979; Siegrist et al., 1989; Ursin, 1978). There is some evidence to suggest that the two CA are differentially sensitive to behavioral and situational factors. NA relates to active, aggressive emotional states, whereas excessive A levels relate to passive, anxious states (Henry, 1982), but also to states involving high mental effort (Fibiger, Singer, \& Miller, 1984; Frankenhaeuser et al., 1971).

Although a positive relation seems to exist between CA levels and adaptive functioning. frequent and excessive elevations and/or long-term elevation of $\mathrm{CA}$ levels are assumed to have damaging effects on various bodily organs and the cardiovascular system (Gruchow, 1979; Madden \& Livnat, 1991; Surwit, Williams, \& Shapiro, 1982). However, in contrast with the numerous studies on acute stress situations, studies concerning habitual CA excretion as related to enduring characteristics of the social environment or of the individual are more scarce. With respect to long-term occupational stress, elevated A excretion during periods of working 'overtime' was observed; not only during working hours but also in the evening at home (Rissler, 1977). In a study by Baum and colleagues (1986), the stress of being unemployed was related to higher levels of both $\mathrm{CA}$, and levels increased as the length of employment increased. Forsman (1980) also found a positive correlation between habitual A excretion and experiences of distress (measuring day-to-day stress) as measured in the field. As another example, the continuing uncertainty and perceived threat of living near the site of the nuclear power plant accident at Three 
Mile Island was associated with chronic elevations in overnight samples of both $\mathrm{A}$ as well as NA (Schaeffer \& Baum, 1984). Finally, in another study investigating the effects of long-term stress on CA, it was found that urinary CA levels in American hostages freed from captivity in Iran were highly elevated and appeared to reflect three affects: distress, anxiety, and elation (Rahe et al., 1990).

In the present study, urinary CA were determined to investigate the relationship between perceived stress and catecholamine excretion. It was hypothesized that high levels of perceived stress, as a measure of more chronic stress, are associated with elevated CA levels, either in general or related to the type of day (work day versus weekend). Additionally, several person characteristics (trait anxiety, depression, anger, and psychosomatic symptoms), and mood states (Fibiger, Singer, Miller, Armstrong, \& Datar, 1984) were explored for their possible relationship with CA levels. As opposed to plasma CA levels, urinary CA levels ( 14 to 24 hour samples) are typically used in studies for the measurement of long-term, chronic stress, and are particularly well-suited for studying psychosocial influences of everyday life (Frankenhaeuser, 1975a). Because urine samples show a slower rate of change than plasma samples, they can be applied to determine long-term changes in CA levels. The collection of urine samples is also relatively easy, non invasive and very well suited for field studies during which subjects' normal habits and ordinary daily activities should nol be changed (Frankenhaeuser \& Gardell, 1976).

\section{METHODS}

\section{Subjects and questlonnaires}

For details about subject recruitment, procedures and questionnaires see section 5.1.

\section{Urine sampling}

Catecholamine (CA) levels were determined in urine. Because the $\mathrm{CA}$ in urine constitute a small but relatively constant fraction of liberated amines in the body, the direction of change or the relative levels are meaningful, but absolute numbers have limited value. NA levels are somewhat difficult to interpret because NA is secreted by both nerves and the adrenal medulla and is also subject to rapid neuronal reuptake, but $\mathrm{A}$ can be considered a reliable estimate of adrenal medullar activity because the adrenal medulla is the sole source of circulating A (Frankenhaeuser, 1975b). The 14-hour samples we used in this study, suppress (not eliminate) the effects of variation between subjects with different circadian thythms and minimize the biasing effects of idiosyncratic events (Baum et al., 1985).

Subjects collected two overnight urine samples (from 6 p.m. to 8 a.m.), one after a workday (Thursday to Friday) and one after a weekend day (Sunday to Monday). Subjects emptied their bladder in the toilet at 6 p.m. and from that moment collected subsequent urine in a 2 liter container (Sarstedt) until 8 a.m. the next day at which time they emptied their bladder for the last time in the container. Subjects refrigerated their samples during the collection period. Immediately after collection of the container by the research staff we added HCL. $(37 \%)$ to the total volume of urine until a $\mathrm{pH}$ of $<3$ was reached, to prevent oxidation. From the total volume, $10 \mathrm{ml}$. samples were extracted and immediately frozen at a temperature of $-20 \mathrm{C}$ until analyses.

Adrenaline and noradrenaline excretion rates were determined by means of high-pressure liquid chromatography (HPLC) with electrochemical detection. ${ }^{3} \mathrm{CA}$ levels were corrected for

Analyses of adrenaline and noradrenaline were performed in the laboratory' of Dr. Rahman and Dr. Duvivier, University Hospital of Liege. Belgium.. 
creatinine excretion, and expressed in $\mu \mathrm{g} / \mathrm{g}$ of creatinine. Samples of subjects with creatinine levels below $0.60 \mathrm{~g} / \mathrm{l}$ were discarded $(\mathrm{n}=9)$, because they might give artificial too high concentrations (low levels could be suspect of e.g. hyperthyroidism, advanced renal disease). Due to practical reasons, three subjects did not collect any urine, and another four subjects collected urine only once.

\section{Statistical analysis}

Analysis of variance was used (SPSS procedure MANOVA) was used to test differences and interactions between HS and LS groups and work day and weekend day samples. Nonparametric Spearman correlations were calculated the various psychological trait and state measures and the catecholamines. Significance tests are two-tailed. Analyses were performed with SPSS-Macintosh version.

\section{RESULTS AND DISCUSSION}

Mean values for adrenaline (A) as well as noradrenaline (NA) after a work day and after a weekend day are shown in Table 5.2.1. As can been seen, CA concentrations were highly comparable in high stress versus low stress subject groups.

Table 5.2.1. Mean adrenaline and noradrenaline concentrations ( $\mu \mathrm{g} / \mathrm{g}$ creatinine) plus standard deviation in high stress (HS) and low stress (LS) groups after a work day and after a weekend day.

\begin{tabular}{lcccc}
\hline & \multicolumn{2}{c}{ Work day } & \multicolumn{2}{c}{ Weekend day } \\
& LS & HS & LS & HS \\
& $\mathrm{n}=42$ & $\mathrm{n}=37$ & $\mathrm{n}=41$ & $\mathrm{n}=37$ \\
\hline & & & & \\
Adrenaline & $4.3(2.4)$ & $4.7(35)$ & $4.3(2.5)$ & $4.3(37)$ \\
Noradrenaline & $21.4(12.4)$ & $19.9(9.3)$ & $19.6(10.5)$ & $19.2(8.3)$ \\
\hline
\end{tabular}

To test for main effects of perceived stress and day type on CA levels as well as interaction effects, analysis of variance were performed with the between-subject factor group (HS, LS) and the within-subject factor day type (workday, weekend). 13 of the 88 subject cases were rejected because of missing data. The remaining 75 subjects included 37 HS and 38 LS subjects. For A, no main effect for group was found $(\mathrm{F}(1,73)=0.34, \mathrm{p}>.05)$. There also was no work day / weekend difference in $\mathrm{A}$ levels, either as a main effect or in interaction with group $(F(1,73)=0.16, p>.05 ; F(1$, $73)=1.59, p>.05$ ). For NA, again no main effects for group or day type were observed, and also no interaction effect (group: $F(1,73)=0.24, p>.05$; day type: $F(1,73)=0.66$, $p>05)$; group $x$ day type: $F(1,73)=0.07, p>.05)$.

Spearman rank order correlations between mean A and NA and several psychological measures (trait anxiety, depression, anger, psychosomatic symptoms) and mean ESM mood states (positive affect, negative affect, agitation) were next computed. None of the correlations reached significance (all correlations <.21). A trend was found for positive affect to be positively related to both $\mathrm{A}$ as well as NA (A: $r h o=.21, \mathrm{p}=.06$; NA: $r h o=.19, \mathrm{p}=.08$ ).

The results do not support our hypothesis that high perceived stress is reiated to high CA levels, either in general or related to the day of the week, and do not 
replicate the findings reviewed above of increased catecholamine excretion in the context of long-term stress. In fact, none of the variables investigated showed any relationship to both adrenaline as well as noradrenaline levels. The trend found for positive affect to be related to both catecholamines is in line with the general finding of a weak but positive correlation between CA secretion and indices of emotional stability and adaptation (Johansson, Frankenhaeuser, \& Magnusson, 1973). In the present study, urine samples were collected between 6 p.m. and 8 a.m. and is comparable in design to the study by Schaeffer and Baum (1984) where, in contrast with our study, they did find elevated catecholamine levels in chronically stressed subjects. Most other studies sampled urine during the day, and in the study by Forsman (1980), where urine was sampled in the morning, afternoon, evening, and at night ( 11 p.m. to 8 a.m.), only the morning and afternoon CA levels were related to the experience of stress as measured in daily life. Therefore it is possible that in our study of healthy subjects, where samples were obtained mostly overnight (constituting a more or less baseline level) which reduces the impact of specific environmental influences during the day, complete unwinding took place during the night. More extreme stress levels are perhaps necessary to observe an impact on CA baseline levels. It would be interesting to investigate whether perceived stress and the other person and mood variables would relate to $\mathrm{CA}$ levels during the day as opposed to overnight, when specific environmental circumstances are more influential.

An important limitation of our study was that no restrictions were put on the subjects concerning feeding and drinking patterns (including alcohol), exercise, and cigarette smoking. This was done to increase compliance because of the already intensive and time consuming nature of our ESM field study. CA levels are, besides stress, sensitive to all the factors mentioned above, and the absence of a control for these factors may have confounded the results. HS and LS groups were therefore compared on the percentage of ESM beeps (see paragraph 'daily experience' of section 5.1) with reported smoking. coffee, food, alcohol intake, and physical exertion reported during both evenings (work and weekend) when urine collection took place (between 6.45 p.m. and 21.45 ). No significant differences were found between groups suggesting that, although we can not rule out the possibility that these factors had an influence on CA excretion, it should not have confounded the group comparisons. 


\section{Chapter 6}

\section{The effects of perceived stress, traits, mood states, and stressful events on salivary cortisol}

\section{Psychosomatic Medicine (in press)}

Marleen van Eck ${ }^{1}$, Hans Berkhof ${ }^{2}$, Nancy Nicolson', Jose Sulon ${ }^{3}$

1 Department of Psychiatry \& Neuropsychology, Social Psychiatry and Psychiatric Epidemiology section, University of Limburg, the Netherlands

2 Department of Statistics and Measurement Theory, University of Groningen, the Netherlands

3 Faculty of Veterinary Medicine, University of Liege, Belgium 

ABSTRACT This study examined the effects of perceived stress and related individual characteristics, mood states, and stressful daily events on salivary cortisol levels. Forty-one 'high stress' and 46 'low stress' subjects were selected on the basis of Perceived Stress Scale scores from a sample of male white collar workers. Subjects completed Experience Sampling self-reports and collected saliva samples ten times a day over five consecutive days. Multilevel analysis revealed that trait anxiety and depression, but not perceived stress, were associated with small but statistically significant cortisol elevation. No effects on cortisol were found for recent life events, chronic difficulties, trait anger, or psychosomatic symptoms. Distress, as reflected by the mood states Negative Affect and Agitation, was associated with higher cortisol levels, whereas Positive Affect had no statistically significant effect. Stressful daily events were associated with increased cortisol secretion, the magnitude of the effect depending on whether the event was still ongoing and on how frequently a similar kind of event had occurred previously. Although perceived stress, anxiety and depression did not increase cortisol reactivity to daily events, we found evidence for reduced habituation to recurrent events in subjects scoring high on these traits. Mood appeared to play a mediating role in the relationship between stressful events and cortisol secretion. These results suggest that negative affectivity is not just a confounder, but is related to elevated cortisol secretion during normal daily activities. The finding that even minor events and fluctuations in mood states were associated with increased adrenocortical activity points to a possible mechanism linking subjective experience to health outcomes.

\section{INTRODUCTION}

The neuroendocrine system has long been thought to play an important role in the causal pathway linking stress and ill health (Cannon, 1929; Selye, 1976; Weiner, 1992). The hypothalamic-pituitary-adrenal (HPA) axis, which is involved in the regulation of a wide range of physiological and behavioral responses to stress, has been implicated in numerous illness processes (Chrousos \& Gold, 1992; De La Torre, 1994; Tsigos \& Chrousos, 1994), including the etiology of psychiatric disorders (Checkley, 1992; Dinan, 1994; Gold et al., 1988). Over the past decade, it has become clear that not only major life events (Brown \& Harris, 1989; Holmes \& Rahe, 1967), but also minor daily stressors or "hassles" can have negative effects on health and well-being (DeLongis et al., 1982; Ivancevich, 1986; Zarski, 1984). In contrast to the wealth of information concerning the neuroendocrine effects of major real-life stressors, there is relatively little known about the effects of chronic stress and even less about the effects of minor daily events. The goal of the current study was to increase our understanding of the impact of daily life stress on the HPA axis.

While a growing number of studies have examined the relationship between daily events and mood (Bolger et al., 1989a; Clark \& Watson, 1988; Goplerud \& Depue, 1985; Lundberg et al., 1989; Neale et al., 1987; Repetti, 1993; Stone, 1987; Stone \& Neale, 1984) or physical symptoms (DeLongis et al., 1988; Goreczny, Brantley, Buss, \& Waters, 1988; Jandorf, Deblinger, Neale, \& Stone, 1986), few have investigated whether stressful daily experiences have an effect on cortisol excretion. Findings have been inconsistent, which is not surprising in light of the large differences in cortisol measurement procedures and definitions of daily stress or distress. Cortisol levels have often been based on a single measurement per subject or per day. To illustrate the diversity of results, greater work demands were associated 
with lower cortisol levels in subjects sampled in the morning (but not in the afternoon) in one study (Caplan et al., 1979); another study found that feelings of irritation, tenseness, and tiredness in assembly line workers were associated with elevated cortisol levels on workdays and that cortisol levels were absolutely higher on 'bad' compared to 'normal' or 'good' workdays (Lundberg et al., 1989). Examining within-subject associations over several days, one study found elevated afternoon urinary cortisol on high stress compared to low stress days (Brantley et al., 1988), while in another, no relationship between the number of undesirable events reported at the end of the day and cortisol levels measured in evening urine could be demonstrated (Cummins \& Gevirtz, 1993). With more frequently measured responses to work stress in air traffic controllers, Rose and colleagues (1993) found that a subgroup of subjects responded to an increase in the number of planes they had to manage with large increases in cortisol.

New approaches are needed for investigating cortisol responses to daily events. Repeated measurement of cortisol not only increases reliability, but provides a clearer picture of the temporal relationship between stressful events and neuroendocrine responses. This is especially important if the events of interest occur unpredictably (as is usually the case in the natural environment) and therefore cannot be directly monitored by the researcher. Additional insights into the stress process can be gained by including measures of the context in which events take place and measures of subjective responses, including mood and cognitive appraisals (Folkman \& Lazarus, 1985).

The current study therefore combines Experience Sampling (ESM (Csikszentmihalyi \& Larson, 1987; de Vries, 1992) methodology and frequent salivary cortisol sampling to address two main questions about daily life stress and cortisol:

(I) Are high perceived stress and distress associated with elevated cortisol levels? Despite the lack of consistent results in the literature on the effects of chronic stress on the HPA axis, we reasoned that healthy individuals experiencing persistent but not overwhelming levels of distress and difficulty in coping with daily demands would have higher overall cortisol than persons who experience fewer problems. In addition to the effects of perceived stress level, we examined the contributions of conceptually related measures of stress exposure and chronic distress. These included recent life events, chronic difficulties, trait anxiety. depression. hostility, and psychosomatic complaints. Several of these variables have previously been linked to cortisol levels (Brandtstädter et al., 1991; Pope \& Smith, 1991; Schaeffer \& Baum, 1984).

In addition to trait-like measures of stress and distress, we assessed the effects of negative and positive mood states on cortisol. Although there is abundant evidence that cortisol increases in response to negative states (Arnetz \& Fjellner, 1986; Lundberg \& Frankenhaeuser, 1980: Mason, 1968; Nicolson. 1992), the effects of positive mood are less clear. Positive affect has been associated with lower (Hubert \& de Jong-Meyer, 1989; Kugler \& Kalveram, 1989) as well as higher (Brown et al., 1993) cortisol levels.

(2) Do stressful daily events influence cortisol levels?

In general, we hypothesized that even minor stressors could result in increased cortisol secretion. We further hypothesized that such effects might be dependent on an individual's chronic level of perceived stress. Based on results of previous studies showing greatei psychological impact of work-related events (Stone, 1987) and negative social interactions (Bolger et al., 1989a), we expected that these categories of events would have the largest impact on cortisol. Similarly, events that subjects 
rated as more unpleasant, more important, less predictable, and less controllable were expected to have larger effects, and ongoing events were expected to have more effect on cortisol than recently terminated events. Finally, we hypothesized that events reported to occur relatively infrequently in daily life would, due to their novelty, have a greater effect on cortisol levels than recurrent events.

The study design compares two groups of male white collar workers, with high versus low levels of perceived stress. A total of 87 subjects completed ESM selfreports and collected saliva for cortisol determination at frequent intervals over a period of five days. A previous analysis of a subset of these data, based on subject mean cortisol levels, provided some support for the hypothesis of elevated cortisol levels in the high stress group, at least on workdays (van Eck \& Nicolson, 1994). The current analysis adds to these preliminary findings by assessing the separate contributions of "trait" (e.g., perceived stress level, trait anxiety, depression) and "state" (e.g., mood, appraisal) variables, at the same time controlling for diurnal and possible confounding influences on cortisol secretion. Most importantly, the application of hierarchical linear modeling, or multilevel analysis (Bryk \& Raudenbush, 1992), enables us to investigate the neuroendocrine effects of the wide variety of stressful events experienced in daily life.

\section{METHODS}

\section{Subjects}

Local industries and government agencies were approached via their personnel departments to participate in the study. A decision as to which categories of employees fell under the definition of 'white-collar' was made by each personnel department on the basis of standard job function descriptions. Questionnaires were distributed among these employees, accompanied by a letter explaining the goals of the study. Participation was voluntary, and care was taken to insure anonymity. 316 male employees from six different industries or agencies completed the screening questionnaire. The mean score on the Perceived Stress Scale (PSS) for this sample was 12.7 (s.d. 6.0), similar to US norms (mean 13.02, s.d. 6.45) (Cohen et al., 1983).

Ninety-two subjects with scores in the upper or lower tertiles of the screening sample distribution (PSS-10 score $\leq 10$ or $\geq 16$ ) were recruited, excluding individuals who reported a history of serious chronic illness, endocrine disorder, medications known to affect cortisol levels, or treatment (past or current) for mental health problems. Fxclusion criteria were reassessed during an initial interview, study aims and procedures were explained, and informed consent obtained. During subject intake, each 'high stress' (HS) subject was matched for age group. marital status, and household composition with a 'low stress' (LS) subject to insure that the two groups did not differ on demographic characteristics that might affect exposure to certain classes of daily stressors. Five subjects were later excluded from analysis: four due to failure to meet ESM compliance criteria (see 'Daily Experience' section, below) and one because he became so acutely stressed that he was unable to work during the sampling period. Of the 87 remaining subjects, 41 subjects comprised the HS group and 46 subjects, the LS group. Mean age was 42.1 years (range 27 to 57 years). $89 \%$ were married, and $81 \%$ had children living at home

\section{Questionnaires}

The following measures were used in the current analysis:

Perceived stress: The 10-item version of the PSS was translated into Dutch. The ittms are rated on a 5-point frequency scale, ranging from 'never' (0) to 'very often' (4) in the last month. 
Life events: Life events were recorded with the questionnaire form of the List of Threatening Experiences (LTE-Q; Brugha et al., 1985). Subjects were asked about the occurrence of 12 categories of events (e.g. death of a partner, child, parent; got divorced) during the last year.

Long-term difficulties: Chronic stress was assessed with the Long-term Difficulties Questionnaire (LLM; Hendriks et al., 1990). This inventory focuses on problems in relation to work/study, housing, physical environment, leisure, finance, and social relationships (partner, family, friends, neighbors). Subjects rate each of the 16 items on a four point intensity scale with the anchors (1) none, (2) some, (3) quite, (4) serious (difficulties). A total score is obtained by summing across all items.

Psychosomatic symptoms: The revised version of the SUNYA. Psychosomatic Symptom Checklist (PSC) (Attanasio et al., 1984) includes 17 common psychosomatic complaints (e.g., headaches, backaches, nausea). Subjects rate each complaint on 5-point scales for frequency (0 "never or rarely occurs " to 4 "occurs daily") and intensity ( 0 "not bothersome" to 4 "extremely bothersome"). A, total score is obtained by summing the cross-products of each item's frequency by intensity.

Depression: Depressive symptomatology was assessed with the Dutch translation (Dijkstra, 1974) of the Zung Self-Rating Depression Scale.

Anxiety: Trait anxiety was measured with the Dutch version (van der Ploeg et al., 1980) of the State-Trait Anxiety Inventory (STAI).

Anger: Trait anger was measured with the Dutch version (van der Ploeg et al., 1982) of the Spielberger Trait Anger Scale. The scale has two subscales: 'anger-temperament' and 'angerreaction'.

\section{Daily experience}

The Experience Sampling Method (ESM) (Csikszentmihalyi \& Larson, 1987; de Vries, 1992) was used to collect data from subjects during their normal daily activities. Subjects received auditory signals ('beeps'), after which they filled in a questionnaire and collected a saliva sample. After receiving detailed instructions, subjects. were sampled for a period of 5 consecutive days (Thursday through Monday). A Seiko wristwatch was programmed to emit. 'beeps' 10 times each day, at semi-random intervals of approximately 90 minutes, between the hours of 8 am and 10 PM. Beeps were clustered around the midpoint of each time block e.g. $8.15 \mathrm{am}, 9.45,11.15$, and so on), with the exact time sequence of 'beeps' varied each day to decrease predictability. in a final 'debriefing' session, subjects were asked to clarify reasons for missing data.

The criteria we set for subject inclusion in the analysis ( $\geq 20$ ESM reports completed within 20 minutes after being signalled and no missing data for entire days) were met by all but foui subjects ( 2 from each group). The remaining 88 subjects completed an average of $83 \%$ of all possible responses within the time limit, for an average of 41 responses per subject. HS and LS groups did not differ in compliance rates (40.1 vs 42.3 responses per subject, Mann-Whitney Utest, $\mathrm{p}>.05$ ). Compliance was lowest for the first 'beep' (at approximately $8.15 \mathrm{am}$ ), with ant average of $73 \%$ valid reports. On Saturdays, response rate for first 'beeps.' was $61 \%$ and on Sundays, $59 \%$. On weekends, $74 \%$ of all missing and invalid responses could be attributed to the fact that subjects were still asleep when signalled.

The ESM form contained open-ended questions concerning thought content, the physical and social context, and what the individual was doing when signalled. The forms also included Likert scales (from 1 'not at all' to 7 'very much') for rating aspects of thoughts, mood, physical well-being, individually defined (psycho)somatic complaints, present activity, and stressful events. Subjects were asked to describe any stressful events or situations which may have taken place in. the interval since the last ESM report and to rate these events on a number of dimensions: unpleasantness, importance, predictability. controllability , and frequency of prior occurrence.. Subjects were also asked to indicate at what time the event had started, and if and when it had 
ended at the moment they were 'beeped'. Information concerning maximum level of physical exertion, smoking, food, coffee, and alcohol intake since the last 'beep' was also obtained.

The 17 ESM mood items were reduced to three mood measures, identified by means of principal component analysis with varimax rotation, which accounted for $78 \%$ of the total variance when subject mean scores were used. Ratings on the items cheerful, satisfied, relaxed, energetic, self-assured, concentrated, and enthusiastic were summed to form a 'Positive Affect' scale (Cronbach's alpha $=.95$ ). Two separate components of negative affect were identified: 'Negative Affect', including the items depressed, anxious, worried, lonely, tired, and miserable (alpha = .87 ), and 'Agitation', with the items restless, irritated, hurried, and nervous (alpha $=.93$ ). The sums of the scale items were divided by the number of items, so that all mood measures have ranges from 1 to 7 .

Subjects' descriptions of stressful daily events were first coded according to content, with categories work, network (events concerning family, friends and acquaintances). household/financial, leisure, personal health-somatic, personal health-psychological, transport, and other. Twelve events coded in the two personal health categories were excluded from analysis because of possible confounding with psychological and somatic state measures. In the current analysis, the remaining events were collapsed into the categories. work ( $48.0 \%$ of events) and nonwork $(50.5 \%)$. Some examples of reported work events were: 'unclear / vague assignment at work', 'too much to organize, not enough time', 'difficult conversation with boss about job performance', 'leading a big meeting'. Reported non-work events included: 'having a fight with: my wife about household duties', 'conflict with spouse about how to raise our son', 'child who will not listen', and 'making dinner, child crying, other child taking a bath, and this all at the same time'. In addition to content, events were classified according to whether or not they involved a social interaction and/or a task demand. ${ }^{4}$

\section{Salivary cortisol}

Salivary cortisol is a reliable indicator of the free cortisol in plasma, which is considered to be the biologically active hormone (Vining et al., 1983). Salivary cortisol increases within minutes in response to acute stressors and has a half-life off approximately one hour (Hellhammer et al., 1987). We have found no differences in cortisol levels in uncentrifuged samples frozen immediately or kept at room temperature for 2 days (Nicolson et al., 1992); others report no differences up to 2-4 weeks (Kahn et al., 1988; Kirschbaum \& Hellhammer, 1989).

At the same time ESM forms were being completed, subjects collected saliva by holding a cotton dental roll in the mouth for approximately 1 minute. The saturated roll was placed in a capped plastic vial ("Salivette", Sarstedt), which subjects stored in their home freezers each evening. At the end of the sampling period, uncentrifuged samples were stored at -20 degrees Celsius until analysis. Compliance with saliva sampling was good in both high and low stress groups, with the same mean response rate $(83 \%)$ as for the ESM reports. Five extreme cortisol values $(>1200 \mathrm{ng} / \mathrm{dl})$ were deleted from the dataset before analysis.

Salivary cortisol levels were determined in duplicate by direct radioimmunoassay (Ansseau et al., 1984), using ${ }^{125}$ I-cortisol (Farmos diagnostica, Finland) and antiserum made against the 3CMO-BSA conjugate by Dr. J. Sulon, University of Liege, Belgium. The lower detection limit of the assay was $12 \mathrm{ng} / \mathrm{dl}$, with a mean intra-assay coefficient of variation of $4.8 \%$ (range: $2.2 \%$ -

4 The reliability of the coding system was assessed by comparing the classifications of 345 events by two independent coders. Interrater agreement was determined by means of Cohen's kappa. On the whole, the qualitative information could be classified with a high degree of agreement (Laundis \& Koch, 1977), especially for the content categories. The overall Kappa for content was .90 , with intra-category Kappa's ranging from .60 to .96 ; Kappa's for social interaction and for task demand were .73 and .65 . respectively. 
$7.5 \%$ for 4 assays). All samples from an individual subject were analysed in the same assay to reduce sources of variability.

\section{Statistical analysis: multilevel or hierarchlcal linear model}

The multilevel model or the hierarchical linear model (Bryik \& Raudenbush, 1992; Goidstein, 1987) is a variant of the multiple linear regression model, applicable for data with a hierarchical nesting structure. In the present dataset, the measurements made at the 'beeps' are nested within persons. The two nesting levels will be called measurement level and person level. At each level of the hierarchy, explanatory variables can be added to the model. The variables that are added at the measurement level (e.g. mood states, events) vary with time, while the added variables at the person level represent characteristics of individual respondents (e.g. high or low perceived stress, trait anxiety).

An advantage of the hierarchical linear model is that it allows for missing observations. In addition, the observations do not need to be evenly spaced over the time interval.

At the measurement level, the relationship between cortisol and time of the day can be modeled explicitly. If, for example, this relation is linear, the model would be of the following form:

$$
(\text { CORT })_{i t}=b_{\mathrm{o} i}+b_{1 i}(\mathrm{TIME})_{i t}+\mathrm{e}_{i t} \text {, }
$$

where $(\text { CORT) })_{i t}$ is the level of cortisol at the th theasurement of person $i$, (TIME) $)_{i r}$ represents the time of the day at which this measurement is taken, $b_{\mathrm{o} i}$ and $b_{1 i}$ are the intercept and slope of person $i$, and the $e_{i l}$ 's are normally distributed error terms with mean zero and variance $\sigma^{2}$. The resulting equation can be extended with time-varying explanatory variables. For example, to study the effect of stressful event occurrence on cortisol, a dummy-coded variable (EVENT) ir can be added to the above equation as follows:

$$
(\mathrm{CORT})_{i t}=b_{\mathrm{o} i}+b_{1 i}(\mathrm{TIME})_{i t}+b_{2 i}(\text { EVENT })_{i t}+\mathrm{e}_{i t} .
$$

where $b_{2 i}$ is the effect of a stressful event for person $i$. The regression coefficients $b_{j i}(j=0,1,2$, ....) are allowed to vary across individuals. Therefore, we can split $b_{j i}$ into two components: a fixed component $d_{0 i}$ that is constant across persons (fixed effect $l$ and a random component $u_{j i}$ that varies across persons (random effeci). This gives the following person-level model for $b_{j i}(j=0,1$. $2, \ldots)$ :

$$
b_{j i}=d_{\mathrm{o} j}+u_{j i}
$$

Instead of estimating $u_{j i}$ for each person, we postulate that the $u_{j i}$ 's $(i=1,2,3, \ldots)$ are randlom draws from a normal distribution (explaining the term random effect). The mean of $u_{j i}$ is zero and the variance of $u_{j i}$ is $\tau_{j j}$. The value of $\tau_{j j}$ indicates how much the value of $u_{j i}$ differ across: persons: the higher the value of $\tau_{j j}$ the more the values of $u_{j i}$ differ. The covariance between $u_{j i}$ and $u_{k i}$ ( $j$ not equal to $k$ ) is $\tau_{j k}$. A positive value of $\tau_{j k}$ implies that a person with a high value of $b_{j}$ tends to have a high value of $b_{k}$ as well.

Suppose that part of the variation in $b_{j i}(j=0,1,2, \ldots)$ across persons can be explained by the person variable (GROUP) that indicates whether person $i$ belongs to the high or low stress group. The regression coefficients $b_{j i}(j=0,1,2)$ can now be modeled as:

$$
b_{j i}=d_{\mathrm{oj}}+d_{1 j}(\text { GROUP })_{i}+\mathrm{u}_{j i},
$$

where $d_{y}(j=0,1,2, \ldots)$ denotes the fixed effect of $(\text { GROUP })_{i}$ on $b_{j i}$. The $u_{j i}(j=0,1,2, \ldots)$ is now a random effect of person $i$ after controlling for (GROUP);

Estimates of the fixed effects $d_{0 j}$ and $d_{1 j}(j=0,1,2)$ as well as of the (co)variances $\sigma^{2}$ and $\tau_{j k}(j, k=0,1,2)$ were obtained with the program ML3e (Prosser, Rasbach, \& Goldstein, 1991). For model selection we started with an empty model and added main and interaction effects of the theoretically important variables. Significance of fixed effects was tested by dividing the estimated effect by its standard error. This ratio is approximately normally distributed. For testing the 
significance of the (co)variances. $\tau_{j k}(j, k=0,1,2)$, we applied the likelihood ratio test (Bryk \& Raudenbush, 1992). This test compares a model with and a model without a random effect. Hence, the (co)variances that are added to the model because of the specification of an extra random effect are tested simultaneously. Non-significant effects were excluded from the models. The postulated normality of the random effects was checked by inspecting normal probability plots of the individual estimates of $b_{j i}(j=0,1,2)$, which were obtained by the empirical Bayes approach (Bryk \& Raudenbush, 1992; Goldstein, 1987). The normality of the measurement level empirical Bayes residuals was also checked by inspection of the normal probability plot. The observed cortisol values for each person were plotted by time of day to check for the presence of outlying cortisol curves (see also Results, below).

The estimation of effects on cortisol entailed four steps, presented here as separate models. Since the analysis of repeated assessments of salivary cortisol is complicated by the hormone's strong diurnal rhythm and secretory peaks (which lead to a decline in variance from moming to evening), the first step involved accurate fitting of the diurnal curve, in order to allow comparisons of cortisol values across the day. Next, possible confounding factors, such as smoking (Kirschbaum \& Hellhammer, 1994), exercise (Cook et al., 1987), coffee (Pincomb et al., 1987) and food intake (Quigley \& Yen, 1979), were included as explanatory variables in the same model. All variables with significant fixed effects identified in this model were included in ail subsequent models.

The remaining three models test our main hypotheses. First, the effects of level of perceived stress, mood states, and individual trait characteristics on overall cortisol level were estimated. Workday vs. weekend differences and interactions between stress level and diurnal variability were also examined. The next model estimated effects of stressful events and event characteristics on cortisol, excluding the contribution of mood variables. In the final model, mood state variables were re-entered in order to evaluate the relative contributions of events, mood, and trait characteristics to cortisol levels.

Instead of a model with two levels, we could have formulated a three-level model, where measurements are nested within days, which in turn are nested within persons. However, when a three-level model was evaluated the p-values were approximately the same as the $p$-values of the two-level model. Because the more complex model did not change the conclusions, we decided to present the simpler model. The similarity of the results of the two models can be explained by the observation that the variance at the day level, although present, was small compared to the variance at the person and the measurement levels. Again, for the sake of simplicity, we did not extend the model with an autocorrelation term, since its inclusion did not change the model results.

\section{RESULTS}

\section{Characteristics of high and low stress groups}

High and low stress groups differed on almost all measures of stress and distress, as assessed with questionnaires and ESM reports (see Table 6.1.). Only the number of life events experienced in the past year did not differentiate the two groups. 
Table 6.1. Characteristics of high and low stress groups.

\begin{tabular}{|c|c|c|c|}
\hline & $\begin{array}{l}\text { LS } \\
\text { mean (st.dev.) }\end{array}$ & $\begin{array}{l}\text { HS } \\
\text { mean (st.dev.) }\end{array}$ & $\frac{D \text {-value }}{\text { (2-tailed) }}$ \\
\hline Total $\mathbf{n}$ & 46 & 42 & \\
\hline Age & $42.7(7.7)$ & $41.5(5.9)$ & ns \\
\hline \multicolumn{4}{|l|}{ Questionnaires } \\
\hline Perceived stress & $7.2(2.2)$ & $18.1(3.4)$ & $<.0001$ \\
\hline Trait anxiety & $28.3(4.4)$ & $39.8(7.6)$ & $<.0001$ \\
\hline Trait anger & $18.8(4.6)$ & $23.0 .(5.2)$ & $<.0001$ \\
\hline Depression & $36.5(5.5)$ & 48.4. (7.7) & $<.0001$ \\
\hline Psychosomatic symptoms & $6.1(5.6)$ & $27.5(23.2)$ & $<.0001$ \\
\hline Life events & $.5(.7)$ & $.8(.9)$ & ns \\
\hline Chronic difficulties & $19.5(2.3)$ & $23.3 \cdot(4.0)$ & $<.0001$ \\
\hline \multicolumn{4}{|l|}{ ESM reports* } \\
\hline Positive affect & $5.7(0.6)$ & 5.3. $(0.3)$ & $<.00 !$ \\
\hline Negative affect & $1.2(0.1)$ & $1.4(0.4)$ & $<.0001$ \\
\hline Agitation & $1.3(0.3)$ & 1.8. $(0.6)$ & $<.0001$ \\
\hline Frequency of stressful events & $12.3 \%(11.9)$ & $22.0 \%(20.0)$ & $<.01$ \\
\hline
\end{tabular}

- subject means, aggregated from 'beep' level data

\section{Controlling for diurnal and external influences on cortisol secretion}

Because the measurement level residuals in a model fitted with raw cortisol data were highly skewed to the right, with decreasing variance from morning to evening, data transformation was necessary to meet standard model assumptions. Log transformation, the usual remedy for cortisol skewness, resulted in increasing variance over the day. We therefore used a fifth root transformation (cortisol ${ }^{0.2}$ ), which gave normally distributed residuals with a homoscedastic pattern throughout the day.

The observed cortisol curve was best described by a two-piece third-degree polynomial (spline function (Smith, 1979)), with the node at $12.25 \mathrm{p} . \mathrm{m}$. The spline was created by adding a truncated term (defined as (TIME -12.25$)^{3}$ after $12.25 \mathrm{pm}$ and as zero before $12.25 \mathrm{pm}$ ) to a third-degree polynomial, which improved fit in the morning hours when cortisol levels dropped most sharply. Random terms were added. to allow each subject to have his own intercept and slope. Random terms for TIME ${ }^{2}$. TIME $^{3}$, and the truncated term did not change the magnitude of the fixed effects and were therefore excluded from the model for the sake of simplicity. All time effects (fixed and random) included in the model were highly significant. As shown in Figure 6.1 , the estimated contisol time curve closely approximates the observed mean values at the "beeps" and is clearly superior to a simple linear curve. Plots of the observed cortisol curve for each individual revealed no outlying curves; further inspection of the normal probability plots of individual cortisol means, slopes, and measurement level residuals, also indicated no significant violation of normality assumptions, supporting the appropriateness of the mixed effects approach.

The model was next extended with the possible confounders (alcohol, coffee, food intake, smoking, and physical exertion). Effects of any food intake and lunch were included separately (Follenius, Brandenberger, Hietter, Simeoni, \& Reinhardt, 1982; Quigley \& Yen, 1979). Maximum recent physical exertion was coded on a 7 point scale. The other variables were coded 1 if reported to have occurred in the interval since the previous 'beep' and 0 otherwise. Recent food, coffee, and alcohol 
intake were reported on $43 \%, 40 \%$, and $12 \%$ (respectively) of total ESM 'beeps', and recent smoking was reported on $16 \%$ of all 'beeps'. The median level of maximum physical exertion in the interval preceding a 'beep' was 3 , with levels of 6 or 7 reported on fewer than $3 \%$ of the total 'beeps'.

In the resulting multilevel model, recent smoking and food intake were associated with higher cortisol levels, with an additional positive effect of lunch on cortisol above food intake in general. No significant effects were found for coffee consumption, alcohol intake, or physical exertion. Model estimates for diurnal and external effects on salivary cortisol levels are shown in Table 6.2. (Column 1).

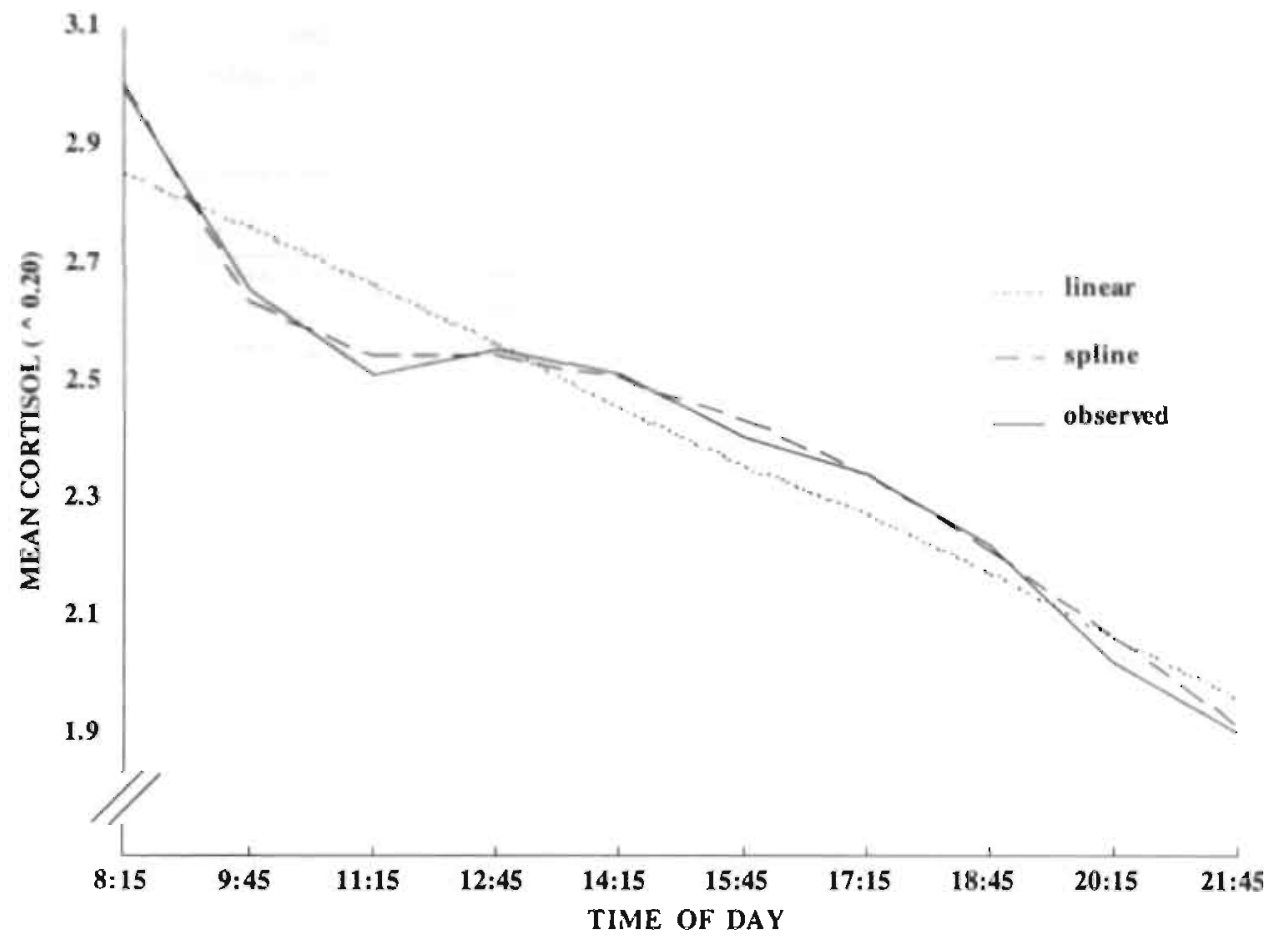

Figure 6.1. Observed and estimated cortisol curves.

Effects of individual characteristics and mood states on cortisol levels In the next model, we tested for effects of psychological trait and state variables on cortisol levels. Results are summarized in Table 6.2. (Column 2). Perceived Stress (PS Group, dummy-coded as 1 for HS and 0 for LS subjects) was entered into the model first, to test our hypothesis that high stress is associated with elevated cortisol. The effect of perceived stress, however, was not significant. The Perceived Stress by Time of Day (TIME) interaction term was also non-significant, indicating that the diurnal pattern of cortisol secretion was similar in HS and LS subjects. Adding Day Type (Work, coded as 1, vs Weekend, coded 0) and its interaction term with Perceived Stress did not improve the model; thus, contrary to expectation, cortisol levels were no higher on workdays than on weekends in either subject group. These variables were therefore excluded from the model. 
We next examined the effects of individual differences in trait anxiety, depression, anger, psychosomatic complaints, number of recent life events, and chronic difficulties. Anger, psychosomatic complaints, life events, and difficulties had no significant effects on cortisol. Trait anxiety and depression were each associated with significant elevations in cortisol levels.

Table 6.2. Effects of individual characteristics " mood states, and stressful daily events on cortisol levels.

The table summarizes the results of steps followed in the development of the full multilevel model. Model 1 provides estimates of diurnal and extraneous effects on cortisol. Model 2 adds estimates for trait characteristics and mood states. Model 3 shows the added contribution of stressful events (with mood effects excluded). In Model 4, all previously significant variables are included simultaneously.

\begin{tabular}{|c|c|c|c|c|}
\hline Fixed Effects ${ }^{\mathrm{a}}$ : & \multicolumn{4}{|c|}{ Estimate + (s.e.) } \\
\hline Interçept & $3.395(0.040) \cdots$ & $3.230(0.080) \cdots$ & $3.260(0.079) \cdots$ & $3.234(0.080)^{* \cdots}$ \\
\hline Time of day (TIME) ${ }^{b}$ & $.0 .577(0.036)^{\cdots}$ & $.0 .584(0.036)^{\cdots}$ & $-0.578(0.037)^{* *}$ & $-0.583(0.036)^{\cdots \cdots}$ \\
\hline TIME $^{2}$ & $0.128(0.010)^{\cdots}$ & $0.130(0.010)^{\cdots *}$ & $0.128(0.010)^{\cdots \cdot}$ & $0.129(0.010)^{\cdots}$ \\
\hline $\mathrm{TIME}^{3}$ & $-0.010(0.001)^{\cdots}$ & $-0.010(0.001)^{* \cdots}$ & $.0 .010(0.001)^{\cdots}$ & $-0.010(0.001)^{\cdots}$ \\
\hline Truncated function & $0.010(0.001) \cdots$ & $0.010(0.001)^{* \cdots}$ & $0.010(0.001)^{\cdots}$ & $0.010(0.001)^{\cdots}$ \\
\hline Food intake & $0.025(0.011)^{\cdots}$ & $0.027(0.010)^{* *}$ & $0.024 ;(0.011)^{\prime \prime}$ & $0.026(0.011)^{* *}$ \\
\hline Lunch & $0.037(0.019)^{\circ}$ & $0.037(0.019)^{*}$ & $0.043(0.019)^{2}$ & $0.043(0.019)^{*}$ \\
\hline Smoking. & $0.046(0.021)^{\circ}$ & $0.048(0.021)^{*}$ & $0.046(0.021)^{*}$ & $0.048(0.021)^{*}$ \\
\hline Trait anxiety & & $0.004(0.002)^{\circ}$ & $0.0039(0.002)^{\circ}$ & $0.004(0.002)^{*}$ \\
\hline Agitation & & $0.027(0.011)^{* *}$ & & $0.022(0.011)^{\prime \prime}$ \\
\hline Stressful event & & & $0.033(0.013)^{*}$ & $0.019(0.014)$ \\
\hline Ongoing event & & & $0.027(0.013)^{*}$ & $0.022(0.013)$ \\
\hline Prior frequency of event & & & $-0.072(0.030)^{*}$ & $-0.076(0.030)^{*}$ \\
\hline Prior frequency $\times$ trait an & & & $0.002(0.0008)^{*}$ & $0.002(0.001)^{\circ}$ \\
\hline
\end{tabular}

Random Effects: (Co)variance + (s.e.)

\begin{tabular}{|c|c|c|c|c|}
\hline \multicolumn{5}{|l|}{ Person level: } \\
\hline Var (Intercept) & $0.031(0.006)^{\cdots}$ & $0.039(0.009)^{2.0 .}$ & $0.031(0.006)^{n \ldots}$ & $0.037(0.009)^{\cdots}$ \\
\hline Var (Time) & $0.0002(0.0001)^{\cdots}$ & $0.0002(0.0001)^{* \cdots}$ & $0.0002(0.0001)^{* \cdots}$ & $0.0002(0.001)^{\cdots}$ \\
\hline Cov (Time, Intercept) & $.0 .001(0.0004)$ & $-0.001(0.0005)$ & $.0 .001(0.0004)$ & $-0.00 !(0.0004)$ \\
\hline \multicolumn{2}{|l|}{ Var (Agitation) } & \multicolumn{2}{|l|}{$0.003(0.001)^{*}$} & $0.003(0.001)^{\cdots *}$ \\
\hline \multicolumn{2}{|c|}{ Cov (Agitation, Intercept) } & \multicolumn{2}{|l|}{$-0.005(0.003)$} & $-0.004(0.003)$ \\
\hline \multicolumn{2}{|c|}{ Cov (Agitation, Time of day) } & \multicolumn{2}{|l|}{$0.0001(0.0002)$} & $0.0001(0.0002)$ \\
\hline \multicolumn{5}{|l|}{ Measurement level: } \\
\hline Var (Residual term) & $0.060(0.0015) \cdots$ & $0.058(0.0015)^{\cdots} \cdots$ & $0.059(0.0015)^{\cdots} \cdots$ & $0.058(0.0015)^{\cdots}$ \\
\hline \multicolumn{5}{|c|}{$\begin{array}{l}\text { Model } 1 \text { and Model 2:3157 observations nested within } 86 \text { subjects ( } 40 \mathrm{HS}, 46 \mathrm{LS} \text { ). } \\
\text { Model } 3 \text { and Model 4:3108 observations, including } 591 \text { stressful events, nested within } 86 \text { subjects ( } 40 \text { HS, } 46 \text { LS). }\end{array}$} \\
\hline \multicolumn{5}{|c|}{$\begin{array}{l}\text { a Non-significant fixed effects were excluded from the model. Model 1: Coffee intake. Alcohol intake, Physical } \\
\text { exertion. Model 2: PS Group, PS Group by Time of day. Day type, PS Group by Day type, Trait anger. Psychosomatic } \\
\text { symptoms, Life-events, Chronic difficulties. Positive affect. Model 3: Event content - Work. Social interaction, Task } \\
\text { demand-; Event appraisal - Unpleasantness. Predictability, Importance. Controllability -- Event by PS Group. Event by } \\
\text { Trait anxiety, Event by Depression. Model 4: Positive affect. Event by Agitation, Event by Negative affect. }\end{array}$} \\
\hline
\end{tabular}


Due to multicollinearity, it was necessary to add these variables separately to the model. In Table 6.2 (Column 2), the effect of trait anxiety is shown. The effect of depression, when entered separately, was of a similar magnitude (estimate Depression: $0.003, \mathrm{p}=.05$ ).

To test the association between mood state and cortisol levels, the variables Positive Affect, Negative Affect and Agitation were next added to the model. Positive Affect was not significantly related to cortisol. In contrast, both Negative Affect and Agitation were associated with higher cortisol. Because Negative Affect and Agitation were intercorrelated ( $r=0.51 ; p<.0001$, over 3569 reports), the effect of Negative Affect was masked when entered simultaneously with Agitation into the equation. When entered separately, standardized effects were approximately the same for both variables. In Table 6.2. (Column 2), the effect of Agitation is shown (estimate Negative Affect: $0.032, \mathrm{p}<.01$ ).

Finally, random effects for Negative Affect and Agitation were included in the model. The random effect for Agitation was significant, meaning that the effect of Agitation on cortisol varied across persons. In Figure 6.2., the individual estimates of the effect of Agitation on cortisol are plotted for HS and LS subjects. Here, it is evident that some individuals displayed a considerably closer relationship between cortisol and Agitation than others, but that these individual differences were not associated with perceived stress level. 


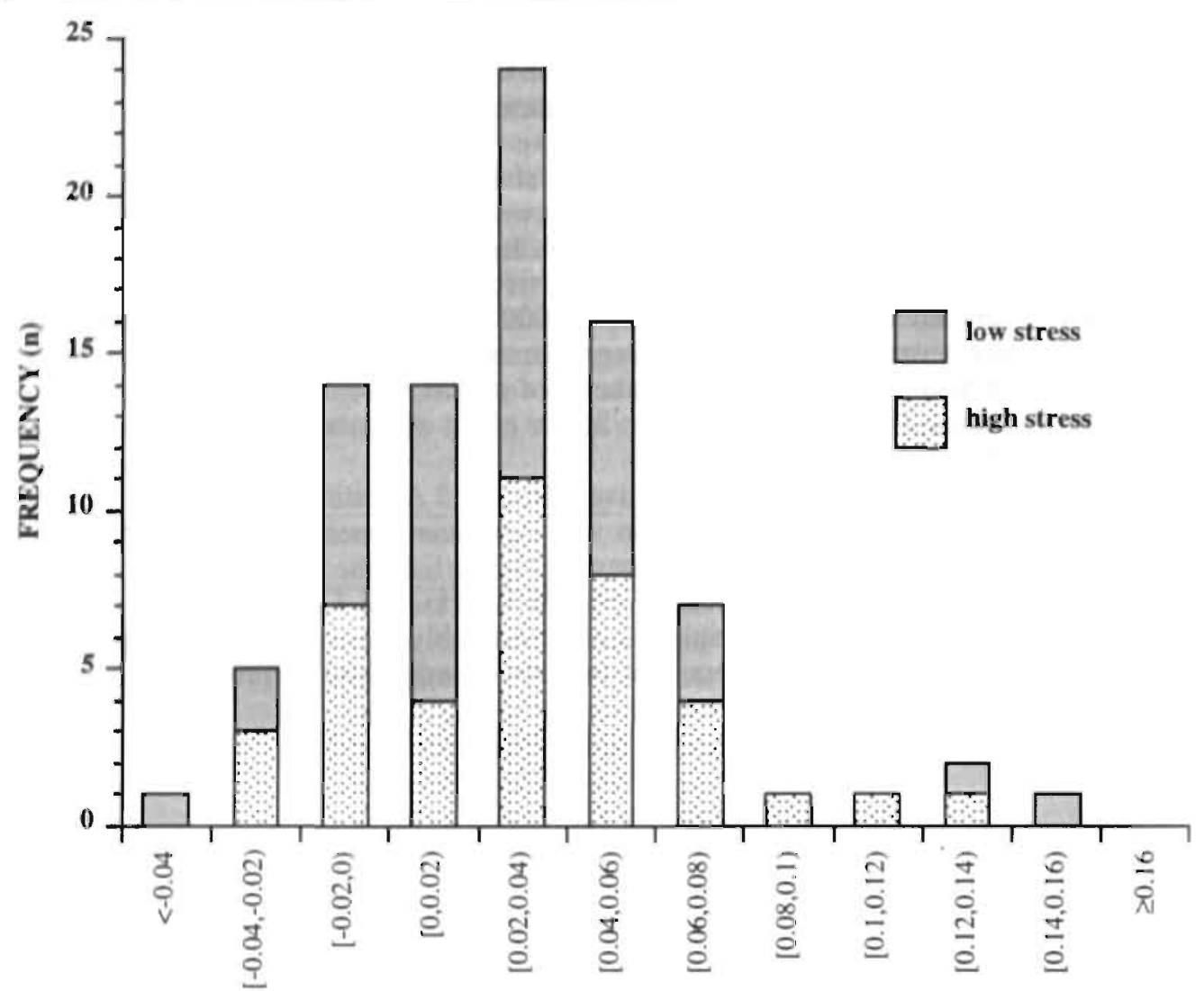

INDIVIDUAL ESTIMATES (SLOPES) OF AGITATION EFFECTS

Figure 6.2. Distribution of the individual estimates of the effects of the mood state Agitation on cortisol for HS and LS subjects. These estimates were calculated by an empirical Bayes approach, using multilevel analyses (see Method section).

To provide a clearer picture of effect magnitudes, the estimate for each variable can be translated back into the original measurement unit. A standard deviation increase in trait anxiety, for example, was associated with a cortisol increase of 5.4 $\mathrm{ng} / \mathrm{dl}$ above the mean level of $81.3 \mathrm{ng} / \mathrm{dl}$. Regarding Agitation, the mood state effect estimate shown in Table 6.2. (Column 2) indicates the increase in transformed cortisol associated with a one unit increase on the mood scale (from 1 to 2 on the scale ranging from 1 to 7). Translated back into raw cortisol, this one unit increase was associated with a cortisol increase of $4.7 \mathrm{ng} / \mathrm{dl}$ above the mean cortisol level. When a subject scores, for instance, 5 on the negative mood scale, the mood estimate (see Table 6.2., Column 2) should be multiplied by 4, resulting in a higher cortisol level. A 4 unit increase on the mood scale was associated with a cortisol increase of $20 \mathrm{ng} / \mathrm{dl}$ above the mean cortisol level. 


\section{Reactivity of cortisol to stressful events}

In the third model, the occurrence of a stressful event since the last ESM report (Event, dummy-coded as $\mathrm{I}$ or 0 ) and variables representing the coded content of the event (work-related or not, social interaction or not, task demand or not; all coded as I or -1 ) and rated characteristics of the event were added to the model. ${ }^{5}$ Results are summarized in Table 6.2. (Column 3). Cortisol levels were significantly elevated following stressful daily events. No random effect for Event was found, meaning that the influence of a stressful event on cortisol did not differ significantly between subjects. Although most of the Event effect was determined by HS subjects, level of perceived stress did not have a significant effect on cortisol reactivity to stressful events. Next, we controlled for the time-frame of the event: was the event already finished when a subject took a saliva sample ( $71 \%$ of all events), or was it still going on ( $29 \%$ of events) ${ }^{6}$ When a stressful event was ongoing at the time the ESM report was completed, the level of cortisol was significantly higher than if the event had already ended. Translated back into the original units, ongoing events were associated with mean cortisol increases of $10.6 \mathrm{ng} / \mathrm{dl}$ and terminated events with increases of $5.7 \mathrm{ng} / \mathrm{dl}$, relative to no event.

The various event content categories (work vs non-work events, social interactions vs non-social events and task demands vs non-task demands) were not significantly associated with cortisol. Contrary to expectation, event unpleasantness, importance, predictability, and controllability contributed no additional effects. Prior frequency did have an independent effect: when similar kinds of events were reported to have occurred relatively frequently in the past, the cortisol response to the current event was lower. Here, significant interactions with trait anxiety were found (see Table 6.2. Column 3). Significant interactions were also found for depression and perceived stress (when entered separately to the model, replacing trait anxiety): cortisol levels were less likely to show this apparent habituation effect to recurrent events in individuals scoring high on these traits (estimate Prior frequency * Depression: $0.0017, \mathrm{p}<.01$; estimate Prior frequency * PSS: $0.033, \mathrm{p}<.01$ ).

\section{Relationship between cortisol reactivity to events and mood state}

In our final model, we examined whether mood states mediated the cortisol response to stressful daily events. Positive Affect had no effect on cortisol and was therefore excluded from the model. When entered separately into the model, the two negative mood states, Negative Affect and Agitation, were each positively related to cortisol. With Agitation in the model, the effect of stressful event occurrence on cortisol was lowered to a non-significant level. A similar pattern was observed for Negative Affect: the effect of Event was reduced (although in this case it remained significant) after addition of Negative Affect to the model. This indicates that negative mood states were stronger predictors of cortisol level than stressful events. Finally, the lack of significant interactions between the two negative mood states and Event indicates that mood effects on cortisol were no greater in the presence than in the absence of an Event. Table 6.2. (Column 4) summarizes model parameters, with

5 For each rated characteristic of the event, the subject's mean was subtracted from the actual rating, thus keeping the Event effect unchanged; this allowed us to assess the withiri-person effects of the various event appraisals above and beyond the effect of the event itself.

6 First coded -0.5 (event finished), 0 (no event), or 0.5 (ongoing event), and then weighted for the number of events that were 'finished' compared to the number of events 'ongoing' in order to keep the effect of Event unchanged after addition. 
Agitation included. Similar results were obtained when Negative Affect was included instead of Agitation (estimate Negative Affect: 0.024, p<.05).

\section{DISCUSSION}

\section{Etfects of tralt measures of stress and distress on salivary cortisol}

The current study provides evidence that trait negative affectivity can result in moderate cortisol elevation. Trait anxiety and depression showed small but statistically significant positive associations with cortisol. To date, evidence for elevated cortisol in association with anxiety and depression has come mainly from studies of more severely or chronically distressed subjects (Rahe et al., 1990; Schaeffer \& Baum, 1984) or psychiatric patients (Linkowski et al., 1985), while several studies of healthy adults have failed to demonstrate an association between basal cortisol and these or other trait measures (Brandtstädter et al., 1991; Ockenfels, Porter, Smyth, Kirschbaum, Hellhammer, \& Stone, 1995). It should be noted that subjects in the current study, having been selected on the basis of extreme perceived stress scores, displayed a broader distribution of depression and anxiety scores than would have been likely in an unselected sample of white collar workers. Moreover, the large number of cortisol samples collected per subject at different times of day provided more reliable estimates of hormone levels than is usually the case. Anxiety and depression were highly correlated in this sample, which made it impossible to assess the relative contributions of each. Further research is necessary to clarify this issue.

We had hypothesized that cortisol levels would be elevated in subjects with high perceived stress (see Introduction, Question 1). A repeated measures analysis of variance of a subset of these data (van Eck \& Nicolson, 1994), with cortisol values aggregated over each subject and time of day, had shown higher cortisol levels in HS compared to LS subjects when only the three workdays were included. Given the lack of a statistically significant effect of PSS on cortisol in the current multilevel analysis, however, we conclude that perceived stress is not a strong predictor of cortisol secretion, at least in this sample. Nor did we find significant interaction effects between PSS and the time of the day that would support a stress-related disruption in the circadian rhythm of secretion, as has been suggested by other authors (Caplan et al. 1979; Ockenfels et al. 1995). Additional measures of life stress, including an objective life event measure as well as a measure of chronic difficulties over a wide range of life domains, similarly showed no statistically significant associations with cortisol. The intercorrelations among the various measures of stress and distress argue for cautious conclusions about the relative influence of each on cortisol levels. The apparently disparate results of a recent study (Ockenfels et al., 1995), which showed an effect of perceived stress, but not of anxiety or depression, on cortisol levels should be seen in this light.

\section{Effects of state affect measures and stressful daily events}

The two negative mood states, Agitation and Negative Affect, were both associated with higher cortisol levels. The estimated effects were relatively small: a one unit increase in Agitation (from 1 to 2 on a scale ranging from 1 to 7 ) was associated with a cortisol increase of $4.7 \mathrm{ng} / \mathrm{dl}$ above the mean level of $81.3 \mathrm{ng} / \mathrm{dl}$. At more extreme scores on the mood scale, however, cortisol increases were more pronounced. Highly negative states were reported infrequently in daily life contexts 
by both HS or LS subjects, resulting in highly skewed distributions and small standard deviations (Agitation was scored as 1 'not at all' on $56 \%$ of all 'beeps', Negative Affect on $51 \%$ of all 'beeps') (see also Table 6.1). The low mean levels of negative states reported by both HS and LS subjects are characteristic of normal samples, with men tending to report even lower, less extreme levels of negative emotions as well as positive emotions than women (Cameron, 1975; Diener et al., 1985).

Positive mood showed no association with cortisol. The results of previous studies are inconsistent with respect to the effects of positive mood states: in some cases, higher positive affect was accompanied by lower cortisol (Hubert \& de JongMeyer, 1990; Hubert \& de Jong-Meyer, 1991), in others, by higher cortisol (Brown et al., 1993; Hubert, Möller, \& de Jong-Meyer, 1993). One of the few naturalistic studies revealed an inverse relationship between positive mood states and cortisol levels (Kugler \& Kalveram, 1989), but this analysis, by aggregating mood states for each subject, tested only between-subject and not within-subject associations. In the present study, the Positive Affect scale included both high (e.g. 'energetic') and low arousal (e.g. "relaxed') items. If, as might be expected on theoretical grounds (Hennessy \& Levine, 1979), high arousal states are associated with elevated cortisol levels and low arousal states with lower cortisol, including both types of items in one scale could cancel out any effect. This possibility should be addressed in future studies of mood effects on cortisol.

Daily events or situations experienced as stressful were associated with increased cortisol secretion (see Introduction, Question 2). Surprisingly, the rated unpleasantness of an event had no additional effect, nor did other appraisal measures. We had expected that events involving negative social interactions might have more pronounced effects on cortisol, but categorization of events on this dimension, or on relationship to work, or task demands, did not improve the model. Significant effects may be difficult to find because of the heterogeneity of responses resulting from the variety of stressful events encountered. Responses will be modulated by all kinds of objective and subjective event characteristics as well as by their interactions with each other.

We did find a significant effect for the time course of an event, with ongoing events having the expected greater effect on cortisol than terminated events. This is in line with temporal patterns of cortisol excretion observed in response to laboratory stressors; after termination of stress exposure, cortisol levels typically return to baseline within 1-2 hours (Kirschbaum \& Hellhammer, 1989). In addition, events that were reported to be recurrent had a smaller effect on cortisol than more novel events, suggesting that habituation can occur. Although perceived stress, trait anxiety, and depression were not significantly associated with elevated cortisol reactivity to stressful events, cortisol habituation to recurrent daily stressors did appear to be less likely to occur in individuals scoring high on these measures. A similar pattern was observed in a recent laboratory study, in which subjects were asked to perform the same stressful task on $\mathrm{S}$ consecutive days. Here, the majority showed a significant cortisol response on the first day only, while a sub-group of individuals with low selfesteem, depressed mood, and physical symptoms showed persistent cortisol responses to the task over all exposures (Kirschbaum, Pruessner, Stone, Federenko, Gaab, Lintz, et al., 1995).

We found no statistically significant individual differences. ("random effects" in the model) in cortisol reactivity to events. While laboratory studies of responses to a battery of stress tasks provide some evidence for individual differences in reactivity 
(Forsman \& Lundberg, 1982), such differences appear to be less stable than differences in basal levels, which have a larger genetic component (Kirschbaum, Wust, Faig, \& Hellhammer, 1992b). It is possible that either the total sample size was too small or that the within-subject samples of events were too heterogeneous in the current study to reveal consistent and reliable individual response patterns.

The effect of stressful events on cortisol appears to be mediated to a large extent by associated increases in negative mood, since addition of Agitation or (to a lesser extent) Negative Affect to the model resulted in a decrease in the Event effect to a non-significant level. This interpretation is consistent with most views of the stress process and is supported by unpublished results from the same study, showing that both Negative Affect and Agitation increase following stressful events. More detailed temporal analyses of these data may help clarify whether an emotional response to an event is necessary to trigger a hormonal response.

\section{LImitations and conclusions}

For practical reasons, the study focused on a relatively homogeneous group of male white collar employees. We are not able to assess the extent to which the results are generalizable to other populations. Given the evidence for sex differences in neuroendocrine reactivity to psychosocial stress (Collins \& Frankenhaeuser, 1978; Kirschbaum, Wust, \& Hellhammer, 1992c), a replication of this study with inclusion of female subjects would be informative. Another limitation is that we did not assess positive daily events, which could theoretically affect cortisol levels via their influences on mood (Stone, 1987), possibly mitigating the effects of negative events. However, since positive events have their greatest effect on positive affect (Stone, 1987), it is important to recall that Positive Affect was not associated with cortisol in the present study.

In conclusion, this study suggests that neuroticism is not simply a confounder in psychosomatic research (McCrae, 1990), but is associated with increased adrenocortical activity during normal daily activities. Self-report measures of anxiety and depression, negative mood states and daily stressors, all known to be strongly related to trait negative affectivity (Watson \& Pennebaker, 1989), were associated not only with increased perceptions of chronic stress, but also with elevated cortisol levels. The finding that even minor everyday events and fluctuations in mood states have an impact on cortisol secretion points to a possible mechanism linking subjective experience to health outcomes. Further research is needed to determine whether mild, chronic cortisol elevation contributes to disease processes in somatic illness or psychiatric disorder, in particular depression.

\section{ACKNOWLEDGEMENTS}

We thank C. Dijkman for assistance in all aspects of the study. We also thank Prof. T.A.B. Snijders and four anonymous reviewers for substantial comments on earlier versions. 


\section{Chapter 7}

\section{Individual differences in cortisol responses to a laboratory speech task and their relationship to responses to stressful daily events}

Biological Psychology 43(1): 69-84 (1996)

Marleen van Eck ${ }^{1}$, Nancy Nicolson ${ }^{1}$, Hans Berkhof ${ }^{2}$, Jose Sulon ${ }^{3}$

Department of Psychiatry \& Neuropsychology, Social Psychiatry and

Psychiatric Epidemiology section, University of Limburg, the Netherlands

2 Department of Statistics and Measurement Theory, University of Groningen, the Netherlands

3 Faculty of Veterinary Medicine, University of Liege, Belgium 

A Stress Inducing Speech Task (SIST) was used to investigate the contribution of perceived stress, individual traits, and current mood states to individual differences in salivary cortisol responses. Additionally, we examined the correspondence between laboratory baseline cortisol levels and overall levels in daily life, and between cortisol responses to the SIST and a measure of stress reactivity to stressful events in daily life. Forty-two 'high stress' and forty-five 'low stress' whitecollar males completed the SIST and an Experience Sampling study, in which stressful daily events and cortisol levels were monitored for five days. No association was found between perceived stress, trait anxiety, anger, depression, psychosomatic symptoms, coping style or personality and cortisol responses to the SIST. Negative mood state at baseline was associated with higher cortisol levels at baseline, just before, and just after the speech. Laboratory and field cortisol levels were moderately correlated, but no association was found between laboratory and field response measures. Laboratory baseline levels, but not responses to the speech task, were significant predictors of field cortisol levels.

\section{INTRODUCTION}

Changes in cortisol levels have been shown to be sensitive indicators of psychosocial stress and coping patterns in both laboratory and natural situations (Frankenhaeuser, 1986; Mason, 1968; Rose, 1984). Although adrenocortical responses to psychological stress have been found to differ between individuals, sources of these differences are far from clear. For a better understanding of the potential link between cortisol responses and disease (Bassett, 1982; Cohen et al., 1991; Gaillard \& Al-Damluji, 1987; Troxler et al., 1977), more insight into individual differences in cortisol reactivity is needed. Since it is reasonable to assume that stress reactions will only lead to disease when they are prolonged or occur very often, it is likely that the pathogenic influence of cortisol hyperreactivity (or hyporeactivity) depends on whether this reflects a stable individual characteristic. Evidence from animal studies indicates that cortisol hyporesponders may be more prone to autoimmune disorders (Sternberg et al., 1989), while cortisol hyperresponders might be more vulnerable to infectious diseases (Mason, 1991). Research on psychological stress and cortisol indicates that the subjective appraisal of apprehension and emotional involvement can lead to increased cortisol secretion (Kirschbaum \& Hellhammer, 1989; Mason, 1975; Rose, 1984). Personality and other traits may influence how stressful situations are appraised and may thus have predictive value for understanding individual differences in emotional and physiological responses to apparently identical situations (Arnetz \& Fjellner, 1986; Vaernes et al., 1982).

The goals of the current study were twofold. Our first objective was to determine whether perceived stress level (as a measure of mild chronic stress) was related to emotional and cortisol responses to an experimental stressor. From existing data, it is not clear whether chĩonic stress affects an individual's pattern of response to acute stressors. There is some evidence for an attenuated cortisol response in chronically stressed human subjects (Bourne et al., 1967; Friedman, Mason, \& Hamburg, 1963) but these studies focused on responses to highly traumatic situations (e.g., acute medical complication in a fatally ill family member, combat exposure). Experiments in rats indicate that the effects of chronic or repeated stress exposure on later glucocorticoid responses depend to a large extent on stressor intensity (Ottenweller, Natelson, Pitman, \& Drastal, 1989). 
There is considerable overlap between perceived stress and other subjective measures of distress, negative affectivity, and ineffective coping. For this reason, we also investigated the contribution of trait anxiety, depression, psychosomatic symptoms, anger, coping style and personality to individual differences in cortisol response. Such traits have been associated with basal cortisol levels (Brandtstädter et al., 1991; Pope \& Smith, 1991; Schaeffer \& Baum, 1984) as well as with stress responses (Bohnen et al., 1991; Demyttenaere et al., 1989; Kirschbaum et al., 1989; Nicolson et al., 1992) in a number of previous studies. Other studies, however, have found no association between personality traits, coping styles, and cortisol responsiveness to laboratory stressors (Bossert et al., 1988; Kirschbaum et al., 1992a).

As noted above, individual characteristics are thought to be important determinants of the emotional response to a given situation; mood states are, in turn, likely to mediate the endocrine response to the situation. Although there is abundant evidence that cortisol increases in response to distress or negative mood (Arnetz \& Fjellner, 1986; Lundberg \& Frankenhaeuser, 1980; Mason, 1968; Nicolson, 1992), the effects of positive mood are less clear. Positive affective states have been associated with decreases (Hubert \& de Jong-Meyer, 1989; Kugler \& Kalveram, 1989) as well as increases (Brown et al., 1993) in cortisol levels.

Our second objective was to compare cortisol responses measured in the laboratory with those measured in real life. As Turner, Ward, Gellman, Johnston, Light, \& van Doornen (Turner et al., 1994) have stated: "if exaggerated responses do play a role in disease development, it is via everyday challenging situations that they will exert their pathogenic influence. The usefulness of laboratory assessment rests, in part, on the assumption that laboratory responses reflect those occurring during natural situations". The widespread availability of ambulatory monitoring techniques has stimulated research on the relationship between laboratory and daily life cardiovascular activity. Evidence from several studies indicates that blood pressure levels obtained in the field are better predictors of subsequent hypertension, complications from hypertension and mortality than measurements obtained in the doctor's office (Devereux, Pickering, Harshfield, 1983; Perloff, Sokolow, \& Cowan, 1983). A recent review (Turner et al., 1994) concluded that moderate evidence exists for laboratory-field generalization of cardiovascular activity. The evidence differs in strength, however, for different types of laboratory-field associations. The associations between basal, chronic levels of cardiovascular activity across settings are the strongest, while inconsistent support has been found for the relationship between laboratory reactivity and field reactivity, and between laboratory reactivity and field levels.

Especially with regard to endocrine reactivity, the generalizability of laboratory studies to field studies has been underexplored. In a study of plasma catecholamine reactivity in a small sample of healthy young men (Dimsdale, 1984), no correlation was observed between endocrine responses to laboratory mental arithmetic and those to real-life public speaking. Although van Doornen and Blokland (1992) observed a correlation between pre-task adrenaline levels in the laboratory (reaction time and tracking task; cold pressor test) and the level preceding a real-life siressor (public defense of $\mathrm{PhD}$ thesis), urinary catecholamine responses to the laboratory tasks did not correlate with responses to the field stressor. To our knowledge, only one siudy has investigated laboratory-field associations of cortisol excretion patterns (Houtman \& Bakker, 1987). In a sample of nine student teachers, saliva cortisol responses were compared in a real and a standardized lecturing situation. Most correlations were low and nonsignificant, but this may have been due 
to the small sample size and the poor standardization of time at which the subjects started lecturing in the real life situation.

In the current study, a Stress Inducing Speech Task (Steiner \& Levine, 1988) was employed to investigate trait and state correlates of salivary cortisol responses to stress. Although studies of psychophysiological reactivity often employ standardized laboratory tasks such as mental arithmetic, computer games or cold pressor tests, one can question whether the reactions observed are relevant or valid reflections of habitual reactivity to naturally occurring psychosocial stressors. Speech tasks have a greater ecological validity and reliably induce endocrine and cardiovascular responses as well as moderate distress (Bassett et al., 1987; Dimsdale, 1984; Kirschbaum \& Hellhammer, 1993; Steiner \& Levine, 1988). In addition to the laboratory experiment, subjects participated in a naturalistic field study of daily stress and cortisol dynamics. This allowed us to evaluate the consistency of laboratory and field cortisol measures, particularly with regard to the usefulness of the laboratory measures in predicting cortisol levels and responses to naturally occurring stressors in the field situation.

\section{METHODS}

\section{Subjects}

Local industries and government agencies were approached via their personnel departments. 316 male white collar workers from six different industries or agencies completed the screening questionnaire. The mean score on the Perceived Stress Scale (PSS) for this sample was 12.7 (s.d. 6.0), similar to US. norms (mean 13.02, s.d. 6.45) (Cohen et al., 1983). Individuals scoring in either the upper or the lower tertile of the screening sample PSS distribution (PSS-10 score $\leq 10$ or $\geq 16$ ), and with no history of serious chronic illness, endocrine disorder, or medications known to affect cortisol levels (as ascertained by self-report) were later approached to participate in the main study, until 92 subjects, balanced for 'low' and 'high' stress states, were recruited ( $85 \%$ of those approached). Of the 92 subjects who took part in the study, 5 were excluded from the analysis due to missing data. The final sample included 42 subjects in the 'high stress' (HS) group and 45 subjects in the 'low stress' (LS) group. Mean age was 42.1 years (range 27 to 57 years): $89 \%$ were married, and $81 \%$ had children living at home.

\section{Experimental stressor: Stress Inducing Speech Task (SIST)}

The SIST took place between 11 am and 1 pm (before lunch), one or two days after subjects completed participation in an Experience Sampling study (see below). On arrival, subjects were unaware that they would be asked to deliver a speech. After completing baseline measures, subjects were asked to prepare ( 10 minutes) and present (5 minutes) a videotaped speech describing their personal strengths and weaknesses for later evaluation by a team of psychologists. Following the preparation period. subjects, received a signal to begin their presentation while looking directly into the camera. After the speech, subjects relaxed for 15 minutes in neutral activities (e.g. reading magazines). At four timepoints subjects filled in a mood questionnaire and provided a saliva sample: (T1) upon arrival, (T2) after the 10 minutes of preparation, (T3) after the 5-minute presentation, and (T4) after 15 minutes of relaxation. During the course of the study, the recovery period was lengthened to provide a clearer picture of the cortisoll response profile; for 49 subjects a fifth saliva sample (T5) was taken on average 50 minutes after the first assessment.

The mood questionnaire consisted of 15 five-point Likert scale items (Folkman \& Lazarus, 1985). Subjects indicated to what extent $(0=$ not at all; $5=$ a great deal $)$ they felt: 
worried, anxious, nervous, tense, confident, hopeful, eager, irritated, sad, disappointed, guilty, exhilarated, pleased, happy and relieved. The 15 mood items formed two distinct dimensions: Negative Affect (NA, 8 items) and Positive Affect (PA, 7 items), with Cronbach's alpha's at T1 of .79 for NA and .75 for PA.

\section{Fleld study: cortisol dynamics In daily life}

The Experience Sampling Method (ESM) (Csikszentmihalyi \& Larson, 1987; de Vries, 1992) was used to collect data from subjects at selected moments during their normal daily activities. Subjects received auditory signals ('beeps') to complete a questionnaire and a saliva sample. Subjects were sampled for a period of 5 consecutive days ( 3 work and 2 non-work days), 10 times each day, at semi-random intervals of approximately 90 minutes between the hours of 8 am and $10 \mathrm{pm}$. Compliance with the procedures was generally good: $83 \%$ of all possible responses were completed within the time limit of 20 minutes. For a more detailed description of the Experience Sampling Method procedure and compliance issues, see van Eck \& Nicolson (1994).

In addition to items concerning current mood, activity, social and physical context, the ESM form contained a 7-point Likert scale (from 1 'not at all stressful' to 7 'very stressful') for the rating of any stressful event or situation which had taken place in the interval since the last ESM report. Subjects reported a total of 626 stressful events during the five days of ESM, with a mean of 7.1 events per subject (median 5.0; st.dev. 7.3). Events were reported on an average of $16.8 \%$ (st.dev. 16.5) of total "beeps". Information concerning smoking, food, coffee, and alcohol intake, and maximum level of physical exertion since the last beep was also obtained.

\section{Paychological moasures}

The following measures were used in the current analysis:

Perceived Stress: The 10-item version of the PSS was translated into Dutch. The PSS is a global measure of the degree to which situations in one's life are appraised as stressful. Items tap the extent to which individuals feel their life to be unpredictable, uncontrollable, and overloaded. The items were rated on a 5-point frequency scale, ranging from 'never' $(0)$ to 'very often' (4) in the last month. All subjects completed the PSS twice, during the initial screening and again immediately preceding Experience Sampling. The two PSS scores were highly correlated (rho=73, $<<.001$ ). The mean of the two PSS scores was 7.2 (st.dev. 2.2) for the low stress group and 18.1 (st.dev. 3.4) for the high stress group.

Psychosomatic symproms: The revised version of the SUNYA Psychosomatic Symptom Checklist (PSC; Attanasio et al., 1984) includes 17 common psychosomatic complaints (e.g., headaches, backaches and nausea). Subjects rated each complaint on 5-point scales for frequency ( 0 'never or rarely occurs' io 4 "occurs daily') and intensity ( 0 'not bothersome' to 4 'extremely bothersome'). A Total Score is obtained by summing the cross-products of each item's frequency by intensity.

Depression: Depressive symptomatology was assessed with the Dutch translation (Dijkstra, 1974) of the Zung Self-Rating Depression Scale (Zung, 1965).

Anxiery: Trait anxiety was measured with the Dutch version (van der Ploeg et al., 1980) of the State-Trait Anxiety Inventor (STAI).

Anger: Trait anger was measured with the Dutch version (van der Ploeg et al., 1982) of the Spielberger Trait Anger Scale. The scale has two subscales: 'anger-temperament' and 'angerreaction :

Personality: Personality characteristics were assessed with the Dutch abridged MMPI (NVM: Luteijn \& Kok, 1985), which consists of five scales: Negativism, Somatization, Timidity, Major Psychopathology, and Extraversion. 
Coping: Coping style was assessed with the 47-item Utrecht Coping List (UCL; Schreurs \& van de Willige, 1988). The seven factor-analytically derived subscales are: 'active problem solving', 'palliative responding', 'avoidance', 'seek social support', 'depressive reaction", 'expression of emotions' and 'comforting cognitions'.

HS subjects scored significantly higher than LS subjects on psychosomatic symptoms ( 27.5 vs $6.1, \mathrm{p}<.001)$, depression $(48.4$ vs $36.5, \mathrm{p}<.001)$, anxiety $(39.8$ vs $28.3, \mathrm{p}<.001)$, anger (18.0 vs 14.6, $\mathrm{p}<.001$ ), the personality scales negativism ( 19.0 vs $10.0, \mathrm{p}<.001)$, somatization $(9.5$ vs $2.8, \mathrm{p}<.001)$, timidity $(11.3$ vs $8.3, \mathrm{p}<.05)$, and psychopathology $(2.8$ vs $1.3, \mathrm{p}<.01)$, and on the coping styles active coping (17.3 vs $21.1, \mathrm{p}<.001)$, palliative reaction (17.2 vs 14.6. $\mathrm{p}<.001$ ), depressive reaction (12.6 vs 8.8, $p<.001$ ), and expressed emotions (6.9 vs 5.8, p<.01). Where possible, mean scores for both samples were compared to published norms for the Dutch population. The general pattern found was that LS subjects scored average or below average compared to the norm scales, while HS subjects scored above average or high. No significant differences between HS and LS groups were found for the personality scale extraversion (16.5 vs $15.4)$ and the coping styles avoidance (15.1 vs 14.1), seek social support (12.5 vs 11.7), and comforting cognitions (11.7 vs 10.8 ).

\section{Saliva sampling and biochemical analysis}

Subjects collected saliva by holding a cotton dental roll in the mouth for approximately 1 minute. The saturated roll was placed in a capped plastic vial ("Salivette", Sarstedt). Uncentrifuged samples were stored at -20 degrees $C$, until analyses. Salivary cortisol levels were determined in duplicate by direct radioimmunoassay (Ansseau, Papart, Pitchot, Timsit-Berthier, Legros, \& von Frenckel, 1992), using ${ }^{125} \mathrm{I}$-cortisol and antiserum made against the 3-CMO-BSA conjugate. The lower detection limit of the assay was $12 \mathrm{ng} / \mathrm{dl}$, with a mean intra-assay coefficient of variation of $4.8 \%$ (range: $2.2 \%-7.5 \%$ for 4 assays).

\section{Statistical analysis}

A fifth root transformation of the raw cortisol data (cortisol ${ }^{0.2}$ ) was used to obtain normally distributed residuals throughout the day in the cortisol field data. For comparability, the same transformation was applied to the laboratory cortisol data. Multivariate analysis of variance for repeated measures (MANOVA procedure, SPSS) was performed to test for effects on cortiso! levels during the SIST; in addition to the repeated measures factor Time (TI through T4 and, for a subsample, T5), Group (PSS high versus low) and Group x Time interaction effects were tested. Baseline cortisol was included as a covariate. A separate MANOVA analyses was repeated on the mood measures. Significance levels are based on multivariate (F) tests. Forward linear stepwise regression analyses were performed to estimate the proportion of variance in cortisol (dependent variable) explained by various (independent) person variables.

Cortisol responses to the SIST were computed as area-under-the-concentration-time curve (AUC), using the trapezoidal integration. The value of the laboratory sample at TI is reported as laboratory baseline (L.BASAL). L.RESPONSE was defined as the portion of the AUC above L.BASAL. Because the SIST always took place between 11.00 a.m. and 1.00 p.m., prior to lunch, field cortisol level (F.BASAL) was defined as the mean level over 5 days with the third ESM beep (at approximately $11.15 \mathrm{a.m}$.) as our reference point. The mean (within-subject) coefficient of variation for the five cortisol values was $4.1 \%$, ranging from $1.5 \%$ to $7.2 \%$.

The model used for the analysis of the cortisol field data has been described in detail elsewhere (van Eck, Berkhof, Nicolson, \& Sulon, in press). The multilevel model is a variant of the multiple linear regression model applicable for data with a hierarchical nesting structure (here: repeated measurements within persons), where the dependent variable (here: cortisol) is defined at the lowest level of the hierarchy. In this model, field cortisol level (F.LEVEL) was defined as the 
mean cortisol level over the five days in the field after controlling for diurnal patterns and the other factors (smoking, lunch and other food intake) found to have significant effects.

Individual cortisol response estimates to stressful events in daily life were calculated by an empirical Bayes, approach, using multilevel analyses (Bryk \& Raudenbush, 1992). These response estimates are weighted averages of an overall regression estimate (based on the whole data set) and a person-specific regression estimate. The weight of the person-specific estimate, relative to the overall estimate, depends on its reliability as an estimate of the individual responses. Events were dummy coded as $\mathrm{l}=$ event, $0=$ no event, since use of the 7-point scale did not improve the prediction of cortisol. Stress response scores in the field (F.RESPONSE) were estimated after diurnal patterns and other factors (listed above) with significant effects on cortisol levels were controlled for in the multilevel model. As reported elsewhere (van Eck et al., in press), stressful daily events were associated with significantly higher cortisol secretion.

The association between laboratory and field cortisol was tested with Spearman rank-order correlations between: 1) L.BASAL and F.BASAL, 2) L.RESPONSE and F.BASAL, and 4) L.RESPONSE and F,RESPONSE. As a last step, the contributions of L.BASAL and L.RESPONSE as predictors of F.LEVEL were evaluated with multilevel analysis.

\section{RESULTS}

\section{Effects of percelved stress level on cortisol and mood response to the experimental stressor}

Salivary cortisol responses to the SIST are shown in Figure 7.1. Most subjects responded to the task with increased cortisol secretion; 76 of the 87 subjects showed a cortisol response greater than L.BASAL (T1). On average, peak cortisol levels were observed at T4, approximately 30 minutes after the SIST preparation period began and 15 minutes after the speech ended. The maximum increase in cortisol during the SIST averaged $159 \mathrm{ng} / \mathrm{dl}$ (a percentual increase of 109\%) above L.BASAL.

MANOVA analysis revealed significant effects for the L.BASAL covariate $(B=$ $82, t(l, 84)=16.25, p<.001$ ) and for the repeated measurement factor Time (T! through T4: $F(3,255)=59.39 ; p<.001)$. The cortisol response pattern of the high stress group did not differ significantly from that of the low stress group, as evidenced by nonsignificant effects for both the PSS main effect $(F(1,84)=.02$; ns $)$ and the PSS by Time interaction $(F(3,255)=.18$; ns). MANOVA analysis for the sub-sample of 49 subjects with a final cortisol measurement at T5 yielded similar results: a significant main effect for the factor Time (T1-T5; $F(4,18.8)=16.83 ; p<.001)$, no main effect for PSS $(F(1,46)=.06$; ns $)$, and no interaction effect for PSS by Time $(F(4,188)=1.46$; ns). On average, cortisol at T5 (268 ng/dl) had significantly declined relative to peak levels $(335 \mathrm{ng} / \mathrm{dl}$ ) (Wilcoxon test, $p<.001$ ), although it was still elevated in relation to L.BASAL ( $167 \mathrm{ng} / \mathrm{dl}$ ). Comparing the recovery process in high vs. low stress groups, post-hoc comparisons showed no difference between the groups in either the magnitude of the cortisol elevation above L.BASAL persisting at T5 (high PSS $(n=26): 109 \mathrm{ng} / \mathrm{dl}$; low PSS $(\mathrm{n}=23): 95 \mathrm{ng} / \mathrm{dl}$; Mann-Whitney test, $\mathrm{n} . \mathrm{s}$.$) or the$ magnitude of the decline in cortisol from maximum response to T5 (high PSS: decline $=54 \mathrm{ng} / \mathrm{dl}$; low PSS: decline $=82 \mathrm{ng} / \mathrm{dl} ;$ Mann-Whitney test, n.s. $)$. 


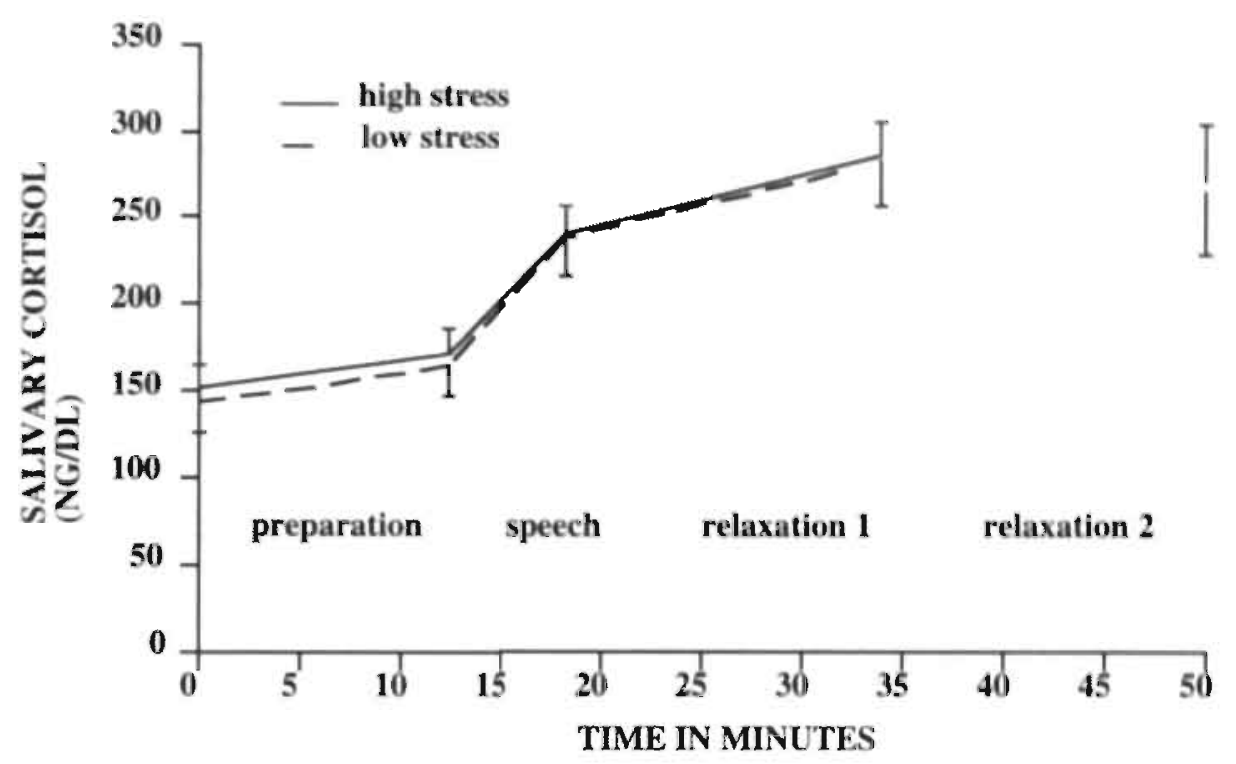

Figure 7.1. Cortisol response to speech task in high and low stress subjects. Data at T1 - T4 based on 87 subjects, data at T5 based on 49 subjects.

Mood states also changed in response to the stress task, with significant Time effects for both PA $(F(3,255)=14.53 ; p<.001)$ and NA $(F(3,255)=42.11 ; p<.001)$. Post-hoc comparisons revealed a significant NA increase $(p<.0001)$ and PA decrease $(p<.001)$ from $\mathrm{T} 1$ to $\mathrm{T} 2$, after preparation but before speech delivery. As can be seen in Figure 7.2., mood had recovered to baseline by 'T3. 'High stress' subjects had a higher overall level of NA than 'low stress' subjects $(F(1,85)=22.15 ; p<.001)$; the level of PA did not differ between groups $(F(1,85)=.55 ; p>.05)$. Time by PSS interactions were not significant; in other words, high stress subjects showed no greater increase in NA or greater decrease in PA in response to the task than low stress subjects. 


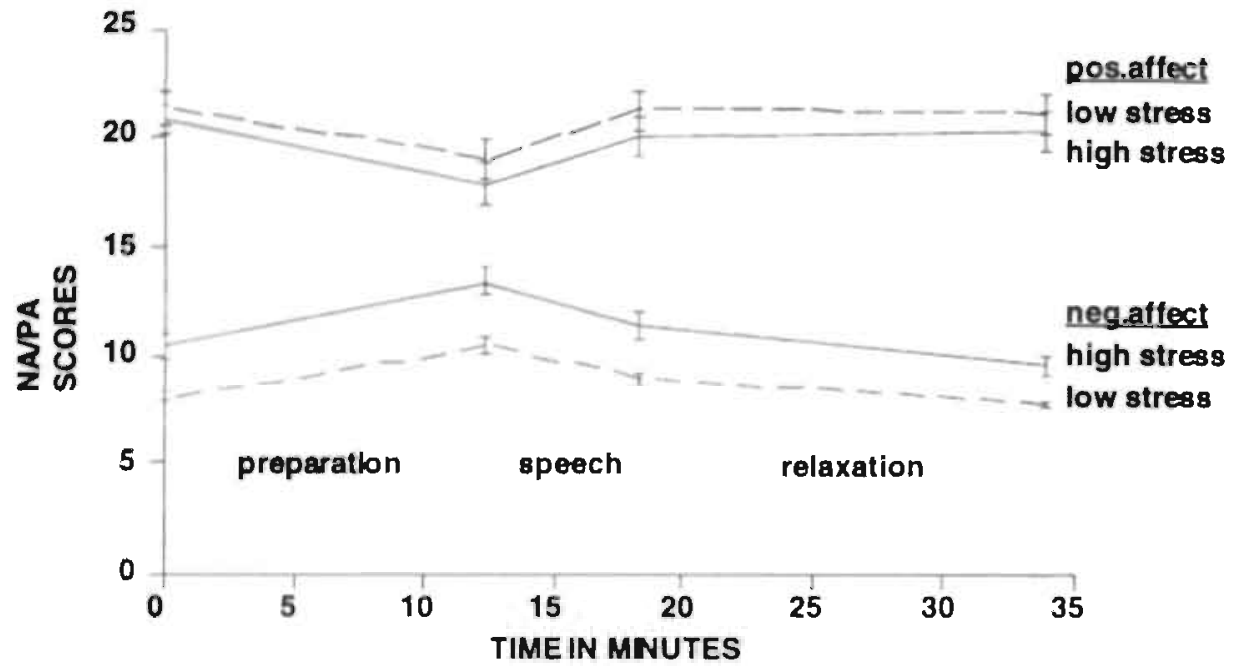

Higure 7.2. Response of NA and PA to speech task in high and low stress subjects.

\section{Relationship between mood state and cortisol response to the SIST}

To investigate whether subjects' emotional responses to the SIST were related to their cortisol responses, Spearman rank correlation coefficients were computed between the four repeated cortisol measurements and the summary measure L.RESPONSE, on the one hand, and the four mood measurements of both mood scales, on the other hand. No significant correlations were found between either NA or PA and L.RESPONSE. Of all correlations between the four PA values and the four cortisol values (T1 through T4), not a single coefficient was significant. However, NA at baseline was positively associated with cortisol at L.BASAL (T1), with cortisol just before the speech task (T2) and with cortisol just after the speech task (T3) (rho $=.29$; $\mathrm{p}<.01, r h o=.31 ; \mathrm{p}<.01, r h o=.25 ; \mathrm{p}<.05$, two-tailed tests).

\section{Relationship between trait characteristics and cortisol response}

Possible influences on cortisol L.RESPONSE of trait anxiety, depression, anger, coping styles, total psychosomatic complaints, and personality scales were tested with forward stepwise multiple regressions. None of the above traits was a significant predictor of L.RESPONSE (all higher $F$ probabilities than .05).

\section{Laboratory to fleld generalization}

L.BASAL was significantly higher than F.BASAL (147 ng/dl versus $116 \mathrm{ng} / \mathrm{dl}$; p<.001, Wilcoxon Matched-Pairs Signed-Rank Test, two-tailed test). The difference beiween these two measures was similar for high and low stress groups (HS: 36.9 ng/dl, LS: $24.7 \mathrm{ng} / \mathrm{dl} ;$ ns). The correlation between L.BASAL and F.BASAL cortisol levels was moderately strong ( $r h o=.56 ; \mathrm{p}<.001)$. No relationship was found between L.RESPONSE and F.BASAL ( $r h o=-.08 ; n s$ ). Next we compared cortisol responses to. the SIST (L.RESPONSE) with estimates of cortisol reactivity to naturally occurring stressful events (F.RESPONSE); no significant relationship was found ( $r h o=-.13$, ns). 
Table 7.1. Effects of lab baseline levels (L.BASAL) and lab response values (L.RESPONSE) on field cortisol level.

\begin{tabular}{lrrr}
\hline Fixed Effects & Estimate & S.E. & $\mathrm{p}<$ \\
\hline Intercept & 2.94 & 0.144 & 0.001 \\
Time of day (TIME) & -0.581 & 0.037 & 0.001 \\
TIME $^{2}$ & 0.129 & 0.010 & 0.001 \\
TIME $^{3}$ & -0.010 & 0.001 & 0.001 \\
Truncated function & 0.010 & 0.001 & 0.001 \\
Lunch & 0.040 & 0.020 & 0.03 \\
Other food intake & 0.025 & 0.01 .1 & 0.01 \\
Smoking & 0.043 & 0.021 & 0.02 \\
\hline L-BASAL & 0.172 & 0.050 & 0.001 \\
LRESPONSE & 0.001 & 0.003 & $n s$ \\
Random Effects & (Co)variance & S.E. & p< \\
\hline Person level: & & & 0.001 \\
intercept & 0.024 & 0.005 & 0.001 \\
Time of day & 0.0002 & 0.00004 & \\
Time of day/Intercept & -0.001 & 0.0004 & 0.001 \\
\hline Measurement level: & & & \\
Residual term & 0.059 & 0.002 & \\
\hline
\end{tabular}

* 3082 observations nested within 85 subjects (41 HS, 44 LS)

Finally, we estimated a multilevel model in which L.BASAL and L.RESPONSE were used to predict cortisol F.LEVEL. This analysis has the advantage of making use of all of the cortisol field data. Controlling for significant external influences should increase both the reliability of the dependent cortisol measure and its comparability to the laboratory assessments. As shown in Table 7.1., when both laboratory measures were entered simultaneously into the model, only L.BASAL explained a significant amount of variance in F.LEVEL.

\section{DISCUSSION}

The first aim of the present study was to evaluate the contribution of perceived stress and trait characteristics to individual differences in cortisol responses to a laboratory stress task. Associations between emotional and cortisol responses to the speech task were also examined. To summarize the main findings, the SiST elicited a strong cortisol response in a large majority of the subjects, which was unrelated to differences in perceived stress level. Trait anxiety, depression, psychosomatic symptoms, anger, coping style and personality similarly failed to predict the cortisol response. Neither negative nor positive emotional responses to the speech task showed any association with the cortisol response. High negative affect at baseline, however, was associated with higher total cortisol levels at baseline, just before the speech task, and again just after the speech task.

This suggests that individual differences in current distress (especially anticipatory distress) may be more important determinants of cortisol secretion than PSS, which is a measure of more chronic distress. Although high PSS was associated 
with high NA, no direct relationship was found between PSS and cortisol secretion. It is worth noting that subjects with the highest NA at lab baseline (T1) had, at the same time, the highest cortisol baselines relative to their own $11 \mathrm{a} . \mathrm{m}$. field levels ( $r h o=.26$, $\mathrm{p}<.01$ ). An elevated laboratory baseline, possibly due to anticipatory anxiety, could explain the lack of a relationship between NA and the additional cortisol response measured during the SIST. Moreover, the fact that high stress subjects had higher baseline NA than low stress subjects suggests that there may be a tendency toward higher cortisol in response to the SIST in this group, which was masked by the anticipation response.

As summarized in an overview of the published data (Kirschbaum et al., 1992a), most studies have found either no association or only a weak association between cortisol responses to stress and personality measures. The hypothesis linking personality factors with cortisol responses rests on the assumption that cortisol reactivity is a stable individual characteristic. There is some evidence in support of this assumption. Forsman and Lundberg (1982) found significant individual consistency in urinary cortisol responses to repeated tasks; correlations were higher between responses to more or less identical tasks (males: $r=65$; females: $r=.38$ ) than between responses to different kind of tasks (males: $r=13$; females: $r=19$ ). Consistency in cortisol responses to different tasks was also noted by Berger et al. (1987). Another study (Kirschbaum et al., 1992c) found significant intercorrelations among maximum cortisol responses to three stimulation procedures (mental arithmetic, public speaking, bicycle ergometry) in males, but not in females. In a more recent study in the same laboratory, significant correlations (ranging from .38 - .60) were found between cortisol responses to the same task repeated on 5 days (Kirschbaum et al., 1995) Although some response consistency seems to exist, at least in males, more research is necessary.

Even if stable individual differences in cortisol reactivity exist, it is not clear that they reflect psychological traits (Bossert et al., 1988). Genetic factors, for instance, have been shown to play a role in cortisol reactivity (Kirschbaum et al., 1992b). Recently, some support for an association between personality factors and cortisol reactivity was reported (Kirschbaum et al., 1995); low self-esteem, negative self-concept, depressed mood and physical health problems were found to differentiate high and low cortisol responders. Twenty healthy males were exposed on five consecutive days to the same psychological stress task (public speaking and mental arithmetic in front of an audience), with low responders showing evidence of habituation after the first day and high responders continuing to show large cortisol responses on successive task exposures. If only Day 1 cortisol responses had been analyzed (as in the present study), only one out of 12 correlations between cortisol responses and personality scales would have reached significance. This indicates that measuring a stress response once may be insufficient for the manifestation of individual differences in cortisol reactivity. This appears to be an important point for further research.

The second aim of the study was to investigate the relationship between laboratory and field cortisol activity. A moderately strong correlation was found between laboratory baseline levels and 11 a.m. field cortisol levels, and laboratory baseline levels also predicted some of the variance in overall field cortisol levels. In contrast, no association was found between laboratory responses and the field reactivity measure, and laboratory responses were not useful in predicting overall cortisol levels in the field. The finding of a relationship between laboratory and field levels of cortisol, but not between. laboratory and field reactivity, is more or less in 
accordance with results of cardiovascular studies, where levels are found to be more stable than reactivity / variability scores across settings (Turner et al., 1994). Similarly, greater consistency has been found for repeated lab baseline cortisol levels than for cortisol reactivity measures to different laboratory tasks (Berger et al., 1987; Forsman. 1982). As with personality traits, the temporal stability of the cortisol response is an important issue. To the extent that 'reactivity' is not a stable trait, a correspondence between laboratory and field cortisol responses will be unlikely. Results of our field study on the effects of stressful daily events on cortisol levels (van Eck et al., in press), did not provide support for stable individual differences in cortisol responses to daily events; cortisol responses were more dependent on the current mood state.

Another possible explanation for the lack of laboratory to field generalization of cortisol responses is the different timing of cortisol response measurement in the lab compared to the field study. Although in the lab cortisol was assessed parallel to the stress task, in the field, stressful events could have occurred at any time in the 90 minutes interval prior to cortisol assessment. Substantial post-stress recovery of cortisol levels might have taken place by the time the saliva sample was collected. However, when we repeated the analysis presented above with a new field response measure based on the subset of stressful events that were still ongoing when cortisol was assessed, again no association was found between laboratory and field reactivity.

Although real life pubiic speaking was reported as a stressful event during ESM sampling by a few subjects, any similarity between lab and field stressors was coincidental. One could argue that the predictive validity of a laboratory stressor would increase if one were to choose a specific field stressor with a strong correspondence in psychological meaning to the laboratory stressor (Houtman \& Bakker, 1987; van Doornen \& Turner, 1992). However, even if the researcher succeeds in matching a field stressor with a relevant laboratory task, a severe drawback of this approach is that only a small subset of the enormous range of stressors people encounter in their daily lives can be simulated in the laboratory. Furthermore, the importance of a laboratory - field association would be lessened dramatically if only linkings of specific lab - field stressors were to show an association (Turner et al., 1994; van Doornen \& Turner, 1992). Significant correlations may be difficult to find because of the heterogeneity of field responses resulting from the variety of stressful experiences encountered. Even more than in the laboratory (Strube, 1989), responses to daily life stressors will be modulated by objective and subjective event characteristics (e.g. novelty, severity, duration, content), interacting with each individual's characteristics and prior experiences (Forsman, 1982). In conclusion, while we found no evidence for an association between cortisol responses in laboratory and field, the paucity of studies on this subject and the difficulties (conceptual, methodological, and statistical) associated with its investigation point to the need for additional research.

\section{ACKNOWLEDGEMENTS}

We thank C. Dijkman for assistance in all aspects of the study. 



\section{Chapter 8}

\section{Concluding remarks}





\section{RECAPITULATION OF THE MAIN RESEARCH AIMS AND FINDINGS}

In an effort to increase our understanding of the stress process as it relates to health, the present research investigated the impact of minor daily events on mood and the HPA system. Our first aim was to describe the nature and scope of daily life stress in a group of white collar men and to contrast the experiences of individuals who perceive themselves to be stressed with those who do not. The second aim was to determine how mood and cortisol levels change in response to stressful daily events. A final aim pertained to the stability and generalizability of individual stress responses. We examined whether the cortisol responses to a laboratory stress task reflect those occurring during stressful situations in real life.

Since it is reasonable to assume that stress responses will only lead to disease when they are prolonged or occur very often, it is likely that the pathogenic influence of these stress responses depends on whether they reflect a stabie individual characteristic or not. Therefore, we also investigated whether more or less stable person characteristics like perceived stress level, trait anxiety, and depressive symptomatology were related to individual differences in responses to daily stress. Special attention was also paid to the possible influence of the context and the appraisal of an event on outcome measures. The Experience Sampling Method was used to collect data from subjects during their normal daily activities. In addition, subjects participated in a laboratory stress task, in which they were unexpectedly asked to deliver a speech. In this final chapter, the main findings of the study are briefly reviewed and discussed, including potential clinical implications and suggestions for future research.

The results showed that work was the major source of daily stress in our sample, followed by problems related to the social network. These event contexts reflect the sample's life stage and demographic characteristics; their most important social roles were those of full-time employee, spouse, and father. In general, stressful daily events could be characterized by their salience and unpleasantness; especially events that were appraised as less predictable and less controllable were experienced as unpleasant. The high stress (HS) group could be characterized as vulnerable, trait anxious individuals, who suffered from a variety of psychological and psychosomatic complaints, experienced chronic environmental and relational difficulties, which they coped with using generally less effective styles. HS subjects reported twice as many events as low stress (LS) subjects, especially more work and social interaction events (involving colleagues or the partner). The HS group differed from the LS group in how both daily activities and stressful events were perceived: HS subjects evaluated their activities as requiring more effort, while they were less motivated and less skillful in doing them, while events were appraised as more stressful and less controllable.

From the data presented in chapter 4,5 , and 6 , we can conclude that high perceived stress was not only related to higher Agitation and higher Negative Affect levels and lower Positive Affect levels across all situations, but also magnified the effects of daily events on negative mood states. Positive mood was only affected by very unpleasant events. The effects of events on negative mood states persisted for at least 90 minutes in both HS and LS groups. A future event also increased current Agitation in both groups, but higher current Negative Affect was only observed in the HS group. The findings also indicated that certain kind of events (task demands) and event appraisals (unpleasantness, controllability, chronicity) were more likely to influence mood states. 
With respect to cortisol, it was not perceived stress but trait anxiety and depressive symptomatology that exhibited small but significant positive associations with cortisol levels during the day. Stressful daily events were found to be associated with increased cortisol secretion, the magnitude of the effect depending on whether the event was still ongoing and on how frequently a similar kind of event had occurred previously. Type of event or type of appraisal had no additional effect on cortisol. Although perceived stress, anxiety, and depression did not increase cortisol reactivity to daily events, evidence was found for reduced habituation to recurrent events in individuals scoring high on these traits. Consistent with most views of the stress process, the effect of stressful events on cortisol appeared to be mediated to a large extent by associated increases in negative mood states.

From the data presented in chapter 7 we conclude that the laboratory response measures do not necessarily reflect those obtained in the field.

The main findings of the study can be briefly summarized as follows:

(1) Individuals with high perceived stress reported twice as many daily events, with significantly more work and social interaction events. Events were also appraised as more stressful and less controllable (Chapter 3 ).

(2) Individuals with high perceived stress not only showed higher negative mood levels and lower positive mood levels throughout the day, but also exhibited higher negative mood states in response to stressful daily events (Chapter 4).

(3) In both groups, minor daily events had small but significant positive effects on salivary cortisol levels, which were largely mediated by negative mood states (Chapter 6).

(4) Individuals scoring high on trait anxiety or depressive symptomatology had higher cortisol secretion throughout the day and showed less habituation of cortisol responses to recurrent daily stressors (Chapter 5 \& 6).

(5) Laboratory and field cortisol levels were found to be moderately correlated, but there was no significant association between laboratory and field cortisol response measures. Laboratory baseline levels, but not responses to the laboratory stressor, were significant predictors of field cortisol levels (Chapter 7).

\section{MEASUREMENT OF STRESS IN DAILY LIFE}

For the measurement of daily stress, various options are available to the researcher, each with its own specific advantages and disadvantages. Laboratory studies try to simulate minor stressful events by exposing subjects to various tasks. like computer games, mental arithmetic, films or speeches. The experimental situation has the major advantage that the stressor can be standardized. thereby controlling for the objective component of the stressor. In addition, laboratory stress models can be of great value for basic research questions (e.g. for studying the mechanisms related to individual differences in response to various standardized stressors, or the influence of coping strategies on response to various stressors). However, one can 
question the relevance of the laboratory stressor for the stress that people experience in everyday life. In the laboratory most stressors are of a voluntary nature, of short duration, often anticipated, and they usually require active, effortful coping. It is not difficult to see that these kind of stressors cannot be as intrinsically motivating, threatening, challenging, or demanding of complex coping strategies as are most of the events people experience in their daily lives. Therefore, the validity of the dominant use of laboratory studies to increase our understanding of stress in daily life is questionable. Although the objective component of psychosocial stress is important for an understanding of the stress process, the study of how people perceive, report, and respond to feelings of stress, irrespective of its objective base, is of great importance in itself. For individuals, their idiosyncratic perceptions of stress and their subjective responses to it are most critical in determining its consequences. They may cause people to alter their work habits or their social relationships, change health behavior, or seek medical or psychological support.

Most studies designed to assess individual's subjective perceptions of daily stress have been limited to checklist questionnaires handed out to large populations at one point in time. Although understandable in light of time and cost factors, both psychological but also physiological stress measures collected at one point in time are subject to various biases. Besides a number of methodological problems with available checkilists (related, for instance, to item content or item sample), event checklists are often handed out long after the events have actually taken place. Therefore, less salient events will be forgotten, and the successful or unsuccessful resolution of the event ('effort after meaning') will determine to a large degree endorsement of an event item and scores on distress measures. These problems may still be profound when events are measured on a daily bases. One-time assessments of physiological parameters will probably be unreliable, for instance in that they include a lot of 'noise' due to normal fluctuations in levels of activity, or in that they reflect test or evaluative anxiety more than daily stress. One-time assessments of most physiological parameters also leave the temporal relationship between psychosocial stress and physiology unclear.

The ESM, in the present study characterized by repeated measurements of events, subjective mood and cortisol, has several advantages over cross-sectional approaches to the study of daily stress. First, it increases assessment reliability and provides better estimates of the frequency, distribution, and intensity of psychological variables. Subjects could not only report events occurring at the moment that they were beeped but also any event that had occurred during the past interval. By using an open-ended approach to elicit stressful events we did not restrict a priori the definition of 'stressful' to certain specific items or classes of events. Since events were assessed not long after their occurrence, we were able to elicit information about the sources of stress and the appraised meaning of an event with a minimum of confounding due to biased recall or forgetting. Using semi-random time intervals, anticipation effects were kept to a minimum.

With the ESM we were able to show, for example, that certain demographic characteristics (age, life stage, marital status) of our study sample were reflected in the pattem of significant events they reported. If one would use a single checklist for various samples or populations, important events with specific salience for a specific population may be missed. Events like school exams or cancelled dates, for instance, may be of great significance for a student population, but of minor relevance for a group of elderly people. Checklists should preferably be developed from data obtained from the population in which the checklist is going to be used. We also 
showed that the experience of stressful events is not static; the frequency of reported events varied according to the day of the week (more events during the week than in the weekend) and the time of the day (relatively more events during work hours). This also indicates the importance of the context, here the work environment, for eliciting events. With ESM, we were also able to compare subjects' immediate level of experience (e.g. ESM events reported during the day) with their short-term retrospective assessments (e.g. events reported at the end of the day). Here we observed that with end-of-day reflections many more events were reported than within days. It would be interesting to further investigate influence of response method (checklist versus open-ended) and possible biases in the process of recollection and appraisals of events, which may be more likely to occur at the end of the day.

Second, ESM provides a clearer picture of the dynamic and temporal nature of the relationship between stressful minor events and affective and neuroendocrine responses. This is especially important if the events of interest occur unpredictably (as is usually the case in the natural environment) and therefore cannot be directly monitored by the researcher. The correspondence between stressful daily events, selfreporis, and physiological measures can only be assessed on a within-subject basis. The results demonstrated the potential value of ESM in studying stress in the field. We were able to relate current mood and cortisol levels in daily life to stressful experiences within the context of stable individual differences, type of event, event appraisal, and time. Since assessments were made close to the moment at which the variables of interest occurred, we increased the possibility of establishing causal relationships. Although we cannot rule out the possibility that, for instance, current mood influenced the reporting of recent stressful events, the finding that prior stressful events were associated with persistent increases in negative mood states supports the assumption that events influenced mood and not vice versa.

Third, ESM provides a means for investigating both state and trait aspects of the daily stress process. In the present study we saw, for example, that a global measure of long-term difficulties was significantly related to the reported number of daily events. Person characteristics were found not only to be related to mean mood levels but also to the magnitude of mood responses to current stressful events. With respect to state aspects of daily stress. ESM is particularlly appropriate for the study of the quality of experiences. Since appraisal processes are thought to be predictive of emotional and physiological outcomes of stress, this is an important feature of ESM. In the present siudy, results indeed revealed that several appraisals were differentially related to the outcome measures. And although subject groups did not differ in the amount of time spent on various activities, important differences between groups were observed in how these activities were appraised and experienced. The multilevel approach used in chapters 4,6 , and 7 is optimally suited for the analyses of the ESM data set, in which both variables at the person level (trait characteristics) and the measurement level (state characteristics) are obtained. Using multilevel analyses we can adequately test the transactional stress model, in which trait and state aspects are intertwined.

A number of possible limitations of the present ESM study should also be considered. The repeated use of a short checklist instead of an open-ended approach to sample events might have had some practical advantages (e.g., no time consuming coding tasks for the researcher). However, most available checklists are too long to be suitable for frequent use, while a short event list limits dramatically the range of events that can be investigated. A checklist might be preferable, however, if one is 
interested in certain kind of events, or when one has knowledge about which kind of events are most important for a particular population. Another limitation of the current study design is the lack of temporal resolution. Many stressful events occurred in the interval between beeps, which means that by the time the measures were taken, some mood and/or cortisol response recovery may have taken place. Theoretically, an event-sampling approach (in which subjects are instructed to fill out a questionnaire and take a saliva sample at the moment or just after they experience a stressful event) might have some advantages, but such a procedure would very likely influence not only the daily routine of subjects but also how events are defined, experienced, and coped with. Only true ambulatory monitoring of cortisol (continuous measures: in practice infeasible) could really solve this problem.

A drawback of every subjective approach to the study of (daily) stress. including ESM, is that we are not able to differentiate between the relative contribution of the person and the environment in the experience and response to stress. In particular, since the present study relied on self-reports of stressful events, it was not possible to determine whether HS subjects were actually more exposed to stressful events or that high perceived stress levels (or other individual characteristics, like trait negative affectivity) merely increased reports of stressful events. Our measurement of daily events might possibly improve if we adapted the approach developed by Brown and colleagues (1974;1978), in which clear definitions have been formulated for what should and what should not be: considered a stressful event. The assessment of events is based as much as possibie on the facts of the event, their salience, and the context in which an event occurred (e.g. loss, or longterm contextual threat), rather than on how the respondent perceived them. In addition, a distinction is made between events which the respondent might have brought upon himself ('dependent' events) and those which simply happened to him ('independent' events). Although interviewers can be trained to reliably code major events, it seems highly unlikely that daily events can reliably be coded as 'dependent' or 'independent'. Another disadvantage is that the interviews necessary for the assessment of events are very time-consuming and therefore costly. One useful approach might be to study the daily stress process in those contexts in which one can anticipate the source(s) of stress and in which the potential stressor is identical for every subject, thereby controlling for the objective stimulus situation (e.g. driving exam, job loss, last weeks of the tax year for accounting firms). But as we can see from these examples, most stressors would then approximate major life events in terms of intensity and in frequency and might therefore not be relevant for an understanding of the much more frequent but minor stresses of day-to-day life.

\section{PERCEIVED STRESS AND NEGATIVE AFFECTIVITY}

Personality factors undoubtedly play an important role in perceived stress. Self-report measures of stress and distress have been found to correlate significantly with measures of trait negative affectivity or neuroticism: the disposition to accentuate the negative aspects of the self, other people, and the world in general, and to experience aversive emotional states. High negative affect individuals are more likely to experience discomfort at all times and across situations, and this trait has been shown to be reflected in higher overall levels of negative mood (Watson \& Clark, 1984). Negative affectivity also appears to be a vulnerability factor for the development of anxiety and depressive disorders, indicates poor prognosis, and seems 
to reflect, both the current influence of state affect and the residual effect of earlier depressive episodes (Clark, Watson, \& Mineka, 1994). Negative affectivity has been found to be highly stable over a 20 year period (Costa \& McCrae, 1980; Costa \& McCrae, 1986; Ormel, 1983), and there is evidence for a genetic compound (Lykken, 1982; Scarr, Webber, Weinberg, \& Wittig, 1981; Shields, 1962). While most theoretical models argue that dysphoric mood states result from an interplay between stressful events and certain vulnerability factors such as dysfunctional cognitions, Watson and colleagues (Watson, Clark, \& Harkness, 1994, p.29) have offered a different explanation: "The same individuals are likely to perceive innocuous or ambiguous events as stressful (negativistic appraisal), to respond poorly to actual stressors in their lives (stress overreactivity), to have a negativistic and dysfunctional cognitive style (e.g. self-criticism and self-blame), and to experience intense episodes of dysphoria (e.g. anxiety, depression, guilt, anger)." In other words, dysphoria, rather than being caused by stress and dysfunctional cognitions, may simply be another subcomponent of the same general construct 'negative affectivity' or neuroticism. It has been shown, for instance, in several prospective studies that a chronic pattern of general emotional and/or physical distress exists in about $25 \%$ of the respondents in the various populations investigated (Depue \& Monroe, 1986). General distress levels in these groups appeared to be determined to a large extent by characteristics of the individual and not by environmental (life events) or psychosocial (social support) variables. It is highly likely that a substantial portion of this chronically distressed group will consist of individuals scoring high on the personality dimension negative affectivity, since most scales of psychological disorder in the stress literature assess general negative affect, life dissatisfaction, and a lack of positive well-being (Depue \& Monroe, 1986).

Perceived stress did not overlap completely with measures of psychological distress in the present study; for example, we found that perceived stress, chronic difficulties, trait anxiety, and depressive symptoms independently predicted psychosomatic symptoms (Appendix I). Level of perceived stress was, however, highly correlated with trait anxiety, which is often used as a measure of trait negative affectivity and may explain at least partly the differences in overall mood found between HS and LS subjects. It is important to note that subjects with high perceived stress levels not only had higher negative mood levels across days, but high perceived stress also magnified the effect of stressful daily events on negative mood states within days. In other words, perceived stress does not just spuriously inflate the relationship among stressors, stress symptoms and affective outcomes, but acts as a moderator variable influencing an individual's reactivity to stressful events. The increased negative mood reactivity to minor events may be an important factor in the maintenance of chronic distress in the high perceived stress group. Therefore it seems important in the future to investigate variables potentially related to stress reactivity. such as coping behavior, and social support.

Global perceived stress was not a strong predictor of cortisol secretion in this sample. However, the intercorrelations among the various measures of stress and distress argue for cautious conclusions about the relative contribution of each to cortisol levels. The fact that self-report measures of anxiety and depression were associated not only with increased perceptions of chronic stress but also with elevated cortisol levels also suggests that negative affectivity is not simply a confounder in psychosomatic research. Several other studies have provided support for a relationship between negative affectivity and objective measures of stress; for instance, trait negative affectivity has been positively associated with salivary 
immunoglobulins (Stone, Cox, Valdimarsdottir, Jandorf, \& Neale, 1987; Ursin, Mykletun, Tonder, Vaernes, Relling, Isaksen, et al., 1984) and systolic blood pressure (Harburg, Julius, McGinn, McLeod, \& Hoobler, 1964). Although perceived stress, trait anxiety, and depression did not increase cortisol reactivity to stressful events, we did find evidence for reduced cortisol habituation to recurrent events in subjects scoring high on these traits. Besides these trait measures, the state measures of negative mood were found to be important mediators of the relationship between stressful events and cortisol secretion. Following the transactional stress model, a subject's appraisal of an event with respect to 'what is at stake' and 'what can be done about it' determines the level of emotional involvement, which in turn influences outcome processes. This study indeed showed that the subjective appraisal of apprehension and emotional involvement can lead to increased cortisol secretion. Since high stress subjects reacted more strongly with negative mood to stressful events, and since the effect of stressful events on cortisol was mediated to a large extent by associated increases in negative mood, the apparent lack of a significant association between perceived stress and cortisol reactivity to stressful events is unclear, although we did observe a trend in the expected direction.

Results of the present study are consistent with three related mechanisms thought to play a role in the modifier effect of negative affectivity on the relationship between life stress and psychobiological distress (Ormel \& Wohlfarth, 1991). First, negative affectivity may influence the appraisal of daily events and therefore their consequences for well-being, with subjects scoring high on measures of negative affectivity appraising identical events as more demanding and threatening (Lazarus \& Folkman, 1984b; Watson \& Clark, 1984). Second, negative affectivity may be associated with differential psychobiological sensitivity to stressors (Bolger \& Schilling, 1991; Eysenck, 1967; Tellegen, 1985). Third, negative affectivity may be related to less adequate coping with daily events (McCrae \& Costa, 1986). Although in the present study we had no control over the kind of events experienced, HS subjects perceived events as equally unpleasant as LS subjects, but these events were appraised as more stressful and less controllable by HS subjects. Moreover, activities (especially work activities) were appraised as more demanding and less enjoyable. With respect to psychobiological sensitivity to stressors, HS subjects showed increased negative mood reactivity to daily events and also exhibited less cortisol habituation to recurrent events when compared to LS subjects. Finally, HS reported using an active coping style less often in general, and the coping styles palliative reaction and expression of emotions more often. These latter coping styles are generally considered to be less effective. HS subjects scored higher on the scale depressive reaction, indicating that they experience more feelings of helplessness in response to daily events than LS subjects. We have to keep in mind, though, that scores on general coping styles are not necessarily predictive of the actual coping strategies used in relation to specific events. However, the fact that HS subjects appraised daily events as less controllable than LS subjects also seems to indicate that they were less certain of their coping skilis in relation to daily events.

The fact that self-reports of stress, symptoms, and negative mood are highly correlated with measures of negative affectivity has important implications for the measurement of stress (Pennebaker \& Watson, 1988). Since there are large individual differences in negative affectivity which seem to have a heritable component and seem to be stable over time and situation, measures of what is presumed to be transient work stress will be strongly correlated with negative affectivity. This means that, for the study of the impact of stressful events, any between-subject design will 
have to gather data from a very large sample to be able to control for individual differences in negative affectivity. Another problem related to the concept of negative affectivity is that of causality; any causal interpretation will be meaningless since the process related to the perception of stress is the same process related to the perception of symptoms. Therefore, the optimal design is a within-subject design with repeated measurements over time, where both within- as well as between-subject relationships can be investigated prospectively, as is the case with ESM. As discussed before, the association between subjective self-reports and physiological measures can also only be determined on a within-subject basis. There is also a great need for longitudinal research, as clearly formulated by Clark et al. (1994, p.113): "Until prospective studies have followed individuals - whose premorbid personality and environmental characteristics are known - over sufficiently long periods of time to yield a sufficient base rate of disorder, we will not be able to distinguish causal from concomitant, confounding, or residual factors."

\section{GENERALIZABILITY FROM THE LABORATORY TO THE FIELD}

The positive relationship between trait anxiety and depressive symptonatology and contisol secretion throughout the day was not icplivated in the laboratory; no association was found between trait anxiety or depression and laboratory baselines (Chapter 7). With respect to stress reactivity, trait characteristics were related neither to elevated cortisol reactivity to stressful daily events in the field nor to the magnitude of the cortisol responses to the laboratory stress task. The field results regarding reduced cortisol habituation to recurrent events in subjects scoring high on perceived stress, trait anxiety, and depression could not be investigated in the laboratory since the laboratory stress task was not a recurrent but a novel event. In the laboratory, only negative mood state at baseline was associated with higher cortisol levels at baseline, just before, and just after the speech. In the field, we also found that current negative mood state was an important mediator of cortisol responses. Since it is reasonable to assume that personality traits influence how stressful situations are appraised and may therefore have predictive value for understanding individual differences in responses to stressful situations (as was the case for emotional responses), the lack of an association between the trait characteristics and cortisol responses, either to the SIST or to naturally occurring events in the field (except for recurrent events), is somewhat disappointing although certainly not an exception in the literature. Several methodological and statistical issues may possibly obscure this relationship. In the laboratory, the absence of a relationship between person characteristics and cortisol baseline levels and cortisol reactivity could be partly explained by an elevated laboratory cortisol baseline in both groups, possibly due to anticipatory anxiety, which also masks the cortisol response. This laboratory baseline was significantly higher than cortisol field levels assessed at the same time. It is also possible that strong novelty or experimental effects of the stressor override any individual differences in cortisol reactivity when laboratory stress responses are assessed only once. The fact that almost $90 \%$ of the subjects reacted with a substantial cortisol response to the SIST task supports this interpretation. Additional support for this argument was also found from a recent study by Kirschbaum and colleagues (1995), where some evidence for an association between personality traits and cortisol reactivity in healthy males was reported. In this study, subjects were exposed on five consecutive days to the same psychological 
stress task; low self-esteem, negative self-concept, depressed mood, and physical health problems differentiated high from low cortisol responders. If only Day 1 cortisol responses had been analyzed, results would have been negative; low responders showed habituation after the first day, while high responders continued to show large cortisol responses over the five days. These results cast great doubt on the value of one-time laboratory stress assessments; they seem to be insufficient for the manifestation of individual differences in cortisol reactivity. Therefore, in future laboratory studies cortisol responses should be measured at least twice; not only to repeated identical tasks, but preferably also to different tasks. In the field, repeated assessments of events were made, but it is possible that the within-subject samples of events were too heterogeneous to reveal consistent and reliable individual response patterns to events in general.

It has been widely assumed that by measuring stress reactivity in the laboratory we can say something about stress reactivity in general, which would help clarify individual vulnerability to real-life stress. The use of laboratory cortisol reactivity as a risk factor for stress-related disease depends on the extent to which reactivity is indeed a reliable, stable, and generalizable individual characteristic. Although several laboratory studies have provided some evidence in support of response consistency across identical and, to a lesser degree, between different kinds of tasks (at least in males) (Forsman, 1982; Kirschbaum et al., 1995; Kirschbaum et al., 1992c), the evidence is far from conclusive, and more research is necessary. Given the above, the generalizability of reactivity from the lab to the field also seems questionable; it is certain that the variety of stressors experienced in real life will be much larger than in the laboratory. Results indeed showed that laboratory and field response measures were not associated.

Even if laboratory response measures have no predictive value for reactivity to stressful events in daily life, it has been hypothesized that they might be good predictors of overall levels of hormonal secretion throughout the day (Manuck et al., 1989). According to this theory, overall levels reflect the cumulative effects of daily life stressors rather than the reactivity to a transient stressful episode. This idea was also not supported, however, by our results; laboratory baseline levels, but not responses to the speech task, were significant predictors of field cortisel levels. Once the pre-task level was known, no additional predictive information was gained from measuring the laboratory stress response. These results are comparable to those for cardiovascular parameters, where levels have been found to be more stable than reactivity scores across settings. Only a weak relationship has been found between laboratory reactivity and daily heart rate and blood pressure levels; once the resting level is known there is no additional gain from task level or stress responses (Turner et al., 1994).

The low predictive validity of laboratory cortisol reactivity may to some extent be ascribed to methodological problems, like elevated baseline cortisol in the lab or the timing of cortisol response measurements in the lab compared to the field. The laboratory procedure could possibly be improved by allowing more time for the 'baseline' level to stabilize. With respect to the timing of response measures, better matching of lab and field measures is probably only possible in a study of predictable real-life stressors. Again, this would drastically curtail the range of real-life stressors that can be investigated. In addition, this study showed that unanticipated events tended to be experienced as particularly unpleasant.

An even more serious problem may be the heterogeneity of stressful experiences in real life. In daily life it is not possible to standardize stressful events to 
control for the influence of factors like novelty, task difficulty, content, duration. These factors could all contribute to low response consistency. In daily life, stressful events, coping, and emotional-behavioral responses are related to each other in an ongoing process. For instance, although a certain work event may be dealt with successfully on Monday, the same event may become stressful again on Friday, if support from colleagues is lacking. Due to the heterogeneity of stressors and the ever changing and complex coping processes, reactivity to stressors in daily life may not show high stability over time. Results of our field study did not provide support for significant individual differences in cortisol responses to daily events; cortisol responses were more dependent on current negative moodl states. Since the current study was one of the first to investigate the generalizability of cortisol reactivity from the lab to the field, more research is necessary to answer this important guestion.

\section{CLINICAL IMPLICATIONS}

Results indicated that the experience of distress in daily life situations can indeed lead to mild HPA hyperactivity. Whether these mild elevations of cortisol levels could be a marker of increased risk for disease processes can only be speculated upon. In general, the physiological function of stress-induced increases in glucocorticoid levels is thought to be adaptive. Although there are several theories linking adrenocortical responses following psychosocial stress to later pathology, there is a general lack of knowledge about the mechanisms involved in the stressillness relationship, and we do not know, for example, how stable the cortisol elevations are, or whether such elevations are substantial enough to have clinical relevance. There are currently no epidemiological studies in which the progressive course and consequences of HPA-axis abnormalities have been investigated. The available data are largely based on animal studies or on correlational designs. There is, for instance, evidence from animal studies which suggests that genetic variation in the stress response plays an important role in illness; while low HPA responsiveness is linked to autoimmune disease, high HPA responsiveness appears to be related to infectious diseases (Mason, 1991; Sternberg et al., 19891).

The costs of chronic elevated cortisol levels may turn out to be more important in predisposing the organism to disease than acute reactivity. Possibly only when stress is frequent and prolonged, without the opportunity to fully recover, will the association between stress and disease become apparent in subsequent processes of adaptation or exhaustion, when suppression of various defense mechanisms proceed far beyond physiologicai needs. With respect to somatic pathology, chronically elevated cortisol levels cause many adverse effects (McEwen \&. Stellar, 1993), with main effectors the immune system (Cohen et al., 1991; Kiecolt-Glaser \&, Glaser, 1991), the cardiovascular system (Brindley \& Rolland, 1989; Jacobs, Friedman, \& Mittleman, 1992), and the adipose tissue and muscle (Cox \& Gonder-Frederick, 1991). With regard to psychiatric disorders, elevated cortisol levels and other HPA disturbances are associated with major depression (Linkowski et al., 1985). In some cases, HPA. abnormalities persist after clinical recovery of depression and are indicative of poor prognosis (Charles, Schittecatte, Rush, Panzer, \& Wilmotte, 1989). On the other hand. low cortisol levels have been associated with post-traumatic stress disorder, which is thought to reflect a chronic adaptation in stress-induced. HPA activation in the form of a heightened feedback sensitivity (Yehuda et al., 1993). It remains unclear. however, whether these HPA disturbances are involved in the etiology of the 
disorder. Interesting in this regard are theories concerning how acute stressful events can result in long lasting biological effects on the organism. It has been postulated that psychosocial stressors may result in alterations at the level of gene regulation and expression; here, the HPA axis is thought to play an important mediating role (Checkley, 1992; Gold, 1988; Holsboer, 1992; Post, 1992). Evidence is mounting from animal studies that the nervous system, especially the hippocampus, is subject to wear and tear as a result of stressful experiences (Jacobson \& Sapolsky, 1991: Sapolsky, Krey, \& McEwen, 1986). Since the hippocampus is very important for mood, learning. and memory processes and subtle regulation of the HPA axis during mental stress, changes in hippocampal functioning may be extremely important for understanding the mechanisms in stress-related disorders, especially psychiatric disorders.

Although chronic levels may be more important than acute reactivity for future disease, the effects of acute stress may synergize with the actions of chronic stress, as has been shown in cardiovascular disease (Jacobs et al., 1992). Persistent increases in cortisol levels may indicate heightened biological vulnerability that increases the probability of dysregulation. This probability of dysregulation is thought to increase substantially when the vulnerable system is challenged by environmental and psychosocial challenges (Axelrod \& Reisine, 1984). Since stressful daily events are very common, especially in the HS group, one might expect frequent provocation of biological instability from these events in vulnerable individuals, and particularly when stress responses fail to habituate upon repeated exposure. Although it seems unlikely that minor events may initiate acute disorder in a stably regulated system, they may lead to disorder in the long run or may help maintain chronic disorder in dysregulated systems (Depue \& Monroe, 1986). Given the relationship between negative affectivity and increased cortisol levels, and between perceived stress (being strongly related to negative affectivity) and increased mood reactivity, it seems possible that stable individual characteristics may contribute to a more general susceptibility or a heightened vulnerability to the development of disorder.

\section{DIRECTIONS FOR FUTURE RESEARCH}

In this final section, a number of suggestions will be made for possible future research into the (daily) stress-distress relationship. As emphasized earlier, there is a general need for prospective longitudinal research, with repeated measurements of the stress-distress relationship over sufficiently long periods of time. It may be useful to combine ESM and traditional research methods (Alliger \& Williams, 1993), since both immediate and long-term assessments of stress variables are meaningful, and ESM can increase our understanding of the mechanisms underlying long-term outcomes as assessed by traditional methods (e.g. prospective surveys). Results obtained in the field should then be investigated in laboratory experiments to see if these results can be replicated. In addition, an interdisciplinary approach will have to be used (McEwen \& Stellar, 1993), involving various psychological, behavioral, neurobiological, endocrine, and immunologic measures, as well as their interactions with each other. Person factors (for example negative affectivity, coping style), stress measures (perceived stress, life events, chronic difficulties, daily events), state. measures (mood states, psychosomatic symptoms), and physiological measures (e.g. neuroendocrine parameters) can than be investigated simultaneously. From the present study, it may seem that all important hormonal effects of psychosocial stress relevant for health are related to cortisol, but this is certainly not true. Stress can not. 
only induce increases in cortisol or catecholamine levels, but has also been related to changes in levels of vasopressin (Axelrod \&. Reisine, 1984), prolactin (Meyerhof, Oleshansky, \& Mougey, 1988), b-endorphin. (Meyerhof et al., 1988), testosterone (Rose, 1984), and other hormones.

Follow-up assessments of the subjects investigated in the present study could shed light on some important but complex questions. For example: How stable is negative affectivity over time and how stable is the negative affectivity - perceived stress, negative mood, cortisol relationship? How stable are cortisol levels and cortisol responses to events and how does stability relate to person characteristics? What are the factors predicting disease, absenteeism, disability versus health? With respect to the health consequences of stress, up till now, most life stress studies included only general indicators of emotional or somatic disorder. Moving away from general models, it may be important to include measures of specific disorders like atherosclerosis, angina pectoris, or respiratory infections, since the mechanisms underlying stress-disorder interactions may vary across disorder and may only be understood by analyzing specific disorders (Depue \& Monroe, 1986). For instance, while personality and coping behavior may play a dominant role in the onset or maintenance of certain clisorders, other disorders may be more strongly related to environmental factors or health behaviors.

In general, representative samples of the general population and, more importantly, of high risk groups (e.g. subjects with a genetic vulnerability for psychiatric disorders, subjects in high risk jobs like health and educational employees) should preferably be observed pre-morbidly and then followed longitudinally. Further research should also focus on women, since there is considerable evidence to suggest that there are differences not only in the kind of daily' stressors women experience (Folkman \& Lazarus, 1980: Frankenhaeuser et al., 1989), but also in physiological (including neuroendocrine) responses to stress (Frankenhaeuser, 1980; Kirschbaum et al., 1992c). In addition, several factors of theoretical importance to the stress process lacking in the present study should ideally be included in such a study: protective factors, such as positive events, social support. and coping strategies; health parameters, such as records of absenteeism, disability and health status, and other physiological measures like cardiovascular measures, day-time catecholamines, and immunological parameters.

Such a study may shed additional light on the relative impact of temperamental and environmental influences on psychological states (anxiety, depression), HPA and immune function, and somatic health (Ormel \& Wohlfarth, 1991). For instance, how do life events, chronic difficulties, and daijy events predict change and stability in psychological distress levels and physiological levels, controiling for important person characteristics like negative affecuvity? Despite a paucity of longitudinal data, several models for the relationship between personality, stressful life events, and well-being have been proposed (Headey \&. Wearing. 1989). Personality models state that wellbeing is solely a function of personality and will therefore be highly stable over time (Costa \& McCrae, 1980). Adaptation level models stipulate that adaptation to events is so rapid and so complete that well-being is not measurably affected by events (Brickman, Coates, \& Janoff-Bulman, 1978; Diener, 1984). Other models have been proposed that treat life events as wholly exogenous stressors with allegedly damaging effects on physical and mental health (Block \& Zautra, 1981). Criticism relating to methodology and model specification as well as meager empirical support for these models have led to the development of other models. A model that has received some empirical support is the dynamic equilibrium model (Duncan-Jones. 
Fergusson, Ormel, \& Horwood, 1990; Headey \& Wearing, 1989). In this model each person is regarded as having 'normal' equilibrium levels of life events and well-being, dependent on stable psychobiological and environmental characteristics. These stable characteristics vary from person to person. Only deviations from normal event levels (internal or external) change the normal level of well-being. Deviations are usually temporary because stable personality traits, time, and support (for example treatment) play an equilibrating function. Another model is the dynamic-vulnerability model (Ormel, 1995), which differs from the previous mentioned model in that it postulates that vulnerability can increase or decrease considerably during the life course following certain internal or external events. Cumulation of vulnerability can occur as a consequence of earlier episodes (Post, 1992). The validity of this model has not yet been thoroughly investigated. Although these models emphasize major life events, they could easily be extended to include chronic difficulties and minor daily events. Only longitudinal studies can test the validity of the various models. It should be noted here that with assessing the relative predictive power of environmental and psychosocial factors on the one hand, and stable person attributes on the other hand, it is not meant that both can be measured independently or that this is a necessity. Although care should be taken that events are not merely reflections of symptomatic impairment (through use of symptom-free event measures and attempts to determine the independence of events), personality factors will influence the generation of most events and vice versa. As formulated by Lazarus and Folkman (1986, p.77): "there is simply no way to define an event as a stressor without referring to the properties of persons that make their well-being in some way vulnerable to that event."

From a practical point of view, knowledge about the relative contribution of personality or environmental factors on well-being may provide important information for intervention programs designed to reduce personal distress. In cases where environmental factors appear to play no role; interventions should primarily focus on strategies that are not tied to stressful situations but that are solely directed at the person (for example relaxation techniques, reducing worry-proness). In the other case, we need to pay more attention to teaching the person how to deal with his psychosocial environment (for example increasing control by teaching adaptive coping strategies, restructuring of dysfunctional cognitions, increasing assertiveness, time-management, and avoiding stressors by stimulus control). Since the evidence so far points to a combination of a vulnerable personality structure and objectively stressful situations as determinants of well-being, intervention programs should be directed at both the person and his environment. By using diaries like those in the present study, valuable information can be obtained about a person's idiosyncratic vulnerabilities relating to his environment (stressful events), personality (event appraisals, stress reactivity), adaptive capacities (coping strategies), and well-being (anxiety, depression, somatic complaints). These personalized diaries provide a rich source of data on which effective interventions for that specific individual can be based, as well as a means of evaluating their effects. Although this last paragraph emphasizes personal responsibility for health and well-being and seems the most sensible and successful strategy in the short term, an interesting question for the future relates to the responsibility of society as a whole. It may be that by increasing our demands on the individual as a result of growing economic pressures, an increasing number of people will not be able to meet the new requirements. The question here is whether these people should be considered ill and deviating from 'normal', or whether society has lost sight of what is 'normal'. 



\section{REFERENCES}

Aagaard, J. (1984). Stressful life events and illness: A review with special reference to a criticism of the life event method. In J. Cullen \& J. Siegriest (Eds.), Breakdown inhuman adaptation to stress. Towards a multidisciplinary approach. Boston: Martinus Nijhof Publishers.

Abelson, J. L.. \& Curtis, G. C. (1996). Hypothalamic-Pituitary-Adrenal axis activity in panic disorder: prediction of long-term outcome by pretreatment cortisol levels. American Journal of Psychiatry, 153(1), 69-73.

Affleck, G.. Tennen, H., Urrows, S., \& Higgins, P. (1994). Person and contextual features of daily stress reactivity: Individual differences in relations of undesirable daily events with mood disturbance and chronic pain intensity. Journal of Personality and Social Psychology, 66(2), 329-340.

Allen. P. I. M., Batty, K. A., Dodd, C. A. S., Herbert, J., Hugh, C. J., Moore, G. F., Seymour, M. J., Shiers, H. M., Stacey, P. M., \& Young. S. K. (1985). Dissociation between emotional and endocrine responses preceding an academic examination in male medical students. Journal of Endocrinology, 107, 163-170.

Alliger, G. M., \& Williams, K. J. (1993). Using signal-contingent experience sampling methodology to study work in the field: A discussion and illustration examining task perceptions and mood. Personnel Psychology, 46, 525-549.

Ansseau, M., Papart, P., Pitchot, W., Timsit-Berthier, M., Legros, J. J., \& von Frenckel, R. (1992). Dexamethasone suppression test and prediction of treatment response to selective antidepressants. European Psychiatry. 7, 191-194.

Ansseau, M., Sulon, J., Doumont. A., Cerfontaine, J. L., Legros, J. J., Sodoyez, J. C., \& DemeyPonsart. E. (1984). The use of saliva cortisol in the dexamethasone suppression test. Psychiatry Research, 13, 201-211.

Ametz, B. B., Brenner, S.-O., Levi, L., Hjelm, R., Petterson, I.-L., Wasserman, J., Petrini, B., Eneroth, P., Kallner, A., Kvetnansky, R., \& Vigas, M. (1991). Neuroendocrine and immunologic effects of unemployment and job insecurity. Psychotherapy and Psychosomatics.

Ametz, B. B., \& Fjellner, B. (1986). Psychological predictors of neuroendocrine responses to mental stress. Journal of Psychosomatic Research, 30, 297-305.

Arrindell, W. A., \& Ettema, H. (1981). Dimensionele structuur, betrouwbaarheid en validiteit van de Nederlandse bewerking van de Symptom Checklist (SCL-90); gegevens gebaseerd op een fobische en een 'normale' populatie. Nederlands Tijdschrift voor Psychologie, 36, 77-108.

Arrindell, W. A., \& Ettema, H. (1986). SCL-90: Handleiding bij een muitidimensionele psychopathologie indicator. Lisse: Swets \& Zeitlinger B.V.

Attanasio, V., Andrasik, F., Bilanchard, E. B., \& Arena, J. G. (1984). Psychometric properties of the SUNYA revision of the Psychosomatic Symptom Checklist. Journal of Behavioral Medicine, $7(2), 247-2.58$.

Averill, J. R. (1973). Personal control over aversive stimuli and its relationship to stress. Psychological Bulletin, 80(4), 286-303.

Axelrod, J., \& Reisine, T. D. (1984). Stress hormones: Their interaction and regulation. Science, 224. 452-459.

Bandura, A., Taylor, C. B., Williams, S. L., Mefford, I. N., \& Barchas, J. D. (1985), Catecholamine secretion as a function of perceived coping self-efficacy. Journal of Consulting and Clinical Psychology, 53(3), 406-414.

Barling, J., \& Kryl, I. P. (1990). Moderators of the relationship between daily work stressors and mood. Work \& Stress, 4(4), 319-329.

Bassett, J. R. (1982). Psychological stress and the coronary artery in ischemic heart disease. In S. Kalsner (Eds.), The coronary artery (pp. 474-500), London: Croom Helm. 
Bassett, J. R., \& Cairncross, K. D. (1977). Changes in the coronary vascular system following prolonged exposure to stress. Pharmacology Biochemistry \& Behavior, 6, 311-318.

Bassett, J. R., Marshall, P. M., \& Spillane, R. (1987). The physiological measurement of acute stress (public speaking) in bank employees. International Journal of Psychophysiology, 5(4), $265-273$.

Baum, A., Fleming, R., \& Reddy, D. M. (1986). Unemployment stress: loss of control, reactance and learned helplessness. Social Science and Medicine, 22(5), 509-516.

Baum, A. S., Lundbeg, U., Grunberg, N. E., Singer. J. E., \& Gatchel, R. J. (1985). Urinary catecholamines in behavioral research on stress. In C. R. Lake \& M. G. Ziegler (Eds.), The Catecholamines in Psychiatric and Neurologic Disorders (pp. 55-72). Stoneham: Butterworth Publishers.

Bebbington, P. E., Tennant, C., \& Hurry, J. (1981). Adversity and the nature of psychiatric disorder in the community, Journal of Affective Disorders, 3, 345-366.

Ben-Aryeh, H., Roll, R., Kahana, L., \& al., e. (1985). Saliva as an indicator of stress. International Journal of Psychosomatics, 32, 3-8.

Berger, M., Bossert, S., Krieg. J. C., Dirlich, G., Ettmeier, W., Schreiber, W., \& von Zerssen, D. (1987). Interindividual differences in the susceptibility of the cortisol system: An important factor for the degree of hypercortisolism in stress situations? Biological Psychiatry, 22, 1327. 1339.

Block, J., \& Zautra, A. (1981). Satisfaction and distress in a community: a test of the effect of life events. American Journal of Community Psychology, 9, 165-180.

Bohnen, N., Nicolson, N., Sulon, Jl., \& Jolles, J. (1991). Coping style, trait anxiety and cortisol reacivity during mental stress. Journal of Psychosomatic Research, 35(2/3), 141- 147.

Bolger, N. (1990). Coping as a personality process: A prospective study. Journal of Personality and Social Psychology, 59, 525-537.

Bolger, N., DeLongis, A.. Kessler, R. C., \& Schilling, E. A. (1989a). Effects of daily stress on negative mood. Journal of Personality and Social Psychology, 57(5), 808-818.

Bolger, N., Delongis. A., Kessler, R. C., \& Wethington, E. (1989b). The contagion of stress across multiple roles. Journal of Marriage and the Family, 51, 175-183.

Bolger, N.. \& Schilling, E. A. (1991). Personality and the problems of everyday life: The role of neuroticism in exposure and reactivity to daily stressors. Journal of Personality, 59(3), 355386.

Bossert, S., Berger, M., Krieg, J. C., Schreiber, W., Junker, M., \& von Zerssen, D. (1988). Cortisol response to various stressful situations: Relationship to personality variables and coping styles. Neuropsychobiology, 20, 36-42.

Bourne, P. G., Rose, K. M., \& Mason, J. W. (1967). Urinary 17-OHCS levels. Data on seven helicopter ambulance medics in combat. Archives of General Psychiatry, 17, 104- I 10.

Bower, G. H. (1981). Mood and memory. American Psychologist, 36(2), 129-148.

Brandstatter. H. (1983). Emotional responses to other persons in everyday life situations. Journal of Personality and Social Psychology. 45(4), 871-883.

Brandtstädter, J., Baltes-Gotz, B., Kirschbaum, C.., \& Hellhammer, D. (1991). Developmental and personality correlates of adrenocortical activity as indexed by salivary cortisol: Observations in the age range of 35 to 65 years. Journal of Psychosomatic Research, 35(2/3), 173- 185.

Brantley, P., Dietz, L., McKnight, G. T., Jones, G. N., \& Tulley, R. (1988). Convergence between the Daily Stress Inventory and endocrine measures of stress. Journal of Consulting and Clinical Psychology, 56, 549-551.

Brantley, P. J., Waggoner, C. D., Jones, G. N., \& Rappaport, N. B. (1987). A daily stress inventory: Development, reliability, and validity. Joumal of Behavioral Medicine, 10(1), 61-74.

Brickman, P.. Coates, D., \& Janoff-Bulman, R. (1978). Lottery winners and accident victims: Is happiness relative? Journal of Personality and Social Psychology, 36, 917-927. 
Brindley, D., \& Rolland, Y. (1989). Possible connections between stress, diabetes, obesity, hypertension and altered lipoprotein metabolism that may result in atherosclerosis. Clinical Science, 77, 453-461.

Brown, G. W. (1974). Meaning, measurement, and stress of life events. In B. S. Dohrenwend \& B. P. Dohrenwend (Eds.), Stressful Life Events: Their Nature and Effects (pp. 217-243). New York: Willey \& Sons.

Brown, G. W. (1989). Life events and measurement. In G. W. Brown \& T. O. Harris (Eds.), Life events and illness (pp. 3-45). London: Guilford Press.

Brown, G. W. (1993). Life events and affective disorder: Replications and limitations. Psychosomatic Medicine, 55, 248-259.

Brown, G. W., \& Harris, T. (1978). Social origins of depression: A study of psychiatric disorder in women. London: Tavistock.

Brown, G. W., \& Harris, T. O. (Ed.). (1989). Life Events and lllness. London: Guilford Press.

Brown, W. A., Sirota, A. D., Niaura, R., \& Engebretson, T. O. (1993). Endocrine correlates of sadness and elation. Psychosomatic Medicine, 55, 458-467.

Brugha, T., Bebbington, P., Tennant, C., \& Hurry, J. (1985). The list of threatening experiences: A subset of 12 life event categories with considerable long-term contextual threat. Psychological Medicine, 15, 189-194.

Brugha, T. S., \& Conroy, R. (1985). Categories of depression: Reported life events in a controlled design. British Journal of Psychiatry, 147, 641-646.

Brugha, T. S., \& Cragg, D. (1990). The list of threatening experiences: the reliability and validity of a brief life events questionnaire. Acta Psychiatrica Scandinavica, 82, 77-81.

Bruijn, J. V. d. (1988). Bedrijfsfitness, vorm van secundaire arbeidsvoorwaarde. Arbeidsomstandigheden, 64(7), 474-477.

Bryk, A. S., \& Raudenbush, S. W. (1987). Application of hierarchical linear models to assessing change. Psychological Bulletin, 101(1), 147-158.

Bryk, A. S., \& Raudenbush, S. W. (1992). Hierarchical Linear Models: Applications and Dafa Analysis Methods. London: Sage Publications.

Bullinger, M., Naber, D., Pickar, D., Cohen, R. M., Kalin, N. H., Pert, A., \& Bunney, W. E. (1984). Endocrine effects of the cold pressor test: Relationship to subjective pain appraisal and coping. Psychiatry Research, 12, 227-233.

Calabrese, J. R., Kling, M. A., \& Gold, P. W. (1987). Alterations in immunocompetence during stress, bereavement, and depression: Focus on neuroendocrine regulation. The American Journal of Psychiatry, 144(9), 1123- 1134.

Cameron, P. (1975). Mood as an indicator of happiness: Age, sex, social class, and situational differences. Journal of Gerontology, 30, 216-224.

Campbell, J. D., Chew, B., \& Scratchley, L. S. (1991). Cognitive and emotional reactions to daily events: The effects of self-esteem and self-complexity. Journal of Personality, 59(3), 473-505.

Cannon, W. B. (1929). Organization for physiological homeostasis. Physiological Review, 9. 399.

Cantor, N., Norem, J., Langston, C., Zirkel, S., Fleeson, W., \& Cook-Flannagan, C. (1991). Life tasks and daily life experience. Journal of Personality. 59(3), 425-451.

Caplan, R. D., Cobb, S., \& French, J. R. P., Jr. (1979). White collar work load and cortisol: Disruption of a circadian rhythm by job stress? Journal of Psychosomatic Research, 23 , $181-192$.

Carroll, B. J. (1985). Dexamethasone suppression test: a review of contemporary confusion. Journal of Clinical Psychiatry, 46(2), 13-24.

Caspi, A., Bolger, N., \& Eckenrode, J. (1987). Linking person and context in the daily stress process. Journal of Personality and Social Psychology, 52(1), 184-195. 
Charles, G. A., Schittecatte, M., Rush, A. J., Panzer, M., \& Wilmotte, J. (1989). Persistent cortisol non-suppression after clinical recovery predicts symptomatic relapse in unipolar depression. Journal of Affective Disorder, 17, 271-278.

Checkley, S. (1992). Neuroendocrine mechanisms and the precipitation of depression by life events. British Journal of Psychiatry, 160(suppl. 15), 7-17.

Chen, P. Y.. \& Spector, P. E. (1991). Negative affectivity as the underlying cause of correlations between stressors and strains. Journal of Applied Psychology. 76(3), 398-407.

Chrousos, G. P., \& Gold, P. W. (1992). The concepts of stress and stress system disorders. Overview of physical and behavioral homeostasis. Journal of the American Medical Association, 267(9), 1244-1252.

Claman, H. N. (1977), Corticosteroids and lymphoid cells. New England Journal of Medicine, $287,388-397$.

Clark, L. A., \& Watson, D. (1988). Mood and the mundane: Relations between daily life events and self-reported mood. Journal of Personality and Social Psychology, 54(2), 296-308.

Clark, L. A., Watson, D., \& Mineka, S. (1994). Temperament, personality, and the mood and anxiety disorders. Journal of Abnormal Psychology, 103(1), 103- 116.

Coeck, C., Jorens, P. G., Vandevivere, J., \& Mahler, C. (1991). ACTH and cortisol levels during residency training. The New England Journal of Medicine, 325(10), 738.

Cohen . S. (1978). Environmental load and the allocation of attention. In A. Baum. J. Singer, \& S. Valins (Eds.), Advances in environmental psychology. Hillsdale: Lawrence Erlbaum.

Cohen. S. (1980). Aftereffects of stress on human performance and social behavior: A review of research and theory. Psychological Bulletin, 82-108.

Cohen, S. (1986). Contrasting the Hassles Scale and the Perceived Stress Scale: Who's really measuring appraised stress? American Psychologist, 41, 716-718.

Cohen, S., Kamarck, T., \& Mermelstein, R. (1983). A global measure of perceived stress. Journal of Health and Social Behavior, 24, 385-396.

Cohen, S., Tyrrell, D. A. J., \& Smith, A. P. (1991). Psychological stress and susceptibility to the common cold. The New England Journal of Medicine, 325(9), 606-612.

Cohen, S., \& Williamson, G. M. (1988). Perceived stress in a probability sample of the United States. In S. Spacepan \& S. Oskamp (Eds.). The Social Psychology of Health (pp. 31-67). Newbury Park, CA: Sage Publications.

Collins, A. C.. \& Frankenhaeuser, M. (1978). Stress responses in male and fernale engineering studenis, Journal of Human Stress, 4(2), 43-48.

Cook, N. I. Ng, A., Read, G. F., Harris, B., \& Riad-Fahmy, D. (1987). Salivary cortisol for monitoring adrenal activity during marathon runs. Hormone Research, 25, 18-23.

Costa, P. T., \& McCrae, R. R. (1980). Influence of extraversion and neuroticism on subjective well-being: Happy and unhappy people. Journal of Personality and Social Psychology, 38(4), 668-678.

Costa, P. T. \& McCrae, R. R. (1986). Personality stability and its implications for clinical psychology. Clinical Psychological Review, 6, 407-423.

Costa, P. T., \& McCrae, R. R. (1987). Neuroticism, somatic complaints, and disease: Is the bark worse than the bite? Journal of Personality, 55(2), 299-316.

Cox, D. J., Freundlich, A., \& Meyer, R. G. (1975). Differential effectiveness of electromyograph feedback, verbal relaxation instructions and medication placebo with tension headaches. Journal of Consulting and Clinical Psychology. 43, 892-898.

Cox, D. J., \& Gonder-Frederick, L. A. (1991). The role of stress in diabetes mellitus. In P. McCabe,N. Schneidermann,T. M. Field, \& J. S. Skyler (Eds.), Stress, Coping and Disease (pp. 118-134). New York: L Erlbaum Assoc,

Cox, T., Gotts, G., Boot, N., \& Kerr, J. (1988). Physical exercise, employee fitness and the management of health at work. Ergonomics, 24, 795-806. 
Csikszentmihalyi, M., \& Larson, R. (1984). Being adolescent: Conflict and growth in the teenage years. New York: Basic Books.

Csikszentmihalyi, M., \& Larson, R. (1987). Validity and reliability of the experience-sampling method. The Journal of Nervous and Mental Disease, 175(9), 526-536.

Cummins, S. E., \& Gevirtz, R. N. (1993). The relationship between daily stress and urinary cortisol in a normal population: An emphasis on individual differences. Behavioral Medicine, 19, 129. 134.

Curtis, G. C. (1972). Psychosomatics and chronobiology: Possible implications of neuroendocrine rhythms. Psychosomatic Medicine 34(3), 235-256.

De La Torre, B. (1994). Psychoendocrinologic mechanisms of life stress. Stress Medicine, 10. 107-114.

de Vries, M. W. (1987). Introduction: Investigating mental disorders in their natural settings. Journal of Nervous and Mental Disease, 175(9), 509-513.

de Vries, M. W. (1992). The Experience of Psychopathology: Investigating Mental Disorders in their Natural Settings. Cambridge: University Press.

de Vries, M. W., \& Delespaul, P. A. E. G. (1992). Variability of schizophrenia symptoms. In M. W. de Vries (Eds.). The experience of psychopathology: Investigating mental disorders in their natural settings (pp. 97-109). Cambridge: Cambridge University Press.

Dekaris, D., Sabioncello, A., Mazuran, R., Rabatic, S., Svoboda-Beusan, I., Racunica, N. L., \& Tomasic, J. (1993). Multiple changes of immunologic parameters in prisoners of war. Assessments after release from a camp in Manjaca, Bosnia. Journal of the American Medical Association, 270(5), 595-599.

Delahunt, J. W., \& Mellsop, G. (1987). Hormone changes in stress. Stress Medicine, 3, 123-134.

Delespaul, P. A. E. G. (1992). Technical note: Devices and time-sampling procedures. In M. W. deVries (Eds.), The experience of psychopathology: Investigating mental disorders in their natural settings (pp. 363-376). Cambridge: Cambridge University Press.

Delespaul, P. A. E. G. (1995) Assessing schizophrenia in daily life-The Experience Sampling Method-. Thesis, University of Limburg, Maastricht, The Netherlands.

DeLongis, A., Coyne, J. C., Dakof, G., Folkman, S., \& Lazarus, R. S. (1982). Relationship of daily hassles, uplifts, and major life events to health status. Health Psychology, I(2), 119-136.

DeLongis, A., Folkman, S., \& Lazarus, R. S. (1988). The impact of daily stress on health and mood: Psychological and social resources as mediators. Journal of Personality and Social Psychology, 54(3), 486-495.

Demyttenaere, K., Nij̧s, P., Evers-Kiebooms, G., \& Koninckx, P. R. (1989). The effect of a specific emotional stressor on prolactin, cortisol, and testosterone concentrations in women varies with their trait anxiety. Fertility and Steriliry, 52(6), 942-948.

Depue, R. A., \& Monroe, S. M. (1986). Conceptualization and measurement of human disorder in life stress research: The problem of chronic disturbance. Psychological Bulletin, 99(1), 36-51.

Derogatis, L. R., Lipman, R. S., \& Cori, L. (1973). SCL-90, an outpatient rating scale -preliminary report. Psychopharmacology Bulletin, 9, 13-27.

Devereux, R. B., Pickering, T. G., Harshfield, G. A., (1983). Left ventricular hypertrophy in patients with hypertension: Importance of blood pressure response to regularly occurring stress. Circulation, 68, 470-476.

Dewe, P. J. (1991). Measuring work stressors: The role of frequency, duration and demand. Work \& Stress, 5, 77-91.

Diener, E. (1984). Subjective well-being. Psychological Bulletin, 45, 542-575.

Diener, E., Sandvikt E., \& Larsen, R. (1985). Age and sex effects for affect intensity. Developmental Psychology, 21, 542-546.

Dienstbier, R. A. (1989). Arousal and physiological toughness: Implications for mental and physical health. Psychological Review, 96(1), 84-100. 
Dijkman-Caes, C., \& deVries, M. W. (1991). Daily life situations and anxiety in panic disorder and agoraphobia. Journal of Anxiety Disorders, 5, 343-357.

Dijkman-Caes, C. I. M. (1993) Panic disorder and agoraphobia in daily life. Thesis, University of Limburg, Maastricht, The Netherlands.

Dijkstra, P. (1974). De zelfbeoordelingsschaal voor depressie van Zung. In: H. M. van Praag \& H. G. M. Rooymans (Eds.), Stemming en Ontstemming (pp. 98-120). Amsterdam; De Erven Bohn.

Dimsdale, J. E. (1984). Generalizing from laboratory to field studies of human stress physiology. Psychosomatic Medicine, 46(5), 463-469.

Dinan, T. G. (1994). Glucocorticoids and the genesis of depressive illness: A psychobiological model. British Journal of Psychiarry, 164, 365-371.

Dohrenwend, B. P., \& Shrout, P. E. (1985). "Hassles" in the conceptualization and measurement of life stress variables. American Psychologist, 40, 780-785.

Dohrenwend, B. S., \& Dohrenwend, B. P. (1974). Overview and prospects, for research on stressful life events. In B. S. Dohrenwend \& B. P. Dohrenwend (Eds.), Stressful Life Events: Their Nature and Effects (pp. 313-331). New York: Wiley, J. \& Sons.

Dohrenwend, B. S.. \& Dohrenwend, B. P. (1978). Some issues in research on stressful life events. The Journal of Nervous and Mental Disease, 166(1),7-15.

Dohrenwend, B. S.. Krassnoff, L., Askenasy, A. R., \& Dohrenwend, B. P. (1978). Exemplification of a method for scaling life events: The PERI Life Events Scale. Journal of Health and Social Behavior, 19, 205-229.

Duncan-Jones, P., Fergusson, D., Ormel, J., \& Horwood, L. (1990). A model of stability and change in minor psychiatric symptoms: results from three longitudinal studies. Psychological Medicine, Monograph Supplements, nr. 18. Cambridge:Cambridge University Press.

Eckenrode, J. (1984). Impact of chronic and acute stressors on daily reports of mood. Journal of Personality and Social Psychology, 46(4), 907-918.

Eckenrode, J., \& Bolger, N. (1995). Daily and within-day event measurement.. In S. Cohen, R. C. Kessler, \& L. Underwood Gordon (Eds.). Measuring stress: A guide for health and social scientists (pp. 80-101). New York: Oxford University Press.

Eysenck, H. J., \& Eysenck, S. B. G. (1985). Personality and' individual differences. New York: Plenum:

Eysenck, H. I.. \& Eysenk, S. B. (1968). Eysenck Personality Inventory. San Diego: CA: Educational and Industrial Testing Service.

Eysenck. H. L. (1967). The Biological Basis of Personality. Springfield, lllinois: Charles C Thomas.

Fulkenberg, L. E. (1987). Employee fitness programs: Their impact on the employee and the prganizationt. Academy Management Review, 12, 511-522.

Fergusson, D. M.. \& Horwood, L. J. (1987). Vulnerability to life event exposure. Psychological Medicine, 17, 739-749.

Fibiger, W., \& Singer, G. (1989). Biochemical assessment and differentiation of mental and physical effort. Work \& Stress. 3(3), 237-247.

Fibiger, W., Singer, G., Miller, A. J., Asmstrong. S., \& Datar, M. (1984). Cortisol and catecholamines changes as functions of time-of-day and self-reported mood. Neuroscience \& Biobehavioral Review.8, 523-530.

Fibiger, W. Singer, G. \& Miller, A. J. (1984). Relationships between catecholamines in urine and physical and mental effort. International Journal of Psychophysiology, I, 325-333.

Folkman. S. (1984). Personal control and stress and coping processes: a theoretical. analysis. Journal of Personality and Social Psychology, 46(4), 839-852.

Folkman, S., \& Lazarus, R. S. (1980). An analysis of coping in a middle-aged community sample. Journal of Health and' Social Behavior, 21, 219-239. 
Folkman, S., \& Lazarus, R. S. (1985). If it changes it must be a process: Study of emotion and coping during three stages of a college examination. Journal of Personality and Social Psychology, 48, 150-170.

Follenius, M., Brandenberger, G., Hietter, B., Simeoni, M., \& Reinhardt, B. (1982). Diurnal cortisol peaks and their relationship to meals. Journal of Clinical Endocrinology and Metabolism, 55, 757-761.

Forsman, L. (1980). Habitual catecholamine excretion and its relation to habitual distress. Biological Psychology, 11, 83-97.

Forsman, L. (1982). Consistency in catecholamine excretion in laboratory and natural settings: Correlational and variance components analysis. Scandinavian Journal of Psychology, 23, 99106.

Forsman, L., \& Lundberg, U. (1982). Consistency in catecholamine and cortisol excretion in males and females. Pharmacology Biochemistry \& Behavior, 17, 555-562.

Frankenhaeuser, M. (1975a), Experimental approaches to the study of catecholamines and emotion. In L. Levi (Eds.), Emotions: Their parameters and measurement (pp. 209-234). New York: Raven Press.

Frankenhaeuser, M. (1975b). Sympathetic-adrenomedullary activity, behavior, and the psychosocial environment. In P. H. Venables \& M. J. Christie (Eds.), Research in psychophysiology (pp. 71-94). New York: Wiley.

Frankenhaeuser, M. (1976). The role of peripheral catecholamines in adaptation to understimulation and overstimulation. In G. Serban (Eds.), Psychopathology of haman adaptation (pp. 173-191). New York: Plenum Press.

Frankenhaeuser, M. (1979). Psychoendocrine approaches to the study of emotion as related to stress and coping. In H. E. Howe \& R. A. Dienstbier (Eds.), Nebraska symposium on motivation 1978. Lincoln: University of Nebraska Press.

Frankenhaeuser, M. (1980). Psychoneuroendocrine approaches to the study of stressful personenvironment transactions. Selye's Guide to Stress Research, 1, 46-70.

Frankenhaeuser, M. (1983). The sympathetic-adrenal and pituitary-adrenal response to challenge: Comparison between the sexes. In T. M. Dembroski (Eds.), Behavioral Bases of Coronary Heart Disease (pp. 91-105). Basel: Karger.

Frankenhaeuser, M. (1986). A psychobiological framework for research on human stress and coping. In M. Appley \& R. Trumbull (Eds.), Dynamics of Stress (pp. 101-116). New York: Plenum.

Frankenhaeuser, M., \& Gardeli, B. (1976). Underload and overload in working life: Outline of a multidisciplinary approach. Journal of Human Stress, 2(3), 35-46.

Frankenhaeuser. M., \& Johansson, G. (1976). Task demand as reflected in catecholamine excretion and heart rate. Journal of Human Stress, 2, 15-23.

Frankenhaeuser, M., Lundberg, U., \& Forsman, L. (1980). Dissociation between sympatheticadrenal and pituitary-adrenal responses to an achievement situation characterized by high controllability. Comparison between type $\mathrm{A}$ and type $\mathrm{B}$ males and females. Biological Psychology, 10, 79-91.

Frankenhaeuser, M., Lundberg, U., Fredrikson, M., Melin, B., Tuomisto, M., Myrsten, A., Hedman, M., Bergman-Losman, B., \& Wallin, L. (1989). Stress on and off the job as related to sex and occupational status in white-collar workers. Journal of Organizational Behavior, 10 , 321-346.

Frankenhaeuser, M., Nordheden, B., Myrsten, A. L., \& Post, B. (1971). Psychophysiological reactions to understimulation and overstimulation. Acta Psychologica, 35, 298-308.

Fredrikson, M., Sundin, O., \& Frankenhaeuser, M. (1985). Cortisol excretion during the defense reaction in humans. Psychosomatic Medicine, 47(4), 313-319. 
Friedman, S. B., Mason, J. W., \& Hamburg, D. A. (1963). Urinary 17-hydroxycooid levels in parents of children with neoplastic disease. Psychosomatic Medicine, 25, 364-376

Gaillard, R.-C., \& Al-Damluji, S. (1987). Stress and the pituitary-adrenal axis. In A. Grossman (Eds.), Balliere's Clinical Endocrinology and Metabolism: Neuroendocrinology of Stress (pp. 319-354). London etc.: Balliere Tindall.

Gibbons, R. D., Hedeker, D., ELkin, I., Waternaux, C., Kraemer, H. C., Greenhouse, J. B., Shea, M. T., Imber, S. D., Sotsky, S. M., \& Watkins, J. T. (1993). Some conceptual and statistical issues in analysis of longitudinal psychiatric data. Archives of General Psychiatry, 50, 739-750.

Goetsch, V. L., Wiebe, D. J., Veltum, L. G., \& Van Dorsten, B. (1990). Case history and shorter communication. Behavior Research and Therapy, 28(6), 531 -537.

Gold, P. W., Goodwin, F. K., \& Chrousos, G. P. (1988). Clinical and biochemical manifestations of depression: Relation to the neurobiology of stress. Part II. New England Journal of Medicine, 3I9(7), 413-420.

Gold. W. (1988). Stress-responsive neuromodulators. Biological Psychiatry, 24" 371-374.

Goldberger, L., \& Breznitz, S. (1982). Handbook of stress. Theoretical and clinical aspects. New York: The Free Press.

Goldstein, H. (1987). Multilevel Models in Educational and Social Research. London: Charles Griffin \& Co.

Goplerud, E., \& Depue, R. A. (1985). Behavioral response to naturally occurring stress in cyclothymia and dysthymia. Journal of Abnormal Psychology, 94(2), 128-139.

Goreczny, A. J., Brantley, P. J., Buss, R. R., \& Waters, F. (1988). Daily stress and anxiety and their relation to daily fluctuations of symptoms in asthma and chronic obstructive pulmonary disease (COPD) patients. Journal of Psychopathology and Behavioral Assessment, 10(3), 259. 267.

Gottman, J. M., \& Levenson, R. W. (1988). The social psychophysiology of marriage. In P. Noller \& M. A. Fitzpatrick (Eds.), Perspectives on marital interaction (pp. 182-200). Philadelphia, PA: Multilingual Matters.

Gruchow, H. W. (1979). Catecholamine activity and infectious disease episodes. Journal of Human Siress, 5, 11-17.

Harburg, E., Julius, S., McGinn, N. F., McLeod, J., \& Hoobler, S. W. (1964). Personality traits and behavioral patterns associated with systolic blood pressure levels in college males. Journal of Chronic Diseases, 17, 405-414.

Headey, B., \& Wearing, A. (1989). Personality, life events, and subjective well-being: Toward' a dynamic equilibrium model. Journal of Personality and Social Psychology, 57(4), 731-739.

Hedeker, D. (1993). MIXREG, a Fortran prograrn for mixed-effects linear regression models. University of Illinois: Prevention Research Center.

Hellhammer, D. H., Kirschbaum, C., \& Belkien, L. (1987). Measurement of salivary cortisol under psychological stimulation. In J. Hingtgen, D. Hellhammer, \& G. Huppmann (Eds.), Advanced Methods in Psychobiology (pp. 281-289). Toronto: C. J. Hogrefe Inc.

Hendriks, A. A. J., Ormel, J., \& van de Willige, G. (1990). Langdurige moeilijkheden gemeten volgens zelfbeoordelingsvragenlijst en semi-gestructureerd interview. Gedrag en Gezondheid. 18(6), 273-283.

Hennessy. J. W., \& Levine, S. (1979). Stress, arousal, and the pituitary-adrenal system: A psychoendocrine hypothesis, Progress in Psychobiology and Physiological Psychology, 8. 133-178.

Hennig, J., Laschefski, U., \& Opper, C. (1994). Biopsychological changes after bungee jumping: beta-endorphin immunoreactivity as a mediator of euphoria? Neuropsychobiology, 29(1), 28-32.

Henry, J. P. (1982). The relation of social to biological processes in disease. Social Science and Medicine, 16, 369-380. 
Henry, J. P., \& Stephens, P. M. (1977). Stress, Health and the Social Environment: a Sociobiologic Approach to Medicine. New York: Springer-Verlag.

Hinkle, L. E. (1974). The concept of 'stress' in the biological and social sciences. International Journal of Psychiatry in Medicine, 5, 335-357.

Hofer, M. A., Wolff, C. T., Friedman, S. B., \& Mason, J. W. (1972a). A psychoendocrine study of bereavement PartI. 17-Hydroxycorticosteroid excretion rates of parents following death of their children from leukemia. Psychosomatic Medicine, 34(6), 481-491.

Hofer, M. A., Wolff, C. T., Friedman, S . B., \& Mason, J. W. (1972b). A psychoendocrine study of bereavement. Part II. Observations on the process of mourning in relation to adrenocortical function. Psychological Medicine, 34(6), 492-504.

Holmes, T. H., \& Masuda, M. (1974). Life change and illness susceptibility. In B. S. Dohrenwend \& B. P. Dohrenwend (Eds.), Stressful life events: Their nature and effects (pp. 45-72). New York: John Wiley \& Sons.

Holmes, T. H., \& Rahe, R. H. (1967). The social readjustment rating scale. Journal of Psychosomatic Research, 11, 213-218.

Holroyd, K. A., \& Lazarus, R. S. (1982). Stress coping and somatic adaptation. In L. Goldberger \& S. Breznitz (Eds.), Handbook of Stress: Theoretical and Clinical' aspects (pp. 21-35). New York: Free Press.

Holsboer, F. (1987). Psychoneuroendocrine strategies. Advances in Psychosomatic Medicine, 17. 185-233.

Holsboer, F. (1992). The hypothalamic-pituitary-adrenocortical system. In E. S. Paykel (Eds.), Handbook of affective disorders (pp. 267-287). New York: Churchill Livingstone Inc.

Hoon, E. F., Hoon, P. W., Rand, K. H., Johnson, J., Hall, N. R., \& Edwards, N. B. (1991). A psychobehavioral model of genital herpes recurrence. Journal of Psychosomatic Research, 35, 25-36.

Hormuth, S. E. (1985). Methoden für psychologische Forschung im Fels (Diskussionspapier Nr. 43). Heidelberg: Psychologischen Institut der Universitat Heidelberg.

Hormuth, S. E. (1986). The sampling of experiences. Journal of Personality, 54(1), 262-293.

Hosman, C. M. H. (1983). Psychosociale problematiek en hulpzoeken. Een sociaalepidemiologische studie ten behoeve van de preventieve geestelijke gezondheidszarg. Lisse: Swets \& Zeitlinger.

Houtman, I. L. D., \& Bakker, F. C. (1987). Stress in student teachers during real and simulated, standardized lectures. Journal of Human Stress, 13(4), 180-187.

Hubert, W., \& de Jong-Meyer, R. (1989). Emotional stress and saliva cortisoll response. J Clin Chem Clin Biochem, 27(4), 235-237.

Hubert, W., \& de Jong-Meyer, R. (1990). Psychophysiological response patterns to positive and negative film stimuli. Biological Psychology, 31, 73-93.

Hubert, W., \& de Jong-Meyer, R. (1991). Autonomic, neuroendocrine, and subjective responses to emotion-inducing film stimuli. Intermational Journal of Psychophysiology, 11, 131-140.

Huber, W. H., Moller, M., \& de Jong-Meyer, R. (1993). Film-induced amusement changes in saliva cortisol levels. Psychoneuroendocrinology, 18(4), 265-272.

Hurlburt, R. T. (1990). Sampling normal and schizophrenic inner experience. New York: Plenum Press.

Isenberg. S. A., Lehrer, P. M., \& Hochron, S. (1992). The effects of suggestion and emotional arousal on pulmonary function in asthma: a review and a hypothesis regarding vagal mediation. Psychosomatic Medicine, 54, 192-216.

Ivancevich, J. M. (1986). Life events and hassles as predictors of health symptoms, job performance, and absenteeism. Journal of Occupationai Behaviour, 7, 39-51.

Jaccard, J., \& Wan, C. K. (1993). Statistical analysis of temporal data with many observations: issues for behavioral medicine data. Annals of Behavioral Medicine, 15(1), 43-49. 
Jacobs, S. C., Friedman, R., \& Mittleman, M. (1992). 9-Fold increased risk of myocardial. infarction following psychological stress as assessed by a case-control study. Circulation, 86 (suppl), 198. Abstract.

Jacobson, L., \& Sapolsky, R. (1991). The role of the hippocampus in feedback regulation of the hypothalamic-pituitary-adrenocortical axis. Endocrine Review, 12, 118-134.

Jandorf, L., Deblinger, E., Neale, J. M.. \& Stone, A. A. (1986). Daily versus major life events as predictors of symptom frequency: A replication study. Journal of General Psychology, II(3), 205-218.

Johansson, G., Frankenhacuser, M., \& Magnusson, D. (1973). Catecholamine output in school children as related to performance and adjustment. Scandinavian Journal of Psychology, 14. 20-28.

Jones, K. V.. Copolev, D. L., \& Outsch, K. H. (1986). Type A, test performance and salivary cortisol. Journal of Psychosomatic Research, 30, 699-707.

Kahn, J.-P., Rubinow, D. R., Davis, C. L., \& al., e. (1988). Salivary cortisol: A practical methodi for evaluation of adrenal function. Biological Psychiatry, 23, 335-349.

Kanner, A. D., Coyne, J. C. Schacfer, C., \& Lazarus, R. S. (1981). Comparison of two modes of stress measurement: Daily hassles and uplifts versus. major life events. Journal of Behavioral Medicine, 4, 1-39.

Kapian, C. D. (1992). Drug craving and drug use in the daily life of heroin addicts, In M. W. deVites (Eds.). The experience of psychopathology: Investigating mental disorders in their natural settings (pp. 193-218). Cambridge: Cambridge Press.

Kaplan, H. D. (1979). Social psychology of disease. In H. G. Freeman,S. Levine, \& L. F. Reeder (Eds.), Handbook of Medical Sociology (pp. 53-70). Englewood Cliffs, N.J: PrenticeHall.

Kathol, R. G., Noyes, R., \& Lopez, A. (1988). Similarities in hypothalamic-pituitary-adrenal axis. acivity between patients with panic disorder and those experiencing external stress. Psychiatric Clinics of North America, II, 335-348.

Katz, R., \& Wykes, T. (1985). The psychological difference between temporally predictable and unpredictable stressiul events: evidence for information control theories. Journal of Personality and Social Psychology: 48(3), 781-790.

Kiecoll-Glaser, J. K. \& Glaser, R. (1991). Stress and immune function in humans. In R. Ader, D. L. Felten, \& N Cohen (Eds.), Psychoneuroimmunology (pp. 849-867), San Diego, California: Academic Press, Inc.

Kirschbaum, C., Bartussek, D., \& Strasburger, C. J. (1992a). Cortisol responses to psychologicar stress and correlations, with personality traits. Personality and Individual Differences, 13(12), 1353-1357.

Kirschbaum, C., \& Hellhammer. D. H. (1989). Salivary cortisol in psychobiological research: An overview. Neuropsychobiology, 22, 150-169.

Kirschbaum, C., \& Hellhammer, D. H. (1990). Methodological aspects of salivary cortisol measurement and its application in stress research (abstract). Journal of Clinical Chemistry and Clinical Biochemistry, 28, 649-066.

Kirschbaum, C., \& Helihammer, D. H. (1993). The 'Trier Social Stress Test' - a tool for investigating psychobiological stress responses in a laboratory setting. Neuropsychobiology, 28, 76-81:

Kirschbaum, C., \& Hellhammer, D. H. (1994). Salivary cortisol in psychoneuroendocrine research: Recent developments and applications. Psychoneuroendocrinology, 19(4), 313-333

Kirschbaum, C., Hellhammer, D. H., Strasburger. C. J., Tiling, E., Kamp, R., \& Luđdecke, H. (1989). Relationships between salivary cortisol, electrodermal activity and anxiety under mild experimental stress in children. In H. Weiner,I. Forin, R. Murison, \& D. Hellhammer (Eds.), Frontiers in Stress Research (pp. 383-387). Toronto: Hans Huber. 
Kirschbaum, C., Pruessner, J., Stone, A. A., Federenko, I., Gaab, J., Lintz, D;, Schommer, N, \& Hellhammer, D. (1995). Persistent high cortisol responses to repeated psychological stress in a subpopulation of healthy men. Psychosomatic Medicine, 57, 468-474.

Kirschbaum, C., Wust, S., Faig, H. G., \& Hellhammer, D. H. (1992b). Heritability of cortisol responses to human corticotrophin-releasing hormone, ergometry, and psychological stress in humans. Journal of Clinical Endocrinology and Metabolism. 75(6), 1520-1530.

Kirschbaum, C., Wust, S., \& Hellhammer, D. (1992c). Consistent sex differences in cortisol responses to psychological stress. Psychosomatic Medicine, 54, 648-657.

Kissinger, P. T., Riggin, R. M., Alcom, R. L., \& Rau, L.-D. (1975), Estimation of catecholamines in urine by high performance liquid chromatography with electrochemical detection. Biochemical Medicine, 13, 299-306.

Klinger, E., Barta, S., \& Maxeimer, M. (1980). Motivational correlates of thought content, frequency and commitment. Journal of Personality and Social Psychology, 39, 1222- 1237.

Kloet, E. R. d., \& Reul, J. M. H. M. (1987). Feedback action and tonic influence of corticosteroids on brain function: a concept arising from the heterogeneity of brain receptor systems. Psychoneuroendocrinology, 12, 85- 105.

Knepper, S. (1991). De epidemiologie van psychische arbeidsongeschiktheid. feiten en achtergronden. In R. Bijl \& D. Bauduin (Eds.), Categorie V. Arbeidsongeschiktheid wegens psychische stoornissen (pp. 7-26). Utrecht: NcGv.

Knight, R. B., Atkins, A., Eagle, C. J., Evans, N., Finkelistein, J. W., Fukushima, D., Katz, J., \& Weiner, H. (1979). Psychological stress, ego defenses, and cortisol production in children hospitalized for elective surgery. Psychosomatic Medicine, 41(1), 40-49.

Koeter, M. W. J., Ormel, J., \& van den Brink, W. (1988). Totaalscore op de SCL-90 als maal voor de ernst van psychopathologie. Nederlands Tijdschrift! voor de Psychologie. 43, 381-391.

Kraan, H., Meertens, H., Hilwig, M., Volovics, L., Dijkman-Caes, C., \& Portegijs, P. (1992). Selecting measures, diagnostic validity and scaling in the study of depression. in M. W. deVries (Eds.), The experience of psychopathology: Investigating mental disorders in their naturat settings (pp. 324-336). Cambridge: Cambridge Press.

Kugler, J., \& Kalveram, K. T. (1989), Is salivary cortisol related to mood states and psychosomatic symptoms? In H. Weiner (Eds.), Frontiers of Stress Research (pp. 389-391). New York Toronto: Hans Huber Publishers.

Laan, M. (1989, 20.04.89). De lange weg naar beter werk (3). Gezondmaking van bedrijven maakt stress tot volksziekte. Het Parool.

Larsen, R. J., Diener, E., \&. Emmons, R. A. (1986). Affect intensity and reactions to daily life events. Journal of Personality and Social Psychology, 5i, 803-814,

Larson, R., \& Delespaul, P. (1992). Analyzing experience sampling data: A guidebook for the perplexed. In M. W. de Vries (Eds.), The experience of psychopathology: Investigating mental disorders in their natural settings (pp. 58-78). Cambridge: Cambridge University Press.

Larson, R. S., \& Csikszentmihalyi, M. (1983). The experience sampling method. In H. R. Reis (Eds.), New directions for methodology of social and behavioral science: naturalistic approaches to studying social interaction. (pp. 41-46). San Francisco: Jossey-Bass.

Laundis, J. R., \& Koch, G. G. (1977). The measurement of observer agreement for categorical data. Biometrics, 33, 396-398.

Lazarus, R. S. (1966). Psychological stress and the coping process. New York: McGraw-Hill.

Lazarus, R. S. (1984). Puzzles in the study of daily hassies. Journal of Behavioral Medicine, 7(4), 375-389.

Lazarus, R. S., De Longis, A., Folkman, S., \&. Gruen, R. (1985). Stress and adaptational outcomes: The problem of confounded. measures. American Psychologist, 40, 770-779.

Lazarus, R. S., \& Folkman, S. (1984a). Coping and adaptation. In. W. D. Gentry (Eds.), Handbook of Behavioral Medicine (pp. 282-325). New York: Guilford Press. 
Lazarus, R. S., \& Folkman, S. (1984b). Stress, appraisal, and coping. New York: Springer.

Lazarus, R. S., \& Folkman, S. (1986). Cognitive theories of stress and the issue of circularity. In M. H. Appley \& R. Trumbull (Eds.), Dynamics of Stress. (pp. 63-80). New York: Plenum.

Lazarus, R. S., \& Launier, R. (1978). Stress-related transactions between person and environment. In L. A. Pervin \& M. Lewis (Eds.), Perspectives in Interactional Psychology (pp. 287-327). New York: Plenum.

Lewinsohn, P. M., \& Amenson, C. S. (1978). Some relations between pleasant and unpleasant mood-related events and depression. Journal of Abnormal Psychology, 87(6), 644-654.

Lewinsohn, P. M., \& Libet, J. (1972). Pleasant events, activity schedules, and depressions. Journal of Abnormal Psychology, 79(3), 291-295.

Lewinsohn, P. M., \& Talkington, J. (1979). Studies on the measurement of unpleasant events and relations with depression. Applied Psychological Measurement, 3, 83-101.

Linkowski, P., Mendlewicz, J., Leclereq, R., Brasseur, M., Hubain, P., Golstein, J., Copinschi, G., \& van Cauter, E. (1985). The 24-hour profile of adrenocorticotropin and cortisol in major depressive illness. Journal of Clinical Endocrinology and Metabolism, 61(3), 429-438.

Lousberg, R., Schmidt, A. J. M., Groenman, N. H., Vendrig, L., \&. Dijkman-Caes, C. (1995). Validating the MPI-DLV with Experience Sampling data. In H. B. R. Lousberg (Eds.), Chronic pain: Multiaxial assessment and behavioral mechanisms. Thesis, University of Limburg, Maastricht, The Netherlands.

Lundberg. U., \& Frankenhaeuser, M. (1980). Pituitary-adrenal and sympathetic-adrenal correlates of distress and effort. Journal of Psychosomatic Research, 24, 125-130.

Lundberg. U., Granqvist, M., Hansson, T., Magnusson, M., \& Wallin. L. (1989). Psychological and physiological stress responses during repetitive work at an assembly line. Work \& Stress, 3(2). 143-153.

Lundberg. U., \& Palm, K. (1989). Workload and catecholamine excretion in parents of preschool children. Work \& Stress, 3(3), 255-260.

Luteijn, F., \& Kok, A. R. (1985). Handleiding NVM. Herziene uitgave. Lisse: Swets \& Zeitlinger.

Lykken, D. T. (1982). Research with twins: the concept of emergenesis. Psychophysiology, 1.1. 249-270.

Madden, K. S., \& Livnat, S. (1991). Catecholamine action and immunologic reactivity. In R. Ader,D. L. Felten, \& N. Cohen (Eds.), Psychoneuroimmunology (pp. 283-310). San Diego. California: Academic Press, Inc.

Manuck, S. B., Kasprowicz, A. L., Monroe, S. M., Larkin, K.. T., \& Kaplan, J. R. (1989). Psychophysiologic reactivity as a dimension of individual differences. In $\mathbf{N}$. Schneiderman, S. M. Weiss, \& P. G. Kaufman (Eds.), Handbook of research methods in cardiovascular behavioral medicine (pp. 365-382), New York: Plenum.

Marcelissen (1989). Onderzoek naar stress in de werksituatie. In Verslag van het symposium 'Stress in de werksituatie: Beleid en preventie.14 december 1988, Den Haag (pp. 43-56). Leiden: NIPG/TNO.

Marco, C. A., \& Suis, J. (1993), Daily stress and the trajectory of mood: Spillover, response assimilation, contrast, and chronic negative affectivity. Journal of Personality and Social Psychology, 64(6), 1053-1063.

Martin, M., Ward, J. C., \& Clark. D. M. (1983). Neuroticism and the recal! of positive and negative personality information. Behaviour Research and Therapy, 21, 495-503.

Mason, D. (1991). Genetic variation in the stress response: susceptibility to experimental allergic encephalomyelitis and implications for human inflammatory disease. Immunology Todiay, 12, 57-60.

Mason. J. W. (1968). A review of psychoendocrine research on the pituitary-adrenal cortical system. Psychosomatic Medicine, 30, 576-607.

Mason, J. W. (1975). A historical view of the stress field. Journal of Human Stress, I(1), 7-12. 
Mason, J. W., Giller, E. L., Kosten, T. R., \& Harkness, L. (1988). Elevation of urinary norepinephrine/cortisol ratio in post-traumatic stress disorder. Journal of Nervous and Mental Disease, I76(8), 498-502.

Mason, J. W., Hartley, H., Kotchen, T. A., Mougey, E. H., Ricketts, P. T., \& Jones, L. G. (1973). Plasma cortisol and norepinephrine responses in anticipation of muscular exercise. Psychosomatic Medicine, 35(5), 406-414.

Massimini, F., Csiksentmihalyi, M., \& Carli, M. (1987). The monitoring of optimal experience. A tool for psychiatric rehabilitation. Journal of Nervous and Mental Disease, 175(9), 545-549.

McCrae, R. R. (1990). Controlling neuroticism in the measurement of stress. Stress Medicine, 6 , 237-241.

McCrae, R. R., \& Costa, P. T. (1986). Personality, coping, and coping effectiveness in an adult sample. Journal of Personality, 54(2), 385-405.

McEwen, B. S., \& Stellar, E. (1993). Stress and the individual. Mechanisms leading to disease. Archives of International Medicine, 153, 2093-2101.

McGrath, J. E., \& Beehr, T. A. (1990). Time and the stress process: Some temporal issues in the conceptualization and measurement of stress. Stress Medicine, 6, 93-104.

Meyerhof, J. L., Oleshansky, M. A., \& Mougey, E. H. (1988). Psychological stress increases plasma levels of prolactin, cortisol, and POMC-derived peptides in man. Psychosomatic Medicine, 50, 295-303.

Miller, S. M. (1979). Controllability and human stress: Method, evidence and theory. Behavior Research and Therapy, 17, 287-305.

Munck, A., Guyre, P. M., \& Holbrook, N. J. (1984). Physiological functions of glucocorticoids in stress and their relation to pharmacological actions. Endocrine Reviews, 5(1), 25-44.

Neale, J. M., Hooley, J. M., Jandorf, L., \& Stone, A. A. (1987). Daily life events and mood. In C. R. Snyder \& C. E. Ford (Eds.), Coping with Negative Life Events: Clinical and Social Psychological Perspectives (pp. 161-189). New York: Plenum.

Nicolson, N. A. (1992). Stress, coping and cortisol dynamics in daily life. In M. W. de Vries (Eds.), The Experience of Psychopathology: Investigating Mental Disorders in their Natural Settings (pp. 219-232). Cambridge: Cambridge University Press.

Nicolson, N. A., van Poll, R., \& deVries, M. (1992). Ambulatory monitoring of salivary cortisol and stress in daily life. In C. Kirschbaum,G. F. Read, \& D. Hellhammer (Eds.), Assessment of Hormones and Drugs in Saliva in Biobehavioral Research (pp. 163-173). Seattle: Hogrefe \& Huber.

Ockenfels, M. C., Porter, L., Smyth, J., Kirschbaum. C., Hellhammer, D. H., \& Stone, A. A. (1995). Effect of chronic stress associated with unemployment on salivary cortisol: Overall cortisol levels, diurnal rhythm, and acute stress reactivity. Psychosomatic Medicine, 57, 460-467.

Ormel, J. (1983). Neuroticism and well-being inventories: Measuring traits or states. Psychological Medicine, 13, 165-176.

Ormel, J. (1985). Her Groningse eerstelijnsproject: Opzet en aanpak. (Intern Rapport), Rijksuniversiteit Groningen, Afdeling Sociale Psychiatrie.

Ormel J. (1995). Etiologie van angst en depressie: kwetsbaarheid en losmakende factoren. In E. R. de Kloet \& V. M. Wiegant (Eds.), Stress en neuropathologie. Leiden: Boerhaave Commissie voor Postacademisch Onderwijs in de Geneeskunde, Rijksuniversiteit Leiden.

Ormel, J., \& Wohlfarth, T. (1991). How neuroticism, long-term difficulties, and life situation. change influence psychological distress. Journal of Personality and Social Psychology, 60(5), 744-755.

Ottenweller, J. E., Natelson, B . H., Pitman, D. L., \& Drastal, S. D . (1989) . Adrenocortical and behavioral responses to repeated stressors: toward an animal model of chronic stress and stressrelated mental illness. Biological Psychiatry, 26(8), 829-41. 
Paykel, E. S. (1974). Life stress and psychiatric disorder: Application of the clinical approach. In B. S. Dohrenwend \& B. P. Dohrenwend (Eds.), Stressful life events: Their narure and effects. (pp. 135-149), New York: John Wiley \& Sons.

Paykel, E. S., \& Dowlatshahi, D. (1988). Life events and mental disorder. In S. Fisher \& J. Reason (Eds.), Handbook of life stress, cognition and health (pp. 241-263). New York: Wiley.

Peeters, M. C. W., Buunk, B. P.. \& Schaufeli, W. B. (1995). A micro-analytic exploration of the cognitive appraisal of daily stressful events at work: the role of controllability. Anxiery, Stress, and Coping, 8, 127-139.

Pennebaker, J. W., \& Watson, D. (1988). Self-reports and physiological measures in the workplace. In J. J. Hurrell, L. R. Murphy, S. L. Sauter, \& C. L. Cooper (Eds.), Occupational Stress: Issues and Developments in Research (pp. 184-199). New York: Taylor \& Francis.

Perloff, D., Sokolow, M., \& Cowan, R. (1983). The prognostic value of ambulatory blood pressures. Journal of the American Medical Association, 249, 2793-2798.

Pfohl, B., Herman, B., \& Schlechte, J. (1985). Pituitary-adrenal axis rhythm disturbances in psychiatric depression. Archives of General Psychiatry, 42, 897-903.

Pincomb, G. A., Lovallo, W. R., Passey, W. R., Brackett, R. B., \& Wilson, M. F. (1987). Caffeine enhances the physiological' response to occupational stress in medical students. Health Psychology, 6, 101-112.

Pitman, R. K., \& Orr, S. P. (1990). Twenty-four hour urinary cortisol and catecholamine excretion in combat-related posttraumatic stress-disorder. Biological Psychiatry, 27, 245-247.

Pope, M. K., \& Smith, T. W. (1991). Cortisol excretion in high and low cynically hostile men. Psychosomatic Medicine, 53, 386-392.

Post, R. M. (1992). Transduction of psychosocial stress into the neurobiology of recurrent affective disorder. American Journal of Psychiatry, 149(8). 999-1011.

Prosser, R., Rasbach, J., \& Goldstein, H. (1991). ML3 Software for Three-level Analyses. Users" Guide for V.2. In London: Institute of Education, University of London.

Quigley, M. E., \& Yen, S. S. C. (1979). A. midday surge in cortisol levels. Journal of Clinical Endocrinology and Metabolism. 49, 945-947.

Rabkin, J. C., \& Streuning. E. L. (1976). Life events, stress, and illness. Science, 194, 1013-1020.

Rahe, R. H., \& Arthur, R. J. (1978). Life change and illness studies: past history and future directions. Journal of Human Stress, 4(2), 3-15.

Rahe, R. H., Karson, S., Howard, N. J., Rubin, R. T., \& Poland, R. E. (1990), Psychological and physiological assessments on American hostages freed from captivity in Iran. Psychosomatic Medicine, 52(1), 1-16.

Rahe, R. H., \& Lind, E. (1971). Psychosocial factors and sudden cardiac death: a pilot study. Journal of Psychosomatic Research, 6, 15-24.

Rehm, L. (1978). Mood, pleasant events, and unpleasant events: Two pilot studies. Journal of Consulting and Clinical Psychology, 46, 854-859.

Repetti, R. L. (1993). Short-term effects of occupational stressors on daily mood and health complaints. Health Psychology, 12(2), 125-131.

Rissler, A. (1977). Stress reactions at work and after work during a period of quantitative overload. Ergonomics, 20, 13-16.

Rose, R. M. (1984). Overview of endocrinology of stress. In G. M. Brown,S. H. Koslow, \& S. Reichlin (Eds.), Neuroendocrinology and Psychiatric Disorder (pp. 95-122), New York: Raven Press.

Rose, R. M., \& Fogg. L. F. (1993). Definition of a responder: analyses of behavioral, cardiovascular, and endocrine responses, to varied workload in air traffic controllers. Psychosomatic Medicine, 55, 325-338.

Rose, R. M., \& Hurst, M. W. (1975). Plasma cortisol and growth hormone responses to intravenous catheterization. Journal of Human Stress, $I(1), 22-36$. 
Rose, R. M., Jenkins, C. D., Hurst, M., Kreger, B. E., Barrett, J., \& Hall, R. P. (1982). Endocrine activity in air traffic controllers at work.III. Relationship to physical and psychiatric morbidity. Psychoneuroendocrinology, 7(2), 125-134.

Sapolsky, R., Krey, L., \& McEwen, B. S. (1986). Neuroendocrinology of stress and aging: the glucocorticoid cascade hypothesis. Endocrine Review, 7, 284-301.

Sarason, 1. G.. Johnson, J. H., \& Siegel, J. M. (1978). Assessing the impact of life changes: Development of the Life Experiences Survey. Journal of Consulting and Clinical Psychology, 5. 932-946.

Scarr, S., Webber, P. L., Weinberg, R. A., \& Wittig, M. A. (1981). Personality resemblance among adolescents and their parents in biologically related and adoptive families. Journal of Personality and Social Psychology, 40, 885-898.

Schaeffer, M. A., \& Baum. A. (1984). Adrenal cortical response to stress at Three Mile Island. The Journal of Nervous and Mental Disease, 46(3), 227-237.

Schaubroeck, J., Ganster, D. C., \& Fox, M. L. (1992). Dispositional affect and work-related stress. Journal of Applied Psychology, 77(3), 322-335.

Schedlowski, M., Jacobs, R., Stratmann, G., Richter, S., Hadicke, A., Tewes, U., Wagner, T. O., \& Schmidt, A. U. (1993). Changes of natural killer cells during acute psychological stress. Journal of Clinical Immunology, 13(2), 119-126.

Schreurs, P. J. G., \& van de Willige, G. (1988). De Utrechtse Copinglijst (UCL). Lisse, the Netherlands: Swets \& Zeitlinger.

Schroeder, D. H., \& Costa, P. T. (1984). Influence of life event stress on physical illness: Substansive effects or methodological flaws. Journal of Personality and Social Psychology, 46. 853-863.

Schroer, C. A. P., Nijhuis, F. J. N., \& van Zutphen, W. (1988). Gebruik en betekenis van de term overspanning in geneeskundige literatuur. Tijdschrift voor Sociale Gezondheidszorg, 60 , 163-167.

Schroer, K. (1993) Verzuim wegens overspanning. Een onderzoek naar de aard van overspanning, de hulpverlening en het verzuimverloop. Thesis, University of Limburg. The Netherlands.

Seligman, M. E. P. (1975). Helplessness: On depression, development and death. San Francisco: Freeman, W.H.

Selye, H. (1936). A syndrome produced by diverse noxious agents. Nature, 138, 32-36.

Selye, H. (1976). The Stress of Life. (revised edition). New York: McGraw Hill.

Shields, J. (1962). Monozygotic twins. Oxford: Oxford University Press.

Siegrist, J., Klein, D., \& Matschinger, H. (1989). Occupational stress, coronary risk factors, and cardiovascular responsiveness. In I. Weiner,R. Florin,Murison, \& D. Hellhammer (Eds.). Frontiers of stress research (pp. 323-335). Toronto: Huber.

Smith, P. L. (1979). Splines as a useful and convenient statistical tool. The American Statistician, 33(2), 57-62.

Solomon, S., Holmes, D. S., \& McCaul, K. D. (1980). Behavioral control over aversive events: Does control that requires effort reduce anxiety and physiological arousal? Journal of Personality and Social Psychology, 39(4), 729-736.

Steiner, H., \& Levine, S. (1988). Acute stress response in anorexia nervosa. A pilot study. Child Psychiatry and Human Development, 18(4), 208-218.

Sternberg, E. M., Hill, J. M., Chrousos, G. P., \& al., e. (1989). Inflammatory mediator-induced hypothalamic-pituitary-adrenal axis activation is defective in streptococcal cell wall arthritissusceptible Lewis rats. Proc Natl Acad Sci USA, 86, 2374-2378.

Stone, A. A. (1987). Event content in a daily survey is differentially associated with concurrent mood. Journal of Personality and Social Psychology, 52(1), 56-58. 
Stone, A. A., Cox, D. S., Valdimarsdottir, H., Jandorf, L., \& Neale, J. M. (1987). Evidence that secretory IgA antibody is associated with daily mood. Journal of Personality and Social Psychology, 52(5), 988-993.

Stone, A. A., Kessler, R. C., \& Haythornthwaite, J. A. (1991). Measuring daily events and experiences: Decisions for the researcher. Journal of Personality, 59(3), 575-607.

Stone, A. A., \& Neale, J. M. (1982). Development of a methodology for assessing daily experiences. In A. Baum \& J. E. Singer (Eds.), Advances in environmental psychology (pp. 49-83). Hillsdale, New Jersey: Lawrence Erlbaum Associates, Publishers.

Stone, A. A., \& Neale, J. M. (1984). Effects of severe daily eventsi on mood. Journal of Personality and Social Psychology, 46(1), 137-144.

Stone, A. A., Neale, J. M., \& Shiffman, S. (1993). Daily assessments of stress and coping and their association with mood. Annals of Behavioral Medicine, 15(1), 8-16.

Stone, A. A., \& Shiffman, S. (1992). Reflections on the intensive measurement of stress, coping, and mood, with emphasis on daily measures. Psychology and Health, 7, 115-129.

Strongman, K., \& Russell, P. (1986). Salience of emotion in recall. Bulletin of the Psychonomic Society, 24, 25-27.

Strube, M. J. (1989). Assessing subjects' construal of the laboratory situation. Ini N. Schneidermann,S. M. Weiss, \& P. G. Kaufmann (Eds.), Handbook of Research Methods in cardiovascular Behavioral Medicine (pp. 527-542). New York / London:" Plenum Press.

Surwit, R. S., Williams, R. B., \& Shapiro, D. (1982), Behavioural approaches to cardiovascular disease. New York: Academic Press.

Szalai, A. (Ed.). (1972). The use of time: Daily activities of urban and suburban populations in rwelve countries. Den Haag: Mouton.

Teasdale, J., \& Forgariy, S. (1979). Differential effects of induced mood on retrieval of pleasant and unpleasant events from episodic memory. Journal of Abnormal Psychology, 88, 248-257.

Tellegen, A. (1985). Structures of mood and personality and their relevance to assessing anxiety; with an emphasis on self-report. In A. H. Tuma \& J. D. Maser (Eds.), Anxiety and the anxiety disorders (pp. 681-706). Hillsdale, NJ: Erlbaum.

Theorell, T. (1989). Spontaneously occurring stressors. In H. e. a. Weiner (Eds.). Frontiers of Stress Research (pp. 111-121). New York, Toronto: Huber, H.

Thompson, S. C. (1981). Will it hurt less if I can control it? A complex answer to a simple question. Psychological Bulletin, 90(1),89-101.

Timio, M., \& Gentili, S. (1976). Adrenosympathetic overactivity under conditions of work stress. British Journal of Preventive and Social Medicine, 30, 262-265.

Tinsley, H. E. A., \& Weiss, D. J. (1975). Interrater reliability and agreement of subjective judgments. Journal of Counseling Psychology, 22(4), 358-376.

Troxler, R. G., Sprague, E. A., Albanese, R. A., Fuchs, R., \& Thompson, A. J. \{1977). The association of elevated plasma cortisol and early atherosclerosis as demonstrated by coronary angiography. Atherosclerosis, 26, 151-162.

Tsigos, C., \& Chrousos, G. P. (1994). Physiology of the hypothalamic-pituitary-adrenal axis, in heaith and dysregulation in psychiatric and autoimmune disorders. Endocrinology and Metabolism Clinics of North America, 23(3), 451-466.

Tumer, J. R., Ward, M. M., Gellman, M. D., Johnston, D. W., Light, K. C., \&: van Doomen, L. J. P. (1994). The relationship between laboratory and ambulatory cardiovascular activity: Current evidence and future directions. Annals of Behavioral Medicine, 16(1), 12-23.

Uhde, T. W., Joffe. R. T., Jimerson, D. C., \& Post. R. M. (1988). Normal urinary free cortisol and plasma MHPG in panic disorder: Clinical and theoretical implications. Biological Psychiatry, 23. 575-585.

Ursin, H. (1978). Activation, coping, and psychosomatics. In H. Ursin. E. Baade, \& S. Levine (Eds.), Psychobiology of Stress (pp. 201-228). New York: Academic Press. 
Urșin, H. (1980). Personality, activation and somatic health. In S. Levine \& H. Ursin (Eds.), Coping and health (pp. 259-279). New York: Plenum Press.

Ursin, H., Mykletun, R., Tonder, O., Vaemes, R., Relling, G., Isaksen, E., \& Murison, R. (1984). Psychological stress factors and concentrations of immunoglobulins and complement components in humans. Scandinavian Journal of Psychology, 25, 340-347.

Vaernes, R., Ursin, H., Darragh, A., \& Lambe, R. (1982). Endocrine response patterns and psychological correlates. Journal of Psychosomatic Research, 26, 123-131.

van der Ploeg, H. M., Defares, P. B., \& Spielberger, C. D. (1980). Handleiding bij de ZelfBeoordelings Vragenlijst - ZBV - een nederlandstalige bewerking van de Spielberger State . Trait Inventory (a manual for the STAI). Lisse: Swets \& Zeitlinger.

van der Ploeg, H. M., Defares, P. B., \& Spielberger, C. D. (1982), Handleiding bij de Zelf-Analyse Vragenlijst - ZAV - een nederlandstalige bewerking van de Spielberger State - Trait Anger Scale. Lisse: Swets \& Zeitlinger.

van Diest, R. (1992). Vital exhaustion or depression: a study of daily mood in exhausted male subjects at risk for myocardial infarction. In M. W. deVries (Eds.), The experience of psychopathology: Investigating mental disorders in their natural settings (pp. 233-239). Cambridge: Cambridge Press.

van Doornen, L. J. P., \& Turner, J. R. (1992). The ecological validity of laboratory testing. In J. R. Turner,A. Sherwood, \& K. C. Light (Eds.), Individual differences in cardiovascular response to stress (pp. 63-83). New York: Plenum.

van Doornen, L. J. P., \& van Blokland, R. W. (1992). The relationship between cardiovascular and catecholamine reactions to laboratory and real-life stress. Psychophysiology, 29(2), 173-181, van Eck, M. M., Berkhof, H., Nicolson, N., \& Sulon, J. (in press). The effects of perceived stress, traits, mood states, and stressful daily events on salivary cortisol. Psychosomatic Medicine.

van Eck, M. M., \& Nicolson, N. A. (1994). Perceived stress and salivary cortisol in daily life. Annals of Behavioral Medicine, 16(3), 221-227.

Vickers, R. R. (1988). Effectiveness of defenses: a significant predictor of cortisol excretion under stress. Journal of Psychosomatic Research, 32, 2! -29.

Vingerhoets, A. J. J. M., \& Menges, L. J. (1989). Psychosocial load and symptoms: An inquiry into their relationship with coping styles. Stress Medicine, 5, 189. 194.

Vining, R. F., McGinley, R. A., \& Symons, R. G. (1983). Hormones in saliva: Mode of entry and consequent implications for clinical interpretation. Clinical Chemistry, 29(i0), 1752-1756.

Wagner, B. M. (i990). Major and daily stress and psychopathology: On the adequacy of the definitions and methods. Stress Medicine, 6, 217-226.

Watson, D. (1988). Intraindividual and interindividual analyses of positive and negative affect: Their relationship to health complaints, perceived stress, and daily activities. Joumal of Personality and Social Psychology, 54(6), 1020- 1030.

Watson, D., \& Clark, L. A. (1984). Negative affectivity: The disposition to experience aversive emotional states. Psychological Bulletin, 96(3), 465-490.

Watson, D., Clark, L. A., \& Harkness, A. R. (1994). Structures of personality and their relevance to psychopathology. Journal of Abnormal Psychology, 103(1), 18-31.

Watson, D., \& Pennebaker, J. W. (1989). Health complaints, stress, and distress: Exploring the central role of negative affectivity. Psychological Review, 96(2), 234-254.

Weiner, H. (1992). Perturbing the Organism: The Biology of Stressful Experience. Chicago: University of Chicago Press.

Wolff, S., \& Goodell, H. (1968). Harold G. Wolffs stress and disease (2 ed.). Springfield (III.): Charles C. Thomas.

Wong, M. M., \& Csikszentmihalyi, M. (1991). Motivation and academic achievement: The effects of personality traits and the quality of experience. Journal of Personality. 59(3), 539-574. 
Wüst, S., Kirschbaum, C., \& Hellhammer, D. (1990). Smoking increases salivary cortisol. In C. Kirschbaum \& D. Hellhammer (Ed.), Proceedings of the 2nd European Symposium on Hormone and Drug Assessment in Saliva. Trier.

Yehuda, R., Resnick, H., Kahana, B., \& Giller, E. L. (1993). Long-lasting hormonal alterations to extreme stress in humans: Normative or maladaptive? Psychosomatic Medicine, 55, 287-297.

Zarski, J. J. (1984). Hassles and health: A replication. Health Psychology, 3(3), 243-251.

Zautra, A. J., \& Guarnaccia, C. A. (1986). Measuring small life events. American Journal of Community Psychology, 14(6), 629-655.

Zautra, A. J., Guarnaccia, C. A., Reich, J. W., \& Dohrenwend, B. P. (1988). The contribution of small events to stress and distress. In L. H. Cohen (Eds.), Life Events and Psychological Functioning. Theoretical and methodological issues (pp. 123-148). Newbury Park, California: Sage Publication.

Zung, W. W. K. (1965). A self-rating depression scale. Archives of General Psychiatry, 12, 63-70. 


\section{Appendices}

Appendix I: Reliability and validity of the Perceived Stress Scale in a sample of Dutch white collar workers.

Appendix II: ESM report.

Appendix III: Interrater agreement on codings for ESM measures. 


\section{Appendix I}

\section{Reliability and validity of the Perceived Stress Scale in a sample of Dutch white collar workers}

\section{INTRODUCTION}

Measures of stress (life events, daily events, or chronic stressful situations), can be broadly divided into subjective and objective stress measures, what points to two conceptually different approaches to the concept of stress. First, the transactional model of psychosocial stress which states that "stress lies not in the environmental input but in the person's appraisal of the relationship between that input and its demands and the person's agendas (e.g. beliefs, commitments, goals) and capabilities to meet, mitigate, or alter these demands in the interest of well-being", p.770 (Folkman \& Lazarus, 1985; Lazarus, De Longis, Folkman, \& Gruen, 1985; Lazarus \& Launier. 1978; Mason, 1975). This view contrast with a second, more objective approach to stress, in which stressors are treated as environmental stimuli, inputs, that are much more independent of the reaction or state of mind of the person (Dohrenwend, Link. Kern, Shrout, \& Markowitz, 1990; Dohrenwend \& Shrout, 1985; Dohrenwend, Dohrenwend, Dodson, \& Shrout, 1984). This approach implies that events are, in and of themselves, the precipitating cause of pathology and illness behavior. From the transactional point of view, the impact of these 'objectively' stressful events is, to a large degree, determined by one's perceptions of their stressfulness.

In research on stress, we would like to be able to identify individuals who are at risk for developing stress-related psychological or somatic problems. We know that only a small percentage of individuals exposed to objective stressful (life) events will fail to cope effectively and thereby develop symptoms. We also know that health complaints (objective and subjective) have multifactorial causes, of which stress is only one. A measure of perceived (appraised) stress would be very useful for screening populations for individuals at risk. Such an instrument should ideally be short; it must also be reliable and have construct validity: that is, it should be able to predict stress symptomatology / pathology, while not simply measuring subjective distress alone.

A subjective stress measure, the Perceived Stress Scale (PSS), was developed by Cohen and colleagues (Cohen, Kamarck, \& Mermelstein, 1983), for the measurement of a global level of perceived stress with the goal of providing additional information about the relation between stress and pathology. The PSS measures cognitions and emotions relating to general stress levels rather than specific events or situations. Items tap the extent to which individuals feel their life to be. unpredictable, uncontrollable and overloaded: important components of the experience of stress (Averill, 1973; Cohen.1978; Lazarus, 1966; Miller, 1979; Seligman, 1975). An example of a PSS item is: 'In the last month, how often have you felt that you were unable to control the important things in your life?' The scale attempts to represent situations where persons perceive that the denands exceed their ability to cope. Because the items are of a general nature they are free of content specific to any subpopulation. Another advantage of the generality of the scale is that it is sensitive to the nonoccurrence of events, to chronic life circumstances, to anticipation of future events and to events occurring in the lives of friends and relatives. In the case of scales measuring event-specific levels of perceived stress, 
respondents are: asked to rate the stressfulness or impact of each experienced event. This means that perceived stress levels will be limited by the specific list of events in the scale. In these scales it is also not possible for a single event to have the impact of three or four less salient ones.

A scale measuring global levels of perceived stress can have various valuable functions (Cohen et al., 1983). At the first place, the PSS can be used when the major aim of the study is the role of appraised stress, as opposed to objective stress. It can, for instance, be used to determine whether appraised stress is an etiologic (or risk) factor in psychological or physical disorders (e.g. when the objective sources of stress are diffuse or difficult to measure). Second, it can be used together with an objective scale to study whether appraised stress mediates the relationship between objective stress and illness. In other words, it can provide information about the processes through which stressful events influence pathology. Similarly, it can be used to look more closely at the process by which various moderators of the objective stressor/pathology relationship operate (e.g. social support, coping, personality). Third, the PSS can be used as an outcome measure, i.e. assessing experienced stress as a function of objective stressful events, coping processes, personality characteristics eIc. A final valuable function of the PSS that will be examined in this article, could be its use as an economical tool for screening purposes. When administered more than

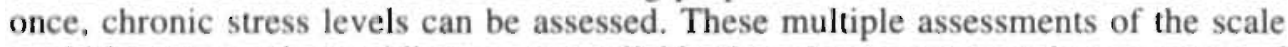
could be averaged, providing a more reliable (based on more samples) measure of chronic stress, and as well a predictor representing a longer time period than the onemonth period covered by the PSS.

Research by Cohen and others showed acceptable levels of validity and reliability of the PSS in samples of the United States. Cronbach's alpha coefficients for the internal reliability of the PSS were high in the various studies (ranging between .75 and .86) (Cohen et al., 1983; Cohen \& Williamson, 1988), and test-retest reliability over a period of two days was also high $(r=85)$. For longer periods (one month, 6 weeks), test-retest correlations were moderate (.50 and .55 respectively) (Cantor, Norem, Langston, Zirkel, Fleeson, \& Cook-Flannagan, 1991; Cohen et al., 1983). Perceived stress seems to be an important mediator of the relationship between stressful life events and symptomatology. Work by Cohen et al. (Cohen. 1986; Cohen et al., 1983; Cohen \& Williamson, 1988) showed for instance that the PSS was a better predictor than life-event scales of psychological symptoms, somatic symptoms and utilization of health services and that stress perception was also related to selfreports of health behaviors. Higher PSS scores were also prospectively associated in a dose-response manner with an increased risk of acute infectious respiratory illness (Cohen. Tyrrell, \& Smith. 19917. The PSS was found to measure a different and independently predictive construct than a depressive symptomatology scale. Cohen (Cohen, 1986) demonstrated that even after controlling for the possible overlap of the PSS and psychopathology, the PSS prospectively predicts psychological symptoms, physical symptoms and health behaviors.

Biy presenting psiychometric and descriptive data on the PSS in a Dutch sample of white collar workers, we want to show that the PSS is a reliable and valid instrument that could be used for screening purposes. The shortness and generality of the scale (containing only 10 items) is a major advantage above the usually long and specific event scales that are used for measuring stress levels, especially in large scale screening where various other instrument are usually included too. As described above, the reliability and validity of the scale has been proven within a culture, i.e. in various samples in the Unites States (students, adult populations, diabetics), but we do 
not have information about the qualities of the scale across cultures. At face value the individual items seem to be aspecific to a particular culture, but this is certainly not enough a base to rely on. Therefore we looked at the reliability and consiruct validity of the scale in a Dutch sample of white collar workers, where the PSS was used as a screening instrument. Besides the internal reliability, we investigated the stability of the PSS over time in a subsample of subjects who filled out the PSS twice. Scores on the scale in the Dutch sample were also compared to US. norms. The construct validity of the PSS was examined through comparison with various indices related to stress: life events, chronic stress, coping style, health behavior, and psychological and somatic complaints. We expected the PSS to be related to the number of life events because perceptions of stress should generally increase with increases of objective stress levels. We expected a higher correlation of the PSS with chronic difficulties though, because in this scale respondents were asked to indicate the intensity of he problems they were facing. The intensity scores should reflect some of the same stress appraisal as measured by the PSS. Perceptions of stress were also expected to be positively related to anxiety, depression, less effective coping styles, and poorer self reported health and health-related behavior. Besides these measures, also cortisol levels were determined in saliva as a physiological measure of subjective stress. Salivary cortisol is a reliable and valid indicator of the free cortisol in plasma, which is considered to be the biologically active hormone. Cortisol concentrations are independent of the flow rate of saliva. Cortisol was measured only once in our respondents, so we did not look for reactive but rather for basal levels of cortisol and the possible influence of (ongoing) perceived stress on that level. Results from studies on cortisol levels in cases of chronic psychosocial load are inconsistent. Sometimes enhanced concentrations have been found (Hofer, Wolff, Friedman, \& Mason, 1972; Jacobs, Mason, Kosten, Kasl, Ostfeld, \& Wahby, 1987, Kiecolt-Glaser, Ricker, George, Messick, Speicher, Gamer, et al., 1984) but decreased levels as well (Caplan, Cobb, \& French, 1979). We also looked at the predictive ability off appraised stress opposed to alternative measures of stress. Parallel to other studies, the PSS was: expected to be the strongest predictor of health variables, because it is presumably the level of appraised stress and not the objective occurrence of an event, that causes one's reaction to a stressor(s).

Subjects were first screened and then a subgroup was selected from various local industries and government agencies according to their perceived stress leve!. Reliability and validity data were collected in these two samples. Although our samples are not representative of the Dutch population in general, we believe that our data should give us a good indication of the usefulness of the Perceived Siress Scale. as a screening instrument in Dutch populations.

\section{METHODS}

\section{Subjects and procedures}

Both the screening sample ( $n=316$ for males, and $n=60$ for females) and the (sub)sample that had been selected for the ESM study ( $n=92$, all males) were used to investigate the reliability and valididty of the PSS. For details about subject recruitment and procedures see: chapter 2 . 


\section{Questionnaires}

\section{Screening sample}

In addition to the PSS, questionnaires concerning coping style (Utrecht Coping List), and psychological and physical symptoms (Symptom Checklist 90; Psychosomatic Symptom Checklist) were completed by the screening sample $(n=376)$. Demographic information was obtained concerning respondents' age, marital status, household composition, completed education, chronic diseases, medications, alcohol use, smoking habits, and participation in active sports. For details about the questionnaires see chapter 2 .

\section{Subsample}

The 92 subjects who participated in the main study completed additional questionnaires concerning psychosocial stress (life events: List of Threatening Experiences; chronic stress: Longterm Difficulties Questionnaire), and psychological symptoms (anxiety: State-Trait Anxiety Inventory; depression: Self-Rating Depression Scale). For details about the questionnaires see chapter 2 .

\section{Cortisol}

When subjects in the screening sample handed in the completed questionnaires, they also provided a single saliva sample for cortisol determination. All samples were taken between 11.00 and 13.00 hours, before lunch. Subjects collected saliva by holding a polyester dental roll in the mouth for 1 to 2 minutes; the roll was then placed in a capped plastic vial (Salivette; Sarstedt). Information about use of coffee and tobacco in the last hour and use of medication in the last 24 hours was simultaneously obtained to control for possible confounding influences.

Uncentrifuged samples were stored at $-20 \mathrm{C}$. Salivary cortisol levels were determined in duplicate by direct radioimmunoassay (Ansseau, Sulon, Doumont, Cerfontaine, Legros, Sodoyez, et al., 1984), using ${ }^{125}$ ]-cortisol (Farmos diagnostica, Finland) and antiserum made against the 3CMO-BSA conjugate by Dr. J. Sulon, University of Liege, Belgium. The lower detection limit of the assay was $12 \mathrm{ng} / 100 \mathrm{ml}$, with a mean intra-assay coefticient of variation of $4.8 \%$ (range: $2.2 \%$ $-7.5 \%$ for 4 assays).

\section{Statistical analysis}

Unless otherwise stated, statistical tests are non-parametric and two-tailed.

\section{RESULTS}

\section{Unldimensionality and reliability of the PSS}

Principal components extraction with varimax rotation was performed on the 10 items of the Perceived Stress Scale. One component was identified which accounted foi $58.7 \%$ of the total variance. All items had positive loadings of .45 or higher. A total scale score was therefore obtained by summation of the 10 item scores.

For the screening sample, Crohnbach's alpha as a measure of the internal reliability of the PSS was .86 , or slightly higher than that of the original US. scale (Cohen \& Williamson, 1988). Item-total correlations fell between .38 and .74. For the 92 subjects who completed the PSS a second time, Crohnbach's alpha for the scale was .85 , with item-total correlations ranging from .29 to .73 .

The PSS was completed for a second time by the subsample of 92 subjects, after an average interval of 116 days (range 13-213 days). We looked at the test-retest reliability of the PSS to examine its stability. PSS scores from the first and second measurements were highly correlated. ( $r h o=.73, p<001$ ), with a mean decrease of 2.0 
(s.d. 4.7, range -9 to +14 points). Given that the PSS is based on reports for the last month, one would expect larger differences in scores as the time interval between test and retest increases. However, linear regression showed no greater change in PSS score with increasing intervals between test and retest $(F(1,90)=.739$; ns).

\section{Cross-cultural generalizability of the PSS: comparison of results with US. normative data}

Table 1 presents the means and standard deviations of scores on the PSS for the various demographic variables represented in our sample. These results can be compared with published norms from a large $(n=2270)$, representative US. sample (Cohen \& Williamson, 1988), to assess the cross-cultural validity of the Dutch version of the PSS.

Table 1. Mean PSS scores and standard deviations for demographic categories.

\begin{tabular}{lrll}
\hline Category & N & PSS mean & SD \\
\hline Sex male & 316 & 12.6 & 5.9 \\
female & 60 & 13.6 & 6.5 \\
Age & & & \\
$20-29$ & 47 & 12.51 & 5.5 \\
$30-39$ & 136 & 12.6 & 6.0 \\
$40-49$ & 128 & 13.2 & 6.1 \\
$50-59$ & 62 & 12.5 & 6.3
\end{tabular}

Household composition

living with parents

single

10.8

12.2

couple/no children

12.6

couple/children

12.9

single parent

23.0

other

$\begin{array}{rr}16 & 10.8 \\ 32 & 12.2 \\ 91 & 12.6 \\ 227 & 12.9 \\ 1 & 23.0 \\ 9 & 13.3\end{array}$

4.8

6.8

5.7

6.1

6.0

Number of people in household

$\begin{array}{lrrr}\text { one } & 33 & 12.1 & 6.7 \\ \text { two } & 98 & 12.5 & 5.7 \\ \text { three } & 77 & 12.2 & 6.0 \\ \text { four or more } & 126 & 14.7 & 7.2 \\ \text { mber of children in household } & & & \\ \text { none } & 189 & 12.3 & 5.7 \\ \leq 2 & 159 & 12.9 & 6.1 \\ >2 & 27 & 14.5 & 6.9\end{array}$

Education

\begin{tabular}{lrrr} 
lower & 7 & 17.7 & 4.2 \\
middle & 132 & 13.1 & 5.7 \\
higher & 232 & 12.5 & 6.2 \\
\hline
\end{tabular}

The mean score for the Dutch sample $(12.7+$ - s.d. 6.0 ; range $2-35)$ is comparable to US. norms (mean $=13.02$, s.d. $=6.45$ ), especially when the selected characteristics of our white collar sample are taken into account. Scores for US. managerial and clerical professions were 12.0 and 13.5, respectively. In agreement 
with US. norms for females and males, women reported somewhat higher mean PSS scores than men, but this difference failed to reach significance in our sample $(F(374)=1.29$, ns; one-way ANOVA). We found no significant association between respondent's age and perceived stress ( $r=-.02$, n.s.), although a negative relationship between these two variables was reported for the US. sample. Respondents still living with their parents had the lowest levels of perceived stress, but one-way ANOVA revealed no significant effect for household composition on level of perceived stress. In the US. sample, PSS was weakly related to household size and the number of children in the household. In our sample a similar pattern was observed for household size $(r h o=10, p<.05)$, but perceived stress level did not increase significantly in households with more children $(<18$ years $)(F(372)=1.86$, ns; one-way ANOVA) probably because of the small number of people with more than 2 children in our sample. As in the US., higher education was associated with lower perceived stress. Probably due to the small number of people with lower education in our sample, however, this effect just failed to reach significance $(F(368)=2.72, p=.06$ : one-way ANOVA.

\section{The construct valldity of the PSS}

\section{Association of PSS with self-reported health and health-related behaviors.}

Fifty-seven respondents (15.2\% of the sample) reported suffering from one or more chronic condition, including high blood pressure $(n=14)$, cardiovascular disease $(n=8)$, respiratory problems $(n=7)$, and diabetes mellitus $(n=1)$. In the open-ended category 'other chronic diceases' $(n=40)$ the most freguently reported conditions were allergies, hay-fever and eczema. As shown in Table 2, subjects with a chronic illness reported no higher perceived stress level than those with no illness.

Table 2. PSS scores in relation to health and health-related behaviors

\begin{tabular}{lcccc}
\hline Measure & & N & PSSinean & SD \\
\hline Do you have a chronic disease? & yes & 57 & 13.3 & 6.3 \\
& no & 319 & 12.6 & 6.0 \\
Do you take medication? & & & & \\
& yes & 53 & $15.0 * *$ & 6.9 \\
Do you exercise regularly? & no & 321 & 12.4 & 5.8 \\
& yes & 248 & 12.5 & 5.8 \\
Do you smoke? & no & 127 & 13.3 & 6.3 \\
& & & & \\
& yes & 104 & 12.1 & 6.0 \\
& no & 270 & 13.0 & 6.0 \\
\hline
\end{tabular}

$* p<0.0$ H, two-tailed; Mann Whitney U-test

Respondents who used medications, however, had significantly higher PSS scores than those who did not. Medications most frequently used were from the categories: circulatory tract $(n=17)$, respiratory tract $(n=12)$, analgesics $(n=10)$, CNS/psychological (e.g. hypnotics, anxiolytics, anti-depressants) $(n=8)$. There was no difference in the PSS scores of respondents who exercised once a week or more 
versus those who reported no regular exercise, and smokers had no higher PSS scores than non-smokers.

Psychosomatic complaints, as measured with the PSC, were examined as another aspect of self-reported health. Frequency, Intensity and Total (frequency $\mathrm{x}$ intensity) scores on the PSC were all positively correlated with perceived stress ( $r h o=.54, .45$, and .59 , respectively; $p<.001$ ). Correlations remained significant even after the more obvious psychological symptoms (fatigue, depression and insomnia) were omitted from the scale ( $r h o=.44, .36$, and .50 , respectively; $p<.001$ ).

\section{Psychological complaints}

Mean scores on the SCL-90 scales $(n=336)$ were within the normal ranges established for the Dutch population (Koeter, OrmeI, \& van den. Brink, 1988). PSS scores were positively correlated with all eight SCL-90 scales: agoraphobia $(r h o=35)$, sleep problems $(r h o=41)$, hostility $(r h o=47)$, sonatic complaints $(r h o=47)$, insufficiency of thought and performance $(r h o=.56)$, distrust and interpersonal sensitivity ( $r h o=.57$ ), anxiety $(r h o=.62)$, depression $(r h o=.66)$, as well as with the total score $(r h o=.68)($ all tests $p<.001)$.

\section{Coping style}

Scores on the Utrecht Coping List scales $(n=215)$, were similar to norms published for the Dutch population (Schreurs \& van de Willige, 1988). The following Spearman correlations between PSS scores and the assessed coping styles were found: 'depressive reactions" ( $r h o=.59, p<.001)$, 'active problem-solving' (rho=-.37. $p<.001$ ), 'palliative' (rho=.22, p<.01), 'expression of emotions' ( $r h o=.13, p<.05$ ), 'avoidance' ( $r h o=11, p<.06$ ), 'seek social support' ( $r h o=.07, \mathrm{~ns}$ ), and 'comforting cognitions' ( $r h o=.06, \mathrm{~ns}$ ).

\section{Cortisol}

We had expected that high perceived stress would be associated with elevated cortisol levels. However, there was no significant correlation between PSS and prelunch salivary cortisol in either males ( $r h o=07$, ns; $n=295$ ) or females ( $r h o=.01$, ns; $\mathrm{n}=58)$. Mean salivary cortisol was $170 \mathrm{ng} / \mathrm{dl}(\mathrm{sd}=80.3)$ for males and $189 \mathrm{ng} / \mathrm{dl}$ for females $(s d=73.9)(p=.05$, Mann-Whitney $U)$.

\section{High PSS vs low PSS comparisons}

The analysis described in the following paragraphs were done in the subsample of our study. In the subsample, high and low perceived stress groups were defined as follows: the mean of the first and second PSS assessments was used to categorize subjects as above or below the screening sample median score (12). For the resulting group of 44 'high stress' subjects, mean PSS score was $18.0(\mathrm{~s} . \mathrm{d}=3.4)$, compared to a mean PSS of $7.3($ s.d. $=2.2)$ in the group of 48 'low stress' subjects.

The construct validity of the PSS was further examined through two indices of distress: anxiety disposition and depressive symptomatology. Perceptions of stress were expected to be positively related to psychological symptoms. Group differences in anxiety (mean: 22.9 high stress vs 18.8 low stress group) and depression (48.4 vs $36.8)$ were both significant $\left(p^{\prime} s<.001\right)$ and in the expected direction. 
Aelationship between PSS and number of life events and chronic difficulties

We found a small but significant correlation between the number of life events and the PSS ( $r h o=.20, p=.03$ ). The difference in mean number of life events between the 'high stress' group (.73 events) and the 'low stress' group. (.46 events) was not significant ( $p=19$; Mann-Whitney U). As expected, the correlation between chronic difficulties and the PSS was markedly higher $(r h o=.56, p<.001)$. There was a significant difference in number of long-term difficulties between the two groups, with respondents in the 'high stress' group experiencing more difficulties than those in the 'low stress' group (23.3 vs $19.5 ; p<, 001)$.

\section{The PSS versus life event and chronic difficulty measures in predicting symptomatology}

Following the analysis presented by Cohen et al. (Cohen et al., 1983), we looked at the PSS compared to the number of life events as predictors of psychosomatic and depressive symptoms. Perceived stress was expected to be a stronger predictor of symptomatology than the number of life events (Lazarus \& Launier, 1978: Mason, 1971), and also, although to a lesser extent, expected to be a stronger predictor than long-term difficulties (though limited by the specific sample of the various difficulties, this measure does account for the appraised severity of difficulties). The data in Table 3 lend support to these assumptions.

Table 3. Spearman correlations of stress measures with psychosomatic and depressive symptomatology.

\begin{tabular}{lcc}
\hline & Psychosomatic Symptoms & Depression \\
\hline Perceived stress & $.60^{* * *}$ & $.76^{* * *}$ \\
Number of life events & .07 & .06 \\
Long-term difficulties & $.47 * * *$ & $.52 * * *$ \\
\hline
\end{tabular}

$\cdots p<.001$

Of the three stress measures, perceived stress showed the highest correlations to both psychosomatic and depressive symptomatology. The number of life-events did not correlate with either psychosomatic or depressive symptomatology. So, although subjects who experienced more life events also experienced more stress, they did not have more symptoms.

Because both perceived stress and chronic difficulties were strongly related to symptomatology it is desirable to show that both stress scales are not measuring the same things. Therefore, chronic difficulties were partialled out of the correlation between perceived stress and psychosomatic symptoms $s_{n}$ and the PSS was partialled out of the correlation between chronic difficulties and psychosomatic symptoms. Partial correlations were calculated with ranked variable scores for reasons of not normally distributed variables. The correlation between the PSS and psychosomatic symptoms, controlling for chronic difficulties, was .45 (p<.001). The correlation between chronic difficulties and psychosomatic symptoms, controlling for perceived stress was .21 $(p<.05)$. Chronic difficulties and perceived stress both independently predicted psychosomatic complaint. Although overlap exists, the scales are not measuring the same thing.

For the validity of the PSS it was also important to demonstrate that perceived stress did not overlap completely with measures of psychological distress. Relations 
among these measures may be due to the common influence of negative affectivity. Self-report stress scales and self-report health measures have been found to reflect a pervasive mood disposition of negative affectivity, thus overestimating the true association between stress and health (Watson \& Clark, 1992; Watson \& Pennebaker. 1989). In our study we found a high correlation between perceived stress and depressive symptomatology $(r h o=.76)$ and between perceived stress and trait-anxiety $(r h o=77)$, probably indicating some overlap again since stress perception could be a symptom of depression or of high trait anxiety. So again partial correlations were calculated on ranked variable scores: results were compared when depressive symptomatology was partialled out of the relation between perceived stress and psychosomatic symptoms and when perceived stress was partialled out of the relation between depressive symptoms and psychosomatic complaints. In the case of the PSS and psychosomatic symptomatology, the correlation was .27 $(\mathrm{p}<.01)$ and in the case of the ZUNG and psychosomatic symptoms, the correlation was .28 (p<.01). It thus appears that both scales independently predict psychosomatic symptomatology. Similarly, both trait anxiety and stress perception independently predicted psychosomatic complaints (partial correlations: $r=.24, p<.05$; and $r=.29, p<.01$. respectively).

\section{DISCUSSION}

The Dutch version of the PSS showed adequate internal reliability and sufficient stability in a sample of white collar workers. The test-retest correlation was high and there was no systematic tendency for scores to fall or rise, reflecting that although the questionnaire is sensitive to temporal change in experienced stress, it also reflects a rather stable individual characteristic. These results are comparable to the results found by Levenstein et al. (Levenstein, Prantera, Varvo, Scribano, Berto, Luzi, et al., 1993) who developed The Perceived Stress Questionnaire (PSQ) which is highly correlated and comparable to the PSS (.73). Here, the sensitivity of this scale to change was tested over 6 months (scale was filled in monthly) and they also found that the mean ratio between a subjects highest and lowest PSQ score was quit small. The fact that the PSS is quite stable but still sensitive to changes in experienced stress is an advantage considering its potential usage as a screening instrument; it is possible to assess chronic levels of perceived stress (when measured more than once) but environmental influences can be studied for its effects as well. Possible causes for the observed test-retest effect are: statistical regression (twice as many people had a lower scale score the second time in stead of a higher score, but this could possibly be explained by the fact that those people with low scale scores the first time were the better adjusted people and therefore less likely to have an increased stress score the second time), and social desirability (subjects selected for the field study wanted to look 'good', stress resistant).

Our sample means and standard deviations of perceived stress were comparable with the US. norms. Especially their means for managerial and clerical professions compared very well with our means for white collar workers. These results give support to the notion that our sample, although not a representative one, does not deviate strongly from a normal probability sample. Means on other crosssectionals like the UCL and the SCL90, when compared to norm scores for the Dutch population, also showed normal population scores. Although correlations between various demographic characteristics and level of perceived stress were in the 
expected direction, most of them did not reach significance. Only household size was positively related to perceived stress. This was probably due to the fact that the correlations described by Cohen and Williamson (Cohen \& Williamson, 1988), which were also small (between .10 and .13), were based on a very large sample $(n=2270)$. Cohen et al.(Cohen et al., 1983) also failed to find a relation between the PSS and sex or age in samples comparable to the size of our white collar sample. It must be said that our sample was also quite homogeneous, in the sense that it consisted of only white collar workers. For instance, differences in socioeconomic status, not measured here, could be very well related to levels of perceived stress.

If the PSS is to be used as a screening instrument it should be able to discriminate subjects on theoretically interesting characteristics. Our data give support for the construct validity of the Dutch version of the PSS; perceived stress was related in the expected way to other stress measures (life events and chronic difficulties), to coping styles, and psychological (anxiety, depression), and psychosomatic complaints. The results on coping are in concordance with studies suggesting that an active-offensive mode of coping (dominated by a tendency of active problem solving and optimism) is more effective in avoiding and solving problems than a reactive-defensive mode of coping (dominated by a tendency of avoidance and depressive reactions).

Our only objective measure of distress, i.e. cortisol, was not related to perceived stress. A major drawback in our study is that we measured cortisol only once. Caplan et al. (Caplan et al., 1979), who studied the effect of perceived white collar workload on cortisol, also did not find a main effect of perceived workload on mean cortisol, but did find an effect of stress on the circadian rhythm of cortisol. Cortisol levels in the morning were significantly lower for employees with high work load. Although our measure of perceived stress is sensitive for more sources of stress than only work load, it would be interesting to study the possible effect of perceived stress on the circadian rhythm. This will be done in our ESM study were we have repeated measures of cortisol.

An important point is, that although the PSS correlates substantially with altemative measures of stress/distress. it assesses a different and independently predictive construct. Some overlap in the various scales is almost inevitable, but the PSS does not measure the same thing as for instance chronic difficulties or depressive symptomatology. The correlation between perceived stress and psychosomatic symptoms, controlling for depressive symptoms, remained significant.

A limitation in our study is that, besides cortisol, we did not have any objective measures of health (e.g. absenteeism at work, visits to health practitioner). Investigating the ability of the PSS to predict such outcomes would be a strong validation. We emphasize that the present correlational analyses do not warrant any inferences of causality. Although perceived stress may have (at least partly) caused both somatic and psychological symptoms, it is also possible that complainis elevated stress perceptions, or that a third factor (e.g. personality) influenced both stress and health. Another important point to be made is that stress scales should be chosen to address specific research questions. The choice for a specific scale will depend on the specific research design used in the study. This also means that the perceived stress scale is not always the appropriate scale. For instance, perceived stress scales should not be used as the only scale in cross-sectional studies of the relationship between stress and psychological distress (Cohen \& Williamson, 1988).

In conclusion, the PSS seems to be a valuable instrument for screening purposes. The scale has high internal reliability. stability, sensitivity and shown 
construct validity. Its subjective character maximizes sensitivity to ongoing stress. Its generality makes this scale less culture and population specific and of special value in populations were the stressful events are hard to pin down. Its short length is also an advantage when used concurrently with various other questionnaires or when used repeatedly over time in longitudinal studies. The PSS seems to be an adequate measure to assess which people are at risk for the development of stress related somatic and mental health problems.

\section{REFERENCES}

Ansseau, M., Sulon, J., Doumont, A., Cerfontaine, J. L., Legros, J. J., Sodoyez, J. C., \& Demey. Ponsart, E. (1984). The use of saliva cortisol in the dexamethasone suppression test. Psychiatry Research, 13, 201-211.

Averill, J. R. (1973). Personal control over aversive stimuli and its relationship to stress.

Psychological Bulletin, 80(4), 286-303.

Cantor, N., Norem, J., Langston, C., Zirkel, S., Fleeson, W., \& Cook-Flannagan, C. (1991). Life tasks and daily life experience. Journal of Personality, 59(3), 425-451.

Caplan, R. D., Cobb, S., \& French, J. R. P., Jr. (1979). White collar work load and cortisol: Disruption of a circadian rhythm by job stress? Journal of Psychosomatic Research, 23, 181. 192.

Cohen, S. (1978). Environmental load and the allocation of attention. In A. Baum, J. Singer, \& S. Valins (Eds.), Advances in environmental psychology. Hillsdale: Lawrence Erlbaum.

Cohen, S. (1986). Contrasting the Hassles Scale and the Perceived Stress Scale: Who's really measuring appraised stress? American Psychologist, 41, 716-718.

Cohen, S., Kamarck, T., \& Mermelstein, R. (1983). A global measure of perceived stress. Journal of Health and Social Behavior, 24, 385-396.

Cohen, S., Tyrrell, D. A. J., \& Smith, A. P. (1991). Psychological stress and susceptibility to the common cold. The New England Journal of Medicine, 325(9), 606-612.

Cohen, S., \& Williamson, G. M. (1988). Perceived stress in a probalitity sample of the United States. In S. Spacepan \& S. Oskamp (Eds.), The Social Psychology of Health (pp. 31-67). Newbury Park, CA: Sage Publications.

Dohrenwend, B. P., Link, B. G., Kern, R., Shrout, P. E., \& Markowitz, J. (1990). Measuring life events: The problem of variability within event categories. Stress Medicine, 6, 179-187.

Dohrenwend, B. P., \& Shrout, P. E. (1985). "Hassles" in the conceptualization and measurement of life stress variables. American Psychologist, 40, 780-785.

Dohrenwend, B. S., Dohrenwend, B. P., Dodson, M., \&. Shrout, P. E. (1984). Symptoms, hassles, social supports, and life events: Problems of confounded measures. Journal of Abnormal Psychology, 93(2), 222-230.

Folkman, S., \& Lazarus, R. S. (1985). If it changes it must be a process: Study of emotion and coping during three stages of a college examination. Journal of Personality and Social Psychology, 48, 150-170.

Hofer, M. A., Wolff, C. T., Friedman, S. B., \& Mason, J. W. (1972). A psychoendocrine study of bereavement PartI. 17-Hydroxycorticosteroid excretion rates of parents following death of their children from leukemia. Psychosomatic Medicine, 34(6), 481-504.

Jacobs, S. C., Mason, J., Kosten, T. R., Kasl, S. V., Ostfeld, A. M., \& Wahby, V. (1987). Urinary free cortisol and separation anxiety early in the course of bereavement and threatened loss. Biological Psychiatry, 22, 148-152. 
Kiecolt-Glaser, J. K., Ricker, D., George, J., Messick, G., Speicher, C. E., Garner, W., \& Glaser, R. (1984). Urinary cortisol levels, cellular immunocompetency, and loneliness in psychiatric inpatients. Psychosomatic Medicine, 46(1), 15-23.

Koeter, M. W. J., Ormel, J., \& van den Brink, W. (1988). Totaalscore op de SCL-90 als maat voof de ernst van psychopathologie. Nederlands Tijdschrift voor de Psychologie, 43, 381-391.

Lazarus, R. S. (1966). Psychological stress and the coping process. New York: McGraw-Hill.

Lazarus, R. S., De Longis, A., Folkman, S., \& Gruen, R. (1985). Stress and adaptational outcomes: The problem of confounded measures. American Psychologisi, 40, 770-779.

Lazarus, R. S., \& Launier, R. (1978). Stress-related transactions between person and environment. In L. A. Pervin \& M. Lewis (Eds.), Perspectives in Interactional Psychology (pp. 287-327). New York: Plenum.

Levenstein, S., Prantera, C., Varvo, V., Scribano, M. L., Berto, E., Luzi, C., \& Andreoli, A. (1993). Development of the perceived stress questionnaire: a new tool for psychosomatic research. Journal of Psychosomatic Research, 37(1), 19-32.

Mason, J. W. (1971). A re-evaluation of the concept of 'non-specificity' in stress theory. Journal of Psychiatric Research, 323-333.

Mason, J, W. (1975). A historical view of the stress field. Journal of Human Stress, 1(1), 7-12.

Miller, S. M. (1979). Controilability and human stress: Method, evidence and theory. Behavior Research and Therapy, 17, 287-305.

Schreurs, P. J. G., \& van de Willige, G. (1988), De Utrechtse Copinglijst (UCL): Lisse, the Netherlands: Swets \& Zeitlinger.

Seligman, M. E. P. (1975). Helplessness: On depression, development and death. San Francisco: Freeman. W.H.

Watson, D., \& Clark, L. A. (1992). Affects separable and inseparable: On the hierarchical arrangement of the negative affects. Journal of Personality and Social Psychology, 62(3), 489-505.

Watson, D., \& Pennebaker, J. W. (1989). Health complaints, stress, and distress: Exploring the central role of negative affectivity. Psychological Review, 96(2), 234-254. 


\section{Appendix II}

\section{ESM report}

\begin{tabular}{lccccccc}
\hline Op het moment van de piep: & niet & \multicolumn{3}{c}{ 'n beetje } & tamelijk & \multicolumn{2}{c}{ zeer } \\
Kon u zich concentreren ? & 1 & 2 & 3 & 4 & 5 & 6 & 7 \\
Piekerde u ? & 1 & 2 & 3 & 4 & 5 & 6 & 7 \\
& & & & & & & \\
Zo ja, waarover..........................................................................................................................................
\end{tabular}

\section{Voelde u zich op dat moment:}

$$
\text { niet 'n beetje tamelijk zeer }
$$

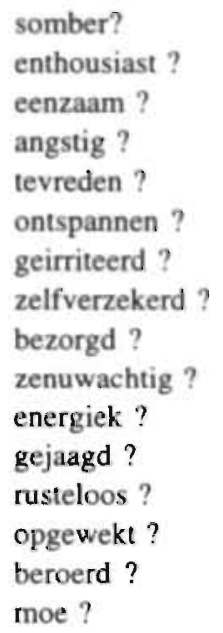

$\begin{array}{lllllll}1 & 2 & 3 & 4 & 5 & 6 & 7 \\ 1 & 2 & 3 & 4 & 5 & 6 & 7 \\ 1 & 2 & 3 & 4 & 5 & 6 & 7 \\ 1 & 2 & 3 & 4 & 5 & 6 & 7 \\ 1 & 2 & 3 & 4 & 5 & 6 & 7 \\ 1 & 2 & 3 & 4 & 5 & 6 & 7 \\ 1 & 2 & 3 & 4 & 5 & 6 & 7 \\ 1 & 2 & 3 & 4 & 5 & 6 & 7 \\ 1 & 2 & 3 & 4 & 5 & 6 & 7 \\ 1 & 2 & 3 & 4 & 5 & 6 & 7 \\ 1 & 2 & 3 & 4 & 5 & 6 & 7 \\ 1 & 2 & 3 & 4 & 5 & 6 & 7 \\ 1 & 2 & 3 & 4 & 5 & 6 & 7 \\ 1 & 2 & 3 & 4 & 5 & 6 & 7 \\ 1 & 2 & 3 & 4 & 5 & 6 & 7 \\ 1 & 2 & 3 & 4 & 5 & 6 & 7\end{array}$

\begin{tabular}{lcccccccc} 
Was uw belangrijkste klacht aanwezig ? & niet & & 'n beetje & tamelijk & \multicolumn{2}{c}{ zeer } \\
Had u last van andere klachten ? & 1 & 2 & 3 & 4 & 5 & 6 & 7 \\
& 1 & 2 & 3 & 4 & 5 & 6 & 7
\end{tabular}




\section{Wat deed u op het moment van de piep ?}

niet 'n beetie tameliik zeer

$\begin{array}{lllllllll}\text { Deed u het graag ? } & 1 & 2 & 3 & 4 & 5 & 6 & 7\end{array}$

$\begin{array}{llllllll}\text { Kostte het u moeite ? } & 1 & 2 & 3 & 4 & 5 & 6 & 7\end{array}$

$\begin{array}{llllllll}\text { Was deze aktiviteit voor u een uitdaging ? } 1 & 2 & 3 & 4 & 5 & 6 & 7\end{array}$

$\begin{array}{llllllll}\text { Kon } u \text { het? } & 1 & 2 & 3 & 4 & 5 & 6 & 7\end{array}$

$\begin{array}{lllllllll}\text { Vond } \mathrm{u} \text { deze aktiviteit belastend? } & 1 & 2 & 3 & 4 & 5 & 6 & 7\end{array}$

\section{Waar was u op het moment van de piep?}

Was u samen met anderen ?

Nee / Ja, met 1_1_1 mensen.

Naam

Wie is dat?

Wilde u nu graagalleen zijn ?

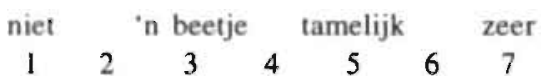

\section{Was er tussen deze en de vorize piep sprake van:}

$\begin{array}{lccccccc} & \text { niet } & \text { 'n beetje } & \text { tamelijk } & \text { zeer } \\ \text { een stressvolle gebeurtenis/situatie ? } & 1 & 2 & 3 & 4 & 5 & 6 & 7\end{array}$

Zo ja, geef een korte omschrijving:

Begintijd:

Eindtijd

Nog niet afgelopen $\mathrm{O}$

Was deze gebeurtenis:

- vervelend?

- verwacht?

- belangrijk voor $\mathrm{u}$ ?

K.on u het verloop ervan beinvloeden ? $\quad \begin{array}{llllllll}2 & 2 & 3 & 4 & 5 & 6 & 7\end{array}$

Heeft deze (of een soortgeliike) situatie zich vaker voor gedaan?

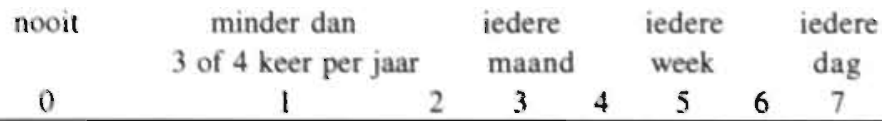




\begin{tabular}{lcccccccc} 
Had $\mathbf{u}$ in de tussentijd last van: & niet & & 'n beetje & \multicolumn{3}{c}{ tamelijk } & zeer \\
Uw belangrijkste klacht? & 1 & 2 & 3 & 4 & 5 & 6 & 7 \\
Andere klachten? & 1 & 2 & 3 & 4 & 5 & 6 & 7
\end{tabular}

Zo ja, welke?

\section{Gebruikte $u$ iets tussen deze en de vorige piep ?}
$\mathrm{O}$ niets
$O$ voedsel
O tabak
O mediciinen, n.I.
O koffie
O alcohol
1_1 glazen

$\begin{array}{lllllllll}\text { Wat was het hoogste niveau van aktiviteit? } & 1 & 2 & 3 & 4 & 5 & 6 & 7\end{array}$

voorbeelden: 1=rusten, 2 =zitten (actief), 3=lopen, $4=$ =stofzuigen, $S=f i e t s e n, 6=t e n n i s s e n, 7=r e n n e n$

Stoorde deze piep u ?

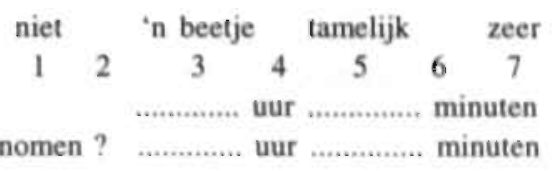

Hoe laat heeft u het speekselmonster genomen ? 


\section{Appendix III}

\section{Interrater agreement on codings for ESM measures}

\section{INTRODUCTION}

An appropriate measure of agreement for nominal data is the Kappa statistic ( $\mathrm{k}$ ) (Cohen, 1960). This statistic is an improvement on the simpler measure, per cent of agreement (Po), because it discounts the proportion of agreement which is expected by chance alone $(\mathrm{Pe})$. The formula for the (unweighted) $k a p p a$ is: $k=(\mathrm{Po}-\mathrm{Pe}) /(1-\mathrm{Pe})$. Because we are dealing with more than two categories we used the intra category kappa (agreement within a category). The 'overall Kappa' is a weighted average of the 'individual Kappa's' for all the alternative dichotomies which can be made by preserving one category and combining all others (Maclure \& Willett, 1987). Values for Kappa vary between 0 and 1.00. When Kappa has a value of 0 there is agreement as expected by chance alone. A Kappa of 1.00 means perfect agreement. We used the criteria given by Landis and Koch (Laundis \& Koch, 1977, p.265) for deciding if the Kappa we found was sufficiently high:

$\begin{array}{ll}\frac{\text { Kappa }}{<0.00} & \text { Degree of Agreement } \\ .00-.20 & \text { 'poor' } \\ .21-.40 & \text { 'flight' } \\ .41-.60 & \text { 'moir' } \\ .61-.80 & \text { 'substante' } \\ .81-1.00 & \text { 'almost perfect' }\end{array}$

\section{RESULTS}

The results on interrater agreement are presented in Table 1 and Table 2. First we will discuss the results for 'activities', 'location' and 'social context', and then for 'stressful events'.

\section{Interrater agreement for the activlty, locatlon and soclal context domains}

Reliability was determined for all the valid cases coded by the two raters. In every domain, the 'can't code' and the 'missing values' category were left out of analysis, because there were only a few observations in these categories: 'can't code' between 0 and 3 observations and 'missing values' between 6 and 14 observations. The fact that so few observations fell into the 'can't code' category means that the qualitative information was generally easy to interpret and to code into one of the available categories. We may conclude that the Kappa's we found were acceptably high (see Table 1). According to the criteria of Laundis and Koch ((Laundis \& Koch, 1977), p.265) the degree of agreement between raters is almost perfect, with all intra category Kappa's $\geq .89$, except for the activity category 'other' (Kappa .58) which had a moderate degree of agreement. So, the qualitative information was coded very reliably and seems easy to interpret. Also the fact that the various 'other' categories 
had so few observations gives support to the notion that the coding system we used was quite exhaustive.

Table 1. Interrater agreement for the activity, location and social context domains.

\begin{tabular}{|c|c|c|}
\hline & Total Observations & Kappa \\
\hline Activity ('what') & 2494 & .91 \\
\hline -leisure/social interaction & 722 & .90 \\
\hline -work & 709 & .95 \\
\hline -household/maintenance & 452 & .94 \\
\hline -transport & 252 & .96 \\
\hline -meals & 188 & .92 \\
\hline -inactivity/rest & 107 & .93 \\
\hline -other & 64 & .58 \\
\hline Location (Where') & 2491 & .94 \\
\hline -at home & 1185 & .97 \\
\hline -work & 788 & .96 \\
\hline -public places & 220 & .90 \\
\hline -transport & 189 & .92 \\
\hline -network & 72 & .91 \\
\hline -other & 37 & .70 \\
\hline Social Context ('Whol') & 2485 & .96 \\
\hline -household members & 964 & .99 \\
\hline -alone & 756 & .99 \\
\hline -colleagues & 546 & .97 \\
\hline -neighbours/acquaintances & 114 & .89 \\
\hline ofriends & 40 & .95 \\
\hline -non resident family & 36 & .89 \\
\hline -strangers & 29 & .90 \\
\hline
\end{tabular}

\section{Interrater agreement for stressful events}

The results for stressful events are presented in table 2. A total of 345 events were described by the 27 subjects. Interrater agreement was determined on all the completed subject responses. The context of events was rated with a high degree of agreement between the two raters. Kappa's varied between .60 and .96 . Only the 'other' category had a moderate degree of agreement, but this category was obviously also the most ambiguous one. It was also possible to use the event information to determine in a reliable way if someone was involved in the event and then who was involved in the event. The Kappa's lay between .76 and .96 . Most of the time no one was involved in the reported event. It was not possible to code in more than a moderately reliable way whether an event was 'external' or 'internal'. Only the 'internal' category had a substantially high Kappa (.76); the other categories had only a moderate degree of agreement. The 'can't code' category contained many observations $(21 \%)$ which suggests that it was very difficult for raters to interpret the qualitative information. Only the more straightforward internal events (e.g. worries, somatic complaints) were rated with a high reliability. The other categories can only be used with caution in further analyses. 
The intra category Kappa's in the social interaction domain were all satisfactory, varying from substantial to almost perfect, except for the can't code category for which the degree of agreement was only moderate. As shown in table 2 . a large number of observations could not be coded: $+10 \%$ of the total. This means that the various categories were not well defined, or that the information gathered was too vague. This second point seems to be more important here; often more detailed information was needed before a reliable judgment could be made. One way to improve on this is by paying more attention to the debriefing session where more information can be obtained on reported information. In the task demands domain, the categories 'problematic task' and 'failure at task' were aggregated into I category and the same accounts for the categories 'a lot of work' and 'time pressure'. This decision was based on the fact that the different subcategories were very interrelated. The Kappa's for the various categories ranged from moderate to almost perfect. Here again, a substantial percentage of responses could not be coded $(+22 \%)$.

Table 2. Interrater agreement for stressful events.

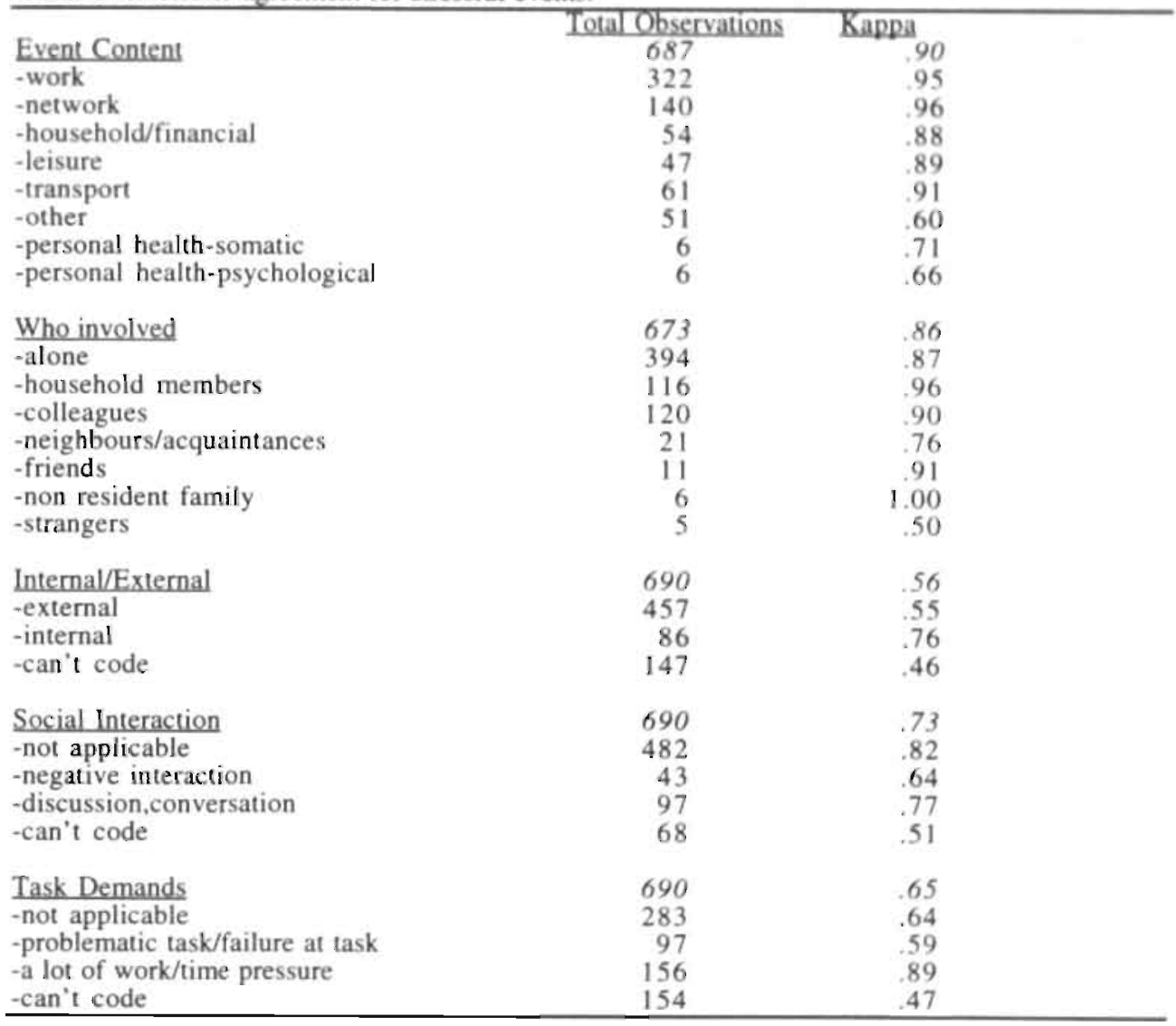




\section{REFERENCES}

Cohen, J. A. (1960). A coefficient of agreement for nominal scales. Educational and Psychological Measurement, 20, 37-46.

Laundis, J. R., \& Koch, G. G. (1977). The measurement of observer agreement for categorical data. Biometrics, 33, 396-398.

Maclure, M., \& Willett, W. C. (1987). Misinterpretation and misuse of the kappa statistic. American Journal of Epidemiology, 126(2), 161-169. 


\section{Summary}

During the last decades, there has been an increasing interest in the stressful aspects of contemporary society and their relationship to the development and maintenance of a wide range of somatic and psychological disorders. While earlier research focused on the impact of major life events or acute experimental stress, little is known about the nature of and the psychological and physiological responses to the minor but much more frequently occurring stresses of daily living. To increase our understanding of the stress process as it relates to health, the present thesis investigated the impact of minor daily events on mood and the HPA system. The aims of the research were 1) to describe the nature and scope of daily life stress in a group of white collar men and to contrast the experiences of individuals who perceive themselves to be stressed with those who do not, 2) to investigate affective and neuroendocrine (cortisol) responses in relation to stressful daily events, and 3) to investigate whether the cortisol responses to a laboratory stress task are generalizable to those occurring during stressful situations in real life. We also investigated whether more or less stable person characteristics like perceived stress level, trait anxiety, and depressive symptomatology were related to individual differences in responses to daily stress.

Chapter I presents an overview of psychological and physiological approaches to the concept of stress and discusses the role of psychosocial stress in health and disease. The existing literature on the impact of daily events on mood and cortisol is briefly reviewed. The following related issues are discussed: individual variability in mood and cortisol, the contexts in which daily events occur, appraisals of events, and the generalizability of laboratory-assessed stress reactivity to real life. Finally, the chapter presents the rationale for the study design and introduces the research questions.

Chapter 2 describes subjects, methods and procedures. Two groups of white collar men, with high versus low levels of perceived stress, were recruited as subjects. The Experience Sampling Method was used to collect data on stressful events and mood from subjects during their normal daily activities. Ten times a day for five consecutive days, subjects received auditory signals (beeps), after which they filled in a questionnaire and collected a saliva sample for cortisol determination. The same subjects also participated in a laboratory stress task, in which they were unexpectedly asked to deliver a speech. Stress responses were operationalized âs increases in cortisol secretion, increases in negative mood, and decreases in positive mood.

Chapter 3 describes quantitative as well as qualitative aspects of the reported stressful daily events. A total of 626 events were reported (on $17 \%$ of all ESM reports). Unpredictable and uncontrollable events were rated as the most unpleasant. Although the majority of stressful events were work-related, events were also frequently reported in a wide variety of life domains. High stress subjects scored high on trait anxiety and depressive symptoms, were bothered by various psychological and psychosomatic complaints, experienced relatively many chronic difficulties, and used more passive and less active coping styles than low stress subjects. High stress subjects reported twice as many stressful events, and they appraised events as more stressful and less controllable. They also reported twice as many work-related events and more events related to their social network. In particular, high stress subjects experienced more negative social interactions, both at work and at home, especially with their colleagues and their wives. 
Chapter 4 investigates the relationship between stressful events and negative and positive mood states, in particular examining the influence of perceived stress level on mood responses. Results showed that stressful events were associated with increases in negative mood. Positive mood only decreased when events were very unpleasant. Subjects scoring high on perceived stress showed significantly stronger negative mood reactivity in response to stressful events. Perceived stress did not influence the duration of the effects of events on mood. Events which involved performance demands and those appraised as more unpleasant, more novel, and less controllable were more likely to influence mood states. Results suggest increased vulnerability to daily events in individuals with high perceived stress.

Chapter 5 addresses the question of whether high perceived stress or related individual characteristics are associated with elevated cortisol or catecholamine levels. Analysis of cortisol values aggregated over each subject and time of day showed higher cortisol levels in high stress compared to low stress subjects when only the workdays were included. Trait anxiety, depressive symptomatology, and negative mood state were also associated with higher cortisol on workdays. Recent life events, chronic difficulties, trait anger, or psychosomatic symptoms, however, showed no relationship to cortisol levels. These results suggest that the kinds of mild chronic or intermittent stress in daily life situations reported during the ESM sampling period were sufficient to increase secretion of cortisol. In contrast, overnight catecholamine levels were unrelated to perceived stress, trait anxiety, depression, anger, psychosomatic symptoms or mood states.

Chapter 6 describes the estimated effects of perceived stress, personality traits, mood states, and stressful daily events on cortisol excretion. A random regression data analysis method revealed that trait anxiety and depression were associated with small but statistically significant cortisol elevations. Although results from the previous chapter had indicated a relationship between perceived stress and mean workday cortisol levels, the random regression analyses, which made use of the full dataset, did not replicate this finding; perceived stress was not associated with cortisol elevation. Cortisol levels were also no higher on workdays than on weekends in either subject group. Stressful daily events were indeed associated with increased cortisol secretion, the magnitude of the effect depending on whether the event was still ongoing and on how frequently a similar kind of event had occurred previously. Type of event or appraisal measures had no additional effects on cortisol. In addition, we found no significant individual differences in cortisol reactivity to events. Although perceived stress, anxiety, and depression did not increase cortisol reactivity to daily events, evidence was found for reduced habituation to recurrent events in subjects scoring high on these traits. Both negative mood states, Agitation and Negative Affect, were also associated with higher cortisol levels. Consistent with most views of the stress process, the effect of stressful events on cortisol appeared to be mediated to a large extent by associated increases in negative mood. The finding that even minor everyday events and fluctuations in mood states have an impact on cortisol secretion may point to a possible mechanism linking subjective experience to health outcomes.

Chapter 7 examines the generalizability of cortisol measures obtained in the laboratory to those obtained in real life. Results showed that the laboratory and reallife (ESM), cortisol levels were moderately correlated, but no association was found between laboratory and real-life stress response measures. Possible implications with respect to the predictive validity of laboratory stress reactivity for stress reactivity in general are discussed. 
In Chapter 8 the major findings of the study are summarized. Both methodological and theoretical issues related to the present research and to stress research in general are discussed, and results are evaluated in the context of possible health implications. Finally, several suggestions for future research are described. 



\section{Samenvatting}

De laatste decennia gaat steeds meer en meer aandacht uit naar de stressvolle elementen van onze huidige maatschappij en de negatieve gevolgen van deze elementen op het ontstaan en het in stand houden van allerlei somatische en psychische stoornissen. Terwijl vroeger onderzoek zich voornamelijk concentreerde op de gevolgen van belangrijke levensgebeurtenissen en acute experimentele stress, is er momenteel nog maar weinig bekend over de aard en de psychologische en fysiologische reacties op de kleine maar meer frequent voorkomende stressvolle gebeurtenissen in het dagelijkse leven. Ter vergroting van ons inzicht in de relatie tussen stress en gezondheid, bestudeert deze dissertatie de effecten vañ stressvolle dagelijkse gebeurtenis op de stemming en het hypofyse-bijnierschors systeem. De doelstellingen van het onderzoek zijn 1) het beschrijven van de aard en de omvang van dagelijkse stress in een groep van werkende mannen ('witte boorden') en het vergelijken van de ervaringen van individuen met een hoog ervaren stressniveau met die van individuen met een laag ervaren stressniveau, 2) het bestuderen van de affectieve en neuro-endocriene (cortisol) reacties op stressvolle dagelijkse gebeurtenissen, en 3 ) het onderzoeken in hoeverre de cortisol reacties op een laboratorium stresstaak corresponderen met de reacties op stressvolle gebeurtenissen in het dagelijkse leven. Tevens onderzochten we of er een relatie was tussen min of meer stabiele persoonskenmerken zoals ervaren stressniveau, angstdispositie en depressieve symptomatologie en individuele verschillen in reacties op dagelijkse. stress.

Hoofdstuk I geeft een overzicht van psychologische en fysiologische benaderingen van het begrip stress en evalueert de rol van psychosociale stress in gezondheid en ziekte. De beschikbare literatuur met betrekking tot de relatie tussen dagelijkse stress enerzijds en stemming en cortisol anderzijds wordt kort besproken, met speciale aandacht voor: individuele variatie in stemming en cortisol, de context waarin stressvolle gebeurtenissen plaatsvinden, de evaluatie (appraisal) van de stressvolle gebeurtenis en de generalisatie van stress-reactiviteit gemeten in het laboratorium naar het veld. Tenslotte wordt de rationale voor het onderzoeksdesign gepresenteerd en worden de onderzoeksvragen geintroduceerd.

In Hoofdstuk 2 worden de subjecten, methoden en procedures beschreven. Er werden twee groepen 'witte boorden' mannen gevormd: een met een hoog en een met een laag ervaren stressniveau. De Experience Sampling Methode werd gebruikt om gegevens van subjecten betreffende stressvolle gebeurtenissen en stemming te verzamelen op geselecteerde momenten tijdens hun normale dagelijkse activiteiten. Subjecten ontvingen tien keer per dag en gedurende vijf dagen signalen (beeps) via een voorgeprogrammeerd horloge, waarna ze een vragenlijst invulden en een speekselmonster namen, voor de cortisolbepaling. Dezelfde subjecten namen ook deel aan een laboratorium stresstaak, waarbij ze onverwacht gevraagd werden een speech te houden. Een stressreactie werd geoperationaliseerd als een stijging in cortisolsecretie, een toename in negatieve stemming en een afname in positieve stemming.

Hoofdstuk 3 beschrijft zowel kwantitatieve als kwalitatieve aspecten van de gerapporteerde stressvolle dagelijkse gebeurtenissen. In totaal werden er 626 gebeurtenissen gerapporteerd (op $17 \%$ van alle ESM beeps) en werden onvoorspelbare en oncontroleerbare gebeurtenissen het meest vervelend bevonden. Alhoewel de voornaamste bron van stress werk-gerelateerd was, kwamen stressvolle gebeurtenissen ook veelvuldig in verschillende andere levensdomeinen voor. 
Subjecten met een hoog stressniveau scoorden hoog op angstdispositie en depressieve symptomen, werden gehinderd door verschillende psychologische en psychosomatische klachten, ervaarden relatief veel langdurige moeilijkheden en gebruikten in het algemeen meer passieve en minder actieve coping stijlen dan subjecten met een laag stressniveau. Hoge stress subjecten rapporteerden twee keer zo veel dagelijkse gebeurtenissen als lage stress subjecten en ervaarden deze als meer stressvol en minder controleerbaar. Zij vermeldden tevens twee keer zoveel werk. problemen en meer problemen in relatie tot hun sociaal netwerk. In het bijzonder ervaarden hoge stress subjecten meer negatieve sociale interacties, zowel thuis als op het werk, en wel speciaal met hun collega's en echtgenotes.

Hoofdstuk 4 onderzoekt de relatie tussen stressvolle gebeurtenissen en negatieve en positieve stemming. Speciale aandacht ging uit naar de invloed van individuele verschillen in ervaren stressniveau op de stemmingsreacties. De resultaten laten zien dat stressvolle gebeurtenissen geassocieerd zijn met een toename in negatieve stemming. Positieve stemming nam alleen af wanneer de gebeurtenis zeer vervelend was. Subjecten met een hoog ervaren stressniveau vertoonden een significant sterkere toename in negatieve stemming als reactie op stressvolle gebeurtenissen. Het ervaren stressniveau had echter geen invloed op de duur van de effecten van stressvolle gebeurtenissen op stemming. Gebeurtenissen, die te maken hadden met de werkdruk of werklast en gebeurtenissen die als vervelender. onbekender en minder controleerbaar werden ervaren, hadden relatief meer invloed op de stemming. De resultaten lijken erop te wijzen dat individuen met een hoog ervaren stressniveau een verhoogde k.wetsbaarheid hebben in relatie tot stressvolle dagelijkse problemen.

Hoofdsıuk 5 is gewijd an de vraag of een hoog ervaren stressniveau of hiermee samenhangende persoonskenmerken geassocieerd zijn met verhoogde cortisol en/of catecholamineniveaus. Analyses van cortisolwaardes geaggregeerd over elk subject en de tijd van de dag heen laten een verhoogd cortisolniveau zien voor hoge stress subjecten in vergelijking met lage stress subjecten, echter alleen tijdens werkdagen. Angst dispositie, depressieve klachten, en negatieve stemming waren ook geassocieerd met hogere cortisolniveaus op werkdagen. Recente levensgebeurtenissen, chronische moeilijkheden, boosheidsdispositie en psychosomatische klachten vertoonde echter geen relatie met cortisol. De resultaten suggereren dat deze vormen van milde chronische of periodieke stress, ervaren in hett dagelijkse leven, in staat zijn de cortisolsecretie te verhogen. Echter, de catecholamineniveaus die werden gemeten gedurende de nacht waren niet geassocieerd met het ervaren stressniveau, angstdispositie, depressie. boosheidsdispositie, psychosomatische klachten, of stemming.

In Hoofdstuk 6 worden de geschatte effecten van het ervaren stressniveau, persoonskenmerken, stemming, en stressvolle gebeurtenissen op de cortisolsecretie beschreven. Een random regression data analyse methode liet zien dat angstdispositie en depressie gerelateerd waren met kleine maar significante verhogingen in. cortisolsecretie. Alhoewel de resultaten van het vorige hoofdstuk een positieve relatie tussen het ervaren stressniveau en het gemiddeld cortisolniveau op werkdagen aangaven, werden deze resultaten niet gerepliceerd wanneer via random regression. analyses gebruik gemaaki werd van alle gegevens; het ervaren stressniveau vertoonde geen relatie met cortisol. De cortisolniveaus waren tevens in beide groepen niet hoger op werkdagen dan in het weekend. De resultaten lieten verder zien dat stressvolle gebeurtenissen inderdaad geassocieerd waren met een verhoogde cortisolsecretie. De grootte van dit effect was afhankelijk van het feit of de stressor 
nog steeds aan de gang was op het moment van de meting en van de frequentie waarmee een gelijksoortige stressor zich reeds eerder had voorgedaan. Het type stressor of de evaluatie van de stressor had geen additioneel effect op cortisol. Tevens vonden we geen individuele verschillen in cortisol reactiviteit op stressvolle gebeurtenissen. Alhoewel het ervaren stressniveau, angstdispositie, en depressieve klachten de cortisol reactiviteit op stressvolle gebeurtenissen niet vergrootten, vonden we wel een verminderde habituatie aan stressoren die reeds herhaaldelijk eerder voorgekomen waren in subjecten die hoog scoorden op deze persoonskenmerken. Beide maten voor negatieve stemming (Agitatie en Negatief Affect) waren ook geassocieerd met hogere cortisolniveaus. In overeenstemming met de meeste opvattingen over het stressproces werd het effect van stressvolle gebeurtenissen op cortisol voornamelijk gemedieerd door de geassocieerde stijging in negatieve stemming. De bevinding dat zelfs kleine, dagelijkse stressoren en fluctuaties in stemming van invloed zijn op de cortisolsecretie wijst op een mogelijk mechanisme wat subjectieve ervaringen met gezondheid en ziekte verbindt.

Hoofdstuk 7 is voornamelijk gewijd aan de vraag of cortisolwaardes gemeten in het laboratorium generaliseerbaar zijn naar cortisolwaardes gemeten in het dagelijkse leven. De resultaten lieten zien dat de cortisolniveaus in het laboratorium en in het dagelijks leven redelijk met elkaar gecorreleerd waren, maar dat er geen relatie was tussen de reactiviteitsmaten in de experimentele setting en de reactiviteitsmaten in het dagelijks leven. De mogelijke implicaties van deze resultaten voor de predictieve validiteit van laboratorium stress-reactiviteit voor stressreactiviteit in het algemeen wordt besproken.

In Hoofdstuk 8 worden de belangrijkste resultaten van het onderzoek samengevat. Zowel methodologische als theoretische aspecten met betrekking tot deze dissertatie en met betrekking tot stress onderzoek in het algemeen worden bediscussieerd en de mogelijke implicaties van de resultaten voor de gezondheid worden nader bekeken. Het hoofdstuk wordt afgesloten met enkele suggesties voor vervolgonderzoek. 



\section{Dank}

Het uitvoeren van dit onderzoek was voor mij van tijd tot tijd vergelijkbaar met een fietstocht door het Limburgse landschap: al fietsend de heuvels op denk je "waar ben ik toch aan begonnen?", maar al snel bij het afdalen verandert deze gedachte in "ik ben blij dat ik het gedaan heb", totdat de volgende heuvel in zicht komt. Mijn dank gaat uit naar diegenen die ervoor gezorgd hebben dat ik in het heuvellandschap bleef en niet in de Alpen terechtkwam.

Daarmee bedoel ik op de eerste plaats de vele proefpersonen die zich bereid verklaarden om zich een kleine week lang door een horloge te laten achtervolgen en hun wel, wee en speeksel aan ons toe te vertrouwen. Zonder hen was dit boek er niet geweest. Op de tweede plaats bedoel ik Nancy Nicolson, mijn co-promotor. Nancy, ik dank je voor je grote betrokkenheid en steun bij het soms eenzame en lange traject van promotie-onderzoek. Met veel deskundigheid, geduld en optimisme voorzag je mijn diverse kladteksten van commentaar. Ik heb veel van je geleerd. Marten de Vries, mijn promotor, wil ik vooral bedanken voor alle vrijheid en vertrouwen die ik van hem kreeg, zowel tijdens als na mijn aanstelling als onderzoeker in opleiding.

Carolien Dijkman dank ik voor de efficiënte en fijne samenwerking tijdens de periode van dataverzameling. Op de vele vroege maandagochtenden was het een troost te weten dat ik niet als enige met de fiets op 'jacht' was naar (urine) monsters. Dr. Jose Sulon, Dr. Rahman en Dr. J. Duvivier ben ik zeer erkentelijk voor de bepalingen van respectievelijk de speeksel -en urinemonsters. De geweldige hulp van Hans Berkhof bij de vele gecompliceerde statistische analyses was onmisbaar. Zonder hem waren vele vraagstellingen onbeantwoord gebleven. Hans heeft laten zien dat een statisticus ook begrip voor de inhoud van de vraagstelling kan hebben, een zeldzaam verschijnsel. Professor Arthur Stone, tevens lid van de beoordelingscommissie, wil ik bedanken voor de motiverende discussies (zowel in Maastricht als in de US) en voor zijn komst naar Maastricht op 18 oktober.

Mijn collega's van de sectie Sociale Psychiatrie en Psychiatrische Epidemiologie wil ik bedanken voor hun morele ondersteuning maar vooral ook voor het gezellig geleuter tijdens de vele lunches en de fanatieke competities als er weer eens een nieuw computerspelletje was. In het bijzonder denk ik hierbij aan: Alex, Bart, Marie-Jose, Monique, Sarah, en Trees. Alhoewel ik 'de club' heb verlaten zal ik ze zeker nog lang missen. Bart Leunissen, mijn kamergenoot, ben ik speciaal erkentelijk voor het herhaaldelijk aanhoren van mijn onderzoeksfrustraties en het (meestal) stilzwijgend accepteren van de wanorde op mijn bureau('s). Bart, ik wens je meer succes bij de volgende kamergenoot. Philip Delespaul wil ik bedanken voor de fantastische hulp bij aspecten van de lay-out.

Mijn paranimfen Monique Pennings en Désirée Nijst bedank ik voor de hulp bij de organisatie van de 18-de. Monique: veel succes bij de 'vernieuwingen' in je leven, zowel privé als in de werksfeer. Desiree: "Hoe zit het nu met de bolderkar?"

Mijn moeder bedank ik voor de vrijheid en het vertrouwen die ze me altijd gegeven heeft om datgene te doen wat ik graag wou doen, om me vervolgens daarin dan ook te stimuleren. Last but not least: Rob. Bedankt voor je steun als ik weer eens met een pestbui thuiskwam. Het feit dat je (gelukkig) vaak niks begreep van mijn onderzoeks -en computer problemen, maakte dat ik de boel weer snel kon relativeren. Je motto "gewoon doorgaan" bracht me steeds weer een stapje verder. 



\section{Curriculum vitae}

Marleen van Eck werd geboren op 10 december 1965 te Wassenaar. In 1984 behaalde zij het VWO diploma aan het Griftland College te Soest. Na een verblijf van een jaar in het buitenland (Israël, Engeland), begon zij met de studie Gezondheidswetenschappen, afstudeerrichting Geestelijke Gezondheidkunde, aan de Rijksuniversiteit Limburg. Deze studie rondde zij in 1990 af. Aansluitend was zij als onderzoeker in opleiding verbonden aan de vakgroep Psychiatrie en Neuropsychologie, sectie Sociale Psychiatrie en Psychiatrische Epidemiologie, van de Rijksuniversiteit Limburg. Hier was zij werkzaam op een project dat gefinancierd werd door de Nederlandse Organisatie voor Wetenschappelijk Onderzoek (NWO), Deze dissertatie is een neerslag van dit werk. Tijdens dit dienstverband volgde zij tevens de opleiding tot gedragstherapeut. Na een tijdelijke aanstelling als toegevoegd onderzoeker bij dezelfde vakgroep is zij sinds 15 oktober 1995 werkzaam bij het RIAGG OZL te Heerlen, afdeling Psychotherapie. 


\section{mood,}

\section{cortisol}
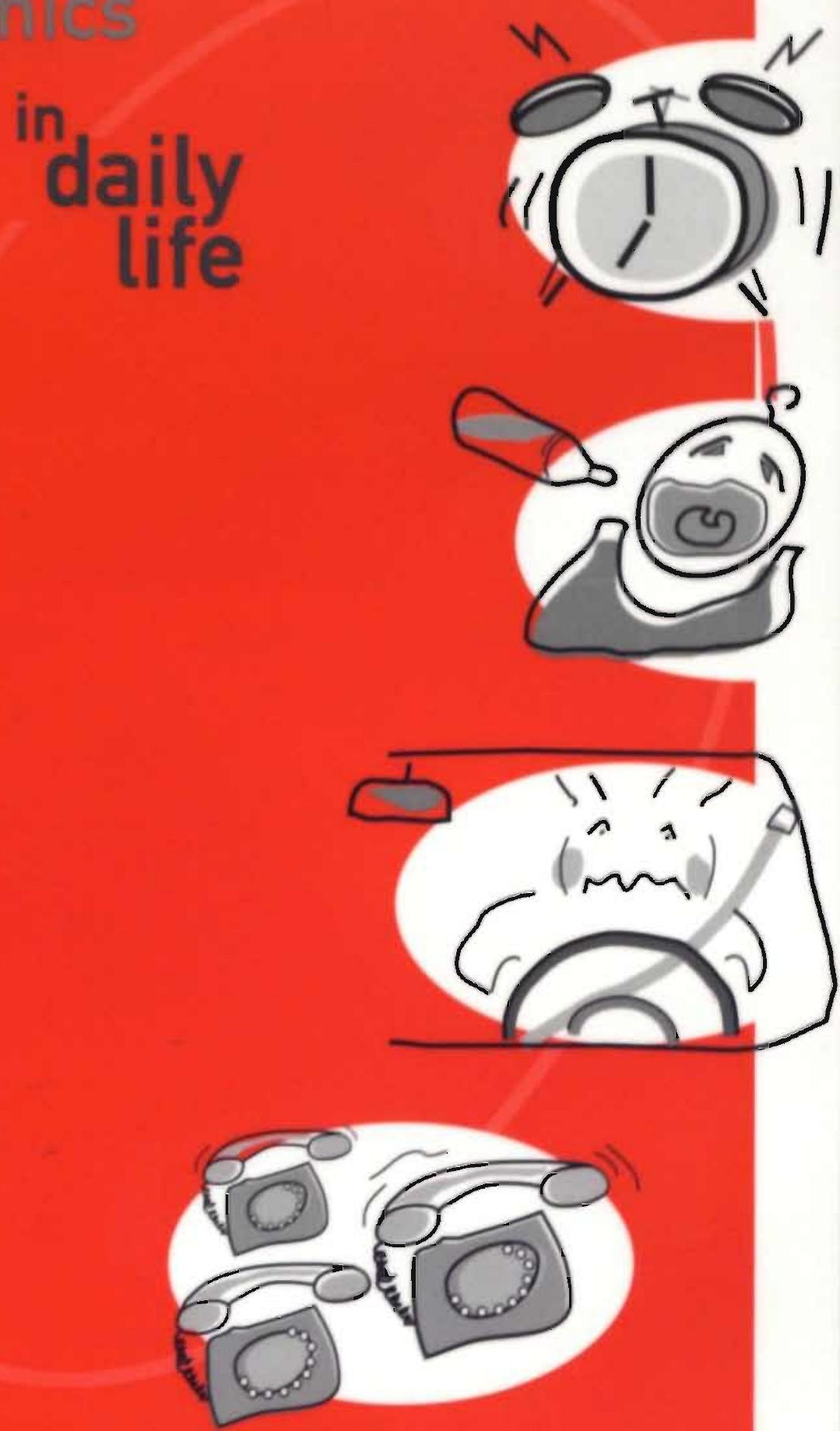

M. van Eck 
STRESS, MOOD, AND CORTISOL DYNAMICS IN DAILY LIFE 


\section{UPSCR}

\section{IPSER Series in Ecological Psychiatry}

M.W. deVries

\section{Editor}

$* * *$

\section{Vol.1 Assessing Schizophrenia in Daily Life}

- The Experience Sampling Method Philippe A.E.G. Delespaul

Vol.2 (Genetic) Epidemiology as a Tool to Identify Risk Factors for Emergence and Persistence of Illness in the Functional Psychosis Jim J. Van Os

Vol.3 Stress, Mood, and Cortisol Dynamics in Daily Life

Marleen M.M. Van Eck

The International Institute for Psycho-social and Socio-ecological Research (IPSER)

Collaborating Center of the World Federation of Mental Health Associated with the Rijksuniversiteit Limburg

Cover design: Mark. Delahay

Druk: Datawyse Maastricht

van Eck. Marleen

Stress, mood, and cortisol dynamics in daily life/Marleen yan Eck.

Thesis University of Limburg. Maastricht. - With ref.-

With summary in Dutch.

ISBN 90-9009783-X

Subject headings: perceived stress/experience sampling/cortisol/mood. 
Promotor

Prof. Dr. M.W. deVries

\section{Co-promotor}

Dr. N.A. Nicolson

\section{Beoordelingscommissie}

Prof. Dr. H.M. van Praag (voorzitter)

Prof. Dr. A.P.W.M. Appels

Prof. Dr. M.A. van den Hout

Prof. Dr. J.F. Orlebeke (Vrije Universiteit Amsterdam)

Prof. Dr. A.A. Stone (State University of New York)

Funding was provided by the Netherlands Organization for Scientific Research (NWO; grant 900-564-024) and the University of Limburg. 
It is not the large things that send a man to the madhouse... No, it's the continuing series of small tragedies that send a man to the madhouse Not the death of his love but a shoelace that snaps with no time left.

Charles Bukowski, 'The shoelace'. Bukowski Reads His Poetry, Takoma Records, Santa Monica, Calif., 1980. 

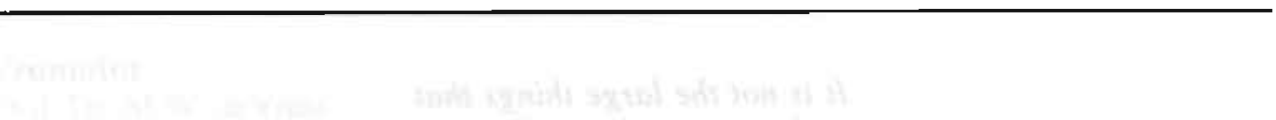

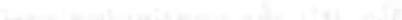




\section{Contents}

page

Chapter 1

Chapter 2

Chapter 3

Chapter 4

Chapter 5

Chapter 5.1

Chapter 5.2

Chapter 6

Chapter 7

Chapter 8

References

Appendices

Summary

Samenvatting

Dankwoord

Curriculum vitae

Introduction $\quad 3$

$\begin{array}{ll}\text { Methods } & 21\end{array}$

The nature of stress in daily life: events, appraisals, and activities

The effects of perceived stress and stressful daily events on mood states

Submitted for publication

Cortisol and catecholamine excretion in relation to perceived stress

Perceived stress and salivary cortisol in daily life Annals of Behavioral Medicine 16(3): 221-227 (1994) Four-teen hour urinary catecholamine excretion in relation to perceived stress

and stressful daily events on salivary cortisol

Psychosomatic Medicine (in press)

Individual differences in cortisol responses to a laboratory 127 speech task and their relationship to responses to stressful daily events

Biological Psychology (43): 09-84 (1996)

Concluding remarks 


\section{Chapter 1}

Introduction 


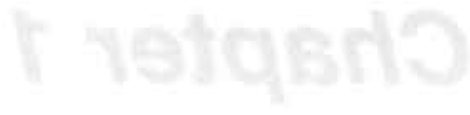

noilsuboyln 
Our lives can be characterized as constant adaptation to sudden or more gradual changes of our environment. Sometimes these changes are small and we can adapt to them without even noticing them, but at other times, these changes can be too frequent or severe and thereby threatening. In any case, at some point in life every human being will experience 'stress'; the day when inexplicably nothing seems to go right is a universal experience. In modern society, stress has become a popular concept and has gained a common everyday usage. We use it, for instance, to explain a wide variety of outcomes, mostly negative: it may serve to explain an upcoming headache or a bad mood, an upset stomach, 'nerves' or a lack of motivation. It is also common practice to attribute eccentric or incomprehensible behavior of friends and acquaintances to the fact that they are 'under a lot of stress'. Stress is even referred to as an 'epidemic disease".

One reason for the popularity of the stress concept may be its increasing linkage to disease and well-being. Research in the field of stress has indeed indicated that in addition to its role in the development and course of a wide range of somatic diseases (e.g. cardiovascular disease, infectious diseases, diabetes mellitus), psychosocial stress plays an important role in the etiology and maintenance of various psychiatric illnesses, including depression, anxiety, and psychosomatic disorders (Brown, 1993; Chrousos \& Gold, 1992; Paykel \& Dowlatshahi, 1988). During the last decade there has been an increase in the Netherlands in the number of mental disorders among those eligible for work disability benefits, as well as an increase in the duration of sickness absences because of mental disorders (Schroer, 1993). Of all disabled workers, more than $28 \%$ (227,000 individuals) received a benefit in 1990 after a mental disorder had been diagnosed; this in contrast to $21 \%$ in 1981. The total percentage of work disability due to mental disorder among civil servants increased over the same period from 35\% to 50\% (Knepper, 1991). In more than $80 \%$ of these cases, the mental disorder was described as "overstrain " (Schroer, Nijhuis, \& van Zutphen, 1988); in 40\% of mental disorder disabilities 'stress at work' was specifically mentioned. These figures are alarming not only for the individual (ex)worker, but also for the corporate community and society as a whole. Companies have to dea! with a considerable loss of work time, resulting in higher production costs and a pressure toward greater work productivity, which may lead again to an increase in absenteeism and disability due to a higher workioad. The costs for society due to stress-related absenteeism and disablement (including medication and health care utilization) have been estimated in 1988 at 9 billion Dutch guilders (Laan, 1989). In an effort to reduce feelings of stress and to increase physical fitness and improve mental health, fitness programs (Bruijn, 1988) and stress management courses (Marcelissen, 1989) at or near the work place have rapidly increased in popularity in the Netherlands, further encouraged by laws placing greater responsibility for the employee's health and well-being on the employer (ARBO-law). The main aims of introducing these programs at the work place are to increase the employees" physical health, thereby reducing absenteeism and work-related stress, and increasing work performance (Cox, Gotts, Boot, \& Kerr, 1988; Falkenberg, 1987).

Although the word stress seems to be implicitly understood by all, it is given substance by an infinite number of different things. For one person stress is having a fight with his or her spouse, for the other it is a busy day at work, and again for another stress may mean having nothing to do. Earlier research emphasized the impact of major disruptions in personal and social life (e.g. death of spouse, unemployment) (Holmes \& Rahe, 1967), but more recent studies, have alerted us to the fact that the minor but much more frequent occurring hassles of daily living also 
play a role in both somatic and psychological health outcomes (DeLongis, Coyne. Dakof, Folkman, \& Lazarus, 1982). These minor but often chronic stresses at work, in the family, and in other social relationships are also much closer to the lay person's concept of stress, as described above, and to problems that may lead to absenteeism and disablement at the work place. Although responses to acute experimental stress have been studied extensively, little is known about the nature and effects of the stresses of daily living. Knowledge about the nature of psychological and physiological responses to daily life stress is possibly of great theoretical importance for an understanding of the etiology and course of both physical and psychological pathology, and is the object of the present thesis. The first goal of the study is to describe the nature and scope of daily life stress, contrasting the experiences of individuals who perceive themselves to be stressed with those who do not. Second, this research investigates affective and neuroendocrine responses in relation to stressful daily events and tries to explain individual differences in such responses. Before explicitly going into the major research questions and how they will be addressed, we will provide a brief overview of various theoretical approaches to stress, followed by a discussion of the stress-illness relationship. Next, the available evidence concerning the influence of daily stress on psychological and physiological functioning will be discussed.

\section{THE CONCEPT OF STRESS: PSYCHOLOGICAL VIEWS}

Although the term 'stress' has been widely used since the beginning of this century (Cannon, 1929), controversy concerning its definition remains. Three distinct definitions of stress can be found in the literature, which vary in their emphasis on stimulus, response or intervening process and which are closely related to ways in which stress can be measured: in terms of a stimulus or a situation (e.g. noise, death of a spouse, mathematics task), in terms of a behavioral or physiological response (e.g. performance decrements, increased heart rate, or elevated cortisol), and stress as a state of imbalance. when the (perceived) demands on the person tax or exceed his (perceived) capabilities to deal with these demands (Goldberger \& Breznitz, 1982). These definitions also vary in their emphasis on physiological/endocrinological versus psychological/behavioral processes. For the sake of simplicity, the various approaches to the concept of stress will be divided below into psychological and physiological stress traditions, starting with the psychological tradition.

\section{Life change approach}

In psychology and medicine, the term stress has traditionally been used to refer to a stimulus or situation which produce certain (behavioral, psychological, and/or physiologicai) responses (Hinkle. 1974; Wolff \& Goodell, 1968). Since the development of a method for the quantification of major life changes by Holmes and Rahe (the Social Readjustment Rating Scale [SRRS]; (Holmes \& Rahe, 1967)), the association between stress and disease has been extensively investigated. The hypothesis guiding the Holnes-Rahe approach was that an increased number of life changes requiring considerable adjustment would precede the onset of illness. This implies that life events are, in and of themselves, the precipitating cause of illness. The SRRS self-report checklist allows the respondent to indicate which events (both positive and negative, such as divorce, birth or death of a close family member, loss of job) were experienced within a specified time period. To assess the significance of 
these events, life change units were obtained by having different groups of subjects indicate the amount of readjustment (independent of the desirability or emotions induced by the event) they thought would be required. The item 'marriage' was used as anchor point in these ratings. In this way, the total number of events or a summation of the life change units could be obtained and then related to measures of illness and health. Since its development, the SRRS has been used in numerous studies to investigate the association between life changes and illness, and life changes have, for instance, been related to sudden cardiac death (Rahe \& Arthur, 1978; Rahe \& Lind, 1971). In general, life events have been shown to be related to various somatic disorders, including heart disease, sudden cardiac death, and infectious diseases (Holmes \& Masuda, 1974), and to various psychiatric disorders, including acute schizophrenia (Brown, 1974) and depression (Paykel, 1974).

\section{Criticism of the life event approach}

The life event approach, in which stressors are basically treated as objective environmental stimuli, has received considerable criticism (Aagaard, 1984; Rabkin \& Streuning, 1976; Schroeder \& Costa, 1984). Some points of criticism are: only low to modest correlations have been found between life event scores and health outcomes: the exclusive use of the category of major life events as a synonym of stress and the use of 'social readjustment' as the sole dimension to define stress; psychometric problems including reliability and content validity; inclusion of many items which may have been confounded with the outcome measures studied; and the use of retrospective study designs, which could lead to an exaggeration of the importance attributed to past events from a need to justify subsequent illness.

Later life event checklists (e.g. the Life Experience Checklist (Sarason, Johnson \& Siegel, 1978) and the PERI lists of life events (Dohrenwend, Krassnoff, Askenasy, \& Dohrenwend, 1978)) addressed some of these issues. Both negative and positive life change scores were included to be able to investigate whether positive and negative life events exhibit the same relationship with physical and emotional outcomes. Also included were additional subjective evaluations of the life events, like desirability, impact, controllability, and predictability. The basic objections, however, remained: retrospective research design, limited and predetermined sample of life events, too little consideration of the subjective evaluation of the event or the ongoing relationship between person and environment. One exception is the laborintensive and time-consuming semi-structured interview (the Bedford Life Events and Difficulties Scale [LEDS] (Brown, 1974)) method developed by Brown and colleagues (Brown \& Harris, 1989) for the measurement of life events and chronic difficulties. Here, in addition to the use of objective criteria for what can be considered a life event or enduring problem, this approach incorporates the influence of psychosocial factors (the biographical context) on the subjective experience of events.

\section{Transactional theory of stress, appraisal and coping}

The transactional stress theory developed by Lazarus and colleagues (Lazarus, 1966) places particular emphasis on the importance of an individual's subjective evaluation ('appraisal') of stressful events. In this now very widely accepted approach to the concept of stress, psychosocial stress is defined as "a relationship between the person and the environment that is appraised by the person as taxing or exceeding his or her resources and endangering his or her well-being"' (Lazarus \& Folkman, 1984b, p. 19). In the present study we will use the term 'stress' 
in this context. This approach states that whether a state of stress develops depends both on external conditions in the environment as well as on the constitutional vulnerability and the adaptive capacity of the person. It therefore may explain why identical events do not lead to stress perceptions in all persons, since differences in personality, social support, prior experience with the stressor, and so on, may effect the person's ability to deal with the stressor. Central to the transactional approach are two processes that act as mediators between the behavior of the person and the environment: first, the subjective evaluation or appraisal of potentially stressful events or situations, and second, the process of coping that follows after an event has been appraised as stressful.

The concept of appraisal refers to three distinct types of appraisal: primary appraisal, secondary appraisal, and reappraisal. Primary appraisal refers to the significance of the situation for a person's well-being ('What is at stake?'). Three broad and basic categories of evaluations of well-being can be distinguished (Lazarus \& Launier, 1978): 'irrelevant', meaning that the situation has no subjective relevance and can therefore be ignored, 'benign-positive' when the person regards an event as signifying a positive state of affairs, and 'harmful-stressful'. Three types of stressful appraisals are mentioned: 'harm/loss', which refers to harm already done, 'threat', which refers to a future potential for harm or loss, and 'challenge', which is defined as a potential for growth or positive gain. Stress can thus have both positive and negative effects. These categories of stressful appraisals do not have to be mutually exclusive, a loss, for example, can at the same time entail a future threat. Secondary appraisal refers to the evaluation of the coping recourses and options available to deal with a stressful transaction ('What can I do about it?'). Consequently, it follows that secondary appraisal determines to a large extent whether one feels threatened as opposed to challenged, and it will shape the coping strategies of the person under stress. Reappraisal points to the feedback process from the ongoing interaction between the person and the environment, which may lead to changes in primary and secondary appraisals.

The concept of coping refers to "the process of managing external and/or internal demands that tax or exceed the resources of the person" (Lazarus \& Launier, 1978. p. 311). Two broad categories of coping nechanisms or strategies have been distinguished, each serving a major adaptive function: problem-focused coping is directed at actively modifying the self or the stressful situation, while emotionfocused coping is directed at managing feelings of distress. Both are used in almost every stressful situation. Coping and appraisal mutually influence each other throughout a stressful encounter, since if the person-environment relation changes as a result of coping efforts the evaluation of the situation will change as well.

Individual differences in stress, appraisal, and coping responses are determined at least in part by an individual's adaptive capacity at the physiological, psychological, and social level (Lazarus \& Folkman, 1984b). Examples at the physiological level are genetic or constitutional factors like physical fitness and genetically determined or acquired immunity. At the psychological level, there are personal goals and commitments, and personality characteristics like self-esteem. neuroticism, and sense of control. At the social level, important antecedent variables are social economic status, social support networks, and sociocultural norms and values. Stressful events or situations can be described with respect to both immediate and long-ternn effects. Short-term effects at the physiological level include somatic or physiological changes and, in extreme cases, acute illness. Effects at the psychological level include positive or negative emotions, and at the social level they 
may entail social disturbances or group alienation. The general categories of longterm outcomes are: physical health (e.g. chronic illness, impaired physiological functioning); subjective well-being (e.g. depression, anxiety, other psychological symptoms); and social functioning (e.g. socoal failure, change in social role). All levels as described ahove are assumed to he interrelated.

\section{Criticism of the transactional approach}

According to the transactional model, stress is best regarded as a complex rubric, a convenient term to refer to the operation of many variables and processes in situations in which the demands tax or exceed the person's resources. This complexity and diversity of aspects related to the transactional definition of stress forces one to make choices about which classes of variables to study. Lazarus and Folkman's definition of stress has also received its share of criticism. The main point of critique has been that it is overly subjective, in that both stress and coping resources are defend and measured in terms of appraisal processes (Dohrenwend \& Shrout, 1985). The choice between obtaining subjective or relatively objective indices of stress is an important one and should depend on the purposes of the study. If the goal is to investigate the etiologic role of life circumstances as precursors of pathology, one should try to reduce the subjectivity of event measures to reduce possible confounding of the stress-pathology relationship. Even then, knowledge about the context of the event, about the circumstances surrounding the event, may still be important for an understanding of why the disorder developed. If not prediction of pathology but understanding the process of stress per se is the main aim. then the subjective experience of stress is worth studying in and of itself (Wagner, 1990). Although a certain degree of circularity (the same process is being measured in the independent and the dependent variables) is inevitable with relational definitions of stress, this can be limited by asking what it is about the person, in interaciion with a given environmental situation, that generates appraisals of harm, threat, or benefit (Lazarus, De Longis, Folkman, \& Gruen, 1985).

\section{THE CONCEPT OF STRESS: PHYSIOLOGICAL VIEWS}

Since most psychological or behavioral signs of stress are often of a subjective nature (such as subjective self-reports of negative affect, low job-satisfaction, anxiety, or agitation), a substantial body of stress research has focused on more objective indicators of stress. Characteristic of both humans and animals is that they respond to stressful events with various well-defined and quantifiable physiological responses, such as the release of stress hormones in the blood, increased heart rate and blood pressure. Moreover, repeated or chronic physiological activation is held to be an important factor in the link between stress and disease. Therefore, we will provide a brief review of the physiological stress tradition.

\section{Short history of early studies}

Early stress research in animals emphasized that responses of the organism to a wide variety of stimuli were non-specific. Selye (1936, p. 32) defined stress as "the nonspecific response of the body to any noxious stimulus". This nonspecific response to stimuli such as heat, cold, or exercise, has been called the General Adaptation Syndrome; it is characterized by increased adreno-cortical activity, degeneration of the thymus and lymphatic structures; and hemorrhage and ulceration of the stomach 
and other parts of the gastrointestinal tract. Three temporal phases can be distinguished: the alarm phase, the stage of resistance, and the stage of exhaustion. According to the theory, conditioning factors (e.g. age, genetic predisposition, diet, drugs) will determine which specific disease of adaptation will manifest itself after prolonged exposure to a stressor. The pituitary-adrenal-cortical axis regulates the release of corticosteroids from the adrenal cortex, with cortisol as the most important glucocorticoid homone in humans, and with the potential of exerting its effects on practically all normal body cells and tissues. Some of the main functions of cortisol are: stimulation of gluconeogenesis, inhibition of glucose uptake, suppression of inflammation and suppression of numerous immune functions (Munck, Guyre, \& Holbrook, 1984). Whereas Selye especially emphasized the activity of the pituitaryadrenal-cortical axis, Cannon (1929) investigated the role of the sympathetic-adrenalmedullary system, which regulates secretion of the catecholamines adrenaline and noradrenaline. Cannon showed that besides physical stimuli, non-physical or emotional stimuli could also activate the physiological system. He formulated the concept of 'physiological homeostasis', which means that those physiological responses will be triggered which meet the demands of the environment. The increased activity of the central nervous system (CNS) and release of catecholamines were interpreted as an emergency' reaction. In this way, the organism is prepared for appropriate action aimed at coping with the stressor and restoring control: "fight or flight'. Enhanced sympathetic activity leads to increases in glucose, respiratory rate, blood pressure, and heart rate, and to a blood flow away from the intestines to the muscles, where oxygen and nutrients are most needed. It was Mason (1968) who pointed out, after reviewing earlier studies on animals, healthy humans, and psychiatric patients, that many of the stressful experiences that had been investigated shared one common characteristic: the induction of emotional arousal. For instance, when an animal is exposed to a novel or unfamiliar environment, it is the psychological relevance of the stressor that determines the stress response and not the particular physical stressor. Important conditions for eliciting responses were found to be novelty, uncertainty, unpredictability, involvement, and anticipation of unpleasant experiences. The importance of emotional arousal as a trigger of the stress response and the fact that those specific responses are triggered that meet the demands of the environment are not compatible with a non-specific stress response as formulated in Selye's theory; the physiological stress response is therefore best regarded as a differentiated response of the organism.

Another important finding was that marked individual differences exist $1 \mathrm{n}$ the adrenal-cortical response to a given stressful situation (Mason, 1968; Rose, 1984). These differences seem to be related to individual coping styles or defense mechanisms and to how the event is appraised. Rose (1984) noted that cortisol responses extinguish rapidly in individuals upon re-exposure to most kind of events, reflecting adaptation.

\section{Recent physiological stress models}

More recent models have integrated the major physiological concepts described above. In the animal model proposed by Henry and Stephens (1977) it was suggested that the nature of behavioral and accompanying physiological reactions are determined by the outcome of the appraisal process, which in turn results in different coping strategies. Two extremes can be differentiated with respect to behavioral, physiological, and CNS activities. When the appraisal process results in fear or anxiety but the animal still tries to eliminate the threat by active effortful 
coping, this will lead to sympathetic activation, for example a pronounced increase in adrenaline release and heart rate. This resembles the 'fight/flight' response described by Cannon (1929), with territorial control (mobility, display, aggression) as behavioral components. However, when the appraisal results in uncertainty or loss of control and the animal sees no escape or has no adequate coping response available, the hypothalamus-pituitary-adrenocortical axis is activated to release ACTH and cortisol, as was originally described by Selye. At the behavioral level this response is accompanied by inhibition (freezing or doing nothing), subordination, and decreased sexual and maternal behavior ('conservation-withdrawal'). Frankenhaeuser's effortdistress model (1983), based on studies of healthy humans (e.g. Frankenhaeuser, Lundberg, \& Forsman, 1980; Lundberg \& Frankenhaeuser, 1980), is consistent with the model of Henry and Stephens (1977). Both focus on two components of psychological arousal, effort and distress (experienced either singly or in combination), which seem to be differentially related to catecholamine and cortisol secretion. A state of 'effort without distress' (a positive affective state) is accompanied by catecholamine secretion, while under this condition cortisol secretion is low or may be actively suppressed. On the other hand, a state of 'effort with distress' (which may characterize many stressful events) is associated with an increased secretion of both catecholamines and cortisol. An example of this state is mental work carried out under conditions of stimulus overload. The state of 'distress without effort', which may be reflected in giving up or feelings of helplessness, is accompanied by an increase in cortisol secretion, and resembles the "conservationwithdrawal' response as described by the model of Henry and Stephens. Personal control seems to be an important modulating factor here, with less control leading to more distress. In summary, cortisol, in comparison to other stress-labile hormones (e.g. catecholamines, growth hormone, prolactin, testosterone), appears to show the most specific relationship to subjective distress as opposed to effort or general arousal (e.g. Lundberg \& Frankenhaeuser, 1980), and is thought to reflect the adequacy of coping behavior (e.g. Vickers, 1988).

\section{STRESS, HEALTH, AND DISEASE}

As described above, in most cases the psychological and physiological responses to stress reflect normal, adaptive processes which prepare the body to cope with the situation. Negative emotions alert people to deal with or avoid difficuli situations, and physiological reactions prepare the body for 'fight or flight' responses (Chrousos \& Gold, 1992). It has also been noted that stress does not always lead to negative effects like poor performance or illness, but may result in training or toughening effects as well (Dienstbier, 1989; Ursin, 1980). Thus in the normal course of events, when coping is successful and the stressful experience is of short duration, the benefits largely outweigh the costs of the stress response. Only when behavioral or cognitive coping strategies for dealing with stressful experiences are not effective or not available and stress endures are psychological and physiological responses likely to become exaggerated or prolonged, thereby increasing the risk for pathology to develop. In humans only extreme stressors will evoke responses like fighting or fleeing, while the majority of events experienced in daily life ask for more subtle solutions. Since rapid availability of oxygen, nutrients, glucose, and so on is not necessary to meet the demands of contemporary daily challenges, disruption of bodily homeostasis may occur. 
question in this regard is whether individual differences in laboratory reactivity reflect those occurring during stressful situation in real-life (Turner, Ward, Gellman, Johnston, Light, \& van Doornen, 1994). The usefulness of laboratory assessments rests, in part, on this assumption. Later in this chapter we will deal with these subjects in more detail.

\section{FROM MAJOR LIFE EVENTS TO MINOR DAILY EVENTS}

As already described previously, the major life event approach has received considerable criticism relating to aspects of both design and method. A basic objection has been the reliance on retrospective research designs. The retrospective assessment of summaries of life events over many months obscures the temporal, dynamic interplay between environmental demands, appraisals of demands, and outcomes (Stone \& Shiffman, 1992), which is central in the transactional theory of stress and coping. More details of the stress process are needed to unravel the mechanisms underlying the relationship between events and disease. The dynamic processes between demands, appraisals, and outcomes are presumed to change so quickly that they can only be captured through frequent assessment. Of course, the frequency of measurement should follow the fluctuations of the phenomena under study; fluctuations in heart rate would ask for minute to minute assessments over periods less than a day, while if we were interested in the relationship between stress and premenstrual symptoms, daily measurements over several months would be more appropriate.

Another disadvantage of retrospective research designs is that the recall of events over relatively long periods may be influenced by biases. Recall of events and mood over long time periods is obviously limited by forgetting, but research suggests that this is not a random process. For instance, it has been shown that recall of past mood and both positive as well as negative events is strongly influenced by the respondent's mood at the time of recall (Bower, 1981; Teasdale \& Forgarty, 1979); the current mood state makes mood-congruent memories more accessible and moodincongruent memories less accessible. Although this applies especialily to global ratings of past experiences, it is also likely that recall of specific events may be affected; emotionally important or salient events are more likely to be remembered than less salient ones (Strongman \& Russell, 1986). By reducing the recall time interval, these biases can be also reduced.

Taken together, these points have lead to the conclusion that other categories of events, such as chronic stressors and the nnilder but much more frequent stresses of daily living, should also be investigated concerning their relationship to health. Since the events of everyday life eccur musch more frequently than the major life events, they might be even more important to well-being. The investigation of minor daily events may also help to delineate the stressful features of chronic experiences, such as work stress. Indeed, an increasing number of studies have shown that daily life events are related to lower psychological well-being and increased somatic symptomatology, even after the possible confounding effect of major life events has been controlled for (e.g. DeLongis et al., 1982; Stone, Neale, \& Shiffman, 1993; Zarski, 1984). Daily events or 'hassles' are defined as "the experiences and conditions of daily living that have been appraised as salient and harmful or threatening to the endorser's wellbeing" (Lazarus, 1984, p.376). Examples of situations that can be hassles are: getting caught in a traffic jam, having to wailt, quarreling with your child. There are several 
question in this regard is whether individual differences in laboratory reactivity reflect those occurring during stressful situation in real-life (Turner, Ward, Gellman, Johnston, Light, \& van Doornen, 1994). The usefulness of laboratory assessments rests, in part, on this assumption. Later in this chapter we will deal with these subjects in more detail.

\section{FROM MAJOR LIFE EVENTS TO MINOR DAILY EVENTS}

As already described previously, the major life event approach has received considerable criticism relating to aspects of both design and method. A basic objection has been the reliance on retrospective research designs. The retrospective assessment of summaries of life events over many months obscures the temporal, dynamic interplay between environmental demands, appraisals of demands, and outcomes (Stone \& Shiffman, 1992), which is central in the transactional theory of stress and coping. More details of the stress process are needed to unravel the mechanisms underlying the relationship between events and disease. The dynamic processes between demands, appraisals, and outcomes are presumed to change so quickly that they can only be captured through frequent assessment. Of course, the frequency of measurement should follow the fluctuations of the phenomena under study; fluctuations in heart rate would ask for minute to minute assessments over periods less than a day, while if we were interested in the relationship between stress and premenstrual symptoms, daily measurements over several months would be more appropriate.

Another disadvantage of retrospective research designs is that the recall of events over relatively long periods may be influenced by biases. Recall of events and mood over long time periods is obviously limited by forgetting, but research suggests that this is not a random process. For instance, it has been shown that recall of past mood and both positive as well as negative events is strongly influenced by the respondent's mood at the time of recall (Bower, 1981; Teasdale \& Forgarty, 1979); the current mood state makes mood-congruent memories more accessible and moodincongruent memories less accessible. Although this applies especialily to global ratings of past experiences, it is also likely that recall of specific events may be affected; emotionally important or salient events are more likely to be remembered than less salient ones (Strongman \& Russell, 1986). By reducing the recall time interval, these biases can be also reduced.

Taken together, these points have lead to the conclusion that other categories of events, such as chronic stressors and the nnilder but much more frequent stresses of daily living, should also be investigated concerning their relationship to health. Since the events of everyday life eccur musch more frequently than the major life events, they might be even more important to well-being. The investigation of minor daily events may also help to delineate the stressful features of chronic experiences, such as work stress. Indeed, an increasing number of studies have shown that daily life events are related to lower psychological well-being and increased somatic symptomatology, even after the possible confounding effect of major life events has been controlled for (e.g. DeLongis et al., 1982; Stone, Neale, \& Shiffman, 1993; Zarski, 1984). Daily events or 'hassles' are defined as "the experiences and conditions of daily living that have been appraised as salient and harmful or threatening to the endorser's wellbeing" (Lazarus, 1984, p.376). Examples of situations that can be hassles are: getting caught in a traffic jam, having to wailt, quarreling with your child. There are several 
forms of daily life stress. Some stressors are chance events, and usually rare in occurrence (e.g. flat tire when in a hurry, out of toilet paper just when needed, unexpected phone calls), while others are repeated, either because a person remains in the same ongoing social situation (e.g. work, marriage) with consistent demands (e.g. high work load, conflicts with spouse), or because of certain personality dispositions, such as a person's ineffective style of coping with common situations (Kanner, Coyne, Schaefer, \& Lazarus, 1981). Since no one lives a life completely without stress, the impact of hassles on physical and mental health is thought to depend on factors such as a chronically high frequency of stressful daily events, an increase in hassles during a given period (the 'last straw'), the presence of hassles with compelling psychological importance, or an underlying (biological) vulnerability to stress or to a specific illness (Kanner et al., 1981; Zautra, Guarnaccia, Reich, \& Dohrenwend, 1988).

The current study investigates the impact of minor daily events on mood and the HPA system. In the next two sections we present a review of the available literature concerning the relationship between daily events, on the one hand, and mood and cortisol responses, on the other hand. The following related subjects will be of special interest: individual differences in mood and cortisol responses, the context of the event, and event appraisa!s.

\section{STRESSFUL DAILY EVENTS AND MOOD STATES}

A common approach to the study of daily stress examines the relationship between stressful events and mood by assessing both once a day for several weeks (Stone \& Shiffman, 1992); others have assessed these phenomena on a monthly basis (e.g. Lewinsohn \& Libet, 1972). The simplest approach is open-ended: subjects are asked to describe the day's most stressful events (Rehm, 1978) or asked to describe anything that went wrong during the day (Caspi, Bolger, \& Eckenrode, 1987; Eckenrode, 1984). Responses are then classified into discrete categories. The most often used method for assessing daily events is the event checklist, where, from a list of events, subjects are asked to identify which events have occurred over a specified time period, ranging from one day to one month. Examples of monthly checklists are the Unpleasant Events Schedule and the Pleasant Events Schedule (Lewinsohn \& Amenson. 1978), the Hassles and Uplifts scale (Kanner et al., 1981), and the Inventory of Small Life Events (Zautra \& Guarnaccia, 1986). Items on these checklists were formulated by the researchers or taken from existing scales and constructed to cover events in major life domains, such as family, work, leisure, and household maintenance. Examples of daily checklists are the Daily Life Experience checklist (Stone \& Neale, 1982), the Daily Stress Inventory (Brantley, Waggoner, Jones, \& Rappaport, 1987), and the Daily Stress Scale (Bolger, DeLongis, Kessler, \& Schiling, 1989a). Here. by using an open-ended format. a more representative domain of events was first obtained by sampling events which conform to certain criteria (e.g. 'stressfulness', (Brantley et al., 1987)) from the participant population in which the checklist was to be used. Elicited events were then grouped into various categories to form the checklist.

Regardless of what approach has been used to study daily stress, several studies have shown a same-day association between daily stress and negative mood (Affleck, Tennen, Urrows, \& Higgins, 1994; Bolger et al., 1989a; Clark \& Watson, 1988; DeLongis, Folkman, \& Lazarus, 1988; Eckenrode, 1984; Larsen, Diener, \& Emmons, 1986; Lewinsohn \& Libet, 1972; Rehm, 1978; Stone \& Neale, 1984; Stone et 
al., 1993). Results with respect to positive mood are inconclusive; most studies have not differentiated between negative and positive mood, but in those that have, positive mood was either lower (Neale, Hooley, Jandorf, \& Stone, 1987; Repetti. 1993; Stone \& Neale, 1984) or unchanged (Watson, 1988) on days when many stressful events occurred.

Evidence for large individual differences in the magnitude and the direction of the association between daily stress and mood has been found in a number of studies. Examples of factors related to such differences are self-esteem (Campbell, Chew, \& Scratchley, 1991), social support (Affleck et al., 1994; Barling \& Kryl, 1990; Caspi et al., 1987; DeLongis et al., 1988), major life events (Affleck et al., 1994; Caspi et al., 1987), and neuroticism (Affleck et al., 1994; Bolger \& Schilling, 1991; Eckenrode, 1984). The moderating role of chronic stress or long-term difficulties on the relationship between daily life stress and mood has received relatively little attention. In a study by Caspi et al. (Caspi et al., 1987), the chronic stress of living in a low quality neighborhood increased the immediate effects of stressful daily events on mood and also increased the likelihood that daily stressors had an enduring effect on next day's mood. Investigating the influence of perceived stress levels on the daily stress-mood relationship may be important, since individuals experiencing high perceived stress feel their lives to be unpredictable, uncontrollable, and overloaded, and can be seen as at risk for the development of stress-related somatic and mental health problems (Cohen, Kamarck, \& Mermelstein, 1983). Personality factors probably play a very important role in perceived stress. Self-report measures of stress have been found to correlate significantly with measures of negative affectivity or neuroticism (Watson, 1988; Watson \& Pennebaker, 1989). Negative affectivity reflects a general disposition to focus on the negative aspects of self and others and to experience events as negative or distressing (Eysenck \& Eysenk, 1968; Watson \& Clark, 1984). In stress research, negative affectivity has been treated in two different ways. First, it is thought of as a confounding variable, spuriously inflating the relationship among stressors, stress symptoms, and affective outcomes (Chen \& Spector, 1991: Costa \& McCrae, 1987; Schaubroeck, Ganster, \& Fox, 1992; Watson \& Pennebaker, 1989). However, negative affectivity has also been investigated as a moderator variable. influencing individuals' reactivity to stressful events. Several studies demonstrated that individuals higher in negative affect show greater negative mood reactivity to stressors (Affleck et al., 1994; Bolger \& Schilling, 1991; Marco \& Suls, 1993). Differences between high and low negative affect individuals in how events are appraised or coped with may possibly account for these differences in mood reactivity.

Little is known about how long the effects of daily events on mood persist. although this would be important for understanding the consequences of minor events. Heaith chances may only occur when daily events are very frequent and negative mood responses persist in time, for instance while coping has been unsuccessful or incomplete. The available literature from end-of-day studies suggests that daily stressors affect same-day mood but do not necessarily affect mood on subsequent days (Bolger et al., 1989a; DeLongis et al., 1988; Lewinsohn \& Libet, 1972; Neale et al., 1987; Rehm, 1978; Stone \& Neale, 1984), but more research is necessary to clarify this subject.

Since people experience a large range of stressful events in daily life, several studies have been employed to try to increase our understanding of what categories of events and which event characteristics are most strongly related to well-being. Several studies indeed seem to indicate that the psychological impact of daily events 
depends on the context in which they occur. Particularly undesirable work events and interpersonal conflicts appear to have the strongest relationship to negative mood states (Bolger et al., 1989a; Bolger \& Schilling, 1991; Clark \& Watson, 1988; Repetti, 1993; Stone, 1987). Work events have also been associated with lower positive mood (Stone, 1987). Despite the important role of the concept of appraisal in contemporary stress theory in determining whether a daily event is experienced as stressful or not, little research has been done in relation to appraisals of naturally occurring daily events. There is evidence from numerous laboratory studies and several naturalistic studies that subjects' personal ratings of events, like frequency, demand, controllability, and predictability of events, improve prediction of outcomes such as performance, anxiety, depression, negative affect, and tension (Averill, 1973; Cohen, 1980; Dewe, 1991; Folkman, 1984; Katz \& Wykes, 1985; McGrath \& Beehr, 1990; Miller, 1979; Peeters, Buunk, \& Schaufeli, 1995; Sarason et al., 1978; Thompson, 1981). Additional research is needed that studies cognitive appraisals of daily events in a natural environment and investigates how these appraisal dimensions relate to mood.

While end-of-day assessments of daily hassles have important advantages compared to the major life event approach, several drawbacks still remain; these will be discussed in the final section of this chapter.

\section{STRESSFUL DAILY EVENTS AND CORTISOL DYNAMICS}

Until recently, the two main approaches to the investigation of the impact of stressful events on the HPA axis in humans have been laboratory reactivity studies and 'natural experiments'. While both approaches have been of great value for our understanding of the stress process, each has limitations. We will see that neither approach is adequate if one adopts the dynamic conceptualization of stress as a process, which changes over time and in relation to the environment.

Most studies of psychophysiological reactivity have used standardized laboratory tasks such as physical strain or exercise, mental stress tasks (e.g. reactiontime tasks, arithmetics, computer games), cold pressor tests, and mood induction, all with questionable relevance to real life (Berger, Bossert, Krieg, Dirlich, Ettmeier, Schreiber, et al., 1987; Brown, Sirota, Niaura, \& Engebretson, 1993; Bullinger. Naber, Pickar, Cohen, Kalin, Pert, et al., 1984; Forsman \& Lundberg. 1982; Mason, Hartley, Kotchen, Mougey, Ricketts, \& Jones, 1973). Ecological valid information about the sources of stress people experience in their daily lives, and the responses to it, seem difficult to obtain in laboratory experiments (Lazarus \& Launier, 1978). One can question whether the responses to such tasks are relevant or valid reflections of habitual reactivity to naturally occurring stressors - in other words, to what extent does laboratory-assessed reactivity generalize to the field? With regard to hormonal reactivity, little is known about the generalizability of laboratory studies to field studies. Results from cardiovascular studies indicate quite strong associations between chronic levels of cardiovascular activity across settings, but little support has been found for the relationship between laboratory reactivity and field reactivity (Turner et al., 1994). Moreover, it is impossible, from a practical and ethical point of view, to generate in the laboratory the stressful events and responses of the type and severity found in real-life (van. Doornen \& Turner, 1992). Although experimental control and the possibility of isolating and manipulating variables have important advantages and may be the optimal strategy for the evaluation of certain aspects of 
stress theory, it seems more important at this stage to investigate and try to describe what is happening in everyday life with regard to stress processes. Some have tried to increase ecological validity by mimicking daily life situations in the laboratory: for example public speaking (Bassett, Marshall, \& Spillane, 1987; Kirschbaum \& Hellhammer, 1993), or stress inducing films (Hubert \& de Jong-Meyer, 1989).

Natural experiments take advantage of the occurrence of real-life, potentially stressful events, of either an acute or of a more chronic nature. With regard to acute stressors, increases in cortisol excretion were, for example, found in situations such as forcing non-swimmers to jump in the deep-end of a swimming pool (Vaernes, Ursin, Darragh, \& Lambe, 1982), an anticipated surgical procedure (Ben-Aryeh, Roll, Kahana, \& al., 1985; Knight, Atkins, Eagle, Evans, Finkelstein, Fukushima, et al., 1979), academic examinations (Allen, Batty, Dodd, Herbert, Hugh, Moore, et al., 1985; Jones, Copolev, \& Outsch, 1986; Nicolson, 1992), and parachute (Schedlowski, Jacobs, Stratmann, Richter, Hadicke, Tewes, et al., 1993; Ursin, 1978) or bungee jumping (Hennig, Laschefski, \& Opper, 1994). Some examples of the kind of chronic stressors studied in the field are living near the damaged power plant at Three Mile Island (Schaeffer \& Baum, 1984), the period of bereavement after the loss of a child (Hofer, Wolff, Friedman, \& Mason, 1972a; Hofer, Wolff, Friedman, \& Mason, 1972b), enforced captivity (Dekaris, Sabioncello, Mazuran, Rabatic, Svoboda-Beusan, Racunica-, et al., 1993; Rahe, Karson, Howard, Rubin. \& Poland, 1990), combal exposure or related post-traumatic stress disorder (Bourne, Rose, \& Mason, 1967; Piman \& Orr, 1990), and work stress (e.g. Arnetz, Brenner, Levi, Hjelm. Petterson, Wasserman, et al., 1991: Caplan, Cobb, \& French, 1979; Coeck, Jorens, Vandevivere, \& Mahler, 1991; Lundberg \& Paim. 1989; Rose \& Fogg, 1993; Theorell, 1989; Timio \& Gentili, 1976). The effects of chronic or intermittent stress has received far less attention in the literature than the effects of acute stress, and data. on cortisol levels during prolonged stress have: been inconsistent, with enhanced (Arnetz et al., 1991; Coeck et al., 1991; Hofer et al., 1972a; Rahe et al., 1990; Rose \& Fogg, 1993; Schaeffer \& Baum, 1984; Timio \& Gentili, 1976) as well as decreased (Bourne et al., 1967; Caplan et al., 1979; Dekaris et al., 1993: Pitman \& Orr, 1990) concentrations reporled, and large variability among individuals.

A major drawback of the natural experiment is that, since the researcher generally has to be present in the field to collect cortisol measures, only anticipated events can be investigated. Additionally, mosi of the studies have examined stress under rather extreme or unusual situations, approaching major life events in terms of severity. Only few studies have investigated whether less severe but much more frequent daily events also have an impact on cortisol secretion, and fundings have been inconsistent, with reports of lowered (Caplan et al., 1979), elevated (Brantley. Dietz, McKnight, Jones, \& 'Tulley, 1988; Lundberg, Granqvist, Hansson, Magnusson, \& Wallin, 1989), and unchanged (Cummins \& Gevirtz, 19913) cortisoll levels.

Since individua! characteristics may influence how stressfu! situations are appraised they are thought to be important determinants of the emotional response to a given situation; mood states are, in turn, likely to mediate the endocrine response to the situation. Available information on the relationship between personality uaits, mood states, and event characteristics on the one hand and on cortisol levels on the other hand has until now been based on the traditional approaches discussed above. Several personality characteristics (e.g. trait anxiety, depression, anger, coping style, personality) have been associated with basal cortisol levels (Brandtstädter. BaltesGotz, Kirschbaum, \& Hellhammer, 1991; Pope \& Smith, 1991; Schaeffer \& Baum, 1984), as well as with cortisol responses, to stress (Bohnen, Nicolson, Sulon, \& Jolles, 
1991; Demyttenaere, Nijs, Evers-Kiebooms, \& Koninckx, 1989; Kirschbaum, Hellhammer, Strasburger, Tiling, Kamp, \& Luddecke, 1989; Nicolson, van Poll, \& deVries, 1992). Other studies, however, have found no association between personality traits, coping styles, and cortisol laboratory baseline levels or responsiveness to laboratory stressors (Bosserts, Berger, Krieg. Schreiber, Junker, \& von Zerssen, 1988; Kirschbaum, Bartussek, \& Strasburger, 1992a). Although there is abundant evidence that cortisol increases in response to distress or negative mood (Arnetz et al., 1991; Lundberg \& Frankenhaeuser, 1980; Mason, 1968), the effects of positive mood are less clear. Positive affective states, have been associated with decreases (Hubert \& de Jong-Meyer, 1989; Kugler \& Kalveram, 1989) as well as increases (Brown et al., 1993) in cortisol levels. Several laboratory studies have investigated the differential effects of various event appraisals (e.g. novelty, predictability, controllability) on cortisol (Mason, 1968). To our knowledge, this has not been done with regard to daily stress yet. In addition, there is no research that investigated whether different types of daily events have a different impact on cortisol. Since the context of an event has been found to relate to the magnitude of its psychological impact, this would be an interesting research question.

A major disadvantage of the studies described above is that often only a small or a few cortisol sample were obtained per subject or per day. In the study by Rose and Fogg (Rose \& Fogg, 1993), where repeated measurements of cortisol responses to work stress in air traffic controllers were obtained, a subgroup of subjects was found to respond to an increase in workload with large increases in cortisol. Repeated measurement of cortisol not only increases reliability, but also allows the investigation of dynamic relationships between stressful daily experiences and neuroendocrine activity. Additional research is necessary to see how the results obtained so far relate to those obtained with repeated measurements designs in the context of real life, and with daily events as the focus of interest.

\section{PRESENT STUDY: AIMS AND RESEARCH QUESTIONS}

Due to the hypothesized role of appraisal as a mediator between the environment and the person in determining the occurrence of a stressful experience and the responses to it, stress should be measured in a context were intrinsically motivating events and emotional involvement are most likely to happen; the natural environment of the subject seems to be the ideal research setting. Here, individuals can be observed in their normal social networks, settings, and activities, and stressful events can be studied in the life setting where they actually occur. Additionally, since stress is currently conceptualized as a dynamic process, which changes over time and in relation to the environment, it is necessary to include repeated measurements of stress, hormone levels, and emotional states to investigate the relationship between stress and affective and neuroendocrine responses (Dimsdale, 1984). The advantages of the repeated measurement approach can be further enhanced by simultaneously collecting information on individual traits, event characteristics and cognitive appraisals, which can then be investigated for their effect on mood and cortisol excretion.

Compared to the major life event approach, end-of-day assessments of minor stressors have important advantages, but even within a day, much of the dynamic interplay between events, appraisals of events, and outcomes remain hidden. Recall biases of events and mood are still likely to occur, and same-day associations between 
daily events and mood remain causally ambiguous. With this we mean that not only daily stress may have an effect on mood, but that a bad mood may increase the likelihood that daily events will occur or will be perceived as stressful.

In the present study, two relatively new approaches were employed to investigate the relationship between stress, affective states, and cortisol: i.e. the Experience Sampling Method (ESM) and ambulatory monitoring of salivary cortisol. The Experience Sampling Method has been specifically designed to study the subjective everyday experiences of people in their natural environments (Csikszentmihalyi \& Larson, 1987; de Vries, 1992). Typically, an electronic signaling device is used to alert subjects to fill in a self-report questionnaire at preselected but randomized time-points, providing information about an individual's mental status or symptoms within the context and flow of experience. When, how often, and for how long subjects are signaled depends on the goal of the study. The prospective assessment procedure is an important characteristic of ESM; since the variables of interest are assessed at frequent intervals during the day and close to the moment at which they actually occur, we are able to look at more dynamic stress processes, with a minimum of confounding due to biased recall or forgetting (Bower, 1981). ESM therefore also provides better estimates of the frequency, distribution, and intensity of psychological variables than cross-sectional designs (Larson \& Csikszentmihalyi. 1983).

The choice of cortisol as a physiological stress indicator was based on both theoretical and practical grounds. From a theoretical point of view, cortisol is a sensitive indicator of the stressfulness and adequacy of psychological responses to person-environment transactions, an important regulator of vital physiological processes, and a possible mediator of physical responses leading to disease. From a practical viewpoint, the opportunity of measuring cortisol reliably in saliva instead of blood makes repeated, stress-free sampling in real-life contexts possible (Kirschbaum \& Hellhammer, 1994; Nicolson et al., 1992). Saliva samples can be reliably collected and stored by subjects themselves, enabling them to carry out their daily routine without undue interference. Although our main emphasis was on cortisol, 14-hour urinary catecholamine levels were also determined twice, as possible indicators of long-term or more chronic stress.

The present study design compares two groups of male white collar workers. with high versus low levels of perceived stress, monitored during their normal daily activities. Subjects completed ESM self-reports concerning activities, mood states, and recent stressful events, and collected saliva samples for cortisol determination in response to signals emitted by a preprogrammed watch ten times a day for five consecutive days. Our study focused on four main topics: (1) the nature and experience of stress in daily life; (2) the relationship between perceived stress, stressful daily events and mood states; (3) the relationship between perceived stress. stressful daily events and cortisol levels; and (4) the generalizability of stress responses from the laboratory to real life. As outlined below, each of these topics will be addressed in separate chapters.

Following a description of the subjects and methods in Chapter 2, Chapter 3 is devoted to the description of quantitative as well as qualitative aspects of stressful daily events. Various questions are addressed, including: How frequently are stressful events experienced? What kind of events are experienced as stressful in daily life? Do stressful experiences differ with respect to frequency, kind, and appraisal in subjects with a high versus low level of perceived stress? How do major life events and chronic difficulties relate to the pattern of stressful daily events? 
Chapter 4 examines the influence of daily events on both negative and positive mood states. In addition to the immediate effects of daily stress on mood, we also examine temporal patterns of the effects of events on mood states. We examine whether average mood levels and mood responses to daily events were related to individual differences in perceived stress level. Finally, various kinds of events (work demands, negative social interactions) and event appraisals (e.g. unpleasantness, predictability, controllability) are investigated for their possible differential effects on mood states.

In Chapter 5 we address the question whether high perceived stress is associated with elevated cortisol levels. We also investigate the extent to which individual characteristics (trait anxiety, depression, anger, psychosomatic complaints, mood states, number of stressful events) contribute to differences in cortisol levels. Lastly, we examine the relationship between perceived stress level and urinary catecholamine excretion following work versus weekend days.

Chapter 6 focuses on the possible impact of minor daily events on cortisol secretion. We also examine the association between an individual's affective state and cortisol changes in response to such events. Finally, we test whether perceived stress and other individual characteristics are related to cortisol reactivity to stressful events.

Chapter 7 describes emotional and cortisol responses to a stress-inducing speech task in the same group of subjects. Beyond assessing the relationship between individual trait characteristics, current mood states, and cortisol responses to the stress task, a main objective of the laboratory experiment was to explore the consistency between laboratory and field cortisol measures, particularly with regard to the usefulness of laboratory measures in predicting cortisol levels and response to stressful events in the field. To this end, cortisol levels and responses measured in the laboratory were compared with those measured in real life.

Chapter 8 summarizes the main research findings and attempts to place them in a larger perspective. In addition it discusses limitations of the study and possible future research directions. 
Chapter 2

Methods 

The research questions presented in Chapter 1 necessitated a diversity of methodological approaches, including questionnaires, the Experience Sampling Method, monitoring of hormone levels in saliva and urine, and an experimental stress task. Chapter 2 describes first the subject selection procedure and demographic characteristics of the sample. Next, the measures and data collection procedures are described in detail. Methodological issues involved in the analysis of ESM data are discussed, including the coding of ESM responses, compliance, and assessment of possible biases and / or experimental effects of ESM procedures.

\section{SUBJECTS}

The sample consisted of 92 male white collar workers who had been first screened and then selected from six local industries and government agencies (see 'procedure' section), based on their perceived stress scores in the upper or lower tertiles of the screening sample distribution (PSS score $<10$ or 216). Subjects who reported a history of serious chronic illness, endocrine disorder, medications known to affect cortisol levels, or treatment (past or current) for mental health problems were excluded. During subject intake, each high stress subject was matched for age group, marital status, and household composition with a low stress subject to insure that the two groups did not differ on demographic characteristics that might affect exposure to certain classes of stressors.

Table 2.1. Demographic data for 'High' and 'Low' stress subjects.

\begin{tabular}{cc}
\hline Low Stress Subjects & High Stress Subjects \\
$\mathrm{N}=46$ & $\mathrm{~N}=42$ \\
\hline
\end{tabular}

Age

mean

s.d.

range
42.7

7.7

27.57
41.5

6.0

28-52

\section{Marital status}

$\begin{array}{lrr}\text { married } & 41(89.1 \%) & 37(88.1 \%) \\ \text { unmarried } & 2(4.3 \%) & 2(4.8 \%) \\ \text { living together } & 2(4.3 \%) & 2(4.8 \%) \\ \text { divorced } & 1(2.2 \%) & 1(2.4 \%)\end{array}$

\section{Household composition}

living alone

couple with children

couple without children
3 (6.5\%)

$37(80.4 \%)$

$6(13.0 \%)$
$3(7.1 \%)$

$34(81.0 \%)$

$5(11.9 \%)$

'High' and 'low' perceived stress groups were defined as follows: the mean of the first and the second PSS assessment was used to categorize subjects as above or below the screening sample median score (12). Four subjects (two from each group). 
One week before the field study, subjects received a second questionnaire battery to be filled in at home and returned at the briefing session. The questionnaires concerned psychosocial stress (including the PSS again), personality and psychological symptoms (depression, anger) (see next section).

\section{CROSS-SECTIONAL INSTRUMENTS}

An overview is presented of the questionnaires used. The questionnaires administered during the screening phase of the study will be described first (Questionnaire I), followed by the questionnaires completed the week before ESM took place (Questionnaire II). Questionnaires which have not been widely used will be described more thoroughly than more familiar ones.

\section{Questionnaire I}

Demographic information: A demographic questionnaire requesıed information concerning respondent's age, marital status, type of household, number of children (for the matching procedure), chronic diseases and medication use (possible exclusion criteria), and alcohol use, smoking habits, and participation in active sports (possible confounders of hormone concentrations).

Perceived Stress: Subjects were selected according to their perceived stress level. Perceived stress was measured by means of the Perceived Stress Scale (PSS: Cohen et al., 1983). The PSS is a global measure of the degree to which situations in one's life are appraised as stressful. ltems were designed to tap the extent to which individuals feel their life to be unpredictable, uncontrollable, and overloaded. These themes are important components of the experience of stress (Averill, 1973; Cohen, 1978; Seligman, 1975). The PSS measures cognitions and emotions relating to general stress levels rather than specific events or situations (see Table 2.2.). The items are rated on a 5-point frequency scale, ranging from 'never' $(0)$ to 'very often' (4). A total score is obtained by reversing the scoring on the positive items and then summing across the 10 items.

Table 2.2. Items Perceived Stress Scale.

1. In the last month, how often have you been upset because of something that happened unexpectedly?

2. In the last month, how often have you felt that you were unable to control the important things in your life?

3. In the last month, how often have you felt nervous and 'stressed'?

4. In the last month, how often have you felt confident about your ability to handle your personal problems?

5. In the last month, how often have you felt that things were going your way?

6. In the last month, how often have you found that you could not cope with all the things that you had to do?

7. In the last month, how often have you been able to control irritations in your life?

8. In the last month, how often have you felt that you were on top of things?

9. In the last month, how often have you been angered because of things that happened that were outside of your control?

10. In the last month, how often have you felt difficulties were piling up so high that you could not overcome them? 
One week before the field study, subjects received a second questionnaire battery to be filled in at home and returned at the briefing session. The questionnaires concerned psychosocial stress (including the PSS again), personality and psychological symptoms (depression, anger) (see next section).

\section{CROSS-SECTIONAL INSTRUMENTS}

An overview is presented of the questionnaires used. The questionnaires administered during the screening phase of the study will be described first (Questionnaire I), followed by the questionnaires completed the week before ESM took place (Questionnaire II). Questionnaires which have not been widely used will be described more thoroughly than more familiar ones.

\section{Questionnaire I}

Demographic information: A demographic questionnaire requesıed information concerning respondent's age, marital status, type of household, number of children (for the matching procedure), chronic diseases and medication use (possible exclusion criteria), and alcohol use, smoking habits, and participation in active sports (possible confounders of hormone concentrations).

Perceived Stress: Subjects were selected according to their perceived stress level. Perceived stress was measured by means of the Perceived Stress Scale (PSS: Cohen et al., 1983). The PSS is a global measure of the degree to which situations in one's life are appraised as stressful. ltems were designed to tap the extent to which individuals feel their life to be unpredictable, uncontrollable, and overloaded. These themes are important components of the experience of stress (Averill, 1973; Cohen, 1978; Seligman, 1975). The PSS measures cognitions and emotions relating to general stress levels rather than specific events or situations (see Table 2.2.). The items are rated on a 5-point frequency scale, ranging from 'never' $(0)$ to 'very often' (4). A total score is obtained by reversing the scoring on the positive items and then summing across the 10 items.

Table 2.2. Items Perceived Stress Scale.

1. In the last month, how often have you been upset because of something that happened unexpectedly?

2. In the last month, how often have you felt that you were unable to control the important things in your life?

3. In the last month, how often have you felt nervous and 'stressed'?

4. In the last month, how often have you felt confident about your ability to handle your personal problems?

5. In the last month, how often have you felt that things were going your way?

6. In the last month, how often have you found that you could not cope with all the things that you had to do?

7. In the last month, how often have you been able to control irritations in your life?

8. In the last month, how often have you felt that you were on top of things?

9. In the last month, how often have you been angered because of things that happened that were outside of your control?

10. In the last month, how often have you felt difficulties were piling up so high that you could not overcome them? 
We used the 10 item version of the scale (PSS10) because it has been shown to have as good or better psychometric properties than the original 14 item version (Cohen \& Williamson, 1988). Research by Cohen and others showed acceptable levels of validity and reliability. See Appendix I for the reliability and validity of the PSS in the present sample.

Coping: Coping conceptualized as a fairly stable characteristic ("coping style') was assessed with the 47-item Utrecht Coping List (UCL), developed for the Dutch population by' Schreurs et al. (Schreurs \& van de Willige, 1988). The seven factor-analytically derived subscales are: 'active problem solving', 'palliative responding', 'avoidance', 'seeking social support', 'depressive reaction', 'expression of emotions', and. 'comforting cognitions'.

Psychological and somatic complaints: The Symptom Checklist (SCL-90; Derogatis, Lipman, \& Cori, 1973) is a 90-item self-report questionnaire developed for measuring psychopathology in ambulatory psychiatric patients, but also in community samples cq non-patients. The questionnaire was adapted and translated into Dutch by Arrindell and Ettema (1981; 1986) and measures, on a five-point scale, both somatic and psychological complaints experienced during the last week. The instrument consists of eight subscales: (1) sleeping problems, (2) hostility, (3) depression, (4) somatic complaints, (5) distrust and interpersonal sensitivity, (6) insufficiency of thought and performance, (7) agoraphobia, and (8) anxiety. The SCL90 total score can be interpreted as an indication of the general level of psychological and/or somatic dysfunctioning during the past week. Reliability and validity data for the Dutch SCL-90 have been reported by Arrindell and Ettema (1986) and by Koeter et al. (1988).

Psychosomatic symptoms: The revised version of the SUNYA Psychosomatic Symptom Checklist (PSC; Attanasio, Andrasik, Blanchard, \& Arena, 1984) contains 17 common psychosomatic complaints, lie headaches, backaches and nausea. Subjects rate each item on frequency $(O=$ not, $4=$ occurs daily) and intensity $(0=$ not a problem, 4 = extremely bothersome) of occurrence, using a five point scale. A Total. score, reflecting the overall level of psychosomatic distress, is obtained by summing the cross-products of each item's frequency by intensity. Factor analyses of PSC data. obtained from college students revealed one general psychosomatic distress factor with little overlap with other commonly used measures of psychological distress such as the Beck depression Inventory or the State-Trait Anxiety Inventory (Attanasio et: al., 1984).

\section{Questionnaire II}

Life events: Life events were recorded with the questionnaire form of the List of Threatening Experiences (LTE.Q: Brugha, Bebbington, Tennant, \& Hurry, 1985). Subjects were asked about the occurrence of 12 categories of events (e.g. death of a partner, child, parent: got divorced, unemployed) during the last year. This list of event categories originated from a large series of life events sampled in a UK community epidemiological survey and in psychiatric outpatients with affective disorders (Bebbington, Tennant, \& Hurry, 1981). Each event in the original list was. rated for long-term contextual threat, according to the methods of Brown and Harris (1978). Reliability and validity data for the LTE-Q have been reported by Brugha and Conroy (1985) and Brugha and Cragg (1990).

Long-rerm Difficulties: Chronic stress was assessed with the Long-term Difficulties Questionnaire (LLM; Hendriks, Ormel, \& van de Willige, 1990; Ormel, 
1985). This inventory is a Dutch adaptation of the Biographical Problem Inventory List (BIOPRO; Hosman, 1983) and focuses on chronic and environmental stress, including problems in relation to work and study, housing, physical environment, leisure, finance and social relationships (partner, family, friends, neighbours). Of the original 32 items, 16 items concerning health were omitted, a question about leisure activities was added. Subjects rate each item on a four-point intensity scale with the anchors (1) none, (2) some, (3) quite, and (4) serious (difficulties). A total score is obtained by summing across all items.

Personality: Personality was assessed with the Dutch abridged MMPI (NVM; Luteijn \& Kok, 1985). This questionnaire consists of 83 items forming 5 scales: Negativism, Somatization. Timidity, Serious Psychopathology and Extraversion. Reliability and validity of the NVM have been described by Luteijn and Kok (1985).

Depression: Depression as a complex of symptoms was assessed with the validated Dutch translation of the Self-Rating Depression Scale (SRDS: Dijkstra 1974: Zung, 1965).

Anxiety: Trait anxiety was measured with the validated Dutch version of the State-Trait Anxiety Inventory (ZBV; van der Ploeg, Defares, \& Spielberger, 1980)

Anger: Trait anger was measured with the validated Dutch version of the Spielberger Trait Anger Scale (ZAV; van der Ploeg, Defares, \& Spielberger, 1982). This questionnaire consists of two subscales: 'anger-temperament' (the general disposition of experiencing anger and giving voice to it) and 'anger-reaction' (the disposition of expressing anger, for instance when provocated or criticized).

\section{THE EXPERIENCE SAMPLING METHOD}

The Experience Sampling Method (ESM) was designed to study the subjective, everyday experiences of people in their natural environments. Typically, an electronic signaling device is used to alert subjects to fill in a self-report questionnaire at preselected but randomized time-points, providing information about an individual's mental status or symptoms within the context and flow of experience. The self-reports request a range of information about subjects' current thoughts, moods, complaints, activities, and physical and social context. Signaling devices (e.g. pagers, wristwatch terminals), schedules of signals (e.g. random, fixed or fixed-random time-sampling; event-sampling) and ESM self-report forms may vary depending on the particular objects and goals of the study (see Csikszentmihalyi \& Larson, 1987; Delespaul, 1992).

During the last two decades, a large number of researchers have contributed, often independently, to the development of the Experience Sampling Method. One of the earliest lines of investigation under the name of ESM was started at the University of Chicago in 1975 by Czikszentimihalyi and associates. They obtained data on daily activities and experiences of adolescents (Csikszentmihalyi \& Larson, 1984). Similar techniques were developed or adapted by Hurlburt (e.g. Hurlburt, 1990) for the study of thought content, by Klinger et al. (1980) who studied the stream of conscious thought in daily life, by Massimini et al. (e.g. Massimini, Csiksentmihalyi, \& Carli, 1987 ) for the study of optimal experience, and by deVries and associates for the study of psychopathology (de Vries, 1987; de Vries, 1992). Many others could be added to this list. (see for a review Delespaul, 1995). The research group in Maastricht has studied a range of psychiatric disorders: schizophrenia (e.g. de Vries \& Delespaul, 1992; Delespaul, 1995), anxiety (e.g. Dijkman-Caes \& deVries, 1991), depression 
(Kraan, Meertens, Hilwig, Volovics, Dijkman-Caes, \&. Portegijs, 1992; van Diest, 1992), and somatization. They have also used ESM to study acute stress (Nicolson et al., 1992), pain (Lousberg, Schmidt, Groenman, Vendrig, \& Dijkman-Caes, 1995), and addiction (Kaplan, 1992).

The above indicates that ESM can be used with a variety of populations, provided that the research subjects can read and write and that a viable research alliance can be established. In spite of the success of ESM in describing mental disorders in context, there are limitations. Difficulties have been encountered in sampling elderly subjects with dementia, and individuals with acute and severe depression.

Early research focused on methodological aspects of ESM such as reactivity effects, self-selection biases, compliance, and validity and reliability of self-reports, and provided important information on the reliability and validity of ESM data (e.g. Csikszentmihalyi \& Larson, 1987; Delespaul, 1995; Hormuth, 1986). These aspects will continue to be of importance in every particular ESM study conducted. Controls and checks on the subjects should be built into the Experience Sampling designs, like checking on missed signals, the timeliness of responses (does the subject respond to the signal on time?) and on the influence of the method on the objective and subjective experiences of the subjects (Are the subject's objective circumstances influenced by participation? Is the subjective perception of a situation influenced by the method?) (see 'compliance and reactivity issues' section).,

\section{Description of the ESM instrument}

The ESM booklets used in the current study consisted of forms to be completed following each signal ('beep level' forms) and forms to be completed at the beginning or the end of each day ('day level' forms). These forms will be described separately below.

\section{ESM form: beep level}

At every 'beep' subjects completed a self-report form (see Appendix II), containing several questions about the subject's mental state and physical and social. context. Subjects were asked what they were brooding about, what they were doing, and where and with whom they were at the moment of the beep. Daily stress or hassles were assessed with the open question 'Did any stressful event or situation take place between the present and the previous beep?'. Thus, the assessment of daily events had a more retrospective character than the other questions at the beeplevel. The ESM forms also included Likert scales (from I 'not at all' to 7 'very much') for the evaluation of thoughts, mood, physical well-being, individually defined psychosomatic complaints, current activity, and stressful events. Activities were evaluated on the dimensions: enjoyed doing, skill, effort, and challenge. The following appraisais of stressful events were also rated on Likert scales: unpleasantness, importance, predictability, controllability, and frequency of prior occurrence. Subjects also reported when the event started and when it ended, or that the event was still going on. This information gives an indication of the duration of the event and the time elapsed since termination. Additional items were included to help in the interpretation of the hormonal data: occurrence and intensity of physical exertion and any ingestion of food, coffee, alcohol, cigarettes or medication which may have taken. place since the last signal. 


\section{ESM form: day level}

At the end of each Experience Sampling day, subjects were asked to rate (Likert-scales) the extent to which they were bothered by their individual complaint that day, how stressful the day had been and how their general mood had been. Also included was a short checklist of 15 events (Daily Stress Scale; Bolger et al., 1989a). Except for transportation and financial problems, this list consisted entirely of items concerning demands (e.g. a lot of work at home, at work, a lot of demands made by family) or interpersonal conflicts (e.g. argument with spouse, child, colleague). Subjects indicated if an event on the list had occurred to them that day and, if so, what the impact of that event had been (from 1 'not at all bothersome' to 7 'extremely bothersome').

Additional information was obtained each day immediately after waking (sleep quality, sleep pattern previous night) and again before going to bed (effects of participation in the study, timing of meals, work and time to bed).

\section{ESM procedures}

Most participants find the ESM rewarding but also taxing. A crucial part of the Experience Sampling process is therefore the creation of an alliance and a mutual understanding about the research procedures and aims of the study. Cooperation and compliance depend on this alliance.

During an initial semi-standardized interview one or two days before the start of ESM, called the 'briefing', the purpose of the ESM research and the additional saliva and urine sampling was explained and informed consent was obtained. We communicated our interest in learning about their daily experiences. At this time we also gathered additional information concerning possible recent diseases and related medication use, and individualized complaints were chosen. Subjects were instructed to select one complaint (either of a psychological or somatic nature) that was important to them and that occurred rather frequently. Instructions about the use of the watch and the ESM booklets were given. Subjects were instructed to fill out selfreports as soon as possible after a signal (within 15 minutes). Anticipated difficulties in the use of ESM (e.g. driving a car, attending a meeting) and possible solutions to it were discussed. The subjects were given control of the signaling device only in that they could turn it off if they did not want to be disturbed (e.g. during a daytime nap). As a reminder, instructions were also printed on the inside cover of the booklet. The subjects were discouraged from showing their completed booklets to others and were asked not to look back through previously completed forms.

The ESM period encompassed five days, from Thursday to Monday (three workdays and two weekend days). Tuesdays and Wednesdays were reserved for (de)briefing sessions. On ESM days, subjects were signaled 10 times a day between 7:30 a.m. and 10:30 p.m., with an average interval of 90 minutes between consecutive 'beeps'. This was done according to a preprogrammed schedule chosen to maximize interpretability of cortisol values without being predictable by the subject. The 'beeps' were randomly distributed around fixed time points (e.g. 8:15 a.m., 9:45 a.m., and so on throughout the day), with a maximum deviation of $20 \mathrm{~min}$. After each signal subjects filled in an ES form, which took about 2 minutes, and collected a saliva sample (see section below).

One or two days after the five days of ESM, a final 'debriefing' session took place, which started with the Stress Inducing Speech Task (SIST; see 'Stress Inducing Speech Task' section). After the stress task, ESM booklets were checked. Subjects 
were asked to explain reasons for any missing responses and to clarify illegible or unclear responses to facilitate later coding. The 'debriefing' session was closed with a structured interview about the possible impact of the study on the daily life of the subject. Subjects received a small financial compensation (fl. 20,-) as well as an information booklet about stress. 'Briefing' and 'debriefing' sessions each took about one hour. An individual subjects was briefed and debriefed by the same person.

\section{SALIVA SAMPLING}

Cortisol levels were determined in saliva. Cortisol in saliva is a reliable and valid indicator of the free cortisol in plasma, which is considered to be the biologically active hormone, and cortisol concentrations are independent of the flow rate of saliva (Vining, McGinley, \& Symons, 1983). The rate of equilibrium of cortisol between saliva and blood is very fast. Cortisol responds within less than five minutes to increases in stress (Vining et al., 1983) and has a half life in blood or saliva of about an hour (Fredrikson, Sundin, \& Frankenhaeuser, 1985). These characteristics of saliva cortisol also provide an appropriate time frame for studying its relationship to experiences in daily life. It is assumed that a sample, collected within 20 minutes after signaling, reflects adrenal cortical activity during the time period between that last signal and the previous signal. Cortisol, in comparison to other stress-labile hormones (e.g. the catecholamines, growth hormone, prolactin, testosterone), shows the most specific relationship to subjective distress, in contrast to general arousal, effort, or trauma (Delahunt \& Mellsop, 1987; Lundberg \& Frankenhaeuser, 1980; Rose, 1984). The ability to measure cortisol reliably in saliva instead of blood, makes repeated, stress-free sampling possible. Measuring cortisol in blood is usually stressful in itself (Rose \& Hurst, 1975) and interrupts ongoing activities, which makes repeated measurement extremely difficult, especially over longer time periods.

Subjects collected saliva by holding a dental cotton roll in the mouth for 1 to 2 minutes. Saturated dental rolls were then placed in a capped plastic vial (Salivette; Sarstedt). which subjects labeled with the time of day and then stored with the ESM booklet in a specially designed wallet. This wallet contained ten Salivette vials and an ESM booklet with ten seli-report forms. Subjects placed their saliva samples in their freezer at the end of each day. We have found no differences in cortisol levels in uncentrifuged samples frozen immediately or kept at room temperature for two days (Nicolson et al., 1992); others report no differences up to two to four weeks (Kahn, Rubinow, Davis, 1988; Kirschbaum \& Hel!hammer, 1989). Uncentrifuged samples were kept at a temperature of $-20 \mathrm{C}$ until they were analyzed. A maximum of 50 samples per subject was thus obtained.

Analysis Salivary cortisol levels were determined in duplicate by direct. radioimmunoassay (Ansseau, Sulon, Doumont, Cerfontaine, Legros, Sodoyez, et al., 1984), using ${ }^{125}$ I-cortisol (Farmos diagnostica, Finland) and antiserum made against the 3-CMO-BSA conjugate by Dr. J. Sulon, University of Liege, Belgium. The lower detection limit of the assay was $12 \mathrm{ng} / \mathrm{dl}$, with a mean intra-assay coefficient of variation of $4.8 \%$ (range: $2.2 \%-7.5 \%$ for 4 assays). Each subject's samples were analyzed in the same assay to reduce sources of variability. 


\section{URINE SAMPLING}

Levels of the catecholamines adrenaline (A) and noradrenaline (NA) were determined in 14-hour urine. Compared to plasma levels which are highly liable with a half-life of less than three minutes, urinary levels represent estimates of sympatheticadrenomedullary activity integrated over extended time periods (usually 1 to 3 hours) and they can be used to determine long-term (14- to 24-hour) changes in levels of catecholamines (CA) (Frankenhaeuser, 1975b). The collection of urine samples is also relatively easy, noninvasive and well-suited for naturalistic field studies (Frankenhaeuser \& Gardell, 1976). Because the catecholamine in urine constitute a small but relatively constant fraction of liberated amines in the body, the direction of change or the relative levels are meaningful, but absolute numbers have limited value. NA levels are somewhat difficult to interpret because NA is secreted by both nerves and the adrenal medulla and is also subject to rapid neuronal reuptake; A levels give a more reliable estimate of adrenal medullary activity because the adrenal medulla is the sole source of circulating A (Frankenhaeuser, 1975b). The 14-hour samples collected in this study were chosen to reduce the effects of variation between subjects with different circadian rhythms and to minimize the biasing effects of idiosyncratic events occurring during the day. For example, a person who runs up the stairs will probably show elevated CA levels at the next voiding, but such one-time elevations should be mitigated in a 14-hour collection. Overnight CA excretions can be regarded as baseline levels and are therefore useful for assessing physiological changes associated with chronic stress (Baum, Lundbeg, Grunberg, Singer, \& Gatchel, 1985).

Subjects collected two overnight urine samples, one after a workday (Thursday to Friday) and one after a weekend day (Sunday to Monday). Urine was collected by the subject from 6.00 p.m. to $8.00 \mathrm{a} . \mathrm{m}$. the following day ( 14 hours), in 2 liter urine containers (Sarstedt). Subjects emptied their bladder in the toilet at 6.00 p.m. and from that moment collected subsequent urine in the container. At $8.00 \mathrm{a} . \mathrm{m}$. the next day they emptied their bladder for the last time in the container. Subjects kept their container in the refrigerator during the collection period. The urine samples were collected by the researcher or research-assistant at $9.00 \mathrm{a} . \mathrm{m}$. at the workplace of the subject. From the time the subject left home until collection of the container at the workplace, samples were kept in an insulated bag with a cooling element. Immediately after the collection of the container we added HCL $(37 \%)$ to the total volume of urine until a $\mathrm{pH}$ of $<3$ was reached, to prevent oxidation. From the total volume, $10 \mathrm{ml}$. samples were extracted and immediately frozen at a temperature of -20 $\mathrm{C}$ until analyses.

Analysis Adrenaline and noradrenaline excretion rates were determined by means of high-pressure liquid chromatography (HPLC) with electrochemical detection (Kissinger, Riggin, Alcorn, \& Rau, 1975). ${ }^{\text {. CA levels were corrected for }}$ creatinine excretion $(g / l)$ and expressed in $\mu \mathrm{g} / \mathrm{g}$ of creatinine. Nine samples with creatinine levels below $0.60 \mathrm{~g} / 1$ were considered unreliable and were not used in the analysis. Due to practical reasons, three subjects did not collect any urine, and another four subjects collected urine only once.

Analyses of adrenaline and noradrenaline were performed under the supervision of Dr. Rahman and Dr. Duvivier, University Hospital of Liege, medical chemistry service, Belgium. 


\section{STRESS INDUCING SPEECH TASK (SIST)}

A disadvantage of conducting a study in the natural environment is the idiosyncratic nature of events and activities and the heterogeneous stressful circumstances subjects encounter in daily life. Individuals' responses to these varying circumstances are difficult to compare. Therefore, all subjects in this study were exposed to a standard stress test in an experimental context in order to get more insight into individual differences in neuroendocrine responses. Additionally, we investigated whether cortisol activity as assessed in the laboratory generalized to cortisol activity as measured in the field. An important consideration was that the information provided by the standardized stressor should be relevant for the understanding of the endocrine response to stimuli in claily life; in other words, the stressor should have 'ecological validity'. We chose to use at speech task, similar to that described by Steiner and associates (1988).

The test took place at the beginning of the ESM debriefing session, between $11 \mathrm{a} . \mathrm{m}$. and $13.00 \mathrm{p} . \mathrm{m}$. This test was unanticipated by the subjects, who believed that the final session would only be used for 'debriefing' the ESM study. The experimenter (blind to whether a subject belonged to the high stress or the low stress group) read the written SIST instructions aloud for the subject. The instructions were to prepare (10 minutes) and present ( 5 minutes) a videotaped speech about their personal strengths and weaknesses for later evaluation by a team of psychologists. After the 10 minutes of preparation, subjects received a signal, the video recorder was started, and the subjects delivered their presentation while looking directly into the camera. Afterwards, subjects relaxed for 15 minutes in neutral activities (e.g. reading magazines). At 4 time points subjects filled in a short mood questionnaire and provided a saliva sample: (T1) upon arrival, (T2) after the 10 minutes of preparation, (T3) after the 5-minute presentation, and (T4) after 15 minutes of relaxation. During the course of the experiment we decided to lengthen the recovery period to get a clearer picture of the cortisol response profile. For 49 subjects, a fifth saliva sample (T5) was taken on average 50 minutes after the first assessment. This way we could look at endocrine responses during the anticipation, reactivity and recovery phase of the stressor.

\section{CODING OF ESM MEASURES}

Information about activities ('What were you doing?'), location ('Where were you?'), social context ('With whom were you?'), and stressful events ('Did any stressful experience or event occur?') was obtained by open-ended questions. In order to be able to identify, for instance, typical patterns of time use or stressful daily events, the total range of activities and daily events has to be reduced. The specific method used for coding these experiences is very important because it entails decisions about which events or experiences can be differentiated and which will be combined. An important question to be answered before using these data in further analyses concerns the reliability of the coding procedure. This was assessed by calculating the agreement between two independent observers on the coding of all variables to be used in the analysis (see Appendix III). 


\section{Coding procedure}

In the literature are examples of ESM studies where responses to open-ended questions were coded by the subjects themselves (e.g. Brandstatter, 1983), by other participants (e.g. Campbell et al., 1991) or by trained coders (e.g. Wong \& Csikszentmihalyi, 1991). In this study we used trained coders for several reasons: first, if coded by subjects themselves they would need more time to fill out the ESM form thereby endangering study compliance (e.g. activities were coded under 57 fine grained but reliable categories which would take up too much time, and too many instructions needed), and second, because we wanted to start without preconceived notions of what a 'stressful event' might be. Pre-coded responses ('menus') require limited numbers of simple categories which are predefined to be of importance.

Responses were coded by two independent raters, according to an established coding system. The two raters first coded three ESM booklets and discussed differences in the results to clarify problematic categories and definitions. Next, they coded a subset of ESM booklets (five booklets for each of 27 subjects) without discussing them with each other. These codings were used for the reliability analysis. All data actually used in further analyses (beyond the reliability analysis) were coded by both raters with differences being replaced by consensus ratings.

\section{Coding of activities, location and social context}

Classification of activities was based on the list of events in the International Time Budget Study (Szalai, 1972). This list contained 96 codes which were later reduced to 47 codes for use in several Experience Sampling Method studies at the University of Limburg. In the current analyses, the 47 activities were collapsed into 8 broader categories: inactivity/rest, work (which also included volunteer work, regular classes, homework, special courses, and study breaks), household/maintenance (also including child care, care to adults, shopping and general services, personal care services, personal hygiene and personal medical services), leisure (including activities like sports, hobbies, attending a film, reading a book, or watching television), social interaction (conversations, visits to or by friends, parties etc.), meals (including regulai meals, special meals and snacks), transport, and other activities (including personal mental or emotional activities, political and civic activities, and other activities not falling in one of the other activities).

With respect to location ('Where are you?') and social context ('Are you together with someone?'), we again used the standard coding manual developed for ESM studies at the University of Limburg, later collapsing the specific codes into six broader categories: at home, nefwork (homes of family, friends, and acquaintances). work, public places (street, shop, cafe, sports hall, health care settings), transport (in car, bus, train, airplane, on bike), and other places.

Responses to the question 'Are you together with someone?' were coded and then collapsed into 7 categories: alone, household members (including partner, children, parents, brothers and sisters and other household members), non-resident family, friends, colleagues, neighbours/acquaintances, and strangers.

Responses were coded missing/invalid if a question was not filled in (missing) or if a response was not legible or invalid (e.g. filled in too late); responses were rated as can't code if something valid had been written down, but it was not clear which category did apply (for instance when important background information is missing). This category can give us a good idea about the quality of the coding system. 


\section{Coding of stressful events}

In contrast to the domains described above, there was no pre-existing coding system for stressful events. Until now, there have been few studies investigating the effects of different types of minor stressors on health and well-being (Bolger et al., $1989 \mathrm{a} ;$ Stone, 1987). It is common practice to aggregate daily stressors into a summary measure for analytic purposes. This may, however, conceal important variation in the microprocesses underlying somatic and psychological well-being. For this reason, we differentiated events reported according to the following domains: context of the event, who was involved in the event (if someone was involved), whether the event was internal or external (observable or not), and whether the event entailed a social interaction or a task demand.

The context categories were based largely on the ESM activity codes, which was expanded to include the categories personal heaith-somatic and personal healthpsychological. We wanted to be able to identify events conceming health, especiaily mental health, because of their possible confounding to psychiatric and somatic states.

The context domain contained the following 8 categories: work (events concerning boss, supervisor, co-worker, clients, general happenings concerning self at work, events concerning study etc.), network (events concerning spouse, partner, children, relatives, friends, neighbours or acquaintances), household/financial (events concerning general housework, family related duties, errands, loans, selling, buying. financial problems etc.), leisure (events concerning hobbies, sports, outings, vacation etc.), personal health-somatic (events concerning illness, injury of the subject himself), personal health-psychological (e.g. nerves, anxiety, worries about health), transport (e.g. missed bus, traffic jam, unusual traffic), and an 'other' category (minor irritants like noise, the weather, broken glass, ESM. cold shower. combinations of different events etc.).

The coding of events under a 'social interaction' domain and a 'task demands' domain paralleled the checklist we used in the ESM booklet at the end of the day, which consisted mainly of events concerning task demands or interpersonal conflicts. A diary study on the influence of daily stress on mental health, based on a community sample of 166 married couples, showed that interpersonal conflicts and tensions were by far the most distressing events (Bolger et al., 1989a). Task demands were also of interest to us because overload is an important theme in a sample of working people. These people have in general more than one social role (family, work eic.) and are therefore more exposed to various role related demands. A study by Stone (1987) showed greater psychological impact of work-related demands.

With respect to the 'social interaction' domain, responses were coded into three categories: not applicable (event is not a social interaction); negative interaction (e.g. argument, conflict) or discussion/conversation.

The 'task demands' domain consisted of five categories: not applicable (clearly no demands involved); problematic task (e.g. difficulties, problems with..); $a$ lot of work/tasks or extra work/tasks; time pressure, deadlines; failure at task.

For coding who was involved in the event we used the same categories as described under the ESM social context domain: alone, household members, nonresident family, friends, colleagues, neighbours/acquaintances and strangers.

Events were coded as external or internal in an effort to separate the more or less 'observable' events from entirely 'subjective' events. Criteria for coding events as external were: 1) they should be observable (i.e. theoretically verifiable) and 2) 
they should have happened in the interval since the last beep. The opposite applies to internal events: they are not observable. Examples of internal events are introspections, worries, internal evaluations, anticipations. The internal/external distinction does not refer to responsibility for causation.

A 'can't code' category was included in every domain for event descriptions which could not be classified due to the absence of sufficient information.

\section{Interrater agreement}

Before using the qualitative information in further analyses, we have to investigate if the coding system we used is a useful one. Generality is important here in demonstrating that the obtained ratings are not the idiosyncratic results of one rater's subjective judgment (Tinsley \& Weiss, 1975). Regarding the interrater agreement we are interested in the following question: Is it possible to classify the information under the various categories and can we do this reliably? Information gathered under the activity, location and company domains is clear and straightforward. The categories belonging to these domains are also very concrete and well-defined. Problems should not be expected here. But with regard to the coding domains for stressful events, we can expect some variance because of interpretation differences and difficulties. Additionally, the subject's description of the event may be lacking in the types of detail necessary to classify it into one of several related categories. In Appendix III we describe how the interrater agreement was calculated (Cohen's unweighted Kappa) and what the Kappa's for the different codes were.

With respect to the reliability of coded responses, we can conclude that the open-ended responses could be classified very reliably (see Appendix III). Even in categories with only a small number of observations the degree of agreement was generally substantial. We did find that some specific event domains were more difficult to rate. This was the case for the domains 'internal/external', 'social interaction' and 'task demands', where Kappa's varied from moderate to almost perfect $(.46-.89)$, but where a substantial number of responses fell into the 'can't code' category. This means that the obtained information was often difficult to interpret, leading to a substantial loss of information. Because these analyses were done after the first 27 subjects were sampled, more attention was paid in subsequent debriefing sessions to the clarification of reported information on stressful events, in order to keep loss of information due to uncodable responses to a minimum.

\section{DATA ANALYSES}

The ESM dataset is characterized by a large number of repeated observations ('beeps') on a relatively small number of individuals, with a large number of variables measured at each beep. As a consequence, Experience Sampling data have a complexity which often make traditional analytic approaches unsatisfactory. Depending on the type of research question, ESM data can be analyzed either at the subject level, at the beep level, or at both levels at the same time (Larson \& Delespaul, 1992). At the beep level, the repeated measurements are used as the unit of analysis, with a maximum of 50 measurements per subject in the current study. At the subject level, the individual is used as the unit of analysis, including one time measurements as well as aggregated data. One time measurements are, for instance, scores from crosssectional questionnaires (e.g. perceived stress score) and briefing and debriefing data. 
Aggregated data are, for instance, mean mood or event scores per subject, or the percentage of total beeps a subject spent on a certain activity (e.g. work).

Although in certain cases beep level analyses may be the only possible approach (e.g. when one is dealing with relatively rarely occurring categories of experience like going to a party'), there are some important problems that are inherent in such an approach (Larson \& Delespaul, 1992). Generally, when beep level analyses are carried out, the number of units in the analyses is inflated and dependency exists between adjacent data points. Significance tests, therefore, do not provide a valid estimate of probabilities. In addition, beep level analyses give: equal weight to all instances of a category of experience. This means that subjects with more valid responses in that category are given more weight and will have more influence: on the outcome. These problems of inflated number of units, dependency between observations, and unequal weighting can be diminished by employing subject level analysis, where aggregate scores are computed for each individual and the subject is used as the unit of analysis (Larson \& Delespaul, 1992). Aggregated scores, which are based on repeated measurements, also have the advantage of increasing the reliability of measurement. Therefore, this is an appropriate approach when research guestions about the chronic level of variables are of interest (see for example Chapter 3 and Chapter 5).

Nonetheless, subject level analyses are also subject to several pitfalls. Besides the fact that they ignore the rich data base available, this approach can introduce aggregation bias, which occurs when unmeasured or unspecified variables exist that distort the causal relationship between variables (e.g. "when heterogeneous groups [or sub-units] are combined into a single group [unit], and the combined group analysis yield conclusions that are misleading about the sub-units") (Jaccard \& Wan, 1993, p.43). Another restriction on the use of subject level analysis is that a substantial number of subjects with at least more than one observation is required when one is interested in making comparisons across several situations (e.g. work. home; work days, weekend days) using a repeated measures analysis of variance, since this technique does not tolerate missing data. Most like $l y$, a number of subjects will not have enough observations in each situation, leading to a substantial loss of information. Two other important shortcomings of this technique are: it may provide misleading estimates of the effects of an independent variable (data are treated as if there were an equal number of observations for each level of the independent variable, but in many occasions this will not be the case), and it ignores the fact that many of the independent variables are correlated in ESM: the data are treated as if the variables are orthogonal. For example, certain stressful events (e.g. network events) tend to occur only in certain locations (e.g. at home rather than at work).

Besides the problems described above, aggregated data obscures the interplay between experientiai and psychological or physiological states and processes which momentary measures try to capture. To gain more insight into the relationship between perceived stress, stressful events, cortisol and / or mood states over time, we will have to apply another analytic method. A more effective approach for analyzing both subject and beep level data is by using random-coefficient regression models (Bryk \& Raudenbush, 1992; Goldstein, 1987). This approach is a variant of the multiple linear regression model, applicable for data with a. hierarchical nesting structure as is the case with ESMI data. This approach can account for the dependency of data within a subject and for residual dependency, it can deal with the problem of missing data, and it allows for individual differences in intercepts, slopes, and error structures. Additionally, by using random regression models, the outcomes 
at the beep level can be modeled as a function of both the beep and the subject level variables. The model allows us to obtain estimates of, for example, cortisol reactivity to events both as a function of psychological state (e.g. mood state) and trait (e.g. level of perceived stress) variables over all subjects and for each individual. The number of observations need not be the same for all persons, and observations may be unequally spaced over the time interval. In the present study, random regression models have been applied in Chapter 4, Chapter 6, and Chapter 7. Greater detail on theoretical and technical aspects of this analytic approach can be found under the method sections of these chapters.

\section{COMPLIANCE AND REACTIVITY ISSUES}

The general purpose of ESM is to study the subjective experiences of persons interacting in their natural environments. This means that the variables measured should be a representative sample of those in the person's environment and that they should be measured with as little distortion in retrospective recall as possible. ESM's primary weaknesses lie mainly in the responsibility given to the subject in collecting not only subjective data, such as feelings and appraisals, but also objective ones like the description of situations. Therefore it is very important to check on compliance of subjects (e.g. How many responses are missing and for what reason?), timeliness of responses (Are responses made on time?), and on effects of ESM on the objective and subjective experiences of the subjects (e.g. Does ESM influence subject's choice of activities, mood?). Because: ESM asks more of its participants than more conventional research methods it is also important to check on how taxing the task of being a subject was fellt to be.

\section{Compliance of subjects}

Particularly for people with relatively high levels of appraised stress, it is important to determine if participants were motivated and able to fill in a sufficient number of ESM booklets within an acceptable time limit after the beep occurred. The choice for this time limit is rather arbitrary; a compromise has to be found between leaving enough time for the subject to comply to his tasks (filling in the booklet and taking a saliva sample) and keeping the delay between the beep and response time as smali as possible.

In this study, a response was considered valid and maintained for analyses when a response was given within 5 minutes before and 20 minutes after the beep occurred. Only subjects with at least 20 valid responses and with not a single day completely without valid responses were included in the analyses. In our sample, 4 subjects did not meet our criteria. Two subjects in the low stress group completed a sufficient number of valid beeps, but both missed a whole day (one subject went abroad for a day with a business client). In the high stress group, one subject had only 16 valid response, and another subject missed all responses at the weekend because he was ill. Analyses were based on 88 subjects. 
Table 2.3. Delay between beep and response time.

ESM formats Saliva Samples

Nr. of responses after: $1 \mathrm{~min}$.

$5 \mathrm{~min}$.

$477 \quad 11 \%$

$168238 \%$

$10 \mathrm{~min}$.

$249857 \%$

$303769 \%$

$15 \mathrm{~min}$.

$328575 \%$

$346479 \%$

$20 \mathrm{~min}$.

$352980 \%$

$360382 \%$

$363283 \%$

$365683 \%$

\begin{tabular}{llllll}
\hline Invalid & 144 & $3 \%$ & 107 & $2 \%$ \\
Missing & 624 & $14 \%$ & 637 & $15 \%$ \\
\hline
\end{tabular}

Total number of responses

4400

4400

Of all programmed beeps, $83 \%$ were responded to within the time limit of 5 minutes before and 20 minutes after the beep occurred (see Table 2.3). For all valid responses the mean delay between beep and response time was 5 minutes $(s, d=4.0)$, and in $3 \%$ of all cases the delay between beep and response time was larger than -5 and +20 minutes (invalid responses). Finally, $14 \%$ of all beeps were not recorded. Although the saliva samples were not included in our 'validity' criteria, we also checked on the compliance of saliva samples. Here we found almost the same distribution of valid, invalid and missing responses: $83 \%$ valid responses, $2 \%$ invalid responses and 15\% missing responses. Mean delay between beep and response time for saliva samples was 3 minutes $(s . d .=4.8)$.

The number of valid, invalid and missing responses in the low versus high stress groups are shown in Table 2.4. The mean number of valid responses completed per subject was 41 . In the low stress group subjects completed an average of 42 (s.d.= 5.7) valid ESM responses compared to $40($ s.d. $=6.8$ ) in the high stress group. The difference between groups was not significant (Mann-Whitney U test; two-sided, $p=.1)$. The mean number of saliva samples was $43(s . d .=5.8)$ in the low stress group and $40($ s.d. $=6.6)$ in the high stress group (Mann-Whitney $U$ test; two-sided, $p=.1$ ).

Table 2.4. Number of valid, invalid, and missing responses in 'High' and 'Low' stress, groups.

\begin{tabular}{|c|c|c|c|c|}
\hline \multirow[b]{2}{*}{ Responses } & \multicolumn{2}{|c|}{$\begin{array}{l}\text { Low Stress Group } \\
\qquad \mathrm{n}=46\end{array}$} & \multicolumn{2}{|c|}{$\begin{array}{c}\text { High Stress Group } \\
n=42\end{array}$} \\
\hline & ESM & Saliva & ESM & Saliva \\
\hline Valid (mean) & $42^{\prime}(84 \%)$ & $43(86 \%)$ & $40(80 \%)$ & $40(80 \%)$ \\
\hline Valid (total) & $1947(85 \%)$ & $1960(85 \%)$ & $1685(80 \%)$ & $1696(81 \%)$ \\
\hline Invalid (total) & $50(2 \%)$ & 32 (1\%) & $94(5 \%)$ & $75(4 \%)$ \\
\hline Missing (total) & 303. (13\%) & $308(13 \%)$ & $321(15 \%)$ & $329(16 \%)$ \\
\hline
\end{tabular}

In summary, four subjects were discarded from the study, because they did not meet the criteria of having filled out at least 20 ESM reports within a time limit of -5 and +20 minutes, and without a complete day missing. The final sample completed $83 \%$ of all possible responses within the time limit, providing an average of 41 
responses per subject. The same applied for compliance of saliva samples. The delay between beep and response time was generally small; $57 \%$ of the responses were given within 5 minutes after the beep. High stress subjects were not less compliant than low stress subjects.

\section{Reasons for missing responses}

Missing responses can be due to a variety of reasons (e.g. sleep, lack of motivation, interference of beeps with important, stressful, or private activities). For the reliability and validity of ESM, it is important to ascertain that there is no systematic underreporting of specific situations. Figure 2.1.a. displays the number of valid responses during the day. The least valid responses were obtained in the morning and late at night, with the first beep (between 8:07 and 8:30 a.m.) receiving the least valid responses $(73 \%)$. One possible reason for missing or out of range responses in the morning and evening hours was that subjects were still or already asleep. Since subjects were asked to fill out the time they woke up in the morning and went to sleep in the evening, the percentage of missing and invalid responses due to sleep could be calculated. Of all missing and invalid responses, $10.4 \%$ were known to be due to the fact that the beep occurred before the subject woke up in the morning. while $0.4 \%$ was due to going to sleep before the last beep occurred. Sleep was the reason for missing and invalid responses in $45 \%$ of all first beeps in the morning (all in the weekend). The small decrease in valid responses at beep 7 was probably related to the fact that subjects were leaving the work place and were on their way home which complicated compliance. Notes in booklets suggested this.

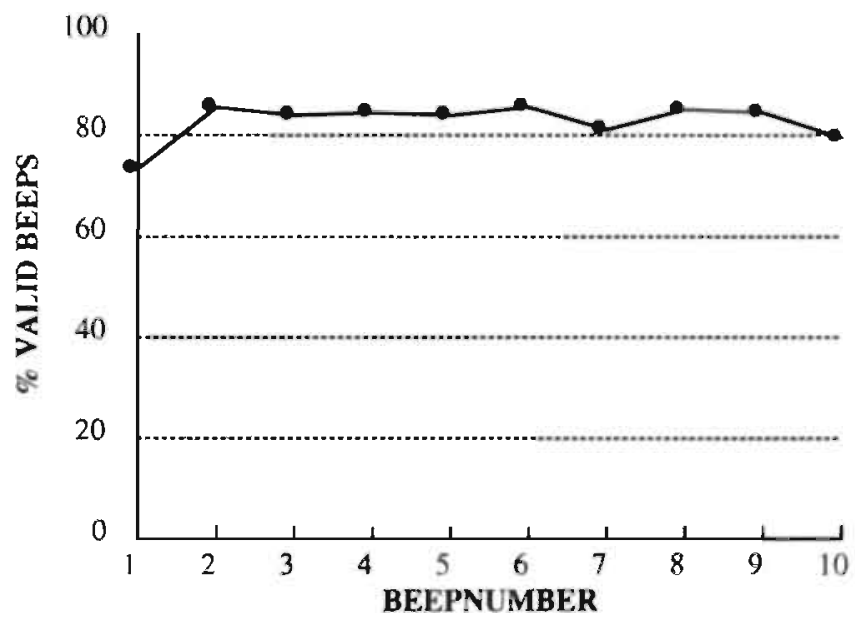

Figure 2.1.a. Percentages of valid responses during the day. 


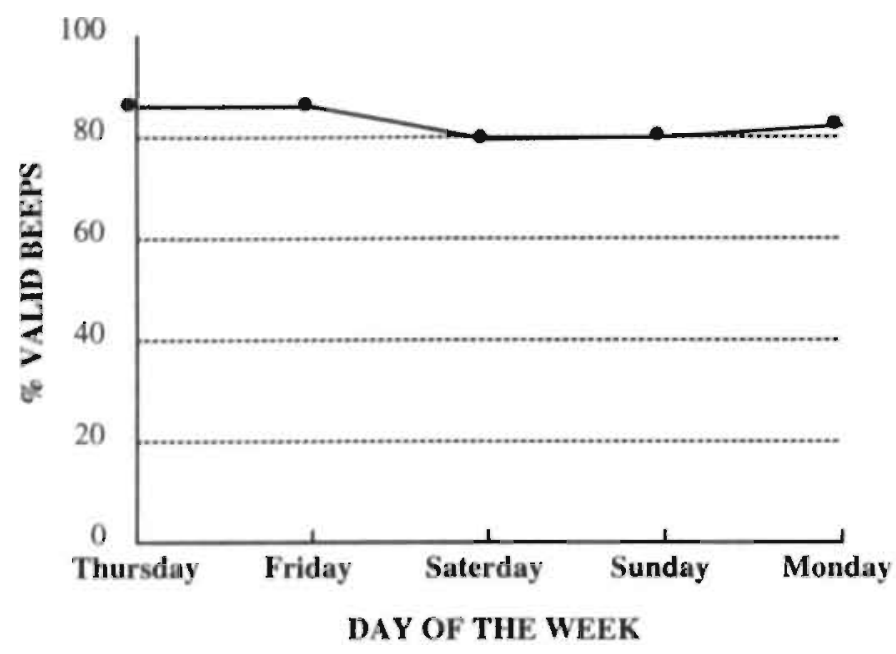

Figure 2.1.b. Percentages of valid responses, at different research days.

Fig. 2.1.b. shows that the percentage of valid responses did not vary much by research day. Although a decline in valid responses might be expected at the end of the research week because of a decrease in motivation, we found this small decline to be most prominent during the weekend. ANOVA-repeated measures over the mean number of valid responses (aggregated by subject) showed a significant withinsubject effect for 'DDay of the Week' $(F(4,344)=4.2 ; p<.01)$, but no berweensubjects effect for 'Group' (high vs. low stress) was found $(\mathrm{F}(1,86)=2.7$; ns), and no significant interaction between 'Day of the Week' and 'Group' $(F(4,344)=.4)$. Significantly more responses were missed during the weekend than during work days $(t(87)=4.4 ; \mathrm{p}<.001)$. In the weekend, $74 \%$ of all missing and invalid responses could be related to the fact that subjects were still asleep.

Notes in booklets revealed various other reasons for missing and out of range responses. Reasons mentioned relatively often were: not hearing the watch in a noisy environment (e.g. sports hall, party) or mistaking the alarm beep in the early morning for the first beep (leading to a missing response at the end of the day), problems with the watch (put off by mistake, not working properly), forgetting to take the ESM format along when leaving the house or work place, engaging in sports and other hobbies, business appointments, meetings or speeches at work, and driving a car or bicycle. In summary, the percentage of missing responses does not vary dramatically during the day and during different research days. The most important reason for missing and out of range responses in the early morning was that subjects were still asleep. The remaining missing and out of range responses were due to a variety of reasons making systematic underreporting of events unlikely.

\section{Representativeness of recorded days}

It is hoped that the five research days provide a representative sample of the subject's daily life. But, since these days were just five days in a subject's daily life, it is quite possible that they, either by chance or as a react of the procedure, differed from 'normal' days. Therefore, we asked subjects during the ESM debriefing 
interview whether the research days had been unusual in some respect, and if so, for what reason. During the ESM period, subjects also evaluated at the end of each day (just before bed time), whether that day could be considered as a normal one. This was measured with a Lickert scale, ranging from 1 ('not at all normal') to 7 ('very much normal').

Fifty one subjects $(58 \%)$ reported during the debriefing that the research days gave a good impression of their normal daily life. Eleven subjects (13\%) were more doubtful, reporting that the research days were a little bit unusual. Twenty-six subjects $(29 \%)$ ) found the research days to be unusual in some respect. No differences were found between the two subject groups. Twenty-one subjects provided explanations for what was unusual: parties, birthdays in family $(n=9)$; more or unusual work $(n=6)$; less work/stress than usual $(n=8)$; health complaints of self or family $(n=4)$; a day off on a usual work day $(n=3)$. 'Less work/stress' as an unusual factor was mentioned by six subjects from the high stress group compared to two subjects from the low stress group. At the day level, the mean rating of how normal the day had been was $4.3(\mathrm{~s} . \mathrm{d}=1.2)$ (aggregated by subject over 5 days). On the whole, then, subjects experienced their research days as normal. High stress subjects appraised research days as being a little less normal than low stress subjects (3.9 versus 4.8 . respectively; $t(84)=4.0 ; p<.001)$, but they still thought that the ESM sampling gave a good impression of their normal daily experiences.

In summary, although both subject groups reported that the research days were occasionally unusual for one reason or another, a majority indicated that the research days were representative of their normal daily routine. In the high stress group, the level of stress or work pressure experienced during the sampling period seems to be particularly salient, as this was frequently mentioned as the reason why the research days were unusual (e.g. "this week was unusual in that I had more work/fewer pressing deadlines than usual" etc.).

\section{Inconvenience and task difficulty of ESM}

To get an indication of how taxing and difficult the ESM procedure was for subjects, we asked during the debriefing interview whether ESM was 'bothersome or annoying' and whether any question in the ESM booklets was difficult to interpret or to answer. Additionally, we inquired whether they were willing to participate in an ESM study again. Subjects gave an evaluation of how disturbing individual beeps were in the ESM reports at the moment they were beeped.

Table 2.5. Annoyance with RSM as, recorded during debriefing interviews.

Low Stress Subjects High Stress Subjects

Did you find ESM

\begin{tabular}{lrr} 
'bothersome'? & $\mathrm{N}=46$ & $\mathrm{~N}=42$ \\
\hline No & $23(50 \%)$ & $21(50 \%)$ \\
A little/sometimes & $20(44 \%)$ & $14(33 \%)$ \\
Yes & $3(6 \%)$ & $7(17 \%)$ \\
\hline
\end{tabular}

As Table 2.5. shows, a vast majority of subjects (89\%) reported that ESM was either slightly or not at all bothersome. Only $6 \%$ of the low stress subjects and $17 \%$ of the high stress subjects said the method was troublesome. The differences between groups were not significant. The ten subjects who found ESM bothersome gave the 
following explanations: the filling in of ESM reports becomes particularly annoying toward the end of the sampling period (three subjects), during weekend leisure time (two subjects), or because it disrupts activities or occurs in the company of other people (five subjects).

Seventy-three percent of the subjects reported to be willing to participate another time with ESM, 14\% would be willing under certain conditions (e.g. only after some time, different questions in the ESM booklet), and $13 \%$ did not want to take part again (e.g. once is enough, too intensive, too long, too many beeps). There were no differences between the two groups in willingness to participate in a future ESM study.

In response to the question whether there were any difficult questions in the ESM forms, $72 \%$ of the subjects had no probiems at all, $19 \%$ had only minor problems, and $9 \%(n=8)$ of the subjects had problems with one or a few specific questions only (e.g. what is a stressful event?; sometimes difficult to score mood items; sometimes appraisals of activities not applicable). Again, there were no group differences.

At the beep-level, subjects rated whether the beep was disturbing on a scale from 1 'not at all' to 7 'very'. Of all valid responses, $48 \%$ were rated as 'not disturbing' (score 1) and $14 \%$ as 'disturbing' (score 5 or higher). 'High' stress subjects rated the beeps as slightly more disturbing on average than low stress subjects (mean score of 2.5 versus 2.2; Mann-Whitney $U$ test; $p=.04$; two-sided). It was further striking, and logical, that invalid beeps were rated as more disturbing on average than valid beeps (mean score $[n=48]$ of 3.0 versus 2.3 ; Mann-Whitney $U$ test; $\mathrm{p}<.01$; two-sided).

In summary, most subjects were not unduly inconvenienced by the ESM procedure and were generally positive in their evaluations of the method. Only $13 \%$ of the subjects stated unwillingness to participate again in an ESM study. Half of the beeps were rated as not disturbing at all. High stress subjects rated the beeps as a little bit more disturbing than low stress subjects, but their average score was still low. These data show that ESM can be used with good results in samples of working men with different levels of perceived stress. Our results compare well with results obtained in other studies. in a study on German adults, $22 \%$ reported that ESM disrupted daily routine and $75 \%$ were willing to participate again with ESM (Hormuth, 1985). In samples of Dutch subjects with a panic or other neurotic disorder, about $28 \%$ said the method was bothersome and $72 \%$ was willing participate again (Dijkman-Caes, 1993).

\section{Study effects}

In order to obtain a representative sample of the experiences of people in their daily life, we made repeated measurements with ESM within a day and during several days. Because of these repeated measurements, which means intense involvement of the subject, we wanted to know if participating in ESM research produced study effects which would reduce the ecological validity of the study. Therefore, debriefing interviews included questions about the possible influence of the method on the subject's daily life. 
Table 2.6. The method's Influence on thoughts, moods, activities and social contacts.

\begin{tabular}{|c|c|c|c|c|}
\hline Study effects & $\begin{array}{l}\text { Low-High. } \\
\text { Subjects } \\
\text { Thoughts } \\
\text { (\%) }\end{array}$ & $\begin{array}{l}\text { Low-High } \\
\text { Subjects } \\
\text { Moods } \\
\text { (\%) }\end{array}$ & $\begin{array}{l}\text { Low-High } \\
\text { Subjects } \\
\text { Activities } \\
\text { (\%) }\end{array}$ & $\begin{array}{l}\text { Low-High } \\
\text { Subjects } \\
\text { Social contacts } \\
\text { (\%) }\end{array}$ \\
\hline Lirtle or none & 13 & 5 & 4 & 9 \\
\hline Yes & 31 & 19 & 4 & 7 \\
\hline No & $\begin{array}{ll}63 & 62\end{array}$ & 76 & 92 & 91 \\
\hline
\end{tabular}

A majority of subjects said the method had no influence on their thoughts, moods, activities and social contacts (see Table 2.6). The small differences found between groups were not significant. The ESM method had the largest influence on subject's thoughts. Effects on thoughts mentioned during debriefing interviews were: thinking about ESM during the day and waiting for beeps to come $(n=10)$, more aware of thoughts $(n=10)$, confrontation with thoughts $(n=3)$, giving a moment thought to things you normally take for granted $(n=l)$, and it helps to relativize experiences $(n=2)$ and to concentrate $(n=1)$. The main effects on mood were: higher awareness of feelings $(n=4)$, and writing down negative feelings makes them disappear $(n=1)$. Irritation $(n=7)$ or being bored $(n=2)$ were mentioned as negative effects of ESM. Effects on activities were: giving more thought to activities than. usual $(n=2)$, less participation in sports $(n=1)$, little adjustments in planned activities during the weekend $(n=3)$, and some people said they waited to engage in a certain activity till after the beep $(n=3)$. The most often mentioned effect of ESM on social contacts was: having to explain ESM all the time to other people ( $n=8$ ), what some people liked and others did not. One subject thought the beeper scared others off, and another subject thought that his family behaved differently (no arguments).

In summary, on average $80 \%$ of the subjects said the method had no influence on daily life. If study effects were reported, there were both positive and negative effects of participating in ESM. 



\section{Chapter 3}

The nature of stress in daily life: events, appraisals, and activities 



\section{INTRODUCTION}

Stressful daily events have been found to be related to lower psychological well-being and increased somatic symptomatology (Del-ongis et al., 1982; Stone et al., 1993). These studies, most of them characterized by an a priori labeling of events as stressors, provided only limited information about the types of events that are experienced as stressful by varying populations and in varying contexts. They did, however, show that large individual differences exist in the vulnerability to minor events as well as in the impact of such events. The meaning and impact of an event is to a large degree determined by one's perceptions and reactions to such events. They may depend, for instance, on earlier experiences, personality social support, personal goals and beliefs. The current transactional approach to psychosocial stress defines minor events or 'hassles' as experiences and conditions of daily living that are experienced as salient and harmful or threatening to the endorser's well-being (Lazarus \& Folkman, 1984b). A central feature of this approach is the importance it places on these cognitive appraisal processes that intervene between the occurrence of an event and the individual's reaction to it. Despite the acknowledged importance of the transactional nature of stress, the significance of the appraisal process, and the many laboratory and animal studies that have been performed on this topic, few studies have investigated the nature of suressful daily events and the meaning individuals give to stressful events in a real-life setting. The present study aims to examine in the natural environment of the individual what types of events are experienced as stressful and how stressful events are appraised. We further investigated whether individuals who perceive themselves to be stressed differ in the types and appraisals of reported stressful events from individuals who do not.

With respect to the nature of stressful events, qualitative measures of stressful daily events may increase our insight into the type of situations or experiences which are most problematic and emotionally difficult for certain populations or for the individual. By exploring recurrent themes or patterns of hassles in reports of everyday events as described by individuals themselves, instead of focusing only on frequencies of event occurrence, we can more reliably characterize those events (e.g. work demands, conflicts with the partner) that form the basis of chronic stressful life conditions (e.g. work problems, family problems) (Wagner, 1990). Research suggests that patterns of hassles vary with developmental stage and sociodemographic characteristics (Kanner et al., 1981). In the study by Kanner et al., groups of middleaged people, students, and health professionals could be differentiated according to certain thematic patterns of hassles. Middle-aged subjects reported more hassles with economic issues (e.g. rising cost of living, property, investments, taxes), health professionals reported more hassles with anxieties and high pressure (e.g. too many things to do, not enough time, too many responsibilities), and college students tended to have more academic and social hassles (e.g. meeting standards, wasting time, and loneliness).

With regard to the meaning and significance of an event, it is possible that although people encounter an enormous range of events in daily life, events are. stressful for only a limited number of reasons. For an understanding of what makes certain events stressful for certain kinds of people, we not only have to know what (is. perceived to have) happened, but also how an individual gives meaning to the event, through cognitive appraisal processes (Lazarus \& Launier, 1978). There is evidence, for instance, that individuals' personal ratings of events improve prediction of outcomes such as anxiety, depression, negative affect, tension, and grade point 
average (Dewe, 1991; Peeters et al., 1995; Sarason et al., 1978). Although the transactional stress theory makes a distinction between primary and secondary appraisal processes ('what is at stake', and 'what can be done about the situation'. respectively), in many cases these processes are not separable (Holroyd \& Lazarus, 1982). For this reason, we have not tried to distinguish between primary and secondary appraisal in the present study. Based on the literature (e.g. Cohen, 1980; Dewe, 1991; Katz \& Wykes, 1985; Lazarus \& Folkman, 1984b; McGrath \& Beehr, 1990; Miller, 1979; Thompson, 1981), we selected five dimensions that appear to be important in how individuals evaluate stressful experiences: Unpleasantness ('How unpleasant was this event for you?'), importance ('How important was this event for you?'), predictability ('To what degree did you foresee that this event was going to happen?'), controllability ('How much controll did you have over the course of the event?'), and frequency of prior event occurrence ('How often has such an event happened to you before?').

The main objectives of this chapter are, firstly, to provide both quantitative and qualitative descriptions of daily life stress in white collar men, and secondly, to contrast the experiences of individuals who perceive themselves to be stressed with those who do not. Considering our first objective, we examine 1) how often stressful situations occurred, 2) what kinds of situations were experienced as stressful, and 3) evaluations of events, as discussed above. Regarding our second objective, we first examine 4) the amount of time spent on different activities in high and low stress subjects. Information on the frequencies of activities that people engage in on a daily basis can provide the necessary framework to understand the experience of stress in daily life. In addition, patterns of daily time use are compared between groups to assess whether the two groups had a similar chance of exposure to various kinds of stressful situations. We next examine 5) whether high stress subjects differed from low stress subjects in how they appraised daily activities in terms of enjoyment, challenge, required skill and effort. Here, we expect that appraisal of activities may reflect the stressfulness of daily life experiences that were not identified by the individual as discrete events. Next, we investigate differences between high and low stress groups in 6) frequency, 7) content and 8) evaluation of stressful experiences. We further examine whether life events and chronic difficulties were related to the frequency of stressful events reported during the five days of ESM. Both life events (for example, divorce) and chronic difficulties (for example, problematic relationship with spouse) could affect the person's pattern of stressful daily events or their personal significance (Kanner et al., 1981). Finally, we investigate a more methodological issue, that is, how do repeated within-day event reports compare with checkilist results obtained at the end of the day? Since most studies measure stress on a daily basis, it is important to investigate the influence of the frequency of measurements on event reports. For instance, do open-ended probes for events within-days elicit more stressful events than end-of-day event checklists (for example, due to forgetting events at the end of the day or increased attention for events within-days), or do we find report of fewer stressful events within-days compared to end-of-day checklist (for example, due to passive recognition of checklist events rather than active recall, or due to a more general evaluation of the demands of the day instead of the more discrete events occurring during the day)?

In the past. several methodological approaches have been used to elicit information about daily events, ranging from open-ended questions to structured checklisis. The advantages and disadvantages of the various methods have been discussed by Stone et al. (1991). The simplest approach has been the open-ended one, 
where subjects were asked about the day's most stressful event (Rehm, 1978) or asked if anything went wrong during the day (Caspi et al., 1987; Eckenrode, 1984). If a respondent answered 'yes', she or he was then asked to describe what had happened. Responses were then classified into discrete categories. Strongly influenced by the work of Holmes and Rahe on major life events (Schedule of Recent Events; Holmes \& Rahe, 1967), the most often used method of assessing daily events is the event checklist, where, from a list of events, subjects are asked to identify those that have occurred to him or her over a specified time period, ranging from one day to one month. Checklists covering the last month include the Unpleasant Events Schedule and the Pleasant Event Schedule (Lewinsohn \& Amenson, 1978; Lewinsohn \& Talkington, 1979), the Hassles and Uplifts scale (Kanner et al., 1981). the Inventory of Small Life Events (Zautra \& Guarnaccia, 1986), and the Everyday Problem Checklist (Vingerhoets \& Menges, 1989). Items on these scales were formulated or taken from existing scales to cover events in major life domains such as family, work, leisure, and household maintenance. The much shorter checklists designed to be used on a daily basis include the Daily Life Experience checklist (DLE; Stone \& Neale, 1982), the Daily Stress Inventory (DSI; Brantley et al., 1987), and the Daily Stress Scale (DSS; Bolger et al., 1989a). To develop daily checklists with representative events, individuals from the population in which the checklist was later intended to be used were asked to provide open-ended descriptions of daily events and situations that fit certain criteria (for example, 'stressfulness", (Brantley et al., 1987)). Elicited events were then grouped into various major life domains (e.g. DLE: work, leisure, family, friends, financial, and other; DSS: overloads. interpersonal conflicts) or chosen on the basis of reported event frequency (DSI).

In the present study, we used the Experience Sampling Method (ESM) (Csikszentmihalyi \& Larson, 1987; de Vries, 1987; de Vries, 1992) to investigate the types and cognitive appraisals of stressful events in the everyday life of white collar men. In the context of their normal social networks, settings and activities, 88 subjects completed ESM self-reports at semi-random intervals ten times a day over 5 consecutive days, including three work and two non-work days. Stressful daily events were assessed by the open-ended question 'did anything stressful take place since the last assessment?' Our choice for the open-ended approach was based on the fact that most daily event checklists are not suitable for frequent use during a single day. Also, we did not want to restrict a priori the definition of 'stressful' to certain categories of problems, being more interested in how white collar men themselves describe their stressful daily events. The ESM affords several advantages for investigating stress from a transactional perspective. Since events are assessed at frequent intervals each day, not long after the occurrence of the event, we are able to look at stress processes more dynamically as they evolve in time, but also more dynamically in the sense that not only the person but also the natural environment of the person is taken into account in the assessment of a stressful experience. These processes can be empirically assessed with a minimum of confounding due to biased recall (e.g. 'effort after meaning') or forgetting (Bower, 1981). In this way, we are able to elicit information about the appraised meaning of an event at the time it occurs, and about the social or physical context at that moment. Using ESM, even very minor sources of stress can be recorded, which might otherwise be forgotten at the end of the day. Therefore, more reliable estimates of rates of event occurrences are possible. 


\section{SUBJECTS}

The subject selection procedure has been described in Chapter 2 (see 'Subjects' and 'Procedure' sections). As shown in Table 3.1., this procedure resulted in two groups (42 High Stress 'HS' subjects and 46 Low Stress 'LS' subjects) that were very similar in sociodemographic characteristics. This means that any differences between the groups in the use of time or in exposure to stressful events are unlikely to reflect differences in age, marital status, or family composition.

Table 3.1. Demographic characteristics of the study participants.

$\begin{array}{ll}\text { LS } & \text { HS } \\ \mathrm{n}=46 & \mathrm{n}=42\end{array}$

p-value

(2-tailed)

\section{demographic variables}

\begin{tabular}{|c|c|c|c|}
\hline age & mean (st.dev.) & mean (st.dev.) & \\
\hline mean & $42.7(7.7)$ & $41.5(6.0)$ & ns \\
\hline range & $27-57$ & $28-52$ & \\
\hline \multicolumn{4}{|l|}{ marital status } \\
\hline married & $41(89.1 \%)$ & $37(88.1 \%)$ & ns \\
\hline unmarried & $2(4.3 \%)$ & $2(4.8 \%)$ & \\
\hline living together & $2(4.3 \%)$ & $2(4.8 \%)$ & \\
\hline divorced & $\mathrm{i}(2.2 \%)$ & $1(2.4 \%)$ & \\
\hline \multicolumn{4}{|l|}{ household composition } \\
\hline living alone & $3(6.5 \%)$ & $3(7.1 \%)$ & ns \\
\hline couple with children & $37(80.4 \%)$ & $34(81.0 \%)$ & \\
\hline couple without children & $6(13.0 \%)$ & $5(11.9 \%)$ & \\
\hline
\end{tabular}

\section{METHODS}

Measures relating to stressful events and to daily activities are derived from ESM reports, as described in Chapter 2 (see 'Description of the ESM instrument' section). End-of-the-day measures used in the current analyses include the Daily Stress Scale (Bolger et al., 1989a) and a global rating of the day's stressfulness. Also included in this analysis are measures of life events, recorded with the List of Threatening Experiences (LTE-Q:Brugha et al., 1985) and chronic difficulties, assessed with the Long-term Difficulties Questionnaire (LLM: Hendriks et al., 1990) (see chapter 2 'Cross-sectional instruments' section).

Analyses were done with SPSS/PC+ 4.0 on Macintosh. Unless stated otherwise, differences in variables were tested with non-parametric tests, using twotailed significance tests. In the analysis of the ESM data, measures were aggregated so that the subject was used as the unit of analysis (Larson \& Delespaul, 1992). 


\section{A CASE EXAMPLE}

The following case study of a subject who experiences high levels of stress in his. life illustrates the level of detail this assessment method provides about the ebb and flow of an individual's stressful experiences during the course of five days.

Jan, 41 years old, married with child (one year old), works for the municipal government as a building and housing inspector. He experiences his life as somewhat overloaded and uncontrollable lately (at least for the past 3 months; PSS score $=17$ ) and describes the following chronic difficulties: work is demanding due to regulatory changes, he has no time to study and is therefore falling behind, there is not enough time for home upkeep and garden maintenance, and too little real leisure time. When asked which psychosomatic complaint bothers him the most, he mentions stomach ache. The five days of ESM for Jan are shown in Figure 3.1. Here we see that on Thursday he has a busy day at work, with difficult assignments. Several work-related stressful events are reported, like settling a petition and dealing with a request for subsidy, with a concurrent increase in feelings of agitation and stomach pain. Positive affect also seems to be below average during that day at work. While driving home he seems to unwind, he starts to feel more relaxed, is less bothered by stomach pain and his mood improves. The next day, Jan describes a similar pattern, with several stressful events in the morning at work, increasing agitation, stomach pain, and a slight dip in positive affect. In the afternoon and evening, several events are described that relate to his child's health and to household maintenance, but these events seem to have hardly any effect on mood state or physical complaint, since after leaving work Jan reports less agitation, stomach pain and higher positive mood. In contrast with the work days, the weekend is spent with relatively few stressful experiences and on the whole he feels relaxed, in a good mood, and not bothered by stomach pain. Most of the time is spent with wife, child and other family members: e.g. playing with son, visiting family, entertaining family, working on car, and watching t.v. Although Monday -back at work- no events are reported, we can see that Jan gets slightly more agitated again and is more bothered by stomach pain than during the weekend. ESM data indicated that work is the major source of stress for Jan, as was also described in the chronic difficulties questionnaire. Household maintenance was also reported as stressful on several occasions. but had less influence on his emotional state or physical complaint. 
Figuur 3.1. Example of one subject's stressful events. emotional states (Agitation, Positive Affect) and psychosomatic complaint reported during five days of ESM
Apitacioa

Complaint

Poritive mood

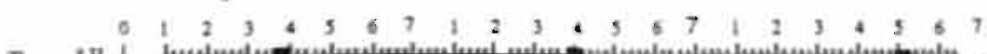

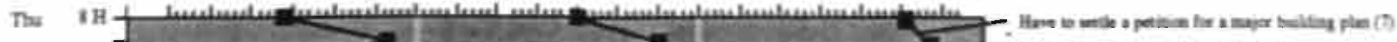
12 H] 年

Fin
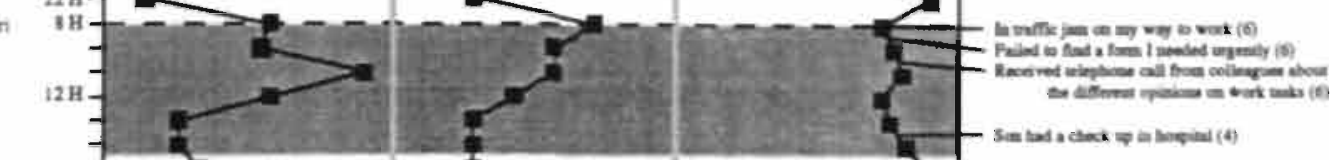

se
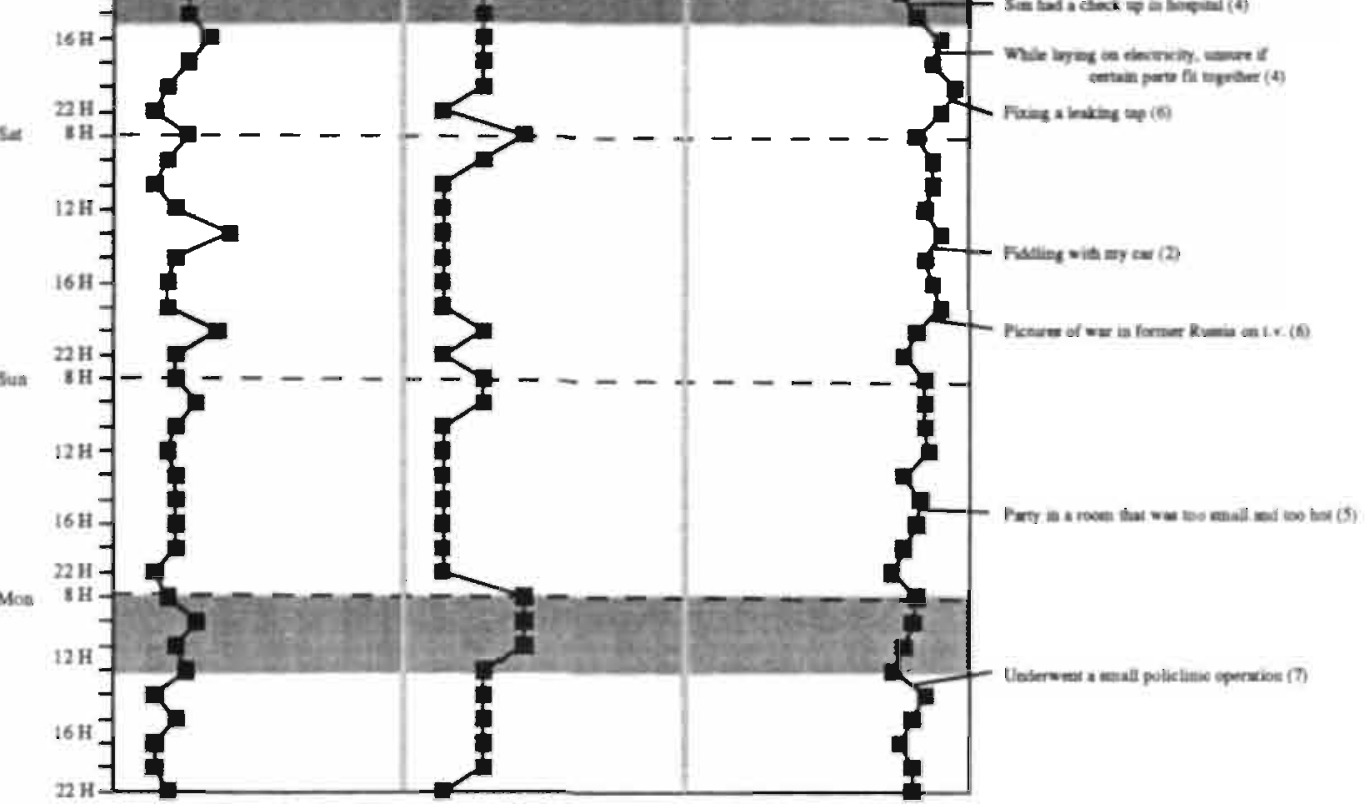


\section{STRESSFUL EVENTS IN DAILY LIFE: FREQUENCY, CONTEXT AND APPRAISAL}

We first examine the frequency, nature, and evaluation of events reported as stressful by this sample of white collar men. The ESM forms asked subjects to describe any stressful events or situations which may have taken place in the interval since the last ESM report (on average 90 minutes). Subjects' descriptions of stressful daily events were coded according to context, with categories work, nefwork (events. concerning family, friends, and acquaintances), household/financial, leisure, personal health (somatic and psychological health), transport, and other. In addition, events were classified according to whether or not they involved a social interaction and/or a task demand (see chapter 2 'Coding of ESM measures section). Subjects also rated the events they reported on the dimensions unpleasantness, imporiance, predictability, controllability, and frequency of prior occurrence.

\section{Event frequency}

Subjects reported a total of 626 stressful events during the five days of ESM, with a mean of 7.1 events per subject (median $5.0 ; \mathrm{s.d} .=7.3)$. Events as a percentage of total beeps averaged $16.8 \%(\mathrm{~s} . \mathrm{d} .=16.5)$. Of all stressful events reported, $33 \%$ were rated as highly stressful (score of $\geq 5$ ).

\section{Event context}

The distribution of events by life domain (expressed as frequency of each type of stressor divided by total beeps x 100) is shown in Figure 3.2. Subjects reported stressful events related to work and to social network most often. Of all events reported, $48 \%$ were work-related. Since the frequency of work events could relate to the amount of time spent at work, we also examined the probability of the occurrence of a work event given the amount of time spent at work. On average, subjects reported being at work on $31 \%$ of total beeps. 52 of the subjects reported a higher percentage of work-related events than would be expected based on the amount of time they spent at work (Wilcoxon Matched-Pairs Signed-Ranks test, $\mathrm{p}<.001$ ). This indicates that work-related events were not only absolutely but also relatively high in frequency. Next, we examined the correlations between the percentages of events reported in the various contexts (Table 3.2). The strongest correlations were between network and household events ( $r h o=.35 ; \mathrm{p}<.01$ ) and between network and work events $(r h o=.35 ; \mathrm{p}<.01)$. These correlations indicate that the experience of stressful events was not constrained to a particular life domain for an individual.

Table 3.2. Correlations (Spearman) between the percentages of events in the various contexts ${ }^{\mathrm{a}}$.

\begin{tabular}{lllllll}
\hline & 1 & 2 & 3 & 4 & 5 & 6 \\
\hline 1. Work & & & & & & \\
2. Network & $.35^{* *}$ & & & & & \\
3. Household & $.29^{* *}$ & $.35^{* *}$ & & & & \\
4. Leisure & $.21^{*}$ & .20 & .14 & & & \\
5. Personal Health & -.18 & .01 & .04 & -.16 & & \\
6. Transport & .15 & .07 & .10 & -.10 & .12 & .12 \\
7. Other & .05 & $.29 * *$ & .20 & .10 & -.10 & \\
\hline
\end{tabular}

*p<.05; * *p<.01; ${ }^{a} \mathrm{n}=88$ 


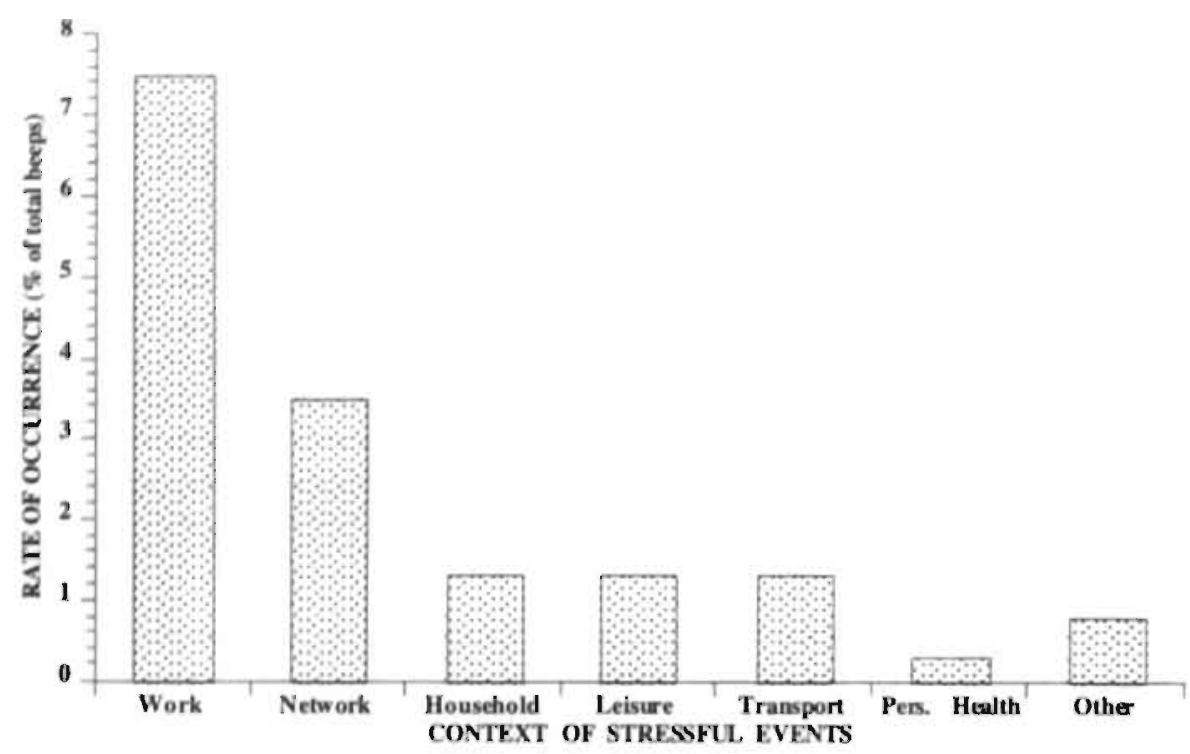

Figure 3.2. Frequency of stressful daily events by life domain.

In Figure 3.3. the percentage of events coded as task demand, as social interaction and as internal or external are shown. A minority of stressful events were characterized as social interaction or task demand events, and the large majority of events were (theoretically) observable or verifiable. Internal events (e.g. worries, anxieties) represented only $6.6 \%$ of all reported events. Examples of reported stressful events for each category are shown in Table 3.3.

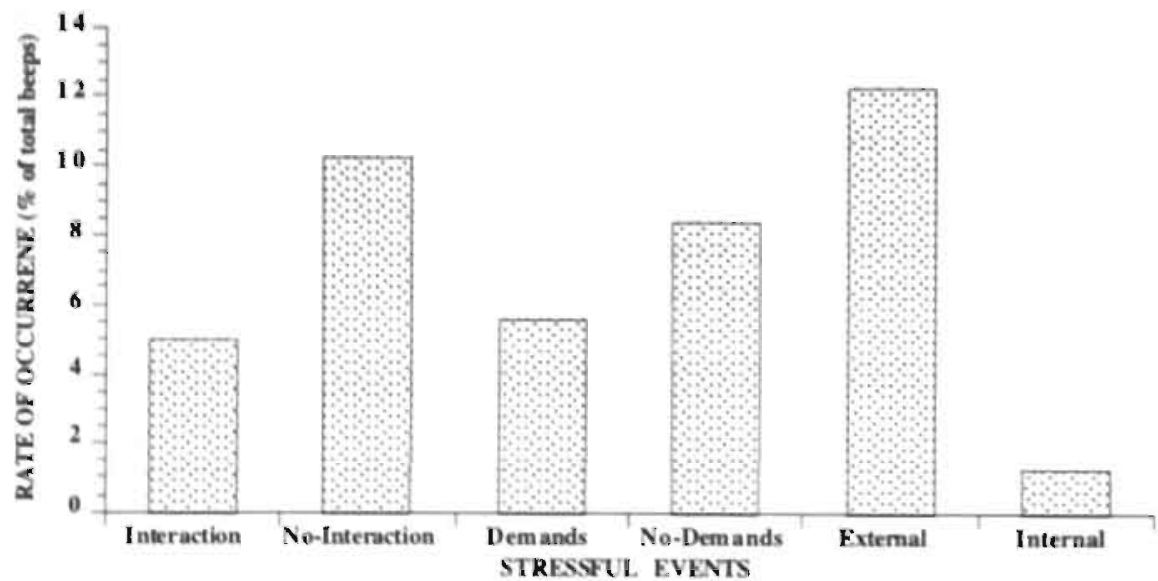

Figure 3.3. Frequency of stressful daily events: social interaction, task demand, and external/internal events. 
Table 3.3. Examples of stressful events.

\section{Work}

(events concerning boss, supervisor, clients. co-worker, general happenings at work)

'behind on computer work'. 'while working getting a lot of crazy telephone calls". "difficult discussion at work meeting'. 'job evaluation conversation'. 'a lot of work, a lot of different things'. 'can not fix computer programme error'. 'discussion about changes in organisation'. 'writing an account that has to be finished today". "making appointments under high time-pressure'.

\section{Network'}

(events concerning spouse, partner, children, relatives, friends, other acquaintances)

'discussion with spouse about arriving too late at friends' house'. 'getting children ready in time for school', 'children are annoying', "argument with spouse about household affairs". 'received message that sister has been in car accident' 'whole family wants attention at the same moment'. 'baby-sitter cancelled appointment:

\section{Houschold}

(events concerning general housework, errands,

family related duties, financial problems, etc.)

having to do a lot of errands in a short time period'. 'have to make a decision about buying a couch'. 'working in garden under high timepressure'. 'having to do a lot of dishes'. 'trying to fix a dripping tab'. 'while repairing bicycle chain breaks'. 'cleaning-up the mess without any support'.

\section{Leisure}

(events concerning hobbies, sports, outings. vacation, etc.)

'playing tennis match'. 'driving in a sports car'. "watching soccer: favoritc team missed a lot of chances'. 'performance with choir'. 'music band played out of tune' 'jogging', 'watching the Euro cup soccer game (Holland-Scotland)'

Personal Health

(events concerning illness, injury of the subject

himself, worries about health, nerves)

'headache'. 'nervous". 'underwent a small operation'.

\section{Transport}

(e.g. missed bus, traffic jam, unusual traffic)

'heavy traffic while driving home'. 'missed bus'. 'cycling in pouring rain'. 'had to hit the brakes suddenly', 'could not find a place to park the car'. "almost run over by a car while on my bike".

\section{Social Interaction}

(c.g. argument, conflict, conversation)

'squabble with children'. 'discussion with colleague about problems in the organisation'. 'conflict with partner about upcoming visit parents'. 'meeting with department'. 'discussion with spouse about furure childcare'. 'argument with boss'. 'had to give bad news to a client'. 'argument with spouse about how to act in certain situation'.

\section{Task Demands}

(e.g. difficult or problematic tasks, lime pressure, deadlines, a lot of work, failure at task)

'pressured by unexpected visit of client at work'. 'have to hurry washing, shaving, and dressing because woke up too late'. 'difficulties painting the wall', 'failed to saw up the wood into pieces'. 'had to take care of a lot of errands during my lunch break'. 'the usual hectic Saturday: running back and forth'. 'too much work to do'.

\section{Event appraisals}

Correlations among event appraisals are shown in Table 3.4. As would be expected, the strongest correlation was between event stressfulness and unpleasantness. Events that were less predictable or less controllable were also more unpleasant. Next we examined whether the various types of stressful events differed on the six appraisal dimensions. Figure 3.4 shows the mean scores for the types of stressful events on the different appraisal dimensions. Surprisingly, Leisure events were rated as most stressful, but they were the least unpleasant by far and the most. expected. An explanation could be that certain Leisure events (e.g. sport games) are experienced as stressful and exciting but that the stress is of a positive and 
challenging nature. Whether the types of events differed significantly from each other with regard to the appraisal dimensions was only tested for a subset of events, since not all event types were reported by all subjects thus resulting in small sample sizes for certain event types. Comparisons were made for Work versus Network, Interactions versus Non-Interaction, and Demand versus Non-Demand events. Work events were reported as occurring significantly with greater regularity than Network events (frequency: Wilcoxon Matched-Pairs Signed-Ranks test, p<.05), but no significant differences were found for the other appraisal dimensions. Interaction events were appraised as significantly more important $(\mathrm{p}<.01)$, more controllable $(\mathrm{p}<.001)$, and occurring more often $(\mathrm{p}=.05)$ than Non-Interaction events. Demands were appraised as significantly less important than Non-Demands events. The other appraisal dimensions showed no significant differences here.

Table 3.4. Correlations (Spearman) between event Appraisais ${ }^{\mathrm{a}}$ and mean appraisal scores ${ }^{\mathrm{b}}$.

\begin{tabular}{|c|c|c|c|c|c|c|c|}
\hline & I & 2 & 3 & 4 & 5 & $\bar{M}$ & $S D$ \\
\hline 1. Unpleasantness & & & & & & 3.6 & 1.4 \\
\hline 2. Stressfulness & $.41 * *$ & & & & & 3.9 & 1.0 \\
\hline 3. Importance & .03 & $.22 * * *$ & & & & 4.1 & 1.5 \\
\hline 4. Controllability & $-.23 * * 0$ & -.03 & .03 & & & 3.7 & 1.5 \\
\hline 5. Chronicity & -.01 & -.03 & $-18 * *$ & $.22 * * *$ & & 3.1 & 1.8 \\
\hline 6. Predictability & $-.31 * *$ & -.03 & $19 * * *$ & .07 & $18 * * *$ & 3.4 & 1.4 \\
\hline
\end{tabular}






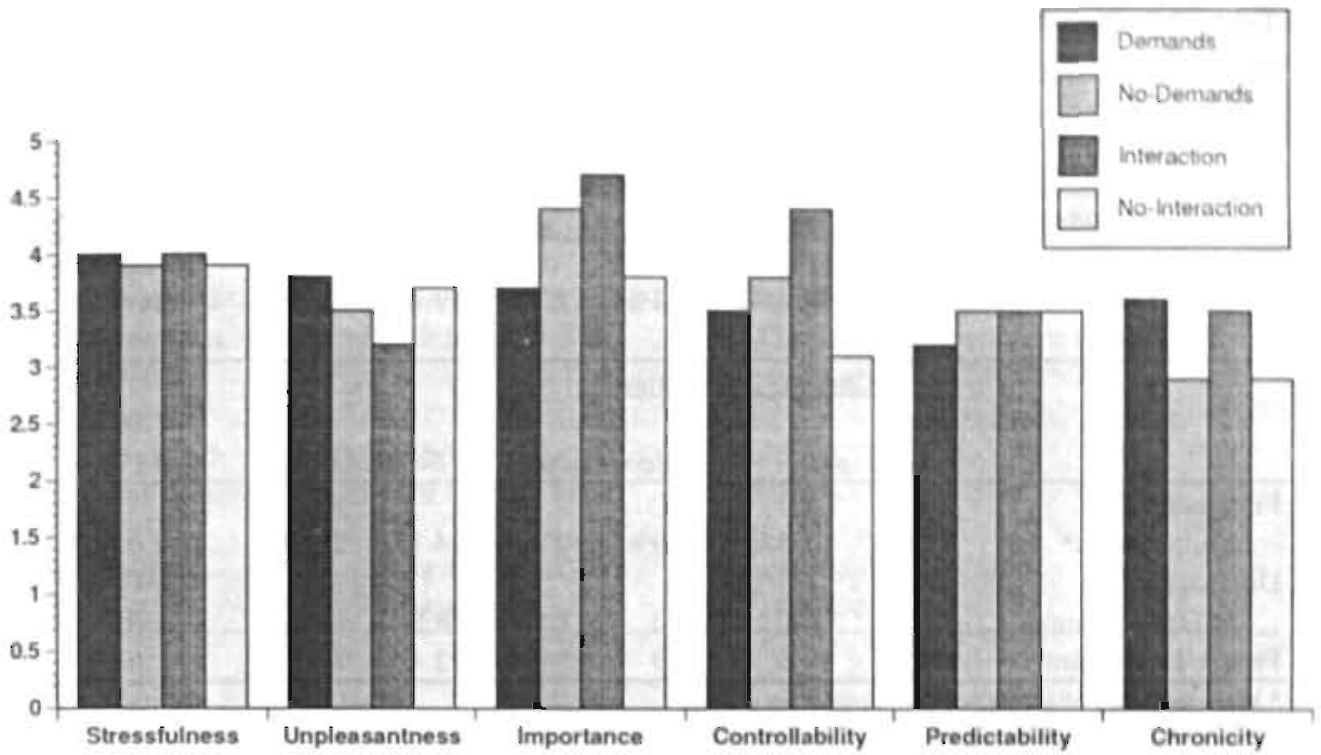

Figure 3.4. Mean appraisal scores calculated for events in the context of $(\mathbf{A})$ work $(n=65)$. network $(n=36)$, household $(n=22)$, leisure $(n=19)$, transport $(n=29)$, and for $(B)$, no-interaction

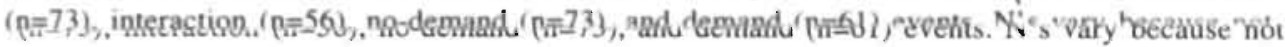
all event types were reported by all subjects.

In summary, subjects reported stressful work events most often, followed by social network events, and the experience of stressful events was not limited to a certain context. In general, events that were appraised as less predictable and less controllable were the most unpleasant. Stressful work events differed from stressful network events only in the appraised frequency of past occurrence, as was confirmed by the current event frequency scores. Negative social interactions were appraised as most important, most controllable, but also as occurring with greater regularity in the past, when compared to all other events, although current frequency scores revealed that social interaction events were actually reported relatively rarely during ESM sampling.

\section{RELATIONSHIP OF DAILY EVENTS TO LIFE EVENTS AND CHRONIC DIFFICULTIES}

It has been suggested (e.g. Hinkle, 1974; Kanner et al., 1981; Kaplan, 1979) that life events may initiate a cascade of daily stressors; for example, a divorce may lead to a series of new hassles in the context of keeping house, making meals, or keeping up social contacts, which did not have to be dealt with previously. As described in Chapter 2, life events were recorded with the questionnaire form of the List of Threatening Experiences (LTE-Q; Brugha et al., 1985), and chronic siress was assessed with the Long-term Difficulties Questionnaire (LLM; Hendriks et al., 1990), which focuses on problems in relation to work/study, housing, physical environment, leisure, finance, and social relationships (see 'Cross-sectional instruments' section). 
Table 3.5. Rate of daily event occurrence by increasing number of life-events and chronic. difficulties.

Number of Life-Events

\begin{tabular}{|c|c|c|c|c|c|c|c|}
\hline & \multicolumn{2}{|c|}{$0(n=51)$} & \multicolumn{2}{|c|}{$!(n=22)$} & \multicolumn{2}{|c|}{$\geq 2(n=15)$} & \multirow[t]{2}{*}{$p$} \\
\hline & mean & sd & mean & sd & mean & sd & \\
\hline $\begin{array}{l}\text { Frequency of } \\
\text { Daily Events }\end{array}$ & $15.7 \%$ & $15.4 \%$ & $17.4 \%$ & $17.9 \%$ & $19.4 \%$ & $18.8 \%$ & ns \\
\hline
\end{tabular}

Chronic Difficulties

\begin{tabular}{|c|c|c|c|c|c|c|c|}
\hline & \multicolumn{2}{|c|}{ None $(n=6)$} & \multicolumn{2}{|c|}{ Minor $(n=39)$} & \multicolumn{2}{|c|}{ Severe $(n=33)$} & p \\
\hline Daily Eventsa & $11.5 \%$ & $17.8 \%$ & $10.9 \%$ & $10.9 \%$ & $24.1 \%$ & $20.3 \%$ & $<.01$ \\
\hline Unpleasantness & & & & & & & \\
\hline of Daily Events ${ }^{b}$ & 2.3 & 1.0 & 3.3 & 1.6 & 4.0 & 1.3 & $<.01$ \\
\hline Freq $\times$ Unpleasant ${ }^{c}$ & 34.2 & 60.6 & 36.9 & 41.4 & 92.4 & 76.0 & $<.01$ \\
\hline
\end{tabular}

a Mean frequency of events as a percentage of total beeps

b Mean unpleasantness rating of events

${ }^{c}$ Mean frequency by unpleasantness rating

When we compared subiects who resoorted no. one. or two or mote life-pevents in the past year on the percentage of stressful daily events reported during the ESM period, however, no differences in rate of stressful daily events occurrences were observed. Subjects with more life-events did not report more daily events (Table 3.5.). Dividing the total scores on the chronic difficulties scale into three categories (none, minor [average or below average in number], or severe [above average in number], we found that subjects with higher scores on the chronic difficulties scale reported more daily events (Kruskal-Wallis one-way anova; p<.001) and rated events as more unpleasant $(\mathrm{p}<.05)$. The sum of frequency by unpleasantness ratings was significantly higher as the chronic difficulties scores increased ( $p<.001)$ (Table 3.5.).

In summary, not life events but chronic difficulties were positively related to the frequency and intensity of stressful daily events reported during the ESM period.

\section{COMPARISON OF WITHIN-DAY EVENT AND END-OF-DAY MEASURES}

We next examined how two different methods of daily event assessment compared in terms of the number and type of stressors reported; in other words, do repeated within-day event reports provide different information than checklist results obtained at the end of the day? Subjects were asked at the end of each Experience Sampling day how stressful the day had been and completed a short checklist, the Daily Stress Scale (Bolger et al., 1989a). This instrument (as described in 'Description of ESM instrument' section chapter 2), consists mainly of items concerning demands (e.g. a lot of work at home, at work) or interpersonal conflicts (e.g. argument with spouse, child, colleague), with two additional items concerning transportation and financial problems. 
Table 3.6. Prevalence of events obtained by within-day (ESM) assessments compared to end-ofday checklist assessments.

Did (at least 1) event occur?

According to:

\begin{tabular}{lccc}
\hline Sources of Stress & ESM & Checklist & Chi-Square \\
\hline $\begin{array}{c}\text { Home stress } \\
\text { Overload }\end{array}$ & $7.1 \%$ & $36.8 \%$ & $<.001$ \\
Argument & $8.6 \%$ & $17.5 \%$ & $<.00 !$ \\
Work stress & & & \\
Overload & $15.3 \%$ & $40.0 \%$ & $<.001$ \\
Argument & $19.6 \%$ & $7.0 \%$ & $<.001$ \\
Home Total & $14.1 \%$ & $43.9 \%$ & $<.001$ \\
Work Total & $26.8 \%$ & $40.7 \%$ & $<.001$ \\
\hline
\end{tabular}

The number of ESM events reported each day was positively correlated with the number of events reported on the checklist at the end of the day ( $r h o=.43$. $\mathrm{p}<.001: \mathrm{n}=439$ ) and with the end of the day global rating of the day's stressfulness ( $r h o=.52, \mathrm{p}<.001 ; \mathrm{n}=404$ ). We compared the frequency of reports of home stress and work stress (rated as overloads or as arguments) within-days with the reports of these events at the end of the day. Scores were rated 0 if no event was reported and 1 if at least one event was reported. Results are shown in Table 3.6. Except for work arguments, a higher frequency of events was reported with the checklist at the end of the day compared to the within-day measurements. All differences were significant. Similar results were found when only checklist events that were at least somewhat unpleasant were analyzed. We can conclude: that by recognition (as with checklists) many more events are reported than when actively asked for (open-ended question). Since some checklist items were of a more global nature (e.g. 'a lot of work at home', 'a lot of work at work'), it is also possible that when summarizing one's experiences at the end of the day these items are more easily endorsed, while during the day events are more discrete and too small in itself to be reported. Perceptual biases (e.g. effort after meaning) may he more likely to occur at the end of the day than at the momentary ESM assessments.

In summary, many more events were reported with the checklist at the end of the day compared to the open-ended, within-day measurements.

\section{DIFFERENCES BETWEEN HIGH AND LOW STRESS GROUPS}

To investigate whether individual perceptions of high versus low levels of stress are associated with personal characteristics and daily life experiences, differences between the two stress groups were tested with respect to psychological characteristics, the distribution of daily activities, and measures of stressful daily events. 


\section{Psychological characteristics}

The two groups were compared on the following measures: life events (LTEQ), chronic difficulties (LLM), psychological and somatic complaints (SCL-90), psychosomatic symptoms (PSC), depressive symptomatology (Zung), trait anxiety (STAI), trait anger (STAS), personality characteristics (Dutch abridged MMPI: NVM), and coping style (UCL). These instruments have been described in Chapter 2 (see section 'Cross-sectional instruments').

Results are shown in Table 3.7. HS subjects reported significantly more chronic difficulties during the last year than LS subjects, but no more life events. HS subjects also exhibited higher scores on both psychological (anxiety, anger, and depression) and psychosomatic complaints questionnaires than LS subjects and scored higher on the personality dimensions negativism, somatization, timidity and serious psychopathology. Additionally, HS subjects seem to use passive coping styles (palliative reaction, depressive reaction, expression of emotion) more often than LS subjects and an active, problem-focused coping style less often. Where possible, mean scores for both samples were compared to published norms for the Dutch population. The general pattern found was that LS subjects scored average or below average compared to norm scales, while HS subjects scored above average or high.

Frequency and intensity scores for the seven different categories of chronic difficulties are shown in Table 3.8. HS subjects reported significantly more chronic difficulties for all categories except for the category Family (i.e. parents, children, brothers / sisters, in-laws). Work and Housing difficulties had the highest prevalence in both groups, and HS subjects reported relational difficulties with the partner relatively frequently. The two groups were similar in the kind and ranking order of frequency of work difficulties mentioned: 1) high work pressure or work load, 2). conflicts or differences in opinion with boss or colleagues, 3) little appreciation and 4) changed or indistinct job functions due to reorganization. With regard to difficulties with partner, again several specific problems were mentioned by both groups: 1) conflicts or differences in opinion (for example, concerning household tasks or children), 2) low frequency of sexual contact (not in the mood, too busy), and 3) diminished interest in each other or doubts about choice of partner. Housing difficulties were more diverse, with problems such as conflicts with neighbours, criminality (vandalism, theft, drugs) and pollution. With regard to the intensity of chronic difficulties, only work difficulties were scored as more intense by HS subjects $($ mean $=2.0$ (s.d. 0.5) for HS subjects versus mean= 1.7 (s.d. 0.4) for LS subjects, Mann-Whitney $U$ test, $\mathrm{p}<.05$ ), and a trend in the same direction was found for difficulties concerning partner (mean=2.1 (s.d. 0.7) for HS subjects versus mean=1.7 (0.3) for LS subjects, $p=.06$ )

As shown in Table 3.9, psychosomatic complaints occurred with regularity (at least once a week) in a higher percentage in HS than in LS subjects and occurred more frequently overall in the HS group. Significant differences between groups in frequency of complaints (using the full range of frequency ratings) were found for: fatigue, depression, backache, headache, insomnia, general weakness, stomach pain, heart palpitations, and nausea. The same psychosomatic complaints were also rated as significantly more severe in HS subjects (p-values all <.01). The nine subjects who reported no complaint at all were all from the LSi group. 
Table 3.7. Characteristics of high and low stress groups.

\begin{tabular}{|c|c|c|c|}
\hline & $\begin{array}{l}\text { LS } \\
\mathrm{n}=46 \\
\text { mean (st.dev) }\end{array}$ & $\begin{array}{l}\text { HS } \\
\mathrm{n}=42 \\
\text { mean (st.dev) }\end{array}$ & $\begin{array}{l}\text { p-value } \\
\text { (2-tailed) }\end{array}$ \\
\hline Life-events & $.5(.7)$ & $.8(.9)$ & ns \\
\hline Chronic difficulties & $19.5(2.3)$ & $23.3(4.0)$ & $\cdots$ \\
\hline SCL-90 (total) & $102.5(11.1)$ & $147.4(39.3)$ & $\cdots$ \\
\hline Trait anxiety & $28.3(4.4)$ & $39.8(7.6)$ & $\cdots$ \\
\hline Depression & $36.5(5.5)$ & $48.4(7.7)$ & $\cdots$ \\
\hline Trait anger & $14.6(4.1)$ & $18.0(4.6)$ & $\cdots$ \\
\hline Psychosomatic symptoms & $6.1(5.6)$ & $27.5(23,2)$ & $\cdots$ \\
\hline \multicolumn{4}{|l|}{ Personality: } \\
\hline Negativism & $10.0(5.5)$ & $19.0(6.8)$ & $\cdots$ \\
\hline Somatization & $2.8(2.8)$ & $9.5(7.3)$ & $\cdots$ \\
\hline Timidity & $8.3(6.3)$ & $11.3(7.4)$ & • \\
\hline Serious Psychopathology & y $1.3(1.9)$ & $2.8(2.5)$ & $\cdots$ \\
\hline Extraversion & $15.4(5.3)$ & $16.5(5.6)$ & ns \\
\hline \multicolumn{4}{|l|}{ Utrecht Coping List: } \\
\hline Active coping & $21.1(4.0)$ & $17.3(3.4)$ & $\cdots$ \\
\hline Palliative reaction & $14.6(3.0)$ & $17.2(3.3)$ & $\cdots$ \\
\hline Avoidance coping & $14.1(2.5)$ & $15.1(3.6)$ & ns \\
\hline Social support & $11.7(2.7)$ & $12.5(2.7)$ & ns \\
\hline Depressive reaction & $8.8(1.6)$ & $12.6(3.4)$ & $* *$ \\
\hline Expression emotions & $5.8(1.3)$ & $6.9(1.7)$ & $* *$ \\
\hline Comforting cognitions & $10.8(2.6)$ & $11.7(2.8)$ & $\mathrm{ns}$ \\
\hline
\end{tabular}

* $p<05 ; \cdots p<01 ; \cdots * p<001$

Table 3.8. Percentage of HS and LS subjects reporting chronic difficulties in each life domain.

\begin{tabular}{|c|c|c|c|}
\hline & $\begin{array}{l}\text { low PSS } \\
\mathrm{n}=40\end{array}$ & $\begin{array}{l}\text { high PSS } \\
n=42\end{array}$ & $\begin{array}{l}\text { p-value } \\
\text { (2-tailed })\end{array}$ \\
\hline 1. Work difficulties & $39 . i \%$ & $88.1 \%$ & $* *$ \\
\hline $\begin{array}{l}\text { 2. Housing/Finance difficulties } \\
\text { Relational difficulties }\end{array}$ & $43.5 \%$ & $71.4 \%$ & $* *$ \\
\hline 3. Partner & $23.9 \%$ & $53.7 \%$ & $*$ \\
\hline 4. Family & $23.9 \%$ & $41.5 \%$ & ns \\
\hline 5. Friends/Acq. & $19.6 \%$ & $38.1 \%$ & $*$ \\
\hline 6. Leisure difficulties & $19.6 \%$ & $39.1 \%$ & * \\
\hline
\end{tabular}

p<05: ** p<.01: "*** p<001; Group differences in the percentage of reported chronic difficulties were tested by
means of chi-square tests. 
Table 3.9. Psychosomatic complaints in HS and LS subjectsa.

\begin{tabular}{|c|c|c|c|}
\hline & low PSS & high PSS & $\begin{array}{l}\text { p-value }{ }^{b} \\
\text { (2-tailed) }\end{array}$ \\
\hline I. Fatigue & $39.2 \%$ & $66.7 \%$ & $* * *$ \\
\hline 2. Depression & $2.2 \%$ & $42.9 \%$ & $* * *$ \\
\hline 3. Backache & $17.3 \%$ & $38.1 \%$ & * \\
\hline 4. Headache & $8.7 \%$ & $35.7 \%$ & $* * *$ \\
\hline 5. Insomnia & $10.9 \%$ & $31.0 \%$ & $* * *$ \\
\hline 6. Weakness & $0.0 \%$ & $19.1 \%$ & $* * *$ \\
\hline 7. Eye pain & $8.7 \%$ & $16.6 \%$ & ns \\
\hline 8. Stomach pain & $2.2 \%$ & $11.9 \%$ & $* * *$ \\
\hline 9. General stiffness & $2.2 \%$ & $11.9 \%$ & ns \\
\hline 10 Heart palpitations & $2.2 \%$ & $11.9 \%$ & $*$ \\
\hline 11 Dizziness & $4.3 \%$ & $11.9 \%$ & ns \\
\hline 12. Diarrhea / constipation & $0.0 \%$ & $9.5 \%$ & ns \\
\hline 13. Nausea & $0.0 \%$ & $7.2 \%$ & $* *$ \\
\hline 14. Spastic colitis & $0.0 \%$ & $7.1 \%$ & ns \\
\hline 15. High blood pressure & $2.2 \%$ & $4.8 \%$ & ns \\
\hline 16. Asthma & $0.0 \%$ & $2.4 \%$ & ns \\
\hline 17. Gastric ulcer & $0.0 \%$ & $0.0 \%$ & $\mathrm{~ns}$ \\
\hline \multicolumn{4}{|l|}{ " $p<05, \cdots p<01, \cdots p<001$} \\
\hline $\begin{array}{l}\text { a In the first two columns, the per } \\
\text { a week are reported. In the th } \\
\text { psychosomatic complatints are sh }\end{array}$ & and LS & $\begin{array}{l}\text { osomatic ci } \\
\text { he differe }\end{array}$ & teported \\
\hline
\end{tabular}

\section{Distrlbution of dally activities}

Next, we examined differences between high and low stress groups in time spent in various activity contexts. One might suspect that HS subjects worked more, or relaxed less, than their LS counterparts; daily stress might be reflected in imbalances in work and maintenance versus rellaxation, recreational and social activities. In addition, if, for instance, HS subjects spent more of their time working, then they would have an increased chance of exposure to stressful work events. This analysis was based on ESM activity codes (see Chapter 2. 'Coding of ESM measures section).

Figure 3.5. shows the mean percentage of time spent in the various activities for LS and HS subjects over all study days. HS subjects did not differ significantly from LS subjects in the percentage of time spent in different activities. When we subdivided the category Leisure into Passive (e.g. watching television) and Active (e.g. sports, hobbies) Leisure, no significant differences were found between the HS and LS group (Passive Leisure: $73.9 \%$ versus $79.1 \%$, Active Leisure: $26.1 \%$ versus $20.9 \%$ respectively; ns). Nor did the percentage of time spent in various activities outside working hours (weekend days and after 5 p.m. during work days) show significant between group differences. In summary, we found no differences between the two groups in patterns of time use. 


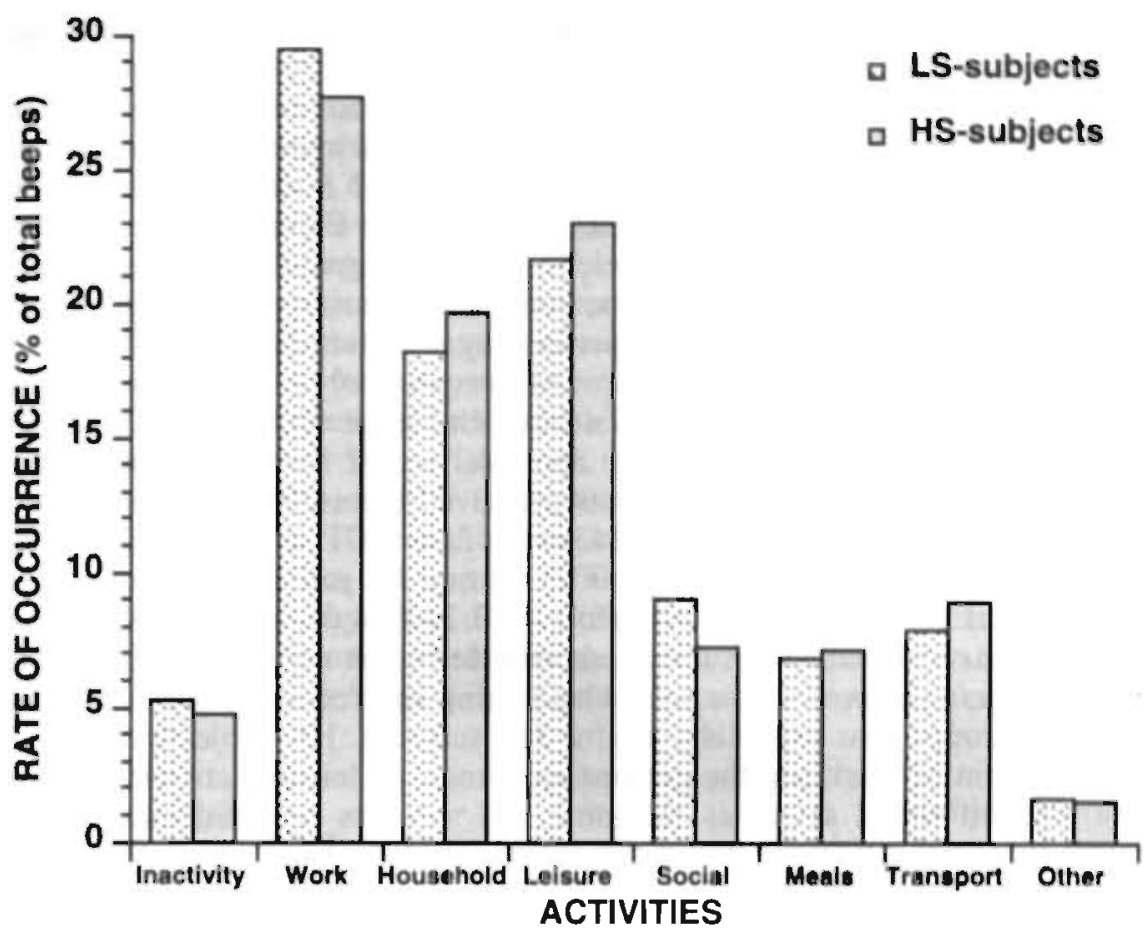

Figure 3.5. Use of time: frequency of activities.

\section{Appraisals of daily activities}

Since appraisals of daily activities were expected to reflect the stressfulness of daily life experiences other than stressful events, we investigated differences between stress groups in how they evaluated their daily activities. Data for this analysis were the ESM ratings of how current activity was experienced in terms of enjoyment, skill, effort, and challenge. As shown in Table 3.10., these dimensions were interrelated.

Table 3.10. Correlations (Pearson) among activity appraisals ${ }^{\mathrm{a}}$.

$1 \quad 2 \quad 3$

\footnotetext{
1. Motivation

2. Skill

3. Effort

$42 * * *$

4. Challenge

$-.32 * * * \quad-.36 * * *$

*...p $<$ <.001

.03

$-.22 * * * \quad .40 * * *$

a Peastson correlations were computed across all beeps $(n=3540)$.
}

Clear differences in how the two group evaluated their activities were found. HS subjects reported that they enjoyed activities less $(4.9$ versus $5.5 ; \mathrm{p}<.001)$, that they were less skillful $(5.7$ versus $6.1 ; \mathrm{p}<.001)$, and that the activities required more 
effort to perform ( 1.8 versus $1.6 ; \mathrm{p}<.01)$ than LS subjects. The two groups did not differ in their assessment of how challenging activities were.

Next, we tested by means of analyses of variance for repeated measures for significant differences in the degree to which the various appraisals were dependent on different activities (Work, Household, Leisure and Social Activities). Mean appraisal scores for the different activities are shown in Figure 3.6a-e. Confirming univariate analyses, the two groups differed in their ratings of the various activities: HS subjects enjoyed doing activities less, perceived themselves as less skillful, and they felt that more effort was required in performing activities $(\mathrm{F}(1,81)=13.46, \mathrm{p}<.001$; $\mathrm{F}(1,81)=6.86, \mathrm{p}<.05 ; \mathrm{F}(1,81)=7.14, \mathrm{p}<.01$, respectively), while there were no differences between groups for the appraisal of challenge $(\mathrm{F}(1,81)=.02$; ns). There were significant differences in the mean appraisal scores for the various activities, with the exception of how skillfull subjects perceived themselves to be in relation to the various activities (enjoyment: $\mathrm{F}(3,243)=45.12, \mathrm{p}<.001$; effort: $\mathrm{F}(3,243)=20.27$, $\mathrm{p}<.001$; challenge: $\mathrm{F}(3,243)=35.81, \mathrm{p}<.001)$. Group by activity interaction effects were significant for enjoyment and effort $(\mathrm{F}(3,243)=4.07, \mathrm{p}<.01 ; \mathrm{F}(3,243)=6.6$, $\mathrm{p}<.001$, respectively), with HS subjects scoring lower on enjoyment and higher on effort. Work was perceived as the most challenging and requiring the most effort in both subject groups, but especially so for HS subjects. HS subjects reported the lowest enjoyment of work and the greatest enjoyment of leisure activities. While LS subjects enjoyed social activities the most, HS subjects reported relatively little enjoyment in social situations and rated social interactions as more demanding in terms of effort than LS subjects.
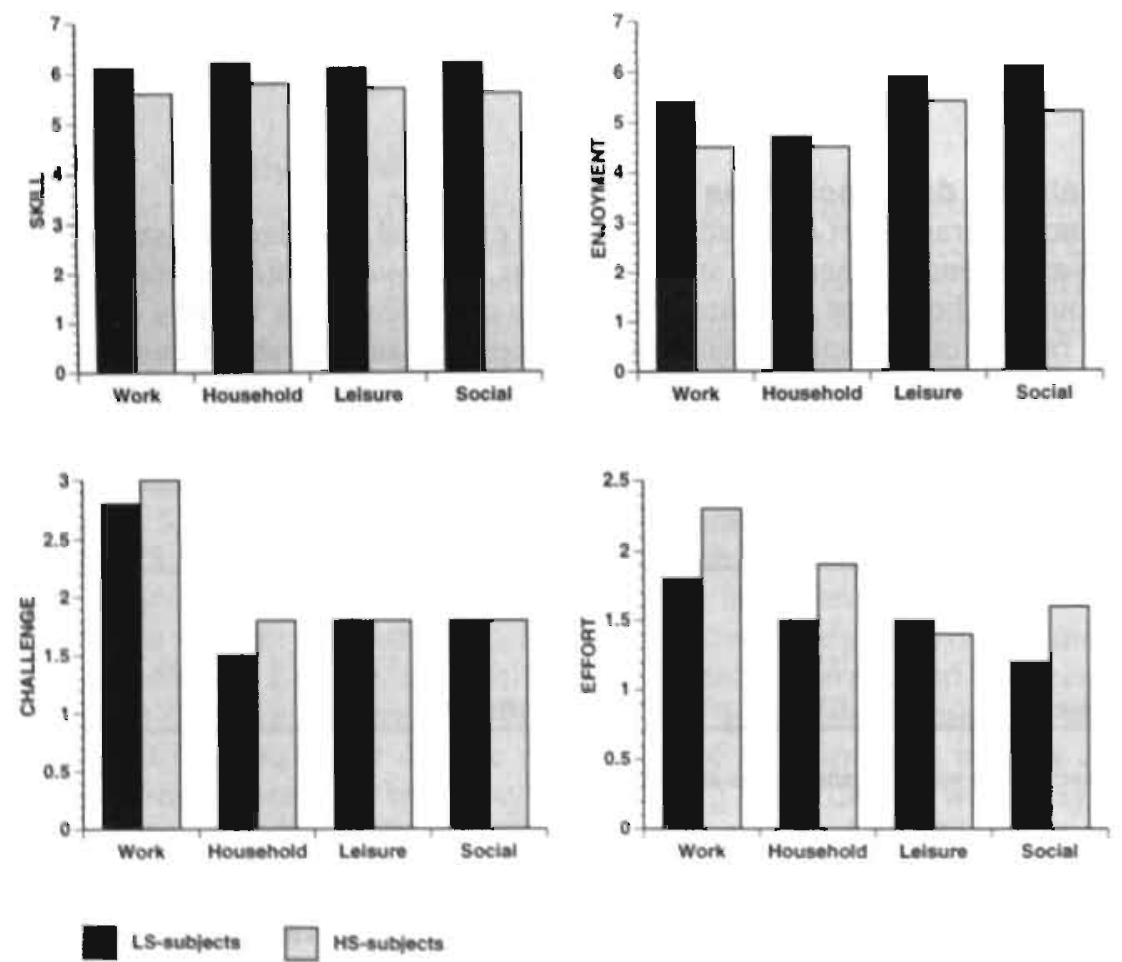

Figure 3.6.a-d Mean appraisal of required skill, enjoyment, effort, and challenge for work. household, leisure, and social activities. 


\section{Frequency, context and appraisal of stressful daily events}

In this final section, differences between stress groups in the frequency, context, and evaluation of stressful daily events are tested.

Event frequency: LS subjects reported a mean of 5.4 events (s.d. $=5.5$ ) and HS subjects 9.0 events (s.d. = 8.6) during the five days of Experience Sampling (MannWhitney U test, p<.05). Seven subjects (all LS) reported no event at all. Event reports as a percentage of total beeps averaged $12.1 \%$ for the LS group (s.d. $=11.5$ ) versus $21.9 \%$ for the HS group (s.d. = 19.5) (Mann-Whitney U test, p<.01). Since a major category of reported events was work-related, we expected more frequent reports of stressful events during weekdays than on weekends. Multivariate analyses of variance for repeated measures indicated a highly significant main effect on event frequency ( $\%$ of total beeps) for the within-subject factor day ( $S$ days) $(\mathrm{F}(4,340)=16.02 ; \mathrm{p}<.001)$, as well as for the between-subject factor group (HS.LS) $(\mathrm{F}(1,85)=8.02 ; \mathrm{p}<.01)$. The interaction of day by group was also significant $(\mathrm{F}(4,340)=3.45 ; \mathrm{p}<.01)$. As can be seen in Figure 3.7., subjects indeed reported more stressful events on workdays than on weekend days. The HS subjects reported more events than LS subjects on each day of the week, but especially so on Thursday, Saturday and Monday. On Sunday, both groups reported relatively few events.

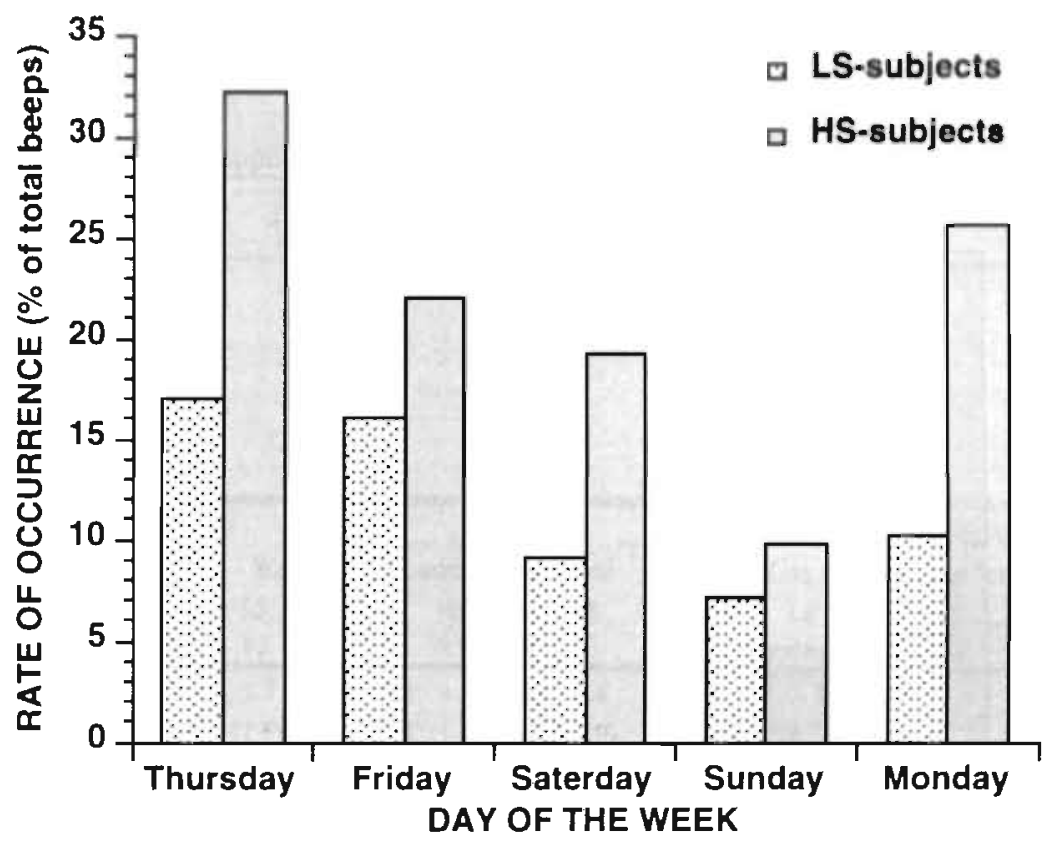

Figure 3.7. Frequency' of stressful daily events for HS and LS groups by day of the week.

Event context: As shown in Figure 3.8, HS subjects reported significantly more stressful work and network events than LS subjects, across all beeps. LS subjects, on the other hand, reported significantly more transportation events. HS subjects aiso reported significantly more social interaction events than LS subjects $(7.0 \%$ versus $3.2 \%, \mathrm{p}<.01)$, but not significantly more task demands $(6.9 \%$ versus $4.4 \%, \mathrm{~ns})$. As 
shown in Table 3.11, task demands as compared to social interactions are reported with equal frequency at home and at work in both groups. Both groups reported more task demands and more social interactions at work than at home. HS subjects reported significantly more events entailing negative social interactions (but not task demands) than LS subjects, both at home and at work.

Social context of event: We also coded who was involved in a reported event (social context) using the following categories: alone, household members, non-resident family, friends, colleagues, neighbours/acquaintances, and strangers (see Chapter 2 'Coding of ESM measures' section). HS subjects reported significantly more daily events concerning household members $(4.1 \%$ versus $1.6 \%, \mathrm{p}<.01)$, colleagues $(4.0 \%$ versus $2.5 \%, \mathrm{p}<.05)$, and other acquaintances $(1.6 \%$ versus $0.5 \%, \mathrm{p}<.05)$ than LS subjects across all beeps. There were no group differences for the categories alone, non-resident family, friends or strangers. Within the category household members, HS subjects reported significantly more events concerning partner (1.6\% versus $0.8 \%$, $\mathrm{p}<.01)$ and children $(2.6 \%$ versus $0.8 \%, \mathrm{p}<.01)$.

Next, we examined who was involved in events coded specifically as social interactions. In both groups, most negative interactions were with colleagues. Although such events were relatively rare, HS subjects had significantly more negative social interactions with colleagues $(3.3 \%$ versus $1.9 \%, \mathrm{p}<.01)$ and with the partner $(1.1 \%$ versus $0.3 \%, \mathrm{p}<.05)$, across all beeps.

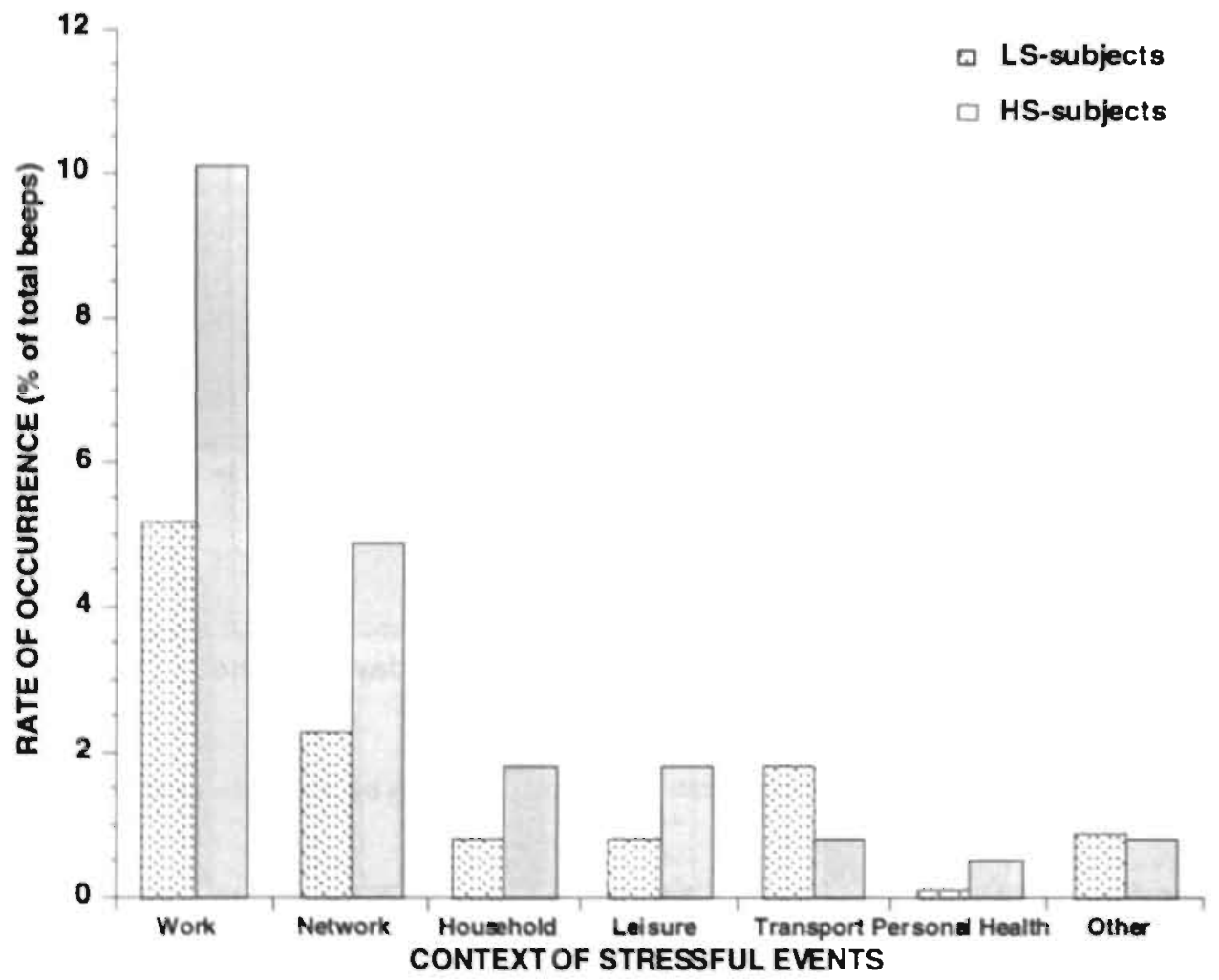

Figure 3.8. Frequency of stressful daily events for HS and LS groups by context. 
Table 3.11. Mean rate of occurrence $(+\mathrm{sd})$ of task demands and social interactions at home and at work (\% of total beeps).

\begin{tabular}{|c|c|c|c|}
\hline & $\begin{array}{l}\mathrm{LS} \\
\mathrm{n}=46\end{array}$ & $\begin{array}{l}\mathrm{HS} \\
\mathrm{n}=42\end{array}$ & $\begin{array}{l}\text { p-value } \\
\text { (2-tailed) }\end{array}$ \\
\hline \multicolumn{4}{|l|}{ at Home } \\
\hline Task demand & $2.6 \%(6.0)$ & $3.5 \%(5.8)$ & ns \\
\hline Social interaction & $1.8 \%(4.4)$ & $4.3 \%(7.5)$ & .05 \\
\hline \multicolumn{4}{|l|}{ at Work } \\
\hline Task demand & $7.3 \%(1[.4)$ & $12.4 \%(16.2)$ & ns \\
\hline Social interaction & $7.7 \%(10.4)$ & $13.2 \%(14.0)$ & .01 \\
\hline
\end{tabular}

Event appraisals: On average. HS subjects appraised events as more stressful and less controllable than LS subjects (Table 3.12). There were no differences between groups in the appraisals of event severity, importance, predictability, and chronicity. Event stressfulness and controllability were further examined for different types of events (work, network, household, leisure, social interactions, task demands). Statistical tests were done only for work events, task demands, and social interactions, because of the small sample sizes related to the other types of events. Results indicated that work events were significantly more stressful for HS than for LS subjects (see Table 3.12.).

Table 3.12. Mean event appraisals (+sd) for HS and LS subjects.

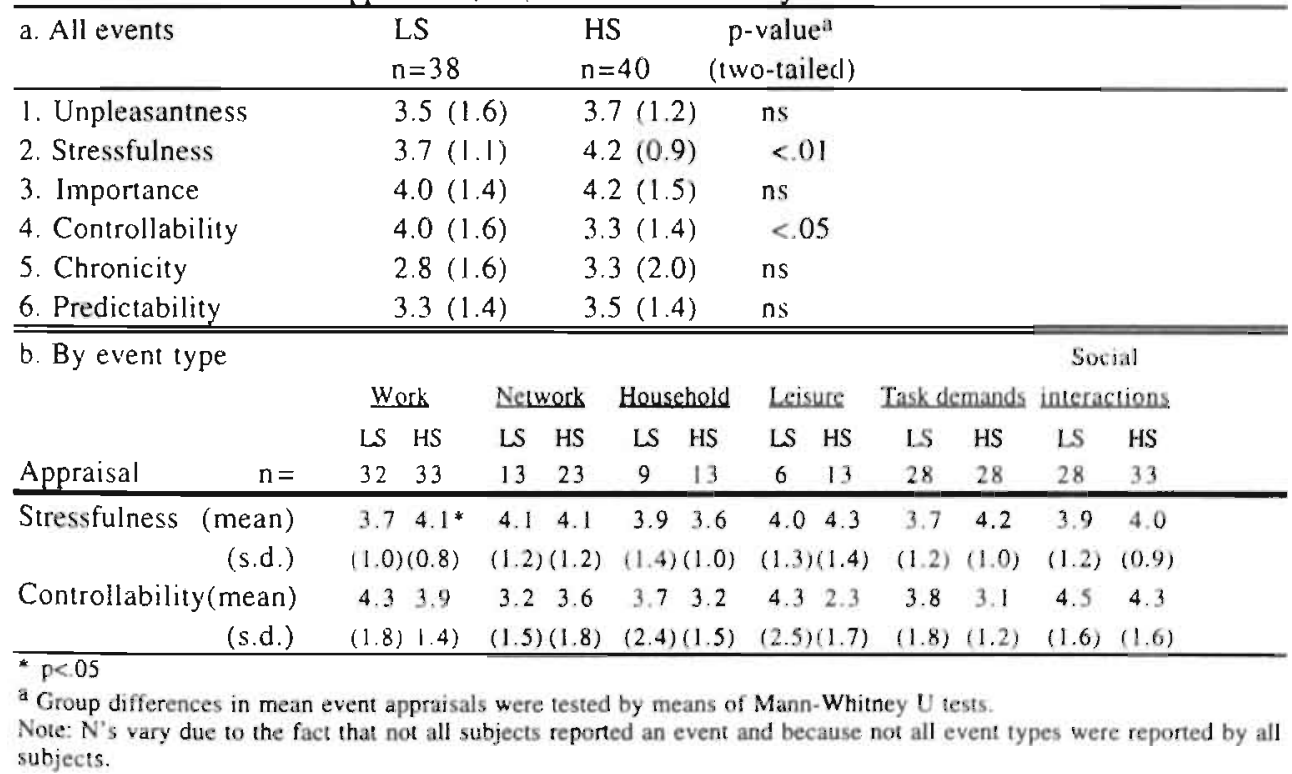

\section{DISCUSSION}

The aim of this chapter was to provide descriptive data on both quantitative and qualitative aspects of stressful events and activities in daily life, to increase our understanding of the construct stress as expressed in daily life. Using ESM, repeated 
measurements of stressful events, activities and their characteristics were made several times a day on several successive days in a sample of male white collar workers with varying stress levels.

Subjects reported a stressful event on $17 \%$ of all beeps. Whether this figure is high or low cannot be said; the frequency of reported stressful daily events cannot be compared to other studies since only a few have used an open-ended format to elicit events (Eckenrode, 1984; Rehm, 1978), and when used, different time-frames (events reported at the end of the day) together with different study populations (students, women) make comparisons irrelevant. Results indicated that in our sample the major source of stress in daily life was work, followed by the social network. These stress contexts make sense, given the developmental and demographic profile of the subjects. their most important social roles were those of employee, husband, and father. In line with the transactional definition of minor stressful events (Lazarus \& Folkman, 1984b, p.376), stressful daily events in this study can be characterized by its salience (importance) and unpleasantness. The finding that unpredictable and uncontrollable events were especially unpleasant is in accordance with results of many laboratory studies (Katz \& Wykes, 1985; Miller, 1979; Solomon, Holmes, \& McCaul, 1980; Thompson, 1981). Although subjective appraisals, as described above, appear to be important factors for understanding the stressfulness of daily events or situations, other dimensions should also be investigated for their relevance to the experience of stressful events, for example the experience of loss associated with an event (e.g. loss of a person, object, status, role, self-esteem) (Brown, 1989), future threat or feelings of uncertainty about how to cope with the event.

As might be expected, a high perceived stress level was related to higher scores on cross-sectional measures of psychological (anxiety, depression, anger) and psychosomatic (fatigue, depression, backache, headache, and insomnia) complaints, to the personality traits negativism, somatization, timidity and psychopathology, and to the more frequent use of passive and less frequent use of active (problem-focused) coping styles. Although HS subjects did not experience significantly more life events in the past year, they did report more chronic difficulties. Since HS subjects were healthy and actively employed, the pronounced group differences are somewhat surprising. From these data, the HS group emerges as a group of vulnerable, somewhat neurotic individuals, troubled by various psychological and psychosomatic complaints, experiencing several chronic environmental and relational difficulties, and using inadequate coping styles. An interesting question in this regard is how these characteristics would influence their lives in the future, if we could follow them, for example, another 10 years. In addition to the subjective measures, we would need to measure objective indices of health and work status (e.g. frequency of absenteeism from work, number of visits to health professionals, health records), to gain insight into the long-term consequences of high perceived stress. The pronounced group differences may also be related to the fact that the LS group contained subjects which seem to be more stress-resistant than on average. Compared to norm scores, LS subjects scored below average on psychological complaints, below average on the personality traits negativism, somatization, timidity, psychopathology, and average on extraversion (NPV). LS subjects also used more adequate coping styles indicated by high (norm) scores on problem-focused coping and low scores on depressive reaction.

Subjects with more chronic difficulties, but not with more life-events, reported significantly more daily events. Since chronic difficulties are much more dependent on the subjective judgment of a person than the more objective life-events, it is not 
surprising that chronic difficulties were related to the highly subjective stressful daily events. With respect to the major life events, it should be noted that life events, as occurred in the past year, were very rare in our sample, making it difficult to find an association between life events and daily events. HS subjects did report more life events, but not significantly more. Chronic difficulties seem not only related to increased perceptions of minor demands, but, as has been suggested for major life events (Kaplan, 1979), also seem related to the affective significance of minor events for the person, since with increasing chronic difficulties, events were rated as increasingly unpleasant. Subjects with high levels of chronic difficulties may experience events that are of themselves more unpleasant, but it is also possible that events. that relate to specific chronic difficulties (for example " an argument with spouse about household chores in the case of chronic marital problems) may be more salient and threatening, and thereby more unpleasant, for the person than the same event occurring in the absence of a chronic problem.

With respect to daily activities, the two groups had similar patterns of time use, and both groups perceived work activities as the most challenging but also requiring. the most effort. HS subjects did differ from LS subjects in how they evaluated their activities. Although in absolute terms HS subjects reported considerable enjoyment in various activities, perceived themselves to be quite skillful, and did not feel that activities required much effort to perform, they evaluated activities significantly less positively on these dimensions than LS subjects. This indicates that perceived stress levels are not only expressed in stressful events, but aiso in the evaluation and experience of daily activities. The differences between stress groups in the appraisal of activities were not restricted to a specific activity but were observed in several contexts.

With respect to daily events, work emerged as the major source of stress. Overall, HS subjects reported twice as many stressful daily events; they were also more likely to report events during work days than LS subjects. They reported twice as many work-related stressful events as LS subjects, as well as significantly more social network events. Within these life domains, HS subjects reported significantly more events related to colleagues, partner, children, and other acquaintances. In addition to an increased (subjective) exposure to stressful daily events, HS subjects appraised events as more stressful and less controllable. Although no group differences were found for task demands, HS subjects had significantly more negative social interactions, both at work and at home, and especially so with their colleagues and their partners. These results suggest that, besides the overall frequency of events, what differentiates HS from LS subjects may not so much be found in the stresses of family or work overloads, time-pressure, recurrent deadlines, but in stress resulting from problems with interpersonal relationships. Although social interaction events were reported too infrequently for firm conclusions, the finding of increased stressful interpersonal events in HS subjects is interesting in light of reports that interpersonal conflicts provoke the strongest negative mood states (Bolger, Delongis, Kessler, \& Wethington, 1989b; Bolger \& Schilling, 1991; Clark \& Watson, 1988; Repetti, 1993). In addition, stressful interpersonal events have been found to be related to neuroticism (Bolger \& Schilling, 1991). It would be interesting to investigate if and through which mechanisms interpersonal conflicts are related to differences in wellbeing between HS and LS subjects. A limitation of the current study is that we did not assess positive events and are thus unable to address the important question of the extent to which positive events influence well-being or compensate for the effects of negative events in HS and LS groups. 
An important next step toward understanding the relationship between stress in daily life and individual health outcomes is to examine short-term psychological and physiological responses to daily events. As we have seen, daily events are heterogeneous with respect to both the context in which they occur and the way in which they are appraised by the individual. Such characteristics of events may be equally if not more important determinants of immediate responses than simple event occurrence. Chapter 4 describes the impact of stressful daily events and their characteristics on negative and positive mood states. 


\section{Chapter 4}

\section{THE EFFECTS OF PERCEIVED STRESS AND STRESSFUL EVENTS ON MOOD STATES}

Submitted for publication

Marleen van Eck ${ }^{1}$. Nancy Nicolson ${ }^{1}$, Hans Berkhof ${ }^{2}$

1 Department of Psychiatry \& Neuropsychology, Social Psychiatry and Psychiatric Epidemiology section, University of Limburg, the Netherlands

2 Department of Statistics and Measurement Theory, University of Groningen, the Netherlands 



\begin{abstract}
This study examined the effects of perceived stress on the relationship between stressful daily events and mood states. Forty 'high stress' (HS) and 46 "low stress' (LS) subjects were selected on the basis of Perceived Stress Scale scores from a. sample of male white collar workers. Subjects completed Experience Sampling self-reports ten times a day over five consecutive days. Multilevel analysis revealed that current stressful events were associated with significant increases in negative mood (Agitation), but not with significant changes in positive mood. HS subjects showed significantly stronger negative mood reactivity (both Agitation and Negative Affect) in response to current events than LS subjects. The effects of events on negative mood persisted for at least $90 \mathrm{~min}$ after an event was reported, both in HS and LS subjects. A future event was associated with higher current Agitation in both subject groups, but with higher Negative Affect in HS subjects only. Results suggest increased vulnerability to daily events in HS subjects, and highlight the need for longitudinal studies for establishing long-term health effects
\end{abstract}

\title{
INTRODUCTION
}

In an effort to understand the effects of environmental stressors on health and psychological distress, there has been a shift from studying the effects of major life events to the effects of minor daily events, or daily hassles (Kanner et al., 1981: Zautra et al., 1988). During the last decade it has become increasingly clear that daily life stress is associated with lower psychological well-being and increased somatic symptomatology (DeLongis et al., 1988; Stone et al., 1993). The recent emphasis on daily life events is due, in part, to the extensive criticism directed at the traditional approaches to the study of psychosocial stress. An important drawback of the major life event approach has been the frequent reliance on retrospective research designs, with related problems of biased recall and forgetting (e.g. Bower, 1981). Additionally, the retrospective assessment of life events over several months obscures the temporal, dynamic interplay between environmental demands, perceptions of demands, and outcomes (Stone \& Shiffman, 1992), a central tenet of current transactional theories of stress and coping (Lazarus \& Folkman, 1984a). End-of-the-day assessments of minor events have been designed to address these problems. Measurement biases can be minimized by measuring the variables related to the stress process closer to the moment they actually occur. Prospective daily designs also offer better opportunities to identify the temporal patterns between events and outcomes, a first step in establishing causal relationships.

But even within the time frame of a day, much of the temporal interplay between events, appraisals of events, and outcomes remains out of sight. In addition, forgetting of less salient events and a bias towards remembering more recent events is still likely to occur. Moreover, the recall of daily mood is probably influenced by the subject's mood at the time of recording (Stone et al., 1991). Same-day associations between daily stress and mood remain causally ambiguous: a bad mood, for example, may increase the likelihood that daily events will occur or will be perceived as stressful. Finally, previous studies suggest that the effects of minor stressful events may be largely confined to the day on which they occur (e.g. Bolger et al., 1989a; Rehm, 1978; Stone \& Neale, 1984). These problems all point to the need to narrow the time frame even further to clarify within-day patterns of association between stressful events and mood. 
In the current study, a prospective within-day design was used, with subjects recording event occurrence and mood states several times a day over several consecutive days (Experience Sampling Method), to examine the effects of daily events on mood. The major issue addressed concerns interindividual variability in the daily stress process. More specifically, we investigated how perceived stress, as a measure of chronic stress, translates into the day-to-day experience of and response to minor daily events. First, we examined whether subjects with a high perceived stress level experience more stressful events. Second, we investigated whether high perceived stress magnifies the effects of daily stressors on mood. Third, we investigated temporal patterns of mood reactivity to events. In this context we hypothesized that the mood responses of high stress subjects might be of a longer duration than those of low stress subjects, and that high stress subjects might show greater mood changes in anticipation of future events. Finally, we explored the relationship between event characteristics, such as the context in which an event occurred and event appraisals, and mood states.

End-of-day diary studies have demonstrated a same-day association between daily stress and negative mood (Affleck et al., 1994; Bolger et al., 1989a; Clark \& Watson, 1988; DeLongis et al., 1988; Eckenrode, 1984; Larsen et al., 1986; Lewinsohn \& Libet, 1972; Rehm, 1978; Stone \& Neale, 1984; Stone et al., 1993). The results with respect to positive mood are less clear. Many studies do not differentiate between positive and negative mood; in those that do, positive mood was either lower (Neale et al., 1987; Repetti, 1993; Stone \& Neale, 1984) or unchanged (Watson, 1988 ) on days when many stressful events occurred. Only one study has previously investigated event and mood associations within days (Marco \& Suls, 1993), and here, daily stress was accompanied by "worse mood". There is evidence for large individual differences in the magnitude and the direction of the association between daily stress and mood. Factors which contribute to such differences include selfesteem and self-complexity (Campbell et al., 1991), social support (Affleck et al., 1994; Barling \& Kryl, 1990; Caspi et al., 1987: DeLongis et al., 1988), recent major life events (Affleck et al., 1994; Caspi et al., 1987) and neuroticism (Affleck et al., 1994; Bolger \& Schilling, 1991; Marco \& Suls, 1993). Little research has been done on the effects of chronic stress or long-term difficulties. In a study by Caspi et al. (1987), the chronic stress of living in a low quality neighborhood exacerbated the immediate effects of daily events on mood and also increased the likelihood that daily stressors had an enduring effect on next day's mood.

In the present study, we investigated the effect of perceived stress level on the relationship between daily events and mood. Individuals with a high perceived stress level feel their life to be unpredictable, uncontrollable and overloaded, and can be seen as at risk for the development of stress-related somatic and mental health problems (Cohen et al., 1983), resulting, for instance, in increased sick leave from work. Personality factors probably play a very important role in perceived stress; research indicates that self-report measures of stress correlate significantly with measures of Negative Affectivity (NA) or neuroticism (Watson, 1988: Watson \& Pennebaker, 1989). Several studies demonstrated that individuals higher in NA exhibit increased negative mood reactivity to minor stressors (Affleck et al., 1994; Bolger \& Schilling, 1991: Marco \& Suls, 1993). Considering the above, we hypothesized that individuals with high perceived stress would show greater mood reactivity to daily events than those with low perceived stress. Differences in mood reactivity might for instance be due to differences in the appraisal of events or differences in coping strategies. In order to examine differences in mood reactivity to 
reported stressful events, it is important to control for possible differences in baseline mood between high and low stress subjects. This way we can distinguish between differences in overall levels of negative mood (when events are absent) and added effects of events.

Little is known about the duration of effects of daily events or about the influence of individual differences on this process, although this would be important for understanding the consequences of daily events and might possibly reveal mechanisms related to the longer-term effects of chronic stressful experiences (Eckenrode \& Bolger, 1995). Possibly only when negative moods persist following stressful events, in addition to a high frequency of events, negative health changes may follow (e.g. accumulation of distress). One interpretation for the persistence of negative mood after an event has ended is that coping has been unsuccessful or still ongoing (e.g. persistence of worrying about event). In the current study we investigated if an event reported one assessment earlier (from now on called "prior event") has an (enduring) effect on current mood, and whether perceived stress influences the relationship between prior event and current mood. Most studies have found no relationship between daily stress and mood on the following day (e.g. Lewinsohn \& Libet, 1972; Neale et al., 1987; Rehm, 1978; Stone \& Neale, 1984); in fact, two studies found that on days following stressful events, mood scores were actually better than on other stress free days (Bolger et al., 1989a; DeLongis et al., 1988). A possible explanation for these findings is that feelings of relief predominated after the resolution of stressful events. Two other studies did document worse moods on the day following a suessful event, but oniy in subjects with a low fevel of social support (Affleck et al., 1994; Caspi et al., 1987). In the only within-day study to date (Marco \& Suls, 1993) prior events had no effect on current mood. We also investigated whether future events have an effect on current mood states and if these effects vary with differences in perceived stress level. Future events may influence current moods due to an increased worrying or attention for future events (anticipation effect). Also for an adequate test of the influence of prior event on current mood state it is necessary to control for possible effects of a future event at $t+1$ on current mood states. In this way, we are able to differentiate between an effect on current mood state due to the influence of a prior event and one duc to an anticipated future event.

Of the enormous range of stressful events people experience in daily life, which categories of events have the strongest psychological impact? Results from end-of-the-day studies indicate that the psychological impact of daily events does indeed depend on the context in which they occur. In particular, undesirable events at work (i.e. feeling under a lot of pressure and having negative interactions with coworkers) were associated with both lower positive and higher negative moods (Repetti, 1993; Stone, 1987). In other studies, interpersonal conflicts were found to provoke the strongest negative mood states (Bolger et al., 1989a; Bolger \& Schilling, 1991; Clark \& Watson, 1988; Repetti, 1993). We therefore hypothesized that, within days, stressful work events, task overloads at work or at home, and negative social interaction events would show a larger effect on mood states than other types of events.

According to contemporary stress theory, the subjective appraisal of a stressful event may be more important than the occurrence of an event per se (Lazarus \& Folkman, 1984b). There is evidence for instance, that subjects' personal ratings of events improve prediction of outcomes such as anxiety, depression, negative affect, tension, and grade point average (Dewe, 1991; Peeters et al., 1995; Sarason et al., 
1978). Despite the theoretical importance of the appraisal process, little research has been done on appraisal dimensions of naturally occurring dailly events in relationship to mood. Results of one study where appraisals of daily events were investigated (Neale et al., 1987) led to the conclusion that a simple score based on the sum of event desirability ratings did as well in predicting mood as more complex appraisal measures. A limitation of this study was that the results were based on summary measures over four days; analysis of measurements within days might reveal more subtle relationships. On theoretical grounds, then, we expected that the more unpleasant and important, and the less predictable and controllable the event, the stronger the mood responses. In addition, we predicted that events that had occurred relatively frequently in the past would have less effect on mood than novel stressors.

\section{METHOD}

\section{Subjects}

Local industries and government agencies were approached via their personnel departments to participate in the study. A decision as to which categories of employees fell under the definition of 'white-collar' was made by each personnel department on the basis of standard job function descriptions. Questionnaires were distributed among these employees, accompanied by a letter explaining the goals of the study. Participation was voluntary, and care was taken to insure anonymity. 316 male employees from six different industries or agencies completed the screening questionnaire. The mean score on the Perceived Siress Scale (PSS) for this sample was 12.7 (s.d. 6.0), similar to US norms (mean 13.02, s.d. 6.45) (48).

Ninety-two subjects with scores in the upper or lower tertiles of the screening sample distribution (PSS-10 score $<10$ or 216 ) were recruited, excluding individuals who reported a history of serious chronic illness or treatment (past or current) for mental health problems. Exclusion criteria were reassessed during an initial interview, study aims and procedures were explained, and informed consent obtained. During subject intake, each 'high stress' (HS) subject was matched for age group, marital status, and household composition with a 'low stress' (LS) subject to insure that the two groups did not differ on demographic characteristics that might affect exposure to certain classes of daily stressors. Six subjects were later excluded from analysis: Four due to failure to meet ESM compliance criteria (see 'Daily Experience' section, below), one because he became so acutely stressed that he was unable to work during the sampling period, and another one was excluded due to missing data on the mood variables. Of the 86 remaining. subjects, 40 subjects comprised the 'high stress' (HS) group and 46 subjects the 'low stress' (LS) group. Mean age was 42.1 years (range 27 to 57 years). $89 \%$ were married, and $81 \%$ had children living at home.

\section{Assessment of perceived stress}

The 10-item version of the PSS was translated into Dutch. The PSS is a global measure of the degree to which situations in one's life are appraised as stressful. Items were designed to tap the extent to which individuals feel their life to be unpredictable, uncontrollable and overloaded. The items were rated on a 5-point frequency scale, ranging from 'never' $(0)$ to 'very often' (4) in the last month. Validity and reliability data have been reported by Cohen and others (Cantor, Norem, Langston, Zirkel, Fleeson, \& Cook-Flannagan, 1991: Cohen, 1986; Cohen et al., 1983; Cohen, Tyrrell, \& Smith, 1991). The mean of the two PSS scores was 7.3 (st.dev. 2.2) for the low stress group and 17.9 (st.dev. 3.3) for the high stress group. 


\section{ESMI procedure}

The Experience Sampling Method (ESM) (Csikszentmihalyi \& Larson, 1987: de Vries, 1992) was used to collect data from subjects at selected moments during their normal daily activities. Subjects received auditory signals ('beeps'), after which they filled in a questionnaire. After a 'briefing' session, in which all procedures were explained in detail and informed consent obtained, subjects were sampled for a period of five consecutive days (Thursday through Monday). A wristwatch was programmed to emit beeps ten times each day, at semi-random intervals of approximately 90 minutes between the hours of 8 am and 10 PM. Beeps were clustered around the midpoint of each time block (i.e., $8.15 \mathrm{am}, 9.45,11.15$, and so on), with the exact time sequence of beeps varied each day to decrease predictability. In a final 'debriefing' session, subjects were asked to clarify reasons for missing data. Compliance with the procedures was generally good. The criteria set for subject inclusion in the analysis (> 20 ESM reports completed within 20 minutes after being signalled and no missing data for entire days) were met by all but four subjects (two from each group). The remaining 88 subjects completed an average of $83 \%$ of all possible responses within the time limit, for an average of 41 responses per subject. HS and LS groups did not differ significantly in compliance rates ( 40.1 vs 42.3 responses per subject, Mann-Whitney U-test, p>.05). Compliance was lowest for the first beep (at approximately $8.15 \mathrm{am})$, with an average of $73 \%$ valid reports. On Saturdays, response rate for first beeps was $61 \%$, and on Sundays, $59 \% .74 \%$ of all missing and invalid responses on weekends could be attributed to the fact that subjects were still asleep when signalled.

\section{ESM measures of streseful evente and mood}

The ESM form contained open-ended questions concerning thought content (worries), the physical and social context, and what the individual was doing when signalled. The forms also included 7-point Likert scales (from 1 'not at all' to 7 'extremely') for rating aspects of thoughts, mood, physical well-being, individually defined (psycho)somatic complaints, current activity, and stressful events.

Stressful events: Subjects were asked to describe any stressful event or situation which may have taken place in the interval since the last ESM report, in order to obtain as complete as possible a record of each subject's stressful experiences. Although we refer to these as 'current' events. it should be noted that they took place during the interval between two beeps (i.e., within a time-interval of approximately 90 minutes) and were not necessarily still ongoing at the moment subjects were signalled. 'Prior' events were defined as events which were reported at beep $t-1$. while 'future' events were defined as events which were reported at beep $t+1$.

Event context: Subjects' descriptions of stressful daily events were coded according to context, with the categories work, network (events concerning family, friends and acquaintances), householdfinancial, leisure, personal health-somatic, personal health-psychological, transport, and other. The twelve events that were coded in one of the personal health categories were excluded from the analysis to avoid possible confounding with the mood outcome measures. Of all reported events, $48 \%$ work-related, with the next most common type (network events) representing $18 \%$ of the total events. For this reason, analysis differentiated between the contexts work (1) vs. non-work (0) events.

Some examples of reported work events are: "unclear $/$ vague assignment at work", 'too much to organize, not enough time', 'difficult conversation with boss about job performance', 'chairing a big meeting'. Reported non-work events included: 'having a fight with my wife about household duties'. 'conflict with spouse about how to raise our son', 'child who will not listen', and 'making dinner, child crying, other child taking a bath, and this all at the same time".

In addition, events were scored according to whether or not they involved a social interaction or a task demand (e.g. overloads, deadlines). The codes for work domain, interaction, 
and demand were not mutually exclusive; for example, an argument with the boss about a missed work deadline would be coded as present for each variable. ${ }^{2}$

Event appraisals: Subjects rated reported events on the following dimensions: unpleasantness, importance, predictability, controllability and frequency of prior occurrence. (chronicity).

Mood measures: The 17 ESM mood items were reduced to three mood measures, based on the results of a principal component analysis with varimax rotation, which accounted for $78 \%$ of the total variance when subject mean scores were used. Ratings on the items cheerful, satisfied, relaxed, energetic, self-assured, concentrated and enthusiastic were summed to form a 'Positive Affect' (PA) scale. (Cronbach's alpha $=.95$ ). Two separate components of negative mood were identified: 'Negative Affect (NA)', including the items depressed, anxious, worried, lonely, tired, and miserable (alpha $=87$ ), and 'Agitation $(\mathrm{Ag})$ ', with the items restless, irritated, hurried, and nervous (alpha $=.93$ ). The sums of the scale items were divided by the number of items in the scale, so that ali mood measures have ranges from i to 7 . Within-subject associations among the mood scales were investigated by first standardizing responses on each scale within a subject ( $\mathrm{z}$ scores) and then calculating Spearman rank correlations over all reports. The resulting correlations were low to negligible (NA - Ag: rho $(3569)=.21, \mathrm{p}<.001 ; \mathrm{NA}-\mathrm{PA}$ : rho (3564) $=$ $-.1 \mathrm{i}, \mathrm{p}<.001 ; \mathrm{Ag}-\mathrm{PA}$; rho $(3563)=-.07, \mathrm{p}<.001$; all tests one-tailed), supporting the notion that the three mood dimensions are relatively independent.

\section{Statistical analysis}

For the estimation of the effects of daily stress on mood we used the hierarchical linear model (HLM) or multilevel model (Bryk \& Raudenbush, 1987; Goldstein, 1987) using the programm MIXREG (Hedeker, 1993). The HLM is a variant of a multiple regression model, for the situation in which the data can be structured in a hierarchical way. As the beep-level observations are nested within persons, the HLM can be used to analyze the present data.

A major advantage of the HLM above more conventional methods like ANOVA for repeated measurements is that HLM deals flexibly with the problem of missing observations, which are inevitable in ESM datasets, and the observations do not need to be evenly spaced over the time interval (Gibbons, Hedeker, Elkin, Waternaux, Kraemer, Greenhouse, et al., 1993). Furthermore, the HLM allows for flexible specification of the dependency among the observations within the same person. In the mood dataset, the dependency consists of more than one component. First, as the average mood level is assumed to vary across persons, two observations are more similar if they are from the same person. Second, two mood observations from the same person tend to be more similar if they are taken at points close in time. For the sake of simplicity, many previous studies have ignored this autocorrelation. This biases the standard errors of the regression coefficients downwards and may consequently lead to incorrect test results. Another attractive feature of the HLM is that both time-varying and time-invariant covariates can be added simultaneously to the model (see below).

The two nesting levels present in the data will be called measurement level and person level. The variables added at the measurement level (e.g. events) vary with time, while the variables added at the person level represent individual characteristics (e.g. perceived stress level). The effect of a time-varying variable can be decomposed in two parts: a fixed effect that is constant across persons and a person-specific random effect that can vary from person to person.

2 The reliability of the coding system was assessed by comparing the classifications of 345 events by two independent coders. Interrater agreement was determined by means of Cohen's kappa. On the whole, the qualitative information could be classified with a high degree of agreement (Laundis \& Koch, 1977), especially for the content categories. The overall Kappa for content was .90. Kappa's for social interaction and for task demand were .73 and .65 , respectively. 
At the measurement level, the relationship between events and mood state for person $i$ can he explicitly modelled. Here, we describe a model with stressful event as the only explanatory variable. The model generalizes in an obvious way to the situation with more time-varying covariates. The model at the measurement level has the following form:

$$
(\text { Mood })_{i j}=\alpha_{i}+\beta_{i} *(\text { Event })_{i j}+\varepsilon_{i j}
$$

where $(\text { Mood })_{i j}$ is the mood state at the $j$-th measurement of person $i$, (Event) ${ }_{i j}$ indicates whether a stressful event occurred to person $i$ at the $j$-th measurement (dummy coded as 1 or 0 ), $\alpha_{i}$ is the intercept of person $i$, the regression coefficient $\beta_{i}$ is the reactivity of person $i$ to a stressful event. and the $\varepsilon_{i j}$ 's are normally distributed residual terms with mean zero and variance $\sigma^{2}$. The residual terms within a person are postulated to be first order autocorrelated. This means that the correlation between two residuals that lie $k$ time units apart equals $\rho^{k}$

The coefficients $\alpha_{i}$ and $\beta_{i}$ are allowed to vary across persons, as indicated by the person index $i$. Instead of modelling the variation across persons by estimating $\alpha_{i}$ and $\beta_{i}$ for each person, these coefficients are written as a function of person-specific covariates. The covariate perceived stress (dummy coded as 1 or 0 ) indicates whether an individual belongs to the high or to the low stress group. The parameters $\alpha_{i}$ and $\beta_{i}$ can be modelled at the person level as:

$$
\begin{aligned}
& \alpha_{i}=\gamma_{\alpha}+\delta_{\alpha} \text { (Perceived stress) } i+u_{\alpha, i} \\
& \beta_{i}=\gamma_{\beta}+\delta_{\beta} \text { (Perceived Stress) } i+u_{\beta, i}
\end{aligned}
$$

where the $\gamma^{\prime}$ 's and $\delta$ 's denote fixed effects, which are constant across persons. Since perceived stress might not explain all variation in the $\alpha_{i}$ 's and $\beta_{i}$ 's across persons, person-specific random terms $u_{\alpha, i}$ and $u_{\beta, i}$ were added to the righthand side of the above equations. The $u_{\alpha, i}$ and $u_{\beta, i}$ are assumed to be normally distributed with mean vector zero, variances $\sigma^{2} \alpha$ and $\sigma^{2} \beta$, and covariance $\sigma_{\alpha \beta}$.

Separate models were built to estimate effects on each of the three mood states; Negative Affect, Agitation, and Positive Affect. In each model, the explanatory variables were selected by a backward selection approach. This means that we started with a model that contained all possible explanatory variables and then excluded variables with a non-significant effect. Two-tailed significance of a fixed effect was tested by dividing the estimated effect by its standard error. This ratio is approximately normally distributed. For testing the significance of the random effects, we applied the likelihood ratio test (one-tailed) (Bryk \& Raudenbush, 1992). Variables with nonsignificant main effects were reiained in the model only when interaction effects were significant. Because the variables prior event (event at beep $t-I$ ) and future event (event at beep $t+l$ ) were missing at the first or the last observation, respectively, of each day, these observations were deleted from the data. This resulted in a reduction from 3452 to 2358 observations. To check that the deletion of observations did not lead to a significant change in the regression coefficients, we started by estimating each model on the basis of all observations, thus without the variables prior and future events. With one exception (for NA; see discussion), deletion of observations did not significantly change the values of the regression coefficients of the remaining variables. We therefore present only the final models, including the prior and future variables. For the eventcontext variable we used effect coding (for example, $0=$ no event, 1 = work event, -1 = non-work event). The event-appraisal variables were assigned the value zero if no event was reported. The other values of the event-appraisal variables were centered around the mean for each subject, so that observed effects represent within-person effects. 


\section{RESULTS}

High stress subjects reported stressful events more frequently and had, on average, higher negative affect and agitation and lower positive affect than low stress subjects (see Table 4.1.).

Table 4.1. Frequency's of stressful events and mood states in low and high stress groups.

\begin{tabular}{|c|c|c|c|}
\hline & $\frac{\text { Low PS }}{(n=46)}$ & $\underset{(n=40)}{\text { High PS }}$ & \\
\hline & mean (st.dev.) & mean (st.dev.) & $\frac{\text { p-value }^{\mathrm{i}}}{(2 \text {-tailed) }}$ \\
\hline $\begin{array}{l}\text { Frequency of stressful events }{ }^{2} \\
\text { Mood States }^{3}\end{array}$ & $12.3 \%(11.9)$ & $20.6 \%(17.8)$ & $<.01$ \\
\hline Positive Affect & $5.7(0.6)$ & $5.3(0.3)$ & $<.001$ \\
\hline Negative Affect & $1.2(0.1)$ & $1.4(0.4)$ & $<.001$ \\
\hline Agitation & $1.3(0.3)$ & $1.7(0.5)$ & $<.001$ \\
\hline
\end{tabular}

${ }^{1}$ Differences in frequency and mood states were tested by means of Mann-Whitney U tests

2 Stressful event reports as a percentage of total ESM reports

${ }^{3}$ Subject means, aggregated fover all ESM reports

For each mood state, results will bo presented in the folivwing orcier: firsi, we will describe the effect of stressful daily events on mood state (both fixed as well as random effect), and whether this effect was related to perceived stress. Second, the results on the relationship between prior and future events on the one hand and current mood state on the other hand will be reported, followed by the effect of perceived stress on this relationship. Third, the effects of event type and event appraisals will be described. For each step, the significant effects of variables will be presented first, followed by the non-significant effects.

\section{Effects of events on Negative Affect}

The effect of daily events on Negative Affect differed significantly between subjects (Random Effects CE in Table 4.2.). Although the occurrence of an event was followed by slight elevations in Negative Affect in both groups, the significant Current Event $\times$ Perceived Stress interaction indicates that stressful events increased Negative Affect mainly in HS subjects. This pattern is shown in Figure 4.1. There was also a positive association between the intercept variance and event variance (Covariance Intercept/CE in Table 4.2.); this indicates that controlling for perceived stress, subjects with the highest average scores on Negative Affect showed the greatest increases in this mood state following events.

A small but marginally significant main effect was present for prior event., showing that the effect of an event on Negative Affect persist on average longer than the interval between two ESM reports. Level of perceived stress did not influence the magnitude of this carryover effect. Similar to the pattern observed for current events, a stressful event occurring in the interval following the current report (future event) had no significant effect over all subjects, but in HS subjects, Negative Affect was elevated preceding a future event. 
Table 4.2. Multilevel Results for Negative Affect (NA).

\begin{tabular}{|c|c|c|c|}
\hline Fixed Effects & Estimate & S.E. & $\mathrm{p}<$ (one-tailed) \\
\hline Intercept (mean NA) & 1.140 & 0.041 & $* * *$ \\
\hline Perceived Stress Group (PS) & 0.170 & 0.055 & $\cdots$ \\
\hline Current Event (CE) & 0.046 & 0.033 & ns \\
\hline CE $\times$ PS & 0.151 & 0.046 & $\cdots$ \\
\hline Prior Event (PE $\left.{ }^{1}\right)$ & 0.032 & 0.018 & .07 \\
\hline Future Event $\left(\mathrm{SE}^{2}\right)$ & 0.030 & 0.026 & ns \\
\hline FE $\times$ PS & 0.066 & 0.037 & .07 \\
\hline Event Unpleasantness & 0.029 & 0.010 & $\cdots$ \\
\hline Controllability Event & -0.019 & 0.009 & - \\
\hline Chronicity Event & -0.019 & 0.010 & * \\
\hline
\end{tabular}

\begin{tabular}{|c|c|c|c|}
\hline Random Effects & Estimate & S.E. & $\mathrm{p}<$ \\
\hline \multicolumn{4}{|l|}{ Person level: } \\
\hline Var (Intercept) & 0.055 & 0.010 & $* *$ \\
\hline Var (Current Event) & 0.012 & 0.006 & • \\
\hline Cov (CE, Intercept) & 0.015 & 0.006 & $\bullet$ \\
\hline \multicolumn{4}{|l|}{ Measurement level: } \\
\hline Var (Residual term) & 0.078 & 0.002 & $* *$ \\
\hline
\end{tabular}

\footnotetext{
Autocorrelation

$0.443 \quad 0.021$

$\mathrm{n}=\mathbf{2 3 5 8}$ observations (including 342 events) nested within 85 subjects ( $39 \mathrm{HS}, 46 \mathrm{LS}$ )

1 event at $1-1 ;{ }^{2}$ event at $1+1 ;{ }^{*} p \leq .05 ;{ }^{* *} p \leq .01 ;{ }^{* \vec{*} *} p \leq .001$

Von-signitican fixed effecis:

Event ryps: Work, Social Interaction, Task Demands

Event appraisals: Importance, Predictability

Interdcrions: PE $\times$ PS

Nore. The variable 'time over days' (automatically estimated when autoconelations are corrected for) was excluded from Table $4.2,4.3$, and 4.4 since estimated effects were negligible (all $<-0.002$ ) and not relevant with regard to hypotheses being tested.
}

As expected, events that were rated as more unpleasant were associated with larger increases in NA, whereas events that were relatively controllable had smaller effects on NA than uncontrollable events. In addition, the more frequently a stressful event had occurred in the past ("chronicity"), the smaller the observed effect on NA. Further differentiation of events by context did not improve the model; in other words, work and non-work events did not differ in their effects on Negative Affect. With respect to the event appraisals, no significant effects were found for event importance or event predictability. 


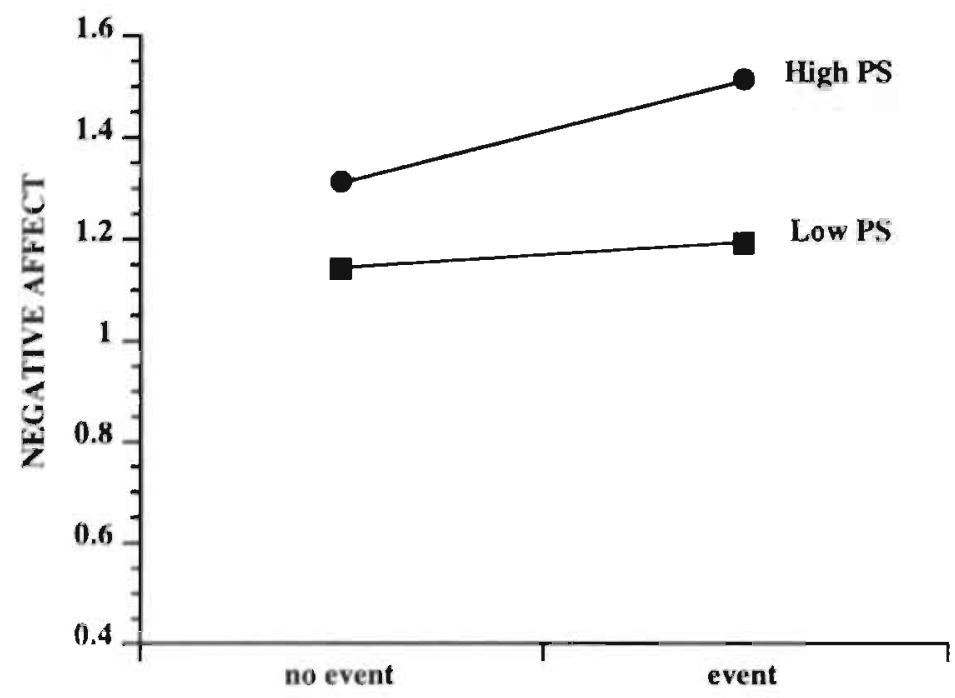

Figure 4.1. Modelled change in Negative Affect in 'high' and 'low' stress subjects in response to a stressful event (controlling for all other variables).

\section{Effects of events on Agitation}

In both HS and LS groups, current stiessful events were associated with significant elevations in Agitation levels (see Table 4.3.). Again we found a random effect for events, meaning that the influence of stressful events on Agitation differed significantly between subjects. Part of this between-subject variance could be explained by perceived stress level: HS subject reacted significantly more strongly to current stressful events than LS subjects, as evidenced by the significant Current Event $x$ Perceived Stress interaction. These results are presented graphically in Figure 4.2. As with Negative Affect, there was a positive association between the intercept variance and event variance (Covariance InterceptiCE in Table 4.3.), indicating that subjects with higher average scores on Agitation showed higher reactivity to current events (after controlling for perceived stress).

With respect to the temporal processes of mood reactivity, the significant effect of prior event on current Agitation indicates a carryover effect of events on mood. The presence of a future event also had a significant positive effect: current Agitation was higher when a stressful event was reported in the interval following the current report. There was no evidence for a moderating effect of perceived stress level on these carryover or anticipation effects.

Current events characterized as task demands were associated with significant increases in Agitation compared to non-task demand events. With respect to the event appraisals we found that, similar to the findings for Negative Affect, when events were more unpleasant than average. Agitation was higher, whereas events that were more controllable than average had a smaller effect on Agitation. Whether or not an event involved a negative social interaction or was work-related had no specific effect on this mood variable. No additional effects were found for event importance, predictability or chronicity. 
Table 4.3. Multilevel Results for Agitation (Ag).

\begin{tabular}{|c|c|c|c|}
\hline Fixed Effects & Estimate & S.E. & ps (one-tailed) \\
\hline Intercept (mean Ag) & 1.247 & 0.058 & $\cdots$ \\
\hline Perceived Stress Group (PS) & 0.323 & 0.077 & $\cdots$ \\
\hline Current Event (CE) & 0.373 & 0.098 & $\cdots$ \\
\hline CE $\times$ PS & 0.433 & 0.140 & $*$ \\
\hline Prior Event (PE ${ }^{l}$ ) & 0.099 & 0.032 & $\cdots$ \\
\hline Future Event $\left(\mathrm{SE}^{2}\right)$ & 0.213 & 0.034 & $\cdots$ \\
\hline Task Demands & 0.132 & 0.033 & $\cdots$ \\
\hline Event Unpleasantness & 0.056 & 0.019 & $\cdots$ \\
\hline Controllability Event & -0.045 & 0.016 & $\cdots$ \\
\hline
\end{tabular}

Random Effects

Estimate S.E. $\mathrm{p}<$

Person level:

$\begin{array}{llll}\text { Var (Intercept) } & 0.101 & 0.019 & * * \\ \text { Var (Current Event) } & 0.255 & 0.058 & * * \\ \text { Cov (CE, Intercept) } & 0.049 & 0.025 & *\end{array}$

Measurement level:

Var (Residual term)

$0.257 \quad 0.008$

Autocorrelation

$0.352 \quad 0.022$

$\mathrm{n}=2358$ observations (including 342 events) nested within 85 subjects ( $39 \mathrm{HS}, 46 \mathrm{~L}$ )

${ }^{1}$ event at $t-1 ;{ }^{2}$ event at $1+1 ;{ }^{*}$ ps.05; ${ }^{* *}$ p $\leq .01 ;{ }^{* * *}$ p $\leq .001$

Non-significant fixed effects:

Event lype: Work, Social Interaction

Event appraisals: Importance, Predictability, Chronicity

Interactions: $\mathrm{PE} \times \mathrm{PS}$. FE $\times$ PS

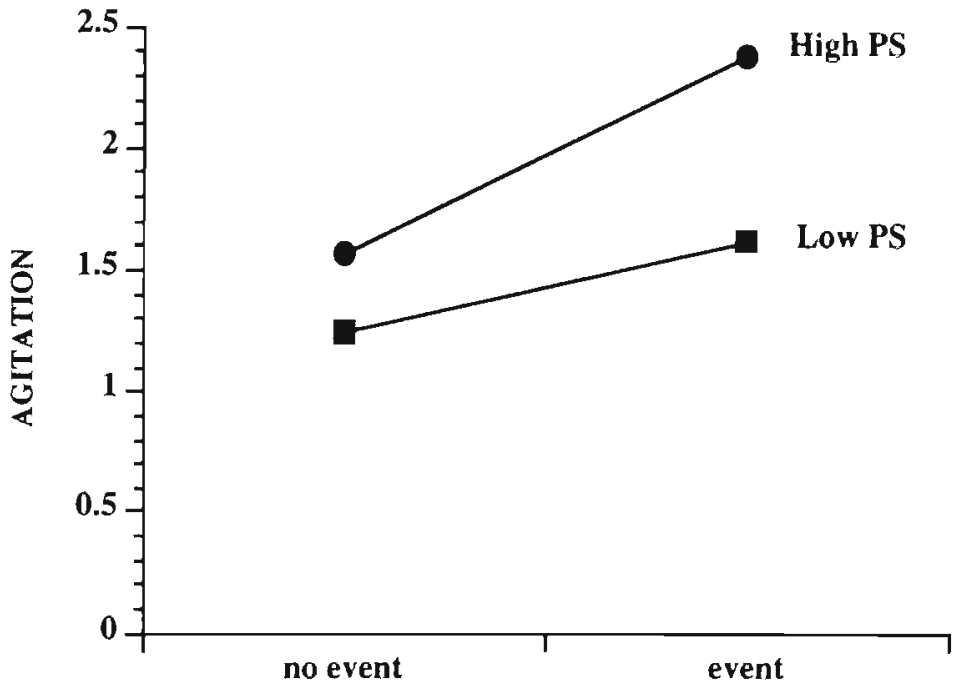

Figure 4.2. Modelled change in Agitation in 'high' and 'low' stress subjects in response to a stressful event (controlling for all other variables). 


\section{Effects of events on Positive Affect}

The multilevel results for Positive Affect are shown in Table 4.4. Occurrence of. a stressful event was not associated with any change in Positive Affect in either HS or LS subjects. In addition, no significant association was found between intercept variance and event variance (Covariance Intercept/CE; see Table 4.4.).

However, a carryover effect for a prior event on current Positive Affect was found: current positive mood was less positive when preceded by a prior event. The significant Prior Event x Perceived Stress interaction indicated a small rebound effect for HS subjects: when a prior event was present, HS subjects had relatively higher current Positive Affect than when a prior event was absent. A future event had no observable effect on current Positive Affect, regardless of perceived stress level. Following events that were more unpleasant than average, a significant decrease in Positive Affect was observed. Positive Affect was no more likely to change in response to a work than to a non-work event, or following a social interaction or a task demand event when compared to no social interaction and non-task demand events.

Table 4.4. Multilevel Results for Positive Affect (PA)

\begin{tabular}{lrcc}
\hline Fixed Effects & Estimate & \multicolumn{1}{c}{ S.E. } & $\mathrm{p}<$ (one-tailed) \\
\hline Intercept (mean PA) & 5.790 & 0.076 & $*$ \\
Perceived Stress Group (PS) & -0.409 & 0.108 & \\
Current Event (CE) & -0.038 & 0.029 & $\mathrm{~ns}$ \\
Prior Event (PE ${ }^{1}$ ) & -0.085 & 0.037 & $*$ \\
$\quad$ PE x PS & 0.101 & 0.052 & $*$ \\
$\quad$ Event Unpleasantness & -0.044 & 0.015 & $* *$
\end{tabular}

\begin{tabular}{lrrr} 
Random Effects & Estimate & S.E. & $\mathrm{p}<$ \\
\hline Person level: & & & \\
$\quad$ Var (Intercept) & 0.241 & 0.039 & $* * *$ \\
$\quad$ Vat (Current Event) & 0.006 & 0.009 & $\mathrm{~ns}$ \\
$\quad$ Cov (CE. Intercept) & -0.017 & 0.015 & $\mathrm{~ns}$ \\
Measurement level: & & & \\
$\quad$ Var (Residual term) & 0.175 & 0.005 & $* *$ \\
& & & \\
\hline Autocorrelation & 0.266 & 0.023 & $* * *$
\end{tabular}

$\mathrm{n}=2358$ observations (including 342 events) nested within 85 subjects ( 39 HS, 46 L.S)

1 event at t-1: "ps.05: "* ps.01: "*at ps.001

Non-significant fixed effects:

Event type: Work, Social Interaction, Task Demands

Event appraisals: Importance. Predictability, Chronicity, Controllability

Future Event

Interactions: $\mathrm{CE} \times \mathrm{PS}, \mathrm{FE} \times \mathrm{PS}$ 


\section{DISCUSSION}

Our main objectives were to investigate whether stressful daily events resulted in short-term changes in mood states, and whether differences in perceived stress level were related to the magnitude of mood changes. Results differed for each of the three mood dimensions investigated. With respect to Agitation, we found that daily events were associated with higher levels of Agitation in both HS and LS groups, with HS subjects showing the strongest mood reactivity. For Negative Affect, events were associated with worse moods in HS subjects only. Current events were not associated with changes in Positive Affect.

These results are consistent with those found in individuals scoring high in trait negative affectivity or neuroticism. Trait negative affectivity has not only been linked to higher overall levels of negative mood (Watson \& Clark, 1984) but also to increased reported exposure to stressful events (e.g. Bolger \& Schilling, 1991: Martin, Ward, \& Clark, 1983) and to increased mood reactivity to stressors (e.g. Bolger \& Schilling, 1991; Eysenck \& Eysenck, 1985; Marco \& Suls, 1993). Level of perceived stress was indeed strongly correlated with trait anxiety in the present study (rho (85) $=.77, \mathrm{p}<.001$ ), and HS subjects not only reported more stressful events, but also higher negative mood levels across all situations and stronger negative mood reactivity to events. Since other analyses indicated that the events reported by HS subjects were rated as no more severe than events reported by LS subjects (mean intensity of events in HS subjects is 3.5 \{st.dev. $=1.3$ \} versus $3.2\{$ st.dev. $=\{.7\}$ in LS subjects; ns), differences in appraised severity of events cannot explain the observed differences in mood responses. A possible explanation for increased mood reactivity among $\mathrm{HS}$ subjects is the use of less effective coping strategies (see (Bolger, 1990)). In our study, as in all studies based on self-report information, we cannot determine whether HS subjects were actually exposed to more stressful situations, or whether they were more likely to perceive events as stressful, had a heightened recall of negative situations, or were more likely to generate stressful situations, for example by failing to anticipate and thereby prevent events from happening. Laboratory studies have shown that neuroticism, which is significantly related to self-report measures of stress (Watson \& Pennebaker, 1989), increases the recall of negatively toned information (Martin et al., 1983), thereby possibly accounting for some of the positive association that has been found between neuroticism and exposure to stressors (e.g. Fergusson \& Horwood, 1987). Contrary to these reports, neuroticism was not related to increased event reporting in a recent within-day field study (Marco \& Suls, 1993). It seems possible that recall biases play less a role when the time intervals between assessments are short. One way of getting more objective event measures is to obtain confirmation of event occurrence from partners (Stone et al., 1991); this option is not realistic, however, when (as in our study) a large percentage of events occur in the workplace. Although it might be possible to obtain confirmation of event occurrence from co-workers or closest colleagues, this option does not seem very practical. In addition, many events (for example, demand situations) are very difficult to observe.

In LS subjects, we found that current events were associated with higher Agitation levels but not with higher Negative Affect levels. Given the specific items of the Agitation scale (restless, irritated, hurried and nervous), it makes sense that this mood scale would be especially sensitive to short-term effects of daily stressors. The Negative Affect scale items (depressed, anxious, worried, lonely, tired and miserable) are more likely to reflect longer term or intense reactions. HS subjects seem to react in 
a more passive and depressive way to stressful events then LS subjects, which may relate to differences in coping strategies, but also to the more chronic nature of stress in HS subjects ('giving up').

In contrast to results of a number of end-of-day diary studies (Neale et. al., 1987; Repetti, 1993; Stone \& Neale, 1984), current stressful events did not lower Positive Affect in the present study. Differences in mood scales and frequency in assessments might account for the lack of an effect of current events on Positive Affect. Associations between stressful events and mood do not have to be the same within and across days. It is possible that an accumulation of several events experienced during the day lowers PA at the end of the day, but that the occurrence of one stressful event does not have an irnmediate effect on Positive. Affect. Lack. of an association between stressful events and Positive Affect is more in line with several studies that suggest that Positive Affect and Negative Affect reflect two separate affective systems and were Positive Affect was found to be unresponsive to perceived daily stress but increased in relation to pleasant social events (Clark. \& Watson, 1988; Kanner et al., 1981; Watson, 1988). A possibility is that people's positive moods are not easily affected by minor stressors, but only, as suggested by the current findings, when events are more unpleasant than average.

To increase our knowledge about how long minor daily events continue to exert their influence on mood we investigated whether stressful events reported on average 90 minutes earlier still influenced current. mood states. Events at beep (t-I) were indeed associated with a persistent elevation in Agitation at beep (t0) and a marginally significant elevation in Negative Affect. In other words, the effects of events on mood states persisted for at least 90 minutes (the average interval between two ESM reports) after a stressful event was reported at (t0). These carryover effects for the negative moods were equivalent in both HS and LS groups. The results contrast with those of Marco and Suls (1993), who found no 'carryover' of the negative mood effects of prior events. Two methodological differences between the two studies may explain the inconsistencies: firstly, Marco and Suls' negative mood scale was composed of different items (tense, unhappy, and angry), and secondly, with eight beeps per day, time intervals between successive reports would have been somewhat longer on average than the 90 minute interval in the current study. Here, the occurrence of a future event was accompanied by higher current Agitation in both HS and LS subjects, but by higher Negative Affect in HS subjects only. The: effect sizes for subsequent events were larger than for prior events (especially for Agitation, and, in HS subjects. Negative Affect), indicating that a future event influenced current negative mood more than prior events. These results confirm the common observation that the anticipation of an event can increase current negative mood. More detailed analysis are necessary to determine whether anticipation effects are limited to certain types of events or certain event appraisals. This was beyond the scope of the present study.

Of the various types of events (work vs non-work events, social interaction vs non-social events and, task demands vs non-task demands), we found that task. demand situations (e.g. overloads, time pressure or deadlines at home or at work) were accompanied by significant higher Agitation levels. This association makes intuitive sense, especially in a white-collar sample. It is not clear, however, why we failed to replicate the finding in several other studies of a strong effect of interpersonal conflicts on negative mood (Bolger et al., 1989a; Bolger \& Schilling, 1991: Clark \& Watson, 1988; Repetti, 1993). The fact that these earlier studies investigated the relationship between interpersonal conflicts and mood across days. 
may in part explain the difference. For instance, the occurrence of repeated social interaction events during the day may be necessary to have a measurable effect on mood. Moreover, retrospective biases may have a greater influence in daily than in within-day studies; for example, a bad mood at the end of the day may be ascribed to a interpersonal conflict earlier that day. Another factor may be that we have coded events as interaction events ourselves, while in the other studies subjects indicated on a checklist whether a personal conflict had occurred or not. It is possible that interpersonal conflicts indicated by subjects on a checklist are of a more serious nature than the events coded by us. Finally, the loss of a considerable number of events reported due to the inclusion of the prior and future event variables (538 events vs. 342 events) may have resulted in a low statistical power. This is supported by the fact that in the full dataset (without the lagged variables), social interaction events and task demands did significantly increase Negative Affect.

Individuals' subjective appraisals of daily events had additional effects on mood responses. The more unpleasant an event was rated, the greater its effect on Agitation and Negative Affect, whereas only events that were more unpleasant than average were associated with lower Positive Affect. The perception of having control over a stressful event lowered its effect on both Agitation and Negative Affect levels. Negative Affect levels were also lower when an event had occurred more frequently than average in the past. No significant effects were found for event importance or event predictability. This does not necessarily mean that these variables are not important. Firstly, within-subject samples of events may have been too heterogeneous or too small in the current study to reveal consistent and reliable effects of all the specific appraisal variables, and secondly, since the appraisal variables were to some degree correlated with each other, the strongest predictor variables will have masked the weaker ones when all variables were entered into the model simultaneously.

Some caveats should be mentioned with respect to interpretation and generalization of these results. Firstly, subjects were well-educated, white collar males; their assessments of what constitutes a 'stressful' event or situation is not necessarily based on the same criteria as other groups (for example females, blue collar workers, students, or retired people) might use. The same applies to their mood ratings. Men tend to report lower, less extreme levels of negative emotions as well as positive emotions than women (Cameron, 1975; Diener, Sandvik, \& Larsen, 1985), and daily stress has been found to be more upsetting to women than to men (Bolger et al., 1989a). Men also appear to expend greater effort in limiting emotional distress (at the expense of physiological distress), resulting in lower emotional reactivity (Gottman \& Levenson, 1988). We also cannot rule out the possibility that current mood influenced the reporting of recent stressful event occurrence. However, the finding that prior events were associated with persistent increases in Agitation and Negative Affect supports the assumption that events influenced mood and not vice versa. Another limitation is that we did not assess positive events and are thus unable to address the important question of the extent to which positive events compensate for, or neutralize, the effects of negative events in HS and LS groups.

In summary, results support the interpretation that individuals with high perceived stress are more vulnerable to 'daily hassles' and therefore may be at greater risk for the development of stress-related health problems. Individuals with high perceived stress levels were more trait anxious, more likely to experience daily stressors, higher in overall negative mood, and more reactive, in terms of negative. mood states, to daily events. This suggests a reciprocal relationship between perceived stress, events and mood reactivity, where increases in event exposure and 
high mood reactivity maintain the appraisal of high stress. Only longitudinal studies with prospective and repeated measurements of both trait and state measures, will be able to clarify causal pathways, including the long-term effects of stressful daily events. Analytic strategies will have to be used that can estimate the relative contribution of each predictor variable, since many variables related to the stress process tend to be highly intercorrelated to each other. Another important subject for future research is the investigation of why HS individuals or trait anxious individuals exhibit greater negative mood reactivity to minor events. Exploration of differences between HS and LS groups in the actual coping strategies used when confronted with stressful events seems to be a good start.

\section{ACKNOWLEDGEMENTS}

We thank C. Dijkman for assistance in all aspects of the study. We also thank Dr. D. Hedeker for his advice regarding the use of the MIXREG program. 


\section{Chapter 5}

\section{Cortisol and catecholamine excretion in relation to perceived stress}

Section S.I: Annals of Behavioral Medicine 16(3): 221-227 (1994)

Marleen van Eck ${ }^{1}$, Nancy Nicolson ${ }^{1}$

Department of Psychiatry \& Neuropsychology, Social Psychiatry and

Psychiatric Epidemiology section, University of Limburg, the Netherlands 
Chapter 5 addresses the relationship between perceived stress and salivary cortisol and urinary catecholamines levels in the context of everyday life. In general we test the hypothesis that high perceived stress is associated with both elevated cortisol as well as elevated catecholamine levels. In section 5.1, two groups of male white collar workers differing in levels of perceived stress were compared on subjective self-report measures of distress and on levels of salivary cortisol, as measured ten times a day over five days. Here, the effect of perceived stress on cortisol is tested, either in general, or during certain phases of the circadian cycle or certain days of the week (work versus non-work days. In section 5.2, the same groups of subjects were compared on 14-hour levels of catecholamines. Urine samples were collected twice overnight, one after a work day and one after a weekend day. Here, we test the effect of perceived stress on catecholamine levels, either in general or during certain days of the week (work versus non-workdays). 



\title{
Section 5.1
}

\section{Perceived stress and salivary cortisol in daily life}

\begin{abstract}
Clarifying the nature of endocrine responses to chronic or intermittent stress in daily life requires repeated measurements of stress, hormone levels and emotional states. In this study, 42 'high stress' (HS) and 46 'low stress' (LS) subjects were selected on the basis of Perceived Stress Scale scores from a larger sample of male white-collar workers. Subjects completed self-reports (Experience Sampling, ESM) and collected saliva samples for cortisol determination 10 times a day over 5 consecutive days, including 3 work and 2 non-work days. Here we test the hypothesis that high perceived stress is associated with elevated cortisol.

The HS group scored higher than the LS group on measures of trait anxiety, depression, anger and psychosomatic complaints, as well as on ESM measures of positive and negative affect and stressful daily events. Although negative affect was higher and stressful events more frequent on workdays than weekends, especially for HS subjects, no difference in workday vs. weekend cortisol levels was found in the subsample of subjects with sufficient data. On workdays, HS subjects had higher mean cortisol levels than LS subjects at each of the 10 sampling times between 8 am. and $10 \mathrm{pm}$ (repeated measures ANOVA, p<.02). Mean workday cortisol was correlated with higher trait anxiety, depression and the low arousal dimension of ESM negative affect.
\end{abstract}

\section{INTRODUCTION}

Over the last 20 years, a wide range of studies has established an association between psychosocial stress and a variety of psychological and physical disorders. The strength of these associations are usually small and inconsistent, but stressful life events, for instance, have been linked to depression, neurotic impairment and other psychological symptomatology (Brown \& Harris, 1989; Dohrenwend \& Dohrenwend, 1974; Dohrenwend \& Dohrenwend, 1978). Stress has also been linked empirically with increased susceptibility to infectious disease (Cohen et al., 1991), with other immune-related disorders like recurrences of herpes infections (Hoon, Hoon, Rand, Johnson, Hall, \& Edwards, 1991) and asthmatic exacerbations (Isenberg, Lehrer, \& Hochron, 1992), with coronary heart disease (Bassett, 1982), and with diabetes mellitus (Goetsch, Wiebe, Veltum, \& Van Dorsten, 1990). The diversity of stress concepts and research methodologies used in these studies makes it difficult to integrate all of the findings.

Since the beginning of this century, the endocrine system has received much attention in stress research (Mason, 1968; Selye, 1936), with an emphasis on the hypothalamic-pituitary-adrenal (HPA) axis, since this system forms a theoretically promising pathway for mediating the relationships between psychosocial stress and subsequent disorder (Curtis, 1972). Cortisol secretion appears to play an important role in the regulation of physiological and behavioral responses under stressful situations (Mason, 1975; Rose, 1984). Cortisol increases after strenuous physical exercise (Mason et al., 1973) and mental task load, specifically in response to distress, as opposed to effort or general arousal (Fibiger \& Singer, 1989; Lundberg \& 
Frankenhaeuser, 1980). Cortisol is also the most important glucocorticoid, with the potential of exerting its effects on practically all normal (body) cells and tissues. Some of the most important effects of cortisol are: stimulation of gluconeogenesis, inhibition of glucose uptake, suppression of inflammation and suppression of numerous immune functions. Glucocorticoids almost invariably suppress rather than enhance biological defense mechanisms. They are thought to protect against the normal defense reactions that are activated by stress, by preventing these defense reactions from overshooting and by' preserving the specificity of immune reactions (Munck et al., 1984).

Indeed, most of the physiological reactions to stress are thought to be adaptive, i.e. they counter the effects of physical stress (trauma, bleeding) or are seen as a preparation for fight or flight responses to acute danger (Cannon, 1929). The question now is, why and when could this adaptive mechanism be a pathway to disease? There are indications that the same suppressive effects of glucocorticoids on several functions of the immune-system may leave a subject more susceptible to infections or even tumors (Claman, 1977). Other studies indicated that increased levels of cortisol may be involved in the etiology of coronary heart diseases (Troxler et al., 1977). The general idea is that stress causes an overproduction of antiinflammatory and immune suppressive action, leading to adverse consequences. (e.g. break down of immune system) (Gaillard \& Al-Damluji, 1987; Selye, 1976). There is also evidence from animal studies for a gradual loss of adaptation within the pituitarycorticoadrenal response. If stress is prolonged, hypophyseal receptors appear to lose their sensitivity to cortisol and as a consequence, prolonged increases in cortisol tend to further slow the recovery of cortisol after stress because of the ineffectiveness of the negative feedback loop (Bassett \& Cairncross, 1977; Kloet \& Reul, 1987). Recent theoretical overviews by Henry and Stephens (1977) and Siegrist (1989) are more or less in line with this concept of loss of adaptation.

It is reasonable to assume that stress reactions will only lead to disease when they are prolonged, or occur very often. The effects of chronic or intermittent stress on the HPA-axis, however, have received far less attention in the literature than the effects of acute stressors, and studies on naturally occurring stressors and their effects are even more rare. Data on cortisol levels during chronic psychosocial stress are inconsistent, with enhanced as well as decreased concentrations reported, and large variability among individuals. Elevated levels of cortisol were found in subjects living near the damaged nuclear power plant at Three Mile Island (Schaeffer \& Baum, 1984). Higher levels of urinary cortisol correlated significantly with urinary catecholamines, self-reports of physical and mental symptoms, and decrements in task performance. A study following parents of leukemic children into the period of bereavement, for as long as 3 years after the death of the child, showed that 17 hydroxycorticosteroid excretion rates were related to the effectiveness of subjects' psychological defenses (Hofer et al., 1972a; Hofer et al., 1972b). Elevated levels of plasma and salivary cortisol were found in Iran hostages after release from captivity, reflecting distress, anxiety and elation. Salivary cortisol was the only physiological measurement that demonstrated a significant correlation with psychiatrists' ratings of the hostages' psychological disturbance (Rahe et al., 1990).

The above studies have examined stress under rather extreme or unusual situations. With respect to more normal settings, studies of air traffic controllers suggest that occupational stress is associated with increased cortisol production (Rose \& Fogg, 1993; Rose, Jenkins, Hurst, Kreger, Barrett, \& Hall, 1982). Caplan et al. (1979) studied white-collar workers in a relatively everyday organizational setting. 
While there was no main effect of work load or personality traits on mean cortisol, high perceived work load was associated with lower morning cortisol values.

The results presented here are part of a larger study concerning emotional and hormonal responses to naturally occurring stress in daily life. Stress is currently conceptualized as a dynamic process, which changes over time and in relation to the environment. It is therefore necessary to include repeated measurements of stress. hormone levels and emotional states to investigate the stress-hormone relationship. This relationship should ideally be studied in naturalistic settings and over time, so that we can begin to understand how adaptation occurs in response to intermittent daily hassles, in individuals who are observed in their normal social networks, settings and activities (Dimsdale, 1984). For these reasons, we chose to use two relatively new techniques, Experience Sampling (ESM) (Csikszentmihalyi \& Larson, 1987; de Vries, 1992) and monitoring of salivary cortisol (Kirschbaum \& Hellhammer, 1989), to assess the relationship between an individual's affective state and neuroendocrine changes over time. Subjects were selected on the basis of Perceived Stress Scale scores (Cohen et al., 1983) from a large sample of male white-collar workers and were then monitored during their daily activities. Subjects completed self-reports. (ESM) and collected saliva samples for cortisol determination at semi-random intervals ten times a day over five consecutive days, including three work and two non-work days.

Here we test the hypothesis that high perceived stress is associated with elevated cortisol, either in general, or during certain phases of the circadian cycle or certain days of the week (e.g. work versus non-work days). With respect to diurnal patterns, no specific hypothesis was formulated. The few studies in which cortisol has been measured at more than one time of day in healthy subjects report significant relationships between cortisol and psychological trait measures at some time points and not at others. For example, in a large German survey study in which salivary cortisol was measured three times on a single day, only 8 am levels showed clear associations with psychological and demographic variables (Brandtstädter et al., 1991). In a similar fashion, perceived work load had an effect on cortisol levels in white-collar workers that was dependent on the time of day when blood was sampled (Caplan et al., 1979), and hostility was associated with high cortisol in daytime, but not in evening or overnight urine samples (Pope \& Smith, 1991). Depressive symptoms could also be related to increased cortisol secretion at specific times of day. Concerning day of the week effects, we hypothesized that cortisol would be elevated on workdays compared to the weekend, possibly in interaction with perceived stress. Although subject groups were selected according to a global measure of stress and not on the basis of work-related stress, we reasoned that work might be a major source of stress. Studies by Frankenhaeuser and colleagues (1989) have demonstrated that cardiovascular and neuroendocrine activity are higher at work than at home, and that slow recovery of responses, or 'unwinding', after work might represent a long-term health risk.

Finally, we examined the extent to which psychological variables (trait anxiety, depression, anger and psychosomatic complaints; ESM measures of mood and stressful events) might contribute to individual differences in workday cortisol level. 


\section{METHOD}

\section{Subjects}

Local industries and government agencies were approached via their personnel departments to participate in the study. A decision as to which categories of employees fell under the definition of 'white-collar' was made by each personnel department on the basis of standard job function descriptions. Questionnaires were distributed among these employees, accompanied by a letter explaining the goals of the study. Participation was voluntary, and care was taken to insure anonymity. 316 male employees from six different industries or agencies completed the screening questionnaire. The mean score on the Perceived Stress Scale (PSS) for this sample was 12.7 (s.d. 6.0), similar to US. norms (mean 13.02, s.d. 6.45) (Cohen et al., 1983).

92 Subjects with scores in the upper or lower tertiles of the screening sample distribution (PSS-10 score $<10$ or 216 ) were recruited to participate in the study, excluding any individuals with a history of endocrine disorder, medications known to affect cortisol levels, psychopathology (major affective disorder, psychoses), or currently in treatment for mental health problems.

'High-' and 'low-' stress subjects were matched for age group, marital status, and household composition. Because it was not possible to match all subjects, numbers of subjects in the final two groups differ slightly.

Four subjects failed to meet Experience Sampling compliance criteria (see 'Daily Experience' section, below) and were therefore excluded from further analysis. Of the 88 remaining subjects, 42 subjects comprised the 'high stress' (HS) group and 46 subjects the 'low stress' (LS) group. Mean age was 42.1 years (range 27 to 57 years), with no significant difference between the two groups. $89 \%$ were married, and $81 \%$ had children living at home.

\section{MEASURES}

\section{Questionnaires}

In addition to the PSS, questionnaires concerning psychosocial stress, coping style, and psychological and physical symptoms were completed. Self-report instruments were chosen on the basis of their theoretical relevance to the stress process and psychometric reliability and validity. The following meastires are used in the cuirent analysis:

Perceived Stress: The 10-item version of the PSS was translated into Dutch by the first author and then back-translated as a check into English by the second author. The items were rated on a 5-point frequency scale, ranging from 'never' (0) to 'very often' (4) in the last month. A total perceived stress score was obtained by reversing the scoring on the positive items and then summing across the 10 items. All subjects completed the PSS twice, during the initial screening and again immediately preceding Experience Sampling. The two PSS scores were highly correlated $(r h o=73, \mathrm{p}<.001)$; the mean score will be used in the analysis.

Psychosomatic symptoms: The SUNYA Psychosomatic Symptom Checklist (PSC) includes 17 common psychosomatic complaints (e.g., headaches, backaches and nausea ). The scale was originally developed by Cox, Freundlich, \& Meyer (1975) and revised by Attanasio et al. (1984). Subjecis rate each complaint on 5 -point scales for frequency ( 0 "never or rarely occurs " to 4 "occurs daily") and intensity ( 0 "not bothersome" to 4 "extremely bothersome"). A Total Score, reflecting the overall level of psychosomatic distress, is obtained by summing the cross-products of each item's frequency by intensity.

Depression: Depressive symptomatology was assessed with the Dutch translation (Dijkstra, 1974) of the Zung Self-Rating Depression Scale (Zung, 1965).

Anxiety; Trait anxiety was measured with the Dutch version (van der Ploeg et al., 1980) of the State-Trait Anxiety Inventory (STAI). 
Anger: Trait anger was measured with the Dutch version (van der Ploeg et al., 1982) of the Spielberger Trait Anger Scale. Although two subscales ('anger-temperament' and 'angerreaction') exist, only the total' score is used here.

\section{Daily experience}

The Experience Sampling Method (ESM) (Csikszentmihalyi \& Larson, 1987; de Vries, 1987; de Vries, 1992) was used to collect data from subjects at selected moments during their normal daily activities. Subjects received auditory signals, after which they filled in a questionnaire and collected a saliva sample. Although additional reports were completed at the end of each day, for the purpose of this analysis we will refer only to the 'beep level' data.

After a 'briefing' session, in which all procedures were explained in detail and informed consent obtained, subjects were sampled for a period of 5 consecutive days, beginning early on a Thursday morning and ending late Monday. In this sample, the weekends were non-workdays. A Seiko wristwatch was programmed to emit beeps 10 times each day, at semi-random intervals of approximately 90 minutes between the hours of 8 am and $10 \mathrm{pm}$. Beeps were clustered around the midpoint of each time block (e.g., 8.15 am, 9.45, 11.15, and so on); the exact time sequence of beeps was varied each day to decrease predictability. In a final 'debriefing' session, ESM forms were checked for legibility, and subjects were asked to clarify reasons for missing data.

Compliance with the procedures was generally good. The criteria we set for inclusion in the analysis ( 220 ESM reports, completed within 20 minutes after being signalled and no missing data for entire days) were met by all but four subjects ( 2 from each group). The remaining 88 subjects completed an average of $83 \%$ of all possible responses within the time limit, for an average of 41 responses per subject. HS and LS groups did not differ in compliance rates (40.1 vs 42.3 responses per subject, Mann-Whitney U-test, p>.05). Compliance was lowest for the first beep (at approximately $8.15 \mathrm{am}$ ), with an average of $73 \%$ valid reports. On Saturdays, response rate for first beeps was $61 \%$, and on Sundays, $59 \%$. $74 \%$ of all missing and invalid responses on weekends could be attributed to the fact that subjects were still asleep.

The ESM form contained open-ended questions concerning thought content, the physical and social context, and what the individual was doing when signalled. The forms also included 7 point Likert scales (from I 'not at all' to 7 'very much') for rating aspects of thoughts, mood, physical well-being, individually defined (psycho)somatic complaints, and present activity. Subjects were asked to describe any stressful events or situations which may have taken place in the interval since the last ESM report and to rate these events on a number of dimensions. Information about smoking (Wust, Kirschbaum, \& Hellhammer, 1990), food (Quigley \& Yen, 1979), coffee (Pincomb, Lovallo, Passey, Brackett, \& Wilson, 1987), and alcohol intake, medications, and physical exertion (Cook, Ng. Read, Harris, \& Riad-Fahmy, 1987) since the last beep was also obtained, to help control for possible confounding influences on cortisol secretion.

\section{Salivary cortisol}

Salivary cortisol is a reliable and valid indicator of the free cortisol in plasma, which is considered to be the biologically active hormone; cortisol concentrations are independent of the flow rate of saliva (Vining et al., 1983). Salivary cortisol increases within minutes in response: to acute stressors (Hellhammer, Kirschbaum, \& Belkien, 1987) and has a half-life of approximately one hour (Fredrikson et al., 1985). We have found no difference in cortisol levels in saliva samples either frozen immediately or kept at room temperature for 2 days (Nicolson et al., 1992); others report no change in unfrozen samples up to 30 days (Kirschbaum \& Hellhammer, 1990).

At the same time ESM forms were being completed, subjects collected saliva by holding a cotton dental roll in the mouth for approximately 1 minute. The saturated roll was placed in a capped plastic vial (Salivette; Sarstedt), which was stored in a specially designed wallet. At the end of each day, subjects placed the vials in their home freezers. At the end of the sampling period, 
uncentrifuged samples were stored at $-20 \mathrm{C}$. Compliance with the saliva sampling procedure was good in both high and low stress groups, with approximately the same mean response rate (83\%) as for the ESM reports.

Salivary cortisol levels were determined in duplicate by direct radioimmunoassay (Ansseau et al., 1984), using ${ }^{125}$ I-cortisol (Farmos diagnostica, Finland) and antiserum made against the 3CMO-BSA conjugate by Dr. J. Sulon, University of Liege, Belgium. The lower detection limit of the assay was $12 \mathrm{ng} / \mathrm{dl}$, with a mean intra-assay coefficient of variation of $4.8 \%$ (range: $2.2 \%$ $7.5 \%$ for 4 assays). Each subject's samples were analysed in the same assay, to reduce sources of variability.

\section{Stallstical analysis}

The 16 ESM mood items were reduced to three mood measures, based on the results of a principal component analysis with varimax rotation, which accounted for $78 \%$ of the total variance when subject mean scores were used. Ratings on the items cheerful, satisfied, relaxed, energetic, self-assured, concentrated and enthusiastic were summed to form ai 'positive affect' (PA) scale (Cronbach's alpha $=.95$ ). Two separate components of negative affect were identified: "NA-low arousal', including the items depressed, anxious, worried, lonely, tired, and miserable (alpha = 87), and 'NA-high arousal', with the items restless, irritated, hurried, and nervous (alpha $=.93$ ). The sums of the scale items were divided by the number of items in the scale, so that all mood measures have ranges from 1 to 7 .

Because the distribution of cortisol values was positively skewed, transformation to natural logarithms of cortisol concentrations was performed prior to statistical analysis. Nonparametric tests were used for univariate group (Mann-Whitney U-test) and within-subject (Wilcoxon matched-pairs signed-rank test) comparisons. Unless noted otherwise, significance tests are twotailed. Analysis of variance (SPSS procedure MANOVA) was used to test differences (and interactions) between HS and LS groups, workdays and weekends, and the 10 times of day sampled. Analyses were performed with SPSS -Macintosh version.

\section{RESULTS}

Given the general nature of the PSS, which was used to define high and low stress groups in this study, it is useful to contrast the two groups on more specific measures of distress. In addition to trait measures, we compared groups on a number of aggregated state measures derived from the ESM data. Mean scores on each of the 3 ESM mood scales were computed across all valid beeps for each subject. Frequency of stressful daily events was calculated as the percentage of total beeps for a subject on which a stressful event was reported, regardless of rated severity. 
Table 5.1.1. Differences in psychological measures between low and high stress groups

$$
\text { low PSS high PSS }
$$

mean (st.dev) mean (st.dev.) D-value

(2-tailed)

Trait measures

Perceived stress (PSS)

Trait Anxiety

Trait Anger

Zung Depression

Psychosomatic

symptoms (PSC)

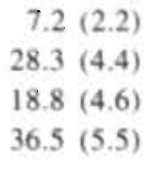

$6.1(5.6)$
$18.1(3.4)$

$39.8(7.6)$

$23.0(5.2)$

$48.4(7.7)$

$27.5(23.2)$
$<.0001$

$<.0001$

$<.000 \|$

$<.0001$

$<.0001$

\section{ESM measures*}

\begin{tabular}{lccl} 
PA & $5.7(0.6)$ & $5.3(0.3)$ & $<.001$ \\
NA-low arousal & $1.2(0.1)$ & $1.4(0.4)$ & $<.0001$ \\
NA-high arousal & $1.3(0.3)$ & $1.8(0.6)$ & $<.0001$ \\
frequency of stressful events & $12.3 \%(11.9)$ & $22.0 \%(20.0)$ & $<.01$ \\
\hline
\end{tabular}

" subject means, aggregated from beep level data

As shown in Table 5.1.1., subjects with a high level of perceived stress were significantly more trait anxious and depressed than subjects with a relatively lower level of perceived stress. They also scored higher on trait anger and reported more psychosomatic symptoms. HS subjects had significantly lower ESM positive affect, higher negative affect-low arousal and higher negative affect-high arousal scores. They were almost twice as likely to report stressful daily events as LS subjects. In absolute terms, the mean number of stressful events reported over the five days of ESM was 9.0 for the HS and 5.3 for the LS group.

We next examined whether high perceived stress was associated with elevated salivary cortisol. Mean cortisol levels were first computed for each subject at each of the 10 time blocks for workdays and again for weekend days. To test for main effects on cortisol levels as well as interactions, analysis of variance was performed with the between-subject factor group (HS, LS) and within-subject factors time of day ( 10 beeps) and day type (workday, weekend). 39 of the 88 subject cases were rejected because of missing data for any of the 20 (10 time blocks $\times 2$ day types) measures. The remaining 49 subjects included $28 \mathrm{LS}$ and $21 \mathrm{HS}$ subjects.

Cortisol concentrations showed the well-defined circadian rhythm, with peak salivary concentrations in the early morning samples. declining values in the afternoon and lowest concentrations at the end of the day; the main ( ffect for time of day was highly significant $(F(9,423)=182.04, p<.001)$, with no significant interactions with either group or day type. Contrary to our hypothesis, no significant main effect for group was found. Further, there was no workday-weekend diffierence in cortisol levels, either as a main effect or in interaction with group. 


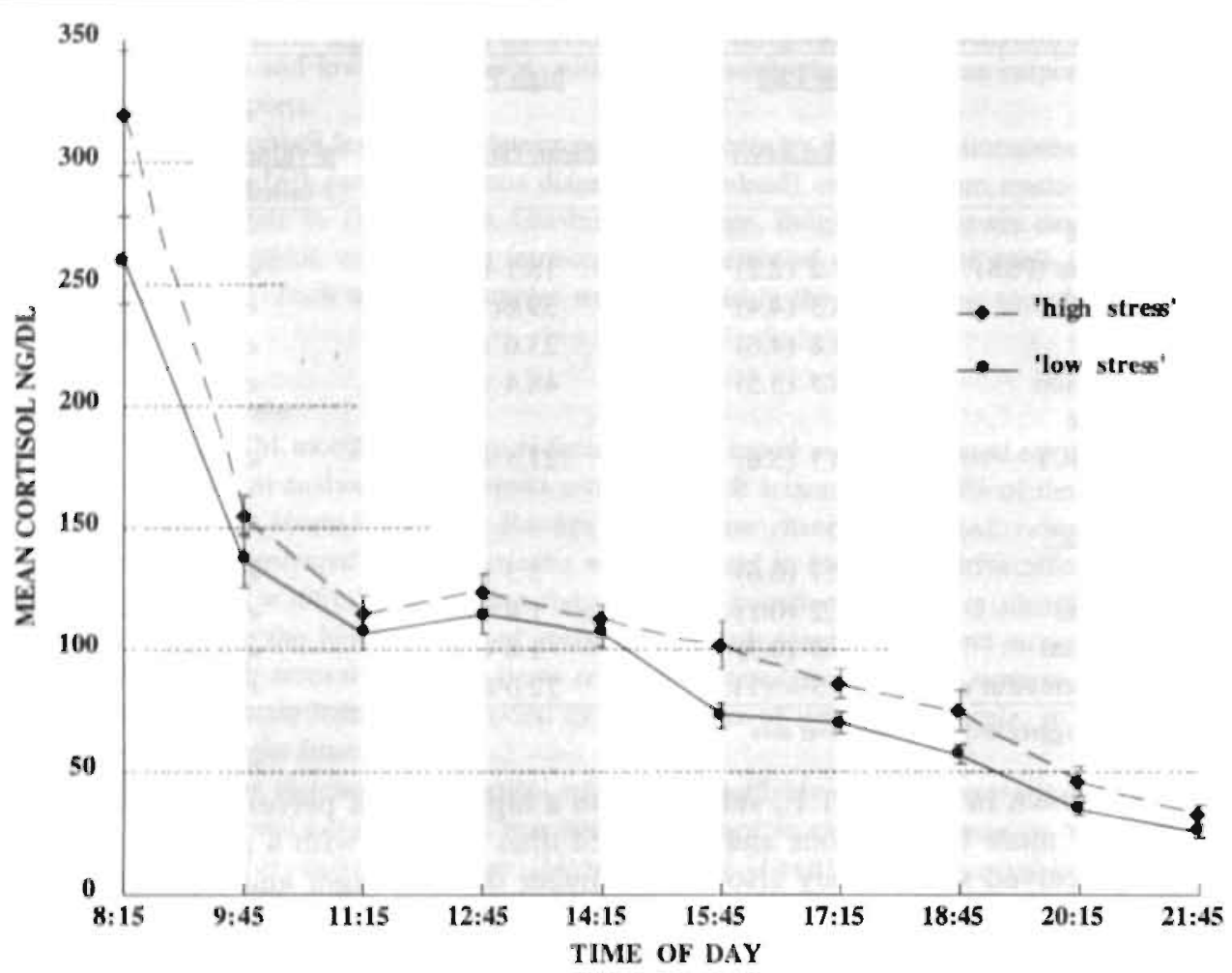

Figure 5.1.1. Mean cortisol (ng/dl; $+1-$ s.e.) during workdays for the 'high' $(n=34)$ and the 'low' stress' group $(\mathrm{n}=39)$.

As noted earlier (see 'Daily Experience', above), the percentage of missing responses was higher on weekends than on workdays, especially for early morning samples. Inclusion of weekend data in the model is thus largely responsible for the considerable data attrition. We therefore chose to examine workdays and weekends again separately. While this does not address the issue of workday versus weekend differences, it allows us to test for group differences in cortisol in a larger sample.

For workdays, 15 subjects were forced out of the analysis due to missing data, leaving $39 \mathrm{LS}$ and $34 \mathrm{HS}$ subjects. Time of day again showed the Iargest effect on cortisol levels $(\mathrm{F}(9.639)=262.98, \mathrm{p}<.001)$, but here the difference between HS and LS groups was also significant $(\mathrm{F}(1.71)=6.02, \mathrm{p}<.02)$. The interaction effect was not significant $(F(9,639)=.67, p>.05)$. Cortiso! concentrations for HS and LS groups on workdays are shown in Figure 5.1.1.

In the analysis of differences in cortisol levels on weekends, only 56 subjects (32 LS and 24 HS) had sufficient data for inclusion. For this subsample, the usual time of day effect was found $(F(9,486)=85.80, p<.001)$, but there was no consistent difference between the two stress groups $(F(1,54)=.79, p>.05)$. The group by time of day interaction approached significance $(F(9,486)=1.83, p=.06)$, with a tendency for the high stress group to have higher cortisol levels in the morning through early afternoon (beeps 2, 3, 4 and 5, between 9.30 am and 2.30 PM) and in the late evening 
(beeps 9 and 10, after 8 PM) only. Again, it is important to note that this (nonsignificant) pattern is based on a relatively small subset of the entire sample.

To summarize, cortisol levels showed the expected strong diurnal pattern in all analyses we performed. More interestingly, 'high stress' subjects had elevated cortisol throughout the day on workdays, confirming our hypothesis, at least for these days. It would seem logical to assume that such a pattern would be mediated by group differences in stress exposure or, more specifically, in emotional response to daily stress. Questions concerning reactivity of mood and cortisol to stressful events are beyond the scope of this paper. However, measures of workday stress and distress could provide additional insights into the perceived stress - cortisol relationship.

Within subjects, workdays in comparison to weekends were associated with a higher rate of stressful events (Wilcoxon matched-pairs test, p<.0001) and greater NA-high arousal ( $<<.0001)$, with no differences in either NA-low arousal or PA. The coded descriptions of reported stressors suggested that the work environment was a major arena for such daily hassles, with stressors more often occurring in work than in household, leisure or social network contexts. $43 \%$ of events in LS subjects and $47 \%$ of events in HS subjects were work-related. While this general pattern of workday distress was found in both groups, within-subject differences in workday vs. weekend NA-high arousal were greater in HS than in LS subjects (Mann-Whitney, $\mathrm{p}<.001)$; that is, HS subjects were disproportionately more negatively aroused on workdays. Workday/weekend differentials in NA-low $(.05<p<.10)$ and stressful events $(.05<p<.10)$ did not distinguish the two groups, although results were in the expected direction of relatively greater work-related stress/distress in HS subjects.

Since HS subjects scored higher than LS subjects on trait anxiety, depression, anger and psychosomatic complaints, as well as on ESM measures of positive and negative affect, we expected positive relationships between these variables and cortisol levels, at least on workdays. Mean levels of cortisol over the three workdays were determined by first calculating the mean at each of the 10 time blocks for every subject and then averaging the 10 values for each subject. This aggregated measure of mean cortisol was slightly higher in the HS (116 ng/dl, sd 37.5) compared to the LS group (101 ng/dl, sd 28.2) (one-tailed Mann-Whitney test, $\mathrm{p}<.05$ ).

Spearman rank order correlations between mean workday cortisol and both psychological measures and ESM mood sciales are shown in Table 5.1.2. Small but significant (one-tailed tests) positive correlations were found between cortisol and trait anxiety, depressive symptomatology and NA-low arousal.

Table 5.1.2. Correlations between psychological variables and mean salivary cortisol during workdays

\begin{tabular}{lcl}
\hline & $\mathrm{r}$ & $\mathrm{n}$ \\
\hline Trait anxiety & $.18^{*}$ & 88 \\
Trait anger & .08 & 88 \\
Zung Depression & $.18^{*}$ & 87 \\
Psychosomatic & & \\
symptoms (PSC) & .14 & 88 \\
PA & -.10 & 87 \\
NA - low arousal & $.20^{*}$ & 87 \\
NA - high arousal & .08 & 87 \\
\hline
\end{tabular}

"pe.05 (Spearman; one-tailed tests) 
Although these results indirectly support the conclusion that daily stress and distress lead to increases in cortisol, the role of possible confounding factors should be explored. If, for example, HS subjects smoked more than LS subjects, this might explain the observed differences in cortisol secretion. HS and LS groups were therefore compared on the percentage of workday beeps with reported smoking, strenuous physical exertion (rated 7 on the 7-point scale), coffee, food, and alcohol intake - all activities that could theoretically result in elevated cortisol secretion (see 'Daily Experience', above). No significant differences were found, with mean rates for HS and LS groups as follows (HS/LS): smoking $17 \% / 16 \%$, exertion $0.7 \% / 1.3 \%$, coffee $46 \% / 41 \%$, food $42 \% / 39 \%$, and alcohol $8.0 \% / 7.8 \%$. Although we cannot conclude that these behaviors had no influence on cortisol secretion, effects seem equally likely to have occurred in either group.

\section{DISCUSSION}

In this study, two groups of male white-collar workers differing in levels of perceived stress were compared on subjective, self-report measures of distress and on levels of salivary cortisol, as measured repeatedly over five days. The 'high stress' group scored higher than the 'low stress' group on measures of trait anxiety, depression, anger and psychosomatic complaints, as well as on ESM measures of positive and negative affect and stressful daily events. Cortisol showed a clear diurnal secretory pattern in both groups, with values in the normal range for healthy men (Kirschbaum \& Hellhammer, 1989). On workdays, cortisol was elevated throughout the day in the 'high stress' relative to the 'low stress' group. Several measures of distress, including both trait (anxiety, depression) and concurrent state (NA-low arousal) measures, correlated significantly with mean workday cortisol.

The observed pattern of higher workday cortisol levels in the 'high stress' group is consistent with our initial hypothesis; a straightforward interpretation would be that mild chronic or intermittent stress in situations like those subjects reported during the ESM sampling period was sufficient to increase secretion of the hormone. Since the half-life of cortisol in saliva is shorter than the intervals between beeps, consistently elevated cortisol from morning through late evening indicates continued secretion. More detailed temporal analyses are needed, however, to understand. cortisol dynamics in response to daily stress.

Of the few studies of stress and cortisol in everyday environments to date. results have been inconsistent. Caplan et al. (1979) tested the effect of perceived work load (a summary measure of quantity, deadlines and calls and office visits) on plasma cortisol and found that high work load was associated with low morning cortisol levels. The authors suggested that this finding might reflect a shift in the circadian rhythm of cortisol as a result of job stress. A major disadvantage of this study is the cross-sectional design, in which blood samples were drawn at various times of day, with only one cortisol determination per subject. In other words, comparisons between morning, midday and afternoon cortisol concentrations were comparisons between subjects and not within subjects. Although perceived stress may differ in important ways from perceived work load, our results clearly contradict those of Caplan and colleagues; we found higher early morning cortisol in high stress subjects and no evidence at all for a circadian shift. Brandtstädter et al. (1991), who measured salivary cortisol concentration in 767 adults three times over the course of one day, found higher morning cortisol concentrations in men with high life 
satisfaction, high psychological well-being and high level of employment. Cortisol levels in the afternoon or early evening were not predicted by psychological variables. Again, these results are difficult to reconcile with our findings.

Trait negative affectivity (NA) is conceptualized as the tendency to experience a wide range of negative emotions (Watson \& Clark, 1984). In studies of stress-illness relationships, trait NA has been held responsible for inflated correlations between stressors and self-reported health symptoms (Costa \& McCrae, 1987; Watson \& Pennebaker, 1989). In general, NA has not been found to predict objective health problems or physiological stress responses and has therefore been considered a nuisance in the analysis of stress data (Schaubroeck et al., 1992). The 'high stress' group in our study was defined on the basis of scores on a very clearly subjective self-report measure, the Perceived Stress Scale, and the fact that HS subjects scored higher on state and trait measures of distress as well as self-reported stress exposure suggests the pervasive influence of trait NA. In this light, our finding of an association between subjective stress, trait and state NA measures, and salivary cortisol levels is particularly noteworthy.

As is usually the case in psychophysiological studies, the psychological variables we measured accounted for only a small percentage of the variability in cortisol. Moreover, the observed elevations in workday cortisol secretion in HS subjects were slight, relative to pathophysiological levels seen in Cushing's disease or some cases of major depression. Since cortisol levels are not routinely measured in prospective epidemiological studies of healthy individuals, we have no guidelines at present for assessing the clinical relevance or health implications of these findings.

Analyses in this study were done on aggregated data, which has the advantage of increasing the reliability of measurement when only the chronic level of variables is of interest. On the other hand, aggregated measures may be biased (Jaccard \& Wan, 1993), and they obscure the important interplay between experiential and physiological states and processes which momentary measures are intended to clarify. To gain more insight into the relationship between perceived stress and cortisol, we need to move to beep-level analyses. We can then investigate fluctuations over time in stressful events, mood, psychosomatic complaints and determine how these fluctuations relate to endocrine activity (concurrent and lagged relationships). One promising method for the analyses of temporal data is hierarchical linear modeling, or multi-level analysis (Bryk \& Raudenbush, 1992). This approach can account for the dependency of data within a subject, can deal with the problem of missing data, and allows for individual differences in intercepts, slopes and error structures. We are currently undertaking multi-level analyses, in the belief that these new methods will help realize the enormous research potential afforded by momentary measures.

\section{ACKNOWLEDGEMENTS}

We thank Dr. J. Sulon, University of Liege, for performing RIA analyses of salivary cortisol, and C. Dijkman for assistance in all aspects of the study. 



\section{Section 5.2}

\section{Four-teen hour urinary catecholamine excretion in relation to perceived stress.}

\section{INTRODUCTION}

It has been well documented that, besides the pituitary and adrenal cortex, the adrenal medullary system also plays a central role in stress and arousal (Cannon, 1929; Mason, 1975). Increased catecholamine (CA) secretion has been associated with exposure to a wide variety of psychological and physical stressors (Frankenhaeuser, 1975a); for example to failure, loss, challenge, mental effort, uncertainty, and threat situations, as well as to physical stimuli such as cold, pain, anoxia, exercise, and heat (Frankenhaeuser, 1976; Frankenhaeuser, 1979). The magnitude of CA responses to potentially stressful situations or events is usually determined by the intensity of the appraisal of threat as related to one's perceived ability to meet the demands of the situation (Lazarus \& Folkman, 1984b).

Personal control appears to be an important factor in relation to CA excretion; high personal control (perceived self-efficacy) was accompanied by low levels of plasma adrenaline $(\mathrm{A})$ and noradrenaline (NA) during interactions with a phobic object, while moderate control resulted in substantial increases in plasma CA (Bandura, Taylor, Williams, Mefford, \& Barchas, 1985). With respect to occupational stress, a lack of control over the pace of one's work and extreme workloads (both high and low) were associated with high levels of CA (Frankenhaeuser \& Johansson, 1976; Frankenhaeuser, Nordheden, Myrsten, \& Post, 1971; Timio \& Gentili, 1976). On the other hand, high CA output has also been associated with successful coping and with the ability to maintain a high performance level during stressful conditions, probably as a result of high mental effort (Frankenhaeuser, 1979; Siegrist et al., 1989; Ursin, 1978). There is some evidence to suggest that the two CA are differentially sensitive to behavioral and situational factors. NA relates to active, aggressive emotional states, whereas excessive A levels relate to passive, anxious states (Henry, 1982), but also to states involving high mental effort (Fibiger, Singer, \& Miller, 1984; Frankenhaeuser et al., 1971).

Although a positive relation seems to exist between CA levels and adaptive functioning. frequent and excessive elevations and/or long-term elevation of $\mathrm{CA}$ levels are assumed to have damaging effects on various bodily organs and the cardiovascular system (Gruchow, 1979; Madden \& Livnat, 1991; Surwit, Williams, \& Shapiro, 1982). However, in contrast with the numerous studies on acute stress situations, studies concerning habitual CA excretion as related to enduring characteristics of the social environment or of the individual are more scarce. With respect to long-term occupational stress, elevated A excretion during periods of working 'overtime' was observed; not only during working hours but also in the evening at home (Rissler, 1977). In a study by Baum and colleagues (1986), the stress of being unemployed was related to higher levels of both $\mathrm{CA}$, and levels increased as the length of employment increased. Forsman (1980) also found a positive correlation between habitual A excretion and experiences of distress (measuring day-to-day stress) as measured in the field. As another example, the continuing uncertainty and perceived threat of living near the site of the nuclear power plant accident at Three 
Mile Island was associated with chronic elevations in overnight samples of both $\mathrm{A}$ as well as NA (Schaeffer \& Baum, 1984). Finally, in another study investigating the effects of long-term stress on CA, it was found that urinary CA levels in American hostages freed from captivity in Iran were highly elevated and appeared to reflect three affects: distress, anxiety, and elation (Rahe et al., 1990).

In the present study, urinary CA were determined to investigate the relationship between perceived stress and catecholamine excretion. It was hypothesized that high levels of perceived stress, as a measure of more chronic stress, are associated with elevated CA levels, either in general or related to the type of day (work day versus weekend). Additionally, several person characteristics (trait anxiety, depression, anger, and psychosomatic symptoms), and mood states (Fibiger, Singer, Miller, Armstrong, \& Datar, 1984) were explored for their possible relationship with CA levels. As opposed to plasma CA levels, urinary CA levels ( 14 to 24 hour samples) are typically used in studies for the measurement of long-term, chronic stress, and are particularly well-suited for studying psychosocial influences of everyday life (Frankenhaeuser, 1975a). Because urine samples show a slower rate of change than plasma samples, they can be applied to determine long-term changes in CA levels. The collection of urine samples is also relatively easy, non invasive and very well suited for field studies during which subjects' normal habits and ordinary daily activities should nol be changed (Frankenhaeuser \& Gardell, 1976).

\section{METHODS}

\section{Subjects and questlonnaires}

For details about subject recruitment, procedures and questionnaires see section 5.1.

\section{Urine sampling}

Catecholamine (CA) levels were determined in urine. Because the $\mathrm{CA}$ in urine constitute a small but relatively constant fraction of liberated amines in the body, the direction of change or the relative levels are meaningful, but absolute numbers have limited value. NA levels are somewhat difficult to interpret because NA is secreted by both nerves and the adrenal medulla and is also subject to rapid neuronal reuptake, but $\mathrm{A}$ can be considered a reliable estimate of adrenal medullar activity because the adrenal medulla is the sole source of circulating A (Frankenhaeuser, 1975b). The 14-hour samples we used in this study, suppress (not eliminate) the effects of variation between subjects with different circadian thythms and minimize the biasing effects of idiosyncratic events (Baum et al., 1985).

Subjects collected two overnight urine samples (from 6 p.m. to 8 a.m.), one after a workday (Thursday to Friday) and one after a weekend day (Sunday to Monday). Subjects emptied their bladder in the toilet at 6 p.m. and from that moment collected subsequent urine in a 2 liter container (Sarstedt) until 8 a.m. the next day at which time they emptied their bladder for the last time in the container. Subjects refrigerated their samples during the collection period. Immediately after collection of the container by the research staff we added HCL. $(37 \%)$ to the total volume of urine until a $\mathrm{pH}$ of $<3$ was reached, to prevent oxidation. From the total volume, $10 \mathrm{ml}$. samples were extracted and immediately frozen at a temperature of $-20 \mathrm{C}$ until analyses.

Adrenaline and noradrenaline excretion rates were determined by means of high-pressure liquid chromatography (HPLC) with electrochemical detection. ${ }^{3} \mathrm{CA}$ levels were corrected for

Analyses of adrenaline and noradrenaline were performed in the laboratory' of Dr. Rahman and Dr. Duvivier, University Hospital of Liege. Belgium.. 
creatinine excretion, and expressed in $\mu \mathrm{g} / \mathrm{g}$ of creatinine. Samples of subjects with creatinine levels below $0.60 \mathrm{~g} / \mathrm{l}$ were discarded $(\mathrm{n}=9)$, because they might give artificial too high concentrations (low levels could be suspect of e.g. hyperthyroidism, advanced renal disease). Due to practical reasons, three subjects did not collect any urine, and another four subjects collected urine only once.

\section{Statistical analysis}

Analysis of variance was used (SPSS procedure MANOVA) was used to test differences and interactions between HS and LS groups and work day and weekend day samples. Nonparametric Spearman correlations were calculated the various psychological trait and state measures and the catecholamines. Significance tests are two-tailed. Analyses were performed with SPSS-Macintosh version.

\section{RESULTS AND DISCUSSION}

Mean values for adrenaline (A) as well as noradrenaline (NA) after a work day and after a weekend day are shown in Table 5.2.1. As can been seen, CA concentrations were highly comparable in high stress versus low stress subject groups.

Table 5.2.1. Mean adrenaline and noradrenaline concentrations ( $\mu \mathrm{g} / \mathrm{g}$ creatinine) plus standard deviation in high stress (HS) and low stress (LS) groups after a work day and after a weekend day.

\begin{tabular}{lcccc}
\hline & \multicolumn{2}{c}{ Work day } & \multicolumn{2}{c}{ Weekend day } \\
& LS & HS & LS & HS \\
& $\mathrm{n}=42$ & $\mathrm{n}=37$ & $\mathrm{n}=41$ & $\mathrm{n}=37$ \\
\hline & & & & \\
Adrenaline & $4.3(2.4)$ & $4.7(35)$ & $4.3(2.5)$ & $4.3(37)$ \\
Noradrenaline & $21.4(12.4)$ & $19.9(9.3)$ & $19.6(10.5)$ & $19.2(8.3)$ \\
\hline
\end{tabular}

To test for main effects of perceived stress and day type on CA levels as well as interaction effects, analysis of variance were performed with the between-subject factor group (HS, LS) and the within-subject factor day type (workday, weekend). 13 of the 88 subject cases were rejected because of missing data. The remaining 75 subjects included 37 HS and 38 LS subjects. For A, no main effect for group was found $(\mathrm{F}(1,73)=0.34, \mathrm{p}>.05)$. There also was no work day / weekend difference in $\mathrm{A}$ levels, either as a main effect or in interaction with group $(F(1,73)=0.16, p>.05 ; F(1$, $73)=1.59, p>.05$ ). For NA, again no main effects for group or day type were observed, and also no interaction effect (group: $F(1,73)=0.24, p>.05$; day type: $F(1,73)=0.66$, $p>05)$; group $x$ day type: $F(1,73)=0.07, p>.05)$.

Spearman rank order correlations between mean A and NA and several psychological measures (trait anxiety, depression, anger, psychosomatic symptoms) and mean ESM mood states (positive affect, negative affect, agitation) were next computed. None of the correlations reached significance (all correlations <.21). A trend was found for positive affect to be positively related to both $\mathrm{A}$ as well as NA (A: $r h o=.21, \mathrm{p}=.06$; NA: $r h o=.19, \mathrm{p}=.08$ ).

The results do not support our hypothesis that high perceived stress is reiated to high CA levels, either in general or related to the day of the week, and do not 
replicate the findings reviewed above of increased catecholamine excretion in the context of long-term stress. In fact, none of the variables investigated showed any relationship to both adrenaline as well as noradrenaline levels. The trend found for positive affect to be related to both catecholamines is in line with the general finding of a weak but positive correlation between CA secretion and indices of emotional stability and adaptation (Johansson, Frankenhaeuser, \& Magnusson, 1973). In the present study, urine samples were collected between 6 p.m. and 8 a.m. and is comparable in design to the study by Schaeffer and Baum (1984) where, in contrast with our study, they did find elevated catecholamine levels in chronically stressed subjects. Most other studies sampled urine during the day, and in the study by Forsman (1980), where urine was sampled in the morning, afternoon, evening, and at night ( 11 p.m. to 8 a.m.), only the morning and afternoon CA levels were related to the experience of stress as measured in daily life. Therefore it is possible that in our study of healthy subjects, where samples were obtained mostly overnight (constituting a more or less baseline level) which reduces the impact of specific environmental influences during the day, complete unwinding took place during the night. More extreme stress levels are perhaps necessary to observe an impact on CA baseline levels. It would be interesting to investigate whether perceived stress and the other person and mood variables would relate to $\mathrm{CA}$ levels during the day as opposed to overnight, when specific environmental circumstances are more influential.

An important limitation of our study was that no restrictions were put on the subjects concerning feeding and drinking patterns (including alcohol), exercise, and cigarette smoking. This was done to increase compliance because of the already intensive and time consuming nature of our ESM field study. CA levels are, besides stress, sensitive to all the factors mentioned above, and the absence of a control for these factors may have confounded the results. HS and LS groups were therefore compared on the percentage of ESM beeps (see paragraph 'daily experience' of section 5.1) with reported smoking. coffee, food, alcohol intake, and physical exertion reported during both evenings (work and weekend) when urine collection took place (between 6.45 p.m. and 21.45 ). No significant differences were found between groups suggesting that, although we can not rule out the possibility that these factors had an influence on CA excretion, it should not have confounded the group comparisons. 


\section{Chapter 6}

\section{The effects of perceived stress, traits, mood states, and stressful events on salivary cortisol}

\section{Psychosomatic Medicine (in press)}

Marleen van Eck ${ }^{1}$, Hans Berkhof ${ }^{2}$, Nancy Nicolson', Jose Sulon ${ }^{3}$

1 Department of Psychiatry \& Neuropsychology, Social Psychiatry and Psychiatric Epidemiology section, University of Limburg, the Netherlands

2 Department of Statistics and Measurement Theory, University of Groningen, the Netherlands

3 Faculty of Veterinary Medicine, University of Liege, Belgium 

ABSTRACT This study examined the effects of perceived stress and related individual characteristics, mood states, and stressful daily events on salivary cortisol levels. Forty-one 'high stress' and 46 'low stress' subjects were selected on the basis of Perceived Stress Scale scores from a sample of male white collar workers. Subjects completed Experience Sampling self-reports and collected saliva samples ten times a day over five consecutive days. Multilevel analysis revealed that trait anxiety and depression, but not perceived stress, were associated with small but statistically significant cortisol elevation. No effects on cortisol were found for recent life events, chronic difficulties, trait anger, or psychosomatic symptoms. Distress, as reflected by the mood states Negative Affect and Agitation, was associated with higher cortisol levels, whereas Positive Affect had no statistically significant effect. Stressful daily events were associated with increased cortisol secretion, the magnitude of the effect depending on whether the event was still ongoing and on how frequently a similar kind of event had occurred previously. Although perceived stress, anxiety and depression did not increase cortisol reactivity to daily events, we found evidence for reduced habituation to recurrent events in subjects scoring high on these traits. Mood appeared to play a mediating role in the relationship between stressful events and cortisol secretion. These results suggest that negative affectivity is not just a confounder, but is related to elevated cortisol secretion during normal daily activities. The finding that even minor events and fluctuations in mood states were associated with increased adrenocortical activity points to a possible mechanism linking subjective experience to health outcomes.

\section{INTRODUCTION}

The neuroendocrine system has long been thought to play an important role in the causal pathway linking stress and ill health (Cannon, 1929; Selye, 1976; Weiner, 1992). The hypothalamic-pituitary-adrenal (HPA) axis, which is involved in the regulation of a wide range of physiological and behavioral responses to stress, has been implicated in numerous illness processes (Chrousos \& Gold, 1992; De La Torre, 1994; Tsigos \& Chrousos, 1994), including the etiology of psychiatric disorders (Checkley, 1992; Dinan, 1994; Gold et al., 1988). Over the past decade, it has become clear that not only major life events (Brown \& Harris, 1989; Holmes \& Rahe, 1967), but also minor daily stressors or "hassles" can have negative effects on health and well-being (DeLongis et al., 1982; Ivancevich, 1986; Zarski, 1984). In contrast to the wealth of information concerning the neuroendocrine effects of major real-life stressors, there is relatively little known about the effects of chronic stress and even less about the effects of minor daily events. The goal of the current study was to increase our understanding of the impact of daily life stress on the HPA axis.

While a growing number of studies have examined the relationship between daily events and mood (Bolger et al., 1989a; Clark \& Watson, 1988; Goplerud \& Depue, 1985; Lundberg et al., 1989; Neale et al., 1987; Repetti, 1993; Stone, 1987; Stone \& Neale, 1984) or physical symptoms (DeLongis et al., 1988; Goreczny, Brantley, Buss, \& Waters, 1988; Jandorf, Deblinger, Neale, \& Stone, 1986), few have investigated whether stressful daily experiences have an effect on cortisol excretion. Findings have been inconsistent, which is not surprising in light of the large differences in cortisol measurement procedures and definitions of daily stress or distress. Cortisol levels have often been based on a single measurement per subject or per day. To illustrate the diversity of results, greater work demands were associated 
with lower cortisol levels in subjects sampled in the morning (but not in the afternoon) in one study (Caplan et al., 1979); another study found that feelings of irritation, tenseness, and tiredness in assembly line workers were associated with elevated cortisol levels on workdays and that cortisol levels were absolutely higher on 'bad' compared to 'normal' or 'good' workdays (Lundberg et al., 1989). Examining within-subject associations over several days, one study found elevated afternoon urinary cortisol on high stress compared to low stress days (Brantley et al., 1988), while in another, no relationship between the number of undesirable events reported at the end of the day and cortisol levels measured in evening urine could be demonstrated (Cummins \& Gevirtz, 1993). With more frequently measured responses to work stress in air traffic controllers, Rose and colleagues (1993) found that a subgroup of subjects responded to an increase in the number of planes they had to manage with large increases in cortisol.

New approaches are needed for investigating cortisol responses to daily events. Repeated measurement of cortisol not only increases reliability, but provides a clearer picture of the temporal relationship between stressful events and neuroendocrine responses. This is especially important if the events of interest occur unpredictably (as is usually the case in the natural environment) and therefore cannot be directly monitored by the researcher. Additional insights into the stress process can be gained by including measures of the context in which events take place and measures of subjective responses, including mood and cognitive appraisals (Folkman \& Lazarus, 1985).

The current study therefore combines Experience Sampling (ESM (Csikszentmihalyi \& Larson, 1987; de Vries, 1992) methodology and frequent salivary cortisol sampling to address two main questions about daily life stress and cortisol:

(I) Are high perceived stress and distress associated with elevated cortisol levels? Despite the lack of consistent results in the literature on the effects of chronic stress on the HPA axis, we reasoned that healthy individuals experiencing persistent but not overwhelming levels of distress and difficulty in coping with daily demands would have higher overall cortisol than persons who experience fewer problems. In addition to the effects of perceived stress level, we examined the contributions of conceptually related measures of stress exposure and chronic distress. These included recent life events, chronic difficulties, trait anxiety. depression. hostility, and psychosomatic complaints. Several of these variables have previously been linked to cortisol levels (Brandtstädter et al., 1991; Pope \& Smith, 1991; Schaeffer \& Baum, 1984).

In addition to trait-like measures of stress and distress, we assessed the effects of negative and positive mood states on cortisol. Although there is abundant evidence that cortisol increases in response to negative states (Arnetz \& Fjellner, 1986; Lundberg \& Frankenhaeuser, 1980: Mason, 1968; Nicolson. 1992), the effects of positive mood are less clear. Positive affect has been associated with lower (Hubert \& de Jong-Meyer, 1989; Kugler \& Kalveram, 1989) as well as higher (Brown et al., 1993) cortisol levels.

(2) Do stressful daily events influence cortisol levels?

In general, we hypothesized that even minor stressors could result in increased cortisol secretion. We further hypothesized that such effects might be dependent on an individual's chronic level of perceived stress. Based on results of previous studies showing greatei psychological impact of work-related events (Stone, 1987) and negative social interactions (Bolger et al., 1989a), we expected that these categories of events would have the largest impact on cortisol. Similarly, events that subjects 
rated as more unpleasant, more important, less predictable, and less controllable were expected to have larger effects, and ongoing events were expected to have more effect on cortisol than recently terminated events. Finally, we hypothesized that events reported to occur relatively infrequently in daily life would, due to their novelty, have a greater effect on cortisol levels than recurrent events.

The study design compares two groups of male white collar workers, with high versus low levels of perceived stress. A total of 87 subjects completed ESM selfreports and collected saliva for cortisol determination at frequent intervals over a period of five days. A previous analysis of a subset of these data, based on subject mean cortisol levels, provided some support for the hypothesis of elevated cortisol levels in the high stress group, at least on workdays (van Eck \& Nicolson, 1994). The current analysis adds to these preliminary findings by assessing the separate contributions of "trait" (e.g., perceived stress level, trait anxiety, depression) and "state" (e.g., mood, appraisal) variables, at the same time controlling for diurnal and possible confounding influences on cortisol secretion. Most importantly, the application of hierarchical linear modeling, or multilevel analysis (Bryk \& Raudenbush, 1992), enables us to investigate the neuroendocrine effects of the wide variety of stressful events experienced in daily life.

\section{METHODS}

\section{Subjects}

Local industries and government agencies were approached via their personnel departments to participate in the study. A decision as to which categories of employees fell under the definition of 'white-collar' was made by each personnel department on the basis of standard job function descriptions. Questionnaires were distributed among these employees, accompanied by a letter explaining the goals of the study. Participation was voluntary, and care was taken to insure anonymity. 316 male employees from six different industries or agencies completed the screening questionnaire. The mean score on the Perceived Stress Scale (PSS) for this sample was 12.7 (s.d. 6.0), similar to US norms (mean 13.02, s.d. 6.45) (Cohen et al., 1983).

Ninety-two subjects with scores in the upper or lower tertiles of the screening sample distribution (PSS-10 score $\leq 10$ or $\geq 16$ ) were recruited, excluding individuals who reported a history of serious chronic illness, endocrine disorder, medications known to affect cortisol levels, or treatment (past or current) for mental health problems. Fxclusion criteria were reassessed during an initial interview, study aims and procedures were explained, and informed consent obtained. During subject intake, each 'high stress' (HS) subject was matched for age group. marital status, and household composition with a 'low stress' (LS) subject to insure that the two groups did not differ on demographic characteristics that might affect exposure to certain classes of daily stressors. Five subjects were later excluded from analysis: four due to failure to meet ESM compliance criteria (see 'Daily Experience' section, below) and one because he became so acutely stressed that he was unable to work during the sampling period. Of the 87 remaining subjects, 41 subjects comprised the HS group and 46 subjects, the LS group. Mean age was 42.1 years (range 27 to 57 years). $89 \%$ were married, and $81 \%$ had children living at home

\section{Questionnaires}

The following measures were used in the current analysis:

Perceived stress: The 10-item version of the PSS was translated into Dutch. The ittms are rated on a 5-point frequency scale, ranging from 'never' (0) to 'very often' (4) in the last month. 
Life events: Life events were recorded with the questionnaire form of the List of Threatening Experiences (LTE-Q; Brugha et al., 1985). Subjects were asked about the occurrence of 12 categories of events (e.g. death of a partner, child, parent; got divorced) during the last year.

Long-term difficulties: Chronic stress was assessed with the Long-term Difficulties Questionnaire (LLM; Hendriks et al., 1990). This inventory focuses on problems in relation to work/study, housing, physical environment, leisure, finance, and social relationships (partner, family, friends, neighbors). Subjects rate each of the 16 items on a four point intensity scale with the anchors (1) none, (2) some, (3) quite, (4) serious (difficulties). A total score is obtained by summing across all items.

Psychosomatic symptoms: The revised version of the SUNYA. Psychosomatic Symptom Checklist (PSC) (Attanasio et al., 1984) includes 17 common psychosomatic complaints (e.g., headaches, backaches, nausea). Subjects rate each complaint on 5-point scales for frequency (0 "never or rarely occurs " to 4 "occurs daily") and intensity ( 0 "not bothersome" to 4 "extremely bothersome"). A, total score is obtained by summing the cross-products of each item's frequency by intensity.

Depression: Depressive symptomatology was assessed with the Dutch translation (Dijkstra, 1974) of the Zung Self-Rating Depression Scale.

Anxiety: Trait anxiety was measured with the Dutch version (van der Ploeg et al., 1980) of the State-Trait Anxiety Inventory (STAI).

Anger: Trait anger was measured with the Dutch version (van der Ploeg et al., 1982) of the Spielberger Trait Anger Scale. The scale has two subscales: 'anger-temperament' and 'angerreaction'.

\section{Daily experience}

The Experience Sampling Method (ESM) (Csikszentmihalyi \& Larson, 1987; de Vries, 1992) was used to collect data from subjects during their normal daily activities. Subjects received auditory signals ('beeps'), after which they filled in a questionnaire and collected a saliva sample. After receiving detailed instructions, subjects. were sampled for a period of 5 consecutive days (Thursday through Monday). A Seiko wristwatch was programmed to emit. 'beeps' 10 times each day, at semi-random intervals of approximately 90 minutes, between the hours of 8 am and 10 PM. Beeps were clustered around the midpoint of each time block e.g. $8.15 \mathrm{am}, 9.45,11.15$, and so on), with the exact time sequence of 'beeps' varied each day to decrease predictability. in a final 'debriefing' session, subjects were asked to clarify reasons for missing data.

The criteria we set for subject inclusion in the analysis ( $\geq 20$ ESM reports completed within 20 minutes after being signalled and no missing data for entire days) were met by all but foui subjects ( 2 from each group). The remaining 88 subjects completed an average of $83 \%$ of all possible responses within the time limit, for an average of 41 responses per subject. HS and LS groups did not differ in compliance rates (40.1 vs 42.3 responses per subject, Mann-Whitney Utest, $\mathrm{p}>.05$ ). Compliance was lowest for the first 'beep' (at approximately $8.15 \mathrm{am}$ ), with ant average of $73 \%$ valid reports. On Saturdays, response rate for first 'beeps.' was $61 \%$ and on Sundays, $59 \%$. On weekends, $74 \%$ of all missing and invalid responses could be attributed to the fact that subjects were still asleep when signalled.

The ESM form contained open-ended questions concerning thought content, the physical and social context, and what the individual was doing when signalled. The forms also included Likert scales (from 1 'not at all' to 7 'very much') for rating aspects of thoughts, mood, physical well-being, individually defined (psycho)somatic complaints, present activity, and stressful events. Subjects were asked to describe any stressful events or situations which may have taken place in. the interval since the last ESM report and to rate these events on a number of dimensions: unpleasantness, importance, predictability. controllability , and frequency of prior occurrence.. Subjects were also asked to indicate at what time the event had started, and if and when it had 
ended at the moment they were 'beeped'. Information concerning maximum level of physical exertion, smoking, food, coffee, and alcohol intake since the last 'beep' was also obtained.

The 17 ESM mood items were reduced to three mood measures, identified by means of principal component analysis with varimax rotation, which accounted for $78 \%$ of the total variance when subject mean scores were used. Ratings on the items cheerful, satisfied, relaxed, energetic, self-assured, concentrated, and enthusiastic were summed to form a 'Positive Affect' scale (Cronbach's alpha $=.95$ ). Two separate components of negative affect were identified: 'Negative Affect', including the items depressed, anxious, worried, lonely, tired, and miserable (alpha = .87 ), and 'Agitation', with the items restless, irritated, hurried, and nervous (alpha $=.93$ ). The sums of the scale items were divided by the number of items, so that all mood measures have ranges from 1 to 7 .

Subjects' descriptions of stressful daily events were first coded according to content, with categories work, network (events concerning family, friends and acquaintances). household/financial, leisure, personal health-somatic, personal health-psychological, transport, and other. Twelve events coded in the two personal health categories were excluded from analysis because of possible confounding with psychological and somatic state measures. In the current analysis, the remaining events were collapsed into the categories. work ( $48.0 \%$ of events) and nonwork $(50.5 \%)$. Some examples of reported work events were: 'unclear / vague assignment at work', 'too much to organize, not enough time', 'difficult conversation with boss about job performance', 'leading a big meeting'. Reported non-work events included: 'having a fight with: my wife about household duties', 'conflict with spouse about how to raise our son', 'child who will not listen', and 'making dinner, child crying, other child taking a bath, and this all at the same time'. In addition to content, events were classified according to whether or not they involved a social interaction and/or a task demand. ${ }^{4}$

\section{Salivary cortisol}

Salivary cortisol is a reliable indicator of the free cortisol in plasma, which is considered to be the biologically active hormone (Vining et al., 1983). Salivary cortisol increases within minutes in response to acute stressors and has a half-life off approximately one hour (Hellhammer et al., 1987). We have found no differences in cortisol levels in uncentrifuged samples frozen immediately or kept at room temperature for 2 days (Nicolson et al., 1992); others report no differences up to 2-4 weeks (Kahn et al., 1988; Kirschbaum \& Hellhammer, 1989).

At the same time ESM forms were being completed, subjects collected saliva by holding a cotton dental roll in the mouth for approximately 1 minute. The saturated roll was placed in a capped plastic vial ("Salivette", Sarstedt), which subjects stored in their home freezers each evening. At the end of the sampling period, uncentrifuged samples were stored at -20 degrees Celsius until analysis. Compliance with saliva sampling was good in both high and low stress groups, with the same mean response rate $(83 \%)$ as for the ESM reports. Five extreme cortisol values $(>1200 \mathrm{ng} / \mathrm{dl})$ were deleted from the dataset before analysis.

Salivary cortisol levels were determined in duplicate by direct radioimmunoassay (Ansseau et al., 1984), using ${ }^{125}$ I-cortisol (Farmos diagnostica, Finland) and antiserum made against the 3CMO-BSA conjugate by Dr. J. Sulon, University of Liege, Belgium. The lower detection limit of the assay was $12 \mathrm{ng} / \mathrm{dl}$, with a mean intra-assay coefficient of variation of $4.8 \%$ (range: $2.2 \%$ -

4 The reliability of the coding system was assessed by comparing the classifications of 345 events by two independent coders. Interrater agreement was determined by means of Cohen's kappa. On the whole, the qualitative information could be classified with a high degree of agreement (Laundis \& Koch, 1977), especially for the content categories. The overall Kappa for content was .90 , with intra-category Kappa's ranging from .60 to .96 ; Kappa's for social interaction and for task demand were .73 and .65 . respectively. 
$7.5 \%$ for 4 assays). All samples from an individual subject were analysed in the same assay to reduce sources of variability.

\section{Statistical analysis: multilevel or hierarchlcal linear model}

The multilevel model or the hierarchical linear model (Bryik \& Raudenbush, 1992; Goidstein, 1987) is a variant of the multiple linear regression model, applicable for data with a hierarchical nesting structure. In the present dataset, the measurements made at the 'beeps' are nested within persons. The two nesting levels will be called measurement level and person level. At each level of the hierarchy, explanatory variables can be added to the model. The variables that are added at the measurement level (e.g. mood states, events) vary with time, while the added variables at the person level represent characteristics of individual respondents (e.g. high or low perceived stress, trait anxiety).

An advantage of the hierarchical linear model is that it allows for missing observations. In addition, the observations do not need to be evenly spaced over the time interval.

At the measurement level, the relationship between cortisol and time of the day can be modeled explicitly. If, for example, this relation is linear, the model would be of the following form:

$$
(\text { CORT })_{i t}=b_{\mathrm{o} i}+b_{1 i}(\mathrm{TIME})_{i t}+\mathrm{e}_{i t} \text {, }
$$

where $(\text { CORT) })_{i t}$ is the level of cortisol at the th theasurement of person $i$, (TIME) $)_{i r}$ represents the time of the day at which this measurement is taken, $b_{\mathrm{o} i}$ and $b_{1 i}$ are the intercept and slope of person $i$, and the $e_{i l}$ 's are normally distributed error terms with mean zero and variance $\sigma^{2}$. The resulting equation can be extended with time-varying explanatory variables. For example, to study the effect of stressful event occurrence on cortisol, a dummy-coded variable (EVENT) ir can be added to the above equation as follows:

$$
(\mathrm{CORT})_{i t}=b_{\mathrm{o} i}+b_{1 i}(\mathrm{TIME})_{i t}+b_{2 i}(\text { EVENT })_{i t}+\mathrm{e}_{i t} .
$$

where $b_{2 i}$ is the effect of a stressful event for person $i$. The regression coefficients $b_{j i}(j=0,1,2$, ....) are allowed to vary across individuals. Therefore, we can split $b_{j i}$ into two components: a fixed component $d_{0 i}$ that is constant across persons (fixed effect $l$ and a random component $u_{j i}$ that varies across persons (random effeci). This gives the following person-level model for $b_{j i}(j=0,1$. $2, \ldots)$ :

$$
b_{j i}=d_{\mathrm{o} j}+u_{j i}
$$

Instead of estimating $u_{j i}$ for each person, we postulate that the $u_{j i}$ 's $(i=1,2,3, \ldots)$ are randlom draws from a normal distribution (explaining the term random effect). The mean of $u_{j i}$ is zero and the variance of $u_{j i}$ is $\tau_{j j}$. The value of $\tau_{j j}$ indicates how much the value of $u_{j i}$ differ across: persons: the higher the value of $\tau_{j j}$ the more the values of $u_{j i}$ differ. The covariance between $u_{j i}$ and $u_{k i}$ ( $j$ not equal to $k$ ) is $\tau_{j k}$. A positive value of $\tau_{j k}$ implies that a person with a high value of $b_{j}$ tends to have a high value of $b_{k}$ as well.

Suppose that part of the variation in $b_{j i}(j=0,1,2, \ldots)$ across persons can be explained by the person variable (GROUP) that indicates whether person $i$ belongs to the high or low stress group. The regression coefficients $b_{j i}(j=0,1,2)$ can now be modeled as:

$$
b_{j i}=d_{\mathrm{oj}}+d_{1 j}(\text { GROUP })_{i}+\mathrm{u}_{j i},
$$

where $d_{y}(j=0,1,2, \ldots)$ denotes the fixed effect of $(\text { GROUP })_{i}$ on $b_{j i}$. The $u_{j i}(j=0,1,2, \ldots)$ is now a random effect of person $i$ after controlling for (GROUP);

Estimates of the fixed effects $d_{0 j}$ and $d_{1 j}(j=0,1,2)$ as well as of the (co)variances $\sigma^{2}$ and $\tau_{j k}(j, k=0,1,2)$ were obtained with the program ML3e (Prosser, Rasbach, \& Goldstein, 1991). For model selection we started with an empty model and added main and interaction effects of the theoretically important variables. Significance of fixed effects was tested by dividing the estimated effect by its standard error. This ratio is approximately normally distributed. For testing the 
significance of the (co)variances. $\tau_{j k}(j, k=0,1,2)$, we applied the likelihood ratio test (Bryk \& Raudenbush, 1992). This test compares a model with and a model without a random effect. Hence, the (co)variances that are added to the model because of the specification of an extra random effect are tested simultaneously. Non-significant effects were excluded from the models. The postulated normality of the random effects was checked by inspecting normal probability plots of the individual estimates of $b_{j i}(j=0,1,2)$, which were obtained by the empirical Bayes approach (Bryk \& Raudenbush, 1992; Goldstein, 1987). The normality of the measurement level empirical Bayes residuals was also checked by inspection of the normal probability plot. The observed cortisol values for each person were plotted by time of day to check for the presence of outlying cortisol curves (see also Results, below).

The estimation of effects on cortisol entailed four steps, presented here as separate models. Since the analysis of repeated assessments of salivary cortisol is complicated by the hormone's strong diurnal rhythm and secretory peaks (which lead to a decline in variance from moming to evening), the first step involved accurate fitting of the diurnal curve, in order to allow comparisons of cortisol values across the day. Next, possible confounding factors, such as smoking (Kirschbaum \& Hellhammer, 1994), exercise (Cook et al., 1987), coffee (Pincomb et al., 1987) and food intake (Quigley \& Yen, 1979), were included as explanatory variables in the same model. All variables with significant fixed effects identified in this model were included in ail subsequent models.

The remaining three models test our main hypotheses. First, the effects of level of perceived stress, mood states, and individual trait characteristics on overall cortisol level were estimated. Workday vs. weekend differences and interactions between stress level and diurnal variability were also examined. The next model estimated effects of stressful events and event characteristics on cortisol, excluding the contribution of mood variables. In the final model, mood state variables were re-entered in order to evaluate the relative contributions of events, mood, and trait characteristics to cortisol levels.

Instead of a model with two levels, we could have formulated a three-level model, where measurements are nested within days, which in turn are nested within persons. However, when a three-level model was evaluated the p-values were approximately the same as the $p$-values of the two-level model. Because the more complex model did not change the conclusions, we decided to present the simpler model. The similarity of the results of the two models can be explained by the observation that the variance at the day level, although present, was small compared to the variance at the person and the measurement levels. Again, for the sake of simplicity, we did not extend the model with an autocorrelation term, since its inclusion did not change the model results.

\section{RESULTS}

\section{Characteristics of high and low stress groups}

High and low stress groups differed on almost all measures of stress and distress, as assessed with questionnaires and ESM reports (see Table 6.1.). Only the number of life events experienced in the past year did not differentiate the two groups. 
Table 6.1. Characteristics of high and low stress groups.

\begin{tabular}{|c|c|c|c|}
\hline & $\begin{array}{l}\text { LS } \\
\text { mean (st.dev.) }\end{array}$ & $\begin{array}{l}\text { HS } \\
\text { mean (st.dev.) }\end{array}$ & $\frac{D \text {-value }}{\text { (2-tailed) }}$ \\
\hline Total $\mathbf{n}$ & 46 & 42 & \\
\hline Age & $42.7(7.7)$ & $41.5(5.9)$ & ns \\
\hline \multicolumn{4}{|l|}{ Questionnaires } \\
\hline Perceived stress & $7.2(2.2)$ & $18.1(3.4)$ & $<.0001$ \\
\hline Trait anxiety & $28.3(4.4)$ & $39.8(7.6)$ & $<.0001$ \\
\hline Trait anger & $18.8(4.6)$ & $23.0 .(5.2)$ & $<.0001$ \\
\hline Depression & $36.5(5.5)$ & 48.4. (7.7) & $<.0001$ \\
\hline Psychosomatic symptoms & $6.1(5.6)$ & $27.5(23.2)$ & $<.0001$ \\
\hline Life events & $.5(.7)$ & $.8(.9)$ & ns \\
\hline Chronic difficulties & $19.5(2.3)$ & $23.3 \cdot(4.0)$ & $<.0001$ \\
\hline \multicolumn{4}{|l|}{ ESM reports* } \\
\hline Positive affect & $5.7(0.6)$ & 5.3. $(0.3)$ & $<.00 !$ \\
\hline Negative affect & $1.2(0.1)$ & $1.4(0.4)$ & $<.0001$ \\
\hline Agitation & $1.3(0.3)$ & 1.8. $(0.6)$ & $<.0001$ \\
\hline Frequency of stressful events & $12.3 \%(11.9)$ & $22.0 \%(20.0)$ & $<.01$ \\
\hline
\end{tabular}

- subject means, aggregated from 'beep' level data

\section{Controlling for diurnal and external influences on cortisol secretion}

Because the measurement level residuals in a model fitted with raw cortisol data were highly skewed to the right, with decreasing variance from morning to evening, data transformation was necessary to meet standard model assumptions. Log transformation, the usual remedy for cortisol skewness, resulted in increasing variance over the day. We therefore used a fifth root transformation (cortisol ${ }^{0.2}$ ), which gave normally distributed residuals with a homoscedastic pattern throughout the day.

The observed cortisol curve was best described by a two-piece third-degree polynomial (spline function (Smith, 1979)), with the node at $12.25 \mathrm{p} . \mathrm{m}$. The spline was created by adding a truncated term (defined as (TIME -12.25$)^{3}$ after $12.25 \mathrm{pm}$ and as zero before $12.25 \mathrm{pm}$ ) to a third-degree polynomial, which improved fit in the morning hours when cortisol levels dropped most sharply. Random terms were added. to allow each subject to have his own intercept and slope. Random terms for TIME ${ }^{2}$. TIME $^{3}$, and the truncated term did not change the magnitude of the fixed effects and were therefore excluded from the model for the sake of simplicity. All time effects (fixed and random) included in the model were highly significant. As shown in Figure 6.1 , the estimated contisol time curve closely approximates the observed mean values at the "beeps" and is clearly superior to a simple linear curve. Plots of the observed cortisol curve for each individual revealed no outlying curves; further inspection of the normal probability plots of individual cortisol means, slopes, and measurement level residuals, also indicated no significant violation of normality assumptions, supporting the appropriateness of the mixed effects approach.

The model was next extended with the possible confounders (alcohol, coffee, food intake, smoking, and physical exertion). Effects of any food intake and lunch were included separately (Follenius, Brandenberger, Hietter, Simeoni, \& Reinhardt, 1982; Quigley \& Yen, 1979). Maximum recent physical exertion was coded on a 7 point scale. The other variables were coded 1 if reported to have occurred in the interval since the previous 'beep' and 0 otherwise. Recent food, coffee, and alcohol 
intake were reported on $43 \%, 40 \%$, and $12 \%$ (respectively) of total ESM 'beeps', and recent smoking was reported on $16 \%$ of all 'beeps'. The median level of maximum physical exertion in the interval preceding a 'beep' was 3 , with levels of 6 or 7 reported on fewer than $3 \%$ of the total 'beeps'.

In the resulting multilevel model, recent smoking and food intake were associated with higher cortisol levels, with an additional positive effect of lunch on cortisol above food intake in general. No significant effects were found for coffee consumption, alcohol intake, or physical exertion. Model estimates for diurnal and external effects on salivary cortisol levels are shown in Table 6.2. (Column 1).

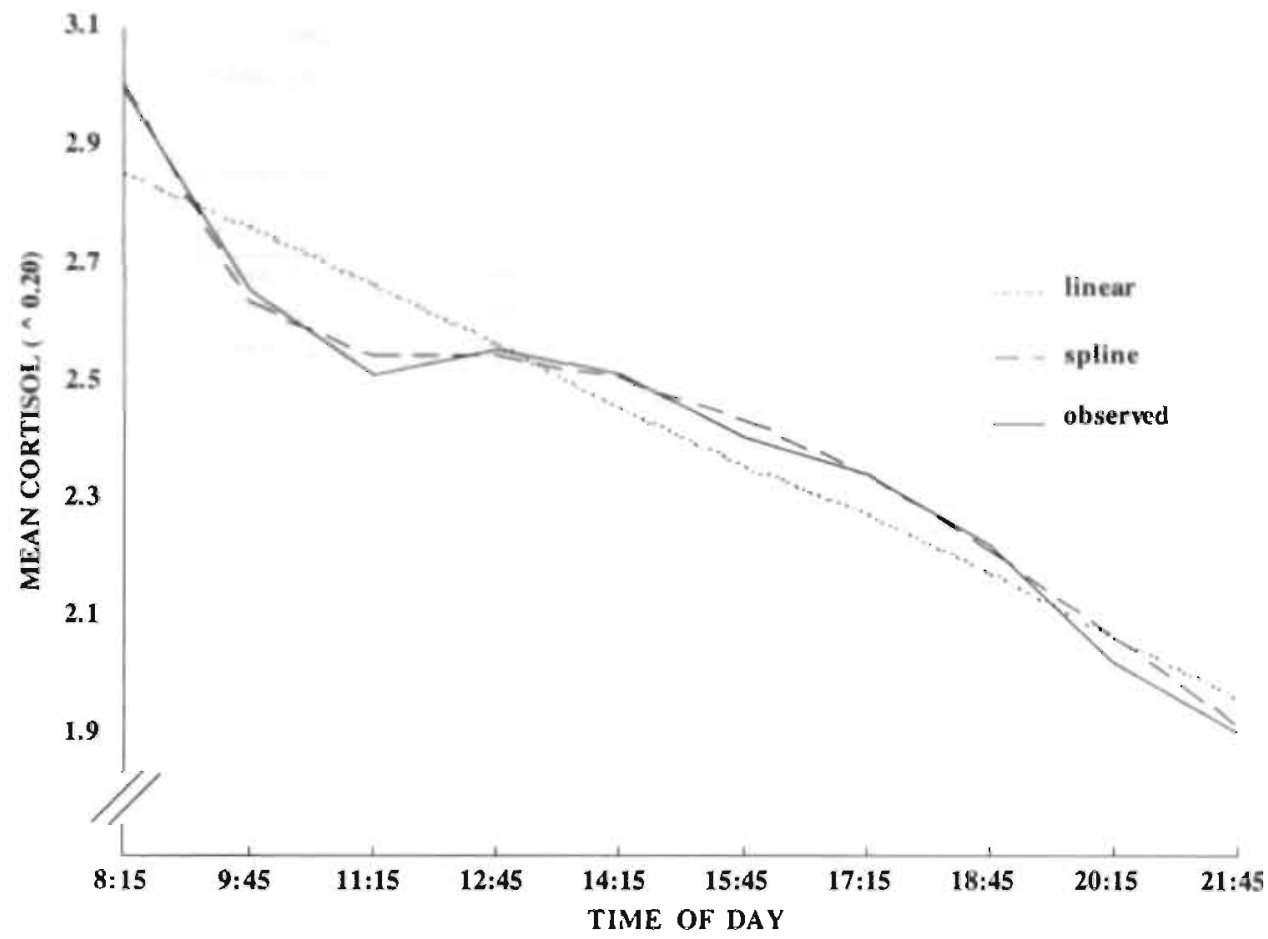

Figure 6.1. Observed and estimated cortisol curves.

Effects of individual characteristics and mood states on cortisol levels In the next model, we tested for effects of psychological trait and state variables on cortisol levels. Results are summarized in Table 6.2. (Column 2). Perceived Stress (PS Group, dummy-coded as 1 for HS and 0 for LS subjects) was entered into the model first, to test our hypothesis that high stress is associated with elevated cortisol. The effect of perceived stress, however, was not significant. The Perceived Stress by Time of Day (TIME) interaction term was also non-significant, indicating that the diurnal pattern of cortisol secretion was similar in HS and LS subjects. Adding Day Type (Work, coded as 1, vs Weekend, coded 0) and its interaction term with Perceived Stress did not improve the model; thus, contrary to expectation, cortisol levels were no higher on workdays than on weekends in either subject group. These variables were therefore excluded from the model. 
We next examined the effects of individual differences in trait anxiety, depression, anger, psychosomatic complaints, number of recent life events, and chronic difficulties. Anger, psychosomatic complaints, life events, and difficulties had no significant effects on cortisol. Trait anxiety and depression were each associated with significant elevations in cortisol levels.

Table 6.2. Effects of individual characteristics " mood states, and stressful daily events on cortisol levels.

The table summarizes the results of steps followed in the development of the full multilevel model. Model 1 provides estimates of diurnal and extraneous effects on cortisol. Model 2 adds estimates for trait characteristics and mood states. Model 3 shows the added contribution of stressful events (with mood effects excluded). In Model 4, all previously significant variables are included simultaneously.

\begin{tabular}{|c|c|c|c|c|}
\hline Fixed Effects ${ }^{\mathrm{a}}$ : & \multicolumn{4}{|c|}{ Estimate + (s.e.) } \\
\hline Interçept & $3.395(0.040) \cdots$ & $3.230(0.080) \cdots$ & $3.260(0.079) \cdots$ & $3.234(0.080)^{* \cdots}$ \\
\hline Time of day (TIME) ${ }^{b}$ & $.0 .577(0.036)^{\cdots}$ & $.0 .584(0.036)^{\cdots}$ & $-0.578(0.037)^{* *}$ & $-0.583(0.036)^{\cdots \cdots}$ \\
\hline TIME $^{2}$ & $0.128(0.010)^{\cdots}$ & $0.130(0.010)^{\cdots *}$ & $0.128(0.010)^{\cdots \cdot}$ & $0.129(0.010)^{\cdots}$ \\
\hline $\mathrm{TIME}^{3}$ & $-0.010(0.001)^{\cdots}$ & $-0.010(0.001)^{* \cdots}$ & $.0 .010(0.001)^{\cdots}$ & $-0.010(0.001)^{\cdots}$ \\
\hline Truncated function & $0.010(0.001) \cdots$ & $0.010(0.001)^{* \cdots}$ & $0.010(0.001)^{\cdots}$ & $0.010(0.001)^{\cdots}$ \\
\hline Food intake & $0.025(0.011)^{\cdots}$ & $0.027(0.010)^{* *}$ & $0.024 ;(0.011)^{\prime \prime}$ & $0.026(0.011)^{* *}$ \\
\hline Lunch & $0.037(0.019)^{\circ}$ & $0.037(0.019)^{*}$ & $0.043(0.019)^{2}$ & $0.043(0.019)^{*}$ \\
\hline Smoking. & $0.046(0.021)^{\circ}$ & $0.048(0.021)^{*}$ & $0.046(0.021)^{*}$ & $0.048(0.021)^{*}$ \\
\hline Trait anxiety & & $0.004(0.002)^{\circ}$ & $0.0039(0.002)^{\circ}$ & $0.004(0.002)^{*}$ \\
\hline Agitation & & $0.027(0.011)^{* *}$ & & $0.022(0.011)^{\prime \prime}$ \\
\hline Stressful event & & & $0.033(0.013)^{*}$ & $0.019(0.014)$ \\
\hline Ongoing event & & & $0.027(0.013)^{*}$ & $0.022(0.013)$ \\
\hline Prior frequency of event & & & $-0.072(0.030)^{*}$ & $-0.076(0.030)^{*}$ \\
\hline Prior frequency $\times$ trait an & & & $0.002(0.0008)^{*}$ & $0.002(0.001)^{\circ}$ \\
\hline
\end{tabular}

Random Effects: (Co)variance + (s.e.)

\begin{tabular}{|c|c|c|c|c|}
\hline \multicolumn{5}{|l|}{ Person level: } \\
\hline Var (Intercept) & $0.031(0.006)^{\cdots}$ & $0.039(0.009)^{2.0 .}$ & $0.031(0.006)^{n \ldots}$ & $0.037(0.009)^{\cdots}$ \\
\hline Var (Time) & $0.0002(0.0001)^{\cdots}$ & $0.0002(0.0001)^{* \cdots}$ & $0.0002(0.0001)^{* \cdots}$ & $0.0002(0.001)^{\cdots}$ \\
\hline Cov (Time, Intercept) & $.0 .001(0.0004)$ & $-0.001(0.0005)$ & $.0 .001(0.0004)$ & $-0.00 !(0.0004)$ \\
\hline \multicolumn{2}{|l|}{ Var (Agitation) } & \multicolumn{2}{|l|}{$0.003(0.001)^{*}$} & $0.003(0.001)^{\cdots *}$ \\
\hline \multicolumn{2}{|c|}{ Cov (Agitation, Intercept) } & \multicolumn{2}{|l|}{$-0.005(0.003)$} & $-0.004(0.003)$ \\
\hline \multicolumn{2}{|c|}{ Cov (Agitation, Time of day) } & \multicolumn{2}{|l|}{$0.0001(0.0002)$} & $0.0001(0.0002)$ \\
\hline \multicolumn{5}{|l|}{ Measurement level: } \\
\hline Var (Residual term) & $0.060(0.0015) \cdots$ & $0.058(0.0015)^{\cdots} \cdots$ & $0.059(0.0015)^{\cdots} \cdots$ & $0.058(0.0015)^{\cdots}$ \\
\hline \multicolumn{5}{|c|}{$\begin{array}{l}\text { Model } 1 \text { and Model 2:3157 observations nested within } 86 \text { subjects ( } 40 \mathrm{HS}, 46 \mathrm{LS} \text { ). } \\
\text { Model } 3 \text { and Model 4:3108 observations, including } 591 \text { stressful events, nested within } 86 \text { subjects ( } 40 \text { HS, } 46 \text { LS). }\end{array}$} \\
\hline \multicolumn{5}{|c|}{$\begin{array}{l}\text { a Non-significant fixed effects were excluded from the model. Model 1: Coffee intake. Alcohol intake, Physical } \\
\text { exertion. Model 2: PS Group, PS Group by Time of day. Day type, PS Group by Day type, Trait anger. Psychosomatic } \\
\text { symptoms, Life-events, Chronic difficulties. Positive affect. Model 3: Event content - Work. Social interaction, Task } \\
\text { demand-; Event appraisal - Unpleasantness. Predictability, Importance. Controllability -- Event by PS Group. Event by } \\
\text { Trait anxiety, Event by Depression. Model 4: Positive affect. Event by Agitation, Event by Negative affect. }\end{array}$} \\
\hline
\end{tabular}


Due to multicollinearity, it was necessary to add these variables separately to the model. In Table 6.2 (Column 2), the effect of trait anxiety is shown. The effect of depression, when entered separately, was of a similar magnitude (estimate Depression: $0.003, \mathrm{p}=.05$ ).

To test the association between mood state and cortisol levels, the variables Positive Affect, Negative Affect and Agitation were next added to the model. Positive Affect was not significantly related to cortisol. In contrast, both Negative Affect and Agitation were associated with higher cortisol. Because Negative Affect and Agitation were intercorrelated ( $r=0.51 ; p<.0001$, over 3569 reports), the effect of Negative Affect was masked when entered simultaneously with Agitation into the equation. When entered separately, standardized effects were approximately the same for both variables. In Table 6.2. (Column 2), the effect of Agitation is shown (estimate Negative Affect: $0.032, \mathrm{p}<.01$ ).

Finally, random effects for Negative Affect and Agitation were included in the model. The random effect for Agitation was significant, meaning that the effect of Agitation on cortisol varied across persons. In Figure 6.2., the individual estimates of the effect of Agitation on cortisol are plotted for HS and LS subjects. Here, it is evident that some individuals displayed a considerably closer relationship between cortisol and Agitation than others, but that these individual differences were not associated with perceived stress level. 


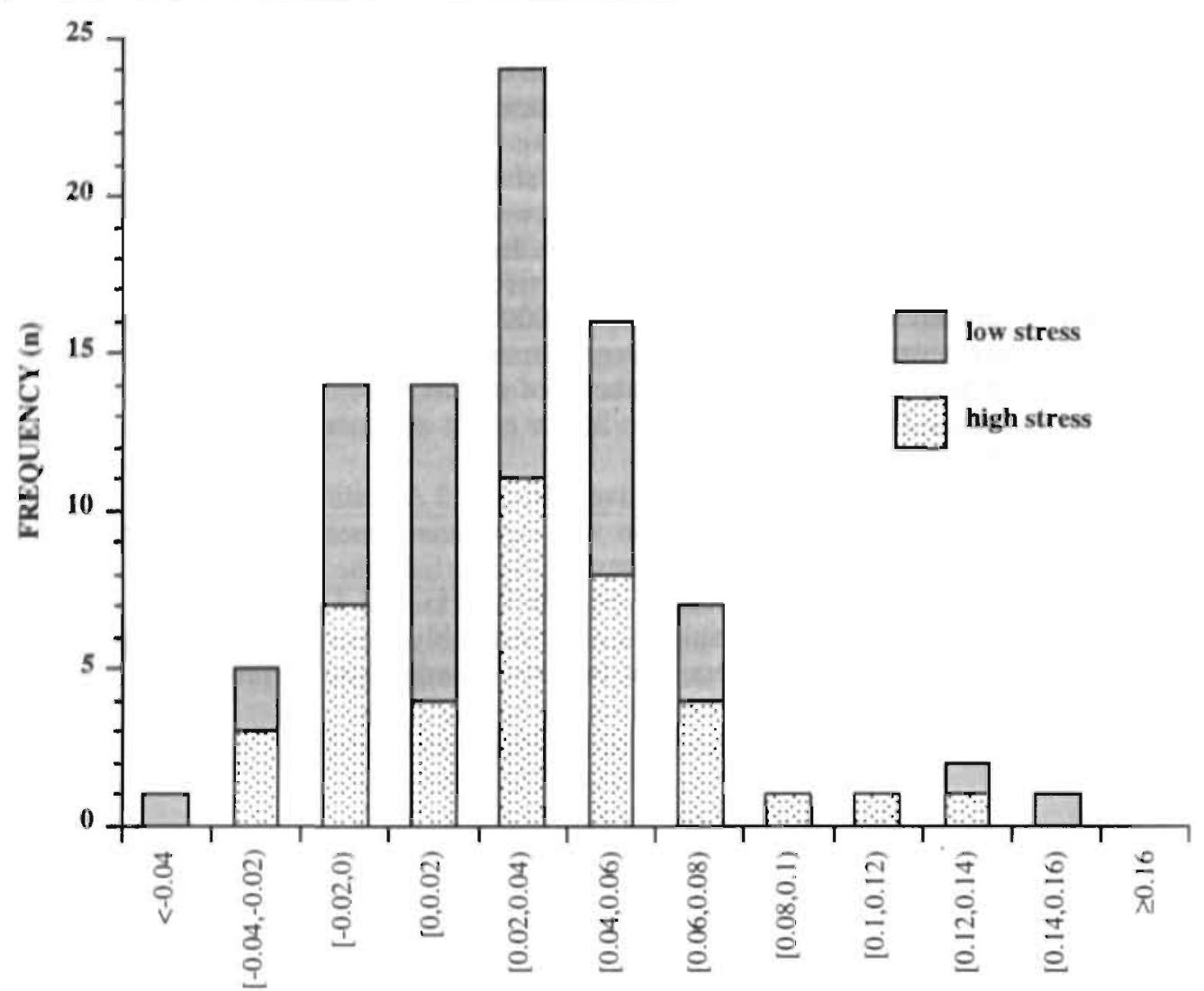

INDIVIDUAL ESTIMATES (SLOPES) OF AGITATION EFFECTS

Figure 6.2. Distribution of the individual estimates of the effects of the mood state Agitation on cortisol for HS and LS subjects. These estimates were calculated by an empirical Bayes approach, using multilevel analyses (see Method section).

To provide a clearer picture of effect magnitudes, the estimate for each variable can be translated back into the original measurement unit. A standard deviation increase in trait anxiety, for example, was associated with a cortisol increase of 5.4 $\mathrm{ng} / \mathrm{dl}$ above the mean level of $81.3 \mathrm{ng} / \mathrm{dl}$. Regarding Agitation, the mood state effect estimate shown in Table 6.2. (Column 2) indicates the increase in transformed cortisol associated with a one unit increase on the mood scale (from 1 to 2 on the scale ranging from 1 to 7). Translated back into raw cortisol, this one unit increase was associated with a cortisol increase of $4.7 \mathrm{ng} / \mathrm{dl}$ above the mean cortisol level. When a subject scores, for instance, 5 on the negative mood scale, the mood estimate (see Table 6.2., Column 2) should be multiplied by 4, resulting in a higher cortisol level. A 4 unit increase on the mood scale was associated with a cortisol increase of $20 \mathrm{ng} / \mathrm{dl}$ above the mean cortisol level. 


\section{Reactivity of cortisol to stressful events}

In the third model, the occurrence of a stressful event since the last ESM report (Event, dummy-coded as $\mathrm{I}$ or 0 ) and variables representing the coded content of the event (work-related or not, social interaction or not, task demand or not; all coded as I or -1 ) and rated characteristics of the event were added to the model. ${ }^{5}$ Results are summarized in Table 6.2. (Column 3). Cortisol levels were significantly elevated following stressful daily events. No random effect for Event was found, meaning that the influence of a stressful event on cortisol did not differ significantly between subjects. Although most of the Event effect was determined by HS subjects, level of perceived stress did not have a significant effect on cortisol reactivity to stressful events. Next, we controlled for the time-frame of the event: was the event already finished when a subject took a saliva sample ( $71 \%$ of all events), or was it still going on ( $29 \%$ of events) ${ }^{6}$ When a stressful event was ongoing at the time the ESM report was completed, the level of cortisol was significantly higher than if the event had already ended. Translated back into the original units, ongoing events were associated with mean cortisol increases of $10.6 \mathrm{ng} / \mathrm{dl}$ and terminated events with increases of $5.7 \mathrm{ng} / \mathrm{dl}$, relative to no event.

The various event content categories (work vs non-work events, social interactions vs non-social events and task demands vs non-task demands) were not significantly associated with cortisol. Contrary to expectation, event unpleasantness, importance, predictability, and controllability contributed no additional effects. Prior frequency did have an independent effect: when similar kinds of events were reported to have occurred relatively frequently in the past, the cortisol response to the current event was lower. Here, significant interactions with trait anxiety were found (see Table 6.2. Column 3). Significant interactions were also found for depression and perceived stress (when entered separately to the model, replacing trait anxiety): cortisol levels were less likely to show this apparent habituation effect to recurrent events in individuals scoring high on these traits (estimate Prior frequency * Depression: $0.0017, \mathrm{p}<.01$; estimate Prior frequency * PSS: $0.033, \mathrm{p}<.01$ ).

\section{Relationship between cortisol reactivity to events and mood state}

In our final model, we examined whether mood states mediated the cortisol response to stressful daily events. Positive Affect had no effect on cortisol and was therefore excluded from the model. When entered separately into the model, the two negative mood states, Negative Affect and Agitation, were each positively related to cortisol. With Agitation in the model, the effect of stressful event occurrence on cortisol was lowered to a non-significant level. A similar pattern was observed for Negative Affect: the effect of Event was reduced (although in this case it remained significant) after addition of Negative Affect to the model. This indicates that negative mood states were stronger predictors of cortisol level than stressful events. Finally, the lack of significant interactions between the two negative mood states and Event indicates that mood effects on cortisol were no greater in the presence than in the absence of an Event. Table 6.2. (Column 4) summarizes model parameters, with

5 For each rated characteristic of the event, the subject's mean was subtracted from the actual rating, thus keeping the Event effect unchanged; this allowed us to assess the withiri-person effects of the various event appraisals above and beyond the effect of the event itself.

6 First coded -0.5 (event finished), 0 (no event), or 0.5 (ongoing event), and then weighted for the number of events that were 'finished' compared to the number of events 'ongoing' in order to keep the effect of Event unchanged after addition. 
Agitation included. Similar results were obtained when Negative Affect was included instead of Agitation (estimate Negative Affect: 0.024, p<.05).

\section{DISCUSSION}

\section{Etfects of tralt measures of stress and distress on salivary cortisol}

The current study provides evidence that trait negative affectivity can result in moderate cortisol elevation. Trait anxiety and depression showed small but statistically significant positive associations with cortisol. To date, evidence for elevated cortisol in association with anxiety and depression has come mainly from studies of more severely or chronically distressed subjects (Rahe et al., 1990; Schaeffer \& Baum, 1984) or psychiatric patients (Linkowski et al., 1985), while several studies of healthy adults have failed to demonstrate an association between basal cortisol and these or other trait measures (Brandtstädter et al., 1991; Ockenfels, Porter, Smyth, Kirschbaum, Hellhammer, \& Stone, 1995). It should be noted that subjects in the current study, having been selected on the basis of extreme perceived stress scores, displayed a broader distribution of depression and anxiety scores than would have been likely in an unselected sample of white collar workers. Moreover, the large number of cortisol samples collected per subject at different times of day provided more reliable estimates of hormone levels than is usually the case. Anxiety and depression were highly correlated in this sample, which made it impossible to assess the relative contributions of each. Further research is necessary to clarify this issue.

We had hypothesized that cortisol levels would be elevated in subjects with high perceived stress (see Introduction, Question 1). A repeated measures analysis of variance of a subset of these data (van Eck \& Nicolson, 1994), with cortisol values aggregated over each subject and time of day, had shown higher cortisol levels in HS compared to LS subjects when only the three workdays were included. Given the lack of a statistically significant effect of PSS on cortisol in the current multilevel analysis, however, we conclude that perceived stress is not a strong predictor of cortisol secretion, at least in this sample. Nor did we find significant interaction effects between PSS and the time of the day that would support a stress-related disruption in the circadian rhythm of secretion, as has been suggested by other authors (Caplan et al. 1979; Ockenfels et al. 1995). Additional measures of life stress, including an objective life event measure as well as a measure of chronic difficulties over a wide range of life domains, similarly showed no statistically significant associations with cortisol. The intercorrelations among the various measures of stress and distress argue for cautious conclusions about the relative influence of each on cortisol levels. The apparently disparate results of a recent study (Ockenfels et al., 1995), which showed an effect of perceived stress, but not of anxiety or depression, on cortisol levels should be seen in this light.

\section{Effects of state affect measures and stressful daily events}

The two negative mood states, Agitation and Negative Affect, were both associated with higher cortisol levels. The estimated effects were relatively small: a one unit increase in Agitation (from 1 to 2 on a scale ranging from 1 to 7 ) was associated with a cortisol increase of $4.7 \mathrm{ng} / \mathrm{dl}$ above the mean level of $81.3 \mathrm{ng} / \mathrm{dl}$. At more extreme scores on the mood scale, however, cortisol increases were more pronounced. Highly negative states were reported infrequently in daily life contexts 
by both HS or LS subjects, resulting in highly skewed distributions and small standard deviations (Agitation was scored as 1 'not at all' on $56 \%$ of all 'beeps', Negative Affect on $51 \%$ of all 'beeps') (see also Table 6.1). The low mean levels of negative states reported by both HS and LS subjects are characteristic of normal samples, with men tending to report even lower, less extreme levels of negative emotions as well as positive emotions than women (Cameron, 1975; Diener et al., 1985).

Positive mood showed no association with cortisol. The results of previous studies are inconsistent with respect to the effects of positive mood states: in some cases, higher positive affect was accompanied by lower cortisol (Hubert \& de JongMeyer, 1990; Hubert \& de Jong-Meyer, 1991), in others, by higher cortisol (Brown et al., 1993; Hubert, Möller, \& de Jong-Meyer, 1993). One of the few naturalistic studies revealed an inverse relationship between positive mood states and cortisol levels (Kugler \& Kalveram, 1989), but this analysis, by aggregating mood states for each subject, tested only between-subject and not within-subject associations. In the present study, the Positive Affect scale included both high (e.g. 'energetic') and low arousal (e.g. "relaxed') items. If, as might be expected on theoretical grounds (Hennessy \& Levine, 1979), high arousal states are associated with elevated cortisol levels and low arousal states with lower cortisol, including both types of items in one scale could cancel out any effect. This possibility should be addressed in future studies of mood effects on cortisol.

Daily events or situations experienced as stressful were associated with increased cortisol secretion (see Introduction, Question 2). Surprisingly, the rated unpleasantness of an event had no additional effect, nor did other appraisal measures. We had expected that events involving negative social interactions might have more pronounced effects on cortisol, but categorization of events on this dimension, or on relationship to work, or task demands, did not improve the model. Significant effects may be difficult to find because of the heterogeneity of responses resulting from the variety of stressful events encountered. Responses will be modulated by all kinds of objective and subjective event characteristics as well as by their interactions with each other.

We did find a significant effect for the time course of an event, with ongoing events having the expected greater effect on cortisol than terminated events. This is in line with temporal patterns of cortisol excretion observed in response to laboratory stressors; after termination of stress exposure, cortisol levels typically return to baseline within 1-2 hours (Kirschbaum \& Hellhammer, 1989). In addition, events that were reported to be recurrent had a smaller effect on cortisol than more novel events, suggesting that habituation can occur. Although perceived stress, trait anxiety, and depression were not significantly associated with elevated cortisol reactivity to stressful events, cortisol habituation to recurrent daily stressors did appear to be less likely to occur in individuals scoring high on these measures. A similar pattern was observed in a recent laboratory study, in which subjects were asked to perform the same stressful task on $\mathrm{S}$ consecutive days. Here, the majority showed a significant cortisol response on the first day only, while a sub-group of individuals with low selfesteem, depressed mood, and physical symptoms showed persistent cortisol responses to the task over all exposures (Kirschbaum, Pruessner, Stone, Federenko, Gaab, Lintz, et al., 1995).

We found no statistically significant individual differences. ("random effects" in the model) in cortisol reactivity to events. While laboratory studies of responses to a battery of stress tasks provide some evidence for individual differences in reactivity 
(Forsman \& Lundberg, 1982), such differences appear to be less stable than differences in basal levels, which have a larger genetic component (Kirschbaum, Wust, Faig, \& Hellhammer, 1992b). It is possible that either the total sample size was too small or that the within-subject samples of events were too heterogeneous in the current study to reveal consistent and reliable individual response patterns.

The effect of stressful events on cortisol appears to be mediated to a large extent by associated increases in negative mood, since addition of Agitation or (to a lesser extent) Negative Affect to the model resulted in a decrease in the Event effect to a non-significant level. This interpretation is consistent with most views of the stress process and is supported by unpublished results from the same study, showing that both Negative Affect and Agitation increase following stressful events. More detailed temporal analyses of these data may help clarify whether an emotional response to an event is necessary to trigger a hormonal response.

\section{LImitations and conclusions}

For practical reasons, the study focused on a relatively homogeneous group of male white collar employees. We are not able to assess the extent to which the results are generalizable to other populations. Given the evidence for sex differences in neuroendocrine reactivity to psychosocial stress (Collins \& Frankenhaeuser, 1978; Kirschbaum, Wust, \& Hellhammer, 1992c), a replication of this study with inclusion of female subjects would be informative. Another limitation is that we did not assess positive daily events, which could theoretically affect cortisol levels via their influences on mood (Stone, 1987), possibly mitigating the effects of negative events. However, since positive events have their greatest effect on positive affect (Stone, 1987), it is important to recall that Positive Affect was not associated with cortisol in the present study.

In conclusion, this study suggests that neuroticism is not simply a confounder in psychosomatic research (McCrae, 1990), but is associated with increased adrenocortical activity during normal daily activities. Self-report measures of anxiety and depression, negative mood states and daily stressors, all known to be strongly related to trait negative affectivity (Watson \& Pennebaker, 1989), were associated not only with increased perceptions of chronic stress, but also with elevated cortisol levels. The finding that even minor everyday events and fluctuations in mood states have an impact on cortisol secretion points to a possible mechanism linking subjective experience to health outcomes. Further research is needed to determine whether mild, chronic cortisol elevation contributes to disease processes in somatic illness or psychiatric disorder, in particular depression.

\section{ACKNOWLEDGEMENTS}

We thank C. Dijkman for assistance in all aspects of the study. We also thank Prof. T.A.B. Snijders and four anonymous reviewers for substantial comments on earlier versions. 


\section{Chapter 7}

\section{Individual differences in cortisol responses to a laboratory speech task and their relationship to responses to stressful daily events}

Biological Psychology 43(1): 69-84 (1996)

Marleen van Eck ${ }^{1}$, Nancy Nicolson ${ }^{1}$, Hans Berkhof ${ }^{2}$, Jose Sulon ${ }^{3}$

Department of Psychiatry \& Neuropsychology, Social Psychiatry and

Psychiatric Epidemiology section, University of Limburg, the Netherlands

2 Department of Statistics and Measurement Theory, University of Groningen, the Netherlands

3 Faculty of Veterinary Medicine, University of Liege, Belgium 

A Stress Inducing Speech Task (SIST) was used to investigate the contribution of perceived stress, individual traits, and current mood states to individual differences in salivary cortisol responses. Additionally, we examined the correspondence between laboratory baseline cortisol levels and overall levels in daily life, and between cortisol responses to the SIST and a measure of stress reactivity to stressful events in daily life. Forty-two 'high stress' and forty-five 'low stress' whitecollar males completed the SIST and an Experience Sampling study, in which stressful daily events and cortisol levels were monitored for five days. No association was found between perceived stress, trait anxiety, anger, depression, psychosomatic symptoms, coping style or personality and cortisol responses to the SIST. Negative mood state at baseline was associated with higher cortisol levels at baseline, just before, and just after the speech. Laboratory and field cortisol levels were moderately correlated, but no association was found between laboratory and field response measures. Laboratory baseline levels, but not responses to the speech task, were significant predictors of field cortisol levels.

\section{INTRODUCTION}

Changes in cortisol levels have been shown to be sensitive indicators of psychosocial stress and coping patterns in both laboratory and natural situations (Frankenhaeuser, 1986; Mason, 1968; Rose, 1984). Although adrenocortical responses to psychological stress have been found to differ between individuals, sources of these differences are far from clear. For a better understanding of the potential link between cortisol responses and disease (Bassett, 1982; Cohen et al., 1991; Gaillard \& Al-Damluji, 1987; Troxler et al., 1977), more insight into individual differences in cortisol reactivity is needed. Since it is reasonable to assume that stress reactions will only lead to disease when they are prolonged or occur very often, it is likely that the pathogenic influence of cortisol hyperreactivity (or hyporeactivity) depends on whether this reflects a stable individual characteristic. Evidence from animal studies indicates that cortisol hyporesponders may be more prone to autoimmune disorders (Sternberg et al., 1989), while cortisol hyperresponders might be more vulnerable to infectious diseases (Mason, 1991). Research on psychological stress and cortisol indicates that the subjective appraisal of apprehension and emotional involvement can lead to increased cortisol secretion (Kirschbaum \& Hellhammer, 1989; Mason, 1975; Rose, 1984). Personality and other traits may influence how stressful situations are appraised and may thus have predictive value for understanding individual differences in emotional and physiological responses to apparently identical situations (Arnetz \& Fjellner, 1986; Vaernes et al., 1982).

The goals of the current study were twofold. Our first objective was to determine whether perceived stress level (as a measure of mild chronic stress) was related to emotional and cortisol responses to an experimental stressor. From existing data, it is not clear whether chĩonic stress affects an individual's pattern of response to acute stressors. There is some evidence for an attenuated cortisol response in chronically stressed human subjects (Bourne et al., 1967; Friedman, Mason, \& Hamburg, 1963) but these studies focused on responses to highly traumatic situations (e.g., acute medical complication in a fatally ill family member, combat exposure). Experiments in rats indicate that the effects of chronic or repeated stress exposure on later glucocorticoid responses depend to a large extent on stressor intensity (Ottenweller, Natelson, Pitman, \& Drastal, 1989). 
There is considerable overlap between perceived stress and other subjective measures of distress, negative affectivity, and ineffective coping. For this reason, we also investigated the contribution of trait anxiety, depression, psychosomatic symptoms, anger, coping style and personality to individual differences in cortisol response. Such traits have been associated with basal cortisol levels (Brandtstädter et al., 1991; Pope \& Smith, 1991; Schaeffer \& Baum, 1984) as well as with stress responses (Bohnen et al., 1991; Demyttenaere et al., 1989; Kirschbaum et al., 1989; Nicolson et al., 1992) in a number of previous studies. Other studies, however, have found no association between personality traits, coping styles, and cortisol responsiveness to laboratory stressors (Bossert et al., 1988; Kirschbaum et al., 1992a).

As noted above, individual characteristics are thought to be important determinants of the emotional response to a given situation; mood states are, in turn, likely to mediate the endocrine response to the situation. Although there is abundant evidence that cortisol increases in response to distress or negative mood (Arnetz \& Fjellner, 1986; Lundberg \& Frankenhaeuser, 1980; Mason, 1968; Nicolson, 1992), the effects of positive mood are less clear. Positive affective states have been associated with decreases (Hubert \& de Jong-Meyer, 1989; Kugler \& Kalveram, 1989) as well as increases (Brown et al., 1993) in cortisol levels.

Our second objective was to compare cortisol responses measured in the laboratory with those measured in real life. As Turner, Ward, Gellman, Johnston, Light, \& van Doornen (Turner et al., 1994) have stated: "if exaggerated responses do play a role in disease development, it is via everyday challenging situations that they will exert their pathogenic influence. The usefulness of laboratory assessment rests, in part, on the assumption that laboratory responses reflect those occurring during natural situations". The widespread availability of ambulatory monitoring techniques has stimulated research on the relationship between laboratory and daily life cardiovascular activity. Evidence from several studies indicates that blood pressure levels obtained in the field are better predictors of subsequent hypertension, complications from hypertension and mortality than measurements obtained in the doctor's office (Devereux, Pickering, Harshfield, 1983; Perloff, Sokolow, \& Cowan, 1983). A recent review (Turner et al., 1994) concluded that moderate evidence exists for laboratory-field generalization of cardiovascular activity. The evidence differs in strength, however, for different types of laboratory-field associations. The associations between basal, chronic levels of cardiovascular activity across settings are the strongest, while inconsistent support has been found for the relationship between laboratory reactivity and field reactivity, and between laboratory reactivity and field levels.

Especially with regard to endocrine reactivity, the generalizability of laboratory studies to field studies has been underexplored. In a study of plasma catecholamine reactivity in a small sample of healthy young men (Dimsdale, 1984), no correlation was observed between endocrine responses to laboratory mental arithmetic and those to real-life public speaking. Although van Doornen and Blokland (1992) observed a correlation between pre-task adrenaline levels in the laboratory (reaction time and tracking task; cold pressor test) and the level preceding a real-life siressor (public defense of $\mathrm{PhD}$ thesis), urinary catecholamine responses to the laboratory tasks did not correlate with responses to the field stressor. To our knowledge, only one siudy has investigated laboratory-field associations of cortisol excretion patterns (Houtman \& Bakker, 1987). In a sample of nine student teachers, saliva cortisol responses were compared in a real and a standardized lecturing situation. Most correlations were low and nonsignificant, but this may have been due 
to the small sample size and the poor standardization of time at which the subjects started lecturing in the real life situation.

In the current study, a Stress Inducing Speech Task (Steiner \& Levine, 1988) was employed to investigate trait and state correlates of salivary cortisol responses to stress. Although studies of psychophysiological reactivity often employ standardized laboratory tasks such as mental arithmetic, computer games or cold pressor tests, one can question whether the reactions observed are relevant or valid reflections of habitual reactivity to naturally occurring psychosocial stressors. Speech tasks have a greater ecological validity and reliably induce endocrine and cardiovascular responses as well as moderate distress (Bassett et al., 1987; Dimsdale, 1984; Kirschbaum \& Hellhammer, 1993; Steiner \& Levine, 1988). In addition to the laboratory experiment, subjects participated in a naturalistic field study of daily stress and cortisol dynamics. This allowed us to evaluate the consistency of laboratory and field cortisol measures, particularly with regard to the usefulness of the laboratory measures in predicting cortisol levels and responses to naturally occurring stressors in the field situation.

\section{METHODS}

\section{Subjects}

Local industries and government agencies were approached via their personnel departments. 316 male white collar workers from six different industries or agencies completed the screening questionnaire. The mean score on the Perceived Stress Scale (PSS) for this sample was 12.7 (s.d. 6.0), similar to US. norms (mean 13.02, s.d. 6.45) (Cohen et al., 1983). Individuals scoring in either the upper or the lower tertile of the screening sample PSS distribution (PSS-10 score $\leq 10$ or $\geq 16$ ), and with no history of serious chronic illness, endocrine disorder, or medications known to affect cortisol levels (as ascertained by self-report) were later approached to participate in the main study, until 92 subjects, balanced for 'low' and 'high' stress states, were recruited ( $85 \%$ of those approached). Of the 92 subjects who took part in the study, 5 were excluded from the analysis due to missing data. The final sample included 42 subjects in the 'high stress' (HS) group and 45 subjects in the 'low stress' (LS) group. Mean age was 42.1 years (range 27 to 57 years): $89 \%$ were married, and $81 \%$ had children living at home.

\section{Experimental stressor: Stress Inducing Speech Task (SIST)}

The SIST took place between 11 am and 1 pm (before lunch), one or two days after subjects completed participation in an Experience Sampling study (see below). On arrival, subjects were unaware that they would be asked to deliver a speech. After completing baseline measures, subjects were asked to prepare ( 10 minutes) and present (5 minutes) a videotaped speech describing their personal strengths and weaknesses for later evaluation by a team of psychologists. Following the preparation period. subjects, received a signal to begin their presentation while looking directly into the camera. After the speech, subjects relaxed for 15 minutes in neutral activities (e.g. reading magazines). At four timepoints subjects filled in a mood questionnaire and provided a saliva sample: (T1) upon arrival, (T2) after the 10 minutes of preparation, (T3) after the 5-minute presentation, and (T4) after 15 minutes of relaxation. During the course of the study, the recovery period was lengthened to provide a clearer picture of the cortisoll response profile; for 49 subjects a fifth saliva sample (T5) was taken on average 50 minutes after the first assessment.

The mood questionnaire consisted of 15 five-point Likert scale items (Folkman \& Lazarus, 1985). Subjects indicated to what extent $(0=$ not at all; $5=$ a great deal $)$ they felt: 
worried, anxious, nervous, tense, confident, hopeful, eager, irritated, sad, disappointed, guilty, exhilarated, pleased, happy and relieved. The 15 mood items formed two distinct dimensions: Negative Affect (NA, 8 items) and Positive Affect (PA, 7 items), with Cronbach's alpha's at T1 of .79 for NA and .75 for PA.

\section{Fleld study: cortisol dynamics In daily life}

The Experience Sampling Method (ESM) (Csikszentmihalyi \& Larson, 1987; de Vries, 1992) was used to collect data from subjects at selected moments during their normal daily activities. Subjects received auditory signals ('beeps') to complete a questionnaire and a saliva sample. Subjects were sampled for a period of 5 consecutive days ( 3 work and 2 non-work days), 10 times each day, at semi-random intervals of approximately 90 minutes between the hours of 8 am and $10 \mathrm{pm}$. Compliance with the procedures was generally good: $83 \%$ of all possible responses were completed within the time limit of 20 minutes. For a more detailed description of the Experience Sampling Method procedure and compliance issues, see van Eck \& Nicolson (1994).

In addition to items concerning current mood, activity, social and physical context, the ESM form contained a 7-point Likert scale (from 1 'not at all stressful' to 7 'very stressful') for the rating of any stressful event or situation which had taken place in the interval since the last ESM report. Subjects reported a total of 626 stressful events during the five days of ESM, with a mean of 7.1 events per subject (median 5.0; st.dev. 7.3). Events were reported on an average of $16.8 \%$ (st.dev. 16.5) of total "beeps". Information concerning smoking, food, coffee, and alcohol intake, and maximum level of physical exertion since the last beep was also obtained.

\section{Paychological moasures}

The following measures were used in the current analysis:

Perceived Stress: The 10-item version of the PSS was translated into Dutch. The PSS is a global measure of the degree to which situations in one's life are appraised as stressful. Items tap the extent to which individuals feel their life to be unpredictable, uncontrollable, and overloaded. The items were rated on a 5-point frequency scale, ranging from 'never' $(0)$ to 'very often' (4) in the last month. All subjects completed the PSS twice, during the initial screening and again immediately preceding Experience Sampling. The two PSS scores were highly correlated (rho=73, $<<.001$ ). The mean of the two PSS scores was 7.2 (st.dev. 2.2) for the low stress group and 18.1 (st.dev. 3.4) for the high stress group.

Psychosomatic symproms: The revised version of the SUNYA Psychosomatic Symptom Checklist (PSC; Attanasio et al., 1984) includes 17 common psychosomatic complaints (e.g., headaches, backaches and nausea). Subjects rated each complaint on 5-point scales for frequency ( 0 'never or rarely occurs' io 4 "occurs daily') and intensity ( 0 'not bothersome' to 4 'extremely bothersome'). A Total Score is obtained by summing the cross-products of each item's frequency by intensity.

Depression: Depressive symptomatology was assessed with the Dutch translation (Dijkstra, 1974) of the Zung Self-Rating Depression Scale (Zung, 1965).

Anxiery: Trait anxiety was measured with the Dutch version (van der Ploeg et al., 1980) of the State-Trait Anxiety Inventor (STAI).

Anger: Trait anger was measured with the Dutch version (van der Ploeg et al., 1982) of the Spielberger Trait Anger Scale. The scale has two subscales: 'anger-temperament' and 'angerreaction :

Personality: Personality characteristics were assessed with the Dutch abridged MMPI (NVM: Luteijn \& Kok, 1985), which consists of five scales: Negativism, Somatization, Timidity, Major Psychopathology, and Extraversion. 
Coping: Coping style was assessed with the 47-item Utrecht Coping List (UCL; Schreurs \& van de Willige, 1988). The seven factor-analytically derived subscales are: 'active problem solving', 'palliative responding', 'avoidance', 'seek social support', 'depressive reaction", 'expression of emotions' and 'comforting cognitions'.

HS subjects scored significantly higher than LS subjects on psychosomatic symptoms ( 27.5 vs $6.1, \mathrm{p}<.001)$, depression $(48.4$ vs $36.5, \mathrm{p}<.001)$, anxiety $(39.8$ vs $28.3, \mathrm{p}<.001)$, anger (18.0 vs 14.6, $\mathrm{p}<.001$ ), the personality scales negativism ( 19.0 vs $10.0, \mathrm{p}<.001)$, somatization $(9.5$ vs $2.8, \mathrm{p}<.001)$, timidity $(11.3$ vs $8.3, \mathrm{p}<.05)$, and psychopathology $(2.8$ vs $1.3, \mathrm{p}<.01)$, and on the coping styles active coping (17.3 vs $21.1, \mathrm{p}<.001)$, palliative reaction (17.2 vs 14.6. $\mathrm{p}<.001$ ), depressive reaction (12.6 vs 8.8, $p<.001$ ), and expressed emotions (6.9 vs 5.8, p<.01). Where possible, mean scores for both samples were compared to published norms for the Dutch population. The general pattern found was that LS subjects scored average or below average compared to the norm scales, while HS subjects scored above average or high. No significant differences between HS and LS groups were found for the personality scale extraversion (16.5 vs $15.4)$ and the coping styles avoidance (15.1 vs 14.1), seek social support (12.5 vs 11.7), and comforting cognitions (11.7 vs 10.8 ).

\section{Saliva sampling and biochemical analysis}

Subjects collected saliva by holding a cotton dental roll in the mouth for approximately 1 minute. The saturated roll was placed in a capped plastic vial ("Salivette", Sarstedt). Uncentrifuged samples were stored at -20 degrees $C$, until analyses. Salivary cortisol levels were determined in duplicate by direct radioimmunoassay (Ansseau, Papart, Pitchot, Timsit-Berthier, Legros, \& von Frenckel, 1992), using ${ }^{125} \mathrm{I}$-cortisol and antiserum made against the 3-CMO-BSA conjugate. The lower detection limit of the assay was $12 \mathrm{ng} / \mathrm{dl}$, with a mean intra-assay coefficient of variation of $4.8 \%$ (range: $2.2 \%-7.5 \%$ for 4 assays).

\section{Statistical analysis}

A fifth root transformation of the raw cortisol data (cortisol ${ }^{0.2}$ ) was used to obtain normally distributed residuals throughout the day in the cortisol field data. For comparability, the same transformation was applied to the laboratory cortisol data. Multivariate analysis of variance for repeated measures (MANOVA procedure, SPSS) was performed to test for effects on cortiso! levels during the SIST; in addition to the repeated measures factor Time (TI through T4 and, for a subsample, T5), Group (PSS high versus low) and Group x Time interaction effects were tested. Baseline cortisol was included as a covariate. A separate MANOVA analyses was repeated on the mood measures. Significance levels are based on multivariate (F) tests. Forward linear stepwise regression analyses were performed to estimate the proportion of variance in cortisol (dependent variable) explained by various (independent) person variables.

Cortisol responses to the SIST were computed as area-under-the-concentration-time curve (AUC), using the trapezoidal integration. The value of the laboratory sample at TI is reported as laboratory baseline (L.BASAL). L.RESPONSE was defined as the portion of the AUC above L.BASAL. Because the SIST always took place between 11.00 a.m. and 1.00 p.m., prior to lunch, field cortisol level (F.BASAL) was defined as the mean level over 5 days with the third ESM beep (at approximately $11.15 \mathrm{a.m}$.) as our reference point. The mean (within-subject) coefficient of variation for the five cortisol values was $4.1 \%$, ranging from $1.5 \%$ to $7.2 \%$.

The model used for the analysis of the cortisol field data has been described in detail elsewhere (van Eck, Berkhof, Nicolson, \& Sulon, in press). The multilevel model is a variant of the multiple linear regression model applicable for data with a hierarchical nesting structure (here: repeated measurements within persons), where the dependent variable (here: cortisol) is defined at the lowest level of the hierarchy. In this model, field cortisol level (F.LEVEL) was defined as the 
mean cortisol level over the five days in the field after controlling for diurnal patterns and the other factors (smoking, lunch and other food intake) found to have significant effects.

Individual cortisol response estimates to stressful events in daily life were calculated by an empirical Bayes, approach, using multilevel analyses (Bryk \& Raudenbush, 1992). These response estimates are weighted averages of an overall regression estimate (based on the whole data set) and a person-specific regression estimate. The weight of the person-specific estimate, relative to the overall estimate, depends on its reliability as an estimate of the individual responses. Events were dummy coded as $\mathrm{l}=$ event, $0=$ no event, since use of the 7-point scale did not improve the prediction of cortisol. Stress response scores in the field (F.RESPONSE) were estimated after diurnal patterns and other factors (listed above) with significant effects on cortisol levels were controlled for in the multilevel model. As reported elsewhere (van Eck et al., in press), stressful daily events were associated with significantly higher cortisol secretion.

The association between laboratory and field cortisol was tested with Spearman rank-order correlations between: 1) L.BASAL and F.BASAL, 2) L.RESPONSE and F.BASAL, and 4) L.RESPONSE and F,RESPONSE. As a last step, the contributions of L.BASAL and L.RESPONSE as predictors of F.LEVEL were evaluated with multilevel analysis.

\section{RESULTS}

\section{Effects of percelved stress level on cortisol and mood response to the experimental stressor}

Salivary cortisol responses to the SIST are shown in Figure 7.1. Most subjects responded to the task with increased cortisol secretion; 76 of the 87 subjects showed a cortisol response greater than L.BASAL (T1). On average, peak cortisol levels were observed at T4, approximately 30 minutes after the SIST preparation period began and 15 minutes after the speech ended. The maximum increase in cortisol during the SIST averaged $159 \mathrm{ng} / \mathrm{dl}$ (a percentual increase of 109\%) above L.BASAL.

MANOVA analysis revealed significant effects for the L.BASAL covariate $(B=$ $82, t(l, 84)=16.25, p<.001$ ) and for the repeated measurement factor Time (T! through T4: $F(3,255)=59.39 ; p<.001)$. The cortisol response pattern of the high stress group did not differ significantly from that of the low stress group, as evidenced by nonsignificant effects for both the PSS main effect $(F(1,84)=.02$; ns $)$ and the PSS by Time interaction $(F(3,255)=.18$; ns). MANOVA analysis for the sub-sample of 49 subjects with a final cortisol measurement at T5 yielded similar results: a significant main effect for the factor Time (T1-T5; $F(4,18.8)=16.83 ; p<.001)$, no main effect for PSS $(F(1,46)=.06$; ns $)$, and no interaction effect for PSS by Time $(F(4,188)=1.46$; ns). On average, cortisol at T5 (268 ng/dl) had significantly declined relative to peak levels $(335 \mathrm{ng} / \mathrm{dl}$ ) (Wilcoxon test, $p<.001$ ), although it was still elevated in relation to L.BASAL ( $167 \mathrm{ng} / \mathrm{dl}$ ). Comparing the recovery process in high vs. low stress groups, post-hoc comparisons showed no difference between the groups in either the magnitude of the cortisol elevation above L.BASAL persisting at T5 (high PSS $(n=26): 109 \mathrm{ng} / \mathrm{dl}$; low PSS $(\mathrm{n}=23): 95 \mathrm{ng} / \mathrm{dl}$; Mann-Whitney test, $\mathrm{n} . \mathrm{s}$.$) or the$ magnitude of the decline in cortisol from maximum response to T5 (high PSS: decline $=54 \mathrm{ng} / \mathrm{dl}$; low PSS: decline $=82 \mathrm{ng} / \mathrm{dl} ;$ Mann-Whitney test, n.s. $)$. 


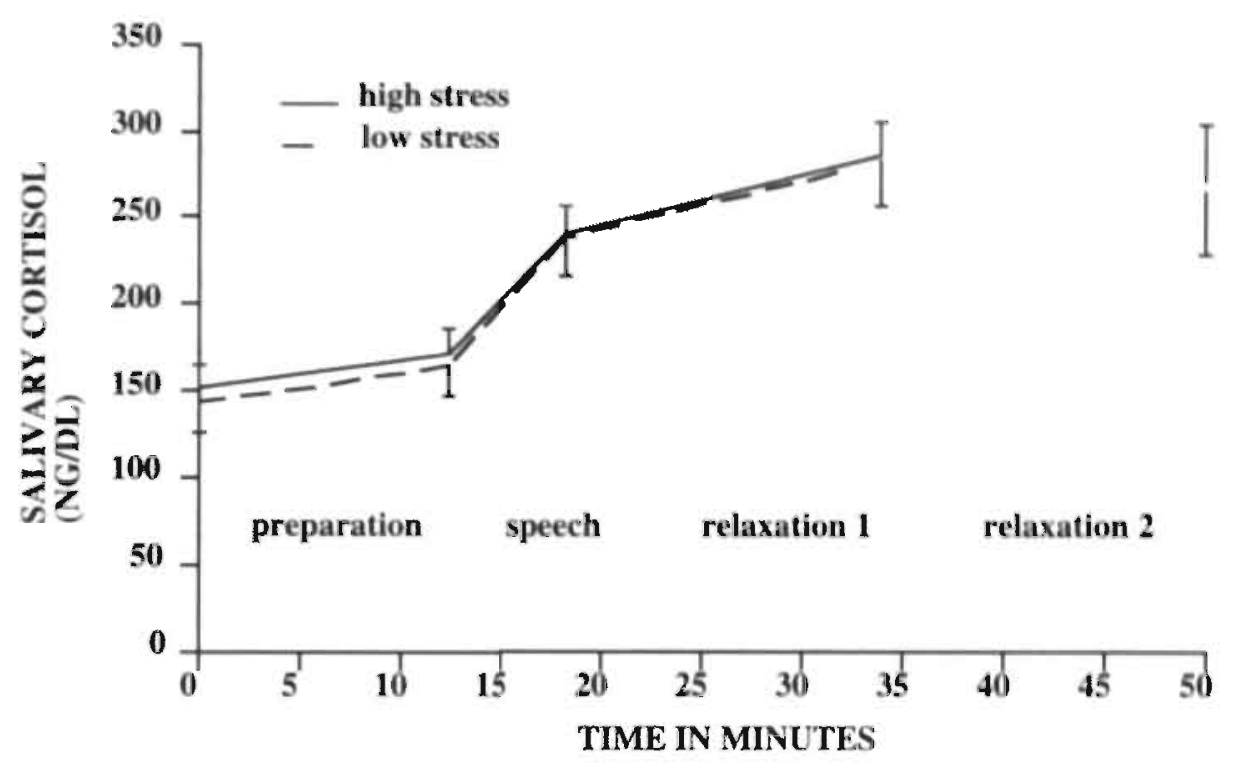

Figure 7.1. Cortisol response to speech task in high and low stress subjects. Data at T1 - T4 based on 87 subjects, data at T5 based on 49 subjects.

Mood states also changed in response to the stress task, with significant Time effects for both PA $(F(3,255)=14.53 ; p<.001)$ and NA $(F(3,255)=42.11 ; p<.001)$. Post-hoc comparisons revealed a significant NA increase $(p<.0001)$ and PA decrease $(p<.001)$ from $\mathrm{T} 1$ to $\mathrm{T} 2$, after preparation but before speech delivery. As can be seen in Figure 7.2., mood had recovered to baseline by 'T3. 'High stress' subjects had a higher overall level of NA than 'low stress' subjects $(F(1,85)=22.15 ; p<.001)$; the level of PA did not differ between groups $(F(1,85)=.55 ; p>.05)$. Time by PSS interactions were not significant; in other words, high stress subjects showed no greater increase in NA or greater decrease in PA in response to the task than low stress subjects. 


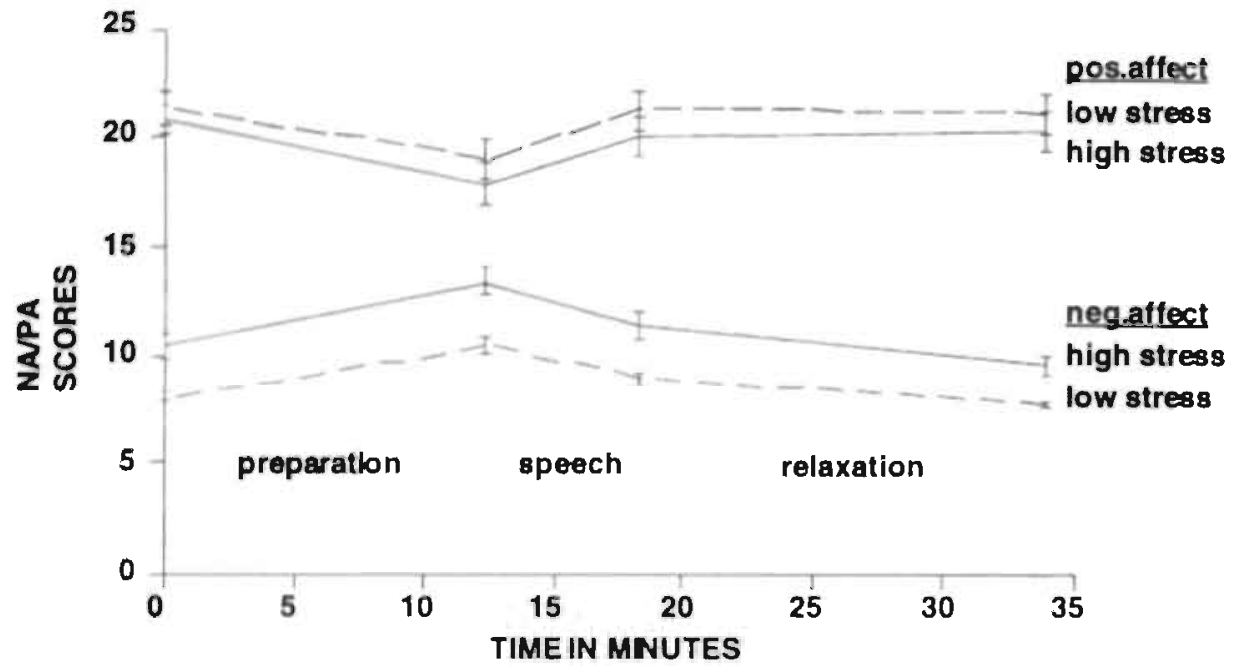

Higure 7.2. Response of NA and PA to speech task in high and low stress subjects.

\section{Relationship between mood state and cortisol response to the SIST}

To investigate whether subjects' emotional responses to the SIST were related to their cortisol responses, Spearman rank correlation coefficients were computed between the four repeated cortisol measurements and the summary measure L.RESPONSE, on the one hand, and the four mood measurements of both mood scales, on the other hand. No significant correlations were found between either NA or PA and L.RESPONSE. Of all correlations between the four PA values and the four cortisol values (T1 through T4), not a single coefficient was significant. However, NA at baseline was positively associated with cortisol at L.BASAL (T1), with cortisol just before the speech task (T2) and with cortisol just after the speech task (T3) (rho $=.29$; $\mathrm{p}<.01, r h o=.31 ; \mathrm{p}<.01, r h o=.25 ; \mathrm{p}<.05$, two-tailed tests).

\section{Relationship between trait characteristics and cortisol response}

Possible influences on cortisol L.RESPONSE of trait anxiety, depression, anger, coping styles, total psychosomatic complaints, and personality scales were tested with forward stepwise multiple regressions. None of the above traits was a significant predictor of L.RESPONSE (all higher $F$ probabilities than .05).

\section{Laboratory to fleld generalization}

L.BASAL was significantly higher than F.BASAL (147 ng/dl versus $116 \mathrm{ng} / \mathrm{dl}$; p<.001, Wilcoxon Matched-Pairs Signed-Rank Test, two-tailed test). The difference beiween these two measures was similar for high and low stress groups (HS: 36.9 ng/dl, LS: $24.7 \mathrm{ng} / \mathrm{dl} ;$ ns). The correlation between L.BASAL and F.BASAL cortisol levels was moderately strong ( $r h o=.56 ; \mathrm{p}<.001)$. No relationship was found between L.RESPONSE and F.BASAL ( $r h o=-.08 ; n s$ ). Next we compared cortisol responses to. the SIST (L.RESPONSE) with estimates of cortisol reactivity to naturally occurring stressful events (F.RESPONSE); no significant relationship was found ( $r h o=-.13$, ns). 
Table 7.1. Effects of lab baseline levels (L.BASAL) and lab response values (L.RESPONSE) on field cortisol level.

\begin{tabular}{lrrr}
\hline Fixed Effects & Estimate & S.E. & $\mathrm{p}<$ \\
\hline Intercept & 2.94 & 0.144 & 0.001 \\
Time of day (TIME) & -0.581 & 0.037 & 0.001 \\
TIME $^{2}$ & 0.129 & 0.010 & 0.001 \\
TIME $^{3}$ & -0.010 & 0.001 & 0.001 \\
Truncated function & 0.010 & 0.001 & 0.001 \\
Lunch & 0.040 & 0.020 & 0.03 \\
Other food intake & 0.025 & 0.01 .1 & 0.01 \\
Smoking & 0.043 & 0.021 & 0.02 \\
\hline L-BASAL & 0.172 & 0.050 & 0.001 \\
LRESPONSE & 0.001 & 0.003 & $n s$ \\
Random Effects & (Co)variance & S.E. & p< \\
\hline Person level: & & & 0.001 \\
intercept & 0.024 & 0.005 & 0.001 \\
Time of day & 0.0002 & 0.00004 & \\
Time of day/Intercept & -0.001 & 0.0004 & 0.001 \\
\hline Measurement level: & & & \\
Residual term & 0.059 & 0.002 & \\
\hline
\end{tabular}

* 3082 observations nested within 85 subjects (41 HS, 44 LS)

Finally, we estimated a multilevel model in which L.BASAL and L.RESPONSE were used to predict cortisol F.LEVEL. This analysis has the advantage of making use of all of the cortisol field data. Controlling for significant external influences should increase both the reliability of the dependent cortisol measure and its comparability to the laboratory assessments. As shown in Table 7.1., when both laboratory measures were entered simultaneously into the model, only L.BASAL explained a significant amount of variance in F.LEVEL.

\section{DISCUSSION}

The first aim of the present study was to evaluate the contribution of perceived stress and trait characteristics to individual differences in cortisol responses to a laboratory stress task. Associations between emotional and cortisol responses to the speech task were also examined. To summarize the main findings, the SiST elicited a strong cortisol response in a large majority of the subjects, which was unrelated to differences in perceived stress level. Trait anxiety, depression, psychosomatic symptoms, anger, coping style and personality similarly failed to predict the cortisol response. Neither negative nor positive emotional responses to the speech task showed any association with the cortisol response. High negative affect at baseline, however, was associated with higher total cortisol levels at baseline, just before the speech task, and again just after the speech task.

This suggests that individual differences in current distress (especially anticipatory distress) may be more important determinants of cortisol secretion than PSS, which is a measure of more chronic distress. Although high PSS was associated 
with high NA, no direct relationship was found between PSS and cortisol secretion. It is worth noting that subjects with the highest NA at lab baseline (T1) had, at the same time, the highest cortisol baselines relative to their own $11 \mathrm{a} . \mathrm{m}$. field levels ( $r h o=.26$, $\mathrm{p}<.01$ ). An elevated laboratory baseline, possibly due to anticipatory anxiety, could explain the lack of a relationship between NA and the additional cortisol response measured during the SIST. Moreover, the fact that high stress subjects had higher baseline NA than low stress subjects suggests that there may be a tendency toward higher cortisol in response to the SIST in this group, which was masked by the anticipation response.

As summarized in an overview of the published data (Kirschbaum et al., 1992a), most studies have found either no association or only a weak association between cortisol responses to stress and personality measures. The hypothesis linking personality factors with cortisol responses rests on the assumption that cortisol reactivity is a stable individual characteristic. There is some evidence in support of this assumption. Forsman and Lundberg (1982) found significant individual consistency in urinary cortisol responses to repeated tasks; correlations were higher between responses to more or less identical tasks (males: $r=65$; females: $r=.38$ ) than between responses to different kind of tasks (males: $r=13$; females: $r=19$ ). Consistency in cortisol responses to different tasks was also noted by Berger et al. (1987). Another study (Kirschbaum et al., 1992c) found significant intercorrelations among maximum cortisol responses to three stimulation procedures (mental arithmetic, public speaking, bicycle ergometry) in males, but not in females. In a more recent study in the same laboratory, significant correlations (ranging from .38 - .60) were found between cortisol responses to the same task repeated on 5 days (Kirschbaum et al., 1995) Although some response consistency seems to exist, at least in males, more research is necessary.

Even if stable individual differences in cortisol reactivity exist, it is not clear that they reflect psychological traits (Bossert et al., 1988). Genetic factors, for instance, have been shown to play a role in cortisol reactivity (Kirschbaum et al., 1992b). Recently, some support for an association between personality factors and cortisol reactivity was reported (Kirschbaum et al., 1995); low self-esteem, negative self-concept, depressed mood and physical health problems were found to differentiate high and low cortisol responders. Twenty healthy males were exposed on five consecutive days to the same psychological stress task (public speaking and mental arithmetic in front of an audience), with low responders showing evidence of habituation after the first day and high responders continuing to show large cortisol responses on successive task exposures. If only Day 1 cortisol responses had been analyzed (as in the present study), only one out of 12 correlations between cortisol responses and personality scales would have reached significance. This indicates that measuring a stress response once may be insufficient for the manifestation of individual differences in cortisol reactivity. This appears to be an important point for further research.

The second aim of the study was to investigate the relationship between laboratory and field cortisol activity. A moderately strong correlation was found between laboratory baseline levels and 11 a.m. field cortisol levels, and laboratory baseline levels also predicted some of the variance in overall field cortisol levels. In contrast, no association was found between laboratory responses and the field reactivity measure, and laboratory responses were not useful in predicting overall cortisol levels in the field. The finding of a relationship between laboratory and field levels of cortisol, but not between. laboratory and field reactivity, is more or less in 
accordance with results of cardiovascular studies, where levels are found to be more stable than reactivity / variability scores across settings (Turner et al., 1994). Similarly, greater consistency has been found for repeated lab baseline cortisol levels than for cortisol reactivity measures to different laboratory tasks (Berger et al., 1987; Forsman. 1982). As with personality traits, the temporal stability of the cortisol response is an important issue. To the extent that 'reactivity' is not a stable trait, a correspondence between laboratory and field cortisol responses will be unlikely. Results of our field study on the effects of stressful daily events on cortisol levels (van Eck et al., in press), did not provide support for stable individual differences in cortisol responses to daily events; cortisol responses were more dependent on the current mood state.

Another possible explanation for the lack of laboratory to field generalization of cortisol responses is the different timing of cortisol response measurement in the lab compared to the field study. Although in the lab cortisol was assessed parallel to the stress task, in the field, stressful events could have occurred at any time in the 90 minutes interval prior to cortisol assessment. Substantial post-stress recovery of cortisol levels might have taken place by the time the saliva sample was collected. However, when we repeated the analysis presented above with a new field response measure based on the subset of stressful events that were still ongoing when cortisol was assessed, again no association was found between laboratory and field reactivity.

Although real life pubiic speaking was reported as a stressful event during ESM sampling by a few subjects, any similarity between lab and field stressors was coincidental. One could argue that the predictive validity of a laboratory stressor would increase if one were to choose a specific field stressor with a strong correspondence in psychological meaning to the laboratory stressor (Houtman \& Bakker, 1987; van Doornen \& Turner, 1992). However, even if the researcher succeeds in matching a field stressor with a relevant laboratory task, a severe drawback of this approach is that only a small subset of the enormous range of stressors people encounter in their daily lives can be simulated in the laboratory. Furthermore, the importance of a laboratory - field association would be lessened dramatically if only linkings of specific lab - field stressors were to show an association (Turner et al., 1994; van Doornen \& Turner, 1992). Significant correlations may be difficult to find because of the heterogeneity of field responses resulting from the variety of stressful experiences encountered. Even more than in the laboratory (Strube, 1989), responses to daily life stressors will be modulated by objective and subjective event characteristics (e.g. novelty, severity, duration, content), interacting with each individual's characteristics and prior experiences (Forsman, 1982). In conclusion, while we found no evidence for an association between cortisol responses in laboratory and field, the paucity of studies on this subject and the difficulties (conceptual, methodological, and statistical) associated with its investigation point to the need for additional research.

\section{ACKNOWLEDGEMENTS}

We thank C. Dijkman for assistance in all aspects of the study. 



\section{Chapter 8}

\section{Concluding remarks}





\section{RECAPITULATION OF THE MAIN RESEARCH AIMS AND FINDINGS}

In an effort to increase our understanding of the stress process as it relates to health, the present research investigated the impact of minor daily events on mood and the HPA system. Our first aim was to describe the nature and scope of daily life stress in a group of white collar men and to contrast the experiences of individuals who perceive themselves to be stressed with those who do not. The second aim was to determine how mood and cortisol levels change in response to stressful daily events. A final aim pertained to the stability and generalizability of individual stress responses. We examined whether the cortisol responses to a laboratory stress task reflect those occurring during stressful situations in real life.

Since it is reasonable to assume that stress responses will only lead to disease when they are prolonged or occur very often, it is likely that the pathogenic influence of these stress responses depends on whether they reflect a stabie individual characteristic or not. Therefore, we also investigated whether more or less stable person characteristics like perceived stress level, trait anxiety, and depressive symptomatology were related to individual differences in responses to daily stress. Special attention was also paid to the possible influence of the context and the appraisal of an event on outcome measures. The Experience Sampling Method was used to collect data from subjects during their normal daily activities. In addition, subjects participated in a laboratory stress task, in which they were unexpectedly asked to deliver a speech. In this final chapter, the main findings of the study are briefly reviewed and discussed, including potential clinical implications and suggestions for future research.

The results showed that work was the major source of daily stress in our sample, followed by problems related to the social network. These event contexts reflect the sample's life stage and demographic characteristics; their most important social roles were those of full-time employee, spouse, and father. In general, stressful daily events could be characterized by their salience and unpleasantness; especially events that were appraised as less predictable and less controllable were experienced as unpleasant. The high stress (HS) group could be characterized as vulnerable, trait anxious individuals, who suffered from a variety of psychological and psychosomatic complaints, experienced chronic environmental and relational difficulties, which they coped with using generally less effective styles. HS subjects reported twice as many events as low stress (LS) subjects, especially more work and social interaction events (involving colleagues or the partner). The HS group differed from the LS group in how both daily activities and stressful events were perceived: HS subjects evaluated their activities as requiring more effort, while they were less motivated and less skillful in doing them, while events were appraised as more stressful and less controllable.

From the data presented in chapter 4,5 , and 6 , we can conclude that high perceived stress was not only related to higher Agitation and higher Negative Affect levels and lower Positive Affect levels across all situations, but also magnified the effects of daily events on negative mood states. Positive mood was only affected by very unpleasant events. The effects of events on negative mood states persisted for at least 90 minutes in both HS and LS groups. A future event also increased current Agitation in both groups, but higher current Negative Affect was only observed in the HS group. The findings also indicated that certain kind of events (task demands) and event appraisals (unpleasantness, controllability, chronicity) were more likely to influence mood states. 
With respect to cortisol, it was not perceived stress but trait anxiety and depressive symptomatology that exhibited small but significant positive associations with cortisol levels during the day. Stressful daily events were found to be associated with increased cortisol secretion, the magnitude of the effect depending on whether the event was still ongoing and on how frequently a similar kind of event had occurred previously. Type of event or type of appraisal had no additional effect on cortisol. Although perceived stress, anxiety, and depression did not increase cortisol reactivity to daily events, evidence was found for reduced habituation to recurrent events in individuals scoring high on these traits. Consistent with most views of the stress process, the effect of stressful events on cortisol appeared to be mediated to a large extent by associated increases in negative mood states.

From the data presented in chapter 7 we conclude that the laboratory response measures do not necessarily reflect those obtained in the field.

The main findings of the study can be briefly summarized as follows:

(1) Individuals with high perceived stress reported twice as many daily events, with significantly more work and social interaction events. Events were also appraised as more stressful and less controllable (Chapter 3 ).

(2) Individuals with high perceived stress not only showed higher negative mood levels and lower positive mood levels throughout the day, but also exhibited higher negative mood states in response to stressful daily events (Chapter 4).

(3) In both groups, minor daily events had small but significant positive effects on salivary cortisol levels, which were largely mediated by negative mood states (Chapter 6).

(4) Individuals scoring high on trait anxiety or depressive symptomatology had higher cortisol secretion throughout the day and showed less habituation of cortisol responses to recurrent daily stressors (Chapter 5 \& 6).

(5) Laboratory and field cortisol levels were found to be moderately correlated, but there was no significant association between laboratory and field cortisol response measures. Laboratory baseline levels, but not responses to the laboratory stressor, were significant predictors of field cortisol levels (Chapter 7).

\section{MEASUREMENT OF STRESS IN DAILY LIFE}

For the measurement of daily stress, various options are available to the researcher, each with its own specific advantages and disadvantages. Laboratory studies try to simulate minor stressful events by exposing subjects to various tasks. like computer games, mental arithmetic, films or speeches. The experimental situation has the major advantage that the stressor can be standardized. thereby controlling for the objective component of the stressor. In addition, laboratory stress models can be of great value for basic research questions (e.g. for studying the mechanisms related to individual differences in response to various standardized stressors, or the influence of coping strategies on response to various stressors). However, one can 
question the relevance of the laboratory stressor for the stress that people experience in everyday life. In the laboratory most stressors are of a voluntary nature, of short duration, often anticipated, and they usually require active, effortful coping. It is not difficult to see that these kind of stressors cannot be as intrinsically motivating, threatening, challenging, or demanding of complex coping strategies as are most of the events people experience in their daily lives. Therefore, the validity of the dominant use of laboratory studies to increase our understanding of stress in daily life is questionable. Although the objective component of psychosocial stress is important for an understanding of the stress process, the study of how people perceive, report, and respond to feelings of stress, irrespective of its objective base, is of great importance in itself. For individuals, their idiosyncratic perceptions of stress and their subjective responses to it are most critical in determining its consequences. They may cause people to alter their work habits or their social relationships, change health behavior, or seek medical or psychological support.

Most studies designed to assess individual's subjective perceptions of daily stress have been limited to checklist questionnaires handed out to large populations at one point in time. Although understandable in light of time and cost factors, both psychological but also physiological stress measures collected at one point in time are subject to various biases. Besides a number of methodological problems with available checkilists (related, for instance, to item content or item sample), event checklists are often handed out long after the events have actually taken place. Therefore, less salient events will be forgotten, and the successful or unsuccessful resolution of the event ('effort after meaning') will determine to a large degree endorsement of an event item and scores on distress measures. These problems may still be profound when events are measured on a daily bases. One-time assessments of physiological parameters will probably be unreliable, for instance in that they include a lot of 'noise' due to normal fluctuations in levels of activity, or in that they reflect test or evaluative anxiety more than daily stress. One-time assessments of most physiological parameters also leave the temporal relationship between psychosocial stress and physiology unclear.

The ESM, in the present study characterized by repeated measurements of events, subjective mood and cortisol, has several advantages over cross-sectional approaches to the study of daily stress. First, it increases assessment reliability and provides better estimates of the frequency, distribution, and intensity of psychological variables. Subjects could not only report events occurring at the moment that they were beeped but also any event that had occurred during the past interval. By using an open-ended approach to elicit stressful events we did not restrict a priori the definition of 'stressful' to certain specific items or classes of events. Since events were assessed not long after their occurrence, we were able to elicit information about the sources of stress and the appraised meaning of an event with a minimum of confounding due to biased recall or forgetting. Using semi-random time intervals, anticipation effects were kept to a minimum.

With the ESM we were able to show, for example, that certain demographic characteristics (age, life stage, marital status) of our study sample were reflected in the pattem of significant events they reported. If one would use a single checklist for various samples or populations, important events with specific salience for a specific population may be missed. Events like school exams or cancelled dates, for instance, may be of great significance for a student population, but of minor relevance for a group of elderly people. Checklists should preferably be developed from data obtained from the population in which the checklist is going to be used. We also 
showed that the experience of stressful events is not static; the frequency of reported events varied according to the day of the week (more events during the week than in the weekend) and the time of the day (relatively more events during work hours). This also indicates the importance of the context, here the work environment, for eliciting events. With ESM, we were also able to compare subjects' immediate level of experience (e.g. ESM events reported during the day) with their short-term retrospective assessments (e.g. events reported at the end of the day). Here we observed that with end-of-day reflections many more events were reported than within days. It would be interesting to further investigate influence of response method (checklist versus open-ended) and possible biases in the process of recollection and appraisals of events, which may be more likely to occur at the end of the day.

Second, ESM provides a clearer picture of the dynamic and temporal nature of the relationship between stressful minor events and affective and neuroendocrine responses. This is especially important if the events of interest occur unpredictably (as is usually the case in the natural environment) and therefore cannot be directly monitored by the researcher. The correspondence between stressful daily events, selfreporis, and physiological measures can only be assessed on a within-subject basis. The results demonstrated the potential value of ESM in studying stress in the field. We were able to relate current mood and cortisol levels in daily life to stressful experiences within the context of stable individual differences, type of event, event appraisal, and time. Since assessments were made close to the moment at which the variables of interest occurred, we increased the possibility of establishing causal relationships. Although we cannot rule out the possibility that, for instance, current mood influenced the reporting of recent stressful events, the finding that prior stressful events were associated with persistent increases in negative mood states supports the assumption that events influenced mood and not vice versa.

Third, ESM provides a means for investigating both state and trait aspects of the daily stress process. In the present study we saw, for example, that a global measure of long-term difficulties was significantly related to the reported number of daily events. Person characteristics were found not only to be related to mean mood levels but also to the magnitude of mood responses to current stressful events. With respect to state aspects of daily stress. ESM is particularlly appropriate for the study of the quality of experiences. Since appraisal processes are thought to be predictive of emotional and physiological outcomes of stress, this is an important feature of ESM. In the present siudy, results indeed revealed that several appraisals were differentially related to the outcome measures. And although subject groups did not differ in the amount of time spent on various activities, important differences between groups were observed in how these activities were appraised and experienced. The multilevel approach used in chapters 4,6 , and 7 is optimally suited for the analyses of the ESM data set, in which both variables at the person level (trait characteristics) and the measurement level (state characteristics) are obtained. Using multilevel analyses we can adequately test the transactional stress model, in which trait and state aspects are intertwined.

A number of possible limitations of the present ESM study should also be considered. The repeated use of a short checklist instead of an open-ended approach to sample events might have had some practical advantages (e.g., no time consuming coding tasks for the researcher). However, most available checklists are too long to be suitable for frequent use, while a short event list limits dramatically the range of events that can be investigated. A checklist might be preferable, however, if one is 
interested in certain kind of events, or when one has knowledge about which kind of events are most important for a particular population. Another limitation of the current study design is the lack of temporal resolution. Many stressful events occurred in the interval between beeps, which means that by the time the measures were taken, some mood and/or cortisol response recovery may have taken place. Theoretically, an event-sampling approach (in which subjects are instructed to fill out a questionnaire and take a saliva sample at the moment or just after they experience a stressful event) might have some advantages, but such a procedure would very likely influence not only the daily routine of subjects but also how events are defined, experienced, and coped with. Only true ambulatory monitoring of cortisol (continuous measures: in practice infeasible) could really solve this problem.

A drawback of every subjective approach to the study of (daily) stress. including ESM, is that we are not able to differentiate between the relative contribution of the person and the environment in the experience and response to stress. In particular, since the present study relied on self-reports of stressful events, it was not possible to determine whether HS subjects were actually more exposed to stressful events or that high perceived stress levels (or other individual characteristics, like trait negative affectivity) merely increased reports of stressful events. Our measurement of daily events might possibly improve if we adapted the approach developed by Brown and colleagues (1974;1978), in which clear definitions have been formulated for what should and what should not be: considered a stressful event. The assessment of events is based as much as possibie on the facts of the event, their salience, and the context in which an event occurred (e.g. loss, or longterm contextual threat), rather than on how the respondent perceived them. In addition, a distinction is made between events which the respondent might have brought upon himself ('dependent' events) and those which simply happened to him ('independent' events). Although interviewers can be trained to reliably code major events, it seems highly unlikely that daily events can reliably be coded as 'dependent' or 'independent'. Another disadvantage is that the interviews necessary for the assessment of events are very time-consuming and therefore costly. One useful approach might be to study the daily stress process in those contexts in which one can anticipate the source(s) of stress and in which the potential stressor is identical for every subject, thereby controlling for the objective stimulus situation (e.g. driving exam, job loss, last weeks of the tax year for accounting firms). But as we can see from these examples, most stressors would then approximate major life events in terms of intensity and in frequency and might therefore not be relevant for an understanding of the much more frequent but minor stresses of day-to-day life.

\section{PERCEIVED STRESS AND NEGATIVE AFFECTIVITY}

Personality factors undoubtedly play an important role in perceived stress. Self-report measures of stress and distress have been found to correlate significantly with measures of trait negative affectivity or neuroticism: the disposition to accentuate the negative aspects of the self, other people, and the world in general, and to experience aversive emotional states. High negative affect individuals are more likely to experience discomfort at all times and across situations, and this trait has been shown to be reflected in higher overall levels of negative mood (Watson \& Clark, 1984). Negative affectivity also appears to be a vulnerability factor for the development of anxiety and depressive disorders, indicates poor prognosis, and seems 
to reflect, both the current influence of state affect and the residual effect of earlier depressive episodes (Clark, Watson, \& Mineka, 1994). Negative affectivity has been found to be highly stable over a 20 year period (Costa \& McCrae, 1980; Costa \& McCrae, 1986; Ormel, 1983), and there is evidence for a genetic compound (Lykken, 1982; Scarr, Webber, Weinberg, \& Wittig, 1981; Shields, 1962). While most theoretical models argue that dysphoric mood states result from an interplay between stressful events and certain vulnerability factors such as dysfunctional cognitions, Watson and colleagues (Watson, Clark, \& Harkness, 1994, p.29) have offered a different explanation: "The same individuals are likely to perceive innocuous or ambiguous events as stressful (negativistic appraisal), to respond poorly to actual stressors in their lives (stress overreactivity), to have a negativistic and dysfunctional cognitive style (e.g. self-criticism and self-blame), and to experience intense episodes of dysphoria (e.g. anxiety, depression, guilt, anger)." In other words, dysphoria, rather than being caused by stress and dysfunctional cognitions, may simply be another subcomponent of the same general construct 'negative affectivity' or neuroticism. It has been shown, for instance, in several prospective studies that a chronic pattern of general emotional and/or physical distress exists in about $25 \%$ of the respondents in the various populations investigated (Depue \& Monroe, 1986). General distress levels in these groups appeared to be determined to a large extent by characteristics of the individual and not by environmental (life events) or psychosocial (social support) variables. It is highly likely that a substantial portion of this chronically distressed group will consist of individuals scoring high on the personality dimension negative affectivity, since most scales of psychological disorder in the stress literature assess general negative affect, life dissatisfaction, and a lack of positive well-being (Depue \& Monroe, 1986).

Perceived stress did not overlap completely with measures of psychological distress in the present study; for example, we found that perceived stress, chronic difficulties, trait anxiety, and depressive symptoms independently predicted psychosomatic symptoms (Appendix I). Level of perceived stress was, however, highly correlated with trait anxiety, which is often used as a measure of trait negative affectivity and may explain at least partly the differences in overall mood found between HS and LS subjects. It is important to note that subjects with high perceived stress levels not only had higher negative mood levels across days, but high perceived stress also magnified the effect of stressful daily events on negative mood states within days. In other words, perceived stress does not just spuriously inflate the relationship among stressors, stress symptoms and affective outcomes, but acts as a moderator variable influencing an individual's reactivity to stressful events. The increased negative mood reactivity to minor events may be an important factor in the maintenance of chronic distress in the high perceived stress group. Therefore it seems important in the future to investigate variables potentially related to stress reactivity. such as coping behavior, and social support.

Global perceived stress was not a strong predictor of cortisol secretion in this sample. However, the intercorrelations among the various measures of stress and distress argue for cautious conclusions about the relative contribution of each to cortisol levels. The fact that self-report measures of anxiety and depression were associated not only with increased perceptions of chronic stress but also with elevated cortisol levels also suggests that negative affectivity is not simply a confounder in psychosomatic research. Several other studies have provided support for a relationship between negative affectivity and objective measures of stress; for instance, trait negative affectivity has been positively associated with salivary 
immunoglobulins (Stone, Cox, Valdimarsdottir, Jandorf, \& Neale, 1987; Ursin, Mykletun, Tonder, Vaernes, Relling, Isaksen, et al., 1984) and systolic blood pressure (Harburg, Julius, McGinn, McLeod, \& Hoobler, 1964). Although perceived stress, trait anxiety, and depression did not increase cortisol reactivity to stressful events, we did find evidence for reduced cortisol habituation to recurrent events in subjects scoring high on these traits. Besides these trait measures, the state measures of negative mood were found to be important mediators of the relationship between stressful events and cortisol secretion. Following the transactional stress model, a subject's appraisal of an event with respect to 'what is at stake' and 'what can be done about it' determines the level of emotional involvement, which in turn influences outcome processes. This study indeed showed that the subjective appraisal of apprehension and emotional involvement can lead to increased cortisol secretion. Since high stress subjects reacted more strongly with negative mood to stressful events, and since the effect of stressful events on cortisol was mediated to a large extent by associated increases in negative mood, the apparent lack of a significant association between perceived stress and cortisol reactivity to stressful events is unclear, although we did observe a trend in the expected direction.

Results of the present study are consistent with three related mechanisms thought to play a role in the modifier effect of negative affectivity on the relationship between life stress and psychobiological distress (Ormel \& Wohlfarth, 1991). First, negative affectivity may influence the appraisal of daily events and therefore their consequences for well-being, with subjects scoring high on measures of negative affectivity appraising identical events as more demanding and threatening (Lazarus \& Folkman, 1984b; Watson \& Clark, 1984). Second, negative affectivity may be associated with differential psychobiological sensitivity to stressors (Bolger \& Schilling, 1991; Eysenck, 1967; Tellegen, 1985). Third, negative affectivity may be related to less adequate coping with daily events (McCrae \& Costa, 1986). Although in the present study we had no control over the kind of events experienced, HS subjects perceived events as equally unpleasant as LS subjects, but these events were appraised as more stressful and less controllable by HS subjects. Moreover, activities (especially work activities) were appraised as more demanding and less enjoyable. With respect to psychobiological sensitivity to stressors, HS subjects showed increased negative mood reactivity to daily events and also exhibited less cortisol habituation to recurrent events when compared to LS subjects. Finally, HS reported using an active coping style less often in general, and the coping styles palliative reaction and expression of emotions more often. These latter coping styles are generally considered to be less effective. HS subjects scored higher on the scale depressive reaction, indicating that they experience more feelings of helplessness in response to daily events than LS subjects. We have to keep in mind, though, that scores on general coping styles are not necessarily predictive of the actual coping strategies used in relation to specific events. However, the fact that HS subjects appraised daily events as less controllable than LS subjects also seems to indicate that they were less certain of their coping skilis in relation to daily events.

The fact that self-reports of stress, symptoms, and negative mood are highly correlated with measures of negative affectivity has important implications for the measurement of stress (Pennebaker \& Watson, 1988). Since there are large individual differences in negative affectivity which seem to have a heritable component and seem to be stable over time and situation, measures of what is presumed to be transient work stress will be strongly correlated with negative affectivity. This means that, for the study of the impact of stressful events, any between-subject design will 
have to gather data from a very large sample to be able to control for individual differences in negative affectivity. Another problem related to the concept of negative affectivity is that of causality; any causal interpretation will be meaningless since the process related to the perception of stress is the same process related to the perception of symptoms. Therefore, the optimal design is a within-subject design with repeated measurements over time, where both within- as well as between-subject relationships can be investigated prospectively, as is the case with ESM. As discussed before, the association between subjective self-reports and physiological measures can also only be determined on a within-subject basis. There is also a great need for longitudinal research, as clearly formulated by Clark et al. (1994, p.113): "Until prospective studies have followed individuals - whose premorbid personality and environmental characteristics are known - over sufficiently long periods of time to yield a sufficient base rate of disorder, we will not be able to distinguish causal from concomitant, confounding, or residual factors."

\section{GENERALIZABILITY FROM THE LABORATORY TO THE FIELD}

The positive relationship between trait anxiety and depressive symptonatology and contisol secretion throughout the day was not icplivated in the laboratory; no association was found between trait anxiety or depression and laboratory baselines (Chapter 7). With respect to stress reactivity, trait characteristics were related neither to elevated cortisol reactivity to stressful daily events in the field nor to the magnitude of the cortisol responses to the laboratory stress task. The field results regarding reduced cortisol habituation to recurrent events in subjects scoring high on perceived stress, trait anxiety, and depression could not be investigated in the laboratory since the laboratory stress task was not a recurrent but a novel event. In the laboratory, only negative mood state at baseline was associated with higher cortisol levels at baseline, just before, and just after the speech. In the field, we also found that current negative mood state was an important mediator of cortisol responses. Since it is reasonable to assume that personality traits influence how stressful situations are appraised and may therefore have predictive value for understanding individual differences in responses to stressful situations (as was the case for emotional responses), the lack of an association between the trait characteristics and cortisol responses, either to the SIST or to naturally occurring events in the field (except for recurrent events), is somewhat disappointing although certainly not an exception in the literature. Several methodological and statistical issues may possibly obscure this relationship. In the laboratory, the absence of a relationship between person characteristics and cortisol baseline levels and cortisol reactivity could be partly explained by an elevated laboratory cortisol baseline in both groups, possibly due to anticipatory anxiety, which also masks the cortisol response. This laboratory baseline was significantly higher than cortisol field levels assessed at the same time. It is also possible that strong novelty or experimental effects of the stressor override any individual differences in cortisol reactivity when laboratory stress responses are assessed only once. The fact that almost $90 \%$ of the subjects reacted with a substantial cortisol response to the SIST task supports this interpretation. Additional support for this argument was also found from a recent study by Kirschbaum and colleagues (1995), where some evidence for an association between personality traits and cortisol reactivity in healthy males was reported. In this study, subjects were exposed on five consecutive days to the same psychological 
stress task; low self-esteem, negative self-concept, depressed mood, and physical health problems differentiated high from low cortisol responders. If only Day 1 cortisol responses had been analyzed, results would have been negative; low responders showed habituation after the first day, while high responders continued to show large cortisol responses over the five days. These results cast great doubt on the value of one-time laboratory stress assessments; they seem to be insufficient for the manifestation of individual differences in cortisol reactivity. Therefore, in future laboratory studies cortisol responses should be measured at least twice; not only to repeated identical tasks, but preferably also to different tasks. In the field, repeated assessments of events were made, but it is possible that the within-subject samples of events were too heterogeneous to reveal consistent and reliable individual response patterns to events in general.

It has been widely assumed that by measuring stress reactivity in the laboratory we can say something about stress reactivity in general, which would help clarify individual vulnerability to real-life stress. The use of laboratory cortisol reactivity as a risk factor for stress-related disease depends on the extent to which reactivity is indeed a reliable, stable, and generalizable individual characteristic. Although several laboratory studies have provided some evidence in support of response consistency across identical and, to a lesser degree, between different kinds of tasks (at least in males) (Forsman, 1982; Kirschbaum et al., 1995; Kirschbaum et al., 1992c), the evidence is far from conclusive, and more research is necessary. Given the above, the generalizability of reactivity from the lab to the field also seems questionable; it is certain that the variety of stressors experienced in real life will be much larger than in the laboratory. Results indeed showed that laboratory and field response measures were not associated.

Even if laboratory response measures have no predictive value for reactivity to stressful events in daily life, it has been hypothesized that they might be good predictors of overall levels of hormonal secretion throughout the day (Manuck et al., 1989). According to this theory, overall levels reflect the cumulative effects of daily life stressors rather than the reactivity to a transient stressful episode. This idea was also not supported, however, by our results; laboratory baseline levels, but not responses to the speech task, were significant predictors of field cortisel levels. Once the pre-task level was known, no additional predictive information was gained from measuring the laboratory stress response. These results are comparable to those for cardiovascular parameters, where levels have been found to be more stable than reactivity scores across settings. Only a weak relationship has been found between laboratory reactivity and daily heart rate and blood pressure levels; once the resting level is known there is no additional gain from task level or stress responses (Turner et al., 1994).

The low predictive validity of laboratory cortisol reactivity may to some extent be ascribed to methodological problems, like elevated baseline cortisol in the lab or the timing of cortisol response measurements in the lab compared to the field. The laboratory procedure could possibly be improved by allowing more time for the 'baseline' level to stabilize. With respect to the timing of response measures, better matching of lab and field measures is probably only possible in a study of predictable real-life stressors. Again, this would drastically curtail the range of real-life stressors that can be investigated. In addition, this study showed that unanticipated events tended to be experienced as particularly unpleasant.

An even more serious problem may be the heterogeneity of stressful experiences in real life. In daily life it is not possible to standardize stressful events to 
control for the influence of factors like novelty, task difficulty, content, duration. These factors could all contribute to low response consistency. In daily life, stressful events, coping, and emotional-behavioral responses are related to each other in an ongoing process. For instance, although a certain work event may be dealt with successfully on Monday, the same event may become stressful again on Friday, if support from colleagues is lacking. Due to the heterogeneity of stressors and the ever changing and complex coping processes, reactivity to stressors in daily life may not show high stability over time. Results of our field study did not provide support for significant individual differences in cortisol responses to daily events; cortisol responses were more dependent on current negative moodl states. Since the current study was one of the first to investigate the generalizability of cortisol reactivity from the lab to the field, more research is necessary to answer this important guestion.

\section{CLINICAL IMPLICATIONS}

Results indicated that the experience of distress in daily life situations can indeed lead to mild HPA hyperactivity. Whether these mild elevations of cortisol levels could be a marker of increased risk for disease processes can only be speculated upon. In general, the physiological function of stress-induced increases in glucocorticoid levels is thought to be adaptive. Although there are several theories linking adrenocortical responses following psychosocial stress to later pathology, there is a general lack of knowledge about the mechanisms involved in the stressillness relationship, and we do not know, for example, how stable the cortisol elevations are, or whether such elevations are substantial enough to have clinical relevance. There are currently no epidemiological studies in which the progressive course and consequences of HPA-axis abnormalities have been investigated. The available data are largely based on animal studies or on correlational designs. There is, for instance, evidence from animal studies which suggests that genetic variation in the stress response plays an important role in illness; while low HPA responsiveness is linked to autoimmune disease, high HPA responsiveness appears to be related to infectious diseases (Mason, 1991; Sternberg et al., 19891).

The costs of chronic elevated cortisol levels may turn out to be more important in predisposing the organism to disease than acute reactivity. Possibly only when stress is frequent and prolonged, without the opportunity to fully recover, will the association between stress and disease become apparent in subsequent processes of adaptation or exhaustion, when suppression of various defense mechanisms proceed far beyond physiologicai needs. With respect to somatic pathology, chronically elevated cortisol levels cause many adverse effects (McEwen \&. Stellar, 1993), with main effectors the immune system (Cohen et al., 1991; Kiecolt-Glaser \&, Glaser, 1991), the cardiovascular system (Brindley \& Rolland, 1989; Jacobs, Friedman, \& Mittleman, 1992), and the adipose tissue and muscle (Cox \& Gonder-Frederick, 1991). With regard to psychiatric disorders, elevated cortisol levels and other HPA disturbances are associated with major depression (Linkowski et al., 1985). In some cases, HPA. abnormalities persist after clinical recovery of depression and are indicative of poor prognosis (Charles, Schittecatte, Rush, Panzer, \& Wilmotte, 1989). On the other hand. low cortisol levels have been associated with post-traumatic stress disorder, which is thought to reflect a chronic adaptation in stress-induced. HPA activation in the form of a heightened feedback sensitivity (Yehuda et al., 1993). It remains unclear. however, whether these HPA disturbances are involved in the etiology of the 
disorder. Interesting in this regard are theories concerning how acute stressful events can result in long lasting biological effects on the organism. It has been postulated that psychosocial stressors may result in alterations at the level of gene regulation and expression; here, the HPA axis is thought to play an important mediating role (Checkley, 1992; Gold, 1988; Holsboer, 1992; Post, 1992). Evidence is mounting from animal studies that the nervous system, especially the hippocampus, is subject to wear and tear as a result of stressful experiences (Jacobson \& Sapolsky, 1991: Sapolsky, Krey, \& McEwen, 1986). Since the hippocampus is very important for mood, learning. and memory processes and subtle regulation of the HPA axis during mental stress, changes in hippocampal functioning may be extremely important for understanding the mechanisms in stress-related disorders, especially psychiatric disorders.

Although chronic levels may be more important than acute reactivity for future disease, the effects of acute stress may synergize with the actions of chronic stress, as has been shown in cardiovascular disease (Jacobs et al., 1992). Persistent increases in cortisol levels may indicate heightened biological vulnerability that increases the probability of dysregulation. This probability of dysregulation is thought to increase substantially when the vulnerable system is challenged by environmental and psychosocial challenges (Axelrod \& Reisine, 1984). Since stressful daily events are very common, especially in the HS group, one might expect frequent provocation of biological instability from these events in vulnerable individuals, and particularly when stress responses fail to habituate upon repeated exposure. Although it seems unlikely that minor events may initiate acute disorder in a stably regulated system, they may lead to disorder in the long run or may help maintain chronic disorder in dysregulated systems (Depue \& Monroe, 1986). Given the relationship between negative affectivity and increased cortisol levels, and between perceived stress (being strongly related to negative affectivity) and increased mood reactivity, it seems possible that stable individual characteristics may contribute to a more general susceptibility or a heightened vulnerability to the development of disorder.

\section{DIRECTIONS FOR FUTURE RESEARCH}

In this final section, a number of suggestions will be made for possible future research into the (daily) stress-distress relationship. As emphasized earlier, there is a general need for prospective longitudinal research, with repeated measurements of the stress-distress relationship over sufficiently long periods of time. It may be useful to combine ESM and traditional research methods (Alliger \& Williams, 1993), since both immediate and long-term assessments of stress variables are meaningful, and ESM can increase our understanding of the mechanisms underlying long-term outcomes as assessed by traditional methods (e.g. prospective surveys). Results obtained in the field should then be investigated in laboratory experiments to see if these results can be replicated. In addition, an interdisciplinary approach will have to be used (McEwen \& Stellar, 1993), involving various psychological, behavioral, neurobiological, endocrine, and immunologic measures, as well as their interactions with each other. Person factors (for example negative affectivity, coping style), stress measures (perceived stress, life events, chronic difficulties, daily events), state. measures (mood states, psychosomatic symptoms), and physiological measures (e.g. neuroendocrine parameters) can than be investigated simultaneously. From the present study, it may seem that all important hormonal effects of psychosocial stress relevant for health are related to cortisol, but this is certainly not true. Stress can not. 
only induce increases in cortisol or catecholamine levels, but has also been related to changes in levels of vasopressin (Axelrod \&. Reisine, 1984), prolactin (Meyerhof, Oleshansky, \& Mougey, 1988), b-endorphin. (Meyerhof et al., 1988), testosterone (Rose, 1984), and other hormones.

Follow-up assessments of the subjects investigated in the present study could shed light on some important but complex questions. For example: How stable is negative affectivity over time and how stable is the negative affectivity - perceived stress, negative mood, cortisol relationship? How stable are cortisol levels and cortisol responses to events and how does stability relate to person characteristics? What are the factors predicting disease, absenteeism, disability versus health? With respect to the health consequences of stress, up till now, most life stress studies included only general indicators of emotional or somatic disorder. Moving away from general models, it may be important to include measures of specific disorders like atherosclerosis, angina pectoris, or respiratory infections, since the mechanisms underlying stress-disorder interactions may vary across disorder and may only be understood by analyzing specific disorders (Depue \& Monroe, 1986). For instance, while personality and coping behavior may play a dominant role in the onset or maintenance of certain clisorders, other disorders may be more strongly related to environmental factors or health behaviors.

In general, representative samples of the general population and, more importantly, of high risk groups (e.g. subjects with a genetic vulnerability for psychiatric disorders, subjects in high risk jobs like health and educational employees) should preferably be observed pre-morbidly and then followed longitudinally. Further research should also focus on women, since there is considerable evidence to suggest that there are differences not only in the kind of daily' stressors women experience (Folkman \& Lazarus, 1980: Frankenhaeuser et al., 1989), but also in physiological (including neuroendocrine) responses to stress (Frankenhaeuser, 1980; Kirschbaum et al., 1992c). In addition, several factors of theoretical importance to the stress process lacking in the present study should ideally be included in such a study: protective factors, such as positive events, social support. and coping strategies; health parameters, such as records of absenteeism, disability and health status, and other physiological measures like cardiovascular measures, day-time catecholamines, and immunological parameters.

Such a study may shed additional light on the relative impact of temperamental and environmental influences on psychological states (anxiety, depression), HPA and immune function, and somatic health (Ormel \& Wohlfarth, 1991). For instance, how do life events, chronic difficulties, and daijy events predict change and stability in psychological distress levels and physiological levels, controiling for important person characteristics like negative affecuvity? Despite a paucity of longitudinal data, several models for the relationship between personality, stressful life events, and well-being have been proposed (Headey \&. Wearing. 1989). Personality models state that wellbeing is solely a function of personality and will therefore be highly stable over time (Costa \& McCrae, 1980). Adaptation level models stipulate that adaptation to events is so rapid and so complete that well-being is not measurably affected by events (Brickman, Coates, \& Janoff-Bulman, 1978; Diener, 1984). Other models have been proposed that treat life events as wholly exogenous stressors with allegedly damaging effects on physical and mental health (Block \& Zautra, 1981). Criticism relating to methodology and model specification as well as meager empirical support for these models have led to the development of other models. A model that has received some empirical support is the dynamic equilibrium model (Duncan-Jones. 
Fergusson, Ormel, \& Horwood, 1990; Headey \& Wearing, 1989). In this model each person is regarded as having 'normal' equilibrium levels of life events and well-being, dependent on stable psychobiological and environmental characteristics. These stable characteristics vary from person to person. Only deviations from normal event levels (internal or external) change the normal level of well-being. Deviations are usually temporary because stable personality traits, time, and support (for example treatment) play an equilibrating function. Another model is the dynamic-vulnerability model (Ormel, 1995), which differs from the previous mentioned model in that it postulates that vulnerability can increase or decrease considerably during the life course following certain internal or external events. Cumulation of vulnerability can occur as a consequence of earlier episodes (Post, 1992). The validity of this model has not yet been thoroughly investigated. Although these models emphasize major life events, they could easily be extended to include chronic difficulties and minor daily events. Only longitudinal studies can test the validity of the various models. It should be noted here that with assessing the relative predictive power of environmental and psychosocial factors on the one hand, and stable person attributes on the other hand, it is not meant that both can be measured independently or that this is a necessity. Although care should be taken that events are not merely reflections of symptomatic impairment (through use of symptom-free event measures and attempts to determine the independence of events), personality factors will influence the generation of most events and vice versa. As formulated by Lazarus and Folkman (1986, p.77): "there is simply no way to define an event as a stressor without referring to the properties of persons that make their well-being in some way vulnerable to that event."

From a practical point of view, knowledge about the relative contribution of personality or environmental factors on well-being may provide important information for intervention programs designed to reduce personal distress. In cases where environmental factors appear to play no role; interventions should primarily focus on strategies that are not tied to stressful situations but that are solely directed at the person (for example relaxation techniques, reducing worry-proness). In the other case, we need to pay more attention to teaching the person how to deal with his psychosocial environment (for example increasing control by teaching adaptive coping strategies, restructuring of dysfunctional cognitions, increasing assertiveness, time-management, and avoiding stressors by stimulus control). Since the evidence so far points to a combination of a vulnerable personality structure and objectively stressful situations as determinants of well-being, intervention programs should be directed at both the person and his environment. By using diaries like those in the present study, valuable information can be obtained about a person's idiosyncratic vulnerabilities relating to his environment (stressful events), personality (event appraisals, stress reactivity), adaptive capacities (coping strategies), and well-being (anxiety, depression, somatic complaints). These personalized diaries provide a rich source of data on which effective interventions for that specific individual can be based, as well as a means of evaluating their effects. Although this last paragraph emphasizes personal responsibility for health and well-being and seems the most sensible and successful strategy in the short term, an interesting question for the future relates to the responsibility of society as a whole. It may be that by increasing our demands on the individual as a result of growing economic pressures, an increasing number of people will not be able to meet the new requirements. The question here is whether these people should be considered ill and deviating from 'normal', or whether society has lost sight of what is 'normal'. 



\section{REFERENCES}

Aagaard, J. (1984). Stressful life events and illness: A review with special reference to a criticism of the life event method. In J. Cullen \& J. Siegriest (Eds.), Breakdown inhuman adaptation to stress. Towards a multidisciplinary approach. Boston: Martinus Nijhof Publishers.

Abelson, J. L.. \& Curtis, G. C. (1996). Hypothalamic-Pituitary-Adrenal axis activity in panic disorder: prediction of long-term outcome by pretreatment cortisol levels. American Journal of Psychiatry, 153(1), 69-73.

Affleck, G.. Tennen, H., Urrows, S., \& Higgins, P. (1994). Person and contextual features of daily stress reactivity: Individual differences in relations of undesirable daily events with mood disturbance and chronic pain intensity. Journal of Personality and Social Psychology, 66(2), 329-340.

Allen. P. I. M., Batty, K. A., Dodd, C. A. S., Herbert, J., Hugh, C. J., Moore, G. F., Seymour, M. J., Shiers, H. M., Stacey, P. M., \& Young. S. K. (1985). Dissociation between emotional and endocrine responses preceding an academic examination in male medical students. Journal of Endocrinology, 107, 163-170.

Alliger, G. M., \& Williams, K. J. (1993). Using signal-contingent experience sampling methodology to study work in the field: A discussion and illustration examining task perceptions and mood. Personnel Psychology, 46, 525-549.

Ansseau, M., Papart, P., Pitchot, W., Timsit-Berthier, M., Legros, J. J., \& von Frenckel, R. (1992). Dexamethasone suppression test and prediction of treatment response to selective antidepressants. European Psychiatry. 7, 191-194.

Ansseau, M., Sulon, J., Doumont. A., Cerfontaine, J. L., Legros, J. J., Sodoyez, J. C., \& DemeyPonsart. E. (1984). The use of saliva cortisol in the dexamethasone suppression test. Psychiatry Research, 13, 201-211.

Ametz, B. B., Brenner, S.-O., Levi, L., Hjelm, R., Petterson, I.-L., Wasserman, J., Petrini, B., Eneroth, P., Kallner, A., Kvetnansky, R., \& Vigas, M. (1991). Neuroendocrine and immunologic effects of unemployment and job insecurity. Psychotherapy and Psychosomatics.

Ametz, B. B., \& Fjellner, B. (1986). Psychological predictors of neuroendocrine responses to mental stress. Journal of Psychosomatic Research, 30, 297-305.

Arrindell, W. A., \& Ettema, H. (1981). Dimensionele structuur, betrouwbaarheid en validiteit van de Nederlandse bewerking van de Symptom Checklist (SCL-90); gegevens gebaseerd op een fobische en een 'normale' populatie. Nederlands Tijdschrift voor Psychologie, 36, 77-108.

Arrindell, W. A., \& Ettema, H. (1986). SCL-90: Handleiding bij een muitidimensionele psychopathologie indicator. Lisse: Swets \& Zeitlinger B.V.

Attanasio, V., Andrasik, F., Bilanchard, E. B., \& Arena, J. G. (1984). Psychometric properties of the SUNYA revision of the Psychosomatic Symptom Checklist. Journal of Behavioral Medicine, $7(2), 247-2.58$.

Averill, J. R. (1973). Personal control over aversive stimuli and its relationship to stress. Psychological Bulletin, 80(4), 286-303.

Axelrod, J., \& Reisine, T. D. (1984). Stress hormones: Their interaction and regulation. Science, 224. 452-459.

Bandura, A., Taylor, C. B., Williams, S. L., Mefford, I. N., \& Barchas, J. D. (1985), Catecholamine secretion as a function of perceived coping self-efficacy. Journal of Consulting and Clinical Psychology, 53(3), 406-414.

Barling, J., \& Kryl, I. P. (1990). Moderators of the relationship between daily work stressors and mood. Work \& Stress, 4(4), 319-329.

Bassett, J. R. (1982). Psychological stress and the coronary artery in ischemic heart disease. In S. Kalsner (Eds.), The coronary artery (pp. 474-500), London: Croom Helm. 
Bassett, J. R., \& Cairncross, K. D. (1977). Changes in the coronary vascular system following prolonged exposure to stress. Pharmacology Biochemistry \& Behavior, 6, 311-318.

Bassett, J. R., Marshall, P. M., \& Spillane, R. (1987). The physiological measurement of acute stress (public speaking) in bank employees. International Journal of Psychophysiology, 5(4), $265-273$.

Baum, A., Fleming, R., \& Reddy, D. M. (1986). Unemployment stress: loss of control, reactance and learned helplessness. Social Science and Medicine, 22(5), 509-516.

Baum, A. S., Lundbeg, U., Grunberg, N. E., Singer. J. E., \& Gatchel, R. J. (1985). Urinary catecholamines in behavioral research on stress. In C. R. Lake \& M. G. Ziegler (Eds.), The Catecholamines in Psychiatric and Neurologic Disorders (pp. 55-72). Stoneham: Butterworth Publishers.

Bebbington, P. E., Tennant, C., \& Hurry, J. (1981). Adversity and the nature of psychiatric disorder in the community, Journal of Affective Disorders, 3, 345-366.

Ben-Aryeh, H., Roll, R., Kahana, L., \& al., e. (1985). Saliva as an indicator of stress. International Journal of Psychosomatics, 32, 3-8.

Berger, M., Bossert, S., Krieg. J. C., Dirlich, G., Ettmeier, W., Schreiber, W., \& von Zerssen, D. (1987). Interindividual differences in the susceptibility of the cortisol system: An important factor for the degree of hypercortisolism in stress situations? Biological Psychiatry, 22, 1327. 1339.

Block, J., \& Zautra, A. (1981). Satisfaction and distress in a community: a test of the effect of life events. American Journal of Community Psychology, 9, 165-180.

Bohnen, N., Nicolson, N., Sulon, Jl., \& Jolles, J. (1991). Coping style, trait anxiety and cortisol reacivity during mental stress. Journal of Psychosomatic Research, 35(2/3), 141- 147.

Bolger, N. (1990). Coping as a personality process: A prospective study. Journal of Personality and Social Psychology, 59, 525-537.

Bolger, N., DeLongis, A.. Kessler, R. C., \& Schilling, E. A. (1989a). Effects of daily stress on negative mood. Journal of Personality and Social Psychology, 57(5), 808-818.

Bolger, N., Delongis. A., Kessler, R. C., \& Wethington, E. (1989b). The contagion of stress across multiple roles. Journal of Marriage and the Family, 51, 175-183.

Bolger, N.. \& Schilling, E. A. (1991). Personality and the problems of everyday life: The role of neuroticism in exposure and reactivity to daily stressors. Journal of Personality, 59(3), 355386.

Bossert, S., Berger, M., Krieg, J. C., Schreiber, W., Junker, M., \& von Zerssen, D. (1988). Cortisol response to various stressful situations: Relationship to personality variables and coping styles. Neuropsychobiology, 20, 36-42.

Bourne, P. G., Rose, K. M., \& Mason, J. W. (1967). Urinary 17-OHCS levels. Data on seven helicopter ambulance medics in combat. Archives of General Psychiatry, 17, 104- I 10.

Bower, G. H. (1981). Mood and memory. American Psychologist, 36(2), 129-148.

Brandstatter. H. (1983). Emotional responses to other persons in everyday life situations. Journal of Personality and Social Psychology. 45(4), 871-883.

Brandtstädter, J., Baltes-Gotz, B., Kirschbaum, C.., \& Hellhammer, D. (1991). Developmental and personality correlates of adrenocortical activity as indexed by salivary cortisol: Observations in the age range of 35 to 65 years. Journal of Psychosomatic Research, 35(2/3), 173- 185.

Brantley, P., Dietz, L., McKnight, G. T., Jones, G. N., \& Tulley, R. (1988). Convergence between the Daily Stress Inventory and endocrine measures of stress. Journal of Consulting and Clinical Psychology, 56, 549-551.

Brantley, P. J., Waggoner, C. D., Jones, G. N., \& Rappaport, N. B. (1987). A daily stress inventory: Development, reliability, and validity. Joumal of Behavioral Medicine, 10(1), 61-74.

Brickman, P.. Coates, D., \& Janoff-Bulman, R. (1978). Lottery winners and accident victims: Is happiness relative? Journal of Personality and Social Psychology, 36, 917-927. 
Brindley, D., \& Rolland, Y. (1989). Possible connections between stress, diabetes, obesity, hypertension and altered lipoprotein metabolism that may result in atherosclerosis. Clinical Science, 77, 453-461.

Brown, G. W. (1974). Meaning, measurement, and stress of life events. In B. S. Dohrenwend \& B. P. Dohrenwend (Eds.), Stressful Life Events: Their Nature and Effects (pp. 217-243). New York: Willey \& Sons.

Brown, G. W. (1989). Life events and measurement. In G. W. Brown \& T. O. Harris (Eds.), Life events and illness (pp. 3-45). London: Guilford Press.

Brown, G. W. (1993). Life events and affective disorder: Replications and limitations. Psychosomatic Medicine, 55, 248-259.

Brown, G. W., \& Harris, T. (1978). Social origins of depression: A study of psychiatric disorder in women. London: Tavistock.

Brown, G. W., \& Harris, T. O. (Ed.). (1989). Life Events and lllness. London: Guilford Press.

Brown, W. A., Sirota, A. D., Niaura, R., \& Engebretson, T. O. (1993). Endocrine correlates of sadness and elation. Psychosomatic Medicine, 55, 458-467.

Brugha, T., Bebbington, P., Tennant, C., \& Hurry, J. (1985). The list of threatening experiences: A subset of 12 life event categories with considerable long-term contextual threat. Psychological Medicine, 15, 189-194.

Brugha, T. S., \& Conroy, R. (1985). Categories of depression: Reported life events in a controlled design. British Journal of Psychiatry, 147, 641-646.

Brugha, T. S., \& Cragg, D. (1990). The list of threatening experiences: the reliability and validity of a brief life events questionnaire. Acta Psychiatrica Scandinavica, 82, 77-81.

Bruijn, J. V. d. (1988). Bedrijfsfitness, vorm van secundaire arbeidsvoorwaarde. Arbeidsomstandigheden, 64(7), 474-477.

Bryk, A. S., \& Raudenbush, S. W. (1987). Application of hierarchical linear models to assessing change. Psychological Bulletin, 101(1), 147-158.

Bryk, A. S., \& Raudenbush, S. W. (1992). Hierarchical Linear Models: Applications and Dafa Analysis Methods. London: Sage Publications.

Bullinger, M., Naber, D., Pickar, D., Cohen, R. M., Kalin, N. H., Pert, A., \& Bunney, W. E. (1984). Endocrine effects of the cold pressor test: Relationship to subjective pain appraisal and coping. Psychiatry Research, 12, 227-233.

Calabrese, J. R., Kling, M. A., \& Gold, P. W. (1987). Alterations in immunocompetence during stress, bereavement, and depression: Focus on neuroendocrine regulation. The American Journal of Psychiatry, 144(9), 1123- 1134.

Cameron, P. (1975). Mood as an indicator of happiness: Age, sex, social class, and situational differences. Journal of Gerontology, 30, 216-224.

Campbell, J. D., Chew, B., \& Scratchley, L. S. (1991). Cognitive and emotional reactions to daily events: The effects of self-esteem and self-complexity. Journal of Personality, 59(3), 473-505.

Cannon, W. B. (1929). Organization for physiological homeostasis. Physiological Review, 9. 399.

Cantor, N., Norem, J., Langston, C., Zirkel, S., Fleeson, W., \& Cook-Flannagan, C. (1991). Life tasks and daily life experience. Journal of Personality. 59(3), 425-451.

Caplan, R. D., Cobb, S., \& French, J. R. P., Jr. (1979). White collar work load and cortisol: Disruption of a circadian rhythm by job stress? Journal of Psychosomatic Research, 23 , $181-192$.

Carroll, B. J. (1985). Dexamethasone suppression test: a review of contemporary confusion. Journal of Clinical Psychiatry, 46(2), 13-24.

Caspi, A., Bolger, N., \& Eckenrode, J. (1987). Linking person and context in the daily stress process. Journal of Personality and Social Psychology, 52(1), 184-195. 
Charles, G. A., Schittecatte, M., Rush, A. J., Panzer, M., \& Wilmotte, J. (1989). Persistent cortisol non-suppression after clinical recovery predicts symptomatic relapse in unipolar depression. Journal of Affective Disorder, 17, 271-278.

Checkley, S. (1992). Neuroendocrine mechanisms and the precipitation of depression by life events. British Journal of Psychiatry, 160(suppl. 15), 7-17.

Chen, P. Y.. \& Spector, P. E. (1991). Negative affectivity as the underlying cause of correlations between stressors and strains. Journal of Applied Psychology. 76(3), 398-407.

Chrousos, G. P., \& Gold, P. W. (1992). The concepts of stress and stress system disorders. Overview of physical and behavioral homeostasis. Journal of the American Medical Association, 267(9), 1244-1252.

Claman, H. N. (1977), Corticosteroids and lymphoid cells. New England Journal of Medicine, $287,388-397$.

Clark, L. A., \& Watson, D. (1988). Mood and the mundane: Relations between daily life events and self-reported mood. Journal of Personality and Social Psychology, 54(2), 296-308.

Clark, L. A., Watson, D., \& Mineka, S. (1994). Temperament, personality, and the mood and anxiety disorders. Journal of Abnormal Psychology, 103(1), 103- 116.

Coeck, C., Jorens, P. G., Vandevivere, J., \& Mahler, C. (1991). ACTH and cortisol levels during residency training. The New England Journal of Medicine, 325(10), 738.

Cohen . S. (1978). Environmental load and the allocation of attention. In A. Baum. J. Singer, \& S. Valins (Eds.), Advances in environmental psychology. Hillsdale: Lawrence Erlbaum.

Cohen. S. (1980). Aftereffects of stress on human performance and social behavior: A review of research and theory. Psychological Bulletin, 82-108.

Cohen, S. (1986). Contrasting the Hassles Scale and the Perceived Stress Scale: Who's really measuring appraised stress? American Psychologist, 41, 716-718.

Cohen, S., Kamarck, T., \& Mermelstein, R. (1983). A global measure of perceived stress. Journal of Health and Social Behavior, 24, 385-396.

Cohen, S., Tyrrell, D. A. J., \& Smith, A. P. (1991). Psychological stress and susceptibility to the common cold. The New England Journal of Medicine, 325(9), 606-612.

Cohen, S., \& Williamson, G. M. (1988). Perceived stress in a probability sample of the United States. In S. Spacepan \& S. Oskamp (Eds.). The Social Psychology of Health (pp. 31-67). Newbury Park, CA: Sage Publications.

Collins, A. C.. \& Frankenhaeuser, M. (1978). Stress responses in male and fernale engineering studenis, Journal of Human Stress, 4(2), 43-48.

Cook, N. I. Ng, A., Read, G. F., Harris, B., \& Riad-Fahmy, D. (1987). Salivary cortisol for monitoring adrenal activity during marathon runs. Hormone Research, 25, 18-23.

Costa, P. T., \& McCrae, R. R. (1980). Influence of extraversion and neuroticism on subjective well-being: Happy and unhappy people. Journal of Personality and Social Psychology, 38(4), 668-678.

Costa, P. T. \& McCrae, R. R. (1986). Personality stability and its implications for clinical psychology. Clinical Psychological Review, 6, 407-423.

Costa, P. T., \& McCrae, R. R. (1987). Neuroticism, somatic complaints, and disease: Is the bark worse than the bite? Journal of Personality, 55(2), 299-316.

Cox, D. J., Freundlich, A., \& Meyer, R. G. (1975). Differential effectiveness of electromyograph feedback, verbal relaxation instructions and medication placebo with tension headaches. Journal of Consulting and Clinical Psychology. 43, 892-898.

Cox, D. J., \& Gonder-Frederick, L. A. (1991). The role of stress in diabetes mellitus. In P. McCabe,N. Schneidermann,T. M. Field, \& J. S. Skyler (Eds.), Stress, Coping and Disease (pp. 118-134). New York: L Erlbaum Assoc,

Cox, T., Gotts, G., Boot, N., \& Kerr, J. (1988). Physical exercise, employee fitness and the management of health at work. Ergonomics, 24, 795-806. 
Csikszentmihalyi, M., \& Larson, R. (1984). Being adolescent: Conflict and growth in the teenage years. New York: Basic Books.

Csikszentmihalyi, M., \& Larson, R. (1987). Validity and reliability of the experience-sampling method. The Journal of Nervous and Mental Disease, 175(9), 526-536.

Cummins, S. E., \& Gevirtz, R. N. (1993). The relationship between daily stress and urinary cortisol in a normal population: An emphasis on individual differences. Behavioral Medicine, 19, 129. 134.

Curtis, G. C. (1972). Psychosomatics and chronobiology: Possible implications of neuroendocrine rhythms. Psychosomatic Medicine 34(3), 235-256.

De La Torre, B. (1994). Psychoendocrinologic mechanisms of life stress. Stress Medicine, 10. 107-114.

de Vries, M. W. (1987). Introduction: Investigating mental disorders in their natural settings. Journal of Nervous and Mental Disease, 175(9), 509-513.

de Vries, M. W. (1992). The Experience of Psychopathology: Investigating Mental Disorders in their Natural Settings. Cambridge: University Press.

de Vries, M. W., \& Delespaul, P. A. E. G. (1992). Variability of schizophrenia symptoms. In M. W. de Vries (Eds.). The experience of psychopathology: Investigating mental disorders in their natural settings (pp. 97-109). Cambridge: Cambridge University Press.

Dekaris, D., Sabioncello, A., Mazuran, R., Rabatic, S., Svoboda-Beusan, I., Racunica, N. L., \& Tomasic, J. (1993). Multiple changes of immunologic parameters in prisoners of war. Assessments after release from a camp in Manjaca, Bosnia. Journal of the American Medical Association, 270(5), 595-599.

Delahunt, J. W., \& Mellsop, G. (1987). Hormone changes in stress. Stress Medicine, 3, 123-134.

Delespaul, P. A. E. G. (1992). Technical note: Devices and time-sampling procedures. In M. W. deVries (Eds.), The experience of psychopathology: Investigating mental disorders in their natural settings (pp. 363-376). Cambridge: Cambridge University Press.

Delespaul, P. A. E. G. (1995) Assessing schizophrenia in daily life-The Experience Sampling Method-. Thesis, University of Limburg, Maastricht, The Netherlands.

DeLongis, A., Coyne, J. C., Dakof, G., Folkman, S., \& Lazarus, R. S. (1982). Relationship of daily hassles, uplifts, and major life events to health status. Health Psychology, I(2), 119-136.

DeLongis, A., Folkman, S., \& Lazarus, R. S. (1988). The impact of daily stress on health and mood: Psychological and social resources as mediators. Journal of Personality and Social Psychology, 54(3), 486-495.

Demyttenaere, K., Nij̧s, P., Evers-Kiebooms, G., \& Koninckx, P. R. (1989). The effect of a specific emotional stressor on prolactin, cortisol, and testosterone concentrations in women varies with their trait anxiety. Fertility and Steriliry, 52(6), 942-948.

Depue, R. A., \& Monroe, S. M. (1986). Conceptualization and measurement of human disorder in life stress research: The problem of chronic disturbance. Psychological Bulletin, 99(1), 36-51.

Derogatis, L. R., Lipman, R. S., \& Cori, L. (1973). SCL-90, an outpatient rating scale -preliminary report. Psychopharmacology Bulletin, 9, 13-27.

Devereux, R. B., Pickering, T. G., Harshfield, G. A., (1983). Left ventricular hypertrophy in patients with hypertension: Importance of blood pressure response to regularly occurring stress. Circulation, 68, 470-476.

Dewe, P. J. (1991). Measuring work stressors: The role of frequency, duration and demand. Work \& Stress, 5, 77-91.

Diener, E. (1984). Subjective well-being. Psychological Bulletin, 45, 542-575.

Diener, E., Sandvikt E., \& Larsen, R. (1985). Age and sex effects for affect intensity. Developmental Psychology, 21, 542-546.

Dienstbier, R. A. (1989). Arousal and physiological toughness: Implications for mental and physical health. Psychological Review, 96(1), 84-100. 
Dijkman-Caes, C., \& deVries, M. W. (1991). Daily life situations and anxiety in panic disorder and agoraphobia. Journal of Anxiety Disorders, 5, 343-357.

Dijkman-Caes, C. I. M. (1993) Panic disorder and agoraphobia in daily life. Thesis, University of Limburg, Maastricht, The Netherlands.

Dijkstra, P. (1974). De zelfbeoordelingsschaal voor depressie van Zung. In: H. M. van Praag \& H. G. M. Rooymans (Eds.), Stemming en Ontstemming (pp. 98-120). Amsterdam; De Erven Bohn.

Dimsdale, J. E. (1984). Generalizing from laboratory to field studies of human stress physiology. Psychosomatic Medicine, 46(5), 463-469.

Dinan, T. G. (1994). Glucocorticoids and the genesis of depressive illness: A psychobiological model. British Journal of Psychiarry, 164, 365-371.

Dohrenwend, B. P., \& Shrout, P. E. (1985). "Hassles" in the conceptualization and measurement of life stress variables. American Psychologist, 40, 780-785.

Dohrenwend, B. S., \& Dohrenwend, B. P. (1974). Overview and prospects, for research on stressful life events. In B. S. Dohrenwend \& B. P. Dohrenwend (Eds.), Stressful Life Events: Their Nature and Effects (pp. 313-331). New York: Wiley, J. \& Sons.

Dohrenwend, B. S.. \& Dohrenwend, B. P. (1978). Some issues in research on stressful life events. The Journal of Nervous and Mental Disease, 166(1),7-15.

Dohrenwend, B. S.. Krassnoff, L., Askenasy, A. R., \& Dohrenwend, B. P. (1978). Exemplification of a method for scaling life events: The PERI Life Events Scale. Journal of Health and Social Behavior, 19, 205-229.

Duncan-Jones, P., Fergusson, D., Ormel, J., \& Horwood, L. (1990). A model of stability and change in minor psychiatric symptoms: results from three longitudinal studies. Psychological Medicine, Monograph Supplements, nr. 18. Cambridge:Cambridge University Press.

Eckenrode, J. (1984). Impact of chronic and acute stressors on daily reports of mood. Journal of Personality and Social Psychology, 46(4), 907-918.

Eckenrode, J., \& Bolger, N. (1995). Daily and within-day event measurement.. In S. Cohen, R. C. Kessler, \& L. Underwood Gordon (Eds.). Measuring stress: A guide for health and social scientists (pp. 80-101). New York: Oxford University Press.

Eysenck, H. J., \& Eysenck, S. B. G. (1985). Personality and' individual differences. New York: Plenum:

Eysenck, H. I.. \& Eysenk, S. B. (1968). Eysenck Personality Inventory. San Diego: CA: Educational and Industrial Testing Service.

Eysenck. H. L. (1967). The Biological Basis of Personality. Springfield, lllinois: Charles C Thomas.

Fulkenberg, L. E. (1987). Employee fitness programs: Their impact on the employee and the prganizationt. Academy Management Review, 12, 511-522.

Fergusson, D. M.. \& Horwood, L. J. (1987). Vulnerability to life event exposure. Psychological Medicine, 17, 739-749.

Fibiger, W., \& Singer, G. (1989). Biochemical assessment and differentiation of mental and physical effort. Work \& Stress. 3(3), 237-247.

Fibiger, W., Singer, G., Miller, A. J., Asmstrong. S., \& Datar, M. (1984). Cortisol and catecholamines changes as functions of time-of-day and self-reported mood. Neuroscience \& Biobehavioral Review.8, 523-530.

Fibiger, W. Singer, G. \& Miller, A. J. (1984). Relationships between catecholamines in urine and physical and mental effort. International Journal of Psychophysiology, I, 325-333.

Folkman. S. (1984). Personal control and stress and coping processes: a theoretical. analysis. Journal of Personality and Social Psychology, 46(4), 839-852.

Folkman, S., \& Lazarus, R. S. (1980). An analysis of coping in a middle-aged community sample. Journal of Health and' Social Behavior, 21, 219-239. 
Folkman, S., \& Lazarus, R. S. (1985). If it changes it must be a process: Study of emotion and coping during three stages of a college examination. Journal of Personality and Social Psychology, 48, 150-170.

Follenius, M., Brandenberger, G., Hietter, B., Simeoni, M., \& Reinhardt, B. (1982). Diurnal cortisol peaks and their relationship to meals. Journal of Clinical Endocrinology and Metabolism, 55, 757-761.

Forsman, L. (1980). Habitual catecholamine excretion and its relation to habitual distress. Biological Psychology, 11, 83-97.

Forsman, L. (1982). Consistency in catecholamine excretion in laboratory and natural settings: Correlational and variance components analysis. Scandinavian Journal of Psychology, 23, 99106.

Forsman, L., \& Lundberg, U. (1982). Consistency in catecholamine and cortisol excretion in males and females. Pharmacology Biochemistry \& Behavior, 17, 555-562.

Frankenhaeuser, M. (1975a), Experimental approaches to the study of catecholamines and emotion. In L. Levi (Eds.), Emotions: Their parameters and measurement (pp. 209-234). New York: Raven Press.

Frankenhaeuser, M. (1975b). Sympathetic-adrenomedullary activity, behavior, and the psychosocial environment. In P. H. Venables \& M. J. Christie (Eds.), Research in psychophysiology (pp. 71-94). New York: Wiley.

Frankenhaeuser, M. (1976). The role of peripheral catecholamines in adaptation to understimulation and overstimulation. In G. Serban (Eds.), Psychopathology of haman adaptation (pp. 173-191). New York: Plenum Press.

Frankenhaeuser, M. (1979). Psychoendocrine approaches to the study of emotion as related to stress and coping. In H. E. Howe \& R. A. Dienstbier (Eds.), Nebraska symposium on motivation 1978. Lincoln: University of Nebraska Press.

Frankenhaeuser, M. (1980). Psychoneuroendocrine approaches to the study of stressful personenvironment transactions. Selye's Guide to Stress Research, 1, 46-70.

Frankenhaeuser, M. (1983). The sympathetic-adrenal and pituitary-adrenal response to challenge: Comparison between the sexes. In T. M. Dembroski (Eds.), Behavioral Bases of Coronary Heart Disease (pp. 91-105). Basel: Karger.

Frankenhaeuser, M. (1986). A psychobiological framework for research on human stress and coping. In M. Appley \& R. Trumbull (Eds.), Dynamics of Stress (pp. 101-116). New York: Plenum.

Frankenhaeuser, M., \& Gardeli, B. (1976). Underload and overload in working life: Outline of a multidisciplinary approach. Journal of Human Stress, 2(3), 35-46.

Frankenhaeuser. M., \& Johansson, G. (1976). Task demand as reflected in catecholamine excretion and heart rate. Journal of Human Stress, 2, 15-23.

Frankenhaeuser, M., Lundberg, U., \& Forsman, L. (1980). Dissociation between sympatheticadrenal and pituitary-adrenal responses to an achievement situation characterized by high controllability. Comparison between type $\mathrm{A}$ and type $\mathrm{B}$ males and females. Biological Psychology, 10, 79-91.

Frankenhaeuser, M., Lundberg, U., Fredrikson, M., Melin, B., Tuomisto, M., Myrsten, A., Hedman, M., Bergman-Losman, B., \& Wallin, L. (1989). Stress on and off the job as related to sex and occupational status in white-collar workers. Journal of Organizational Behavior, 10 , 321-346.

Frankenhaeuser, M., Nordheden, B., Myrsten, A. L., \& Post, B. (1971). Psychophysiological reactions to understimulation and overstimulation. Acta Psychologica, 35, 298-308.

Fredrikson, M., Sundin, O., \& Frankenhaeuser, M. (1985). Cortisol excretion during the defense reaction in humans. Psychosomatic Medicine, 47(4), 313-319. 
Friedman, S. B., Mason, J. W., \& Hamburg, D. A. (1963). Urinary 17-hydroxycooid levels in parents of children with neoplastic disease. Psychosomatic Medicine, 25, 364-376

Gaillard, R.-C., \& Al-Damluji, S. (1987). Stress and the pituitary-adrenal axis. In A. Grossman (Eds.), Balliere's Clinical Endocrinology and Metabolism: Neuroendocrinology of Stress (pp. 319-354). London etc.: Balliere Tindall.

Gibbons, R. D., Hedeker, D., ELkin, I., Waternaux, C., Kraemer, H. C., Greenhouse, J. B., Shea, M. T., Imber, S. D., Sotsky, S. M., \& Watkins, J. T. (1993). Some conceptual and statistical issues in analysis of longitudinal psychiatric data. Archives of General Psychiatry, 50, 739-750.

Goetsch, V. L., Wiebe, D. J., Veltum, L. G., \& Van Dorsten, B. (1990). Case history and shorter communication. Behavior Research and Therapy, 28(6), 531 -537.

Gold, P. W., Goodwin, F. K., \& Chrousos, G. P. (1988). Clinical and biochemical manifestations of depression: Relation to the neurobiology of stress. Part II. New England Journal of Medicine, 3I9(7), 413-420.

Gold. W. (1988). Stress-responsive neuromodulators. Biological Psychiatry, 24" 371-374.

Goldberger, L., \& Breznitz, S. (1982). Handbook of stress. Theoretical and clinical aspects. New York: The Free Press.

Goldstein, H. (1987). Multilevel Models in Educational and Social Research. London: Charles Griffin \& Co.

Goplerud, E., \& Depue, R. A. (1985). Behavioral response to naturally occurring stress in cyclothymia and dysthymia. Journal of Abnormal Psychology, 94(2), 128-139.

Goreczny, A. J., Brantley, P. J., Buss, R. R., \& Waters, F. (1988). Daily stress and anxiety and their relation to daily fluctuations of symptoms in asthma and chronic obstructive pulmonary disease (COPD) patients. Journal of Psychopathology and Behavioral Assessment, 10(3), 259. 267.

Gottman, J. M., \& Levenson, R. W. (1988). The social psychophysiology of marriage. In P. Noller \& M. A. Fitzpatrick (Eds.), Perspectives on marital interaction (pp. 182-200). Philadelphia, PA: Multilingual Matters.

Gruchow, H. W. (1979). Catecholamine activity and infectious disease episodes. Journal of Human Siress, 5, 11-17.

Harburg, E., Julius, S., McGinn, N. F., McLeod, J., \& Hoobler, S. W. (1964). Personality traits and behavioral patterns associated with systolic blood pressure levels in college males. Journal of Chronic Diseases, 17, 405-414.

Headey, B., \& Wearing, A. (1989). Personality, life events, and subjective well-being: Toward' a dynamic equilibrium model. Journal of Personality and Social Psychology, 57(4), 731-739.

Hedeker, D. (1993). MIXREG, a Fortran prograrn for mixed-effects linear regression models. University of Illinois: Prevention Research Center.

Hellhammer, D. H., Kirschbaum, C., \& Belkien, L. (1987). Measurement of salivary cortisol under psychological stimulation. In J. Hingtgen, D. Hellhammer, \& G. Huppmann (Eds.), Advanced Methods in Psychobiology (pp. 281-289). Toronto: C. J. Hogrefe Inc.

Hendriks, A. A. J., Ormel, J., \& van de Willige, G. (1990). Langdurige moeilijkheden gemeten volgens zelfbeoordelingsvragenlijst en semi-gestructureerd interview. Gedrag en Gezondheid. 18(6), 273-283.

Hennessy. J. W., \& Levine, S. (1979). Stress, arousal, and the pituitary-adrenal system: A psychoendocrine hypothesis, Progress in Psychobiology and Physiological Psychology, 8. 133-178.

Hennig, J., Laschefski, U., \& Opper, C. (1994). Biopsychological changes after bungee jumping: beta-endorphin immunoreactivity as a mediator of euphoria? Neuropsychobiology, 29(1), 28-32.

Henry, J. P. (1982). The relation of social to biological processes in disease. Social Science and Medicine, 16, 369-380. 
Henry, J. P., \& Stephens, P. M. (1977). Stress, Health and the Social Environment: a Sociobiologic Approach to Medicine. New York: Springer-Verlag.

Hinkle, L. E. (1974). The concept of 'stress' in the biological and social sciences. International Journal of Psychiatry in Medicine, 5, 335-357.

Hofer, M. A., Wolff, C. T., Friedman, S. B., \& Mason, J. W. (1972a). A psychoendocrine study of bereavement PartI. 17-Hydroxycorticosteroid excretion rates of parents following death of their children from leukemia. Psychosomatic Medicine, 34(6), 481-491.

Hofer, M. A., Wolff, C. T., Friedman, S . B., \& Mason, J. W. (1972b). A psychoendocrine study of bereavement. Part II. Observations on the process of mourning in relation to adrenocortical function. Psychological Medicine, 34(6), 492-504.

Holmes, T. H., \& Masuda, M. (1974). Life change and illness susceptibility. In B. S. Dohrenwend \& B. P. Dohrenwend (Eds.), Stressful life events: Their nature and effects (pp. 45-72). New York: John Wiley \& Sons.

Holmes, T. H., \& Rahe, R. H. (1967). The social readjustment rating scale. Journal of Psychosomatic Research, 11, 213-218.

Holroyd, K. A., \& Lazarus, R. S. (1982). Stress coping and somatic adaptation. In L. Goldberger \& S. Breznitz (Eds.), Handbook of Stress: Theoretical and Clinical' aspects (pp. 21-35). New York: Free Press.

Holsboer, F. (1987). Psychoneuroendocrine strategies. Advances in Psychosomatic Medicine, 17. 185-233.

Holsboer, F. (1992). The hypothalamic-pituitary-adrenocortical system. In E. S. Paykel (Eds.), Handbook of affective disorders (pp. 267-287). New York: Churchill Livingstone Inc.

Hoon, E. F., Hoon, P. W., Rand, K. H., Johnson, J., Hall, N. R., \& Edwards, N. B. (1991). A psychobehavioral model of genital herpes recurrence. Journal of Psychosomatic Research, 35, 25-36.

Hormuth, S. E. (1985). Methoden für psychologische Forschung im Fels (Diskussionspapier Nr. 43). Heidelberg: Psychologischen Institut der Universitat Heidelberg.

Hormuth, S. E. (1986). The sampling of experiences. Journal of Personality, 54(1), 262-293.

Hosman, C. M. H. (1983). Psychosociale problematiek en hulpzoeken. Een sociaalepidemiologische studie ten behoeve van de preventieve geestelijke gezondheidszarg. Lisse: Swets \& Zeitlinger.

Houtman, I. L. D., \& Bakker, F. C. (1987). Stress in student teachers during real and simulated, standardized lectures. Journal of Human Stress, 13(4), 180-187.

Hubert, W., \& de Jong-Meyer, R. (1989). Emotional stress and saliva cortisoll response. J Clin Chem Clin Biochem, 27(4), 235-237.

Hubert, W., \& de Jong-Meyer, R. (1990). Psychophysiological response patterns to positive and negative film stimuli. Biological Psychology, 31, 73-93.

Hubert, W., \& de Jong-Meyer, R. (1991). Autonomic, neuroendocrine, and subjective responses to emotion-inducing film stimuli. Intermational Journal of Psychophysiology, 11, 131-140.

Huber, W. H., Moller, M., \& de Jong-Meyer, R. (1993). Film-induced amusement changes in saliva cortisol levels. Psychoneuroendocrinology, 18(4), 265-272.

Hurlburt, R. T. (1990). Sampling normal and schizophrenic inner experience. New York: Plenum Press.

Isenberg. S. A., Lehrer, P. M., \& Hochron, S. (1992). The effects of suggestion and emotional arousal on pulmonary function in asthma: a review and a hypothesis regarding vagal mediation. Psychosomatic Medicine, 54, 192-216.

Ivancevich, J. M. (1986). Life events and hassles as predictors of health symptoms, job performance, and absenteeism. Journal of Occupationai Behaviour, 7, 39-51.

Jaccard, J., \& Wan, C. K. (1993). Statistical analysis of temporal data with many observations: issues for behavioral medicine data. Annals of Behavioral Medicine, 15(1), 43-49. 
Jacobs, S. C., Friedman, R., \& Mittleman, M. (1992). 9-Fold increased risk of myocardial. infarction following psychological stress as assessed by a case-control study. Circulation, 86 (suppl), 198. Abstract.

Jacobson, L., \& Sapolsky, R. (1991). The role of the hippocampus in feedback regulation of the hypothalamic-pituitary-adrenocortical axis. Endocrine Review, 12, 118-134.

Jandorf, L., Deblinger, E., Neale, J. M.. \& Stone, A. A. (1986). Daily versus major life events as predictors of symptom frequency: A replication study. Journal of General Psychology, II(3), 205-218.

Johansson, G., Frankenhacuser, M., \& Magnusson, D. (1973). Catecholamine output in school children as related to performance and adjustment. Scandinavian Journal of Psychology, 14. 20-28.

Jones, K. V.. Copolev, D. L., \& Outsch, K. H. (1986). Type A, test performance and salivary cortisol. Journal of Psychosomatic Research, 30, 699-707.

Kahn, J.-P., Rubinow, D. R., Davis, C. L., \& al., e. (1988). Salivary cortisol: A practical methodi for evaluation of adrenal function. Biological Psychiatry, 23, 335-349.

Kanner, A. D., Coyne, J. C. Schacfer, C., \& Lazarus, R. S. (1981). Comparison of two modes of stress measurement: Daily hassles and uplifts versus. major life events. Journal of Behavioral Medicine, 4, 1-39.

Kapian, C. D. (1992). Drug craving and drug use in the daily life of heroin addicts, In M. W. deVites (Eds.). The experience of psychopathology: Investigating mental disorders in their natural settings (pp. 193-218). Cambridge: Cambridge Press.

Kaplan, H. D. (1979). Social psychology of disease. In H. G. Freeman,S. Levine, \& L. F. Reeder (Eds.), Handbook of Medical Sociology (pp. 53-70). Englewood Cliffs, N.J: PrenticeHall.

Kathol, R. G., Noyes, R., \& Lopez, A. (1988). Similarities in hypothalamic-pituitary-adrenal axis. acivity between patients with panic disorder and those experiencing external stress. Psychiatric Clinics of North America, II, 335-348.

Katz, R., \& Wykes, T. (1985). The psychological difference between temporally predictable and unpredictable stressiul events: evidence for information control theories. Journal of Personality and Social Psychology: 48(3), 781-790.

Kiecoll-Glaser, J. K. \& Glaser, R. (1991). Stress and immune function in humans. In R. Ader, D. L. Felten, \& N Cohen (Eds.), Psychoneuroimmunology (pp. 849-867), San Diego, California: Academic Press, Inc.

Kirschbaum, C., Bartussek, D., \& Strasburger, C. J. (1992a). Cortisol responses to psychologicar stress and correlations, with personality traits. Personality and Individual Differences, 13(12), 1353-1357.

Kirschbaum, C., \& Hellhammer. D. H. (1989). Salivary cortisol in psychobiological research: An overview. Neuropsychobiology, 22, 150-169.

Kirschbaum, C., \& Hellhammer, D. H. (1990). Methodological aspects of salivary cortisol measurement and its application in stress research (abstract). Journal of Clinical Chemistry and Clinical Biochemistry, 28, 649-066.

Kirschbaum, C., \& Helihammer, D. H. (1993). The 'Trier Social Stress Test' - a tool for investigating psychobiological stress responses in a laboratory setting. Neuropsychobiology, 28, 76-81:

Kirschbaum, C., \& Hellhammer, D. H. (1994). Salivary cortisol in psychoneuroendocrine research: Recent developments and applications. Psychoneuroendocrinology, 19(4), 313-333

Kirschbaum, C., Hellhammer, D. H., Strasburger. C. J., Tiling, E., Kamp, R., \& Luđdecke, H. (1989). Relationships between salivary cortisol, electrodermal activity and anxiety under mild experimental stress in children. In H. Weiner,I. Forin, R. Murison, \& D. Hellhammer (Eds.), Frontiers in Stress Research (pp. 383-387). Toronto: Hans Huber. 
Kirschbaum, C., Pruessner, J., Stone, A. A., Federenko, I., Gaab, J., Lintz, D;, Schommer, N, \& Hellhammer, D. (1995). Persistent high cortisol responses to repeated psychological stress in a subpopulation of healthy men. Psychosomatic Medicine, 57, 468-474.

Kirschbaum, C., Wust, S., Faig, H. G., \& Hellhammer, D. H. (1992b). Heritability of cortisol responses to human corticotrophin-releasing hormone, ergometry, and psychological stress in humans. Journal of Clinical Endocrinology and Metabolism. 75(6), 1520-1530.

Kirschbaum, C., Wust, S., \& Hellhammer, D. (1992c). Consistent sex differences in cortisol responses to psychological stress. Psychosomatic Medicine, 54, 648-657.

Kissinger, P. T., Riggin, R. M., Alcom, R. L., \& Rau, L.-D. (1975), Estimation of catecholamines in urine by high performance liquid chromatography with electrochemical detection. Biochemical Medicine, 13, 299-306.

Klinger, E., Barta, S., \& Maxeimer, M. (1980). Motivational correlates of thought content, frequency and commitment. Journal of Personality and Social Psychology, 39, 1222- 1237.

Kloet, E. R. d., \& Reul, J. M. H. M. (1987). Feedback action and tonic influence of corticosteroids on brain function: a concept arising from the heterogeneity of brain receptor systems. Psychoneuroendocrinology, 12, 85- 105.

Knepper, S. (1991). De epidemiologie van psychische arbeidsongeschiktheid. feiten en achtergronden. In R. Bijl \& D. Bauduin (Eds.), Categorie V. Arbeidsongeschiktheid wegens psychische stoornissen (pp. 7-26). Utrecht: NcGv.

Knight, R. B., Atkins, A., Eagle, C. J., Evans, N., Finkelistein, J. W., Fukushima, D., Katz, J., \& Weiner, H. (1979). Psychological stress, ego defenses, and cortisol production in children hospitalized for elective surgery. Psychosomatic Medicine, 41(1), 40-49.

Koeter, M. W. J., Ormel, J., \& van den Brink, W. (1988). Totaalscore op de SCL-90 als maal voor de ernst van psychopathologie. Nederlands Tijdschrift! voor de Psychologie. 43, 381-391.

Kraan, H., Meertens, H., Hilwig, M., Volovics, L., Dijkman-Caes, C., \& Portegijs, P. (1992). Selecting measures, diagnostic validity and scaling in the study of depression. in M. W. deVries (Eds.), The experience of psychopathology: Investigating mental disorders in their naturat settings (pp. 324-336). Cambridge: Cambridge Press.

Kugler, J., \& Kalveram, K. T. (1989), Is salivary cortisol related to mood states and psychosomatic symptoms? In H. Weiner (Eds.), Frontiers of Stress Research (pp. 389-391). New York Toronto: Hans Huber Publishers.

Laan, M. (1989, 20.04.89). De lange weg naar beter werk (3). Gezondmaking van bedrijven maakt stress tot volksziekte. Het Parool.

Larsen, R. J., Diener, E., \&. Emmons, R. A. (1986). Affect intensity and reactions to daily life events. Journal of Personality and Social Psychology, 5i, 803-814,

Larson, R., \& Delespaul, P. (1992). Analyzing experience sampling data: A guidebook for the perplexed. In M. W. de Vries (Eds.), The experience of psychopathology: Investigating mental disorders in their natural settings (pp. 58-78). Cambridge: Cambridge University Press.

Larson, R. S., \& Csikszentmihalyi, M. (1983). The experience sampling method. In H. R. Reis (Eds.), New directions for methodology of social and behavioral science: naturalistic approaches to studying social interaction. (pp. 41-46). San Francisco: Jossey-Bass.

Laundis, J. R., \& Koch, G. G. (1977). The measurement of observer agreement for categorical data. Biometrics, 33, 396-398.

Lazarus, R. S. (1966). Psychological stress and the coping process. New York: McGraw-Hill.

Lazarus, R. S. (1984). Puzzles in the study of daily hassies. Journal of Behavioral Medicine, 7(4), 375-389.

Lazarus, R. S., De Longis, A., Folkman, S., \&. Gruen, R. (1985). Stress and adaptational outcomes: The problem of confounded. measures. American Psychologist, 40, 770-779.

Lazarus, R. S., \& Folkman, S. (1984a). Coping and adaptation. In. W. D. Gentry (Eds.), Handbook of Behavioral Medicine (pp. 282-325). New York: Guilford Press. 
Lazarus, R. S., \& Folkman, S. (1984b). Stress, appraisal, and coping. New York: Springer.

Lazarus, R. S., \& Folkman, S. (1986). Cognitive theories of stress and the issue of circularity. In M. H. Appley \& R. Trumbull (Eds.), Dynamics of Stress. (pp. 63-80). New York: Plenum.

Lazarus, R. S., \& Launier, R. (1978). Stress-related transactions between person and environment. In L. A. Pervin \& M. Lewis (Eds.), Perspectives in Interactional Psychology (pp. 287-327). New York: Plenum.

Lewinsohn, P. M., \& Amenson, C. S. (1978). Some relations between pleasant and unpleasant mood-related events and depression. Journal of Abnormal Psychology, 87(6), 644-654.

Lewinsohn, P. M., \& Libet, J. (1972). Pleasant events, activity schedules, and depressions. Journal of Abnormal Psychology, 79(3), 291-295.

Lewinsohn, P. M., \& Talkington, J. (1979). Studies on the measurement of unpleasant events and relations with depression. Applied Psychological Measurement, 3, 83-101.

Linkowski, P., Mendlewicz, J., Leclereq, R., Brasseur, M., Hubain, P., Golstein, J., Copinschi, G., \& van Cauter, E. (1985). The 24-hour profile of adrenocorticotropin and cortisol in major depressive illness. Journal of Clinical Endocrinology and Metabolism, 61(3), 429-438.

Lousberg, R., Schmidt, A. J. M., Groenman, N. H., Vendrig, L., \&. Dijkman-Caes, C. (1995). Validating the MPI-DLV with Experience Sampling data. In H. B. R. Lousberg (Eds.), Chronic pain: Multiaxial assessment and behavioral mechanisms. Thesis, University of Limburg, Maastricht, The Netherlands.

Lundberg. U., \& Frankenhaeuser, M. (1980). Pituitary-adrenal and sympathetic-adrenal correlates of distress and effort. Journal of Psychosomatic Research, 24, 125-130.

Lundberg. U., Granqvist, M., Hansson, T., Magnusson, M., \& Wallin. L. (1989). Psychological and physiological stress responses during repetitive work at an assembly line. Work \& Stress, 3(2). 143-153.

Lundberg. U., \& Palm, K. (1989). Workload and catecholamine excretion in parents of preschool children. Work \& Stress, 3(3), 255-260.

Luteijn, F., \& Kok, A. R. (1985). Handleiding NVM. Herziene uitgave. Lisse: Swets \& Zeitlinger.

Lykken, D. T. (1982). Research with twins: the concept of emergenesis. Psychophysiology, 1.1. 249-270.

Madden, K. S., \& Livnat, S. (1991). Catecholamine action and immunologic reactivity. In R. Ader,D. L. Felten, \& N. Cohen (Eds.), Psychoneuroimmunology (pp. 283-310). San Diego. California: Academic Press, Inc.

Manuck, S. B., Kasprowicz, A. L., Monroe, S. M., Larkin, K.. T., \& Kaplan, J. R. (1989). Psychophysiologic reactivity as a dimension of individual differences. In $\mathbf{N}$. Schneiderman, S. M. Weiss, \& P. G. Kaufman (Eds.), Handbook of research methods in cardiovascular behavioral medicine (pp. 365-382), New York: Plenum.

Marcelissen (1989). Onderzoek naar stress in de werksituatie. In Verslag van het symposium 'Stress in de werksituatie: Beleid en preventie.14 december 1988, Den Haag (pp. 43-56). Leiden: NIPG/TNO.

Marco, C. A., \& Suis, J. (1993), Daily stress and the trajectory of mood: Spillover, response assimilation, contrast, and chronic negative affectivity. Journal of Personality and Social Psychology, 64(6), 1053-1063.

Martin, M., Ward, J. C., \& Clark. D. M. (1983). Neuroticism and the recal! of positive and negative personality information. Behaviour Research and Therapy, 21, 495-503.

Mason, D. (1991). Genetic variation in the stress response: susceptibility to experimental allergic encephalomyelitis and implications for human inflammatory disease. Immunology Todiay, 12, 57-60.

Mason. J. W. (1968). A review of psychoendocrine research on the pituitary-adrenal cortical system. Psychosomatic Medicine, 30, 576-607.

Mason, J. W. (1975). A historical view of the stress field. Journal of Human Stress, I(1), 7-12. 
Mason, J. W., Giller, E. L., Kosten, T. R., \& Harkness, L. (1988). Elevation of urinary norepinephrine/cortisol ratio in post-traumatic stress disorder. Journal of Nervous and Mental Disease, I76(8), 498-502.

Mason, J. W., Hartley, H., Kotchen, T. A., Mougey, E. H., Ricketts, P. T., \& Jones, L. G. (1973). Plasma cortisol and norepinephrine responses in anticipation of muscular exercise. Psychosomatic Medicine, 35(5), 406-414.

Massimini, F., Csiksentmihalyi, M., \& Carli, M. (1987). The monitoring of optimal experience. A tool for psychiatric rehabilitation. Journal of Nervous and Mental Disease, 175(9), 545-549.

McCrae, R. R. (1990). Controlling neuroticism in the measurement of stress. Stress Medicine, 6 , 237-241.

McCrae, R. R., \& Costa, P. T. (1986). Personality, coping, and coping effectiveness in an adult sample. Journal of Personality, 54(2), 385-405.

McEwen, B. S., \& Stellar, E. (1993). Stress and the individual. Mechanisms leading to disease. Archives of International Medicine, 153, 2093-2101.

McGrath, J. E., \& Beehr, T. A. (1990). Time and the stress process: Some temporal issues in the conceptualization and measurement of stress. Stress Medicine, 6, 93-104.

Meyerhof, J. L., Oleshansky, M. A., \& Mougey, E. H. (1988). Psychological stress increases plasma levels of prolactin, cortisol, and POMC-derived peptides in man. Psychosomatic Medicine, 50, 295-303.

Miller, S. M. (1979). Controllability and human stress: Method, evidence and theory. Behavior Research and Therapy, 17, 287-305.

Munck, A., Guyre, P. M., \& Holbrook, N. J. (1984). Physiological functions of glucocorticoids in stress and their relation to pharmacological actions. Endocrine Reviews, 5(1), 25-44.

Neale, J. M., Hooley, J. M., Jandorf, L., \& Stone, A. A. (1987). Daily life events and mood. In C. R. Snyder \& C. E. Ford (Eds.), Coping with Negative Life Events: Clinical and Social Psychological Perspectives (pp. 161-189). New York: Plenum.

Nicolson, N. A. (1992). Stress, coping and cortisol dynamics in daily life. In M. W. de Vries (Eds.), The Experience of Psychopathology: Investigating Mental Disorders in their Natural Settings (pp. 219-232). Cambridge: Cambridge University Press.

Nicolson, N. A., van Poll, R., \& deVries, M. (1992). Ambulatory monitoring of salivary cortisol and stress in daily life. In C. Kirschbaum,G. F. Read, \& D. Hellhammer (Eds.), Assessment of Hormones and Drugs in Saliva in Biobehavioral Research (pp. 163-173). Seattle: Hogrefe \& Huber.

Ockenfels, M. C., Porter, L., Smyth, J., Kirschbaum. C., Hellhammer, D. H., \& Stone, A. A. (1995). Effect of chronic stress associated with unemployment on salivary cortisol: Overall cortisol levels, diurnal rhythm, and acute stress reactivity. Psychosomatic Medicine, 57, 460-467.

Ormel, J. (1983). Neuroticism and well-being inventories: Measuring traits or states. Psychological Medicine, 13, 165-176.

Ormel, J. (1985). Her Groningse eerstelijnsproject: Opzet en aanpak. (Intern Rapport), Rijksuniversiteit Groningen, Afdeling Sociale Psychiatrie.

Ormel J. (1995). Etiologie van angst en depressie: kwetsbaarheid en losmakende factoren. In E. R. de Kloet \& V. M. Wiegant (Eds.), Stress en neuropathologie. Leiden: Boerhaave Commissie voor Postacademisch Onderwijs in de Geneeskunde, Rijksuniversiteit Leiden.

Ormel, J., \& Wohlfarth, T. (1991). How neuroticism, long-term difficulties, and life situation. change influence psychological distress. Journal of Personality and Social Psychology, 60(5), 744-755.

Ottenweller, J. E., Natelson, B . H., Pitman, D. L., \& Drastal, S. D . (1989) . Adrenocortical and behavioral responses to repeated stressors: toward an animal model of chronic stress and stressrelated mental illness. Biological Psychiatry, 26(8), 829-41. 
Paykel, E. S. (1974). Life stress and psychiatric disorder: Application of the clinical approach. In B. S. Dohrenwend \& B. P. Dohrenwend (Eds.), Stressful life events: Their narure and effects. (pp. 135-149), New York: John Wiley \& Sons.

Paykel, E. S., \& Dowlatshahi, D. (1988). Life events and mental disorder. In S. Fisher \& J. Reason (Eds.), Handbook of life stress, cognition and health (pp. 241-263). New York: Wiley.

Peeters, M. C. W., Buunk, B. P.. \& Schaufeli, W. B. (1995). A micro-analytic exploration of the cognitive appraisal of daily stressful events at work: the role of controllability. Anxiery, Stress, and Coping, 8, 127-139.

Pennebaker, J. W., \& Watson, D. (1988). Self-reports and physiological measures in the workplace. In J. J. Hurrell, L. R. Murphy, S. L. Sauter, \& C. L. Cooper (Eds.), Occupational Stress: Issues and Developments in Research (pp. 184-199). New York: Taylor \& Francis.

Perloff, D., Sokolow, M., \& Cowan, R. (1983). The prognostic value of ambulatory blood pressures. Journal of the American Medical Association, 249, 2793-2798.

Pfohl, B., Herman, B., \& Schlechte, J. (1985). Pituitary-adrenal axis rhythm disturbances in psychiatric depression. Archives of General Psychiatry, 42, 897-903.

Pincomb, G. A., Lovallo, W. R., Passey, W. R., Brackett, R. B., \& Wilson, M. F. (1987). Caffeine enhances the physiological' response to occupational stress in medical students. Health Psychology, 6, 101-112.

Pitman, R. K., \& Orr, S. P. (1990). Twenty-four hour urinary cortisol and catecholamine excretion in combat-related posttraumatic stress-disorder. Biological Psychiatry, 27, 245-247.

Pope, M. K., \& Smith, T. W. (1991). Cortisol excretion in high and low cynically hostile men. Psychosomatic Medicine, 53, 386-392.

Post, R. M. (1992). Transduction of psychosocial stress into the neurobiology of recurrent affective disorder. American Journal of Psychiatry, 149(8). 999-1011.

Prosser, R., Rasbach, J., \& Goldstein, H. (1991). ML3 Software for Three-level Analyses. Users" Guide for V.2. In London: Institute of Education, University of London.

Quigley, M. E., \& Yen, S. S. C. (1979). A. midday surge in cortisol levels. Journal of Clinical Endocrinology and Metabolism. 49, 945-947.

Rabkin, J. C., \& Streuning. E. L. (1976). Life events, stress, and illness. Science, 194, 1013-1020.

Rahe, R. H., \& Arthur, R. J. (1978). Life change and illness studies: past history and future directions. Journal of Human Stress, 4(2), 3-15.

Rahe, R. H., Karson, S., Howard, N. J., Rubin, R. T., \& Poland, R. E. (1990), Psychological and physiological assessments on American hostages freed from captivity in Iran. Psychosomatic Medicine, 52(1), 1-16.

Rahe, R. H., \& Lind, E. (1971). Psychosocial factors and sudden cardiac death: a pilot study. Journal of Psychosomatic Research, 6, 15-24.

Rehm, L. (1978). Mood, pleasant events, and unpleasant events: Two pilot studies. Journal of Consulting and Clinical Psychology, 46, 854-859.

Repetti, R. L. (1993). Short-term effects of occupational stressors on daily mood and health complaints. Health Psychology, 12(2), 125-131.

Rissler, A. (1977). Stress reactions at work and after work during a period of quantitative overload. Ergonomics, 20, 13-16.

Rose, R. M. (1984). Overview of endocrinology of stress. In G. M. Brown,S. H. Koslow, \& S. Reichlin (Eds.), Neuroendocrinology and Psychiatric Disorder (pp. 95-122), New York: Raven Press.

Rose, R. M., \& Fogg. L. F. (1993). Definition of a responder: analyses of behavioral, cardiovascular, and endocrine responses, to varied workload in air traffic controllers. Psychosomatic Medicine, 55, 325-338.

Rose, R. M., \& Hurst, M. W. (1975). Plasma cortisol and growth hormone responses to intravenous catheterization. Journal of Human Stress, $I(1), 22-36$. 
Rose, R. M., Jenkins, C. D., Hurst, M., Kreger, B. E., Barrett, J., \& Hall, R. P. (1982). Endocrine activity in air traffic controllers at work.III. Relationship to physical and psychiatric morbidity. Psychoneuroendocrinology, 7(2), 125-134.

Sapolsky, R., Krey, L., \& McEwen, B. S. (1986). Neuroendocrinology of stress and aging: the glucocorticoid cascade hypothesis. Endocrine Review, 7, 284-301.

Sarason, 1. G.. Johnson, J. H., \& Siegel, J. M. (1978). Assessing the impact of life changes: Development of the Life Experiences Survey. Journal of Consulting and Clinical Psychology, 5. 932-946.

Scarr, S., Webber, P. L., Weinberg, R. A., \& Wittig, M. A. (1981). Personality resemblance among adolescents and their parents in biologically related and adoptive families. Journal of Personality and Social Psychology, 40, 885-898.

Schaeffer, M. A., \& Baum. A. (1984). Adrenal cortical response to stress at Three Mile Island. The Journal of Nervous and Mental Disease, 46(3), 227-237.

Schaubroeck, J., Ganster, D. C., \& Fox, M. L. (1992). Dispositional affect and work-related stress. Journal of Applied Psychology, 77(3), 322-335.

Schedlowski, M., Jacobs, R., Stratmann, G., Richter, S., Hadicke, A., Tewes, U., Wagner, T. O., \& Schmidt, A. U. (1993). Changes of natural killer cells during acute psychological stress. Journal of Clinical Immunology, 13(2), 119-126.

Schreurs, P. J. G., \& van de Willige, G. (1988). De Utrechtse Copinglijst (UCL). Lisse, the Netherlands: Swets \& Zeitlinger.

Schroeder, D. H., \& Costa, P. T. (1984). Influence of life event stress on physical illness: Substansive effects or methodological flaws. Journal of Personality and Social Psychology, 46. 853-863.

Schroer, C. A. P., Nijhuis, F. J. N., \& van Zutphen, W. (1988). Gebruik en betekenis van de term overspanning in geneeskundige literatuur. Tijdschrift voor Sociale Gezondheidszorg, 60 , 163-167.

Schroer, K. (1993) Verzuim wegens overspanning. Een onderzoek naar de aard van overspanning, de hulpverlening en het verzuimverloop. Thesis, University of Limburg. The Netherlands.

Seligman, M. E. P. (1975). Helplessness: On depression, development and death. San Francisco: Freeman, W.H.

Selye, H. (1936). A syndrome produced by diverse noxious agents. Nature, 138, 32-36.

Selye, H. (1976). The Stress of Life. (revised edition). New York: McGraw Hill.

Shields, J. (1962). Monozygotic twins. Oxford: Oxford University Press.

Siegrist, J., Klein, D., \& Matschinger, H. (1989). Occupational stress, coronary risk factors, and cardiovascular responsiveness. In I. Weiner,R. Florin,Murison, \& D. Hellhammer (Eds.). Frontiers of stress research (pp. 323-335). Toronto: Huber.

Smith, P. L. (1979). Splines as a useful and convenient statistical tool. The American Statistician, 33(2), 57-62.

Solomon, S., Holmes, D. S., \& McCaul, K. D. (1980). Behavioral control over aversive events: Does control that requires effort reduce anxiety and physiological arousal? Journal of Personality and Social Psychology, 39(4), 729-736.

Steiner, H., \& Levine, S. (1988). Acute stress response in anorexia nervosa. A pilot study. Child Psychiatry and Human Development, 18(4), 208-218.

Sternberg, E. M., Hill, J. M., Chrousos, G. P., \& al., e. (1989). Inflammatory mediator-induced hypothalamic-pituitary-adrenal axis activation is defective in streptococcal cell wall arthritissusceptible Lewis rats. Proc Natl Acad Sci USA, 86, 2374-2378.

Stone, A. A. (1987). Event content in a daily survey is differentially associated with concurrent mood. Journal of Personality and Social Psychology, 52(1), 56-58. 
Stone, A. A., Cox, D. S., Valdimarsdottir, H., Jandorf, L., \& Neale, J. M. (1987). Evidence that secretory IgA antibody is associated with daily mood. Journal of Personality and Social Psychology, 52(5), 988-993.

Stone, A. A., Kessler, R. C., \& Haythornthwaite, J. A. (1991). Measuring daily events and experiences: Decisions for the researcher. Journal of Personality, 59(3), 575-607.

Stone, A. A., \& Neale, J. M. (1982). Development of a methodology for assessing daily experiences. In A. Baum \& J. E. Singer (Eds.), Advances in environmental psychology (pp. 49-83). Hillsdale, New Jersey: Lawrence Erlbaum Associates, Publishers.

Stone, A. A., \& Neale, J. M. (1984). Effects of severe daily eventsi on mood. Journal of Personality and Social Psychology, 46(1), 137-144.

Stone, A. A., Neale, J. M., \& Shiffman, S. (1993). Daily assessments of stress and coping and their association with mood. Annals of Behavioral Medicine, 15(1), 8-16.

Stone, A. A., \& Shiffman, S. (1992). Reflections on the intensive measurement of stress, coping, and mood, with emphasis on daily measures. Psychology and Health, 7, 115-129.

Strongman, K., \& Russell, P. (1986). Salience of emotion in recall. Bulletin of the Psychonomic Society, 24, 25-27.

Strube, M. J. (1989). Assessing subjects' construal of the laboratory situation. Ini N. Schneidermann,S. M. Weiss, \& P. G. Kaufmann (Eds.), Handbook of Research Methods in cardiovascular Behavioral Medicine (pp. 527-542). New York / London:" Plenum Press.

Surwit, R. S., Williams, R. B., \& Shapiro, D. (1982), Behavioural approaches to cardiovascular disease. New York: Academic Press.

Szalai, A. (Ed.). (1972). The use of time: Daily activities of urban and suburban populations in rwelve countries. Den Haag: Mouton.

Teasdale, J., \& Forgariy, S. (1979). Differential effects of induced mood on retrieval of pleasant and unpleasant events from episodic memory. Journal of Abnormal Psychology, 88, 248-257.

Tellegen, A. (1985). Structures of mood and personality and their relevance to assessing anxiety; with an emphasis on self-report. In A. H. Tuma \& J. D. Maser (Eds.), Anxiety and the anxiety disorders (pp. 681-706). Hillsdale, NJ: Erlbaum.

Theorell, T. (1989). Spontaneously occurring stressors. In H. e. a. Weiner (Eds.). Frontiers of Stress Research (pp. 111-121). New York, Toronto: Huber, H.

Thompson, S. C. (1981). Will it hurt less if I can control it? A complex answer to a simple question. Psychological Bulletin, 90(1),89-101.

Timio, M., \& Gentili, S. (1976). Adrenosympathetic overactivity under conditions of work stress. British Journal of Preventive and Social Medicine, 30, 262-265.

Tinsley, H. E. A., \& Weiss, D. J. (1975). Interrater reliability and agreement of subjective judgments. Journal of Counseling Psychology, 22(4), 358-376.

Troxler, R. G., Sprague, E. A., Albanese, R. A., Fuchs, R., \& Thompson, A. J. \{1977). The association of elevated plasma cortisol and early atherosclerosis as demonstrated by coronary angiography. Atherosclerosis, 26, 151-162.

Tsigos, C., \& Chrousos, G. P. (1994). Physiology of the hypothalamic-pituitary-adrenal axis, in heaith and dysregulation in psychiatric and autoimmune disorders. Endocrinology and Metabolism Clinics of North America, 23(3), 451-466.

Tumer, J. R., Ward, M. M., Gellman, M. D., Johnston, D. W., Light, K. C., \&: van Doomen, L. J. P. (1994). The relationship between laboratory and ambulatory cardiovascular activity: Current evidence and future directions. Annals of Behavioral Medicine, 16(1), 12-23.

Uhde, T. W., Joffe. R. T., Jimerson, D. C., \& Post. R. M. (1988). Normal urinary free cortisol and plasma MHPG in panic disorder: Clinical and theoretical implications. Biological Psychiatry, 23. 575-585.

Ursin, H. (1978). Activation, coping, and psychosomatics. In H. Ursin. E. Baade, \& S. Levine (Eds.), Psychobiology of Stress (pp. 201-228). New York: Academic Press. 
Urșin, H. (1980). Personality, activation and somatic health. In S. Levine \& H. Ursin (Eds.), Coping and health (pp. 259-279). New York: Plenum Press.

Ursin, H., Mykletun, R., Tonder, O., Vaemes, R., Relling, G., Isaksen, E., \& Murison, R. (1984). Psychological stress factors and concentrations of immunoglobulins and complement components in humans. Scandinavian Journal of Psychology, 25, 340-347.

Vaernes, R., Ursin, H., Darragh, A., \& Lambe, R. (1982). Endocrine response patterns and psychological correlates. Journal of Psychosomatic Research, 26, 123-131.

van der Ploeg, H. M., Defares, P. B., \& Spielberger, C. D. (1980). Handleiding bij de ZelfBeoordelings Vragenlijst - ZBV - een nederlandstalige bewerking van de Spielberger State . Trait Inventory (a manual for the STAI). Lisse: Swets \& Zeitlinger.

van der Ploeg, H. M., Defares, P. B., \& Spielberger, C. D. (1982), Handleiding bij de Zelf-Analyse Vragenlijst - ZAV - een nederlandstalige bewerking van de Spielberger State - Trait Anger Scale. Lisse: Swets \& Zeitlinger.

van Diest, R. (1992). Vital exhaustion or depression: a study of daily mood in exhausted male subjects at risk for myocardial infarction. In M. W. deVries (Eds.), The experience of psychopathology: Investigating mental disorders in their natural settings (pp. 233-239). Cambridge: Cambridge Press.

van Doornen, L. J. P., \& Turner, J. R. (1992). The ecological validity of laboratory testing. In J. R. Turner,A. Sherwood, \& K. C. Light (Eds.), Individual differences in cardiovascular response to stress (pp. 63-83). New York: Plenum.

van Doornen, L. J. P., \& van Blokland, R. W. (1992). The relationship between cardiovascular and catecholamine reactions to laboratory and real-life stress. Psychophysiology, 29(2), 173-181, van Eck, M. M., Berkhof, H., Nicolson, N., \& Sulon, J. (in press). The effects of perceived stress, traits, mood states, and stressful daily events on salivary cortisol. Psychosomatic Medicine.

van Eck, M. M., \& Nicolson, N. A. (1994). Perceived stress and salivary cortisol in daily life. Annals of Behavioral Medicine, 16(3), 221-227.

Vickers, R. R. (1988). Effectiveness of defenses: a significant predictor of cortisol excretion under stress. Journal of Psychosomatic Research, 32, 2! -29.

Vingerhoets, A. J. J. M., \& Menges, L. J. (1989). Psychosocial load and symptoms: An inquiry into their relationship with coping styles. Stress Medicine, 5, 189. 194.

Vining, R. F., McGinley, R. A., \& Symons, R. G. (1983). Hormones in saliva: Mode of entry and consequent implications for clinical interpretation. Clinical Chemistry, 29(i0), 1752-1756.

Wagner, B. M. (i990). Major and daily stress and psychopathology: On the adequacy of the definitions and methods. Stress Medicine, 6, 217-226.

Watson, D. (1988). Intraindividual and interindividual analyses of positive and negative affect: Their relationship to health complaints, perceived stress, and daily activities. Joumal of Personality and Social Psychology, 54(6), 1020- 1030.

Watson, D., \& Clark, L. A. (1984). Negative affectivity: The disposition to experience aversive emotional states. Psychological Bulletin, 96(3), 465-490.

Watson, D., Clark, L. A., \& Harkness, A. R. (1994). Structures of personality and their relevance to psychopathology. Journal of Abnormal Psychology, 103(1), 18-31.

Watson, D., \& Pennebaker, J. W. (1989). Health complaints, stress, and distress: Exploring the central role of negative affectivity. Psychological Review, 96(2), 234-254.

Weiner, H. (1992). Perturbing the Organism: The Biology of Stressful Experience. Chicago: University of Chicago Press.

Wolff, S., \& Goodell, H. (1968). Harold G. Wolffs stress and disease (2 ed.). Springfield (III.): Charles C. Thomas.

Wong, M. M., \& Csikszentmihalyi, M. (1991). Motivation and academic achievement: The effects of personality traits and the quality of experience. Journal of Personality. 59(3), 539-574. 
Wüst, S., Kirschbaum, C., \& Hellhammer, D. (1990). Smoking increases salivary cortisol. In C. Kirschbaum \& D. Hellhammer (Ed.), Proceedings of the 2nd European Symposium on Hormone and Drug Assessment in Saliva. Trier.

Yehuda, R., Resnick, H., Kahana, B., \& Giller, E. L. (1993). Long-lasting hormonal alterations to extreme stress in humans: Normative or maladaptive? Psychosomatic Medicine, 55, 287-297.

Zarski, J. J. (1984). Hassles and health: A replication. Health Psychology, 3(3), 243-251.

Zautra, A. J., \& Guarnaccia, C. A. (1986). Measuring small life events. American Journal of Community Psychology, 14(6), 629-655.

Zautra, A. J., Guarnaccia, C. A., Reich, J. W., \& Dohrenwend, B. P. (1988). The contribution of small events to stress and distress. In L. H. Cohen (Eds.), Life Events and Psychological Functioning. Theoretical and methodological issues (pp. 123-148). Newbury Park, California: Sage Publication.

Zung, W. W. K. (1965). A self-rating depression scale. Archives of General Psychiatry, 12, 63-70. 


\section{Appendices}

Appendix I: Reliability and validity of the Perceived Stress Scale in a sample of Dutch white collar workers.

Appendix II: ESM report.

Appendix III: Interrater agreement on codings for ESM measures. 


\section{Appendix I}

\section{Reliability and validity of the Perceived Stress Scale in a sample of Dutch white collar workers}

\section{INTRODUCTION}

Measures of stress (life events, daily events, or chronic stressful situations), can be broadly divided into subjective and objective stress measures, what points to two conceptually different approaches to the concept of stress. First, the transactional model of psychosocial stress which states that "stress lies not in the environmental input but in the person's appraisal of the relationship between that input and its demands and the person's agendas (e.g. beliefs, commitments, goals) and capabilities to meet, mitigate, or alter these demands in the interest of well-being", p.770 (Folkman \& Lazarus, 1985; Lazarus, De Longis, Folkman, \& Gruen, 1985; Lazarus \& Launier. 1978; Mason, 1975). This view contrast with a second, more objective approach to stress, in which stressors are treated as environmental stimuli, inputs, that are much more independent of the reaction or state of mind of the person (Dohrenwend, Link. Kern, Shrout, \& Markowitz, 1990; Dohrenwend \& Shrout, 1985; Dohrenwend, Dohrenwend, Dodson, \& Shrout, 1984). This approach implies that events are, in and of themselves, the precipitating cause of pathology and illness behavior. From the transactional point of view, the impact of these 'objectively' stressful events is, to a large degree, determined by one's perceptions of their stressfulness.

In research on stress, we would like to be able to identify individuals who are at risk for developing stress-related psychological or somatic problems. We know that only a small percentage of individuals exposed to objective stressful (life) events will fail to cope effectively and thereby develop symptoms. We also know that health complaints (objective and subjective) have multifactorial causes, of which stress is only one. A measure of perceived (appraised) stress would be very useful for screening populations for individuals at risk. Such an instrument should ideally be short; it must also be reliable and have construct validity: that is, it should be able to predict stress symptomatology / pathology, while not simply measuring subjective distress alone.

A subjective stress measure, the Perceived Stress Scale (PSS), was developed by Cohen and colleagues (Cohen, Kamarck, \& Mermelstein, 1983), for the measurement of a global level of perceived stress with the goal of providing additional information about the relation between stress and pathology. The PSS measures cognitions and emotions relating to general stress levels rather than specific events or situations. Items tap the extent to which individuals feel their life to be. unpredictable, uncontrollable and overloaded: important components of the experience of stress (Averill, 1973; Cohen.1978; Lazarus, 1966; Miller, 1979; Seligman, 1975). An example of a PSS item is: 'In the last month, how often have you felt that you were unable to control the important things in your life?' The scale attempts to represent situations where persons perceive that the denands exceed their ability to cope. Because the items are of a general nature they are free of content specific to any subpopulation. Another advantage of the generality of the scale is that it is sensitive to the nonoccurrence of events, to chronic life circumstances, to anticipation of future events and to events occurring in the lives of friends and relatives. In the case of scales measuring event-specific levels of perceived stress, 
respondents are: asked to rate the stressfulness or impact of each experienced event. This means that perceived stress levels will be limited by the specific list of events in the scale. In these scales it is also not possible for a single event to have the impact of three or four less salient ones.

A scale measuring global levels of perceived stress can have various valuable functions (Cohen et al., 1983). At the first place, the PSS can be used when the major aim of the study is the role of appraised stress, as opposed to objective stress. It can, for instance, be used to determine whether appraised stress is an etiologic (or risk) factor in psychological or physical disorders (e.g. when the objective sources of stress are diffuse or difficult to measure). Second, it can be used together with an objective scale to study whether appraised stress mediates the relationship between objective stress and illness. In other words, it can provide information about the processes through which stressful events influence pathology. Similarly, it can be used to look more closely at the process by which various moderators of the objective stressor/pathology relationship operate (e.g. social support, coping, personality). Third, the PSS can be used as an outcome measure, i.e. assessing experienced stress as a function of objective stressful events, coping processes, personality characteristics eIc. A final valuable function of the PSS that will be examined in this article, could be its use as an economical tool for screening purposes. When administered more than


could be averaged, providing a more reliable (based on more samples) measure of chronic stress, and as well a predictor representing a longer time period than the onemonth period covered by the PSS.

Research by Cohen and others showed acceptable levels of validity and reliability of the PSS in samples of the United States. Cronbach's alpha coefficients for the internal reliability of the PSS were high in the various studies (ranging between .75 and .86) (Cohen et al., 1983; Cohen \& Williamson, 1988), and test-retest reliability over a period of two days was also high $(r=85)$. For longer periods (one month, 6 weeks), test-retest correlations were moderate (.50 and .55 respectively) (Cantor, Norem, Langston, Zirkel, Fleeson, \& Cook-Flannagan, 1991; Cohen et al., 1983). Perceived stress seems to be an important mediator of the relationship between stressful life events and symptomatology. Work by Cohen et al. (Cohen. 1986; Cohen et al., 1983; Cohen \& Williamson, 1988) showed for instance that the PSS was a better predictor than life-event scales of psychological symptoms, somatic symptoms and utilization of health services and that stress perception was also related to selfreports of health behaviors. Higher PSS scores were also prospectively associated in a dose-response manner with an increased risk of acute infectious respiratory illness (Cohen. Tyrrell, \& Smith. 19917. The PSS was found to measure a different and independently predictive construct than a depressive symptomatology scale. Cohen (Cohen, 1986) demonstrated that even after controlling for the possible overlap of the PSS and psychopathology, the PSS prospectively predicts psychological symptoms, physical symptoms and health behaviors.

Biy presenting psiychometric and descriptive data on the PSS in a Dutch sample of white collar workers, we want to show that the PSS is a reliable and valid instrument that could be used for screening purposes. The shortness and generality of the scale (containing only 10 items) is a major advantage above the usually long and specific event scales that are used for measuring stress levels, especially in large scale screening where various other instrument are usually included too. As described above, the reliability and validity of the scale has been proven within a culture, i.e. in various samples in the Unites States (students, adult populations, diabetics), but we do 
not have information about the qualities of the scale across cultures. At face value the individual items seem to be aspecific to a particular culture, but this is certainly not enough a base to rely on. Therefore we looked at the reliability and consiruct validity of the scale in a Dutch sample of white collar workers, where the PSS was used as a screening instrument. Besides the internal reliability, we investigated the stability of the PSS over time in a subsample of subjects who filled out the PSS twice. Scores on the scale in the Dutch sample were also compared to US. norms. The construct validity of the PSS was examined through comparison with various indices related to stress: life events, chronic stress, coping style, health behavior, and psychological and somatic complaints. We expected the PSS to be related to the number of life events because perceptions of stress should generally increase with increases of objective stress levels. We expected a higher correlation of the PSS with chronic difficulties though, because in this scale respondents were asked to indicate the intensity of he problems they were facing. The intensity scores should reflect some of the same stress appraisal as measured by the PSS. Perceptions of stress were also expected to be positively related to anxiety, depression, less effective coping styles, and poorer self reported health and health-related behavior. Besides these measures, also cortisol levels were determined in saliva as a physiological measure of subjective stress. Salivary cortisol is a reliable and valid indicator of the free cortisol in plasma, which is considered to be the biologically active hormone. Cortisol concentrations are independent of the flow rate of saliva. Cortisol was measured only once in our respondents, so we did not look for reactive but rather for basal levels of cortisol and the possible influence of (ongoing) perceived stress on that level. Results from studies on cortisol levels in cases of chronic psychosocial load are inconsistent. Sometimes enhanced concentrations have been found (Hofer, Wolff, Friedman, \& Mason, 1972; Jacobs, Mason, Kosten, Kasl, Ostfeld, \& Wahby, 1987, Kiecolt-Glaser, Ricker, George, Messick, Speicher, Gamer, et al., 1984) but decreased levels as well (Caplan, Cobb, \& French, 1979). We also looked at the predictive ability off appraised stress opposed to alternative measures of stress. Parallel to other studies, the PSS was: expected to be the strongest predictor of health variables, because it is presumably the level of appraised stress and not the objective occurrence of an event, that causes one's reaction to a stressor(s).

Subjects were first screened and then a subgroup was selected from various local industries and government agencies according to their perceived stress leve!. Reliability and validity data were collected in these two samples. Although our samples are not representative of the Dutch population in general, we believe that our data should give us a good indication of the usefulness of the Perceived Siress Scale. as a screening instrument in Dutch populations.

\section{METHODS}

\section{Subjects and procedures}

Both the screening sample ( $n=316$ for males, and $n=60$ for females) and the (sub)sample that had been selected for the ESM study ( $n=92$, all males) were used to investigate the reliability and valididty of the PSS. For details about subject recruitment and procedures see: chapter 2 . 


\section{Questionnaires}

\section{Screening sample}

In addition to the PSS, questionnaires concerning coping style (Utrecht Coping List), and psychological and physical symptoms (Symptom Checklist 90; Psychosomatic Symptom Checklist) were completed by the screening sample $(n=376)$. Demographic information was obtained concerning respondents' age, marital status, household composition, completed education, chronic diseases, medications, alcohol use, smoking habits, and participation in active sports. For details about the questionnaires see chapter 2 .

\section{Subsample}

The 92 subjects who participated in the main study completed additional questionnaires concerning psychosocial stress (life events: List of Threatening Experiences; chronic stress: Longterm Difficulties Questionnaire), and psychological symptoms (anxiety: State-Trait Anxiety Inventory; depression: Self-Rating Depression Scale). For details about the questionnaires see chapter 2 .

\section{Cortisol}

When subjects in the screening sample handed in the completed questionnaires, they also provided a single saliva sample for cortisol determination. All samples were taken between 11.00 and 13.00 hours, before lunch. Subjects collected saliva by holding a polyester dental roll in the mouth for 1 to 2 minutes; the roll was then placed in a capped plastic vial (Salivette; Sarstedt). Information about use of coffee and tobacco in the last hour and use of medication in the last 24 hours was simultaneously obtained to control for possible confounding influences.

Uncentrifuged samples were stored at $-20 \mathrm{C}$. Salivary cortisol levels were determined in duplicate by direct radioimmunoassay (Ansseau, Sulon, Doumont, Cerfontaine, Legros, Sodoyez, et al., 1984), using ${ }^{125}$ ]-cortisol (Farmos diagnostica, Finland) and antiserum made against the 3CMO-BSA conjugate by Dr. J. Sulon, University of Liege, Belgium. The lower detection limit of the assay was $12 \mathrm{ng} / 100 \mathrm{ml}$, with a mean intra-assay coefticient of variation of $4.8 \%$ (range: $2.2 \%$ $-7.5 \%$ for 4 assays).

\section{Statistical analysis}

Unless otherwise stated, statistical tests are non-parametric and two-tailed.

\section{RESULTS}

\section{Unldimensionality and reliability of the PSS}

Principal components extraction with varimax rotation was performed on the 10 items of the Perceived Stress Scale. One component was identified which accounted foi $58.7 \%$ of the total variance. All items had positive loadings of .45 or higher. A total scale score was therefore obtained by summation of the 10 item scores.

For the screening sample, Crohnbach's alpha as a measure of the internal reliability of the PSS was .86 , or slightly higher than that of the original US. scale (Cohen \& Williamson, 1988). Item-total correlations fell between .38 and .74. For the 92 subjects who completed the PSS a second time, Crohnbach's alpha for the scale was .85 , with item-total correlations ranging from .29 to .73 .

The PSS was completed for a second time by the subsample of 92 subjects, after an average interval of 116 days (range 13-213 days). We looked at the test-retest reliability of the PSS to examine its stability. PSS scores from the first and second measurements were highly correlated. ( $r h o=.73, p<001$ ), with a mean decrease of 2.0 
(s.d. 4.7, range -9 to +14 points). Given that the PSS is based on reports for the last month, one would expect larger differences in scores as the time interval between test and retest increases. However, linear regression showed no greater change in PSS score with increasing intervals between test and retest $(F(1,90)=.739$; ns).

\section{Cross-cultural generalizability of the PSS: comparison of results with US. normative data}

Table 1 presents the means and standard deviations of scores on the PSS for the various demographic variables represented in our sample. These results can be compared with published norms from a large $(n=2270)$, representative US. sample (Cohen \& Williamson, 1988), to assess the cross-cultural validity of the Dutch version of the PSS.

Table 1. Mean PSS scores and standard deviations for demographic categories.

\begin{tabular}{lrll}
\hline Category & N & PSS mean & SD \\
\hline Sex male & 316 & 12.6 & 5.9 \\
female & 60 & 13.6 & 6.5 \\
Age & & & \\
$20-29$ & 47 & 12.51 & 5.5 \\
$30-39$ & 136 & 12.6 & 6.0 \\
$40-49$ & 128 & 13.2 & 6.1 \\
$50-59$ & 62 & 12.5 & 6.3
\end{tabular}

Household composition

living with parents

single

10.8

12.2

couple/no children

12.6

couple/children

12.9

single parent

23.0

other

$\begin{array}{rr}16 & 10.8 \\ 32 & 12.2 \\ 91 & 12.6 \\ 227 & 12.9 \\ 1 & 23.0 \\ 9 & 13.3\end{array}$

4.8

6.8

5.7

6.1

6.0

Number of people in household

$\begin{array}{lrrr}\text { one } & 33 & 12.1 & 6.7 \\ \text { two } & 98 & 12.5 & 5.7 \\ \text { three } & 77 & 12.2 & 6.0 \\ \text { four or more } & 126 & 14.7 & 7.2 \\ \text { mber of children in household } & & & \\ \text { none } & 189 & 12.3 & 5.7 \\ \leq 2 & 159 & 12.9 & 6.1 \\ >2 & 27 & 14.5 & 6.9\end{array}$

Education

\begin{tabular}{lrrr} 
lower & 7 & 17.7 & 4.2 \\
middle & 132 & 13.1 & 5.7 \\
higher & 232 & 12.5 & 6.2 \\
\hline
\end{tabular}

The mean score for the Dutch sample $(12.7+$ - s.d. 6.0 ; range $2-35)$ is comparable to US. norms (mean $=13.02$, s.d. $=6.45$ ), especially when the selected characteristics of our white collar sample are taken into account. Scores for US. managerial and clerical professions were 12.0 and 13.5, respectively. In agreement 
with US. norms for females and males, women reported somewhat higher mean PSS scores than men, but this difference failed to reach significance in our sample $(F(374)=1.29$, ns; one-way ANOVA). We found no significant association between respondent's age and perceived stress ( $r=-.02$, n.s.), although a negative relationship between these two variables was reported for the US. sample. Respondents still living with their parents had the lowest levels of perceived stress, but one-way ANOVA revealed no significant effect for household composition on level of perceived stress. In the US. sample, PSS was weakly related to household size and the number of children in the household. In our sample a similar pattern was observed for household size $(r h o=10, p<.05)$, but perceived stress level did not increase significantly in households with more children $(<18$ years $)(F(372)=1.86$, ns; one-way ANOVA) probably because of the small number of people with more than 2 children in our sample. As in the US., higher education was associated with lower perceived stress. Probably due to the small number of people with lower education in our sample, however, this effect just failed to reach significance $(F(368)=2.72, p=.06$ : one-way ANOVA.

\section{The construct valldity of the PSS}

\section{Association of PSS with self-reported health and health-related behaviors.}

Fifty-seven respondents (15.2\% of the sample) reported suffering from one or more chronic condition, including high blood pressure $(n=14)$, cardiovascular disease $(n=8)$, respiratory problems $(n=7)$, and diabetes mellitus $(n=1)$. In the open-ended category 'other chronic diceases' $(n=40)$ the most freguently reported conditions were allergies, hay-fever and eczema. As shown in Table 2, subjects with a chronic illness reported no higher perceived stress level than those with no illness.

Table 2. PSS scores in relation to health and health-related behaviors

\begin{tabular}{lcccc}
\hline Measure & & N & PSSinean & SD \\
\hline Do you have a chronic disease? & yes & 57 & 13.3 & 6.3 \\
& no & 319 & 12.6 & 6.0 \\
Do you take medication? & & & & \\
& yes & 53 & $15.0 * *$ & 6.9 \\
Do you exercise regularly? & no & 321 & 12.4 & 5.8 \\
& yes & 248 & 12.5 & 5.8 \\
Do you smoke? & no & 127 & 13.3 & 6.3 \\
& & & & \\
& yes & 104 & 12.1 & 6.0 \\
& no & 270 & 13.0 & 6.0 \\
\hline
\end{tabular}

$* p<0.0$ H, two-tailed; Mann Whitney U-test

Respondents who used medications, however, had significantly higher PSS scores than those who did not. Medications most frequently used were from the categories: circulatory tract $(n=17)$, respiratory tract $(n=12)$, analgesics $(n=10)$, CNS/psychological (e.g. hypnotics, anxiolytics, anti-depressants) $(n=8)$. There was no difference in the PSS scores of respondents who exercised once a week or more 
versus those who reported no regular exercise, and smokers had no higher PSS scores than non-smokers.

Psychosomatic complaints, as measured with the PSC, were examined as another aspect of self-reported health. Frequency, Intensity and Total (frequency $\mathrm{x}$ intensity) scores on the PSC were all positively correlated with perceived stress ( $r h o=.54, .45$, and .59 , respectively; $p<.001$ ). Correlations remained significant even after the more obvious psychological symptoms (fatigue, depression and insomnia) were omitted from the scale ( $r h o=.44, .36$, and .50 , respectively; $p<.001$ ).

\section{Psychological complaints}

Mean scores on the SCL-90 scales $(n=336)$ were within the normal ranges established for the Dutch population (Koeter, OrmeI, \& van den. Brink, 1988). PSS scores were positively correlated with all eight SCL-90 scales: agoraphobia $(r h o=35)$, sleep problems $(r h o=41)$, hostility $(r h o=47)$, sonatic complaints $(r h o=47)$, insufficiency of thought and performance $(r h o=.56)$, distrust and interpersonal sensitivity ( $r h o=.57$ ), anxiety $(r h o=.62)$, depression $(r h o=.66)$, as well as with the total score $(r h o=.68)($ all tests $p<.001)$.

\section{Coping style}

Scores on the Utrecht Coping List scales $(n=215)$, were similar to norms published for the Dutch population (Schreurs \& van de Willige, 1988). The following Spearman correlations between PSS scores and the assessed coping styles were found: 'depressive reactions" ( $r h o=.59, p<.001)$, 'active problem-solving' (rho=-.37. $p<.001$ ), 'palliative' (rho=.22, p<.01), 'expression of emotions' ( $r h o=.13, p<.05$ ), 'avoidance' ( $r h o=11, p<.06$ ), 'seek social support' ( $r h o=.07, \mathrm{~ns}$ ), and 'comforting cognitions' ( $r h o=.06, \mathrm{~ns}$ ).

\section{Cortisol}

We had expected that high perceived stress would be associated with elevated cortisol levels. However, there was no significant correlation between PSS and prelunch salivary cortisol in either males ( $r h o=07$, ns; $n=295$ ) or females ( $r h o=.01$, ns; $\mathrm{n}=58)$. Mean salivary cortisol was $170 \mathrm{ng} / \mathrm{dl}(\mathrm{sd}=80.3)$ for males and $189 \mathrm{ng} / \mathrm{dl}$ for females $(s d=73.9)(p=.05$, Mann-Whitney $U)$.

\section{High PSS vs low PSS comparisons}

The analysis described in the following paragraphs were done in the subsample of our study. In the subsample, high and low perceived stress groups were defined as follows: the mean of the first and second PSS assessments was used to categorize subjects as above or below the screening sample median score (12). For the resulting group of 44 'high stress' subjects, mean PSS score was $18.0(\mathrm{~s} . \mathrm{d}=3.4)$, compared to a mean PSS of $7.3($ s.d. $=2.2)$ in the group of 48 'low stress' subjects.

The construct validity of the PSS was further examined through two indices of distress: anxiety disposition and depressive symptomatology. Perceptions of stress were expected to be positively related to psychological symptoms. Group differences in anxiety (mean: 22.9 high stress vs 18.8 low stress group) and depression (48.4 vs $36.8)$ were both significant $\left(p^{\prime} s<.001\right)$ and in the expected direction. 
Aelationship between PSS and number of life events and chronic difficulties

We found a small but significant correlation between the number of life events and the PSS ( $r h o=.20, p=.03$ ). The difference in mean number of life events between the 'high stress' group (.73 events) and the 'low stress' group. (.46 events) was not significant ( $p=19$; Mann-Whitney U). As expected, the correlation between chronic difficulties and the PSS was markedly higher $(r h o=.56, p<.001)$. There was a significant difference in number of long-term difficulties between the two groups, with respondents in the 'high stress' group experiencing more difficulties than those in the 'low stress' group (23.3 vs $19.5 ; p<, 001)$.

\section{The PSS versus life event and chronic difficulty measures in predicting symptomatology}

Following the analysis presented by Cohen et al. (Cohen et al., 1983), we looked at the PSS compared to the number of life events as predictors of psychosomatic and depressive symptoms. Perceived stress was expected to be a stronger predictor of symptomatology than the number of life events (Lazarus \& Launier, 1978: Mason, 1971), and also, although to a lesser extent, expected to be a stronger predictor than long-term difficulties (though limited by the specific sample of the various difficulties, this measure does account for the appraised severity of difficulties). The data in Table 3 lend support to these assumptions.

Table 3. Spearman correlations of stress measures with psychosomatic and depressive symptomatology.

\begin{tabular}{lcc}
\hline & Psychosomatic Symptoms & Depression \\
\hline Perceived stress & $.60^{* * *}$ & $.76^{* * *}$ \\
Number of life events & .07 & .06 \\
Long-term difficulties & $.47 * * *$ & $.52 * * *$ \\
\hline
\end{tabular}

$\cdots p<.001$

Of the three stress measures, perceived stress showed the highest correlations to both psychosomatic and depressive symptomatology. The number of life-events did not correlate with either psychosomatic or depressive symptomatology. So, although subjects who experienced more life events also experienced more stress, they did not have more symptoms.

Because both perceived stress and chronic difficulties were strongly related to symptomatology it is desirable to show that both stress scales are not measuring the same things. Therefore, chronic difficulties were partialled out of the correlation between perceived stress and psychosomatic symptoms $s_{n}$ and the PSS was partialled out of the correlation between chronic difficulties and psychosomatic symptoms. Partial correlations were calculated with ranked variable scores for reasons of not normally distributed variables. The correlation between the PSS and psychosomatic symptoms, controlling for chronic difficulties, was .45 (p<.001). The correlation between chronic difficulties and psychosomatic symptoms, controlling for perceived stress was .21 $(p<.05)$. Chronic difficulties and perceived stress both independently predicted psychosomatic complaint. Although overlap exists, the scales are not measuring the same thing.

For the validity of the PSS it was also important to demonstrate that perceived stress did not overlap completely with measures of psychological distress. Relations 
among these measures may be due to the common influence of negative affectivity. Self-report stress scales and self-report health measures have been found to reflect a pervasive mood disposition of negative affectivity, thus overestimating the true association between stress and health (Watson \& Clark, 1992; Watson \& Pennebaker. 1989). In our study we found a high correlation between perceived stress and depressive symptomatology $(r h o=.76)$ and between perceived stress and trait-anxiety $(r h o=77)$, probably indicating some overlap again since stress perception could be a symptom of depression or of high trait anxiety. So again partial correlations were calculated on ranked variable scores: results were compared when depressive symptomatology was partialled out of the relation between perceived stress and psychosomatic symptoms and when perceived stress was partialled out of the relation between depressive symptoms and psychosomatic complaints. In the case of the PSS and psychosomatic symptomatology, the correlation was .27 $(\mathrm{p}<.01)$ and in the case of the ZUNG and psychosomatic symptoms, the correlation was .28 (p<.01). It thus appears that both scales independently predict psychosomatic symptomatology. Similarly, both trait anxiety and stress perception independently predicted psychosomatic complaints (partial correlations: $r=.24, p<.05$; and $r=.29, p<.01$. respectively).

\section{DISCUSSION}

The Dutch version of the PSS showed adequate internal reliability and sufficient stability in a sample of white collar workers. The test-retest correlation was high and there was no systematic tendency for scores to fall or rise, reflecting that although the questionnaire is sensitive to temporal change in experienced stress, it also reflects a rather stable individual characteristic. These results are comparable to the results found by Levenstein et al. (Levenstein, Prantera, Varvo, Scribano, Berto, Luzi, et al., 1993) who developed The Perceived Stress Questionnaire (PSQ) which is highly correlated and comparable to the PSS (.73). Here, the sensitivity of this scale to change was tested over 6 months (scale was filled in monthly) and they also found that the mean ratio between a subjects highest and lowest PSQ score was quit small. The fact that the PSS is quite stable but still sensitive to changes in experienced stress is an advantage considering its potential usage as a screening instrument; it is possible to assess chronic levels of perceived stress (when measured more than once) but environmental influences can be studied for its effects as well. Possible causes for the observed test-retest effect are: statistical regression (twice as many people had a lower scale score the second time in stead of a higher score, but this could possibly be explained by the fact that those people with low scale scores the first time were the better adjusted people and therefore less likely to have an increased stress score the second time), and social desirability (subjects selected for the field study wanted to look 'good', stress resistant).

Our sample means and standard deviations of perceived stress were comparable with the US. norms. Especially their means for managerial and clerical professions compared very well with our means for white collar workers. These results give support to the notion that our sample, although not a representative one, does not deviate strongly from a normal probability sample. Means on other crosssectionals like the UCL and the SCL90, when compared to norm scores for the Dutch population, also showed normal population scores. Although correlations between various demographic characteristics and level of perceived stress were in the 
expected direction, most of them did not reach significance. Only household size was positively related to perceived stress. This was probably due to the fact that the correlations described by Cohen and Williamson (Cohen \& Williamson, 1988), which were also small (between .10 and .13), were based on a very large sample $(n=2270)$. Cohen et al.(Cohen et al., 1983) also failed to find a relation between the PSS and sex or age in samples comparable to the size of our white collar sample. It must be said that our sample was also quite homogeneous, in the sense that it consisted of only white collar workers. For instance, differences in socioeconomic status, not measured here, could be very well related to levels of perceived stress.

If the PSS is to be used as a screening instrument it should be able to discriminate subjects on theoretically interesting characteristics. Our data give support for the construct validity of the Dutch version of the PSS; perceived stress was related in the expected way to other stress measures (life events and chronic difficulties), to coping styles, and psychological (anxiety, depression), and psychosomatic complaints. The results on coping are in concordance with studies suggesting that an active-offensive mode of coping (dominated by a tendency of active problem solving and optimism) is more effective in avoiding and solving problems than a reactive-defensive mode of coping (dominated by a tendency of avoidance and depressive reactions).

Our only objective measure of distress, i.e. cortisol, was not related to perceived stress. A major drawback in our study is that we measured cortisol only once. Caplan et al. (Caplan et al., 1979), who studied the effect of perceived white collar workload on cortisol, also did not find a main effect of perceived workload on mean cortisol, but did find an effect of stress on the circadian rhythm of cortisol. Cortisol levels in the morning were significantly lower for employees with high work load. Although our measure of perceived stress is sensitive for more sources of stress than only work load, it would be interesting to study the possible effect of perceived stress on the circadian rhythm. This will be done in our ESM study were we have repeated measures of cortisol.

An important point is, that although the PSS correlates substantially with altemative measures of stress/distress. it assesses a different and independently predictive construct. Some overlap in the various scales is almost inevitable, but the PSS does not measure the same thing as for instance chronic difficulties or depressive symptomatology. The correlation between perceived stress and psychosomatic symptoms, controlling for depressive symptoms, remained significant.

A limitation in our study is that, besides cortisol, we did not have any objective measures of health (e.g. absenteeism at work, visits to health practitioner). Investigating the ability of the PSS to predict such outcomes would be a strong validation. We emphasize that the present correlational analyses do not warrant any inferences of causality. Although perceived stress may have (at least partly) caused both somatic and psychological symptoms, it is also possible that complainis elevated stress perceptions, or that a third factor (e.g. personality) influenced both stress and health. Another important point to be made is that stress scales should be chosen to address specific research questions. The choice for a specific scale will depend on the specific research design used in the study. This also means that the perceived stress scale is not always the appropriate scale. For instance, perceived stress scales should not be used as the only scale in cross-sectional studies of the relationship between stress and psychological distress (Cohen \& Williamson, 1988).

In conclusion, the PSS seems to be a valuable instrument for screening purposes. The scale has high internal reliability. stability, sensitivity and shown 
construct validity. Its subjective character maximizes sensitivity to ongoing stress. Its generality makes this scale less culture and population specific and of special value in populations were the stressful events are hard to pin down. Its short length is also an advantage when used concurrently with various other questionnaires or when used repeatedly over time in longitudinal studies. The PSS seems to be an adequate measure to assess which people are at risk for the development of stress related somatic and mental health problems.

\section{REFERENCES}

Ansseau, M., Sulon, J., Doumont, A., Cerfontaine, J. L., Legros, J. J., Sodoyez, J. C., \& Demey. Ponsart, E. (1984). The use of saliva cortisol in the dexamethasone suppression test. Psychiatry Research, 13, 201-211.

Averill, J. R. (1973). Personal control over aversive stimuli and its relationship to stress.

Psychological Bulletin, 80(4), 286-303.

Cantor, N., Norem, J., Langston, C., Zirkel, S., Fleeson, W., \& Cook-Flannagan, C. (1991). Life tasks and daily life experience. Journal of Personality, 59(3), 425-451.

Caplan, R. D., Cobb, S., \& French, J. R. P., Jr. (1979). White collar work load and cortisol: Disruption of a circadian rhythm by job stress? Journal of Psychosomatic Research, 23, 181. 192.

Cohen, S. (1978). Environmental load and the allocation of attention. In A. Baum, J. Singer, \& S. Valins (Eds.), Advances in environmental psychology. Hillsdale: Lawrence Erlbaum.

Cohen, S. (1986). Contrasting the Hassles Scale and the Perceived Stress Scale: Who's really measuring appraised stress? American Psychologist, 41, 716-718.

Cohen, S., Kamarck, T., \& Mermelstein, R. (1983). A global measure of perceived stress. Journal of Health and Social Behavior, 24, 385-396.

Cohen, S., Tyrrell, D. A. J., \& Smith, A. P. (1991). Psychological stress and susceptibility to the common cold. The New England Journal of Medicine, 325(9), 606-612.

Cohen, S., \& Williamson, G. M. (1988). Perceived stress in a probalitity sample of the United States. In S. Spacepan \& S. Oskamp (Eds.), The Social Psychology of Health (pp. 31-67). Newbury Park, CA: Sage Publications.

Dohrenwend, B. P., Link, B. G., Kern, R., Shrout, P. E., \& Markowitz, J. (1990). Measuring life events: The problem of variability within event categories. Stress Medicine, 6, 179-187.

Dohrenwend, B. P., \& Shrout, P. E. (1985). "Hassles" in the conceptualization and measurement of life stress variables. American Psychologist, 40, 780-785.

Dohrenwend, B. S., Dohrenwend, B. P., Dodson, M., \&. Shrout, P. E. (1984). Symptoms, hassles, social supports, and life events: Problems of confounded measures. Journal of Abnormal Psychology, 93(2), 222-230.

Folkman, S., \& Lazarus, R. S. (1985). If it changes it must be a process: Study of emotion and coping during three stages of a college examination. Journal of Personality and Social Psychology, 48, 150-170.

Hofer, M. A., Wolff, C. T., Friedman, S. B., \& Mason, J. W. (1972). A psychoendocrine study of bereavement PartI. 17-Hydroxycorticosteroid excretion rates of parents following death of their children from leukemia. Psychosomatic Medicine, 34(6), 481-504.

Jacobs, S. C., Mason, J., Kosten, T. R., Kasl, S. V., Ostfeld, A. M., \& Wahby, V. (1987). Urinary free cortisol and separation anxiety early in the course of bereavement and threatened loss. Biological Psychiatry, 22, 148-152. 
Kiecolt-Glaser, J. K., Ricker, D., George, J., Messick, G., Speicher, C. E., Garner, W., \& Glaser, R. (1984). Urinary cortisol levels, cellular immunocompetency, and loneliness in psychiatric inpatients. Psychosomatic Medicine, 46(1), 15-23.

Koeter, M. W. J., Ormel, J., \& van den Brink, W. (1988). Totaalscore op de SCL-90 als maat voof de ernst van psychopathologie. Nederlands Tijdschrift voor de Psychologie, 43, 381-391.

Lazarus, R. S. (1966). Psychological stress and the coping process. New York: McGraw-Hill.

Lazarus, R. S., De Longis, A., Folkman, S., \& Gruen, R. (1985). Stress and adaptational outcomes: The problem of confounded measures. American Psychologisi, 40, 770-779.

Lazarus, R. S., \& Launier, R. (1978). Stress-related transactions between person and environment. In L. A. Pervin \& M. Lewis (Eds.), Perspectives in Interactional Psychology (pp. 287-327). New York: Plenum.

Levenstein, S., Prantera, C., Varvo, V., Scribano, M. L., Berto, E., Luzi, C., \& Andreoli, A. (1993). Development of the perceived stress questionnaire: a new tool for psychosomatic research. Journal of Psychosomatic Research, 37(1), 19-32.

Mason, J. W. (1971). A re-evaluation of the concept of 'non-specificity' in stress theory. Journal of Psychiatric Research, 323-333.

Mason, J, W. (1975). A historical view of the stress field. Journal of Human Stress, 1(1), 7-12.

Miller, S. M. (1979). Controilability and human stress: Method, evidence and theory. Behavior Research and Therapy, 17, 287-305.

Schreurs, P. J. G., \& van de Willige, G. (1988), De Utrechtse Copinglijst (UCL): Lisse, the Netherlands: Swets \& Zeitlinger.

Seligman, M. E. P. (1975). Helplessness: On depression, development and death. San Francisco: Freeman. W.H.

Watson, D., \& Clark, L. A. (1992). Affects separable and inseparable: On the hierarchical arrangement of the negative affects. Journal of Personality and Social Psychology, 62(3), 489-505.

Watson, D., \& Pennebaker, J. W. (1989). Health complaints, stress, and distress: Exploring the central role of negative affectivity. Psychological Review, 96(2), 234-254. 


\section{Appendix II}

\section{ESM report}

\begin{tabular}{lccccccc}
\hline Op het moment van de piep: & niet & \multicolumn{3}{c}{ 'n beetje } & tamelijk & \multicolumn{2}{c}{ zeer } \\
Kon u zich concentreren ? & 1 & 2 & 3 & 4 & 5 & 6 & 7 \\
Piekerde u ? & 1 & 2 & 3 & 4 & 5 & 6 & 7 \\
& & & & & & & \\
Zo ja, waarover..........................................................................................................................................
\end{tabular}

\section{Voelde u zich op dat moment:}

$$
\text { niet 'n beetje tamelijk zeer }
$$

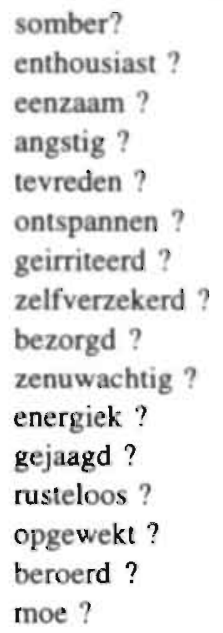

$\begin{array}{lllllll}1 & 2 & 3 & 4 & 5 & 6 & 7 \\ 1 & 2 & 3 & 4 & 5 & 6 & 7 \\ 1 & 2 & 3 & 4 & 5 & 6 & 7 \\ 1 & 2 & 3 & 4 & 5 & 6 & 7 \\ 1 & 2 & 3 & 4 & 5 & 6 & 7 \\ 1 & 2 & 3 & 4 & 5 & 6 & 7 \\ 1 & 2 & 3 & 4 & 5 & 6 & 7 \\ 1 & 2 & 3 & 4 & 5 & 6 & 7 \\ 1 & 2 & 3 & 4 & 5 & 6 & 7 \\ 1 & 2 & 3 & 4 & 5 & 6 & 7 \\ 1 & 2 & 3 & 4 & 5 & 6 & 7 \\ 1 & 2 & 3 & 4 & 5 & 6 & 7 \\ 1 & 2 & 3 & 4 & 5 & 6 & 7 \\ 1 & 2 & 3 & 4 & 5 & 6 & 7 \\ 1 & 2 & 3 & 4 & 5 & 6 & 7 \\ 1 & 2 & 3 & 4 & 5 & 6 & 7\end{array}$

\begin{tabular}{lcccccccc} 
Was uw belangrijkste klacht aanwezig ? & niet & & 'n beetje & tamelijk & \multicolumn{2}{c}{ zeer } \\
Had u last van andere klachten ? & 1 & 2 & 3 & 4 & 5 & 6 & 7 \\
& 1 & 2 & 3 & 4 & 5 & 6 & 7
\end{tabular}




\section{Wat deed u op het moment van de piep ?}

niet 'n beetie tameliik zeer

$\begin{array}{lllllllll}\text { Deed u het graag ? } & 1 & 2 & 3 & 4 & 5 & 6 & 7\end{array}$

$\begin{array}{llllllll}\text { Kostte het u moeite ? } & 1 & 2 & 3 & 4 & 5 & 6 & 7\end{array}$

$\begin{array}{llllllll}\text { Was deze aktiviteit voor u een uitdaging ? } 1 & 2 & 3 & 4 & 5 & 6 & 7\end{array}$

$\begin{array}{llllllll}\text { Kon } u \text { het? } & 1 & 2 & 3 & 4 & 5 & 6 & 7\end{array}$

$\begin{array}{lllllllll}\text { Vond } \mathrm{u} \text { deze aktiviteit belastend? } & 1 & 2 & 3 & 4 & 5 & 6 & 7\end{array}$

\section{Waar was u op het moment van de piep?}

Was u samen met anderen ?

Nee / Ja, met 1_1_1 mensen.

Naam

Wie is dat?

Wilde u nu graagalleen zijn ?



\section{Was er tussen deze en de vorize piep sprake van:}

$\begin{array}{lccccccc} & \text { niet } & \text { 'n beetje } & \text { tamelijk } & \text { zeer } \\ \text { een stressvolle gebeurtenis/situatie ? } & 1 & 2 & 3 & 4 & 5 & 6 & 7\end{array}$

Zo ja, geef een korte omschrijving:

Begintijd:

Eindtijd

Nog niet afgelopen $\mathrm{O}$

Was deze gebeurtenis:

- vervelend?

- verwacht?

- belangrijk voor $\mathrm{u}$ ?

K.on u het verloop ervan beinvloeden ? $\quad \begin{array}{llllllll}2 & 2 & 3 & 4 & 5 & 6 & 7\end{array}$

Heeft deze (of een soortgeliike) situatie zich vaker voor gedaan?

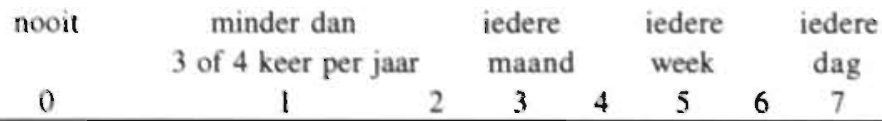




\begin{tabular}{lcccccccc} 
Had $\mathbf{u}$ in de tussentijd last van: & niet & & 'n beetje & \multicolumn{3}{c}{ tamelijk } & zeer \\
Uw belangrijkste klacht? & 1 & 2 & 3 & 4 & 5 & 6 & 7 \\
Andere klachten? & 1 & 2 & 3 & 4 & 5 & 6 & 7
\end{tabular}

Zo ja, welke?

\section{Gebruikte $u$ iets tussen deze en de vorige piep ?}
$\mathrm{O}$ niets
$O$ voedsel
O tabak
O mediciinen, n.I.
O koffie
O alcohol
1_1 glazen

$\begin{array}{lllllllll}\text { Wat was het hoogste niveau van aktiviteit? } & 1 & 2 & 3 & 4 & 5 & 6 & 7\end{array}$

voorbeelden: 1=rusten, 2 =zitten (actief), 3=lopen, $4=$ =stofzuigen, $S=f i e t s e n, 6=t e n n i s s e n, 7=r e n n e n$

Stoorde deze piep u ?

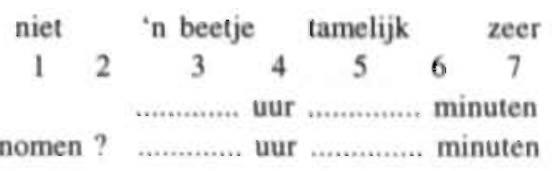

Hoe laat heeft u het speekselmonster genomen ? 


\section{Appendix III}

\section{Interrater agreement on codings for ESM measures}

\section{INTRODUCTION}

An appropriate measure of agreement for nominal data is the Kappa statistic ( $\mathrm{k}$ ) (Cohen, 1960). This statistic is an improvement on the simpler measure, per cent of agreement (Po), because it discounts the proportion of agreement which is expected by chance alone $(\mathrm{Pe})$. The formula for the (unweighted) $k a p p a$ is: $k=(\mathrm{Po}-\mathrm{Pe}) /(1-\mathrm{Pe})$. Because we are dealing with more than two categories we used the intra category kappa (agreement within a category). The 'overall Kappa' is a weighted average of the 'individual Kappa's' for all the alternative dichotomies which can be made by preserving one category and combining all others (Maclure \& Willett, 1987). Values for Kappa vary between 0 and 1.00. When Kappa has a value of 0 there is agreement as expected by chance alone. A Kappa of 1.00 means perfect agreement. We used the criteria given by Landis and Koch (Laundis \& Koch, 1977, p.265) for deciding if the Kappa we found was sufficiently high:

$\begin{array}{ll}\frac{\text { Kappa }}{<0.00} & \text { Degree of Agreement } \\ .00-.20 & \text { 'poor' } \\ .21-.40 & \text { 'flight' } \\ .41-.60 & \text { 'moir' } \\ .61-.80 & \text { 'substante' } \\ .81-1.00 & \text { 'almost perfect' }\end{array}$

\section{RESULTS}

The results on interrater agreement are presented in Table 1 and Table 2. First we will discuss the results for 'activities', 'location' and 'social context', and then for 'stressful events'.

\section{Interrater agreement for the activlty, locatlon and soclal context domains}

Reliability was determined for all the valid cases coded by the two raters. In every domain, the 'can't code' and the 'missing values' category were left out of analysis, because there were only a few observations in these categories: 'can't code' between 0 and 3 observations and 'missing values' between 6 and 14 observations. The fact that so few observations fell into the 'can't code' category means that the qualitative information was generally easy to interpret and to code into one of the available categories. We may conclude that the Kappa's we found were acceptably high (see Table 1). According to the criteria of Laundis and Koch ((Laundis \& Koch, 1977), p.265) the degree of agreement between raters is almost perfect, with all intra category Kappa's $\geq .89$, except for the activity category 'other' (Kappa .58) which had a moderate degree of agreement. So, the qualitative information was coded very reliably and seems easy to interpret. Also the fact that the various 'other' categories 
had so few observations gives support to the notion that the coding system we used was quite exhaustive.

Table 1. Interrater agreement for the activity, location and social context domains.

\begin{tabular}{|c|c|c|}
\hline & Total Observations & Kappa \\
\hline Activity ('what') & 2494 & .91 \\
\hline -leisure/social interaction & 722 & .90 \\
\hline -work & 709 & .95 \\
\hline -household/maintenance & 452 & .94 \\
\hline -transport & 252 & .96 \\
\hline -meals & 188 & .92 \\
\hline -inactivity/rest & 107 & .93 \\
\hline -other & 64 & .58 \\
\hline Location (Where') & 2491 & .94 \\
\hline -at home & 1185 & .97 \\
\hline -work & 788 & .96 \\
\hline -public places & 220 & .90 \\
\hline -transport & 189 & .92 \\
\hline -network & 72 & .91 \\
\hline -other & 37 & .70 \\
\hline Social Context ('Whol') & 2485 & .96 \\
\hline -household members & 964 & .99 \\
\hline -alone & 756 & .99 \\
\hline -colleagues & 546 & .97 \\
\hline -neighbours/acquaintances & 114 & .89 \\
\hline ofriends & 40 & .95 \\
\hline -non resident family & 36 & .89 \\
\hline -strangers & 29 & .90 \\
\hline
\end{tabular}

\section{Interrater agreement for stressful events}

The results for stressful events are presented in table 2. A total of 345 events were described by the 27 subjects. Interrater agreement was determined on all the completed subject responses. The context of events was rated with a high degree of agreement between the two raters. Kappa's varied between .60 and .96 . Only the 'other' category had a moderate degree of agreement, but this category was obviously also the most ambiguous one. It was also possible to use the event information to determine in a reliable way if someone was involved in the event and then who was involved in the event. The Kappa's lay between .76 and .96 . Most of the time no one was involved in the reported event. It was not possible to code in more than a moderately reliable way whether an event was 'external' or 'internal'. Only the 'internal' category had a substantially high Kappa (.76); the other categories had only a moderate degree of agreement. The 'can't code' category contained many observations $(21 \%)$ which suggests that it was very difficult for raters to interpret the qualitative information. Only the more straightforward internal events (e.g. worries, somatic complaints) were rated with a high reliability. The other categories can only be used with caution in further analyses. 
The intra category Kappa's in the social interaction domain were all satisfactory, varying from substantial to almost perfect, except for the can't code category for which the degree of agreement was only moderate. As shown in table 2 . a large number of observations could not be coded: $+10 \%$ of the total. This means that the various categories were not well defined, or that the information gathered was too vague. This second point seems to be more important here; often more detailed information was needed before a reliable judgment could be made. One way to improve on this is by paying more attention to the debriefing session where more information can be obtained on reported information. In the task demands domain, the categories 'problematic task' and 'failure at task' were aggregated into I category and the same accounts for the categories 'a lot of work' and 'time pressure'. This decision was based on the fact that the different subcategories were very interrelated. The Kappa's for the various categories ranged from moderate to almost perfect. Here again, a substantial percentage of responses could not be coded $(+22 \%)$.

Table 2. Interrater agreement for stressful events.






\section{REFERENCES}

Cohen, J. A. (1960). A coefficient of agreement for nominal scales. Educational and Psychological Measurement, 20, 37-46.

Laundis, J. R., \& Koch, G. G. (1977). The measurement of observer agreement for categorical data. Biometrics, 33, 396-398.

Maclure, M., \& Willett, W. C. (1987). Misinterpretation and misuse of the kappa statistic. American Journal of Epidemiology, 126(2), 161-169. 


\section{Summary}

During the last decades, there has been an increasing interest in the stressful aspects of contemporary society and their relationship to the development and maintenance of a wide range of somatic and psychological disorders. While earlier research focused on the impact of major life events or acute experimental stress, little is known about the nature of and the psychological and physiological responses to the minor but much more frequently occurring stresses of daily living. To increase our understanding of the stress process as it relates to health, the present thesis investigated the impact of minor daily events on mood and the HPA system. The aims of the research were 1) to describe the nature and scope of daily life stress in a group of white collar men and to contrast the experiences of individuals who perceive themselves to be stressed with those who do not, 2) to investigate affective and neuroendocrine (cortisol) responses in relation to stressful daily events, and 3) to investigate whether the cortisol responses to a laboratory stress task are generalizable to those occurring during stressful situations in real life. We also investigated whether more or less stable person characteristics like perceived stress level, trait anxiety, and depressive symptomatology were related to individual differences in responses to daily stress.

Chapter I presents an overview of psychological and physiological approaches to the concept of stress and discusses the role of psychosocial stress in health and disease. The existing literature on the impact of daily events on mood and cortisol is briefly reviewed. The following related issues are discussed: individual variability in mood and cortisol, the contexts in which daily events occur, appraisals of events, and the generalizability of laboratory-assessed stress reactivity to real life. Finally, the chapter presents the rationale for the study design and introduces the research questions.

Chapter 2 describes subjects, methods and procedures. Two groups of white collar men, with high versus low levels of perceived stress, were recruited as subjects. The Experience Sampling Method was used to collect data on stressful events and mood from subjects during their normal daily activities. Ten times a day for five consecutive days, subjects received auditory signals (beeps), after which they filled in a questionnaire and collected a saliva sample for cortisol determination. The same subjects also participated in a laboratory stress task, in which they were unexpectedly asked to deliver a speech. Stress responses were operationalized âs increases in cortisol secretion, increases in negative mood, and decreases in positive mood.

Chapter 3 describes quantitative as well as qualitative aspects of the reported stressful daily events. A total of 626 events were reported (on $17 \%$ of all ESM reports). Unpredictable and uncontrollable events were rated as the most unpleasant. Although the majority of stressful events were work-related, events were also frequently reported in a wide variety of life domains. High stress subjects scored high on trait anxiety and depressive symptoms, were bothered by various psychological and psychosomatic complaints, experienced relatively many chronic difficulties, and used more passive and less active coping styles than low stress subjects. High stress subjects reported twice as many stressful events, and they appraised events as more stressful and less controllable. They also reported twice as many work-related events and more events related to their social network. In particular, high stress subjects experienced more negative social interactions, both at work and at home, especially with their colleagues and their wives. 
Chapter 4 investigates the relationship between stressful events and negative and positive mood states, in particular examining the influence of perceived stress level on mood responses. Results showed that stressful events were associated with increases in negative mood. Positive mood only decreased when events were very unpleasant. Subjects scoring high on perceived stress showed significantly stronger negative mood reactivity in response to stressful events. Perceived stress did not influence the duration of the effects of events on mood. Events which involved performance demands and those appraised as more unpleasant, more novel, and less controllable were more likely to influence mood states. Results suggest increased vulnerability to daily events in individuals with high perceived stress.

Chapter 5 addresses the question of whether high perceived stress or related individual characteristics are associated with elevated cortisol or catecholamine levels. Analysis of cortisol values aggregated over each subject and time of day showed higher cortisol levels in high stress compared to low stress subjects when only the workdays were included. Trait anxiety, depressive symptomatology, and negative mood state were also associated with higher cortisol on workdays. Recent life events, chronic difficulties, trait anger, or psychosomatic symptoms, however, showed no relationship to cortisol levels. These results suggest that the kinds of mild chronic or intermittent stress in daily life situations reported during the ESM sampling period were sufficient to increase secretion of cortisol. In contrast, overnight catecholamine levels were unrelated to perceived stress, trait anxiety, depression, anger, psychosomatic symptoms or mood states.

Chapter 6 describes the estimated effects of perceived stress, personality traits, mood states, and stressful daily events on cortisol excretion. A random regression data analysis method revealed that trait anxiety and depression were associated with small but statistically significant cortisol elevations. Although results from the previous chapter had indicated a relationship between perceived stress and mean workday cortisol levels, the random regression analyses, which made use of the full dataset, did not replicate this finding; perceived stress was not associated with cortisol elevation. Cortisol levels were also no higher on workdays than on weekends in either subject group. Stressful daily events were indeed associated with increased cortisol secretion, the magnitude of the effect depending on whether the event was still ongoing and on how frequently a similar kind of event had occurred previously. Type of event or appraisal measures had no additional effects on cortisol. In addition, we found no significant individual differences in cortisol reactivity to events. Although perceived stress, anxiety, and depression did not increase cortisol reactivity to daily events, evidence was found for reduced habituation to recurrent events in subjects scoring high on these traits. Both negative mood states, Agitation and Negative Affect, were also associated with higher cortisol levels. Consistent with most views of the stress process, the effect of stressful events on cortisol appeared to be mediated to a large extent by associated increases in negative mood. The finding that even minor everyday events and fluctuations in mood states have an impact on cortisol secretion may point to a possible mechanism linking subjective experience to health outcomes.

Chapter 7 examines the generalizability of cortisol measures obtained in the laboratory to those obtained in real life. Results showed that the laboratory and reallife (ESM), cortisol levels were moderately correlated, but no association was found between laboratory and real-life stress response measures. Possible implications with respect to the predictive validity of laboratory stress reactivity for stress reactivity in general are discussed. 
In Chapter 8 the major findings of the study are summarized. Both methodological and theoretical issues related to the present research and to stress research in general are discussed, and results are evaluated in the context of possible health implications. Finally, several suggestions for future research are described. 



\section{Samenvatting}

De laatste decennia gaat steeds meer en meer aandacht uit naar de stressvolle elementen van onze huidige maatschappij en de negatieve gevolgen van deze elementen op het ontstaan en het in stand houden van allerlei somatische en psychische stoornissen. Terwijl vroeger onderzoek zich voornamelijk concentreerde op de gevolgen van belangrijke levensgebeurtenissen en acute experimentele stress, is er momenteel nog maar weinig bekend over de aard en de psychologische en fysiologische reacties op de kleine maar meer frequent voorkomende stressvolle gebeurtenissen in het dagelijkse leven. Ter vergroting van ons inzicht in de relatie tussen stress en gezondheid, bestudeert deze dissertatie de effecten vañ stressvolle dagelijkse gebeurtenis op de stemming en het hypofyse-bijnierschors systeem. De doelstellingen van het onderzoek zijn 1) het beschrijven van de aard en de omvang van dagelijkse stress in een groep van werkende mannen ('witte boorden') en het vergelijken van de ervaringen van individuen met een hoog ervaren stressniveau met die van individuen met een laag ervaren stressniveau, 2) het bestuderen van de affectieve en neuro-endocriene (cortisol) reacties op stressvolle dagelijkse gebeurtenissen, en 3 ) het onderzoeken in hoeverre de cortisol reacties op een laboratorium stresstaak corresponderen met de reacties op stressvolle gebeurtenissen in het dagelijkse leven. Tevens onderzochten we of er een relatie was tussen min of meer stabiele persoonskenmerken zoals ervaren stressniveau, angstdispositie en depressieve symptomatologie en individuele verschillen in reacties op dagelijkse. stress.

Hoofdstuk I geeft een overzicht van psychologische en fysiologische benaderingen van het begrip stress en evalueert de rol van psychosociale stress in gezondheid en ziekte. De beschikbare literatuur met betrekking tot de relatie tussen dagelijkse stress enerzijds en stemming en cortisol anderzijds wordt kort besproken, met speciale aandacht voor: individuele variatie in stemming en cortisol, de context waarin stressvolle gebeurtenissen plaatsvinden, de evaluatie (appraisal) van de stressvolle gebeurtenis en de generalisatie van stress-reactiviteit gemeten in het laboratorium naar het veld. Tenslotte wordt de rationale voor het onderzoeksdesign gepresenteerd en worden de onderzoeksvragen geintroduceerd.

In Hoofdstuk 2 worden de subjecten, methoden en procedures beschreven. Er werden twee groepen 'witte boorden' mannen gevormd: een met een hoog en een met een laag ervaren stressniveau. De Experience Sampling Methode werd gebruikt om gegevens van subjecten betreffende stressvolle gebeurtenissen en stemming te verzamelen op geselecteerde momenten tijdens hun normale dagelijkse activiteiten. Subjecten ontvingen tien keer per dag en gedurende vijf dagen signalen (beeps) via een voorgeprogrammeerd horloge, waarna ze een vragenlijst invulden en een speekselmonster namen, voor de cortisolbepaling. Dezelfde subjecten namen ook deel aan een laboratorium stresstaak, waarbij ze onverwacht gevraagd werden een speech te houden. Een stressreactie werd geoperationaliseerd als een stijging in cortisolsecretie, een toename in negatieve stemming en een afname in positieve stemming.

Hoofdstuk 3 beschrijft zowel kwantitatieve als kwalitatieve aspecten van de gerapporteerde stressvolle dagelijkse gebeurtenissen. In totaal werden er 626 gebeurtenissen gerapporteerd (op $17 \%$ van alle ESM beeps) en werden onvoorspelbare en oncontroleerbare gebeurtenissen het meest vervelend bevonden. Alhoewel de voornaamste bron van stress werk-gerelateerd was, kwamen stressvolle gebeurtenissen ook veelvuldig in verschillende andere levensdomeinen voor. 
Subjecten met een hoog stressniveau scoorden hoog op angstdispositie en depressieve symptomen, werden gehinderd door verschillende psychologische en psychosomatische klachten, ervaarden relatief veel langdurige moeilijkheden en gebruikten in het algemeen meer passieve en minder actieve coping stijlen dan subjecten met een laag stressniveau. Hoge stress subjecten rapporteerden twee keer zo veel dagelijkse gebeurtenissen als lage stress subjecten en ervaarden deze als meer stressvol en minder controleerbaar. Zij vermeldden tevens twee keer zoveel werk. problemen en meer problemen in relatie tot hun sociaal netwerk. In het bijzonder ervaarden hoge stress subjecten meer negatieve sociale interacties, zowel thuis als op het werk, en wel speciaal met hun collega's en echtgenotes.

Hoofdstuk 4 onderzoekt de relatie tussen stressvolle gebeurtenissen en negatieve en positieve stemming. Speciale aandacht ging uit naar de invloed van individuele verschillen in ervaren stressniveau op de stemmingsreacties. De resultaten laten zien dat stressvolle gebeurtenissen geassocieerd zijn met een toename in negatieve stemming. Positieve stemming nam alleen af wanneer de gebeurtenis zeer vervelend was. Subjecten met een hoog ervaren stressniveau vertoonden een significant sterkere toename in negatieve stemming als reactie op stressvolle gebeurtenissen. Het ervaren stressniveau had echter geen invloed op de duur van de effecten van stressvolle gebeurtenissen op stemming. Gebeurtenissen, die te maken hadden met de werkdruk of werklast en gebeurtenissen die als vervelender. onbekender en minder controleerbaar werden ervaren, hadden relatief meer invloed op de stemming. De resultaten lijken erop te wijzen dat individuen met een hoog ervaren stressniveau een verhoogde k.wetsbaarheid hebben in relatie tot stressvolle dagelijkse problemen.

Hoofdsıuk 5 is gewijd an de vraag of een hoog ervaren stressniveau of hiermee samenhangende persoonskenmerken geassocieerd zijn met verhoogde cortisol en/of catecholamineniveaus. Analyses van cortisolwaardes geaggregeerd over elk subject en de tijd van de dag heen laten een verhoogd cortisolniveau zien voor hoge stress subjecten in vergelijking met lage stress subjecten, echter alleen tijdens werkdagen. Angst dispositie, depressieve klachten, en negatieve stemming waren ook geassocieerd met hogere cortisolniveaus op werkdagen. Recente levensgebeurtenissen, chronische moeilijkheden, boosheidsdispositie en psychosomatische klachten vertoonde echter geen relatie met cortisol. De resultaten suggereren dat deze vormen van milde chronische of periodieke stress, ervaren in hett dagelijkse leven, in staat zijn de cortisolsecretie te verhogen. Echter, de catecholamineniveaus die werden gemeten gedurende de nacht waren niet geassocieerd met het ervaren stressniveau, angstdispositie, depressie. boosheidsdispositie, psychosomatische klachten, of stemming.

In Hoofdstuk 6 worden de geschatte effecten van het ervaren stressniveau, persoonskenmerken, stemming, en stressvolle gebeurtenissen op de cortisolsecretie beschreven. Een random regression data analyse methode liet zien dat angstdispositie en depressie gerelateerd waren met kleine maar significante verhogingen in. cortisolsecretie. Alhoewel de resultaten van het vorige hoofdstuk een positieve relatie tussen het ervaren stressniveau en het gemiddeld cortisolniveau op werkdagen aangaven, werden deze resultaten niet gerepliceerd wanneer via random regression. analyses gebruik gemaaki werd van alle gegevens; het ervaren stressniveau vertoonde geen relatie met cortisol. De cortisolniveaus waren tevens in beide groepen niet hoger op werkdagen dan in het weekend. De resultaten lieten verder zien dat stressvolle gebeurtenissen inderdaad geassocieerd waren met een verhoogde cortisolsecretie. De grootte van dit effect was afhankelijk van het feit of de stressor 
nog steeds aan de gang was op het moment van de meting en van de frequentie waarmee een gelijksoortige stressor zich reeds eerder had voorgedaan. Het type stressor of de evaluatie van de stressor had geen additioneel effect op cortisol. Tevens vonden we geen individuele verschillen in cortisol reactiviteit op stressvolle gebeurtenissen. Alhoewel het ervaren stressniveau, angstdispositie, en depressieve klachten de cortisol reactiviteit op stressvolle gebeurtenissen niet vergrootten, vonden we wel een verminderde habituatie aan stressoren die reeds herhaaldelijk eerder voorgekomen waren in subjecten die hoog scoorden op deze persoonskenmerken. Beide maten voor negatieve stemming (Agitatie en Negatief Affect) waren ook geassocieerd met hogere cortisolniveaus. In overeenstemming met de meeste opvattingen over het stressproces werd het effect van stressvolle gebeurtenissen op cortisol voornamelijk gemedieerd door de geassocieerde stijging in negatieve stemming. De bevinding dat zelfs kleine, dagelijkse stressoren en fluctuaties in stemming van invloed zijn op de cortisolsecretie wijst op een mogelijk mechanisme wat subjectieve ervaringen met gezondheid en ziekte verbindt.

Hoofdstuk 7 is voornamelijk gewijd aan de vraag of cortisolwaardes gemeten in het laboratorium generaliseerbaar zijn naar cortisolwaardes gemeten in het dagelijkse leven. De resultaten lieten zien dat de cortisolniveaus in het laboratorium en in het dagelijks leven redelijk met elkaar gecorreleerd waren, maar dat er geen relatie was tussen de reactiviteitsmaten in de experimentele setting en de reactiviteitsmaten in het dagelijks leven. De mogelijke implicaties van deze resultaten voor de predictieve validiteit van laboratorium stress-reactiviteit voor stressreactiviteit in het algemeen wordt besproken.

In Hoofdstuk 8 worden de belangrijkste resultaten van het onderzoek samengevat. Zowel methodologische als theoretische aspecten met betrekking tot deze dissertatie en met betrekking tot stress onderzoek in het algemeen worden bediscussieerd en de mogelijke implicaties van de resultaten voor de gezondheid worden nader bekeken. Het hoofdstuk wordt afgesloten met enkele suggesties voor vervolgonderzoek. 



\section{Dank}

Het uitvoeren van dit onderzoek was voor mij van tijd tot tijd vergelijkbaar met een fietstocht door het Limburgse landschap: al fietsend de heuvels op denk je "waar ben ik toch aan begonnen?", maar al snel bij het afdalen verandert deze gedachte in "ik ben blij dat ik het gedaan heb", totdat de volgende heuvel in zicht komt. Mijn dank gaat uit naar diegenen die ervoor gezorgd hebben dat ik in het heuvellandschap bleef en niet in de Alpen terechtkwam.

Daarmee bedoel ik op de eerste plaats de vele proefpersonen die zich bereid verklaarden om zich een kleine week lang door een horloge te laten achtervolgen en hun wel, wee en speeksel aan ons toe te vertrouwen. Zonder hen was dit boek er niet geweest. Op de tweede plaats bedoel ik Nancy Nicolson, mijn co-promotor. Nancy, ik dank je voor je grote betrokkenheid en steun bij het soms eenzame en lange traject van promotie-onderzoek. Met veel deskundigheid, geduld en optimisme voorzag je mijn diverse kladteksten van commentaar. Ik heb veel van je geleerd. Marten de Vries, mijn promotor, wil ik vooral bedanken voor alle vrijheid en vertrouwen die ik van hem kreeg, zowel tijdens als na mijn aanstelling als onderzoeker in opleiding.

Carolien Dijkman dank ik voor de efficiënte en fijne samenwerking tijdens de periode van dataverzameling. Op de vele vroege maandagochtenden was het een troost te weten dat ik niet als enige met de fiets op 'jacht' was naar (urine) monsters. Dr. Jose Sulon, Dr. Rahman en Dr. J. Duvivier ben ik zeer erkentelijk voor de bepalingen van respectievelijk de speeksel -en urinemonsters. De geweldige hulp van Hans Berkhof bij de vele gecompliceerde statistische analyses was onmisbaar. Zonder hem waren vele vraagstellingen onbeantwoord gebleven. Hans heeft laten zien dat een statisticus ook begrip voor de inhoud van de vraagstelling kan hebben, een zeldzaam verschijnsel. Professor Arthur Stone, tevens lid van de beoordelingscommissie, wil ik bedanken voor de motiverende discussies (zowel in Maastricht als in de US) en voor zijn komst naar Maastricht op 18 oktober.

Mijn collega's van de sectie Sociale Psychiatrie en Psychiatrische Epidemiologie wil ik bedanken voor hun morele ondersteuning maar vooral ook voor het gezellig geleuter tijdens de vele lunches en de fanatieke competities als er weer eens een nieuw computerspelletje was. In het bijzonder denk ik hierbij aan: Alex, Bart, Marie-Jose, Monique, Sarah, en Trees. Alhoewel ik 'de club' heb verlaten zal ik ze zeker nog lang missen. Bart Leunissen, mijn kamergenoot, ben ik speciaal erkentelijk voor het herhaaldelijk aanhoren van mijn onderzoeksfrustraties en het (meestal) stilzwijgend accepteren van de wanorde op mijn bureau('s). Bart, ik wens je meer succes bij de volgende kamergenoot. Philip Delespaul wil ik bedanken voor de fantastische hulp bij aspecten van de lay-out.

Mijn paranimfen Monique Pennings en Désirée Nijst bedank ik voor de hulp bij de organisatie van de 18-de. Monique: veel succes bij de 'vernieuwingen' in je leven, zowel privé als in de werksfeer. Desiree: "Hoe zit het nu met de bolderkar?"

Mijn moeder bedank ik voor de vrijheid en het vertrouwen die ze me altijd gegeven heeft om datgene te doen wat ik graag wou doen, om me vervolgens daarin dan ook te stimuleren. Last but not least: Rob. Bedankt voor je steun als ik weer eens met een pestbui thuiskwam. Het feit dat je (gelukkig) vaak niks begreep van mijn onderzoeks -en computer problemen, maakte dat ik de boel weer snel kon relativeren. Je motto "gewoon doorgaan" bracht me steeds weer een stapje verder. 



\section{Curriculum vitae}

Marleen van Eck werd geboren op 10 december 1965 te Wassenaar. In 1984 behaalde zij het VWO diploma aan het Griftland College te Soest. Na een verblijf van een jaar in het buitenland (Israël, Engeland), begon zij met de studie Gezondheidswetenschappen, afstudeerrichting Geestelijke Gezondheidkunde, aan de Rijksuniversiteit Limburg. Deze studie rondde zij in 1990 af. Aansluitend was zij als onderzoeker in opleiding verbonden aan de vakgroep Psychiatrie en Neuropsychologie, sectie Sociale Psychiatrie en Psychiatrische Epidemiologie, van de Rijksuniversiteit Limburg. Hier was zij werkzaam op een project dat gefinancierd werd door de Nederlandse Organisatie voor Wetenschappelijk Onderzoek (NWO), Deze dissertatie is een neerslag van dit werk. Tijdens dit dienstverband volgde zij tevens de opleiding tot gedragstherapeut. Na een tijdelijke aanstelling als toegevoegd onderzoeker bij dezelfde vakgroep is zij sinds 15 oktober 1995 werkzaam bij het RIAGG OZL te Heerlen, afdeling Psychotherapie. 
